\title{
Enantioselective Type II Cycloaddition of Alkynes via C-C Activation of Cyclobutanones: Rapid and Asymmetric Construction of [3.3.1] Bridged Bicycles
}

Si-Hua Hou, Xuan Yu, ${ }^{\dagger}$ Rui Zhang, ${ }^{\dagger}$ Lin Deng, Mengxi Zhang, Adriana Y. Prichina and Guangbin Dong* Department of Chemistry, University of Chicago, Chicago, Illinois 60637, United States;

Email: gbdong@uchicago.edu

\section{Table of Contents}

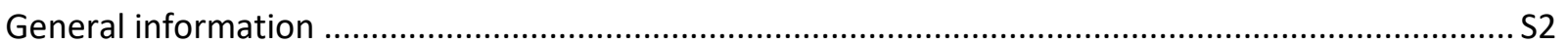

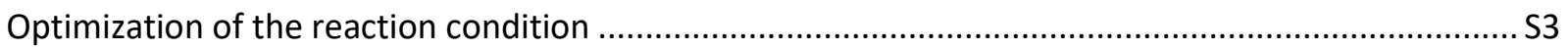

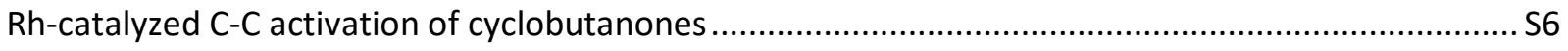

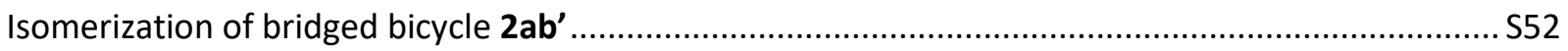

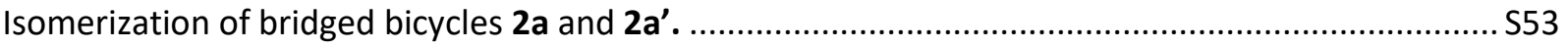

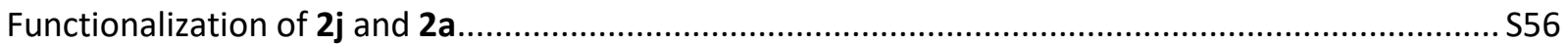

Substrates with low yield, low conversion or decomposition ........................................................ S61

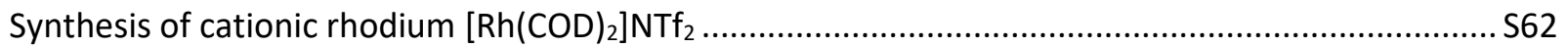

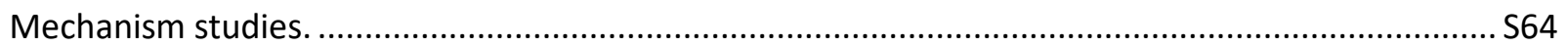

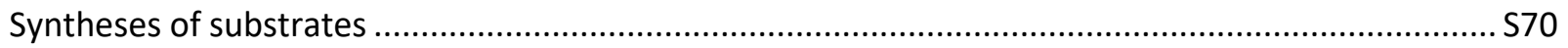

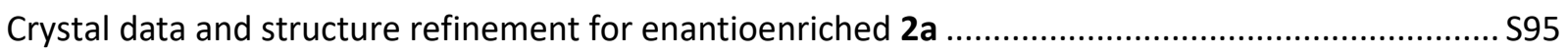

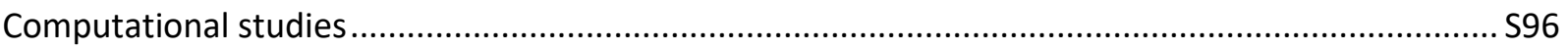

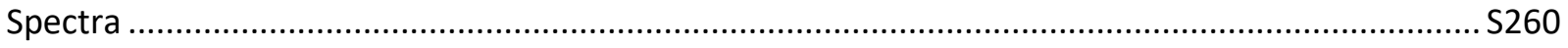

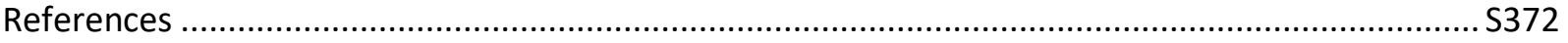




\section{General information}

Unless noted otherwise, all solvents were dried by filtration through a Pure-Solv MD-5 Solvent Purification System (Innovative Technology), all reactions were carried out under nitrogen atmosphere, all commercially available substrates were used without further purification. Thin layer chromatography (TLC) analysis was run on silica gel plates purchased from EMD Chemical (silica gel 60, F254). Infrared spectrum was recorded on a Nicolet iS5 FT-IR Spectrometer. Samples were scanned as neat liquids or dissolved in DCM on potassium bromide (KBr) salt plates. Frequencies were reported in reciprocal centimeters $\left(\mathrm{cm}^{-1}\right)$. High-resolution mass spectra (HRMS) were obtained on an Agilent 6224 TOF-MS spectrometer and were reported for the molecular ion $[\mathrm{M}]^{+},[\mathrm{M}+\mathrm{Na}]^{+}, \mathrm{or}[\mathrm{M}+\mathrm{H}]^{+} . \mathrm{Nuclear}$ magnetic resonance (NMR) spectrum $\left({ }^{1} \mathrm{H} N M R,{ }^{2} \mathrm{H} N M R,{ }^{13} \mathrm{C}\right.$ NMR, 135 DEPAT NMR, H-H COSY, C-H COSY, NOEY ) were recorded with a Bruker Model DMX 400 instrument (400 MHz for ${ }^{1} \mathrm{H}, 101 \mathrm{MHz}$ for ${ }^{13} \mathrm{C}$ ) unless noted. For $\mathrm{CDCl}_{3}$ solutions, the chemical shifts were reported as parts per million (ppm) referenced to residual protium or carbon of the solvents: $\mathrm{CHCl}_{3} \delta \mathrm{H}(7.27 \mathrm{ppm})$ and $\mathrm{CDCl}_{3} \delta \mathrm{C}(77.00 \mathrm{ppm})$. Coupling constants were reported in Hertz (Hz). Data for ${ }^{1} \mathrm{H}$ NMR spectra were reported as following: chemical shift $(\delta, \mathrm{ppm})$, multiplicity $(b r=b r o a d, s=s i n g l e t, d=$ doublet, $\mathrm{t}$ = triplet, $\mathrm{q}=$ quartet, $\mathrm{dd}=$ doublet of doublets, $\mathrm{td}=$ triplet of doublets, $\mathrm{ddd}=$ doublet of doublet of doublets, $\mathrm{m}=$ multiplet), coupling constant $(\mathrm{Hz})$, and integration. Analytical HPLC was carried out on an Angilent 1260 infinity HPLC with DAD, Chiralpak IA, IC, IF, and Chiralcel OD-H, served as columns, and mixtures of $n$-hexane and $i$-PrOH were used for elution. 


\section{Optimization of the reaction condition}

Table S1. Optimization of the Reaction with Cyclobutanone $1 \mathbf{a}^{a}$

\section{Changing ligand}

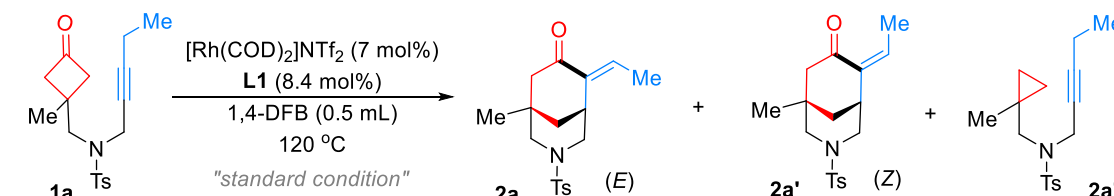

1a Ts

"standard condition"

2a Ts $(E$

2a' 's $(Z)$

$2 a^{\prime \prime}$

\begin{tabular}{|c|c|}
\hline entry & modification from "standard condition" \\
\hline 1 & None \\
\hline 2 & L2 instead of L1 \\
\hline 3 & L3 instead of L1 \\
\hline 4 & L4 instead of L1 \\
\hline 5 & L5 instead of L1 \\
\hline 6 & L6 instead of L1 \\
\hline 7 & $\mathbf{L 7}$ insead of $\mathbf{L 1}$ \\
\hline 8 & L8 insead of L1 \\
\hline 9 & L9 insead of L1 \\
\hline 10 & L10 insead of L1 \\
\hline 11 & L11 insead of L1 \\
\hline $12^{c}$ & $\mathrm{PCy}_{3}$ insead of L1 \\
\hline $13^{c}$ & $\mathrm{PPh}_{3}$ insead of $\mathbf{L 1}$ \\
\hline $14^{c}$ & IMes insead of L1 \\
\hline $15^{c}$ & L12 insead of L1 \\
\hline
\end{tabular}

(R)-Difluorophos (L4)

conversion $/ \%$

yield $/ \%(\mathrm{er})^{b}$

$\begin{array}{cccc}\mathbf{2 a} & \mathbf{2 a} & \mathbf{2 a} \\ 100 & 70(96.5: 3.5) & 11(97: 3) & 1\end{array}$

98

$33(89: 11)$

13

\section{7}

100

85

73

95

92

95

94

84

10

\section{0}

10

10

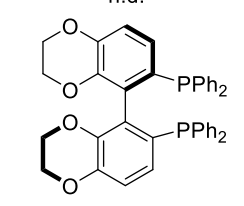

(R)-synphos (L5)

(R)-Tol-MeO-biphep (L6)

$$
\begin{aligned}
& \mathrm{Ar}=\mathrm{DTBM},(R)-\mathrm{DTBM} \text {-segphos }(\mathrm{L} 1) \\
& \mathrm{Ar}=\mathrm{DM},(R)-\mathrm{DM}-\text { segphos L2 } \\
& \mathrm{Ar}=\mathrm{Ph},(R) \text {-segphos L3 }
\end{aligned}
$$

DTBM:
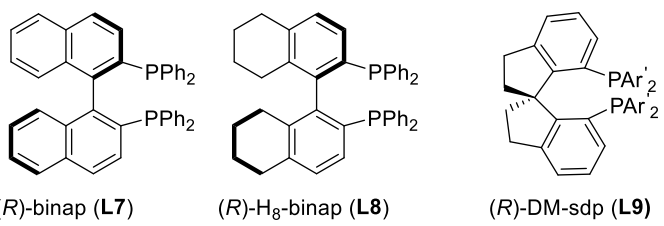

(R)-DM-sdp (L9)
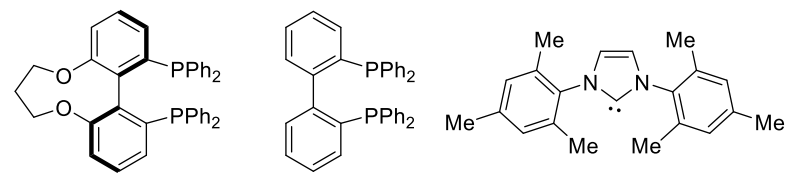

(R)- $\mathrm{C}_{3}$-TunePhos (L10)

biphep (L11)

IMes

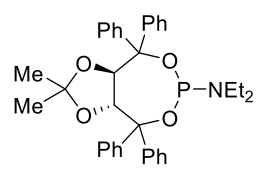

L12

${ }^{a}$ All reactions were run on a $0.1 \mathrm{mmol}$ scale for $48 \mathrm{~h} .{ }^{b}$ Yields of $2 \mathrm{a}$ are isolated yields; the er was determined by chiral HPLC. ${ }^{c}$ The ratio of ligand was $14.8 \mathrm{~mol} \%$. 
Table S2. Optimization of the Reaction with Cyclobutanone $1 \mathbf{a}^{a}$

Changing rhodium catalyst, temperature, and reaction time

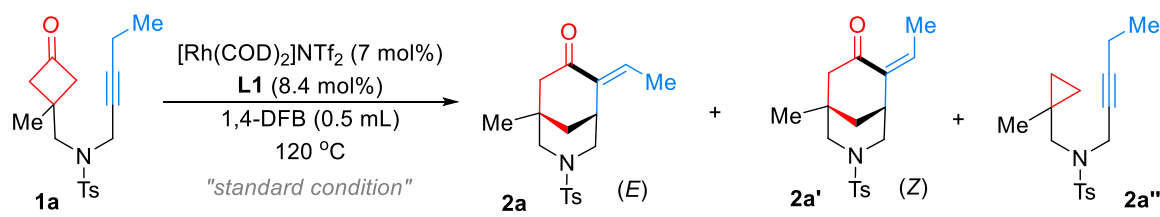

\begin{tabular}{|c|c|c|c|c|c|}
\hline \multirow[t]{2}{*}{ entry } & \multirow[t]{2}{*}{ modification from "standard condition" } & \multirow[t]{2}{*}{ conversion $/ \%$} & \multicolumn{3}{|c|}{ yield $/ \%(e r)^{b}$} \\
\hline & & & $2 a$ & $2 a^{\prime}$ & 2a" \\
\hline 1 & None & 100 & $70(96.5: 3.5)$ & $11(97: 3)$ & 1 \\
\hline $2^{c}$ & {$[\mathrm{Rh}(\mathrm{COD}) \mathrm{Cl}]_{2}$ instead of $\left[\mathrm{Rh}(\mathrm{COD})_{2}\right] \mathrm{NTf}_{2}$} & 40 & n.d. & n.d. & 35 \\
\hline $3^{c}$ & {$\left[\mathrm{Rh}(\mathrm{COE})_{2} \mathrm{Cl}\right]_{2}$ instead of $\left[\mathrm{Rh}(\mathrm{COD})_{2}\right] \mathrm{NTf}_{2}$} & 37 & n.d. & n.d. & 32 \\
\hline $4^{c}$ & {$\left[\mathrm{Rh}\left(\mathrm{C}_{2} \mathrm{H}_{4}\right)_{2} \mathrm{Cl}\right]_{2}$ instead of $\left[\mathrm{Rh}(\mathrm{COD})_{2}\right] \mathrm{NTf}_{2}$} & 88 & n.d. & n.d. & 86 \\
\hline $5^{c}$ & $\mathrm{RhCl}\left(\mathrm{PPh}_{3}\right)_{3}$ instead of $\left[\mathrm{Rh}(\mathrm{COD})_{2}\right] \mathrm{NTf}_{2}$ & 74 & n.d. & n.d. & 66 \\
\hline 6 & $\mathrm{Rh}(\mathrm{acac})(\mathrm{COD})$ instead of $\left[\mathrm{Rh}(\mathrm{COD})_{2}\right] \mathrm{NTf}_{2}$ & 7 & n.d. & n.d. & 5 \\
\hline 7 & $110^{\circ} \mathrm{C}$ instead of $120^{\circ} \mathrm{C}$ & 90 & $45(98.5: 1.5)$ & $25(90.5: 9.5)$ & 17 \\
\hline 8 & $100^{\circ} \mathrm{C}$ instead of $120^{\circ} \mathrm{C}$ & 47 & 8 (n.a.) & 20 (n.a.) & 17 \\
\hline 9 & $130^{\circ} \mathrm{C}$ instead of $120^{\circ} \mathrm{C}$ & 100 & $58(96: 4)$ & $8(95.5: 4.5)$ & n.d. \\
\hline 10 & $24 \mathrm{~h}$ instead of $48 \mathrm{~h}$ & 91 & $64(96.5: 3.5)$ & $10(92.5: 7.5)$ & 8 \\
\hline
\end{tabular}

${ }^{a}$ All reactions were run on a $0.1 \mathrm{mmol}$ scale for $48 \mathrm{~h} .{ }^{b}$ Yields of $\mathbf{2 a}$ are isolated yields; the er was determined by chiral HPLC. ${ }^{c}$ The ratio of neutral rhodium was $3.5 \mathrm{~mol} \%$.

Table S3. Optimization of the Reaction with Cyclobutanone $1 \mathbf{a}^{a}$

Changing cationic rhodium to neutral rhodium with silver salts

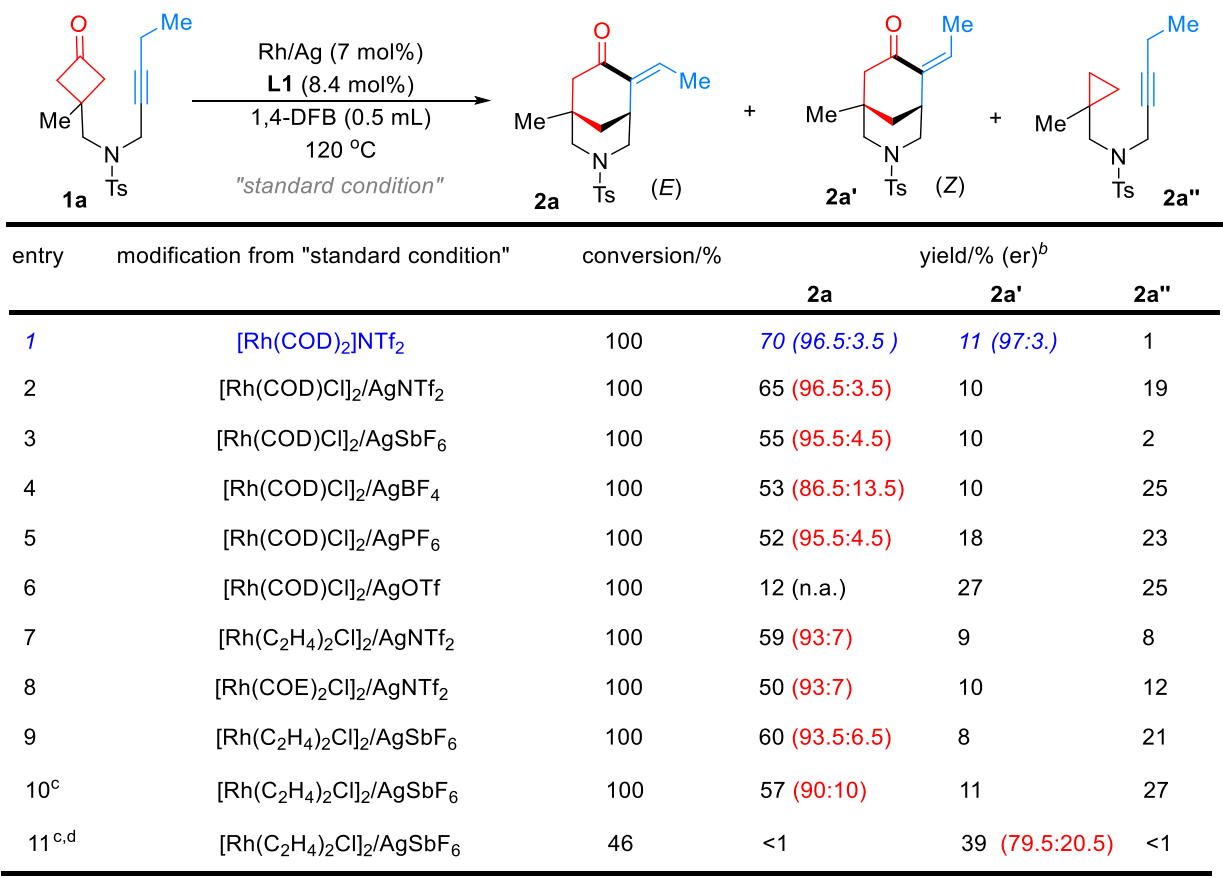


${ }^{a}$ All reactions were run on a $0.1 \mathrm{mmol}$ scale for $48 \mathrm{~h} .{ }^{b}$ Yields of $\mathbf{2 a}$ are isolated yields; the er was determined by chiral $\mathrm{HPLC}^{c} 1,4$-Dioxane $(0.5 \mathrm{~mL})$ was used insead of 1,4 -DFB. ${ }^{d}$ The reaction was run at room temperaure for 4 days.

Table S4. Selected Optimization of the Reaction with Cyclobutanone $1 \mathbf{a b}^{a}$

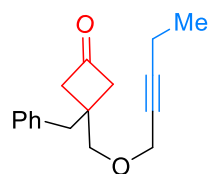

$1 a b$

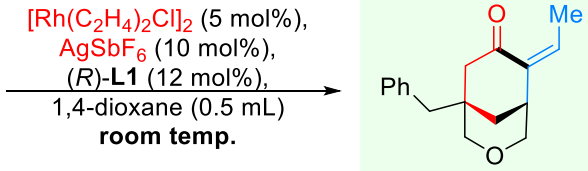

2ab'

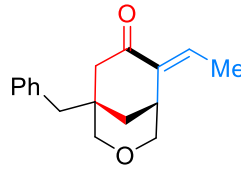

$2 a b$

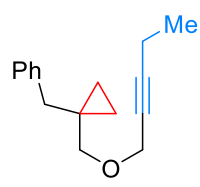

2ab"

\begin{tabular}{|c|c|c|c|c|c|}
\hline \multirow[b]{2}{*}{ entry } & \multirow[b]{2}{*}{ modification from "standard condition" } & \multirow[b]{2}{*}{ conversion $/ \%$} & \multicolumn{3}{|c|}{ yield $/ \%$} \\
\hline & & & 2ab' $(\mathrm{er})^{b}$ & $2 \mathbf{a b}^{b}$ & $2 a b^{\prime \prime b}$ \\
\hline 1 & none & 56 & $38(99.5: 0.5)$ & 1 & 2 \\
\hline 2 & 11 days instead of 3 days & 75 & $54(99.5: 0.5)$ & 2 & 2 \\
\hline 3 & $50^{\circ} \mathrm{C}$ instead of $\mathrm{rt}$ & 69 & $39(>99.5: 0.5)$ & 3 & 10 \\
\hline 4 & $65^{\circ} \mathrm{C}$ instead of $\mathrm{rt}$ & 56 & $35(>99.5: 0.5)$ & 5 & 15 \\
\hline 5 & $55^{\circ} \mathrm{C}$ instead of $\mathrm{rt}$ & 69 & $45(>99.5: 0.5)$ & 4 & 11 \\
\hline 6 & $55^{\circ} \mathrm{C}$ instead of rt, 4 days & 70 & 42 & 3 & 10 \\
\hline 7 & $55^{\circ} \mathrm{C}$ instead of $\mathrm{rt}, 5$ days & 74 & 45 & 4 & 12 \\
\hline 8 & $55^{\circ} \mathrm{C}$ instead of rt, 6 days & 77 & 45 & 5 & 12 \\
\hline 9 & {$\left[\mathrm{Rh}(\mathrm{COE})_{2} \mathrm{Cl}\right]_{2}$ instead of $\left[\mathrm{Rh}\left(\mathrm{C}_{2} \mathrm{H}_{4}\right)_{2} \mathrm{Cl}\right]_{2}$} & $<10$ & n.d. & n.d. & n.d. \\
\hline 10 & {$[\mathrm{Rh}(\mathrm{COD}) \mathrm{Cl}]_{2}$ instead of $\left[\mathrm{Rh}\left(\mathrm{C}_{2} \mathrm{H}_{4}\right)_{2} \mathrm{Cl}\right]_{2}$} & $<10$ & n.d. & n.d. & n.d. \\
\hline 11 & $\mathrm{AgNTf}_{2}$ instead of $\mathrm{AgSbF}_{6}$ & 25 & 23 & $<1$ & $<1$ \\
\hline 12 & $\mathrm{AgBF}_{4}$ instead of $\mathrm{AgSbF}_{6}$ & 27 & 23 & $<1$ & $<1$ \\
\hline 13 & $\mathrm{AgPF}_{6}$ instead of $\mathrm{AgSbF}_{6}$ & 25 & 18 & $<1$ & $<1$ \\
\hline 14 & AgOTf instead of $\mathrm{AgSbF}_{6}$ & 34 & 28 & $<1$ & $<1$ \\
\hline 15 & $(R)$-Difluorphos instead of $(\mathrm{R})-\mathrm{L} 1$ & $<10$ & n.d. & n.d. & n.d. \\
\hline 16 & without $\left[\mathrm{Rh}\left(\mathrm{C}_{2} \mathrm{H}_{4}\right)_{2} \mathrm{Cl}\right]_{2}$ & $<20$ & n.d. & n.d. & n.d. \\
\hline 17 & without $\mathrm{AgSbF}_{6}$ & $<10$ & n.d. & n.d. & n.d. \\
\hline 18 & 1,4-dioxane $(0.25 \mathrm{~mL})$ & 35 & $28(99.5: 0.5)$ & $<1$ & 1 \\
\hline 19 & 1,4-dioxane (1 mL) & 30 & 23 & n.d. & n.d. \\
\hline $20^{c}$ & THF instead of 1,4-dioxane & 25 & 18 & $<1$ & $<1$ \\
\hline $21^{c}$ & toluene instead of 1,4-dioxane & $<5$ & 2 & n.d. & n.d. \\
\hline $22^{c}$ & DCE instead of 1,4-dioxane & 32 & 23 & $<1$ & 3 \\
\hline $23^{c}$ & 1,4 -DFB instead of 1,4-dioxane & 37 & 28 & $<1$ & 2 \\
\hline $24^{c}$ & EtOAc instead of 1,4-dioxane & 27 & 23 & $<1$ & 2 \\
\hline $25^{c}$ & TBME instead of 1,4-dioxane & 34 & 26 & $<1$ & 4 \\
\hline $26^{c}$ & DCM instead of 1,4-dioxane & 25 & 20 & $<1$ & 4 \\
\hline $27^{c}$ & MeCN instead of 1,4-dioxane & $<5$ & n.d. & n.d. & n.d. \\
\hline
\end{tabular}

${ }^{a}$ All reactions were run on a $0.1 \mathrm{mmol}$ scale for 3 days. ${ }^{b}$ Yields of $\mathbf{2 a b}$ ' are isolated yields; the er was determined by chiral HPLC. ${ }^{c}$ The reaction was run for 4 days. 


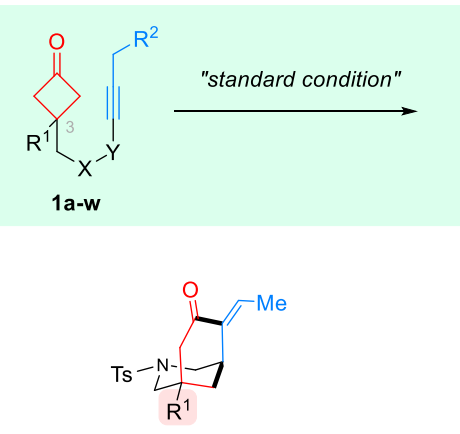

2a, $\mathbf{R}^{1}=\mathrm{Me}, 70 \%(\operatorname{er} 96.5: 3.5)+11 \% 2 \mathrm{a}^{\prime}$ $\mathbf{2 b}, \mathbf{R}^{1}=\mathrm{Et}, \mathbf{7 1 \%}(\mathrm{er} 95: 5)+12 \% \mathbf{2 b}$ 2c, $R^{1}={ }^{\prime} \mathrm{Pr}, 83 \%($ er $98: 2)+16 \% 2 \mathrm{c}^{\prime}$ 2d, $\mathbf{R}^{1}=\mathrm{CH}_{2}$ OTIPS, $69 \%($ er $97.5: 2.5)+11 \% 2 d^{\prime \prime}$
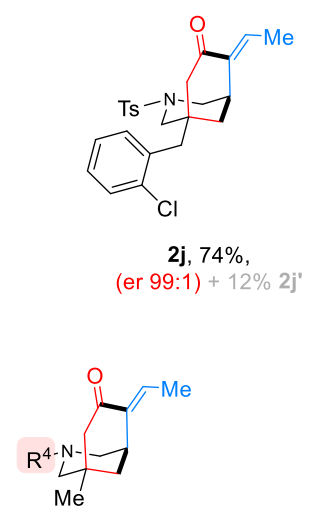

$\mathbf{2 m}, \mathrm{R}^{4}=$ Bs, $\quad 63 \%($ er $95: 5)+10 \% 2 \mathrm{~m}^{\prime}$ 2n, $\mathrm{R}^{4}=p$-Ns, $53 \%($ er $94.5: 5.5)+9 \% 2 n^{\prime}$ 2o, $R^{4}=$ Ms, $\quad 60 \%($ er $96: 4)+10 \% 20$ 2p, $R^{4}=o-N s, 32 \%($ er $90: 10)+5 \% 2 p^{\prime}$ 2q, $R^{4}=$ Piv, $\quad<2 \%$
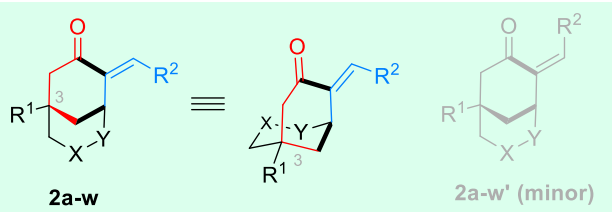

$$
\mathrm{R}^{3}
$$

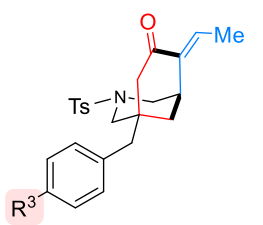

2e, $R^{3}=H, \quad 76 \%(\operatorname{er} 95.5: 4.5)+12 \% 2 e^{\prime}$ 2f, $R^{3}=F, \quad 78 \%($ er $94.5: 5.5)+13 \% 2 f$ $\mathbf{2 g}, \mathbf{R}^{3}=\mathrm{CF}_{3}, \mathbf{7 8 \%}(\mathrm{er} 95.5: 4.5)+13 \% 2 \mathrm{~g}^{\prime}$ $\mathbf{2 h}, \mathbf{R}^{3}=$ OMe, $80 \%($ er $94.5: 5.5)+13 \% 2 h$ $\mathbf{2 i}, \mathbf{R}^{3}=\mathbf{M e}, \quad \mathbf{7 1} \%($ er $96: 4)+12 \% 2 i^{\prime}$

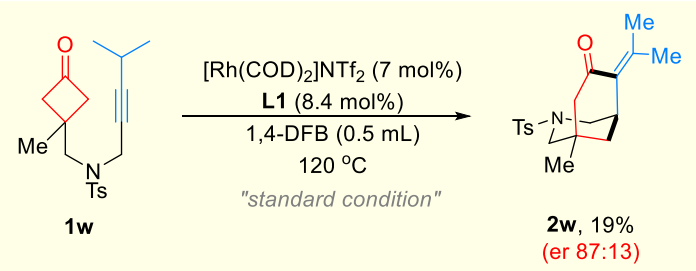

General procedure of preparing 2a-s and $\mathbf{2 w}$ : A flame dried $8 \mathrm{~mL}$ tube was charged with the substrate (1a-s or $\mathbf{1 w}$, $0.1 \mathrm{mmol}, 1$ equiv) and ( $R$ )-(-)-DTBM-segphos (L1, $9.9 \mathrm{mg}, 0.0084 \mathrm{mmol}, 8.4 \mathrm{~mol} \%$ ). The tube was then transferred to a nitrogen-filled glove box. After addition of $\left[\mathrm{Rh}(\mathrm{COD})_{2}\right] \mathrm{NTf} \mathrm{f}_{2}(4.2 \mathrm{mg}, 0.007 \mathrm{mmol}, 7 \mathrm{~mol} \%)$ and 1,4-diflorobenzene $(0.5 \mathrm{~mL})$, the tube was sealed and taken out of glove box. The reaction was stirred at $120^{\circ} \mathrm{C}$ in an oil bath for $48 \mathrm{~h}$, before it was then cooled to room temperature. The products were directly purified by silica gel flash chromatography to yield bridged bicycles $\mathbf{2 a - s}$ and $\mathbf{2 w}$. 
<smiles>[R]CC#CCN([As])CC1(C)CC(=O)C1</smiles>

1t-v
$\left[\mathrm{Rh}(\mathrm{COD})_{2}\right] \mathrm{NTf}_{2}(7 \mathrm{~mol} \%)$

L1 (8.4 mol\%), 1.4-DFB

then $\mathrm{Pd} / \mathrm{C}, \mathrm{H}_{2}$, EtOH

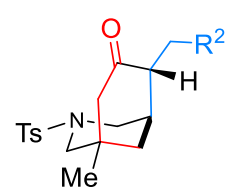

$2 t-v$ 2t, $\mathbf{R}^{2}={ }^{n} \operatorname{Pr}, 71 \%($ er $95: 5) d r>20: 1$

$\mathbf{2 u}, \mathbf{R}^{2}=$ Et, $65 \%$ (er 95.5:4.5) dr $>20: 1$

2v, $R^{2}=B n, 64 \%($ er 94:6) $d r>20: 1$

General procedure of preparing $2 \mathrm{t}-\mathbf{v}$ : A flame dried $8 \mathrm{~mL}$ tube was charged with the substrate $(\mathbf{1 t}-\mathbf{v}, 0.1 \mathrm{mmol}, 1$ equiv) and (R)-(-)-DTBM-segphos (L1, $9.9 \mathrm{mg}, 0.0084 \mathrm{mmol}, 8.4 \mathrm{~mol} \%)$. The tube was then transferred to a nitrogenfilled glove box. After addition of $\left[\mathrm{Rh}(\mathrm{COD})_{2}\right] \mathrm{NTf}_{2}(4.2 \mathrm{mg}, 0.007 \mathrm{mmol}, 7 \mathrm{~mol} \%)$ and 1,4-diflorobenzene $(0.5 \mathrm{~mL})$, the tube was sealed and taken out of glove box. The reaction was stirred at $120^{\circ} \mathrm{C}$ in an oil bath for $48 \mathrm{~h}$, before it was then cooled to room temperature. The reaction mixture was concentrated under reduced pressure. The residue was dissolved in $\mathrm{EtOH}(1 \mathrm{~mL})$, and $\mathrm{Pd} / \mathrm{C}(6.7 \mathrm{mg}, 20 \% \mathrm{wt})$ was added to the mixture. The reaction vessel was evacuated and refilled with $\mathrm{H}_{2}$ three times. After stirred at $\mathrm{rt}$ under $\mathrm{H}_{2}$ atmosphere for $3 \mathrm{~h}$, the reaction mixture was filtrated through a short of silica gel column and washed with EtOAc. The filtrate was concentrated under reduced pressure. The products were purified by silica gel flash chromatography to yield bridged bicycles $\mathbf{2 t - v}$. The $d r$ ratio of compound 2t-v was determined by ${ }^{1} \mathrm{H}$ NMR.
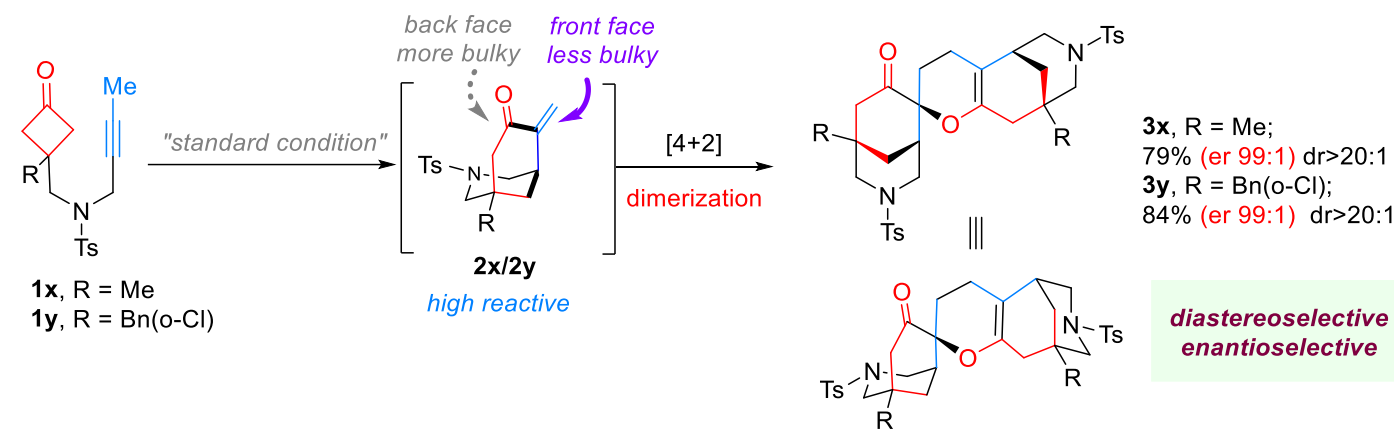

General procedure of preparing $\mathbf{3 x}-\mathbf{y}$ : A flame dried $8 \mathrm{~mL}$ tube was charged with the substrate $(\mathbf{1 x}-\mathbf{y}, 0.1 \mathrm{mmol}, 1$ equiv) and (R)-(-)-DTBM-segphos (L1, $9.9 \mathrm{mg}, 0.0084 \mathrm{mmol}, 8.4 \mathrm{~mol} \%)$. The tube was then transferred to a nitrogenfilled glove box. After addition of $\left[\mathrm{Rh}(\mathrm{COD})_{2}\right] \mathrm{NTf}_{2}(4.2 \mathrm{mg}, 0.007 \mathrm{mmol}, 7 \mathrm{~mol} \%)$ and 1,4-diflorobenzene $(0.5 \mathrm{~mL})$, the tube was sealed and taken out of glove box. The reaction was stirred at $120^{\circ} \mathrm{C}$ in an oil bath for $48 \mathrm{~h}$, before it was then cooled to room temperature. The products were directly purified by silica gel flash chromatography to yield bridged bicycles $\mathbf{3 x}-\mathbf{y}$. The $d r$ ratio of compound $\mathbf{3} \mathbf{x}-\mathbf{y}$ was determined by ${ }^{1} \mathrm{H}$ NMR. 

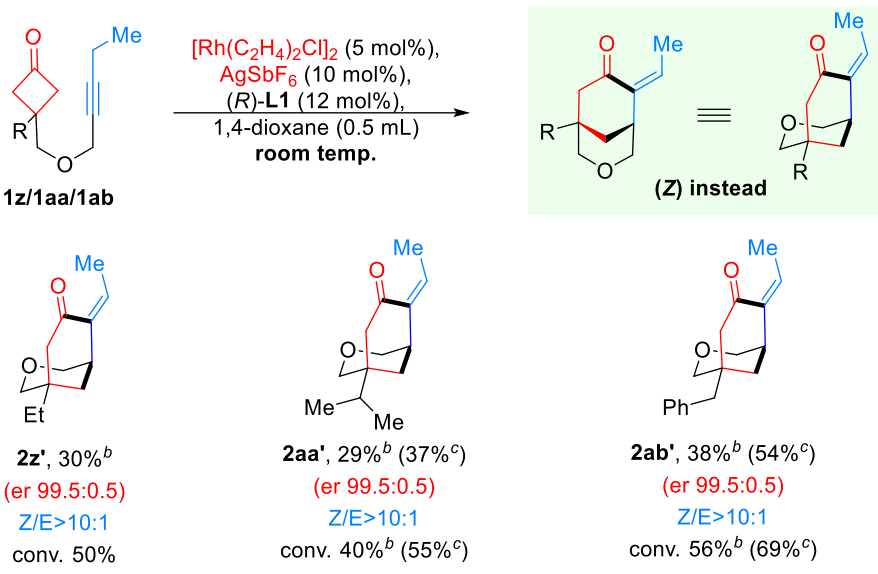

General procedure of preparing $2 \mathbf{z}^{\prime}$-ab': A flame dried $4 \mathrm{~mL}$ vial was charged with (R)-(-)-DTBM-segphos (L1, $9.9 \mathrm{mg}$, $0.0084 \mathrm{mmol}, 8.4 \mathrm{~mol} \%)$. The vial was then transferred to a nitrogen-filled glove box. After addition of $\left[\mathrm{Rh}\left(\mathrm{C}_{2} \mathrm{H}_{4}\right)_{2} \mathrm{Cl}\right]_{2}$ (2.0 mg, $0.005 \mathrm{mmol}, 5 \mathrm{~mol} \%$ ), AgSbF 6 (3.4 mg, $0.01 \mathrm{mmol}, 10 \mathrm{~mol} \%$ ) and 1,4-dioxane (0.5 mL), the reaction was stirred at room temperature for $2 \mathrm{~min}$. After addition of the substrate $(\mathbf{1} \mathbf{x}-\mathbf{y}, 0.1 \mathrm{mmol}, 1$ equiv), the vial was sealed and taken out of glove box. The reaction was stirred at room temperature for 3 days or 11 days. The reaction mixture was concentrated under reduced pressure. The products were purified by silica gel flash chromatography to yield bridged bicycles $2 z^{\prime}-a b^{\prime}$. The $Z / E$ ratio of compound $\mathbf{2}^{\prime} / \mathbf{2}$ was determined by ${ }^{1} \mathrm{H} N M R$.

Note: Following the above procedure, all racemic samples could be synthesized by changing chiral ligand $\mathbf{L}$ to racemic $\mathbf{L 1}$.

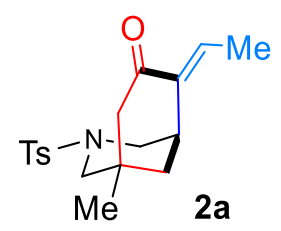

2a (23.1 $\mathrm{mg})$ was isolated as a white solid in $70 \%$ yield.

$\mathrm{Rf}=0.2$, Hexane/ EtOAc 2:1.

Mp: $194.6-195.8^{\circ} \mathrm{C}$.

${ }^{1} \mathrm{H}$ NMR (400 MHz, Chloroform-d) $\delta 7.63-7.57(\mathrm{~m}, 2 \mathrm{H}), 7.34-7.29(\mathrm{~m}, 2 \mathrm{H}), 6.73(\mathrm{q}, J=7.4,6.9 \mathrm{~Hz}, 1 \mathrm{H}), 3.58-3.50$ $(\mathrm{m}, 1 \mathrm{H}), 3.42(\mathrm{dt}, J=11.4,2.1 \mathrm{~Hz}, 1 \mathrm{H}), 3.17(\mathrm{t}, J=3.0 \mathrm{~Hz}, 1 \mathrm{H}), 2.50-2.44(\mathrm{~m}, 2 \mathrm{H}), 2.43(\mathrm{~s}, 3 \mathrm{H}), 2.23-2.13(\mathrm{~m}, 2 \mathrm{H})$, $1.77(\mathrm{~d}, J=7.3 \mathrm{~Hz}, 3 \mathrm{H}), 1.72(\mathrm{ddt}, J=13.2,3.6,1.9 \mathrm{~Hz}, 1 \mathrm{H}), 1.49$ (dt, $J=13.2,3.1 \mathrm{~Hz}, 1 \mathrm{H}), 0.97(\mathrm{~s}, 3 \mathrm{H})$.

${ }^{13} \mathrm{C}$ NMR (101 MHz, CDCl $) \delta$ 197.2, 143.7, 138.0, 133.6, 133.0, 129.6, 127.9, 57.3, 51.5, 51.2, 37.5, 32.4, 31.1, 27.1, $21.5,13.2$

HRMS ESI Calcd for $\mathrm{C}_{18} \mathrm{H}_{24} \mathrm{NO}_{3} \mathrm{~S}[\mathrm{M}+\mathrm{H}]^{+}:$334.1471, Found: 334.1470.

IR (KBr): 2923, 2848, 1686, 1617, 1457, 1338, 1248, 1165, 1089, $1029 \mathrm{~cm}^{-1}$. 
Chiral HPLC (Chiralpak IA, Hexane/Isopropanol $=90 / 10$, flow rate $=1.0 \mathrm{~mL} / \mathrm{min}, 230 \mathrm{~nm}$ ), $\mathrm{t}_{\mathrm{minor}}=25.3 \mathrm{~min}, \mathrm{t}_{\mathrm{major}}=$ $30.2 \min .[\alpha]^{21.5}=-65.6\left(c 1.2, \mathrm{CHCl}_{3}\right)$ at $96.5: 3.5 \mathrm{er}$.

\section{Racemic sample $2 a$}

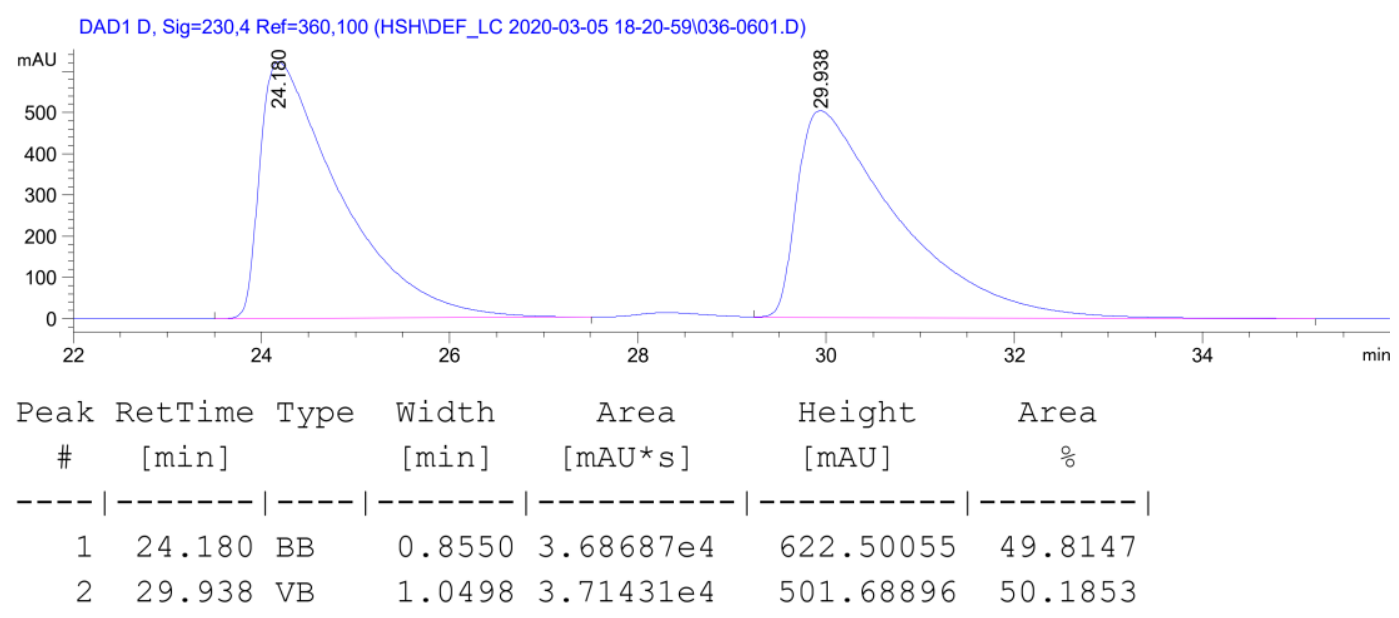

\section{Enantiomeric sample 2a}

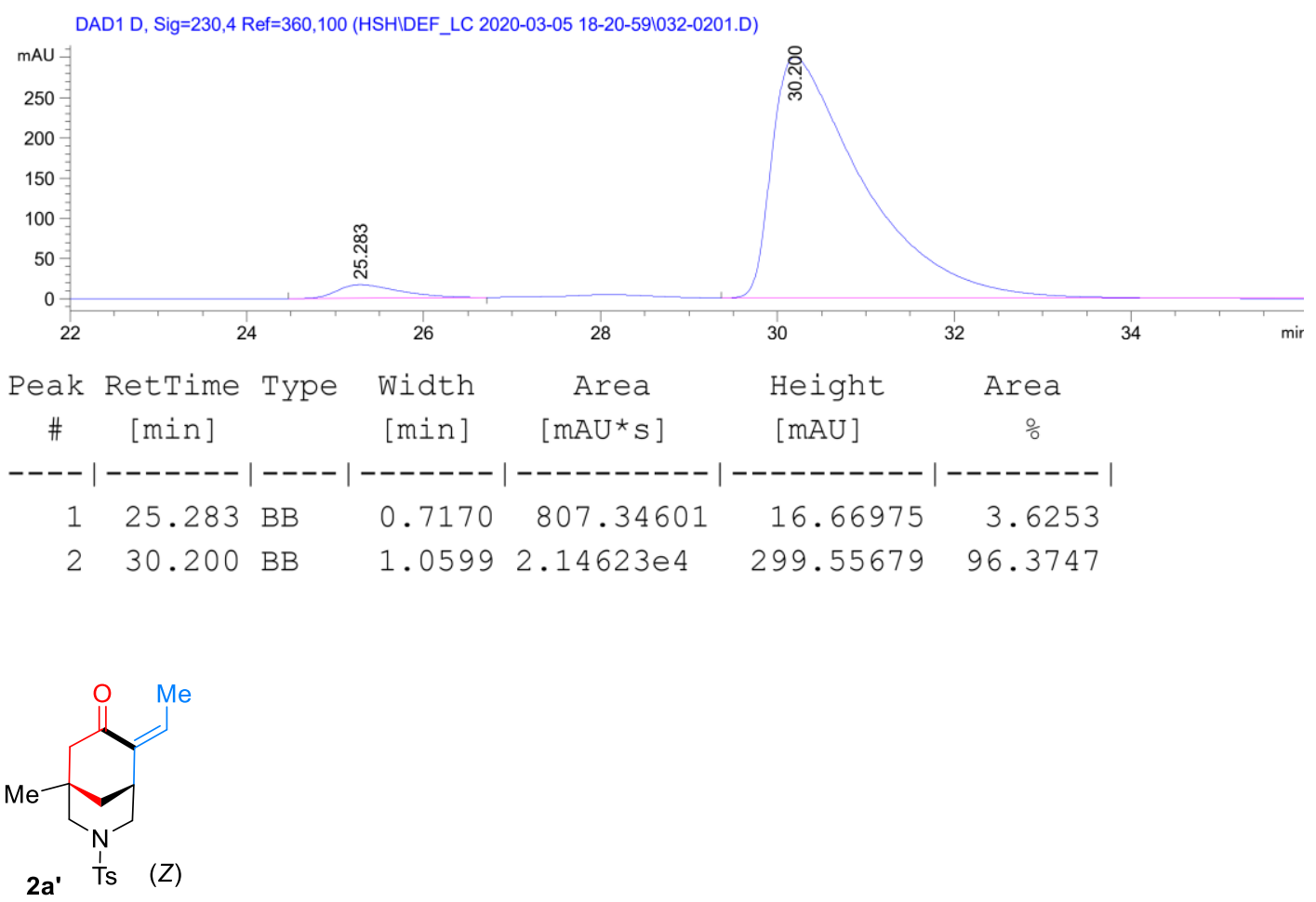

2a' (3.9 mg) was isolated as a foam in $11 \%$ yield.

Rf $=0.25$, Hexane/ EtOAc 2:1.

${ }^{1} \mathrm{H}$ NMR (400 MHz, Benzene- $\left.d_{6}\right) \delta 7.77-7.68(\mathrm{~m}, 2 \mathrm{H}), 6.90-6.81(\mathrm{~m}, 2 \mathrm{H}), 5.36(\mathrm{q}, J=7.2 \mathrm{~Hz}, 1 \mathrm{H}), 3.52(\mathrm{ddt}, J=10.9$, 3.3, $1.8 \mathrm{~Hz}, 1 \mathrm{H}), 3.38(\mathrm{dt}, J=11.2,2.1 \mathrm{~Hz}, 1 \mathrm{H}), 2.32-2.18(\mathrm{~m}, 2 \mathrm{H}), 2.11-2.05(\mathrm{~m}, 1 \mathrm{H}), 2.04-1.95(\mathrm{~m}, 4 \mathrm{H}), 1.88(\mathrm{~s}$, $3 \mathrm{H}), 1.70(\mathrm{dd}, J=16.9,1.4 \mathrm{~Hz}, 1 \mathrm{H}), 1.16(\mathrm{ddt}, J=13.0,3.7,2.0 \mathrm{~Hz}, 1 \mathrm{H}), 0.80(\mathrm{dt}, J=13.0,3.1 \mathrm{~Hz}, 1 \mathrm{H}), 0.37(\mathrm{~s}, 3 \mathrm{H})$. 
${ }^{13} \mathrm{C}$ NMR (101 MHz, C6 $\left.\mathrm{D}_{6}\right) \delta 197.1,143.3,138.3,134.69,134.66,129.6,128.6,57.0,53.6,52.5,40.6,38.5,33.1$,

26.3, 21.1, 15.5 .

HRMS ESI Calcd for $\mathrm{C}_{18} \mathrm{H}_{24} \mathrm{NO}_{3} \mathrm{~S}[\mathrm{M}+\mathrm{H}]^{+}: 334.1471$, Found: 334.1472.

IR (KBr): 2927, 2854, 1734, 1688, 1628,1598, 1457, 1345, 1230, 1165, $1092 \mathrm{~cm}^{-1}$.

Chiral HPLC (Chiralpak IA, Hexane/Isopropanol =90/10, flow rate $=1.0 \mathrm{~mL} / \mathrm{min}, 230 \mathrm{~nm}$ ), $\mathrm{t}_{\mathrm{minor}}=19.1 \mathrm{~min}, \mathrm{t}_{\mathrm{major}}=$ $26.7 \min .[\alpha]^{21.5}=-27.3\left(c 0.7, \mathrm{CHCl}_{3}\right)$ at $97: 3 \mathrm{er}$

\section{Racemic sample 2a'}

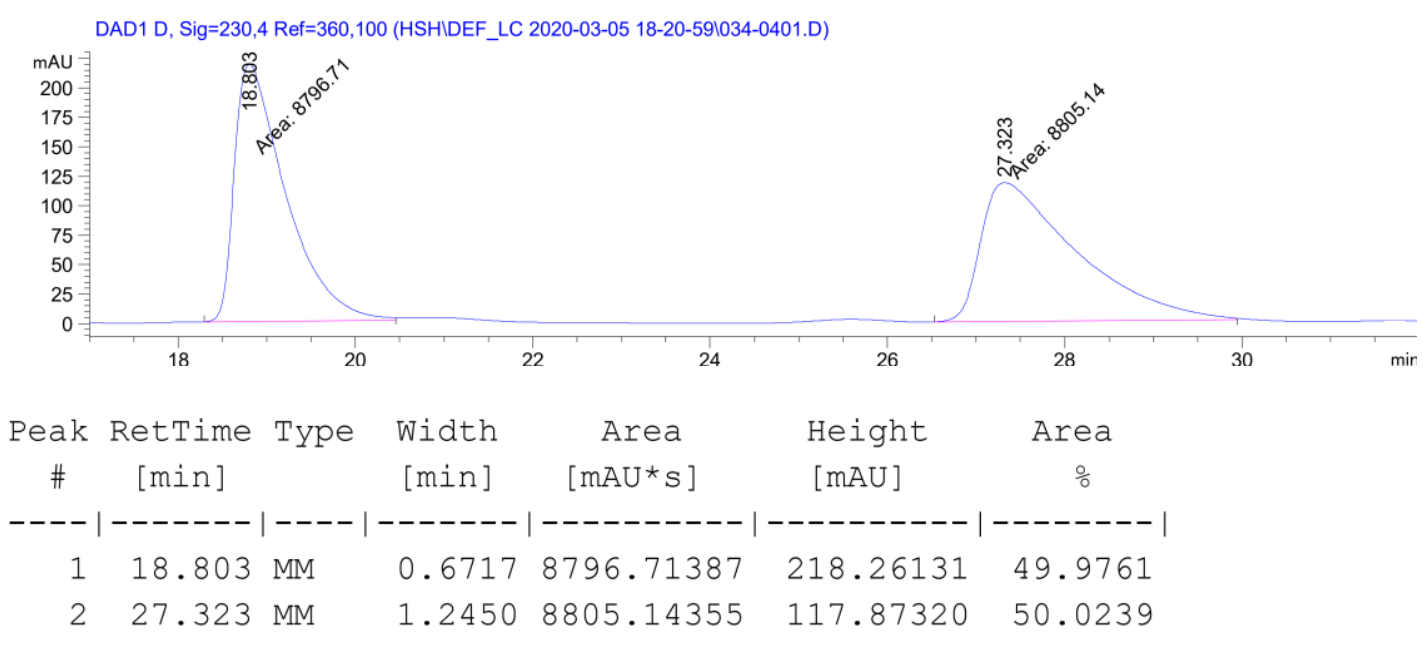

\section{Enantiomeric sample 2a'}

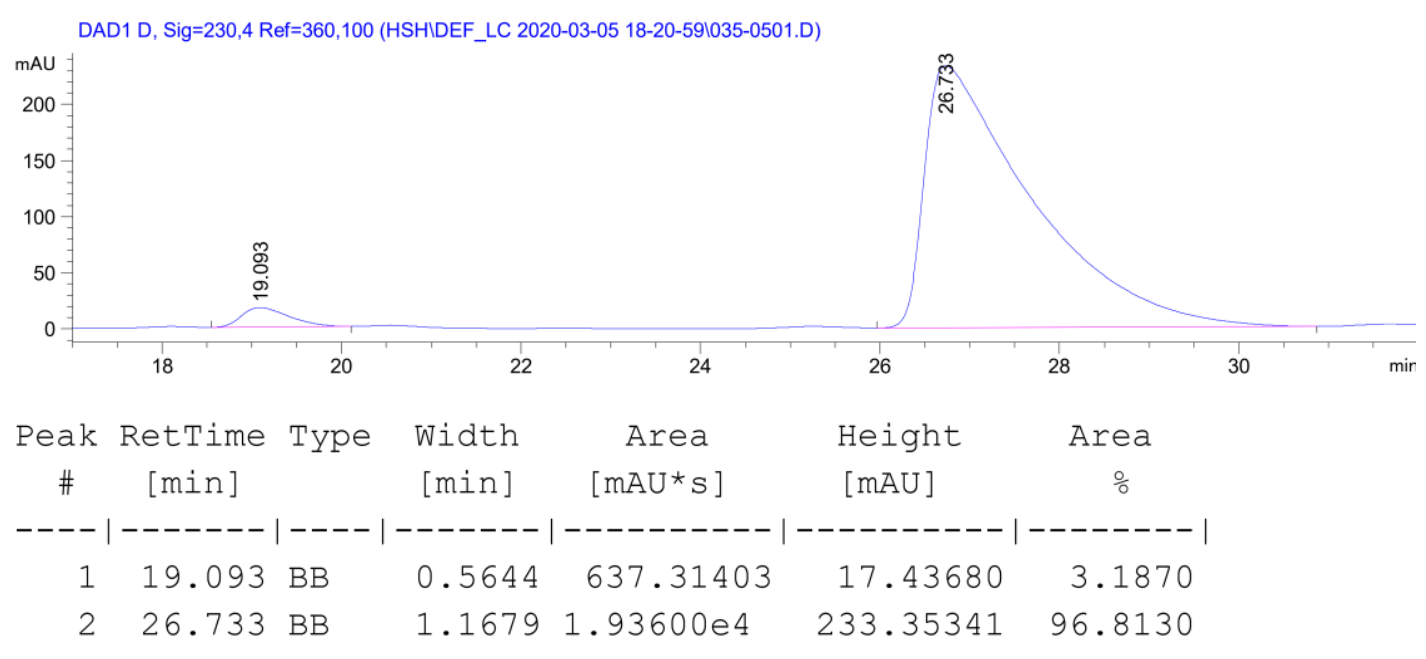<smiles>CCC#CCN([13CH3])CC1(C)CC1</smiles> 


\section{Compound 2a".}

$\mathrm{Rf}=0.45$, Hexane/ EtOAc 8:1.

${ }^{1} \mathrm{H}$ NMR (400 MHz, Chloroform-d) $\delta 7.77-7.68(\mathrm{~m}, 2 \mathrm{H}), 7.33-7.20(\mathrm{~m}, 2 \mathrm{H}), 4.23(\mathrm{t}, J=2.2 \mathrm{~Hz}, 2 \mathrm{H}), 3.02(\mathrm{~s}, 2 \mathrm{H}), 2.40$ $(\mathrm{s}, 3 \mathrm{H}), 1.92-1.82(\mathrm{~m}, 2 \mathrm{H}), 1.10(\mathrm{~s}, 3 \mathrm{H}), 0.85(\mathrm{t}, J=7.5 \mathrm{~Hz}, 3 \mathrm{H}), 0.44-0.37(\mathrm{~m}, 2 \mathrm{H}), 0.37-0.31(\mathrm{~m}, 2 \mathrm{H})$.

${ }^{13} \mathrm{C}$ NMR (101 MHz, CDCl $) \delta$ 142.9, 136.3, 129.1, 127.8, 87.7, 71.7, 53.5, 36.2, 21.4, 20.7, 13.5, 12.9, 12.0, 11.0.

HRMS ESI Calcd for $\mathrm{C}_{17} \mathrm{H}_{24} \mathrm{NO}_{2} \mathrm{~S}[\mathrm{M}+\mathrm{H}]^{+}:$306.1522, Found: 306.1524.

IR (KBr): 2975,2937, 2879, 1598, 1495, 1435, 1349, 1163, 1121, 1091, $1032 \mathrm{~cm}^{-1}$.

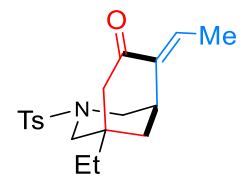

2b

2b (24.6 mg) was isolated as a white solid in $71 \%$ yield.

$\mathrm{Rf}=0.2$, Hexane/ EtOAc 2:1.

Mp: $149.6-150.7^{\circ} \mathrm{C}$.

${ }^{1} \mathrm{H}$ NMR (400 MHz, Chloroform-d) $\delta 7.63-7.58(\mathrm{~m}, 2 \mathrm{H}), 7.32$ (d, J= $\left.7.7 \mathrm{~Hz}, 2 \mathrm{H}\right), 6.73(\mathrm{q}, J=7.3 \mathrm{~Hz}, 1 \mathrm{H}), 3.58-3.46$ $(\mathrm{m}, 2 \mathrm{H}), 3.17(\mathrm{t}, J=3.0 \mathrm{~Hz}, 1 \mathrm{H}), 2.46-2.39(\mathrm{~m}, 4 \mathrm{H}), 2.21(\mathrm{~s}, 1 \mathrm{H}), 2.18(\mathrm{dd}, J=6.3,1.3 \mathrm{~Hz}, 1 \mathrm{H}), 1.77(\mathrm{~d}, J=7.3 \mathrm{~Hz}, 3 \mathrm{H})$, $1.71(\mathrm{ddt}, J=13.2,3.6,1.9 \mathrm{~Hz}, 1 \mathrm{H}), 1.45(\mathrm{dt}, J=13.2,3.1 \mathrm{~Hz}, 1 \mathrm{H}), 1.42-1.24(\mathrm{~m}, 4 \mathrm{H}), 0.85(\mathrm{t}, J=7.6 \mathrm{~Hz}, 3 \mathrm{H})$.

${ }^{13} \mathrm{C}$ NMR (101 MHz, $\left.\mathrm{CDCl}_{3}\right) \delta 197.5,143.7,138.4,133.5,133.1,129.6,127.9,56.1,51.9,48.4,35.6,35.1,32.9,30.9$, $21.5,13.2,7.1$.

HRMS ESI Calcd for $\mathrm{C}_{19} \mathrm{H}_{26} \mathrm{NO}_{3} \mathrm{~S}[\mathrm{M}+\mathrm{H}]^{+}: 348.1628$, Found: 348.1631.

IR (KBr): 2964, 2915, 2854, 1688, 1624, 1339, 1232, 1166, $1090 \mathrm{~cm}^{-1}$.

Chiral HPLC (Chiralpak IF, Hexane/Isopropanol $=70 / 30$, flow rate $=1.0 \mathrm{~mL} / \mathrm{min}, 230 \mathrm{~nm}$ ), $\mathrm{t}_{\mathrm{minor}}=26.8 \mathrm{~min}, \mathrm{t}_{\mathrm{major}}=$ $38.1 \min .[\alpha]^{21.5} \mathrm{D}=-56.5\left(\mathrm{c} 1.2, \mathrm{CHCl}_{3}\right)$ at $95: 5 \mathrm{er}$.

\section{Racemic sample $\mathbf{2 b}$}

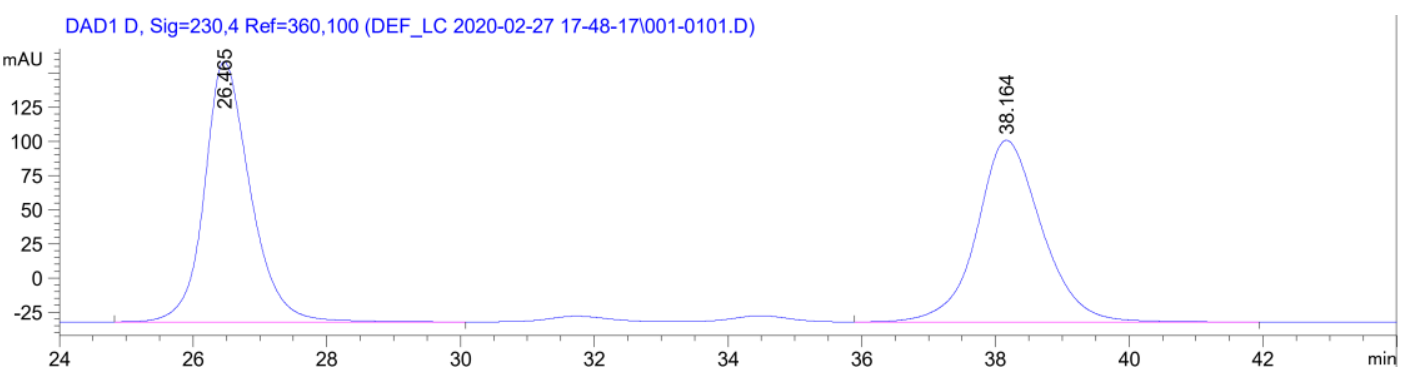




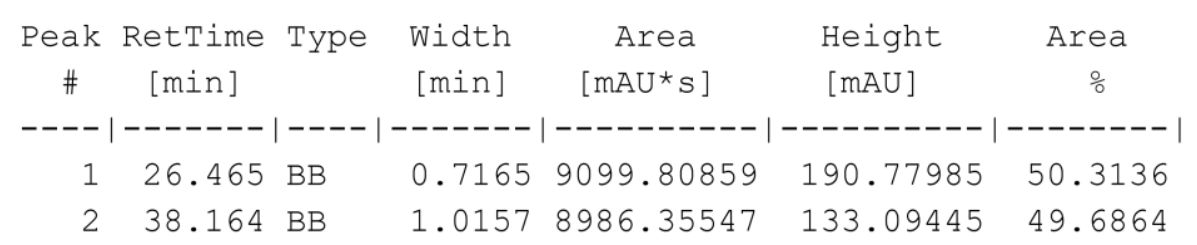

\section{Enantiomeric sample 2b}
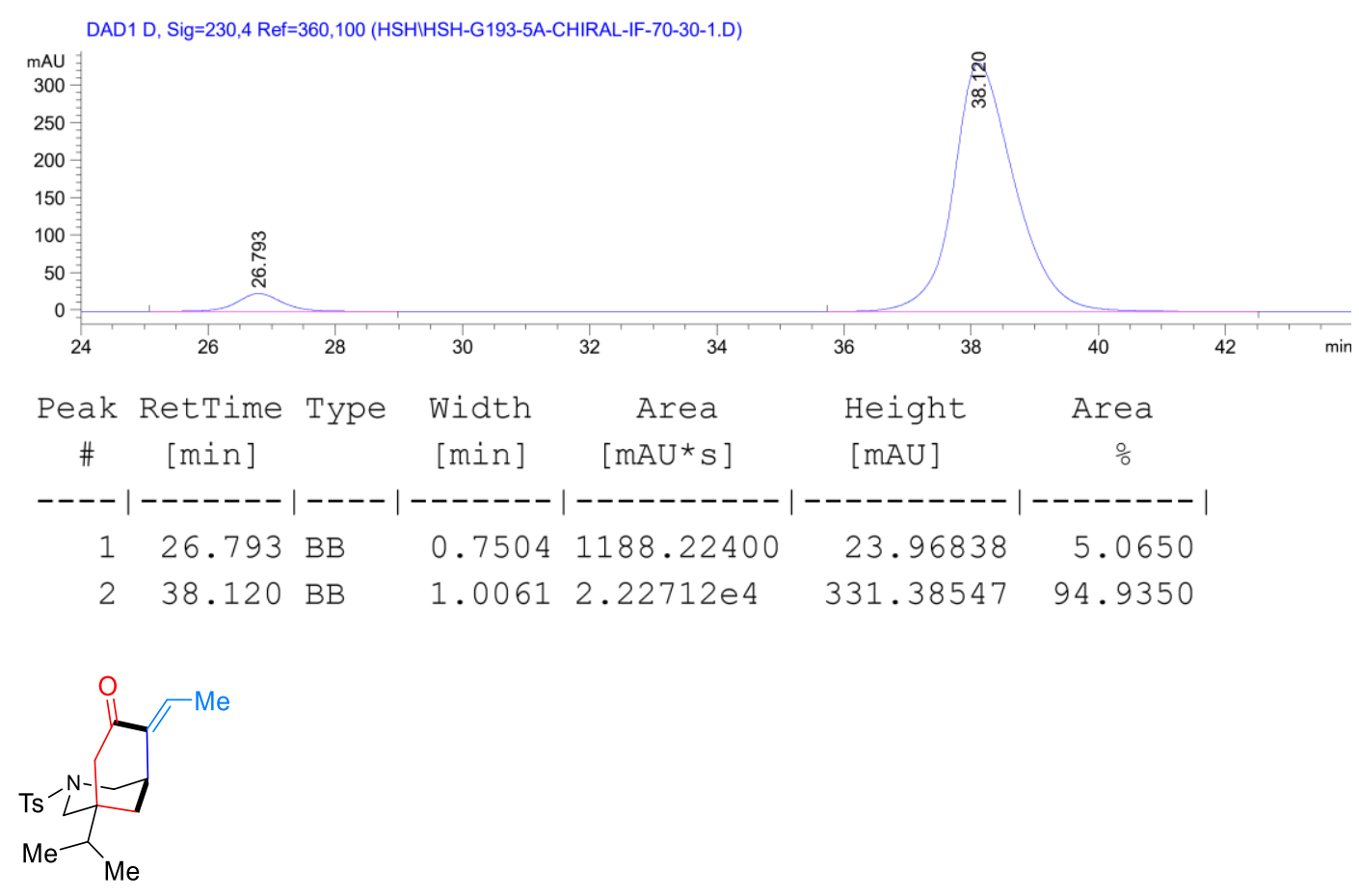

2c

2c (30 mg) was isolated as a foam in $83 \%$ yield.

$\mathrm{Rf}=0.33$, Hexane/ EtOAc 2:1.

${ }^{1} \mathrm{H}$ NMR $\left(400 \mathrm{MHz}\right.$, Benzene- $\left.d_{6}\right) \delta 7.73(\mathrm{~d}, J=8.3 \mathrm{~Hz}, 2 \mathrm{H}), 6.86(\mathrm{~d}, J=8.1 \mathrm{~Hz}, 2 \mathrm{H}), 6.81(\mathrm{q}, J=7.3 \mathrm{~Hz}, 1 \mathrm{H}), 3.73-3.65$ (m, 1H), $3.59-3.50(\mathrm{~m}, 1 \mathrm{H}), 2.61(\mathrm{p}, J=3.0 \mathrm{~Hz}, 1 \mathrm{H}), 2.41(\mathrm{dd}, J=17.9,3.0 \mathrm{~Hz}, 1 \mathrm{H}), 2.26(\mathrm{dd}, J=10.8,2.2 \mathrm{~Hz}, 1 \mathrm{H}), 2.14$ (dd, $J=11.3,1.4 \mathrm{~Hz}, 1 \mathrm{H}), 1.94(\mathrm{dd}, J=17.9,1.4 \mathrm{~Hz}, 1 \mathrm{H}), 1.88(\mathrm{~s}, 3 \mathrm{H}), 1.28$ (d, J = 7.3 Hz, 3H), 1.21 (ddt, J=13.1, 3.7, $1.9 \mathrm{~Hz}, 1 \mathrm{H}), 0.99-0.90(\mathrm{~m}, 1 \mathrm{H}), 0.87(\mathrm{dt}, J=13.1,3.2 \mathrm{~Hz}, 1 \mathrm{H}), 0.51-0.44(\mathrm{~m}, 6 \mathrm{H})$.

${ }^{13}$ C NMR (101 MHz, C6 $\left.\mathrm{D}_{6}\right) \delta$ 195.9, 143.4, 139.0, 134.8, 132.3, 129.6, 128.4, 54.8, 51.8, 45.6, 37.4, 35.3, 33.9, 31.1, 21.1, 16.3, 16.2, 12.7 .

HRMS ESI Calcd for $\mathrm{C}_{20} \mathrm{H}_{27} \mathrm{NNaO}_{3} \mathrm{~S}[\mathrm{M}+\mathrm{Na}]^{+}: 384.1604$, Found: 384.1599 .

IR (KBr): 2964, 2876, 1687, 1626, 1466, 1340, 1293, 1234, 1164, $1090 \mathrm{~cm}^{-1}$.

Chiral HPLC (Chiralpak IF, Hexane/Isopropanol $=70 / 30$, flow rate $=1.0 \mathrm{~mL} / \mathrm{min}, 230 \mathrm{~nm}$ ), $t_{\text {minor }}=25.4 \mathrm{~min}, \mathrm{t}_{\mathrm{major}}=$ $35.4 \min .[\alpha]^{21.5}=-70.1\left(c 0.7, \mathrm{CHCl}_{3}\right)$ at $98: 2 \mathrm{er}$.

\section{Racemic sample 2c}




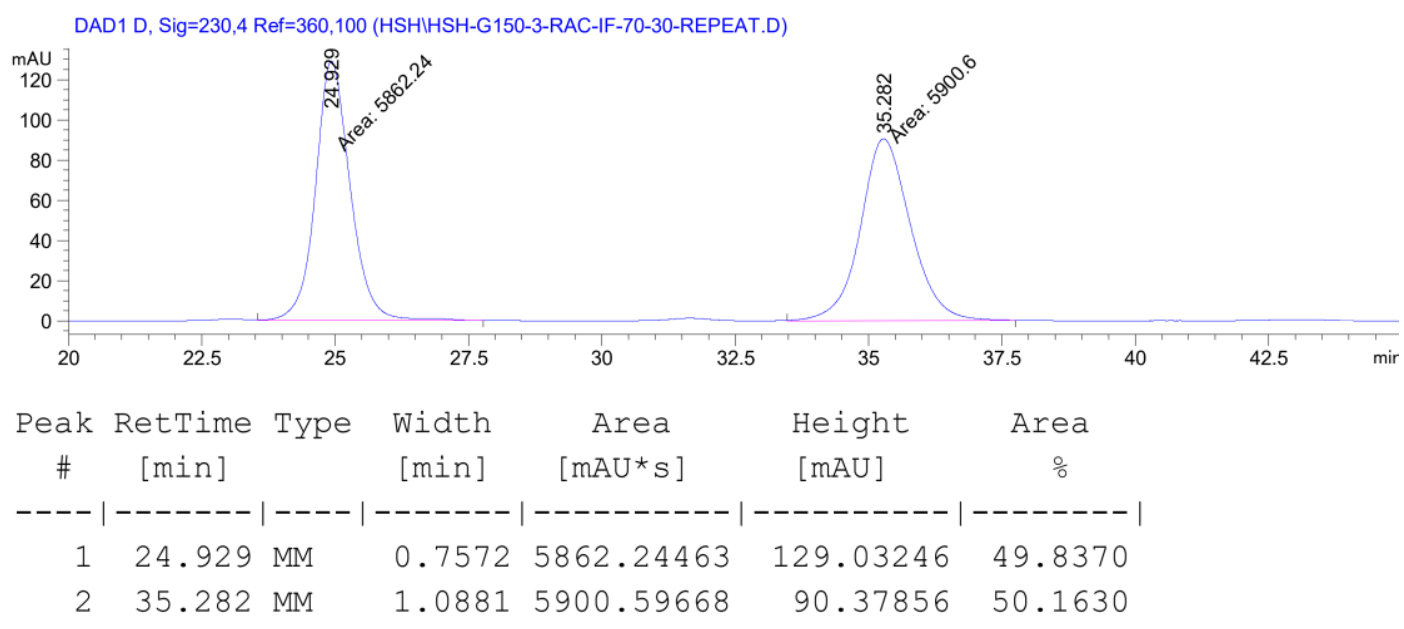

\section{Enantiomeric sample 2c}

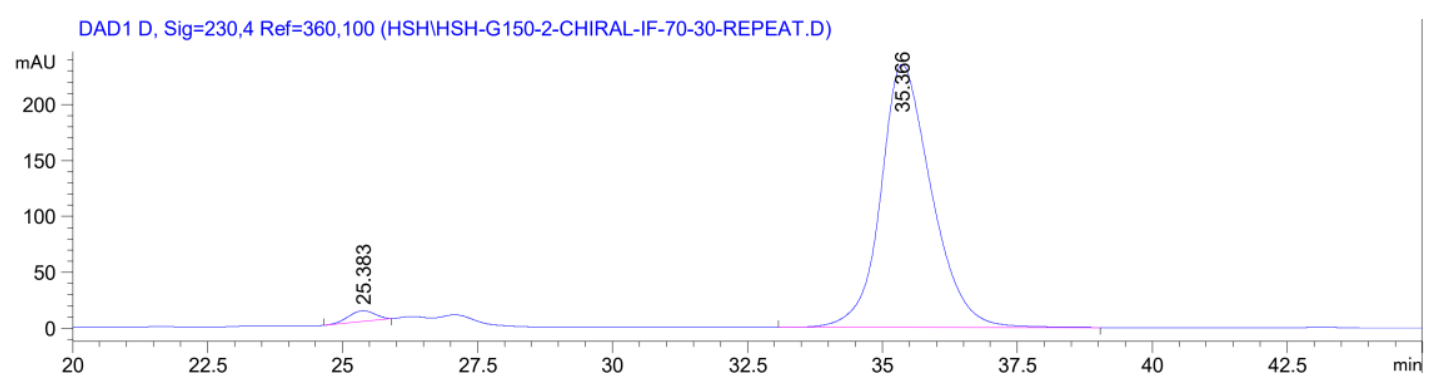

\begin{tabular}{|c|c|c|c|c|c|c|}
\hline $\begin{array}{c}\text { Peak } \\
\text { \# }\end{array}$ & $\begin{array}{c}\text { RetTime } \\
\text { [min] }\end{array}$ & Type & $\begin{array}{l}\text { Width } \\
\text { [min] }\end{array}$ & $\begin{array}{c}\text { Area } \\
{\left[\mathrm{mAU}{ }^{*} \mathrm{~s}\right]}\end{array}$ & $\begin{array}{l}\text { Height } \\
{[\mathrm{mAU}]}\end{array}$ & $\begin{array}{c}\text { Area } \\
\quad \%\end{array}$ \\
\hline-- & - & & ------ & --------- & --------- & ------- \\
\hline 1 & 25.383 & BB & 0.5444 & 323.93460 & 9.43901 & 2.0617 \\
\hline 2 & 35.366 & BB & 0.9840 & $1.53879 e 4$ & 234.99232 & 97.9383 \\
\hline
\end{tabular}

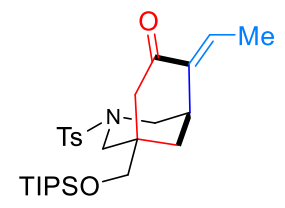

2d

$\mathbf{2 d}(35 \mathrm{mg})$ was isolated as a colorless oil in $69 \%$ yield.

$\mathrm{Rf}=0.16$, Hexane/ EtOAc 4:1.

${ }^{1} \mathrm{H}$ NMR $\left(400 \mathrm{MHz}\right.$, Benzene- $\left.d_{6}\right) \delta 7.75(\mathrm{~d}, J=8.3 \mathrm{~Hz}, 2 \mathrm{H}), 6.91-6.80(\mathrm{~m}, 3 \mathrm{H}), 3.68-3.59(\mathrm{~m}, 2 \mathrm{H}), 3.14-3.04(\mathrm{~m}$, $2 \mathrm{H}), 2.68(\mathrm{t}, J=2.9 \mathrm{~Hz}, 1 \mathrm{H}), 2.53(\mathrm{dd}, J=18.0,2.9 \mathrm{~Hz}, 1 \mathrm{H}), 2.37(\mathrm{dd}, J=11.4,1.3 \mathrm{~Hz}, 1 \mathrm{H}), 2.32(\mathrm{dd}, J=11.0,2.2 \mathrm{~Hz}$, $1 \mathrm{H}), 2.17(\mathrm{dd}, J=18.0,1.3 \mathrm{~Hz}, 1 \mathrm{H}), 1.90(\mathrm{~s}, 3 \mathrm{H}), 1.38-1.31(\mathrm{~m}, 1 \mathrm{H}), 1.29(\mathrm{~d}, J=7.3 \mathrm{~Hz}, 3 \mathrm{H}), 1.16(\mathrm{dt}, J=13.1,3.2 \mathrm{~Hz}$, $1 \mathrm{H}), 0.98(\mathrm{~d}, J=5.7 \mathrm{~Hz}, 18 \mathrm{H}), 0.96-0.89(\mathrm{~m}, 3 \mathrm{H})$.

${ }^{13} \mathrm{C}$ NMR (101 MHz, C6 $\left.\mathrm{D}_{6}\right) \delta 195.4,143.4,138.9,134.5,132.9,129.6,129.6,128.5,70.4,54.3,51.9,47.0,38.1,32.7$, 31.0, 21.1, 18.1, 12.8, 12.1. 
HRMS ESI Calcd for $\mathrm{C}_{27} \mathrm{H}_{44} \mathrm{NO}_{4} \mathrm{SSi}[\mathrm{M}+\mathrm{H}]^{+}:$506.2755, Found: 506.2759.

IR (KBr): 2924, 2865, 1690, 1624, 1436, 1355, 1343, 1168, $1092 \mathrm{~cm}^{-1}$.

Chiral HPLC (Chiralcel OB-H, Hexane/Isopropanol =90/10, flow rate $=1.0 \mathrm{~mL} / \mathrm{min}, 230 \mathrm{~nm}$ ), $\mathrm{t}_{\text {minor }}=11.7 \mathrm{~min}, \mathrm{t}_{\mathrm{major}}=$ $12.8 \min .[\alpha]^{21.5}{ }_{D}=-40\left(c 1.2, \mathrm{CHCl}_{3}\right)$ at $97.5: 2.5$ er.

\section{Racemic sample $2 d$}

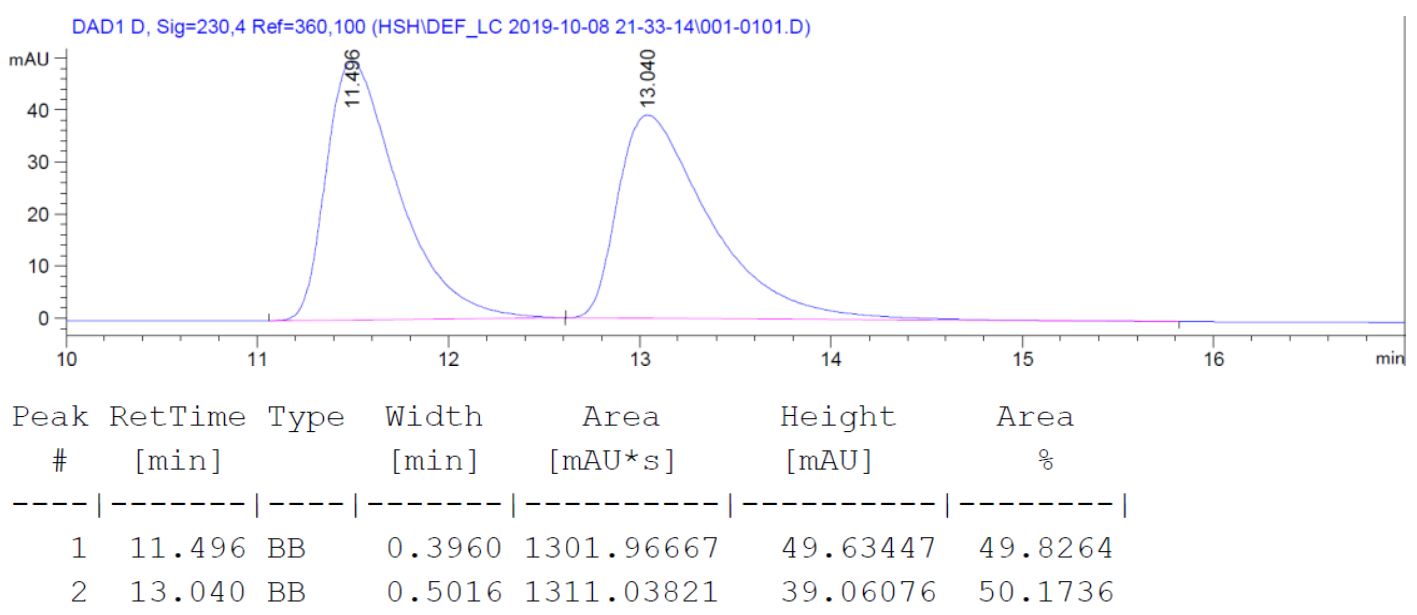

\section{Enantiomeric sample 2d}

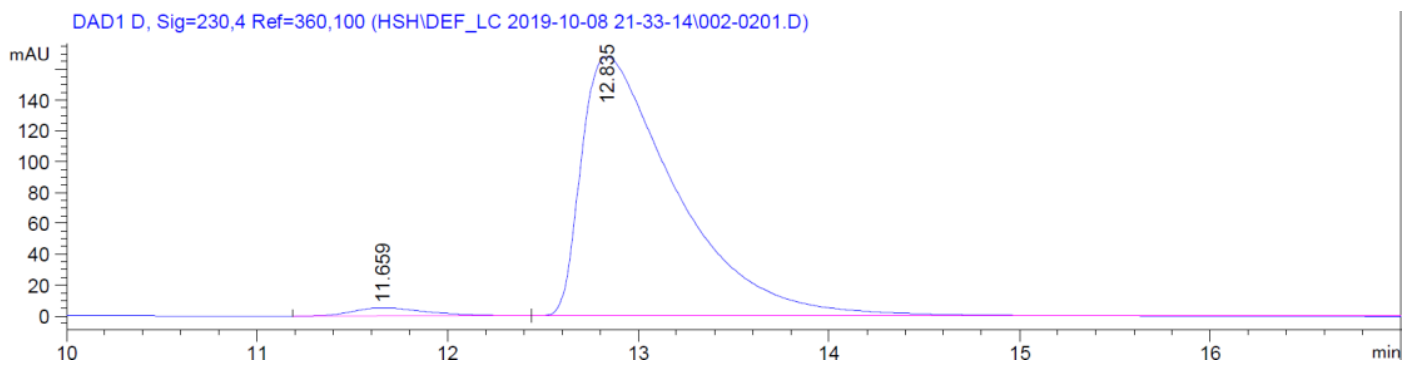

\begin{tabular}{|c|c|c|c|c|c|c|}
\hline $\begin{array}{c}\text { Peak } \\
\quad \#\end{array}$ & $\begin{array}{c}\text { RetTime } \\
\text { [min] }\end{array}$ & Type & $\begin{array}{l}\text { Width } \\
\text { [min] }\end{array}$ & $\begin{array}{c}\text { Area } \\
{\left[\mathrm{mAU}{ }^{*} \mathrm{~s}\right]}\end{array}$ & $\begin{array}{l}\text { Height } \\
\text { [mAU] }\end{array}$ & $\begin{array}{c}\text { Area } \\
\quad \frac{\circ}{0}\end{array}$ \\
\hline--1 & -------- & & - - - - - - & ---------- & ----- & -------- \\
\hline 1 & 11.659 & BB & 0.4066 & 139.13046 & 5.12725 & 2.3304 \\
\hline 2 & 12.835 & BB & 0.5085 & 5831.11523 & 168.20187 & 97.6696 \\
\hline
\end{tabular}

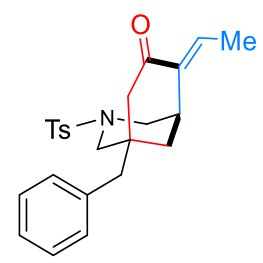

$2 e$

2 e (31 mg) was isolated as a white solid in 76\% yield. 
$\mathrm{Rf}=0.28$, Hexane/ EtOAc 2:1.

$\mathrm{Mp}: 169.4-172.6^{\circ} \mathrm{C}$.

${ }^{1} \mathrm{H}$ NMR (400 MHz, Chloroform-d) $\delta 7.63-7.54(\mathrm{~m}, 2 \mathrm{H}), 7.36-7.21(\mathrm{~m}, 5 \mathrm{H}), 7.09-7.00(\mathrm{~m}, 2 \mathrm{H}), 6.66(\mathrm{q}, J=7.2 \mathrm{~Hz}$, $1 \mathrm{H}), 3.51(\mathrm{dt}, J=11.3,1.9 \mathrm{~Hz}, 2 \mathrm{H}), 3.16(\mathrm{t}, J=3.0 \mathrm{~Hz}, 1 \mathrm{H}), 2.61-2.46(\mathrm{~m}, 3 \mathrm{H}), 2.44(\mathrm{~s}, 3 \mathrm{H}), 2.41-2.24(\mathrm{~m}, 3 \mathrm{H}), 1.73$ $(\mathrm{d}, J=7.2 \mathrm{~Hz}, 3 \mathrm{H}), 1.70-1.64(\mathrm{~m}, 1 \mathrm{H}), 1.56(\mathrm{dt}, J=13.2,3.1 \mathrm{~Hz}, 1 \mathrm{H})$.

${ }^{13} \mathrm{C}$ NMR (101 MHz, CDCl$)$ ) $\delta$ 197.2, 143.8, 138.1, 135.4, 133.4, 133.0, 130.4, 129.6, 128.2, 127.9, 126.8, 56.6, 51.7, $48.8,46.4,36.2,35.3,30.9,21.6,13.2$.

HRMS ESI Calcd for $\mathrm{C}_{24} \mathrm{H}_{28} \mathrm{NO}_{3} \mathrm{~S}[\mathrm{M}+\mathrm{H}]^{+}:$410.1784, Found: 410.1784 .

IR (KBr): 3027, 2929, 2863, 1688, 1625, 1452, 1340, 1167, 1090, $1009 \mathrm{~cm}^{-1}$.

Chiral HPLC (Chiralpak IB, Hexane/Isopropanol =90/10, flow rate $=1.0 \mathrm{~mL} / \mathrm{min}, 230 \mathrm{~nm}$ ), $\mathrm{t}_{\mathrm{minor}}=46.9 \mathrm{~min}, \mathrm{t}_{\mathrm{major}}=50$ $\min .[\alpha]^{21.5}=-46.4\left(c 1.5, \mathrm{CHCl}_{3}\right)$ at 95.5:4.5 er.

\section{Racemic sample $\mathbf{2 e}$}

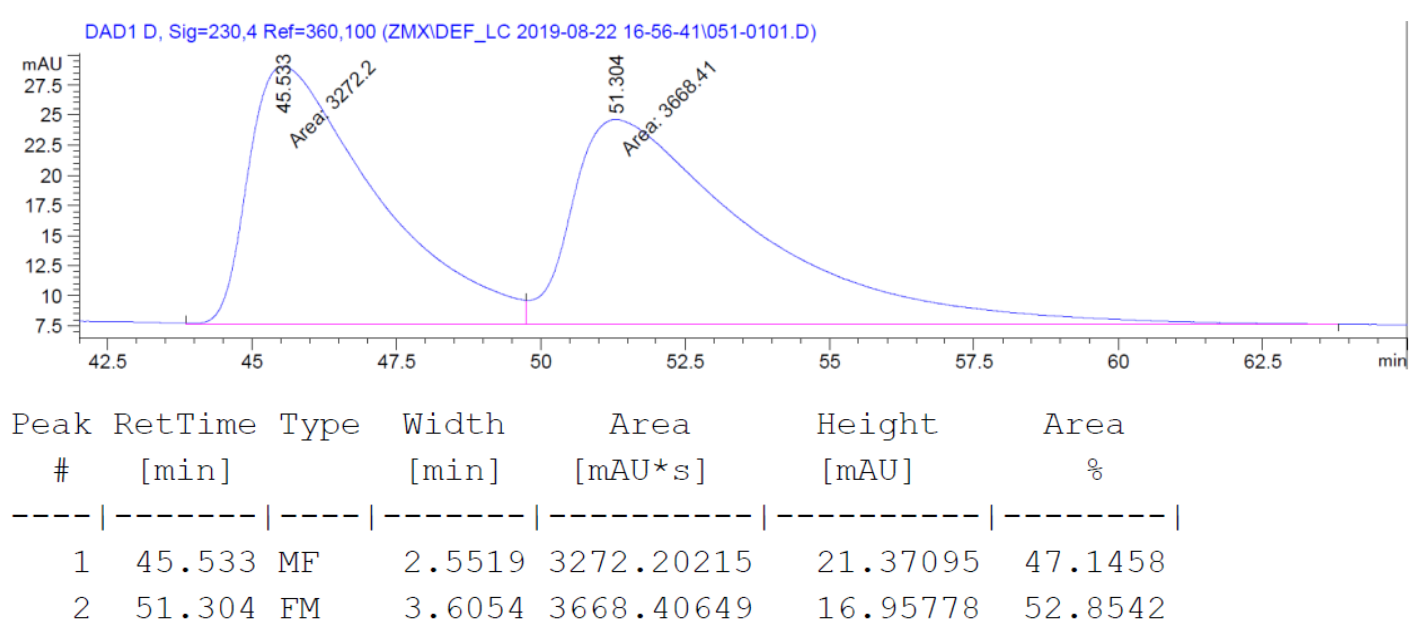

\section{Enantiomeric sample 2e}

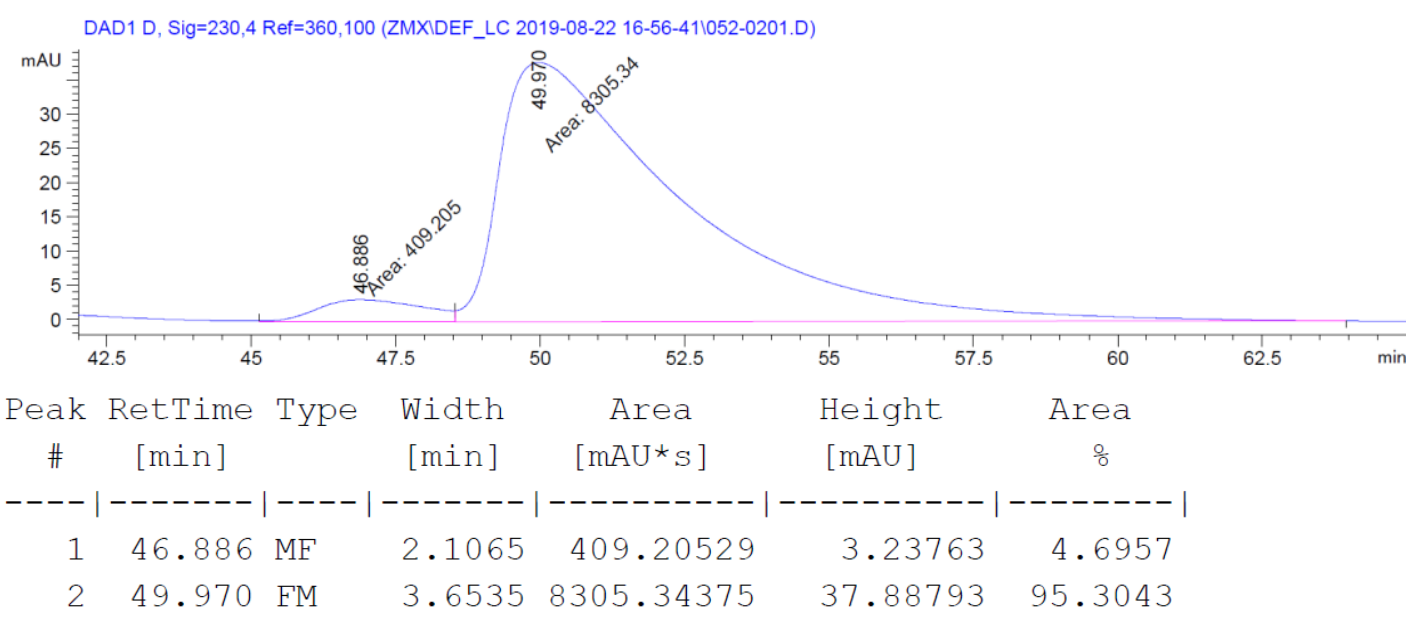




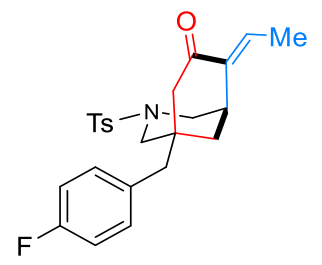

$2 \mathbf{f}$

$2 \mathrm{f}(33.3 \mathrm{mg})$ was isolated as a white solid in $78 \%$ yield.

Rf $=0.25$, Hexane/ EtOAc 2:1.

Mp: $165.0-167.0^{\circ} \mathrm{C}$.

${ }^{1} \mathrm{H}$ NMR $\left(400 \mathrm{MHz}\right.$, Benzene- $\left.d_{6}\right) \delta 7.77-7.69(\mathrm{~m}, 2 \mathrm{H}), 6.86(\mathrm{~d}, J=8.1 \mathrm{~Hz}, 2 \mathrm{H}), 6.76-6.64(\mathrm{~m}, 3 \mathrm{H}), 6.51-6.44(\mathrm{~m}$, $2 \mathrm{H}), 3.53-3.41(\mathrm{~m}, 2 \mathrm{H}), 2.60-2.52(\mathrm{~m}, 1 \mathrm{H}), 2.30(\mathrm{dd}, J=17.6,3.0 \mathrm{~Hz}, 1 \mathrm{H}), 2.23(\mathrm{dd}, J=10.8,2.2 \mathrm{~Hz}, 1 \mathrm{H}), 2.15(\mathrm{dd}, J$ $=11.2,1.4 \mathrm{~Hz}, 1 \mathrm{H}), 1.97-1.80(\mathrm{~m}, 6 \mathrm{H}), 1.20(\mathrm{~d}, J=7.3 \mathrm{~Hz}, 3 \mathrm{H}), 1.11-1.04(\mathrm{~m}, 1 \mathrm{H}), 0.86(\mathrm{dt}, J=13.0,3.2 \mathrm{~Hz}, 1 \mathrm{H})$.

${ }^{13} \mathrm{C}$ NMR $\left(101 \mathrm{MHz}, \mathrm{C}_{6} \mathrm{D}_{6}\right) \delta 195.4,162.2\left(\mathrm{~d},{ }^{1} \mathrm{~J}_{\mathrm{C}-\mathrm{F}}=245.4 \mathrm{~Hz}\right), 143.6,138.5,134.6,132.5,132.1\left(\mathrm{~d},{ }^{3} J_{\mathrm{C}-\mathrm{F}}=8.1 \mathrm{~Hz}\right), 131.7$ $\left(d,{ }^{4} J_{C-F}=4.0 \mathrm{~Hz}\right), 129.6,128.5,115.1\left(d,{ }^{2} J_{C-F}=21.2 \mathrm{~Hz}\right), 56.5,51.8,49.0,45.1,36.0\left(d, J_{C-F}=1.0 \mathrm{~Hz}\right), 35.0,31.1,21.1$, 12.7.

HRMS ESI Calcd for $\mathrm{C}_{24} \mathrm{H}_{27} \mathrm{FNO}_{3} \mathrm{~S}[\mathrm{M}+\mathrm{H}]^{+}:$428.1690, Found: 428.1694.

IR (KBr): 2964, 2876, 1687, 1626, 1598, 1466, 1340, 1234, 1164, $1090 \mathrm{~cm}^{-1}$.

Chiral HPLC (Chiralpak IA, Hexane/Isopropanol $=90 / 10$, flow rate $=1.0 \mathrm{~mL} / \mathrm{min}, 230 \mathrm{~nm}$ ), $\mathrm{t}_{\text {minor }}=49.6 \mathrm{~min}, \mathrm{t}_{\mathrm{major}}=$ $64.7 \min .[\alpha]^{21.5}=-39.5\left(c 1.2, \mathrm{CHCl}_{3}\right)$ at $94.5: 5.5 \mathrm{er}$.

\section{Racemic sample $2 f$}

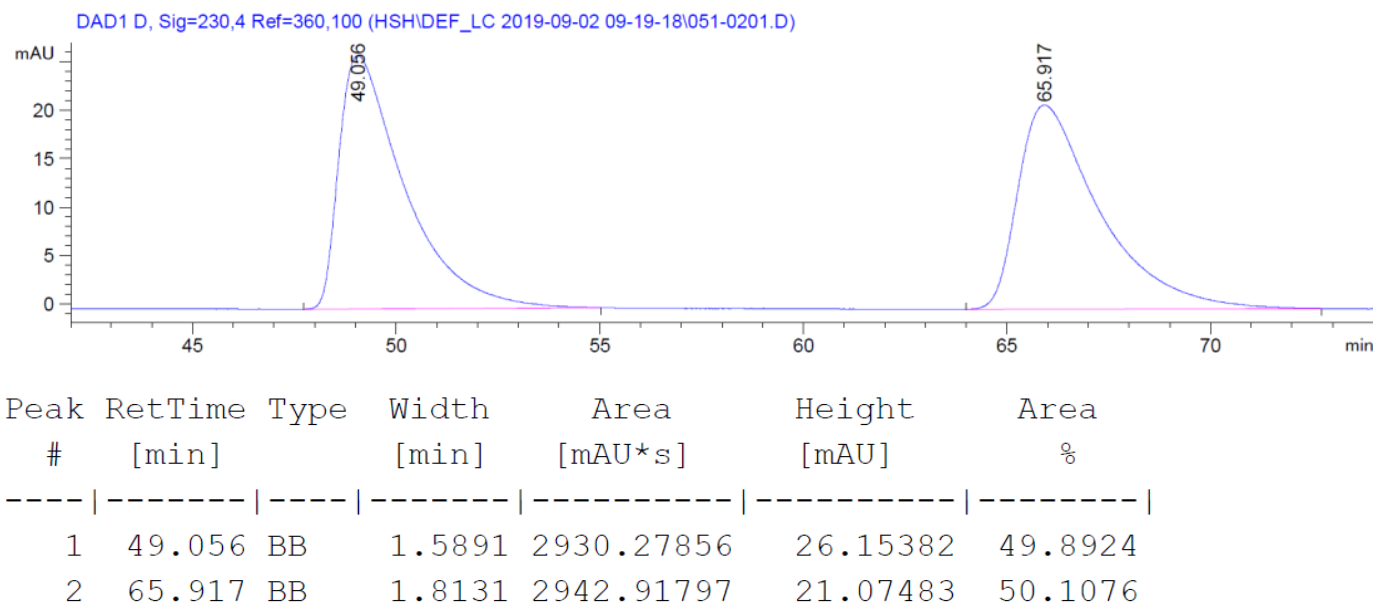

Enantiomeric sample $2 f$ 

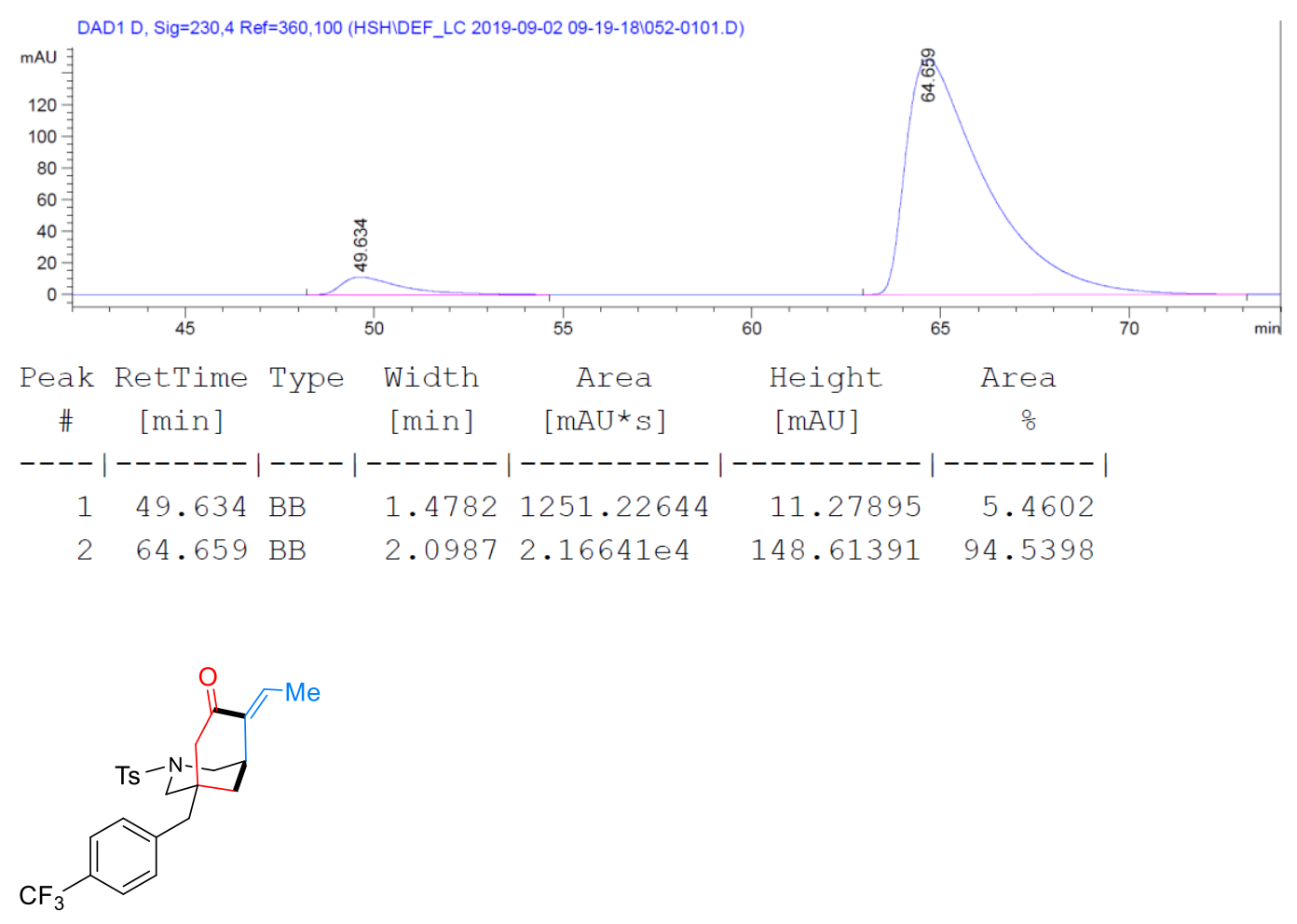

$2 \mathrm{~g}$

$2 \mathrm{~g}(37.4 \mathrm{mg})$ was isolated as a white solid in $78 \%$ yield.

$\mathrm{Rf}=0.3$, Hexane/ EtOAc 2:1.

Mp: $214.3-215.7^{\circ} \mathrm{C}$.

${ }^{1} \mathrm{H}$ NMR $\left(400 \mathrm{MHz}\right.$, Benzene- $\left.d_{6}\right) \delta 7.74(\mathrm{~d}, J=8.3 \mathrm{~Hz}, 2 \mathrm{H}), 7.29(\mathrm{~d}, J=8.0 \mathrm{~Hz}, 2 \mathrm{H}), 6.92(\mathrm{~d}, J=8.0 \mathrm{~Hz}, 2 \mathrm{H}), 6.72(\mathrm{q}, J=$ $7.2 \mathrm{~Hz}, 1 \mathrm{H}), 6.63(\mathrm{~d}, J=7.9 \mathrm{~Hz}, 2 \mathrm{H}), 3.57-3.43(\mathrm{~m}, 2 \mathrm{H}), 2.61(\mathrm{t}, J=2.9 \mathrm{~Hz}, 1 \mathrm{H}), 2.35(\mathrm{dd}, J=17.6,3.0 \mathrm{~Hz}, 1 \mathrm{H}), 2.21$ (dd, $J=11.0,2.1 \mathrm{~Hz}, 1 \mathrm{H}), 2.12(\mathrm{dd}, J=11.5,1.4 \mathrm{~Hz}, 1 \mathrm{H}), 2.00-1.84(\mathrm{~m}, 6 \mathrm{H}), 1.25(\mathrm{~d}, J=7.3 \mathrm{~Hz}, 3 \mathrm{H}), 1.10-1.00(\mathrm{~m}$, $1 \mathrm{H}), 0.87(\mathrm{dt}, J=13.1,3.2 \mathrm{~Hz}, 1 \mathrm{H})$.

${ }^{19}$ F NMR (376 MHz, $\left.\mathrm{C}_{6} \mathrm{D}_{6}\right) \delta-62.1$.

${ }^{13} \mathrm{C}$ NMR (101 MHz, C6 $\left.\mathrm{D}_{6}\right) \delta$ 195.2, 143.7, 140.3, 138.4, 134.3, 132.7, 131.0, 129.7, $129.0\left(\mathrm{q},{ }^{3} \mathrm{~J}_{\mathrm{C}-\mathrm{F}}=4.0 \mathrm{~Hz}\right), 128.5$, $125.2\left(q,{ }^{2} J_{C-F}=32.3 \mathrm{~Hz}\right), 125.0\left(q,{ }^{1} J_{C-F}=272.7 \mathrm{~Hz}\right), 56.6,51.8,48.9,45.5,36.1,34.8,31.1,21.1,12.7$.

HRMS ESI Calcd for $\mathrm{C}_{25} \mathrm{H}_{27} \mathrm{~F}_{3} \mathrm{NO}_{3} \mathrm{~S}[\mathrm{M}+\mathrm{H}]^{+}: 478.1658$, Found: 478.1657.

IR (KBr): 2930, 2854, 1689, 1619, 1598, 1449, 1325, 1166, 1123, 1066, $1020 \mathrm{~cm}^{-1}$.

Chiral HPLC (Chiralpak IF, Hexane/Isopropanol $=60 / 40$, flow rate $=1.0 \mathrm{~mL} / \mathrm{min}, 230 \mathrm{~nm}$ ), $\mathrm{t}_{\text {minor }}=17.4 \mathrm{~min}, \mathrm{t}_{\mathrm{major}}=$ $27.6 \min .[\alpha]^{21.5}=-56.7\left(c 1.7, \mathrm{CHCl}_{3}\right)$ at 95.5:4.5 er.

\section{Racemic sample $\mathbf{2 g}$}




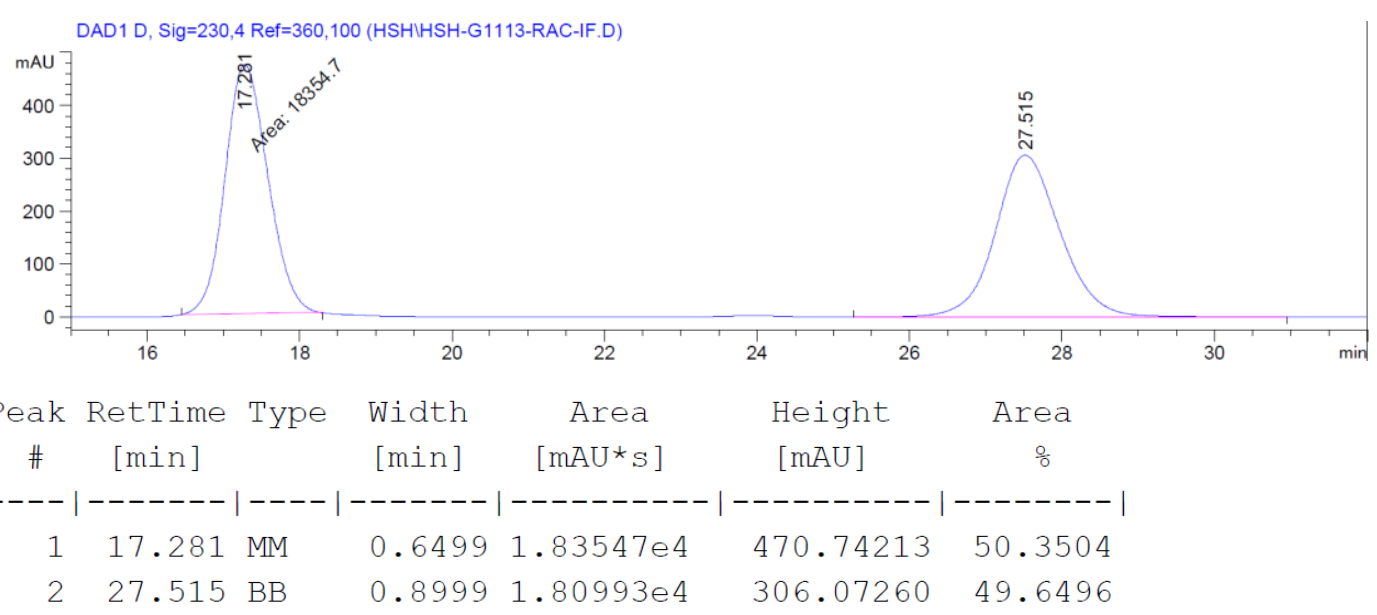

\section{Enantiomeric sample 2g}

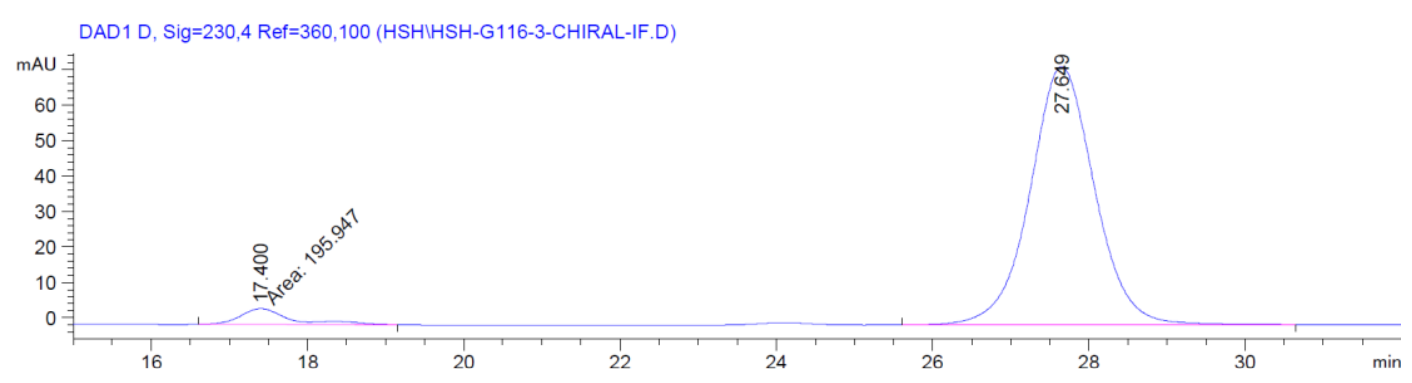

\begin{tabular}{|c|c|c|c|c|c|c|}
\hline $\begin{array}{c}\text { Peak } \\
\#\end{array}$ & $\begin{array}{c}\text { RetTime } \\
\text { [min] }\end{array}$ & Type & $\begin{array}{l}\text { Width } \\
\text { [min] }\end{array}$ & $\begin{array}{c}\text { Area } \\
{\left[\mathrm{mAU}^{\star} \mathrm{s}\right]}\end{array}$ & $\begin{array}{c}\text { Height } \\
{[\mathrm{mAU}]}\end{array}$ & $\begin{array}{c}\text { Area } \\
\frac{\circ}{0}\end{array}$ \\
\hline-- & & & -- & ------ & ------- & ---- \\
\hline 1 & 17.400 & MM & 0.7359 & 195.94733 & 4.43808 & 4.4694 \\
\hline 2 & 27.649 & $\mathrm{BB}$ & 0.8725 & 4188.22705 & 72.87016 & 95.5306 \\
\hline
\end{tabular}

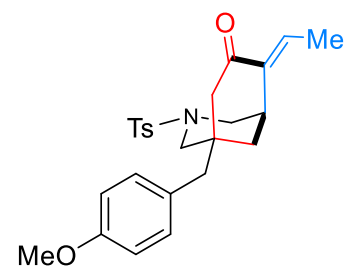

2h

$2 \mathrm{~h}(35 \mathrm{mg})$ was isolated as a foam in $80 \%$ yield.

$\mathrm{Rf}=0.4$, Hexane/ EtOAc 2:1.

${ }^{1} \mathrm{H}$ NMR (400 MHz, Benzene- $\left.d_{6}\right) \delta 7.78-7.70(\mathrm{~m}, 2 \mathrm{H}), 6.92-6.83(\mathrm{~m}, 2 \mathrm{H}), 6.77-6.66(\mathrm{~m}, 5 \mathrm{H}), 3.60-3.46(\mathrm{~m}, 2 \mathrm{H})$, $3.33(\mathrm{~s}, 3 \mathrm{H}), 2.63-2.55(\mathrm{~m}, 1 \mathrm{H}), 2.40(\mathrm{dd}, J=17.6,3.0 \mathrm{~Hz}, 1 \mathrm{H}), 2.29-2.19(\mathrm{~m}, 2 \mathrm{H}), 2.09(\mathrm{dd}, J=17.7,1.4 \mathrm{~Hz}, 1 \mathrm{H})$, $2.03(\mathrm{~d}, J=13.5 \mathrm{~Hz}, 1 \mathrm{H}), 1.97(\mathrm{~d}, J=13.4 \mathrm{~Hz}, 1 \mathrm{H}), 1.89(\mathrm{~s}, 3 \mathrm{H}), 1.25-1.16(\mathrm{~m}, 4 \mathrm{H}), 0.97(\mathrm{dt}, J=13.1,3.2 \mathrm{~Hz}, 1 \mathrm{H})$.

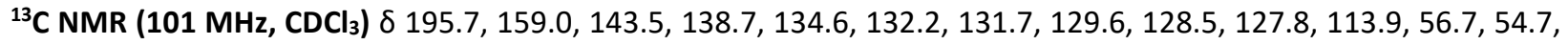
$51.8,49.1,45.3,36.2,35.0,31.2,21.1,12.7$. 
HRMS ESI Calcd for $\mathrm{C}_{25} \mathrm{H}_{30} \mathrm{NO}_{4} \mathrm{~S}[\mathrm{M}+\mathrm{H}]^{+}:$440.1890, Found: 440.1887.

IR (KBr): 2931, 2849, 1687, 1624, 1512, 1339, 1248, 1166, 1137, $1034 \mathrm{~cm}^{-1}$.

Chiral HPLC (Chiralpak IF, Hexane/Isopropanol $=60 / 40$, flow rate $=1.0 \mathrm{~mL} / \mathrm{min}, 230 \mathrm{~nm}$ ) , $\mathrm{t}_{\text {minor }}=23.3 \mathrm{~min}, \mathrm{t}_{\mathrm{major}}=$ $36.3 \min .[\alpha]^{21.5}=-50.3\left(c 1.5, \mathrm{CHCl}_{3}\right)$ at $94.5: 5.5 \mathrm{er}$.

\section{Racemic sample $\mathbf{2 h}$}

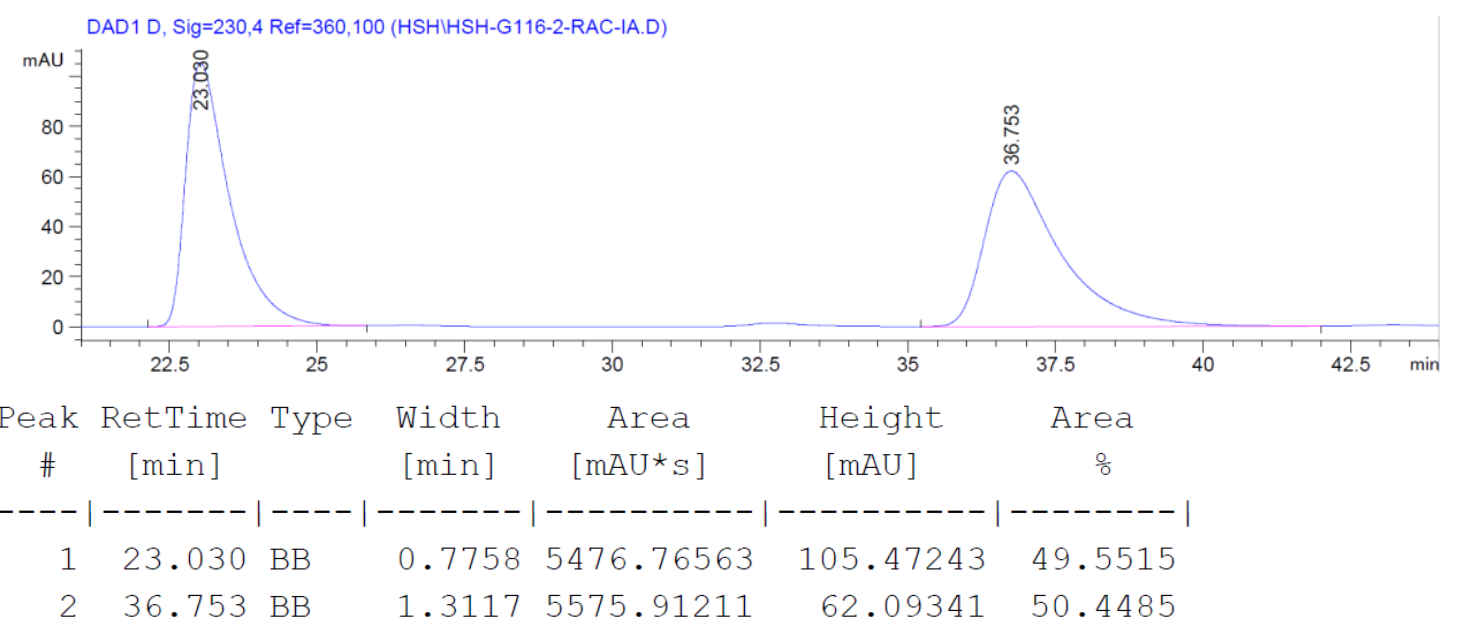

\section{Enantiomeric sample $\mathbf{2 h}$}
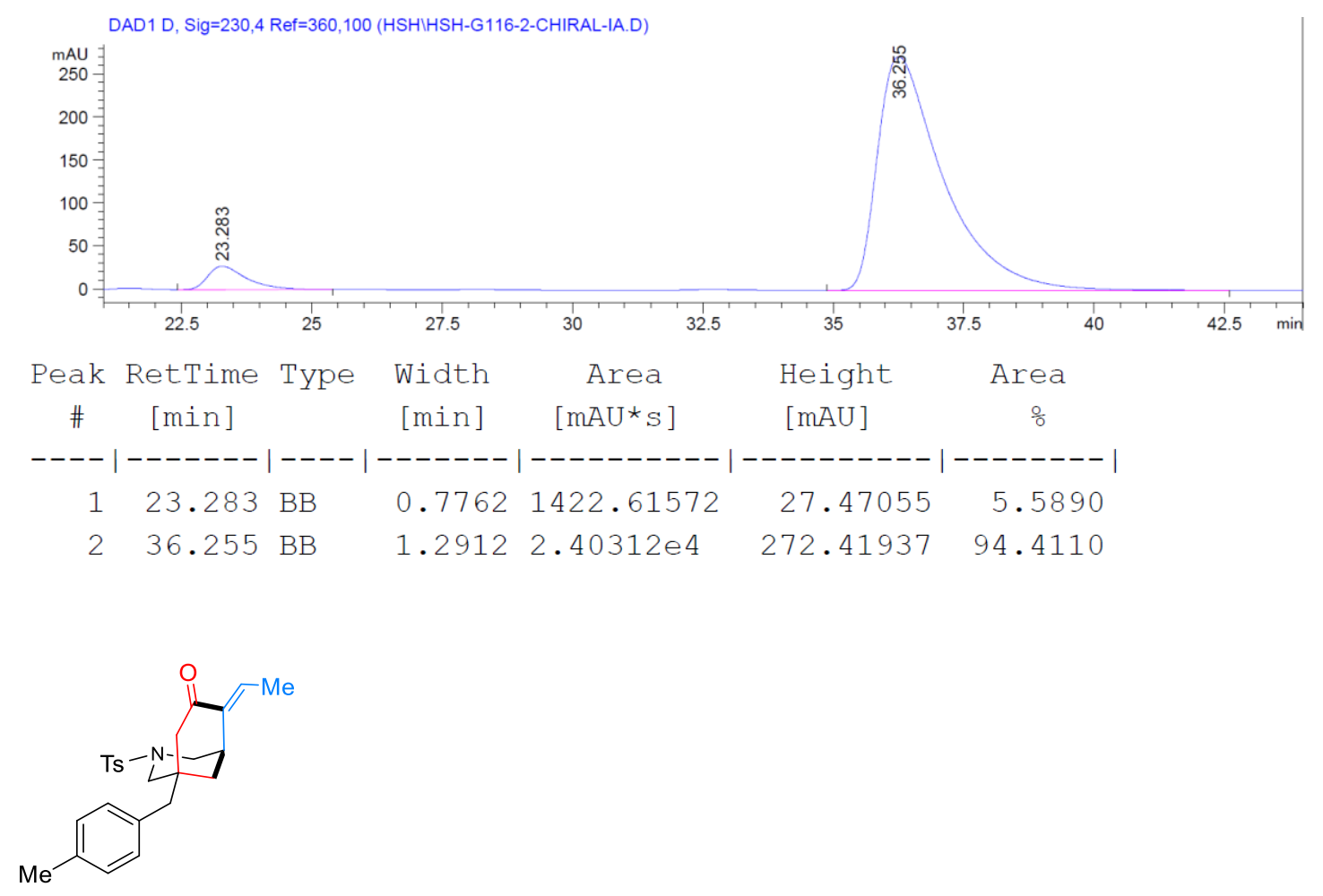

2i 
$2 \mathbf{i}$ (30 mg) was isolated as a white solid in $71 \%$ yield.

Rf $=0.26$, Hexane/ EtOAc 2:1.

Mp: $193.5-195.1^{\circ} \mathrm{C}$.

${ }^{1} \mathrm{H}$ NMR (400 MHz, Benzene-d $\left.)\right) \delta 7.74(\mathrm{~d}, J=8.3 \mathrm{~Hz}, 2 \mathrm{H}), 6.92(\mathrm{~d}, J=7.7 \mathrm{~Hz}, 2 \mathrm{H}), 6.89(\mathrm{~d}, J=8.0 \mathrm{~Hz}, 2 \mathrm{H}), 6.76-6.60$ $(\mathrm{m}, 3 \mathrm{H}), 3.59-3.46(\mathrm{~m}, 2 \mathrm{H}), 2.58(\mathrm{q}, J=2.9 \mathrm{~Hz}, 1 \mathrm{H}), 2.40(\mathrm{dd}, J=17.7,3.0 \mathrm{~Hz}, 1 \mathrm{H}), 2.23(\mathrm{dt}, J=10.8,1.8 \mathrm{~Hz}, 2 \mathrm{H}), 2.13$ $-2.01(\mathrm{~m}, 5 \mathrm{H}), 1.98(\mathrm{~d}, J=13.3 \mathrm{~Hz}, 1 \mathrm{H}), 1.90(\mathrm{~s}, 3 \mathrm{H}), 1.25-1.14(\mathrm{~m}, 4 \mathrm{H}), 0.98(\mathrm{dt}, J=13.1,3.2 \mathrm{~Hz}, 1 \mathrm{H})$.

${ }^{13}$ C NMR (101 MHz, C6 6 ) $\delta$ 195.6, 143.4, 138.7, 136.2, 134.7, 133.0, 132.2, 130.7, 129.6, 129.0, 128.5, 56.7, 51.8, $49.2,45.7,36.2,35.1,31.2,21.1,21.0,12.6$.

HRMS ESI Calcd for $\mathrm{C}_{25} \mathrm{H}_{30} \mathrm{NO}_{3} \mathrm{~S}[\mathrm{M}+\mathrm{H}]^{+}:$424.1941, Found: 424.1939.

IR (KBr): 3021, 2924, 2852, 1688, 1625, 1514, 1448, 1339, 1166, $1090 \mathrm{~cm}^{-1}$.

Chiral HPLC (Chiralpak IA, Hexane/Isopropanol $=50 / 50$, flow rate $=1.0 \mathrm{~mL} / \mathrm{min}, 230 \mathrm{~nm}$ ) , $\mathrm{t}_{\text {minor }}=15.9 \mathrm{~min}, \mathrm{t}_{\mathrm{major}}=$ $21.2 \min .[\alpha]^{21.5}=-67.9\left(c 1.5, \mathrm{CHCl}_{3}\right)$ at $96: 4$ er.

\section{Racemic sample $\mathbf{2 i}$}

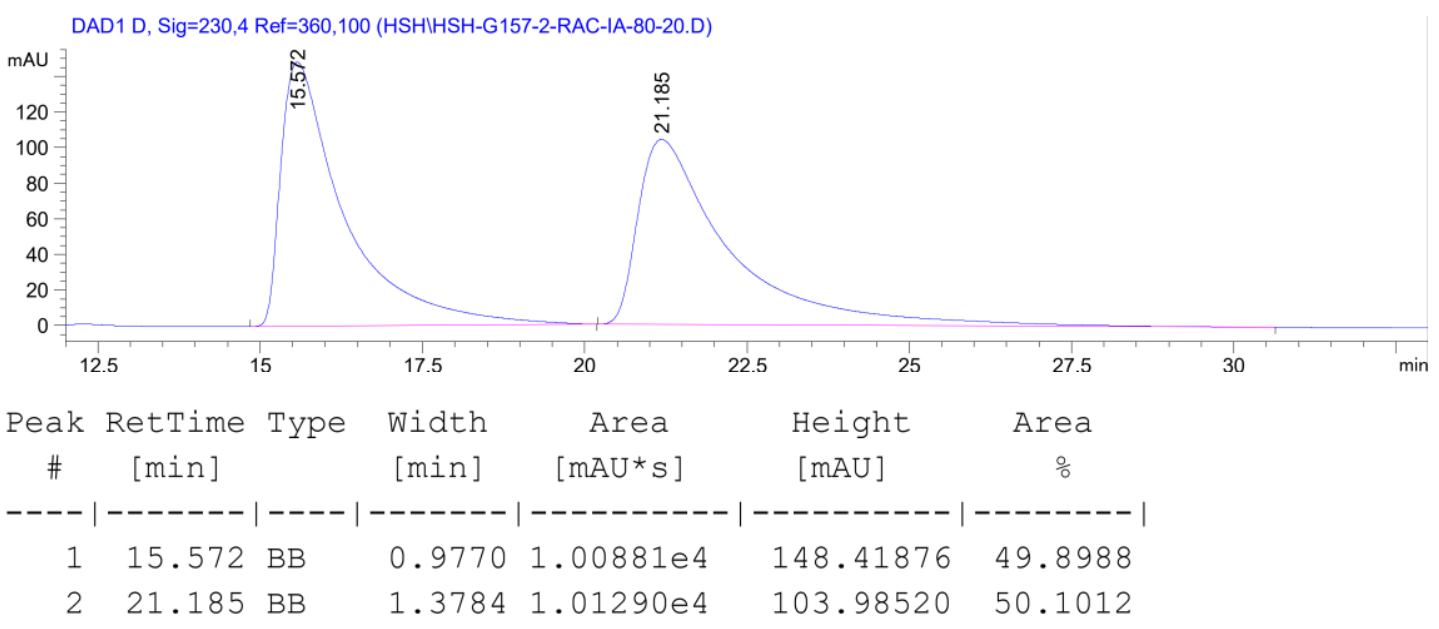

Enantiomeric sample $\mathbf{2 i}$

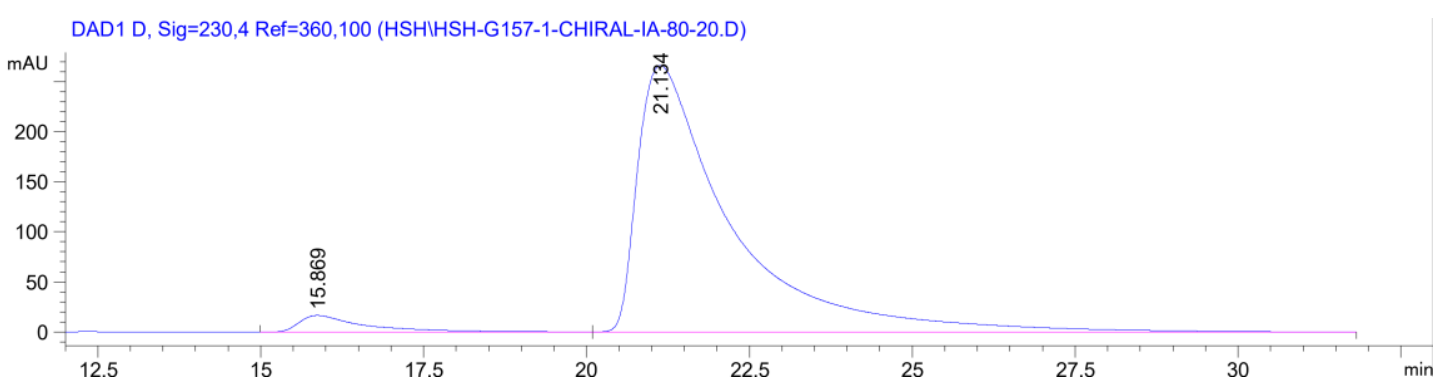




\begin{tabular}{|c|c|c|c|c|c|c|}
\hline $\begin{array}{c}\text { Peak } \\
\quad \#\end{array}$ & $\begin{array}{c}\text { RetTime } \\
\text { [min] }\end{array}$ & Type & $\begin{array}{l}\text { Width } \\
\text { [min] }\end{array}$ & $\begin{array}{c}\text { Area } \\
{\left[\mathrm{mAU}{ }^{*} \mathrm{~S}\right]}\end{array}$ & $\begin{array}{l}\text { Height } \\
{[\mathrm{mAU}]}\end{array}$ & $\begin{array}{c}\text { Area } \\
\quad \circ\end{array}$ \\
\hline & & & & $|----------|$ & $----------\mid$ & $--------\mid$ \\
\hline 1 & 15.869 & $\mathrm{BB}$ & 0.9674 & 1159.29712 & 16.62881 & 4.1949 \\
\hline 2 & 21.134 & BBA & 1.3982 & $2.64768 e 4$ & 266.24255 & 95.8051 \\
\hline
\end{tabular}

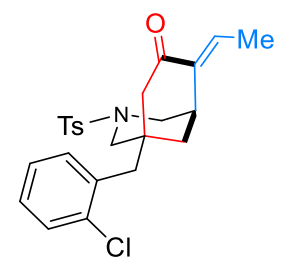

2j

$2 \mathbf{j}$ (32.6 mg) was isolated as a white solid in $74 \%$ yield.

$\mathrm{Rf}=0.4$, Hexane/ EtOAc 2:1.

Mp: $185.3-186.3^{\circ} \mathrm{C}$

${ }^{1} \mathrm{H}$ NMR $\left(400 \mathrm{MHz}\right.$, Benzene- $\left.d_{6}\right) \delta 7.76-7.71(\mathrm{~m}, 2 \mathrm{H}), 7.13(\mathrm{dd}, J=7.9,1.5 \mathrm{~Hz}, 1 \mathrm{H}), 6.86(\mathrm{~d}, J=8.1 \mathrm{~Hz}, 2 \mathrm{H}), 6.78(\mathrm{td}$, $J=7.4,1.5 \mathrm{~Hz}, 1 \mathrm{H}), 6.74-6.66(\mathrm{~m}, 2 \mathrm{H}), 6.64(\mathrm{dd}, J=7.5,1.9 \mathrm{~Hz}, 1 \mathrm{H}), 3.65(\mathrm{dt}, J=11.3,2.1 \mathrm{~Hz}, 1 \mathrm{H}), 3.47(\mathrm{ddt}, J=10.8$, 3.3, $1.7 \mathrm{~Hz}, 1 \mathrm{H}), 2.56-2.47(\mathrm{~m}, 2 \mathrm{H}), 2.39-2.32(\mathrm{~m}, 2 \mathrm{H}), 2.25(\mathrm{~d}, J=13.5 \mathrm{~Hz}, 1 \mathrm{H}), 2.19-2.08(\mathrm{~m}, 2 \mathrm{H}), 1.88(\mathrm{~s}, 3 \mathrm{H})$, $1.28(\mathrm{ddt}, J=13.1,3.5,1.8 \mathrm{~Hz}, 1 \mathrm{H}), 1.19$ (d, $J=7.3 \mathrm{~Hz}, 4 \mathrm{H})$.

${ }^{13}$ C NMR (101 MHz, C6 $\left.\mathrm{D}_{6}\right) \delta 195.1,143.4,138.6,135.3,134.6,134.4,133.0,132.3,130.0,129.6,128.5,128.4,126.5$, $56.5,51.8,49.5,42.5,37.5,35.2,31.1,21.1,12.6$.

HRMS ESI Calcd for $\mathrm{C}_{24} \mathrm{H}_{27} \mathrm{CINO}_{3} \mathrm{~S}[\mathrm{M}+\mathrm{H}]^{+}:$444.1395, Found: 444.1398.

IR (KBr): 2925, 2850, 1687, 1624, 1467, 1444, 1340, 1122, $1037 \mathrm{~cm}^{-1}$.

Chiral HPLC (Chiralpak IF, Hexane/Isopropanol $=50 / 50$, flow rate $=1.0 \mathrm{~mL} / \mathrm{min}, 230 \mathrm{~nm}$ ) , $\mathrm{t}_{\text {minor }}=19.4 \mathrm{~min}, \mathrm{t}_{\mathrm{major}}=$ $47.8 \min .[\alpha]^{21.5}=-53.3\left(c 0.6, \mathrm{CHCl}_{3}\right)$ at $99: 1 \mathrm{er}$.

\section{Racemic sample $\mathbf{2} \mathbf{j}$}

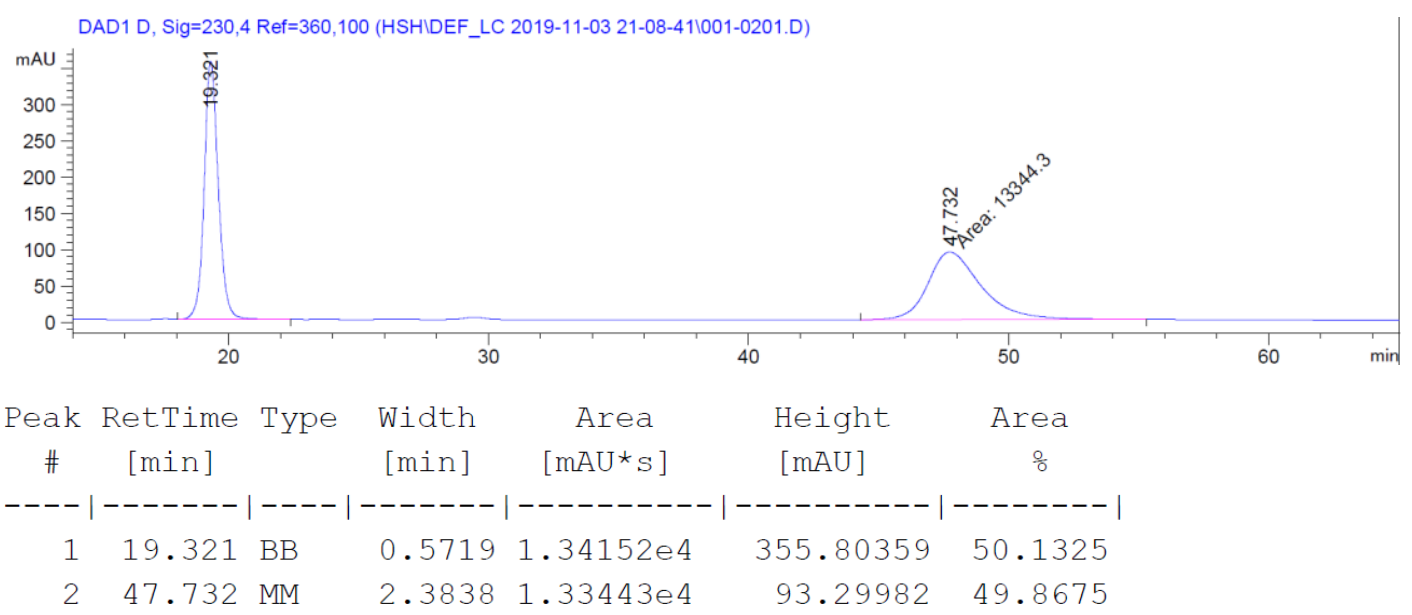




\section{Enantiomeric sample 2j}

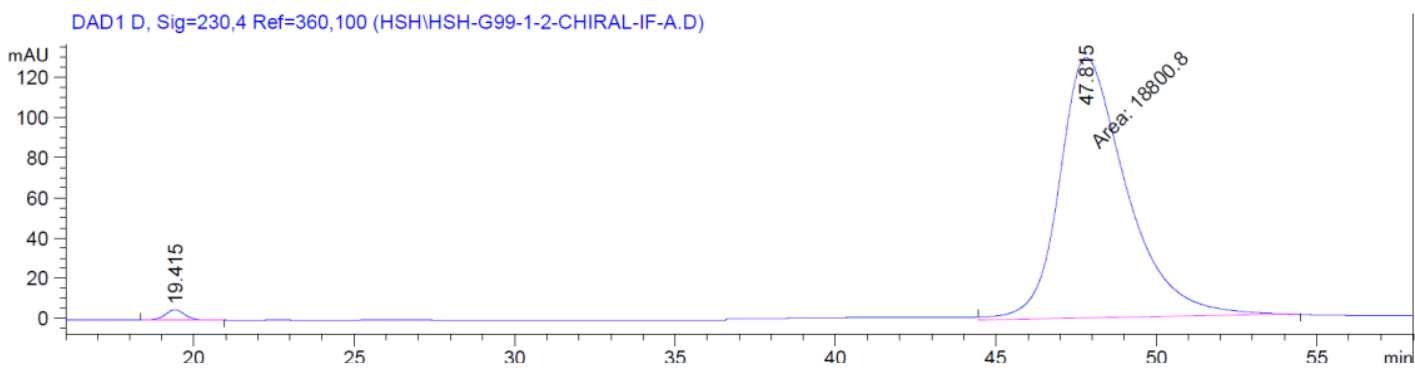

\begin{tabular}{|c|c|c|c|c|c|c|}
\hline $\begin{array}{c}\text { Peak } \\
\quad \#\end{array}$ & $\begin{array}{c}\text { RetTime } \\
\text { [min] }\end{array}$ & Type & $\begin{array}{l}\text { Width } \\
\text { [min] }\end{array}$ & $\begin{array}{c}\text { Area } \\
{\left[\mathrm{mAU}^{\star} \mathrm{s}\right]}\end{array}$ & $\begin{array}{c}\text { Height } \\
{[\mathrm{mAU}]}\end{array}$ & $\begin{array}{c}\text { Area } \\
\frac{\circ}{0}\end{array}$ \\
\hline 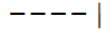 & ----- & & - & ---------- & ----ー---ー- & 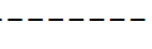 \\
\hline 1 & 19.415 & $\mathrm{BB}$ & 0.6439 & 219.39745 & 5.14056 & 1.1535 \\
\hline 2 & 47.815 & MM & 2.4216 & $1.88008 \mathrm{e} 4$ & 129.39648 & 98.8465 \\
\hline
\end{tabular}

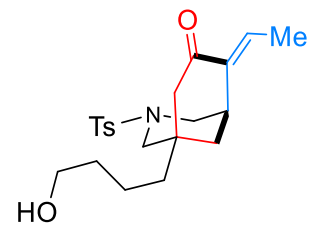

$2 \mathbf{k}$

$2 \mathbf{k}(22 \mathrm{mg})$ was isolated as a foam in $56 \%$ yield.

$\mathrm{Rf}=0.15$, Hexane/ EtOAc 1:3.

${ }^{1} \mathrm{H}$ NMR $\left(500 \mathrm{MHz}\right.$, Benzene- $\left.d_{6}\right) \delta 7.77-7.71(\mathrm{~m}, 2 \mathrm{H}), 6.88(\mathrm{~d}, J=8.0 \mathrm{~Hz}, 2 \mathrm{H}), 6.83(\mathrm{q}, J=7.3 \mathrm{~Hz}, 1 \mathrm{H}), 3.62-3.52(\mathrm{~m}$, $2 \mathrm{H}), 3.37(\mathrm{t}, J=6.3 \mathrm{~Hz}, 2 \mathrm{H}), 2.62(\mathrm{t}, J=3.0 \mathrm{~Hz}, 1 \mathrm{H}), 2.48(\mathrm{dd}, J=17.9,3.0 \mathrm{~Hz}, 1 \mathrm{H}), 2.24(\mathrm{dd}, J=10.7,2.2 \mathrm{~Hz}, 1 \mathrm{H}), 2.10$ (dd, $J=11.2,1.3 \mathrm{~Hz}, 1 \mathrm{H}), 1.97(\mathrm{dd}, J=17.9,1.3 \mathrm{~Hz}, 1 \mathrm{H}), 1.89(\mathrm{~s}, 3 \mathrm{H}), 1.30(\mathrm{~d}, J=7.2 \mathrm{~Hz}, 3 \mathrm{H}), 1.27-1.20(\mathrm{~m}, 2 \mathrm{H}), 1.20$ $-1.14(\mathrm{~m}, 1 \mathrm{H}), 1.04-0.95(\mathrm{~m}, 2 \mathrm{H}), 0.87(\mathrm{dt}, J=13.2,3.1 \mathrm{~Hz}, 1 \mathrm{H}), 0.82-0.76(\mathrm{~m}, 2 \mathrm{H})$.

${ }^{13}$ C NMR (101 MHz, C6 $\left.D_{6}\right) \delta$ 196.1, 143.4, 138.8, 134.6, 132.6, 129.7, 128.4, 62.2, 56.5, 52.0, 49.2, 40.4, 35.8, 35.0, $33.5,31.2,21.1,19.1,12.7$.

HRMS ESI Calcd for $\mathrm{C}_{21} \mathrm{H}_{30} \mathrm{NO}_{4} \mathrm{~S}[\mathrm{M}+\mathrm{H}]^{+}: 392.1890$, Found: 392.1888.

IR (KBr): 3436, 2937, 2860,1687, 1623, 1598, 1494, 1448, 1339, 1166, 1090, $1059 \mathrm{~cm}^{-1}$.

Chiral HPLC (Chiralpak IA, Hexane/Isopropanol $=90 / 10$, flow rate $=1.0 \mathrm{~mL} / \mathrm{min}, 230 \mathrm{~nm}$ ) , $\mathrm{t}_{\text {minor }}=28.1 \mathrm{~min}, \mathrm{t}_{\mathrm{major}}=$ $39 \min .[\alpha]^{21.5}=-61.3\left(c 0.6, \mathrm{CHCl}_{3}\right)$ at 92.5:7.5 er.

\section{Racemic sample $2 \mathbf{k}$}




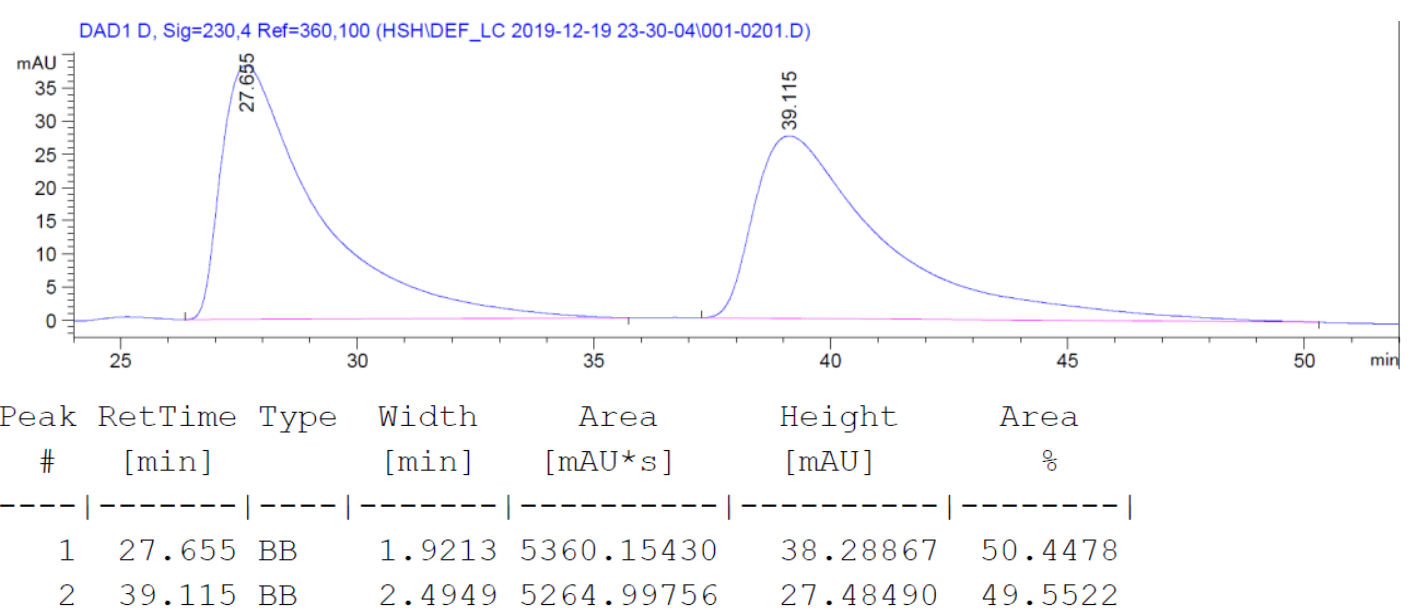

\section{Enantiomeric sample 2k}
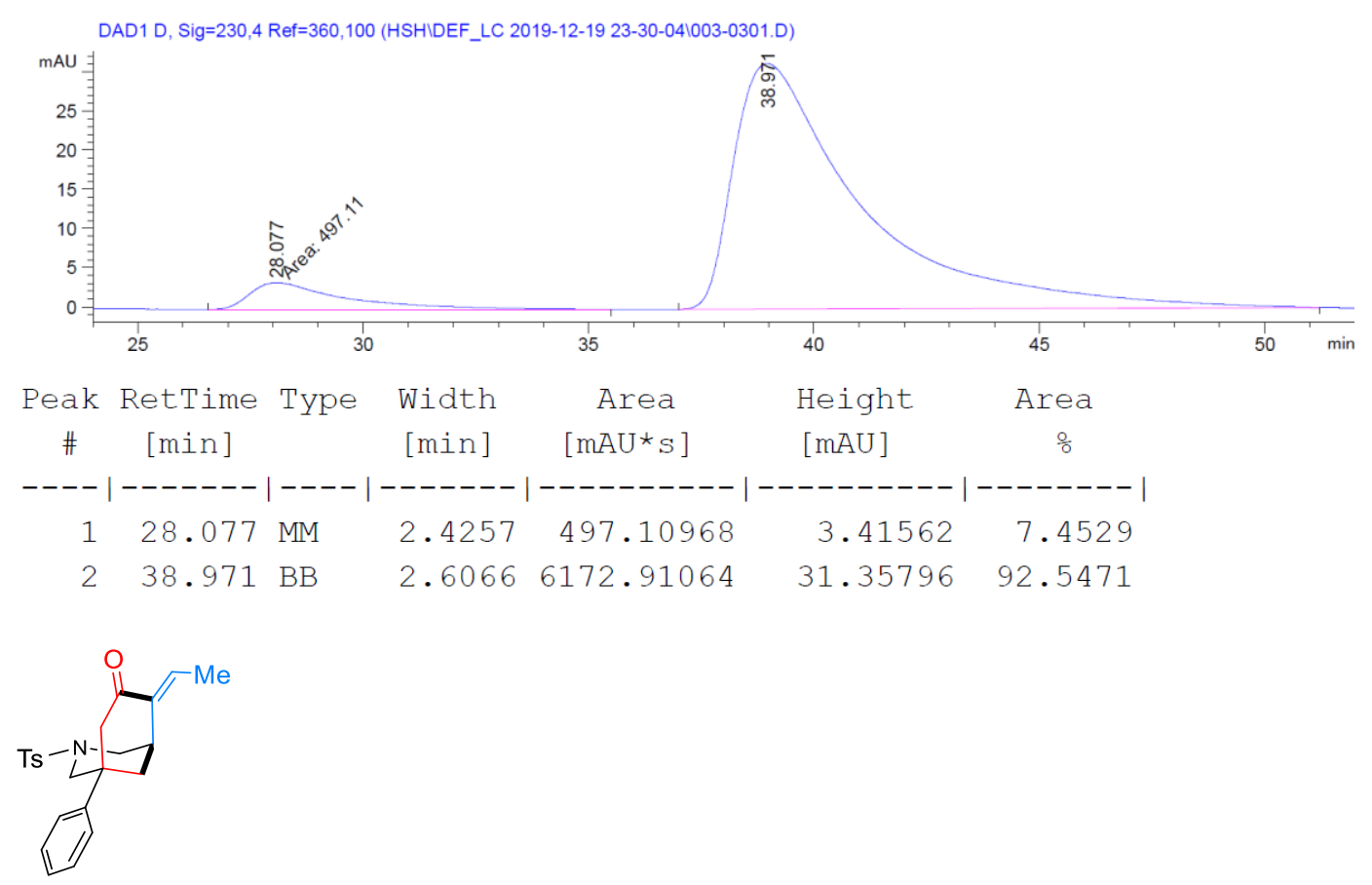

2)

2l (11.9 mg) was isolated as a foam in $30 \%$ yield.

$\mathrm{Rf}=0.25$, Hexane/ EtOAc 2:1.

${ }^{1} \mathrm{H}$ NMR $\left(400 \mathrm{MHz}\right.$, Benzene- $\left.d_{6}\right) \delta 7.67-7.60(\mathrm{~m}, 2 \mathrm{H}), 7.11-6.99(\mathrm{~m}, 3 \mathrm{H}), 6.90-6.81(\mathrm{~m}, 3 \mathrm{H}), 6.77(\mathrm{~d}, J=8.1 \mathrm{~Hz}$, 2H), $3.95(\mathrm{dt}, J=11.4,2.0 \mathrm{~Hz}, 1 \mathrm{H}$ ), $3.61(\mathrm{ddt}, J=10.8,3.2,1.7 \mathrm{~Hz}, 1 \mathrm{H}), 3.05(\mathrm{dd}, J=17.8,2.9 \mathrm{~Hz}, 1 \mathrm{H}), 2.69(\mathrm{p}, J=3.0$ $\mathrm{Hz}, 1 \mathrm{H}), 2.34(\mathrm{dd}, J=11.4,1.3 \mathrm{~Hz}, 1 \mathrm{H}), 2.25(\mathrm{dd}, J=6.0,1.8 \mathrm{~Hz}, 1 \mathrm{H}), 2.24-2.18(\mathrm{~m}, 1 \mathrm{H}), 1.86(\mathrm{~s}, 3 \mathrm{H}), 1.53(\mathrm{ddt}, J=$ $12.9,3.5,1.8 \mathrm{~Hz}, 1 \mathrm{H}), 1.46(\mathrm{dt}, J=13.0,3.2 \mathrm{~Hz}, 1 \mathrm{H}), 1.29(\mathrm{~d}, J=7.3 \mathrm{~Hz}, 3 \mathrm{H})$.

${ }^{13} \mathrm{C}$ NMR $\left(101 \mathrm{MHz}, \mathrm{C}_{6} \mathrm{D}_{6}\right) \delta$ 194.5, 145.3, 143.3, 138.2, 134.5, 132.9, 129.6, 128.9, 127.1, 125.1, 57.6, 51.7, 51.1, 38.9, 34.6, 31.1, 21.1, 12.7.

HRMS ESI Calcd for $\mathrm{C}_{23} \mathrm{H}_{26} \mathrm{NO}_{3} \mathrm{~S}[\mathrm{M}+\mathrm{H}]^{+}$: 396.1628, Found: 396.1626. 
IR (KBr): 2928, 2853, 1736, 1689, 1627, 1598, 1497, 1446, 1341, 1233, 1166, 1091, $1027 \mathrm{~cm}^{-1}$.

Chiral HPLC (Chiralpak IA, Hexane/Isopropanol $=90 / 10$, flow rate $=1.0 \mathrm{~mL} / \mathrm{min}, 230 \mathrm{~nm}$ ) , $\mathrm{t}_{\text {minor }}=45 \mathrm{~min}, \mathrm{t}_{\text {major }}=54.1$ $\min .[\alpha]^{21.5} \mathrm{D}=-58.9\left(c 1.1, \mathrm{CHCl}_{3}\right)$ at 99:1 er.

\section{Racemic sample 21}

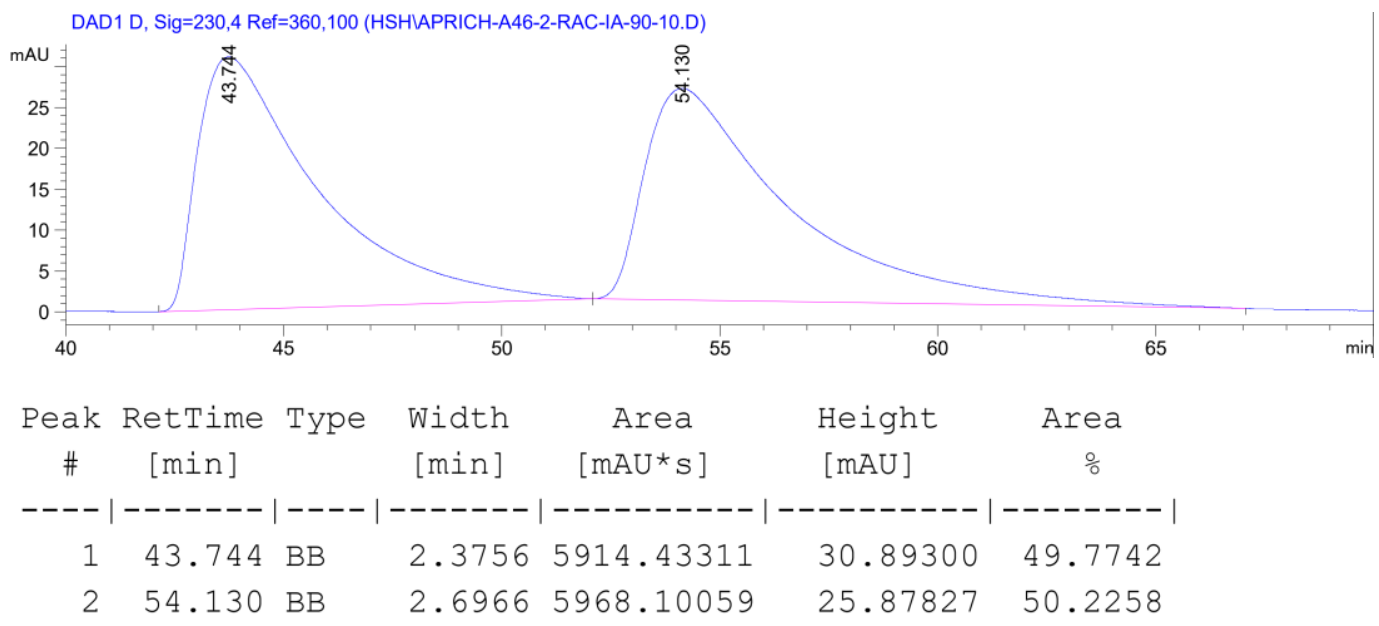

\section{Enantiomeric sample 21}
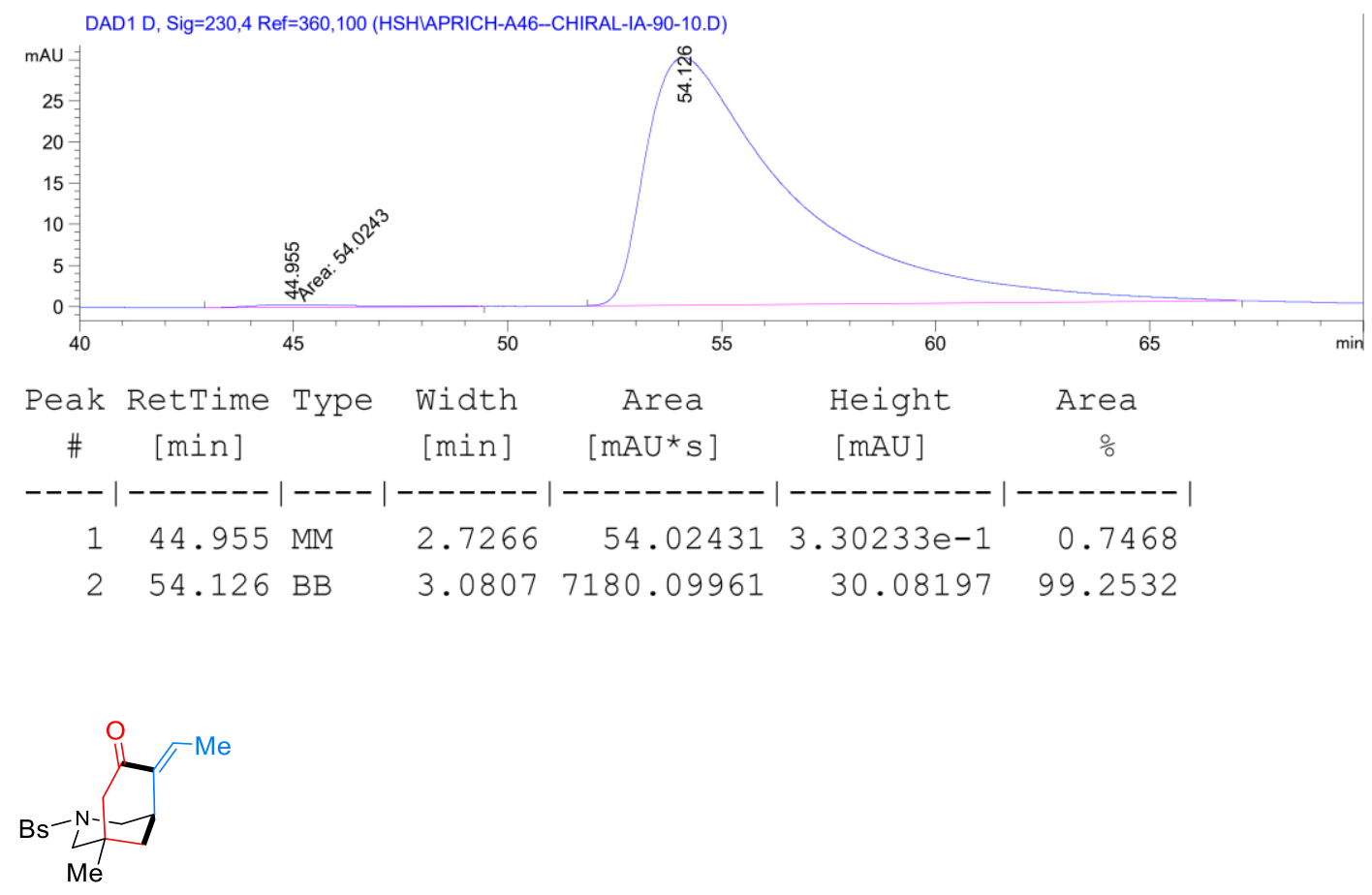

$2 \mathrm{~m}$

$2 \mathrm{~m}(20 \mathrm{mg})$ was isolated as a foam in $63 \%$ yield.

$\mathrm{Rf}=0.3$, Hexane/ EtOAc 2:1. 
${ }^{1} \mathrm{H}$ NMR (400 MHz, Benzene-d $\left.d_{6}\right) \delta 77-7.69(\mathrm{~m}, 2 \mathrm{H}), 7.06-6.95(\mathrm{~m}, 3 \mathrm{H}), 6.79(\mathrm{q}, J=7.3 \mathrm{~Hz}, 1 \mathrm{H}), 3.47(\mathrm{ddd}, J=10.8$, 3.1, $1.7 \mathrm{~Hz}, 1 \mathrm{H}), 3.38(\mathrm{dt}, J=11.3,2.1 \mathrm{~Hz}, 1 \mathrm{H}), 2.55(\mathrm{t}, J=3.0 \mathrm{~Hz}, 1 \mathrm{H}), 2.38(\mathrm{dd}, J=17.9,3.0 \mathrm{~Hz}, 1 \mathrm{H}), 2.12(\mathrm{dd}, J=10.9$, $2.2 \mathrm{~Hz}, 1 \mathrm{H}), 1.95(\mathrm{dd}, J=11.3,1.4 \mathrm{~Hz}, 1 \mathrm{H}), 1.81(\mathrm{dd}, J=17.9,1.4 \mathrm{~Hz}, 1 \mathrm{H}), 1.25(\mathrm{~d}, J=7.3 \mathrm{~Hz}, 3 \mathrm{H}), 1.08(\mathrm{ddt}, J=13.1$, 3.7, $2.0 \mathrm{~Hz}, 1 \mathrm{H}), 0.79(\mathrm{dt}, J=13.1,3.1 \mathrm{~Hz}, 1 \mathrm{H}), 0.39(\mathrm{~s}, 3 \mathrm{H})$.

${ }^{13} \mathrm{C}$ NMR (101 MHz, C6 $\left.\mathrm{D}_{6}\right) \delta$ 195.3, 138.3, 137.6, 132.7, 132.5, 128.9, 57.2, 51.6, 51.3, 37.3, 32.1, 31.3, 26.7, 12.7. HRMS ESI Calcd for $\mathrm{C}_{17} \mathrm{H}_{22} \mathrm{NO}_{3} \mathrm{~S}[\mathrm{M}+\mathrm{H}]^{+}: 320.1315$, Found: 320.1319.

IR (KBr): 2927, 2854, 1688, 1623, 1446, 1341, 1248, 1172, $1029 \mathrm{~cm}^{-1}$.

Chiral HPLC (Chiralpak IF, Hexane/Isopropanol $=70 / 30$, flow rate $=1.0 \mathrm{~mL} / \mathrm{min}, 230 \mathrm{~nm}$ ), $t_{\text {minor }}=24.8 \mathrm{~min}, \mathrm{t}_{\mathrm{major}}=$ $34.8 \min .[\alpha]^{21.5}=-57.3\left(c 0.8, \mathrm{CHCl}_{3}\right)$ at $95: 5 \mathrm{er}$.

\section{Racemic sample $2 \mathrm{~m}$}

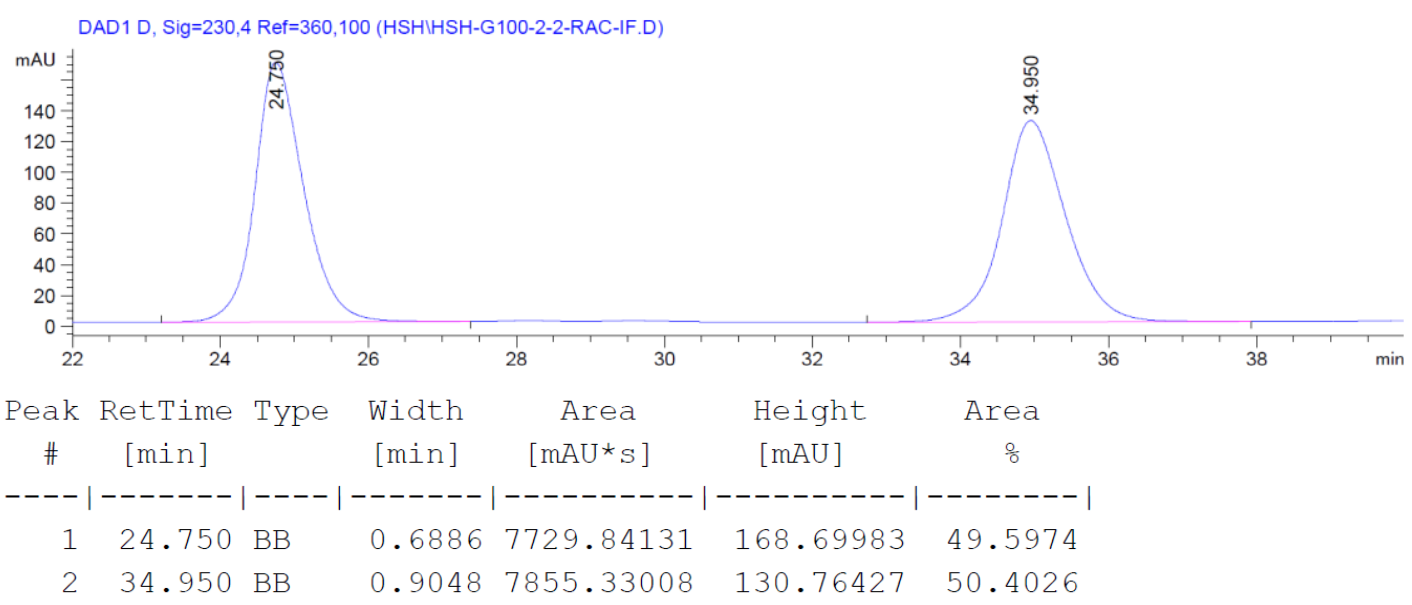

\section{Enantiomeric sample 2m}

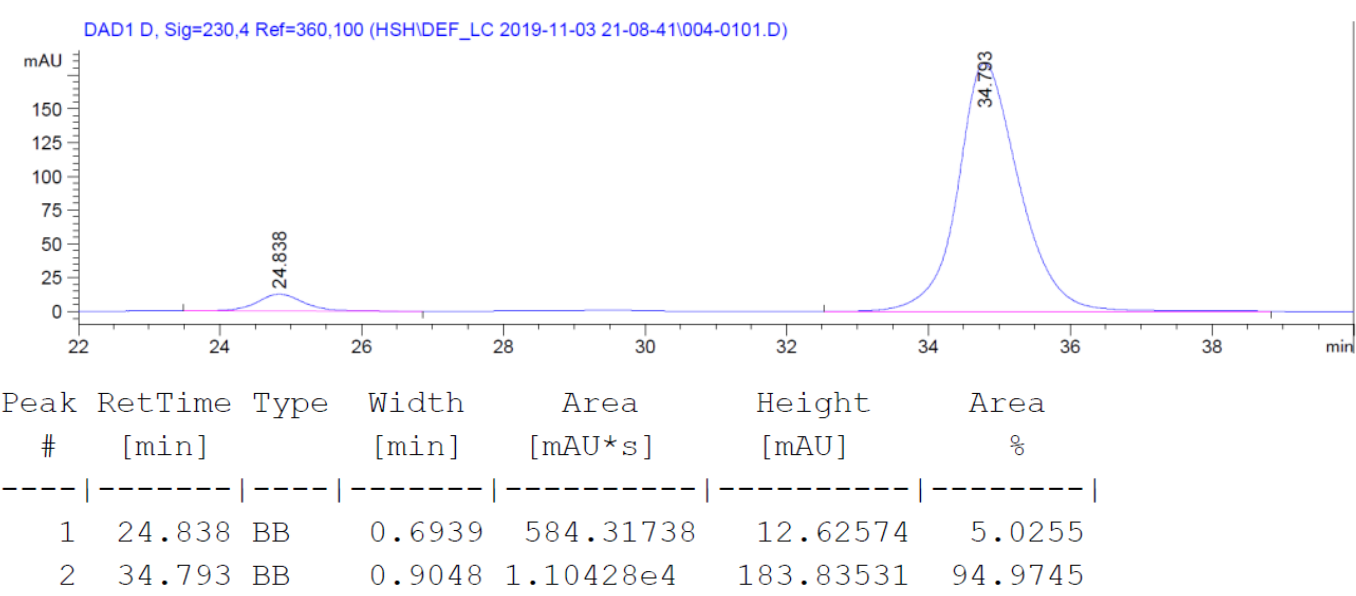




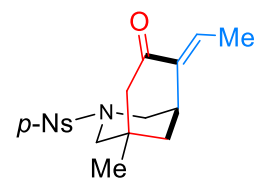

2n

2n (19.5 mg) was isolated as a white solid in $53 \%$ yield.

$\mathrm{Rf}=0.1$, Hexane/ EtOAc 2:1.

Mp: n.a. (decomposed at $158^{\circ} \mathrm{C}$ )

${ }^{1} \mathrm{H}$ NMR (400 MHz, Benzene-d $\left.\boldsymbol{d}_{6}\right) \delta 7.72-7.65(\mathrm{~m}, 2 \mathrm{H}), 7.43-7.36(\mathrm{~m}, 2 \mathrm{H}), 6.70(\mathrm{q}, J=7.3 \mathrm{~Hz}, 1 \mathrm{H}), 3.35-3.28(\mathrm{~m}$, $1 \mathrm{H}), 3.25(\mathrm{dt}, J=11.3,2.1 \mathrm{~Hz}, 1 \mathrm{H}), 2.55-2.46(\mathrm{~m}, 1 \mathrm{H}), 2.26(\mathrm{dd}, J=17.9,3.0 \mathrm{~Hz}, 1 \mathrm{H}), 2.05(\mathrm{dd}, J=11.1,2.2 \mathrm{~Hz}, 1 \mathrm{H})$, $1.88(\mathrm{dd}, J=11.4,1.5 \mathrm{~Hz}, 1 \mathrm{H}), 1.76(\mathrm{dd}, J=17.9,1.5 \mathrm{~Hz}, 1 \mathrm{H}), 1.22(\mathrm{~d}, J=7.3 \mathrm{~Hz}, 3 \mathrm{H}), 1.06(\mathrm{ddt}, J=13.3,3.7,1.9 \mathrm{~Hz}$, $1 \mathrm{H}), 0.82(\mathrm{dt}, J=13.2,3.1 \mathrm{~Hz}, 1 \mathrm{H}), 0.41(\mathrm{~s}, 3 \mathrm{H})$.

${ }^{13} \mathrm{C}$ NMR (101 MHz, C6 $\left.\mathrm{D}_{6}\right) \delta 194.9,142.6,137.9,133.1,128.8,123.9,57.0,51.5,51.2,37.2,32.1,31.2,26.6,12.7$.

HRMS ESI Calcd for $\mathrm{C}_{17} \mathrm{H}_{21} \mathrm{~N}_{2} \mathrm{O}_{5} \mathrm{~S}[\mathrm{M}+\mathrm{H}]^{+}:$365.1166, Found: 365.1168.

IR (KBr): 2930, 2857, 1687, 1625, 1457, 1333, 1193, 1161, 1059, $1031 \mathrm{~cm}^{-1}$.

Chiral HPLC (Chiralpak IA, Hexane/Isopropanol $=80 / 20$, flow rate $=1.0 \mathrm{~mL} / \mathrm{min}, 230 \mathrm{~nm}$ ), $\mathrm{t}_{\text {minor }}=20.7 \mathrm{~min}, \mathrm{t}_{\mathrm{major}}=$ $31.8 \min .[\alpha]^{21.5}=-48.2\left(c 1.0, \mathrm{CHCl}_{3}\right)$ at $94.5: 5.5 \mathrm{er}$.

\section{Racemic sample $\mathbf{2 n}$}

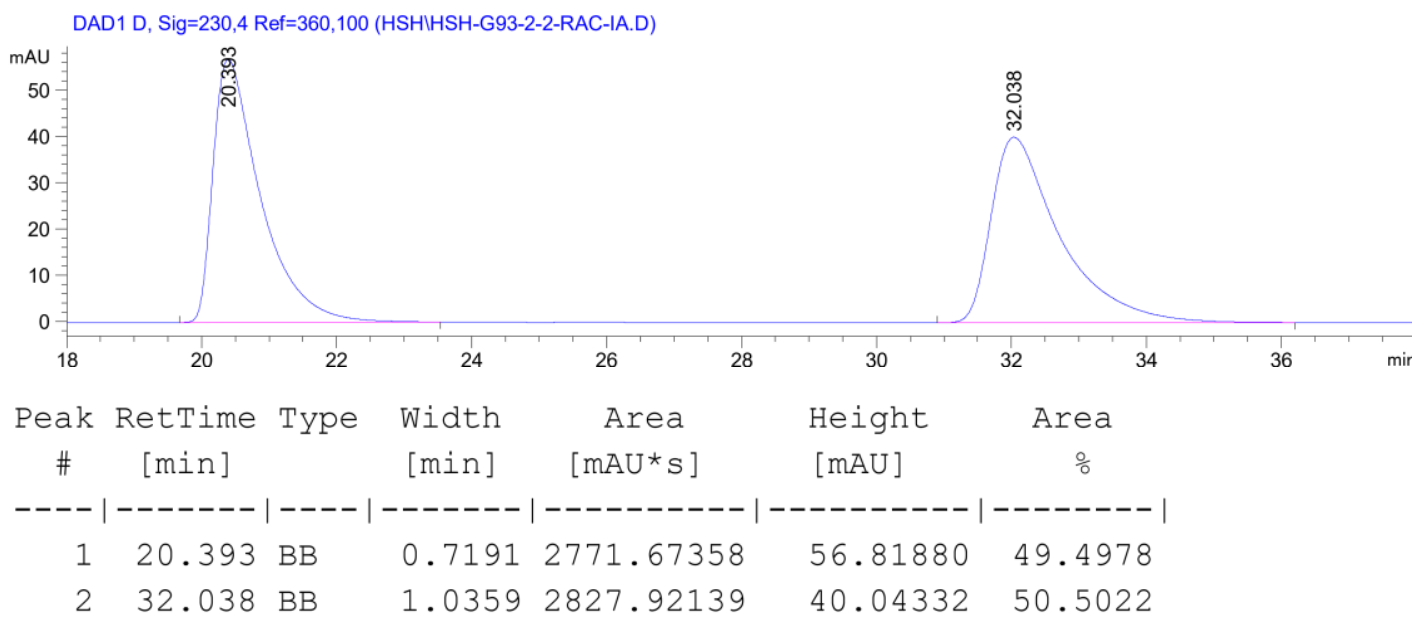

Enantiomeric sample 2n 

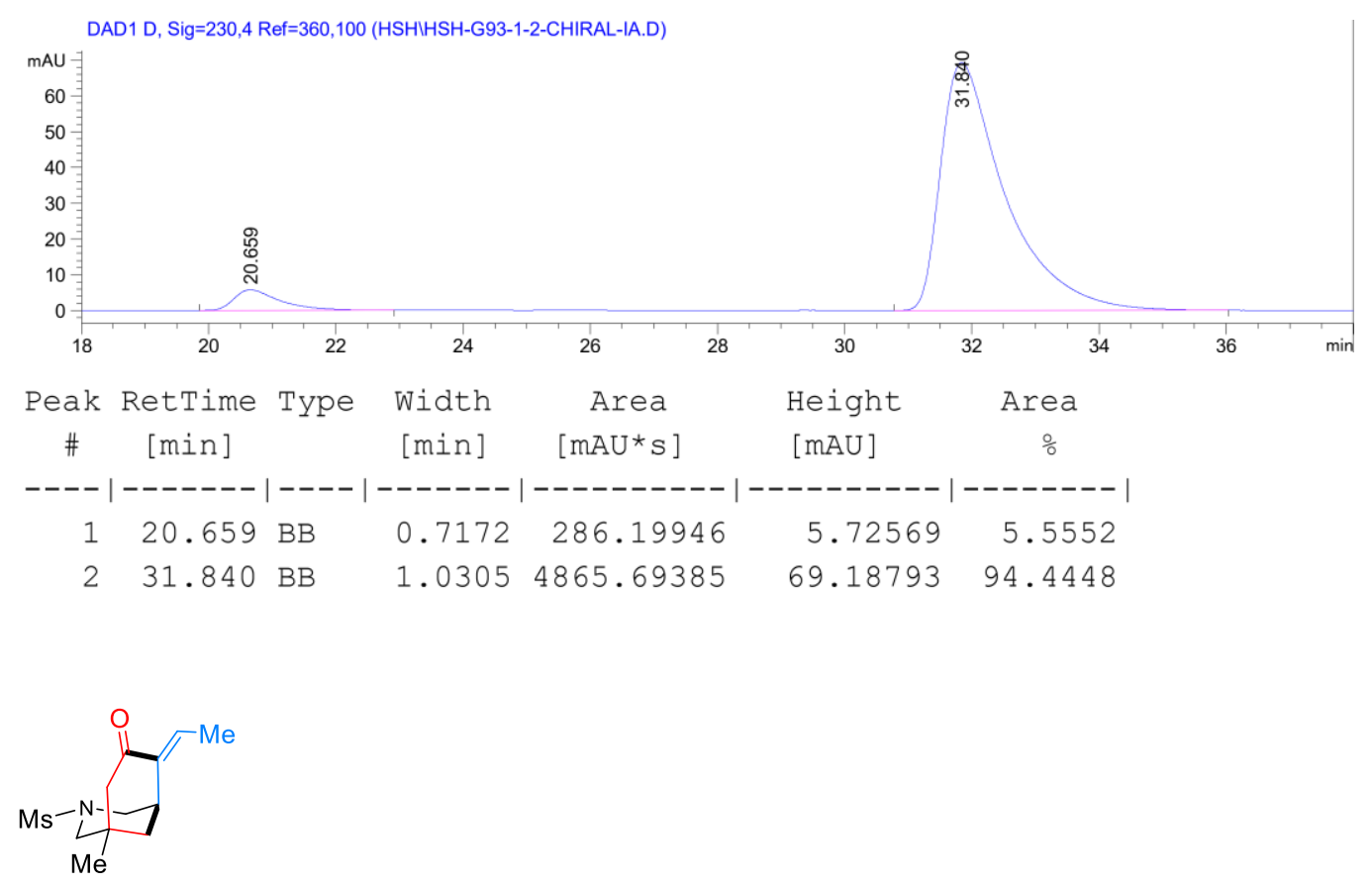

20

20 (15.6 mg) was isolated as a colorless oil in $60 \%$ yield.

$\mathrm{Rf}=0.2$, Hexane/ EtOAc 2:1.

${ }^{1} \mathrm{H}$ NMR (400 MHz, Benzene-d6) $\delta 6.67(\mathrm{q}, J=7.2 \mathrm{~Hz}, 1 \mathrm{H}), 3.35-3.23(\mathrm{~m}, 2 \mathrm{H}), 2.56-2.50(\mathrm{~m}, 1 \mathrm{H}), 2.31(\mathrm{dd}, J=11.4$, $2.1 \mathrm{~Hz}, 1 \mathrm{H}), 2.29-2.23(\mathrm{~m}, 4 \mathrm{H}), 2.12(\mathrm{dd}, J=11.7,1.4 \mathrm{~Hz}, 1 \mathrm{H}), 1.78(\mathrm{dd}, J=17.6,1.4 \mathrm{~Hz}, 1 \mathrm{H}), 1.27(\mathrm{~d}, J=7.2 \mathrm{~Hz}, 3 \mathrm{H})$, $1.10(\mathrm{ddt}, J=13.1,3.7,1.9 \mathrm{~Hz}, 1 \mathrm{H}), 0.91(\mathrm{dt}, J=13.1,3.1 \mathrm{~Hz}, 1 \mathrm{H}), 0.46(\mathrm{~s}, 3 \mathrm{H})$.

${ }^{13} \mathrm{C}$ NMR (101 MHz, C6 $\left.\mathrm{D}_{6}\right) \delta$ 196.5, 139.1, 132.0, 57.0, 51.8, 51.6, 37.4, 36.8, 32.7, 31.6, 26.4, 12.7.

HRMS ESI Calcd for $\mathrm{C}_{12} \mathrm{H}_{20} \mathrm{NO}_{3} \mathrm{~S}[\mathrm{M}+\mathrm{H}]^{+}:$258.1158, Found: 258.1159 .

IR (KBr): 2929, 1859, 1687, 1624, 1530, 1350, 1170, $1030 \mathrm{~cm}^{-1}$.

Chiral HPLC (Chiralpak IF, Hexane/Isopropanol $=80 / 20$, flow rate $=1.0 \mathrm{~mL} / \mathrm{min}, 230 \mathrm{~nm}$ ), $\mathrm{t}_{\operatorname{minor}}=52.9 \mathrm{~min}, \mathrm{t}_{\mathrm{major}}=43$ $\min .[\alpha]^{21.5}=-95.1\left(c 0.8, \mathrm{CHCl}_{3}\right)$ at $96: 4 \mathrm{er}$.

\section{Racemic sample 20}

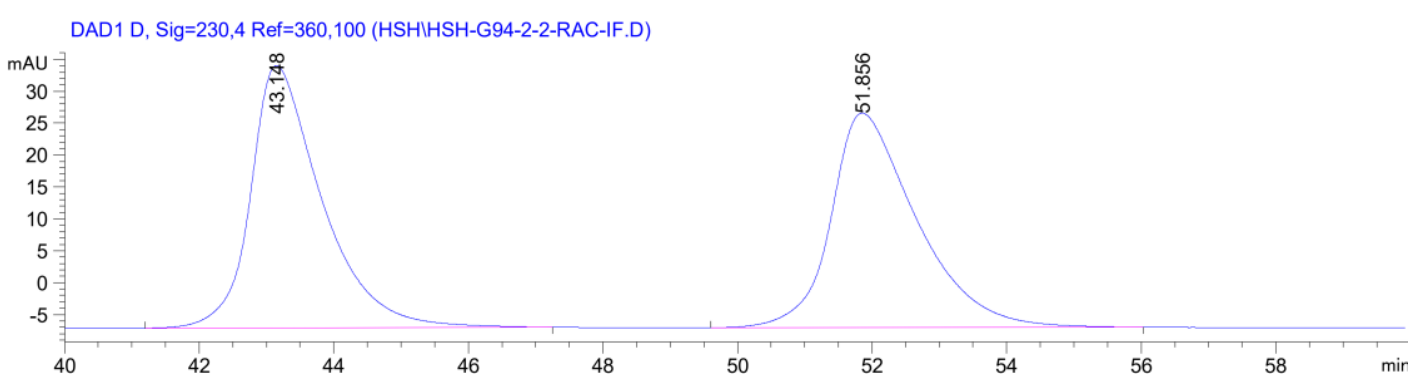




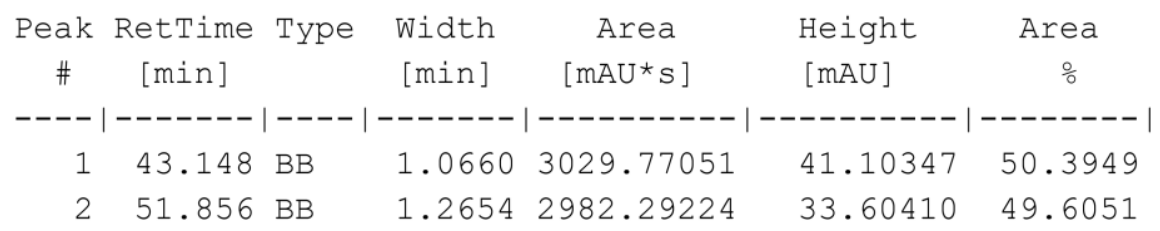

\section{Enantiomeric sample 20}

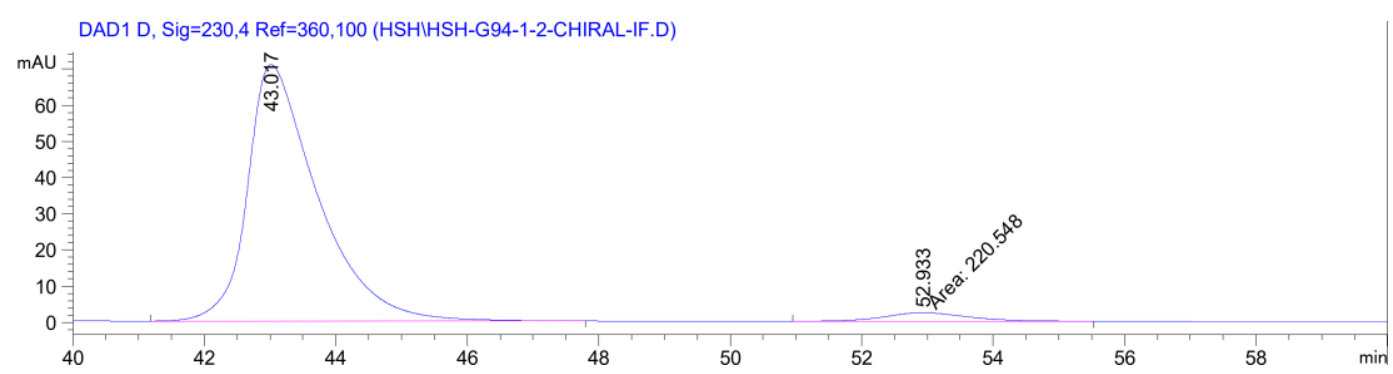

\begin{tabular}{|c|c|c|c|c|c|c|}
\hline $\begin{array}{c}\text { Peak } \\
\#\end{array}$ & $\begin{array}{c}\text { RetTime } \\
\text { [min] }\end{array}$ & Type & $\begin{array}{l}\text { Width } \\
\text { [min] }\end{array}$ & $\begin{array}{c}\text { Area } \\
{\left[\mathrm{mAU}{ }^{*} \mathrm{~s}\right]}\end{array}$ & $\begin{array}{l}\text { Height } \\
\text { [mAU] }\end{array}$ & $\begin{array}{c}\text { Area } \\
\quad \%\end{array}$ \\
\hline & & & & ---------- & --------- & \\
\hline 1 & 43.017 & $\mathrm{BB}$ & 1.0747 & 5277.15576 & 70.86942 & 95.9884 \\
\hline 2 & 52.933 & MM & 1.5323 & 220.54784 & 2.39894 & 4.0116 \\
\hline
\end{tabular}

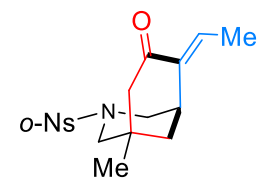

$2 p$

2 p (11.6 mg) was isolated as a foam in $32 \%$ yield.

$\mathrm{Rf}=0.13$, Hexane/ EtOAc 2:1.

${ }^{1} \mathrm{H}$ NMR (400 MHz, Benzene-d6) $\delta 7.56(\mathrm{dd}, J=7.9,1.3 \mathrm{~Hz}, 1 \mathrm{H}), 6.79(\mathrm{td}, J=7.7,1.3 \mathrm{~Hz}, 1 \mathrm{H}), 6.76-6.67(\mathrm{~m}, 2 \mathrm{H}), 6.60$ (td, $J=7.7,1.4 \mathrm{~Hz}, 1 \mathrm{H}), 3.67-3.57(\mathrm{~m}, 1 \mathrm{H}), 3.52(\mathrm{dt}, J=12.0,2.1 \mathrm{~Hz}, 1 \mathrm{H}), 2.54(\mathrm{p}, J=2.9 \mathrm{~Hz}, 1 \mathrm{H}), 2.50(\mathrm{dd}, J=11.6$, $2.2 \mathrm{~Hz}, 1 \mathrm{H}), 2.42(\mathrm{dd}, J=18.1,3.0 \mathrm{~Hz}, 1 \mathrm{H}), 2.20$ (dd, $J=12.0,1.5 \mathrm{~Hz}, 1 \mathrm{H}), 1.77(\mathrm{dd}, J=18.0,1.5 \mathrm{~Hz}, 1 \mathrm{H}), 1.26(\mathrm{~d}, J=$ $7.3 \mathrm{~Hz}, 3 \mathrm{H}), 1.07(\mathrm{ddt}, J=13.2,3.7,1.9 \mathrm{~Hz}, 1 \mathrm{H}), 0.87(\mathrm{dt}, J=13.2,3.1 \mathrm{~Hz}, 1 \mathrm{H}), 0.44(\mathrm{~s}, 3 \mathrm{H})$.

${ }^{13} \mathrm{C}$ NMR (101 MHz, C6 $\left.\mathrm{D}_{6}\right) \delta 195.0,137.8,133.4,133.2,131.7,131.0,130.8,123.7,56.9,51.7,51.0,37.2,32.2,31.4$, $26.7,12.7$.

HRMS ESI Calcd for $\mathrm{C}_{17} \mathrm{H}_{21} \mathrm{~N}_{2} \mathrm{O}_{5} \mathrm{~S}[\mathrm{M}+\mathrm{H}]^{+}:$365.1166, Found: 365.1169.

IR (KBr): 2928, 1686, 1624, 1545, 1464, 1374, 1359, 1175,1031cm $\mathrm{cm}^{-1}$.

Chiral HPLC (Chiralpak IF, Hexane/Isopropanol $=80 / 20$, flow rate $=1.0 \mathrm{~mL} / \mathrm{min}, 230 \mathrm{~nm}$ ), $\mathrm{t}_{\text {minor }}=55.4 \mathrm{~min}, \mathrm{t}_{\mathrm{major}}=$ $75.4 \min .[\alpha]^{21.5}=-47.1\left(c 0.6, \mathrm{CHCl}_{3}\right)$ at $90: 10 \mathrm{er}$. 


\section{Racemic sample $\mathbf{2 p}$}

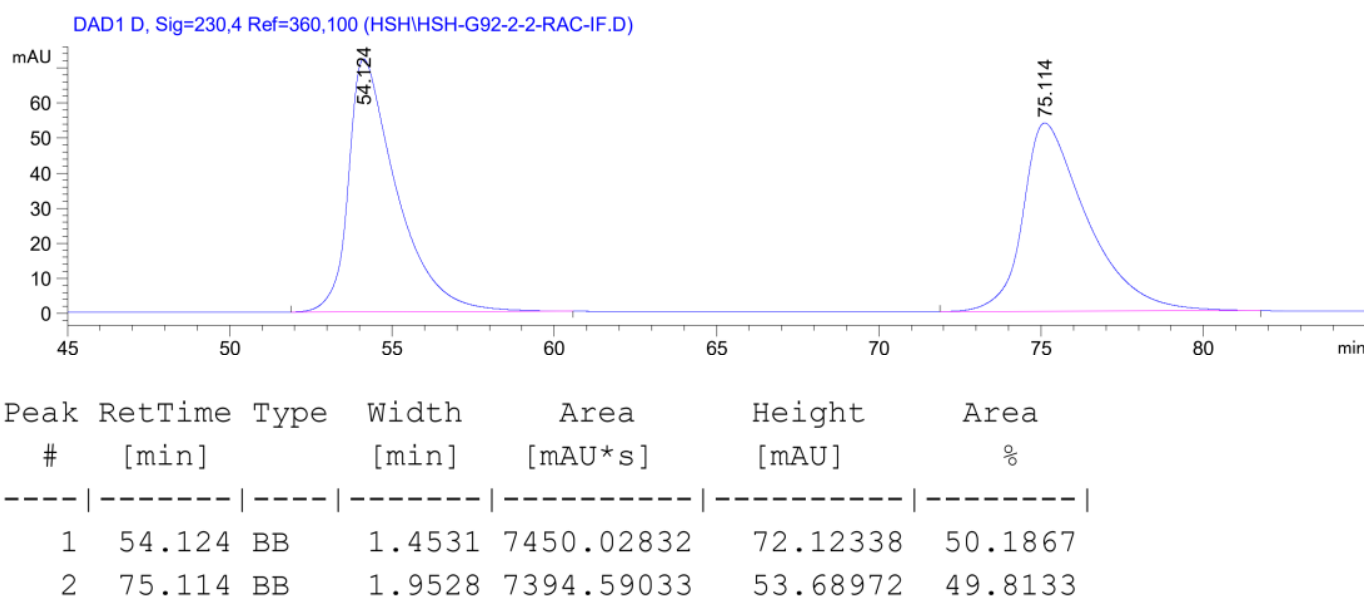

\section{Enantiomeric sample 2p}
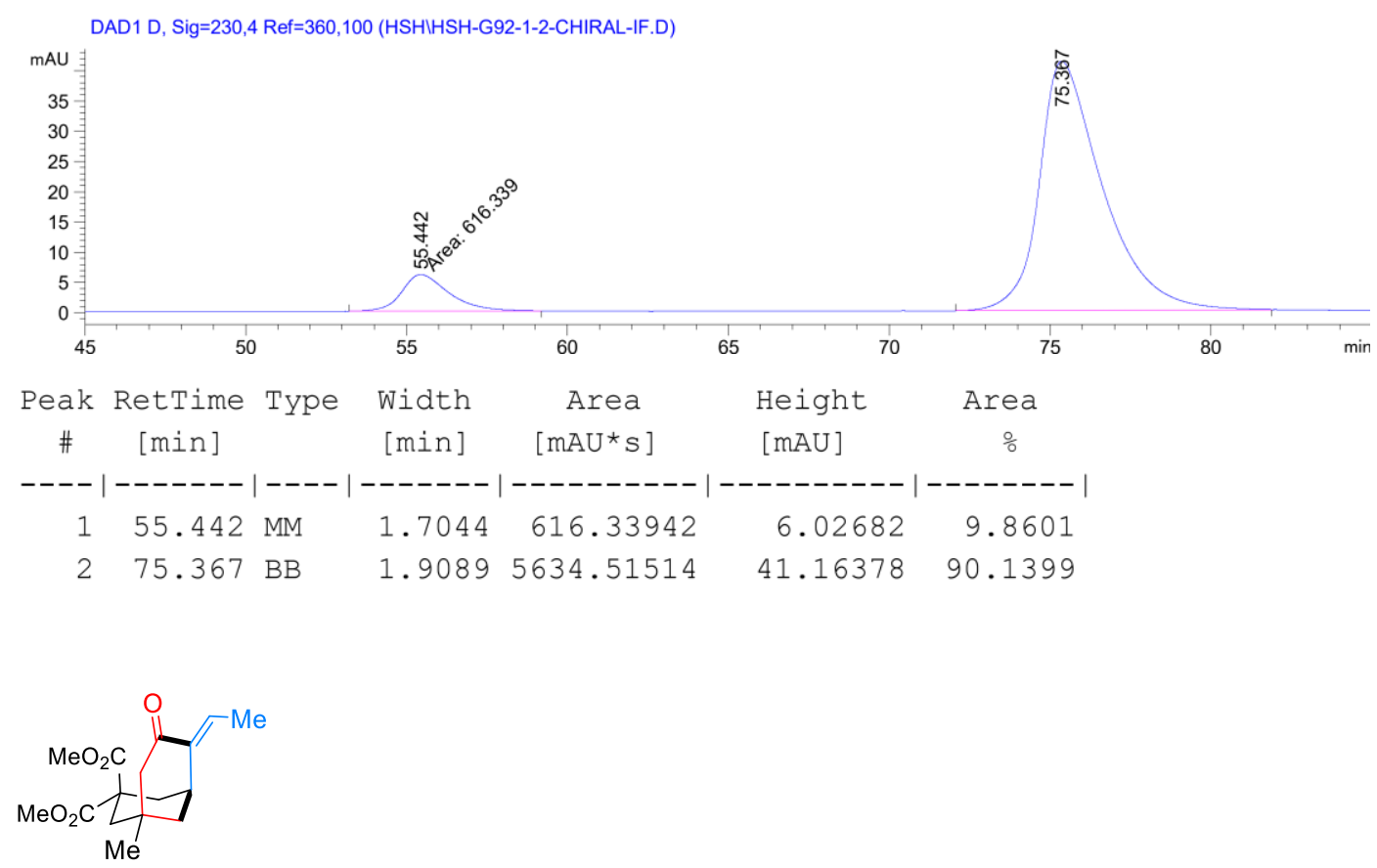

$2 r$

2 r (10 mg) was isolated as an oil in $34 \%$ yield.

$\mathrm{Rf}=0.25$, Hexane/ EtOAc 4:1.

${ }^{1} \mathrm{H}$ NMR (400 MHz, Chloroform-d) $\delta 6.87(\mathrm{qd}, J=7.3,1.2 \mathrm{~Hz}, 1 \mathrm{H}), 3.68(\mathrm{~s}, 3 \mathrm{H}), 3.56(\mathrm{~s}, 3 \mathrm{H}), 3.25-3.20(\mathrm{~m}, 1 \mathrm{H}), 2.67$ (dd, $J=19.0,3.0 \mathrm{~Hz}, 1 \mathrm{H}), 2.63-2.51(\mathrm{~m}, 2 \mathrm{H}), 2.15(\mathrm{dd}, J=13.7,3.9 \mathrm{~Hz}, 1 \mathrm{H}), 2.08(\mathrm{dd}, J=19.0,1.6 \mathrm{~Hz}, 1 \mathrm{H}), 1.80(\mathrm{~d}, J$ $=7.4 \mathrm{~Hz}, 3 \mathrm{H}), 1.70-1.56(\mathrm{~m}, 3 \mathrm{H}), 1.06(\mathrm{~s}, 3 \mathrm{H})$.

${ }^{13} \mathrm{C}$ NMR (101 MHz, $\left.\mathrm{CDCl}_{3}\right) \delta$ 198.5, 172.8, 171.5, 137.7, 137.1, 53.0, 52.8, 52.7, 51.6, 41.8, 38.9, 35.3, 32.6, 31.2, $30.0,13.5$.

HRMS ESI Calcd for $\mathrm{C}_{16} \mathrm{H}_{23} \mathrm{O}_{5}[\mathrm{M}+\mathrm{H}]^{+}:$295.1540, Found: 295.1544. 
IR (KBr): 2952, 2871, 1733, 1687, 1614, 1435, 1238, 1158, 1110, 1094, $1033 \mathrm{~cm}^{-1}$.

Chiral HPLC (Chiralpak IA, Hexane/Isopropanol $=90 / 10$, flow rate $=1.0 \mathrm{~mL} / \mathrm{min}, 230 \mathrm{~nm}$ ) , $\mathrm{t}_{\text {minor }}=13.4 \mathrm{~min}, \mathrm{t}_{\text {major }}=$ 14.7min. $[\alpha]^{21.5} \mathrm{D}=-44.8\left(c 0.9, \mathrm{CHCl}_{3}\right)$ at 87:13 er.

\section{Racemic sample $2 r$}

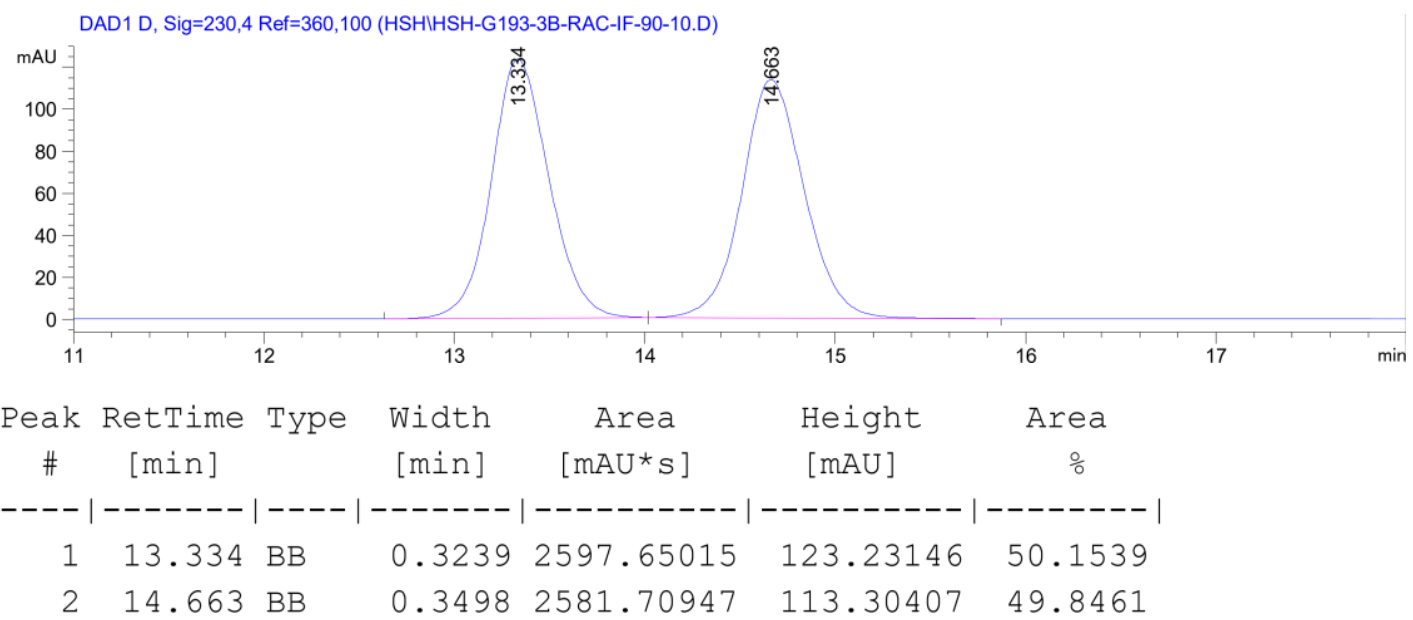

\section{Enantiomeric sample 2r}
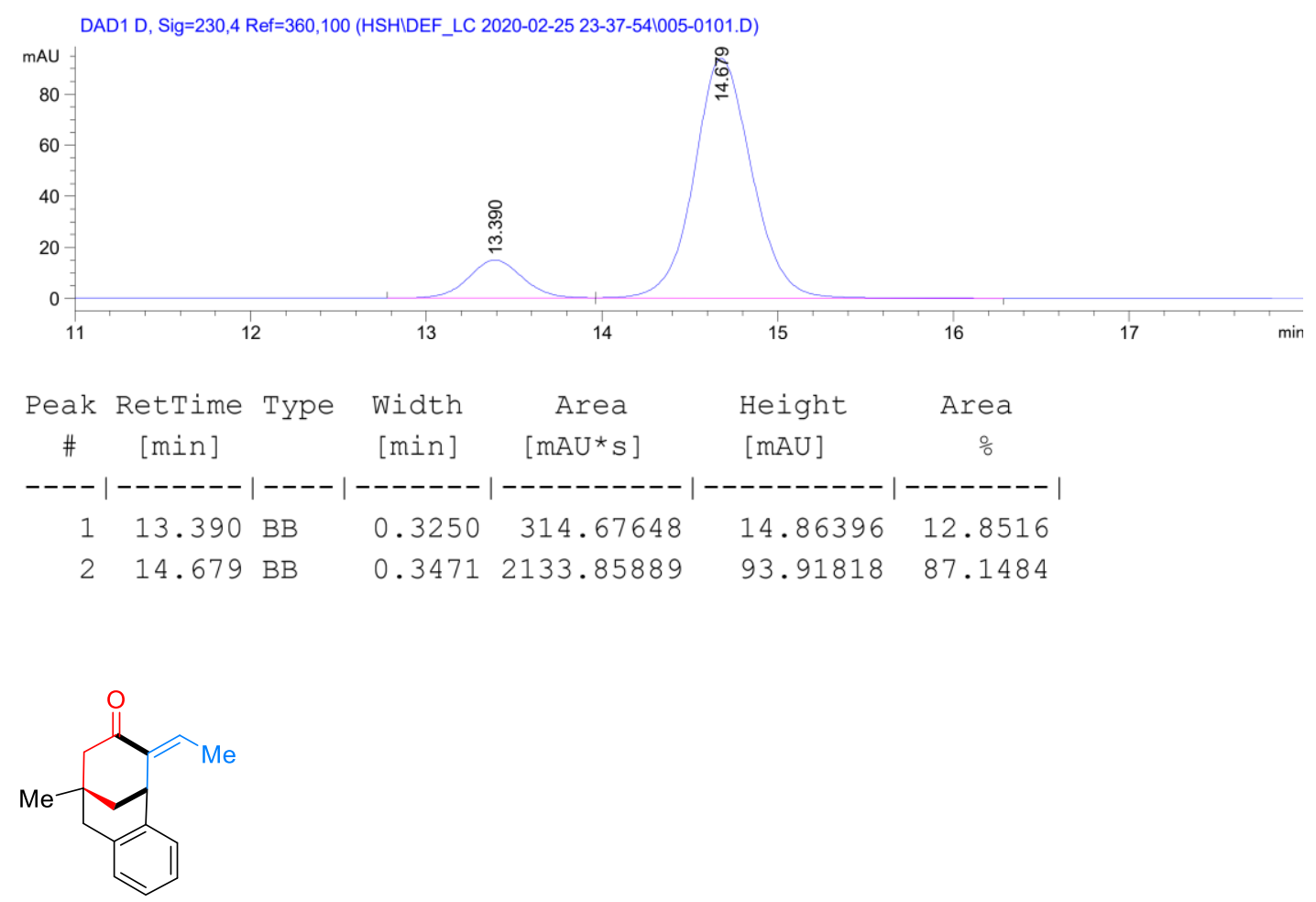

$2 s$

2s (4 mg) was isolated as an oil in $18 \%$ yield. 
$\mathrm{Rf}=0.24$, Hexane/ EtOAc 10:1.

${ }^{1} \mathrm{H}$ NMR (400 MHz, Chloroform-d) $\delta 7.13-6.98(\mathrm{~m}, 4 \mathrm{H}), 6.33(\mathrm{q}, J=7.2 \mathrm{~Hz}, 1 \mathrm{H}), 4.27(\mathrm{t}, J=3.2 \mathrm{~Hz}, 1 \mathrm{H}), 2.86(\mathrm{~d}, J=$ $17.1 \mathrm{~Hz}, 1 \mathrm{H}), 2.72(\mathrm{dd}, J=17.4,1.9 \mathrm{~Hz}, 1 \mathrm{H}), 2.48(\mathrm{dd}, J=17.9,2.5 \mathrm{~Hz}, 1 \mathrm{H}), 2.35(\mathrm{dd}, J=17.9,1.7 \mathrm{~Hz}, 1 \mathrm{H}), 2.11-2.04$ $(\mathrm{m}, 1 \mathrm{H}), 2.04-1.94(\mathrm{~m}, 4 \mathrm{H}), 1.21(\mathrm{~s}, 3 \mathrm{H})$.

${ }^{13} \mathrm{C}$ NMR (101 MHz, CDCl$)$ ) $\delta$ 201.7, 142.0, 138.6, 134.0, 129.4, 128.3, 128.2, 126.7, 126.5, 54.0, 43.5, 37.5, 36.9, 32.0, $31.9,13.7$.

HRMS ESI Calcd for $\mathrm{C}_{16} \mathrm{H}_{19} \mathrm{O}[\mathrm{M}+\mathrm{H}]^{+}:$227.1430, Found: 227.1432.

IR (KBr): 2950, 2924, 2869, 1691, 1630, 1488, 1454, 1378, 1274, 1226, $1115 \mathrm{~cm}^{-1}$.

Chiral HPLC (Chiralpak IB, Hexane/Isopropanol $=99 / 1$, flow rate $=1.0 \mathrm{~mL} / \mathrm{min}, 230 \mathrm{~nm}$ ) $, t_{\text {minor }}=8.9 \mathrm{~min}, \mathrm{t}_{\text {major }}=8$ $\min .[\alpha]^{21.5}=-40\left(c 0.4, \mathrm{CHCl}_{3}\right)$ at $61: 39 \mathrm{er}$.

\section{Racemic sample $2 \mathbf{s}$}

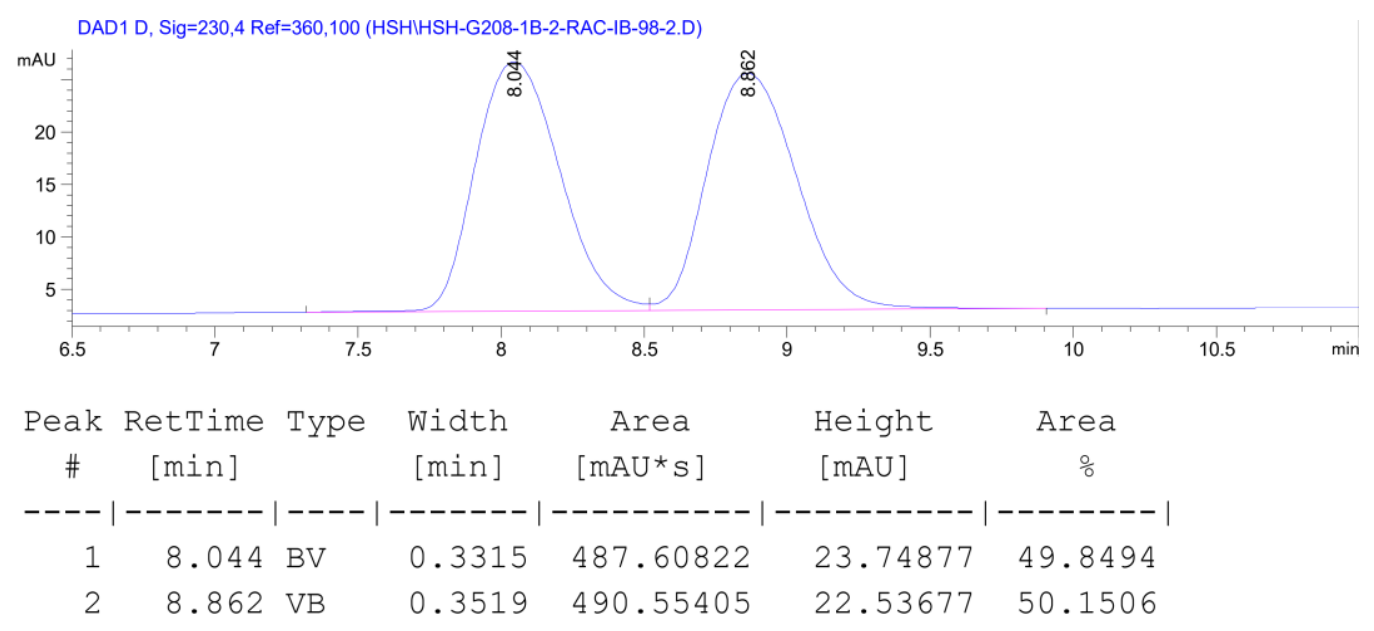

\section{Enantiomeric sample 2s}

UAD1 D, Sig=230,4 Ret=360, 100 (HSHIHSH-G208-1A-2-CHIRAL-IB-9y-1.D)

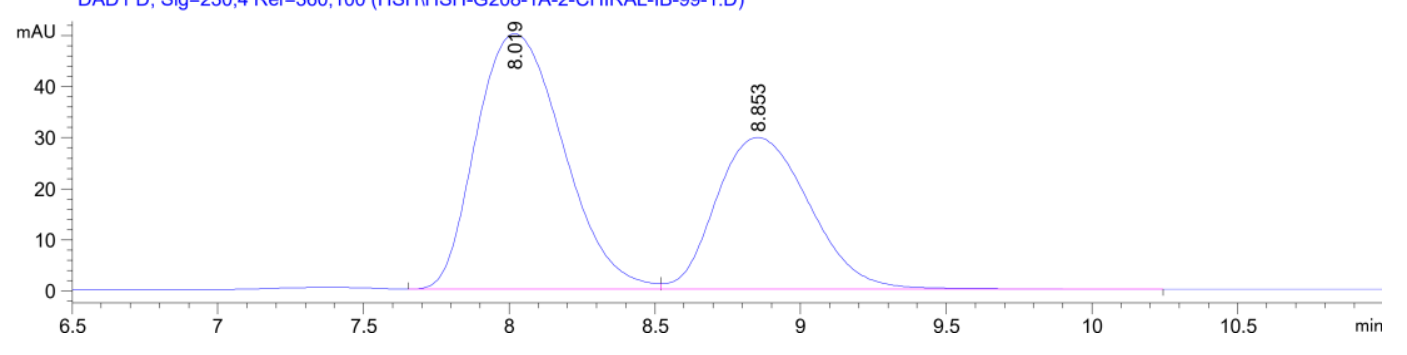

\begin{tabular}{|c|c|c|c|c|c|c|}
\hline $\begin{array}{c}\text { Peak } \\
\quad \#\end{array}$ & $\begin{array}{c}\text { RetTime } \\
\text { [min] }\end{array}$ & Type & $\begin{array}{l}\text { Width } \\
\text { [min] }\end{array}$ & $\begin{array}{c}\text { Area } \\
{\left[\mathrm{mAU}^{*} \mathrm{~s}\right]}\end{array}$ & $\begin{array}{l}\text { Height } \\
\text { [mAU] }\end{array}$ & $\begin{array}{c}\text { Area } \\
\quad \%\end{array}$ \\
\hline & & & & $|-----------|$ & $---2---1$ & \\
\hline 1 & 8.019 & BV & 0.3340 & 1037.01428 & 50.00335 & 61.0372 \\
\hline 2 & 8.853 & VB & 0.3600 & 661.97418 & 29.69953 & 38.9628 \\
\hline
\end{tabular}




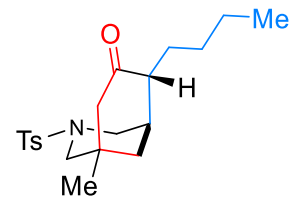

2t

$2 t(25.6 \mathrm{mg})$ was isolated as a white solid in $71 \%$ yield. $d r>20: 1$.

$\mathrm{Rf}=0.2$, Hexane/ EtOAc 2:1.

Mp: $157-158.3^{\circ} \mathrm{C}$.

${ }^{1} \mathrm{H}$ NMR (400 MHz, Chloroform-d) $\delta 7.66-7.59(\mathrm{~m}, 2 \mathrm{H}), 7.32(\mathrm{~d}, J=8.1 \mathrm{~Hz}, 2 \mathrm{H}), 3.69$ (dq $\left.J=11.5,2.1 \mathrm{~Hz}, 1 \mathrm{H}\right), 3.40$ $(\mathrm{dt}, J=11.3,2.1 \mathrm{~Hz}, 1 \mathrm{H}), 2.43(\mathrm{~s}, 3 \mathrm{H}), 2.38-2.22(\mathrm{~m}, 3 \mathrm{H}), 2.21-2.08(\mathrm{~m}, 3 \mathrm{H}), 1.98(\mathrm{ttd}, J=8.6,5.5,2.2 \mathrm{~Hz}, 1 \mathrm{H}), 1.81$ $(\mathrm{dq}, J=13.2,2.2 \mathrm{~Hz}, 1 \mathrm{H}), 1.54(\mathrm{dt}, J=13.3,3.2 \mathrm{~Hz}, 1 \mathrm{H}), 1.37-1.16(\mathrm{~m}, 5 \mathrm{H}), 0.97(\mathrm{~s}, 3 \mathrm{H}), 0.89(\mathrm{t}, J=7.1 \mathrm{~Hz}, 3 \mathrm{H})$.

${ }^{13} \mathrm{C}$ NMR (101 MHz, CDCl 3 ) $\delta$ 207.5, 143.8, 133.0, 129.5, 128.0, 57.1, 52.8, 50.9, 46.3, 39.8, 34.8, 34.3, 29.4, 26.6, 25.5, 22.7, 21.5, 14.0.

HRMS ESI Calcd for $\mathrm{C}_{20} \mathrm{H}_{30} \mathrm{NO}_{3} \mathrm{~S}[\mathrm{M}+\mathrm{H}]^{+}:$364.1941, Found: 364.1942.

IR (KBr): 2954, 2929, 2870, 1706, 1457, 1363, 1346, 1219, 1165, 1107, 1094, 1021 $\mathrm{cm}^{-1}$.

Chiral HPLC (Chiralpak IF, Hexane/Isopropanol $=80 / 20$, flow rate $=1.0 \mathrm{~mL} / \mathrm{min}, 230 \mathrm{~nm}$ ) , $\mathrm{t}_{\text {minor }}=28.2 \mathrm{~min}, \mathrm{t}_{\text {major }}=$ $39.8 \min .[\alpha]^{21.5} \mathrm{D}=+0.7\left(\mathrm{c} 1.1, \mathrm{CHCl}_{3}\right)$ a $95: 5 \mathrm{er}$.

\section{Racemic sample $2 \mathrm{t}$}

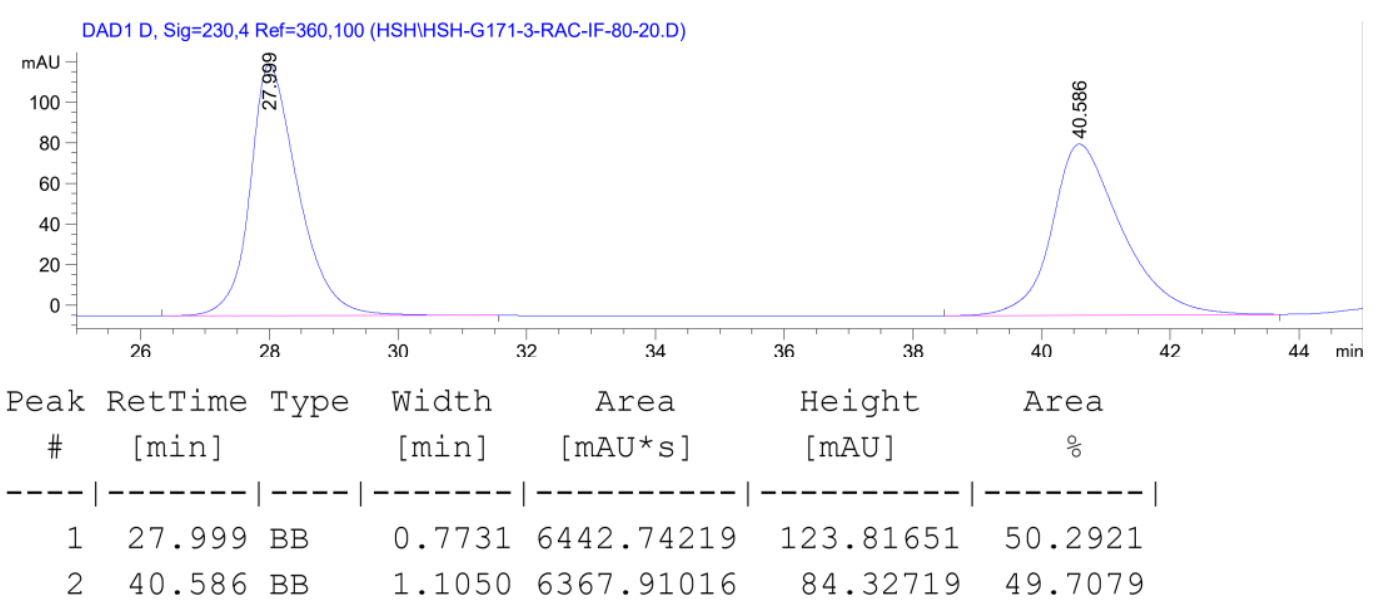

Enantiomeric sample 2t 

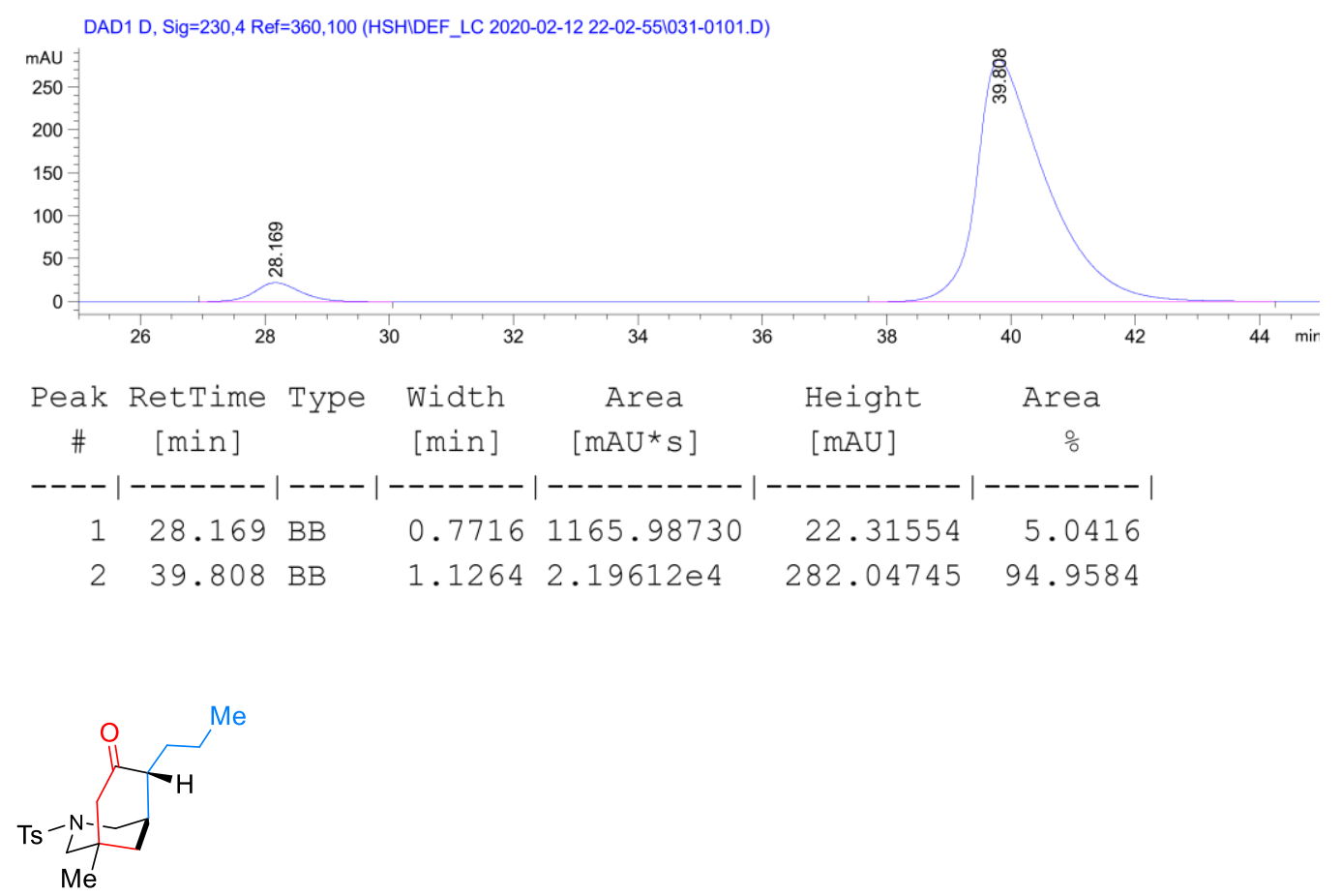

$2 u$

2 u (22.7 mg) was isolated as a white solid in 65\% yield. $d r>20: 1$.

$\mathrm{Rf}=0.28$, Hexane/ EtOAc 2:1.

$\mathrm{Mp}: 163.4-165.6^{\circ} \mathrm{C}$.

${ }^{1} \mathrm{H}$ NMR (400 MHz, Chloroform-d) $\delta 7.64-7.58(\mathrm{~m}, 2 \mathrm{H}), 7.36-7.29(\mathrm{~m}, 2 \mathrm{H}), 3.70(\mathrm{dq}, J=11.7,2.1 \mathrm{~Hz}, 1 \mathrm{H}), 3.39(\mathrm{dt}$, $J=11.3,2.1 \mathrm{~Hz}, 1 \mathrm{H}), 2.43(\mathrm{~s}, 3 \mathrm{H}), 2.37-2.27(\mathrm{~m}, 2 \mathrm{H}), 2.24(\mathrm{dd}, J=11.6,2.3 \mathrm{~Hz}, 1 \mathrm{H}), 2.21-2.15(\mathrm{~m}, 1 \mathrm{H}), 2.02-1.91$ $(\mathrm{m}, 1 \mathrm{H}), 1.81(\mathrm{dq}, J=13.2,2.4 \mathrm{~Hz}, 1 \mathrm{H}), 1.53(\mathrm{dt}, J=13.3,3.2 \mathrm{~Hz}, 1 \mathrm{H}), 1.38-1.14(\mathrm{~m}, 4 \mathrm{H}), 0.96(\mathrm{~s}, 3 \mathrm{H}), 0.91(\mathrm{t}, J=7.2$ $\mathrm{Hz}, 3 \mathrm{H})$.

${ }^{13} \mathrm{C}$ NMR (101 MHz, $\left.\mathrm{CDCl}_{3}\right) \delta 207.5,143.7,132.9,129.5,127.9,57.1,52.7,50.6,46.4,39.8,34.8,34.3,28.0,26.5$, $21.5,20.4,14.1$.

HRMS ESI Calcd for $\mathrm{C}_{19} \mathrm{H}_{28} \mathrm{NO}_{3} \mathrm{~S}[\mathrm{M}+\mathrm{H}]^{+}: 350.1784$, Found: 350.1788 .

IR (KBr): 2954, 2929, 2870, 1705, 1494, 1456, 1361, 1341, 1164, 1105, 1093, $1020 \mathrm{~cm}^{-1}$.

Chiral HPLC (Chiralpak IF, Hexane/Isopropanol $=80 / 20$, flow rate $=1.0 \mathrm{~mL} / \mathrm{min}, 230 \mathrm{~nm}$ ), $\mathrm{t}_{\text {minor }}=30.2 \mathrm{~min}, \mathrm{t}_{\mathrm{major}}=$ 42.4min. $[\alpha]^{21.5}=+3.2\left(c 1.1, \mathrm{CHCl}_{3}\right)$ at 95.5:4.5 er.

\section{Racemic sample $2 \mathbf{u}$}




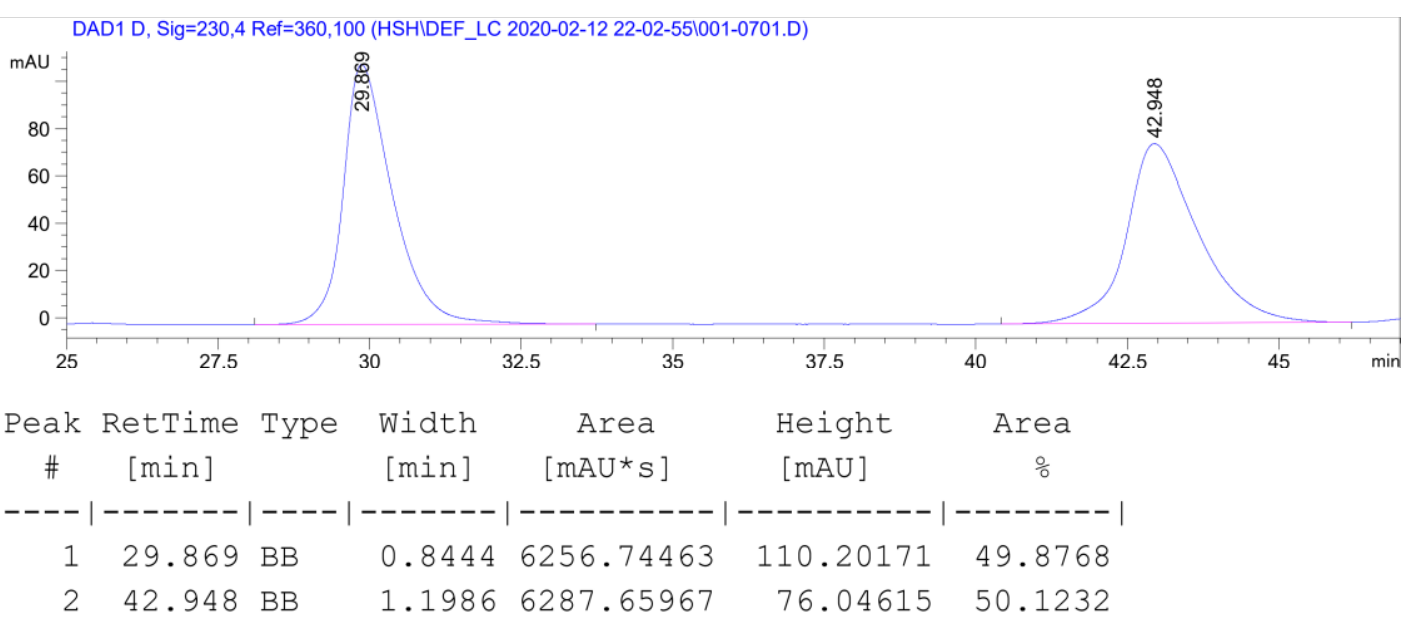

\section{Enantiomeric sample 2u}
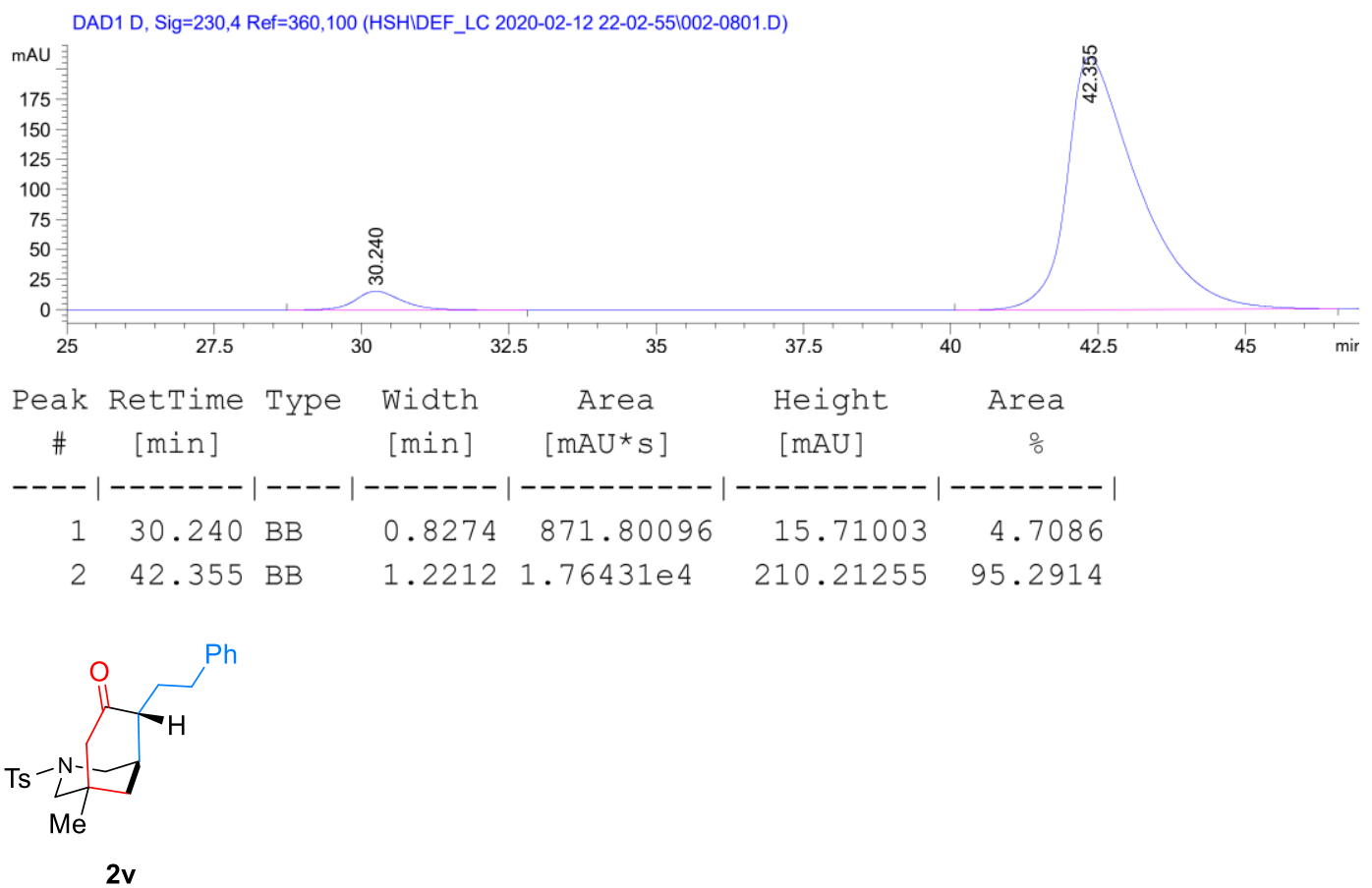

2v (26.2mg) was isolated as a white solid in 64\% yield. $d r>20: 1$.

$\mathrm{Rf}=0.3$, Hexane/ EtOAc 2:1.

Mp: $174.9-175.3^{\circ} \mathrm{C}$.

${ }^{1} \mathrm{H}$ NMR (400 MHz, Chloroform-d) $\delta 7.66$ - $7.60(\mathrm{~m}, 2 \mathrm{H}), 7.35-7.30(\mathrm{~m}, 2 \mathrm{H}), 7.30-7.25(\mathrm{~m}, 2 \mathrm{H}), 7.23-7.15(\mathrm{~m}, 3 \mathrm{H})$, $3.76-3.65(\mathrm{~m}, 1 \mathrm{H}), 3.43(\mathrm{dt}, J=11.3,2.0 \mathrm{~Hz}, 1 \mathrm{H}), 2.77-2.60(\mathrm{~m}, 2 \mathrm{H}), 2.42(\mathrm{~s}, 3 \mathrm{H}), 2.40-2.24(\mathrm{~m}, 4 \mathrm{H}), 2.20-2.08$ $(\mathrm{m}, 3 \mathrm{H}), 1.78(\mathrm{dq}, J=13.1,2.2 \mathrm{~Hz}, 1 \mathrm{H}), 1.56-1.40(\mathrm{~m}, 2 \mathrm{H}), 0.97(\mathrm{~s}, 3 \mathrm{H})$.

${ }^{13} \mathrm{C}$ NMR $\left(101 \mathrm{MHz} \mathrm{CDCl}_{3}\right) \delta$ 207.3, 143.8, 141.8, 132.9, 129.6, 128.4, 128.3, 128.0, 125.8, 57.0, 52.8, 49.8, 46.5, 39.7, $34.9,34.8,33.4,27.8,26.5,21.5$.

HRMS ESI Calcd for $\mathrm{C}_{24} \mathrm{H}_{30} \mathrm{NO}_{3} \mathrm{~S}[\mathrm{M}+\mathrm{H}]^{+}:$412.1941, Found: 412.1945.

IR (KBr): 2925, 2852, 1705, 1598, 1495, 1454, 1363, 1346, 1218, 1165, 1094, 1021 $\mathrm{cm}^{-1}$. 
Chiral HPLC (Chiralpak IA, Hexane/Isopropanol $=80 / 20$, flow rate $=1.0 \mathrm{~mL} / \mathrm{min}, 230 \mathrm{~nm}$ ) , $\mathrm{t}_{\text {minor }}=16 \mathrm{~min}, \mathrm{t}_{\mathrm{major}}=21.8$ $\min .[\alpha]_{D}^{21.5_{D}}=+5.7\left(c 1.3, \mathrm{CHCl}_{3}\right)$ at $94: 6 \mathrm{er}$.

\section{Racemic sample 2v}

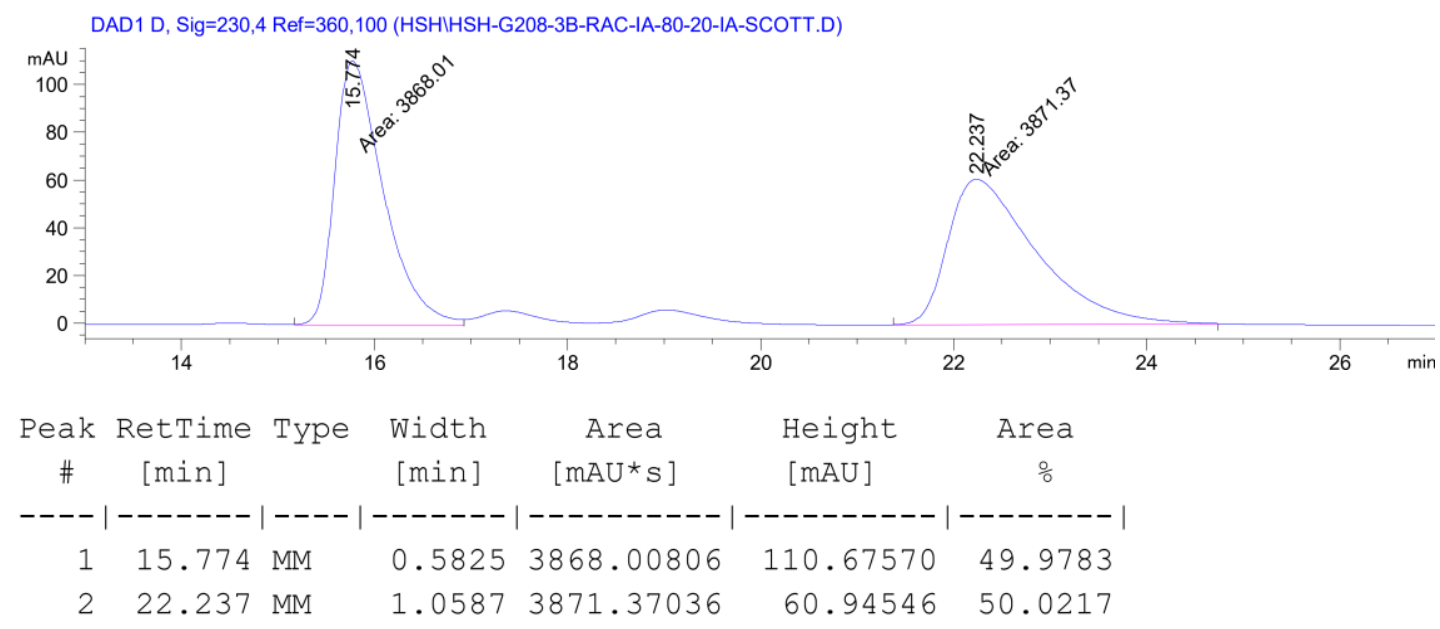

\section{Enantiomeric sample 2v}
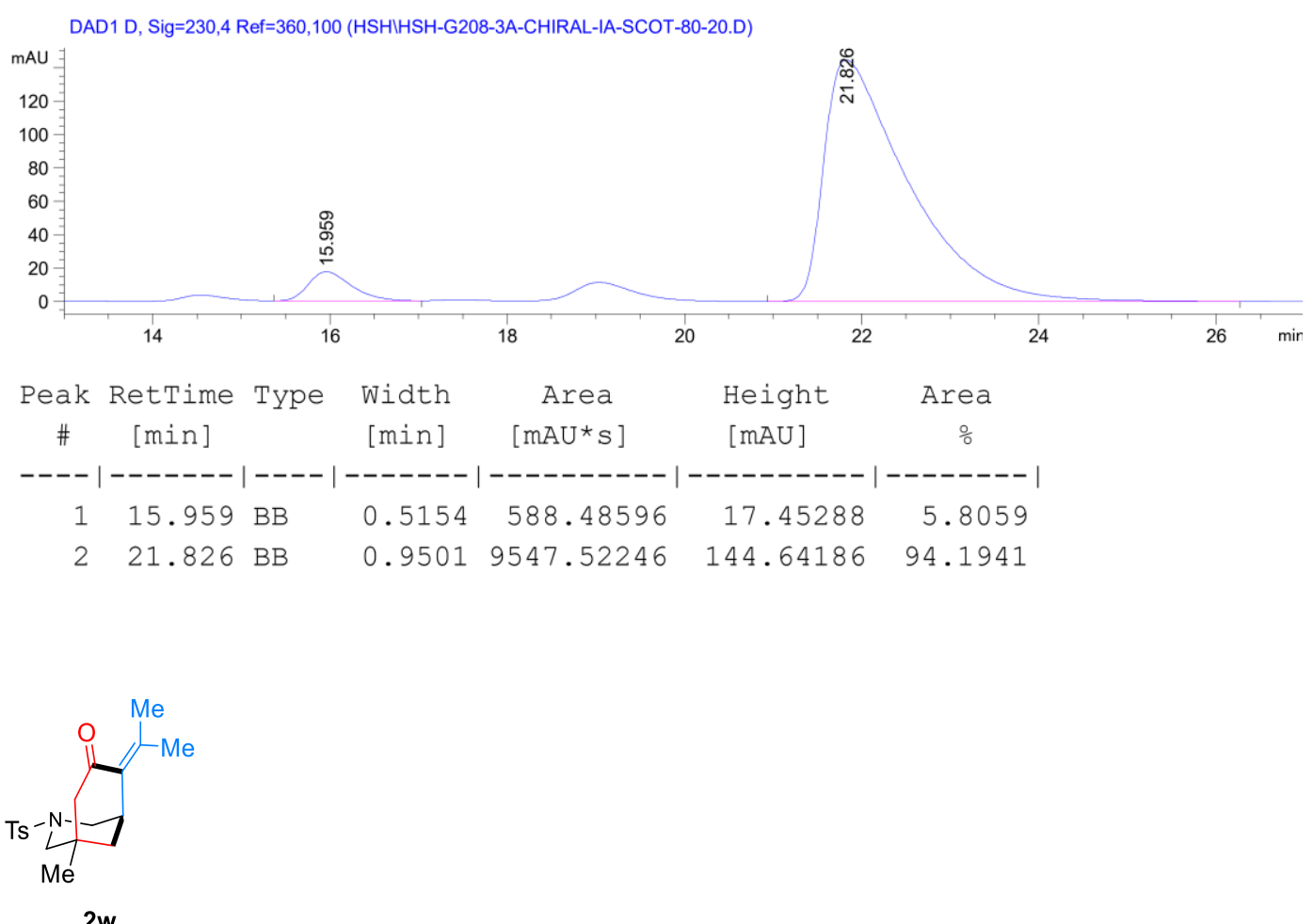

$2 \mathbf{w}(6.6 \mathrm{mg})$ was isolated as a white solid in $19 \%$ yield.

$\mathrm{Rf}=0.1$, Hexane/ EtOAc 2:1.

Mp: $134.0-134.6^{\circ} \mathrm{C}$. 
${ }^{1} \mathrm{H}$ NMR (400 MHz, Benzene- $\left.\boldsymbol{d}_{6}\right) \delta 7.79-7.70(\mathrm{~m}, 2 \mathrm{H}), 6.91-6.80(\mathrm{~m}, 2 \mathrm{H}), 3.60-3.53(\mathrm{~m}, 1 \mathrm{H}), 3.40(\mathrm{dt}, J=11.2,2.1$ $\mathrm{Hz}, 1 \mathrm{H}), 2.71-2.63(\mathrm{~m}, 1 \mathrm{H}), 2.34(\mathrm{dd}, J=16.6,3.1 \mathrm{~Hz}, 1 \mathrm{H}), 2.27(\mathrm{dd}, J=10.9,2.3 \mathrm{~Hz}, 1 \mathrm{H}), 2.11-2.05(\mathrm{~m}, 4 \mathrm{H}), 1.88$ (s, 3H), 1.77 (dd, $J=16.6,1.4 \mathrm{~Hz}, 1 \mathrm{H}), 1.44(\mathrm{~d}, J=31.1 \mathrm{~Hz}, 1 \mathrm{H}), 1.35(\mathrm{~s}, 3 \mathrm{H}), 1.15$ (ddt, J=13.0, 3.6, $2.0 \mathrm{~Hz}, 1 \mathrm{H}), 0.86$ $(\mathrm{dt}, J=13.0,3.2 \mathrm{~Hz}, 1 \mathrm{H}), 0.41(\mathrm{~s}, 3 \mathrm{H})$.

${ }^{13} \mathrm{C}$ NMR (101 MHz, $\left.\mathrm{CDCl}_{3}\right) \delta$ 197.2, 143.3, 143.1, 134.8, 133.0, 129.5, 128.6, 57.1, 53.8, 51.1, 38.3, 34.1, 33.0, 26.5, $23.4,21.8,21.1$.

HRMS ESI Calcd for $\mathrm{C}_{19} \mathrm{H}_{26} \mathrm{NO}_{3} \mathrm{~S}[\mathrm{M}+\mathrm{H}]^{+}: 348.1628$, Found: 348.1624 .

IR (KBr): 2957, 2871, 1999, 1680, 1616, 1560, 1446, 1348, 1164, 1093, $1054 \mathrm{~cm}^{-1}$.

Chiral HPLC (Chiralpak IF, Hexane/Isopropanol $=70 / 30$, flow rate $=1.0 \mathrm{~mL} / \mathrm{min}, 254 \mathrm{~nm}$ ), $\mathrm{t}_{\text {minor }}=18.8 \mathrm{~min}, \mathrm{t}_{\mathrm{major}}=$ $29.4 \min .[\alpha]^{25}=-27.2\left(c 0.2, \mathrm{CHCl}_{3}\right)$ at $87: 13 \mathrm{er}$.

\section{Racemic sample $2 \mathbf{w}$}

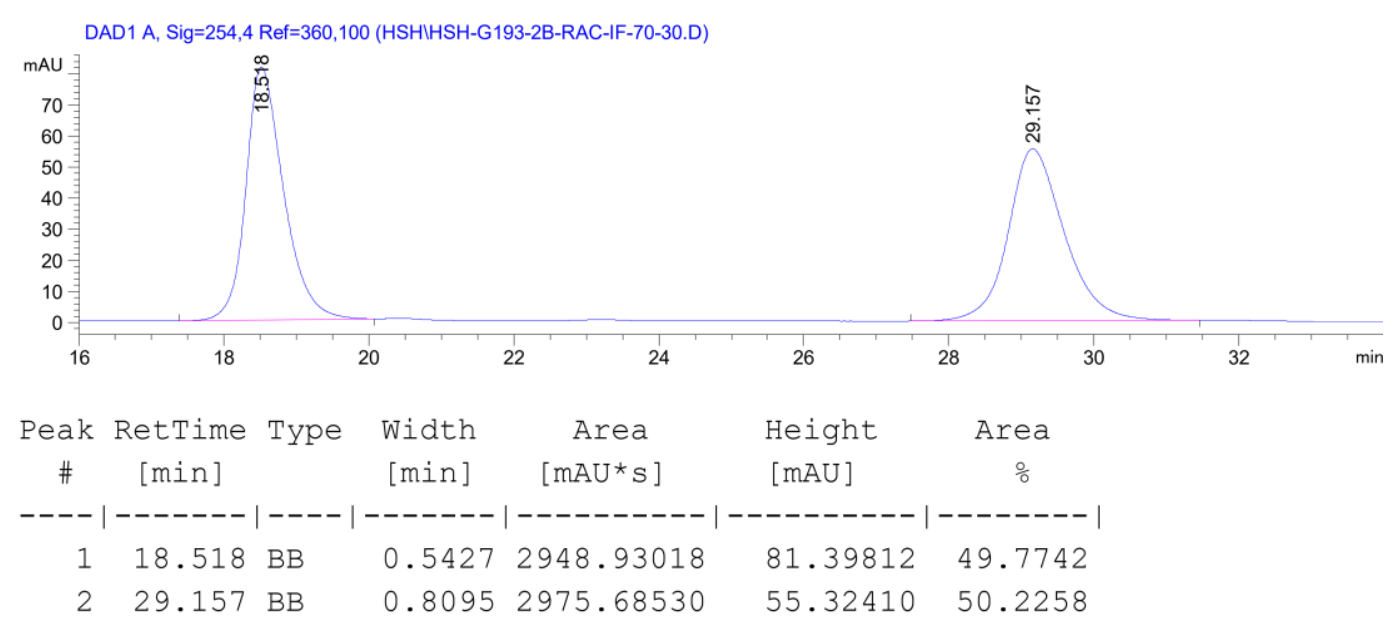

\section{Enantiomeric sample 2w}

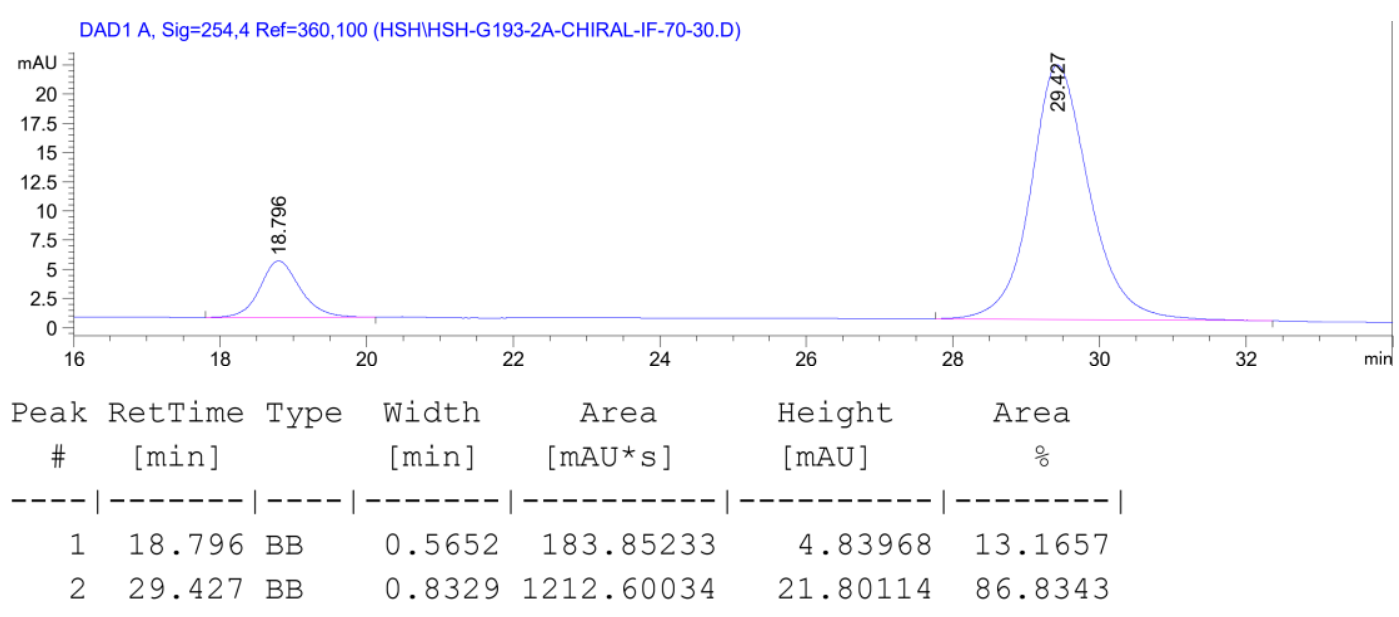




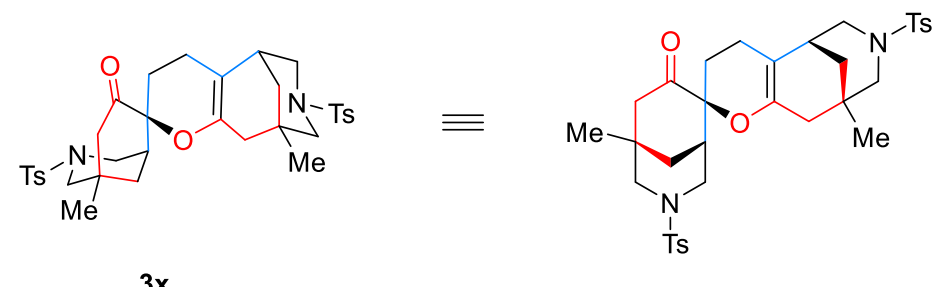

$3 \mathbf{x}(25 \mathrm{mg}$ ) was isolated as an oil in 79\% yield. $d r>20: 1$.

$\mathrm{Rf}=0.2$, Hexane/ EtOAc 4:1.

${ }^{1} \mathrm{H}$ NMR (400 MHz, Chloroform-d) $\delta 7.63-7.58(\mathrm{~m}, 2 \mathrm{H}), 7.58-7.54(\mathrm{~m}, 2 \mathrm{H}), 7.31(\mathrm{dd}, J=8.1,4.3 \mathrm{~Hz}, 4 \mathrm{H}), 3.78-$ $3.69(\mathrm{~m}, 1 \mathrm{H}), 3.64-3.55(\mathrm{~m}, 1 \mathrm{H}), 3.50-3.38(\mathrm{~m}, 2 \mathrm{H}), 2.80-2.72(\mathrm{~m}, 1 \mathrm{H}), 2.63(\mathrm{dd}, J=15.2,1.2 \mathrm{~Hz}, 1 \mathrm{H}), 2.42(\mathrm{~s}, 6 \mathrm{H})$, $2.37-2.19(\mathrm{~m}, 6 \mathrm{H}), 2.14-1.93(\mathrm{~m}, 7 \mathrm{H}), 1.68-1.59(\mathrm{~m}, 2 \mathrm{H}), 1.31(\mathrm{dt}, J=13.4,3.0 \mathrm{~Hz}, 1 \mathrm{H}), 1.20(\mathrm{dd}, J=12.4,2.6 \mathrm{~Hz}$, $1 \mathrm{H}), 0.99(\mathrm{~s}, 3 \mathrm{H}), 0.89(\mathrm{~s}, 3 \mathrm{H})$.

${ }^{13} \mathrm{C}$ NMR (101 MHz, CDCl$)$ ) $203.4,146.0,143.7,143.1,134.74$ 132.7, 129.7, 129.6, 127.8, 126.8, 104.4, 78.3, 58.2, $57.3,50.0,47.6,47.5,39.8,37.4,35.3,35.0,34.7,34.4,31.3,27.6,26.5,21.9,21.5,21.5,20.5$.

HRMS ESI Calcd for $\mathrm{C}_{34} \mathrm{H}_{43} \mathrm{~N}_{2} \mathrm{O}_{6} \mathrm{~S}_{2}[\mathrm{M}+\mathrm{H}]^{+}: 639.2557$, Found: 639.2559.

IR (KBr): 2953, 2924, 2838, 1723, 1693, 1598, 1457, 1344, 1166, 1091 $\mathrm{cm}^{-1}$.

Chiral HPLC (Chiralpak IA, Hexane/Isopropanol $=80 / 20$, flow rate $=1.0 \mathrm{~mL} / \mathrm{min}, 230 \mathrm{~nm}$ ) , $\mathrm{t}_{\text {minor }}=37.1 \mathrm{~min}, \mathrm{t}_{\text {major }}=$ $47.4 \min .[\alpha]^{21.5} \mathrm{D}=-3.7\left(\mathrm{c} 0.9, \mathrm{CHCl}_{3}\right)$ at $99: 1 \mathrm{er}$.

\section{Racemic sample $3 x$}

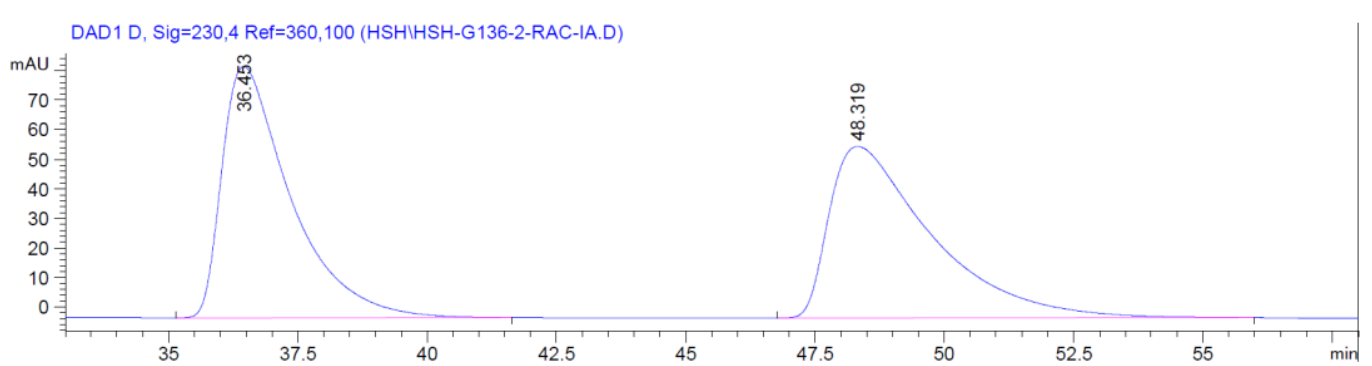

\begin{tabular}{|c|c|c|c|c|c|c|}
\hline $\begin{array}{c}\text { Peak } \\
\#\end{array}$ & $\begin{array}{l}\text { RetTime } \\
\text { [min] }\end{array}$ & Type & $\begin{array}{l}\text { Width } \\
{[\mathrm{min}]}\end{array}$ & $\begin{array}{c}\text { Area } \\
{\left[\mathrm{mAU}^{\star} \mathrm{s}\right]}\end{array}$ & $\begin{array}{l}\text { Height } \\
\text { [mAU] }\end{array}$ & $\begin{array}{c}\text { Area } \\
\quad \frac{\circ}{0}\end{array}$ \\
\hline & & & -- & -------- & -------- & --- \\
\hline 1 & 36.453 & BB & 1.3452 & 7846.10059 & 85.13452 & 643 \\
\hline 2 & 48.319 & $\mathrm{BB}$ & 1.9235 & 7984.05273 & 58.09049 & 50.4357 \\
\hline
\end{tabular}

\section{Enantiomeric sample $3 x$}




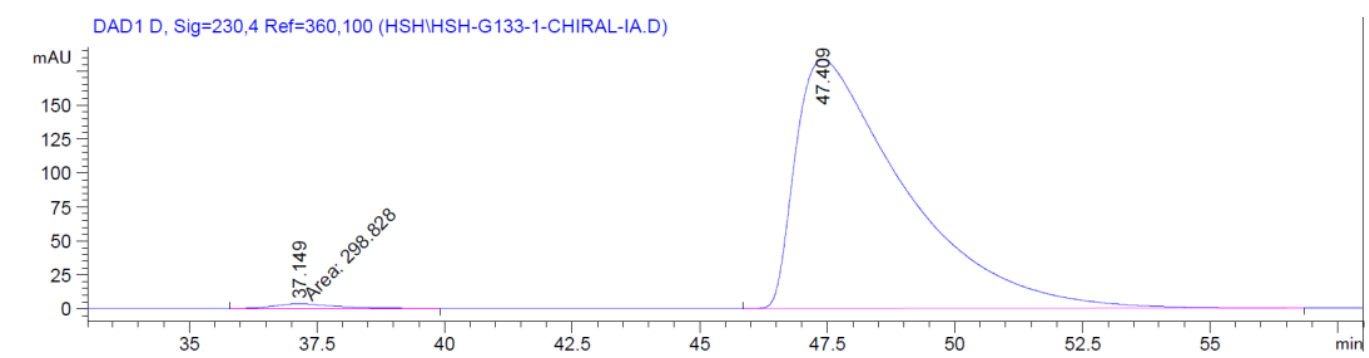

\begin{tabular}{|c|c|c|c|c|c|c|}
\hline $\begin{array}{c}\text { Peak } \\
\quad \#\end{array}$ & $\begin{array}{c}\text { RetTime } \\
\text { [min] }\end{array}$ & Type & $\begin{array}{l}\text { Width } \\
\text { [min] }\end{array}$ & $\begin{array}{c}\text { Area } \\
{\left[\mathrm{mAU}^{*} \mathrm{~s}\right]}\end{array}$ & $\begin{array}{l}\text { Height } \\
{[\mathrm{mAU}]}\end{array}$ & $\begin{array}{c}\text { Area } \\
\frac{\circ}{0}\end{array}$ \\
\hline-1 & |------- & & $-0----$ & $-----------\mid$ & --------- & $-------\mid$ \\
\hline 1 & 37.149 & MM & 1.4763 & 298.82822 & 3.37353 & 1.0826 \\
\hline 2 & 47.409 & $\mathrm{BB}$ & 2.1175 & $2.73040 e 4$ & 183.12833 & 98.9174 \\
\hline
\end{tabular}

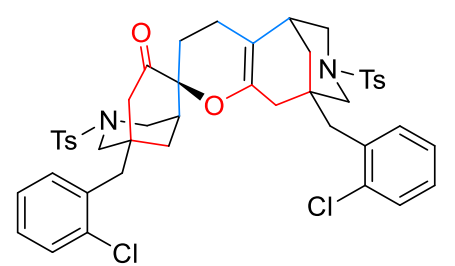

$3 y$

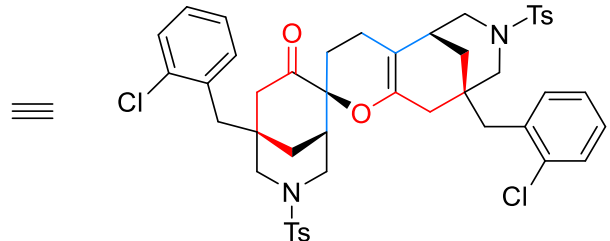

Ts

3y (36 mg) was isolated as a foam in 84\% yield. $d r>20: 1$.

$\mathrm{Rf}=0.5$, Hexane/ EtOAc 2:1.

${ }^{1} \mathrm{H}$ NMR (400 MHz, Chloroform-d) $87.61-7.53(\mathrm{~m}, 4 \mathrm{H}), 7.41-7.34(\mathrm{~m}, 2 \mathrm{H}), 7.34-7.28(\mathrm{~m}, 4 \mathrm{H}), 7.23-7.15(\mathrm{~m}, 5 \mathrm{H})$, $7.15-7.09(\mathrm{~m}, 1 \mathrm{H}), 3.76-3.67(\mathrm{~m}, 1 \mathrm{H}), 3.61(\mathrm{dt}, J=11.3,2.0 \mathrm{~Hz}, 1 \mathrm{H}), 3.54(\mathrm{dq}, J=11.3,2.2 \mathrm{~Hz}, 2 \mathrm{H}), 2.87-2.64(\mathrm{~m}$, $6 \mathrm{H}), 2.45-2.35(\mathrm{~m}, 8 \mathrm{H}), 2.33-2.14(\mathrm{~m}, 7 \mathrm{H}), 2.03-1.84(\mathrm{~m}, 4 \mathrm{H}), 1.74-1.67(\mathrm{~m}, 1 \mathrm{H}), 1.45(\mathrm{dt}, J=13.8,3.1 \mathrm{~Hz}, 1 \mathrm{H})$, $1.36(\mathrm{dd}, J=12.5,2.9 \mathrm{~Hz}, 1 \mathrm{H})$.

${ }^{13} \mathrm{C}$ NMR $\left(101 \mathrm{MHz}, \mathrm{CDCl}_{3}\right)$ $\delta$ 203.0, 145.9, 143.7, 143.2, 135.0, 134.9, 134.5, 134.4, 133.8, 132.7, 132.6, 132.4, 129.9, 129.7, 129.6, 128.3, 128.1, 127.8, 126.9, 126.5, 126.4, 104.3, 78.7, 57.1, 55.9, 47.7, 47.63, 47.59, 43.2, 42.6, 39.6, 37.6, 36.3, 35.3, 34.9, 34.4, 33.3, 21.9, 21.5, 21.5, 20.4.

HRMS ESI Calcd for $\mathrm{C}_{46} \mathrm{H}_{49} \mathrm{Cl}_{2} \mathrm{~N}_{2} \mathrm{O}_{6} \mathrm{~S}_{2}[\mathrm{M}+\mathrm{H}]^{+}:$859.2404, Found: 859.2407.

IR (KBr): 2924, 2854, 1723, 1695, 1597, 1445, 1167, 1090, 1052, $1037 \mathrm{~cm}^{-1}$.

Ciral HPLC (Chiralpak IA, Hexane/Isopropanol $=70 / 30$, flow rate $=1.0 \mathrm{~mL} / \mathrm{min}, 230 \mathrm{~nm}$ ) , $\mathrm{t}_{\text {minor }}=30 \mathrm{~min}, \mathrm{t}_{\text {major }}=57.4$ $\min .[\alpha]^{21.5}=-11.1\left(c 0.9, \mathrm{CHCl}_{3}\right)$ at 99:1 er.

\section{Racemic sample 3y}




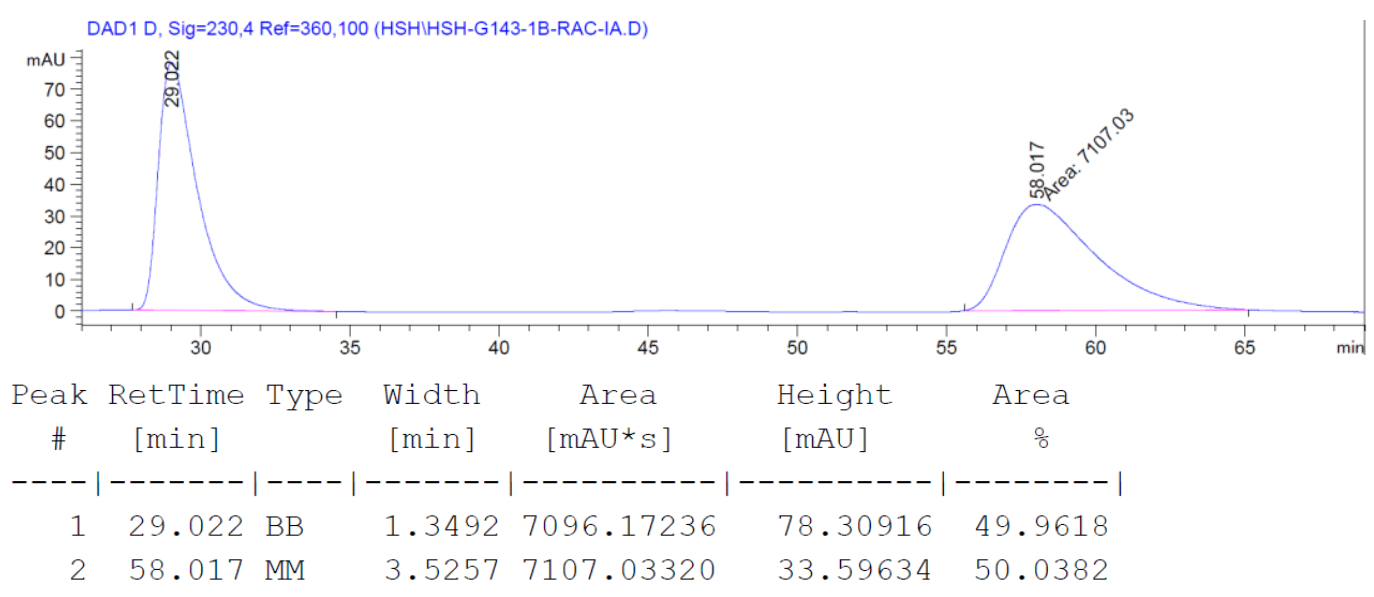

\section{Enantiomeric sample 3y}
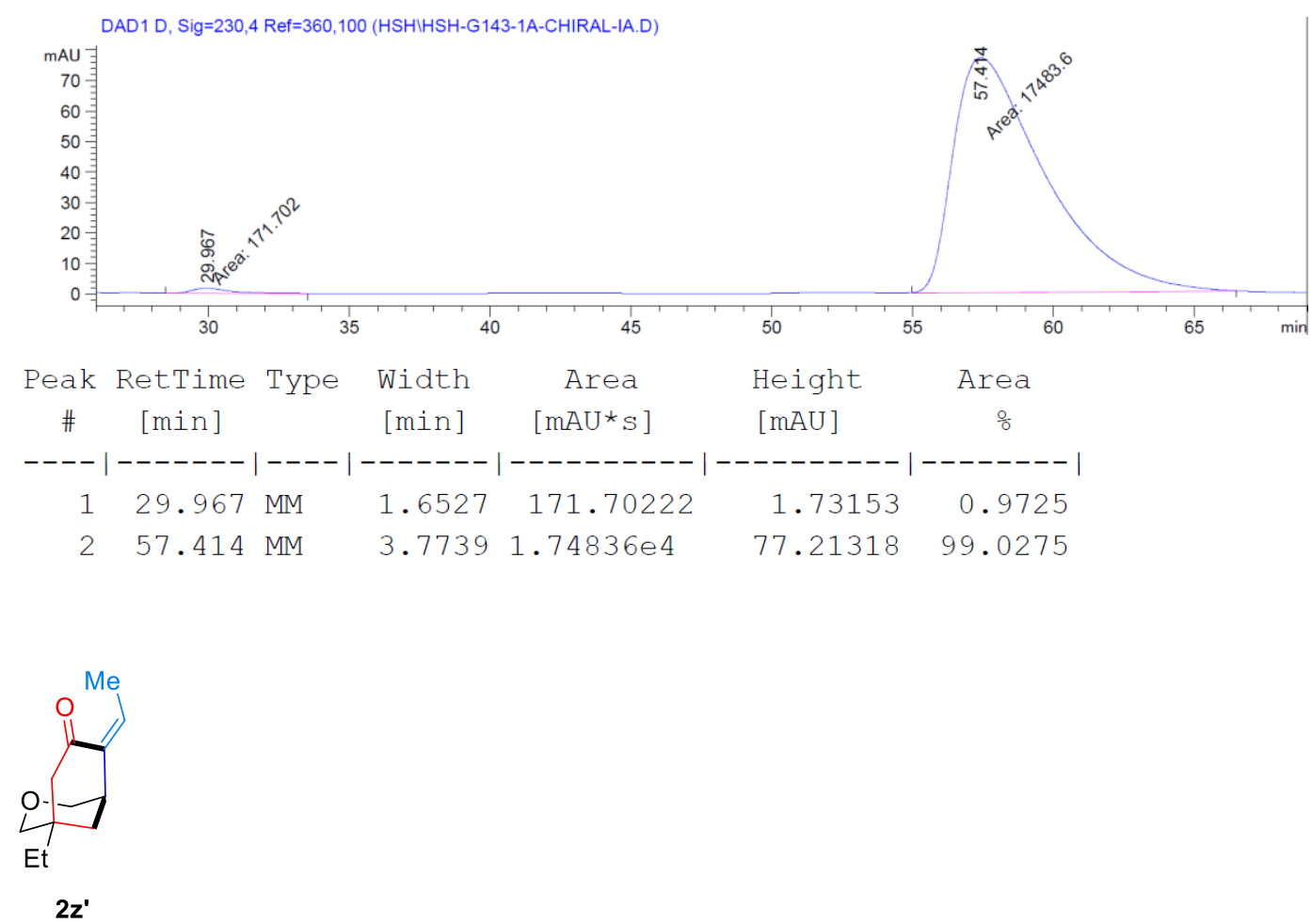

$\mathbf{2 z}$ (5.8 mg) was isolated as an oil in $30 \%$ yield after running the reaction at room temperature for 3 days.

$\mathrm{Rf}=0.4$, Hexane/ EtOAc 3:1.

${ }^{1} \mathrm{H}$ NMR (400 MHz, Benzene-d6) $\delta 5.39(\mathrm{q}, J=7.2 \mathrm{~Hz}, 1 \mathrm{H}), 3.61(\mathrm{dtd}, J=10.7,2.2,0.8 \mathrm{~Hz}, 1 \mathrm{H}), 3.47$ (dd, $J=11.0,2.8$ $\mathrm{Hz}, 1 \mathrm{H}), 3.13(\mathrm{dd}, J=10.6,1.7 \mathrm{~Hz}, 1 \mathrm{H}), 2.93(\mathrm{dd}, J=10.9,1.6 \mathrm{~Hz}, 1 \mathrm{H}), 2.36(\mathrm{dd}, J=16.4,3.0 \mathrm{~Hz}, 1 \mathrm{H}), 2.06(\mathrm{~d}, J=7.2$ $\mathrm{Hz}, 3 \mathrm{H}), 2.01(\mathrm{tt}, J=3.4,1.9 \mathrm{~Hz}, 1 \mathrm{H}), 1.90(\mathrm{dd}, J=16.4,1.6 \mathrm{~Hz}, 1 \mathrm{H}), 1.42(\mathrm{dq}, J=12.6,2.8 \mathrm{~Hz}, 1 \mathrm{H}), 1.17(\mathrm{dt}, J=12.6$, $3.1 \mathrm{~Hz}, 1 \mathrm{H}), 0.72(\mathrm{qd}, J=7.5,7.1,1.7 \mathrm{~Hz}, 2 \mathrm{H}), 0.45(\mathrm{t}, J=7.6 \mathrm{~Hz}, 3 \mathrm{H})$.

${ }^{13}$ C NMR (101 MHz, C6 $\left.\mathrm{D}_{6}\right) \delta 199.2,140.3,132.3,77.8,74.3,51.4,42.7,36.8,36.8,30.7,15.4,6.9$.

HRMS ESI Calcd for $\mathrm{C}_{12} \mathrm{H}_{19} \mathrm{O}_{2}[\mathrm{M}+\mathrm{H}]^{+}:$195.1380, Found: 195.1385.

IR (KBr): 2962, 2915, 2852, 1781, 1689, 1632, 1463, 1447, 1383, 1226, 1110, $1079 \mathrm{~cm}^{-1}$. 
Chiral HPLC (Chiralpak IF, Hexane/Isopropanol $=90 / 10$, flow rate $=1.0 \mathrm{~mL} / \mathrm{min}, 230 \mathrm{~nm}$ ), $t_{\text {minor }}=18.6 \mathrm{~min}, \mathrm{t}_{\mathrm{major}}=$ $14.1 \min .[\alpha]^{21.5}=-137.5\left(c 0.6, \mathrm{CHCl}_{3}\right)$ at 99.7:0.3 er.

\section{Racemic sample $2 z^{\prime}$}

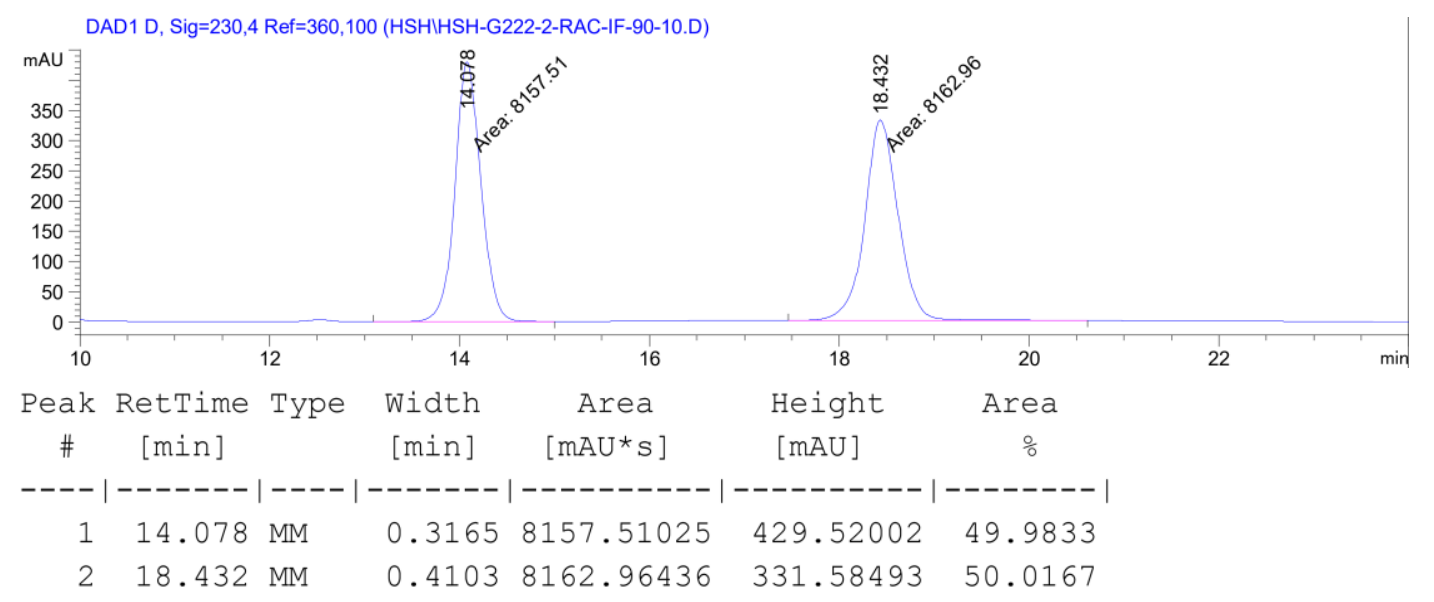

\section{Enantiomeric sample 2z'}
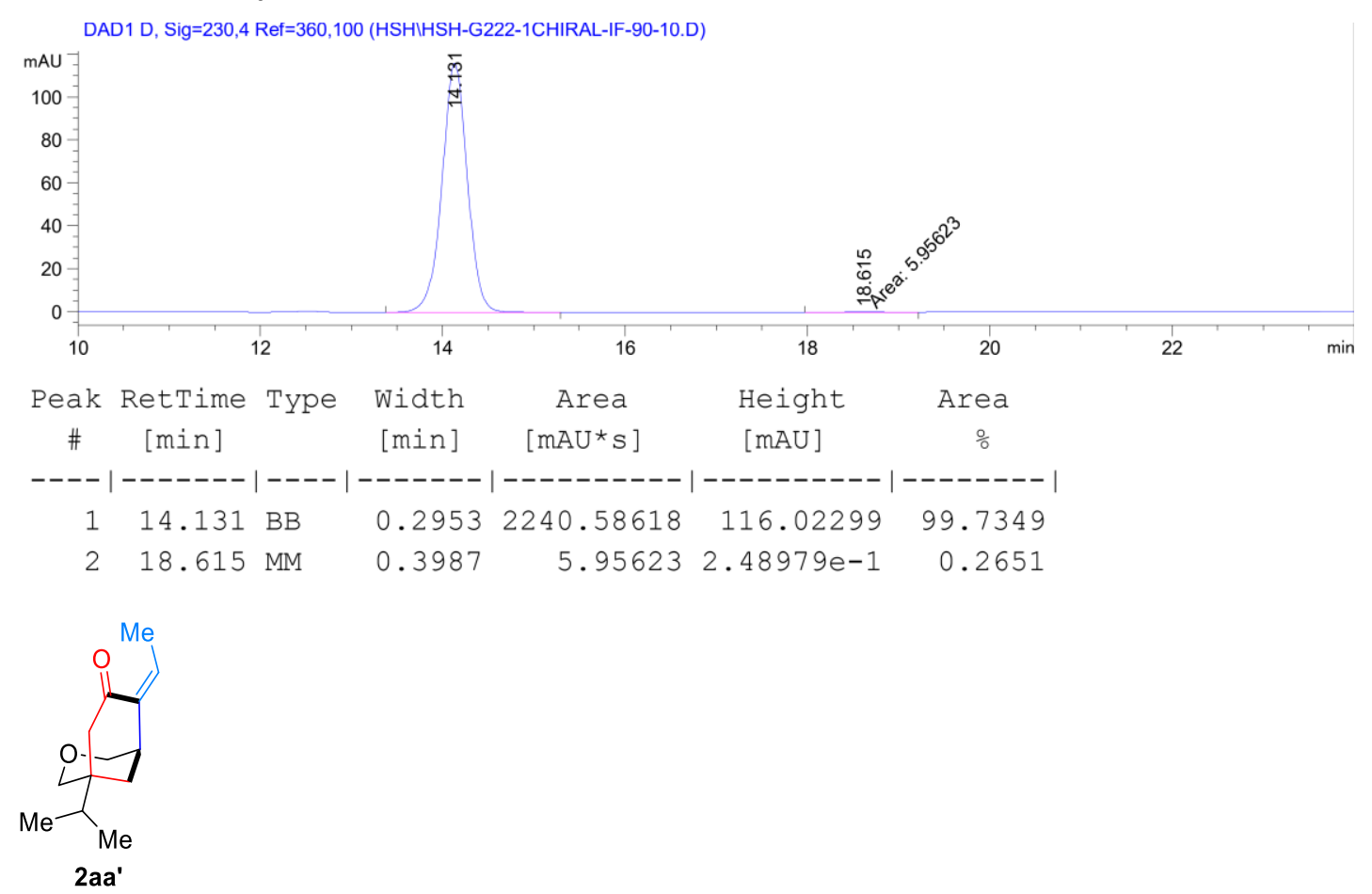

2aa' (6.1 mg) was isolated as an oil in 29\% yield after running the reaction at room temperature for 3 days. $\mathrm{Rf}=0.4$, Hexane/ EtOAc 3:1.

${ }^{1} \mathrm{H}$ NMR (400 MHz, Benzene-d 6$) \delta 5.39(\mathrm{qd}, J=7.2,0.6 \mathrm{~Hz}, 1 \mathrm{H}), 3.67-3.58(\mathrm{~m}, 2 \mathrm{H}), 3.12(\mathrm{dd}, J=10.6,1.7 \mathrm{~Hz}, 1 \mathrm{H})$, $2.99(\mathrm{dd}, J=11.0,1.6 \mathrm{~Hz}, 1 \mathrm{H}), 2.42(\mathrm{dd}, J=16.4,3.1 \mathrm{~Hz}, 1 \mathrm{H}), 2.07(\mathrm{~d}, J=7.1 \mathrm{~Hz}, 3 \mathrm{H}), 2.03(\mathrm{t}, J=1.8 \mathrm{~Hz}, 1 \mathrm{H}), 1.94(\mathrm{dd}$, 
$J=16.4,1.7 \mathrm{~Hz}, 1 \mathrm{H}), 1.51(\mathrm{dt}, J=12.7,2.8 \mathrm{~Hz}, 2 \mathrm{H}), 1.20(\mathrm{dt}, J=12.7,3.2 \mathrm{~Hz}, 1 \mathrm{H}), 0.99-0.89(\mathrm{~m}, 1 \mathrm{H}), 0.57-0.52(\mathrm{~m}$, $1 \mathrm{H}), 0.50(\mathrm{~d}, J=6.9 \mathrm{~Hz}, 3 \mathrm{H}), 0.46(\mathrm{~d}, J=6.9 \mathrm{~Hz}, 3 \mathrm{H})$.

${ }^{13}$ C NMR (101 MHz, C6 $\left.D_{6}\right) \delta 199.4,140.5,132.1,76.7,74.1,48.2,42.6,39.2,35.2,33.8,16.4,16.3,15.4$.

HRMS ESI Calcd for $\mathrm{C}_{13} \mathrm{H}_{21} \mathrm{O}_{2}[\mathrm{M}+\mathrm{H}]^{+}:$209.1536, Found: 209.1538.

IR (KBr): 2960, 2862, 1780, 1689, 1632, 1468, 1445, 1383, 1370, 1227, 1133, $1086 \mathrm{~cm}^{-1}$.

Chiral HPLC (Chiralpak IF, Hexane/Isopropanol $=90 / 10$, flow rate $=0.8 \mathrm{~mL} / \mathrm{min}, 230 \mathrm{~nm}$ ), $\mathrm{t}_{\mathrm{minor}}=23.9 \mathrm{~min}, \mathrm{t}_{\mathrm{major}}=$ $22.6 \min .[\alpha]^{21.5}=-166.8\left(c 0.5, \mathrm{CHCl}_{3}\right)$ at 99.5:0.5 er.

\section{Racemic sample 2aa'}

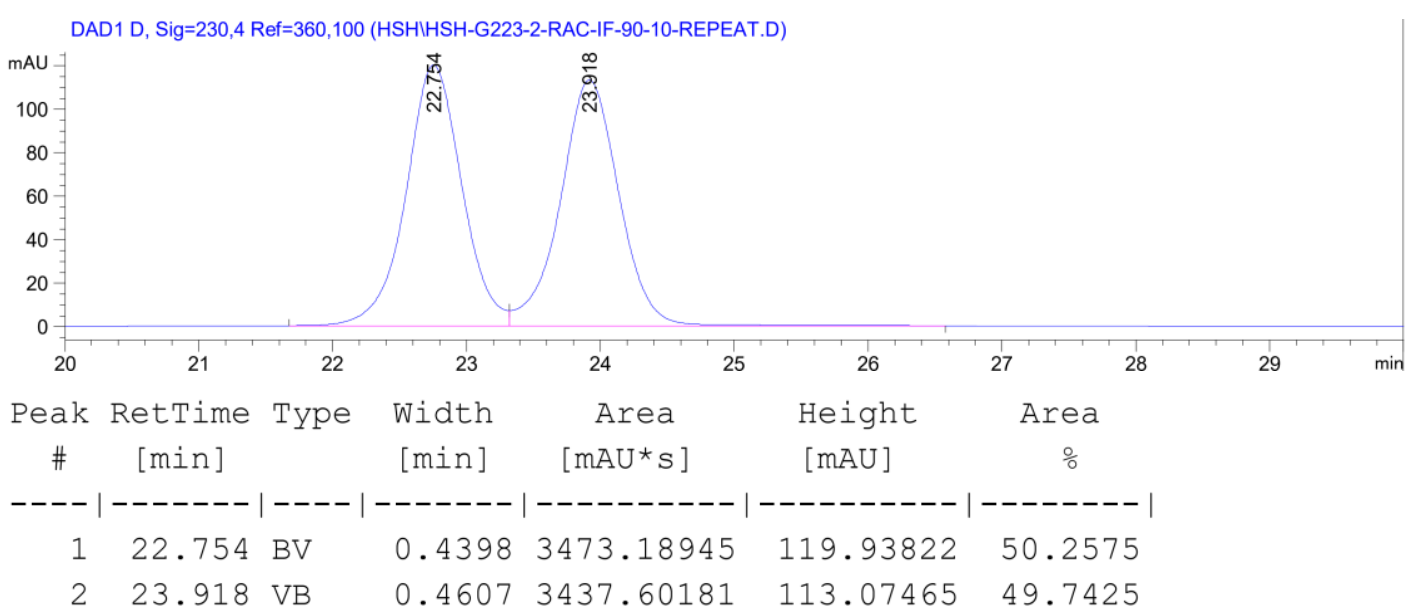

\section{Enantiomeric sample 2aa'}

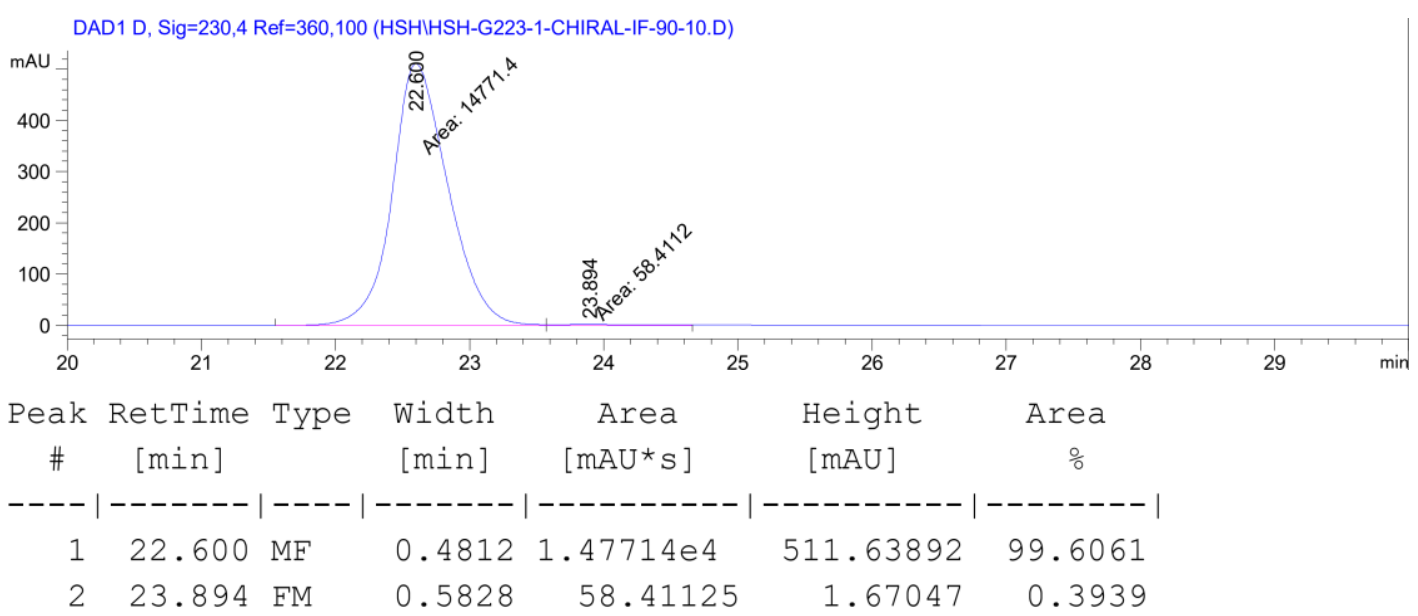




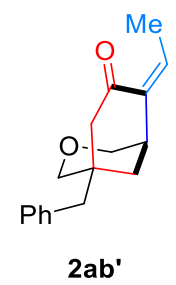

2ab' (9.7 mg) was isolated as an oil in 38\% yield after running the reaction at room temperature for 3 days. $\mathrm{Rf}=0.24$, Hexane/ EtOAc 4:1.

${ }^{1} \mathrm{H}$ NMR (400 MHz, Benzene- $\left.d_{6}\right) \delta 7.13-7.02(\mathrm{~m}, 3 \mathrm{H}), 6.79-6.73(\mathrm{~m}, 2 \mathrm{H}), 5.30(\mathrm{q}, J=7.2 \mathrm{~Hz}, 1 \mathrm{H}), 3.59-3.53(\mathrm{~m}$, 1H), 3.50 (dd, $J=10.9,2.8 \mathrm{~Hz}, 1 \mathrm{H}), 3.05$ (ddd, $J=13.9,10.8,1.7 \mathrm{~Hz}, 2 \mathrm{H}), 2.39$ (dd, $J=16.3,3.1 \mathrm{~Hz}, 1 \mathrm{H}), 2.05-1.95$ $(\mathrm{m}, 6 \mathrm{H}), 1.92(\mathrm{~d}, J=13.2 \mathrm{~Hz}, 1 \mathrm{H}), 1.46-1.40(\mathrm{~m}, 1 \mathrm{H}), 1.31-1.25(\mathrm{~m}, 1 \mathrm{H})$.

${ }^{13} \mathrm{C}$ NMR (101 MHz, C6 6 ) $\delta$ 198.8, 139.9, 136.3, 132.4, 130.6, 128.3, 126.7, 78.0, 74.1, 52.0, 44.5, 42.6, 37.9, 36.7, 15.3.

HRMS ESI Calcd for $\mathrm{C}_{17} \mathrm{H}_{21} \mathrm{O}_{2}[\mathrm{M}+\mathrm{H}]^{+}:$257.1536, Found: 257.1540 .

IR (KBr): 2912, 2850, 1778, 1689, 1631, 1495, 1452, 1383, 1226, 1201, 1097, $1079 \mathrm{~cm}^{-1}$.

Chiral HPLC (Chiralpak IF, Hexane/Isopropanol $=90 / 10$, flow rate $=1.0 \mathrm{~mL} / \mathrm{min}, 230 \mathrm{~nm}$ ), $t_{\text {minor }}=24.6 \mathrm{~min}, \mathrm{t}_{\mathrm{major}}=$ $19.3 \min .[\alpha]^{21.5}{ }_{D}=-152.5\left(c 0.3, \mathrm{CHCl}_{3}\right)$ at 99.5:0.5 er.

\section{Racemic sample 2ab'}

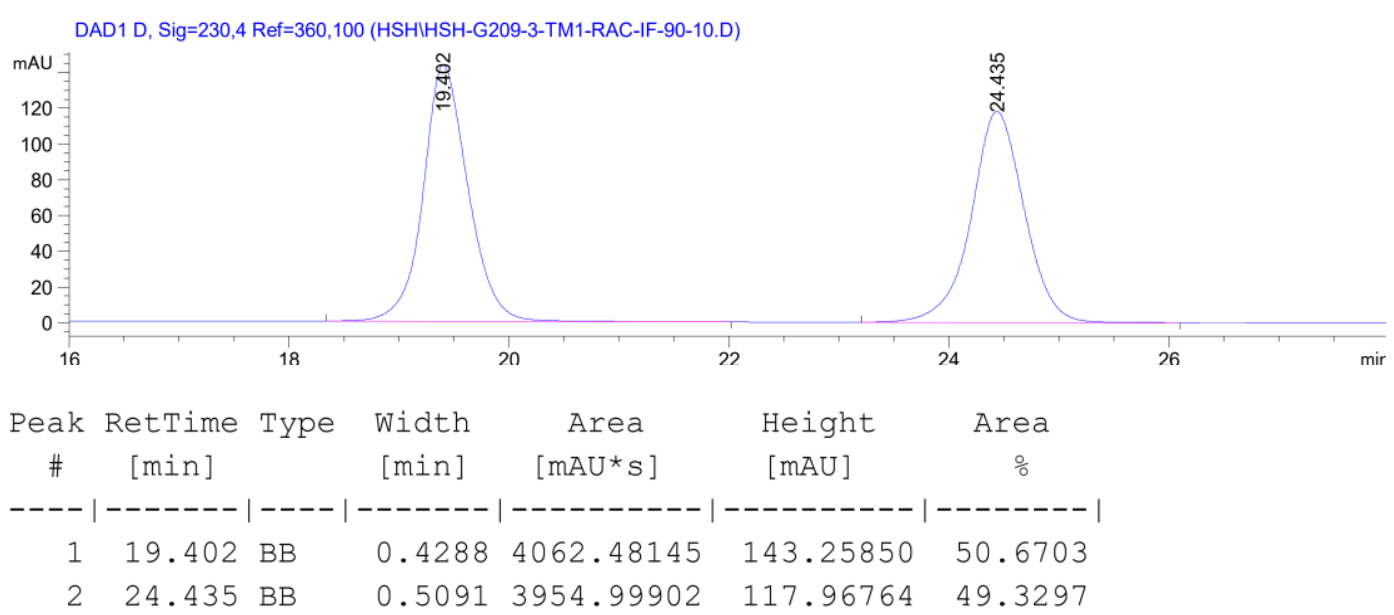

\section{Enantiomeric sample 2ab'}



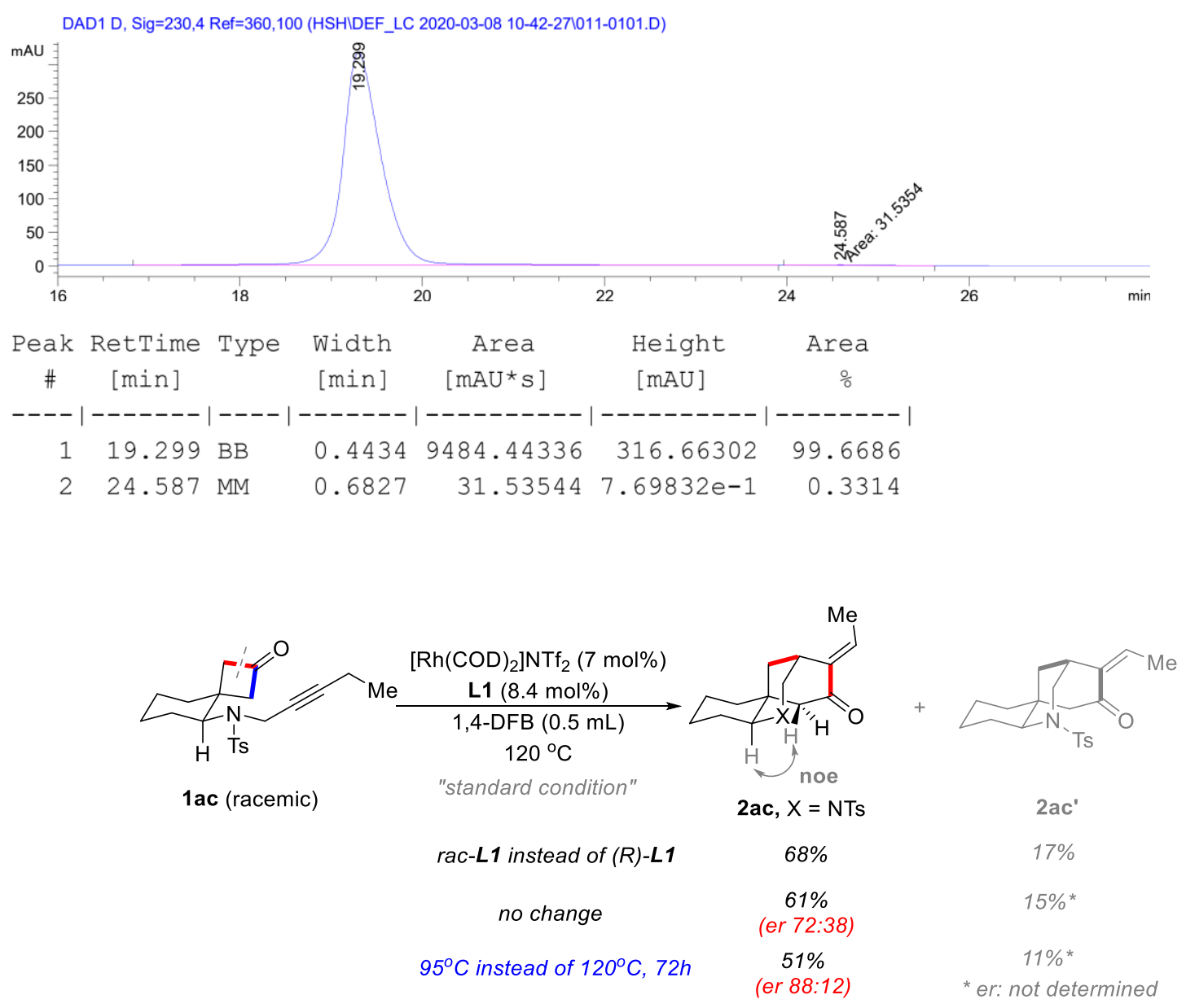

Compound 2ac. A flame dried $8 \mathrm{~mL}$ tube was charged with the substrate 1ac (37.3 mg, $0.1 \mathrm{mmol}, 1$ equiv) and $(R)$ (-)-DTBM-segphos (L1, $9.9 \mathrm{mg}, 0.0084 \mathrm{mmol}, 8.4 \mathrm{~mol} \%$ ). The tube was then transferred to a nitrogen-filled glove box. After addition of $\left[\mathrm{Rh}(\mathrm{COD})_{2}\right] \mathrm{NTf}_{2}(4.2 \mathrm{mg}, 0.007 \mathrm{mmol}, 7 \mathrm{~mol} \%)$ and 1,4-diflorobenzene $(0.5 \mathrm{~mL})$, the tube was sealed and taken out of glove box. The reaction was stirred at $95^{\circ} \mathrm{C}$ in an oil bath for $72 \mathrm{~h}$, before it was then cooled to room temperature. The product was directly purified by silica gel flash chromatography to yield $2 \mathrm{ac}(19 \mathrm{mg})$ as a foam in $51 \%$ yield. The yield of compound $2 \mathrm{ac}^{\prime}$ was determined by ${ }^{1} \mathrm{H}$ NMR.

Note: The pure sample of $2 a c^{\prime}$ cannot be obtained. Alternative method to confirm the structure of $2 a c^{\prime}$ is isomerization of $\mathbf{2 a c}$ ' to $\mathbf{2 a c}$ under PTS/CDCl 3 condition (For detail of the procedure, see isomerization of compound

$\left.2 a b^{\prime}\right)$.

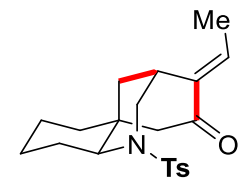

2ac

$\mathrm{Rf}=0.33$, Hexane/ EtOAc 2:1. 
${ }^{1} \mathrm{H}$ NMR (400 MHz, Benzene-d $\left.\mathbf{d}_{6}\right) \delta 7.81-7.71(\mathrm{~m}, 2 \mathrm{H}), 6.92-6.82(\mathrm{~m}, 2 \mathrm{H}), 6.55(\mathrm{q}, J=7.3 \mathrm{~Hz}, 1 \mathrm{H}), 3.73(\mathrm{dd}, J=12.1$, $4.4 \mathrm{~Hz}, 1 \mathrm{H}), 3.05$ (dt, J = 11.9, $2.2 \mathrm{~Hz}, 1 \mathrm{H}), 2.93$ (dd, J = 12.0, $2.3 \mathrm{~Hz}, 1 \mathrm{H}), 2.49$ (t, J=3.0 Hz, $1 \mathrm{H}), 2.32(\mathrm{dd}, J=18.2,3.1$ $\mathrm{Hz}, 1 \mathrm{H}), 1.88(\mathrm{~s}, 4 \mathrm{H}), 1.79(\mathrm{~d}, J=18.1 \mathrm{~Hz}, 1 \mathrm{H}), 1.68(\mathrm{dt}, J=13.5,3.3 \mathrm{~Hz}, 1 \mathrm{H}), 1.61-1.40(\mathrm{~m}, 3 \mathrm{H}), 1.16(\mathrm{~d}, J=7.3 \mathrm{~Hz}$, $3 \mathrm{H}), 1.11-1.05(\mathrm{~m}, 1 \mathrm{H}), 1.05-0.88(\mathrm{~m}, 3 \mathrm{H}), 0.82(\mathrm{td}, J=13.3,4.0 \mathrm{~Hz}, 1 \mathrm{H}), 0.76-0.67(\mathrm{~m}, 1 \mathrm{H})$.

${ }^{13}$ C NMR (101 MHz, C6 $\left.D_{6}\right) \delta$ 195.0, 143.0, 139.4, 137.2, 132.3, 129.4, 128.3, 61.0, 54.6, 45.6, 39.5, 35.4, 31.3, 29.3, 25.3, 25.0, 21.1, 21.0, 12.6 .

HRMS ESI Calcd for $\mathrm{C}_{21} \mathrm{H}_{28} \mathrm{NO}_{3} \mathrm{~S}[\mathrm{M}+\mathrm{H}]^{+}: 374.1784$, Found: 374.1784 .

IR (KBr): 2927, 2865, 1742, 1688, 1623, 1598, 1447, 1375, 1336, 1304, 1270, 1236, 1164, 1090, $1022,1009 \mathrm{~cm}^{-1}$.

Chiral HPLC (Chiralpak IF, Hexane/Isopropanol $=70 / 30$, flow rate $=1.0 \mathrm{~mL} / \mathrm{min}, 230 \mathrm{~nm}$ ), $t_{\text {minor }}=35.2 \mathrm{~min}, \mathrm{t}_{\mathrm{major}}=$ $32.2 \min .[\alpha]^{21.5}=-86.5\left(c 0.8, \mathrm{CHCl}_{3}\right)$ at 88:12 er.

\section{Racemic sample 2ac}
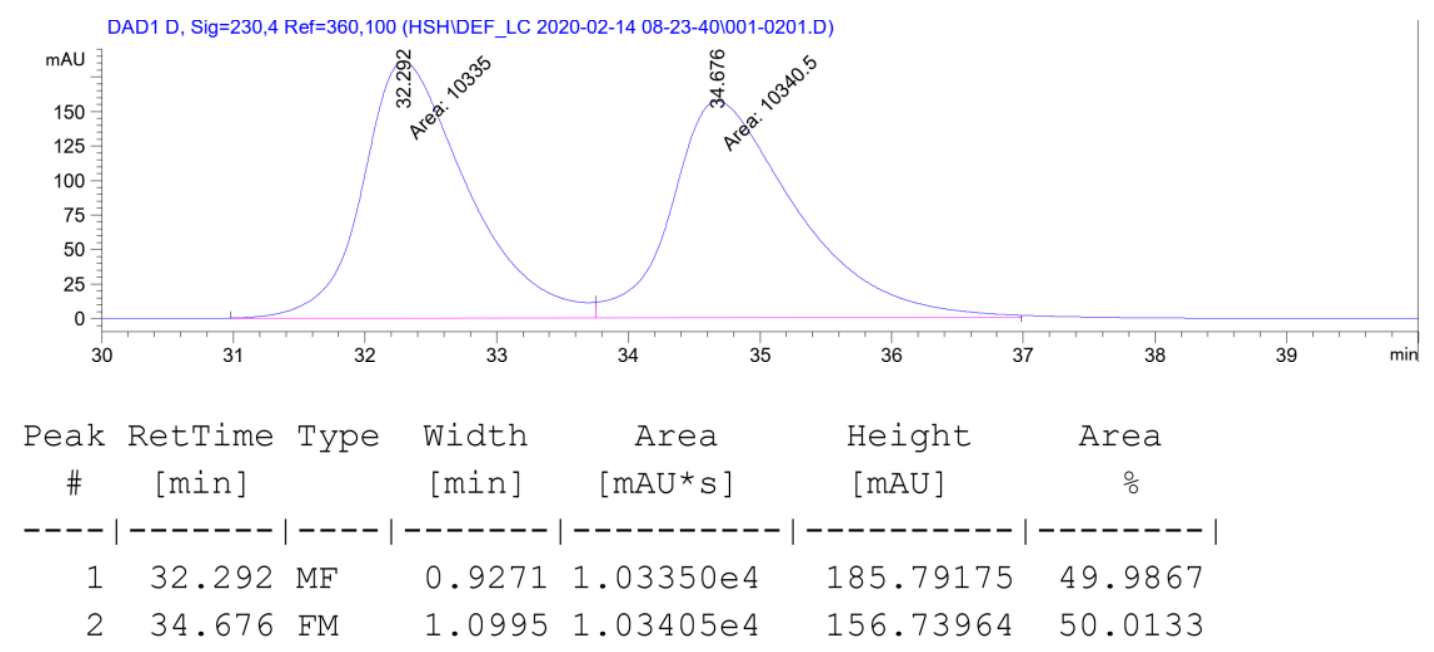

\section{Enantiomeric sample 2ac}

DAD1 D, Sig=230,4 Ref=360, 100 (HSHIDEF_LC 2020-02-25 23-37-541006-0301.D)

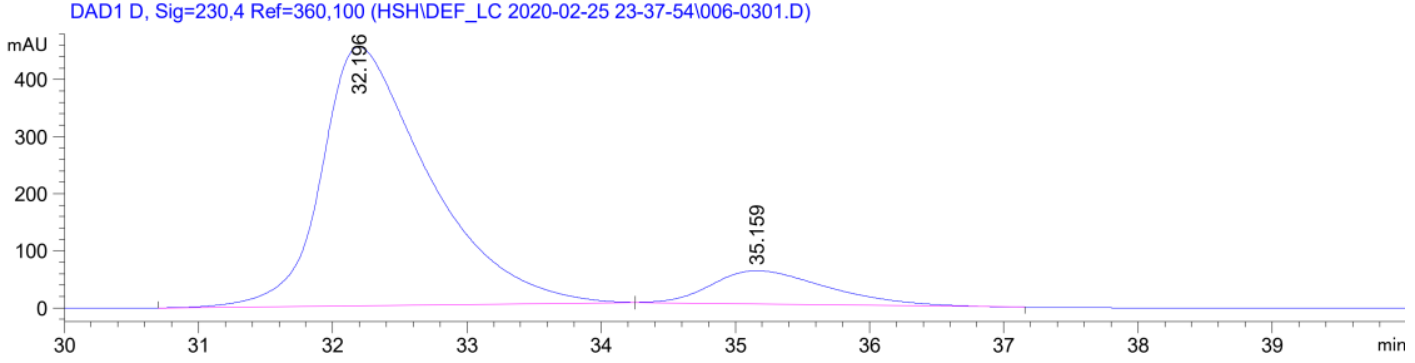

\begin{tabular}{|c|c|c|c|c|c|c|}
\hline $\begin{array}{c}\text { Peak } \\
\#\end{array}$ & $\begin{array}{c}\text { RetTime } \\
\text { [min] }\end{array}$ & Type & $\begin{array}{l}\text { Width } \\
\text { [min] }\end{array}$ & $\begin{array}{c}\text { Area } \\
{\left[\mathrm{mAU}^{*} \mathrm{~s}\right]}\end{array}$ & $\begin{array}{l}\text { Height } \\
\text { [mAU] }\end{array}$ & $\begin{array}{c}\text { Area } \\
\frac{\circ}{0}\end{array}$ \\
\hline-1 & |------- & & ---- & ---------- & --------- & ------- \\
\hline 1 & 32.196 & BB & 0.8196 & $2.52217 e 4$ & 452.97824 & 87.8520 \\
\hline 2 & 35.159 & $\mathrm{BB}$ & 0.9159 & 3487.58984 & 57.96559 & 12.1480 \\
\hline
\end{tabular}




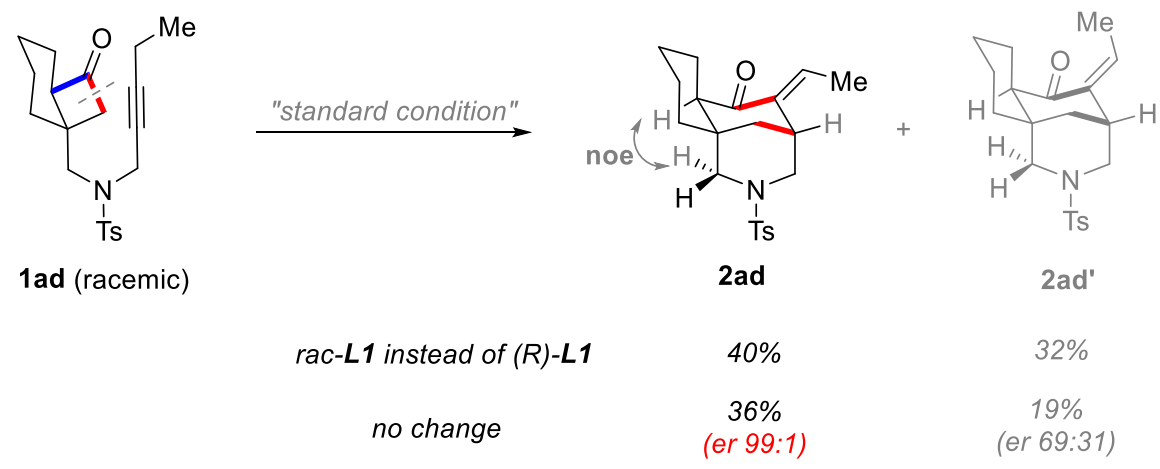

(3)

Compound 2ad and 2ad'. A flame dried $8 \mathrm{~mL}$ tube was charged with the substrate 1ad ( $37.3 \mathrm{mg}, 0.1 \mathrm{mmol}, 1$ equiv) and (R)-(-)-DTBM-segphos (L1, $9.9 \mathrm{mg}, 0.0084 \mathrm{mmol}, 8.4 \mathrm{~mol} \%$ ). The tube was then transferred to a nitrogen-filled glove box. After addition of [Rh(COD) 2$] \mathrm{NTf}_{2}(4.2 \mathrm{mg}, 0.007 \mathrm{mmol}, 7 \mathrm{~mol} \%)$ and 1,4-diflorobenzene $(0.5 \mathrm{~mL})$, the tube was sealed and taken out of glove box. The reaction was stirred at $120^{\circ} \mathrm{C}$ in an oil bath for $48 \mathrm{~h}$, before it was then cooled to room temperature. The products were directly purified by silica gel flash chromatography to yield 2ad $(13.5 \mathrm{mg})$ as a foam in $36 \%$ yield, and $\mathbf{2 a d}$ ' $(7.1 \mathrm{mg}$ ) as a foam in $19 \%$ yield.

Note: The structure of $\mathbf{2 a d}$ was confirmed by 2D NMR. The E/Z isomers of $\mathbf{2 a d} / \mathbf{2 a d}$ ' was confirmed by hydrogenation, which gave same product 2ad-1 (See blew for detail).

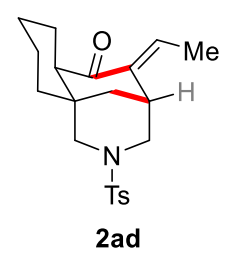

$\mathrm{Rf}=0.33$, Hexane/ EtOAc 2:1.

${ }^{1} \mathrm{H}$ NMR $\left(400 \mathrm{MHz}\right.$, Methylene Chloride- $\left.d_{2}\right) \delta 7.63-7.57(\mathrm{~m}, 2 \mathrm{H}), 7.41-7.34(\mathrm{~m}, 2 \mathrm{H}), 6.55(\mathrm{q}, J=7.1 \mathrm{~Hz}, 1 \mathrm{H}), 3.50$ (ddt, $J=10.6,3.2,1.8 \mathrm{~Hz}, 1 \mathrm{H}), 3.36(\mathrm{dt}, J=11.3,2.1 \mathrm{~Hz}, 1 \mathrm{H}), 3.19(\mathrm{p}, J=3.0 \mathrm{~Hz}, 1 \mathrm{H}), 2.47(\mathrm{~s}, 3 \mathrm{H}), 2.41(\mathrm{dd}, J=10.7$, $2.1 \mathrm{~Hz}, 1 \mathrm{H}$ ), 2.31 (ddd, $J=13.3,3.8,1.9 \mathrm{~Hz}, 1 \mathrm{H}), 2.26$ (ddd, $J=12.5,4.8,2.0 \mathrm{~Hz}, 1 \mathrm{H}$ ), 2.13 (d, $J=11.3 \mathrm{~Hz}, 1 \mathrm{H}$ ), 1.80 (d, $J=7.2 \mathrm{~Hz}, 4 \mathrm{H}), 1.67-1.60(\mathrm{~m}, 1 \mathrm{H}), 1.60-1.53(\mathrm{~m}, 1 \mathrm{H}), 1.49-1.22(\mathrm{~m}, 6 \mathrm{H}), 1.18-1.08(\mathrm{~m}, 2 \mathrm{H})$.

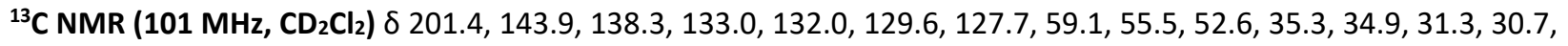
27.8, 25.9, 21.3, 20.5, 13.1.

HRMS ESI Calcd for $\mathrm{C}_{21} \mathrm{H}_{28} \mathrm{NO}_{3} \mathrm{~S}[\mathrm{M}+\mathrm{H}]^{+}: 374.1784$, Found: 374.1791.

IR (KBr): 2931, 2856, 1742, 1686, 1629, 1598, 1453, 1351, 1340, 1224, 1166, 1090, $1039 \mathrm{~cm}^{-1}$.

Chiral HPLC (Chiralpak IF, Hexane/Isopropanol $=60 / 40$, flow rate $=1.0 \mathrm{~mL} / \mathrm{min}, 230 \mathrm{~nm}$ ), $\mathrm{t}_{\text {minor }}=17.8 \mathrm{~min}, \mathrm{t}_{\text {major }}=$ $24.6 \min .[\alpha]^{21.5} \mathrm{D}=-104.9\left(c 1.1, \mathrm{CHCl}_{3}\right)$ at 99:1 er. 


\section{Racemic sample 2 ad}

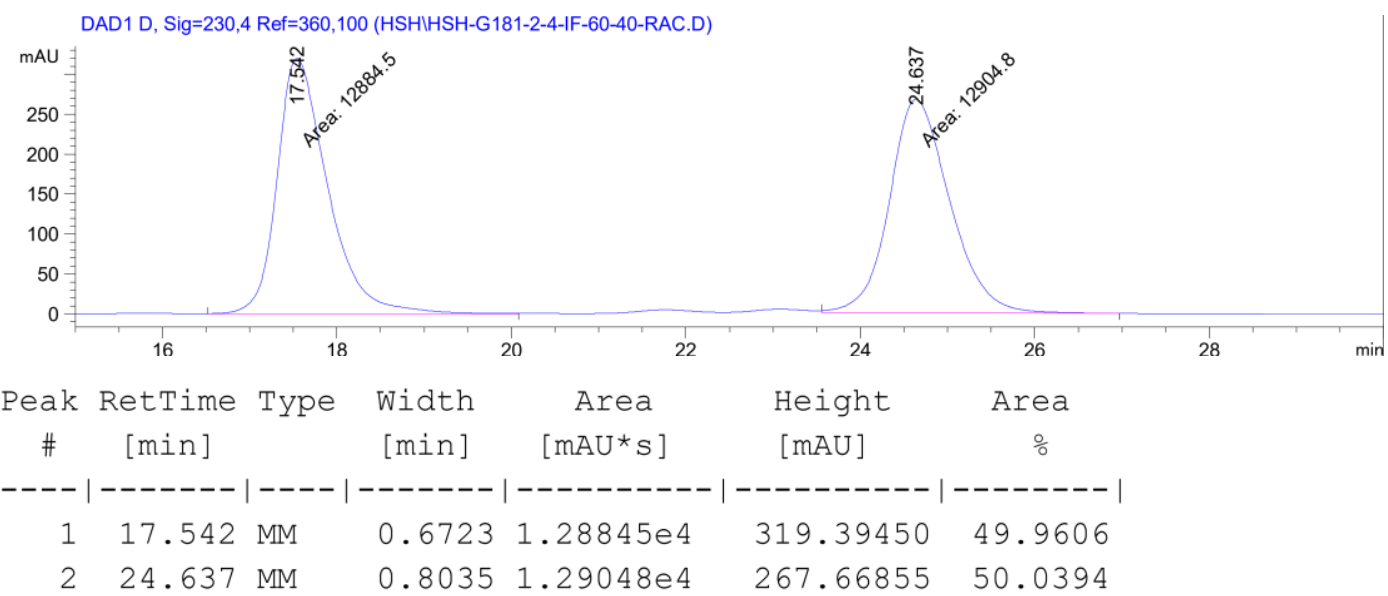

\section{Enantiomeric sample 2ad}
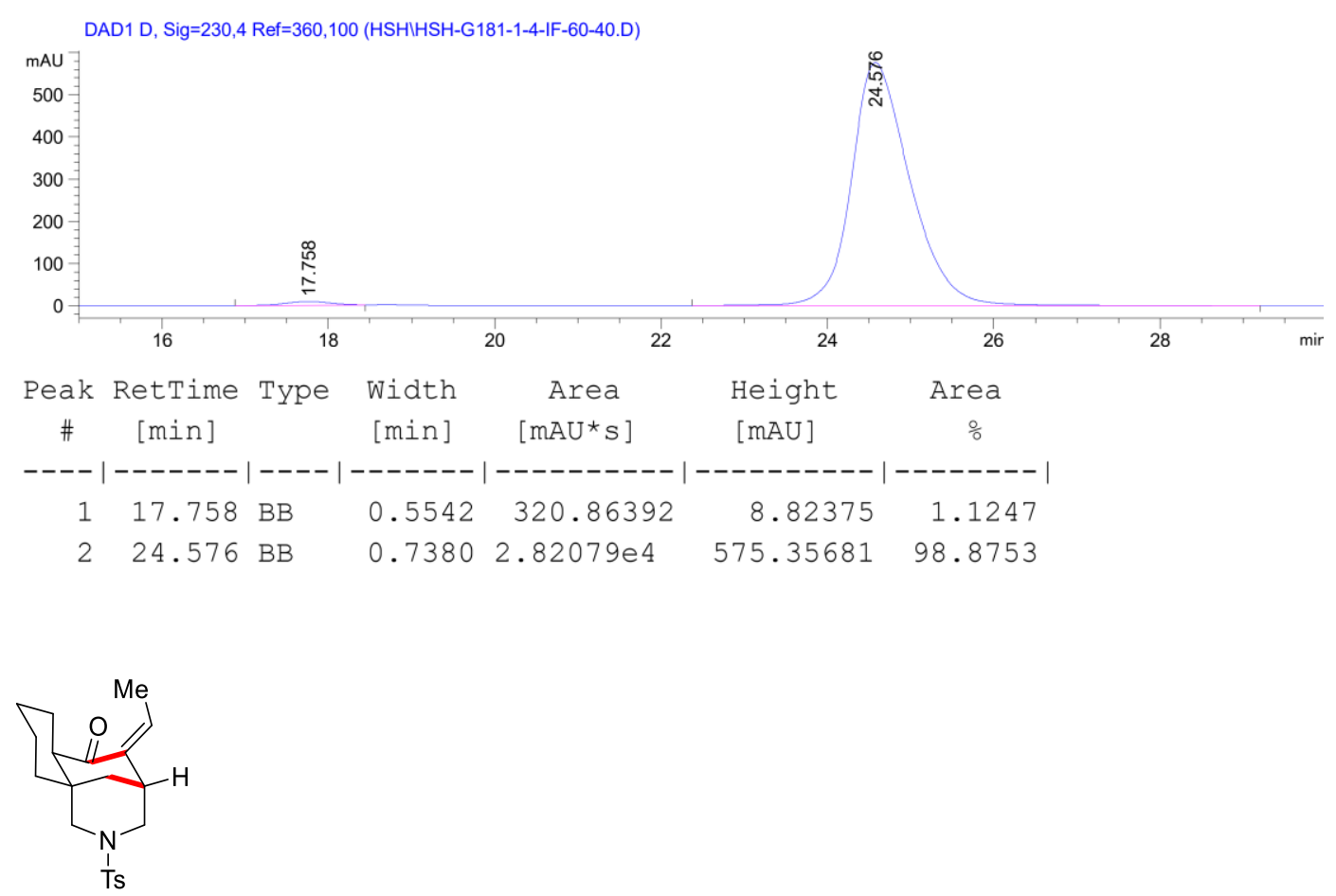

2ad'

$\mathrm{Rf}=0.4$, Hexane/ EtOAc 2:1.

${ }^{1} \mathrm{H}$ NMR (400 MHz, Benzene- $\left.d_{6}\right) \delta 7.84-7.76(\mathrm{~m}, 2 \mathrm{H}), 6.89-6.84(\mathrm{~m}, 2 \mathrm{H}), 5.39(\mathrm{q}, J=7.1 \mathrm{~Hz}, 1 \mathrm{H}), 3.58(\mathrm{ddt}, J=10.8$, 3.3, $1.8 \mathrm{~Hz}, 1 \mathrm{H}), 3.33(\mathrm{dt}, J=11.2,2.1 \mathrm{~Hz}, 1 \mathrm{H}), 2.32(\mathrm{dd}, J=10.8,2.1 \mathrm{~Hz}, 1 \mathrm{H}), 2.15-2.08(\mathrm{~m}, 3 \mathrm{H}), 1.92(\mathrm{~d}, J=7.1 \mathrm{~Hz}$, $3 \mathrm{H}), 1.88(\mathrm{~s}, 3 \mathrm{H}), 1.81(\mathrm{dt}, J=13.1,1.9 \mathrm{~Hz}, 1 \mathrm{H}), 1.56-1.49(\mathrm{~m}, 1 \mathrm{H}), 1.42-1.34(\mathrm{~m}, 2 \mathrm{H}), 1.17-1.09(\mathrm{~m}, 1 \mathrm{H}), 1.06-$ $0.92(\mathrm{~m}, 2 \mathrm{H}), 0.89-0.75(\mathrm{~m}, 2 \mathrm{H}), 0.57(\mathrm{td}, J=13.4,4.3 \mathrm{~Hz}, 1 \mathrm{H}), 0.49(\mathrm{dt}, J=13.1,2.4 \mathrm{~Hz}, 1 \mathrm{H})$.

${ }^{13}$ C NMR (126 MHz, C $\left.6 D_{6}\right) \delta 200.7,143.3,137.8,134.8,134.1,129.5,128.7,58.9,56.9,52.7,40.8,35.5,35.1,31.9$, 27.0, 25.7, 21.1, 20.6, 15.3.

HRMS ESI Calcd for $\mathrm{C}_{21} \mathrm{H}_{28} \mathrm{NO}_{3} \mathrm{~S}[\mathrm{M}+\mathrm{H}]^{+}: 374.1784$, Found: 374.1781. 
IR (KBr): 2928, 2855, 1743, 1688, 1635, 1597, 1454, 1346, 1262, 1165, 1090, $1041 \mathrm{~cm}^{-1}$.

Chiral HPLC (Chiralpak IF, Hexane/Isopropanol $=60 / 40$, flow rate $=1.0 \mathrm{~mL} / \mathrm{min}, 230 \mathrm{~nm}$ ), $\mathrm{t}_{\text {minor }}=13.3 \mathrm{~min}, \mathrm{t}_{\text {major }}=$ $18.6 \min .[\alpha]^{21.5} \mathrm{D}=-28.5\left(c 0.3, \mathrm{CHCl}_{3}\right)$ at 69:31 er.

\section{Racemic sample 2ad'}

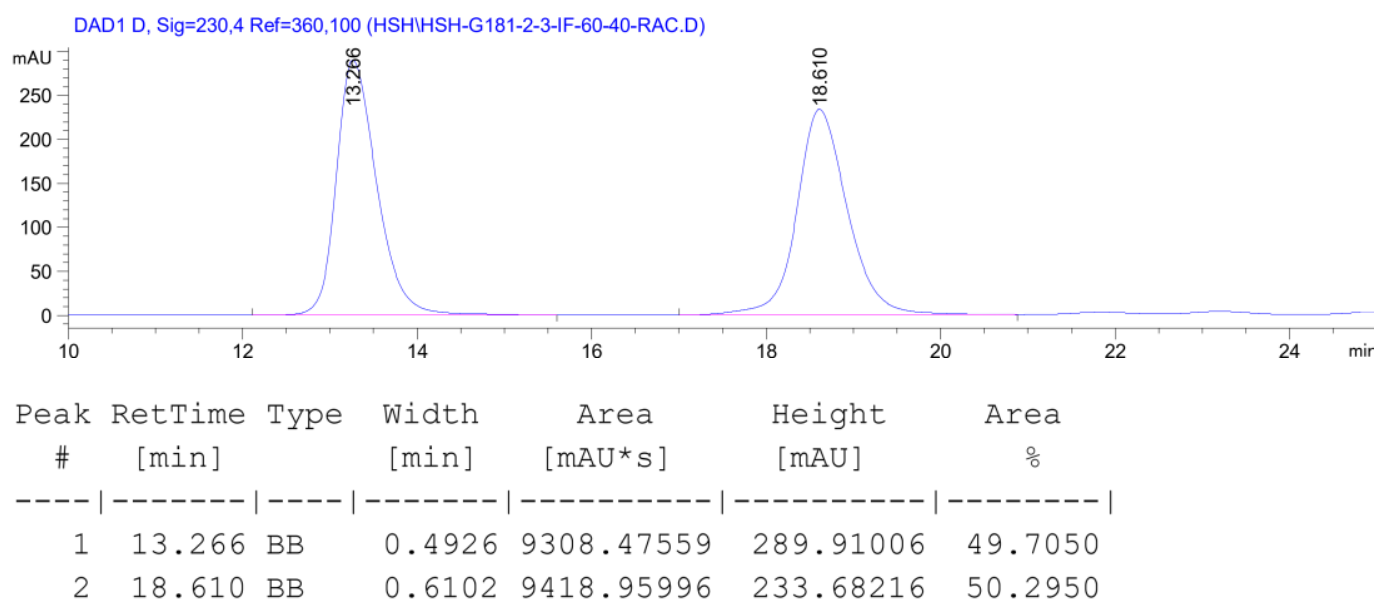

\section{Enantiomeric sample 2ad'}
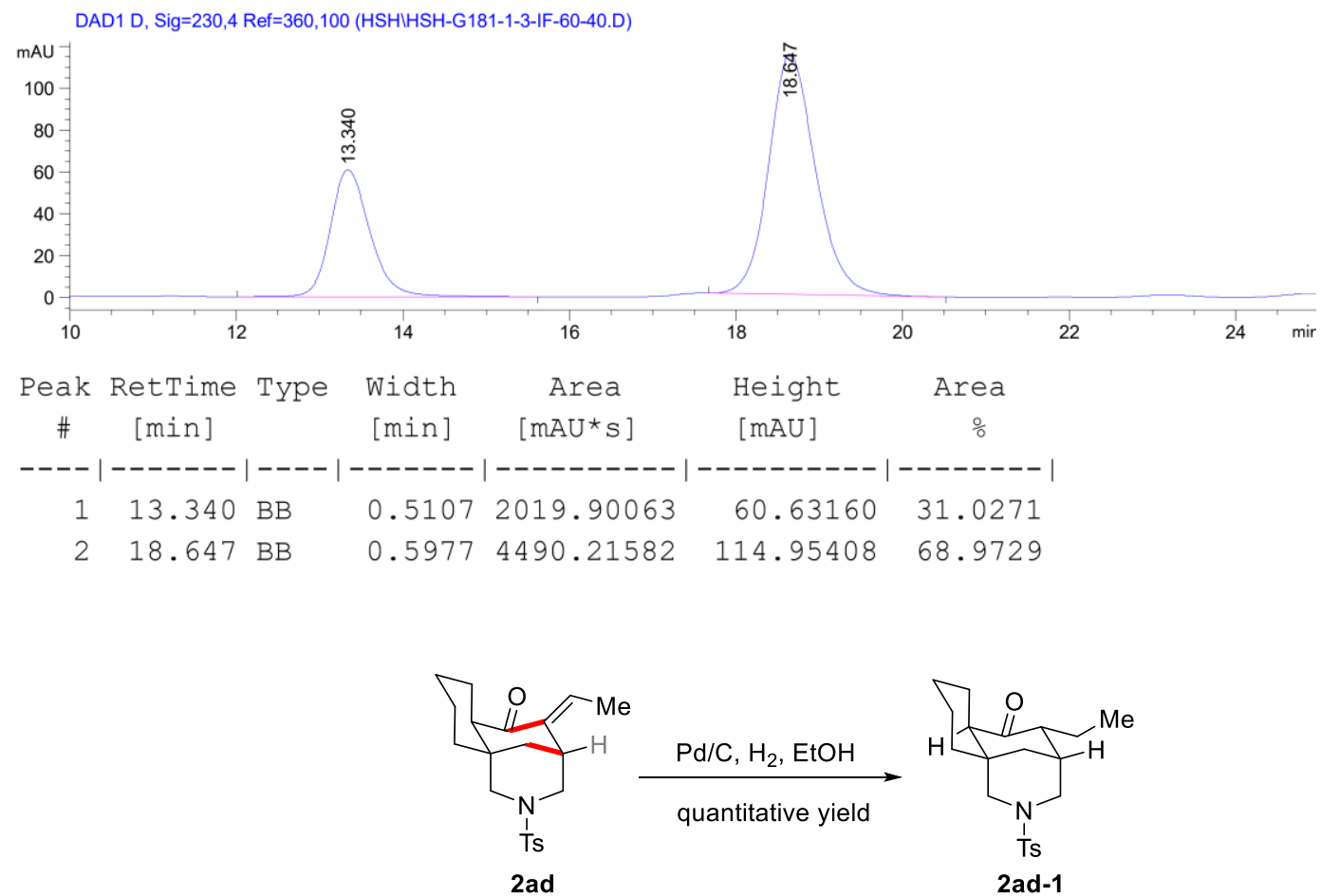

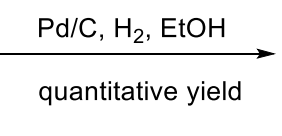

quantitative yield

2ad

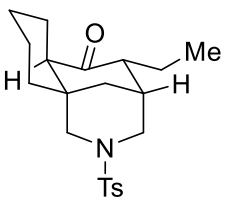

2ad-1

Compound 2ad-1. Compound 2ad (5 mg) and Pd/C (1 mg, 20\%wt) was dissolved in $\mathrm{EtOH}(1 \mathrm{~mL})$ at room temperature.

The reaction mixture was evacuated and refilled with $\mathrm{H}_{2}$ three times. After stirred at room temperature under $\mathrm{H}_{2}$ atmosphere for $3 \mathrm{~h}$, the reaction mixture was filtrated through a short of silica gel column and washed with EtOAc. 
The filtrate was concentrated under reduced pressure. The product was purified by silica gel flash chromatography to yield $5 \mathrm{mg}$ of $\mathbf{2 a d}-\mathbf{1}$ in quantitative yield as a foam.

$\mathrm{Rf}=0.35$, Hexane/ EtOAc 2:1.

${ }^{1} \mathrm{H}$ NMR (400 MHz, Chloroform-d) $\delta 7.65-7.60(\mathrm{~m}, 2 \mathrm{H}), 7.36-7.29(\mathrm{~m}, 2 \mathrm{H}), 3.75-3.70(\mathrm{~m}, 1 \mathrm{H}), 3.25(\mathrm{dt}, \mathrm{J}=11.1$, $2.1 \mathrm{~Hz}, 1 \mathrm{H}), 2.44(\mathrm{~s}, 3 \mathrm{H}), 2.42-2.36(\mathrm{~m}, 1 \mathrm{H}), 2.36-2.28(\mathrm{~m}, 1 \mathrm{H}), 2.28-2.15(\mathrm{~m}, 3 \mathrm{H}), 2.12(\mathrm{~d}, J=11.2 \mathrm{~Hz}, 1 \mathrm{H}), 2.10$ $-1.99(\mathrm{~m}, 1 \mathrm{H}), 1.85-1.76(\mathrm{~m}, 1 \mathrm{H}), 1.76-1.68(\mathrm{~m}, 1 \mathrm{H}), 1.58(\mathrm{dt}, J=13.5,3.9 \mathrm{~Hz}, 1 \mathrm{H}), 1.52-1.25(\mathrm{~m}, 6 \mathrm{H}), 1.22(\mathrm{dt}, J$ $=13.0,3.8 \mathrm{~Hz}, 1 \mathrm{H}), 1.15$ (ddd, $J=13.5,3.1,2.0 \mathrm{~Hz}, 1 \mathrm{H}), 1.07(\mathrm{td}, J=13.4,4.4 \mathrm{~Hz}, 1 \mathrm{H}), 0.91(\mathrm{t}, J=7.5 \mathrm{~Hz}, 3 \mathrm{H})$.

${ }^{13} \mathrm{C}$ NMR (126 MHz, $\left.\mathrm{CDCl}_{3}\right) \delta$ 211.2, 143.7, 133.1, 129.5, 128.0, 59.2, 56.9, 48.7, 46.9, 36.1, 35.4, 33.6, 32.4, 28.4, $25.9,21.5,20.7,18.8,11.8$.

HRMS ESI Calcd for $\mathrm{C}_{21} \mathrm{H}_{30} \mathrm{NO}_{3} \mathrm{~S}[\mathrm{M}+\mathrm{H}]^{+}: 376.1941$, Found: 376.1942.

IR (KBr): 2931, 2856, 1742, 1686, 1629, 1598, 1453, 1351, 1340, 1224, 1166, 1090, $1039 \mathrm{~cm}^{-1}$.

Chiral HPLC (Chiralpak IF, Hexane/Isopropanol $=60 / 40$, flow rate $=1.0 \mathrm{~mL} / \mathrm{min}, 230 \mathrm{~nm}$ ), $t_{\text {minor }}=16.8 \mathrm{~min}, \mathrm{t}_{\mathrm{major}}=$ $19.1 \min .[\alpha]^{21.5} \mathrm{D}=-24.5\left(\mathrm{c} 0.5, \mathrm{CHCl}_{3}\right)$ at $98: 2 \mathrm{er}$.

\section{Racemic sample 2ad-1}

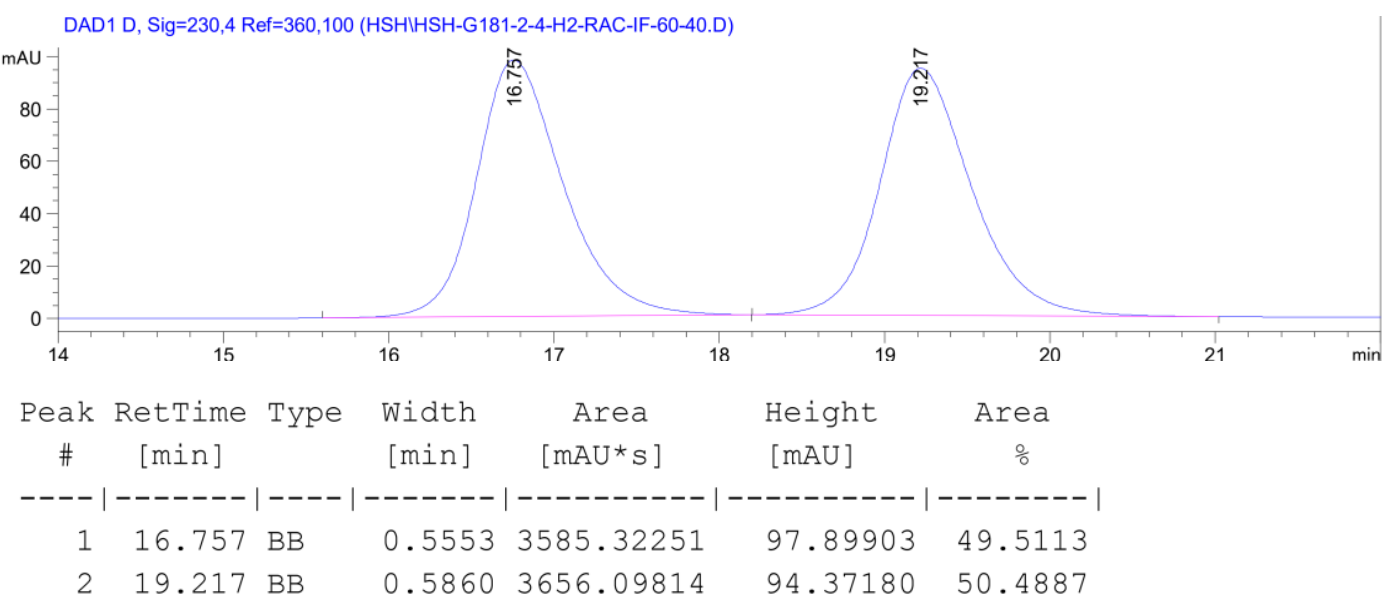

\section{Enantiomeric sample 2ad-1 (er 98:2)}

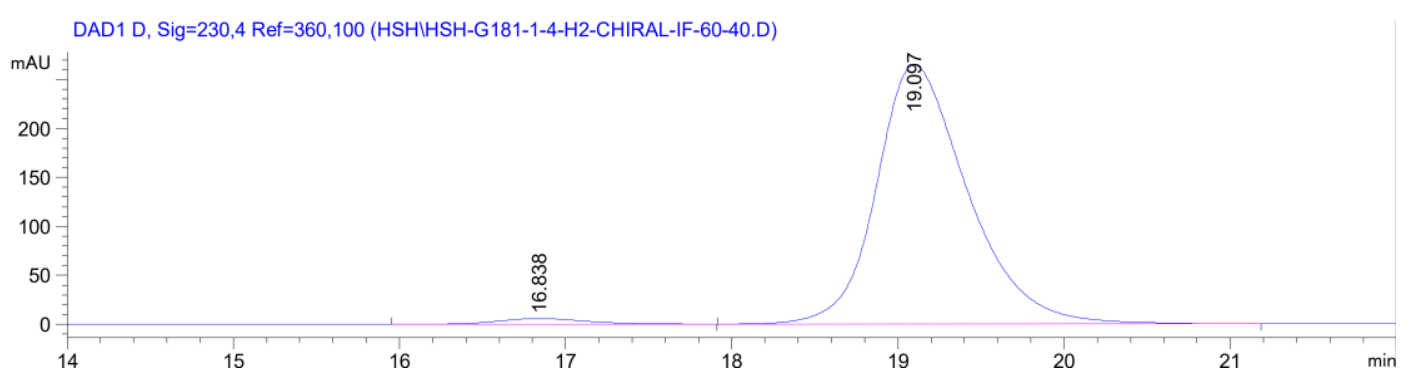




\begin{tabular}{|c|c|c|c|c|c|c|}
\hline $\begin{array}{c}\text { Peak } \\
\#\end{array}$ & $\begin{array}{c}\text { RetTime } \\
\text { [min] }\end{array}$ & Type & $\begin{array}{l}\text { Width } \\
\text { [min] }\end{array}$ & $\begin{array}{c}\text { Area } \\
{\left[\mathrm{mAU}^{*} \mathrm{~s}\right]}\end{array}$ & $\begin{array}{l}\text { Height } \\
\text { [mAU] }\end{array}$ & $\begin{array}{c}\text { Area } \\
\frac{\circ}{0}\end{array}$ \\
\hline & & 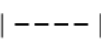 & & ---------- & -------1 & $--------\mid$ \\
\hline 1 & 16.838 & $\mathrm{BB}$ & 0.5569 & 214.56458 & 5.78289 & 2.0727 \\
\hline 2 & 19.097 & BB & 0.5789 & $1.01373 \mathrm{e} 4$ & 264.64441 & 97.9273 \\
\hline
\end{tabular}
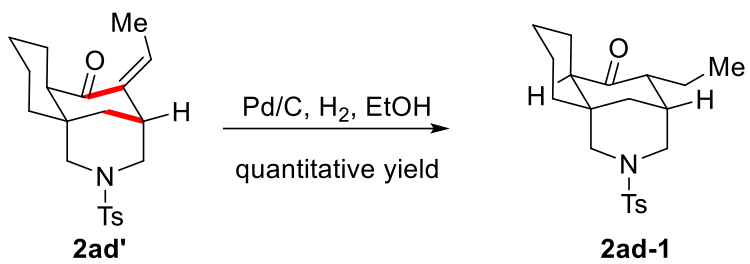

Compound 2ad-1. Compound 2ad' (5 mg) and Pd/C (1 mg, 20\%wt) was dissolved in EtOH (1 mL) at room temperature. The reaction mixture was evacuated and refilled with $\mathrm{H}_{2}$ three times. After stirred at room temperature under $\mathrm{H}_{2}$ atmosphere for $3 \mathrm{~h}$, the reaction mixture was filtrated through a short of silica gel column and washed with EtOAc. The filtrate was concentrated under reduced pressure. The product was purified by silica gel flash chromatography to yield 5 mg of $\mathbf{2 a d}-\mathbf{1}$ in quantitative yield as a foam.

Chiral HPLC (Chiralpak IF, Hexane/Isopropanol $=60 / 40$, flow rate $=1.0 \mathrm{~mL} / \mathrm{min}, 230 \mathrm{~nm}$ ), $t_{\text {minor }}=16.8 \mathrm{~min}, \mathrm{t}_{\mathrm{major}}=$ $19.3 \mathrm{~min}$.

\section{Racemic sample 2ad-1}

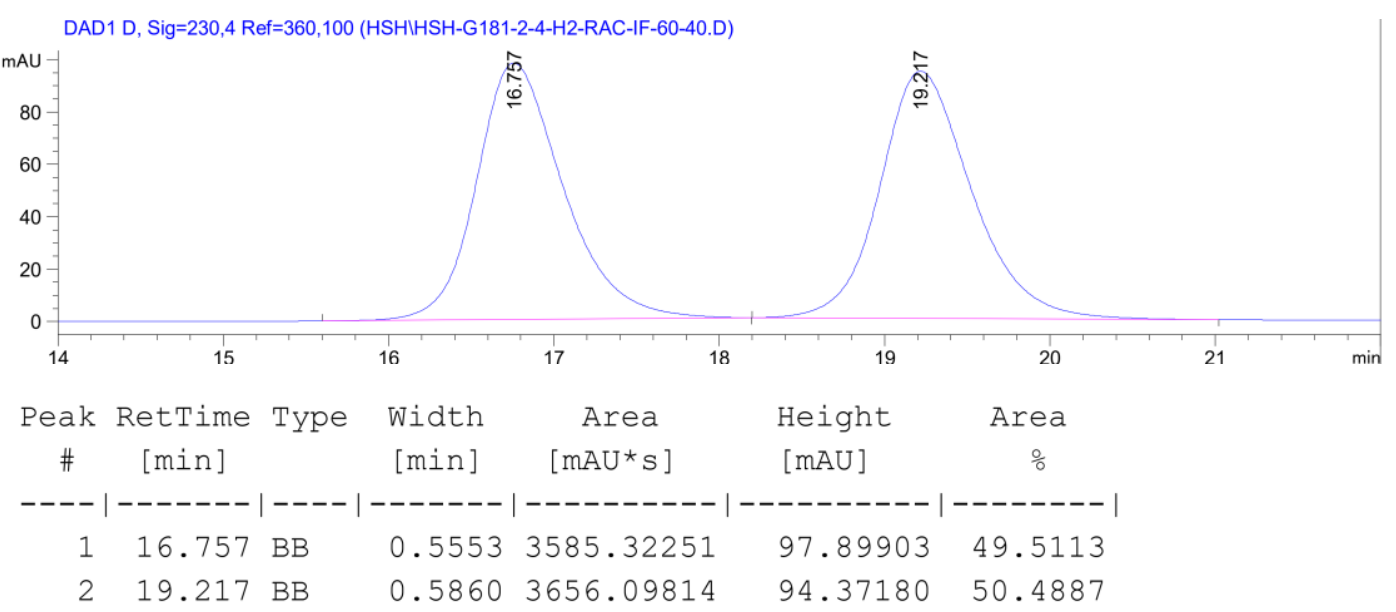

\section{Enantiomeric sample 2ad-1 (er 70:30)}

DAD1 D, Sig=230,4 Ref=360,100 (HSHIHSH-G181-1-3-H2-CHIRAL-IF-60-40.D)
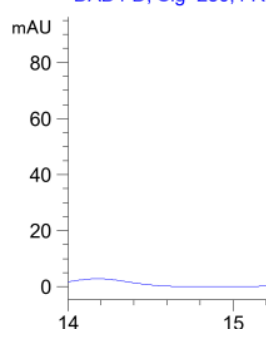

5

16

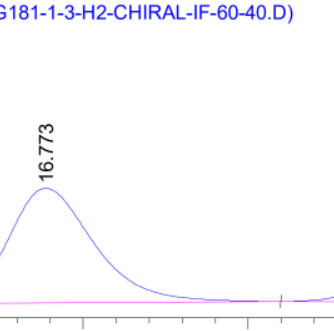

18 


\begin{tabular}{|c|c|c|c|c|c|c|}
\hline $\begin{array}{c}\text { Peak } \\
\#\end{array}$ & $\begin{array}{c}\text { RetTime } \\
\text { [min] }\end{array}$ & Type & $\begin{array}{l}\text { Width } \\
\text { [min] }\end{array}$ & $\begin{array}{c}\text { Area } \\
{\left[\mathrm{mAU}{ }^{\star} \mathrm{s}\right]}\end{array}$ & $\begin{array}{l}\text { Height } \\
{[\mathrm{mAU}]}\end{array}$ & $\begin{array}{c}\text { Area } \\
\frac{\circ}{0}\end{array}$ \\
\hline & 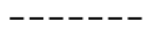 & & & ---------- & ------ & $-----\mid$ \\
\hline 1 & 16.773 & BB & 0.5589 & 1503.82312 & 40.90724 & 29.9692 \\
\hline 2 & 19.235 & BB & 0.5855 & 3514.07959 & 90.80247 & 70.0308 \\
\hline
\end{tabular}

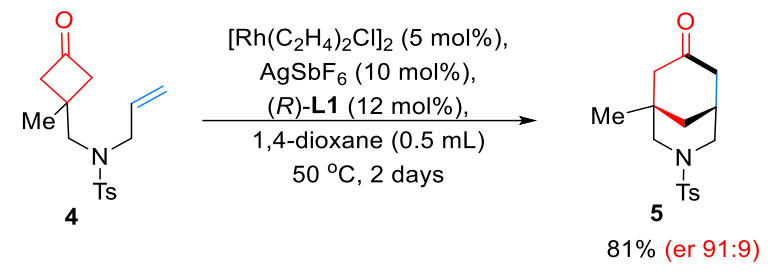

Compound 5. A flame dried $4 \mathrm{~mL}$ vial was charged with the substrate $4(30.7 \mathrm{mg}, 0.1 \mathrm{mmol}, 1$ equiv) and (R)-(-)DTBM-segphos (L1, 9.9 mg, $0.0084 \mathrm{mmol}, 8.4 \mathrm{~mol} \%$ ). The vial was then transferred to a nitrogen-filled glove box. After addition of $\left[\mathrm{Rh}\left(\mathrm{C}_{2} \mathrm{H}_{4}\right)_{2} \mathrm{Cl}\right]_{2}(2.0 \mathrm{mg}, 0.005 \mathrm{mmol}, 5 \mathrm{~mol} \%), \mathrm{AgSbF}_{6}(3.4 \mathrm{mg}, 0.01 \mathrm{mmol}, 10 \mathrm{~mol} \%)$ and 1,4-dioxane $(0.5 \mathrm{~mL})$, the vial was sealed and taken out of glove box. The reaction was stirred at room temperature for $5 \mathrm{~min}$ and $50^{\circ} \mathrm{C}$ for 2 days. The reaction mixture was concentrated under reduced pressure. The product was purified by silica gel flash chromatography to yield bridged bicycle $5(25 \mathrm{mg})$ in $81 \%$ yield as a colorless oil.

$\mathrm{Rf}=0.3$, Hexane/ EtOAc 1:1.

${ }^{1} \mathrm{H}$ NMR (400 MHz, Chloroform-d) $\delta 7.65-7.60(\mathrm{~m}, 2 \mathrm{H}), 7.37-7.30(\mathrm{~m}, 2 \mathrm{H}), 3.63(\mathrm{dq}, J=11.1,2.1 \mathrm{~Hz}, 1 \mathrm{H}), 3.43(\mathrm{dt}$, $J=11.3,2.1 \mathrm{~Hz}, 1 \mathrm{H}), 2.49-2.32(\mathrm{~m}, 8 \mathrm{H}), 2.19-2.10(\mathrm{~m}, 2 \mathrm{H}), 1.75(\mathrm{dq}, J=13.3,2.3 \mathrm{~Hz}, 1 \mathrm{H}), 1.46(\mathrm{dq}, J=13.4,2.8 \mathrm{~Hz}$, $1 \mathrm{H}), 0.98(\mathrm{~s}, 3 \mathrm{H})$.

${ }^{13} \mathrm{C}$ NMR (101 MHz, $\left.\mathrm{CDCl}_{3}\right) \delta$ 206.5, 143.8, 133.1, 129.6, 127.9, 77.3, 77.0, 76.7, 57.1, 52.6, 51.2, 44.7, 37.9, 34.0, $30.5,26.7,21.5$.

Note: The ${ }^{1} \mathrm{H}$ and ${ }^{13} \mathrm{C}-\mathrm{NMR}$ spectra of compound 5 are identical to the reported spectra. ${ }^{1}$

Chiral HPLC (Chiralpak IF, Hexane/Isopropanol $=60 / 40$, flow rate $=1.0 \mathrm{~mL} / \mathrm{min}, 230 \mathrm{~nm}$ ), $\mathrm{t}_{\text {minor }}=21.2 \mathrm{~min}, \mathrm{t}_{\text {major }}=$ $22.5 \min .[\alpha]^{21.5}=-6.9\left(c 1.1, \mathrm{CHCl}_{3}\right)$ at $91: 9$ er.

\section{Racemic Sample 5}

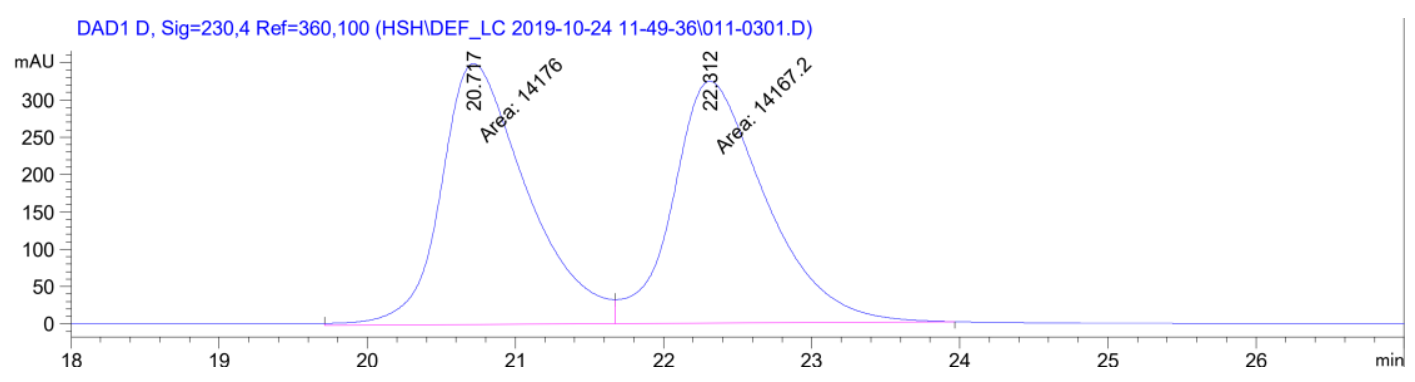




\begin{tabular}{|c|c|c|c|c|c|c|}
\hline $\begin{array}{c}\text { Peak } \\
\#\end{array}$ & $\begin{array}{c}\text { RetTime } \\
\text { [min] }\end{array}$ & Type & $\begin{array}{l}\text { Width } \\
\text { [min] }\end{array}$ & $\begin{array}{c}\text { Area } \\
{\left[\mathrm{mAU}{ }^{\star} \mathrm{s}\right]}\end{array}$ & $\begin{array}{l}\text { Height } \\
\text { [mAU] }\end{array}$ & $\begin{array}{c}\text { Area } \\
\frac{\circ}{0}\end{array}$ \\
\hline-- & ----- & & ------ & ---------- & ---------- & ------- \\
\hline 1 & 20.717 & $\mathrm{MF}$ & 0.6758 & $1.41760 \mathrm{e} 4$ & 349.59460 & 50.0155 \\
\hline 2 & 22.312 & FM & 0.7285 & $1.41672 \mathrm{e} 4$ & 324.11008 & 49.9845 \\
\hline
\end{tabular}

\section{Enantiomeric Sample 5}

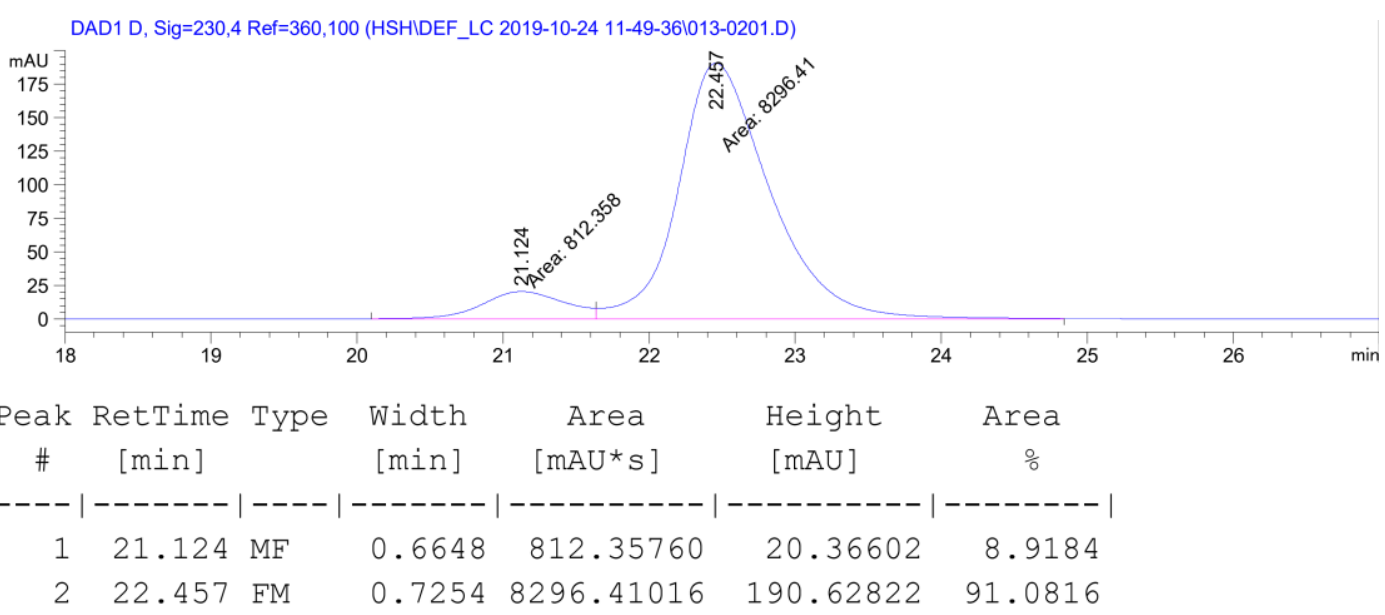


Isomerization of bridged bicycle 2ab'

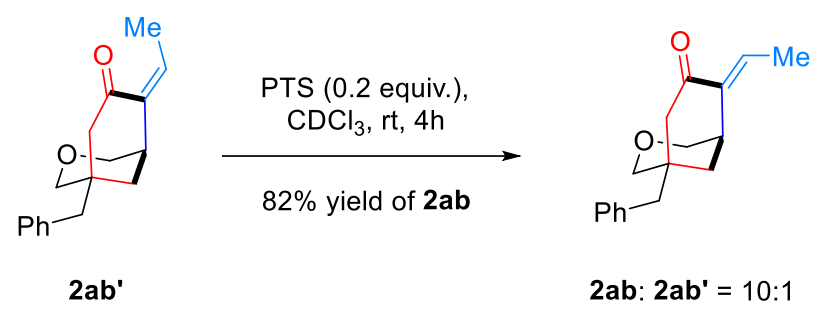

Compound 2ab. PTS. $\mathrm{H}_{2} \mathrm{O}$ (1.8 mg, $0.001 \mathrm{mmol}, 0.2$ equiv) was added to a solution of compound 2ab' (12.8 mg, 0.05 mmol, 1 equiv) in $\mathrm{CDCl}_{3}(1 \mathrm{~mL})$. After stirred at room temperature for $4 \mathrm{~h}$, the ratio of $\mathbf{2} \mathbf{a b}$ and $\mathbf{2} \mathbf{a} \mathbf{b}^{\prime}$ ' was determined by ${ }^{1} \mathrm{H}$ NMR. Then the product was directly purified by silica gel flash chromatography to give $10.5 \mathrm{mg}$ of compound 2 ab as a foam in $82 \%$ yield.

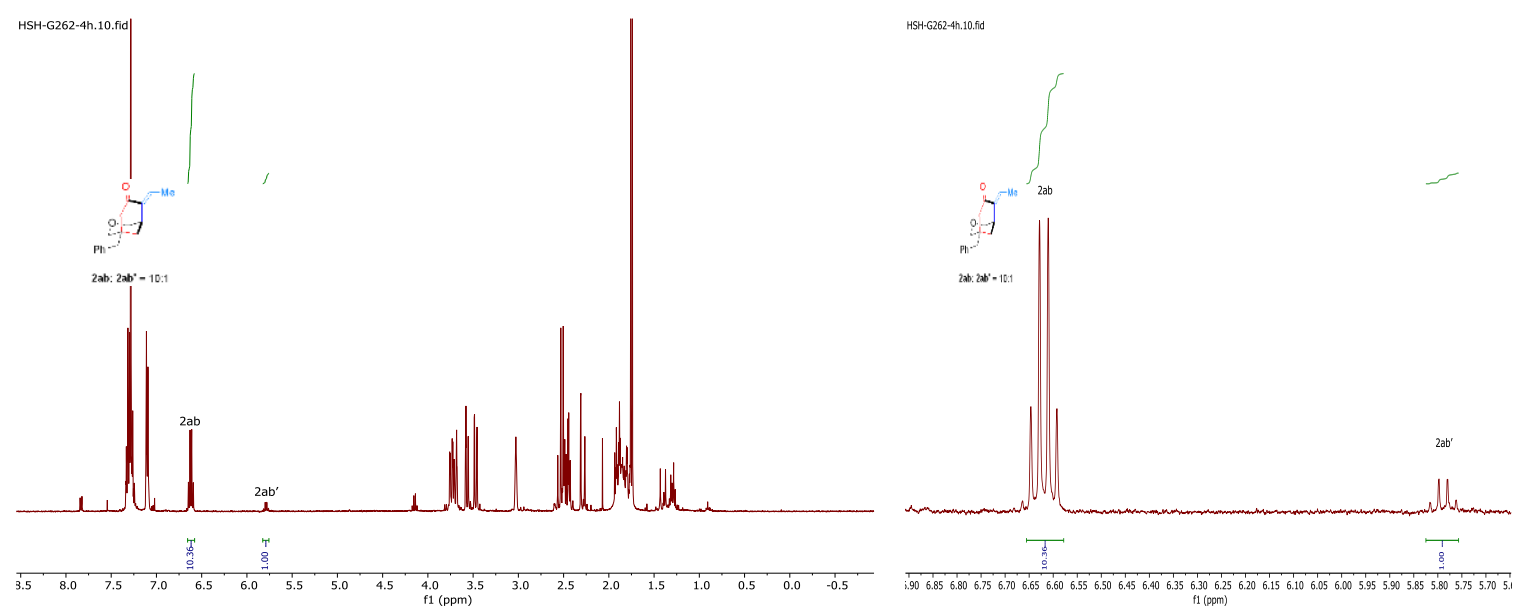

\section{Compound 2ab}

$\mathrm{Rf}=0.16$, Hexane/ EtOAc 4:1.

$[\alpha]^{21.5} \mathrm{D}=-111.0\left(c 1.0, \mathrm{CHCl}_{3}\right)$.

${ }^{1} \mathrm{H}$ NMR $\left(400 \mathrm{MHz}\right.$, Benzene- $\left.d_{6}\right) \delta 7.13-7.04(\mathrm{~m}, 3 \mathrm{H}), 6.80-6.74(\mathrm{~m}, 2 \mathrm{H}), 6.70(\mathrm{qd}, J=7.2,0.7 \mathrm{~Hz}, 1 \mathrm{H}), 3.49$ (ddd, $J$ $=14.3,10.6,1.7 \mathrm{~Hz}, 2 \mathrm{H}), 3.12-3.04(\mathrm{~m}, 2 \mathrm{H}), 2.51-2.37(\mathrm{~m}, 2 \mathrm{H}), 2.09(\mathrm{dd}, J=17.3,1.7 \mathrm{~Hz}, 1 \mathrm{H}), 2.06-1.91(\mathrm{~m}, 2 \mathrm{H})$, $1.32(\mathrm{dd}, J=3.3,1.7 \mathrm{~Hz}, 2 \mathrm{H}), 1.23(\mathrm{~d}, J=7.2 \mathrm{~Hz}, 3 \mathrm{H})$.

${ }^{13}$ C NMR (101 MHz, $\left.C_{6} D_{6}\right) \delta$ 197.1, 140.1, 136.3, 130.6, 130.6, 126.7, 78.2, 73.2, 49.8, 44.8, 36.5, 35.5, 33.0, 12.7.

HRMS ESI Calcd for $\mathrm{C}_{17} \mathrm{H}_{21} \mathrm{O}_{2}[\mathrm{M}+\mathrm{H}]^{+}:$257.1536, Found: 257.1533.

IR (KBr): 2928, 2849, 1688, 1628, 1453, 1306, 1288, 1263, 1204, 1084, $1063 \mathrm{~cm}^{-1}$. 
Isomerization of bridged bicycles $\mathbf{2} \mathbf{a}$ and $\mathbf{2} \mathbf{a}^{\prime}$

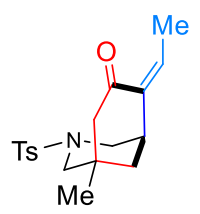

2a'

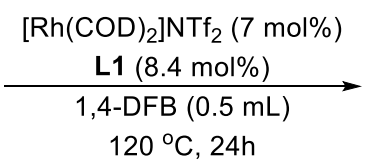

quantitative yield

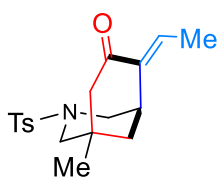

2a: $2 \mathbf{a}^{\prime}=6: 1$

Isomerization of 2a'. A flame dried $8 \mathrm{~mL}$ tube was charged with the substrate $2 a^{\prime}$ ( $33.3 \mathrm{mg}, 0.1 \mathrm{mmol}, 1 \mathrm{equiv}$ ) and $(R)$-(-)-DTBM-segphos (L1, $9.9 \mathrm{mg}, 0.0084 \mathrm{mmol}, 8.4 \mathrm{~mol} \%$ ). The tube was then transferred to a nitrogen-filled glove box. After addition of $\left[\mathrm{Rh}(\mathrm{COD})_{2}\right] \mathrm{NTf}_{2}(4.2 \mathrm{mg}, 0.007 \mathrm{mmol}, 7 \mathrm{~mol} \%)$ and 1,4-diflorobenzene $(0.5 \mathrm{~mL})$, the tube was sealed and taken out of glove box. The reaction was stirred at $120^{\circ} \mathrm{C}$ in an oil bath for $24 \mathrm{~h}$, before it was then cooled to room temperature. The reaction mixture was concentrated under reduced pressure, the $E / Z$ ratio of compound 2a and $2 a^{\prime}$ was determined by ${ }^{1} \mathrm{H} N M R$.

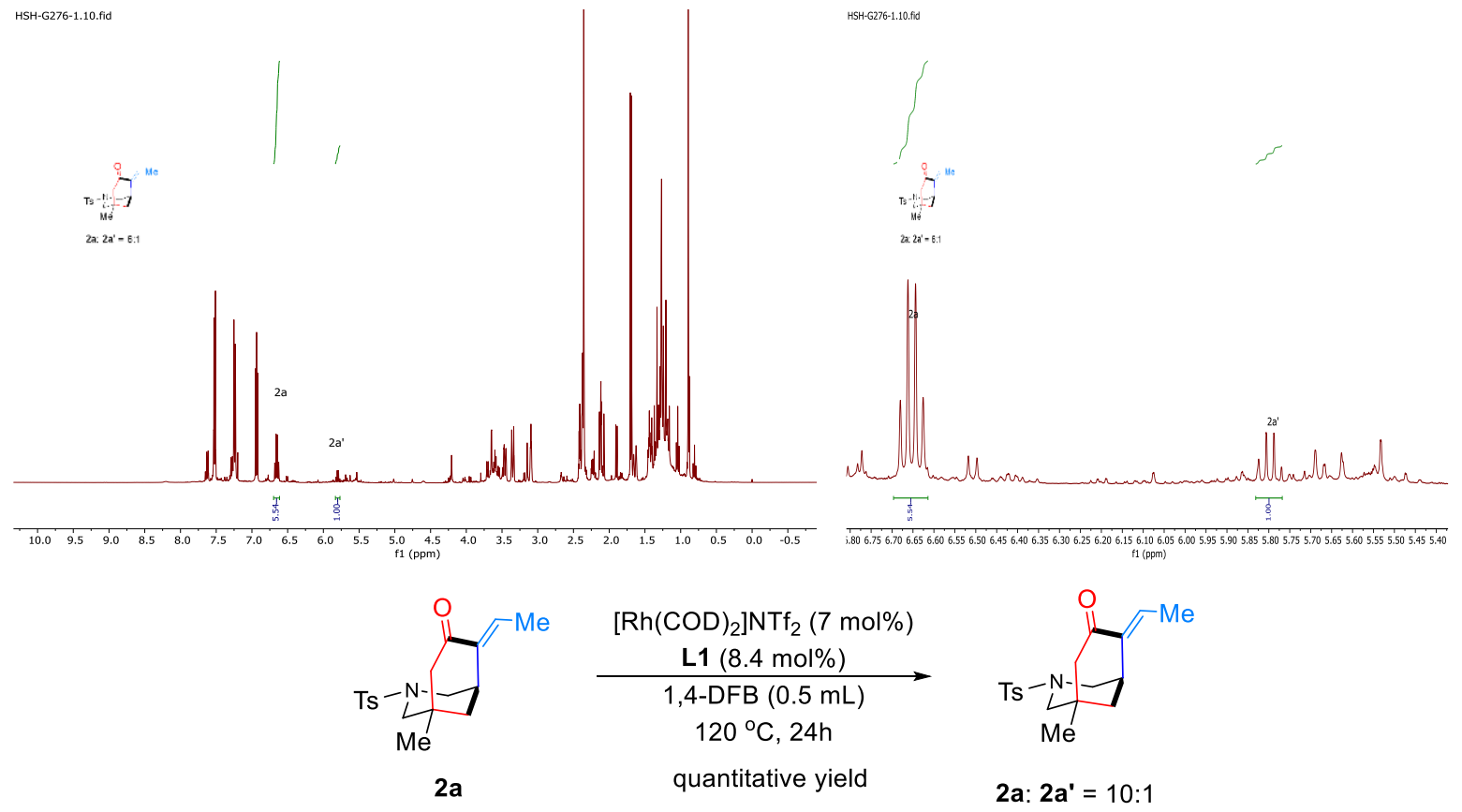

Isomerization of $2 \mathrm{a}$. A flame dried $8 \mathrm{~mL}$ tube was charged with the substrate $2 \mathrm{a}(33.3 \mathrm{mg}, 0.1 \mathrm{mmol}, 1 \mathrm{equiv})$ and $(R)$-(-)-DTBM-segphos (L1, $9.9 \mathrm{mg}, 0.0084 \mathrm{mmol}, 8.4 \mathrm{~mol} \%$ ). The tube was then transferred to a nitrogen-filled glove box. After addition of $\left[\mathrm{Rh}(\mathrm{COD})_{2}\right] \mathrm{NTf}_{2}(4.2 \mathrm{mg}, 0.007 \mathrm{mmol}, 7 \mathrm{~mol} \%)$ and 1,4-diflorobenzene $(0.5 \mathrm{~mL})$, the tube was sealed and taken out of glove box. The reaction was stirred at $120^{\circ} \mathrm{C}$ in an oil bath for $24 \mathrm{~h}$, before it was then cooled to room temperature. The reaction mixture was concentrated under reduced pressure, the $E / Z$ ratio of compound 2a and $2 a^{\prime}$ was determined by ${ }^{1} \mathrm{H}$ NMR. 

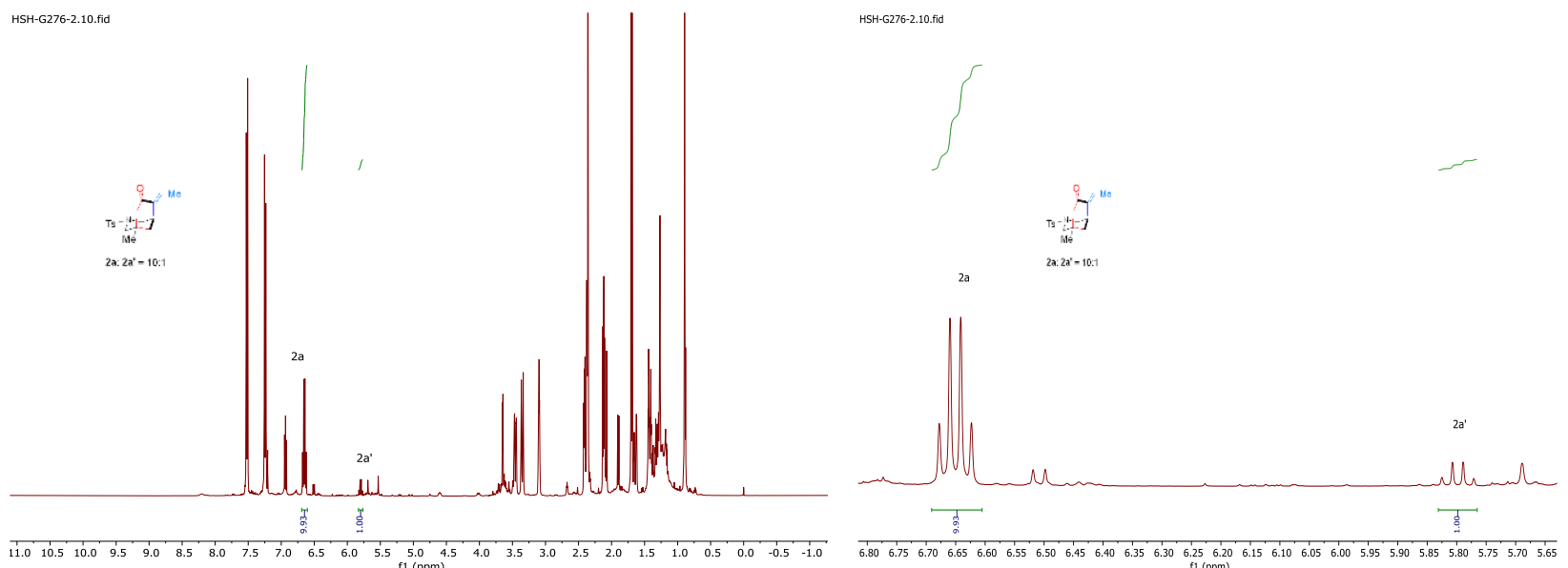

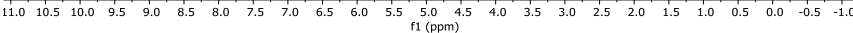
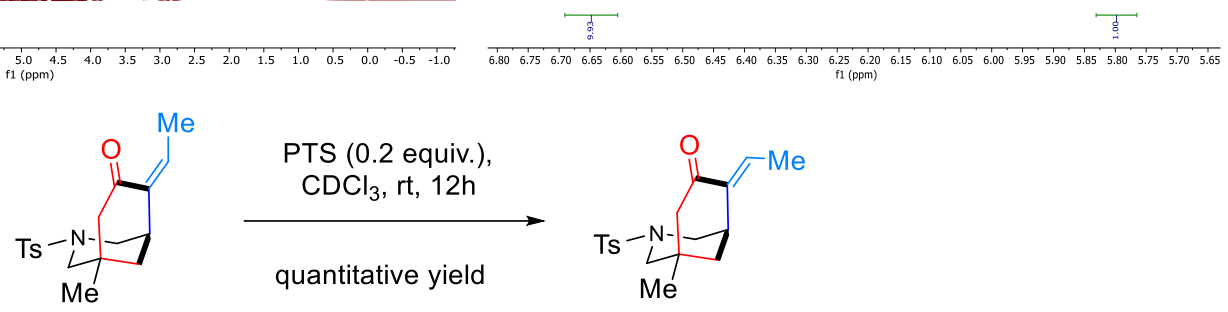

2a'

2a: $\mathbf{2} \mathbf{a}^{\prime}=17: 1$

Isomerization of $2 \mathrm{a}^{\prime}$. PTS· $\mathrm{H}_{2} \mathrm{O}$ ( $3.8 \mathrm{mg}, 0.02 \mathrm{mmol}, 0.2$ equiv) was added to a solution of compound $\mathbf{2 a}$ ' $(33.3 \mathrm{mg}, 0.1$ mmol, 1 equiv) in $\mathrm{CDCl}_{3}(1 \mathrm{~mL})$. After stirred at room temperature for $12 \mathrm{~h}$, the $E / Z$ ratio of compound $\mathbf{2 a}$ and $\mathbf{2} \mathbf{a}^{\prime}$ was determined by ${ }^{1} \mathrm{H}$ NMR.
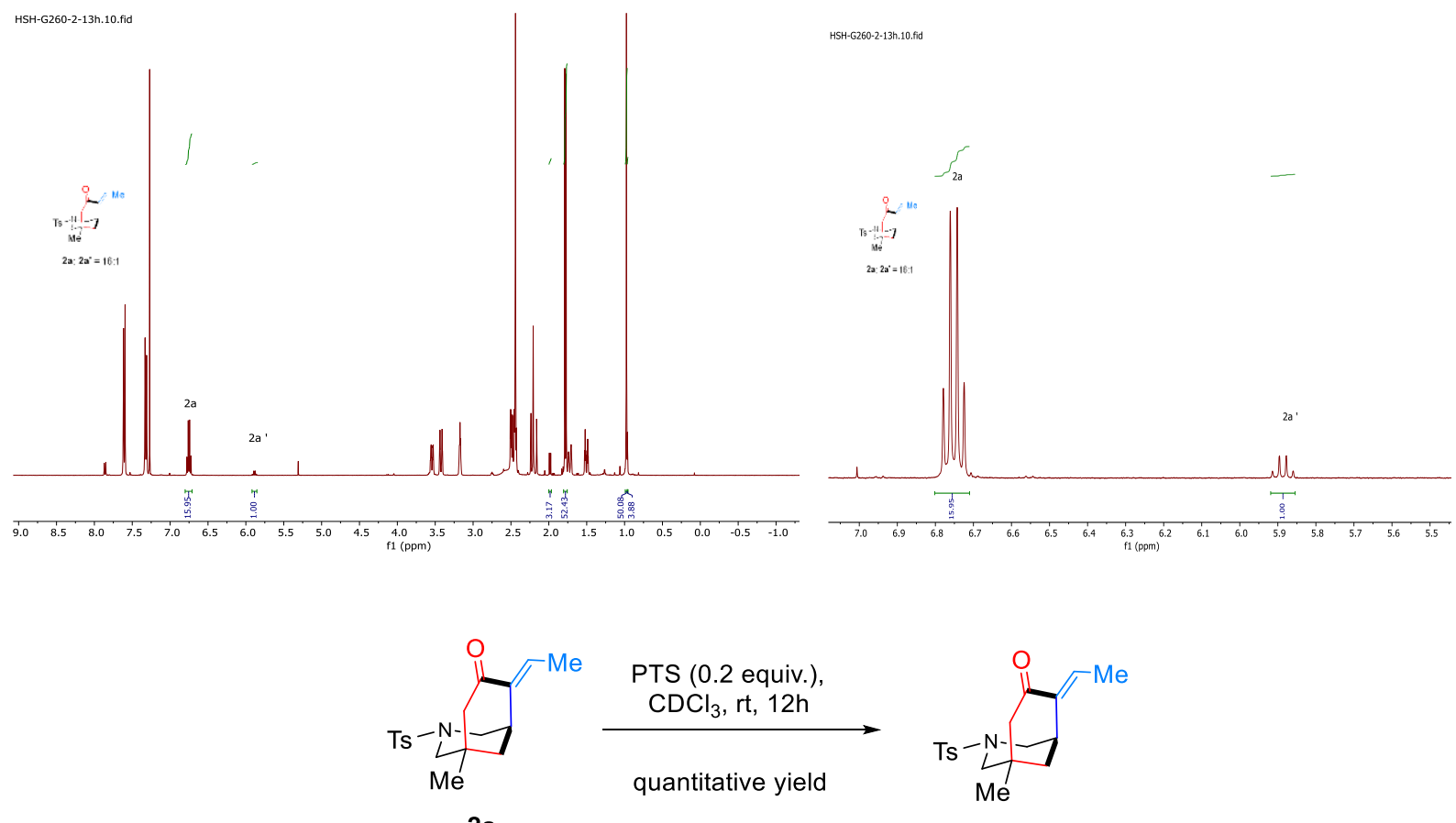

2a: $\mathbf{2} \mathbf{a}^{\prime}=16: 1$

Isomerization of $2 \mathrm{a}$. PTS $\mathrm{H}_{2} \mathrm{O}$ ( $3.8 \mathrm{mg}, 0.02 \mathrm{mmol}, 0.2$ equiv) was added to a solution of compound $2 \mathrm{a}$ ( $33.3 \mathrm{mg}, 0.1$ mmol, 1 equiv) in $\mathrm{CDCl}_{3}(1 \mathrm{~mL})$. After stirred at room temperature for $12 \mathrm{~h}$, the $E / Z$ ratio of compound $\mathbf{2 a}$ and $\mathbf{2 a}$ ' was determined by ${ }^{1} \mathrm{H}$ NMR. 


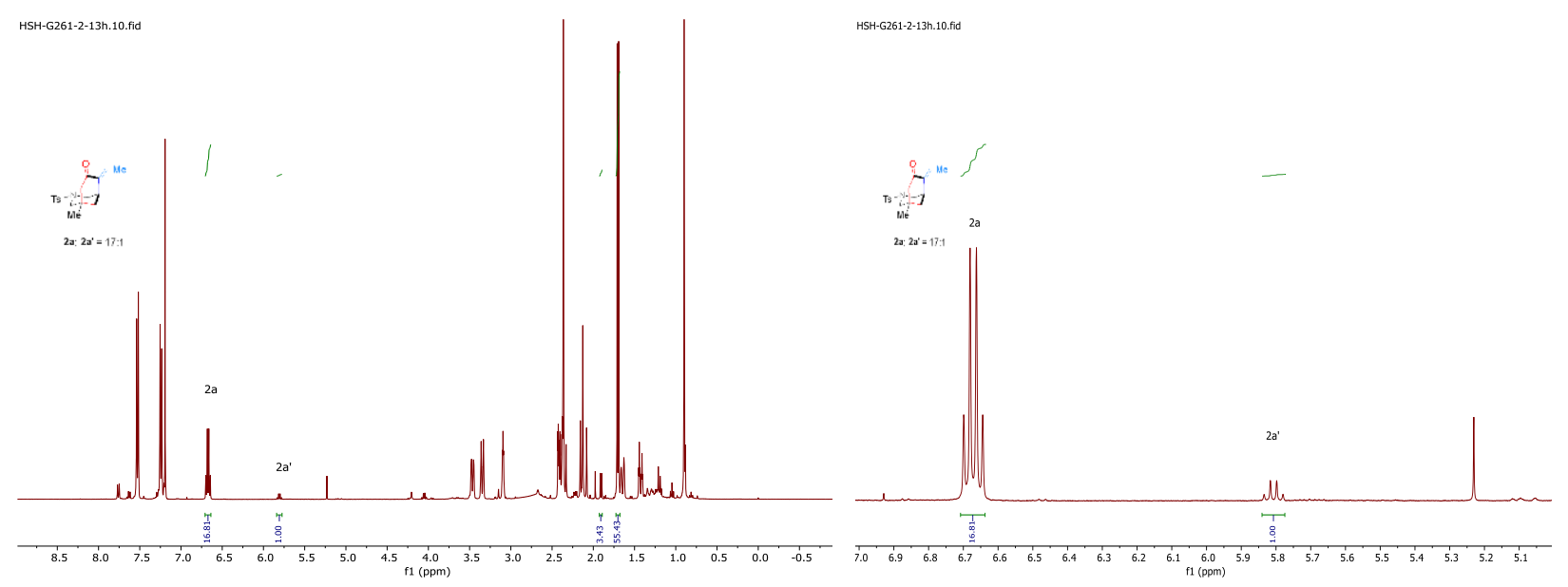




\section{Functionalization of $\mathbf{2} \mathbf{j}$ and $\mathbf{2 a}$}

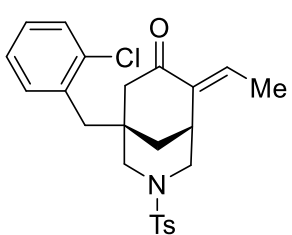

$2 \mathrm{j}$

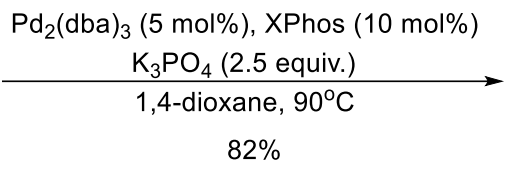

$82 \%$

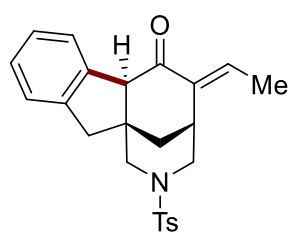

$2 \mathrm{j}-1$

Compound $\mathbf{2 j - 1}$. A flame dried $8 \mathrm{~mL}$ tube was charged with the substrate $2 \mathbf{j}\left(44.3 \mathrm{mg}, 0.1 \mathrm{mmol}, 1\right.$ equiv), $\mathrm{Pd}_{2}\left(\mathrm{dba}_{3}\right.$ (4.6 mg, $0.005 \mathrm{mmol} 5 \mathrm{~mol} \%$ ), XPhos ( $4.8 \mathrm{mg}, 0.01 \mathrm{mmol}, 10 \mathrm{~mol} \%$ ) and $\mathrm{K}_{3} \mathrm{PO}_{4}(53 \mathrm{mg}, 0.25 \mathrm{mmol}, 2.5$ equiv). The tube was then transferred to a nitrogen-filled glove box. After addition of 1,4-dioxane (1.0 mL), the tube was sealed and taken out of glove box. The reaction was stirred at $90^{\circ} \mathrm{C}$ in an oil bath for $24 \mathrm{~h}$, before it was then cooled to room temperature. The reaction mixture was filtered over a plug of silica gel and washed with $20 \mathrm{~mL}$ EtOAc. The filtrate was concentrated under reduced pressure. The product was purified by silica gel flash chromatography to yield $33.3 \mathrm{mg}$ of compound $\mathbf{2 j - 1}$ as a yellow foam in $82 \%$ yield.

$\mathrm{Rf}=0.55$, Hexane/ EtOAc 2:1.

$[\alpha]^{21.5}=-249\left(c 0.8, \mathrm{CHCl}_{3}\right)$.

${ }^{1} \mathrm{H}$ NMR (400 MHz, Benzene- $\left.d_{6}\right) \delta 7.90-7.83(\mathrm{~m}, 1 \mathrm{H}), 7.74-7.67(\mathrm{~m}, 2 \mathrm{H}), 7.04(\mathrm{ddt}, J=11.8,7.3,4.1 \mathrm{~Hz}, 3 \mathrm{H}), 7.00$ $-6.94(\mathrm{~m}, 1 \mathrm{H}), 6.89-6.82(\mathrm{~m}, 2 \mathrm{H}), 3.80(\mathrm{~s}, 1 \mathrm{H}), 3.71-3.58(\mathrm{~m}, 2 \mathrm{H}), 2.46-2.39(\mathrm{~m}, 1 \mathrm{H}), 2.34(\mathrm{~d}, J=11.1 \mathrm{~Hz}, 1 \mathrm{H})$, $2.28(\mathrm{dd}, J=11.0,2.5 \mathrm{~Hz}, 1 \mathrm{H}), 2.22(\mathrm{~d}, J=15.4 \mathrm{~Hz}, 1 \mathrm{H}), 2.08(\mathrm{dd}, J=14.1,1.6 \mathrm{~Hz}, 1 \mathrm{H}), 1.91(\mathrm{~s}, 3 \mathrm{H}), 1.43-1.34(\mathrm{~m}$, $1 \mathrm{H}), 1.22(\mathrm{~d}, J=7.4 \mathrm{~Hz}, 3 \mathrm{H}), 0.81-0.72(\mathrm{~m}, 1 \mathrm{H})$.

${ }^{13} \mathrm{C}$ NMR (101 MHz, C6 $\left.\mathrm{D}_{6}\right) \delta$ 195.1, 143.4, 140.6, 140.4, 137.5, 136.2, 135.0, 129.7, 127.5, 127.2, 125.5, 125.1, 61.2, 55.7, 51.1, 43.8, 43.0, 33.7, 31.1, 21.1, 12.9 .

HRMS ESI Calcd for $\mathrm{C}_{24} \mathrm{H}_{26} \mathrm{NO}_{3} \mathrm{~S}[\mathrm{M}+\mathrm{H}]^{+}:$408.1628, Found: 408.1628.

IR (KBr): 3025, 2927, 2852, 1740, 1682, 1614, 1466, 1446, 1354, 1339, 1165, 1090, $1010 \mathrm{~cm}^{-1}$.
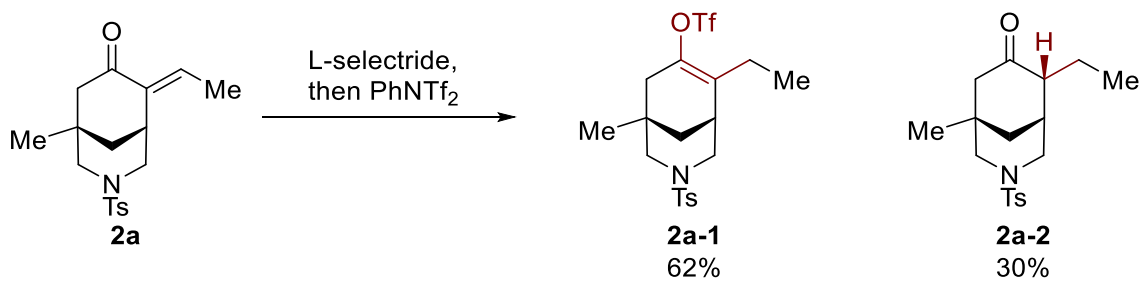

Compound2a-1. L-selectride (0.11 mL, $1 \mathrm{M}$ in THF, $0.11 \mathrm{mmol}, 1.1$ equiv) was added to a solution of compound $2 \mathrm{a}$ (33.3 mg, $0.1 \mathrm{mmol}, 1$ equiv) in THF $(2 \mathrm{~mL})$ at $-78^{\circ} \mathrm{C}$. After stirred at $-78^{\circ} \mathrm{C}$ for $1 \mathrm{~h}$, a solution of PhNTf $2(39.3 \mathrm{mg}$, $0.11 \mathrm{mmol}, 1.1$ equiv) in THF $(1 \mathrm{~mL})$ was added to the reaction mixture. The reaction mixture was stirred at $-78^{\circ} \mathrm{C}$ for $1 \mathrm{~h}$ and then warmed to room temperature. After stirred at room temperature overnight, the reaction mixture was quenched with saturated $\mathrm{NH}_{4} \mathrm{Cl}$ aqueous solution, extracted with DCM. The combined organic extract was washed with saturated $\mathrm{NaHCO}_{3}$ aqueous solution and brine, dried with $\mathrm{Na}_{2} \mathrm{SO}_{4}$ and concentrated under reduced 
pressure. The residue was purified by silica gel flash chromatography to give $29 \mathrm{mg}$ of compound $\mathbf{2 a - 1}$ as a white solid in $62 \%$ yield and $10 \mathrm{mg}$ of ketone $\mathbf{2 a - 2}$ in $30 \%$ yield.

$\mathrm{Rf}=0.42$, Hexane/ EtOAc 4:1.

$\mathrm{Mp}: 84-86^{\circ} \mathrm{C}$.

$[\alpha]^{21.5}=-2.2\left(c 1.0, \mathrm{CHCl}_{3}\right)$.

${ }^{1} \mathrm{H}$ NMR (400 MHz, Chloroform-d) $\delta 7.65-7.58(\mathrm{~m}, 2 \mathrm{H}), 7.35-7.29(\mathrm{~m}, 2 \mathrm{H}), 3.66(\mathrm{dq}, J=11.6,1.9 \mathrm{~Hz}, 1 \mathrm{H}), 3.52(\mathrm{dt}$, $J=11.6,2.0 \mathrm{~Hz}, 1 \mathrm{H}), 2.59-2.54(\mathrm{~m}, 1 \mathrm{H}), 2.47-2.41(\mathrm{~m}, 4 \mathrm{H}), 2.41-2.33(\mathrm{~m}, 1 \mathrm{H}), 2.32-2.20(\mathrm{~m}, 2 \mathrm{H}), 2.18(\mathrm{dd}, J=$ $11.7,1.1 \mathrm{~Hz}, 1 \mathrm{H}), 2.04-1.90(\mathrm{~m}, 1 \mathrm{H}), 1.68-1.60(\mathrm{~m}, 1 \mathrm{H}), 1.31(\mathrm{dd}, J=12.7,2.8 \mathrm{~Hz}, 1 \mathrm{H}), 1.05(\mathrm{t}, J=7.6 \mathrm{~Hz}, 3 \mathrm{H}), 0.98$ $(\mathrm{s}, 3 \mathrm{H})$.

${ }^{19} \mathrm{~F}$ NMR (376 MHz, $\left.\mathrm{CDCl}_{3}\right) \delta$ - 74.9 .

${ }^{13} \mathrm{C}$ NMR $\left(101 \mathrm{MHz}, \mathrm{CDCl}_{3}\right) \delta$ 143.5, 143.3, 134.3, 131.0, 129.6, 127.4, 57.8, 47.1, 39.5, 36.8, 33.6, 31.8, 27.2, 22.1, 21.5, 11.7.

HRMS ESI Calcd for $\mathrm{C}_{19} \mathrm{H}_{25} \mathrm{~F}_{3} \mathrm{NO}_{5} \mathrm{~S}_{2}[\mathrm{M}+\mathrm{H}]^{+}: 468.1121$, Found: 468.1126.

IR (KBr): 2971, 2930, 2857, 1465, 1410, 1346, 1247, 1211, 1164, 1099, 1047, $1023 \mathrm{~cm}^{-1}$.

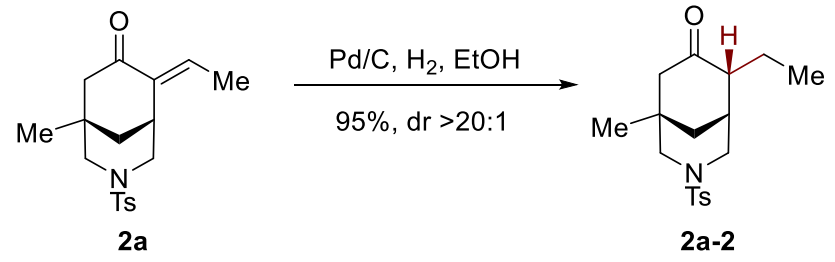

Compound 2a-2. Compound 2a (33.3 mg, $0.1 \mathrm{mmol})$ and $\mathrm{Pd} / \mathrm{C}(3.3 \mathrm{mg}, 10 \% \mathrm{wt})$ was dissolved in $\mathrm{EtOH}(1 \mathrm{~mL})$ at room temperature. The reaction mixture was evacuated and refilled with $\mathrm{H}_{2}$ three times. After stirred at room temperature under $\mathrm{H}_{2}$ atmosphere for $3 \mathrm{~h}$, the reaction mixture was filtrated through a short of silica gel column and washed with EtOAc. The filtrate was concentrated under reduced pressure. The residue was purified by silica gel flash chromatography to give $32 \mathrm{mg}$ of $\mathbf{2 a - 2}$ as a whit solid in $95 \%$ yield. The $d r$ ratio of compound $\mathbf{2 a - 2}$ was determined by ${ }^{1} \mathrm{H} N M R$.

$\mathrm{Rf}=0.22$, Hexane/ EtOAc 2:1.

$\mathrm{Mp}: 134-136^{\circ} \mathrm{C}$.

$[\alpha]^{21.5}=-3.2\left(c 1.6, \mathrm{CHCl}_{3}\right)$.

${ }^{1} \mathrm{H}$ NMR (400 MHz, Chloroform-d) $\delta 7.64-7.57(\mathrm{~m}, 2 \mathrm{H}), 7.35-7.28(\mathrm{~m}, 2 \mathrm{H}), 3.71(\mathrm{dq}, J=11.6,2.1 \mathrm{~Hz}, 1 \mathrm{H}), 3.39(\mathrm{dt}$, $J=11.1,2.1 \mathrm{~Hz}, 1 \mathrm{H}), 2.43(\mathrm{~s}, 3 \mathrm{H}), 2.37-2.29(\mathrm{~m}, 2 \mathrm{H}), 2.22(\mathrm{dd}, J=11.6,2.3 \mathrm{~Hz}, 1 \mathrm{H}), 2.19-2.01(\mathrm{~m}, 4 \mathrm{H}), 1.83-1.76$ $(\mathrm{m}, 1 \mathrm{H}), 1.53(\mathrm{dt}, J=13.3,3.3 \mathrm{~Hz}, 1 \mathrm{H}), 1.37-1.24(\mathrm{~m}, 1 \mathrm{H}), 0.96(\mathrm{~s}, 3 \mathrm{H}), 0.93(\mathrm{t}, J=7.4 \mathrm{~Hz}, 3 \mathrm{H})$.

${ }^{13} \mathrm{C}$ NMR $\left(101 \mathrm{MHz}, \mathrm{CDCl}_{3}\right.$ ) $\delta$ 207.5, 143.7, 132.9, 129.6, 127.8, 57.3, 52.7, 52.5, 46.3, 39.7, 34.8, 33.8, 26.5, 21.5, 18.9, 11.8 .

HRMS ESI Calcd for $\mathrm{C}_{18} \mathrm{H}_{26} \mathrm{NO}_{3} \mathrm{~S}[\mathrm{M}+\mathrm{H}]^{+}: 336.1628$, Found: 336.1627.

IR (KBr): 2959, 2928, 2873, 1706, 1598, 1457, 1363, 1345, 1166, 1093, $1021 \mathrm{~cm}^{-1}$. 


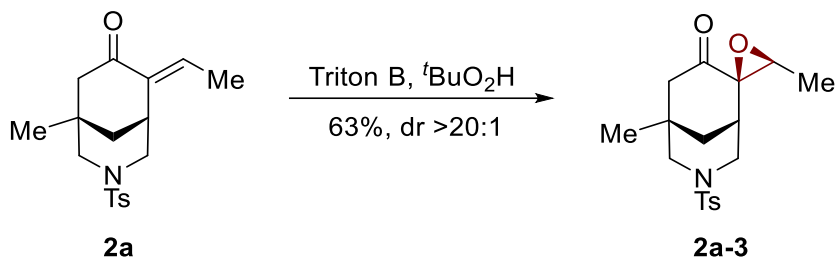

Compound 2a-3. Triton $\mathrm{B}\left(83.5 \mathrm{mg}, 0.2 \mathrm{mmol}, 40 \%\right.$ wt in $\mathrm{MeOH}, 2$ equiv) and $t \mathrm{BuO}_{2} \mathrm{H}(0.4 \mathrm{~mL}, 2 \mathrm{mmol}, 5-6 \mathrm{M}$ in decane, 20 equiv) were added to a solution of compound 2 a ( $33.3 \mathrm{mg}, 0.1 \mathrm{mmol}, 1$ equiv) in THF ( $2 \mathrm{~mL})$ at room temperature. After stirred at room temperature for 30 mins, the reaction was quenched by adding saturated $\mathrm{NH}_{4} \mathrm{Cl}$ aqueous solution and extracted with EtOAc. The combined organic extract was washed with saturated $\mathrm{NaHCO}_{3}$ aqueous solution and brine, dried with $\mathrm{Na}_{2} \mathrm{SO}_{4}$ and concentrated under reduced pressure. The residue was purified by silica gel flash chromatography to give $22 \mathrm{mg}$ of compound $\mathbf{2 a}-\mathbf{3}$ as a white solid in $63 \%$ yield. The $d r$ ratio of compound $2 \mathrm{a}-3$ was determined by ${ }^{1} \mathrm{H}$ NMR.

$\mathrm{Rf}=0.22$, Hexane/ EtOAc 2:1.

$\mathrm{Mp}: 155-157^{\circ} \mathrm{C}$.

$[\alpha]^{21.5} \mathrm{D}=-55.3\left(c 0.6, \mathrm{CHCl}_{3}\right)$.

${ }^{1} \mathrm{H}$ NMR (400 MHz, Chloroform-d) $\delta 7.65-7.59(\mathrm{~m}, 2 \mathrm{H}), 7.38-7.32(\mathrm{~m}, 2 \mathrm{H}), 3.92(\mathrm{q}, J=5.7 \mathrm{~Hz}, 1 \mathrm{H}), 3.68(\mathrm{dq}, J=$ 11.4, $2.0 \mathrm{~Hz}, 1 \mathrm{H}), 3.49(\mathrm{dt}, J=11.3,2.1 \mathrm{~Hz}, 1 \mathrm{H}), 2.55-2.45(\mathrm{~m}, 5 \mathrm{H}), 2.35(\mathrm{dd}, J=17.1,1.3 \mathrm{~Hz}, 1 \mathrm{H}), 2.23(\mathrm{dd}, J=11.3$, $1.2 \mathrm{~Hz}, 1 \mathrm{H}), 2.11-2.03(\mathrm{~m}, 2 \mathrm{H}), 1.52(\mathrm{dt}, J=14.1,3.5 \mathrm{~Hz}, 1 \mathrm{H}), 1.31(\mathrm{~d}, J=5.7 \mathrm{~Hz}, 3 \mathrm{H}), 1.05(\mathrm{~s}, 3 \mathrm{H})$.

${ }^{13} \mathrm{C}$ NMR (101 MHz, $\left.\mathrm{CDCl}_{3}\right) \delta$ 202.1, 144.1, 132.7, 129.7, 128.0, 62.0, 56.89, 56.2, 51.3, 48.6, 36.2, 33.8, 33.4, 26.5, 21.6, 12.8

HRMS ESI Calcd for $\mathrm{C}_{18} \mathrm{H}_{24} \mathrm{NO}_{4} \mathrm{~S}[\mathrm{M}+\mathrm{H}]^{+}: 350.1421$, Found:350.1420.

IR (KBr): 2928, 2855, 1716, 1597, 1466, 1343, 1166, 1108, 1092, $1014 \mathrm{~cm}^{-1}$.

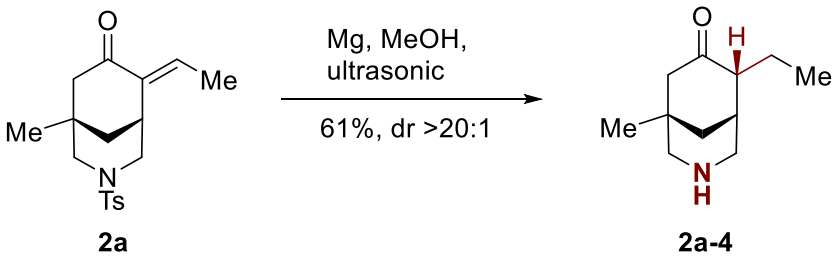

Compound 2a-4. Magnesium dust ( $25 \mathrm{mg}, 1 \mathrm{mmol}, 10$ equiv) was added in one portion to a suspension of $\mathbf{2 a}$ ( 33.3 $\mathrm{mg}, 0.1 \mathrm{mmol}, 1$ equiv) in $\mathrm{MeOH}(3 \mathrm{~mL})$. After stirred under sonication for $4 \mathrm{~h}$, the reaction was diluted with ether and quenched by adding saturated $\mathrm{NH}_{4} \mathrm{Cl}$ aqueous solution and extracted with EtOAc. The combined organic extract was washed with saturated $\mathrm{NaHCO}_{3}$ aqueous solution and brine, dried with $\mathrm{Na}_{2} \mathrm{SO}_{4}$ and concentrated under reduced pressure. The residue was purified by short silica gel flash chromatography to give $11 \mathrm{mg}$ of compound $2 \mathrm{a}-\mathbf{4}$ as an oil in $60 \%$ yield. The $d r$ ratio of compound $2 \mathrm{a}-4$ was determined by ${ }^{1} \mathrm{H}$ NMR.

$\mathrm{Rf}=0.38$, Hexane/ EtOAc 1:1. 
$[\alpha]^{21.5}=+2.1\left(c 0.5, \mathrm{CHCl}_{3}\right)$.

${ }^{1} \mathrm{H}$ NMR $\left(500 \mathrm{MHz}\right.$, Benzene- $\left.d_{6}\right) \delta 2.57(\mathrm{dd}, J=11.5,2.8 \mathrm{~Hz}, 1 \mathrm{H}), 2.39-2.28(\mathrm{~m}, 1 \mathrm{H}), 2.25$ (ddd, $J=12.7,9.2,2.4 \mathrm{~Hz}$, $2 \mathrm{H}), 2.04(\mathrm{dd}, J=11.4,2.2 \mathrm{~Hz}, 1 \mathrm{H}$ ), $2.00(\mathrm{dd}, J=10.9,1.0 \mathrm{~Hz}, 1 \mathrm{H}), 1.77(\mathrm{dt}, J=15.1,1.2 \mathrm{~Hz}, 1 \mathrm{H}), 1.71(\mathrm{dq}, J=5.6,2.7$ $\mathrm{Hz}, 1 \mathrm{H}$ ), 1.62 (dddd, $J=7.4,5.9,4.5,1.2 \mathrm{~Hz}, 1 \mathrm{H}$ ), $1.31-1.17(\mathrm{~m}, 3 \mathrm{H}), 0.94(\mathrm{t}, J=7.5 \mathrm{~Hz}, 3 \mathrm{H}), 0.57(\mathrm{~s}, 3 \mathrm{H}$ ).

${ }^{13} \mathrm{C}$ NMR $\left(126 \mathrm{MHz}, \mathrm{C}_{6} \mathrm{D}_{6}\right) \delta$ 205.9, 59.1, 53.4, 52.7, 46.8, 41.3, 35.6, 34.9, 26.9, 20.0, 12.5.

HRMS ESI Calcd for $\mathrm{C}_{11} \mathrm{H}_{20} \mathrm{NO}[\mathrm{M}+\mathrm{H}]^{+}:$182.1539, Found: 182.1537.

IR (KBr): 3354, 2953, 2926, 2871, 2737, 1699, 1457, 1373, 1273, $1115 \mathrm{~cm}^{-1}$.
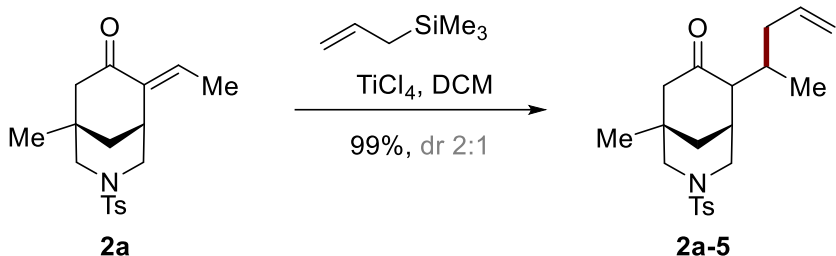

Compound 2a-5. $\mathrm{TiCl}_{4}$ (1 M in toluene, $0.12 \mathrm{~mL}, 0.12 \mathrm{mmol}, 1.2$ equiv) was added to a solution of compound $2 \mathrm{a}$ (33.3 $\mathrm{mg}, 0.1 \mathrm{mmol}, 1$ equiv) in $\mathrm{DCM}(1 \mathrm{~mL})$ at room temperature. After stirred at room temperature for $10 \mathrm{~min}$, the reaction mixture was cooled to $0{ }^{\circ} \mathrm{C}$, and allyltrimethylsilane $(20 \mu \mathrm{L}, 0.13 \mathrm{mmol}, 1.3$ equiv) was added to the reaction mixture at $0^{\circ} \mathrm{C}$. After stirred at room temperature for $2 \mathrm{~h}$, the reaction was quenched by adding a solution of $\mathrm{NEt}_{3}$ $(0.4 \mathrm{~mL})$ and $\mathrm{MeOH}(0.4 \mathrm{~mL})$ and extracted with $\mathrm{DCM}$. The combined organic extract was washed with $1 \mathrm{M} \mathrm{HCl}$ aqueous solution, saturated $\mathrm{NaHCO}_{3}$ aqueous solution and brine, dried with $\mathrm{Na}_{2} \mathrm{SO}_{4}$ and concentrated under reduced pressure. The residue was purified by silica gel flash chromatography to give 37 mg of compound $\mathbf{2 a - 5}$ ( $d r$ 2:1) as a foam in $99 \%$ yield. The $d r$ ratio of compound $2 \mathrm{a}-5$ was determined by ${ }^{1} \mathrm{H}$ NMR.

$\mathrm{Rf}=0.5$, Hexane/ EtOAc 2:1.

$[\alpha]^{21.5}=+2.5\left(c\right.$ 1.7, $\left.\mathrm{CHCl}_{3}\right)$.

${ }^{1}$ H NMR (400 MHz, Chloroform-d) $\delta 7.64-7.58$ (m, 2H), 7.32 (d, J = 8.1 Hz, 2H), $5.88-5.67(\mathrm{~m}, 1 \mathrm{H}), 5.09-4.90$ (m, 2H), $3.74-3.55(\mathrm{~m}, 1 \mathrm{H}), 3.48-3.35(\mathrm{~m}, 1 \mathrm{H}), 2.55-2.45(\mathrm{~m}, 1 \mathrm{H}), 2.43(\mathrm{~s}, 4 \mathrm{H}), 2.37-2.25(\mathrm{~m}, 2 \mathrm{H}), 2.20-2.10(\mathrm{~m}$, $2 \mathrm{H}), 2.09-2.02(\mathrm{~m}, 1 \mathrm{H}), 2.01-1.95(\mathrm{~m}, 1 \mathrm{H}), 1.95-1.84(\mathrm{~m}, 1 \mathrm{H}), 1.84-1.76(\mathrm{~m}, 1 \mathrm{H}), 1.51(\mathrm{dq}, J=13.3,3.5 \mathrm{~Hz}, 1 \mathrm{H})$, $1.08(\mathrm{~d}, J=6.3 \mathrm{~Hz}, 1 \mathrm{H}), 0.98-0.92(\mathrm{~m}, 3 \mathrm{H}), 0.84(\mathrm{~d}, J=6.7 \mathrm{~Hz}, 2 \mathrm{H})$.

${ }^{13} \mathrm{C}$ NMR (101 MHz, CDCl$)$ ) $\delta$ 206.7, 206.6, 143.7, 137.1, 136.1, 133.0, 133.0, 129.5, 127.9, 116.6, 116.1, 56.9, 56.0, $54.8,53.8,53.7,46.6,40.8,40.4,37.3,35.2,35.2,33.4,33.3,28.7,28.6,26.4,21.5,19.6,16.0$.

HRMS ESI Calcd for $\mathrm{C}_{21} \mathrm{H}_{30} \mathrm{NO}_{3} \mathrm{~S}[\mathrm{M}+\mathrm{H}]^{+}: 376.1941$, Found: 376.1944 .

IR (KBr): 2966, 2928, 2871, 2651, 1707, 1456, 1364, 1346, 1165, $1092 \mathrm{~cm}^{-1}$.

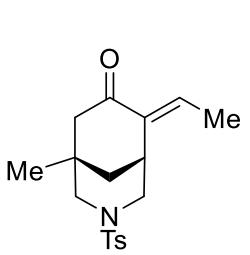

$2 \mathbf{a}$

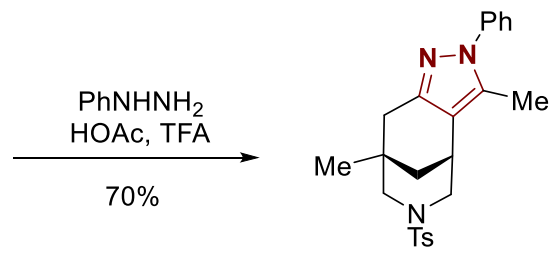

2a-6 
Compound 2a-6. TFA $(0.2 \mathrm{~mL})$ was added to a solution of compound $2 \mathrm{a}(33.3 \mathrm{mg}, 0.1 \mathrm{mmol}, 1$ equiv), and phenylhydrazine $\left(28.2 \mathrm{mg}, 0.26 \mathrm{mmol}, 2.6\right.$ equiv) in $\mathrm{HOAc}(0.8 \mathrm{~mL})$ at room temperature. After stirred at $100{ }^{\circ} \mathrm{C}$ for $24 \mathrm{~h}$, the reaction mixture was cooled to room temperature and then poured into water. The mixture was extracted with DCM. The combined organic extract was washed with saturated $\mathrm{NaHCO}_{3}$ aqueous solution and brine, dried with $\mathrm{Na}_{2} \mathrm{SO}_{4}$ and concentrated under reduced pressure. The residue was purified by silica gel flash chromatography to give $30 \mathrm{mg}$ of compound $\mathbf{2 a - 6}$ as a foam in $70 \%$ yield.

Rf $=0.25$, Hexane/ EtOAc 2:1.

$[\alpha]^{21.5}=+43.5\left(c 1.3, \mathrm{CHCl}_{3}\right)$.

${ }^{1} \mathrm{H}$ NMR (400 MHz, Benzene- $\left.d_{6}\right) \delta 7.55-7.50(\mathrm{~m}, 2 \mathrm{H}), 7.50-7.43(\mathrm{~m}, 2 \mathrm{H}), 7.14-7.06(\mathrm{~m}, 2 \mathrm{H}), 6.98-6.92(\mathrm{~m}, 1 \mathrm{H})$, $6.74-6.68(\mathrm{~m}, 2 \mathrm{H}), 3.64-3.56(\mathrm{~m}, 1 \mathrm{H}), 3.48(\mathrm{dt}, J=12.0,2.0 \mathrm{~Hz}, 1 \mathrm{H}), 2.52-2.49(\mathrm{~m}, 1 \mathrm{H}), 2.49-2.44(\mathrm{~m}, 1 \mathrm{H}), 2.40$ $(\mathrm{d}, J=16.9 \mathrm{~Hz}, 1 \mathrm{H}), 2.25(\mathrm{dd}, J=12.1,1.5 \mathrm{~Hz}, 1 \mathrm{H}), 2.20-2.13(\mathrm{~m}, 4 \mathrm{H}), 1.82(\mathrm{~s}, 3 \mathrm{H}), 1.23-1.12(\mathrm{~m}, 1 \mathrm{H}), 0.91(\mathrm{dd}, J=$ $12.7,2.8 \mathrm{~Hz}, 1 \mathrm{H}), 0.51(\mathrm{~s}, 3 \mathrm{H})$.

${ }^{13}$ C NMR (101 MHz, C6 $\left.\mathrm{D}_{6}\right) \delta$ 144.8, 142.6, 141.3, 139.3, 137.0, 129.4, 129.0, 127.5, 125.7, 122.3, 118.0, 57.8, 49.2, $38.0,36.2,30.8,27.9,27.7,21.0,11.5$.

HRMS ESI Calcd for $\mathrm{C}_{24} \mathrm{H}_{28} \mathrm{~N}_{3} \mathrm{O}_{2} \mathrm{~S}[\mathrm{M}+\mathrm{H}]^{+}:$422.1897, Found: 422.1896.

IR (KBr): 3061, 2955, 2923, 2851, 1599, 1505, 1457, 1390, 1343, 1210, 1159, 1052, $1006 \mathrm{~cm}^{-1}$. 
Substrates with low yield, low conversion or decomposition

low yield
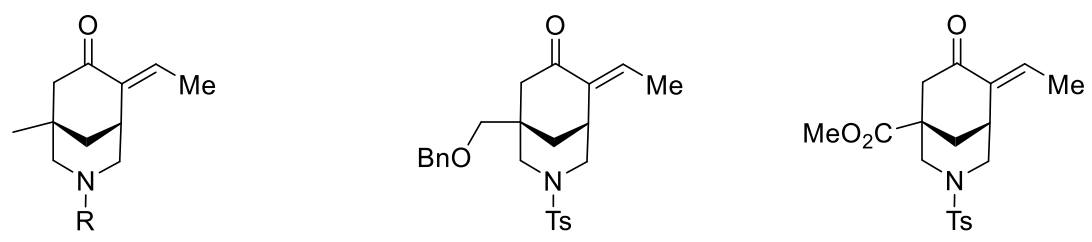

$\mathrm{R}=\mathrm{CO}_{2} \mathrm{Me}, 22 \%$ yield

$34 \%$ yield, er $75: 25$

$<5 \%$ yield

$\mathrm{R}=\mathrm{Ac}, 14 \%$ yield

$\mathrm{R}=\mathrm{Boc},<2 \%$ yield

low conversion

(c)
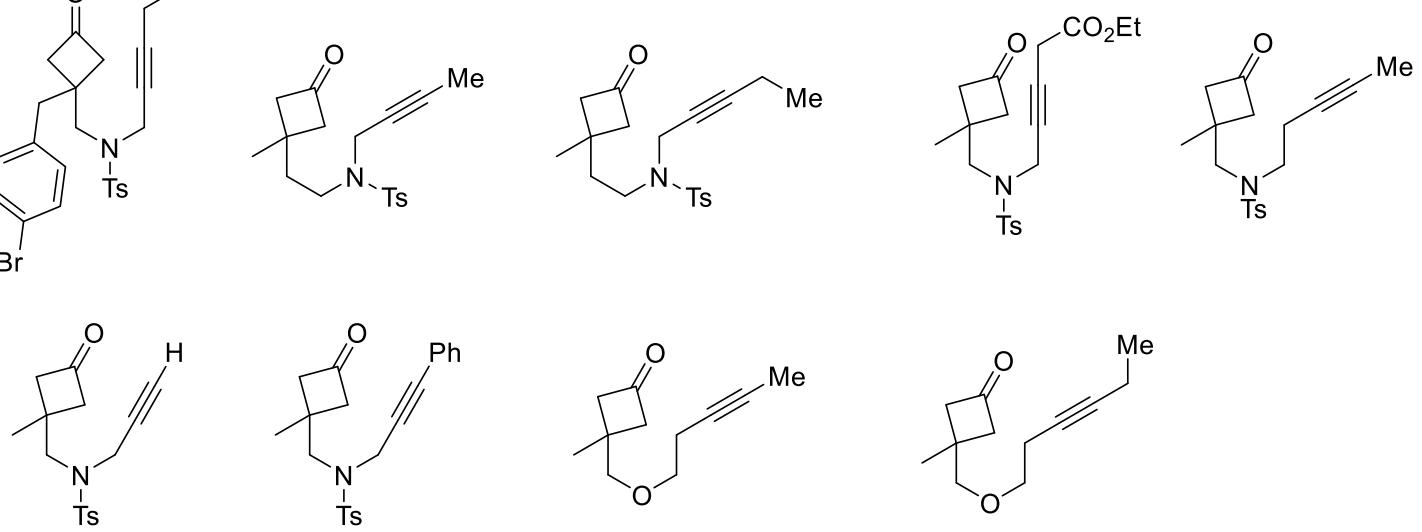

decomposition<smiles>CCC#CCN([13CH3])CC1CC(=O)C1</smiles><smiles>[3H]N(CC#CCCCOCc1ccccc1)CC1(C)CC(=O)C1</smiles><smiles>[3H]N(CC#CCCCO[SnH])CC1(C)CC(=O)C1</smiles><smiles>[3H]N(CC#CCCOC)CC1(C)CC(=O)C1</smiles><smiles>CCC#CN(S)CCC1(C)CC(=O)C1</smiles><smiles>CCC#CCN(Cc1ccccc1)CC1(C)CC(=O)C1</smiles><smiles>[3H]N(CC#CCC)CC1(CO)CC(=O)C1</smiles><smiles>CCC#CCN([13CH3])C(C)(C)C1(C)CC(=O)C1</smiles><smiles>CCC#CCN(Cc1ccccc1)C(=O)C1(C)CC(=O)C1</smiles><smiles>CC#CCCN(C)CC1(C)CC(=O)C1</smiles><smiles>CCC#CCCN(S)CC1(C)CC(=O)C1</smiles> 
Synthesis of cationic rhodium $\left[\mathrm{Rh}(\mathrm{COD})_{2}\right] \mathrm{NTf}_{2}$

$$
\begin{array}{cccc}
{\left[\mathrm{Rh}(\mathrm{COD}) \mathrm{Cl}_{2}+\mathrm{AgNTf}_{2}\right.} & +\operatorname{COD} \underset{\mathrm{mCM}, \mathrm{rt}}{\stackrel{83 \% \text { yield }}{\longrightarrow}} & {\left[\mathrm{Rh}(\mathrm{COD})_{2}\right] \mathrm{NTf}_{2}} \\
0.5 \mathrm{mmol} & 1 \mathrm{mmol} & 3 \mathrm{mmol} & 500 \mathrm{mg}
\end{array}
$$

In a nitrogen-filled glove box, a solution of $\mathrm{AgNTf}_{2}$ (388 mg, $1 \mathrm{mmol}, 2$ equiv) in DCM (10 mL) was added to a solution of $[\mathrm{Rh}(\mathrm{COD}) \mathrm{Cl}]_{2}$ (248 mg, $0.5 \mathrm{mmol}, 1$ equiv) and 1,5-cyclooctadiene (COD) (0.37 mL, $3 \mathrm{mmol}, 6$ equiv) in DCM (5 mL). After stirred at room temperature for $1 \mathrm{~h}$, the reaction mixture was filtered through Celite and washed with DCM (3 $\mathrm{mL}$ ) and THF ( $3 \mathrm{~mL})$. The filtrate was concentrated under reduced pressure until left around $1 \mathrm{~mL}$ solvent. After Et ${ }_{2} \mathrm{O}$ $(50 \mathrm{~mL})$ was added, the mixture was filtered and washed with $\mathrm{Et}_{2} \mathrm{O}(2 \mathrm{X} 10 \mathrm{~mL})$ to give $500 \mathrm{mg}$ of $\left[\mathrm{Rh}(\mathrm{COD})_{2}\right] \mathrm{NTf} \mathrm{f}_{2}$ as a red solid in $83 \%$ yield.

Note: All procedure was conducted in the nitrogen-filled glove box.

${ }^{1} \mathrm{H}$ NMR (400 MHz, Chloroform-d) $\delta 5.41-5.25$ (m, 8H), $2.69-2.41$ (m, 16H).

${ }^{13}$ C NMR (101 MHz, CDCl $) \delta 107.6,107.5,29.7$.

${ }^{19}$ F NMR (376 MHz, $\left.\mathrm{CDCl}_{3}\right) \delta-78.7$.

${ }^{1} \mathrm{H}-\mathrm{NMR}$ for $\left[\mathrm{Rh}(\mathrm{COD})_{2}\right] \mathrm{NTf}_{2}$ in $\mathrm{CDCl}_{3}, 400 \mathrm{MHz}$

liux3-130-3.10.fid

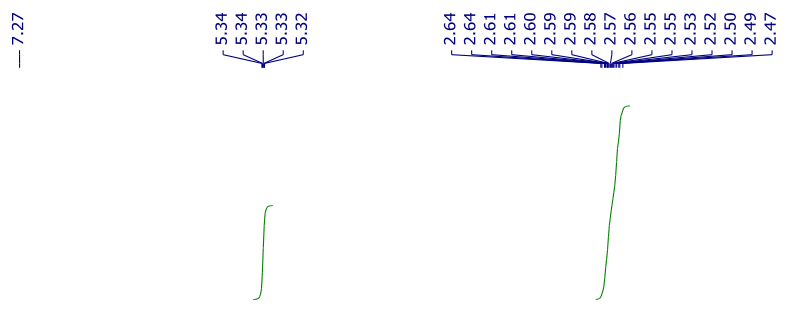

$\left[\mathrm{Rh}(\mathrm{COD})_{2} \mathrm{NTf}_{2}\right.$

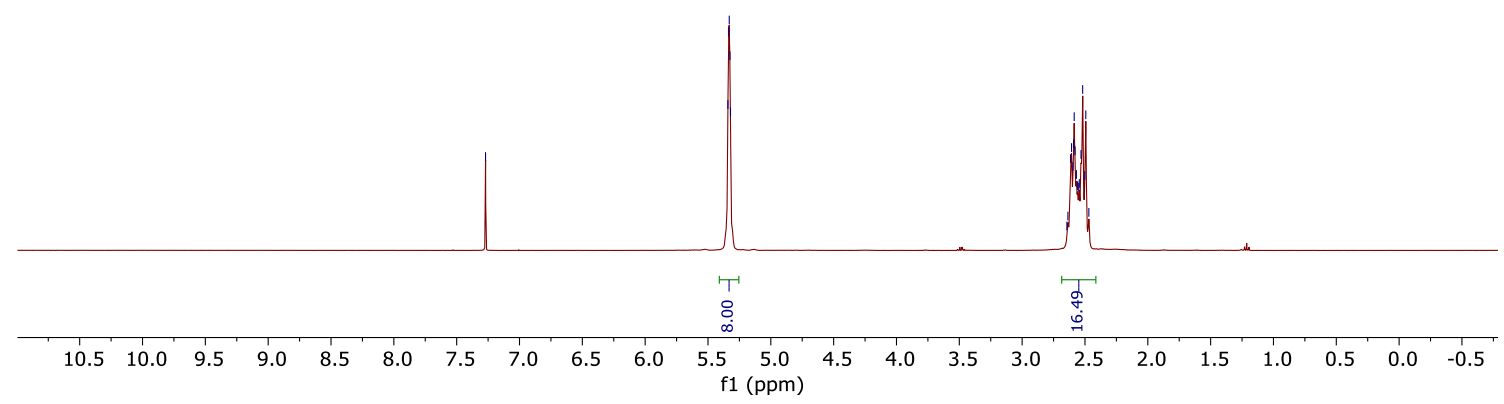


${ }^{13} \mathrm{C}-\mathrm{NMR}$ for $\left[\mathrm{Rh}(\mathrm{COD})_{2}\right] \mathrm{NTf} f_{2}$ in $\mathrm{CDCl}_{3}, 101 \mathrm{MHz}$

liux3-130-3.11.fid

$\left[\mathrm{Rh}(\mathrm{COD})_{2}\right] \mathrm{NTf}$

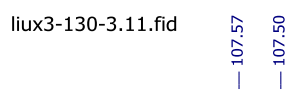

।

107.9107.8107.7107.6107.5107.4 $\mathrm{f} 1(\mathrm{ppm})$

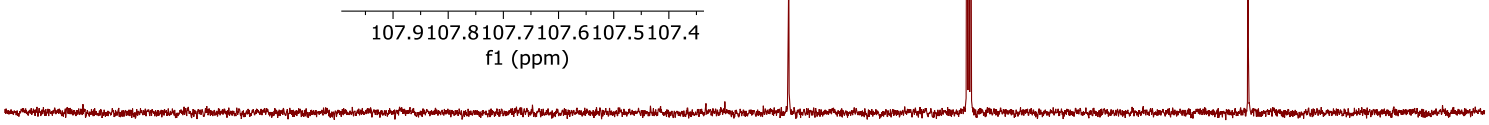

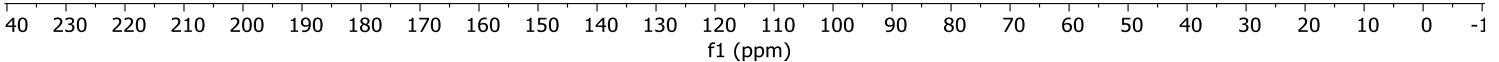

${ }^{19} \mathrm{~F}-\mathrm{NMR}$ for $\left[\mathrm{Rh}(\mathrm{COD})_{2}\right] \mathrm{NTf}_{2}$ in $\mathrm{CDCl}_{3}, 376 \mathrm{MHz}$

liux3-130-3.12.fid

$\left[\mathrm{Rh}(\mathrm{COD})_{2} \mathrm{NTf}_{2}\right.$

$\begin{array}{lllllllllllllllllllllll}10 & 0 & -10 & -20 & -30 & -40 & -50 & -60 & -70 & -80 & -90 & -100 & -110 & -120 & -130 & -140 & -150 & -160 & -170 & -180 & -190 & -200 & -21 c\end{array}$ 


\section{Mechanism studies.}

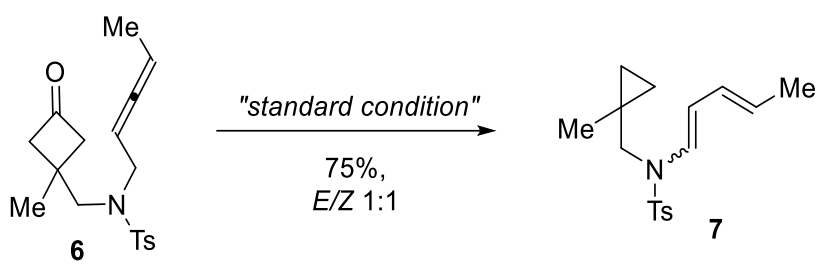

Compound 7. A flame dried $8 \mathrm{~mL}$ tube was charged with the substrate $6(33.3 \mathrm{mg}, 0.1 \mathrm{mmol}, 1$ equiv) and (R)-(-)DTBM-segphos (L1, $9.9 \mathrm{mg}, 0.0084 \mathrm{mmol}, 8.4 \mathrm{~mol} \%$ ). The tube was then transferred to a nitrogen-filled glove box. After addition of $\left[\mathrm{Rh}(\mathrm{COD})_{2}\right] \mathrm{NTf}_{2}(4.2 \mathrm{mg}, 0.007 \mathrm{mmol}, 7 \mathrm{~mol} \%)$ and 1,4-diflorobenzene $(0.5 \mathrm{~mL})$, the tube was sealed and taken out of glove box. The reaction was stirred at $120^{\circ} \mathrm{C}$ in an oil bath for $48 \mathrm{~h}$, before it was then cooled to room temperature. The product was directly purified by silica gel flash chromatography to yield compound 7 (22.9 $\mathrm{mg}$ ) in $75 \%$ yield. The $E / Z$ ratio of compound 7 was determined by crude ${ }^{1} \mathrm{H}$ NMR.

$\mathrm{Rf}=0.7$, Hexane/ EtOAc 10:1.

$Z$ isomer: ${ }^{1}$ H NMR (400 MHz, Chloroform-d) $\delta$ 7.66-7.62 (m, 2H), 7.30-7.27 (m, 2H), $6.78-6.62(\mathrm{~m}, 1 \mathrm{H}), 6.05-5.89$ $(\mathrm{m}, 2 \mathrm{H}), 5.45-5.34(\mathrm{~m}, 1 \mathrm{H}), 3.30(\mathrm{~s}, 2 \mathrm{H}), 2.41(\mathrm{~s}, 3 \mathrm{H}), 1.71(\mathrm{dd}, J=7.1,1.4 \mathrm{~Hz}, 3 \mathrm{H}), 1.09(\mathrm{~s}, 3 \mathrm{H}), 0.58-0.46(\mathrm{~m}, 2 \mathrm{H})$, $0.39-0.26(m, 2 H)$.

$E$ isomer: ${ }^{1} \mathrm{H}$ NMR $(400 \mathrm{MHz}$, Chloroform- $d) \delta$ 7.66-7.62 (m, 2H), 7.30-7.27 (m, 2H), $6.58(\mathrm{~d}, J=13.9 \mathrm{~Hz}, 1 \mathrm{H}), 5.95-$ $5.85(\mathrm{~m}, 1 \mathrm{H}), 5.69(\mathrm{dd}, J=14.0,10.4 \mathrm{~Hz}, 1 \mathrm{H}), 5.57(\mathrm{dq}, J=15.1,6.6 \mathrm{~Hz}, 1 \mathrm{H}), 3.24(\mathrm{~s}, 2 \mathrm{H}), 2.41(\mathrm{~d}, J=1.7 \mathrm{~Hz}, 3 \mathrm{H}), 1.75$ (dd, $J=6.8,1.6 \mathrm{~Hz}, 3 \mathrm{H}), 1.07(\mathrm{~s}, 3 \mathrm{H}), 0.58-0.46(\mathrm{~m}, 2 \mathrm{H}), 0.39-0.26(\mathrm{~m}, 2 \mathrm{H})$.

HRMS ESI Calcd for $\mathrm{C}_{17} \mathrm{H}_{24} \mathrm{NO}_{2} \mathrm{~S}[\mathrm{M}+\mathrm{H}]^{+}:$306.1522, Found: 306.1526.

IR (KBr): 2962, 1673, 1616, 1452, 1360, 1262, 1165, $1090 \mathrm{~cm}^{-1}$.

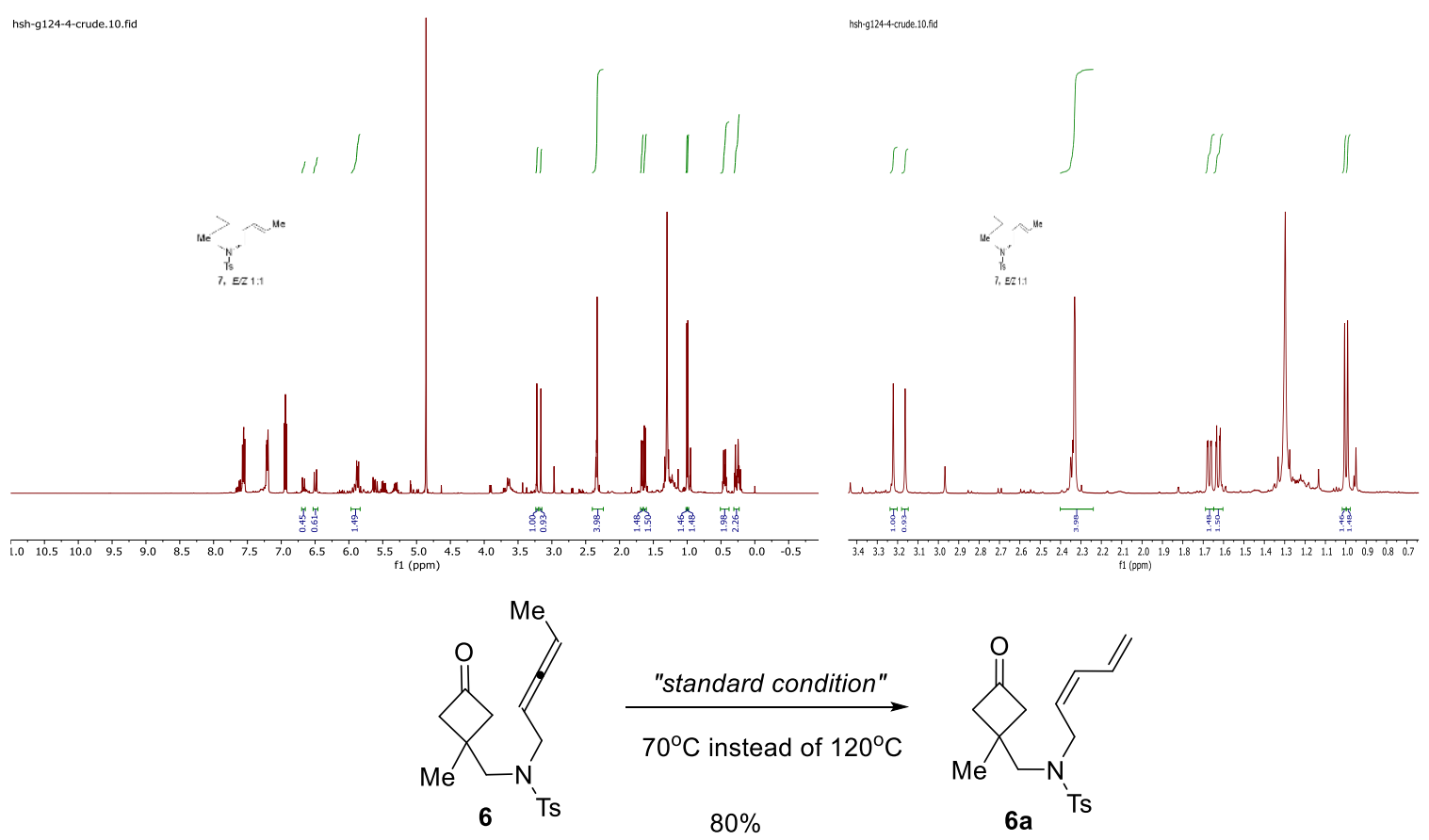


Compound 6a. A flame dried $8 \mathrm{~mL}$ tube was charged with the substrate $6(33.3 \mathrm{mg}, 0.1 \mathrm{mmol}, 1$ equiv) and (R)-(-)DTBM-segphos (L1, $9.9 \mathrm{mg}, 0.0084 \mathrm{mmol}, 8.4 \mathrm{~mol} \%$ ). The tube was then transferred to a nitrogen-filled glove box. After addition of $\left[\mathrm{Rh}(\mathrm{COD})_{2}\right] \mathrm{NTf}_{2}(4.2 \mathrm{mg}, 0.007 \mathrm{mmol}, 7 \mathrm{~mol} \%)$ and 1,4-diflorobenzene $(0.5 \mathrm{~mL})$, the tube was sealed and taken out of glove box. The reaction was stirred at $70^{\circ} \mathrm{C}$ in an oil bath for $48 \mathrm{~h}$, before it was then cooled to room temperature. The product was directly purified by silica gel flash chromatography to yield compound 6 a ( 26.7 $\mathrm{mg})$ as a colorless oil in $80 \%$ yield.

Rf $=0.36$, Hexane/ EtOAc 4:1.

${ }^{1} \mathrm{H}$ NMR (400 MHz, Chloroform-d) $\delta 7.75-7.67(\mathrm{~m}, 2 \mathrm{H}), 7.36-7.29(\mathrm{~m}, 2 \mathrm{H}), 6.43$ (dddd, J = 16.7, 11.2, 10.1, 1.1 Hz, 1H), 6.05 (ddt, $J=11.1,10.0,0.9 \mathrm{~Hz}, 1 \mathrm{H}), 5.30-5.18(\mathrm{~m}, 2 \mathrm{H}), 5.09$ (dddd, $J=11.1,8.0,5.7,1.2 \mathrm{~Hz}, 1 \mathrm{H}), 4.06(\mathrm{dd}, J=$ 6.9, $1.8 \mathrm{~Hz}, 2 \mathrm{H}), 3.41(\mathrm{~s}, 2 \mathrm{H}), 3.13-2.99(\mathrm{~m}, 2 \mathrm{H}), 2.77-2.64(\mathrm{~m}, 2 \mathrm{H}), 2.45(\mathrm{~s}, 3 \mathrm{H}), 1.39(\mathrm{~s}, 3 \mathrm{H})$.

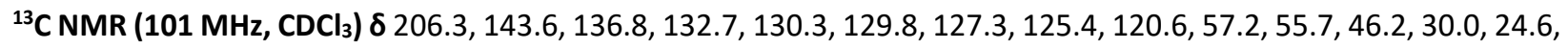
21.5.

HRMS ESI Calcd for $\mathrm{C}_{18} \mathrm{H}_{24} \mathrm{NO}_{3} \mathrm{~S}[\mathrm{M}+\mathrm{H}]^{+}: 334.1471$, Found: 334.1461.

IR (KBr): 2958, 2925, 1781, 1725, 1455, 1381, 1339, 1290, 1159, $1090 \mathrm{~cm}^{-1}$.

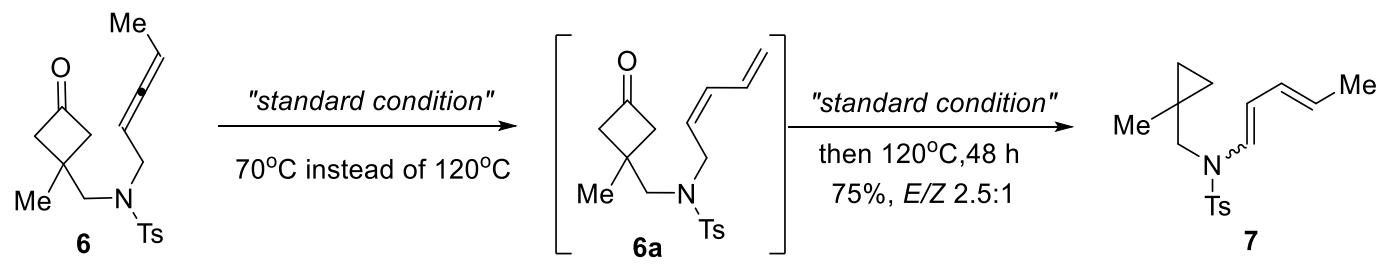

Compound 7. A flame dried $8 \mathrm{~mL}$ tube was charged with the substrate $6(33.3 \mathrm{mg}, 0.1 \mathrm{mmol}, 1$ equiv) and $(R)-(-)-$ DTBM-segphos (L1, $9.9 \mathrm{mg}, 0.0084 \mathrm{mmol}, 8.4 \mathrm{~mol} \%$ ). The tube was then transferred to a nitrogen-filled glove box. After addition of $\left[\mathrm{Rh}(\mathrm{COD})_{2}\right] \mathrm{NTf}_{2}(4.2 \mathrm{mg}, 0.007 \mathrm{mmol}, 7 \mathrm{~mol} \%)$ and 1,4-diflorobenzene $(0.5 \mathrm{~mL})$, the tube was sealed and taken out of glove box. The reaction was stirred at $70^{\circ} \mathrm{C}$ for $24 \mathrm{~h}$ and $120^{\circ} \mathrm{C}$ for $48 \mathrm{~h}$, before it was then cooled to room temperature. The product was directly purified by silica gel flash chromatography to yield compound 7 ( 22.9 $\mathrm{mg}$ ) in $75 \%$ yield with $2.5: 1 \mathrm{E} / \mathrm{Z}$ ratio.

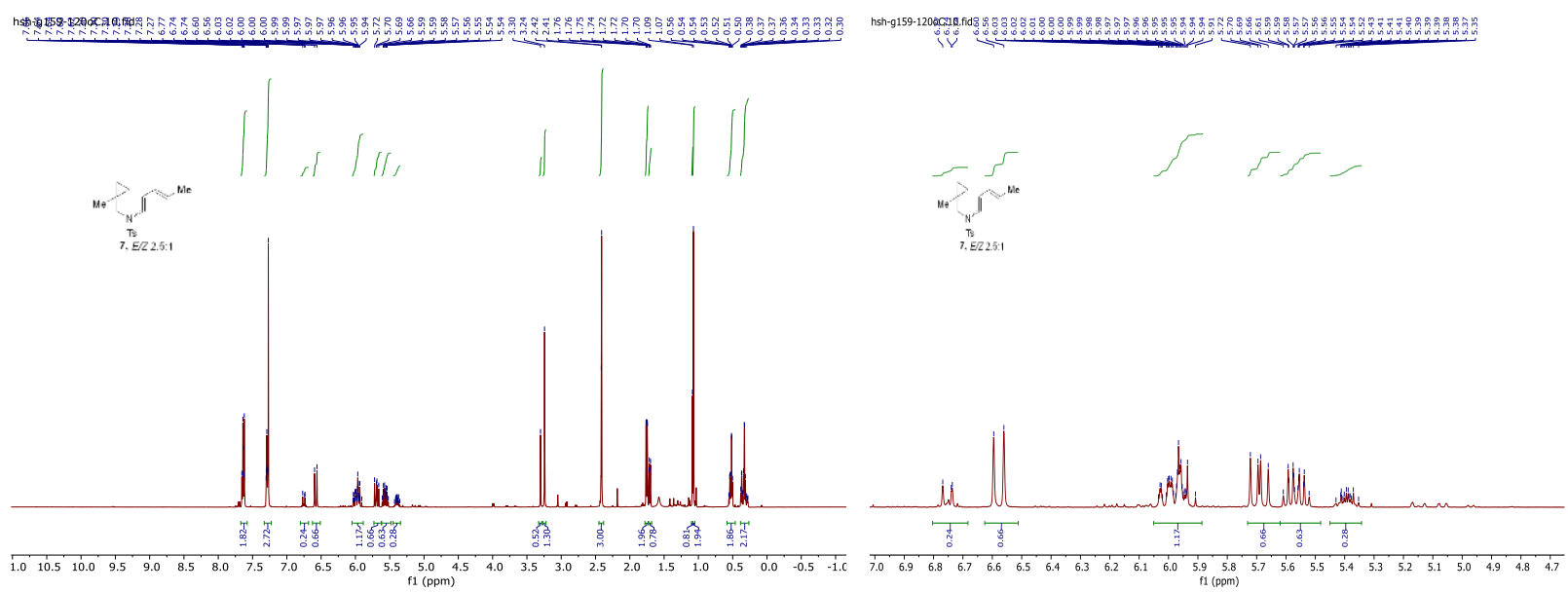



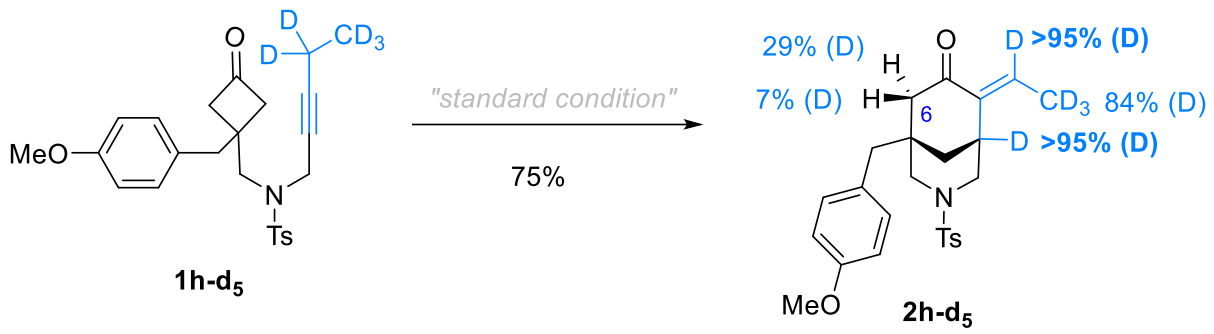

(5)

Compound 2h-d5. A flame dried $8 \mathrm{~mL}$ tube was charged with the substrate $\mathbf{1 h}$-d5 ( $44.5 \mathrm{mg}, 0.1 \mathrm{mmol}, 1$ equiv) and (R)-(-)-DTBM-segphos (L1, $9.9 \mathrm{mg}, 0.0084 \mathrm{mmol}, 8.4 \mathrm{~mol} \%$ ). The tube was then transferred to a nitrogen-filled glove box. After addition of $\left[\mathrm{Rh}(\mathrm{COD})_{2}\right] \mathrm{NTf}_{2}(4.2 \mathrm{mg}, 0.007 \mathrm{mmol}, 7 \mathrm{~mol} \%)$ and 1,4-diflorobenzene $(0.5 \mathrm{~mL})$, the tube was sealed and taken out of glove box. The reaction was stirred at $120^{\circ} \mathrm{C}$ in an oil bath for $48 \mathrm{~h}$, before it was then cooled to room temperature. The product was directly purified by silica gel flash chromatography to yield bridged bicycle $2 \mathrm{~h}-\mathrm{d5}(33 \mathrm{mg})$ as a foam in $75 \%$ yield.

${ }^{1} \mathrm{H}$ NMR $\left(400 \mathrm{MHz}\right.$, Benzene- $\left.d_{6}\right) \delta 7.80-7.71(\mathrm{~m}, 2 \mathrm{H}), 6.93-6.85(\mathrm{~m}, 2 \mathrm{H}), 6.76-6.67(\mathrm{~m}, 4 \mathrm{H}), 3.61-3.47(\mathrm{~m}, 2 \mathrm{H})$, $3.33(\mathrm{~s}, 3 \mathrm{H}), 2.41(\mathrm{dd}, J=17.8,3.0 \mathrm{~Hz}, 0.93 \mathrm{H}), 2.30-2.17(\mathrm{~m}, 2 \mathrm{H}), 2.10(\mathrm{dd}, J=17.6,1.4 \mathrm{~Hz}, 0.71 \mathrm{H}), 2.04(\mathrm{~d}, J=13.5$ $\mathrm{Hz}, 1 \mathrm{H}), 1.98(\mathrm{~d}, J=13.4 \mathrm{~Hz}, 1 \mathrm{H}), 1.90(\mathrm{~s}, 3 \mathrm{H}), 1.24-1.16(\mathrm{~m}, 1.47 \mathrm{H}), 0.96(\mathrm{dd}, J=13.1,2.9 \mathrm{~Hz}, 1 \mathrm{H})$.

${ }^{13} \mathrm{C}$ NMR (101 MHz, C6 $\left.\mathrm{D}_{6}\right) \delta 195.7,159.0,143.5,138.6,134.7,131.7,129.6,113.9,56.7,54.7,51.8,49.1,45.3,45.2$, $36.2,36.2,34.9,21.1$.
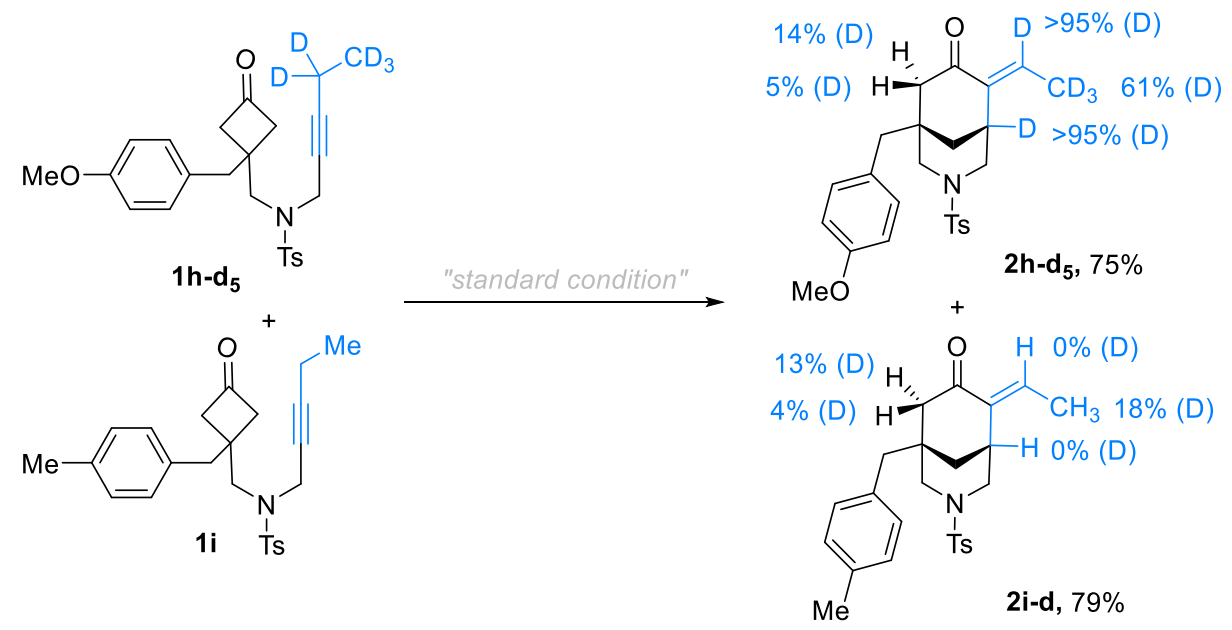

(6)

Compounds $\mathbf{2 h - d 5}$ and $\mathbf{2 i - d}$. A flame dried $8 \mathrm{~mL}$ tube was charged with the substrate $\mathbf{1 h}-\mathbf{d} \mathbf{5}(22.3 \mathrm{mg}, 0.05 \mathrm{mmol}$, 0.5 equiv), $1 \mathbf{i}$ (21.2 mg, $0.05 \mathrm{mmol}, 0.5$ equiv) and (R)-(-)-DTBM-segphos (L1, $9.9 \mathrm{mg}, 0.0084 \mathrm{mmol}, 8.4 \mathrm{~mol} \%$ ). The tube was then transferred to a nitrogen-filled glove box. After addition of [Rh(COD) $\left.{ }_{2}\right] \mathrm{NTf}_{2}(4.2 \mathrm{mg}, 0.007 \mathrm{mmol}, 7$ mol\%) and 1,4-diflorobenzene $(0.5 \mathrm{~mL})$, the tube was sealed and taken out of glove box. The reaction was stirred at $120^{\circ} \mathrm{C}$ in an oil bath for $48 \mathrm{~h}$, before it was then cooled to room temperature. The products were directly purified 
by silica gel flash chromatography to yield bridged bicycles $\mathbf{2} \mathbf{h}-\mathbf{d}_{\mathbf{5}}(\mathbf{1 6 . 4} \mathbf{m g})$ in $\mathbf{7 5 \%}$ yield as a foam and $\mathbf{2 i - 1}$ (18.9 $\left.\mathrm{mg}\right)$ in $79 \%$ yield as a foam.

\section{Compound $2 \mathrm{~h}-\mathrm{d} 5$.}

${ }^{1} \mathrm{H}$ NMR (400 MHz, Benzene- $\left.d_{6}\right) \delta 7.79-7.69(\mathrm{~m}, 2 \mathrm{H}), 6.87(\mathrm{~d}, J=8.0 \mathrm{~Hz}, 2 \mathrm{H}), 6.75-6.62(\mathrm{~m}, 4 \mathrm{H}), 3.52(\mathrm{td}, J=10.1$, $2.0 \mathrm{~Hz}, 2 \mathrm{H}$ ), $3.32(\mathrm{~s}, 3 \mathrm{H}), 2.39(\mathrm{dd}, J=17.7,3.0 \mathrm{~Hz}, 0.95 \mathrm{H}), 2.24(\mathrm{dd}, J=11.2,3.1 \mathrm{~Hz}, 2 \mathrm{H}), 2.08(\mathrm{dd}, J=17.6,1.4 \mathrm{~Hz}$, $0.86 \mathrm{H}), 2.02(\mathrm{~d}, J=13.5 \mathrm{~Hz}, 1 \mathrm{H}), 1.96(\mathrm{~d}, J=13.4 \mathrm{~Hz}, 1 \mathrm{H}), 1.88(\mathrm{~s}, 3 \mathrm{H}), 1.23-1.14(\mathrm{~m}, 2.18 \mathrm{H}), 0.95(\mathrm{dd}, J=13.1,3.0$ $\mathrm{Hz}, 1 \mathrm{H})$.

\section{Compound 2i-d.}

${ }^{1} \mathrm{H}$ NMR (400 MHz, Benzene-d6) $\delta 7.78-7.69(\mathrm{~m}, 2 \mathrm{H}), 6.92(\mathrm{~d}, J=7.7 \mathrm{~Hz}, 2 \mathrm{H}), 6.86(\mathrm{~d}, J=8.1 \mathrm{~Hz}, 2 \mathrm{H}), 6.72-6.64(\mathrm{~m}$, $3 \mathrm{H}), 3.51(\mathrm{tt}, J=10.9,1.8 \mathrm{~Hz}, 2 \mathrm{H}), 2.56(\mathrm{t}, J=3.0 \mathrm{~Hz}, 1 \mathrm{H}), 2.38(\mathrm{dd}, J=17.6,3.0 \mathrm{~Hz}, 0.96 \mathrm{H}), 2.24(\mathrm{ddd}, J=10.8,3.8$, $1.7 \mathrm{~Hz}, 2 \mathrm{H}), 2.13-2.00(\mathrm{~m}, 4.87 \mathrm{H}), 1.96(\mathrm{~d}, J=13.3 \mathrm{~Hz}, 1 \mathrm{H}), 1.88(\mathrm{~s}, 3 \mathrm{H}), 1.23-1.14(\mathrm{~m}, 3.45 \mathrm{H}), 0.97(\mathrm{dt}, J=13.1$, $3.2 \mathrm{~Hz}, 1 \mathrm{H})$.

${ }^{13} \mathrm{C}$ NMR (101 MHz, C6 $\left.\mathrm{D}_{6}\right) \delta$ 195.5, 143.4, 138.6, 136.2, 134.8, 133.0, 132.2, 130.6, 129.6, 129.0, 128.5, 56.7, 51.7, $49.2,45.8,36.2,35.1,31.2,21.1,21.0,12.6$.

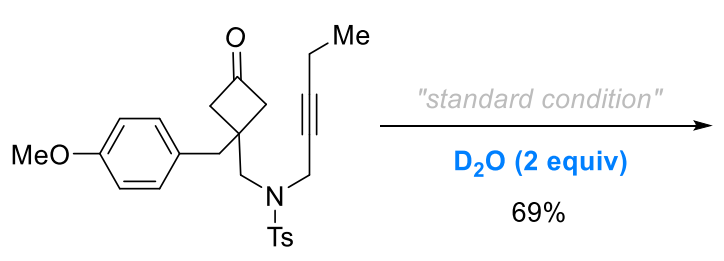

$1 \mathrm{~h}$

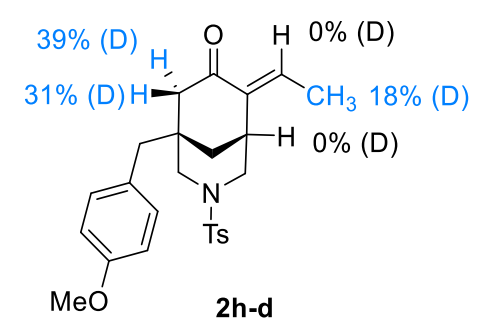

(7)

Compound $2 \mathrm{~h}$-d. A flame dried $8 \mathrm{~mL}$ tube was charged with the substrate $1 \mathrm{~h}(43.9 \mathrm{mg}, 0.1 \mathrm{mmol}, 1 \mathrm{equiv})$ and $(R)$ (-)-DTBM-segphos (L1, $9.9 \mathrm{mg}, 0.0084 \mathrm{mmol}, 8.4 \mathrm{~mol} \%$ ). The tube was then transferred to a nitrogen-filled glove box. After addition of $\left[\mathrm{Rh}(\mathrm{COD})_{2}\right] \mathrm{NTf}_{2}(4.2 \mathrm{mg}, 0.007 \mathrm{mmol}, 7 \mathrm{~mol} \%), 1,4$-diflorobenzene $(0.5 \mathrm{~mL})$ and $\mathrm{D}_{2} \mathrm{O}(3.6 \mu \mathrm{L}$, $0.2 \mathrm{mmol}, 2$ equiv), the tube was sealed and taken out of glove box. The reaction was stirred at $120^{\circ} \mathrm{C}$ in an oil bath for $48 \mathrm{~h}$, before it was cooled to room temperature. The product was directly purified by silica gel flash chromatography to yield bridged bicycle $\mathbf{2 h - d}(30 \mathrm{mg})$ in $69 \%$ yield as a foam.

${ }^{1} \mathrm{H}$ NMR $\left(400 \mathrm{MHz}\right.$, Benzene- $\left.d_{6}\right) \delta 7.80-7.68(\mathrm{~m}, 2 \mathrm{H}), 6.94-6.84(\mathrm{~m}, 2 \mathrm{H}), 6.79-6.63(\mathrm{~m}, 5 \mathrm{H}), 3.53(\mathrm{tt}, J=10.7,1.7$ $\mathrm{Hz}, 2 \mathrm{H}$ ), $3.33(\mathrm{~s}, 3 \mathrm{H}), 2.60(\mathrm{t}, J=3.0 \mathrm{~Hz}, 1 \mathrm{H}), 2.41(\mathrm{dd}, J=17.7,3.0 \mathrm{~Hz}, 0.69 \mathrm{H}), 2.24$ (ddd, J = 11.0, 7.2, $4.1 \mathrm{~Hz}, 2 \mathrm{H}$ ), $2.10(\mathrm{dd}, J=17.7,1.4 \mathrm{~Hz}, 0.61 \mathrm{H}), 2.04(\mathrm{~d}, J=13.5 \mathrm{~Hz}, 1 \mathrm{H}), 1.98(\mathrm{~d}, J=13.5 \mathrm{~Hz}, 1 \mathrm{H}), 1.90(\mathrm{~s}, 3 \mathrm{H}), 1.26-1.16(\mathrm{~m}, 3.45 \mathrm{H})$, $0.97(\mathrm{dt}, J=12.9,2.9 \mathrm{~Hz}, 1 \mathrm{H})$. 


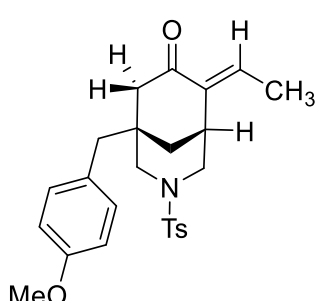

$2 \mathrm{~h}$

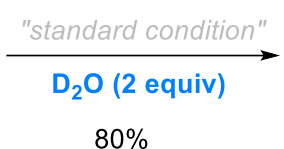

$80 \%$

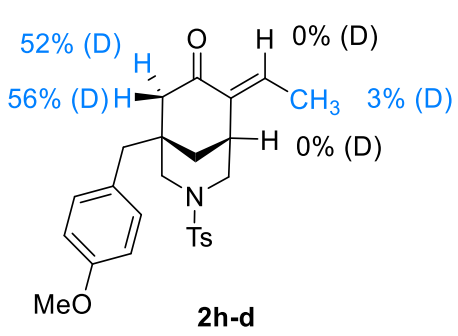

(8)

Compound 2 h-d. A flame dried $8 \mathrm{~mL}$ tube was charged with the substrate $2 \mathrm{~h}$ ( $25 \mathrm{mg}, 0.057 \mathrm{mmol}, 1$ equiv) and $(R)$ (-)-DTBM-segphos (L1, $5.7 \mathrm{mg}, 0.0048 \mathrm{mmol}, 8.4 \mathrm{~mol} \%)$. The tube was then transferred to a nitrogen-filled glove box. After addition of $\left[\mathrm{Rh}(\mathrm{COD})_{2}\right] \mathrm{NTf}_{2}(2.3 \mathrm{mg}, 0.004 \mathrm{mmol}, 7 \mathrm{~mol} \%)$, 1,4-diflorobenzene $(0.5 \mathrm{~mL})$ and $\mathrm{D}_{2} \mathrm{O}(2.1 \mu \mathrm{L}$, $0.114 \mathrm{mmol}, 2$ equiv), the tube was sealed and taken out of glove box. The reaction was stirred at $120^{\circ} \mathrm{C}$ in an oil bath for $48 \mathrm{~h}$, before it was then cooled to room temperature. The product was directly purified by silica gel flash chromatography to yield bridged bicycle $\mathbf{2} \mathbf{h}-\mathbf{d}(20 \mathrm{mg}$ ) in $80 \%$ yield as a foam.

${ }^{1} \mathrm{H}$ NMR (400 MHz, Chloroform-d) $87.79-7.70(\mathrm{~m}, 2 \mathrm{H}), 6.92-6.85(\mathrm{~m}, 2 \mathrm{H}), 6.76-6.65(\mathrm{~m}, 5 \mathrm{H}), 3.54(\mathrm{tt}, J=9.9,2.2$ $\mathrm{Hz}, 2 \mathrm{H}$ ), 2.41 (dd, $J=17.7,3.0 \mathrm{~Hz}, 0.48 \mathrm{H}$ ), $3.34(\mathrm{~s}, 3 \mathrm{H}), 2.60$ (q, $J=2.9 \mathrm{~Hz}, 1 \mathrm{H}$ ), 2.25 (ddd, $J=11.3,5.7,2.0 \mathrm{~Hz}, 2 \mathrm{H}$ ), $2.10(\mathrm{dd}, J=17.7,1.4 \mathrm{~Hz}, 0.44 \mathrm{H}), 2.04(\mathrm{~d}, J=13.5 \mathrm{~Hz}, 1 \mathrm{H}), 1.98(\mathrm{~d}, J=13.5 \mathrm{~Hz}, 1 \mathrm{H}), 1.90(\mathrm{~s}, 3 \mathrm{H}), 1.25-1.16(\mathrm{~m}, 3.88 \mathrm{H})$, $1.01-0.93(\mathrm{~m}, 1 \mathrm{H})$.
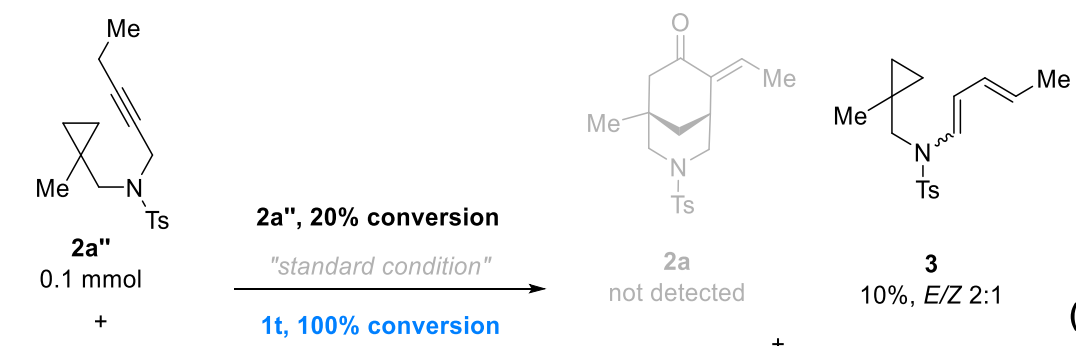

(9)
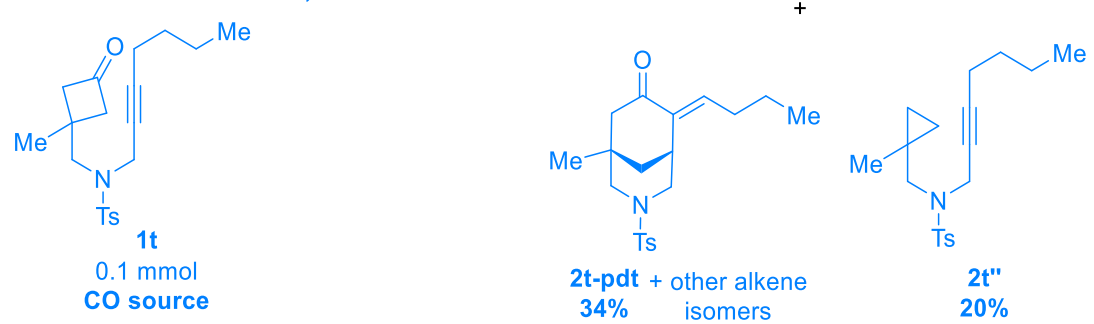

Control experiments. A flame dried $8 \mathrm{~mL}$ tube was charged with compound $2 \mathrm{a}^{\prime \prime}$ ( $30.5 \mathrm{mg}, 0.1 \mathrm{mmol}, 1$ equiv), 1t (36.1 mg, $0.1 \mathrm{mmol}, 1$ equiv) and (R)-(-)-DTBM-segphos (L1, $9.9 \mathrm{mg}, 0.0084 \mathrm{mmol}, 8.4 \mathrm{~mol} \%$ ). The tube was then transferred to a nitrogen-filled glove box. After addition of [Rh(COD) $\left.)_{2}\right] \mathrm{NTf}_{2}(4.2 \mathrm{mg}, 0.007 \mathrm{mmol}, 7 \mathrm{~mol} \%)$ and 1,4diflorobenzene $(0.5 \mathrm{~mL})$, the tube was sealed and taken out of glove box. The reaction was stirred at $120^{\circ} \mathrm{C}$ in an oil bath for $48 \mathrm{~h}$, before it was cooled to room temperature. The yields were determined by ${ }^{1} \mathrm{H} N M R$ using dibromomethane as the internal standard in $\mathrm{CDCl}_{3}$. The product was purified by silica gel flash chromatography to yield bridged bicycle $\mathbf{2 t - p d t}(12 \mathrm{mg}$ ) in $34 \%$ yield as a foam. 


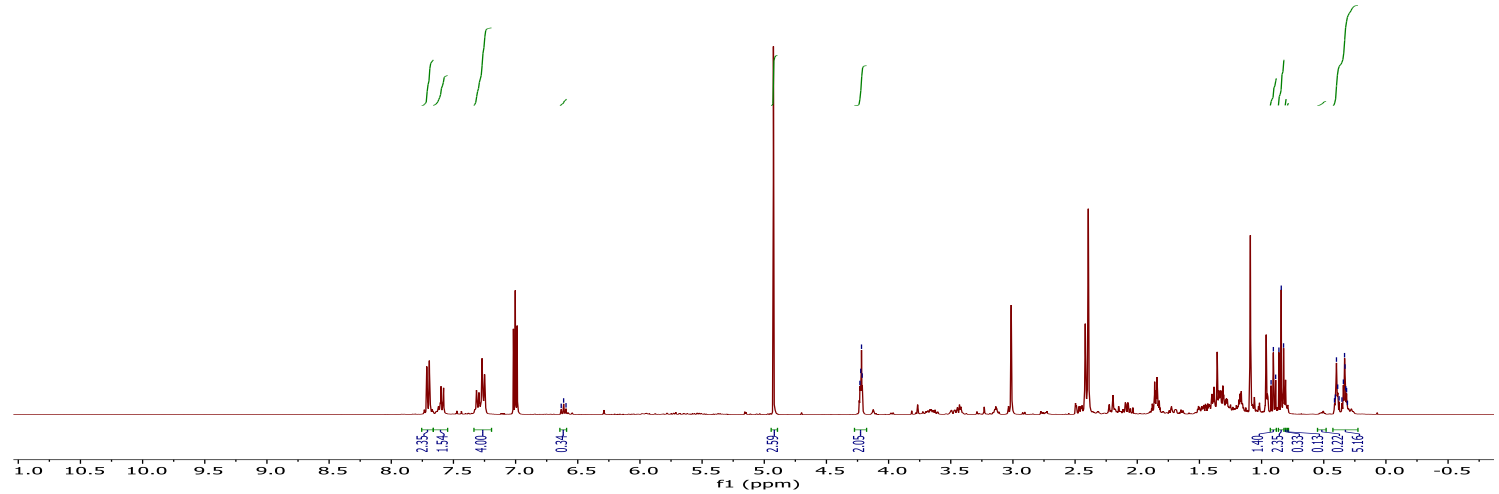

\section{Compound 2t-pdt}

$\mathrm{Rf}=0.2$, Hexane/ EtOAc 2:1.

${ }^{1} \mathrm{H}$ NMR $\left(400 \mathrm{MHz}\right.$, Benzene- $\left.d_{6}\right) \delta 7.75-7.68(\mathrm{~m}, 2 \mathrm{H}), 6.85(\mathrm{~d}, J=8.0 \mathrm{~Hz}, 2 \mathrm{H}), 6.77(\mathrm{t}, J=7.7 \mathrm{~Hz}, 1 \mathrm{H}), 3.51(\mathrm{ddt}, J=$ 10.8, 3.4, $1.8 \mathrm{~Hz}, 1 \mathrm{H}), 3.41(\mathrm{dt}, J=11.3,2.1 \mathrm{~Hz}, 1 \mathrm{H}), 2.62(\mathrm{p}, J=2.9 \mathrm{~Hz}, 1 \mathrm{H}), 2.39(\mathrm{dd}, J=17.9,3.1 \mathrm{~Hz}, 1 \mathrm{H}), 2.28(\mathrm{dd}, J$ $=10.9,2.2 \mathrm{~Hz}, 1 \mathrm{H}), 2.08(\mathrm{dd}, J=11.3,1.4 \mathrm{~Hz}, 1 \mathrm{H}), 1.88(\mathrm{~s}, 3 \mathrm{H}), 1.81(\mathrm{dd}, J=17.9,1.4 \mathrm{~Hz}, 1 \mathrm{H}), 1.76-1.63(\mathrm{~m}, 2 \mathrm{H}), 1.20$ $-1.08(\mathrm{~m}, 3 \mathrm{H}), 0.87(\mathrm{dt}, J=13.1,3.1 \mathrm{~Hz}, 1 \mathrm{H}), 0.72(\mathrm{t}, J=7.4 \mathrm{~Hz}, 3 \mathrm{H}), 0.40(\mathrm{~d}, J=2.1 \mathrm{~Hz}, 3 \mathrm{H})$.

${ }^{13} \mathrm{C}$ NMR $\left(101 \mathrm{MHz}, \mathrm{CDCl}_{3}\right) \delta$ 195.6, 143.4, 137.8, 137.7, 134.7, 129.6, 128.5, 57.2, 52.0, 51.5, 37.5, 32.2, 31.8, 29.4, 26.7, 22.2, 21.1, 13.9.

HRMS ESI Calcd for $\mathrm{C}_{20} \mathrm{H}_{27} \mathrm{NNaO}_{3} \mathrm{~S}$ [M+Na] ${ }^{+}: 384.1608$, Found: 384.1606.

IR (KBr): 2958, 2929, 2871, 1688, 1623, 1464, 1343, 1166, $1093 \mathrm{~cm}^{-1}$. 


\section{Syntheses of substrates}

Compounds S1-S15 $\left(\mathbf{S} 1^{1}, \mathbf{S 2}-\mathbf{S} 7^{2}, \mathbf{S} 8^{3}, \mathbf{S} 9^{4}, \mathbf{S 1 0 ^ { 5 }}, \mathbf{S 1 1 ^ { 6 }}, \mathbf{S 1 2}{ }^{7}, \mathbf{S 1 3 ^ { 8 }}, \mathbf{S 1 4 ^ { 9 }}, \mathbf{S 1 5 ^ { 1 0 }}\right)$ were prepared according to literature methods, and their characterization data match the reported data.

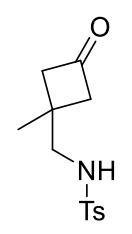

S1<smiles>COCC(CC1(C)CC(=O)C1)C(=O)OC</smiles>

S7

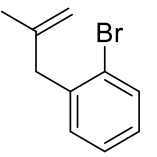

S13

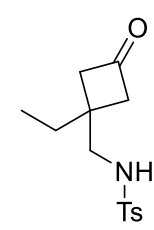

S2

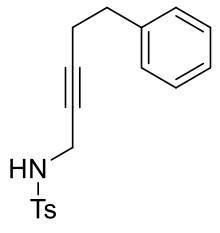

S8

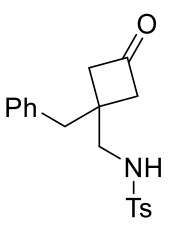

S3

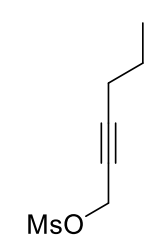

S9<smiles>CNCC1(c2ccccc2)CC(=O)C1</smiles>

S4<smiles>CCCCC#CCOC</smiles>

S10<smiles>CNC1CCCCC12CC(=O)C2</smiles>

S5<smiles>COC#CC(C)C</smiles>

S11<smiles>O=C1C[C@]2(CN[As])CCCC[C@@H]12</smiles>

S6<smiles>[OH+]CCCCI</smiles>

S12

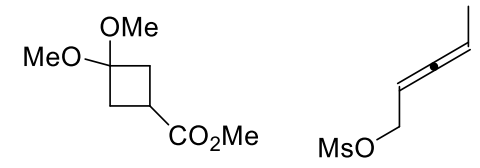

S14

S15

Note: Two synthetic routes of preparation substrate $1 a$ were developed (General procedures $\boldsymbol{A}$ and $\boldsymbol{B}$ ).

General procedure A.

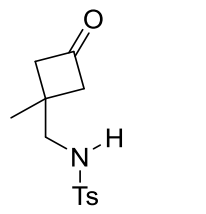

S1

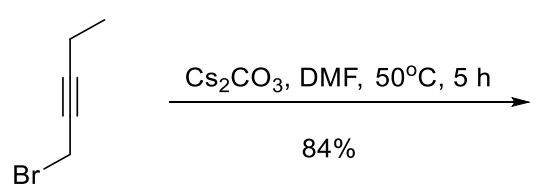

$\mathrm{Br}^{\prime}$<smiles>[CH]C1CC1</smiles>

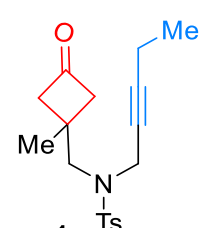

$1 a^{\mathrm{Ts}}$

Synthesis of substrate $1 \mathrm{a} . \mathrm{Cs}_{2} \mathrm{CO}_{3}(3.91 \mathrm{~g}, 12 \mathrm{mmol}, 1.2$ equiv) was added to a solution of compound $\mathbf{S 1}(2.67 \mathrm{~g}, 10$ $\mathrm{mmol}, 1$ equiv) and 1-bromo-2-pentyne (1.22 mL, $12 \mathrm{mmol}, 1.2$ equiv) in DMF (20 mL) at room temperature. After stirred at $50^{\circ} \mathrm{C}$ for $5 \mathrm{~h}$, the reaction mixture was cooled to room temperature, quenched by adding saturated $\mathrm{NH}_{4} \mathrm{Cl}$ aqueous solution. The reaction mixture was extracted with $\mathrm{Et}_{2} \mathrm{O}$, and the organic phase was washed with $\mathrm{H}_{2} \mathrm{O}$, saturated $\mathrm{NaHCO}_{3}$ aqueous solution and brine, dried with $\mathrm{Na}_{2} \mathrm{SO}_{4}$ and concentrated under reduced pressure. The residue was purified by column chromatography on silica gel to give $2.8 \mathrm{~g}$ of compound $1 \mathrm{a}$ in $84 \%$ yield as a white solid.

$\mathrm{Rf}=0.33$, Hexane/ EtOAc 4:1.

Mp: $69.1-70.9^{\circ} \mathrm{C}$ 
${ }^{1} \mathrm{H}$ NMR (400 MHz, Chloroform-d) $87.77-7.71(\mathrm{~m}, 2 \mathrm{H}), 7.36-7.28(\mathrm{~m}, 2 \mathrm{H}), 4.11(\mathrm{t}, J=2.3 \mathrm{~Hz}, 2 \mathrm{H}), 3.43(\mathrm{~s}, 2 \mathrm{H}), 3.19$ $-3.06(\mathrm{~m}, 2 \mathrm{H}), 2.82-2.72(\mathrm{~m}, 2 \mathrm{H}), 2.43(\mathrm{~s}, 3 \mathrm{H}), 1.91(\mathrm{qt}, J=7.5,2.2 \mathrm{~Hz}, 2 \mathrm{H}), 1.40(\mathrm{~s}, 3 \mathrm{H}), 0.88(\mathrm{t}, J=7.5 \mathrm{~Hz}, 3 \mathrm{H})$.

${ }^{13} \mathrm{C}$ NMR $\left(101 \mathrm{MHz}, \mathrm{CDCl}_{3}\right) \delta 206.4,143.5,135.8,129.4,127.8,88.9,71.2,57.4,54.0,37.8,29.3,24.1,21.5,13.4$, 12.0.

HRMS ESI Calcd for $\mathrm{C}_{18} \mathrm{H}_{24} \mathrm{NO}_{3} \mathrm{~S}[\mathrm{M}+\mathrm{H}]^{+}: 334.1471$, Found: 334.1472.

IR (KBr): 2974, 2926, 2878, 1782, 1598, 1452, 1383, 1347, 1160, 1091, $1056 \mathrm{~cm}^{-1}$.<smiles>CCC#CCN(CCCC)CC1(CC)CCC(=O)C1</smiles>

Synthesis of substrate $\mathbf{1 b}$. Following the general procedure $\mathbf{A}$ of preparation of substrate $\mathbf{1 a}, 359 \mathrm{mg}$ of substrate 1b was synthesized from $\mathbf{S 2}(2 \mathrm{mmol})$ and 1-bromo-2-pentyne $(2.4 \mathrm{mmol})$ in $52 \%$ yield as a white solid.

$\mathrm{Rf}=0.40$, Hexane/ EtOAc 4:1.

$\mathrm{Mp}: 82.9-83.9^{\circ} \mathrm{C}$

${ }^{1} \mathrm{H}$ NMR (400 MHz, Chloroform-d) $\delta 7.74(\mathrm{~d}, J=8.4 \mathrm{~Hz}, 2 \mathrm{H}), 7.31(\mathrm{~d}, J=8.0 \mathrm{~Hz}, 2 \mathrm{H}), 4.09(\mathrm{~s}, 2 \mathrm{H}), 3.42(\mathrm{~s}, 2 \mathrm{H}), 3.13-$ $2.96(\mathrm{~m}, 2 \mathrm{H}), 2.86-2.72(\mathrm{~m}, 2 \mathrm{H}), 2.43(\mathrm{~s}, 3 \mathrm{H}), 1.93-1.81(\mathrm{~m}, 2 \mathrm{H}), 1.72(\mathrm{q}, J=7.4 \mathrm{~Hz}, 2 \mathrm{H}), 1.03(\mathrm{t}, J=7.3 \mathrm{~Hz}, 3 \mathrm{H})$, $0.86(\mathrm{t}, J=7.5 \mathrm{~Hz}, 3 \mathrm{H})$.

${ }^{13} \mathrm{C}$ NMR (101 MHz, $\left.\mathrm{CDCl}_{3}\right) \delta$ 206.6, 143.5, 135.5, 129.4, 128.0, 89.3, 71.0, 55.4, 50.8, 37.6, 32.8, 29.0, 21.5, 13.4, 12.0, 8.8.

HRMS ESI Calcd for $\mathrm{C}_{19} \mathrm{H}_{26} \mathrm{NO}_{3} \mathrm{~S}[\mathrm{M}+\mathrm{H}]^{+}: 348.1628$, Found: 348.1627.

IR (KBr): 2974, 2924, 2878, 1782, 1598, 1448, 1348, 1330, 1163, $1091 \mathrm{~cm}^{-1}$.

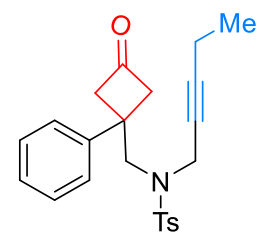

11

Synthesis of substrate 11. Following the general procedure A of preparation of substrate 1a, $359 \mathrm{mg}$ of substrate $\mathbf{1 l}$ was synthesized from $\mathbf{S 4}(1 \mathrm{mmol})$ and 1-bromo-2-pentyne $(1.3 \mathrm{mmol})$ in $92 \%$ yield as a white solid.

$\mathrm{Rf}=0.40$, Hexane/ EtOAc 4:1.

Mp: $85.1-87.8^{\circ} \mathrm{C}$

${ }^{1} \mathrm{H}$ NMR (400 MHz, Chloroform-d) $87.72-7.65(\mathrm{~m}, 2 \mathrm{H}), 7.43-7.29(\mathrm{~m}, 5 \mathrm{H}), 7.29-7.25(\mathrm{~m}, 2 \mathrm{H}), 3.67-3.57(\mathrm{~m}, 4 \mathrm{H})$, $3.47-3.38(\mathrm{~m}, 2 \mathrm{H}), 3.35(\mathrm{t}, J=2.2 \mathrm{~Hz}, 2 \mathrm{H}), 2.41(\mathrm{~s}, 3 \mathrm{H}), 1.81(\mathrm{qt}, J=7.5,2.2 \mathrm{~Hz}, 2 \mathrm{H}), 0.82(\mathrm{t}, J=7.5 \mathrm{~Hz}, 3 \mathrm{H})$. 
${ }^{13} \mathrm{C}$ NMR $\left(101 \mathrm{MHz}, \mathrm{CDCl}_{3}\right) \delta$ 205.2, 143.7, 143.5, 135.9, 129.3, 128.8, 128.0, 127.1, 127.1, 87.8, 71.5, 56.5, 55.8, 38.6, $38.5,21.5,13.4,12.0$.

HRMS ESI Calcd for $\mathrm{C}_{23} \mathrm{H}_{26} \mathrm{NO}_{3} \mathrm{~S}[\mathrm{M}+\mathrm{H}]^{+}:$396.1628, Found: 396.1629.

IR (KBr): 3027, 2977, 2937, 1786, 1599, 1496, 1447, 1381, 1347, 1161, 1094, $1006 \mathrm{~cm}^{-1}$.

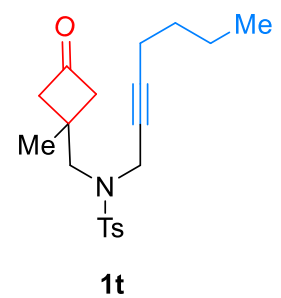

Synthesis of substrate 1t. Following the general procedure A of preparation of substrate 1a, 329 mg of substrate 1t was synthesized from S1 (1 mmol) and S10 (1.3 mmol) in 91\% yield as a white solid.

$\mathrm{Rf}=0.5$, Hexane/ EtOAc 4:1.

Mp: $49.7-51.4^{\circ} \mathrm{C}$.

${ }^{1} \mathrm{H}$ NMR (400 MHz, Chloroform-d) $\delta 7.77-7.70(\mathrm{~m}, 2 \mathrm{H}), 7.31(\mathrm{~d}, J=8.1 \mathrm{~Hz}, 2 \mathrm{H}), 4.13(\mathrm{t}, J=2.3 \mathrm{~Hz}, 2 \mathrm{H}), 3.43(\mathrm{~s}, 2 \mathrm{H})$, $3.19-3.08(\mathrm{~m}, 2 \mathrm{H}), 2.82-2.69(\mathrm{~m}, 2 \mathrm{H}), 2.43(\mathrm{~s}, 3 \mathrm{H}), 1.90(\mathrm{tq}, J=4.5,2.3 \mathrm{~Hz}, 2 \mathrm{H}), 1.40(\mathrm{~s}, 3 \mathrm{H}), 1.26-1.12(\mathrm{~m}, 4 \mathrm{H})$, $0.89-0.79(\mathrm{~m}, 3 \mathrm{H})$.

${ }^{13} \mathrm{C}$ NMR (101 MHz, CDCl 3$) \delta 206.3,143.4,135.9,129.4,127.8,87.7,71.7,57.4,54.0,37.8,30.3,29.3,24.1,21.8$, $21.5,18.0,13.5$.

HRMS ESI Calcd for $\mathrm{C}_{20} \mathrm{H}_{28} \mathrm{NO}_{3} \mathrm{~S}[\mathrm{M}+\mathrm{H}]^{+}: 362.1784$, Found: 362.1792.

IR (KBr): 2958, 2871, 1782, 1598, 1494, 1454, 1383, 1348, 1285, 1186, 1163, 1090, $1057 \mathrm{~cm}^{-1}$.

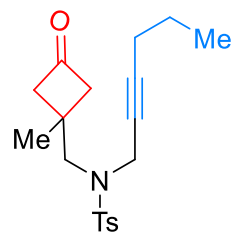

1u

Synthesis of substrate $1 \mathbf{u}$. Following the general procedure A of preparation of substrate $1 \mathrm{a}, 310 \mathrm{mg}$ of substrate 1u was synthesized from $\mathbf{S 1}(1 \mathrm{mmol})$ and $\mathbf{S 9}(1.3 \mathrm{mmol})$ in $90 \%$ yield as a white solid.

$\mathrm{Rf}=0.4$, Hexane/ EtOAc 4:1.

Mp: $51.4-52.8^{\circ} \mathrm{C}$.

${ }^{1} \mathrm{H}$ NMR (400 MHz, Chloroform-d) $\delta 7.77-7.70(\mathrm{~m}, 2 \mathrm{H}), 7.36-7.29(\mathrm{~m}, 2 \mathrm{H}), 4.14(\mathrm{t}, \mathrm{J}=2.3 \mathrm{~Hz}, 2 \mathrm{H}), 3.44(\mathrm{~s}, 2 \mathrm{H}), 3.21$ $-3.08(\mathrm{~m}, 2 \mathrm{H}), 2.83-2.70(\mathrm{~m}, 2 \mathrm{H}), 2.43(\mathrm{~s}, 3 \mathrm{H}), 1.89(\mathrm{tt}, J=7.0,2.3 \mathrm{~Hz}, 2 \mathrm{H}), 1.41(\mathrm{~s}, 3 \mathrm{H}), 1.27(\mathrm{~h}, J=7.3 \mathrm{~Hz}, 2 \mathrm{H}), 0.80$ $(\mathrm{t}, J=7.4 \mathrm{~Hz}, 3 \mathrm{H})$.

${ }^{13} \mathrm{C}$ NMR (101 MHz, CDCl$)$ ) $\delta$ 206.4, 143.5, 135.9, 129.4, 127.8, 87.5, 71.9, 57.4, 54.0, 37.8, 29.3, 24.1, 21.7, 21.5, $20.4,13.4$ 
HRMS ESI Calcd for $\mathrm{C}_{19} \mathrm{H}_{26} \mathrm{NO}_{3} \mathrm{~S}[\mathrm{M}+\mathrm{H}]^{+}: 348.1628$, Found: 348.1636.

IR (KBr): 2963, 2931, 2872, 1784, 1598, 1455, 1383, 1348, 1285, 1161, 1091, $1059 \mathrm{~cm}^{-1}$.

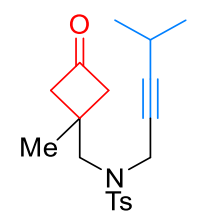

$1 w$

Synthesis of substrate 1w. Following the general procedure A of preparation of substrate $1 \mathrm{a}, 300 \mathrm{mg}$ of substrate 1w was synthesized from S1 $(1 \mathrm{mmol})$ and $\mathbf{S 1 1}(1.3 \mathrm{mmol})$ in $86 \%$ yield as a white solid.

$\mathrm{Rf}=0.4$, Hexane/ EtOAc 4:1.

Mp: $85.0-86.6^{\circ} \mathrm{C}$.

${ }^{1} \mathrm{H}$ NMR (400 MHz, Chloroform-d) $\delta 7.77-7.70(\mathrm{~m}, 2 \mathrm{H}), 7.35-7.28(\mathrm{~m}, 2 \mathrm{H}), 4.13(\mathrm{~d}, J=2.1 \mathrm{~Hz}, 2 \mathrm{H}), 3.43(\mathrm{~s}, 2 \mathrm{H})$, $3.18-3.08(\mathrm{~m}, 2 \mathrm{H}), 2.84-2.69(\mathrm{~m}, 2 \mathrm{H}), 2.43(\mathrm{~s}, 3 \mathrm{H}), 2.34-2.20(\mathrm{~m}, 1 \mathrm{H}), 1.41(\mathrm{~s}, 3 \mathrm{H}), 0.91(\mathrm{~d}, J=6.9 \mathrm{~Hz}, 6 \mathrm{H})$.

${ }^{13} \mathrm{C}$ NMR (101 MHz, $\left.\mathrm{CDCl}_{3}\right) \delta$ 206.4, 143.5, 135.9, 129.5, 127.8, 93.2, 71.0, 57.4, 53.9, 37.7, 29.2, 24.1, 22.5, 21.5, 20.2 .

HRMS ESI Calcd for $\mathrm{C}_{19} \mathrm{H}_{26} \mathrm{NO}_{3} \mathrm{~S}[\mathrm{M}+\mathrm{H}]^{+}: 348.1628$, Found: 348.1633.

IR (KBr): 2970, 2929, 2871, 1784, 1598, 1494, 1451, 1383, 1348, 1185, 1161, $1091 \mathrm{~cm}^{-1}$.

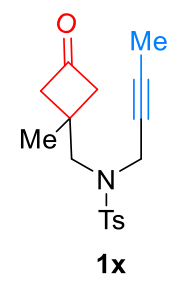

Synthesis of substrate 1x. Following the general procedure A of preparation of substrate $1 \mathbf{a}, 300$ mg of substrate 1x was synthesized from S1 (1 mmol) and 1-Bromo-2-butyne $(1.5 \mathrm{mmol})$ in 94\% yield as a white solid.

$\mathrm{Rf}=0.4$, Hexane/ EtOAc 4:1.

${ }^{1} \mathrm{H}$ NMR (400 MHz, Chloroform-d) $\delta 7.78-7.70(\mathrm{~m}, 2 \mathrm{H}), 7.35-7.28(\mathrm{~m}, 2 \mathrm{H}), 4.09(\mathrm{q}, J=2.4 \mathrm{~Hz}, 2 \mathrm{H}), 3.42(\mathrm{~s}, 2 \mathrm{H})$, $3.19-3.08(\mathrm{~m}, 2 \mathrm{H}), 2.83-2.69(\mathrm{~m}, 2 \mathrm{H}), 2.44(\mathrm{~s}, 3 \mathrm{H}), 1.54(\mathrm{t}, J=2.4 \mathrm{~Hz}, 3 \mathrm{H}), 1.39(\mathrm{~s}, 3 \mathrm{H})$.

${ }^{13} \mathrm{C}$ NMR (101 MHz, $\left.\mathrm{CDCl}_{3}\right) \delta$ 206.4, 143.5, 135.8, 129.4, 129.3, 127.9, 82.9, 71.1, 57.4, 54.2, 37.9, 29.3, 24.2, 24.1, 21.5, 3.2.

HRMS ESI Calcd for $\mathrm{C}_{17} \mathrm{H}_{22} \mathrm{NO}_{3} \mathrm{~S}[\mathrm{M}+\mathrm{H}]^{+}:$320.1315, Found: 320.1315.

IR (KBr): 2961, 2922, 1869, 1783, 1598, 1494, 1442, 1383, 1347, 1161, 1117, 1091, $1057 \mathrm{~cm}^{-1}$. 


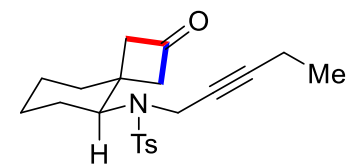

1 ac (racemic)

Synthesis of substrate 1ac. Following the general procedure A of preparation of substrate 1a, $407 \mathrm{mg}$ of substrate 1ac was synthesized from $\mathbf{S 5}(1.24 \mathrm{mmol})$ and 1-Bromo-2-pentyne $(1.87 \mathrm{mmol})$ in $88 \%$ yield as a white solid.

$\mathrm{Rf}=0.44$, Hexane/ EtOAc 3:1.

Mp: $130.4-131.7^{\circ} \mathrm{C}$.

${ }^{1} \mathrm{H}$ NMR (400 MHz, Chloroform-d) $\delta 7.80-7.69(\mathrm{~m}, 2 \mathrm{H}), 7.33-7.27(\mathrm{~m}, 2 \mathrm{H}), 4.13(\mathrm{dt}, J=18.6,2.3 \mathrm{~Hz}, 1 \mathrm{H}), 3.96-$ $3.83(\mathrm{~m}, 2 \mathrm{H}), 3.58(\mathrm{dt}, J=17.5,2.8 \mathrm{~Hz}, 1 \mathrm{H}), 3.34(\mathrm{dq}, J=17.2,2.6 \mathrm{~Hz}, 1 \mathrm{H}), 2.59$ (ddd, $J=17.2,6.4,2.4 \mathrm{~Hz}, 1 \mathrm{H}), 2.47-$ $2.38(\mathrm{~m}, 4 \mathrm{H}), 2.09-1.98(\mathrm{~m}, 2 \mathrm{H}), 1.82-1.62(\mathrm{~m}, 4 \mathrm{H}), 1.49-1.14(\mathrm{~m}, 4 \mathrm{H}), 1.00(\mathrm{t}, J=7.5 \mathrm{~Hz}, 3 \mathrm{H})$.

${ }^{13} \mathrm{C}$ NMR $\left(101 \mathrm{MHz}, \mathrm{CDCl}_{3}\right) \delta$ 207.1, 143.5, 137.3, 129.5, 127.4, 87.0, 75.1, 61.5, 55.3, 51.5, 39.2, 35.9, 34.6, 28.2, 26.3, 23.0, 21.5, 13.3, 12.3 .

HRMS ESI Calcd for $\mathrm{C}_{21} \mathrm{H}_{28} \mathrm{NO}_{3} \mathrm{~S}[\mathrm{M}+\mathrm{H}]^{+}: 374.1784$, Found: 374.1789 .

IR (KBr): 2976, 2935, 2859, 1781, 1598, 1450, 1479, 1340, 1164, 1110, 1090, $1029 \mathrm{~cm}^{-1}$.

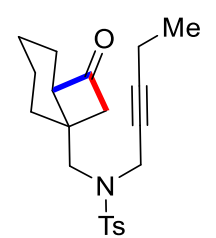

1ad (racemic)

Synthesis of substrate 1ad. Following the general procedure A of preparation of substrate 1a, $335 \mathrm{mg}$ of substrate 1ad was synthesized from $\mathbf{S 6}(1.48 \mathrm{mmol})$ and 1-Bromo-2-pentyne $(2.23 \mathrm{mmol})$ in $61 \%$ yield as a white solid.

$\mathrm{Rf}=0.31$, Hexane/ EtOAc 5:1.

Mp: $99.5-101.2^{\circ} \mathrm{C}$.

${ }^{1} \mathrm{H}$ NMR (400 MHz, Chloroform-d) $87.78-7.72(\mathrm{~m}, 2 \mathrm{H}), 7.34-7.28(\mathrm{~m}, 2 \mathrm{H}), 4.33(\mathrm{dtd}, J=18.5,2.3,1.0 \mathrm{~Hz}, 1 \mathrm{H}), 3.86$ $(\mathrm{dt}, J=18.5,2.3 \mathrm{~Hz}, 1 \mathrm{H}), 3.69(\mathrm{dd}, J=14.0,1.1 \mathrm{~Hz}, 1 \mathrm{H}), 3.20(\mathrm{~d}, J=14.1 \mathrm{~Hz}, 1 \mathrm{H}), 3.15-3.05(\mathrm{~m}, 2 \mathrm{H}), 2.53(\mathrm{~d}, J=16.7$ $\mathrm{Hz}, 1 \mathrm{H}), 2.43(\mathrm{~s}, 3 \mathrm{H}), 2.28-2.18(\mathrm{~m}, 1 \mathrm{H}), 2.06-1.97(\mathrm{~m}, 1 \mathrm{H}), 1.90(\mathrm{qt}, J=7.5,2.2 \mathrm{~Hz}, 2 \mathrm{H}), 1.67-1.52(\mathrm{~m}, 3 \mathrm{H}), 1.46$ $-1.33(\mathrm{~m}, 1 \mathrm{H}), 1.29-1.14(\mathrm{~m}, 3 \mathrm{H}), 0.88(\mathrm{t}, J=7.5 \mathrm{~Hz}, 3 \mathrm{H})$.

${ }^{13} \mathrm{C}$ NMR (101 MHz, CDCl 3 ) $\delta$ 207.1, 143.5, 135.9, 129.4, 127.9, 88.6, 71.3, 60.1, 57.1, 51.6, 38.3, 31.4, 31.3, 22.4, 21.5, 20.8, 20.2, 13.4, 12.1 .

HRMS ESI Calcd for $\mathrm{C}_{21} \mathrm{H}_{28} \mathrm{NO}_{3} \mathrm{~S}[\mathrm{M}+\mathrm{H}]^{+}: 374.1784$, Found: 374.1792 .

IR (KBr): 2976, 2933, 2856, 1777, 1452, 1372, 1347, 1306, 1161, $1090 \mathrm{~cm}^{-1}$. 
Anothe synthetic route of preparing substrate $\mathbf{1 a}$ was developed and applied to synthesize substrates $\mathbf{1 c}$ and $\mathbf{1 e - 1 \mathbf { k }}$ (General procedure B). S16 could be obtained from S14 by alkylation/reduction/mesylation. Then a one-pot procedure of preparing 1a from S16 was realized in high yield (see below).
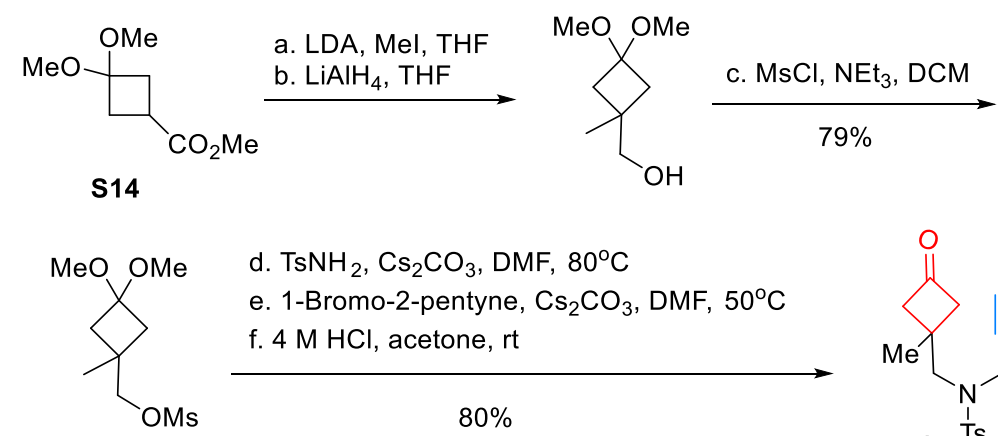

d. $\mathrm{TsNH}_{2}, \mathrm{Cs}_{2} \mathrm{CO}_{3}, \mathrm{DMF}, 80^{\circ} \mathrm{C}$

e. 1-Bromo-2-pentyne, $\mathrm{Cs}_{2} \mathrm{CO}_{3}, \mathrm{DMF}, 50^{\circ} \mathrm{C}$

f. $4 \mathrm{M} \mathrm{HCl}$, acetone, rt

S16

$80 \%$

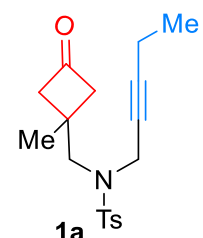

General procedure B:

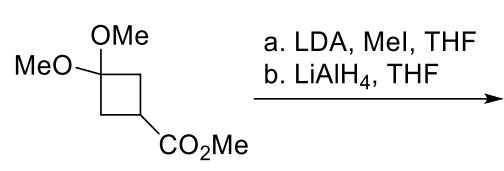

S14

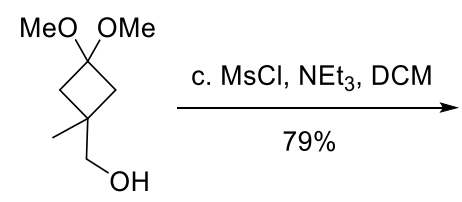

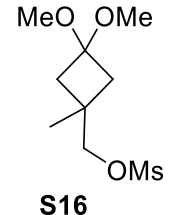

Synthesis of compound S16. $n$-BuLi (56 mL, $2.5 \mathrm{M}$ in hexane, $140 \mathrm{mmol}, 1.4$ equiv) was added to a solution of diisopropylamine $\left(21 \mathrm{~mL}, 150 \mathrm{mmol}, 1.5\right.$ equiv) in THF $(100 \mathrm{~mL})$ at $-78^{\circ} \mathrm{C}$. After stirred at $-78^{\circ} \mathrm{C}$ for $0.5 \mathrm{~h}$, a solution of ester S14 (17.4 g, $100 \mathrm{mmol}, 1$ equiv) in THF $(20 \mathrm{~mL})$ was added to the reaction mixture. After stirred at $-78^{\circ} \mathrm{C}$ for $1 \mathrm{~h}$, Mel (15.6 mL, $250 \mathrm{mmol}, 2$ equiv) was added to the reaction mixture. After stirred at $-78^{\circ} \mathrm{C}$ for $0.5 \mathrm{~h}$, the reaction mixture was warmed to room temperature and stirred overnight. The reaction mixture was quenched by adding saturated $\mathrm{NH}_{4} \mathrm{Cl}$ aqueous solution. The reaction mixture was extracted with $\mathrm{Et}_{2} \mathrm{O}$, and the organic phase was washed with $\mathrm{H}_{2} \mathrm{O}$, saturated $\mathrm{NaHCO}_{3}$ aqueous solution and brine, dried with $\mathrm{Na}_{2} \mathrm{SO}_{4}$ and concentrated under reduced pressure to give the crude ester that was used for the next step without further purification. A solution of the above crude ester in THF (100 mL) was added to a solution of $\mathrm{LiAlH}_{4}\left(3.8 \mathrm{~g}, 100 \mathrm{mmol}, 1.0\right.$ equiv) in $\mathrm{THF}(60 \mathrm{~mL})$ at $0^{\circ} \mathrm{C}$. After stirred at room temperature for $3 \mathrm{~h}$, the reaction mixture was cooled to $0^{\circ} \mathrm{C}$, quenched by adding $\mathrm{H}_{2} \mathrm{O}(3.8 \mathrm{~mL})$, $20 \% \mathrm{NaOH}$ aqueous solution $(7.6 \mathrm{~mL})$, and $\mathrm{H}_{2} \mathrm{O}(11.4 \mathrm{~mL})$. After stirred at room temperature for $0.5 \mathrm{~h}, \mathrm{Na}_{2} \mathrm{SO}_{4} \mathrm{was}$ added to the reaction mixture. After stirred at room temperature for $0.5 \mathrm{~h}$, the reaction mixture was filtered with funnel and washed with EtOAc. The filtrate was concentrated under reduced pressure to give the crude alcohol which was used for the next step without further purification. $\mathrm{MsCl}(7.7 \mathrm{~mL}, 100 \mathrm{mmol}, 1$ equiv) was added to a solution of the above alcohol and $\mathrm{NEt}_{3}\left(28 \mathrm{~mL}, 200 \mathrm{mmol}, 2\right.$ equiv) in $\mathrm{DCM}(200 \mathrm{ml})$ at $0^{\circ} \mathrm{C}$. After stirred at $0^{\circ} \mathrm{C}$ for 1 $h$, the reaction mixture was quenched by adding saturated $\mathrm{NH}_{4} \mathrm{Cl}$ aqueous solution. The reaction mixture was extracted with DCM, and the organic phase was washed with $\mathrm{H}_{2} \mathrm{O}$, saturated $\mathrm{NaHCO}_{3}$ aqueous solution and brine, dried with $\mathrm{Na}_{2} \mathrm{SO}_{4}$ and concentrated under reduced pressure. The residue was purified by column chromatography on silica gel to give $18.8 \mathrm{~g}$ of $\mathbf{S} 16$ in $79 \%$ yield as a colorless oil.

$\mathrm{Rf}=0.25$, Hexane/ EtOAc 2:1. 
${ }^{1} \mathrm{H}$ NMR (400 MHz, Chloroform-d) $\delta 4.13(\mathrm{~s}, 2 \mathrm{H}), 3.14(\mathrm{~s}, 3 \mathrm{H}), 3.13(\mathrm{~s}, 3 \mathrm{H}), 3.02(\mathrm{~s}, 3 \mathrm{H}), 2.16-2.09(\mathrm{~m}, 2 \mathrm{H}), 1.97-$ $1.89(\mathrm{~m}, 2 \mathrm{H}), 1.26(\mathrm{~s}, 3 \mathrm{H})$.

${ }^{13} \mathrm{C}$ NMR (101 MHz, CDCl 3$) \delta 98.5,76.6,48.3,48.2,40.2,37.1,29.0,24.3$.

HRMS ESI Calcd for $\mathrm{C}_{9} \mathrm{H}_{19} \mathrm{O}_{5} \mathrm{~S}[\mathrm{M}+\mathrm{H}]^{+}:$239.0948, Found: 239.0949.

IR (KBr): 2941, 1458, 1355, 1274, 1175, 1109, $1040 \mathrm{~cm}^{-1}$.

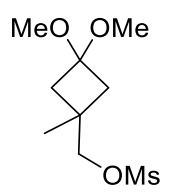

d. $\mathrm{TsNH}_{2}, \mathrm{Cs}_{2} \mathrm{CO}_{3}, \mathrm{DMF}, 80^{\circ} \mathrm{C}$

e. 1-Bromo-2-pentyne, $\mathrm{Cs}_{2} \mathrm{CO}_{3}, \mathrm{DMF}, 50^{\circ} \mathrm{C}$

f. $4 \mathrm{M} \mathrm{HCl}$, acetone, rt

S16

$80 \%$

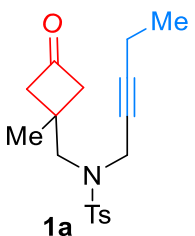

Synthesis of substrate 1a. $\mathrm{Cs}_{2} \mathrm{CO}_{3}(6.52 \mathrm{~g}, 20 \mathrm{mmol}, 2$ equiv) was added to a solution of compound S16 (2.38 g, 10 mmol, 1 equiv) and $\mathrm{TsNH}_{2}\left(1.71 \mathrm{~g}, 10 \mathrm{mmol}, 10\right.$ equiv) in $\mathrm{DMF}(30 \mathrm{~mL})$ at room temperature. After stirred at $80^{\circ} \mathrm{C}$ overnight, the reaction mixture was cooled to room temperature. $\mathrm{Cs}_{2} \mathrm{CO}_{3}(6.52 \mathrm{~g}, 20 \mathrm{mmol}, 2$ equiv) and 1-bromo2-pentyne $\left(1.53 \mathrm{ml}, 15 \mathrm{mmol}, 1.5\right.$ equiv) were successively added to the reaction mixture. After stirred at $50^{\circ} \mathrm{C}$ for $12 \mathrm{~h}$, the reaction mixture was cooled to $0^{\circ} \mathrm{C}$. Then $4 \mathrm{M} \mathrm{HCl}(40 \mathrm{~mL})$ and acetone $(40 \mathrm{~mL})$ were successively added to the reaction mixture. After stirred at room temperature for $2 \mathrm{~h}$, the reaction mixture was extracted with $\mathrm{Et}{ }_{2} \mathrm{O}$, and the organic phase was washed with $\mathrm{H}_{2} \mathrm{O}$, saturated $\mathrm{NaHCO}_{3}$ aqueous solution and brine, dried with $\mathrm{Na}_{2} \mathrm{SO}_{4}$ and concentrated under reduced pressure. The residue was purified by column chromatography on silica gel to give 2.66 g of compound $1 \mathrm{a}$ in $80 \%$ yield as a white solid.

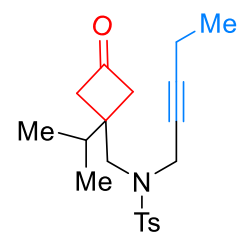

1c

Synthesis of substrate 1c. Following the general procedure B of preparation of substrate $\mathbf{1 a}$, substrate $\mathbf{1 c}$ was synthesized from S14, $\mathbf{i P r I}, \mathrm{TsNH}_{2}$ and 1-Bromo-2-pentyne as a white solid.

$\mathrm{Rf}=0.43$, Hexane/ EtOAc 4:1.

Mp: $95.8-96.8^{\circ} \mathrm{C}$

${ }^{1} \mathrm{H}$ NMR (400 MHz, Chloroform-d) $\delta 7.79-7.70(\mathrm{~m}, 2 \mathrm{H}), 7.38-7.30(\mathrm{~m}, 2 \mathrm{H}), 4.10(\mathrm{t}, J=2.3 \mathrm{~Hz}, 2 \mathrm{H}), 3.46(\mathrm{~s}, 2 \mathrm{H}), 3.11$ $-2.99(\mathrm{~m}, 2 \mathrm{H}), 2.99-2.88(\mathrm{~m}, 2 \mathrm{H}), 2.45(\mathrm{~s}, 3 \mathrm{H}), 2.03(\mathrm{p}, J=6.8 \mathrm{~Hz}, 1 \mathrm{H}), 1.89$ (qt, J = 7.5, $2.2 \mathrm{~Hz}, 2 \mathrm{H}), 1.05(\mathrm{~d}, J=6.8$ $\mathrm{Hz}, 6 \mathrm{H}), 0.87(\mathrm{t}, J=7.5 \mathrm{~Hz}, 3 \mathrm{H})$.

${ }^{13} \mathrm{C}$ NMR (101 MHz, CDCl$)$ ) $\delta$ 206.8, 143.5, 135.3, 129.3, 128.0, 89.3, 71.0, 53.1, 50.5, 38.0, 35.9, 32.1, 21.5, 17.28, 13.3, 12.0.

HRMS ESI Calcd for $\mathrm{C}_{20} \mathrm{H}_{27} \mathrm{NNaO}_{3} \mathrm{~S}[\mathrm{M}+\mathrm{Na}]^{+}: 384.1604$, Found: 384.1604 . 
IR (KBr): 2974, 2938, 2878, 1782, 1598, 1448, 1382, 1347, 1162, 1114, 1091 $\mathrm{cm}^{-1}$.

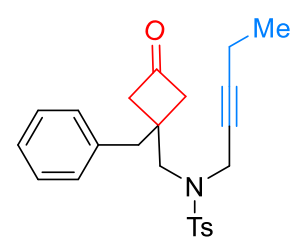

$1 e$

Synthesis of substrate 1e. Following the general procedure B of preparation of substrate 1a, substrate 1e was synthesized from S14, $\mathrm{BnBr}$, $\mathrm{TsNH}_{2}$ and 1-Bromo-2-pentyne as a white solid.

Rf $=0.28$, Hexane/ EtOAc 4:1.

Mp: $89.1-91.2{ }^{\circ} \mathrm{C}$

${ }^{1} \mathrm{H}$ NMR (400 MHz, Chloroform-d) $\delta 7.67-7.62(\mathrm{~m}, 2 \mathrm{H}), 7.29-7.15(\mathrm{~m}, 8 \mathrm{H}), 4.05(\mathrm{t}, \mathrm{J}=2.2 \mathrm{~Hz}, 2 \mathrm{H}), 3.41(\mathrm{~s}, 2 \mathrm{H}), 3.04$ $-2.96(\mathrm{~m}, 2 \mathrm{H}), 2.95-2.86(\mathrm{~m}, 4 \mathrm{H}), 2.36(\mathrm{~s}, 3 \mathrm{H}), 1.79$ (dddd, J = 9.7, 7.5, 4.8, 2.2 Hz, 2H), $0.78(\mathrm{t}, J=7.5 \mathrm{~Hz}, 3 \mathrm{H})$.

${ }^{13} \mathrm{C}$ NMR (101 MHz, CDCl$)$ ) $\delta$ 205.5, 143.6, 136.9, 135.4, 130.5, 129.4, 128.5, 128.0, 126.9, 89.3, 71.1, 54.5, 52.8, 41.6, $38.2,33.2,21.5,13.4,12.0$.

HRMS ESI Calcd for $\mathrm{C}_{24} \mathrm{H}_{28} \mathrm{NO}_{3} \mathrm{~S}[\mathrm{M}+\mathrm{H}]^{+}:$410.1784, Found: 410.1784 .

IR (KBr): 2942, 2866, 1787, 1463, 1350, 1163, 1091, 1069, $1015 \mathrm{~cm}^{-1}$.

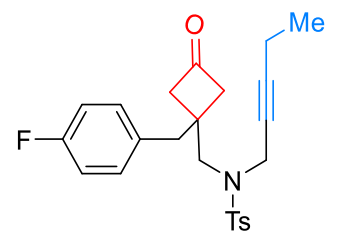

$1 f$

Synthesis of substrate 1f. Following the general procedure B of preparation of substrate 1a, substrate $\mathbf{1 f}$ was synthesized from S14, 4-Fluorobenzyl bromide, $\mathrm{TsNH}_{2}$ and 1-Bromo-2-pentyne as a white solid.

$\operatorname{Rf}=0.27$, Hexane/ EtOAc 4:1.

Mp: $128.3-129.9^{\circ} \mathrm{C}$

${ }^{1} \mathrm{H}$ NMR (400 MHz, Chloroform-d) $\delta 7.75-7.68(\mathrm{~m}, 2 \mathrm{H}), 7.36-7.29(\mathrm{~m}, 2 \mathrm{H}), 7.26-7.20(\mathrm{~m}, 2 \mathrm{H}), 7.07-6.99(\mathrm{~m}, 2 \mathrm{H})$, $4.12(\mathrm{t}, J=2.2 \mathrm{~Hz}, 2 \mathrm{H}), 3.46(\mathrm{~s}, 2 \mathrm{H}), 3.12-3.03(\mathrm{~m}, 2 \mathrm{H}), 2.99(\mathrm{~s}, 2 \mathrm{H}), 2.98-2.91(\mathrm{~m}, 2 \mathrm{H}), 2.44(\mathrm{~s}, 3 \mathrm{H}), 1.87(\mathrm{qt}, J=7.5$, $2.2 \mathrm{~Hz}, 2 \mathrm{H}), 0.86(\mathrm{t}, J=7.5 \mathrm{~Hz}, 3 \mathrm{H})$.

${ }^{13} \mathrm{C}$ NMR (101 MHz, CDCl $) \delta 205.1,161.9\left(\mathrm{~d},{ }^{1} \mathrm{~J}_{\mathrm{C}-\mathrm{F}}=246.4 \mathrm{~Hz}\right), 143.7,135.3,132.6\left(\mathrm{~d},{ }^{4} \mathrm{~J}_{\mathrm{C}-\mathrm{F}}=3.0 \mathrm{~Hz}\right), 131.9\left(\mathrm{~d},{ }^{3} \mathrm{JC}_{\mathrm{C}-\mathrm{F}}=\right.$ $8.1 \mathrm{~Hz}), 129.4,128.0,115.3\left(\mathrm{~d},{ }^{2} J_{\mathrm{C}-\mathrm{F}}=21.2 \mathrm{~Hz}\right), 89.5,71.0,54.7,52.6,40.7,38.1,33.1\left(\mathrm{~d},{ }^{5} J_{\mathrm{C}-\mathrm{F}}=2.0 \mathrm{~Hz}\right), 21.5,13.4$, 12.0 .

HRMS ESI Calcd for $\mathrm{C}_{24} \mathrm{H}_{27} \mathrm{FNO}_{3} \mathrm{~S}[\mathrm{M}+\mathrm{H}]^{+}:$428.1690, Found: 428.1688 .

IR (KBr): 2978, 2921, 1784, 1599, 1510, 1448, 1380, 1348, 1222, 1163, 1109, $1092 \mathrm{~cm}^{-1}$. 


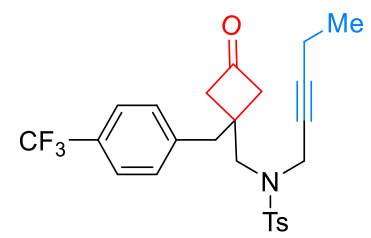

$1 \mathrm{~g}$

Synthesis of substrate $\mathbf{1 g}$. Following the general procedure B of preparation of substrate $1 \mathbf{a}$, substrate $\mathbf{1 g}$ was synthesized from S14, 4-(Trifluoromethyl)benzyl bromide, $\mathrm{TsNH}_{2}$ and 1-Bromo-2-pentyne as a white solid.

$\mathrm{Rf}=0.27$, Hexane/ EtOAc 4:1.

Mp: $138.1-140.4{ }^{\circ} \mathrm{C}$

${ }^{1}$ H NMR (400 MHz, Chloroform-d) $\delta 7.74-7.68(\mathrm{~m}, 2 \mathrm{H}), 7.60$ (d, J= $\left.7.8 \mathrm{~Hz}, 2 \mathrm{H}\right), 7.41(\mathrm{~d}, J=8.0 \mathrm{~Hz}, 2 \mathrm{H}), 7.35-7.28$ $(\mathrm{m}, 2 \mathrm{H}), 4.12(\mathrm{t}, J=2.3 \mathrm{~Hz}, 2 \mathrm{H}), 3.47(\mathrm{~s}, 2 \mathrm{H}), 3.17-3.04(\mathrm{~m}, 4 \mathrm{H}), 3.02-2.90(\mathrm{~m}, 2 \mathrm{H}), 2.43(\mathrm{~s}, 3 \mathrm{H}), 1.86(\mathrm{qt}, J=7.5,2.2$ $\mathrm{Hz}, 2 \mathrm{H}), 0.85(\mathrm{t}, J=7.5 \mathrm{~Hz}, 3 \mathrm{H})$.

${ }^{19}$ F NMR (376 MHz, $\left.\mathrm{CDCl}_{3}\right) \delta-62.45$.

${ }^{13} \mathrm{C}$ NMR (101 MHz, CDCl 3$) \delta 204.6,143.8,141.0,135.1,130.8,129.4,129.2\left(q,{ }^{2} J_{C-F}=32.3 \mathrm{~Hz}\right), 128.0,125.4\left(q,{ }^{3} J_{C-F}\right.$ $=4.0 \mathrm{~Hz}), 124.1\left(\mathrm{q},{ }^{1} \mathrm{~J}_{\mathrm{C}-\mathrm{F}}=272.7 \mathrm{~Hz}\right), 89.6,70.8,54.9,52.5,41.3,38.1,33.1,21.5,13.3,12.0$.

HRMS ESI Calcd for $\mathrm{C}_{25} \mathrm{H}_{27} \mathrm{~F}_{3} \mathrm{NO}_{3} \mathrm{~S}[\mathrm{M}+\mathrm{H}]^{+}:$: 478.1658, Found: 478.1655 .

IR (KBr): 2979, 2923, 1785, 1619, 1448, 1379, 1348, 1326, 1163, 1119, $1068 \mathrm{~cm}^{-1}$.

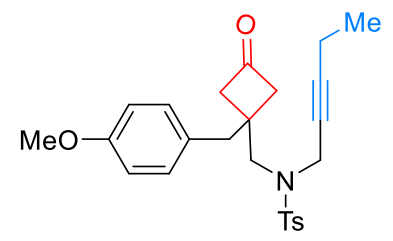

1h

Synthesis of substrate $\mathbf{1 h}$. Following the general procedure B of preparation of substrate $\mathbf{1 a}$, substrate $\mathbf{1 h}$ was synthesized from S14, 4-Methoxybenzyl bromide, $\mathbf{T s N H}_{2}$ and 1-Bromo-2-pentyne as a white solid.

$\mathrm{Rf}=0.22$, Hexane/ EtOAc 4:1.

Mp: $112.7-113.6^{\circ} \mathrm{C}$

${ }^{1} \mathrm{H}$ NMR (400 MHz, Chloroform-d) $\delta 7.76-7.69(\mathrm{~m}, 2 \mathrm{H}), 7.34-7.28(\mathrm{~m}, 2 \mathrm{H}), 7.21-7.14(\mathrm{~m}, 2 \mathrm{H}), 6.90-6.83(\mathrm{~m}, 2 \mathrm{H})$, $4.12(\mathrm{t}, J=2.3 \mathrm{~Hz}, 2 \mathrm{H}), 3.80(\mathrm{~s}, 3 \mathrm{H}), 3.47(\mathrm{~s}, 2 \mathrm{H}), 3.09-2.93(\mathrm{~m}, 6 \mathrm{H}), 2.43(\mathrm{~s}, 3 \mathrm{H}), 1.87(q \mathrm{t}, J=7.5,2.2 \mathrm{~Hz}, 2 \mathrm{H}), 0.86(\mathrm{t}$, $J=7.5 \mathrm{~Hz}, 3 \mathrm{H})$.

${ }^{13} \mathrm{C}$ NMR $\left(101 \mathrm{MHz} \mathrm{CDCl}_{3}\right) \delta$ 205.6, 158.5, 143.6, 135.4, 131.5, 129.4, 128.8, 128.0, 113.8, 89.3, 71.2, 55.2, 54.5, 52.7, 40.7, 38.2, 33.2, 21.5, 13.4, 12.0 .

HRMS ESI Calcd for $\mathrm{C}_{25} \mathrm{H}_{30} \mathrm{NO}_{4} \mathrm{~S}[\mathrm{M}+\mathrm{H}]^{+}:$440.1890, Found: 440.1888.

IR (KBr): 2976, 2936, 2838, 1782, 1611, 1513, 1443, 1250, 1162, 1091, $1035 \mathrm{~cm}^{-1}$. 


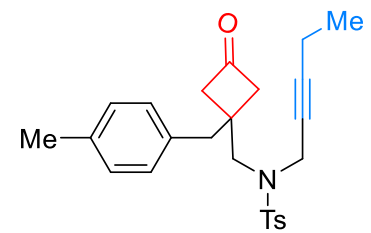

$1 i$

Synthesis of substrate 1i. Following the general procedure B of preparation of substrate $1 \mathbf{a}$, substrate $\mathbf{1 i}$ was synthesized from S14, 4-Methylbenzyl bromide, $\mathrm{TsNH}_{2}$ and 1-Bromo-2-pentyne as a white solid.

$\operatorname{Rf}=0.28$, Hexane/ EtOAc 5:1.

Mp: $132.6-133.9^{\circ} \mathrm{C}$.

${ }^{1} \mathrm{H}$ NMR (400 MHz, Chloroform-d) $\delta 7.75-7.70(\mathrm{~m}, 2 \mathrm{H}), 7.34-7.29(\mathrm{~m}, 2 \mathrm{H}), 7.14(\mathrm{~s}, 4 \mathrm{H}), 4.13(\mathrm{t}, \mathrm{J}=2.3 \mathrm{~Hz}, 2 \mathrm{H}), 3.48$ (s, 2H), $3.09-3.02(\mathrm{~m}, 2 \mathrm{H}), 3.02-2.93(\mathrm{~m}, 4 \mathrm{H}), 2.44(\mathrm{~s}, 3 \mathrm{H}), 2.34(\mathrm{~s}, 3 \mathrm{H}), 1.87(\mathrm{qt}, J=7.5,2.2 \mathrm{~Hz}, 2 \mathrm{H}), 0.86(\mathrm{t}, J=7.5$ $\mathrm{Hz}, 3 \mathrm{H})$.

${ }^{13} \mathrm{C}$ NMR $\left(101 \mathrm{MHz} \mathrm{CDCl}_{3}\right) \delta$ 205.7, 143.6, 136.4, 135.4, 133.7, 130.4, 129.4, 129.1, 128.0, 89.3, 71.1, 54.4, 52.8, 41.1, $38.2,33.2,21.5,21.0,13.4,12.0$.

HRMS ESI Calcd for $\mathrm{C}_{25} \mathrm{H}_{30} \mathrm{NO}_{3} \mathrm{~S}[\mathrm{M}+\mathrm{H}]^{+}:$424.1941, Found: 424.1946.

IR (KBr): 3023, 2978, 2920, 1782, 1598, 1516, 1447, 1379, 1348, 1161, 1090, $1044 \mathrm{~cm}^{-1}$.

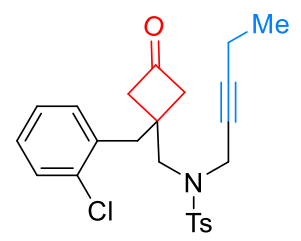

1j

Synthesis of substrate $\mathbf{1 j}$. Following the general procedure $\mathbf{B}$ of preparation of substrate $\mathbf{1 a}$, substrate $\mathbf{1} \mathbf{j}$ was synthesized from S14, 2-Chlorobenzyl bromide, $\mathrm{TsNH}_{\mathbf{2}}$ and 1-Bromo-2-pentyne as a white solid.

Rf $=0.30$, Hexane/ EtOAc 4:1.

$\mathrm{Mp}: 79.9-80.7^{\circ} \mathrm{C}$

${ }^{1} \mathrm{H}$ NMR (400 MHz, Chloroform-d) $\delta 7.78-7.71(\mathrm{~m}, 2 \mathrm{H}), 7.44-7.39(\mathrm{~m}, 1 \mathrm{H}), 7.37-7.31(\mathrm{~m}, 3 \mathrm{H}), 7.29-7.20(\mathrm{~m}, 2 \mathrm{H})$, $4.21(\mathrm{t}, J=2.3 \mathrm{~Hz}, 2 \mathrm{H}), 3.55(\mathrm{~s}, 2 \mathrm{H}), 3.21(\mathrm{~s}, 2 \mathrm{H}), 3.04(\mathrm{~s}, 4 \mathrm{H}), 2.44(\mathrm{~s}, 3 \mathrm{H}), 1.89(\mathrm{qt}, J=7.5,2.2 \mathrm{~Hz}, 2 \mathrm{H}), 0.87(\mathrm{t}, J=7.5$ $\mathrm{Hz}, 3 \mathrm{H})$.

${ }^{13}$ C NMR (101 MHz, $\left.\mathrm{CDCl}_{3}\right) \delta$ 204.9, 143.8, 135.9, 135.2, 132.1, 131.6, 129.4, 128.0, 121.0, 89.6, 70.9, 54.8, 52.5, $40.9,38.1,33.0,21.5,13.4,12.0$.

HRMS ESI Calcd for $\mathrm{C}_{24} \mathrm{H}_{27} \mathrm{CINO}_{3} \mathrm{~S}[\mathrm{M}+\mathrm{H}]^{+}: 444.1395$, Found: 444.1395 .

IR (KBr): 2977, 2923, 1784, 1598, 1442, 1379, 1348, 1163, 1091, $1052 \mathrm{~cm}^{-1}$. 


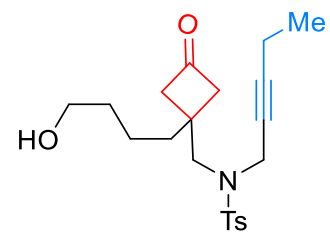

$1 \mathbf{k}$

Synthesis of substrate $\mathbf{1 k}$. Following the general procedure B of preparation of substrate $\mathbf{1 a}$, substrate $\mathbf{1 k}$ was synthesized from S14, 4-Methylbenzyl bromide, $\mathbf{T s N H}_{2}$ and S12 as a colorless oil.

$\mathrm{Rf}=0.45$, Hexane/ EtOAc 1:3.

${ }^{1} \mathrm{H}$ NMR (400 MHz, Chloroform-d) $\delta 7.75-7.68(\mathrm{~m}, 2 \mathrm{H}), 7.34-7.28(\mathrm{~m}, 2 \mathrm{H}), 4.09(\mathrm{t}, J=2.3 \mathrm{~Hz}, 2 \mathrm{H}), 3.68(\mathrm{t}, J=6.2$ $\mathrm{Hz}, 2 \mathrm{H}), 3.41(\mathrm{~s}, 2 \mathrm{H}), 3.11-3.00(\mathrm{~m}, 2 \mathrm{H}), 2.87-2.76(\mathrm{~m}, 2 \mathrm{H}), 2.42(\mathrm{~s}, 3 \mathrm{H}), 1.87$ (qt, J = 7.5, $2.2 \mathrm{~Hz}, 2 \mathrm{H}), 1.72-1.64$ (m, 2H), $1.64-1.57(\mathrm{~m}, 2 \mathrm{H}), 1.57-1.45(\mathrm{~m}, 2 \mathrm{H}), 0.85(\mathrm{t}, J=7.5 \mathrm{~Hz}, 3 \mathrm{H})$.

${ }^{13} \mathrm{C}$ NMR (101 MHz, $\left.\mathrm{CDCl}_{3}\right) \delta$ 206.4, 143.6, 135.3, 129.4, 129.3, 127.9, 89.4, 70.9, 62.2, 55.8, 51.0, 37.5, 35.7, 32.7, $32.3,21.5,20.5,13.4,12.0$.

HRMS ESI Calcd for $\mathrm{C}_{21} \mathrm{H}_{30} \mathrm{NO}_{4} \mathrm{~S}[\mathrm{M}+\mathrm{H}]^{+}: 392.1890$, Found: 392.1889.

IR (KBr): 3539, 2975, 2937, 1866, 1781, 1735, 1598, 1451, 1347, 1161, $1091 \mathrm{~cm}^{-1}$.

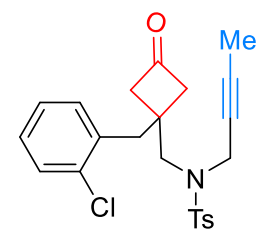

$1 y$

Synthesis of substrate 1y. Following the general procedure B of preparation of substrate $\mathbf{1 a}$, substrate $\mathbf{1 y}$ was synthesized from S14, 2-chlorobenzyl bromide, $\mathrm{TsNH}_{2}$ and 1-bromo-2-butyne as a white solid.

$\mathrm{Rf}=0.30$, Hexane/ EtOAc 4:1.

${ }^{1} \mathrm{H}$ NMR (400 MHz, Chloroform-d) $\delta 7.78-7.71(\mathrm{~m}, 2 \mathrm{H}), 7.40$ (dd, J = 7.5, $\left.1.8 \mathrm{~Hz}, 1 \mathrm{H}\right), 7.36-7.29$ (m, 3H), $7.29-$ $7.19(\mathrm{~m}, 2 \mathrm{H}), 4.18(\mathrm{q}, J=2.4 \mathrm{~Hz}, 2 \mathrm{H}), 3.54(\mathrm{~s}, 2 \mathrm{H}), 3.20(\mathrm{~s}, 2 \mathrm{H}), 3.03(\mathrm{~s}, 4 \mathrm{H}), 2.45(\mathrm{~s}, 3 \mathrm{H}), 1.52(\mathrm{t}, J=2.4 \mathrm{~Hz}, 3 \mathrm{H})$.

${ }^{13}$ C NMR (101 MHz, CDCl $)$ ) 205.4, 143.7, 135.4, 135.0, 134.9, 132.3, 130.1, 129.4, 128.5, 128.0, 127.0, 83.5, 70.9, $54.3,53.4,37.9,37.4,33.4,21.5,3.2$.

HRMS ESI Calcd for $\mathrm{C}_{23} \mathrm{H}_{25} \mathrm{ClNO}_{3} \mathrm{~S}[\mathrm{M}+\mathrm{H}]^{+}:$430.1238, Found: 430.1237.

IR (KBr): 2921, 2857, 1784, 1707, 1597, 1474, 1442, 1379, 1347, 1163, 1090, $1052 \mathrm{~cm}^{-1}$.

\section{Synthetic route of substrate $\mathbf{1 d}$.}



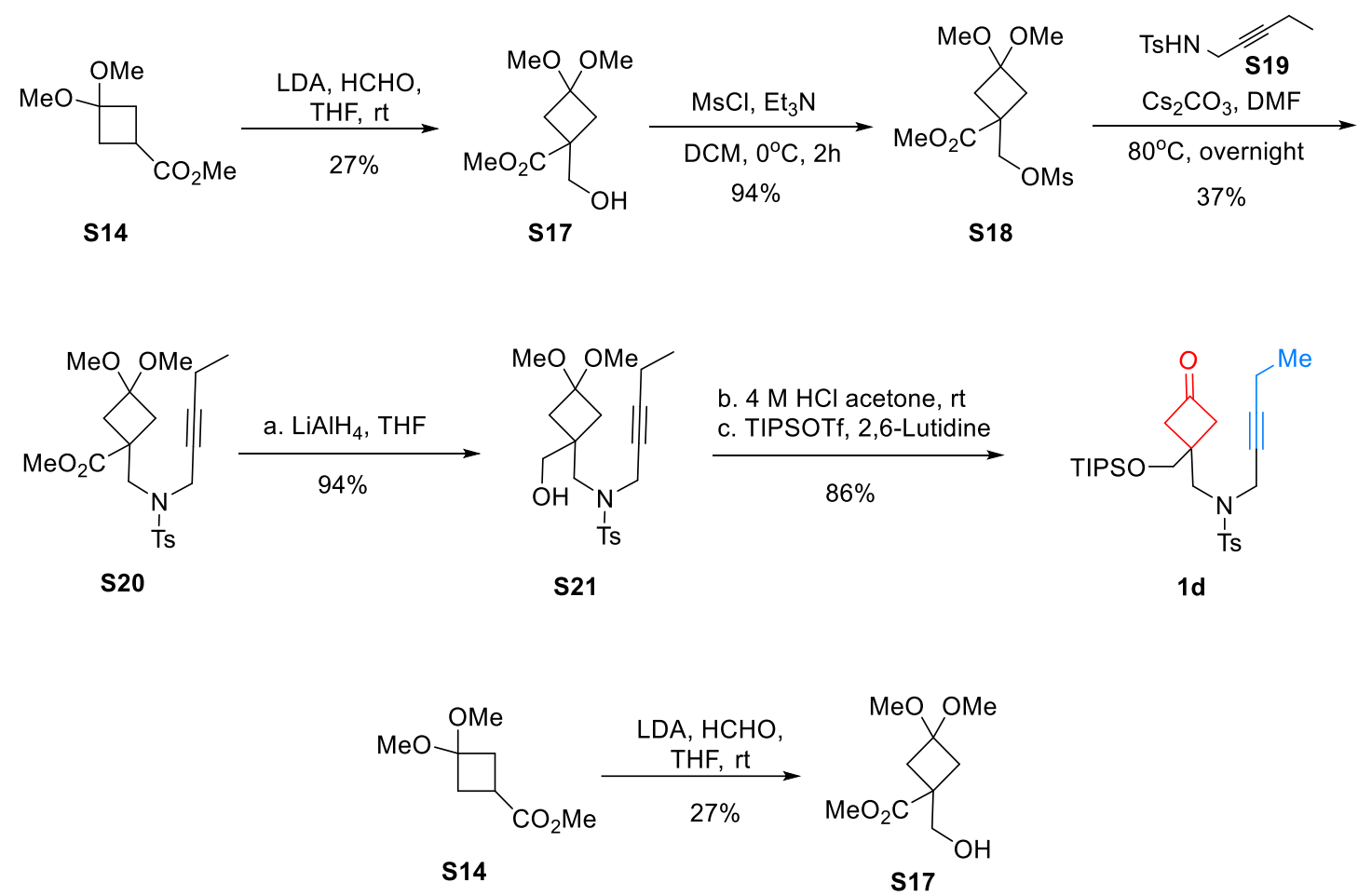

Synthesis of alcohol S17. $n$-BuLi ( $56 \mathrm{~mL}, 2.5 \mathrm{M}$ in hexane, $140 \mathrm{mmol}, 1.4$ equiv) was added to a solution of diisopropylamine $\left(21 \mathrm{~mL}, 150 \mathrm{mmol}, 1.5\right.$ equiv) in THF $(100 \mathrm{~mL})$ at $-78^{\circ} \mathrm{C}$. After stirred at $-78^{\circ} \mathrm{C}$ for $0.5 \mathrm{~h}$, a solution of ester $\mathbf{S 1 4}\left(17.4 \mathrm{~g}, 100 \mathrm{mmol}, 1\right.$ equiv) in THF $(20 \mathrm{~mL})$ was added to the reaction mixture. After stirred at $-78^{\circ} \mathrm{C}$ for $1 \mathrm{~h}$, paraformaldehyde $\left(6.0 \mathrm{~g}, 200 \mathrm{mmol}, 2\right.$ equiv) was added to the reaction mixture. After stirred at $-78^{\circ} \mathrm{C}$ for 0.5 $h$, the reaction mixture was warmed to room temperature and stirred for overnight. The reaction mixture was quenched by adding saturated $\mathrm{NH}_{4} \mathrm{Cl}$ aqueous solution. The reaction mixture was extracted with $\mathrm{Et}_{2} \mathrm{O}$, and the organic phase was washed with $\mathrm{H}_{2} \mathrm{O}$, saturated $\mathrm{NaHCO}_{3}$ aqueous solution, brine, dried with $\mathrm{Na}_{2} \mathrm{SO}_{4}$ and concentrated under reduced pressure. The residue was purified by column chromatography on silica gel to give $5.54 \mathrm{~g}$ of alcohol S17 in 27\% yield as a yellow oil.

$\mathrm{Rf}=0.2$, Hexane/ EtOAc 2:1.

${ }^{1} \mathrm{H}$ NMR (400 MHz, Chloroform-d) $\delta 3.81(\mathrm{~s}, 2 \mathrm{H}), 3.74(\mathrm{~s}, 3 \mathrm{H}), 3.14(\mathrm{~s}, 3 \mathrm{H}), 3.14(\mathrm{~s}, 3 \mathrm{H}), 2.57-2.48(\mathrm{~m}, 2 \mathrm{H}), 2.47-$ $2.24(\mathrm{~m}, 1 \mathrm{H}), 2.23-2.17(\mathrm{~m}, 2 \mathrm{H})$.

${ }^{13} \mathrm{C}$ NMR (101 MHz, $\mathrm{CDCl}_{3}$ ) $\delta$ 176.1, 98.8, 66.6, 52.3, 48.4, 48.3, 39.5, 37.7.

HRMS ESI Calcd for $\mathrm{C}_{9} \mathrm{H}_{17} \mathrm{O}_{5}[\mathrm{M}+\mathrm{H}]^{+}:$205.1071, Found: 205.1068.

IR (KBr): 3459, 2994, 2953, 2833, 1732, 1438, 1329, 1279, 1219, 1188, 1096, $1041 \mathrm{~cm}^{-1}$.

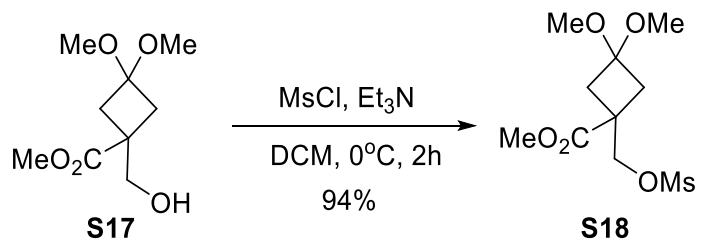


Synthesis of alcohol S18. $\mathrm{MsCl}(2.5 \mathrm{~mL}, 32.5 \mathrm{mmol}, 1.2$ equiv) was added to a solution of alcohol S17 (5.54 g, 27 mmol, 1 equiv) and $\mathrm{NEt}_{3}\left(7.5 \mathrm{~mL}, 54 \mathrm{mmol}, 2\right.$ equiv) in $\mathrm{DCM}(50 \mathrm{~mL})$ at $0^{\circ} \mathrm{C}$. After stirred at $0^{\circ} \mathrm{C}$ for $1.5 \mathrm{~h}$, the reaction mixture was quenched by adding saturated $\mathrm{NH}_{4} \mathrm{Cl}$ aqueous solution. The reaction mixture was extracted with $\mathrm{DCM}$, and the organic phase was washed with $\mathrm{H}_{2} \mathrm{O}$, saturated $\mathrm{NaHCO}_{3}$ aqueous solution and brine, dried with $\mathrm{Na}_{2} \mathrm{SO}_{4}$ and concentrated under reduced pressure. The residue was purified by column chromatography on silica gel to give 7.25 g of compound $\mathbf{S 1 8}$ in $94 \%$ yield as a yellow solid.

Rf $=0.3$, Hexane/ EtOAc/DCM 3:1:1.

Mp: $47-49^{\circ} \mathrm{C}$.

${ }^{1} \mathrm{H}$ NMR (400 MHz, Chloroform-d) $\delta 4.51(\mathrm{~s}, 2 \mathrm{H}), 3.77(\mathrm{~s}, 3 \mathrm{H}), 3.16(\mathrm{t}, J=2.8 \mathrm{~Hz}, 6 \mathrm{H}), 3.03(\mathrm{~s}, 3 \mathrm{H}), 2.66-2.55(\mathrm{~m}, 2 \mathrm{H})$, $2.31-2.21(\mathrm{~m}, 2 \mathrm{H})$.

${ }^{13} \mathrm{C} \mathrm{NMR}\left(101 \mathrm{MHz}, \mathrm{CDCl}_{3}\right) \delta 173.5,98.3,72.1,52.7,48.51,48.47,38.2,37.7,37.3$.

HRMS ESI Calcd for $\mathrm{C}_{10} \mathrm{H}_{19} \mathrm{O}_{7} \mathrm{~S}[\mathrm{M}+\mathrm{H}]^{+}:$283.0846, Found: 283.0854.

IR (KBr): 2956, 1735, 1438, 1358, 1283, 1227, 1177, 1104, $1040 \mathrm{~cm}^{-1}$.

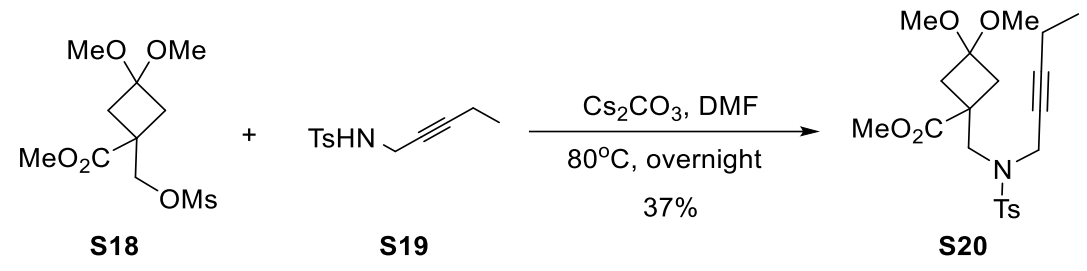

Synthesis of alcohol S20. $\mathrm{Cs}_{2} \mathrm{CO}_{3}(1.30 \mathrm{~g}, 4 \mathrm{mmol}, 2$ equiv) was added to a solution of compound S18 (846 mg, 3 mmol, 1.5 equiv) and compound $\mathbf{S 1 9 ^ { 1 1 }}$ (546 mg, 2 mmol, 1 equiv) in DMF ( $5 \mathrm{~mL}$ ) at room temperature. After stirred at $80^{\circ} \mathrm{C}$ for overnight, the reaction mixture was cooled to room temperature, quenched by adding saturated $\mathrm{NH}_{4} \mathrm{Cl}$ aqueous solution. The reaction mixture was extracted with $\mathrm{Et}_{2} \mathrm{O}$, and the organic phase was washed with $\mathrm{H}_{2} \mathrm{O}$, saturated $\mathrm{NaHCO}_{3}$ aqueous solution and brine, dried with $\mathrm{Na}_{2} \mathrm{SO}_{4}$ and concentrated under reduced pressure. The residue was purified by column chromatography on silica gel to give $313 \mathrm{mg}$ of compound $\mathbf{S 2 0}$ in $37 \%$ yield as a yellow oil.

$\mathrm{Rf}=0.3$, Hexane/ EtOAc 4:1.

${ }^{1} \mathrm{H}$ NMR (400 MHz, Chloroform-d) $\delta 7.75-7.68(\mathrm{~m}, 2 \mathrm{H}), 7.32-7.27(\mathrm{~m}, 2 \mathrm{H}), 4.04(\mathrm{t}, \mathrm{J}=2.3 \mathrm{~Hz}, 2 \mathrm{H}), 3.74(\mathrm{~s}, 3 \mathrm{H}), 3.59$ $(\mathrm{s}, 2 \mathrm{H}), 3.16(\mathrm{~s}, 3 \mathrm{H}), 3.12(\mathrm{~s}, 3 \mathrm{H}), 2.69-2.60(\mathrm{~m}, 2 \mathrm{H}), 2.42(\mathrm{~s}, 3 \mathrm{H}), 2.35-2.27(\mathrm{~m}, 2 \mathrm{H}), 1.94-1.86(\mathrm{~m}, 2 \mathrm{H}), 0.88(\mathrm{t}, \mathrm{J}$ $=7.5 \mathrm{~Hz}, 3 \mathrm{H})$.

${ }^{13} \mathrm{C}$ NMR (101 MHz, $\left.\mathrm{CDCl}_{3}\right) \delta 174.9,143.3,135.9,129.3,127.9,98.7,88.3,71.4,52.4,51.7,48.6,48.5,39.5,39.2$, $37.4,21.5,13.4,12.1$.

HRMS ESI Calcd for $\mathrm{C}_{21} \mathrm{H}_{30} \mathrm{NO}_{6} \mathrm{~S}[\mathrm{M}+\mathrm{H}]^{+}:$424.1788, Found:424.1780.

IR (KBr): 2950, 1735, 1598, 1444, 1351, 1281, 1163, 1090, $1042 \mathrm{~cm}^{-1}$. 


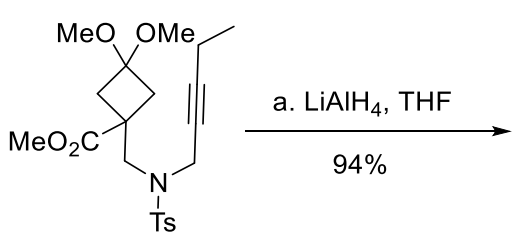

S20

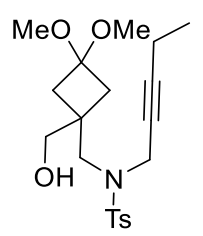

S21

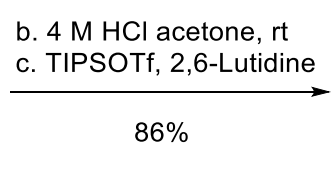

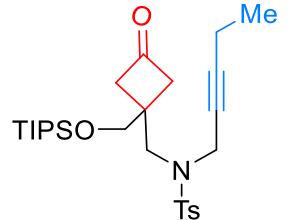

$1 d$

Synthesis of substrate 1d. A solution of ester $\mathbf{S 2 0}(1.4 \mathrm{~g}, 3.3 \mathrm{mmol}, 1$ equiv) in THF ( $5 \mathrm{~mL}$ ) was added to a solution of $\mathrm{LiAlH}_{4}\left(150 \mathrm{mg}, 3.95 \mathrm{mmol}, 1.2\right.$ equiv) in THF $(10 \mathrm{~mL})$ at $0^{\circ} \mathrm{C}$. After stirred at room temperature for $2 \mathrm{~h}$, the reaction mixture was cooled to $0^{\circ} \mathrm{C}$, quenched by adding $\mathrm{H}_{2} \mathrm{O}(0.15 \mathrm{~mL}), 20 \% \mathrm{NaOH}$ aqueous solution $(0.30 \mathrm{~mL})$, and $\mathrm{H}_{2} \mathrm{O}(0.45$ $\mathrm{mL}$ ). After stirred at room temperature for $0.5 \mathrm{~h}, \mathrm{Na}_{2} \mathrm{SO}_{4}$ was added to the reaction mixture. After stirred at room temperature for $0.5 \mathrm{~h}$, the reaction mixture was filtered with funnel and washed with EtOAc. The filtrate was concentrated under reduced pressure to give $1.22 \mathrm{~g}$ of crude alcohol S21 in 94\% yield as a yellow oil which was used for the next step without further purification. $4 \mathrm{M} \mathrm{HCl}(5 \mathrm{~mL}$ ) was added to a solution of alcohol $\mathbf{S 2 1}$ (198 $\mathrm{mg}, 0.5$ mmol, 1 equiv) in acetone $(5 \mathrm{~mL})$ at $0^{\circ} \mathrm{C}$. After stirred at room temperature for $1 \mathrm{~h}$, the reaction mixture was extracted with $\mathrm{Et}_{2} \mathrm{O}$, and the organic phase was washed with saturated $\mathrm{NaHCO}_{3}$ aqueous solution, brine, dried with $\mathrm{Na}_{2} \mathrm{SO}_{4}$ and concentrated under reduced pressure to give of crude ketone which was used for the next step without further purification. TIPSOTf ( $0.16 \mathrm{~mL}, 0.6 \mathrm{mmol}, 1.2$ equiv) was added to a solution of above crude ketone and 2,6-lutidine ( $0.0875 \mathrm{~mL}, 0.75 \mathrm{mmol}, 1.5$ equiv) in $\mathrm{DCM}(3 \mathrm{~mL})$ at $0^{\circ} \mathrm{C}$. After stirred at room temperature for $2 \mathrm{~h}$, the reaction mixture was quenched by adding saturated $\mathrm{NH}_{4} \mathrm{Cl}$ aqueous solution. The reaction mixture was extracted with DCM, and the organic phase was washed with $\mathrm{H}_{2} \mathrm{O}$, saturated $\mathrm{NaHCO}_{3}$ aqueous solution and brine, dried with $\mathrm{Na}_{2} \mathrm{SO}_{4}$ and concentrated under reduced pressure. The residue was purified by column chromatography on silica gel to give 217 mg of substrate $1 \mathbf{d}$ in $86 \%$ yield as a colorless oil.

$\mathrm{Rf}=0.52$, Hexane/ EtOAc 4:1.

${ }^{1} \mathrm{H}$ NMR (400 MHz, Chloroform-d) $87.78-7.71(\mathrm{~m}, 2 \mathrm{H}), 7.35-7.28(\mathrm{~m}, 2 \mathrm{H}), 4.13(\mathrm{t}, \mathrm{J}=2.2 \mathrm{~Hz}, 2 \mathrm{H}), 3.85(\mathrm{~s}, 2 \mathrm{H}), 3.50$ $(\mathrm{s}, 2 \mathrm{H}), 3.14-2.94(\mathrm{~m}, 4 \mathrm{H}), 2.43(\mathrm{~s}, 3 \mathrm{H}), 1.88(\mathrm{qt}, J=7.5,2.2 \mathrm{~Hz}, 2 \mathrm{H}), 1.17-1.03(\mathrm{~m}, 21 \mathrm{H}), 0.87(\mathrm{t}, J=7.5 \mathrm{~Hz}, 3 \mathrm{H})$. ${ }^{13} \mathrm{C} \mathrm{NMR}\left(101 \mathrm{MHz}, \mathrm{CDCl}_{3}\right) \delta$ 205.7, 143.5, 135.7, 129.4, 128.0, 88.6, 71.5, 66.7, 52.6, 50.6, 38.7, 35.0, 21.5, 18.0, 13.4, 12.0, 11.9.

HRMS ESI Calcd for $\mathrm{C}_{27} \mathrm{H}_{44} \mathrm{NO}_{4} \mathrm{SSi}[\mathrm{M}+\mathrm{H}]^{+}: 506.2755$, Found: 506.2758.

IR (KBr): 2942, 2866, 1787, 1688, 1628, 1598, 1457, 1345, 1230, 1165, $1092 \mathrm{~cm}^{-1}$.

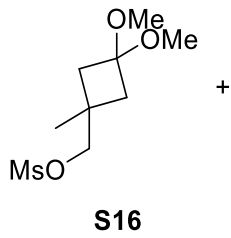

$\mathbf{S 1 6}$

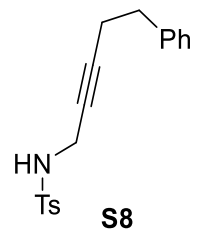

S8
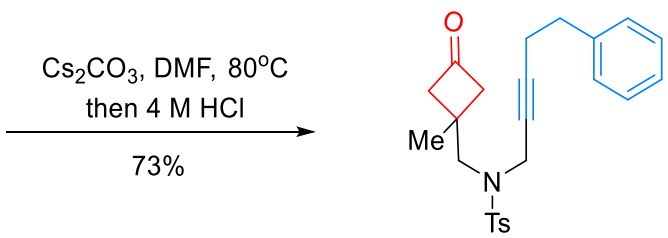

$1 \mathrm{v}$ 
Synthesis of substrate $1 \mathbf{v} . \mathrm{Cs}_{2} \mathrm{CO}_{3}(874 \mathrm{mg}, 2.68 \mathrm{mmol}, 4$ equiv) was added to a solution of compound S16 (319 mg, $1.34 \mathrm{mmol}, 2$ equiv) and $\mathbf{S} 8(210 \mathrm{mg}, 0.67 \mathrm{mmol}, 1$ equiv) in DMF ( $4 \mathrm{~mL})$ at room temperature. After stirred at $80^{\circ} \mathrm{C}$ for $12 \mathrm{~h}$, the reaction mixture was cooled to $0^{\circ} \mathrm{C}$. Then $4 \mathrm{M} \mathrm{HCl}(6 \mathrm{~mL})$ and acetone $(6 \mathrm{~mL})$ were successively added to the reaction mixture. After stirred at room temperature for $2 \mathrm{~h}$, the reaction mixture was extracted with $\mathrm{Et}_{2} \mathrm{O}$, and the organic phase was washed with $\mathrm{H}_{2} \mathrm{O}$, saturated $\mathrm{NaHCO}_{3}$ aqueous solution and brine, dried with $\mathrm{Na}_{2} \mathrm{SO}_{4}$ and concentrated under reduced pressure. The residue was purified by column chromatography on silica gel to give 200 $\mathrm{mg}$ of compound $\mathbf{1 v}$ in $73 \%$ yield as a white solid.

$\mathrm{Rf}=0.4$, Hexane/ EtOAc 4:1.

$\mathrm{Mp}: 89.8-90.5^{\circ} \mathrm{C}$.

${ }^{1} \mathrm{H}$ NMR (400 MHz, Chloroform-d) $\delta 7.79-7.68(\mathrm{~m}, 2 \mathrm{H}), 7.32(\mathrm{~d}, J=8.0 \mathrm{~Hz}, 2 \mathrm{H}), 7.30-7.24(\mathrm{~m}, 2 \mathrm{H}), 7.24-7.18(\mathrm{~m}$, 1H), $7.07-6.98(\mathrm{~m}, 2 \mathrm{H}), 4.10(\mathrm{t}, J=2.2 \mathrm{~Hz}, 2 \mathrm{H}), 3.25(\mathrm{~s}, 2 \mathrm{H}), 3.04-2.94(\mathrm{~m}, 2 \mathrm{H}), 2.75-2.64(\mathrm{~m}, 2 \mathrm{H}), 2.55(\mathrm{t}, J=7.3$ $\mathrm{Hz}, 2 \mathrm{H}), 2.46(\mathrm{~s}, 3 \mathrm{H}), 2.26(\mathrm{tt}, J=7.3,2.3 \mathrm{~Hz}, 2 \mathrm{H}), 1.35(\mathrm{~s}, 3 \mathrm{H})$.

${ }^{13}$ C NMR (101 MHz, CDCl $)$ ) $206.3,143.5,140.0,135.9,129.4,128.4,128.2,127.9,126.4,86.6,72.7,57.3,53.8,37.5$, $34.2,29.0,23.9,21.5,20.0$.

HRMS ESI Calcd for $\mathrm{C}_{24} \mathrm{H}_{28} \mathrm{NO}_{3} \mathrm{~S}[\mathrm{M}+\mathrm{H}]^{+}: 410.1784$, Found:410.1792

IR (KBr): 3028, 2958, 2866, 1783, 1598, 1495, 1454, 1383, 1348, 1161, 1090, $1057 \mathrm{~cm}^{-1}$.
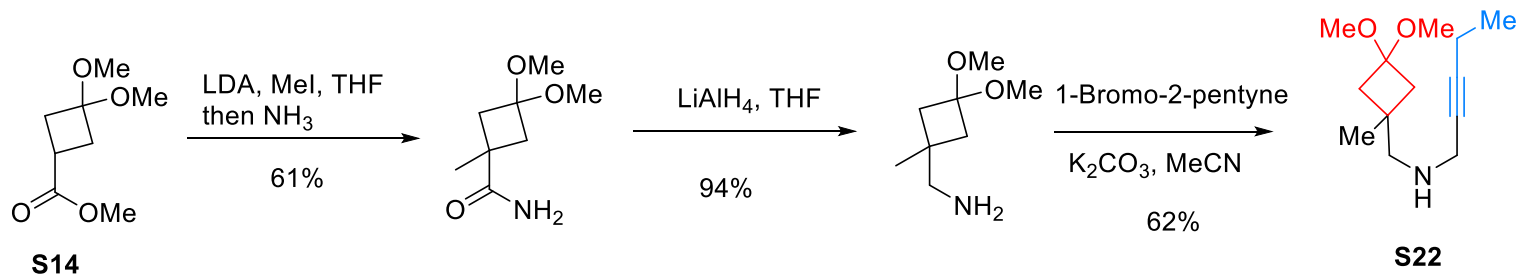

S14

Synthesis of secondary amine S22. $n$-BuLi (56 mL, $2.5 \mathrm{M}$ in hexane, $140 \mathrm{mmol}, 1.4$ equiv) was added to a solution of diisopropylamine $\left(21 \mathrm{~mL}, 150 \mathrm{mmol}, 1.5\right.$ equiv) in THF $(100 \mathrm{~mL})$ at $-78^{\circ} \mathrm{C}$. After stirred at $-78^{\circ} \mathrm{C}$ for $0.5 \mathrm{~h}$, a solution of S14 (17.4 g, $100 \mathrm{mmol}, 1$ equiv) in THF $(20 \mathrm{~mL})$ was added to the reaction mixture. After stirred at $-78^{\circ} \mathrm{C}$ for $1 \mathrm{~h}$, Mel (12.46 mL, $200 \mathrm{mmol}, 2$ equiv) in THF $(10 \mathrm{~mL})$ was added to the reaction mixture. After stirred at $-78^{\circ} \mathrm{C}$ for 0.5 $h$, the reaction mixture was warmed to room temperature and stirred for overnight. The reaction mixture was quenched by adding saturated $\mathrm{NH}_{4} \mathrm{Cl}$ aqueous solution. The reaction mixture was extracted with $\mathrm{Et}_{2} \mathrm{O}$, and the organic phase was washed with $\mathrm{H}_{2} \mathrm{O}$, saturated $\mathrm{NaHCO}_{3}$ aqueous solution and brine, dried with $\mathrm{Na}_{2} \mathrm{SO}_{4}$ and concentrated under reduced pressure to give the crude product which was used for the next step without further purification. The above crude product was dissolved in $30 \% \mathbf{N H}_{3}(230 \mathrm{~mL})$ aqueous solution at room temperature. After stirred at room temperature for overnight, the reaction mixture was extracted with DCM, and the organic phase was washed with brine, dried with $\mathrm{Na}_{2} \mathrm{SO}_{4}$ and concentrated under reduced pressure to give $10.6 \mathrm{~g}$ of the amide intermediate in $61 \%$ yield as a yellow solid which was used for the next step without further purification. A solution of the amide $\left(5.20 \mathrm{~g}, 30 \mathrm{mmol}, 1\right.$ equiv) in THF $(20 \mathrm{~mL})$ was added to a solution of $\mathrm{LiAlH}_{4}(1.48 \mathrm{~g}, 39 \mathrm{mmol}$, 
1.3 equiv) in $\mathrm{THF}$ at $0^{\circ} \mathrm{C}$. After stirred at room temperature for overnight, the reaction mixture was cooled to $0^{\circ} \mathrm{C}$, quenched by adding $\mathrm{H}_{2} \mathrm{O}(1.48 \mathrm{~mL}), 20 \% \mathrm{NaOH}$ aqueous solution $(2.96 \mathrm{~mL})$ and $\mathrm{H}_{2} \mathrm{O}(4.44 \mathrm{~mL})$. After stirred at room temperature for $0.5 \mathrm{~h}, \mathrm{Na}_{2} \mathrm{SO}_{4}$ was added to the reaction mixture. After stirred at room temperature for $0.5 \mathrm{~h}$, the reaction mixture was filtered with funnel and washed with EtOAc. The filtrate was concentrated under reduced pressure to give the crude amine $(4.5 \mathrm{~g}, 94 \%$ yield) as a yellow oil which was used for the next step without further purification. $\mathrm{K}_{2} \mathrm{CO}_{3}(2.07 \mathrm{~g}, 15 \mathrm{mmol}, 3$ equiv) was added to a solution of the above amine $(1.33 \mathrm{~g}, 8.4 \mathrm{mmol}, 1.7$ equiv) and 1-bromo-2-pentyne $(0.51 \mathrm{ml}, 5 \mathrm{mmol}, 1$ equiv) in $\mathrm{MeCN}(40 \mathrm{~mL})$ at room temperature. After stirred at room temperature for $10 \mathrm{~h}$, the reaction mixture was quenched by adding $\mathrm{H}_{2} \mathrm{O}$. The reaction mixture was extracted with $\mathrm{DCM}$, and the organic phase was washed with brine, dried with $\mathrm{Na}_{2} \mathrm{SO}_{4}$ and concentrated under reduced pressure. The residue was purified by column chromatography on silica gel to give $700 \mathrm{mg}$ of secondary amine $\mathbf{S 2 2}$ in $62 \%$ yield as a yellow oil.

$\mathrm{Rf}=0.3$, Hexane/ EtOAc 1:1.

${ }^{1} \mathrm{H}$ NMR $(400 \mathrm{MHz}$, Chloroform-d) $\delta 3.40(\mathrm{t}, J=2.2 \mathrm{~Hz}, 2 \mathrm{H}), 3.13(\mathrm{~s}, 3 \mathrm{H}), 3.12(\mathrm{~s}, 3 \mathrm{H}), 2.66(\mathrm{~s}, 2 \mathrm{H}), 2.19$ (qt, $J=7.5,2.2$ $\mathrm{Hz}, 2 \mathrm{H}), 2.05-1.99(\mathrm{~m}, 2 \mathrm{H}), 1.91-1.85(\mathrm{~m}, 2 \mathrm{H}), 1.19(\mathrm{~s}, 3 \mathrm{H}), 1.13(\mathrm{t}, J=7.5 \mathrm{~Hz}, 3 \mathrm{H})$.

${ }^{13} \mathrm{C}$ NMR (101 MHz, CDCl 3 ) $\delta$ 99.2, 85.2, 59.2, 48.1, 41.7, 39.2, 28.9, 25.4, 14.0, 12.4.

HRMS ESI Calcd for $\mathrm{C}_{13} \mathrm{H}_{24} \mathrm{NO}_{2}[\mathrm{M}+\mathrm{H}]^{+}: 226.1802$, Found: 226.1802.

IR (KBr): 3332, 2975, 2935, 2928, 1454, 1320, 1272, 1191, 1103, $1042 \mathrm{~cm}^{-1}$.

\section{General procedure C.}

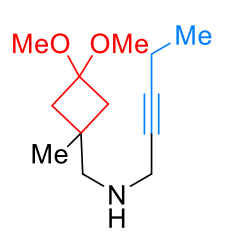

S22

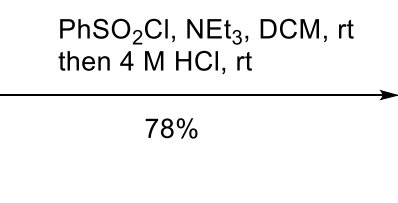

$\mathrm{PhSO}_{2} \mathrm{Cl}, \mathrm{NEt}_{3}, \mathrm{DCM}, \mathrm{r}$ $78 \%$

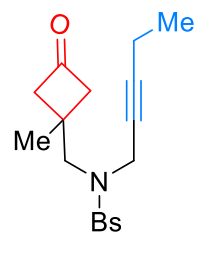

$1 \mathrm{~m}$

Synthesis of substrate $1 \mathrm{~m}$. NEt $3(0.28 \mathrm{~mL}, 2 \mathrm{mmol}, 2$ equiv) was added to a solution of secondary amine S22 (225 $\mathrm{mg}, 1 \mathrm{mmol}, 1$ equiv) and $\mathrm{PhSO}_{2} \mathrm{Cl}\left(0.15 \mathrm{~mL}, 1.2 \mathrm{mmol}, 1.2\right.$ equiv) in $\mathrm{DCM}(2 \mathrm{ml})$ at $0^{\circ} \mathrm{C}$. After stirred at room temperature for overnight, the reaction mixture was cooled to $0^{\circ} \mathrm{C}$. Then $4 \mathrm{M} \mathrm{HCl}(4 \mathrm{~mL})$ was added to the reaction mixture. After stirred at room temperature for $4 \mathrm{~h}$, the reaction mixture was extracted with $\mathrm{Et}_{2} \mathrm{O}$, and the organic phase was washed with $\mathrm{H}_{2} \mathrm{O}$, saturated $\mathrm{NaHCO}_{3}$ aqueous solution and brine, dried with $\mathrm{Na}_{2} \mathrm{SO}_{4}$ and concentrated under reduced pressure. The residue was purified by column chromatography on silica gel to give $248 \mathrm{mg}$ of compound $1 \mathrm{~m}$ in $78 \%$ yield as a colorless oil.

$\mathrm{Rf}=0.27$, Hexane/ EtOAc 4:1.

Mp: $68.3-69.6^{\circ} \mathrm{C}$. 
${ }^{1} \mathrm{H}$ NMR (400 MHz, Chloroform-d) $\delta 7.89-7.82(\mathrm{~m}, 2 \mathrm{H}), 7.63-7.56(\mathrm{~m}, 1 \mathrm{H}), 7.56-7.49(\mathrm{~m}, 2 \mathrm{H}), 4.14(\mathrm{t}, J=2.2 \mathrm{~Hz}$, $2 \mathrm{H}), 3.46(\mathrm{~s}, 2 \mathrm{H}), 3.20-3.08(\mathrm{~m}, 2 \mathrm{H}), 2.84-2.71(\mathrm{~m}, 2 \mathrm{H}), 1.89(\mathrm{qt}, J=7.5,2.2 \mathrm{~Hz}, 2 \mathrm{H}), 1.41(\mathrm{~s}, 3 \mathrm{H}), 0.87(\mathrm{t}, J=7.5 \mathrm{~Hz}$, $3 \mathrm{H})$.

${ }^{13} \mathrm{C}$ NMR (101 MHz, CDCl$)$ ) $206.3,138.8,132.7,128.8,127.8,89.0,71.0,57.4,54.0,37.8,29.3,24.1,13.5,12.0$. HRMS ESI Calcd for $\mathrm{C}_{17} \mathrm{H}_{22} \mathrm{NO}_{3} \mathrm{~S}[\mathrm{M}+\mathrm{H}]^{+}: 320.1315$, Found: 320.1318.

IR (KBr): 2975, 2926, 2877, 1782, 1447, 1383, 1349, 1166, 1092, $1056 \mathrm{~cm}^{-1}$.

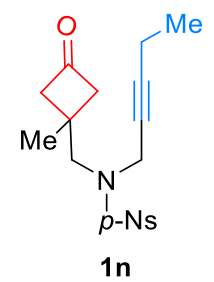

Synthesis of substrate $\mathbf{1 n}$. Following the general procedure $\mathbf{C}$ of preparation of substrate $\mathbf{1 m}, 338 \mathrm{mg}$ of substrate 1n was synthesized from secondary amine $\mathbf{S 2 2}(1 \mathrm{mmol})$ and $\mathrm{NsCl}(1.2 \mathrm{mmol})$ in $93 \%$ yield as a white solid.

$\mathrm{Rf}=0.33$, Hexane/ EtOAc 4:1.

Mp: $83.2-84.6^{\circ} \mathrm{C}$

${ }^{1} \mathrm{H}$ NMR (400 MHz, Chloroform-d) $\delta 8.42-8.35(\mathrm{~m}, 1 \mathrm{H}), 8.10-8.03(\mathrm{~m}, 1 \mathrm{H}), 4.19(\mathrm{t}, J=2.3 \mathrm{~Hz}, 1 \mathrm{H}), 3.48(\mathrm{~s}, 1 \mathrm{H}), 3.19$ - $3.07(\mathrm{~m}, 1 \mathrm{H}), 2.87-2.76(\mathrm{~m}, 1 \mathrm{H}), 1.90$ (qt, $J=7.5,2.2 \mathrm{~Hz}, 1 \mathrm{H}), 1.43(\mathrm{~s}, 2 \mathrm{H}), 0.87(\mathrm{t}, J=7.5 \mathrm{~Hz}, 2 \mathrm{H})$.

${ }^{13} \mathrm{C}$ NMR $\left(101 \mathrm{MHz}, \mathrm{CDCl}_{3}\right) \delta$ 205.5, 150.2, 144.7, 129.1, 124.0, 89.6, 70.5, 57.5, 54.1, 37.8, 29.3, 24.1, 13.4, 12.0.

HRMS ESI Calcd for $\mathrm{C}_{17} \mathrm{H}_{21} \mathrm{~N}_{2} \mathrm{O}_{5} \mathrm{~S}[\mathrm{M}+\mathrm{H}]^{+}: 365.1166$, Found: 365.1165 .

IR (KBr): 3106, 2976, 2936, 1784, 1606, 1531, 1454, 1352, 1312, 1147, 1108, 1090, $1057 \mathrm{~cm}^{-1}$.

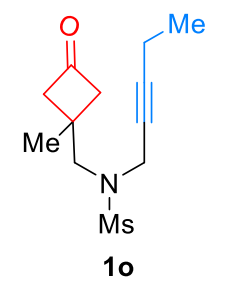

Synthesis of substrate 10. Following the general procedure C of preparation of substrate $1 \mathrm{~m}, 200 \mathrm{mg}$ of substrate 10 was synthesized from secondary amine $\mathbf{S 2 2}(1 \mathrm{mmol})$ and $\mathrm{MsCl}(1.2 \mathrm{mmol})$ in $78 \%$ yield as a colorless oil.

$\mathrm{Rf}=0.1$, Hexane/ EtOAc 4:1.

${ }^{1} \mathrm{H}$ NMR (400 MHz, Chloroform-d) $\delta 4.11(\mathrm{t}, J=2.3 \mathrm{~Hz}, 2 \mathrm{H}), 3.49(\mathrm{~s}, 2 \mathrm{H}), 3.20-3.10(\mathrm{~m}, 2 \mathrm{H}), 2.95(\mathrm{~s}, 3 \mathrm{H}), 2.82-2.72$ $(\mathrm{m}, 2 \mathrm{H}), 2.24$ (qt, $J=7.5,2.2 \mathrm{~Hz}, 2 \mathrm{H}), 1.38(\mathrm{~s}, 3 \mathrm{H}), 1.15(\mathrm{t}, J=7.5 \mathrm{~Hz}, 3 \mathrm{H})$.

${ }^{13} \mathrm{C}$ NMR $\left(101 \mathrm{MHz}, \mathrm{CDCl}_{3}\right) \delta$ 206.0, 89.4, 71.9, 57.2, 54.0, 37.8, 37.6, 29.5, 24.2, 13.7, 12.2.

HRMS ESI Calcd for $\mathrm{C}_{12} \mathrm{H}_{20} \mathrm{NO}_{3} \mathrm{~S}[\mathrm{M}+\mathrm{H}]^{+}:$258.1158, Found: 258.1156 .

IR (KBr): 2975, 2932, 2878, 1783, 1456, 1380, 1329, 1228, 1153,, 1118, $1059 \mathrm{~cm}^{-1}$. 


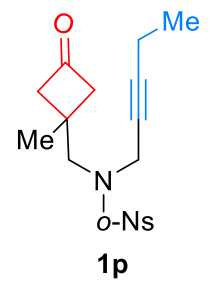

Synthesis of substrate 1p. Following the general procedure $\mathrm{C}$ of preparation of substrate $1 \mathrm{~m}, 309 \mathrm{mg}$ of substrate 1p was synthesized from secondary amine S22 (1 mmol) and 2-Nitrobenzenesulfonyl chloride $(1.2 \mathrm{mmol})$ in $82 \%$ yield as a colorless oil.

$\mathrm{Rf}=0.4$, Hexane/ EtOAc 2:1.

${ }^{1} \mathrm{H}$ NMR (400 MHz, Chloroform-d) $\delta 8.11-8.04(\mathrm{~m}, 1 \mathrm{H}), 7.76-7.68(\mathrm{~m}, 2 \mathrm{H}), 7.67-7.62(\mathrm{~m}, 1 \mathrm{H}), 4.16(\mathrm{t}, J=2.2 \mathrm{~Hz}$, 2H), $3.70(\mathrm{~s}, 2 \mathrm{H}), 3.22-3.09(\mathrm{~m}, 2 \mathrm{H}), 2.86-2.72(\mathrm{~m}, 2 \mathrm{H}), 2.01(\mathrm{qt}, J=7.5,2.2 \mathrm{~Hz}, 2 \mathrm{H}), 1.36(\mathrm{~s}, 3 \mathrm{H}), 0.95(\mathrm{t}, J=7.5 \mathrm{~Hz}$, $3 \mathrm{H})$.

${ }^{13} \mathrm{C}$ NMR (101 MHz, $\left.\mathrm{CDCl}_{3}\right) \delta$ 205.9, 148.4, 133.7, 132.5, 131.5, 131.1, 124.1, 89.2, 71.2, 57.6, 54.6, 37.4, 29.3, 23.8, 13.6, 12.1 .

HRMS ESI Calcd for $\mathrm{C}_{17} \mathrm{H}_{21} \mathrm{~N}_{2} \mathrm{O}_{5} \mathrm{~S}[\mathrm{M}+\mathrm{H}]^{+}:$365.1166, Found: 365.1174.

IR (KBr): 2976, 1783, 1545, 1373, 1358, 1171, 1142, 1126, $1066 \mathrm{~cm}^{-1}$.

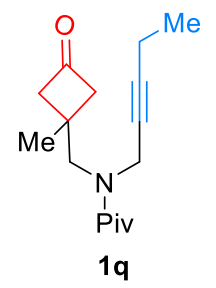

Synthesis of substrate 1q. Following the general procedure $\mathbf{C}$ of preparation of substrate $1 \mathrm{~m}, 260 \mathrm{mg}$ of substrate 1q was synthesized from secondary amine $\mathbf{S 2 2}(1 \mathrm{mmol})$ and $\mathrm{PivCl}(1.3 \mathrm{mmol})$ in 99\% yield as a colorless oil. Rf $=0.4$, Hexane/ EtOAc 4:1.

${ }^{1} \mathrm{H}$ NMR (400 MHz, Chloroform-d) $\delta 4.22(\mathrm{t}, \mathrm{J}=2.2 \mathrm{~Hz}, 2 \mathrm{H}), 3.77(\mathrm{~s}, 2 \mathrm{H}), 3.20-3.07$ (m, 2H), $2.77-2.64(\mathrm{~m}, 2 \mathrm{H}), 2.19$ (qt, $J=7.5,2.2 \mathrm{~Hz}, 2 \mathrm{H}), 1.31(\mathrm{~s}, 9 \mathrm{H}), 1.27(\mathrm{~s}, 3 \mathrm{H}), 1.12(\mathrm{t}, J=7.5 \mathrm{~Hz}, 3 \mathrm{H})$.

${ }^{13} \mathrm{C}$ NMR (101 MHz, CDCl 3$) \delta$ 207.4, 178.1, 86.9, 74.1, 57.7, 54.1, 39.1, 29.8, 28.6, 24.3, 13.7, 12.3.

HRMS ESI Calcd for $\mathrm{C}_{16} \mathrm{H}_{26} \mathrm{NO}_{2}[\mathrm{M}+\mathrm{H}]^{+}:$264.1958, Found: 264.1961.

IR (KBr): 2975, 2929, 1784, 1637, 1478, 1410, 1379, 1183, 1141, $1018 \mathrm{~cm}^{-1}$. 


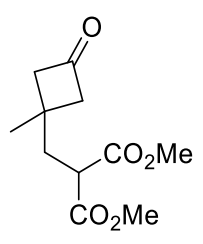

S13

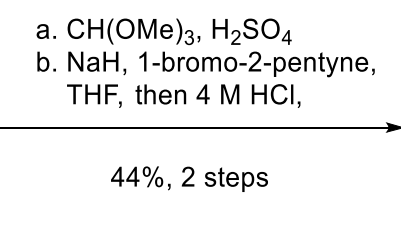

steps

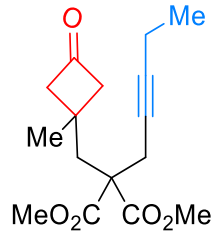

$1 \mathrm{r}$

Synthesis of substrate $1 \mathrm{r} . \mathrm{H}_{2} \mathrm{SO}_{4}(8 \mathrm{mg}, 0.083 \mathrm{mmol}, 5 \mathrm{~mol} \%$ ) was added to a solution of S13 (376 mg, $1.65 \mathrm{mmol}, 1$ equiv) in $\mathrm{CH}(\mathrm{OMe})_{3}(0.6 \mathrm{~mL}, 3 \mathrm{mmol}, 1.2$ equiv) at room temperature. After stirred at room temperature for overnight, the reaction mixture was quenched by adding saturated $\mathrm{NaHCO}_{3}$ aqueous solution, extracted with $\mathrm{Et}_{2} \mathrm{O}$. the organic phase was washed with $\mathrm{H}_{2} \mathrm{O}$, saturated $\mathrm{NaHCO}_{3}$ aqueous solution and brine, dried with $\mathrm{Na}_{2} \mathrm{SO}_{4}$ and concentrated under reduced pressure to give $405 \mathrm{mg}$ of crude ketal in $92 \%$ yield which was used for the next step without further purification. A solution of above crude ketal ( $405 \mathrm{mg}, 1.5 \mathrm{mmol}, 1$ equiv) was added to a solution of $\mathrm{NaH}$ (115 mg, 60\% in oil, $3 \mathrm{mmol}, 2$ equiv) in THF $(3 \mathrm{~mL})$ at $0^{\circ} \mathrm{C}$. After stirred at room temperature for $1 \mathrm{~h}, 1-\mathrm{bromo}-$ 2-pentyne ( $265 \mathrm{mg}, 1.8 \mathrm{mmol}, 1.2$ equiv) was added to the reaction mixture. After stirred at room temperature for overnight, the reaction mixture was cooled to $0^{\circ} \mathrm{C}$. Then $4 \mathrm{M} \mathrm{HCl}(4 \mathrm{~mL})$ was added to the reaction mixture. After stirred at room temperature for $4 \mathrm{~h}$, the reaction mixture was extracted with $\mathrm{Et}_{2} \mathrm{O}$, and the organic phase was washed with $\mathrm{H}_{2} \mathrm{O}$, saturated $\mathrm{NaHCO}_{3}$ aqueous solution and brine, dried with $\mathrm{Na}_{2} \mathrm{SO}_{4}$ and concentrated under reduced pressure. The residue was purified by column chromatography on silica gel to give $210 \mathrm{mg}$ of compound $1 \mathrm{r}$ in $48 \%$ yield as a white solid.

$\mathrm{Rf}=0.34$, Hexane/ EtOAc 5:1.

Mp: $39.3-41.8^{\circ} \mathrm{C}$.

${ }^{1} \mathrm{H}$ NMR (400 MHz, Chloroform-d) $\delta 3.74(\mathrm{~s}, 6 \mathrm{H}), 3.21-3.10(\mathrm{~m}, 2 \mathrm{H}), 2.87-2.80(\mathrm{~m}, 2 \mathrm{H}), 2.78-2.67(\mathrm{~m}, 2 \mathrm{H}), 2.59$ $(\mathrm{s}, 2 \mathrm{H}), 2.19-2.08(\mathrm{~m}, 2 \mathrm{H}), 1.21(\mathrm{~s}, 3 \mathrm{H}), 1.09(\mathrm{t}, J=7.5 \mathrm{~Hz}, 3 \mathrm{H})$.

${ }^{13} \mathrm{C}$ NMR (101 MHz, CDCl 3$) \delta 207.8,170.9,86.7,73.7,60.0,56.6,52.8,41.7,27.8,24.2,23.2,14.1,12.3$.

HRMS ESI Calcd for $\mathrm{C}_{16} \mathrm{H}_{23} \mathrm{O}_{5}[\mathrm{M}+\mathrm{H}]^{+}:$295.1540, Found: 295.1542.

IR (KBr): 2955, 1782, 1732, 1438, 1383, 1336, 1310, 1275, 1211, 1142, 1114, 1063, $1034 \mathrm{~cm}^{-1}$.

\section{Synthetic route of substrate $1 \mathrm{~s}$.}

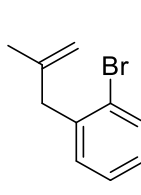

S15

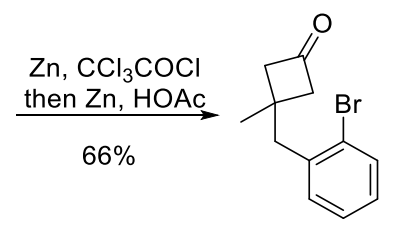

S15-1

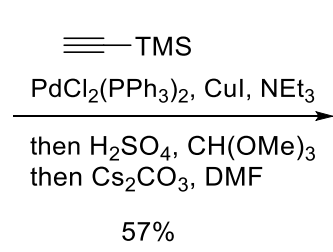

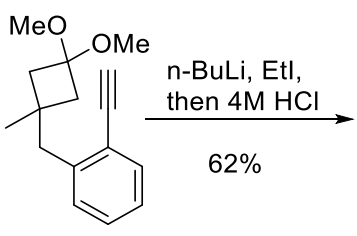

S15-2

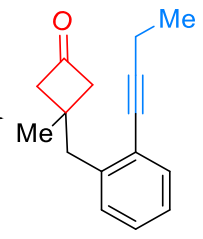

$1 \mathrm{~s}$ 


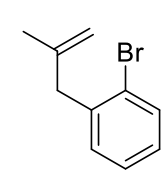

S15

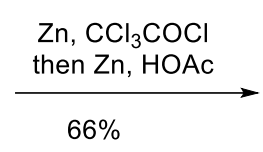

$66 \%$

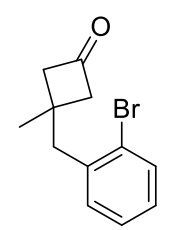

S15-1

Synthesis of compound S15-1. ${ }^{1}$ Trichloroacetyl chloride ( $0.58 \mathrm{~mL}, 5 \mathrm{mmol}, 2$ equiv) was added in one portion to a suspension of $\mathbf{S} 15$ (528 mg, $2.5 \mathrm{mmol}, 1$ equiv) and zinc dust (488 mg, $7.5 \mathrm{mmol}, 3$ equiv) in $\mathrm{Et}_{2} \mathrm{O}(25 \mathrm{~mL}$ ) at room temperature. The reaction mixture was stirred under sonication for $2 \mathrm{~h}$. Then the reaction was diluted with ether and filtered through a pad of celite. The residue was filtered again through a pad of silica gel and the filtrate was concentrated under reduced pressure to give a yellow oil, which was used directly without further purification. Zinc dust ( $488 \mathrm{mg}, 7.5 \mathrm{mmol}, 3$ equiv) was added to a solution of the yellow oil in acetic acid ( $20 \mathrm{~mL}$ ) at room temperature. After stirred at $70{ }^{\circ} \mathrm{C}$ overnight, the reaction mixture was cooled to room temperature and concentrated under reduced pressure to eliminate most acetic acid. The residue was dissolved in EtOAc $(50 \mathrm{~mL})$ and the organic phase was washed with $\mathrm{H}_{2} \mathrm{O}$, saturated $\mathrm{NaHCO}_{3}$ aqueous solution and brine, dried with $\mathrm{Na}_{2} \mathrm{SO}_{4}$ and concentrated under reduced pressure. The residue was purified by column chromatography on silica gel to give $415 \mathrm{mg}$ of compound S15-1 in 66\% yield as a colorless oil.

$\mathrm{Rf}=0.26$, Hexane/ EtOAc 10:1.

${ }^{1} \mathrm{H}$ NMR (400 MHz, Chloroform-d) $\delta 7.61$ (dd, J = 8.0, $1.3 \mathrm{~Hz}, 1 \mathrm{H}$ ), $7.33-7.24(\mathrm{~m}, 2 \mathrm{H}), 7.14$ (ddd, J = 7.9, 6.9, $2.2 \mathrm{~Hz}$, $1 \mathrm{H}), 3.17(\mathrm{~s}, 2 \mathrm{H}), 3.15-3.07(\mathrm{~m}, 2 \mathrm{H}), 2.74-2.66(\mathrm{~m}, 2 \mathrm{H}), 1.41(\mathrm{~s}, 3 \mathrm{H})$.

${ }^{13} \mathrm{C}$ NMR (101 MHz, CDCl$)$ ) $\delta$ 207.3, 138.1, 133.3, 131.3, 128.3, 127.3, 125.6, 57.2, 44.7, 30.4, 26.9.

HRMS ESI Calcd for $\mathrm{C}_{12} \mathrm{H}_{14} \mathrm{BrO}[\mathrm{M}+\mathrm{H}]^{+}:$253.0223, Found: 253.0224.

IR (KBr): 2955, 2919, 2867, 1781, 1469, 1439, 1379, 1253, 1138, $1026 \mathrm{~cm}^{-1}$.

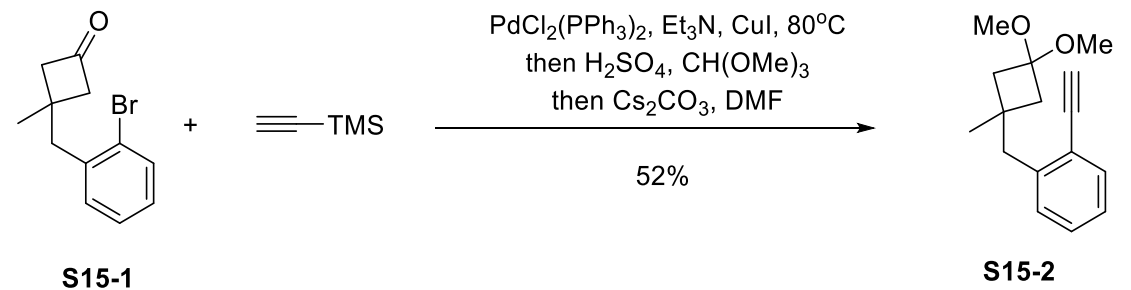

Synthesis of compound $\mathbf{S 1 5 - 2 ^ { 1 2 }}$. Trimethylsilylacetylene (366 mg, $3.73 \mathrm{mmol}, 1.2$ equiv) was added to a solution of compound S15-1 (787 mg, 3.1 mmol, 1 equiv), $\mathrm{PdCl}_{2}\left(\mathrm{PPh}_{3}\right)_{2}$ (43.5mg, $0.062 \mathrm{mmol}, 2 \mathrm{~mol} \%$ ) and Cul (5.9 mg, 0.031 $\mathrm{mmol}, 1 \mathrm{~mol} \%)$ in degassed $\mathrm{Et}_{3} \mathrm{~N}(9 \mathrm{~mL})$ at room temperature. After stirred at $80^{\circ} \mathrm{C}$ for overnight, the reaction mixture was filtered and washed with EtOAc. The filtrate was concentrated under reduced pressure to give a yellow oil, which was dissolved in triethyl orthoformate $(1.1 \mathrm{~mL}, 9.3 \mathrm{mmol}, 3$ equiv), and conc. sulfuric acid ( $16 \mathrm{mg}, 0.15 \mathrm{mmol}, 5$ mol\%) was added to the solution at room temperature. After stirred at room temperature for $3 \mathrm{~h}$, the mixture was diluted with water, extracted with $\mathrm{Et}_{2} \mathrm{O}$, dried with $\mathrm{Na}_{2} \mathrm{SO}_{4}$ and concentrated under reduced pressure to give the 
residue. $\mathrm{Cs}_{2} \mathrm{CO}_{3}(2.1 \mathrm{~g}, 6.2 \mathrm{mmol}, 2$ equiv) was added to a solution of the above residue in $\mathrm{DMF}(7 \mathrm{~mL})$ at room temperature. After stirred at room temperature for overnight, the reaction mixture was quenched by adding saturated $\mathrm{NH}_{4} \mathrm{Cl}$ aqueous solution. The reaction mixture was extracted with $\mathrm{Et}_{2} \mathrm{O}$, and the organic phase was washed with $\mathrm{H}_{2} \mathrm{O}$, saturated $\mathrm{NaHCO}_{3}$ aqueous solution and brine, dried with $\mathrm{Na}_{2} \mathrm{SO}_{4}$ and concentrated under reduced pressure. The residue was purified by column chromatography on silica gel to give $393 \mathrm{mg}$ of compound $\mathbf{5 1 5 - 2}$ in $52 \%$ yield as a yellow oil.

$\mathrm{Rf}=0.36$, Hexane/ EtOAc 10:1.

${ }^{1} \mathrm{H}$ NMR (400 MHz, Chloroform-d) $\delta 7.51$ (dd, $\left.J=7.6,1.5 \mathrm{~Hz}, 1 \mathrm{H}\right), 7.29(\mathrm{td}, J=7.5,1.5 \mathrm{~Hz}, 1 \mathrm{H}), 7.24-7.16(\mathrm{~m}, 2 \mathrm{H})$, $3.25(\mathrm{~s}, 1 \mathrm{H}), 3.16(\mathrm{~s}, 3 \mathrm{H}), 3.14(\mathrm{~s}, 3 \mathrm{H}), 3.03(\mathrm{~s}, 2 \mathrm{H}), 2.29-2.23(\mathrm{~m}, 2 \mathrm{H}), 1.99-1.91(\mathrm{~m}, 2 \mathrm{H}), 1.19(\mathrm{~s}, 3 \mathrm{H})$.

${ }^{13} \mathrm{C} \mathrm{NMR}$ (101 MHz, CDCl $)$ ) $142.1,133.1,129.8,128.4,125.9,122.7,99.1,83.4,80.5,48.12,48.10,45.3,43.0,30.6$, 26.7.

HRMS ESI Calcd for $\mathrm{C}_{16} \mathrm{H}_{21} \mathrm{O}_{2}[\mathrm{M}+\mathrm{H}]^{+}: 245.1536$, Found: 245.1536 .

IR (KBr): 3294, 2936, 2828, 1446, 1274, 110, 1102, $1041 \mathrm{~cm}^{-1}$.

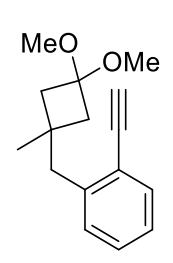

S15-2

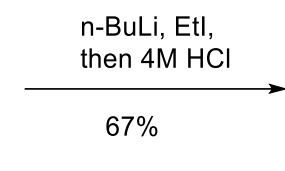

$67 \%$

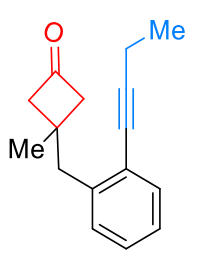

1s

Synthesis of substrate 1s. $n B$ BuLi $(0.5 \mathrm{~mL}, 2.5 \mathrm{M}$ in hexane, $1.2 \mathrm{mmol}, 1.2$ equiv) was added to a solution of compound S15-2 (244 mg, 1 mmol, 1 equiv) and HMPA (0.45 mL, $2.6 \mathrm{mmol}, 2.6$ equiv) in THF ( $2 \mathrm{~mL})$ at $-78^{\circ} \mathrm{C}$. After stirred at $78^{\circ} \mathrm{C}$ for $1 \mathrm{~h}$, Etl $\left(0.1 \mathrm{~mL}, 1.2 \mathrm{mmol}, 1.2\right.$ equiv) was added to the reaction mixture. After stirred at $-78^{\circ} \mathrm{C}$ for $0.5 \mathrm{~h}$, the reaction mixture was warmed to room temperature and stirred at room temperature for overnight. Then the reaction mixture was cooled to $0{ }^{\circ} \mathrm{C}$, and $4 \mathrm{M} \mathrm{HCl}(4 \mathrm{~mL})$ and acetone $(4 \mathrm{~mL})$ were successively added to the reaction mixture. After stirred at room temperature for $2 \mathrm{~h}$, the reaction mixture was extracted with Et ${ }_{2} \mathrm{O}$, and the organic phase was washed with $\mathrm{H}_{2} \mathrm{O}$, saturated $\mathrm{NaHCO}_{3}$ aqueous solution and brine, dried with $\mathrm{Na}_{2} \mathrm{SO}_{4}$ and concentrated under reduced pressure. The residue was purified by column chromatography on silica gel to give $120 \mathrm{mg}$ of compound $1 \mathrm{~s}$ in $67 \%$ yield as a colorless oil.

$\mathrm{Rf}=0.23$, Hexane/ EtOAc 20:1.

${ }^{1} \mathrm{H}$ NMR (400 MHz, Chloroform-d) $\delta 7.46-7.41(\mathrm{~m}, 1 \mathrm{H}), 7.25-7.15(\mathrm{~m}, 3 \mathrm{H}), 3.19-3.10(\mathrm{~m}, 4 \mathrm{H}), 2.69-2.60(\mathrm{~m}, 2 \mathrm{H})$, $2.45(\mathrm{q}, J=7.5 \mathrm{~Hz}, 2 \mathrm{H}), 1.38(\mathrm{~s}, 3 \mathrm{H}), 1.24(\mathrm{t}, J=7.5 \mathrm{~Hz}, 3 \mathrm{H})$.

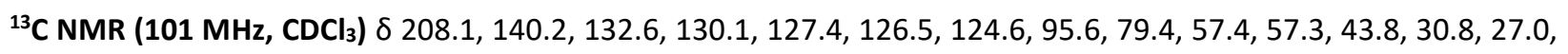
$13.9,13.1$.

HRMS ESI Calcd for $\mathrm{C}_{16} \mathrm{H}_{29} \mathrm{O}[\mathrm{M}+\mathrm{H}]^{+}:$227.1430, Found: 227.1433.

IR (KBr): 2973, 2916, 2869, 2233, 1780, 1484, 1448, 1378, 1319, 1256, 1137, $1072 \mathrm{~cm}^{-1}$. 


\section{General procedure D.}

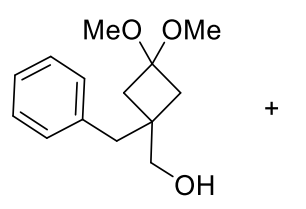

S23
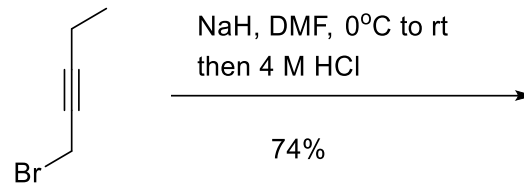

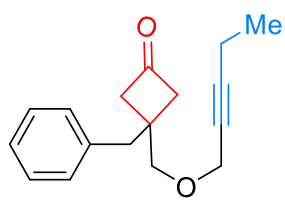

$1 \mathrm{ab}$

Synthesis of substrate 1ab. $\mathrm{NaH}(1.2 \mathrm{~g}, 30 \mathrm{mmol}, 1.5$ equiv) was added to a solution of the corresponding alcohol $\mathrm{S}^{13} 3^{13}\left(4.44 \mathrm{~g}, 20 \mathrm{mmol}, 1\right.$ equiv) in DMF $(20 \mathrm{~mL})$ at $0^{\circ} \mathrm{C}$. After stirred at $0^{\circ} \mathrm{C}$ for $20 \mathrm{~min}, 1$-bromo-2-pentyne $(3.23 \mathrm{~g}$, $22 \mathrm{mmol}, 1.1$ equiv) was added to the reaction mixture. The reaction mixture was warmed to room temperature and stirred for overnight. Then $4 \mathrm{M} \mathrm{HCl}(40 \mathrm{~mL})$ and acetone $(40 \mathrm{~mL})$ were successively added to the reaction mixture at $0^{\circ} \mathrm{C}$. After stirred at room temperature for $2 \mathrm{~h}$, the reaction mixture was extracted with $\mathrm{Et}_{2} \mathrm{O}$, and the organic phase was washed with $\mathrm{H}_{2} \mathrm{O}$, saturated $\mathrm{NaHCO}_{3}$ aqueous solution and brine, dried with $\mathrm{Na}_{2} \mathrm{SO}_{4}$ and concentrated under reduced pressure. The residue was purified by column chromatography on silica gel to give $3.8 \mathrm{~g}$ of compound $1 \mathrm{ab}$ in $73 \%$ yield as a colorless oil.

$\mathrm{Rf}=0.38$, Hexane/ EtOAc 10:1.

${ }^{1} \mathrm{H}$ NMR (400 MHz, Chloroform-d) $\delta 7.36-7.29(\mathrm{~m}, 2 \mathrm{H}), 7.29-7.25(\mathrm{~m}, 1 \mathrm{H}), 7.25-7.20(\mathrm{~m}, 2 \mathrm{H}), 4.19(\mathrm{t}, \mathrm{J}=2.2 \mathrm{~Hz}$, $2 \mathrm{H}), 3.46(\mathrm{~s}, 2 \mathrm{H}), 2.99(\mathrm{~s}, 2 \mathrm{H}), 2.97-2.82(\mathrm{~m}, 4 \mathrm{H}), 2.24$ (qt, $J=7.5,2.1 \mathrm{~Hz}, 2 \mathrm{H}), 1.15(\mathrm{t}, J=7.5 \mathrm{~Hz}, 3 \mathrm{H})$.

${ }^{13} \mathrm{C}$ NMR $\left(101 \mathrm{MHz}, \mathrm{CDCl}_{3}\right) \delta$ 206.8, 137.9, 129.9, 128.4, 126.6, 88.7, 75.0, 73.3, 59.0, 53.9, 41.6, 33.8, $13.8,12.4$.

HRMS ESI Calcd for $\mathrm{C}_{17} \mathrm{H}_{21} \mathrm{O}_{2}[\mathrm{M}+\mathrm{H}]^{+}:$257.1536, Found: 257.1536.

IR (KBr): 3062, 3028, 2977, 2919, 2236, 1780, 1716, 1496, 1455, 1377, 1260, 1175, 1089, $1022 \mathrm{~cm}^{-1}$.

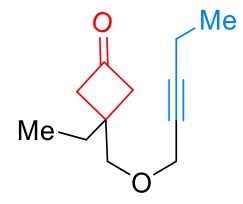

$1 z$

Synthesis of substrate 1z. Following the general procedure D of preparation of substrate 1ab, $330 \mathrm{mg}$ of substrate $1 z$ was synthesized from corresponding alcohol $(2 \mathrm{mmol})$ and 1-bromo-2-pentyne $(2.4 \mathrm{mmol})$ in $85 \%$ yield as a colorless oil.

$\mathrm{Rf}=0.5$, Hexane/ EtOAc 10:1.

${ }^{1} \mathrm{H}$ NMR (400 MHz, Chloroform-d) $\delta 4.17(\mathrm{t}, J=2.2 \mathrm{~Hz}, 2 \mathrm{H}), 3.55(\mathrm{~s}, 2 \mathrm{H}), 3.01-2.90(\mathrm{~m}, 2 \mathrm{H}), 2.76-2.66(\mathrm{~m}, 2 \mathrm{H}), 2.24$ (qt, $J=7.5,2.2 \mathrm{~Hz}, 2 \mathrm{H}), 1.72(\mathrm{q}, J=7.4 \mathrm{~Hz}, 2 \mathrm{H}), 1.15(\mathrm{t}, J=7.5 \mathrm{~Hz}, 3 \mathrm{H}), 0.94(\mathrm{t}, J=7.4 \mathrm{~Hz}, 3 \mathrm{H})$.

${ }^{13} \mathrm{C}$ NMR $\left(101 \mathrm{MHz}, \mathrm{CDCl}_{3}\right) \delta 207.8,88.5,75.2,73.1,59.0,53.7,33.3,29.1,13.8,12.4,9.2$.

HRMS ESI Calcd for $\mathrm{C}_{12} \mathrm{H}_{19} \mathrm{O}_{2}[\mathrm{M}+\mathrm{H}]^{+}:$195.1380, Found: 195.1374.

IR (KBr): 2971, 2939, 2881, 2236, 1780, 1712, 1461, 1380, 1245, 1183, 1090, $1020 \mathrm{~cm}^{-1}$. 


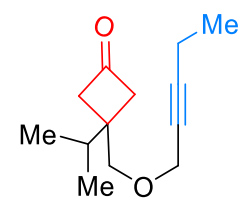

1aa

Synthesis of substrate 1aa. Following the general procedure D of preparation of substrate 1aa, $660 \mathrm{mg}$ of substrate 1aa was synthesized from corresponding alcohol $(4 \mathrm{mmol})$ and 1-bromo-2-pentyne $(4.8 \mathrm{mmol})$ in $80 \%$ yield as a colorless oil.

$\mathrm{Rf}=0.5$, Hexane/ EtOAc 10:1.

${ }^{1} \mathrm{H}$ NMR (400 MHz, Chloroform-d) $\delta 4.14(\mathrm{q}, J=1.9 \mathrm{~Hz}, 2 \mathrm{H}), 3.59(\mathrm{~d}, J=1.3 \mathrm{~Hz}, 2 \mathrm{H}), 2.99-2.88(\mathrm{~m}, 2 \mathrm{H}), 2.84-2.72$ (m, 2H), 2.23 (qt, $J=7.5,2.1 \mathrm{~Hz}, 2 \mathrm{H}), 1.97(\mathrm{pd}, J=6.9,1.3 \mathrm{~Hz}, 1 \mathrm{H}), 1.14(\mathrm{td}, J=7.5,1.3 \mathrm{~Hz}, 3 \mathrm{H}), 0.98(\mathrm{dd}, J=6.9,1.3$ $\mathrm{Hz}, 6 \mathrm{H})$.

${ }^{13} \mathrm{C}$ NMR $\left(101 \mathrm{MHz}, \mathrm{CDCl}_{3}\right) \delta 207.8,88.5,77.3,77.0,76.7,75.2,72.0,59.0,52.2,36.6,33.3,17.8,13.8,12.4$.

HRMS ESI Calcd for $\mathrm{C}_{13} \mathrm{H}_{21} \mathrm{O}_{2}[\mathrm{M}+\mathrm{H}]^{+}:$209.1536, Found: 209.1535 .

IR (KBr): 2964, 2878, 2228, 1782, 1735, 1465, 1376, 1319, 1264, 1177, 1136, 1092, $1028 \mathrm{~cm}^{-1}$.

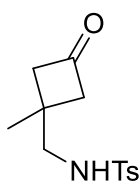

s1

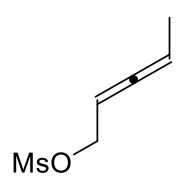

S15

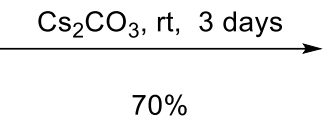

$70 \%$

$70 \%$

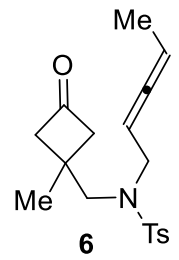

6 Ts

Synthesis of compound 6. $\mathrm{Cs}_{2} \mathrm{CO}_{3}(489 \mathrm{mg}, 1.5 \mathrm{mmol}, 1.5$ equiv) was added to a solution of compound $\mathrm{S} 1$ (267 mg, $1 \mathrm{mmol}, 1$ equiv) and $\mathbf{S} 15$ (194.4 mg, $1.2 \mathrm{mmol}, 1.2$ equiv) in DMF (2 mL) at room temperature. After stirred at room temperature for 3 days, the reaction mixture was quenched by adding saturated $\mathrm{NH}_{4} \mathrm{Cl}$ aqueous solution. The reaction mixture was extracted with $\mathrm{Et}_{2} \mathrm{O}$, and the organic phase was washed with $\mathrm{H}_{2} \mathrm{O}$, saturated $\mathrm{NaHCO}_{3}$ aqueous solution and brine, dried with $\mathrm{Na}_{2} \mathrm{SO}_{4}$ and concentrated under reduced pressure. The residue was purified by column chromatography on silica gel to give $230 \mathrm{mg}$ of compound 6 in $70 \%$ yield as white solid.

$\mathrm{Rf}=0.35$, Hexane/ EtOAc 4:1.

$\mathrm{Mp}: 71.9-73.3^{\circ} \mathrm{C}$

${ }^{1} \mathrm{H}$ NMR (400 MHz, Chloroform-d) $\delta 7.75-7.68(\mathrm{~m}, 2 \mathrm{H}), 7.36-7.29(\mathrm{~m}, 2 \mathrm{H}), 5.16-5.00(\mathrm{~m}, 1 \mathrm{H}), 4.70-4.54(\mathrm{~m}, 1 \mathrm{H})$, $3.96-3.82(\mathrm{~m}, 2 \mathrm{H}), 3.49(\mathrm{~s}, 2 \mathrm{H}), 3.19-3.01(\mathrm{~m}, 2 \mathrm{H}), 2.79-2.68(\mathrm{~m}, 2 \mathrm{H}), 2.44(\mathrm{~s}, 3 \mathrm{H}), 1.64-1.57(\mathrm{~m}, 3 \mathrm{H}), 1.38(\mathrm{~s}$, $3 \mathrm{H})$.

${ }^{13} \mathrm{C}$ NMR (101 MHz, CDCl$)$ ) $\delta$ 206.4, 206.3, 143.5, 137.2, 129.8, 127.2, 87.4, 85.2, 57.3, 54.4, 47.7, 29.9, 24.5, 21.5, 13.9.

HRMS ESI Calcd for $\mathrm{C}_{18} \mathrm{H}_{24} \mathrm{NO}_{3} \mathrm{~S}[\mathrm{M}+\mathrm{H}]^{+}: 334.1471$, Found: 334.1476. 
IR (KBr): 2959, 2926, 2869, 1965, 1782, 1598, 1494, 1450, 1344, 1159, 1091, $912 \mathrm{~cm}^{-1}$.

Synthetic route of substrate $1 \mathrm{~h}-\mathrm{d}_{5}$.

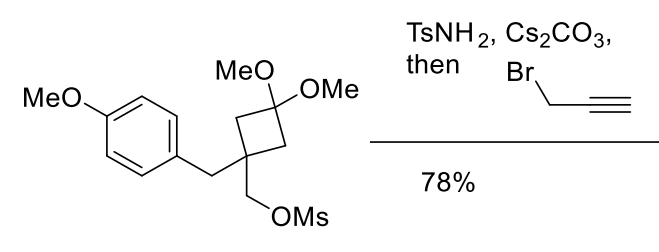

S24

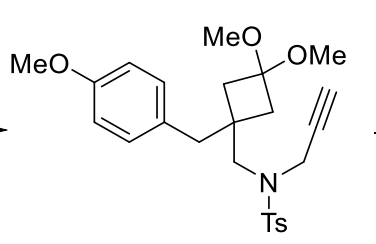

S25

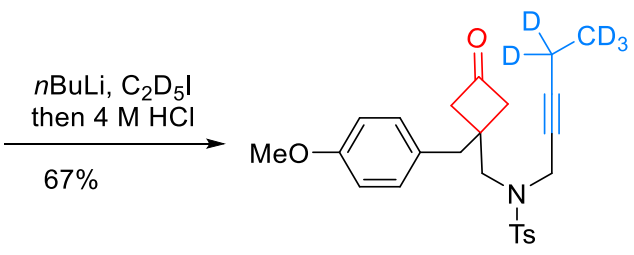

$1 \mathrm{~h}-\mathrm{d}_{5}$

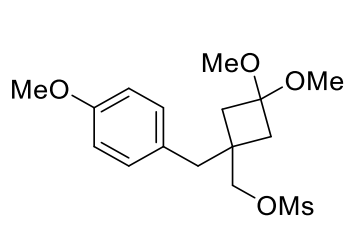

S24

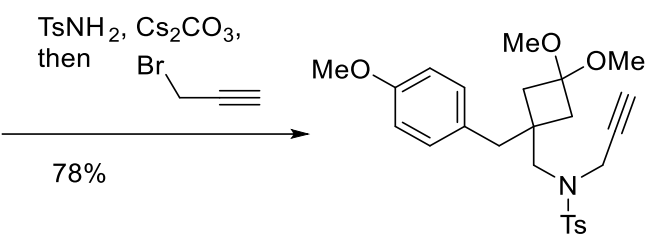

S25

Synthesis of compound $\mathbf{S 2 5} . \mathrm{Cs}_{2} \mathrm{CO}_{3}(2.04 \mathrm{~g}, 6.26 \mathrm{mmol}, 2$ equiv) was added to a solution of compound $\mathbf{S 2 4}$ (1.08 g, $3.13 \mathrm{mmol}, 1$ equiv) and $\mathrm{TsNH}_{2}$ ( $0.56 \mathrm{~g}, 3.29 \mathrm{mmol}, 1.05$ equiv) in DMF ( $6 \mathrm{~mL}$ ) at room temperature. After stirred at $80^{\circ} \mathrm{C}$ for $36 \mathrm{~h}$, the reaction mixture was cooled to room temperature. $\mathrm{Cs}_{2} \mathrm{CO}_{3}(2.04 \mathrm{~g}, 6.26 \mathrm{mmol}, 2$ equiv) and propargyl bromide (932 $\mathrm{mg}, 6.26 \mathrm{mmol}, 2$ equiv) were successively added to the reaction mixture. After stirred at $50^{\circ} \mathrm{C}$ for $12 \mathrm{~h}$, the reaction mixture was cooled to room temperature, quenched by adding saturated $\mathrm{NH}_{4} \mathrm{Cl}$ aqueous solution. The reaction mixture was extracted with $\mathrm{Et}_{2} \mathrm{O}$, and the organic phase was washed with $\mathrm{H}_{2} \mathrm{O}$, saturated $\mathrm{NaHCO}_{3}$ aqueous solution and brine, dried with $\mathrm{Na}_{2} \mathrm{SO}_{4}$ and concentrated under reduced pressure. The residue was purified by column chromatography on silica gel to give $1.12 \mathrm{~g}$ of compound $\mathbf{S 2 5}$ in $78 \%$ yield as a white solid.

$\mathrm{Rf}=0.3$, Hexane/ EtOAc 4:1.

Mp: $130.9-132.3^{\circ} \mathrm{C}$.

${ }^{1} \mathrm{H}$ NMR (400 MHz, Chloroform-d) $\delta 7.75-7.67(\mathrm{~m}, 2 \mathrm{H}), 7.29(\mathrm{~d}, J=7.9 \mathrm{~Hz}, 2 \mathrm{H}), 7.26-7.19(\mathrm{~m}, 2 \mathrm{H}), 6.91-6.82(\mathrm{~m}$, $2 \mathrm{H}), 4.08(\mathrm{~d}, J=2.5 \mathrm{~Hz}, 2 \mathrm{H}), 3.80(\mathrm{~s}, 3 \mathrm{H}), 3.30(\mathrm{~s}, 2 \mathrm{H}), 3.11(\mathrm{~s}, 3 \mathrm{H}), 3.10(\mathrm{~s}, 3 \mathrm{H}), 2.86(\mathrm{~s}, 2 \mathrm{H}), 2.43(\mathrm{~s}, 3 \mathrm{H}), 2.18(\mathrm{~d}, J=$ $13.1 \mathrm{~Hz}, 2 \mathrm{H}), 2.12-2.05(\mathrm{~m}, 2 \mathrm{H}), 1.97(\mathrm{t}, J=2.4 \mathrm{~Hz}, 1 \mathrm{H})$.

${ }^{13} \mathrm{C}$ NMR (101 MHz, CDCl $)$ ) 158.1, 143.6, 135.3, 131.6, 130.0, 129.3, 128.1, 113.4, 99.1, 76.2, 75.0, 55.2, 53.0, 48.3, 48.2, 40.9, 39.8, 37.3, 32.9, 21.5.

HRMS ESI Calcd for $\mathrm{C}_{25} \mathrm{H}_{31} \mathrm{NNaO}_{5} \mathrm{~S}[\mathrm{M}+\mathrm{Na}]^{+}: 480.1815$, Found: 480.1812

IR (KBr): 3241, 2991, 2939, 2831, 1611, 1513, 1447, 1349, 1277, 1248, 1181, 1163, $1038 \mathrm{~cm}^{-1}$. 


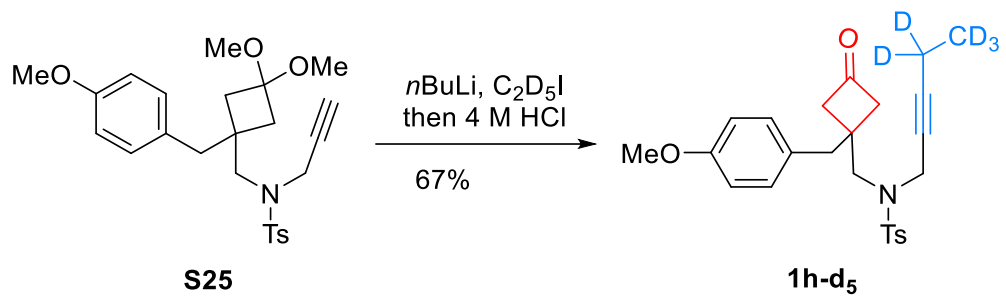

Synthesis of substrate $\mathbf{1 h}-\mathbf{d}_{5}$. $n B$ BuLi $(0.52 \mathrm{~mL}, 2.5 \mathrm{M}$ in hexane, $1.3 \mathrm{mmol}, 1.3$ equiv) was added to a solution of compound S25 (457 mg, $1 \mathrm{mmol}, 1$ equiv) and HMPA (0.45 mL, $2.6 \mathrm{mmol}, 2.6$ equiv) in THF (5 mL) at $-78^{\circ} \mathrm{C}$. After stirred at $-78^{\circ} \mathrm{C}$ for $1 \mathrm{~h}, \mathrm{C}_{2} \mathrm{D}_{5} \mathrm{l}(209.3 \mathrm{mg}, 1.3 \mathrm{mmol}, 1.3$ equiv) was added to the reaction mixture. After stirred at $78^{\circ} \mathrm{C}$ for $0.5 \mathrm{~h}$, the reaction mixture was warmed to room temperature and stirred at room temperature for overnight. Then the reaction mixture was cooled to $0{ }^{\circ} \mathrm{C}$, and $4 \mathrm{M} \mathrm{HCl}(10 \mathrm{~mL})$ and acetone $(10 \mathrm{~mL})$ were successively added to the reaction mixture. After stirred at room temperature for another $2 \mathrm{~h}$, the reaction mixture was extracted with $\mathrm{Et}_{2} \mathrm{O}$, and the organic phase was washed with $\mathrm{H}_{2} \mathrm{O}$, saturated $\mathrm{NaHCO}_{3}$ aqueous solution and brine, dried with $\mathrm{Na}_{2} \mathrm{SO}_{4}$ and concentrated under reduced pressure. The residue was purified by column chromatography on silica gel to give $300 \mathrm{mg}$ of compound $\mathbf{1} \mathbf{h}-\mathbf{d}_{5}$ in $67 \%$ yield as a white solid.

$\mathrm{Rf}=0.22$, Hexane/ EtOAc 4:1.

Mp: $113.8-115.1^{\circ} \mathrm{C}$

${ }^{1} \mathrm{H}$ NMR (400 MHz, Chloroform-d) $\delta 7.76-7.68(\mathrm{~m}, 2 \mathrm{H}), 7.35-7.28(\mathrm{~m}, 2 \mathrm{H}), 7.22-7.14(\mathrm{~m}, 2 \mathrm{H}), 6.90-6.82(\mathrm{~m}, 2 \mathrm{H})$, $4.12(\mathrm{~s}, 2 \mathrm{H}), 3.80(\mathrm{~s}, 3 \mathrm{H}), 3.47(\mathrm{~s}, 2 \mathrm{H}), 3.09-2.91(\mathrm{~m}, 6 \mathrm{H}), 2.43(\mathrm{~s}, 3 \mathrm{H})$.

${ }^{2} \mathrm{H}$ NMR (77 MHz, Chloroform-d) $\delta 1.83$ (brs, 2H), 0.82 (brs, 3H).

${ }^{13} \mathrm{C}$ NMR $\left(101 \mathrm{MHz}, \mathrm{CDCl}_{3}\right) \delta$ 205.6, 158.5, 143.6, 135.4, 131.5, 129.4, 128.8, 128.0, 113.8, 89.3, 71.1, 55.2, 54.4, 52.7, $40.6,38.1,33.2,21.5$.

HRMS ESI Calcd for $\mathrm{C}_{25} \mathrm{H}_{25} \mathrm{D}_{5} \mathrm{NO}_{4} \mathrm{~S}[\mathrm{M}+\mathrm{H}]^{+}$: 445.2204, Found: 445.2206.

IR (KBr): 2919, 1782, 1611, 1513, 1443, 1347, 1250, 1180, 1162, 1117, 1091, $1035 \mathrm{~cm}^{-1}$. 
Crystal data and structure refinement for enantioenriched $2 \mathrm{a}$

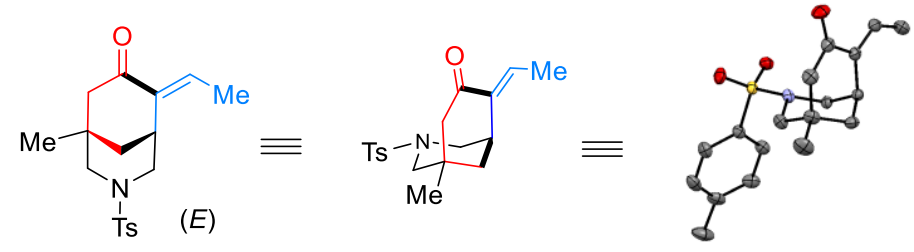

$2 a$
X-ray structure $\quad$ CCDC 2003640

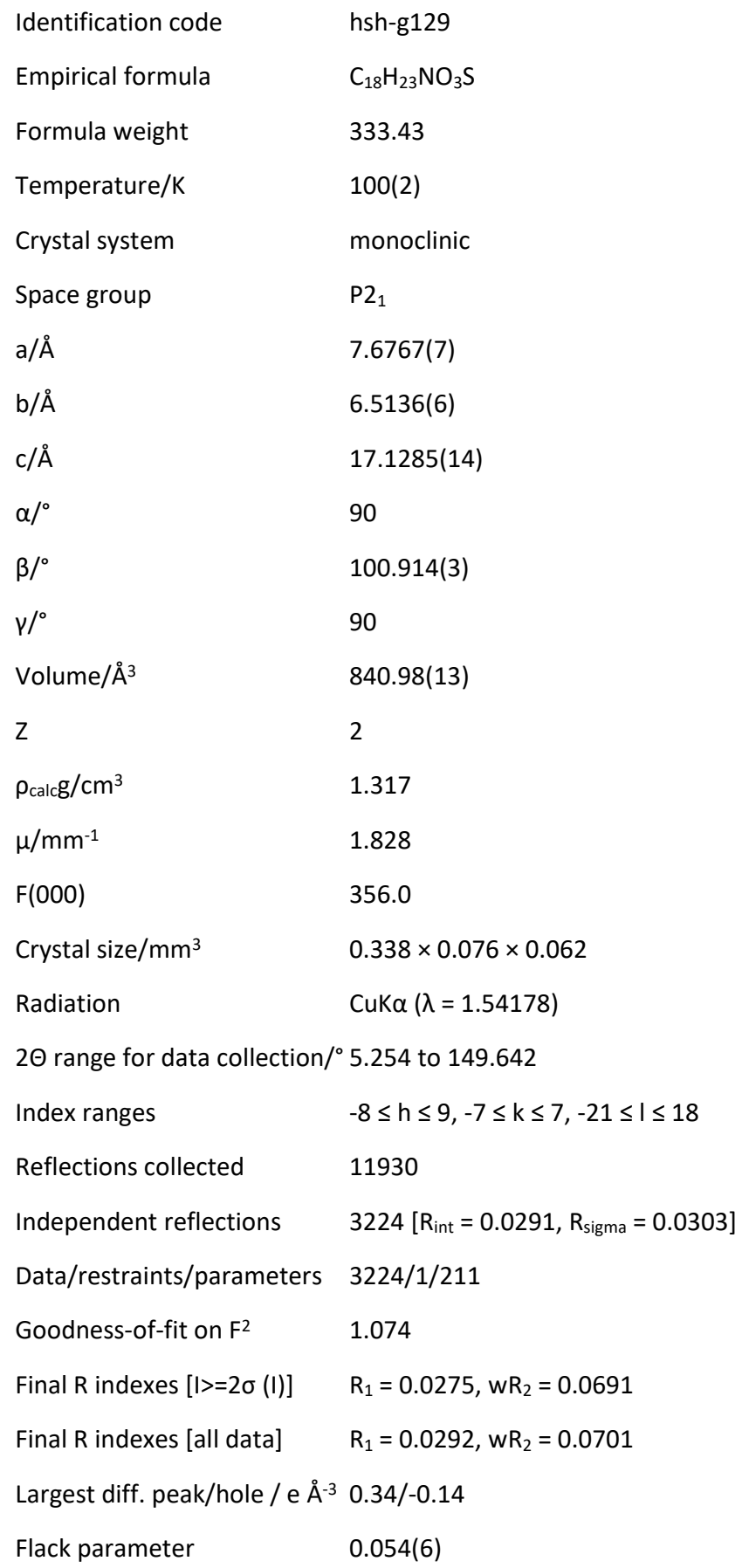

$\gamma /{ }^{\circ}$ 90

Volume $/ \AA^{3}$

$840.98(13)$

Z

2

$\rho_{\text {calc }} \mathrm{g} / \mathrm{cm}^{3} \quad 1.317$

$\mu / \mathrm{mm}^{-1} \quad 1.828$

$\mathrm{F}(000) \quad 356.0$

Crystal size $/ \mathrm{mm}^{3} \quad 0.338 \times 0.076 \times 0.062$

Radiation $\quad$ CuK $\alpha(\lambda=1.54178)$

$2 \Theta$ range for data collection $/{ }^{\circ} 5.254$ to 149.642 


\section{Computational studies}

\section{Computational details}

All calculations were performed with Gaussian $16^{14}$. Geometry optimizations were performed with the B3LYP functional ${ }^{15}$ with a mixed basis set of LANL2DZ ${ }^{16}$ for Rh and 6-31G(d) for other atoms in gas phase. Frequency analysis was conducted at the same level of theory to verify the stationary points to be minima or saddle points and to obtain zero-point energy (ZPE) and thermal energy corrections at $298.15 \mathrm{~K}$. Single-point energy calculations on optimized geometries were performed with the M06-L functional ${ }^{17}$, with a mixed basis set of SDD ${ }^{18}$ for Rh and 6$311+\mathrm{G}(\mathrm{d}, \mathrm{p})$ for other atoms and the $\mathrm{SMD}^{19}$ solvation model with toluene as the solvent. Computed structures were illustrated using CYLView ${ }^{20}$.

\section{Isomers of the Rh-substrate complex and oxidative addition transition states}

Rh-substrate complex. Rh-substrate complexes with different coordination modes were carefully studied. Their structures and energies are summarized in Figure S1. IntA1 with alkyne and sulfonyl coordination is the most stable one. IntA2 is the reactive complex with carbonyl and C-C bond coordination that will lead to subsequent $\mathrm{C}-\mathrm{C}$ activation. IntA6 and IntA7 both have the Rh center coordinated by alkyne and carbonyl, albeit with different orientations.
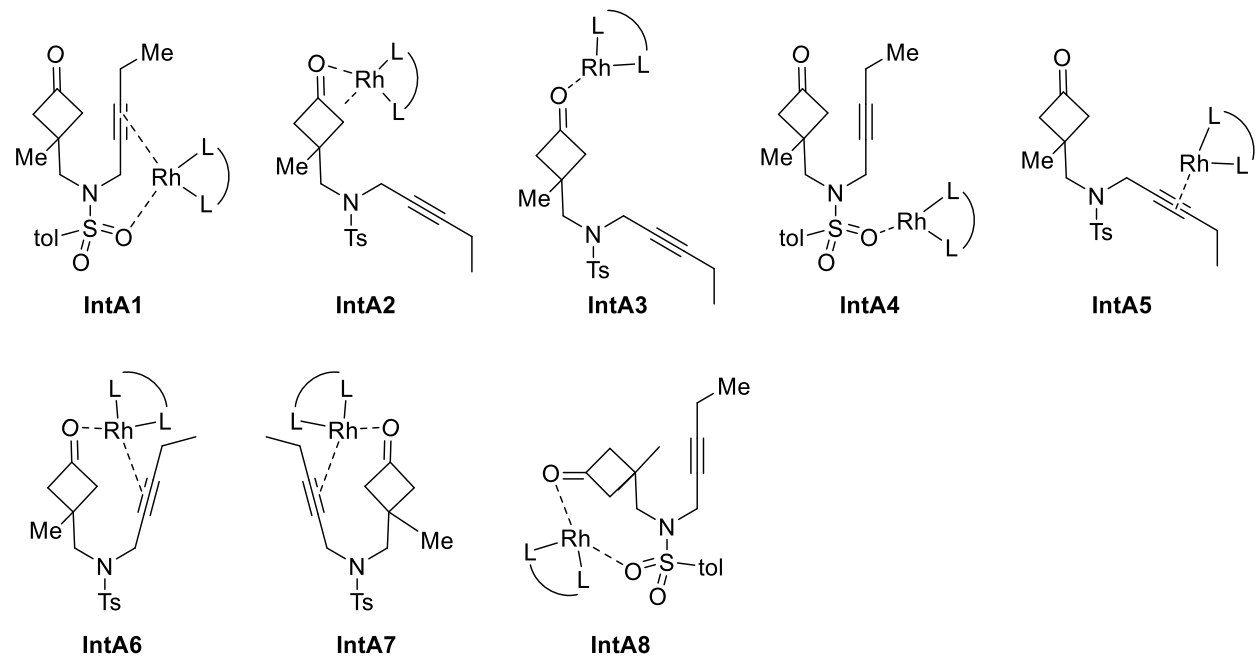

\begin{tabular}{cccc}
\hline Complex & Coordination & $\Delta H(\mathrm{kcal} / \mathrm{mol})$ & $\Delta G(\mathrm{kcal} / \mathrm{mol})$ \\
\hline IntA1 & Alkyne + sulfonyl & -36.4 & -19.3 \\
IntA2 & Carbonyl + C-C & -21.8 & -8.8 \\
IntA3 & Carbonyl & -14.7 & -3.3 \\
IntA4 & Sulfonyl & -21.4 & -9.7 \\
IntA5 & Alkyne & -29.2 & -16.1 \\
IntA6 & Alkyne + Carbonyl & -30.2 & -12.7 \\
IntA7 & Alkyne + Carbonyl & -28.2 & -10.4 \\
IntA8 & Carbonyl + Sulfonyl & -28.3 & -13.9 \\
\hline
\end{tabular}


Figure S1 Structures and energies of different Rh-substrate complex isomers. All energies are with respect to Rh- $(R)$ segphos and 1a.

$\mathrm{C}-\mathrm{C}$ activation transition states and enantioselectivity analysis. In the $\mathrm{C}-\mathrm{C}$ activation transition states, the $\mathrm{Rh}$ center could either remain open (TS1a) or be coordinated by the alkyne (TS1b), as shown in Figure S2. TS1a is favored by $2.7 \mathrm{kcal} / \mathrm{mol}$. In consideration of the fact that the $\mathrm{C}-\mathrm{C}$ activation is irreversible and the rate determining step of this transformation, the enantioselectivity is also controlled in this step. The transition states leading to the other enantiomer (TS1a-ee, TS1b-ee) were calculated. Compared with TS1a-ee, TS1a is slightly favored by 0.7 $\mathrm{kcal} / \mathrm{mol}$. In view of the high enantioselectivity observed, the energy difference is to some extent underestimated likely due to the use of $(R)$-sephos instead of $(R)$-DTBM-segphos as the ligand. TS1b, with a much compact structure, shows a large energy difference compared with TS1b-ee.
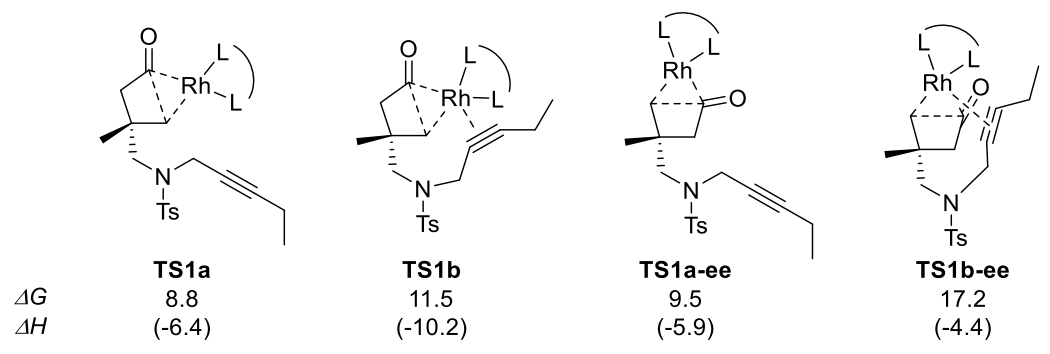

Figure S2 Structures and energies of different C-C activation transition states. All energies are with respect to Rh$(R)$-segphos and 1a.

\section{Isomers of the Rh-alkyne complex after $\mathrm{C}$ - $\mathrm{C}$ activation and migratory insertion transition states}
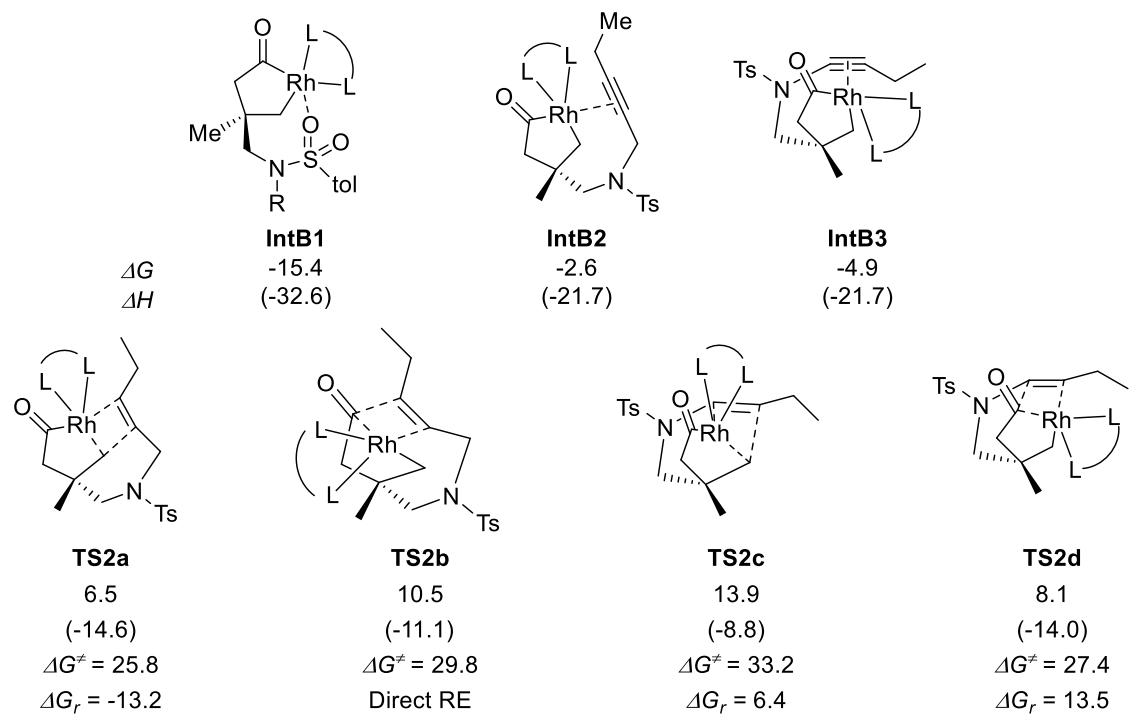

$$
\begin{gathered}
\text { TS2d } \\
8.1 \\
(-14.0) \\
\Delta G^{\neq}=27.4 \\
\Delta G_{r}=13.5
\end{gathered}
$$

Figure S3 Structures and energies of different Rh-alkyne complexes after C-C activation and migratory insertion transition states. $\Delta G$ and $\Delta H$ energies are with respect to Rh- $(R)$-segphos and 1a. $\Delta G^{\neq}$is with respect to IntA1. $\Delta G_{r}$ denotes the free energy change of the migration insertion step, therefore with regard to IntB1. 
As illustrated in Figure S3, IntB1 with sulfonyl group coordination is $12.8 \mathrm{kcal} / \mathrm{mol}$ more stable than IntB2 with alkyne coordination. In IntB3, which is $2.3 \mathrm{kcal} / \mathrm{mol}$ more stable than IntB2, the alkyne chain could rotate and coordinate with $\mathrm{Rh}$ center in a different orientation. Both IntB2 and IntB3 are reactive complexes and will lead to subsequent migratory insertion. For each complex, the alkyne could insert into either Rh-alkyl bond or Rh-carbonyl bond. Among all the four possible transition states, TS2a, derived from IntB2 with Rh-alkyl insertion, is expected to lead to the final product and also is kinetically favored. TS2b, with Rh-carbonyl insertion is more distorted, rendering the forming intermediate highly unstable. The following reductive elimination is barrierless and will finally lead to the same product as TS2a. In TS2c and TS2d, alkynes are approaching in the opposite direction and will give a different product. Although the activation barriers are comparable (especially for TS2d), these insertion reactions are significantly endergonic and therefore reversible, while the insertion with TS2a is exergonic by $13.2 \mathrm{kcal} / \mathrm{mol}$. As a result, the normal insertion product will be thermodynamically favored.

\section{Decarbonylation pathway analysis}

DFT calculations were also performed to study the decarbonylation side reactions. The energy profile is shown in Figure S4. The decarbonylation step (TS-deCO) has similar activation barrier $\left(\Delta G^{\neq}=25.4 \mathrm{kcal} / \mathrm{mol}\right.$ with respect to IntA1) compared with the migratory insertion. The resulting intermediate will undergo a fast reductive elimination to give cyclopropane product $\mathbf{2 a}$ '. The results indicate that the decarbonylation is a non-negligible side reaction, which is consistent with the experimental observations of the decarbonylation byproducts.

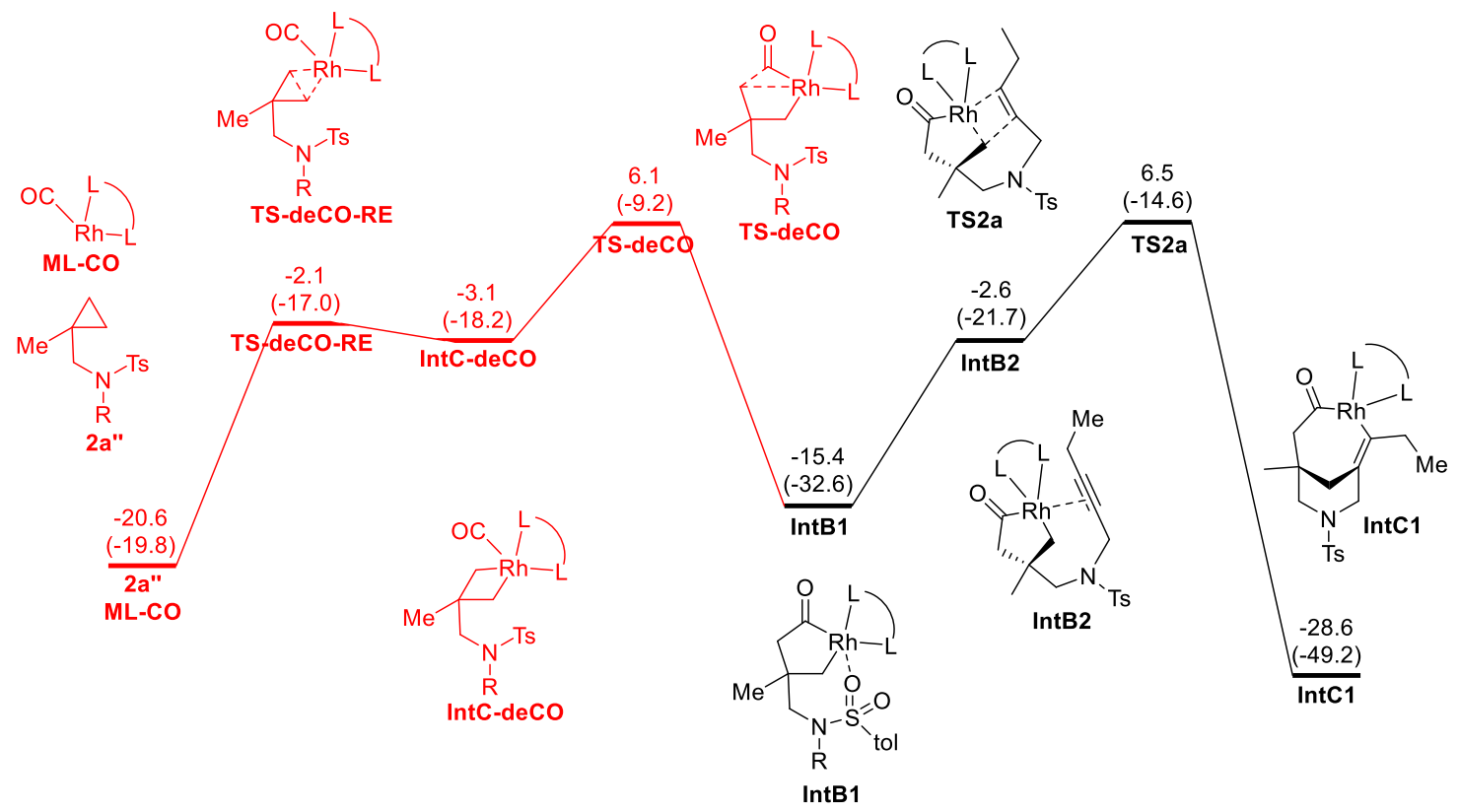

Figure S4 Energy profile for the decarbonylation pathway. All energies are with respect to Rh- $(R)$-segphos and 1a. 


\section{Cartesian coordinates of optimized structures}

ML

B3LYP SCF energy: $\quad 2557.79024534$ a.u.

B3LYP enthalpy: $\quad 2557.187746$ a.u.

B3LYP free energy: 2557.303705 a.u.

M06-L SCF energy in solution: $\quad 2559.23415640$ a.u.

M06-L enthalpy in solution: $\quad 2558.631657$ a.u.

M06-L free energy in solution: $\quad 2558.747616$ a.u.

Cartesian coordinates
$\begin{array}{llll}\text { ATOM } & \mathrm{X} & \mathrm{Y} & \mathrm{Z}\end{array}$
$\begin{array}{llll}\mathrm{P} & -1.633729 & -0.822082 & 0.133185\end{array}$
$\begin{array}{llll}\mathrm{P} & 1.633815 & -0.821937 & -0.133265\end{array}$
$\begin{array}{llll}\text { O } & -0.780144 & 3.721646 & -1.344365\end{array}$
$\begin{array}{llll}\text { O } & 0.269670 & 3.949211 & -3.396370\end{array}$
$\begin{array}{llll}\text { O } & 0.779761 & 3.721598 & 1.344725\end{array}$
$\begin{array}{llll}\text { O } & -0.270135 & 3.948902 & 3.396716\end{array}$
$\begin{array}{llll}\text { C } & 1.296645 & 0.672997 & -1.128422\end{array}$
C $\quad 0.340286 \quad 1.633883 \quad-0.665431$
$\begin{array}{llll}\text { C } & 0.093201 & 2.686486 & -1.532279\end{array}$
$\begin{array}{llll}\text { C } & 0.720308 & 2.825599 & -2.772809\end{array}$
$\begin{array}{llll}\text { C } & 1.647804 & 1.910991 & -3.222795\end{array}$
$\begin{array}{llll}\text { C } & 1.920503 & 0.825923 & -2.372376\end{array}$
$\begin{array}{llll}\text { C } & -0.657743 & 4.570090 & -2.495700\end{array}$
$\begin{array}{llll}\text { C } & -1.296740 & 0.672778 & 1.128488\end{array}$
$\begin{array}{llll}\text { C } & -1.920641 & 0.825536 & 2.372439\end{array}$
C $\quad-1.648076 \quad 1.910569 \quad 3.222945$
$\begin{array}{llll}\text { C } & -0.720666 & 2.825307 & 2.773046\end{array}$
$\begin{array}{llll}\text { C } & -0.093512 & 2.686356 & 1.532521\end{array}$
$\begin{array}{llll}\text { C } & -0.340474 & 1.633798 & 0.665585\end{array}$
$\begin{array}{llll}\text { C } & 0.657041 & 4.570072 & 2.496006\end{array}$ 


$$
\begin{aligned}
& \begin{array}{llll}
\text { C } & 2.337049 & -0.232389 & 1.453339
\end{array} \\
& \begin{array}{llll}
\text { C } & 2.175848 & -1.011257 & 2.609317
\end{array} \\
& \begin{array}{llll}
\text { C } & 3.089667 & 0.951906 & 1.517249
\end{array} \\
& \begin{array}{llll}
\text { C } & 2.967807 & -1.782710 & -0.950377
\end{array} \\
& \begin{array}{llll}
\text { C } & 4.292539 & -1.782846 & -0.482818
\end{array} \\
& \begin{array}{llll}
\text { C } & 2.622756 & -2.605928 & -2.041198
\end{array} \\
& \begin{array}{llll}
\text { C } & -2.337036 & -0.232435 & -1.453351
\end{array} \\
& \begin{array}{llll}
\text { C } & -3.089893 & 0.951721 & -1.517074
\end{array} \\
& \begin{array}{llll}
\text { C } & -2.175629 & -1.011056 & -2.609463
\end{array} \\
& \begin{array}{llll}
\text { C } & -2.622425 & -2.606155 & 2.041176
\end{array} \\
& \begin{array}{llll}
\text { C } & -2.967556 & -1.783143 & 0.950222
\end{array} \\
& \begin{array}{llll}
\text { C } & -4.292225 & -1.783702 & 0.482475
\end{array} \\
& \text { H } \quad 2.141384 \quad 2.022839 \quad-4.181337 \\
& \text { H } \quad 2.645889 \quad 0.089884 \quad-2.698792 \\
& \text { H } \quad-1.632449 \quad 4.663711 \quad-2.984017 \\
& \mathrm{H} \quad-0.270684 \quad 5.548382 \quad-2.190711 \\
& \begin{array}{llll}
\mathrm{H} & -2.645953 & 0.089388 & 2.698781
\end{array} \\
& \begin{array}{llll}
\mathrm{H} & -2.141684 & 2.022290 & 4.181487
\end{array} \\
& \mathrm{H} \quad 1.631710 \quad 4.664094 \quad 2.984311 \\
& \text { H } \quad 0.269611 \quad 5.548201 \quad 2.190950 \\
& \mathrm{H} \quad 1.583237 \quad-1.921676 \quad 2.563007 \\
& \text { H } \quad 3.213346 \quad 1.569783 \quad 0.632950 \\
& \text { H } \quad 4.573904 \quad-1.163552 \quad 0.362148 \\
& \text { H } \quad 1.598460 \quad-2.611591 \quad-2.408056 \\
& \text { H } \quad-3.213741 \quad 1.569403 \quad-0.632660 \\
& \text { H } \quad-1.582841 \quad-1.921367 \quad-2.563291 \\
& \mathrm{H} \quad-1.598187 \quad-2.611483 \quad 2.408192 \\
& \text { H } \quad-4.573653 \quad-1.164550 \quad-0.362572 \\
& \begin{array}{llll}
\text { Rh } & 0.000095 & -2.309953 & -0.000103
\end{array} \\
& \begin{array}{llll}
\text { C } & 3.676462 & 1.342001 & 2.722080
\end{array} \\
& \begin{array}{llll}
\mathrm{H} & 4.262759 & 2.255670 & 2.763684
\end{array} \\
& \begin{array}{llll}
\text { C } & 3.513766 & 0.560288 & 3.868545
\end{array} \\
& \begin{array}{llll}
\mathrm{H} & 3.970850 & 0.867614 & 4.804787
\end{array}
\end{aligned}
$$




$\begin{array}{lrrr}\mathrm{C} & 2.762033 & -0.615787 & 3.811673 \\ \mathrm{H} & 2.630983 & -1.223227 & 4.702447 \\ \mathrm{C} & 5.253135 & -2.580631 & -1.105078 \\ \mathrm{H} & 6.275930 & -2.571579 & -0.739871 \\ \mathrm{C} & 4.904523 & -3.389187 & -2.189862 \\ \mathrm{H} & 5.656406 & -4.010930 & -2.667048 \\ \mathrm{C} & 3.588701 & -3.401082 & -2.658269 \\ \mathrm{H} & 3.313605 & -4.029162 & -3.500521 \\ \mathrm{C} & -2.761838 & -0.615477 & -3.811774 \\ \mathrm{H} & -2.630624 & -1.222727 & -4.702654 \\ \mathrm{C} & -3.513804 & 0.560455 & -3.868461 \\ \mathrm{H} & -3.970911 & 0.867865 & -4.804665 \\ \mathrm{C} & -3.676717 & 1.341920 & -2.721854 \\ \mathrm{H} & -4.263207 & 2.255472 & -2.763314 \\ \mathrm{C} & -5.252673 & -2.581730 & 1.104652 \\ \mathrm{H} & -6.275418 & -2.573010 & 0.739297 \\ \mathrm{C} & -4.903979 & -3.390097 & 2.189551 \\ \mathrm{H} & -5.655747 & -4.012023 & 2.666678 \\ \mathrm{C} & -3.588225 & -3.401552 & 2.658161 \\ \mathrm{H} & -3.313067 & -4.029479 & 3.500507\end{array}$

1a

B3LYP SCF energy: $\quad 1378.22414897$ a.u.

B3LYP enthalpy: 1377.819134 a.u.

B3LYP free energy: 1377.903711 a.u.

M06-L SCF energy in solution: $\quad 1378.40383689$ a.u.

M06-L enthalpy in solution: $\quad 1377.998822$ a.u.

M06-L free energy in solution: $\quad 1378.083399$ a.u.

Cartesian coordinates

$\begin{array}{llll}\text { ATOM } & \mathrm{X} & \mathrm{Y} & \mathrm{Z}\end{array}$ 


$$
\begin{aligned}
& \begin{array}{llll}
\text { O } & 5.548524 & -1.278119 & -1.633605
\end{array} \\
& \begin{array}{llll}
\text { O } & 1.124684 & -2.031465 & 1.126124
\end{array} \\
& \begin{array}{llll}
\text { C } & 4.594773 & -0.668790 & -1.220534
\end{array} \\
& \text { S } \quad 0.194396 \quad-0.954390 \quad 1.480263 \\
& \begin{array}{llll}
\text { C } & 3.671997 & 0.389285 & -1.846329
\end{array} \\
& \begin{array}{llll}
\mathrm{N} & 0.912474 & 0.474098 & 0.922462
\end{array} \\
& \begin{array}{llll}
\text { O } & -0.194633 & -0.687731 & 2.867837
\end{array} \\
& \begin{array}{llll}
\text { C } & -1.313818 & -1.260680 & 0.550739
\end{array} \\
& \begin{array}{llll}
\text { C } & 3.065195 & 0.631218 & -0.416222
\end{array} \\
& \begin{array}{llll}
\mathrm{H} & 4.175488 & 1.235928 & -2.325917
\end{array} \\
& \mathrm{H} \quad 2.970040 \quad-0.047669-2.568866 \\
& \begin{array}{llll}
\text { C } & 0.331678 & 1.749287 & 1.394573
\end{array} \\
& \begin{array}{llll}
\text { C } & -2.518812 & -0.703156 & 0.983482
\end{array} \\
& \begin{array}{llll}
\text { C } & -1.271299 & -2.072375 & -0.585583
\end{array} \\
& \begin{array}{llll}
\mathrm{H} & -0.063739 & 1.568973 & 2.397564
\end{array} \\
& \text { H } \quad 1.159103 \quad 2.457136 \quad 1.512766 \\
& \mathrm{H} \quad-2.546996 \quad-0.105765 \quad 1.887925 \\
& \begin{array}{llll}
\text { C } & -3.682879 & -0.954377 & 0.260440
\end{array} \\
& \begin{array}{llll}
\text { C } & -2.444527 & -2.307550 & -1.299669
\end{array} \\
& \mathrm{H} \quad-0.338616 \quad-2.540882 \quad-0.880267 \\
& \text { H } \quad-4.624669-0.536262 \quad 0.607658 \\
& \begin{array}{llll}
\text { C } & -3.665329 & -1.750351 & -0.894288
\end{array} \\
& \text { H } \quad-2.413365 \quad-2.948185 \quad-2.177633 \\
& \text { C } \quad-4.927628-1.995649-1.685920 \\
& \text { H } \quad-5.035703 \quad-1.258670 \quad-2.492875 \\
& \text { H } \quad-4.922603 \quad-2.986805-2.151127 \\
& \text { H } \quad-5.818437 \quad-1.920224 \quad-1.054182 \\
& \begin{array}{llll}
\text { C } & 1.536564 & 0.460439 & -0.415471
\end{array} \\
& \text { H } \quad 1.073768 \quad 1.256111 \quad-1.014689 \\
& \text { H } \quad 1.291864 \quad-0.484974 \quad-0.907572 \\
& \begin{array}{llll}
\text { C } & 3.882451 & -0.592276 & 0.130471
\end{array} \\
& \begin{array}{llll}
\mathrm{H} & 3.270659 & -1.473830 & 0.351031
\end{array} \\
& \text { H } \quad 4.532350 \quad-0.405485 \quad 0.992648
\end{aligned}
$$




$$
\begin{array}{llll}
\mathrm{C} & -0.690697 & 2.340192 & 0.519054 \\
\mathrm{C} & -1.502480 & 2.859580 & -0.213917 \\
\mathrm{C} & 3.526334 & 1.956971 & 0.193820 \\
\mathrm{H} & 4.610590 & 2.077539 & 0.082204 \\
\mathrm{H} & 3.046047 & 2.815093 & -0.293594 \\
\mathrm{H} & 3.305754 & 1.995808 & 1.266177 \\
\mathrm{C} & -2.517337 & 3.467844 & -1.077690 \\
\mathrm{H} & -2.360963 & 4.554808 & -1.110358 \\
\mathrm{H} & -2.372979 & 3.112373 & -2.107171 \\
\mathrm{C} & -3.958483 & 3.165856 & -0.626331 \\
\mathrm{H} & -4.676372 & 3.634449 & -1.308110 \\
\mathrm{H} & -4.142014 & 2.086990 & -0.615854 \\
\mathrm{H} & -4.139675 & 3.550809 & 0.382195
\end{array}
$$

IntA1

B3LYP SCF energy: $\quad 3936.04313275$ a.u.

B3LYP enthalpy: 3935.033862 a.u.

B3LYP free energy: 3935.207105 a.u.

M06-L SCF energy in solution: $\quad 3937.69781897$ a.u.

M06-L enthalpy in solution: $\quad 3936.688548$ a.u.

M06-L free energy in solution: $\quad 3936.861791$ a.u.

Cartesian coordinates

\begin{tabular}{lccc}
\multicolumn{1}{l}{ ATOM X } & Y \\
P & -0.975312 & -1.378507 & 0.829310 \\
P & -1.572246 & 1.599811 & -0.612336 \\
O & -5.143673 & -2.265633 & -1.296337 \\
O & -5.146748 & -2.133946 & -3.608161 \\
O & -6.026119 & 0.140508 & 0.523266 \\
O & -6.459992 & -0.123091 & 2.783464 \\
C & -2.707158 & 0.505191 & -1.578169
\end{tabular}




$$
\begin{aligned}
& \begin{array}{llll}
\text { C } & -3.521531 & -0.453977 & -0.893632
\end{array} \\
& \text { C } \quad-4.286531 \quad-1.278621 \quad-1.702411 \\
& \begin{array}{llll}
\text { C } & -4.291716 & -1.203508 & -3.095589
\end{array} \\
& \begin{array}{llll}
\text { C } & -3.531016 & -0.272276 & -3.766376
\end{array} \\
& \begin{array}{llll}
\text { C } & -2.736943 & 0.577413 & -2.976297
\end{array} \\
& \begin{array}{llll}
\text { C } & -5.623336 & -2.896526 & -2.490969
\end{array} \\
& \begin{array}{llll}
\text { C } & -2.665557 & -1.066355 & 1.481765
\end{array} \\
& \begin{array}{llll}
\text { C } & -2.946309 & -1.227847 & 2.842989
\end{array} \\
& \begin{array}{llll}
\text { C } & -4.207617 & -0.934465 & 3.392554
\end{array} \\
& \begin{array}{llll}
\text { C } & -5.167422 & -0.471883 & 2.519183
\end{array} \\
& \begin{array}{llll}
\text { C } & -4.903030 & -0.315706 & 1.157755
\end{array} \\
& \begin{array}{llll}
\text { C } & -3.672250 & -0.592908 & 0.585430
\end{array} \\
& \begin{array}{llll}
\text { C } & -7.011691 & 0.342876 & 1.544512
\end{array} \\
& \begin{array}{llll}
\text { C } & -2.721497 & 2.436927 & 0.561997
\end{array} \\
& \begin{array}{llll}
\text { C } & -2.432341 & 2.488847 & 1.931850
\end{array} \\
& \begin{array}{llll}
\text { C } & -3.915189 & 3.012736 & 0.092830
\end{array} \\
& \begin{array}{llll}
\text { C } & -1.035018 & 2.915962 & -1.792673
\end{array} \\
& \begin{array}{llll}
\text { C } & -1.415305 & 4.258501 & -1.661720
\end{array} \\
& \begin{array}{llll}
\text { C } & -0.147543 & 2.562229 & -2.826969
\end{array} \\
& \begin{array}{llll}
\text { C } & -1.205068 & -2.745423 & -0.380141
\end{array} \\
& \begin{array}{llll}
\text { C } & -2.165311 & -3.748412 & -0.162813
\end{array} \\
& \begin{array}{llll}
\text { C } & 0.512524 & -1.254408 & 3.196098
\end{array} \\
& \begin{array}{llll}
\text { C } & -0.049280 & -2.120278 & 2.241717
\end{array} \\
& \begin{array}{llll}
\text { C } & 0.135916 & -3.502425 & 2.388021
\end{array} \\
& \text { H } \quad-3.544452-0.194215 \quad-4.847539 \\
& \text { H } \quad-2.140430 \quad 1.321159 \quad-3.487486 \\
& \text { H } \quad-5.224443 \quad-3.916247 \quad-2.549645 \\
& \text { H } \quad-6.716877 \quad-2.896163 \quad-2.490569 \\
& \mathrm{H} \quad-2.174107 \quad-1.596980 \quad 3.507051 \\
& \mathrm{H} \quad-4.414597 \quad-1.070340 \quad 4.448081 \\
& \begin{array}{llll}
\mathrm{H} & -7.236982 & 1.412617 & 1.625749
\end{array} \\
& \mathrm{H} \quad-7.909592 \quad-0.234701 \quad 1.305583 \\
& \begin{array}{llll}
\mathrm{H} & -1.532797 & 2.008836 & 2.302853
\end{array}
\end{aligned}
$$




\begin{tabular}{|c|c|c|c|}
\hline & 4.169846 & 2.955805 & -0.961566 \\
\hline & -2.085928 & 4.564197 & -0.866677 \\
\hline & 0.179597 & 1.530677 & -2.927532 \\
\hline & -2.808904 & -3.706621 & 0.710932 \\
\hline & 0.393699 & -0.179958 & 3.079775 \\
\hline & -0.278401 & -4.189192 & 1.657907 \\
\hline & 0.261470 & 0.533622 & 0.167362 \\
\hline & 2.967438 & 1.768150 & -1.262537 \\
\hline & 3.883349 & 0.667729 & -0.849735 \\
\hline & 3.583263 & 2.666782 & -1.359349 \\
\hline & 2.583702 & 1.526020 & -2.254182 \\
\hline & 4.674299 & 0.926559 & 0.378502 \\
\hline & 3.049961 & -0.814926 & -0.867232 \\
\hline & 4.028683 & 1.343631 & 1.164459 \\
\hline & 5.040441 & -0.038924 & 0.739543 \\
\hline & 5.896231 & 1.829719 & 0.145186 \\
\hline & 2.162911 & -0.922622 & 0.334503 \\
\hline & 2.420099 & -0.911455 & -2.190162 \\
\hline & 4.319185 & -2.048481 & -0.694053 \\
\hline & 6.724445 & 2.034043 & 1.464418 \\
\hline & 5.203257 & -2.250683 & -1.760695 \\
\hline & 4.377806 & -2.828983 & 0.462227 \\
\hline & 7.708758 & 1.557854 & 1.521057 \\
\hline & 6.166228 & 1.789798 & 2.378543 \\
\hline & 5.130260 & -1.646517 & -2.658782 \\
\hline & 6.169776 & -3.243790 & -1.647888 \\
\hline & 5.353837 & -3.821644 & 0.548850 \\
\hline & 3.670327 & -2.666368 & 1.268047 \\
\hline & 6.862522 & -3.406704 & -2.469219 \\
\hline & 6.261379 & -4.045000 & -0.495837 \\
\hline & 5.408226 & -4.434222 & 1.444506 \\
\hline & 7.301016 & -5.134411 & -0.404107 \\
\hline & 1.023291 & -5.990021 & -1.032845 \\
\hline
\end{tabular}




$$
\begin{aligned}
& \text { H } \quad 8.278413 \quad-4.783079 \quad-0.752098 \\
& \mathrm{H} \quad 7.413352 \quad-5.498981 \quad 0.620871 \\
& \begin{array}{llll}
\text { C } & -2.304968 & -4.796394 & -1.073510
\end{array} \\
& \text { H } \quad-3.044128 \quad-5.572103 \quad-0.891965 \\
& \begin{array}{llll}
\text { C } & -1.496622 & -4.848275 & -2.212715
\end{array} \\
& \text { H } \quad-1.608601 \quad-5.663623 \quad-2.922114 \\
& \begin{array}{llll}
\text { C } & -0.547676 & -3.849591 & -2.438007
\end{array} \\
& \text { H } \quad 0.079380 \quad-3.880926 \quad-3.324511 \\
& \begin{array}{llll}
\text { C } & -0.399643 & -2.801415 & -1.527141
\end{array} \\
& \text { H } \quad 0.338497 \quad-2.029002 \quad-1.715565 \\
& \begin{array}{llll}
\text { C } & 0.858187 & -4.006568 & 3.472547
\end{array} \\
& \text { H } \quad 0.991851 \quad-5.079984 \quad 3.574617 \\
& \begin{array}{llll}
\text { C } & 1.400999 & -3.139655 & 4.422340
\end{array} \\
& \text { H } \quad 1.957640 \quad-3.535404 \quad 5.267267 \\
& \begin{array}{llll}
\text { C } & 1.225558 & -1.760273 & 4.282670
\end{array} \\
& \text { H } \quad 1.646945 \quad-1.079204 \quad 5.017028 \\
& \begin{array}{llll}
\text { C } & -4.782974 & 3.653550 & 0.977206
\end{array} \\
& \text { H } \quad-5.696344 \quad 4.106461 \quad 0.601399 \\
& \begin{array}{llll}
\text { C } & -4.479275 & 3.711170 & 2.340753
\end{array} \\
& \mathrm{H} \quad \begin{array}{llll}
-5.156811 & 4.209152 & 3.028659
\end{array} \\
& \begin{array}{llll}
\text { C } & -3.307260 & 3.121543 & 2.817426
\end{array} \\
& \mathrm{H} \quad-3.075800 \quad 3.148888 \quad 3.878463 \\
& \begin{array}{llll}
\text { C } & -0.933510 & 5.221888 & -2.553611
\end{array} \\
& \mathrm{H} \quad-1.238195 \quad 6.258072 \quad-2.436634 \\
& \begin{array}{llll}
\text { C } & -0.074201 & 4.857413 & -3.588905
\end{array} \\
& \mathrm{H} \quad 0.291430 \quad 5.606685 \quad-4.285183 \\
& \begin{array}{llll}
\text { C } & 0.316720 & 3.521873 & -3.724989
\end{array} \\
& \mathrm{H} \quad 0.985963 \quad 3.228280 \quad-4.529191 \\
& \begin{array}{llll}
\text { C } & 1.867764 & 1.999507 & -0.292389
\end{array} \\
& \begin{array}{llll}
\text { C } & 1.270629 & 2.452836 & 0.703553
\end{array} \\
& \begin{array}{llll}
\text { C } & 0.917390 & 3.416283 & 1.769288
\end{array} \\
& \begin{array}{llll}
\mathrm{H} & -0.063025 & 3.852034 & 1.543882
\end{array} \\
& \text { H } \quad 0.800344 \quad 2.885449 \quad 2.722079
\end{aligned}
$$




$\begin{array}{llll}\mathrm{C} & 1.961449 & 4.537838 & 1.916820 \\ \mathrm{H} & 2.074323 & 5.093998 & 0.980813 \\ \mathrm{H} & 1.648033 & 5.240469 & 2.695826 \\ \mathrm{H} & 2.939206 & 4.135225 & 2.200692 \\ \mathrm{C} & 6.775336 & 1.336749 & -1.007697 \\ \mathrm{H} & 7.098695 & 0.302270 & -0.841325 \\ \mathrm{H} & 6.238686 & 1.372777 & -1.960986 \\ \mathrm{H} & 7.674430 & 1.956543 & -1.101755 \\ \mathrm{C} & 5.649092 & 3.382125 & 0.081672 \\ \mathrm{H} & 4.660355 & 3.693670 & 0.441255 \\ \mathrm{H} & 5.858568 & 3.893997 & -0.863676 \\ \mathrm{C} & 6.700300 & 3.543781 & 1.190635 \\ \mathrm{O} & 7.305404 & 4.482131 & 1.634386\end{array}$

IntA2

B3LYP SCF energy: 3936.03926182 a.u.

B3LYP enthalpy: 3935.029277 a.u.

B3LYP free energy: $\quad 3935.209115$ a.u.

M06-L SCF energy in solution: $\quad 3937.67513281$ a.u.

M06-L enthalpy in solution: $\quad 3936.665148$ a.u.

M06-L free energy in solution: $\quad 3936.844986$ a.u.

Cartesian coordinates

\begin{tabular}{lccc}
\multicolumn{2}{l}{ ATOM X } & Y \\
P & -1.823480 & 0.300233 & -1.677840 \\
P & -2.105851 & -1.213001 & 1.214050 \\
O & -4.298333 & 3.578510 & 0.966418 \\
O & -3.274488 & 4.370599 & 2.888268 \\
O & -6.279045 & 1.172528 & 1.079969 \\
O & -7.692431 & 0.602463 & -0.664062 \\
C & -2.502829 & 0.465542 & 1.833097
\end{tabular}




$$
\begin{aligned}
& \text { C } \quad-3.368150 \quad 1.295764 \quad 1.054503 \\
& \begin{array}{llll}
\text { C } & -3.537130 & 2.587500 & 1.525499
\end{array} \\
& \begin{array}{llll}
\text { C } & -2.920209 & 3.068752 & 2.681076
\end{array} \\
& \begin{array}{llll}
\text { C } & -2.097440 & 2.272867 & 3.447667
\end{array} \\
& \begin{array}{llll}
\text { C } & -1.898270 & 0.956309 & 2.995145
\end{array} \\
& \begin{array}{llll}
\text { C } & -4.153219 & 4.726521 & 1.813119
\end{array} \\
& \begin{array}{llll}
\text { C } & -3.637901 & 0.464151 & -1.411913
\end{array} \\
& \begin{array}{llll}
\text { C } & -4.511440 & 0.126589 & -2.453909
\end{array} \\
& \begin{array}{llll}
\text { C } & -5.909291 & 0.142446 & -2.309482
\end{array} \\
& \begin{array}{llll}
\text { C } & -6.394752 & 0.504397 & -1.072432
\end{array} \\
& \begin{array}{llll}
\text { C } & -5.538955 & 0.850206 & -0.026118
\end{array} \\
& \begin{array}{llll}
\text { C } & -4.156915 & 0.852716 & -0.134595
\end{array} \\
& \begin{array}{llll}
\text { C } & -7.650764 & 0.953398 & 0.725527
\end{array} \\
& \begin{array}{llll}
\text { C } & -3.688792 & -2.141626 & 1.265580
\end{array} \\
& \text { C } \quad-3.917883 \quad-3.151662 \quad 0.319666 \\
& \begin{array}{llll}
\text { C } & -4.652526 & -1.893096 & 2.256368
\end{array} \\
& \begin{array}{llll}
\text { C } & -1.012589 & -2.017690 & 2.455328
\end{array} \\
& \begin{array}{llll}
\text { C } & -1.436441 & -3.084102 & 3.265459
\end{array} \\
& \begin{array}{llll}
\text { C } & 0.330094 & -1.591073 & 2.527550
\end{array} \\
& \begin{array}{llll}
\text { C } & -1.134017 & 1.963532 & -1.297668
\end{array} \\
& \begin{array}{llll}
\text { C } & -1.853503 & 3.133404 & -1.594289
\end{array} \\
& \begin{array}{llll}
\text { C } & 0.142410 & 2.067331 & -0.724124
\end{array} \\
& \begin{array}{llll}
\text { C } & -1.749767 & -1.213344 & -4.030118
\end{array} \\
& \begin{array}{llll}
\text { C } & -1.613321 & 0.079279 & -3.495178
\end{array} \\
& \mathrm{H} \quad-1.627197 \quad 2.641817 \quad 4.352046 \\
& \mathrm{H} \quad-1.251680 \quad 0.308515 \quad 3.575056 \\
& \begin{array}{llll}
\mathrm{H} & -3.712323 & 5.548684 & 1.239168
\end{array} \\
& \mathrm{H} \quad-5.130806 \quad 5.008126 \quad 2.218971 \\
& \text { H } \quad-4.105992 \quad-0.157683 \quad-3.416871 \\
& \text { H } \quad-6.569419 \quad-0.116506 \quad-3.129464 \\
& \mathrm{H} \quad-8.054160 \quad 0.126990 \quad 1.322333 \\
& \mathrm{H} \quad \begin{array}{llll}
-8.221530 & 1.872979 & 0.885765
\end{array} \\
& \text { H } \quad-3.176561 \quad-3.333072 \quad-0.454816
\end{aligned}
$$




$$
\begin{aligned}
& \text { H } \quad-4.491605 \quad-1.105803 \quad 2.987147 \\
& \text { H } \quad-2.463277 \quad-3.430939 \quad 3.218006 \\
& \begin{array}{llll}
\mathrm{H} & -2.851422 & 3.067830 & -2.017930
\end{array} \\
& \begin{array}{llll}
\mathrm{H} & 0.695827 & 1.167867 & -0.468380
\end{array} \\
& \text { H } \quad-1.953512 \quad-2.048266 \quad-3.364836 \\
& \begin{array}{llll}
\text { Rh } & -0.799961 & -1.354145 & -0.597992
\end{array} \\
& \begin{array}{llll}
\text { O } & 2.858637 & 0.046780 & 0.692556
\end{array} \\
& \begin{array}{llll}
\text { C } & 1.841414 & -2.256868 & -1.935661
\end{array} \\
& \begin{array}{llll}
\text { S } & 4.199330 & -0.120122 & 1.280371
\end{array} \\
& \begin{array}{llll}
\text { O } & 0.773675 & -1.690347 & -2.123710
\end{array} \\
& \mathrm{~N} \quad 4.910381 \quad-1.384945 \quad 0.402602 \\
& \begin{array}{llll}
\text { O } & 4.363330 & -0.475871 & 2.693747
\end{array} \\
& \begin{array}{llll}
\text { C } & 5.099625 & 1.400331 & 0.990938
\end{array} \\
& \begin{array}{llll}
\text { C } & 6.229890 & -1.865512 & 0.880176
\end{array} \\
& \begin{array}{llll}
\text { C } & 6.141763 & 1.753933 & 1.851904
\end{array} \\
& \begin{array}{llll}
\text { C } & 4.763249 & 2.209381 & -0.098819
\end{array} \\
& \begin{array}{llll}
\text { H } & 6.236573 & -1.753205 & 1.968018
\end{array} \\
& \begin{array}{llll}
\text { H } & 6.268215 & -2.943148 & 0.687768
\end{array} \\
& \begin{array}{llll}
\mathrm{H} & 6.378121 & 1.129863 & 2.706519
\end{array} \\
& \text { C } \quad 6.851293 \quad 2.927976 \quad 1.610175 \\
& \begin{array}{llll}
\text { C } & 5.487584 & 3.377966 & -0.325017
\end{array} \\
& \text { H } \quad 3.934536 \quad 1.941637 \quad-0.745475 \\
& \begin{array}{llll}
\mathrm{H} & 7.657662 & 3.208259 & 2.282896
\end{array} \\
& \begin{array}{llll}
\text { C } & 6.539991 & 3.757147 & 0.521981
\end{array} \\
& \begin{array}{llll}
\mathrm{H} & 5.227097 & 4.010032 & -1.170175
\end{array} \\
& \begin{array}{llll}
\text { C } & 7.294997 & 5.043533 & 0.290087
\end{array} \\
& \mathrm{H} \quad 7.292433 \quad 5.326410 \quad-0.767254 \\
& \begin{array}{llll}
\mathrm{H} & 6.838678 & 5.869782 & 0.850908
\end{array} \\
& \text { H } \quad 8.335292 \quad 4.963520 \quad 0.621182 \\
& \begin{array}{llll}
\text { C } & 3.072212 & -2.420579 & -2.807261
\end{array} \\
& \text { H } \quad 3.360558 \quad-1.557100 \quad-3.416202 \\
& \mathrm{H} \quad 2.988000 \quad-3.297571 \quad-3.461138 \\
& \begin{array}{llll}
\text { C } & 3.870311 & -2.724876 & -1.480297
\end{array}
\end{aligned}
$$




$$
\begin{aligned}
& \text { C } \quad 2.501532 \quad-2.876441 \quad-0.725303 \\
& \mathrm{H} \quad 0.679557 \quad-0.769935 \quad 1.906475 \\
& \text { H } \quad 2.209832 \quad-3.927593 \quad-0.599390 \\
& \text { C } \quad 4.737353 \quad-3.981936-1.513215 \\
& \text { H } \quad 5.056644 \quad-4.273477 \quad-0.506954 \\
& \text { H } \quad 4.181765 \quad-4.828235 \quad-1.933744 \\
& \text { H } \quad 5.634418 \quad-3.832473 \quad-2.125616 \\
& \text { C } \quad 4.634458 \quad-1.451736 \quad-1.041855 \\
& \text { H } \quad 5.581863 \quad-1.379337 \quad-1.591315 \\
& \text { H } \quad 4.039695 \quad-0.577483 \quad-1.315598 \\
& \text { H } \quad 2.371384 \quad-2.348029 \quad 0.222062 \\
& \text { C } \quad 7.389842 \quad-1.209608 \quad 0.265714 \\
& \text { C } \quad 8.351536 \quad-0.692941 \quad-0.257829 \\
& \text { C } \quad 9.535770 \quad-0.066932 \quad-0.851456 \\
& \text { H } \quad 9.492588 \quad 1.017254 \quad-0.680564 \\
& \text { H } \quad 9.513860 \quad-0.205284 \quad-1.940855 \\
& \text { C } \quad 10.855909 \quad-0.628184 \quad-0.287488 \\
& \text { H } \quad 10.937565 \quad-1.702542 \quad-0.478702 \\
& \text { H } \quad 11.708071 \quad-0.128559 \quad-0.759320 \\
& \text { H } \quad 10.918323 \quad-0.469674 \quad 0.793502 \\
& \text { C } \quad-1.618125 \quad-1.429718 \quad-5.401113 \\
& \text { H } \quad-1.733247 \quad-2.432665 \quad-5.802708 \\
& \text { C } \quad-1.330313 \quad-0.360523 \quad-6.253443 \\
& \text { H } \quad-1.222290 \quad-0.529087 \quad-7.321242 \\
& \text { C } \quad \begin{array}{llll}
-1.176911 & 0.923558 & -5.728724
\end{array} \\
& \text { H } \quad-0.948129 \quad 1.757595 \quad-6.386247 \\
& \text { C } \quad-1.318080 \quad 1.145885 \quad-4.357210 \\
& \text { H } \quad-1.195494 \quad 2.149481 \quad-3.964605 \\
& \text { C } \quad-1.296032 \quad 4.385740 \quad-1.332294 \\
& \text { H } \quad \begin{array}{llll}
-1.857578 & 5.285119 & -1.570295
\end{array} \\
& \text { C } \quad-0.023426 \quad 4.481959 \quad-0.763276 \\
& \text { H } \quad 0.404774 \quad 5.458229 \quad-0.552909 \\
& \text { C } \quad 0.692016 \quad 3.322546 \quad-0.457015
\end{aligned}
$$




$$
\begin{array}{llll}
\mathrm{H} & 1.675112 & 3.389750 & 0.000601 \\
\mathrm{C} & -0.539223 & -3.698475 & 4.140092 \\
\mathrm{H} & -0.878423 & -4.518333 & 4.767080 \\
\mathrm{C} & 0.785663 & -3.260596 & 4.215463 \\
\mathrm{H} & 1.476849 & -3.740578 & 4.902743 \\
\mathrm{C} & 1.221315 & -2.205876 & 3.409743 \\
\mathrm{H} & 2.247475 & -1.852809 & 3.463717 \\
\mathrm{C} & -5.823151 & -2.651614 & 2.300244 \\
\mathrm{H} & -6.561674 & -2.458632 & 3.073615 \\
\mathrm{C} & -6.043426 & -3.658001 & 1.355748 \\
\mathrm{H} & -6.956340 & -4.245923 & 1.391456 \\
\mathrm{C} & -5.090863 & -3.906551 & 0.365147 \\
\mathrm{H} & -5.262050 & -4.685069 & -0.372925
\end{array}
$$

IntA3

B3LYP SCF energy: $\quad 3936.02969046$ a.u.

B3LYP enthalpy: $\quad 3935.020230$ a.u.

B3LYP free energy: 3935.202667 a.u.

M06-L SCF energy in solution: $\quad 3937.66329429$ a.u.

M06-L enthalpy in solution: $\quad 3936.653834$ a.u.

M06-L free energy in solution: $\quad 3936.836271$ a.u.

Cartesian coordinates

\begin{tabular}{lrrr}
\multicolumn{1}{l}{ ATOM X } & Y \\
P & -2.072340 & 1.614958 & -0.655120 \\
P & -2.401192 & -1.486381 & 0.396926 \\
O & -6.302578 & 1.915906 & 1.607549 \\
O & -6.240376 & 1.468184 & 3.878659 \\
O & -7.012906 & -0.219176 & -0.566284 \\
O & -7.518606 & 0.455010 & -2.723121 \\
C & -3.613290 & -0.621172 & 1.479162
\end{tabular}




$$
\begin{array}{lrrr}
\mathrm{C} & -4.536230 & 0.331709 & 0.937873 \\
\mathrm{C} & -5.361062 & 0.954723 & 1.861789 \\
\mathrm{C} & -5.323380 & 0.693747 & 3.231915 \\
\mathrm{C} & -4.444712 & -0.223089 & 3.764891 \\
\mathrm{C} & -3.590836 & -0.873020 & 2.857593 \\
\mathrm{C} & -6.939844 & 2.197058 & 2.860534 \\
\mathrm{C} & -3.773701 & 1.405832 & -1.300272 \\
\mathrm{C} & -4.099414 & 1.818116 & -2.596386 \\
\mathrm{C} & -5.350709 & 1.547031 & -3.178745 \\
\mathrm{C} & -6.246959 & 0.838752 & -2.408429 \\
\mathrm{C} & -5.938278 & 0.433633 & -1.108383 \\
\mathrm{C} & -4.720743 & 0.692577 & -0.500639 \\
\mathrm{C} & -7.990044 & -0.317276 & -1.612130 \\
\mathrm{C} & -3.417814 & -2.495126 & -0.758964 \\
\mathrm{H} & -8.095380 & -1.366234 & -1.914757 \\
\mathrm{C} & -2.853795 & -2.910737 & -1.976171 \\
\mathrm{C} & -4.721614 & -2.900313 & -0.431454 \\
\mathrm{H} & -1.566764 & -2.716137 & 1.488382 \\
\mathrm{H} & --3.368936 & 2.364104 & -3.182092 \\
\mathrm{C} & -1.977903 & -4.055383 & 1.561365 \\
\mathrm{C} & -0.477453 & -2.291802 & 2.268888 \\
\mathrm{C} & -2.205673 & 2.718518 & 0.802405 \\
\mathrm{C} & -3.199191 & 3.707252 & 0.886595 \\
\mathrm{C} & -1.256684 & 2.603923 & 1.829059 \\
\mathrm{H} & -0.678526 & 1.871289 & -3.059858 \\
\mathrm{H} & -1.101246 & 2.556998 & -1.901723 \\
\mathrm{H} & -0.669972 & 3.877319 & -1.695257 \\
\mathrm{H} & -4.872579 & -0.433955 & 4.827717 \\
\mathrm{H} & -1.261867
\end{array}
$$




$$
\begin{aligned}
& \mathrm{H} \quad-1.845176 \quad-2.601472 \quad-2.233686 \\
& \mathrm{H} \quad-5.175023 \quad-2.581414 \quad 0.501695 \\
& \text { H } \quad-2.812776 \quad-4.403852 \quad 0.962642 \\
& \begin{array}{llll}
\mathrm{H} & -0.146862 & -1.257991 & 2.207038
\end{array} \\
& \begin{array}{llll}
\text { H } & -3.945759 & 3.797839 & 0.102834
\end{array} \\
& \mathrm{H} \quad-0.492940 \quad 1.832845 \quad 1.766319 \\
& \text { H } \quad-1.016269 \quad 0.851576 \quad-3.235782 \\
& \text { H } \quad-0.977061 \quad 4.417313 \quad-0.805934 \\
& \begin{array}{llll}
\text { Rh } & -0.856524 & -0.256432 & -0.632274
\end{array} \\
& \begin{array}{llll}
\text { O } & 0.497046 & -1.934866 & -1.109212
\end{array} \\
& \begin{array}{lllll}
\text { O } & 5.298840 & 0.920676 & -0.522816
\end{array} \\
& \begin{array}{llll}
\text { C } & 1.696861 & -2.015317 & -0.866422
\end{array} \\
& \begin{array}{llll}
\text { S } & 5.704371 & 0.092443 & 0.626806
\end{array} \\
& \begin{array}{llll}
\text { C } & 2.729268 & -3.061414 & -1.227305
\end{array} \\
& \begin{array}{llll}
\mathrm{N} & 6.091651 & -1.512404 & 0.095345
\end{array} \\
& \begin{array}{llll}
\text { O } & 4.756440 & -0.078957 & 1.738534
\end{array} \\
& \begin{array}{llll}
\text { C } & 7.234839 & 0.725063 & 1.293979
\end{array} \\
& \begin{array}{llll}
\text { C } & 3.692538 & -2.387275 & -0.176295
\end{array} \\
& \text { H } \quad 2.439609-4.104052-1.058706 \\
& \text { H } \quad 3.060512 \quad-2.954440 \quad-2.269483 \\
& \begin{array}{llll}
\text { C } & 7.460933 & -1.710693 & -0.452671
\end{array} \\
& \begin{array}{llll}
\text { C } & 7.590458 & 0.397631 & 2.606086
\end{array} \\
& \begin{array}{llll}
\text { C } & 8.013805 & 1.591442 & 0.524309
\end{array} \\
& \begin{array}{llll}
\mathrm{H} & 8.176634 & -1.342370 & 0.286485
\end{array} \\
& \text { H } \quad 7.596366 \quad-2.798926 \quad-0.490488 \\
& \text { H } \quad 6.952159-0.249469 \quad 3.198063 \\
& \begin{array}{llll}
\text { C } & 8.759156 & 0.934441 & 3.139058
\end{array} \\
& \begin{array}{llll}
\text { C } & 9.176915 & 2.121202 & 1.080233
\end{array} \\
& \mathrm{H} \quad 7.705500 \quad 1.845852-0.483493 \\
& \text { H } \quad 9.040830 \quad 0.688687 \quad 4.159663 \\
& \begin{array}{llll}
\text { C } & 9.571553 & 1.798859 & 2.387105
\end{array} \\
& \text { H } \quad 9.785555 \quad 2.802021 \quad 0.491063 \\
& \begin{array}{llll}
\text { C } & 10.847680 & 2.355358 & 2.970177
\end{array}
\end{aligned}
$$




$$
\begin{aligned}
& \mathrm{H} \quad 11.142507 \quad 3.286259 \quad 2.476440 \\
& \text { H } \quad 10.745353 \quad 2.553348 \quad 4.042089 \\
& \text { H } \quad 11.674470 \quad 1.643145 \quad 2.848896 \\
& \begin{array}{llll}
\text { C } & 5.054436 & -2.064960 & -0.810231
\end{array} \\
& \text { H } \quad 5.476665 \quad-3.010191 \quad-1.170492 \\
& \text { H } \quad 4.921398 \quad-1.418672 \quad-1.690085 \\
& \text { C } \quad 2.664532 \quad-1.197590 \quad-0.051710 \\
& \text { H } \quad 2.981989-0.285297 \quad-0.573050 \\
& \text { H } \quad 2.338300 \quad-0.913584 \quad 0.952155 \\
& \begin{array}{llll}
\text { C } & 7.733127 & -1.124173 & -1.770563
\end{array} \\
& \begin{array}{llll}
\text { C } & 7.956360 & -0.657568 & -2.865249
\end{array} \\
& \begin{array}{llll}
\text { C } & 8.253205 & -0.080191 & -4.178491
\end{array} \\
& \text { H } \quad 8.213569-0.870508 \quad-4.940402 \\
& \text { H } \quad 7.462312 \quad 0.635517 \quad-4.441089 \\
& \begin{array}{llll}
\text { C } & 9.624069 & 0.621166 & -4.234794
\end{array} \\
& \text { H } \quad 9.796054 \quad 1.033075 \quad-5.234542 \\
& \text { H } \quad 9.673627 \quad 1.441065 \quad-3.511324 \\
& \text { H } \quad 10.432146 \quad-0.081213 \quad-4.008277 \\
& \begin{array}{llll}
\text { C } & 3.813077 & -3.208232 & 1.108673
\end{array} \\
& \mathrm{H} \quad 4.319981 \quad-2.629251 \quad 1.883622 \\
& \text { H } \quad 2.822882 \quad-3.495876 \quad 1.482772 \\
& \text { H } \quad 4.384129 \quad-4.127486 \quad 0.930707 \\
& \begin{array}{llll}
\text { C } & 0.175370 & -3.184953 & 3.118749
\end{array} \\
& \text { H } \quad 1.008291 \quad-2.841694 \quad 3.726105 \\
& \begin{array}{llll}
\text { C } & -0.241382 & -4.517844 & 3.185373
\end{array} \\
& \text { H } \quad 0.269315 \quad-5.215483 \quad 3.843117 \\
& \begin{array}{llll}
\text { C } & -1.314564 & -4.949916 & 2.404658
\end{array} \\
& \text { H } \quad-1.640724 \quad-5.985074 \quad 2.451287 \\
& \begin{array}{llll}
\text { C } & -5.446332 & -3.709209 & -1.309253
\end{array} \\
& \text { H } \quad-6.452778 \quad-4.022047 \quad-1.044561 \\
& \begin{array}{llll}
\text { C } & -4.879564 & -4.118224 & -2.518239
\end{array} \\
& \text { H } \quad-5.446136 \quad-4.746294 \quad-3.200021 \\
& \begin{array}{llll}
\text { C } & -3.582533 & -3.718619 & -2.849422
\end{array}
\end{aligned}
$$




$$
\begin{array}{llll}
\mathrm{H} & -3.138137 & -4.034190 & -3.789145 \\
\mathrm{C} & -3.234931 & 4.570592 & 1.982414 \\
\mathrm{H} & -4.003381 & 5.336554 & 2.040170 \\
\mathrm{C} & -2.284924 & 4.452509 & 3.000479 \\
\mathrm{H} & -2.316251 & 5.125417 & 3.852807 \\
\mathrm{C} & -1.296545 & 3.468716 & 2.923465 \\
\mathrm{H} & -0.558752 & 3.373150 & 3.714971 \\
\mathrm{C} & 0.154611 & 4.500582 & -2.632271 \\
\mathrm{H} & 0.477712 & 5.524151 & -2.465523 \\
\mathrm{C} & 0.565722 & 3.815624 & -3.778644 \\
\mathrm{H} & 1.209763 & 4.306223 & -4.502771 \\
\mathrm{C} & 0.148077 & 2.500585 & -3.992295 \\
\mathrm{H} & 0.461032 & 1.964968 & -4.884064
\end{array}
$$

IntA4

B3LYP SCF energy: $\quad 3936.03250221$ a.u.

B3LYP enthalpy: 3935.022969 a.u.

B3LYP free energy: 3935.204989 a.u.

M06-L SCF energy in solution: $\quad 3937.67403994$ a.u.

M06-L enthalpy in solution: $\quad 3936.664507$ a.u.

M06-L free energy in solution: $\quad 3936.846527$ a.u.

Cartesian coordinates

\begin{tabular}{lccc}
\multicolumn{1}{l}{ ATOM X } & Y \\
P & -1.255472 & -1.152098 & 0.792749 \\
P & -1.997805 & 1.670699 & -0.638591 \\
O & -5.560900 & -2.172238 & -1.275388 \\
O & -5.684780 & -1.948516 & -3.577352 \\
O & -6.402110 & 0.138548 & 0.629491 \\
O & -6.732692 & -0.112975 & 2.907821 \\
C & -3.199080 & 0.656068 & -1.579328
\end{tabular}




$$
\begin{aligned}
& \begin{array}{llll}
\text { C } & -3.954653 & -0.340485 & -0.885517
\end{array} \\
& \begin{array}{llll}
\text { C } & -4.745926 & -1.150850 & -1.683395
\end{array} \\
& \begin{array}{llll}
\text { C } & -4.821808 & -1.020194 & -3.071221
\end{array} \\
& \begin{array}{llll}
\text { C } & -4.111629 & -0.052670 & -3.747912
\end{array} \\
& \begin{array}{llll}
\text { C } & -3.291349 & 0.783167 & -2.969358
\end{array} \\
& \begin{array}{llll}
\text { C } & -6.104008 & -2.754024 & -2.468710
\end{array} \\
& \begin{array}{llll}
\text { C } & -2.950052 & -0.895516 & 1.463150
\end{array} \\
& \begin{array}{llll}
\text { C } & -3.170259 & -1.035851 & 2.839375
\end{array} \\
& \begin{array}{llll}
\text { C } & -4.420759 & -0.800631 & 3.436369
\end{array} \\
& \begin{array}{llll}
\text { C } & -5.440642 & -0.415854 & 2.594276
\end{array} \\
& \text { C } \quad-5.238106 \quad-0.272866 \quad 1.221060 \\
& \begin{array}{llll}
\text { C } & -4.020220 & -0.497709 & 0.598477
\end{array} \\
& \begin{array}{llll}
\text { C } & -7.392167 & 0.169740 & 1.666034
\end{array} \\
& \begin{array}{llll}
\text { C } & -2.995578 & 2.661581 & 0.540832
\end{array} \\
& \begin{array}{llll}
\text { C } & -2.415633 & 3.058878 & 1.754689
\end{array} \\
& \begin{array}{llll}
\text { C } & -4.301585 & 3.075350 & 0.232392
\end{array} \\
& \text { C } \quad-1.267150 \quad 2.891190 \quad-1.806842 \\
& \begin{array}{llll}
\text { C } & -1.647912 & 4.242742 & -1.836886
\end{array} \\
& \begin{array}{llll}
\text { C } & -0.221397 & 2.453845 & -2.645031
\end{array} \\
& \text { C } \quad-1.443283 \quad-2.501373 \quad-0.445453 \\
& \text { C } \quad-2.405882-3.511563 \quad-0.280255 \\
& \begin{array}{llll}
\text { C } & -0.585869 & -2.544383 & -1.555506
\end{array} \\
& \begin{array}{llll}
\text { C } & 0.324204 & -1.035374 & 3.102657
\end{array} \\
& \begin{array}{llll}
\text { C } & -0.293241 & -1.898241 & 2.180448
\end{array} \\
& \begin{array}{llll}
\text { C } & -0.153042 & -3.284364 & 2.345196
\end{array} \\
& \text { H } \quad-4.181214 \quad 0.058139 \quad-4.824049 \\
& \mathrm{H} \quad-2.714051 \quad 1.548979 \quad-3.474212 \\
& \mathrm{H} \quad-5.713006 \quad-3.771453 \quad-2.588269 \\
& \text { H } \quad-7.196377 \quad-2.753736 \quad-2.410773 \\
& \text { H } \quad-2.353612 \quad-1.343462 \quad 3.480639 \\
& \text { H } \quad-4.573591 \quad-0.917036 \quad 4.503254 \\
& \text { H } \quad-7.840863 \quad 1.166433 \quad 1.711024 \\
& \text { H } \quad-8.150570 \quad-0.597858 \quad 1.472756
\end{aligned}
$$




$$
\begin{aligned}
& \text { H } \quad-1.408963 \quad 2.727409 \quad 1.997219 \\
& \text { H } \quad-4.766526 \quad 2.763807 \quad-0.698464 \\
& \text { H } \quad-2.443653 \quad 4.599363 \quad-1.191503 \\
& \text { H } \quad 0.085816 \quad 1.409949-2.631258
\end{aligned}
$$

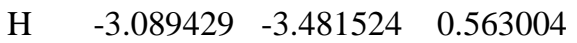

$$
\begin{aligned}
& \text { H } \quad 0.147387 \quad-1.756001 \quad-1.701192 \\
& \begin{array}{llll}
\mathrm{H} & 0.236655 & 0.039512 & 2.967749
\end{array} \\
& \text { H } \quad-0.610946 \quad-3.968062 \quad 1.638477 \\
& \begin{array}{llll}
\mathrm{Rh} & -0.157124 & 0.629947 & 0.053605
\end{array} \\
& \begin{array}{llll}
\text { O } & 7.237031 & 4.742922 & 2.410351
\end{array} \\
& \begin{array}{llll}
\text { O } & 1.979409 & 0.037498 & 0.457556
\end{array} \\
& \begin{array}{llll}
\text { C } & 6.458220 & 3.941224 & 1.968691
\end{array} \\
& \text { S } \quad 2.973535 \quad-0.222499 \quad-0.628292 \\
& \begin{array}{llll}
\text { C } & 6.064873 & 3.506459 & 0.550604
\end{array} \\
& \begin{array}{llll}
\mathrm{N} & 4.083093 & 1.028030 & -0.485004
\end{array} \\
& \begin{array}{llll}
\text { O } & 2.524274 & -0.239619 & -2.023830
\end{array} \\
& \begin{array}{llll}
\text { C } & 3.721828 & -1.797541 & -0.242001
\end{array} \\
& \begin{array}{llll}
\text { C } & 4.810029 & 2.789345 & 1.172813
\end{array} \\
& \text { H } \quad 5.883913 \quad 4.315056-0.165945 \\
& \text { H } \quad 6.805566 \quad 2.817193 \quad 0.125780 \\
& \begin{array}{llll}
\text { C } & 4.975788 & 1.269365 & -1.647198
\end{array} \\
& \text { C } \quad 4.387685 \quad-2.490636 \quad-1.258945 \\
& \begin{array}{llll}
\text { C } & 3.650227 & -2.308741 & 1.056772
\end{array} \\
& \text { H } \quad 4.420817 \quad 0.988489-2.545856 \\
& \text { H } \quad 5.119961 \quad 2.354307 \quad-1.700326 \\
& \text { H } \quad 4.415828 \quad-2.094415 \quad-2.267843 \\
& \begin{array}{llll}
\text { C } & 4.988274 & -3.709852 & -0.959835
\end{array} \\
& \begin{array}{llll}
\text { C } & 4.261501 & -3.530798 & 1.331818
\end{array} \\
& \text { H } \quad 3.100463 \quad-1.780971 \quad 1.827913 \\
& \text { H } \quad 5.492396 \quad-4.260190 \quad-1.749937 \\
& \begin{array}{llll}
\text { C } & 4.943000 & -4.246809 & 0.337311
\end{array} \\
& \begin{array}{llll}
\mathrm{H} & 4.194478 & -3.939354 & 2.336365
\end{array} \\
& \begin{array}{llll}
\text { C } & 5.621802 & -5.557380 & 0.651648
\end{array}
\end{aligned}
$$




$$
\begin{aligned}
& \text { H } \quad 6.688555 \quad-5.402980 \quad 0.860293 \\
& \begin{array}{llll}
\mathrm{H} & 5.182707 & -6.036749 & 1.531707
\end{array} \\
& \text { H } \quad \begin{array}{llll}
5.554191 & -6.255524 & -0.188944
\end{array} \\
& \begin{array}{llll}
\text { C } & 4.636498 & 1.294151 & 0.861579
\end{array} \\
& \begin{array}{llll}
\mathrm{H} & 5.596272 & 0.770898 & 0.971888
\end{array} \\
& \text { H } \quad 3.934844 \quad 0.877875 \quad 1.587909 \\
& \begin{array}{llll}
\text { C } & 5.414114 & 3.012629 & 2.605457
\end{array} \\
& \text { H } \quad 5.886795 \quad 2.116849 \quad 3.030420 \\
& \mathrm{H} \quad 4.760823 \quad 3.455004 \quad 3.364851 \\
& \begin{array}{llll}
\text { C } & -5.009861 & 3.880963 & 1.125287
\end{array} \\
& \mathrm{H} \quad-6.018173 \quad 4.201634 \quad 0.878225 \\
& \text { C } \quad-4.424503 \quad 4.275295 \quad 2.331251 \\
& \text { H } \quad-4.978920 \quad 4.900993 \quad 3.025041 \\
& \begin{array}{llll}
\text { C } & -3.127783 & 3.862619 & 2.645740
\end{array} \\
& \text { H } \quad-2.672498 \quad 4.163955 \quad 3.584911 \\
& \begin{array}{llll}
\text { C } & -1.006143 & 5.133432 & -2.697871
\end{array} \\
& \text { H } \quad-1.312814 \quad 6.175346 \quad-2.718074 \\
& \begin{array}{llll}
\text { C } & 0.025089 & 4.691125 & -3.530672
\end{array} \\
& \mathrm{H} \quad 0.520938 \quad 5.389829 \quad-4.198582 \\
& \begin{array}{llll}
\text { C } & 0.417142 & 3.351387 & -3.502749
\end{array} \\
& \text { H } \quad 1.217158 \quad 3.000571 \quad-4.148419 \\
& \begin{array}{llll}
\text { C } & -0.684457 & -3.585434 & -2.480168
\end{array} \\
& \text { H } \quad-0.019143 \quad-3.606636 \quad-3.338650 \\
& \begin{array}{llll}
\text { C } & -1.637738 & -4.590872 & -2.306252
\end{array} \\
& \text { H } \quad-1.713571 \quad-5.399893 \quad-3.027490 \\
& \text { C } \quad-2.497826-4.552298 \quad-1.205795 \\
& \text { H } \quad-3.241684-5.332092-1.066202 \\
& \begin{array}{llll}
\text { C } & 0.578457 & -3.795699 & 3.419717
\end{array} \\
& \mathrm{H} \quad 0.675513 \quad-4.871273 \quad 3.538431 \\
& \begin{array}{llll}
\text { C } & 1.176278 & -2.932070 & 4.339571
\end{array} \\
& \text { H } \quad 1.737461 \quad-3.333140 \quad 5.179118 \\
& \begin{array}{llll}
\text { C } & 1.048288 & -1.549141 & 4.179001
\end{array} \\
& \begin{array}{llll}
\mathrm{H} & 1.510619 & -0.871513 & 4.891581
\end{array}
\end{aligned}
$$




$\begin{array}{llll}\mathrm{C} & 6.276391 & 0.594557 & -1.588240 \\ \mathrm{C} & 7.374350 & 0.084775 & -1.545539 \\ \mathrm{C} & 3.515078 & 3.578371 & 0.963202 \\ \mathrm{H} & 3.645687 & 4.625982 & 1.257911 \\ \mathrm{H} & 3.201632 & 3.560067 & -0.085989 \\ \mathrm{H} & 2.699008 & 3.158996 & 1.564632 \\ \mathrm{C} & 8.689725 & -0.560343 & -1.520061 \\ \mathrm{H} & 9.422653 & 0.108035 & -1.991214 \\ \mathrm{H} & 9.013577 & -0.674459 & -0.476822 \\ \mathrm{C} & 8.708788 & -1.930803 & -2.223283 \\ \mathrm{H} & 9.710836 & -2.367914 & -2.168084 \\ \mathrm{H} & 8.003644 & -2.621102 & -1.749261 \\ \mathrm{H} & 8.434519 & -1.832501 & -3.278229\end{array}$

IntA5

B3LYP SCF energy: $\quad 3936.03061028$ a.u.

B3LYP enthalpy: 3935.021554 a.u.

B3LYP free energy: 3935.201252 a.u.

M06-L SCF energy in solution: $\quad 3937.68609563$ a.u.

M06-L enthalpy in solution: $\quad 3936.677039$ a.u.

M06-L free energy in solution: $\quad 3936.856737$ a.u.

Cartesian coordinates

\begin{tabular}{lccc}
\multicolumn{1}{l}{ ATOM X } & Y \\
P & -1.428904 & -1.590808 & -0.424587 \\
P & -1.844081 & 1.645695 & 0.231901 \\
O & -6.254294 & -1.142971 & -0.655085 \\
O & -7.099569 & 0.330384 & -2.229703 \\
O & -5.781260 & -0.261279 & 2.295382 \\
O & -5.430470 & -1.805744 & 3.984587 \\
C & -3.504790 & 1.322353 & -0.477556
\end{tabular}




$$
\begin{array}{lrrr}
\mathrm{C} & -4.206043 & 0.124302 & -0.128512 \\
\mathrm{C} & -5.404020 & -0.079656 & -0.794327 \\
\mathrm{C} & -5.916294 & 0.803585 & -1.746625 \\
\mathrm{C} & -5.254970 & 1.963678 & -2.084336 \\
\mathrm{C} & -4.034603 & 2.202876 & -1.428747 \\
\mathrm{C} & -7.374213 & -0.880546 & -1.513357 \\
\mathrm{C} & -2.686264 & -1.735815 & 0.908648 \\
\mathrm{C} & -2.495476 & -2.665209 & 1.939527 \\
\mathrm{C} & -3.371494 & -2.777895 & 3.032847 \\
\mathrm{C} & -4.445790 & -1.916313 & 3.049693 \\
\mathrm{C} & -4.654578 & -0.990250 & 2.025909 \\
\mathrm{C} & -3.814344 & -0.852904 & 0.932561 \\
\mathrm{C} & -6.342691 & -0.813622 & 3.494482 \\
\mathrm{C} & -2.070838 & 1.761710 & 2.048577 \\
\mathrm{H} & -6.455872 & -0.022665 & 4.241385 \\
\mathrm{C} & -0.992762 & 1.452358 & 2.892159 \\
\mathrm{C} & -3.278764 & 2.206676 & 2.609231 \\
\mathrm{H} & -1.375580 & 3.340320 & -0.310001 \\
\mathrm{H} & --3.277691 & -0.749299 & -0.907360 \\
\mathrm{C} & -1.496268 & 4.454206 & 0.534940 \\
\mathrm{C} & -0.858364 & 3.517645 & -1.606006 \\
\mathrm{C} & -2.327179 & -1.899014 & -1.994165 \\
\mathrm{C} & -3.445915 & -2.747956 & -2.028332 \\
\mathrm{C} & -1.854028 & -1.334839 & -3.188104 \\
\mathrm{H} & -0.664168 & -2.996391 & 0.807088 \\
\mathrm{H} & -0.302673 & -3.032496 & -0.213884 \\
\mathrm{H} & -0.362817 & -4.152333 & -1.056067 \\
\mathrm{H} & -3.49586868 & 2.657541 & -2.814972 \\
\mathrm{H} & 3.110052 & -1.673412 \\
\mathrm{H} & -3.266165
\end{array}
$$




$$
\begin{aligned}
& \begin{array}{llll}
\mathrm{H} & -0.057213 & 1.105472 & 2.460308
\end{array} \\
& \text { H } \quad-4.124486 \quad 2.439004 \quad 1.969148 \\
& \mathrm{H} \quad-1.886979 \quad 4.339308 \quad 1.540161 \\
& \text { H } \quad-3.828726 \quad-3.184940 \quad-1.111241 \\
& \text { H } \quad-0.990374 \quad-0.677350 \quad-3.164307 \\
& \text { H } \quad 0.726856 \quad-2.129086 \quad 1.459015 \\
& \text { H } \quad-1.095669-4.198844 \quad-1.853939 \\
& \begin{array}{llll}
\text { Rh } & -0.094267 & 0.266212 & -0.437377
\end{array} \\
& \begin{array}{llll}
\text { O } & 4.269680 & -1.093955 & -1.947114
\end{array} \\
& \begin{array}{llll}
\text { C } & 6.144719 & 4.476706 & 0.286385
\end{array} \\
& \text { S } \quad \begin{array}{llll}
5.272244 & -0.484444 & -1.067949
\end{array} \\
& \begin{array}{llll}
\text { O } & 6.698170 & 5.511684 & 0.022834
\end{array} \\
& \begin{array}{llll}
\mathrm{N} & 4.388441 & 0.468457 & 0.024542
\end{array} \\
& \begin{array}{lllll}
\text { O } & 6.337893 & 0.379056 & -1.580680
\end{array} \\
& \begin{array}{llll}
\text { C } & 6.041208 & -1.801170 & -0.127382
\end{array} \\
& \begin{array}{llll}
\text { C } & 3.081869 & -0.059622 & 0.448615
\end{array} \\
& \begin{array}{llll}
\text { C } & 7.318888 & -1.611354 & 0.410037
\end{array} \\
& \begin{array}{llll}
\text { C } & 5.354774 & -3.003559 & 0.067011
\end{array} \\
& \text { H } \quad 3.114061 \quad-1.150495 \quad 0.583333 \\
& \text { H } \quad 2.866725 \quad 0.363266 \quad 1.433120 \\
& \text { H } \quad 7.862153 \quad-0.693930 \quad 0.210814 \\
& \begin{array}{llll}
\text { C } & 7.896568 & -2.629028 & 1.164642
\end{array} \\
& \begin{array}{llll}
\text { C } & 5.951734 & -4.009691 & 0.825895
\end{array} \\
& \text { H } \quad 4.386475 \quad-3.159810 \quad-0.396661 \\
& \text { H } \quad 8.892210 \quad-2.485261 \quad 1.576476 \\
& \begin{array}{llll}
\text { C } & 7.225250 & -3.841515 & 1.387881
\end{array} \\
& \mathrm{H} \quad 5.425904 \quad-4.950200 \quad 0.970593 \\
& \begin{array}{llll}
\text { C } & 7.879973 & -4.948071 & 2.178715
\end{array} \\
& \text { H } \quad 7.146296 \quad-5.677982 \quad 2.534128 \\
& \text { H } \quad 8.610127 \quad-5.488142 \quad 1.562269 \\
& \begin{array}{llll}
\mathrm{H} & 8.419427 & -4.554549 & 3.046810
\end{array} \\
& \begin{array}{llll}
\text { C } & 5.323270 & 3.470963 & -0.526887
\end{array} \\
& \text { H } \quad 5.950987 \quad 2.806982 \quad-1.132921
\end{aligned}
$$




\begin{tabular}{|c|c|c|c|}
\hline & 4.548243 & 3.899463 & -1.171346 \\
\hline & 4.858303 & 2.829450 & 0.826649 \\
\hline & 5.910621 & 3.707544 & 1.597259 \\
\hline & -0.750475 & 2.660726 & -2.265220 \\
\hline & 5.550212 & 4.300109 & 2.444803 \\
\hline & 5.143019 & 1.325899 & 0.962534 \\
\hline & 6.207156 & 1.160483 & 0.780563 \\
\hline & 4.941319 & 0.998329 & 1.993227 \\
\hline & 3.421079 & 3.209949 & 1.192501 \\
\hline & 3.285719 & 4.296860 & 1.145390 \\
\hline & 2.697326 & 2.763211 & 0.502041 \\
\hline & 3.171275 & 2.893930 & 2.214359 \\
\hline & 6.807069 & 3.158959 & 1.914681 \\
\hline & 1.978278 & 0.246238 & -0.502294 \\
\hline & 1.552912 & 0.492603 & -1.674791 \\
\hline & 1.732798 & 0.752057 & -3.123027 \\
\hline & 2.204818 & -0.143263 & -3.548146 \\
\hline & 0.764895 & 0.881773 & -3.620590 \\
\hline & 2.630094 & 1.976653 & -3.385134 \\
\hline & 2.192265 & 2.886200 & -2.960528 \\
\hline & 3.620652 & 1.829076 & -2.947690 \\
\hline & 2.748804 & 2.121510 & -4.463813 \\
\hline & -3.399170 & 2.343507 & 3.992949 \\
\hline & -4.334728 & 2.694875 & 4.419143 \\
\hline & -2.321591 & 2.034438 & 4.826700 \\
\hline & -2.419221 & 2.141329 & 5.903250 \\
\hline & -1.118802 & 1.587666 & 4.275114 \\
\hline & -0.279953 & 1.344957 & 4.921206 \\
\hline & -1.111146 & 5.719943 & 0.088000 \\
\hline & -1.207054 & 6.574459 & 0.751624 \\
\hline & -0.607406 & 5.888583 & -1.202604 \\
\hline & -0.309290 & 6.874829 & -1.546314 \\
\hline & -0.484805 & 4.785067 & -2.051340 \\
\hline
\end{tabular}




$$
\begin{array}{lrrr}
\mathrm{H} & -0.094655 & 4.910707 & -3.057332 \\
\mathrm{C} & -2.488514 & -1.614633 & -4.398994 \\
\mathrm{H} & -2.115862 & -1.172488 & -5.318462 \\
\mathrm{C} & -3.598446 & -2.461444 & -4.427645 \\
\mathrm{H} & -4.090927 & -2.680243 & -5.370779 \\
\mathrm{C} & -4.074628 & -3.027449 & -3.242266 \\
\mathrm{H} & -4.935191 & -3.690346 & -3.261476 \\
\mathrm{C} & 0.522767 & -5.217368 & -0.874803 \\
\mathrm{H} & 0.468024 & -6.077428 & -1.535759 \\
\mathrm{C} & 1.468731 & -5.180447 & 0.149746 \\
\mathrm{H} & 2.149777 & -6.014823 & 0.291238 \\
\mathrm{C} & 1.536402 & -4.068121 & 0.994302 \\
\mathrm{H} & 2.268751 & -4.034489 & 1.796260
\end{array}
$$

IntA6

B3LYP SCF energy: $\quad 3936.03740804$ a.u.

B3LYP enthalpy: $\quad 3935.027660$ a.u.

B3LYP free energy: $\quad 3935.200281$ a.u.

M06-L SCF energy in solution: $\quad 3937.68841728$ a.u.

M06-L enthalpy in solution: $\quad 3936.678669$ a.u.

M06-L free energy in solution: $\quad 3936.851290$ a.u.

Cartesian coordinates

\begin{tabular}{lccl}
\multicolumn{2}{l}{ ATOM X } & Y \\
P & 1.143408 & 1.602941 & -0.202770 \\
P & 1.968777 & -1.672794 & 0.030440 \\
O & 5.971193 & 1.775713 & -0.211859 \\
O & 7.037197 & 0.723711 & -1.979741 \\
O & 5.512905 & 0.311070 & 2.532008 \\
O & 4.925238 & 1.503155 & 4.429570 \\
C & 3.572055 & -1.012928 & -0.577868
\end{tabular}




$$
\begin{aligned}
& \begin{array}{llll}
\text { C } & 4.097272 & 0.185580 & -0.005727
\end{array} \\
& \begin{array}{llll}
\text { C } & 5.270661 & 0.656629 & -0.572788
\end{array} \\
& \begin{array}{llll}
\text { C } & 5.914470 & 0.027509 & -1.638625
\end{array} \\
& \begin{array}{llll}
\text { C } & 5.422333 & -1.132681 & -2.194938
\end{array} \\
& \begin{array}{llll}
\text { C } & 4.233587 & -1.639433 & -1.640905
\end{array} \\
& \begin{array}{llll}
\text { C } & 7.084087 & 1.863922 & -1.111563
\end{array} \\
& \begin{array}{llll}
\text { C } & 2.324219 & 1.662166 & 1.223974
\end{array} \\
& \begin{array}{llll}
\text { C } & 2.000162 & 2.389653 & 2.376034
\end{array} \\
& \begin{array}{llll}
\text { C } & 2.812148 & 2.407001 & 3.523656
\end{array} \\
& \begin{array}{llll}
\text { C } & 3.965893 & 1.657632 & 3.472339
\end{array} \\
& \begin{array}{llll}
\text { C } & 4.314413 & 0.941975 & 2.327516
\end{array} \\
& \begin{array}{llll}
\text { C } & 3.542232 & 0.909416 & 1.176976
\end{array} \\
& \begin{array}{llll}
\text { C } & 5.897431 & 0.602407 & 3.882321
\end{array} \\
& \begin{array}{llll}
\text { C } & 2.289507 & -2.176739 & 1.772133
\end{array} \\
& \begin{array}{llll}
\text { C } & 1.215057 & -2.234087 & 2.673894
\end{array} \\
& \begin{array}{llll}
\text { C } & 3.573353 & -2.536571 & 2.211968
\end{array} \\
& \begin{array}{llll}
\text { C } & 1.748845 & -3.259557 & -0.885867
\end{array} \\
& \begin{array}{llll}
\text { C } & 2.163668 & -4.494957 & -0.368180
\end{array} \\
& \text { C } \quad \begin{array}{llll}
1.140638 & -3.221871 & -2.152356
\end{array} \\
& \begin{array}{llll}
\text { C } & 2.186464 & 2.120822 & -1.631897
\end{array} \\
& \text { C } \quad 3.042472 \quad 3.230465 \quad-1.521342 \\
& \begin{array}{llll}
\text { C } & 2.130104 & 1.421163 & -2.844220
\end{array} \\
& \begin{array}{llll}
\text { C } & -0.938978 & 2.961508 & 1.116454
\end{array} \\
& \begin{array}{llll}
\text { C } & -0.010449 & 3.025593 & 0.061099
\end{array} \\
& \begin{array}{llll}
\text { C } & -0.035830 & 4.143303 & -0.784409
\end{array} \\
& \text { H } \quad \begin{array}{llll}
5.926227 & -1.631135 & -3.015274
\end{array} \\
& \text { H } \quad 3.826875 \quad-2.552073 \quad-2.058634 \\
& \text { H } \quad 6.998528 \quad 2.779119 \quad-1.707807 \\
& \begin{array}{llll}
\mathrm{H} & 8.018112 & 1.849259 & -0.540817
\end{array} \\
& \mathrm{H} \quad 1.097846 \quad 2.985525 \quad 2.396580 \\
& \mathrm{H} \quad 2.544560 \quad 2.986120 \quad 4.400182 \\
& \text { H } \quad 5.904852 \quad-0.324516 \quad 4.467182 \\
& \begin{array}{llll}
\mathrm{H} & 6.881159 & 1.081752 & 3.887148
\end{array}
\end{aligned}
$$




$$
\begin{aligned}
& \mathrm{H} \quad 0.218429 \quad-1.965950 \quad 2.337264 \\
& \mathrm{H} \quad 4.416747 \quad-2.485199 \quad 1.530379 \\
& \text { H } \quad 2.629460-4.547906 \quad 0.610275 \\
& \text { H } \quad 3.113914 \quad 3.771468 \quad-0.582184 \\
& \begin{array}{llll}
\mathrm{H} & 1.491771 & 0.547174 & -2.923828
\end{array} \\
& \text { H } \quad-0.955143 \quad 2.091474 \quad 1.767182 \\
& \text { H } \quad 0.654716 \quad 4.217536 \quad-1.616379 \\
& \begin{array}{llll}
\mathrm{Rh} & 0.014227 & -0.364172 & -0.329886
\end{array} \\
& \begin{array}{llll}
\text { O } & -4.922659 & 1.690686 & -1.236529
\end{array} \\
& \begin{array}{llll}
\text { C } & -2.132371 & -2.860891 & -0.193325
\end{array} \\
& \begin{array}{llll}
\text { S } & -5.501678 & 0.393299 & -0.876438
\end{array} \\
& \begin{array}{llll}
\text { O } & -1.089297 & -2.308771 & 0.127676
\end{array} \\
& \mathrm{~N} \quad-4.356232 \quad-0.339935 \quad 0.127680 \\
& \begin{array}{llll}
\text { O } & -5.881501 & -0.596027 & -1.891821
\end{array} \\
& \begin{array}{llll}
\text { C } & -6.937486 & 0.681149 & 0.154560
\end{array} \\
& \begin{array}{llll}
\text { C } & -3.143780 & 0.406676 & 0.498846
\end{array} \\
& \begin{array}{llll}
\text { C } & -8.036109 & -0.176440 & 0.072531
\end{array} \\
& \begin{array}{llll}
\text { C } & -6.946385 & 1.778980 & 1.022172
\end{array} \\
& \begin{array}{llll}
\mathrm{H} & -3.409501 & 1.450809 & 0.690437
\end{array} \\
& \mathrm{H} \quad-2.786805 \quad-0.018312 \quad 1.442124 \\
& \mathrm{H} \quad-8.027893 \quad-1.001276 \quad-0.631577 \\
& \begin{array}{llll}
\text { C } & -9.146468 & 0.063512 & 0.882805
\end{array} \\
& \begin{array}{llll}
\text { C } & -8.063293 & 2.001588 & 1.820791
\end{array} \\
& \begin{array}{llll}
\text { H } & -6.101888 & 2.459701 & 1.051298
\end{array} \\
& \begin{array}{llll}
\mathrm{H} & -10.004441 & -0.600417 & 0.818201
\end{array} \\
& \begin{array}{llll}
\text { C } & -9.179996 & 1.150100 & 1.766602
\end{array} \\
& \text { H } \quad-8.076196 \quad 2.856604 \quad 2.492001 \\
& \begin{array}{llll}
\text { C } & -10.396903 & 1.424976 & 2.616077
\end{array} \\
& \text { H } \quad-10.117086 \quad 1.766770 \quad 3.618278 \\
& \begin{array}{llll}
\mathrm{H} & -11.015501 & 2.212458 & 2.166426
\end{array} \\
& \text { H } \quad-11.023871 \quad 0.534544 \quad 2.720696 \\
& \begin{array}{llll}
\text { C } & -3.074785 & -3.716879 & 0.626725
\end{array}
\end{aligned}
$$

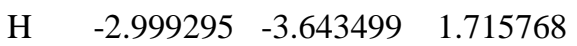




$$
\begin{aligned}
& \text { H } \quad-3.043822 \quad-4.769521 \quad 0.323900 \\
& \begin{array}{llll}
\text { C } & -4.216253 & -2.936894 & -0.140880
\end{array} \\
& \begin{array}{llll}
\text { C } & -3.169105 & -2.609551 & -1.263895
\end{array} \\
& \text { H } \quad \begin{array}{llll}
0.801849 & -2.270352 & -2.554877
\end{array} \\
& \text { H } \quad-3.164187 \quad-3.413397 \quad-2.009623 \\
& \begin{array}{llll}
\text { C } & -4.653543 & -1.664748 & 0.675204
\end{array} \\
& \mathrm{H} \quad \begin{array}{llll}
-5.734678 & -1.724198 & 0.838878
\end{array} \\
& \text { H } \quad-4.204529-1.695261 \quad 1.674753 \\
& \text { C } \quad-5.421011 \quad-3.767911 \quad-0.579293 \\
& \text { H } \quad-5.102965 \quad-4.675142 \quad-1.104982 \\
& \text { H } \quad-6.023432-4.075485 \quad 0.284243 \\
& \text { H } \quad-6.054892 \quad-3.183760 \quad-1.254166 \\
& \text { H } \quad-3.191040 \quad-1.640869-1.756341 \\
& \begin{array}{llll}
\text { C } & -2.059321 & 0.401092 & -0.527410
\end{array} \\
& \begin{array}{llll}
\text { C } & -1.559696 & 0.510861 & -1.662655
\end{array} \\
& \begin{array}{llll}
\text { C } & -1.399036 & 0.845537 & -3.093694
\end{array} \\
& \text { H } \quad-0.727143 \quad 1.709191 \quad-3.177066 \\
& \begin{array}{llll}
\mathrm{H} & -0.897494 & 0.019319 & -3.613517
\end{array} \\
& \begin{array}{llll}
\text { C } & -2.741033 & 1.164002 & -3.781242
\end{array} \\
& \mathrm{H} \quad-3.415586 \quad 0.302660 \quad-3.760823 \\
& \mathrm{H} \quad-3.250563 \quad 1.992780 \quad-3.284968 \\
& \text { H } \quad-2.558068 \quad 1.432691 \quad-4.827202 \\
& \begin{array}{llll}
\text { C } & 3.774829 & -2.950313 & 3.530170
\end{array} \\
& \text { H } \quad 4.771257 \quad-3.233857 \quad 3.858310 \\
& \begin{array}{llll}
\text { C } & 2.701036 & -3.003929 & 4.421736
\end{array} \\
& \text { H } \quad 2.860561 \quad-3.323961 \quad 5.447612 \\
& \text { C } \quad 1.421744 \quad-2.645310 \quad 3.991252 \\
& \mathrm{H} \quad 0.584304 \quad-2.684219 \quad 4.682457 \\
& \text { C } \quad \begin{array}{llll}
1.978205 & -5.666962 & -1.105561
\end{array} \\
& \text { H } \quad 2.302835 \quad-6.617734 \quad-0.692147 \\
& \begin{array}{llll}
\text { C } & 1.381898 & -5.618723 & -2.366521
\end{array} \\
& \text { H } \quad 1.242268 \quad-6.531557 \quad-2.938758 \\
& \begin{array}{llll}
\text { C } & 0.964717 & -4.392120 & -2.890729
\end{array}
\end{aligned}
$$




$$
\begin{array}{llll}
\mathrm{H} & 0.500921 & -4.347493 & -3.872452 \\
\mathrm{C} & 2.904290 & 1.827468 & -3.933297 \\
\mathrm{H} & 2.856470 & 1.273583 & -4.866598 \\
\mathrm{C} & 3.739413 & 2.939995 & -3.821115 \\
\mathrm{H} & 4.337605 & 3.259410 & -4.669843 \\
\mathrm{C} & 3.807940 & 3.640283 & -2.612838 \\
\mathrm{H} & 4.456931 & 4.506885 & -2.520100 \\
\mathrm{C} & -0.952983 & 5.176776 & -0.569764 \\
\mathrm{H} & -0.959300 & 6.032287 & -1.239101 \\
\mathrm{C} & -1.849926 & 5.114271 & 0.494664 \\
\mathrm{H} & -2.556438 & 5.921807 & 0.663000 \\
\mathrm{C} & -1.838667 & 4.001949 & 1.341191 \\
\mathrm{H} & -2.533960 & 3.943375 & 2.174436
\end{array}
$$

IntA7

B3LYP SCF energy: $\quad 3936.03282545$ a.u.

B3LYP enthalpy: $\quad 3935.022918$ a.u.

B3LYP free energy: 3935.195008 a.u.

M06-L SCF energy in solution: $\quad 3937.68534715$ a.u.

M06-L enthalpy in solution: $\quad 3936.675440$ a.u.

M06-L free energy in solution: $\quad 3936.847530$ a.u.

Cartesian coordinates

\begin{tabular}{lccc}
\multicolumn{1}{l}{ ATOM X } & Y \\
P & -1.261364 & -1.585272 & -0.153900 \\
P & -1.914692 & 1.715789 & -0.098477 \\
O & -6.107086 & -1.503533 & 0.018777 \\
O & -7.185637 & -0.497266 & -1.766658 \\
O & -5.460742 & 0.069811 & 2.652613 \\
O & -4.846541 & -1.034039 & 4.593506 \\
C & -3.570051 & 1.109270 & -0.613702
\end{tabular}




$$
\begin{aligned}
& \begin{array}{llll}
\text { C } & -4.141096 & -0.014890 & 0.057326
\end{array} \\
& \begin{array}{llll}
\text { C } & -5.364918 & -0.444513 & -0.430163
\end{array} \\
& \begin{array}{llll}
\text { C } & -6.015750 & 0.155858 & -1.508425
\end{array} \\
& \begin{array}{llll}
\text { C } & -5.477340 & 1.242747 & -2.161542
\end{array} \\
& \text { C } \quad-4.237072 \quad 1.707296 \quad-1.689482 \\
& \begin{array}{llll}
\text { C } & -7.314498 & -1.504424 & -0.754087
\end{array} \\
& \begin{array}{llll}
\text { C } & -2.394298 & -1.506099 & 1.305372
\end{array} \\
& \begin{array}{llll}
\text { C } & -2.053116 & -2.178603 & 2.485625
\end{array} \\
& \begin{array}{llll}
\text { C } & -2.815714 & -2.088896 & 3.663053
\end{array} \\
& \begin{array}{llll}
\text { C } & -3.935187 & -1.288872 & 3.610988
\end{array} \\
& \text { C } \quad-4.300026 \quad-0.625840 \quad 2.439321 \\
& \begin{array}{llll}
\text { C } & -3.576588 & -0.698458 & 1.259275
\end{array} \\
& \begin{array}{llll}
\text { C } & -5.775784 & -0.092076 & 4.042157
\end{array} \\
& \text { C } \quad-2.156386 \quad 2.342910 \quad 1.615373 \\
& \begin{array}{llll}
\text { C } & -1.064381 & 2.369576 & 2.497089
\end{array} \\
& \begin{array}{llll}
\text { C } & -3.401663 & 2.824656 & 2.050591
\end{array} \\
& \begin{array}{llll}
\text { C } & -1.630688 & 3.223973 & -1.123055
\end{array} \\
& \begin{array}{llll}
\text { C } & -1.920391 & 4.516730 & -0.663895
\end{array} \\
& \begin{array}{llll}
\text { C } & -1.092671 & 3.063831 & -2.411789
\end{array} \\
& \begin{array}{llll}
\text { C } & -2.361931 & -2.103793 & -1.537528
\end{array} \\
& \begin{array}{llll}
\text { C } & -3.310547 & -3.123726 & -1.348504
\end{array} \\
& \text { C } \quad-2.244498 \quad-1.506013 \quad-2.799029 \\
& \begin{array}{llll}
\text { C } & 0.830699 & -2.968515 & 1.121810
\end{array} \\
& \begin{array}{llll}
\text { C } & -0.172897 & -3.050729 & 0.139153
\end{array} \\
& \begin{array}{llll}
\text { C } & -0.262399 & -4.219718 & -0.630912
\end{array} \\
& \text { H } \quad-5.984039 \quad 1.717243 \quad-2.994247 \\
& \text { H } \quad-3.795066 \quad 2.565323 \quad-2.180822 \\
& \mathrm{H} \quad \begin{array}{llll}
-7.442601 & -2.482054 & -1.227689
\end{array} \\
& \text { H } \quad-8.165463 \quad-1.263849-0.105875 \\
& \text { H } \quad-1.175549 \quad-2.811085 \quad 2.505575 \\
& \text { H } \quad-2.537501 \quad-2.626107 \quad 4.562618 \\
& \text { H } \quad-5.666243 \quad 0.870906 \quad 4.555145 \\
& \text { H } \quad-6.792512 \quad-0.482277 \quad 4.143293
\end{aligned}
$$




$$
\begin{aligned}
& \text { H } \quad-0.096936 \quad 2.006431 \quad 2.165338 \\
& \mathrm{H} \quad-4.258661 \quad 2.798665 \quad 1.384643 \\
& \mathrm{H} \quad-2.329835 \quad 4.663775 \quad 0.329971 \\
& \text { H } \quad-0.852868 \quad 2.065765 \quad-2.770537 \\
& \text { H } \quad-3.427105 \quad-3.584717 \quad-0.371963 \\
& \text { H } \quad-1.528968 \quad-0.701650 \quad-2.939906 \\
& \text { H } \quad 0.925766 \quad-2.065440 \quad 1.717745 \\
& \text { H } \quad-1.017795 \quad-4.313410 \quad-1.402480 \\
& \begin{array}{llll}
\mathrm{Rh} & -0.046234 & 0.308651 & -0.420473
\end{array} \\
& \begin{array}{llll}
\text { O } & 1.246554 & 2.239861 & 0.022090
\end{array} \\
& \text { O } 4.856346-2.159605 \quad 1.406565 \\
& \begin{array}{llll}
\text { C } & 2.304580 & 2.602548 & -0.468424
\end{array} \\
& \begin{array}{llll}
\text { S } & 5.379155 & -1.411105 & 0.256373
\end{array} \\
& \begin{array}{llll}
\text { C } & 3.656179 & 2.910730 & 0.161594
\end{array} \\
& \begin{array}{llll}
\mathrm{N} & 4.397056 & 0.019087 & 0.154037
\end{array} \\
& \begin{array}{llll}
\text { O } & 5.482264 & -2.054102 & -1.061057
\end{array} \\
& \begin{array}{llll}
\text { C } & 6.978041 & -0.733304 & 0.678417
\end{array} \\
& \begin{array}{llll}
\text { C } & 4.278570 & 2.289972 & -1.145417
\end{array} \\
& \text { H } \quad 3.852895 \quad 3.988075 \quad 0.208268 \\
& \mathrm{H} \quad 3.876026 \quad 2.460085 \quad 1.131631 \\
& \begin{array}{llll}
\text { C } & 2.991151 & -0.231845 & 0.557195
\end{array} \\
& \begin{array}{llll}
\text { C } & 8.031517 & -0.856129 & -0.227910
\end{array} \\
& \begin{array}{llll}
\text { C } & 7.167169 & -0.148715 & 1.935038
\end{array} \\
& \begin{array}{llll}
\mathrm{H} & 3.012690 & -1.085225 & 1.239503
\end{array} \\
& \mathrm{H} \quad 2.648540 \quad 0.618170 \quad 1.149023 \\
& \text { H } \quad 7.865390 \quad-1.332112-1.188396 \\
& \begin{array}{llll}
\text { C } & 9.290092 & -0.371083 & 0.130172
\end{array} \\
& \begin{array}{llll}
\text { C } & 8.428950 & 0.329122 & 2.271630
\end{array} \\
& \begin{array}{llll}
\text { H } & 6.342190 & -0.078528 & 2.636235
\end{array} \\
& \text { H } \quad 10.114696 \quad-0.464488 \quad-0.571411 \\
& \begin{array}{llll}
\text { C } & 9.510162 & 0.224797 & 1.378861
\end{array} \\
& \begin{array}{llll}
\mathrm{H} & 8.583491 & 0.784742 & 3.246390
\end{array} \\
& \begin{array}{llll}
\text { C } & 10.880447 & 0.718708 & 1.774267
\end{array}
\end{aligned}
$$




$$
\begin{aligned}
& \text { H } \quad 10.822981 \quad 1.667949 \quad 2.317726 \\
& \begin{array}{llll}
\text { H } & 11.377602 & -0.002719 & 2.435530
\end{array} \\
& \text { H } \quad 11.5239390 .862159 \quad 0.901262 \\
& \begin{array}{llll}
\text { C } & 1.534340 & -0.783690 & -1.641639
\end{array} \\
& \begin{array}{llll}
\text { C } & 2.030108 & -0.499066 & -0.538125
\end{array} \\
& \begin{array}{llll}
\text { C } & 2.885112 & 2.493543 & -1.856789
\end{array} \\
& \begin{array}{llll}
\text { H } & 2.850773 & 3.458757 & -2.376347
\end{array} \\
& \text { H } \quad 2.485737 \quad 1.710756 \quad-2.503982 \\
& \begin{array}{llll}
\text { C } & 5.422343 & 3.102560 & -1.760348
\end{array} \\
& \begin{array}{llll}
\mathrm{H} & 6.305757 & 3.084567 & -1.111942
\end{array} \\
& \text { H } \quad 5.712979 \quad 2.695372 \quad-2.736284 \\
& \mathrm{H} \quad 5.128610 \quad 4.147871 \quad-1.907295 \\
& \begin{array}{llll}
\text { C } & 4.701651 & 0.786245 & -1.074055
\end{array} \\
& \text { H } \quad 4.311346 \quad 0.271099 \quad-1.960619 \\
& \text { H } \quad 5.790994 \quad 0.761736 \quad-1.158130 \\
& \text { C } \quad 1.369629-1.414616 \quad-2.968100 \\
& \text { H } \quad 0.668802 \quad-2.252506 \quad-2.865072 \\
& \text { H } \quad 0.902694 \quad-0.710665 \quad-3.667921 \\
& \begin{array}{llll}
\text { C } & 2.706089 & -1.925267 & -3.541150
\end{array} \\
& \text { H } \quad 3.385480 \quad-1.095622 \quad-3.761772 \\
& \text { H } \quad 3.210588 \quad-2.592561 \quad-2.837325 \\
& \text { H } \quad 2.522854 \quad-2.468996 \quad-4.473837 \\
& \begin{array}{llll}
\text { C } & -0.865179 & 4.171160 & -3.228960
\end{array} \\
& \text { H } \quad-0.459832 \quad 4.032337 \quad-4.227690 \\
& \begin{array}{llll}
\text { C } & -1.157767 & 5.455900 & -2.762674
\end{array} \\
& \text { H } \quad-0.978213 \quad 6.319736 \quad-3.396535 \\
& \begin{array}{llll}
\text { C } & -1.682107 & 5.625134 & -1.480421
\end{array} \\
& \text { H } \quad-1.910189 \quad 6.621367 \quad-1.112023 \\
& \begin{array}{llll}
\text { C } & -3.547394 & 3.328805 & 3.344231
\end{array} \\
& \text { H } \quad-4.513406 \quad 3.706481 \quad 3.668443 \\
& \begin{array}{llll}
\text { C } & -2.456340 & 3.351323 & 4.216326
\end{array} \\
& \mathrm{H} \quad-2.572515 \quad 3.741701 \quad 5.223514 \\
& \begin{array}{llll}
\text { C } & -1.216041 & 2.871162 & 3.790614
\end{array}
\end{aligned}
$$




$$
\begin{array}{lrrr}
\mathrm{H} & -0.365575 & 2.885032 & 4.466557 \\
\mathrm{C} & -3.052148 & -1.924255 & -3.858727 \\
\mathrm{H} & -2.956397 & -1.449829 & -4.831373 \\
\mathrm{C} & -3.982649 & -2.946644 & -3.667050 \\
\mathrm{H} & -4.608859 & -3.274488 & -4.491993 \\
\mathrm{C} & -4.111091 & -3.544885 & -2.410070 \\
\mathrm{H} & -4.834905 & -4.340393 & -2.255641 \\
\mathrm{C} & 1.711940 & -4.026059 & 1.337575 \\
\mathrm{H} & 2.491347 & -3.934783 & 2.087782 \\
\mathrm{C} & 1.611212 & -5.185974 & 0.564151 \\
\mathrm{H} & 2.304303 & -6.006947 & 0.722506 \\
\mathrm{C} & 0.624832 & -5.278851 & -0.416214 \\
\mathrm{H} & 0.541590 & -6.175687 & -1.023668
\end{array}
$$

IntA8

B3LYP SCF energy: 3936.04022180 a.u.

B3LYP enthalpy: 3935.030148 a.u.

B3LYP free energy: $\quad 3935.207818$ a.u.

M06-L SCF energy in solution: $\quad 3937.68561759$ a.u.

M06-L enthalpy in solution: $\quad 3936.675544$ a.u.

M06-L free energy in solution: $\quad 3936.853214$ a.u.

Cartesian coordinates

\begin{tabular}{lccc}
\multicolumn{1}{l}{ ATOM X } & Y \\
P & -0.806047 & 1.406934 & 0.448665 \\
P & -1.779718 & -1.472689 & -0.818882 \\
O & -5.081153 & 0.787471 & 2.656914 \\
O & -5.284214 & -1.090435 & 3.998636 \\
O & -6.022483 & 0.771540 & -0.310340 \\
O & -6.293906 & 2.732873 & -1.512150 \\
C & -2.908018 & -1.426492 & 0.640205
\end{tabular}




$$
\begin{aligned}
& \begin{array}{llll}
\text { C } & -3.587054 & -0.212393 & 0.968002
\end{array} \\
& \begin{array}{llll}
\text { C } & -4.347643 & -0.240353 & 2.126183
\end{array} \\
& \begin{array}{llll}
\text { C } & -4.471083 & -1.366901 & 2.937968
\end{array} \\
& \begin{array}{llll}
\text { C } & -3.841426 & -2.550686 & 2.623464
\end{array} \\
& \begin{array}{llll}
\text { C } & -3.055242 & -2.554163 & 1.458118
\end{array} \\
& \begin{array}{llll}
\text { C } & -5.646226 & 0.291704 & 3.877203
\end{array} \\
& \begin{array}{llll}
\text { C } & -2.503141 & 1.900397 & -0.098360
\end{array} \\
& \begin{array}{llll}
\text { C } & -2.692921 & 3.087641 & -0.816665
\end{array} \\
& \begin{array}{llll}
\text { C } & -3.940052 & 3.469354 & -1.342119
\end{array} \\
& \begin{array}{llll}
\text { C } & -4.990960 & 2.607111 & -1.122678
\end{array} \\
& \begin{array}{llll}
\text { C } & -4.823244 & 1.428821 & -0.397054
\end{array} \\
& \begin{array}{llll}
\text { C } & -3.609995 & 1.028154 & 0.140602
\end{array} \\
& \begin{array}{llll}
\text { C } & -6.952289 & 1.530338 & -1.094265
\end{array} \\
& \begin{array}{llll}
\text { C } & -2.872432 & -1.142528 & -2.263668
\end{array} \\
& \begin{array}{llll}
\text { C } & -2.335014 & -0.501197 & -3.389577
\end{array} \\
& \begin{array}{llll}
\text { C } & -4.214043 & -1.557427 & -2.283369
\end{array} \\
& \begin{array}{llll}
\text { C } & -1.327546 & -3.258588 & -0.986890
\end{array} \\
& \begin{array}{llll}
\text { C } & -1.844988 & -4.077317 & -2.000496
\end{array} \\
& \begin{array}{llll}
\text { C } & -0.413835 & -3.810287 & -0.071066
\end{array} \\
& \begin{array}{llll}
\text { C } & -0.939520 & 1.249435 & 2.277582
\end{array} \\
& \begin{array}{llll}
\text { C } & -1.824000 & 2.046335 & 3.023176
\end{array} \\
& \begin{array}{llll}
\text { C } & -0.104577 & 0.342206 & 2.944967
\end{array} \\
& \begin{array}{llll}
\text { C } & 0.608037 & 3.246949 & -1.124785
\end{array} \\
& \begin{array}{llll}
\text { C } & 0.199177 & 2.934283 & 0.183975
\end{array} \\
& \begin{array}{llll}
\text { C } & 0.553548 & 3.798506 & 1.229567
\end{array} \\
& \text { H } \quad-3.947227 \quad-3.435735 \quad 3.240562 \\
& \text { H } \quad-2.556662 \quad-3.476934 \quad 1.192471 \\
& \mathrm{H} \quad-5.233476 \quad 0.851813 \quad 4.724156 \\
& \text { H } \quad-6.736471 \quad 0.380330 \quad 3.838236 \\
& \text { H } \quad-1.855338 \quad 3.753034 \quad-0.979501 \\
& \text { H } \quad-4.066036 \quad 4.396213 \quad-1.890275 \\
& \text { H } \quad-7.244326 \quad 0.948729 \quad-1.977124 \\
& \text { H } \quad-7.822746 \quad 1.785546 \quad-0.482852
\end{aligned}
$$




$$
\begin{aligned}
& \text { H } \quad-1.299562 \quad-0.172038 \quad-3.369842 \\
& \text { H } \quad-4.645860 \quad-2.046092 \quad-1.414943 \\
& \text { H } \quad-2.546272 \quad-3.674284 \quad-2.722708 \\
& \text { H } \quad 0.005098 \quad-3.181999 \quad 0.710808 \\
& \text { H } \quad-2.484476 \quad 2.745445 \quad 2.518770 \\
& \text { H } \quad 0.582343 \quad-0.271000 \quad 2.370072 \\
& \text { H } \quad 0.357874 \quad 2.572214 \quad-1.939336 \\
& \begin{array}{llll}
\mathrm{H} & 0.262107 & 3.570231 & 2.248644
\end{array} \\
& \mathrm{Rh} \quad 0.124646 \quad-0.283340 \quad-0.736141 \\
& \begin{array}{llll}
\text { O } & 1.141297 & -1.558879 & -2.266472
\end{array} \\
& \begin{array}{llll}
\text { O } & 2.347627 & 0.542468 & -0.760601
\end{array} \\
& \begin{array}{llll}
\text { C } & 2.146169 & -2.224076 & -2.069841
\end{array} \\
& \begin{array}{llll}
\text { S } & 3.404436 & 0.755981 & 0.261057
\end{array} \\
& \begin{array}{llll}
\text { C } & 3.379577 & -2.455872 & -2.924487
\end{array} \\
& \begin{array}{llll}
\mathrm{N} & 4.401219 & -0.599317 & 0.148639
\end{array} \\
& \begin{array}{llll}
\text { O } & 3.061235 & 0.912378 & 1.673233
\end{array} \\
& \begin{array}{llll}
\text { C } & 4.351781 & 2.176778 & -0.266986
\end{array} \\
& \begin{array}{llll}
\text { C } & 4.169919 & -2.511518 & -1.556023
\end{array} \\
& \text { H } \quad 3.361276 \quad-3.436090 \quad-3.414589 \\
& \text { H } \quad 3.623719 \quad-1.685996 \quad-3.662929 \\
& \begin{array}{llll}
\text { C } & 5.368618 & -0.821420 & 1.249928
\end{array} \\
& \begin{array}{llll}
\text { C } & 5.065674 & 2.899098 & 0.694534
\end{array} \\
& \begin{array}{llll}
\text { C } & 4.420201 & 2.518779 & -1.620889
\end{array} \\
& \begin{array}{llll}
\mathrm{H} & 6.385027 & -0.627692 & 0.877089
\end{array} \\
& \begin{array}{llll}
\mathrm{H} & 5.161324 & -0.084945 & 2.030952
\end{array} \\
& \text { H } \quad 4.972103 \quad 2.650250 \quad 1.746116 \\
& \begin{array}{llll}
\text { C } & 5.865414 & 3.963100 & 0.284782
\end{array} \\
& \begin{array}{llll}
\text { C } & 5.230289 & 3.584134 & -2.008610
\end{array} \\
& \mathrm{H} \quad 3.824556 \quad 1.984512-2.352648 \\
& \text { H } \quad 6.411626 \quad 4.533672 \quad 1.031272 \\
& \begin{array}{llll}
\text { C } & 5.971138 & 4.316961 & -1.069271
\end{array} \\
& \text { H } \quad 5.280016 \quad 3.857630 \quad-3.059315 \\
& \begin{array}{llll}
\text { C } & 6.868814 & 5.449893 & -1.502961
\end{array}
\end{aligned}
$$




\begin{tabular}{|c|c|c|c|}
\hline & 7.898113 & 5.096585 & -1.648189 \\
\hline & 6.537036 & 5.886520 & -2.449883 \\
\hline & 6.902635 & 6.245065 & -0.751409 \\
\hline & 4.740261 & -1.099538 & -1.195761 \\
\hline & 5.832981 & -1.118561 & -1.288206 \\
\hline & 4.382389 & -0.370970 & -1.925821 \\
\hline & 2.806030 & -2.786179 & -0.832548 \\
\hline & 2.601195 & -2.304535 & 0.123671 \\
\hline & 2.620631 & -3.863447 & -0.754295 \\
\hline & -5.000555 & -1.336002 & -3.414852 \\
\hline & -6.036135 & -1.665484 & -3.423761 \\
\hline & -4.457835 & -0.697465 & -4.533320 \\
\hline & -5.072630 & -0.524864 & -5.412373 \\
\hline & -3.125246 & -0.280517 & -4.519035 \\
\hline & -2.701061 & 0.219167 & -5.385483 \\
\hline & -1.464897 & -5.419440 & -2.090011 \\
\hline & -1.874386 & -6.039975 & -2.882183 \\
\hline & -0.573645 & -5.963047 & -1.164865 \\
\hline & -0.289019 & -7.009485 & -1.230423 \\
\hline & -0.050438 & -5.154742 & -0.151251 \\
\hline & 0.639067 & -5.571811 & 0.578349 \\
\hline & -0.151432 & 0.233368 & 4.335878 \\
\hline & 0.500182 & -0.472210 & 4.843670 \\
\hline & -1.031582 & 1.028994 & \\
\hline & -1.067573 & 0.943601 & 6.154232 \\
\hline & -1.866589 & 1.936239 & 4.413606 \\
\hline & -2.549709 & 2.561203 & 4.982770 \\
\hline & 1.282631 & 4.961261 & 0.967983 \\
\hline & 1.547998 & 5.622589 & 1.788172 \\
\hline & 1.660774 & 5.276972 & -0.337167 \\
\hline & 2.218938 & 6.187075 & -0.538520 \\
\hline & 1.322451 & 4.415823 & -1.384481 \\
\hline & 1.617700 & 4.652603 & -2.402960 \\
\hline
\end{tabular}




$$
\begin{array}{llll}
\mathrm{C} & 5.290247 & -2.162945 & 1.832497 \\
\mathrm{C} & 5.262468 & -3.246852 & 2.371060 \\
\mathrm{C} & 5.228624 & -3.608556 & -1.455316 \\
\mathrm{H} & 4.807379 & -4.583804 & -1.724569 \\
\mathrm{H} & 6.065073 & -3.407753 & -2.135999 \\
\mathrm{H} & 5.624526 & -3.676585 & -0.437165 \\
\mathrm{C} & 5.254996 & -4.556408 & 3.029117 \\
\mathrm{H} & 4.667854 & -4.491226 & 3.954891 \\
\mathrm{H} & 4.731752 & -5.279160 & 2.388184 \\
\mathrm{C} & 6.669127 & -5.079178 & 3.348579 \\
\mathrm{H} & 6.606199 & -6.055149 & 3.840280 \\
\mathrm{H} & 7.261826 & -5.190964 & 2.435296 \\
\mathrm{H} & 7.198103 & -4.391706 & 4.015560
\end{array}
$$

TS1a

B3LYP SCF energy: $\quad 3935.99789622$ a.u.

B3LYP enthalpy: 3934.989210 a.u.

B3LYP free energy: 3935.165441 a.u.

M06-L SCF energy in solution: $\quad 3937.64937359$ a.u.

M06-L enthalpy in solution: $\quad 3936.640687$ a.u.

M06-L free energy in solution: 3936.816918 a.u.

Imaginary frequency: \$TAB $\$-174.6651 \mathrm{~cm}-1$

Cartesian coordinates

\begin{tabular}{lccc}
\multicolumn{2}{l}{ ATOM } & X & Y \\
P & 1.686171 & -1.704442 & -0.483969 \\
P & 1.730952 & 1.649952 & 0.228512 \\
O & 5.890474 & -1.312948 & 1.720634 \\
O & 5.819729 & -0.638630 & 3.935837 \\
O & 6.383523 & 0.719693 & -0.641553 \\
O & 6.956493 & -0.084563 & -2.737439
\end{tabular}




$$
\begin{aligned}
& \begin{array}{llll}
\text { C } & 3.019075 & 0.995553 & 1.379991
\end{array} \\
& \begin{array}{llll}
\text { C } & 3.998773 & 0.054024 & 0.928956
\end{array} \\
& \begin{array}{llll}
\text { C } & 4.877888 & -0.408217 & 1.895802
\end{array} \\
& \begin{array}{llll}
\text { C } & 4.838855 & -0.008903 & 3.231398
\end{array} \\
& \begin{array}{llll}
\text { C } & 3.908196 & 0.902078 & 3.679023
\end{array} \\
& \begin{array}{llll}
\text { C } & 3.001983 & 1.393205 & 2.724831
\end{array} \\
& \begin{array}{llll}
\text { C } & 6.525195 & -1.463489 & 2.998212
\end{array} \\
& \begin{array}{llll}
\text { C } & 3.337420 & -1.305911 & -1.184430
\end{array} \\
& \begin{array}{llll}
\text { C } & 3.707382 & -1.792740 & -2.444183
\end{array} \\
& \begin{array}{llll}
\text { C } & 4.919281 & -1.442400 & -3.064751
\end{array} \\
& \begin{array}{llll}
\text { C } & 5.741512 & -0.583239 & -2.369463
\end{array} \\
& \text { C } \quad 5.392782 \quad-0.102207 \quad-1.107344 \\
& \begin{array}{llll}
\text { C } & 4.207354 & -0.427125 & -0.468257
\end{array} \\
& \begin{array}{llll}
\text { C } & 7.419647 & 0.704706 & -1.633775
\end{array} \\
& \begin{array}{llll}
\text { C } & 2.654693 & 2.364023 & -1.187650
\end{array} \\
& \begin{array}{llll}
\text { C } & 2.135396 & 2.254800 & -2.485178
\end{array} \\
& \begin{array}{llll}
\text { C } & 3.858620 & 3.059797 & -0.984451
\end{array} \\
& \begin{array}{llll}
\text { C } & 1.015526 & 3.094327 & 1.127307
\end{array} \\
& \begin{array}{llll}
\text { C } & 1.308374 & 4.420341 & 0.776362
\end{array} \\
& \begin{array}{llll}
\text { C } & 0.123485 & 2.853208 & 2.189720
\end{array} \\
& \begin{array}{llll}
\text { C } & 1.984142 & -2.591540 & 1.093461
\end{array} \\
& \begin{array}{llll}
\text { C } & 3.120477 & -3.392042 & 1.292173
\end{array} \\
& \begin{array}{llll}
\text { C } & 1.016442 & -2.502603 & 2.106531
\end{array} \\
& \begin{array}{llll}
\text { C } & 0.507006 & -2.566790 & -2.884024
\end{array} \\
& \begin{array}{llll}
\text { C } & 0.958331 & -2.962921 & -1.612249
\end{array} \\
& \mathrm{H} \quad 3.876946 \quad 1.228711 \quad 4.712126 \\
& \text { H } \quad 2.275393 \quad 2.121186 \quad 3.058664 \\
& \text { H } \quad 6.462826 \quad-2.509324 \quad 3.315186 \\
& \text { H } \quad 7.566632 \quad-1.130759 \quad 2.931934 \\
& \text { H } \quad 3.048577 \quad-2.473293 \quad-2.968277 \\
& \text { H } \quad \begin{array}{llll}
5.193538 & -1.833161 & -4.038047
\end{array} \\
& \text { H } \quad 7.612975 \quad 1.726900 \quad-1.973178 \\
& \text { H } \quad 8.323453 \quad 0.249604 \quad-1.213144
\end{aligned}
$$




\begin{tabular}{|c|c|c|c|}
\hline & .217918 & 1.695817 & -2.6 \\
\hline & 4.282261 & 3.137555 & 0.012377 \\
\hline & 1.980381 & 4.635022 & -0.046893 \\
\hline & 3.879287 & -3.462770 & 0.518584 \\
\hline & 0.130770 & -1.888127 & 1.959620 \\
\hline & 0.601291 & -1.529689 & -3.193100 \\
\hline & 0.116450 & 0.074373 & -0.323829 \\
\hline & -3.346240 & -0.090870 & 1.774881 \\
\hline & -1.375913 & 0.220601 & -1.596194 \\
\hline & -4.720010 & 0.423777 & 1.684020 \\
\hline & -1.630813 & -0.950914 & -1.331209 \\
\hline & -4.707824 & 1.514341 & 0.373545 \\
\hline & -5.336483 & 1.154451 & 2.793306 \\
\hline & -5.769255 & -0.969665 & 1.276762 \\
\hline & -5.880739 & 2.414738 & 0.254151 \\
\hline & -7.134001 & -0.910836 & 1.573398 \\
\hline & -5.214251 & -2.099727 & 0.669610 \\
\hline & -6.205023 & 2.649605 & \\
\hline & -5.521002 & 3.354293 & -0.179168 \\
\hline & -7.547412 & -0.038469 & 2.066799 \\
\hline & -7.944723 & -1.995176 & 1.248052 \\
\hline & -6.044404 & -3.172297 & 0.347460 \\
\hline & -4.147786 & -2.146950 & 0.476503 \\
\hline & -9.004678 & -1.956184 & \\
\hline & -7.417259 & -3.140168 & 0.630575 \\
\hline & -5.615414 & -4.053924 & -0.121983 \\
\hline & -8.301459 & -4.322669 & 0.315610 \\
\hline & -7.896391 & -4.919814 & -0.507403 \\
\hline & -8.392329 & -4.984704 & 1.186749 \\
\hline & -9.313652 & -4.006110 & 0.043422 \\
\hline & -2.123973 & 1.153710 & \\
\hline & -2.782029 & 0.655987 & -3.209823 \\
\hline & -1.452926 & 1.849513 & -2.999462 \\
\hline
\end{tabular}




$$
\begin{aligned}
& \begin{array}{llll}
\text { C } & -2.789762 & 1.847609 & -1.252763
\end{array} \\
& \begin{array}{lllll}
\text { C } & -1.649845 & 1.648291 & -0.210928
\end{array} \\
& \mathrm{H} \quad-0.141340 \quad 1.833647 \quad 2.457559 \\
& \text { H } \quad-1.013015 \quad 2.526566 \quad-0.195260 \\
& \begin{array}{llll}
\text { C } & -3.069784 & 3.337145 & -1.500777
\end{array} \\
& \text { H } \quad-3.389204 \quad 3.824611 \quad-0.574538 \\
& \mathrm{H} \quad-2.166115 \quad 3.849430 \quad-1.849081 \\
& \text { H } \quad-3.852424 \quad 3.475536 \quad-2.256572 \\
& \begin{array}{llll}
\text { C } & -4.078154 & 1.066050 & -0.878766
\end{array} \\
& \begin{array}{llll}
\mathrm{H} & -4.811138 & 1.169253 & -1.689038
\end{array} \\
& \text { H } \quad-3.843469 \quad 0.001699 \quad-0.804616 \\
& \text { H } \quad-1.994674 \quad 1.366941 \quad 0.782204 \\
& \begin{array}{llll}
\text { C } & -6.992277 & 1.892262 & -0.550630
\end{array} \\
& \begin{array}{llll}
\text { C } & -7.900514 & 1.471658 & -1.232110
\end{array} \\
& \begin{array}{llll}
\text { C } & -9.029832 & 0.968003 & -2.017800
\end{array} \\
& \text { H } \quad-9.207546 \quad-0.083248 \quad-1.752712 \\
& \text { H } \quad-8.766564 \quad 0.973439 \quad-3.084203 \\
& \begin{array}{llll}
\text { C } & -10.320960 & 1.781564 & -1.801962
\end{array} \\
& \begin{array}{llll}
\mathrm{H} & -10.177645 & 2.826904 & -2.092317
\end{array} \\
& \mathrm{H} \quad-11.133599 \quad 1.364689-2.405716 \\
& \text { H } \quad-10.625615 \quad 1.760127 \quad-0.751074 \\
& \begin{array}{llll}
\text { C } & -0.051258 & -3.496818 & -3.759134
\end{array} \\
& \text { H } \quad-0.386630 \quad-3.178581 \quad-4.742213 \\
& \begin{array}{llll}
\text { C } & -0.187464 & -4.831382 & -3.368642
\end{array} \\
& \mathrm{H} \quad-0.628577 \quad-5.555070 \quad-4.048061 \\
& \begin{array}{llll}
\text { C } & 0.242727 & -5.230409 & -2.103009
\end{array} \\
& \text { H } \quad 0.138796 \quad-6.266156-1.792609 \\
& \text { C } \quad 0.814747 \quad-4.304406 \quad-1.227991 \\
& \text { H } \quad 1.149622 \quad-4.632198 \quad-0.250208 \\
& \begin{array}{llll}
\text { C } & 3.280206 & -4.093672 & 2.487736
\end{array} \\
& \begin{array}{llll}
\mathrm{H} & 4.157737 & -4.718010 & 2.632448
\end{array} \\
& \text { C } \quad 2.313051 \quad-4.000680 \quad 3.492555 \\
& \text { H } \quad 2.441295 \quad-4.548068 \quad 4.422074
\end{aligned}
$$




$$
\begin{array}{llll}
\mathrm{C} & 1.181530 & -3.205131 & 3.301282 \\
\mathrm{H} & 0.427444 & -3.129851 & 4.079288 \\
\mathrm{C} & 0.735688 & 5.480074 & 1.484986 \\
\mathrm{H} & 0.972496 & 6.501797 & 1.202159 \\
\mathrm{C} & -0.131350 & 5.230377 & 2.548663 \\
\mathrm{H} & -0.573089 & 6.056751 & 3.097960 \\
\mathrm{C} & -0.437388 & 3.912688 & 2.900437 \\
\mathrm{H} & -1.121313 & 3.707976 & 3.718874 \\
\mathrm{C} & 4.519909 & 3.645134 & -2.064243 \\
\mathrm{H} & 5.446301 & 4.187681 & -1.897350 \\
\mathrm{C} & 3.993226 & 3.535866 & -3.354315 \\
\mathrm{H} & 4.511746 & 3.990866 & -4.193419 \\
\mathrm{C} & 2.802628 & 2.837781 & -3.563906 \\
\mathrm{H} & 2.395389 & 2.743662 & -4.566583
\end{array}
$$

TS1b

B3LYP SCF energy: $\quad 3935.99187906$ a.u.

B3LYP enthalpy: 3934.983558 a.u.

B3LYP free energy: 3935.149509 a.u.

M06-L SCF energy in solution: $\quad 3937.65503454$ a.u.

M06-L enthalpy in solution: $\quad 3936.646713$ a.u.

M06-L free energy in solution: $\quad 3936.812664$ a.u.

Imaginary frequency: \$TAB $\$-332.6681 \mathrm{~cm}-1$

Cartesian coordinates

\begin{tabular}{lccc} 
ATOM X & Y & \multicolumn{2}{l}{ Z } \\
P & -1.573473 & 1.758643 & -0.207676 \\
P & -1.034205 & -1.726772 & -0.270302 \\
O & -4.287467 & 0.352063 & 3.377987 \\
O & -3.223077 & -0.626407 & 5.188064 \\
O & -5.582748 & -1.452362 & 1.108355
\end{tabular}




$$
\begin{aligned}
& \begin{array}{llll}
\text { O } & -7.039970 & -0.624423 & -0.490933
\end{array} \\
& \begin{array}{llll}
\text { C } & -1.716700 & -1.470412 & 1.433530
\end{array} \\
& \begin{array}{llll}
\text { C } & -2.838004 & -0.602739 & 1.627083
\end{array} \\
& \begin{array}{llll}
\text { C } & -3.236450 & -0.408071 & 2.939646
\end{array} \\
& \begin{array}{llll}
\text { C } & -2.601979 & -0.998557 & 4.031865
\end{array} \\
& \begin{array}{llll}
\text { C } & -1.533388 & -1.850286 & 3.862779
\end{array} \\
& \begin{array}{llll}
\text { C } & -1.106322 & -2.072190 & 2.541497
\end{array} \\
& \begin{array}{llll}
\text { C } & -4.249053 & 0.301228 & 4.810931
\end{array} \\
& \begin{array}{llll}
\text { C } & -3.267650 & 1.038647 & -0.343382
\end{array} \\
& \begin{array}{llll}
\text { C } & -4.165312 & 1.525412 & -1.304278
\end{array} \\
& \begin{array}{llll}
\text { C } & -5.472584 & 1.029290 & -1.442194
\end{array} \\
& \begin{array}{llll}
\text { C } & -5.847490 & 0.029066 & -0.572497
\end{array} \\
& \begin{array}{llll}
\text { C } & -4.967814 & -0.462102 & 0.391716
\end{array} \\
& \begin{array}{llll}
\text { C } & -3.668571 & -0.006523 & 0.542857
\end{array} \\
& \begin{array}{llll}
\text { C } & -6.921933 & -1.544219 & 0.603808
\end{array} \\
& \text { C } \quad-2.455936-2.337272 \quad-1.265300 \\
& \begin{array}{llll}
\text { C } & -2.569456 & -1.975980 & -2.615020
\end{array} \\
& \begin{array}{llll}
\text { C } & -3.384648 & -3.232507 & -0.706536
\end{array} \\
& \begin{array}{llll}
\text { C } & 0.055007 & -3.216572 & -0.131113
\end{array} \\
& \text { C } \quad-0.245584 \quad-4.404937 \quad-0.815816 \\
& \begin{array}{llll}
\text { C } & 1.244578 & -3.158124 & 0.619607
\end{array} \\
& \begin{array}{llll}
\text { C } & -1.519917 & 2.442280 & 1.500742
\end{array} \\
& \begin{array}{llll}
\text { C } & -2.663313 & 3.027815 & 2.071868
\end{array} \\
& \begin{array}{llll}
\text { C } & -0.331370 & 2.399337 & 2.243711
\end{array} \\
& \begin{array}{llll}
\text { C } & -1.560709 & 3.014655 & -2.713412
\end{array} \\
& \begin{array}{llll}
\text { C } & -1.671573 & 3.224158 & -1.326746
\end{array} \\
& \begin{array}{llll}
\text { C } & -1.867588 & 4.528253 & -0.848849
\end{array} \\
& \text { H } \quad-1.049905 \quad-2.336003 \quad 4.702775 \\
& \text { H } \quad-0.283001 \quad-2.757249 \quad 2.394477 \\
& \mathrm{H} \quad-4.003725 \quad 1.295153 \quad 5.203281 \\
& \text { H } \quad-5.213692 \quad-0.048873 \quad 5.189592 \\
& \text { H } \quad-3.862988 \quad 2.325564 \quad-1.965865 \\
& \text { H } \quad-6.151613 \quad 1.420120 \quad-2.191353
\end{aligned}
$$




$$
\begin{aligned}
& \text { H } \quad-7.107166 \quad-2.561034 \quad 0.244988 \\
& \mathrm{H} \quad \begin{array}{llll}
-7.630552 & -1.265808 & 1.391798
\end{array} \\
& \text { H } \quad-1.852319-1.291802-3.057348 \\
& \begin{array}{llll}
\mathrm{H} & -3.306628 & -3.518362 & 0.338134
\end{array} \\
& \text { H } \quad-1.143672 \quad-4.480634 \quad-1.416808 \\
& \text { H } \quad-3.597788 \quad 3.048935 \quad 1.519770 \\
& \begin{array}{llll}
\mathrm{H} & 0.551633 & 1.939910 & 1.814344
\end{array} \\
& \text { H } \quad-1.400280 \quad 2.013995 \quad-3.103950 \\
& \begin{array}{llll}
\mathrm{H} & -1.951610 & 4.715643 & 0.215928
\end{array} \\
& \begin{array}{lllll}
\mathrm{Rh} & 0.218941 & 0.329806 & -0.985842
\end{array} \\
& \begin{array}{llll}
\text { O } & 4.084465 & -1.695962 & 1.865731
\end{array} \\
& \begin{array}{llll}
\text { S } & 4.804366 & -1.407648 & 0.618775
\end{array} \\
& \mathrm{~N} \quad 3.792495 \quad-0.344958 \quad-0.233825 \\
& \begin{array}{llll}
\text { O } & 5.158112 & -2.459774 & -0.335369
\end{array} \\
& \begin{array}{llll}
\text { C } & 6.294911 & -0.512547 & 1.039573
\end{array} \\
& \begin{array}{llll}
\text { C } & 6.336712 & 0.247948 & 2.212361
\end{array} \\
& \begin{array}{llll}
\text { C } & 7.397026 & -0.569640 & 0.180879
\end{array} \\
& \text { H } \quad 5.491143 \quad 0.249717 \quad 2.892054 \\
& \begin{array}{llll}
\text { C } & 7.491892 & 0.967054 & 2.512419
\end{array} \\
& \begin{array}{llll}
\text { C } & 8.542255 & 0.156218 & 0.500213
\end{array} \\
& \text { H } \quad 7.365392 \quad-1.195806 \quad-0.704266 \\
& \mathrm{H} \quad 7.530725 \quad 1.553301 \quad 3.426854 \\
& \begin{array}{llll}
\text { C } & 8.610054 & 0.935404 & 1.665430
\end{array} \\
& \text { H } \quad 9.403204 \quad 0.107976-0.161449 \\
& \begin{array}{llll}
\text { C } & 9.870124 & 1.687388 & 2.018519
\end{array} \\
& \text { H } \quad 10.561345 \quad 1.044780 \quad 2.579296 \\
& \begin{array}{llll}
\mathrm{H} & 10.398608 & 2.028143 & 1.122488
\end{array} \\
& \begin{array}{llll}
\mathrm{H} & 9.656092 & 2.559810 & 2.643675
\end{array} \\
& \mathrm{H} \quad 1.532297 \quad-2.248578 \quad 1.138323 \\
& \begin{array}{llll}
\text { C } & 3.102118 & 0.688888 & 0.554598
\end{array} \\
& \begin{array}{llll}
\mathrm{H} & 3.790510 & 1.507013 & 0.830660
\end{array} \\
& \begin{array}{llll}
\mathrm{H} & 2.743008 & 0.224417 & 1.475328
\end{array} \\
& \begin{array}{llll}
\text { C } & 1.760693 & -1.163170 & -1.978971
\end{array}
\end{aligned}
$$




$$
\begin{aligned}
& \begin{array}{llll}
\text { C } & 3.020361 & -0.584015 & -2.665327
\end{array} \\
& \begin{array}{llll}
\mathrm{H} & 1.345470 & -2.046004 & -2.469022
\end{array} \\
& \begin{array}{llll}
\text { C } & 2.295970 & 0.537123 & -3.468654
\end{array} \\
& \begin{array}{llll}
\text { C } & 0.904775 & 0.133807 & -2.982574
\end{array} \\
& \text { H } \quad 2.590016 \quad 1.554763 \quad-3.204995 \\
& \text { H } \quad 2.350715 \quad 0.433305 \quad-4.557389 \\
& \begin{array}{llll}
\text { O } & -0.088998 & -0.018899 & -3.662312
\end{array} \\
& \text { H } \quad 1.928842 \quad-1.411070 \quad-0.931849 \\
& \text { C } \quad 4.094591 \quad-0.054046-1.641168 \\
& \text { H } \quad 5.050345 \quad-0.521945-1.881615 \\
& \mathrm{H} \quad 4.228678 \quad 1.027394 \quad-1.757430 \\
& \begin{array}{llll}
\text { C } & 3.673128 & -1.627781 & -3.587834
\end{array} \\
& \text { H } \quad 4.055845 \quad-2.468678 \quad-3.000026 \\
& \text { H } \quad 2.955425 \quad-2.014472 \quad-4.320136 \\
& \text { H } \quad 4.511929 \quad-1.188701 \quad-4.142103 \\
& \begin{array}{llll}
\text { C } & 2.001169 & 1.303923 & -0.222713
\end{array} \\
& \begin{array}{llll}
\text { C } & 1.537720 & 2.206231 & -0.955303
\end{array} \\
& \begin{array}{llll}
\text { C } & 1.572153 & 3.541214 & -1.595834
\end{array} \\
& \text { H } \quad 0.809816 \quad 4.180869-1.139995 \\
& \text { H } \quad 1.284491 \quad 3.455299 \quad-2.649937 \\
& \begin{array}{llll}
\text { C } & 2.953499 & 4.209046 & -1.467238
\end{array} \\
& \text { H } \quad 2.931372 \quad 5.199833 \quad-1.932889 \\
& \text { H } \quad 3.235235 \quad 4.334304 \quad-0.416568 \\
& \text { H } \quad 3.734581 \quad 3.621310 \quad-1.961464 \\
& \text { C } \quad-1.951078 \quad 5.601385-1.740464 \\
& \text { Н } \quad-2.100229 \quad 6.606341 \quad-1.355784 \\
& \begin{array}{llll}
\text { C } & -1.849590 & 5.384843 & -3.115329
\end{array} \\
& \text { H } \quad-1.920310 \quad 6.220335 \quad-3.805852 \\
& \begin{array}{llll}
\text { C } & -1.657796 & 4.088131 & -3.599729
\end{array} \\
& \text { H } \quad-1.579929 \quad 3.909629 \quad-4.668568 \\
& \begin{array}{llll}
\text { C } & -0.281593 & 2.940610 & 3.529538
\end{array} \\
& \text { H } \quad 0.643242 \quad 2.896883 \quad 4.097733 \\
& \begin{array}{llll}
\text { C } & -1.416967 & 3.533288 & 4.084838
\end{array}
\end{aligned}
$$




$$
\begin{array}{llll}
\mathrm{H} & -1.375851 & 3.958819 & 5.083557 \\
\mathrm{C} & -2.607275 & 3.575947 & 3.353653 \\
\mathrm{H} & -3.494499 & 4.035795 & 3.780184 \\
\mathrm{C} & -3.603494 & -2.502097 & -3.394615 \\
\mathrm{H} & -3.685273 & -2.216718 & -4.439588 \\
\mathrm{C} & -4.523151 & -3.390568 & -2.836655 \\
\mathrm{H} & -5.323404 & -3.800732 & -3.446333 \\
\mathrm{C} & -4.410968 & -3.756365 & -1.491445 \\
\mathrm{H} & -5.118580 & -4.456304 & -1.055033 \\
\mathrm{C} & 0.607664 & -5.508529 & -0.729235 \\
\mathrm{H} & 0.355359 & -6.419135 & -1.265318 \\
\mathrm{C} & 1.770924 & -5.444352 & 0.036528 \\
\mathrm{H} & 2.431929 & -6.303798 & 0.100705 \\
\mathrm{C} & 2.089311 & -4.263339 & 0.711242 \\
\mathrm{H} & 3.000497 & -4.183691 & 1.294509
\end{array}
$$

TS1a-ee

B3LYP SCF energy: 3935.99331398 a.u.

B3LYP enthalpy: 3934.985410 a.u.

B3LYP free energy: 3935.161334 a.u.

M06-L SCF energy in solution: $\quad 3937.64778748$ a.u.

M06-L enthalpy in solution: $\quad 3936.639883$ a.u.

M06-L free energy in solution: 3936.815807 a.u.

Imaginary frequency: $\$$ TAB $\$-304.4862 \mathrm{~cm}-1$

Cartesian coordinates
ATOM $\mathrm{X} \quad \mathrm{Y} \quad \mathrm{Z}$
$\begin{array}{llll}\text { O } & -3.851492 & -1.889711 & -0.620498\end{array}$
$\begin{array}{llll}\text { C } & -1.277378 & 0.808718 & 0.603404\end{array}$
S $\quad-5.140090 \quad-1.336272 \quad-1.055967$
$\begin{array}{llll}\text { O } & -1.563811 & 0.126255 & 1.562644\end{array}$ 


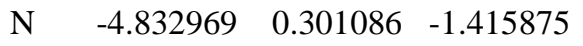

$$
\begin{aligned}
& \begin{array}{llll}
\text { O } & -5.846980 & -1.865821 & -2.224408
\end{array} \\
& \begin{array}{llll}
\text { C } & -6.242563 & -1.426813 & 0.353719
\end{array} \\
& \begin{array}{llll}
\text { C } & -5.890327 & 1.020170 & -2.166563
\end{array} \\
& \begin{array}{llll}
\text { C } & -7.625138 & -1.413896 & 0.149038
\end{array} \\
& \begin{array}{llll}
\text { C } & -5.708366 & -1.513399 & 1.642373
\end{array} \\
& \text { H } \quad-6.343185 \quad 0.295972 \quad-2.848524 \\
& \text { H } \quad-5.389086 \quad 1.763844 \quad-2.795606 \\
& \text { H } \quad-8.026588 \quad-1.371948 \quad-0.857177 \\
& \begin{array}{llll}
\text { C } & -8.473534 & -1.479339 & 1.251354
\end{array} \\
& \begin{array}{llll}
\text { C } & -6.575003 & -1.572969 & 2.732695
\end{array} \\
& \text { H } \quad-4.633808 \quad-1.559184 \quad 1.783945 \\
& \text { H } \quad-9.549306 \quad-1.479084 \quad 1.094603 \\
& \begin{array}{llll}
\text { C } & -7.966067 & -1.558368 & 2.557714
\end{array} \\
& \text { H } \quad-6.162964 \quad-1.643610 \quad 3.736184 \\
& \begin{array}{llll}
\text { C } & -8.897159 & -1.665026 & 3.741471
\end{array} \\
& \text { H } \quad-8.429145 \quad-1.288319 \quad 4.656430 \\
& \text { H } \quad-9.177970 \quad-2.710604 \quad 3.924142 \\
& \text { H } \quad-9.823789-1.105867 \quad 3.575406 \\
& \begin{array}{llll}
\text { C } & -1.890450 & 2.175651 & 0.250540
\end{array} \\
& \begin{array}{llll}
\mathrm{H} & -2.446687 & 2.631225 & 1.076975
\end{array} \\
& \text { H } \quad-1.152290 \quad 2.905783 \quad-0.115990 \\
& \begin{array}{llll}
\text { C } & -2.677016 & 1.551965 & -0.944214
\end{array} \\
& \begin{array}{llll}
\text { C } & -1.700446 & 0.362178 & -1.178227
\end{array} \\
& \text { H } \quad-1.124406 \quad 0.485451 \quad-2.102542 \\
& \begin{array}{llll}
\text { C } & -2.781549 & 2.484115 & -2.156652
\end{array} \\
& \text { H } \quad-3.199555 \quad 1.953951 \quad-3.018189 \\
& \mathrm{H} \quad-1.790793 \quad 2.855372 \quad-2.446830 \\
& \mathrm{H} \quad-3.415178 \quad 3.352984 \quad-1.941354 \\
& \begin{array}{llll}
\text { C } & -4.061788 & 1.080683 & -0.433329
\end{array} \\
& \text { H } \quad-4.657852 \quad 1.954747 \quad-0.140821 \\
& \begin{array}{llll}
\mathrm{H} & -3.915484 & 0.484441 & 0.471087
\end{array} \\
& \text { H } \quad-2.120686 \quad-0.640628-1.137372
\end{aligned}
$$




$$
\begin{aligned}
& \begin{array}{llll}
\text { C } & -6.909694 & 1.679832 & -1.340205
\end{array} \\
& \begin{array}{llll}
\text { C } & -7.739092 & 2.241879 & -0.660228
\end{array} \\
& \begin{array}{llll}
\text { C } & -8.778243 & 2.894337 & 0.140234
\end{array} \\
& \text { H } \quad-9.086710 \quad 2.214220 \quad 0.946022 \\
& \begin{array}{llll}
\text { H } & -8.359000 & 3.781644 & 0.633738
\end{array} \\
& \begin{array}{llll}
\text { C } & -10.009502 & 3.301884 & -0.692412
\end{array} \\
& \text { H } \quad-9.731760 \quad 4.007070 \quad-1.481860 \\
& \begin{array}{llll}
\text { H } & -10.756567 & 3.780082 & -0.050544
\end{array} \\
& \begin{array}{llll}
\mathrm{H} & -10.468781 & 2.427980 & -1.164661
\end{array} \\
& \begin{array}{llll}
\mathrm{P} & 2.444475 & 1.758482 & 0.473116
\end{array} \\
& \text { P } \quad \begin{array}{llll}
1.328664 & -1.274701 & -0.674873
\end{array} \\
& \begin{array}{llll}
\text { O } & 6.550726 & -0.344609 & -0.472812
\end{array} \\
& \begin{array}{llll}
\text { O } & 6.934647 & -0.914104 & -2.685085
\end{array} \\
& \begin{array}{llll}
\text { O } & 5.403513 & -2.447823 & 1.601756
\end{array} \\
& \begin{array}{llll}
\text { O } & 5.519625 & -2.017815 & 3.874588
\end{array} \\
& \begin{array}{llll}
\text { C } & 3.055969 & -1.219960 & -1.311873
\end{array} \\
& \begin{array}{llll}
\text { C } & 4.128361 & -0.788091 & -0.462795
\end{array} \\
& \begin{array}{llll}
\text { C } & 5.376947 & -0.729416 & -1.060196
\end{array} \\
& \begin{array}{llll}
\text { C } & 5.614755 & -1.070271 & -2.392917
\end{array} \\
& \begin{array}{llll}
\text { C } & 4.599470 & -1.511031 & -3.212573
\end{array} \\
& \begin{array}{llll}
\text { C } & 3.316753 & -1.575373 & -2.642905
\end{array} \\
& \begin{array}{llll}
\text { C } & 7.561577 & -0.434951 & -1.486390
\end{array} \\
& \begin{array}{llll}
\text { C } & 3.433857 & 0.678224 & 1.567929
\end{array} \\
& \begin{array}{llll}
\text { C } & 3.526406 & 0.933004 & 2.940420
\end{array} \\
& \begin{array}{llll}
\text { C } & 4.213003 & 0.075663 & 3.818353
\end{array} \\
& \begin{array}{llll}
\text { C } & 4.794385 & -1.041312 & 3.259187
\end{array} \\
& \begin{array}{llll}
\text { C } & 4.721101 & -1.297982 & 1.888612
\end{array} \\
& \begin{array}{llll}
\text { C } & 4.052343 & -0.476094 & 0.996689
\end{array} \\
& \begin{array}{llll}
\text { C } & 5.849142 & -2.975582 & 2.859917
\end{array} \\
& \begin{array}{llll}
\text { C } & 1.396770 & -2.414719 & 0.761440
\end{array} \\
& \begin{array}{llll}
\text { C } & 0.530823 & -2.261470 & 1.852844
\end{array} \\
& \begin{array}{llll}
\text { C } & 2.278775 & -3.509680 & 0.718190
\end{array} \\
& \begin{array}{llll}
\text { C } & 0.312749 & -2.138665 & -1.937841
\end{array}
\end{aligned}
$$




$$
\begin{aligned}
& \text { C } \quad-0.349753 \quad-3.337130 \quad-1.632344 \\
& \text { C } \quad 0.119382 \quad-1.556932 \quad-3.206198 \\
& \text { C } \quad 3.633024 \quad 2.500037 \quad-0.712786 \\
& \begin{array}{llll}
\text { C } & 4.954309 & 2.794415 & -0.338872
\end{array} \\
& \begin{array}{llll}
\text { C } & 3.198326 & 2.814672 & -2.009265
\end{array} \\
& \begin{array}{llll}
\text { C } & 0.852458 & 2.910882 & 2.477155
\end{array} \\
& \begin{array}{llll}
\text { C } & 1.839061 & 3.159233 & 1.502348
\end{array} \\
& \begin{array}{llll}
\text { C } & 2.293031 & 4.474013 & 1.310184
\end{array} \\
& \text { H } \quad 4.779145 \quad-1.800567 \quad-4.241593 \\
& \text { H } \quad 2.510852-1.937680 \quad-3.266535 \\
& \text { H } \quad 7.983828 \quad 0.558668 \quad-1.669595 \\
& \text { H } \quad 8.334075 \quad-1.143647 \quad-1.171807 \\
& \text { H } \quad 3.060859 \quad 1.819751 \quad 3.352955 \\
& \text { H } \quad 4.283177 \quad 0.284632 \quad 4.879706 \\
& \text { H } \quad 5.327503 \quad-3.917511 \quad 3.064535 \\
& \begin{array}{llll}
\mathrm{H} & 6.933059 & -3.118510 & 2.832034
\end{array} \\
& \text { H } \quad-0.172587 \quad-1.436807 \quad 1.883044 \\
& \mathrm{H} \quad 2.952740 \quad-3.640024 \quad-0.122384 \\
& \text { H } \quad-0.235392 \quad-3.793189-0.655952 \\
& \text { H } \quad \begin{array}{llll}
0.601208 & -0.614409 & -3.453515
\end{array} \\
& \text { H } \quad 5.306004 \quad 2.546464 \quad 0.658172 \\
& \text { H } \quad 2.178643 \quad 2.580587 \quad-2.307345 \\
& \begin{array}{llll}
\mathrm{H} & 0.479585 & 1.902286 & 2.631245
\end{array} \\
& \text { H } \quad 3.049595 \quad 4.687458 \quad 0.563049 \\
& \begin{array}{llll}
\text { Rh } & 0.484594 & 0.838071 & -0.398086
\end{array} \\
& \begin{array}{llll}
\text { C } & 2.292690 & -4.435316 & 1.760617
\end{array} \\
& \mathrm{H} \quad 2.968354 \quad-5.285058 \quad 1.714540 \\
& \begin{array}{llll}
\text { C } & 1.436311 & -4.274491 & 2.853836
\end{array} \\
& \text { H } \quad 1.449410 \quad-4.996935 \quad 3.665194 \\
& \begin{array}{llll}
\text { C } & 0.558758 & -3.190416 & 2.896067
\end{array} \\
& \text { H } \quad-0.114281 \quad-3.065544 \quad 3.739372 \\
& \text { C } \quad-1.174341-3.946803-2.580412 \\
& \text { H } \quad-1.691057 \quad-4.866820 \quad-2.325246
\end{aligned}
$$




$$
\begin{array}{lrrr}
\mathrm{C} & -1.341806 & -3.375402 & -3.840811 \\
\mathrm{H} & -1.986797 & -3.851091 & -4.573465 \\
\mathrm{C} & -0.689837 & -2.178883 & -4.153846 \\
\mathrm{H} & -0.822570 & -1.723784 & -5.131149 \\
\mathrm{C} & 4.067688 & 3.419414 & -2.918176 \\
\mathrm{H} & 3.722799 & 3.657939 & -3.920141 \\
\mathrm{C} & 5.379016 & 3.713734 & -2.538468 \\
\mathrm{H} & 6.055964 & 4.185395 & -3.245061 \\
\mathrm{C} & 5.820305 & 3.401022 & -1.249634 \\
\mathrm{H} & 6.838710 & 3.633689 & -0.950865 \\
\mathrm{C} & 1.777747 & 5.516058 & 2.083802 \\
\mathrm{H} & 2.138553 & 6.528472 & 1.926805 \\
\mathrm{C} & 0.810028 & 5.259872 & 3.056464 \\
\mathrm{H} & 0.414725 & 6.072939 & 3.658505 \\
\mathrm{C} & 0.349862 & 3.954998 & 3.253060 \\
\mathrm{H} & -0.402711 & 3.748401 & 4.008665
\end{array}
$$

TS1b-ee

B3LYP SCF energy: 3935.98273596 a.u.

B3LYP enthalpy: 3934.974319 a.u.

B3LYP free energy: 3935.140541 a.u.

M06-L SCF energy in solution: $\quad 3937.64583263$ a.u.

M06-L enthalpy in solution: $\quad 3936.637416$ a.u.

M06-L free energy in solution: $\quad 3936.803638$ a.u.

Imaginary frequency: \$TAB \$ $-343.2055 \mathrm{~cm}-1$

Cartesian coordinates

$\begin{array}{lccc}\text { ATOM } & \text { X } & \text { Y } & \text { Z } \\ \text { Rh } & 0.274104 & 0.334322 & -0.738352 \\ \text { O } & 4.133748 & -0.178555 & 2.118519 \\ \text { S } & 4.768759 & 0.636659 & 1.077563\end{array}$




\begin{tabular}{|c|c|c|c|}
\hline & 909302 & 0.307960 & \\
\hline & 4.816977 & 2.097963 & 1.162284 \\
\hline & 5.438409 & 0.039457 & 0.846989 \\
\hline & 6.724126 & -1.312037 & 1.067225 \\
\hline & 7.435414 & 0.926092 & 0.431958 \\
\hline & 5.951084 & -1.983961 & 1.425529 \\
\hline & 8.020131 & -1.773326 & 0.852624 \\
\hline & 8.726577 & 0.443679 & 0.223969 \\
\hline & 7.206722 & 1.978243 & 0.301461 \\
\hline & 8.247355 & -2.821761 & 1.027728 \\
\hline & 9.040854 & -0.907666 & 0.428013 \\
\hline & 9.505650 & 1.131868 & -0.093242 \\
\hline & 10.449725 & -1.413140 & 0.234348 \\
\hline & 11.007561 & -1.380189 & 1.179333 \\
\hline & 10.999089 & -0.804009 & -0.490013 \\
\hline & 10.459763 & -2.4 & -0.1 \\
\hline & 3.342793 & -1.023757 & -0.564427 \\
\hline & & -1.646899 & -1.168125 \\
\hline & & -1.503402 & \\
\hline & 1.786771 & 2.13 & -0.84 \\
\hline & 2.958544 & 2.145665 & -1.856858 \\
\hline & 1.381002 & 3.127207 & -0.647282 \\
\hline & 2.137976 & 1.717321 & -3.108636 \\
\hline & & & \\
\hline & & & \\
\hline & 2.141609 & 2.428 & -3.941380 \\
\hline & -0.184747 & 2.348606 & -2.750984 \\
\hline & 2.022001 & 1.653970 & 0.113612 \\
\hline & 4.134478 & 1.168964 & -1.502474 \\
\hline & 5.026108 & 1.767681 & -1.308773 \\
\hline & & & -2.367587 \\
\hline & 3.522287 & 3.569663 & -2.01577 \\
\hline & 3.958202 & 3.914018 & -1.07196 \\
\hline
\end{tabular}




$$
\begin{array}{lrrr}
\mathrm{H} & 2.739536 & 4.276029 & -2.314145 \\
\mathrm{H} & 4.304655 & 3.594944 & -2.784147 \\
\mathrm{C} & 2.048759 & -0.945551 & -1.284352 \\
\mathrm{C} & 1.224031 & -1.074190 & -2.206235 \\
\mathrm{C} & 0.584484 & -1.406540 & -3.496923 \\
\mathrm{H} & -0.373105 & -1.902710 & -3.311831 \\
\mathrm{H} & 0.343753 & -0.472588 & -4.019897 \\
\mathrm{C} & 1.479250 & -2.291596 & -4.384179 \\
\mathrm{H} & 0.970812 & -2.497332 & -5.331422 \\
\mathrm{H} & 1.693414 & -3.247533 & -3.897316 \\
\mathrm{H} & 2.431446 & -1.799490 & -4.609027 \\
\mathrm{P} & -1.507999 & 1.759349 & 0.074325 \\
\mathrm{P} & -0.878099 & -1.647045 & 0.225840 \\
\mathrm{C} & -5.854237 & -0.002786 & 1.358446 \\
\mathrm{C} & -1.270185 & -3.039463 & -0.936255 \\
\mathrm{O} & -5.935153 & -1.076122 & 3.406362 \\
\mathrm{O} & -5.514728 & -1.458184 & -1.315344 \\
\mathrm{O} & -5.849328 & -0.463138 & -3.375938 \\
\mathrm{C} & -2.445706 & -1.447946 & 1.214758 \\
\mathrm{C} & -3.571631 & -0.701647 & 0.739294 \\
\mathrm{C} & -4.681200 & -0.680095 & 1.570537 \\
\mathrm{C} & -4.732500 & -1.313532 & 2.809849 \\
\mathrm{C} & -3.653690 & -2.019124 & 3.293034 \\
\mathrm{C} & -2.517104 & -2.070074 & 2.470155 \\
\mathrm{C} & -6.723018 & -0.370512 & 2.437828 \\
\mathrm{C} & -2.901611 & 1.144753 & -0.965374 \\
\mathrm{C} & -4.086328 & 1.695078 & -2.244457 \\
\mathrm{C} & -656149 & 1.235748 & -3.138285 \\
\mathrm{C} & -1.652671 & -0.397353 & -1.460413 \\
\mathrm{C} & 0.017057 & -0.560682 \\
\mathrm{C} & -1.427066
\end{array}
$$




$$
\begin{aligned}
& \text { C } \quad-2.584712 \quad-3.318836-1.337520 \\
& \text { C } \quad 0.269892 \quad-2.465285 \quad 1.423739 \\
& \begin{array}{llll}
\text { C } & 0.163179 & -3.837326 & 1.722173
\end{array} \\
& \begin{array}{llll}
\text { C } & 1.222403 & -1.700924 & 2.112447
\end{array} \\
& \begin{array}{llll}
\text { C } & -2.012450 & 1.737144 & 1.849820
\end{array} \\
& \begin{array}{llll}
\text { C } & -3.242449 & 2.287143 & 2.247139
\end{array} \\
& \begin{array}{llll}
\text { C } & -1.089665 & 1.348351 & 2.827653
\end{array} \\
& \begin{array}{llll}
\text { C } & -0.378321 & 4.233958 & 0.632659
\end{array} \\
& \begin{array}{llll}
\text { C } & -1.408433 & 3.605050 & -0.091044
\end{array} \\
& \begin{array}{llll}
\text { C } & -2.351159 & 4.409627 & -0.746263
\end{array} \\
& \mathrm{H} \quad-3.674001 \quad-2.506006 \quad 4.261341 \\
& \text { H } \quad-1.666222 \quad-2.621705 \quad 2.843397 \\
& \text { H } \quad-7.138955 \quad 0.531296 \quad 2.893890 \\
& \text { H } \quad-7.516772 \quad-1.029293 \quad 2.063859 \\
& \text { H } \quad-2.430451 \quad 2.483667 \quad-2.581101 \\
& \text { H } \quad-4.190527 \quad 1.679915 \quad-4.119377 \\
& \text { Н } \quad-6.500639-2.369462-2.894920 \\
& \text { H } \quad-7.386945-1.011604 \quad-2.094864
\end{aligned}
$$

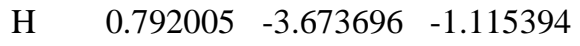

$$
\begin{aligned}
& \text { H } \quad-3.416307 \quad-2.7446688-0.950452 \\
& \mathrm{H} \quad-0.568080 \quad-4.452360 \quad 1.209048 \\
& \text { H } \quad 1.345285 \quad-0.647581 \quad 1.880739 \\
& \begin{array}{llll}
\mathrm{H} & -3.963124 & 2.608866 & 1.500633
\end{array} \\
& \begin{array}{llll}
\mathrm{H} & -0.131678 & 0.931277 & 2.533005
\end{array} \\
& \begin{array}{llll}
\mathrm{H} & 0.323812 & 3.641294 & 1.212414
\end{array} \\
& \text { H } \quad-3.191172 \quad 3.967221 \quad-1.266391 \\
& \text { C } \quad-2.850242-4.370255-2.218845 \\
& \text { H } \quad-3.876694 \quad-4.572831 \quad-2.511214 \\
& \begin{array}{llll}
\text { C } & -1.813287 & -5.165740 & -2.704411
\end{array} \\
& \text { H } \quad-2.023940 \quad-5.988118-3.381984 \\
& \begin{array}{llll}
\text { C } & -0.501850 & -4.907053 & -2.298541
\end{array} \\
& \begin{array}{llll}
\mathrm{H} & 0.312721 & -5.532488 & -2.653481
\end{array} \\
& \begin{array}{llll}
\text { C } & 0.984367 & -4.418679 & 2.687778
\end{array}
\end{aligned}
$$




$$
\begin{array}{lrrr}
\mathrm{H} & 0.886818 & -5.478481 & 2.906056 \\
\mathrm{C} & 1.923363 & -3.642784 & 3.372530 \\
\mathrm{H} & 2.561011 & -4.097921 & 4.125202 \\
\mathrm{C} & 2.043409 & -2.283785 & 3.082197 \\
\mathrm{H} & 2.785103 & -1.671891 & 3.583135 \\
\mathrm{C} & -1.390685 & 1.496591 & 4.184311 \\
\mathrm{H} & -0.664554 & 1.192719 & 4.932895 \\
\mathrm{C} & -2.616949 & 2.034819 & 4.572036 \\
\mathrm{H} & -2.851107 & 2.154338 & 5.625993 \\
\mathrm{C} & -3.543305 & 2.427118 & 3.600273 \\
\mathrm{H} & -4.496751 & 2.854438 & 3.898437 \\
\mathrm{C} & -2.237063 & 5.801876 & -0.716865 \\
\mathrm{H} & -2.978148 & 6.406718 & -1.231852 \\
\mathrm{C} & -1.190526 & 6.413270 & -0.027739 \\
\mathrm{H} & -1.105597 & 7.495888 & -0.009266 \\
\mathrm{C} & -0.265437 & 5.623365 & 0.657806 \\
\mathrm{H} & 0.538689 & 6.085938 & 1.222955
\end{array}
$$

IntB1

B3LYP SCF energy: $\quad 3936.04112013$ a.u.

B3LYP enthalpy: $\quad 3935.030718$ a.u.

B3LYP free energy: 3935.203856 a.u.

M06-L SCF energy in solution: $\quad 3937.69280503$ a.u.

M06-L enthalpy in solution: $\quad 3936.682403$ a.u.

M06-L free energy in solution: $\quad 3936.855541$ a.u.

Cartesian coordinates

$$
\begin{array}{lccc}
\text { ATOM X } & \text { Y } & \multicolumn{2}{l}{\text { Z }} \\
\text { P } & -0.614856 & 1.547926 & -0.384498 \\
\text { P } & -1.915180 & -1.792146 & 0.093314 \\
\text { O } & -4.667181 & 2.465814 & 1.726881
\end{array}
$$




$$
\begin{aligned}
& \begin{array}{llll}
\text { O } & -4.896851 & 1.726685 & 3.909468
\end{array} \\
& \begin{array}{llll}
\text { O } & -5.832241 & 0.800154 & -0.712292
\end{array} \\
& \begin{array}{llll}
\text { O } & -6.069389 & 1.831385 & -2.774015
\end{array} \\
& \begin{array}{llll}
\text { C } & -2.849397 & -0.727858 & 1.292013
\end{array} \\
& \begin{array}{llll}
\text { C } & -3.389744 & 0.537749 & 0.881671
\end{array} \\
& \begin{array}{llll}
\text { C } & -4.054527 & 1.249727 & 1.868024
\end{array} \\
& \begin{array}{llll}
\text { C } & -4.199347 & 0.810697 & 3.183759
\end{array} \\
& \begin{array}{llll}
\text { C } & -3.695341 & -0.404308 & 3.589702
\end{array} \\
& \begin{array}{llll}
\text { C } & -3.021425 & -1.158721 & 2.615879
\end{array} \\
& \begin{array}{llll}
\text { C } & -5.171557 & 2.819607 & 3.020520
\end{array} \\
& \begin{array}{llll}
\text { C } & -2.277993 & 1.726519 & -1.151837
\end{array} \\
& \begin{array}{llll}
\text { C } & -2.442676 & 2.359245 & -2.388501
\end{array} \\
& \begin{array}{llll}
\text { C } & -3.689297 & 2.445674 & -3.034126
\end{array} \\
& \begin{array}{llll}
\text { C } & -4.761040 & 1.875853 & -2.383303
\end{array} \\
& \begin{array}{llll}
\text { C } & -4.614127 & 1.252468 & -1.144865
\end{array} \\
& \begin{array}{llll}
\text { C } & -3.398058 & 1.133621 & -0.490904
\end{array} \\
& \begin{array}{llll}
\text { C } & -6.775450 & 1.136391 & -1.739039
\end{array} \\
& \begin{array}{llll}
\text { C } & -3.071446 & -1.901691 & -1.339590
\end{array} \\
& \begin{array}{llll}
\text { C } & -2.660003 & -1.738057 & -2.668492
\end{array} \\
& \begin{array}{llll}
\text { C } & -4.419581 & -2.207470 & -1.072516
\end{array} \\
& \begin{array}{llll}
\text { C } & -1.962501 & -3.508841 & 0.795698
\end{array} \\
& \begin{array}{llll}
\text { C } & -2.580063 & -4.564309 & 0.106246
\end{array} \\
& \begin{array}{llll}
\text { C } & -1.308766 & -3.799950 & 2.009667
\end{array} \\
& \begin{array}{llll}
\text { C } & -0.759484 & 2.448880 & 1.220614
\end{array} \\
& \begin{array}{llll}
\text { C } & -1.571843 & 3.586850 & 1.356735
\end{array} \\
& \begin{array}{llll}
\text { C } & -0.007344 & 2.014154 & 2.323078
\end{array} \\
& \begin{array}{llll}
\text { C } & 0.909138 & 2.227541 & -2.663709
\end{array} \\
& \begin{array}{llll}
\text { C } & 0.506107 & 2.636485 & -1.379321
\end{array} \\
& \text { H } \quad-3.820760 \quad-0.768003 \quad 4.603090 \\
& \mathrm{H} \quad-2.656735 \quad-2.129747 \quad 2.916238 \\
& \text { H } \quad-4.656614 \quad 3.716833 \quad 3.380331 \\
& \text { H } \quad-6.252905 \quad 2.976824 \quad 2.961574 \\
& \text { H } \quad-1.586622 \quad 2.812117 \quad-2.873292
\end{aligned}
$$




$$
\begin{aligned}
& \text { H } \quad-3.801481 \quad 2.944326 \quad-3.990270 \\
& \begin{array}{llll}
\mathrm{H} & -7.209838 & 0.217726 & -2.148271
\end{array} \\
& \mathrm{H} \quad \begin{array}{llll}
-7.550130 & 1.788983 & -1.322881
\end{array} \\
& \text { H } \quad-1.639370 \quad-1.468583 \quad-2.899773 \\
& \mathrm{H} \quad-4.760301 \quad-2.324065 \quad-0.048357 \\
& \text { H } \quad-3.070709 \quad-4.387415 \quad-0.842886 \\
& \begin{array}{llll}
\mathrm{H} & -2.163940 & 3.934272 & 0.515547
\end{array} \\
& \begin{array}{llll}
\text { H } & 0.644153 & 1.150148 & 2.235709
\end{array} \\
& \begin{array}{llll}
\mathrm{H} & 0.561462 & 1.283271 & -3.064885
\end{array} \\
& \begin{array}{llll}
\mathrm{Rh} & 0.239668 & -0.872355 & -0.045628
\end{array} \\
& \begin{array}{llll}
\text { O } & 2.166896 & 0.235831 & 0.271332
\end{array} \\
& \begin{array}{llll}
\text { C } & 0.708102 & -1.533083 & -1.865040
\end{array} \\
& \begin{array}{llll}
\text { S } & 3.372688 & -0.010502 & 1.126175
\end{array} \\
& \begin{array}{llll}
\text { O } & 0.462270 & -0.881334 & -2.847514
\end{array} \\
& \begin{array}{llll}
\mathrm{N} & 3.894159 & -1.521827 & 0.678490
\end{array} \\
& \begin{array}{llll}
\text { O } & 3.250877 & 0.011653 & 2.584731
\end{array} \\
& \begin{array}{llll}
\text { C } & 4.546930 & 1.242355 & 0.642002
\end{array} \\
& \begin{array}{llll}
\text { C } & 5.047594 & -2.114477 & 1.391746
\end{array} \\
& \text { C } \quad 5.543234 \quad 1.610110 \quad 1.552336 \\
& \begin{array}{llll}
\text { C } & 4.485615 & 1.807419 & -0.635004
\end{array} \\
& \text { H } \quad 5.073878 \quad-1.677932 \quad 2.395222 \\
& \text { H } \quad 4.822641 \quad-3.178060 \quad 1.533942 \\
& \begin{array}{llll}
\mathrm{H} & 5.561983 & 1.182071 & 2.548544
\end{array} \\
& \begin{array}{llll}
\text { C } & 6.493395 & 2.550643 & 1.165840
\end{array} \\
& \begin{array}{llll}
\text { C } & 5.446722 & 2.748700 & -0.996897
\end{array} \\
& \text { H } \quad 3.687755 \quad 1.545150 \quad-1.320502 \\
& \text { H } \quad 7.268032 \quad 2.841823 \quad 1.870262 \\
& \begin{array}{llll}
\text { C } & 6.463682 & 3.133471 & -0.110779
\end{array} \\
& \text { H } \quad 5.395681 \quad 3.200048 \quad-1.983839 \\
& \begin{array}{llll}
\text { C } & 7.481417 & 4.176042 & -0.504321
\end{array} \\
& \begin{array}{llll}
\mathrm{H} & 7.595038 & 4.237137 & -1.590922
\end{array} \\
& \text { H } \quad 7.178141 \quad 5.169639 \quad-0.149133 \\
& \text { H } \quad 8.462659 \quad 3.962795 \quad-0.068088
\end{aligned}
$$




$$
\begin{aligned}
& \begin{array}{llll}
\text { C } & 1.488800 & -2.849476 & -1.906941
\end{array} \\
& \begin{array}{llll}
\mathrm{H} & 2.103223 & -2.875799 & -2.816347
\end{array}
\end{aligned}
$$

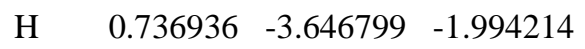

$$
\begin{aligned}
& \begin{array}{llll}
\text { C } & 2.265608 & -3.011661 & -0.589606
\end{array} \\
& \begin{array}{llll}
\text { C } & 1.241512 & -2.646997 & 0.493505
\end{array} \\
& \mathrm{H} \quad-0.766170 \quad-3.022191 \quad 2.538169 \\
& \text { H } \quad 0.549358 \quad-3.479785 \quad 0.595223 \\
& \begin{array}{llll}
\text { C } & 2.749941 & -4.467359 & -0.417574
\end{array} \\
& \text { H } \quad 3.263616 \quad-4.600470 \quad 0.541349 \\
& \text { H } \quad 1.905864 \quad-5.165515 \quad-0.434556 \\
& \mathrm{H} \quad 3.446124 \quad-4.758760 \quad-1.214210 \\
& \begin{array}{llll}
\text { C } & 3.496431 & -2.047358 & -0.635783
\end{array} \\
& \text { H } \quad 4.368554 \quad-2.560739-1.055034 \\
& \text { H } \quad 3.291788 \quad-1.204874 \quad-1.300734 \\
& \text { H } \quad 1.703260 \quad-2.463109 \quad 1.471044 \\
& \begin{array}{llll}
\text { C } & 6.330974 & -1.958398 & 0.701746
\end{array} \\
& \begin{array}{llll}
\text { C } & 7.393270 & -1.860129 & 0.129709
\end{array} \\
& \begin{array}{llll}
\text { C } & 8.682030 & -1.752735 & -0.558318
\end{array} \\
& \text { H } \quad 9.466549 \quad-1.555867 \quad 0.184783 \\
& \text { H } \quad 8.663718 \quad-0.874463 \quad-1.217789 \\
& \begin{array}{llll}
\text { C } & 9.044632 & -3.008541 & -1.373812
\end{array} \\
& \text { H } \quad 8.295031 \quad-3.203621 \quad-2.147298 \\
& \text { H } \quad 10.014970 \quad-2.872825 \quad-1.862065 \\
& \text { H } \quad 9.105115 \quad-3.889990 \quad-0.728125 \\
& \begin{array}{llll}
\text { C } & 1.755139 & 3.030148 & -3.431343
\end{array} \\
& \text { H } \quad 2.042814 \quad 2.704249 \quad-4.427403 \\
& \begin{array}{llll}
\text { C } & 2.226279 & 4.244375 & -2.925467
\end{array} \\
& \mathrm{H} \quad 2.880005 \quad 4.870907 \quad-3.526325 \\
& \begin{array}{llll}
\text { C } & 1.847112 & 4.648700 & -1.645239
\end{array} \\
& \text { H } \quad 2.209025 \quad 5.589406 \quad-1.239518 \\
& \begin{array}{llll}
\text { C } & 0.993410 & 3.853581 & -0.877071
\end{array} \\
& \begin{array}{llll}
\mathrm{H} & 0.709105 & 4.190814 & 0.112709
\end{array} \\
& \begin{array}{llll}
\text { C } & -1.626250 & 4.273755 & 2.570429
\end{array}
\end{aligned}
$$




$$
\begin{array}{lrrr}
\mathrm{H} & -2.247930 & 5.161016 & 2.658809 \\
\mathrm{C} & -0.877948 & 3.829712 & 3.664436 \\
\mathrm{H} & -0.922418 & 4.365624 & 4.608486 \\
\mathrm{C} & -0.068985 & 2.699217 & 3.538513 \\
\mathrm{H} & 0.520012 & 2.349903 & 4.381680 \\
\mathrm{C} & -2.578499 & -5.858911 & 0.633075 \\
\mathrm{H} & -3.066251 & -6.658116 & 0.082355 \\
\mathrm{C} & -1.963002 & -6.123439 & 1.855255 \\
\mathrm{H} & -1.970492 & -7.128667 & 2.266482 \\
\mathrm{C} & -1.323757 & -5.088102 & 2.541544 \\
\mathrm{H} & -0.823785 & -5.283734 & 3.485816 \\
\mathrm{C} & -5.327156 & -2.366428 & -2.117772 \\
\mathrm{H} & -6.361197 & -2.616406 & -1.896632 \\
\mathrm{C} & -4.907723 & -2.207247 & -3.442063 \\
\mathrm{H} & -5.615678 & -2.330730 & -4.256805 \\
\mathrm{C} & -3.577580 & -1.887419 & -3.712153 \\
\mathrm{H} & -3.245845 & -1.750611 & -4.737302
\end{array}
$$

IntB2

B3LYP SCF energy: $\quad 3936.01264904$ a.u.

B3LYP enthalpy: 3935.002509 a.u.

B3LYP free energy: 3935.172690 a.u.

M06-L SCF energy in solution: $\quad 3937.67512262$ a.u.

M06-L enthalpy in solution: $\quad 3936.664983$ a.u.

M06-L free energy in solution: $\quad 3936.835164$ a.u.

Cartesian coordinates

$$
\begin{array}{lccc}
\text { ATOM } & \text { X } & \text { Y } & \multicolumn{2}{l}{\text { Z }} \\
\text { P } & -1.674265 & 1.853025 & -0.232985 \\
\text { P } & -1.057641 & -1.732669 & -0.050328 \\
\text { O } & -5.146986 & 0.372189 & 2.613911
\end{array}
$$




$$
\begin{aligned}
& \text { O } \quad-4.516416 \quad-0.491213 \quad 4.666948 \\
& \begin{array}{llll}
\text { O } & -5.751142 & -1.538069 & 0.138301
\end{array} \\
& \text { O } \quad-6.851857 \quad-0.735886-1.735985 \\
& \begin{array}{llll}
\text { C } & -2.099080 & -1.362032 & 1.434650
\end{array} \\
& \begin{array}{llll}
\text { C } & -3.271851 & -0.552733 & 1.308056
\end{array} \\
& \begin{array}{llll}
\text { C } & -3.996067 & -0.356924 & 2.473138
\end{array} \\
& \begin{array}{llll}
\text { C } & -3.620875 & -0.872590 & 3.712973
\end{array} \\
& \begin{array}{llll}
\text { C } & -2.489244 & -1.644924 & 3.853687
\end{array} \\
& \begin{array}{llll}
\text { C } & -1.742002 & -1.881710 & 2.688015
\end{array} \\
& \begin{array}{llll}
\text { C } & -5.564768 & 0.200635 & 3.974392
\end{array} \\
& \begin{array}{llll}
\text { C } & -3.304426 & 1.110429 & -0.698756
\end{array} \\
& \begin{array}{llll}
\text { C } & -3.995127 & 1.587864 & -1.819902
\end{array} \\
& \begin{array}{llll}
\text { C } & -5.205229 & 1.029485 & -2.265998
\end{array} \\
& \begin{array}{llll}
\text { C } & -5.701281 & -0.029315 & -1.538676
\end{array} \\
& \begin{array}{llll}
\text { C } & -5.033930 & -0.510147 & -0.413896
\end{array} \\
& \begin{array}{llll}
\text { C } & -3.831818 & 0.007542 & 0.043196
\end{array} \\
& \begin{array}{llll}
\text { C } & -6.877197 & -1.751296 & -0.724685
\end{array} \\
& \text { C } \quad-2.223622-2.513656-1.239259 \\
& \begin{array}{llll}
\text { C } & -2.138551 & -2.244045 & -2.611965
\end{array} \\
& \begin{array}{llll}
\text { C } & -3.172874 & -3.443128 & -0.777750
\end{array} \\
& \begin{array}{llll}
\text { C } & 0.027729 & -3.127390 & 0.498646
\end{array} \\
& \begin{array}{llll}
\text { C } & -0.104466 & -4.425674 & -0.014034
\end{array} \\
& \begin{array}{llll}
\text { C } & 1.068238 & -2.868716 & 1.411021
\end{array} \\
& \begin{array}{llll}
\text { C } & -1.869411 & 2.349792 & 1.532292
\end{array} \\
& \begin{array}{llll}
\text { C } & -3.084859 & 2.845323 & 2.033916
\end{array} \\
& \begin{array}{llll}
\text { C } & -0.759451 & 2.279576 & 2.387397
\end{array} \\
& \begin{array}{llll}
\text { C } & -1.421498 & 3.524352 & -2.510707
\end{array} \\
& \begin{array}{llll}
\text { C } & -1.664324 & 3.484146 & -1.124570
\end{array} \\
& \begin{array}{llll}
\text { C } & -1.854384 & 4.699130 & -0.445867
\end{array} \\
& \text { H } \quad-2.196449 \quad-2.063306 \quad 4.809930 \\
& \mathrm{H} \quad-0.874007 \quad-2.520328 \quad 2.776090 \\
& \text { H } \quad-5.718621 \quad 1.180132 \quad 4.434706 \\
& \text { H } \quad-6.481089 \quad-0.401103 \quad 4.004127
\end{aligned}
$$




$$
\begin{aligned}
& \mathrm{H} \quad-3.603232 \quad 2.431795 \quad-2.371206 \\
& \text { H } \quad-5.724631 \quad 1.421064 \quad-3.133252 \\
& \text { H } \quad-6.792095 \quad-2.736924 \quad-1.196281 \\
& \text { H } \quad-7.801553 \quad-1.666177 \quad-0.145465 \\
& \text { H } \quad-1.434446 \quad-1.509753 \quad-2.981599 \\
& \begin{array}{llll}
\text { H } & -3.258207 & -3.653848 & 0.283819
\end{array} \\
& \text { H } \quad-0.883009-4.657056 \quad-0.731230 \\
& \text { H } \quad-3.957460 \quad 2.895523 \quad 1.389902 \\
& \begin{array}{llll}
\mathrm{H} & 0.187846 & 1.900289 & 2.013787
\end{array} \\
& \text { H } \quad-1.271514 \quad 2.604933 \quad-3.063388 \\
& \mathrm{H} \quad-2.038851 \quad 4.708654 \quad 0.621715 \\
& \begin{array}{llll}
\mathrm{Rh} & 0.397833 & 0.245799 & -0.618262
\end{array} \\
& \begin{array}{llll}
\text { O } & 4.873178 & -1.925170 & 1.519991
\end{array} \\
& \begin{array}{llll}
\text { S } & 5.406242 & -1.430128 & 0.244791
\end{array} \\
& \text { N } \quad 4.265732 \quad-0.331300 \quad-0.325892 \\
& \begin{array}{llll}
\text { O } & 5.703354 & -2.322352 & -0.877557
\end{array} \\
& \begin{array}{llll}
\text { C } & 6.881549 & -0.484988 & 0.607964
\end{array} \\
& \begin{array}{llll}
\text { C } & 7.059053 & 0.056512 & 1.883544
\end{array} \\
& \begin{array}{llll}
\text { C } & 7.833146 & -0.284072 & -0.396730
\end{array} \\
& \text { H } \quad 6.332047 \quad-0.140066 \quad 2.664344 \\
& \begin{array}{llll}
\text { C } & 8.197692 & 0.817864 & 2.144287
\end{array} \\
& \begin{array}{llll}
\text { C } & 8.963750 & 0.478051 & -0.115247
\end{array} \\
& \text { H } \quad 7.700094 \quad-0.739874 \quad-1.372096 \\
& \mathrm{H} \quad 8.342378 \quad 1.234953 \quad 3.137522 \\
& \begin{array}{llll}
\text { C } & 9.165531 & 1.041679 & 1.154819
\end{array} \\
& \begin{array}{llll}
\text { H } & 9.709018 & 0.629907 & -0.891879
\end{array} \\
& \begin{array}{llll}
\text { C } & 10.413633 & 1.836012 & 1.454631
\end{array} \\
& \mathrm{H} \quad 11.252323 \quad 1.168511 \quad 1.691514 \\
& \text { H } \quad 10.717413 \quad 2.444493 \quad 0.596360 \\
& \text { H } \quad 10.271472 \quad 2.500344 \quad 2.312439 \\
& \begin{array}{llll}
\mathrm{H} & 1.209591 & -1.864603 & 1.803549
\end{array} \\
& \begin{array}{llll}
\text { C } & 3.501856 & 0.437932 & 0.661585
\end{array} \\
& \text { H } \quad 4.110738 \quad 1.252842 \quad 1.089494
\end{aligned}
$$




$$
\begin{aligned}
& \mathrm{H} \quad 3.219522 \quad-0.225784 \quad 1.482981 \\
& \begin{array}{llll}
\text { C } & 1.817922 & -1.273370 & -1.235431
\end{array} \\
& \begin{array}{llll}
\text { C } & 2.757044 & -0.755494 & -2.335511
\end{array} \\
& \text { H } \quad 1.195484 \quad-2.065029-1.658003 \\
& \begin{array}{llll}
\text { C } & 1.917693 & 0.246455 & -3.176297
\end{array} \\
& \begin{array}{llll}
\text { C } & 0.510677 & 0.388141 & -2.598268
\end{array} \\
& \text { H } \quad 2.349452 \quad 1.256978 \quad-3.178321 \\
& \text { H } \quad 1.806907 \quad-0.052474 \quad-4.224784 \\
& \begin{array}{llll}
\text { O } & -0.485335 & 0.577433 & -3.240589
\end{array} \\
& \begin{array}{llll}
\mathrm{H} & 2.345157 & -1.690310 & -0.382055
\end{array} \\
& \begin{array}{llll}
\text { C } & 4.062126 & -0.087176 & -1.749994
\end{array} \\
& \text { H } \quad 4.931392 \quad-0.482290 \quad-2.277219 \\
& \text { H } \quad 4.053079 \quad 0.995982 \quad-1.914898 \\
& \begin{array}{llll}
\text { C } & 3.190845 & -1.945909 & -3.224713
\end{array} \\
& \text { H } \quad 3.756497 \quad-2.674484 \quad-2.636279 \\
& \text { H } \quad 2.322129 \quad-2.449541 \quad-3.664250 \\
& \text { H } \quad 3.834515 \quad-1.606882 \quad-4.046545 \\
& \begin{array}{llll}
\text { C } & 2.316724 & 1.087623 & 0.057023
\end{array} \\
& \begin{array}{llll}
\text { C } & 1.699921 & 2.074523 & -0.401903
\end{array} \\
& \begin{array}{llll}
\text { C } & 1.604437 & 3.515565 & -0.732572
\end{array} \\
& \text { H } \quad 0.840697 \quad 3.982201 \quad-0.101752 \\
& \text { H } \quad 1.243971 \quad 3.640055 \quad-1.759480 \\
& \begin{array}{llll}
\text { C } & 2.953564 & 4.232008 & -0.545071
\end{array} \\
& \text { H } \quad 2.845235 \quad 5.293635 \quad-0.789682 \\
& \begin{array}{llll}
\mathrm{H} & 3.302587 & 4.156177 & 0.489973
\end{array} \\
& \text { H } \quad 3.724508 \quad 3.809659-1.197942 \\
& \text { C } \quad-1.805460 \quad 5.915424 \quad-1.131866 \\
& \text { H } \quad-1.955699 \quad 6.842172 \quad-0.585105 \\
& \begin{array}{llll}
\text { C } & -1.572102 & 5.942414 & -2.506592
\end{array} \\
& \text { H } \quad-1.538728 \quad 6.888957 \quad-3.038383 \\
& \begin{array}{llll}
\text { C } & -1.384745 & 4.741291 & -3.193788
\end{array} \\
& \text { H } \quad-1.207438 \quad 4.747125 \quad-4.265808 \\
& \begin{array}{llll}
\text { C } & -0.858604 & 2.697300 & 3.716571
\end{array}
\end{aligned}
$$




$$
\begin{array}{lrrr}
\mathrm{H} & 0.007870 & 2.634513 & 4.368917 \\
\mathrm{C} & -2.068471 & 3.192810 & 4.204412 \\
\mathrm{H} & -2.146308 & 3.519768 & 5.237386 \\
\mathrm{C} & -3.180278 & 3.266553 & 3.360331 \\
\mathrm{H} & -4.123048 & 3.657062 & 3.733925 \\
\mathrm{C} & -2.984091 & -2.898439 & -3.511104 \\
\mathrm{H} & -2.913510 & -2.676680 & -4.572005 \\
\mathrm{C} & -3.914983 & -3.829322 & -3.049270 \\
\mathrm{H} & -4.566415 & -4.342482 & -3.751051 \\
\mathrm{C} & -4.008075 & -4.100119 & -1.680562 \\
\mathrm{H} & -4.727674 & -4.827600 & -1.314818 \\
\mathrm{C} & 0.770513 & -5.438768 & 0.389714 \\
\mathrm{H} & 0.652949 & -6.438073 & -0.019611 \\
\mathrm{C} & 1.790193 & -5.170625 & 1.301251 \\
\mathrm{H} & 2.474575 & -5.957654 & 1.603790 \\
\mathrm{C} & 1.939503 & -3.878637 & 1.813239 \\
\mathrm{H} & 2.751088 & -3.647398 & 2.495453
\end{array}
$$

IntB3

B3LYP SCF energy: $\quad 3936.01969559$ a.u.

B3LYP enthalpy: 3935.009359 a.u.

B3LYP free energy: 3935.177638 a.u.

M06-L SCF energy in solution: $\quad 3937.68083364$ a.u.

M06-L enthalpy in solution: $\quad 3936.670497$ a.u.

M06-L free energy in solution: $\quad 3936.838776$ a.u.

Cartesian coordinates

$\begin{array}{lrrr}\text { ATOM } & \text { X } & \text { Y } & \text { Z } \\ \text { P } & 1.409539 & 1.812190 & 0.218328 \\ \text { P } & 1.248196 & -1.755357 & -0.403240 \\ \text { O } & 5.904674 & 0.789290 & -0.618496\end{array}$




$$
\begin{aligned}
& \begin{array}{llll}
\text { O } & 6.401865 & 0.129188 & -2.782554
\end{array} \\
& \begin{array}{llll}
\text { O } & 5.395823 & -1.310262 & 1.727241
\end{array} \\
& \begin{array}{llll}
\text { O } & 5.431479 & -0.573734 & 3.923863
\end{array} \\
& \begin{array}{llll}
\text { C } & 2.807008 & -1.143070 & -1.201501
\end{array} \\
& \begin{array}{llll}
\text { C } & 3.713694 & -0.322342 & -0.456605
\end{array} \\
& \begin{array}{llll}
\text { C } & 4.886268 & 0.020416 & -1.111179
\end{array} \\
& \begin{array}{llll}
\text { C } & 5.190731 & -0.372156 & -2.414351
\end{array} \\
& \begin{array}{llll}
\text { C } & 4.328826 & -1.161233 & -3.142463
\end{array} \\
& \begin{array}{llll}
\text { C } & 3.134308 & -1.539375 & -2.505903
\end{array} \\
& \begin{array}{llll}
\text { C } & 6.890295 & 0.872302 & -1.655602
\end{array} \\
& \begin{array}{llll}
\text { C } & 2.678715 & 1.180125 & 1.398828
\end{array} \\
& \begin{array}{llll}
\text { C } & 2.728907 & 1.627191 & 2.722648
\end{array} \\
& \begin{array}{llll}
\text { C } & 3.625464 & 1.095669 & 3.667443
\end{array} \\
& \begin{array}{llll}
\text { C } & 4.466904 & 0.097648 & 3.227712
\end{array} \\
& \begin{array}{llll}
\text { C } & 4.438952 & -0.348197 & 1.906884
\end{array} \\
& \begin{array}{llll}
\text { C } & 3.562892 & 0.147008 & 0.954245
\end{array} \\
& \begin{array}{llll}
\text { C } & 5.999258 & -1.521983 & 3.010997
\end{array} \\
& \begin{array}{llll}
\text { C } & 1.951640 & -2.733710 & 1.004149
\end{array} \\
& \begin{array}{llll}
\text { C } & 1.605871 & -2.515262 & 2.344529
\end{array} \\
& \begin{array}{llll}
\text { C } & 2.920776 & -3.711129 & 0.707324
\end{array} \\
& \text { C } \quad 0.606525 \quad-3.028988-1.581624 \\
& \text { C } \quad \begin{array}{llll}
0.485271 & -4.385374 & -1.248321
\end{array} \\
& \begin{array}{llll}
\text { C } & 0.125855 & -2.601093 & -2.834617
\end{array} \\
& \text { C } \quad 2.431065 \quad 2.427103 \quad-1.195596 \\
& \begin{array}{llll}
\text { C } & 3.559604 & 3.234610 & -0.970725
\end{array} \\
& \begin{array}{llll}
\text { C } & 2.081990 & 2.107518 & -2.515154
\end{array} \\
& \begin{array}{llll}
\text { C } & -0.069373 & 3.306404 & 2.109615
\end{array} \\
& \begin{array}{llll}
\text { C } & 0.727927 & 3.372836 & 0.951043
\end{array} \\
& \begin{array}{llll}
\text { C } & 0.900249 & 4.621818 & 0.331185
\end{array} \\
& \text { H } \quad 4.561687 \quad-1.485528 \quad-4.150344 \\
& \text { H } \quad 2.465414 \quad-2.186935 \quad-3.055297 \\
& \text { H } \quad 7.028201 \quad 1.919109-1.944301 \\
& \text { H } \quad \begin{array}{llll}
7.828523 & 0.428687 & -1.306343
\end{array}
\end{aligned}
$$




$$
\begin{aligned}
& \begin{array}{llll}
\text { H } & 2.065048 & 2.419318 & 3.044626
\end{array} \\
& \mathrm{H} \quad 3.656368 \quad 1.459887 \quad 4.688145 \\
& \text { H } \quad 5.774903 \quad-2.538740 \quad 3.353293 \\
& \begin{array}{llll}
\mathrm{H} & 7.078613 & -1.356633 & 2.938437
\end{array} \\
& \text { H } \quad 0.928664 \quad-1.719140 \quad 2.619394 \\
& \text { H } \quad 3.237972 \quad-3.869126 \quad-0.318782 \\
& \text { H } \quad \begin{array}{llll}
0.822111 & -4.746463 & -0.283693
\end{array} \\
& \text { H } \quad 3.856550 \quad 3.479071 \quad 0.044912 \\
& \text { H } \quad 1.232199 \quad 1.458789-2.707886 \\
& \begin{array}{llll}
\mathrm{H} & -0.245216 & 2.350959 & 2.589405
\end{array} \\
& \text { H } \quad 1.496127 \quad 4.711806 \quad-0.569182 \\
& \begin{array}{llll}
\mathrm{Rh} & -0.457024 & -0.009816 & -0.161312
\end{array} \\
& \begin{array}{llll}
\text { O } & -5.277416 & 2.102823 & -0.761243
\end{array} \\
& \begin{array}{llll}
\text { C } & -0.935005 & -0.471632 & 1.708268
\end{array} \\
& \begin{array}{llll}
\text { S } & -5.366138 & 0.637969 & -0.698582
\end{array} \\
& \begin{array}{llll}
\text { O } & -0.517143 & 0.172744 & 2.635279
\end{array} \\
& \begin{array}{llll}
\mathrm{N} & -4.405315 & 0.205033 & 0.638565
\end{array} \\
& \begin{array}{llll}
\text { O } & -5.010747 & -0.181867 & -1.864561
\end{array} \\
& \begin{array}{llll}
\text { C } & -7.011631 & 0.159972 & -0.188434
\end{array} \\
& \begin{array}{llll}
\text { C } & -3.453523 & 1.199450 & 1.116697
\end{array} \\
& \begin{array}{llll}
\text { C } & -7.669293 & -0.864098 & -0.869467
\end{array} \\
& \begin{array}{llll}
\text { C } & -7.636173 & 0.850480 & 0.856128
\end{array} \\
& \begin{array}{llll}
\mathrm{H} & -3.964117 & 2.160325 & 1.228155
\end{array} \\
& \text { H } \quad-3.114533 \quad 0.892222 \quad 2.109295 \\
& \text { H } \quad-7.174326-1.372474-1.689670 \\
& \begin{array}{llll}
\text { C } & -8.966544 & -1.207513 & -0.485327
\end{array} \\
& \begin{array}{llll}
\text { C } & -8.928693 & 0.493834 & 1.222704
\end{array} \\
& \text { H } \quad \begin{array}{llll}
-7.121290 & 1.658137 & 1.366249
\end{array} \\
& \mathrm{H} \quad-9.482846 \quad-2.005120 \quad-1.012736 \\
& \begin{array}{llll}
\text { C } & -9.615594 & -0.538842 & 0.559962
\end{array} \\
& \begin{array}{llll}
\text { H } & -9.418699 & 1.027233 & 2.033290
\end{array} \\
& \begin{array}{llll}
\text { C } & -11.027216 & -0.895814 & 0.957338
\end{array} \\
& \text { H } \quad-11.126806 \quad-0.978726 \quad 2.045365
\end{aligned}
$$




$$
\begin{aligned}
& \text { H } \quad-11.733012 \quad-0.123054 \quad 0.626979 \\
& \mathrm{H} \quad-11.342680 \quad-1.844145 \quad 0.512793 \\
& \begin{array}{llll}
\text { C } & -1.849748 & -1.681892 & 1.859489
\end{array} \\
& \text { H } \quad-2.357462 \quad-1.628314 \quad 2.831871 \\
& \text { H } \quad-1.160142 \quad-2.535411 \quad 1.915668 \\
& \begin{array}{llll}
\text { C } & -2.794508 & -1.875305 & 0.651142
\end{array} \\
& \begin{array}{llll}
\text { C } & -2.055422 & -1.355466 & -0.603803
\end{array} \\
& \text { H } \quad 0.165054 \quad-1.547916 \quad-3.102083 \\
& \text { H } \quad-1.630700 \quad-2.190959-1.154376 \\
& \begin{array}{llll}
\text { C } & -4.193985 & -1.220330 & 0.910994
\end{array} \\
& \text { H } \quad-4.928743 \quad-1.755056 \quad 0.304859 \\
& \text { H } \quad-4.468684 \quad-1.406159 \quad 1.959200 \\
& \begin{array}{llll}
\text { C } & -3.090274 & -3.389503 & 0.511167
\end{array} \\
& \text { H } \quad-2.161258 \quad-3.958100 \quad 0.397932 \\
& \text { H } \quad-3.621838 \quad-3.781715 \quad 1.388156 \\
& \text { H } \quad-3.709246 \quad-3.580865 \quad-0.371823 \\
& \mathrm{H} \quad-2.723997 \quad-0.814720 \quad-1.277583 \\
& \begin{array}{llll}
\text { C } & -2.325201 & 1.430587 & 0.176538
\end{array} \\
& \begin{array}{llll}
\text { C } & -1.766539 & 1.868396 & -0.829917
\end{array} \\
& \begin{array}{llll}
\text { C } & -1.443820 & 2.622263 & -2.051698
\end{array} \\
& \text { H } \quad-0.430449 \quad 3.031181 \quad-1.992891 \\
& \text { H } \quad-1.464845 \quad 1.938319 \quad-2.910748 \\
& \begin{array}{llll}
\text { C } & -2.459248 & 3.766231 & -2.264390
\end{array} \\
& \text { H } \quad-3.482810 \quad 3.384616 \quad-2.283471 \\
& \text { H } \quad-2.377934 \quad 4.502672 \quad-1.459478 \\
& \mathrm{H} \quad-2.245172 \quad 4.265464 \quad-3.214927 \\
& \begin{array}{llll}
\text { C } & 3.484287 & -4.483911 & 1.721028
\end{array} \\
& \text { H } \quad 4.220798 \quad-5.242118 \quad 1.470555 \\
& \text { C } \quad 3.106334 \quad-4.280541 \quad 3.051393 \\
& \text { H } \quad 3.540365 \quad-4.887447 \quad 3.841138 \\
& \begin{array}{llll}
\text { C } & 2.177844 & -3.286732 & 3.360103
\end{array} \\
& \mathrm{H} \quad 1.898107 \quad-3.100472 \quad 4.392931 \\
& \begin{array}{llll}
\text { C } & -0.070104 & -5.293165 & -2.155868
\end{array}
\end{aligned}
$$




$$
\begin{array}{lrrr}
\mathrm{H} & -0.152206 & -6.340381 & -1.879268 \\
\mathrm{C} & -0.512815 & -4.861854 & -3.405053 \\
\mathrm{H} & -0.939886 & -5.570001 & -4.108995 \\
\mathrm{C} & -0.415467 & -3.508819 & -3.742305 \\
\mathrm{H} & -0.771775 & -3.157987 & -4.706462 \\
\mathrm{C} & 2.829972 & 2.593706 & -3.589868 \\
\mathrm{H} & 2.548533 & 2.333474 & -4.606295 \\
\mathrm{C} & 3.938087 & 3.409156 & -3.356014 \\
\mathrm{H} & 4.516990 & 3.793668 & -4.190917 \\
\mathrm{C} & 4.302487 & 3.726720 & -2.043992 \\
\mathrm{H} & 5.163514 & 4.362459 & -1.855685 \\
\mathrm{C} & 0.311960 & 5.771331 & 0.865675 \\
\mathrm{H} & 0.461300 & 6.726357 & 0.369695 \\
\mathrm{C} & -0.452672 & 5.696987 & 2.029025 \\
\mathrm{H} & -0.902463 & 6.592968 & 2.447174 \\
\mathrm{C} & -0.638981 & 4.459586 & 2.649316 \\
\mathrm{H} & -1.235534 & 4.387896 & 3.554819
\end{array}
$$

TS2a

B3LYP SCF energy: $\quad 3936.00020119$ a.u.

B3LYP enthalpy: 3934.991284 a.u.

B3LYP free energy: 3935.158321 a.u.

M06-L SCF energy in solution: $\quad 3937.66261532$ a.u.

M06-L enthalpy in solution: $\quad 3936.653698$ a.u.

M06-L free energy in solution: $\quad 3936.820735$ a.u.

Imaginary frequency: \$TAB $\$-284.7862 \mathrm{~cm}-1$

Cartesian coordinates

$$
\begin{array}{lrcrl}
\multicolumn{2}{l}{\text { ATOM } \mathrm{X}} & \mathrm{Y} & \multicolumn{2}{l}{\mathrm{Z}} \\
\mathrm{P} & -1.702993 & 1.832918 & -0.265635 \\
\mathrm{P} & -1.161009 & -1.696361 & 0.102029
\end{array}
$$




$$
\begin{aligned}
& \begin{array}{llll}
\text { O } & -5.510810 & 0.432560 & 2.251003
\end{array} \\
& \begin{array}{llll}
\text { O } & -5.131472 & -0.409669 & 4.371930
\end{array} \\
& \begin{array}{llll}
\text { O } & -5.822586 & -1.526568 & -0.253820
\end{array} \\
& \begin{array}{llll}
\text { O } & -6.688495 & -0.768456 & -2.262155
\end{array} \\
& \begin{array}{llll}
\text { C } & -2.355553 & -1.329301 & 1.461715
\end{array} \\
& \begin{array}{llll}
\text { C } & -3.501706 & -0.522074 & 1.183717
\end{array} \\
& \begin{array}{llll}
\text { C } & -4.358763 & -0.309706 & 2.251656
\end{array} \\
& \begin{array}{llll}
\text { C } & -4.132829 & -0.811930 & 3.532899
\end{array} \\
& \begin{array}{llll}
\text { C } & -3.026961 & -1.582252 & 3.817808
\end{array} \\
& \begin{array}{llll}
\text { C } & -2.145811 & -1.834125 & 2.751940
\end{array} \\
& \begin{array}{llll}
\text { C } & -6.102696 & 0.241615 & 3.542781
\end{array} \\
& \begin{array}{llll}
\text { C } & -3.285348 & 1.098151 & -0.859218
\end{array} \\
& \begin{array}{llll}
\text { C } & -3.838072 & 1.552250 & -2.063272
\end{array} \\
& \begin{array}{llll}
\text { C } & -4.990245 & 0.986834 & -2.634108
\end{array} \\
& \begin{array}{llll}
\text { C } & -5.566914 & -0.058955 & -1.948231
\end{array} \\
& \text { C } \quad-5.037986 \quad-0.515020 \quad-0.741036 \\
& \begin{array}{llll}
\text { C } & -3.899642 & 0.014133 & -0.152878
\end{array} \\
& \begin{array}{llll}
\text { C } & -6.796457 & -1.798601 & -1.270755
\end{array} \\
& \begin{array}{llll}
\text { C } & -2.166122 & -2.667916 & -1.100547
\end{array} \\
& \begin{array}{llll}
\text { C } & -1.922084 & -2.571281 & -2.478025
\end{array} \\
& \begin{array}{llll}
\text { C } & -3.146992 & -3.564472 & -0.641122
\end{array} \\
& \begin{array}{llll}
\text { C } & -0.010302 & -2.951176 & 0.826389
\end{array} \\
& \begin{array}{llll}
\text { C } & -0.006427 & -4.294849 & 0.425427
\end{array} \\
& \begin{array}{llll}
\text { C } & 0.945342 & -2.526870 & 1.769064
\end{array} \\
& \begin{array}{llll}
\text { C } & -2.007329 & 2.308426 & 1.487749
\end{array} \\
& \begin{array}{llll}
\text { C } & -3.251143 & 2.815671 & 1.900339
\end{array} \\
& \begin{array}{llll}
\text { C } & -0.966570 & 2.216126 & 2.423699
\end{array} \\
& \begin{array}{llll}
\text { C } & -1.178879 & 3.530714 & -2.484850
\end{array} \\
& \begin{array}{llll}
\text { C } & -1.569929 & 3.470124 & -1.133777
\end{array} \\
& \text { C } \quad-1.813500 \quad 4.676051 \quad-0.456255 \\
& \begin{array}{llll}
\mathrm{H} & -2.850181 & -1.984668 & 4.808843
\end{array} \\
& \text { H } \quad-1.286806 \quad-2.463361 \quad 2.946339 \\
& \text { H } \quad-6.356272 \quad 1.212875 \quad 3.974344
\end{aligned}
$$




$$
\begin{aligned}
& \text { H } \quad-6.990722 \quad-0.396027 \quad 3.448901 \\
& \text { H } \quad-3.375431 \quad 2.378979 \quad-2.585417 \\
& \mathrm{H} \quad \begin{array}{llll}
-5.404257 & 1.358827 & -3.564295
\end{array} \\
& \text { H } \quad-6.580135 \quad-2.770587 \quad-1.730395 \\
& \text { H } \quad-7.797373 \quad-1.777426 \quad-0.831906 \\
& \text { H } \quad-1.190600 \quad-1.868180 \quad-2.855337 \\
& \text { H } \quad-3.354264 \quad-3.646340 \quad 0.421273 \\
& \text { H } \quad-0.724281 \quad-4.650248 \quad-0.304930 \\
& \text { H } \quad-4.072752 \quad 2.880566 \quad 1.193632 \\
& \begin{array}{llll}
\mathrm{H} & 0.002265 & 1.825753 & 2.122731
\end{array} \\
& \text { H } \quad-0.991255 \quad 2.620706 \quad-3.040628 \\
& \text { H } \quad-2.109266 \quad 4.671101 \quad 0.585744 \\
& \begin{array}{llll}
\mathrm{Rh} & 0.252982 & 0.368951 & -0.437658
\end{array} \\
& \begin{array}{llll}
\text { O } & 5.079307 & -2.005366 & 1.048535
\end{array} \\
& \begin{array}{llll}
\text { S } & 5.647332 & -1.336515 & -0.130855
\end{array} \\
& \text { N } \quad 4.587383 \quad-0.085446 \quad-0.558564 \\
& \begin{array}{llll}
\text { O } & 5.925394 & -2.069182 & -1.369530
\end{array} \\
& \begin{array}{llll}
\text { C } & 7.147284 & -0.512715 & 0.387241
\end{array} \\
& \begin{array}{llll}
\text { C } & 7.376237 & -0.283047 & 1.744696
\end{array} \\
& \begin{array}{llll}
\text { C } & 8.074128 & -0.108722 & -0.578768
\end{array} \\
& \text { H } \quad 6.662478 \quad-0.631475 \quad 2.483327 \\
& \begin{array}{llll}
\text { C } & 8.544818 & 0.372457 & 2.132439
\end{array} \\
& \begin{array}{llll}
\text { C } & 9.234169 & 0.542339 & -0.170822
\end{array} \\
& \text { H } \quad \begin{array}{llll}
7.897399 & -0.320245 & -1.628070
\end{array} \\
& \begin{array}{llll}
\mathrm{H} & 8.729478 & 0.549097 & 3.188835
\end{array} \\
& \begin{array}{llll}
\text { C } & 9.489563 & 0.794606 & 1.187312
\end{array} \\
& \text { H } \quad 9.959916 \quad 0.853319 \quad-0.917947 \\
& \text { C } \quad 10.768159 \quad 1.473396 \quad 1.614999 \\
& \begin{array}{llll}
\mathrm{H} & 11.603928 & 0.761767 & 1.620870
\end{array} \\
& \text { H } \quad 11.039474 \quad 2.284139 \quad 0.930435 \\
& \begin{array}{llll}
\mathrm{H} & 10.684658 & 1.890288 & 2.623141
\end{array} \\
& \text { H } \quad 0.972426 \quad-1.485260 \quad 2.081719 \\
& \begin{array}{llll}
\text { C } & 3.786178 & 0.534588 & 0.505511
\end{array}
\end{aligned}
$$




$$
\begin{aligned}
& \begin{array}{llll}
\mathrm{H} & 4.244619 & 1.478791 & 0.833793
\end{array} \\
& \text { H } \quad 3.754757 \quad-0.142153 \quad 1.364362 \\
& \begin{array}{llll}
\text { C } & 2.038893 & -1.029775 & -0.909462
\end{array} \\
& \begin{array}{llll}
\text { C } & 2.736225 & -0.636901 & -2.214399
\end{array} \\
& \text { H } \quad 1.274247 \quad-1.771470 \quad-1.132740 \\
& \begin{array}{llll}
\text { C } & 1.765326 & 0.295567 & -2.994557
\end{array} \\
& \begin{array}{llll}
\text { C } & 0.337123 & 0.359269 & -2.423160
\end{array} \\
& \begin{array}{llll}
\text { H } & 2.112598 & 1.337217 & -2.970086
\end{array} \\
& \text { H } \quad 1.670088 \quad 0.013632 \quad-4.048504 \\
& \begin{array}{llll}
\text { O } & -0.642709 & 0.430160 & -3.114562
\end{array} \\
& \text { H } \quad 2.674037 \quad-1.477467 \quad-0.151356 \\
& \begin{array}{llll}
\text { C } & 4.116602 & 0.068996 & -1.935033
\end{array} \\
& \text { H } \quad 4.883615 \quad-0.336351 \quad-2.595205 \\
& \text { H } \quad 4.048961 \quad 1.144149 \quad-2.136293 \\
& \begin{array}{llll}
\text { C } & 2.983899 & -1.925898 & -3.035191
\end{array} \\
& \text { H } \quad 3.644082-2.610255 \quad-2.495189 \\
& \text { H } \quad 2.041775 \quad-2.443534 \quad-3.251811 \\
& \text { H } \quad 3.463961 \quad-1.686947 \quad-3.992135 \\
& \begin{array}{llll}
\text { C } & 2.407417 & 0.888734 & 0.052725
\end{array} \\
& \begin{array}{llll}
\text { C } & 1.642535 & 1.892067 & -0.180964
\end{array} \\
& \begin{array}{llll}
\text { C } & 1.657455 & 3.385803 & -0.185419
\end{array} \\
& \text { H } \quad 0.877196 \quad 3.750460 \quad 0.492663 \\
& \begin{array}{llll}
\mathrm{H} & 1.348828 & 3.732081 & -1.179617
\end{array} \\
& \begin{array}{llll}
\text { C } & 3.010534 & 4.007024 & 0.191751
\end{array} \\
& \text { H } \quad 2.944154 \quad 5.099050 \quad 0.146192 \\
& \text { H } \quad 3.309073 \quad 3.733525 \quad 1.209691 \\
& \text { H } \quad 3.804002 \quad 3.691380-0.494288 \\
& \text { C } \quad-1.673902 \quad 5.903163 \quad-1.109330 \\
& \text { H } \quad-1.867375 \quad 6.822200 \quad-0.563325 \\
& \begin{array}{llll}
\text { C } & -1.292383 & 5.950522 & -2.449500
\end{array} \\
& \text { H } \quad-1.187058 \quad 6.905453 \quad-2.956243 \\
& \begin{array}{llll}
\text { C } & -1.049156 & 4.758661 & -3.135411
\end{array} \\
& \text { H } \quad-0.757648 \quad 4.780304 \quad-4.181874
\end{aligned}
$$




$$
\begin{array}{lrrr}
\mathrm{C} & -1.160971 & 2.624184 & 3.745175 \\
\mathrm{H} & -0.347332 & 2.543880 & 4.460454 \\
\mathrm{C} & -2.397527 & 3.133128 & 4.143954 \\
\mathrm{H} & -2.549217 & 3.452936 & 5.170870 \\
\mathrm{C} & -3.441146 & 3.228081 & 3.219120 \\
\mathrm{H} & -4.405074 & 3.626028 & 3.524009 \\
\mathrm{C} & -2.640628 & -3.359384 & -3.380001 \\
\mathrm{H} & -2.445670 & -3.269928 & -4.444789 \\
\mathrm{C} & -3.606375 & -4.253321 & -2.916165 \\
\mathrm{H} & -4.160412 & -4.869807 & -3.618506 \\
\mathrm{C} & -3.858120 & -4.354211 & -1.544637 \\
\mathrm{H} & -4.604401 & -5.052737 & -1.175828 \\
\mathrm{C} & 0.924300 & -5.190915 & 0.960117 \\
\mathrm{H} & 0.915420 & -6.227225 & 0.634616 \\
\mathrm{C} & 1.859966 & -4.760127 & 1.899068 \\
\mathrm{H} & 2.587020 & -5.456291 & 2.306392 \\
\mathrm{C} & 1.867496 & -3.422729 & 2.306012 \\
\mathrm{H} & 2.604061 & -3.075574 & 3.024173
\end{array}
$$

TS2b

B3LYP SCF energy: 3935.99054534 a.u.

B3LYP enthalpy: 3934.982158 a.u.

B3LYP free energy: 3935.148207 a.u.

M06-L SCF energy in solution: $\quad 3937.65658179$ a.u.

M06-L enthalpy in solution: $\quad 3936.648194$ a.u.

M06-L free energy in solution: $\quad 3936.814243$ a.u.

Imaginary frequency: $\$$ TAB $\$-300.2344 \mathrm{~cm}-1$

Cartesian coordinates

\begin{tabular}{lccc} 
ATOM & X & Y & \multicolumn{2}{l}{ Z } \\
P & -1.601239 & 1.807018 & -0.086523
\end{tabular}




$$
\begin{aligned}
& \begin{array}{llll}
\mathrm{P} & -0.937681 & -1.749942 & -0.268741
\end{array} \\
& \begin{array}{llll}
\text { O } & -4.710689 & 0.104975 & 3.007309
\end{array} \\
& \begin{array}{llll}
\text { O } & -3.857291 & -0.948414 & 4.885598
\end{array} \\
& \begin{array}{llll}
\text { O } & -5.643424 & -1.580002 & 0.460775
\end{array} \\
& \begin{array}{llll}
\text { O } & -7.011171 & -0.529229 & -1.084762
\end{array} \\
& \text { C } \quad-1.862033 \quad-1.542231 \quad 1.318471 \\
& \begin{array}{llll}
\text { C } & -3.026692 & -0.714755 & 1.405616
\end{array} \\
& \begin{array}{llll}
\text { C } & -3.596952 & -0.615836 & 2.666113
\end{array} \\
& \begin{array}{llll}
\text { C } & -3.088682 & -1.246156 & 3.800404
\end{array} \\
& \begin{array}{llll}
\text { C } & -1.968666 & -2.044293 & 3.732227
\end{array} \\
& \begin{array}{llll}
\text { C } & -1.371036 & -2.177745 & 2.468785
\end{array} \\
& \begin{array}{llll}
\text { C } & -4.921848 & -0.114372 & 4.408319
\end{array} \\
& \begin{array}{llll}
\text { C } & -3.316167 & 1.173724 & -0.372975
\end{array} \\
& \begin{array}{llll}
\text { C } & -4.168068 & 1.804122 & -1.288630
\end{array} \\
& \begin{array}{llll}
\text { C } & -5.446018 & 1.311720 & -1.606010
\end{array} \\
& \begin{array}{llll}
\text { C } & -5.838024 & 0.158259 & -0.964536
\end{array} \\
& \begin{array}{llll}
\text { C } & -5.010020 & -0.471592 & -0.037027
\end{array} \\
& \begin{array}{llll}
\text { C } & -3.742496 & -0.018192 & 0.293098
\end{array} \\
& \begin{array}{llll}
\text { C } & -6.957227 & -1.582798 & -0.114964
\end{array} \\
& \begin{array}{llll}
\text { C } & -2.201531 & -2.222835 & -1.517115
\end{array} \\
& \begin{array}{llll}
\text { C } & -2.121800 & -1.706684 & -2.819329
\end{array} \\
& \begin{array}{llll}
\text { C } & -3.228829 & -3.128242 & -1.201521
\end{array} \\
& \begin{array}{llll}
\text { C } & 0.079442 & -3.268228 & -0.014376
\end{array} \\
& \begin{array}{llll}
\text { C } & -0.245136 & -4.492072 & -0.617418
\end{array} \\
& \begin{array}{llll}
\text { C } & 1.235707 & -3.192809 & 0.784296
\end{array} \\
& \begin{array}{llll}
\text { C } & -1.527132 & 2.096031 & 1.732586
\end{array} \\
& \begin{array}{llll}
\text { C } & -2.609009 & 2.674460 & 2.418445
\end{array} \\
& \begin{array}{llll}
\text { C } & -0.362665 & 1.776155 & 2.443950
\end{array} \\
& \begin{array}{llll}
\text { C } & -1.701141 & 3.700091 & -2.180204
\end{array} \\
& \begin{array}{lllll}
\text { C } & -1.594777 & 3.520008 & -0.787953
\end{array} \\
& \begin{array}{llll}
\text { C } & -1.423088 & 4.654346 & 0.022941
\end{array} \\
& \mathrm{H} \quad-1.572348 \quad-2.552811 \quad 4.603666 \\
& \text { H } \quad-0.505656 \quad-2.821803 \quad 2.395838
\end{aligned}
$$




$$
\begin{aligned}
& \mathrm{H} \quad-4.895740 \quad 0.844641 \quad 4.935124 \\
& \mathrm{H} \quad-5.879983 \quad-0.623680 \quad 4.559699 \\
& \mathrm{H} \quad-3.855551 \quad 2.723087 \quad-1.765920 \\
& \text { H } \quad-6.091310 \quad 1.821399 \quad-2.312498 \\
& \text { H } \quad-7.135593 \quad-2.541877 \quad-0.609399 \\
& \text { H } \quad-7.701094 \quad-1.397256 \quad 0.669116 \\
& \text { H } \quad-1.340514 \quad-0.996679 \quad-3.078935 \\
& \mathrm{H} \quad-3.317220 \quad-3.521450 \quad-0.193403 \\
& \text { H } \quad-1.117044 \quad-4.578537 \quad-1.254949 \\
& \mathrm{H} \quad-3.522265 \quad 2.918435 \quad 1.883930 \\
& \begin{array}{llll}
\mathrm{H} & 0.472382 & 1.315808 & 1.925175
\end{array} \\
& \text { H } \quad-1.785516 \quad 2.842471 \quad-2.835972 \\
& \text { H } \quad-1.329333 \quad 4.551118 \quad 1.097498 \\
& \begin{array}{lllll}
\mathrm{Rh} & 0.267139 & 0.260475 & -1.075079
\end{array} \\
& \begin{array}{llll}
\text { O } & 4.148900 & -1.720673 & 1.877422
\end{array} \\
& \begin{array}{llll}
\text { S } & 4.837772 & -1.478818 & 0.602904
\end{array} \\
& \text { N } \quad 3.796351-0.467003 \quad-0.267060 \\
& \begin{array}{llll}
\text { O } & 5.186248 & -2.566546 & -0.311884
\end{array} \\
& \begin{array}{llll}
\text { C } & 6.324911 & -0.547801 & 0.955172
\end{array} \\
& \begin{array}{llll}
\text { C } & 6.387529 & 0.245663 & 2.104709
\end{array} \\
& \begin{array}{llll}
\text { C } & 7.401567 & -0.608247 & 0.065023
\end{array} \\
& \begin{array}{llll}
\mathrm{H} & 5.562055 & 0.251384 & 2.808630
\end{array} \\
& \begin{array}{llll}
\text { C } & 7.537981 & 0.992744 & 2.350680
\end{array} \\
& \begin{array}{llll}
\text { C } & 8.542387 & 0.145664 & 0.330105
\end{array} \\
& \text { H } \quad 7.354606 \quad-1.257541 \quad-0.802656 \\
& \text { H } \quad 7.593135 \quad 1.604476 \quad 3.247443 \\
& \begin{array}{llll}
\text { C } & 8.630907 & 0.957114 & 1.471861
\end{array} \\
& \begin{array}{llll}
\text { H } & 9.383994 & 0.094554 & -0.355847
\end{array} \\
& \begin{array}{llll}
\text { C } & 9.887515 & 1.739586 & 1.766505
\end{array} \\
& \text { H } \quad 10.612471 \quad 1.119703 \quad 2.309992 \\
& \text { H } \quad 10.375201 \quad 2.077187 \quad 0.846378 \\
& \text { H } \quad 9.679006 \quad 2.617039 \quad 2.386440 \\
& \begin{array}{llll}
\mathrm{H} & 1.538038 & -2.252129 & 1.234533
\end{array}
\end{aligned}
$$




$$
\begin{aligned}
& \begin{array}{llll}
\text { C } & 3.084880 & 0.573442 & 0.486958
\end{array} \\
& \text { H } \quad 3.743907 \quad 1.424304 \quad 0.737388 \\
& \begin{array}{llll}
\mathrm{H} & 2.712722 & 0.135801 & 1.415056
\end{array} \\
& \begin{array}{llll}
\text { C } & 1.624435 & -1.192202 & -1.892174
\end{array} \\
& \begin{array}{llll}
\text { C } & 2.845251 & -0.641821 & -2.645030
\end{array} \\
& \text { H } \quad 0.990989-1.735902 \quad-2.604625 \\
& \begin{array}{llll}
\text { C } & 2.306508 & 0.520715 & -3.496385
\end{array} \\
& \begin{array}{llll}
\text { C } & 1.066504 & 1.168891 & -2.876268
\end{array} \\
& \text { H } \quad 3.045220 \quad 1.309262-3.696272 \\
& \text { H } \quad 1.969963 \quad 0.159936 \quad-4.475861 \\
& \begin{array}{llll}
\text { O } & 0.209107 & 1.735527 & -3.505446
\end{array} \\
& \text { H } \quad \begin{array}{lll}
1.931005 & -1.878440 & -1.109886
\end{array} \\
& \begin{array}{llll}
\text { C } & 4.036538 & -0.217764 & -1.693988
\end{array} \\
& \text { H } \quad 4.917002 \quad-0.797344 \quad-1.972299 \\
& \text { H } \quad 4.292049 \quad 0.840315 \quad-1.839816 \\
& \begin{array}{llll}
\text { C } & 3.395943 & -1.749300 & -3.578451
\end{array} \\
& \text { H } \quad 3.749876 \quad-2.599018-2.985412 \\
& \text { H } \quad 2.625335 \quad-2.108042 \quad-4.269472 \\
& \text { H } \quad 4.239426 \quad-1.380343 \quad-4.176651 \\
& \begin{array}{llll}
\text { C } & 1.992613 & 1.073753 & -0.380915
\end{array} \\
& \begin{array}{llll}
\text { C } & 1.702606 & 1.977142 & -1.260100
\end{array} \\
& \text { C } \quad 1.902046 \quad 3.431009-1.576793 \\
& \text { H } \quad 0.938592 \quad 3.857162-1.870772 \\
& \text { H } \quad 2.547839 \quad 3.513560 \quad-2.462924 \\
& \begin{array}{llll}
\text { C } & 2.508286 & 4.220516 & -0.409511
\end{array} \\
& \text { H } \quad 2.624766 \quad 5.271007 \quad-0.694989 \\
& \text { H } \quad 1.860004 \quad 4.176867 \quad 0.471230 \\
& \text { H } \quad 3.496523 \quad 3.838464 \quad-0.131927 \\
& \text { C } \quad-1.378072 \quad 5.931732-0.541152 \\
& \text { H } \quad-1.247982 \quad 6.795861 \quad 0.104372 \\
& \begin{array}{llll}
\text { C } & -1.508125 & 6.099732 & -1.919969
\end{array} \\
& \text { H } \quad-1.479865 \quad 7.094591 \quad-2.355186 \\
& \begin{array}{llll}
\text { C } & -1.670298 & 4.978992 & -2.737723
\end{array}
\end{aligned}
$$




$$
\begin{array}{lrrr}
\mathrm{H} & -1.763301 & 5.095283 & -3.813780 \\
\mathrm{C} & -0.275407 & 2.030493 & 3.814601 \\
\mathrm{H} & 0.629928 & 1.771472 & 4.356350 \\
\mathrm{C} & -1.351291 & 2.612188 & 4.486497 \\
\mathrm{H} & -1.283406 & 2.813149 & 5.551892 \\
\mathrm{C} & -2.517562 & 2.934258 & 3.785699 \\
\mathrm{H} & -3.355817 & 3.392474 & 4.303709 \\
\mathrm{C} & -3.046987 & -2.091183 & -3.791796 \\
\mathrm{H} & -2.976991 & -1.680663 & -4.794906 \\
\mathrm{C} & -4.056544 & -3.000585 & -3.473360 \\
\mathrm{H} & -4.773511 & -3.304365 & -4.230689 \\
\mathrm{C} & -4.145515 & -3.517641 & -2.177785 \\
\mathrm{H} & -4.929055 & -4.227194 & -1.926532 \\
\mathrm{C} & 0.556760 & -5.617033 & -0.406113 \\
\mathrm{H} & 0.291076 & -6.557229 & -0.881045 \\
\mathrm{C} & 1.690198 & -5.535098 & 0.401909 \\
\mathrm{H} & 2.313286 & -6.410756 & 0.558889 \\
\mathrm{C} & 2.030885 & -4.317533 & 0.996661 \\
\mathrm{H} & 2.923626 & -4.225307 & 1.606284
\end{array}
$$

$\mathrm{TS} 2 \mathrm{c}$

B3LYP SCF energy: $\quad 3935.98358833$ a.u.

B3LYP enthalpy: 3934.975223 a.u.

B3LYP free energy: 3935.139544 a.u.

M06-L SCF energy in solution: $\quad 3937.65288470$ a.u.

M06-L enthalpy in solution: $\quad 3936.644519$ a.u.

M06-L free energy in solution: $\quad 3936.808840$ a.u.

Imaginary frequency: \$TAB \$ $-335.3927 \mathrm{~cm}-1$

Cartesian coordinates

ATOM $\mathrm{X} \quad \mathrm{Y} \quad \mathrm{Z}$ 


$$
\begin{array}{lrrr}
\mathrm{P} & -0.644203 & -1.719928 & -0.285570 \\
\mathrm{P} & -1.647730 & 1.732775 & -0.039552 \\
\mathrm{O} & -5.460148 & -1.889966 & 0.520895 \\
\mathrm{O} & -6.888352 & -0.950593 & -1.035307 \\
\mathrm{O} & -4.557597 & -0.210883 & 3.025611 \\
\mathrm{O} & -3.587958 & -1.204146 & 4.877526 \\
\mathrm{C} & -3.269704 & 0.935783 & -0.427328 \\
\mathrm{C} & -3.631649 & -0.249129 & 0.284892 \\
\mathrm{C} & -4.886224 & -0.761101 & -0.006434 \\
\mathrm{C} & -5.749727 & -0.202516 & -0.947735 \\
\mathrm{C} & -5.408695 & 0.932832 & -1.649137 \\
\mathrm{C} & -4.151962 & 1.493299 & -1.362406 \\
\mathrm{C} & -6.806651 & -1.908522 & 0.027923 \\
\mathrm{C} & -1.633303 & -1.644771 & 1.265551 \\
\mathrm{C} & -1.072525 & -2.247441 & 2.401230 \\
\mathrm{C} & -0.161287 & -4.505017 & -0.217727 \\
\mathrm{C} & -1.650191 & -2.160204 & 3.677694 \\
\mathrm{C} & -2.817511 & -1.435245 & 3.775390 \\
\mathrm{C} & -3.394475 & -0.835213 & 2.657201 \\
\mathrm{C} & -2.848389 & -0.893743 & 1.382837 \\
\mathrm{C} & -4.626943 & -0.311708 & 4.453868 \\
\mathrm{C} & -1.893608 & 2.280243 & 1.708010 \\
\mathrm{C} & -0.889896 & 2.111694 & 2.671599 \\
\mathrm{C} & -3.103911 & 2.889468 & 2.084908 \\
\mathrm{C} & -1.657459 & 3.316172 & -0.998534 \\
\mathrm{C} & -1.589433 & 4.572629 & -0.379244 \\
\mathrm{C} & -1.635410 & 3.259347 & -2.405393 \\
\mathrm{C} & -1.777495 & -2.236655 & -1.644722 \\
\mathrm{C} & -2.965206 & -2.947268 & -1.409435 \\
\mathrm{C} & -1.996000 & -2.970414 \\
\mathrm{C} & -3.129422 & 0.192628 \\
\mathrm{C} & -3.227099 & -0.085356 \\
\mathrm{C} & -375065
\end{array}
$$




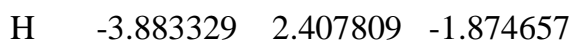

$$
\begin{aligned}
& \text { H } \quad-7.039717 \quad-2.902959 \quad-0.358778 \\
& \text { H } \quad-7.495502 \quad-1.621937 \quad 0.832826 \\
& \text { H } \quad-0.149458 \quad-2.804177 \quad 2.302263 \\
& \text { H } \quad-1.200316 \quad-2.640298 \quad 4.539242 \\
& \mathrm{H} \quad-4.460603 \quad 0.678205 \quad 4.896527 \\
& \text { H } \quad-5.598214 \quad-0.720718 \quad 4.744266 \\
& \begin{array}{llll}
\mathrm{H} & 0.030659 & 1.602152 & 2.417776
\end{array} \\
& \text { H } \quad-3.899814 \quad 3.012440 \quad 1.356488 \\
& \begin{array}{llll}
\mathrm{H} & -1.599771 & 4.649802 & 0.702225
\end{array} \\
& \text { H } \quad-3.291700 \quad-3.147908 \quad-0.394323 \\
& \text { H } \quad-0.454296 \quad-1.469247 \quad-3.171030 \\
& \text { H } \quad 2.245198 \quad-2.159401 \quad 0.312956 \\
& \text { H } \quad-1.216293 \quad-4.613236-0.443360 \\
& \begin{array}{llll}
\text { Rh } & 0.392672 & 0.443571 & -0.886232
\end{array} \\
& \begin{array}{llll}
\text { O } & 5.093624 & -2.459267 & -0.793117
\end{array} \\
& \begin{array}{llll}
\text { C } & 1.577990 & 0.470589 & 0.731333
\end{array} \\
& \begin{array}{llll}
\text { S } & 5.427466 & -1.037422 & -0.921743
\end{array} \\
& \begin{array}{llll}
\text { O } & 1.428134 & -0.300715 & 1.639383
\end{array} \\
& \text { N } \quad 3.894893 \quad-0.243771 \quad-1.135295 \\
& \begin{array}{llll}
\text { O } & 6.373594 & -0.580918 & -1.947042
\end{array} \\
& \begin{array}{llll}
\text { C } & 5.979678 & -0.416131 & 0.662212
\end{array} \\
& \begin{array}{llll}
\text { C } & 3.077239 & -0.979466 & -2.154605
\end{array} \\
& \begin{array}{llll}
\text { C } & 7.102253 & 0.412294 & 0.709290
\end{array} \\
& \begin{array}{llll}
\text { C } & 5.335337 & -0.827236 & 1.834187
\end{array} \\
& \text { H } \quad 3.570094 \quad-0.971772 \quad-3.139426 \\
& \text { H } \quad 2.963606 \quad-2.009673 \quad-1.824638 \\
& \text { H } \quad 7.603426 \quad 0.694671 \quad-0.210476 \\
& \begin{array}{llll}
\text { C } & 7.570519 & 0.848108 & 1.949207
\end{array} \\
& \begin{array}{llll}
\text { C } & 5.816627 & -0.378516 & 3.060713
\end{array} \\
& \begin{array}{llll}
\mathrm{H} & 4.476282 & -1.488378 & 1.788380
\end{array} \\
& \begin{array}{llll}
\mathrm{H} & 8.445241 & 1.491681 & 1.990198
\end{array} \\
& \begin{array}{llll}
\text { C } & 6.940948 & 0.461603 & 3.140162
\end{array}
\end{aligned}
$$




$$
\begin{aligned}
& \mathrm{H} \quad 5.319491 \quad-0.694001 \quad 3.974407 \\
& \begin{array}{llll}
\text { C } & 7.475200 & 0.905696 & 4.479937
\end{array} \\
& \begin{array}{llll}
\mathrm{H} & 6.666597 & 1.079844 & 5.197477
\end{array} \\
& \text { H } \quad 8.129991 \quad 0.136575 \quad 4.909776 \\
& \begin{array}{llll}
\mathrm{H} & 8.062897 & 1.824643 & 4.394365
\end{array} \\
& \begin{array}{llll}
\text { C } & 2.629416 & 1.580870 & 0.760021
\end{array} \\
& \begin{array}{llll}
\mathrm{H} & 3.496627 & 1.194678 & 1.304060
\end{array} \\
& \begin{array}{llll}
\mathrm{H} & 2.184311 & 2.376587 & 1.374910
\end{array} \\
& \begin{array}{llll}
\text { C } & 2.971495 & 2.139241 & -0.620823
\end{array} \\
& \begin{array}{llll}
\text { C } & 1.629004 & 2.373946 & -1.330548
\end{array} \\
& \text { H } \quad-1.675546 \quad 2.296882 \quad-2.909245 \\
& \text { H } \quad 0.939377 \quad 2.925029 \quad-0.685990 \\
& \begin{array}{llll}
\text { C } & 4.018485 & 1.221802 & -1.349016
\end{array} \\
& \begin{array}{llll}
\mathrm{H} & 4.053647 & 1.460340 & -2.420191
\end{array} \\
& \begin{array}{llll}
\mathrm{H} & 4.992277 & 1.510179 & -0.946890
\end{array} \\
& \begin{array}{llll}
\text { C } & 3.652296 & 3.523414 & -0.470962
\end{array} \\
& \begin{array}{llll}
\mathrm{H} & 2.962786 & 4.261267 & -0.046809
\end{array} \\
& \begin{array}{llll}
\mathrm{H} & 4.525983 & 3.459648 & 0.188514
\end{array} \\
& \text { H } \quad 3.9918693 .900761 \quad-1.442423 \\
& \mathrm{H} \quad 1.721723 \quad 2.975893 \quad-2.232274 \\
& \text { C } \quad \begin{array}{llll}
1.811535 & -0.213121 & -2.210929
\end{array} \\
& \begin{array}{llll}
\text { C } & 1.391611 & 0.874823 & -2.773121
\end{array} \\
& \begin{array}{llll}
\text { C } & 1.310921 & 1.598169 & -4.082103
\end{array} \\
& \text { H } \quad 0.548712 \quad 2.382456-4.032673 \\
& \begin{array}{llll}
\mathrm{H} & 2.267378 & 2.111022 & -4.263547
\end{array} \\
& \begin{array}{llll}
\text { C } & 1.013784 & 0.644191 & -5.249973
\end{array} \\
& \text { H } \quad 1.774847 \quad-0.137984 \quad-5.331046 \\
& \text { H } \quad 0.038631 \quad 0.161726 \quad-5.128109 \\
& \text { H } \quad 1.002919 \quad 1.203312-6.191315 \\
& \begin{array}{llll}
\text { C } & -3.292010 & 3.338857 & 3.391423
\end{array} \\
& \text { H } \quad-4.228126 \quad 3.817289 \quad 3.665844 \\
& \begin{array}{llll}
\text { C } & -2.280179 & 3.175585 & 4.342836
\end{array} \\
& \text { H } \quad-2.427034 \quad 3.527491 \quad 5.360171
\end{aligned}
$$




$$
\begin{array}{lrrr}
\mathrm{C} & -1.083012 & 2.556575 & 3.981943 \\
\mathrm{H} & -0.298618 & 2.412277 & 4.719388 \\
\mathrm{C} & -1.516320 & 5.740809 & -1.145652 \\
\mathrm{H} & -1.465588 & 6.704390 & -0.646634 \\
\mathrm{C} & -1.518421 & 5.672744 & -2.537699 \\
\mathrm{H} & -1.472101 & 6.581563 & -3.130558 \\
\mathrm{C} & -1.582009 & 4.425489 & -3.167272 \\
\mathrm{H} & -1.591601 & 4.362203 & -4.252103 \\
\mathrm{C} & -2.159342 & -2.449979 & -4.037445 \\
\mathrm{H} & -1.837155 & -2.261962 & -5.057958 \\
\mathrm{C} & -3.340971 & -3.151460 & -3.793901 \\
\mathrm{H} & -3.945341 & -3.507411 & -4.623280 \\
\mathrm{C} & -3.739068 & -3.400447 & -2.478716 \\
\mathrm{H} & -4.653109 & -3.954089 & -2.282468 \\
\mathrm{C} & 0.620413 & -5.649918 & -0.073226 \\
\mathrm{H} & 0.160991 & -6.628586 & -0.179745 \\
\mathrm{C} & 1.987339 & -5.539921 & 0.192887 \\
\mathrm{H} & 2.597454 & -6.433339 & 0.290578 \\
\mathrm{C} & 2.567234 & -4.278592 & 0.323211 \\
\mathrm{H} & 3.632336 & -4.174504 & 0.501545
\end{array}
$$

TS2d

B3LYP SCF energy: $\quad 3935.99318717$ a.u.

B3LYP enthalpy: 3934.984509 a.u.

B3LYP free energy: 3935.149881 a.u.

M06-L SCF energy in solution: $\quad 3937.66141353$ a.u.

M06-L enthalpy in solution: $\quad 3936.652735$ a.u.

M06-L free energy in solution: $\quad 3936.818107$ a.u.

Imaginary frequency: \$TAB \$ $-348.8524 \mathrm{~cm}-1$

Cartesian coordinates 


$$
\begin{aligned}
& \begin{array}{llll}
\text { ATOM } & \mathrm{X} & \mathrm{Y} & \mathrm{Z}
\end{array} \\
& \begin{array}{llll}
\mathrm{P} & -1.417867 & 1.800156 & -0.058612
\end{array} \\
& \begin{array}{llll}
\mathrm{P} & -1.189578 & -1.715803 & 0.323368
\end{array} \\
& \begin{array}{llll}
\text { O } & -5.949554 & 0.522171 & 1.031500
\end{array} \\
& \begin{array}{llll}
\text { O } & -6.289856 & -0.380958 & 3.133057
\end{array} \\
& \begin{array}{llll}
\text { O } & -5.508337 & -1.276791 & -1.509809
\end{array} \\
& \begin{array}{llll}
\text { O } & -5.659340 & -0.391807 & -3.643734
\end{array} \\
& \text { C } \quad-2.729793 \quad-1.282780 \quad 1.252195 \\
& \begin{array}{llll}
\text { C } & -3.720392 & -0.457132 & 0.638754
\end{array} \\
& \begin{array}{llll}
\text { C } & -4.874153 & -0.253334 & 1.378107
\end{array} \\
& \begin{array}{llll}
\text { C } & -5.080598 & -0.787008 & 2.649606
\end{array} \\
& \begin{array}{llll}
\text { C } & -4.129949 & -1.575883 & 3.258481
\end{array} \\
& \begin{array}{llll}
\text { C } & -2.952177 & -1.814565 & 2.530183
\end{array} \\
& \begin{array}{llll}
\text { C } & -6.933148 & 0.306783 & 2.051099
\end{array} \\
& \begin{array}{llll}
\text { C } & -2.795742 & 1.214927 & -1.138870
\end{array} \\
& \begin{array}{llll}
\text { C } & -2.915719 & 1.745255 & -2.429901
\end{array} \\
& \begin{array}{llll}
\text { C } & -3.853702 & 1.273600 & -3.363388
\end{array} \\
& \begin{array}{llll}
\text { C } & -4.665549 & 0.239121 & -2.952994
\end{array} \\
& \begin{array}{llll}
\text { C } & -4.567756 & -0.293749 & -1.668589
\end{array} \\
& \begin{array}{llll}
\text { C } & -3.651759 & 0.144695 & -0.724393
\end{array} \\
& \text { C } \quad-6.125156-1.447353 \quad-2.792693 \\
& \begin{array}{llll}
\text { C } & -1.868759 & -2.631103 & -1.126530
\end{array} \\
& \begin{array}{llll}
\text { C } & -1.491933 & -2.280856 & -2.429981
\end{array} \\
& \begin{array}{llll}
\text { C } & -2.816772 & -3.653438 & -0.942132
\end{array} \\
& \begin{array}{llll}
\text { C } & -0.391456 & -2.972478 & 1.419208
\end{array} \\
& \begin{array}{llll}
\text { C } & -0.384673 & -4.348537 & 1.155156
\end{array} \\
& \text { C } \quad 0.286762 \quad-2.498296 \quad 2.557136 \\
& \begin{array}{llll}
\text { C } & -2.163680 & 2.077196 & 1.604845
\end{array} \\
& \begin{array}{llll}
\text { C } & -3.417058 & 2.696632 & 1.752269
\end{array} \\
& \begin{array}{llll}
\text { C } & -1.443361 & 1.724332 & 2.754418
\end{array} \\
& \begin{array}{llll}
\text { C } & -0.318325 & 3.735088 & -1.817584
\end{array} \\
& \begin{array}{llll}
\text { C } & -1.038619 & 3.532902 & -0.625411
\end{array} \\
& \begin{array}{llll}
\text { C } & -1.412583 & 4.659948 & 0.126583
\end{array}
\end{aligned}
$$




$$
\begin{aligned}
& \text { H } \quad-4.282176 \quad-1.997266 \quad 4.245644 \\
& \text { H } \quad-2.205561 \quad-2.452617 \quad 2.983214 \\
& \begin{array}{llll}
\mathrm{H} & -7.308799 & 1.269847 & 2.404491
\end{array} \\
& \text { H } \quad-7.744907 \quad-0.315737 \quad 1.653920 \\
& \mathrm{H} \quad-2.271865 \quad 2.559209 \quad-2.734774 \\
& \text { H } \quad-3.934449 \quad 1.706066 \quad-4.354156 \\
& \text { H } \quad-5.825688 \quad-2.414983 \quad-3.213817 \\
& \text { H } \quad-7.211161 \quad-1.377257 \quad-2.687846 \\
& \mathrm{H} \quad-0.826333 \quad-1.441845 \quad-2.591656 \\
& \text { H } \quad-3.160208 \quad-3.905101 \quad 0.056742 \\
& \text { H } \quad-0.877027 \quad-4.741487 \quad 0.273016 \\
& \text { H } \quad-4.000058 \quad 2.957812 \quad 0.873946 \\
& \text { H } \quad-0.482056 \quad 1.226049 \quad 2.657673 \\
& \begin{array}{llll}
\mathrm{H} & 0.004427 & 2.884158 & -2.405472
\end{array} \\
& \begin{array}{llll}
\mathrm{H} & -1.947921 & 4.548062 & 1.060926
\end{array} \\
& \begin{array}{llll}
\mathrm{Rh} & 0.529967 & 0.060719 & -0.148116
\end{array} \\
& \begin{array}{llll}
\text { O } & 4.944766 & 1.829817 & 1.009584
\end{array} \\
& \begin{array}{llll}
\text { C } & 1.172906 & 0.294095 & -2.159515
\end{array} \\
& \begin{array}{llll}
\text { S } & 5.101298 & 0.398582 & 0.717795
\end{array} \\
& \begin{array}{llll}
\text { O } & 0.451891 & 0.910455 & -2.915294
\end{array} \\
& \begin{array}{llll}
\mathrm{N} & 4.419581 & 0.191737 & -0.824993
\end{array} \\
& \begin{array}{llll}
\text { O } & 4.561945 & -0.613590 & 1.634934
\end{array} \\
& \begin{array}{llll}
\text { C } & 6.831612 & 0.031988 & 0.469498
\end{array} \\
& \begin{array}{llll}
\text { C } & 3.644837 & 1.269210 & -1.397966
\end{array} \\
& \begin{array}{llll}
\text { C } & 7.366799 & -1.144439 & 0.994032
\end{array} \\
& \begin{array}{llll}
\text { C } & 7.633318 & 0.944259 & -0.225814
\end{array} \\
& \mathrm{H} \quad 4.083091 \quad 2.224957 \quad-1.108435 \\
& \text { H } \quad 3.703793 \quad 1.186549 \quad-2.487118 \\
& \text { H } \quad 6.733934 \quad-1.826920 \quad 1.550599 \\
& \begin{array}{llll}
\text { C } & 8.723291 & -1.412751 & 0.804725
\end{array} \\
& \begin{array}{llll}
\text { C } & 8.982190 & 0.658657 & -0.401815
\end{array} \\
& \text { H } \quad 7.210401 \quad 1.866907 \quad-0.609789 \\
& \text { H } \quad 9.145266 \quad-2.327423 \quad 1.212289
\end{aligned}
$$




$$
\begin{aligned}
& \begin{array}{llll}
\text { C } & 9.550000 & -0.522404 & 0.108181
\end{array} \\
& \text { H } \quad 9.610064 \quad 1.364988 \quad-0.938882 \\
& \text { C } \quad \begin{array}{llll}
11.021176 & -0.804335 & -0.075797
\end{array} \\
& \text { H } \quad 11.331743 \quad-0.640186 \quad-1.113744 \\
& \text { H } \quad 11.627843 \quad-0.138653 \quad 0.551174 \\
& \text { H } \quad \begin{array}{llll}
11.270538 & -1.834117 & 0.195647
\end{array} \\
& \begin{array}{llll}
\text { C } & 2.008088 & -0.897026 & -2.635660
\end{array} \\
& \text { H } \quad 2.671316 \quad-0.565057 \quad-3.447259 \\
& \text { H } \quad 1.279890 \quad-1.561450 \quad-3.114452 \\
& \begin{array}{llll}
\text { C } & 2.747448 & -1.652076 & -1.511695
\end{array} \\
& \begin{array}{llll}
\text { C } & 1.922868 & -1.553989 & -0.206277
\end{array} \\
& \text { H } \quad 0.317933 \quad-1.431450 \quad 2.765855 \\
& \text { H } \quad 1.391253 \quad-2.489633 \quad-0.054008 \\
& \begin{array}{llll}
\text { C } & 4.216190 & -1.161229 & -1.343447
\end{array} \\
& \text { H } \quad 4.734723 \quad-1.850711 \quad-0.673320 \\
& \text { H } \quad 4.715871 \quad-1.228287 \quad-2.320743 \\
& \begin{array}{llll}
\text { C } & 2.868298 & -3.136654 & -1.937507
\end{array} \\
& \text { H } \quad 1.876502-3.568754 \quad-2.108766 \\
& \text { H } \quad 3.446006 \quad-3.247172 \quad-2.863992 \\
& \text { H } \quad 3.362307 \quad-3.727762-1.158591 \\
& \mathrm{H} \quad 2.540580 \quad-1.376021 \quad 0.678936 \\
& \begin{array}{llll}
\text { C } & 2.184548 & 1.311729 & -0.937680
\end{array} \\
& \begin{array}{llll}
\text { C } & 1.637993 & 1.790445 & 0.129819
\end{array} \\
& \begin{array}{llll}
\text { C } & 1.735613 & 2.779641 & 1.233128
\end{array} \\
& \begin{array}{llll}
\text { H } & 0.736750 & 3.029769 & 1.601357
\end{array} \\
& \begin{array}{llll}
\mathrm{H} & 2.253571 & 2.276072 & 2.061465
\end{array} \\
& \begin{array}{llll}
\text { C } & 2.487292 & 4.065860 & 0.848889
\end{array} \\
& \begin{array}{llll}
\mathrm{H} & 3.528285 & 3.847660 & 0.601818
\end{array} \\
& \begin{array}{llll}
\mathrm{H} & 2.005105 & 4.565610 & 0.004298
\end{array} \\
& \mathrm{H} \quad 2.482051 \quad 4.751050 \quad 1.702978 \\
& \begin{array}{llll}
\text { C } & -3.331961 & -4.343224 & -2.039417
\end{array} \\
& \mathrm{H} \quad \begin{array}{llll}
-4.053530 & -5.140112 & -1.882517
\end{array} \\
& \begin{array}{llll}
\text { C } & -2.926890 & -4.006278 & -3.334543
\end{array}
\end{aligned}
$$




$$
\begin{array}{lrrr}
\mathrm{H} & -3.327625 & -4.546065 & -4.188039 \\
\mathrm{C} & -2.017261 & -2.965673 & -3.528264 \\
\mathrm{H} & -1.721233 & -2.678144 & -4.533068 \\
\mathrm{C} & 0.269241 & -5.231087 & 2.020494 \\
\mathrm{H} & 0.267037 & -6.294766 & 1.799948 \\
\mathrm{C} & 0.921094 & -4.752563 & 3.156231 \\
\mathrm{H} & 1.427325 & -5.441031 & 3.826584 \\
\mathrm{C} & 0.929190 & -3.380373 & 3.423318 \\
\mathrm{H} & 1.444994 & -2.996086 & 4.298492 \\
\mathrm{C} & -1.951515 & 1.998650 & 4.026380 \\
\mathrm{H} & -1.381166 & 1.719518 & 4.907774 \\
\mathrm{C} & -3.189605 & 2.627123 & 4.161841 \\
\mathrm{H} & -3.585322 & 2.843868 & 5.149909 \\
\mathrm{C} & -3.923174 & 2.970934 & 3.022535 \\
\mathrm{H} & -4.890025 & 3.456336 & 3.123189 \\
\mathrm{C} & -1.099108 & 5.947915 & -0.313776 \\
\mathrm{H} & -1.400398 & 6.802885 & 0.284985 \\
\mathrm{C} & -0.409126 & 6.137443 & -1.510806 \\
\mathrm{H} & -0.172143 & 7.140519 & -1.853889 \\
\mathrm{C} & -0.019899 & 5.025453 & -2.259536 \\
\mathrm{H} & 0.523811 & 5.157113 & -3.191097
\end{array}
$$

IntC1

B3LYP SCF energy: $\quad 3936.06119544$ a.u.

B3LYP enthalpy: 3935.050207 a.u.

B3LYP free energy: 3935.217899 a.u.

M06-L SCF energy in solution: $\quad 3937.71982717$ a.u.

M06-L enthalpy in solution: $\quad 3936.708839$ a.u.

M06-L free energy in solution: $\quad 3936.876531$ a.u.

Cartesian coordinates 


$$
\begin{aligned}
& \begin{array}{llll}
\text { ATOM } & \mathrm{X} & \mathrm{Y} & \mathrm{Z}
\end{array} \\
& \begin{array}{llll}
\mathrm{P} & -1.562578 & 1.835576 & -0.374699
\end{array} \\
& \text { P } \quad-1.480262 \quad-1.723151 \quad 0.288160 \\
& \begin{array}{llll}
\text { O } & -5.776748 & 1.003723 & 1.702026
\end{array} \\
& \begin{array}{llll}
\text { O } & -5.792373 & 0.263213 & 3.894050
\end{array} \\
& \begin{array}{llll}
\text { O } & -5.945814 & -1.155180 & -0.711936
\end{array} \\
& \text { O } \quad-6.433624 \quad-0.506346-2.878485 \\
& \begin{array}{llll}
\text { C } & -2.787787 & -1.161158 & 1.457144
\end{array} \\
& \begin{array}{llll}
\text { C } & -3.774453 & -0.245144 & 0.980162
\end{array} \\
& \begin{array}{llll}
\text { C } & -4.735253 & 0.136214 & 1.902110
\end{array} \\
& \begin{array}{llll}
\text { C } & -4.747221 & -0.306184 & 3.225197
\end{array} \\
& \begin{array}{llll}
\text { C } & -3.796178 & -1.182333 & 3.701078
\end{array} \\
& \begin{array}{llll}
\text { C } & -2.816638 & -1.605005 & 2.784894
\end{array} \\
& \begin{array}{llll}
\text { C } & -6.555318 & 0.967759 & 2.906401
\end{array} \\
& \begin{array}{llll}
\text { C } & -3.104529 & 1.178295 & -1.121769
\end{array} \\
& \begin{array}{llll}
\text { C } & -3.432981 & 1.570119 & -2.427150
\end{array} \\
& \begin{array}{llll}
\text { C } & -4.541992 & 1.060607 & -3.119985
\end{array} \\
& \begin{array}{llll}
\text { C } & -5.308312 & 0.130422 & -2.452813
\end{array} \\
& \begin{array}{llll}
\text { C } & -5.004024 & -0.261816 & -1.147911
\end{array} \\
& \begin{array}{llll}
\text { C } & -3.918535 & 0.218448 & -0.433639
\end{array} \\
& \text { C } \quad-6.774306-1.443047 \quad-1.846108 \\
& \begin{array}{llll}
\text { C } & -2.374134 & -2.827698 & -0.885095
\end{array} \\
& \begin{array}{llll}
\text { C } & -1.886187 & -3.013755 & -2.187520
\end{array} \\
& \begin{array}{llll}
\text { C } & -3.515165 & -3.538247 & -0.475069
\end{array} \\
& \begin{array}{llll}
\text { C } & -0.425999 & -2.872841 & 1.276217
\end{array} \\
& \begin{array}{llll}
\text { C } & -0.370272 & -4.251431 & 1.023593
\end{array} \\
& \begin{array}{llll}
\text { C } & 0.414274 & -2.332396 & 2.268759
\end{array} \\
& \begin{array}{llll}
\text { C } & -2.013241 & 2.445639 & 1.296973
\end{array} \\
& \begin{array}{llll}
\text { C } & -3.232703 & 3.109148 & 1.516259
\end{array} \\
& \begin{array}{llll}
\text { C } & -1.118879 & 2.286591 & 2.365281
\end{array} \\
& \begin{array}{llll}
\text { C } & -0.534514 & 3.250194 & -2.605498
\end{array} \\
& \begin{array}{llll}
\text { C } & -1.139680 & 3.355510 & -1.339521
\end{array} \\
& \begin{array}{llll}
\text { C } & -1.393442 & 4.635107 & -0.818843
\end{array}
\end{aligned}
$$




$$
\begin{aligned}
& \mathrm{H} \quad-3.807770 \quad-1.536362 \quad 4.725738 \\
& \mathrm{H} \quad-2.071000 \quad-2.311980 \quad 3.127427 \\
& \begin{array}{llll}
\mathrm{H} & -6.741277 & 1.988138 & 3.250121
\end{array} \\
& \text { H } \quad-7.495265 \quad 0.433652 \quad 2.719182 \\
& \mathrm{H} \quad-2.817815 \quad 2.299567 \quad-2.936274 \\
& \text { H } \quad-4.779776 \quad 1.382504 \quad-4.127249 \\
& \text { H } \quad-6.569779 \quad-2.461575 \quad-2.197571 \\
& \text { H } \quad-7.824569-1.315522 \quad-1.572900 \\
& \text { H } \quad-1.018863 \quad-2.462671 \quad-2.529959 \\
& \text { H } \quad-3.908381 \quad-3.404392 \quad 0.527556 \\
& \text { H } \quad-1.005913 \quad-4.693112 \quad 0.263996 \\
& \begin{array}{llll}
\text { H } & -3.946322 & 3.221968 & 0.705917
\end{array} \\
& \text { H } \quad-0.171889 \quad 1.776581 \quad 2.209086 \\
& \begin{array}{llll}
\mathrm{H} & -0.334297 & 2.277685 & -3.037407
\end{array} \\
& \text { H } \quad-1.844914 \quad 4.751835 \quad 0.158954 \\
& \begin{array}{llll}
\mathrm{Rh} & 0.103416 & 0.229170 & -0.216954
\end{array} \\
& \begin{array}{llll}
\text { O } & 5.319686 & -1.534079 & 1.386625
\end{array} \\
& \begin{array}{llll}
\text { S } & 5.905586 & -1.311114 & 0.055026
\end{array} \\
& \text { N } \quad 4.943859 \quad-0.195885 \quad-0.801977 \\
& \begin{array}{llll}
\text { O } & 6.121036 & -2.435413 & -0.862759
\end{array} \\
& \begin{array}{llll}
\text { C } & 7.445172 & -0.434004 & 0.288033
\end{array} \\
& \begin{array}{llll}
\text { C } & 7.820654 & -0.033298 & 1.569627
\end{array} \\
& \begin{array}{llll}
\text { C } & 8.271525 & -0.194170 & -0.815151
\end{array} \\
& \begin{array}{llll}
\mathrm{H} & 7.175914 & -0.249267 & 2.414424
\end{array} \\
& \begin{array}{llll}
\text { C } & 9.037119 & 0.629475 & 1.741918
\end{array} \\
& \begin{array}{llll}
\text { C } & 9.479391 & 0.466184 & -0.622233
\end{array} \\
& \text { H } \quad \begin{array}{llll}
7.976813 & -0.528358 & -1.804613
\end{array} \\
& \begin{array}{llll}
\mathrm{H} & 9.334578 & 0.942847 & 2.739111
\end{array} \\
& \begin{array}{llll}
\text { C } & 9.883044 & 0.888737 & 0.656684
\end{array} \\
& \text { H } \quad 10.125889 \quad 0.653661 \quad-1.475832 \\
& \begin{array}{llll}
\text { C } & 11.209530 & 1.582849 & 0.849433
\end{array} \\
& \begin{array}{llll}
\text { H } & 12.040397 & 0.872937 & 0.748629
\end{array} \\
& \begin{array}{llll}
\text { H } & 11.363037 & 2.366429 & 0.099016
\end{array}
\end{aligned}
$$




\begin{tabular}{|c|c|c|c|}
\hline & 1.283776 & 2.040558 & 1.840211 \\
\hline & 0.387205 & -1.265935 & 2.484858 \\
\hline & 4.258891 & 0.851113 & -0.020034 \\
\hline & 4.458483 & 1.828389 & -0.479978 \\
\hline & 4.690650 & 0.869683 & 0.985132 \\
\hline & 2.481685 & -0.895489 & -0.267938 \\
\hline & 2.773231 & -1.155059 & -1.762201 \\
\hline & 1.453210 & -1.263860 & -0.039345 \\
\hline & 1.799899 & -0.317726 & -2.629962 \\
\hline & 0.337239 & -0.092680 & -2.166700 \\
\hline & 2.169559 & 0.712467 & -2.713010 \\
\hline & 1.732662 & -0.722882 & -3.645779 \\
\hline & -0.567089 & -0.041930 & -2.956538 \\
\hline & 3.094417 & -1.536445 & 0.375984 \\
\hline & 4.251625 & -0.652797 & -2.016596 \\
\hline & 4.852339 & -1.446737 & -2.459405 \\
\hline & 4.253721 & 0.191802 & -2.716791 \\
\hline & 2.640238 & -2.652559 & -2.085197 \\
\hline & 3.350160 & -3.237952 & -1.492105 \\
\hline & 1.629052 & -3.020529 & -1.869169 \\
\hline & 2.848266 & -2.847222 & -3.143855 \\
\hline & 2.760558 & 0.566010 & 0.018873 \\
\hline & 1.751427 & 1.457432 & 0.012788 \\
\hline & 1.952823 & 2.949103 & 0.151960 \\
\hline & 1.027003 & 3.490269 & -0.049582 \\
\hline & 2.662849 & 3.284778 & -0.621860 \\
\hline & 2.488824 & 3.385078 & 1.529396 \\
\hline & 2.624257 & 4.472101 & 1.557492 \\
\hline & 1.793092 & 3.114228 & 2.330622 \\
\hline & 3.453846 & 2.921457 & 1.757854 \\
\hline & -1.056805 & 5.777575 & -1.548385 \\
\hline & -1.259076 & 6.757999 & -1.126931 \\
\hline & -0.464041 & 5.662253 & -2.805137 \\
\hline
\end{tabular}




$$
\begin{array}{lrrr}
\mathrm{H} & -0.202983 & 6.552111 & -3.370490 \\
\mathrm{C} & -0.207044 & 4.394489 & -3.332002 \\
\mathrm{H} & 0.250747 & 4.292956 & -4.311914 \\
\mathrm{C} & -1.432570 & 2.784553 & 3.631211 \\
\mathrm{H} & -0.733289 & 2.652203 & 4.451687 \\
\mathrm{C} & -2.640283 & 3.452627 & 3.838742 \\
\mathrm{H} & -2.883216 & 3.844051 & 4.822352 \\
\mathrm{C} & -3.538106 & 3.614169 & 2.780002 \\
\mathrm{H} & -4.479055 & 4.133858 & 2.937305 \\
\mathrm{C} & -2.523208 & -3.896158 & -3.062101 \\
\mathrm{H} & -2.136743 & -4.028045 & -4.068581 \\
\mathrm{C} & -3.654104 & -4.600776 & -2.646520 \\
\mathrm{H} & -4.147474 & -5.289093 & -3.326988 \\
\mathrm{C} & -4.148162 & -4.420432 & -1.351580 \\
\mathrm{H} & -5.022592 & -4.973108 & -1.018693 \\
\mathrm{C} & 0.502901 & -5.067714 & 1.747805 \\
\mathrm{H} & 0.535473 & -6.133226 & 1.539041 \\
\mathrm{C} & 1.327246 & -4.522234 & 2.731956 \\
\mathrm{H} & 2.007158 & -5.159189 & 3.289898 \\
\mathrm{C} & 1.278823 & -3.150294 & 2.994825 \\
\mathrm{H} & 1.919300 & -2.716735 & 3.757295
\end{array}
$$

IntC3

B3LYP SCF energy: $\quad 3936.02690368$ a.u.

B3LYP enthalpy: $\quad 3935.016500$ a.u.

B3LYP free energy: 3935.181599 a.u.

M06-L SCF energy in solution: $\quad 3937.69066851$ a.u.

M06-L enthalpy in solution: $\quad 3936.680265$ a.u.

M06-L free energy in solution: $\quad 3936.845364$ a.u.

Cartesian coordinates 


\begin{tabular}{|c|c|c|c|}
\hline & & $\mathrm{Y}$ & Z \\
\hline & -0.582493 & -1.566616 & -0.594068 \\
\hline & -1.792169 & 1.671912 & 0.362005 \\
\hline & -5.390933 & -2.151146 & -0.056803 \\
\hline & -6.839557 & -0.953395 & -1.40356 \\
\hline & -4.654776 & -0.970502 & 2.808605 \\
\hline & -3.668370 & -2.250695 & 4.464576 \\
\hline & -3.374133 & 0.943191 & -0.232193 \\
\hline & -3.670325 & -0.394831 & 0.166938 \\
\hline & -4.879219 & -0.899679 & -0.283056 \\
\hline & -5.754510 & -0.182012 & -1.098382 \\
\hline & -5.475292 & 1.106580 & -1.498420 \\
\hline & -4.264546 & 1.657666 & -1.042061 \\
\hline & -6.719875 & -2.131933 & -0.597439 \\
\hline & -1.593638 & -1.839471 & 0.918196 \\
\hline & -1.028958 & -2.626655 & 1.933449 \\
\hline & -1.644983 & -2.833773 & 3.177050 \\
\hline & -2.858490 & -2.2 & 3.371498 \\
\hline & -3.444657 & -1.437432 & 2.369711 \\
\hline & -2.866218 & -1.207234 & 1.131034 \\
\hline & -4.759262 & -1.361456 & 4.183602 \\
\hline & -2.079743 & 1.886870 & 2.169817 \\
\hline & -1.061580 & 1.599994 & 3.090445 \\
\hline & -3.321212 & & \\
\hline & -1.770805 & 3.391000 & -0.315031 \\
\hline & -1.782465 & 4.527618 & 0.507107 \\
\hline & -1.647506 & 3.565683 & -1.707246 \\
\hline & -1.696169 & -1.769097 & -2.041576 \\
\hline & -2.763626 & -2.681929 & -2.000510 \\
\hline & -1.424275 & -1.086935 & -3.237475 \\
\hline & 1.685541 & -3.110468 & \\
\hline & 0.563428 & -3.004566 & -0.720695 \\
\hline & 0.319975 & -4.023636 & -1.658820 \\
\hline
\end{tabular}




$$
\begin{aligned}
& \mathrm{H} \quad-6.157830 \quad 1.670758 \quad-2.123900 \\
& \text { H } \quad-4.033970 \quad 2.678698 \quad-1.320386 \\
& \begin{array}{llll}
\mathrm{H} & -6.872575 & -3.016394 & -1.219843
\end{array} \\
& \text { H } \quad-7.448002 \quad-2.091169 \quad 0.222668 \\
& \text { H } \quad-0.079301 \quad-3.113881 \quad 1.762438 \\
& \text { H } \quad-1.188481 \quad-3.453234 \quad 3.940386 \\
& \text { H } \quad-4.678233 \quad-0.473687 \quad 4.822192 \\
& \text { H } \quad-5.704804 \quad-1.885643 \quad 4.344171 \\
& \mathrm{H} \quad-0.112258 \quad 1.204411 \quad 2.749305 \\
& \mathrm{H} \quad-4.125084 \quad 2.557306 \quad 1.943053 \\
& \mathrm{H} \quad-1.873431 \quad 4.421859 \quad 1.582611 \\
& \text { H } \quad-2.988172 \quad-3.221001 \quad-1.085780 \\
& \text { H } \quad-0.584811 \quad-0.399948 \quad-3.292852 \\
& \text { H } \quad 1.908601 \quad-2.321919 \quad 0.829345 \\
& \text { H } \quad-0.525487 \quad-3.965006 \quad-2.332609 \\
& \begin{array}{llll}
\text { Rh } & 0.277715 & 0.584495 & -0.670494
\end{array} \\
& \begin{array}{llll}
\text { O } & 4.833848 & -2.429759 & -0.957472
\end{array} \\
& \begin{array}{llll}
\text { C } & 1.551969 & 0.524037 & 0.834424
\end{array} \\
& \begin{array}{llll}
\text { S } & 5.258677 & -1.027339 & -0.935385
\end{array} \\
& \begin{array}{llll}
\text { O } & 1.598978 & -0.296483 & 1.714771
\end{array} \\
& \text { N } \quad 3.797961 \quad-0.098952 \quad-1.022910 \\
& \begin{array}{llll}
\text { O } & 6.224278 & -0.534684 & -1.927936
\end{array} \\
& \begin{array}{llll}
\text { C } & 5.886551 & -0.618028 & 0.692785
\end{array} \\
& \begin{array}{llll}
\text { C } & 2.887785 & -0.652840 & -2.103953
\end{array} \\
& \begin{array}{llll}
\text { C } & 7.174215 & -0.089078 & 0.796618
\end{array} \\
& \begin{array}{llll}
\text { C } & 5.131373 & -0.906058 & 1.834059
\end{array} \\
& \text { H } \quad 3.388008 \quad-0.626854 \quad-3.083304 \\
& \mathrm{H} \quad 2.669352 \quad-1.685271 \quad-1.845214 \\
& \text { H } \quad 7.744679 \quad 0.118373 \quad-0.102343 \\
& \begin{array}{llll}
\text { C } & 7.705232 & 0.159050 & 2.062519
\end{array} \\
& \begin{array}{llll}
\text { C } & 5.676816 & -0.644084 & 3.087993
\end{array} \\
& \begin{array}{llll}
\text { H } & 4.129723 & -1.311805 & 1.744997
\end{array} \\
& \begin{array}{llll}
\mathrm{H} & 8.707812 & 0.570161 & 2.146549
\end{array}
\end{aligned}
$$




$$
\begin{aligned}
& \begin{array}{llll}
\text { C } & 6.971586 & -0.115517 & 3.224587
\end{array} \\
& \text { H } \quad \begin{array}{llll}
5.090899 & -0.859599 & 3.977981
\end{array} \\
& \begin{array}{llll}
\text { C } & 7.566400 & 0.123182 & 4.591228
\end{array} \\
& \text { H } \quad 6.799872 \quad 0.406586 \quad 5.319885 \\
& \text { H } \quad 8.051989 \quad-0.786731 \quad 4.967488 \\
& \text { H } \quad 8.325481 \quad 0.911139 \quad 4.567336 \\
& \begin{array}{llll}
\text { C } & 2.456927 & 1.787422 & 0.856840
\end{array} \\
& \text { H } \quad 3.284346 \quad 1.530505 \quad 1.527278 \\
& \begin{array}{llll}
\mathrm{H} & 1.863208 & 2.566881 & 1.355457
\end{array} \\
& \begin{array}{llll}
\text { C } & 3.000108 & 2.340763 & -0.474867
\end{array} \\
& \begin{array}{llll}
\text { C } & 1.810105 & 2.628269 & -1.486394
\end{array} \\
& \text { H } \quad-1.627764 \quad 2.699356-2.364778 \\
& \text { H } \quad 0.865825 \quad 2.699697 \quad-0.903494 \\
& \begin{array}{llll}
\text { C } & 4.089816 & 1.363545 & -1.051542
\end{array} \\
& \begin{array}{llll}
\mathrm{H} & 4.399958 & 1.689009 & -2.051439
\end{array} \\
& \text { H } \quad 4.964119 \quad 1.498963 \quad-0.409136 \\
& \begin{array}{llll}
\text { C } & 3.714740 & 3.677173 & -0.177920
\end{array} \\
& \mathrm{H} \quad 3.000245 \quad 4.434934 \quad 0.163148 \\
& \text { H } \quad 4.478739 \quad 3.561786 \quad 0.598954 \\
& \text { H } \quad 4.207243 \quad 4.063370 \quad-1.077831 \\
& \text { H } \quad 1.890257 \quad 3.626900 \quad-1.930255 \\
& \begin{array}{llll}
\text { C } & 1.728548 & 0.289776 & -2.042205
\end{array} \\
& \begin{array}{llll}
\text { C } & 1.871935 & 1.542541 & -2.553958
\end{array} \\
& \begin{array}{llll}
\text { C } & 2.460623 & 1.909805 & -3.898922
\end{array} \\
& \text { H } \quad 3.308693 \quad 2.597137 \quad-3.767604 \\
& \text { H } \quad 2.844875 \quad 1.016782-4.402988 \\
& \begin{array}{llll}
\text { C } & 1.415621 & 2.591178 & -4.805059
\end{array} \\
& \text { H } \quad 0.583992 \quad 1.911892 \quad-5.021759 \\
& \text { H } \quad 1.003476 \quad 3.492841 \quad-4.336831 \\
& \text { H } \quad 1.871238 \quad 2.886199 \quad-5.756030 \\
& \begin{array}{llll}
\text { C } & -3.528939 & 2.535041 & 4.008835
\end{array} \\
& \mathrm{H} \quad-4.488954 \quad 2.901524 \quad 4.361935 \\
& \text { C } \quad-2.504956 \quad 2.255621 \quad 4.919469
\end{aligned}
$$




$$
\begin{array}{llll}
\mathrm{H} & -2.667805 & 2.403517 & 5.983417 \\
\mathrm{C} & -1.274744 & 1.784542 & 4.458754 \\
\mathrm{H} & -0.479930 & 1.553901 & 5.162293 \\
\mathrm{C} & -1.684404 & 5.806075 & -0.049917 \\
\mathrm{H} & -1.697163 & 6.675392 & 0.601400 \\
\mathrm{C} & -1.579945 & 5.968128 & -1.431337 \\
\mathrm{H} & -1.511594 & 6.963245 & -1.861165 \\
\mathrm{C} & -1.564379 & 4.842783 & -2.260765 \\
\mathrm{H} & -1.487731 & 4.959111 & -3.338326 \\
\mathrm{C} & -2.212903 & -1.304681 & -4.369229 \\
\mathrm{H} & -1.992335 & -0.771747 & -5.289710 \\
\mathrm{C} & -3.272817 & -2.210990 & -4.320502 \\
\mathrm{H} & -3.883062 & -2.383571 & -5.202314 \\
\mathrm{C} & -3.543383 & -2.900785 & -3.135837 \\
\mathrm{H} & -4.361215 & -3.614615 & -3.095196 \\
\mathrm{C} & 1.167239 & -5.129887 & -1.733195 \\
\mathrm{H} & 0.964651 & -5.907574 & -2.463962 \\
\mathrm{C} & 2.266132 & -5.235380 & -0.880881 \\
\mathrm{H} & 2.927323 & -6.094406 & -0.945401 \\
\mathrm{C} & 2.523589 & -4.222170 & 0.043298 \\
\mathrm{H} & 3.396030 & -4.278247 & 0.685106
\end{array}
$$

IntC4

B3LYP SCF energy: $\quad 3936.01121779$ a.u.

B3LYP enthalpy: 3935.000513 a.u.

B3LYP free energy: 3935.165562 a.u.

M06-L SCF energy in solution: $\quad 3937.67975257$ a.u.

M06-L enthalpy in solution: $\quad 3936.669048$ a.u.

M06-L free energy in solution: $\quad 3936.834097$ a.u.

Cartesian coordinates 


$$
\begin{aligned}
& \text { ATOM } \mathrm{X} \quad \mathrm{Y} \quad \mathrm{Z} \\
& \begin{array}{llll}
\text { P } & -1.415548 & 1.770838 & -0.009984
\end{array} \\
& \begin{array}{llll}
\mathrm{P} & -1.115501 & -1.641813 & 0.229728
\end{array} \\
& \begin{array}{llll}
\text { O } & -5.895505 & 0.323091 & 1.428710
\end{array} \\
& \begin{array}{llll}
\text { O } & -6.019381 & -0.699804 & 3.498146
\end{array} \\
& \begin{array}{llll}
\text { O } & -5.646169 & -1.246456 & -1.215610
\end{array} \\
& \begin{array}{llll}
\text { O } & -5.953952 & -0.285253 & -3.298868
\end{array} \\
& \begin{array}{llll}
\text { C } & -2.594068 & -1.338468 & 1.291742
\end{array} \\
& \begin{array}{llll}
\text { C } & -3.668091 & -0.535313 & 0.802757
\end{array} \\
& \text { C } \quad-4.764466 \quad-0.421662 \quad 1.641410 \\
& \begin{array}{llll}
\text { C } & -4.838645 & -1.025513 & 2.896553
\end{array} \\
& \begin{array}{llll}
\text { C } & -3.804155 & -1.791058 & 3.387868
\end{array} \\
& \begin{array}{llll}
\text { C } & -2.680514 & -1.936198 & 2.556324
\end{array} \\
& \begin{array}{llll}
\text { C } & -6.783883 & -0.002503 & 2.505060
\end{array} \\
& \begin{array}{llll}
\text { C } & -2.885535 & 1.206336 & -0.974388
\end{array} \\
& \begin{array}{llll}
\text { C } & -3.098195 & 1.779509 & -2.235975
\end{array} \\
& \begin{array}{llll}
\text { C } & -4.111338 & 1.349536 & -3.108028
\end{array} \\
& \begin{array}{llll}
\text { C } & -4.904352 & 0.313011 & -2.666701
\end{array} \\
& \text { C } \quad-4.713548 \quad-0.263353 \quad-1.411591 \\
& \begin{array}{llll}
\text { C } & -3.720474 & 0.132519 & -0.527730
\end{array} \\
& \text { C } \quad-6.366873-1.364182 \quad-2.449499 \\
& \begin{array}{llll}
\text { C } & -1.855711 & -2.466175 & -1.246960
\end{array} \\
& \begin{array}{llll}
\text { C } & -1.468605 & -2.079326 & -2.538544
\end{array} \\
& \begin{array}{llll}
\text { C } & -2.842150 & -3.456706 & -1.099272
\end{array} \\
& \begin{array}{llll}
\text { C } & -0.191091 & -2.935982 & 1.167166
\end{array} \\
& \begin{array}{llll}
\text { C } & -0.290861 & -4.306700 & 0.892733
\end{array} \\
& \begin{array}{llll}
\text { C } & 0.665358 & -2.508502 & 2.196577
\end{array} \\
& \begin{array}{llll}
\text { C } & -1.939104 & 1.903643 & 1.751215
\end{array} \\
& \begin{array}{llll}
\text { C } & -3.187343 & 2.447770 & 2.100679
\end{array} \\
& \begin{array}{llll}
\text { C } & -1.053667 & 1.525453 & 2.770131
\end{array} \\
& \begin{array}{llll}
\text { C } & -0.537947 & 3.841638 & -1.726205
\end{array} \\
& \begin{array}{llll}
\text { C } & -1.179118 & 3.547878 & -0.509746
\end{array} \\
& \begin{array}{llll}
\text { C } & -1.623398 & 4.613481 & 0.290449
\end{array}
\end{aligned}
$$




$$
\begin{aligned}
& \text { H } \quad-3.853448 \quad-2.261386 \quad 4.363345 \\
& \begin{array}{llll}
\mathrm{H} & -1.865127 & -2.550760 & 2.914551
\end{array} \\
& \begin{array}{llll}
\mathrm{H} & -7.187282 & 0.916859 & 2.935313
\end{array} \\
& \begin{array}{llll}
\mathrm{H} & -7.585437 & -0.655273 & 2.136315
\end{array} \\
& \mathrm{H} \quad-2.468292 \quad 2.594846 \quad-2.566233 \\
& \text { H } \quad-4.260824 \quad 1.813173 \quad-4.076382 \\
& \text { H } \quad-6.116966 \quad-2.319581 \quad-2.926604 \\
& \text { H } \quad-7.439707 \quad-1.283709 \quad-2.255122 \\
& \text { H } \quad-0.743044 \quad-1.281668 \quad-2.666979 \\
& \text { H } \quad-3.187051 \quad-3.739531 \quad-0.109277 \\
& \text { H } \quad-0.927439 \quad-4.662539 \quad 0.090483 \\
& \text { H } \quad-3.899990 \quad 2.718302 \quad 1.326844 \\
& \text { H } \quad-0.100092 \quad 1.072566 \quad 2.513249 \\
& \text { H } \quad-0.146466 \quad 3.044194 \quad-2.350178 \\
& \text { H } \quad-2.096191 \quad 4.429619 \quad 1.246664 \\
& \begin{array}{llll}
\mathrm{Rh} & 0.492156 & 0.192883 & -0.386442
\end{array} \\
& \begin{array}{llll}
\text { O } & 4.492251 & 1.040630 & 1.671769
\end{array} \\
& \begin{array}{llll}
\text { C } & 1.655770 & 0.886663 & -2.567583
\end{array} \\
& \begin{array}{llll}
\text { S } & 4.865389 & -0.190461 & 0.960660
\end{array} \\
& \begin{array}{llll}
\text { O } & 0.815845 & 1.486847 & -3.228729
\end{array} \\
& \mathrm{~N} \quad 4.154123 \quad-0.056526 \quad-0.565433 \\
& \text { O } \quad 4.496756 \quad-1.513734 \quad 1.469572 \\
& \begin{array}{llll}
\text { C } & 6.637168 & -0.168389 & 0.719133
\end{array} \\
& \begin{array}{llll}
\text { C } & 3.774687 & 1.230993 & -1.100230
\end{array} \\
& \begin{array}{llll}
\text { C } & 7.321712 & -1.374104 & 0.546187
\end{array} \\
& \begin{array}{llll}
\text { C } & 7.313641 & 1.054693 & 0.685175
\end{array} \\
& \text { H } \quad 4.134203 \quad 2.014853-0.435866 \\
& \mathrm{H} \quad 4.242330 \quad 1.380884 \quad-2.082735 \\
& \begin{array}{llll}
\mathrm{H} & 6.789631 & -2.317239 & 0.608009
\end{array} \\
& \begin{array}{llll}
\text { C } & 8.697376 & -1.345045 & 0.325230
\end{array} \\
& \begin{array}{llll}
\text { C } & 8.688413 & 1.061013 & 0.464012
\end{array} \\
& \begin{array}{llll}
\mathrm{H} & 6.776335 & 1.981635 & 0.854851
\end{array} \\
& \text { H } \quad 9.233957 \quad-2.281172 \quad 0.194961
\end{aligned}
$$




$$
\begin{aligned}
& \begin{array}{llll}
\text { C } & 9.402417 & -0.133412 & 0.279423
\end{array} \\
& \begin{array}{llll}
\mathrm{H} & 9.218798 & 2.009530 & 0.443203
\end{array} \\
& \begin{array}{llll}
\text { C } & 10.897617 & -0.114422 & 0.074534
\end{array} \\
& \text { H } \quad 11.216431 \quad 0.778757 \quad-0.472275 \\
& \text { H } \quad 11.422135 \quad-0.110118 \quad 1.038983 \\
& \text { H } \quad 11.238599 \quad-0.995064 \quad-0.478449 \\
& \begin{array}{llll}
\text { C } & 2.284654 & -0.394307 & -3.109457
\end{array} \\
& \text { H } \quad 3.160230 \quad-0.128999 \quad-3.721546 \\
& \text { H } \quad 1.549362 \quad-0.799657 \quad-3.812185 \\
& \begin{array}{llll}
\text { C } & 2.665667 & -1.454509 & -2.047338
\end{array} \\
& \text { C } \quad \begin{array}{llll}
1.627872 & -1.569116 & -0.895555
\end{array} \\
& \begin{array}{llll}
\text { H } & 0.781863 & -1.447162 & 2.399765
\end{array} \\
& \text { H } \quad 0.934352 \quad-2.370473 \quad-1.149710 \\
& \begin{array}{llll}
\text { C } & 4.080310 & -1.208828 & -1.454180
\end{array} \\
& \text { H } \quad 4.364353 \quad-2.088547 \quad-0.874507 \\
& \text { H } \quad 4.804518 \quad-1.095968 \quad-2.276344 \\
& \begin{array}{llll}
\text { C } & 2.750458 & -2.820683 & -2.772930
\end{array}
\end{aligned}
$$

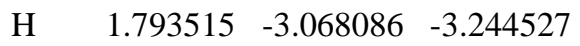

$$
\begin{aligned}
& \mathrm{H} \quad 3.517650 \quad-2.810320 \quad-3.557608 \\
& \mathrm{H} \quad 2.996522 \quad-3.622718 \quad-2.068352 \\
& \text { H } \quad 2.116520 \quad-1.836662 \quad 0.043975 \\
& \begin{array}{llll}
\text { C } & 2.236366 & 1.387892 & -1.258540
\end{array} \\
& \begin{array}{llll}
\text { C } & 1.529413 & 1.827125 & -0.179015
\end{array} \\
& \begin{array}{llll}
\text { C } & 1.828963 & 2.781726 & 0.922985
\end{array} \\
& \begin{array}{llll}
\mathrm{H} & 0.958624 & 2.903152 & 1.571767
\end{array} \\
& \mathrm{H} \quad 2.593110 \quad 2.271530 \quad 1.532883 \\
& \begin{array}{llll}
\text { C } & 2.356358 & 4.158403 & 0.481104
\end{array} \\
& \text { H } \quad 3.259346 \quad 4.070601 \quad-0.131369 \\
& \text { H } \quad 1.606831 \quad 4.705415 \quad-0.094778 \\
& \text { H } \quad 2.610269 \quad 4.748080 \quad 1.367690 \\
& \begin{array}{llll}
\text { C } & -3.396594 & -4.072800 & -2.221871
\end{array} \\
& \text { H } \quad-4.147993 \quad-4.847012 \quad-2.093416 \\
& \begin{array}{llll}
\text { C } & -2.990158 & -3.693422 & -3.504122
\end{array}
\end{aligned}
$$




$$
\begin{array}{lrrr}
\mathrm{H} & -3.423288 & -4.173562 & -4.377153 \\
\mathrm{C} & -2.033007 & -2.689008 & -3.660439 \\
\mathrm{H} & -1.729330 & -2.373718 & -4.654595 \\
\mathrm{C} & 0.441254 & -5.230374 & 1.643691 \\
\mathrm{H} & 0.357632 & -6.289536 & 1.417075 \\
\mathrm{C} & 1.278083 & -4.797668 & 2.672454 \\
\mathrm{H} & 1.848489 & -5.518263 & 3.251354 \\
\mathrm{C} & 1.390106 & -3.432337 & 2.947803 \\
\mathrm{H} & 2.055418 & -3.083571 & 3.731760 \\
\mathrm{C} & -1.393565 & 1.711068 & 4.112113 \\
\mathrm{H} & -0.696368 & 1.414537 & 4.890536 \\
\mathrm{C} & -2.626202 & 2.270502 & 4.449396 \\
\mathrm{H} & -2.891113 & 2.417332 & 5.492502 \\
\mathrm{C} & -3.524872 & 2.631331 & 3.441508 \\
\mathrm{H} & -4.490844 & 3.056525 & 3.699242 \\
\mathrm{C} & -1.452478 & 5.935197 & -0.128028 \\
\mathrm{H} & -1.800088 & 6.743752 & 0.508858 \\
\mathrm{C} & -0.842382 & 6.216996 & -1.350476 \\
\mathrm{H} & -0.717398 & 7.245967 & -1.675537 \\
\mathrm{C} & -0.384061 & 5.165243 & -2.145438 \\
\mathrm{H} & 0.104312 & 5.368260 & -3.094549
\end{array}
$$

TS3a

B3LYP SCF energy: $\quad 3936.04761031$ a.u.

B3LYP enthalpy: 3935.037507 a.u.

B3LYP free energy: 3935.202843 a.u.

M06-L SCF energy in solution: $\quad 3937.70858501$ a.u.

M06-L enthalpy in solution: $\quad 3936.698482$ a.u.

M06-L free energy in solution: $\quad 3936.863818$ a.u.

Imaginary frequency: \$TAB \$ $-225.2374 \mathrm{~cm}-1$ 


$$
\begin{aligned}
& \text { Cartesian coordinates } \\
& \begin{array}{llll}
\text { ATOM } & \mathrm{X} & \mathrm{Y} & \mathrm{Z}
\end{array} \\
& \begin{array}{llll}
\mathrm{P} & -1.595135 & 1.745915 & -0.240031
\end{array} \\
& \begin{array}{llll}
\mathrm{P} & -1.562614 & -1.636392 & 0.135261
\end{array} \\
& \begin{array}{llll}
\text { O } & -5.834212 & 0.784887 & 2.050113
\end{array} \\
& \begin{array}{llll}
\text { O } & -5.788578 & -0.280757 & 4.103517
\end{array} \\
& \text { O } \quad-6.197331 \quad-0.949986-0.504420 \\
& \begin{array}{llll}
\text { O } & -6.804672 & -0.066639 & -2.556803
\end{array} \\
& \begin{array}{llll}
\text { C } & -2.870736 & -1.323446 & 1.387602
\end{array} \\
& \begin{array}{llll}
\text { C } & -3.872000 & -0.353832 & 1.079781
\end{array} \\
& \begin{array}{llll}
\text { C } & -4.795230 & -0.106864 & 2.082612
\end{array} \\
& \begin{array}{llll}
\text { C } & -4.769044 & -0.744059 & 3.323419
\end{array} \\
& \begin{array}{llll}
\text { C } & -3.814209 & -1.689016 & 3.628660
\end{array} \\
& \begin{array}{llll}
\text { C } & -2.862365 & -1.965382 & 2.631249
\end{array} \\
& \begin{array}{llll}
\text { C } & -6.571043 & 0.577278 & 3.263356
\end{array} \\
& \begin{array}{llll}
\text { C } & -3.240721 & 1.239857 & -0.919032
\end{array} \\
& \begin{array}{llll}
\text { C } & -3.644360 & 1.769125 & -2.154084
\end{array} \\
& \begin{array}{llll}
\text { C } & -4.833227 & 1.398243 & -2.801735
\end{array} \\
& \begin{array}{llll}
\text { C } & -5.614732 & 0.463115 & -2.161419
\end{array} \\
& \text { C } \quad-5.242366 \quad-0.063580 \quad-0.925313 \\
& \begin{array}{llll}
\text { C } & -4.076947 & 0.280798 & -0.256167
\end{array} \\
& \begin{array}{llll}
\text { C } & -7.167232 & -1.030020 & -1.557489
\end{array} \\
& \begin{array}{llll}
\text { C } & -2.457746 & -2.309691 & -1.322184
\end{array} \\
& \begin{array}{llll}
\text { C } & -2.103123 & -1.895958 & -2.614155
\end{array} \\
& \text { C } \quad-3.488206-3.251251 \quad-1.158251 \\
& \begin{array}{llll}
\text { C } & -0.578765 & -3.047997 & 0.801079
\end{array} \\
& \begin{array}{llll}
\text { C } & -0.550296 & -4.307025 & 0.182390
\end{array} \\
& \begin{array}{llll}
\text { C } & 0.228375 & -2.831404 & 1.935044
\end{array} \\
& \begin{array}{llll}
\text { C } & -1.900068 & 2.155941 & 1.527872
\end{array} \\
& \begin{array}{llll}
\text { C } & -3.089876 & 2.788918 & 1.925263
\end{array} \\
& \begin{array}{llll}
\text { C } & -0.910851 & 1.893763 & 2.487748
\end{array} \\
& \begin{array}{llll}
\text { C } & -0.928027 & 3.470609 & -2.394423
\end{array}
\end{aligned}
$$

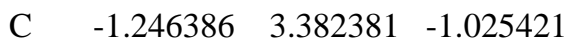




$$
\begin{aligned}
& \begin{array}{llll}
\text { C } & -1.292348 & 4.564710 & -0.267010
\end{array} \\
& \text { H } \quad-3.799830 \quad-2.1983694 .585517 \\
& \mathrm{H} \quad-2.111620 \quad-2.716264 \quad 2.842373 \\
& \text { H } \quad-6.724636 \quad 1.536244 \quad 3.764328 \\
& \begin{array}{llll}
\mathrm{H} & -7.527277 & 0.091350 & 3.033082
\end{array} \\
& \mathrm{H} \quad-3.034531 \quad 2.516452 \quad-2.640185 \\
& \mathrm{H} \quad-5.119767 \quad 1.831830 \quad-3.752960 \\
& \text { H } \quad-7.147317 \quad-2.034080 \quad-1.996321 \\
& \text { H } \quad-8.158137 \quad-0.788264 \quad-1.162362 \\
& \mathrm{H} \quad \begin{array}{llll}
-1.330710 & -1.144355 & -2.747147
\end{array} \\
& \text { H } \quad-3.784009 \quad-3.566788 \quad-0.161804 \\
& \text { H } \quad-1.157537 \quad-4.499052 \quad-0.695201 \\
& \text { H } \quad-3.869744 \quad 2.991910 \quad 1.197773 \\
& \begin{array}{llll}
\mathrm{H} & 0.016434 & 1.411433 & 2.187422
\end{array} \\
& \mathrm{H} \quad-0.823393 \quad 2.573632 \quad-2.990100 \\
& \mathrm{H} \quad-1.519580 \quad 4.532393 \quad 0.791319 \\
& \begin{array}{llll}
\mathrm{Rh} & 0.051316 & 0.116577 & -0.258577
\end{array} \\
& \begin{array}{llll}
\text { O } & 5.560922 & -1.625057 & 1.490812
\end{array} \\
& \begin{array}{llll}
\text { S } & 6.127199 & -1.337474 & 0.161731
\end{array} \\
& \begin{array}{llll}
\mathrm{N} & 5.081610 & -0.301848 & -0.693398
\end{array} \\
& \begin{array}{llll}
\text { O } & 6.438333 & -2.431241 & -0.764919
\end{array} \\
& \begin{array}{llll}
\text { C } & 7.579331 & -0.325568 & 0.406449
\end{array} \\
& \begin{array}{llll}
\text { C } & 7.955936 & 0.031589 & 1.700453
\end{array} \\
& \begin{array}{llll}
\text { C } & 8.345283 & 0.055345 & -0.700906
\end{array} \\
& \begin{array}{llll}
\text { H } & 7.358017 & -0.290406 & 2.545915
\end{array} \\
& \begin{array}{llll}
\text { C } & 9.111809 & 0.792969 & 1.882392
\end{array} \\
& \begin{array}{llll}
\text { C } & 9.492946 & 0.812671 & -0.498246
\end{array} \\
& \text { H } \quad 8.049255 \quad-0.242088 \quad-1.701624 \\
& \begin{array}{llll}
\mathrm{H} & 9.409922 & 1.073583 & 2.889060
\end{array} \\
& \begin{array}{llll}
\text { C } & 9.896276 & 1.193516 & 0.794018
\end{array} \\
& \text { H } \quad 10.091911 \quad 1.111303 \quad-1.354853 \\
& \begin{array}{llll}
\text { C } & 11.158154 & 1.997100 & 0.994777
\end{array} \\
& \begin{array}{llll}
\mathrm{H} & 12.047231 & 1.383598 & 0.801250
\end{array}
\end{aligned}
$$




\begin{tabular}{|c|c|c|c|}
\hline & 11.201021 & 2.850725 & 0.30890 \\
\hline & 11.234278 & 2.377780 & 2.017432 \\
\hline & 0.230644 & -1.859441 & 2.423270 \\
\hline & 4.339464 & 0.714544 & 0.087184 \\
\hline & 4.573708 & 1.713875 & -0.294097 \\
\hline & 4.660583 & 0.666361 & 1.132890 \\
\hline & 2.619764 & -1.086037 & -0.042733 \\
\hline & 2.982567 & -1.536886 & -1.481808 \\
\hline & 1.570434 & -1.380326 & 0.166729 \\
\hline & 1.839275 & -1.036745 & -2.390004 \\
\hline & 1.160000 & 0.326979 & -2.040903 \\
\hline & 2.165821 & -0.925503 & -3.430296 \\
\hline & 1.036665 & -1.785962 & -2.391396 \\
\hline & 0.828819 & 1.082446 & -2.925546 \\
\hline & 3.222150 & -1.600423 & 0.712870 \\
\hline & 4.344848 & -0.837610 & -1.853499 \\
\hline & 5.001079 & -1.532159 & -2.378157 \\
\hline & 4.166465 & 0.015593 & -2.518762 \\
\hline & 3.107380 & -3.066326 & -1.578152 \\
\hline & 3.964118 & -3.423299 & -0.999178 \\
\hline & 2.202996 & -3.560598 & -1.204653 \\
\hline & 3.258518 & -3.377405 & -2.618972 \\
\hline & 2.865154 & 0.394750 & -0.041365 \\
\hline & 1.893149 & 1.227284 & -0.497053 \\
\hline & 2.114850 & 2.700393 & -0.737679 \\
\hline & 1.327186 & 3.085525 & -1.384491 \\
\hline & 3.051171 & 2.841739 & -1.298830 \\
\hline & 2.180031 & 3.529817 & 0.555405 \\
\hline & 2.322657 & 4.589942 & 0.320467 \\
\hline & 1.256512 & 3.436508 & 1.135636 \\
\hline & 3.009772 & & 1.197525 \\
\hline & -1.049117 & 5.801750 & -0.866890 \\
\hline & -1.092913 & 6.704091 & -0.263751 \\
\hline
\end{tabular}




$$
\begin{array}{lrrr}
\mathrm{C} & -0.755243 & 5.880260 & -2.228161 \\
\mathrm{H} & -0.569548 & 6.844461 & -2.692599 \\
\mathrm{C} & -0.693608 & 4.710760 & -2.988398 \\
\mathrm{H} & -0.451271 & 4.757521 & -4.046140 \\
\mathrm{C} & -1.106973 & 2.254576 & 3.822433 \\
\mathrm{H} & -0.334734 & 2.044872 & 4.556956 \\
\mathrm{C} & -2.289658 & 2.887161 & 4.208379 \\
\mathrm{H} & -2.441056 & 3.171655 & 5.245697 \\
\mathrm{C} & -3.278658 & 3.154705 & 3.257787 \\
\mathrm{H} & -4.199042 & 3.650644 & 3.552968 \\
\mathrm{C} & -2.759079 & -2.425096 & -3.727871 \\
\mathrm{H} & -2.480417 & -2.095098 & -4.724497 \\
\mathrm{C} & -3.772384 & -3.370180 & -3.559377 \\
\mathrm{H} & -4.279597 & -3.784010 & -4.426314 \\
\mathrm{C} & -4.137026 & -3.781350 & -2.273512 \\
\mathrm{H} & -4.924781 & -4.517546 & -2.138895 \\
\mathrm{C} & 0.258441 & -5.326587 & 0.692880 \\
\mathrm{H} & 0.269400 & -6.296038 & 0.203058 \\
\mathrm{C} & 1.044778 & -5.104730 & 1.823666 \\
\mathrm{H} & 1.672438 & -5.899019 & 2.216667 \\
\mathrm{C} & 1.026586 & -3.853456 & 2.446514 \\
\mathrm{H} & 1.638869 & -3.671519 & 3.324977
\end{array}
$$

TS3b

B3LYP SCF energy: $\quad 3936.01788626$ a.u.

B3LYP enthalpy: $\quad 3935.011929$ a.u.

B3LYP free energy: 3935.178842 a.u.

M06-L SCF energy in solution: $\quad 3937.67941371$ a.u.

M06-L enthalpy in solution: $\quad 3936.673456$ a.u.

M06-L free energy in solution: $\quad 3936.840369$ a.u.

Imaginary frequency: $\$ \mathrm{TAB} \$-650.6151 \mathrm{~cm}-1$ 
Cartesian coordinates

\begin{tabular}{|c|c|c|c|}
\hline & $\mathrm{X}$ & $\mathrm{Y}$ & Z \\
\hline $\mathrm{P}$ & -2.377539 & 1.844042 & -0.188787 \\
\hline $\mathrm{P}$ & -0.847530 & -1.505225 & 0.210888 \\
\hline $\mathrm{O}$ & -5.881812 & -0.537675 & 1.593225 \\
\hline $\mathrm{O}$ & -5.652431 & -1.315656 & 3.762720 \\
\hline $\mathrm{O}$ & -5.283362 & -2.504526 & -0.916803 \\
\hline $\mathrm{O}$ & -6.063156 & -1.893136 & -3.010064 \\
\hline $\mathrm{C}$ & -2.320719 & -1.504299 & 1.332490 \\
\hline $\mathrm{C}$ & -3.565432 & -0.962144 & 0.870347 \\
\hline $\mathrm{C}$ & -4.598567 & -0.967779 & 1.794490 \\
\hline $\mathrm{C}$ & -4.467827 & -1.439956 & 3.101112 \\
\hline $\mathrm{C}$ & -3.279839 & -1.966662 & 3.556808 \\
\hline $\mathrm{C}$ & -2.211740 & -1.989167 & 2.642954 \\
\hline $\mathrm{C}$ & -6.560797 & -0.687057 & 2.847001 \\
\hline $\mathrm{C}$ & -3.534202 & 0.747976 & -1.106151 \\
\hline $\mathrm{C}$ & -4.036627 & 1.111418 & -2.360136 \\
\hline $\mathrm{C}$ & -4.897548 & 0.282079 & -3.100313 \\
\hline $\mathrm{C}$ & -5.234649 & -0.923532 & -2.526440 \\
\hline $\mathrm{C}$ & -4.756420 & -1.290969 & -1.268241 \\
\hline $\mathrm{C}$ & -3.897251 & -0.505176 & -0.517197 \\
\hline $\mathrm{C}$ & -6.043724 & -2.954603 & -2.046722 \\
\hline $\mathrm{C}$ & -1.412056 & -2.503948 & -1.233945 \\
\hline $\mathrm{C}$ & -0.980481 & -2.222435 & -2.537718 \\
\hline $\mathrm{C}$ & -2.215645 & -3.636248 & -1.009067 \\
\hline $\mathrm{C}$ & 0.393405 & -2.624727 & 1.012646 \\
\hline $\mathrm{C}$ & 0.779200 & -3.840920 & 0.426940 \\
\hline $\mathrm{C}$ & 1.017724 & -2.234850 & 2.211345 \\
\hline $\mathrm{C}$ & -3.326111 & 2.289679 & 1.328796 \\
\hline $\mathrm{C}$ & -4.703949 & 2.555388 & 1.256856 \\
\hline $\mathrm{C}$ & -2.673534 & 2.417847 & 2.561935 \\
\hline $\mathrm{C}$ & -1.793854 & 3.504311 & -2.429468 \\
\hline
\end{tabular}




$$
\begin{aligned}
& \begin{array}{llll}
\text { C } & -2.290673 & 3.447631 & -1.113217
\end{array} \\
& \text { C } \quad-2.635711 \quad 4.651321 \quad-0.476486 \\
& \mathrm{H} \quad-3.176230 \quad-2.358821 \quad 4.562223 \\
& \text { H } \quad-1.279753 \quad-2.428779 \quad 2.973280 \\
& \begin{array}{llll}
\mathrm{H} & -6.837098 & 0.301237 & 3.230844
\end{array} \\
& \text { H } \quad-7.440438 \quad-1.323501 \quad 2.712524 \\
& \text { H } \quad-3.773932 \quad 2.072002 \quad-2.783680 \\
& \text { H } \quad-5.283283 \quad 0.582040 \quad-4.068005 \\
& \text { H } \quad-5.558078 \quad-3.834263 \quad-2.484505 \\
& \text { H } \quad-7.066574 \quad-3.179160 \quad-1.731067 \\
& \mathrm{H} \quad-0.378151 \quad-1.346427 \quad-2.736244 \\
& \text { H } \quad-2.560644 \quad-3.869324-0.006728 \\
& \text { H } \quad 0.331835 \quad-4.167766 \quad-0.503827 \\
& \mathrm{H} \quad-5.227340 \quad 2.456148 \quad 0.310944 \\
& \mathrm{H} \quad-1.609467 \quad 2.216294 \quad 2.630427 \\
& \text { H } \quad-1.510284 \quad 2.596561 \quad-2.947412 \\
& \begin{array}{llll}
\mathrm{H} & -3.018683 & 4.645747 & 0.537218
\end{array} \\
& \begin{array}{llll}
\text { Rh } & -0.132666 & 0.905221 & 0.291135
\end{array} \\
& \begin{array}{llll}
\text { O } & 5.894624 & -1.962488 & -0.141395
\end{array} \\
& \text { S } \quad 6.323893 \quad-0.735200 \quad-0.825937 \\
& \begin{array}{llll}
\mathrm{N} & 5.155139 & 0.480220 & -0.546688
\end{array} \\
& \begin{array}{llll}
\text { O } & 6.573971 & -0.722017 & -2.270978
\end{array} \\
& \begin{array}{llll}
\text { C } & 7.777083 & -0.127027 & 0.018962
\end{array} \\
& \begin{array}{llll}
\text { C } & 8.180194 & -0.726342 & 1.211806
\end{array} \\
& \begin{array}{llll}
\text { C } & 8.506973 & 0.925677 & -0.542924
\end{array} \\
& \text { H } \quad 7.616280-1.559501 \quad 1.616736 \\
& \begin{array}{llll}
\text { C } & 9.324317 & -0.251279 & 1.854749
\end{array} \\
& \begin{array}{llll}
\text { C } & 9.644303 & 1.383723 & 0.112743
\end{array} \\
& \text { H } \quad 8.196740 \quad 1.366940 \quad-1.484483 \\
& \begin{array}{llll}
\mathrm{H} & 9.644217 & -0.717342 & 2.782991
\end{array} \\
& \begin{array}{llll}
\text { C } & 10.072414 & 0.805100 & 1.320218
\end{array} \\
& \text { H } \quad 10.216811 \quad 2.199406 \quad-0.321597 \\
& \begin{array}{llll}
\text { C } & 11.325505 & 1.297261 & 2.002992
\end{array}
\end{aligned}
$$




\begin{tabular}{|c|c|c|c|}
\hline & 2.221358 & 0.950394 & 1.47218 \\
\hline & 11.366593 & 2.391951 & 2.022544 \\
\hline & 11.391186 & 0.934829 & 3.033107 \\
\hline & 0.763978 & -1.285833 & 2.671950 \\
\hline & 4.366789 & 0.394430 & 0.690983 \\
\hline & 4.420705 & 1.347830 & 1.236796 \\
\hline & 4.801366 & -0.380799 & 1.328428 \\
\hline & 2.795983 & -0.648150 & -0.975081 \\
\hline & 3.057893 & 0.481063 & -2.014748 \\
\hline & 1.804377 & -1.080141 & -1.118904 \\
\hline & 1.998169 & 1.631832 & -1.832583 \\
\hline & 0.572535 & 1.126781 & -1.576557 \\
\hline & 2.293043 & 2.300808 & -1.025155 \\
\hline & 1.943245 & 2.220765 & -2.755433 \\
\hline & -0.165718 & 0.827633 & -2.475491 \\
\hline & 3.532727 & -1.447989 & -1.090779 \\
\hline & 4.485070 & 1.090704 & -1.703049 \\
\hline & 5.140782 & 0.965268 & -2.563969 \\
\hline & 4.405196 & 2.167687 & -1.505368 \\
\hline & 3.009369 & -0.078320 & -3.444127 \\
\hline & 3.750074 & -0.876064 & -3.562493 \\
\hline & 2.020492 & -0.485490 & -3.682123 \\
\hline & 3.235881 & 0.701780 & -4.180704 \\
\hline & 2.920721 & 0.058649 & 0.342368 \\
\hline & 1.881784 & 0.646281 & 0.925136 \\
\hline & 1.548044 & 1.826361 & 1.607104 \\
\hline & 0.204941 & 2.448573 & 0.740124 \\
\hline & 2.031611 & 2.745345 & 1.262540 \\
\hline & 1.100796 & 1.875852 & 3.052902 \\
\hline & 0.556058 & 2.796561 & 3.280627 \\
\hline & 0.482461 & 1.015836 & 3.326021 \\
\hline & 1.996986 & 1.852784 & 3.688029 \\
\hline & -2.495026 & 5.873564 & $-1.15 / 04 c$ \\
\hline
\end{tabular}




$$
\begin{array}{lrrr}
\mathrm{H} & -2.770427 & 6.791301 & -0.625668 \\
\mathrm{C} & -2.013354 & 5.917155 & -2.445380 \\
\mathrm{H} & -1.910663 & 6.868055 & -2.960194 \\
\mathrm{C} & -1.665910 & 4.727515 & -3.088467 \\
\mathrm{H} & -1.293388 & 4.747190 & -4.108967 \\
\mathrm{C} & -3.377252 & 2.804227 & 3.704485 \\
\mathrm{H} & -2.858811 & 2.896236 & 4.654719 \\
\mathrm{C} & -4.744275 & 3.073898 & 3.622402 \\
\mathrm{H} & -5.292399 & 3.382046 & 4.508252 \\
\mathrm{C} & -5.404970 & 2.950570 & 2.396347 \\
\mathrm{H} & -6.467200 & 3.168148 & 2.324980 \\
\mathrm{C} & -1.346703 & -3.056226 & -3.597287 \\
\mathrm{H} & -1.010080 & -2.823054 & -4.603337 \\
\mathrm{C} & -2.137668 & -4.181784 & -3.366097 \\
\mathrm{H} & -2.413538 & -4.833284 & -4.190535 \\
\mathrm{C} & -2.570374 & -4.470589 & -2.068486 \\
\mathrm{H} & -3.178538 & -5.350732 & -1.877625 \\
\mathrm{C} & 1.747252 & -4.647028 & 1.031277 \\
\mathrm{H} & 2.035528 & -5.580384 & 0.556546 \\
\mathrm{C} & 2.337870 & -4.260478 & 2.233138 \\
\mathrm{H} & 3.086848 & -4.891313 & 2.702727 \\
\mathrm{C} & 1.966362 & -3.050914 & 2.824924 \\
\mathrm{H} & 2.423270 & -2.736500 & 3.759199
\end{array}
$$

IntD1

B3LYP SCF energy: $\quad 3936.07772329$ a.u.

B3LYP enthalpy: 3935.065406 a.u.

B3LYP free energy: 3935.232946 a.u.

M06-L SCF energy in solution: $\quad 3937.73536387$ a.u.

M06-L enthalpy in solution: $\quad 3936.723047$ a.u.

M06-L free energy in solution: $\quad 3936.890587$ a.u. 
Cartesian coordinates

\begin{tabular}{|c|c|c|c|}
\hline & $X$ & $\mathrm{Y}$ & Z \\
\hline $\mathrm{P}$ & -1.536588 & 1.657994 & -0.181212 \\
\hline $\mathrm{P}$ & -1.305206 & -1.660964 & -0.143750 \\
\hline $\mathrm{O}$ & -4.790195 & 0.443103 & 3.234791 \\
\hline $\mathrm{O}$ & -3.987851 & -0.692285 & 5.087566 \\
\hline $\mathrm{O}$ & -5.985511 & -1.090661 & 0.815711 \\
\hline $\mathrm{O}$ & -7.205498 & -0.101385 & -0.885990 \\
\hline $\mathrm{C}$ & -2.145706 & -1.455030 & 1.479193 \\
\hline $\mathrm{C}$ & -3.235397 & -0.537456 & 1.590884 \\
\hline $\mathrm{C}$ & -3.762731 & -0.380460 & 2.862041 \\
\hline $\mathrm{C}$ & -3.280754 & -1.059760 & 3.981382 \\
\hline $\mathrm{C}$ & -2.240498 & -1.957944 & 3.886092 \\
\hline $\mathrm{C}$ & -1.681807 & -2.140153 & 2.609058 \\
\hline $\mathrm{C}$ & -5.005603 & 0.210189 & 4.633834 \\
\hline $\mathrm{C}$ & -3.303139 & 1.153767 & -0.393524 \\
\hline $\mathrm{C}$ & -4.062194 & 1.736112 & -1.418987 \\
\hline $\mathrm{C}$ & -5.398898 & 1.386658 & -1.672517 \\
\hline $\mathrm{C}$ & -5.950287 & 0.426904 & -0.852955 \\
\hline $\mathrm{C}$ & -5.212641 & -0.159691 & 0.175126 \\
\hline $\mathrm{C}$ & -3.891463 & 0.157978 & 0.448441 \\
\hline $\mathrm{C}$ & -7.286526 & -1.014932 & 0.216784 \\
\hline $\mathrm{C}$ & -2.596583 & -2.244199 & -1.310960 \\
\hline $\mathrm{C}$ & -2.528591 & -1.858104 & -2.657619 \\
\hline $\mathrm{C}$ & -3.618706 & -3.112632 & -0.893356 \\
\hline $\mathrm{C}$ & -0.174313 & -3.101389 & 0.070910 \\
\hline $\mathrm{C}$ & -0.416868 & -4.333644 & -0.556212 \\
\hline $\mathrm{C}$ & 0.986256 & -2.954333 & 0.853664 \\
\hline $\mathrm{C}$ & -1.392004 & 2.120894 & 1.591025 \\
\hline $\mathrm{C}$ & -2.430262 & 2.806595 & 2.244658 \\
\hline $\mathrm{C}$ & -0.222948 & 1.810531 & 2.300675 \\
\hline $\mathrm{C}$ & -1.298295 & 3.153243 & -2.531000 \\
\hline
\end{tabular}




$$
\begin{aligned}
& \text { C } \quad-1.435150 \quad 3.230065 \quad-1.134799 \\
& \begin{array}{llll}
\text { C } & -1.539272 & 4.491317 & -0.527177
\end{array} \\
& \begin{array}{llll}
\mathrm{H} & -1.873505 & -2.501061 & 4.749501
\end{array} \\
& \mathrm{H} \quad-0.871715 \quad-2.850905 \quad 2.509941 \\
& \text { H } \quad-4.921792 \quad 1.155991 \quad 5.177155 \\
& \text { H } \quad-5.990868 \quad-0.246418 \quad 4.782350 \\
& \text { H } \quad-3.620362 \quad 2.500161 \quad-2.043914 \\
& \text { H } \quad-5.965904 \quad 1.853563 \quad-2.469730 \\
& \text { H } \quad-7.576359-2.003565 \quad-0.150484 \\
& \text { H } \quad-8.007673 \quad-0.637130 \quad 0.950765 \\
& \text { H } \quad-1.747731 \quad-1.173876 \quad-2.981239 \\
& \text { H } \quad-3.688551 \quad-3.411104 \quad 0.148580 \\
& \text { H } \quad-1.299981 \quad-4.473045 \quad-1.169304 \\
& \begin{array}{llll}
\mathrm{H} & -3.352825 & 3.030841 & 1.717700
\end{array} \\
& \begin{array}{llll}
\mathrm{H} & 0.570574 & 1.259612 & 1.803886
\end{array} \\
& \text { H } \quad-1.175179 \quad 2.191093 \quad-3.016601 \\
& \begin{array}{llll}
\mathrm{H} & -1.628968 & 4.577872 & 0.549768
\end{array} \\
& \begin{array}{llll}
\mathrm{Rh} & 0.012294 & 0.126544 & -0.905641
\end{array} \\
& \begin{array}{llll}
\text { O } & 4.361025 & -1.769456 & 1.285527
\end{array} \\
& \begin{array}{llll}
\text { S } & 5.285728 & -1.299858 & 0.240160
\end{array} \\
& \mathrm{~N} \quad 4.467635 \quad-0.132244 \quad-0.679788 \\
& \begin{array}{llll}
\text { O } & 5.883105 & -2.235256 & -0.718145
\end{array} \\
& \begin{array}{llll}
\text { C } & 6.603758 & -0.407444 & 1.053439
\end{array} \\
& \begin{array}{llll}
\text { C } & 6.429309 & 0.043503 & 2.362469
\end{array} \\
& \begin{array}{llll}
\text { C } & 7.796595 & -0.172940 & 0.362503
\end{array} \\
& \text { H } \quad 5.509745 \quad-0.173684 \quad 2.894905 \\
& \begin{array}{llll}
\text { C } & 7.462904 & 0.749148 & 2.978116
\end{array} \\
& \begin{array}{llll}
\text { C } & 8.815593 & 0.532181 & 0.995276
\end{array} \\
& \text { H } \quad \begin{array}{lll}
7.929781 & -0.553953 & -0.644478
\end{array} \\
& \text { H } \quad 7.333578 \quad 1.098076 \quad 3.999290 \\
& \begin{array}{llll}
\text { C } & 8.667761 & 1.005180 & 2.309658
\end{array} \\
& \begin{array}{llll}
\mathrm{H} & 9.746388 & 0.712503 & 0.463642
\end{array} \\
& \begin{array}{llll}
\text { C } & 9.795048 & 1.741562 & 2.991808
\end{array}
\end{aligned}
$$




\begin{tabular}{|c|c|c|c|}
\hline & 10.559850 & 1.039531 & 3.3483 \\
\hline & 10.290558 & 2.437781 & 2.306727 \\
\hline & 9.439693 & 2.308391 & 3.857540 \\
\hline & 1.203960 & -2.003837 & 1.333102 \\
\hline & 3.334980 & 0.596092 & -0.045660 \\
\hline & 3.615245 & 1.633451 & 0.137410 \\
\hline & 3.105703 & 0.111404 & 0.905561 \\
\hline & 2.165198 & -0.951445 & -1.583598 \\
\hline & 3.185435 & -0.932127 & -2.750511 \\
\hline & 1.179589 & -1.275007 & -2.003296 \\
\hline & 2.522728 & -0.099526 & -3.866518 \\
\hline & 1.664064 & 1.087097 & -3.381704 \\
\hline & 3.265975 & 0.323195 & -4.555979 \\
\hline & 1.877582 & -0.739118 & -4.482189 \\
\hline & 1.038489 & 1.752858 & -4.190060 \\
\hline & 2.439132 & -1.673659 & -0.816060 \\
\hline & 4.469402 & -0.222645 & -2.155116 \\
\hline & 5.367664 & -0.761673 & -2.454548 \\
\hline & 4.554826 & 0.800080 & -2.541357 \\
\hline & 3.512169 & -2.350636 & -3.237002 \\
\hline & 4.012963 & -2.927849 & -2.454128 \\
\hline & 2.602063 & -2.883067 & -3.538373 \\
\hline & 4.180464 & -2.314424 & -4.105864 \\
\hline & 2.201800 & 0.468093 & -1.047446 \\
\hline & 1.805659 & 1.474676 & -1.926878 \\
\hline & 2.017172 & 2.975017 & -1.753547 \\
\hline & 1.271936 & 3.459859 & -2.387550 \\
\hline & 2.982921 & 3.221314 & -2.225484 \\
\hline & 1.978202 & 3.603806 & -0.353749 \\
\hline & 1.836148 & 4.684963 & -0.449735 \\
\hline & 1.152741 & 3.216411 & 0.248358 \\
\hline & 2.902682 & 3.454632 & 0.211075 \\
\hline & -1.520975 & 5.649431 & -1.306227 \\
\hline
\end{tabular}




$$
\begin{aligned}
& \text { H } \quad-1.600484 \quad 6.619729 \quad-0.824266 \\
& \begin{array}{llll}
\text { C } & -1.402708 & 5.563729 & -2.695267
\end{array} \\
& \text { H } \quad-1.390856 \quad 6.467753 \quad-3.297400 \\
& \begin{array}{llll}
\text { C } & -1.288850 & 4.313358 & -3.306470
\end{array} \\
& \text { H } \quad-1.171845 \quad 4.233738 \quad-4.382861 \\
& \begin{array}{llll}
\text { C } & -0.086337 & 2.189825 & 3.637134
\end{array} \\
& \text { H } \quad 0.821446 \quad 1.940614 \quad 4.179160 \\
& \begin{array}{llll}
\text { C } & -1.115540 & 2.883706 & 4.275821
\end{array} \\
& \mathrm{H} \quad-1.008016 \quad 3.181020 \quad 5.315079 \\
& \begin{array}{llll}
\text { C } & -2.287138 & 3.190105 & 3.578221
\end{array} \\
& \mathrm{H} \quad-3.091833 \quad 3.727599 \quad 4.072125 \\
& \text { C } \quad-3.464055 \quad-2.336958 \quad-3.576595 \\
& \mathrm{H} \quad-3.404955 \quad-2.030166 \quad-4.616885 \\
& \text { C } \quad-4.474155 \quad-3.204368 \quad-3.156524 \\
& \text { H } \quad-5.201887 \quad-3.577672-3.871395 \\
& \begin{array}{llll}
\text { C } & -4.550387 & -3.590515 & -1.815195
\end{array} \\
& \text { H } \quad-5.334053 \quad-4.267599-1.486674 \\
& \begin{array}{llll}
\text { C } & 0.477822 & -5.394181 & -0.393231
\end{array} \\
& \mathrm{H} \quad 0.276580-6.342266 \quad-0.883857 \\
& \begin{array}{llll}
\text { C } & 1.620861 & -5.239756 & 0.391986
\end{array} \\
& \text { H } \quad 2.316003 \quad-6.065721 \quad 0.511345 \\
& \begin{array}{llll}
\text { C } & 1.875515 & -4.016163 & 1.017331
\end{array} \\
& \text { H } \quad 2.774891 \quad-3.870200 \quad 1.606192
\end{aligned}
$$

IntD2

B3LYP SCF energy: $\quad 3936.02614515$ a.u.

B3LYP enthalpy: 3935.017888 a.u.

B3LYP free energy: 3935.185209 a.u.

M06-L SCF energy in solution: $\quad 3937.68402142$ a.u.

M06-L enthalpy in solution: $\quad 3936.675764$ a.u.

M06-L free energy in solution: $\quad 3936.843085$ a.u. 
Cartesian coordinates

\begin{tabular}{|c|c|c|c|}
\hline & $\mathrm{X}$ & $\mathrm{Y}$ & Z \\
\hline $\mathrm{P}$ & -2.470652 & 1.808760 & -0.038584 \\
\hline $\mathrm{P}$ & -0.766178 & -1.531441 & -0.147921 \\
\hline $\mathrm{O}$ & -5.498786 & -0.904805 & 2.171319 \\
\hline $\mathrm{O}$ & -4.927022 & -2.020260 & 4.116935 \\
\hline $\mathrm{O}$ & -5.296824 & -2.642875 & -0.563509 \\
\hline $\mathrm{O}$ & -6.440823 & -1.928553 & -2.446530 \\
\hline $\mathrm{C}$ & -2.060053 & -1.818755 & 1.149088 \\
\hline $\mathrm{C}$ & -3.348581 & -1.203895 & 1.001723 \\
\hline $\mathrm{C}$ & -4.205348 & -1.346814 & 2.083235 \\
\hline $\mathrm{C}$ & -3.871127 & -2.030166 & 3.253226 \\
\hline $\mathrm{C}$ & -2.649949 & -2.650003 & 3.395329 \\
\hline $\mathrm{C}$ & -1.753318 & -2.524882 & 2.318890 \\
\hline $\mathrm{C}$ & -5.931856 & -1.200466 & 3.506441 \\
\hline $\mathrm{C}$ & -3.696303 & 0.678687 & -0.811310 \\
\hline $\mathrm{C}$ & -4.413737 & 1.098132 & -1.939706 \\
\hline $\mathrm{C}$ & -5.368380 & 0.290148 & -2.579291 \\
\hline $\mathrm{C}$ & -5.579469 & -0.957047 & -2.035057 \\
\hline $\mathrm{C}$ & -4.885607 & -1.382211 & -0.902108 \\
\hline $\mathrm{C}$ & -3.928237 & -0.618773 & -0.252262 \\
\hline $\mathrm{C}$ & -6.301122 & -3.012873 & -1.517926 \\
\hline $\mathrm{C}$ & -1.564299 & -2.143332 & -1.694942 \\
\hline $\mathrm{C}$ & -1.404250 & -1.460646 & -2.907860 \\
\hline $\mathrm{C}$ & -2.273321 & -3.357853 & -1.677177 \\
\hline $\mathrm{C}$ & 0.566027 & -2.799558 & 0.115989 \\
\hline $\mathrm{C}$ & 0.889806 & -3.724996 & -0.891262 \\
\hline $\mathrm{C}$ & 1.345121 & -2.803349 & 1.287982 \\
\hline $\mathrm{C}$ & -3.229993 & 2.241414 & 1.589772 \\
\hline $\mathrm{C}$ & -4.624045 & 2.194679 & 1.755353 \\
\hline $\mathrm{C}$ & -2.437495 & 2.708258 & 2.650783 \\
\hline $\mathrm{C}$ & -2.181844 & 3.440543 & -2.343842 \\
\hline
\end{tabular}




$$
\begin{aligned}
& \begin{array}{llll}
\text { C } & -2.547712 & 3.394299 & -0.985861
\end{array} \\
& \text { C } \quad-2.926132 \quad 4.586693 \quad-0.348152 \\
& \text { H } \quad-2.397281 \quad-3.218711 \quad 4.283160 \\
& \text { H } \quad-0.798335 \quad-3.027032 \quad 2.402522 \\
& \text { H } \quad-6.033372 \quad-0.265594 \quad 4.071059 \\
& \text { H } \quad-6.876573 \quad-1.749083 \quad 3.469516 \\
& \text { H } \quad-4.248336 \quad 2.090149 \quad-2.338300 \\
& \text { H } \quad-5.914246 \quad 0.635363 \quad-3.449853 \\
& \text { H } \quad-5.980667 \quad-3.911204 \quad-2.054570 \\
& \text { H } \quad-7.253519 \quad-3.176691 \quad-1.002425 \\
& \text { H } \quad-0.868301 \quad-0.519723 \quad-2.934989 \\
& \text { H } \quad-2.405538 \quad-3.897486 \quad-0.744235 \\
& \text { H } \quad 0.330976 \quad-3.743944 \quad-1.818456 \\
& \mathrm{H} \quad-5.257144 \quad 1.843711 \quad 0.948080 \\
& \mathrm{H} \quad-1.361505 \quad 2.775824 \quad 2.527932 \\
& \text { H } \quad-1.882952 \quad 2.537421 \quad-2.860804 \\
& \begin{array}{llll}
\mathrm{H} & -3.217956 & 4.583425 & 0.695438
\end{array} \\
& \begin{array}{llll}
\mathrm{Rh} & -0.225782 & 0.964881 & 0.358265
\end{array} \\
& \begin{array}{llll}
\text { O } & 5.952022 & -1.671231 & -0.936452
\end{array} \\
& \begin{array}{llll}
\mathrm{S} & 6.369478 & -0.264909 & -1.026562
\end{array} \\
& \begin{array}{llll}
\mathrm{N} & 5.171370 & 0.706671 & -0.295790
\end{array} \\
& \begin{array}{llll}
\text { O } & 6.649852 & 0.360122 & -2.323357
\end{array} \\
& \begin{array}{llll}
\text { C } & 7.792218 & -0.056270 & 0.035728
\end{array} \\
& \begin{array}{llll}
\text { C } & 8.181037 & -1.097781 & 0.877459
\end{array} \\
& \text { C } \quad 8.513128 \quad 1.141523 \quad-0.011289 \\
& \text { H } \quad 7.624861 \quad-2.028776 \quad 0.879281 \\
& \begin{array}{llll}
\text { C } & 9.301290 & -0.926950 & 1.692251
\end{array} \\
& \begin{array}{llll}
\text { C } & 9.626621 & 1.291593 & 0.807775
\end{array} \\
& \mathrm{H} \quad 8.214721 \quad 1.934844 \quad-0.688813 \\
& \begin{array}{llll}
\mathrm{H} & 9.610109 & -1.737155 & 2.347535
\end{array} \\
& \begin{array}{llll}
\text { C } & 10.039733 & 0.262840 & 1.672071
\end{array} \\
& \text { H } \quad 10.192094 \quad 2.2193620 .772499 \\
& \begin{array}{llll}
\text { C } & 11.267535 & 0.433564 & 2.533484
\end{array}
\end{aligned}
$$




\begin{tabular}{|c|c|c|c|}
\hline & 12.181510 & 0.352660 & 1.931218 \\
\hline & 11.284925 & 1.417947 & 3.014244 \\
\hline & 11.319229 & -0.329243 & 3.315958 \\
\hline & 1.160656 & -2.077909 & 2.070402 \\
\hline & 4.362914 & 0.107416 & 0.779496 \\
\hline & 4.441117 & 0.708575 & 1.694331 \\
\hline & 4.747375 & -0.895019 & 0.989080 \\
\hline & 2.829099 & -0.141831 & -1.190354 \\
\hline & 3.144348 & 1.270929 & -1.756428 \\
\hline & 1.847273 & -0.487415 & -1.517474 \\
\hline & 2.014223 & 2.269350 & -1.302695 \\
\hline & 0.632036 & 1.615194 & -1.334020 \\
\hline & 2.209651 & 2.658408 & -0.304832 \\
\hline & 1.967038 & 3.117361 & -1.996468 \\
\hline & 0.044362 & 1.398024 & -2.358094 \\
\hline & 3.568695 & -0.866453 & -1.542835 \\
\hline & 4.509014 & 1.741162 & -1.104454 \\
\hline & 5.208039 & 2.052945 & -1.879692 \\
\hline & 4.342007 & 2.602195 & -0.445199 \\
\hline & 3.251224 & 1.227020 & -3.287614 \\
\hline & 4.047618 & 0.540798 & -3.593053 \\
\hline & 2.310697 & 0.896324 & -3.741210 \\
\hline & 3.490522 & 2.217767 & -3.692204 \\
\hline & 2.914924 & 0.032319 & 0.299089 \\
\hline & 1.904828 & 0.417596 & 1.053517 \\
\hline & 1.339007 & 1.103531 & 2.081931 \\
\hline & -0.143458 & 2.490397 & 0.775768 \\
\hline & 1.573688 & 2.166384 & 2.160236 \\
\hline & 0.687200 & 0.510010 & 3.310751 \\
\hline & -0.090778 & 1.167905 & 3.708222 \\
\hline & 0.243613 & -0.470748 & 3.125328 \\
\hline & 1.450112 & 0.395629 & 4.094203 \\
\hline & -2.935214 & 5.794178 & -1.049109 \\
\hline
\end{tabular}




$$
\begin{array}{lrrr}
\mathrm{H} & -3.232089 & 6.705665 & -0.538880 \\
\mathrm{C} & -2.574039 & 5.829914 & -2.396178 \\
\mathrm{H} & -2.585480 & 6.769578 & -2.940882 \\
\mathrm{C} & -2.200813 & 4.648648 & -3.040836 \\
\mathrm{H} & -1.921983 & 4.663608 & -4.090705 \\
\mathrm{C} & -3.021924 & 3.111328 & 3.852591 \\
\mathrm{H} & -2.394944 & 3.473279 & 4.662604 \\
\mathrm{C} & -4.408297 & 3.056808 & 4.009172 \\
\mathrm{H} & -4.864269 & 3.374559 & 4.942525 \\
\mathrm{C} & -5.206088 & 2.601713 & 2.957063 \\
\mathrm{H} & -6.286817 & 2.571244 & 3.065131 \\
\mathrm{C} & -1.947608 & -1.983038 & -4.085274 \\
\mathrm{H} & -1.819836 & -1.444487 & -5.020024 \\
\mathrm{C} & -2.646634 & -3.189776 & -4.062536 \\
\mathrm{H} & -3.062304 & -3.596687 & -4.980063 \\
\mathrm{C} & -2.807506 & -3.877277 & -2.855208 \\
\mathrm{H} & -3.341308 & -4.823733 & -2.832762 \\
\mathrm{C} & 1.936146 & -4.634280 & -0.720101 \\
\mathrm{H} & 2.167312 & -5.335278 & -1.516886 \\
\mathrm{C} & 2.676469 & -4.646116 & 0.460654 \\
\mathrm{H} & 3.486148 & -5.357521 & 0.593399 \\
\mathrm{C} & 2.374825 & -3.726483 & 1.466748 \\
\mathrm{H} & 2.947323 & -3.718818 & 2.390234
\end{array}
$$

TS4a-cis

B3LYP SCF energy: $\quad 3936.05008311$ a.u.

B3LYP enthalpy: 3935.042882 a.u.

B3LYP free energy: 3935.208451 a.u.

M06-L SCF energy in solution: $\quad 3937.71100823$ a.u.

M06-L enthalpy in solution: $\quad 3936.703807$ a.u.

M06-L free energy in solution: $\quad 3936.869376$ a.u. 
Imaginary frequency: \$TAB \$ $-632.5872 \mathrm{~cm}-1$

Cartesian coordinates

\begin{tabular}{|c|c|c|c|}
\hline & $X$ & $\mathrm{Y}$ & Z \\
\hline$P$ & -1.856508 & 1.858447 & -0.081801 \\
\hline $\mathrm{P}$ & -0.891815 & -1.445904 & 0.315279 \\
\hline $\mathrm{O}$ & -5.756385 & -0.098559 & 1.928066 \\
\hline $\mathrm{O}$ & -5.528888 & -0.986961 & 4.054290 \\
\hline $\mathrm{O}$ & -5.511110 & -1.961049 & -0.606881 \\
\hline $\mathrm{O}$ & -6.214074 & -1.268936 & -2.701795 \\
\hline $\mathrm{C}$ & -2.303848 & -1.358489 & 1.504800 \\
\hline $\mathrm{C}$ & -3.515815 & -0.716385 & 1.104969 \\
\hline $\mathrm{C}$ & -4.518872 & -0.669745 & 2.059296 \\
\hline $\mathrm{C}$ & -4.385635 & -1.200342 & 3.341898 \\
\hline $\mathrm{C}$ & -3.227859 & -1.831277 & 3.739153 \\
\hline $\mathrm{C}$ & -2.191016 & -1.899410 & 2.792007 \\
\hline $\mathrm{C}$ & -6.461049 & -0.389344 & 3.143279 \\
\hline $\mathrm{C}$ & -3.251191 & 0.955044 & -0.882473 \\
\hline $\mathrm{C}$ & -3.704508 & 1.364406 & -2.144468 \\
\hline $\mathrm{C}$ & -4.706339 & 0.680632 & -2.853340 \\
\hline $\mathrm{C}$ & -5.236330 & -0.436858 & -2.247057 \\
\hline $\mathrm{C}$ & -4.807468 & -0.850128 & -0.985370 \\
\hline $\mathrm{C}$ & -3.821601 & -0.200129 & -0.260183 \\
\hline $\mathrm{C}$ & -6.343504 & -2.308602 & -1.721501 \\
\hline $\mathrm{C}$ & -1.577636 & -2.383897 & -1.111930 \\
\hline $\mathrm{C}$ & -1.390340 & -1.903915 & -2.416400 \\
\hline $\mathrm{C}$ & -2.308963 & -3.567658 & -0.915743 \\
\hline $\mathrm{C}$ & 0.317370 & -2.569167 & 1.132351 \\
\hline $\mathrm{C}$ & 0.630954 & -3.839975 & 0.629231 \\
\hline $\mathrm{C}$ & 0.987527 & -2.112785 & 2.283674 \\
\hline $\mathrm{C}$ & -2.436326 & 2.290501 & 1.605304 \\
\hline $\mathrm{C}$ & -3.769880 & 2.673314 & 1.830637 \\
\hline $\mathrm{C}$ & -1.531903 & 2.297506 & 2.676656 \\
\hline
\end{tabular}




$$
\begin{aligned}
& \begin{array}{llll}
\text { C } & -1.247751 & 3.498043 & -2.280066
\end{array} \\
& \begin{array}{llll}
\text { C } & -1.772854 & 3.470589 & -0.975772
\end{array} \\
& \begin{array}{llll}
\text { C } & -2.227875 & 4.667223 & -0.400576
\end{array} \\
& \text { H } \quad-3.121080 \quad-2.261012 \quad 4.728674 \\
& \mathrm{H} \quad-1.283062 \quad-2.410647 \quad 3.081818 \\
& \text { H } \quad-6.844309 \quad 0.540189 \quad 3.572769 \\
& \text { H } \quad-7.273736 \quad-1.095759 \quad 2.936030 \\
& \text { H } \quad-3.286024 \quad 2.250073 \quad-2.602747 \\
& \mathrm{H} \quad-5.046898 \quad 1.021026 \quad-3.824550 \\
& \text { H } \quad-6.000500 \quad-3.257333 \quad-2.150451 \\
& \text { H } \quad-7.385072 \quad-2.372203 \quad-1.394718 \\
& \text { H } \quad-0.864177 \quad-0.966196 \quad-2.575076 \\
& \begin{array}{llll}
\text { H } & -2.484944 & -3.938534 & 0.089862
\end{array} \\
& \text { H } \quad \begin{array}{llll}
0.138161 & -4.214640 & -0.260802
\end{array} \\
& \text { H } \quad-4.486172 \quad 2.659488 \quad 1.014636 \\
& \begin{array}{llll}
\mathrm{H} & -0.503284 & 1.988079 & 2.513474
\end{array} \\
& \text { H } \quad-0.845993 \quad 2.595245 \quad-2.730530 \\
& \begin{array}{llll}
\mathrm{H} & -2.627368 & 4.676689 & 0.606878
\end{array} \\
& \begin{array}{llll}
\text { Rh } & 0.192938 & 0.681607 & -0.254041
\end{array} \\
& \begin{array}{llll}
\text { O } & 5.118187 & -2.173935 & 1.773093
\end{array} \\
& \begin{array}{llll}
\text { S } & 5.729980 & -1.619946 & 0.562251
\end{array} \\
& \text { N } \quad 4.436065 \quad-1.129113 \quad-0.404906 \\
& \begin{array}{llll}
\text { O } & 6.604235 & -2.408201 & -0.307218
\end{array} \\
& \begin{array}{llll}
\text { C } & 6.615124 & -0.133068 & 1.029970
\end{array} \\
& \begin{array}{llll}
\text { C } & 6.254759 & 0.548173 & 2.196041
\end{array} \\
& \begin{array}{llll}
\text { C } & 7.645037 & 0.343325 & 0.212951
\end{array} \\
& \mathrm{H} \quad 5.482578 \quad 0.144130 \quad 2.842155 \\
& \begin{array}{llll}
\text { C } & 6.927666 & 1.721824 & 2.533551
\end{array} \\
& \begin{array}{llll}
\text { C } & 8.305755 & 1.517283 & 0.567510
\end{array} \\
& \text { H } \quad 7.940834 \quad-0.215002 \quad-0.668927 \\
& \begin{array}{llll}
\mathrm{H} & 6.656250 & 2.247958 & 3.445358
\end{array} \\
& \begin{array}{llll}
\text { C } & 7.960489 & 2.225216 & 1.729248
\end{array} \\
& \begin{array}{llll}
\mathrm{H} & 9.112863 & 1.883949 & -0.061715
\end{array}
\end{aligned}
$$




$$
\begin{aligned}
& \begin{array}{llll}
\text { C } & 8.711982 & 3.474099 & 2.122681
\end{array} \\
& \text { H } \quad 9.628187 \quad 3.220288 \quad 2.671562 \\
& \text { H } \quad 9.011892 \quad 4.055716 \quad 1.244765 \\
& \text { H } \quad 8.110655 \quad 4.118296 \quad 2.771749 \\
& \mathrm{H} \quad 0.776376 \quad-1.120928 \quad 2.676430 \\
& \begin{array}{llll}
\text { C } & 3.253417 & -0.541189 & 0.271041
\end{array} \\
& \begin{array}{llll}
\text { H } & 3.498972 & 0.416141 & 0.752066
\end{array} \\
& \text { H } \quad 2.931553 \quad-1.242266 \quad 1.038653 \\
& \begin{array}{llll}
\text { C } & 2.307632 & -1.536368 & -1.808561
\end{array} \\
& \begin{array}{llll}
\text { C } & 3.507817 & -1.166392 & -2.721527
\end{array} \\
& \mathrm{H} \quad 1.404534-1.664564 \quad-2.412553 \\
& \begin{array}{llll}
\text { C } & 3.004305 & -0.008206 & -3.627575
\end{array} \\
& \begin{array}{llll}
\text { C } & 1.936380 & 0.8774448 & -2.968007
\end{array} \\
& \text { H } \quad 3.828873 \quad 0.648705 \quad-3.941269 \\
& \text { H } \quad 2.559897 \quad-0.397577 \quad-4.550653 \\
& \begin{array}{llll}
\text { O } & 1.142179 & 1.528557 & -3.621781
\end{array} \\
& \text { H } \quad 2.487864 \quad-2.472739 \quad-1.277007 \\
& \begin{array}{llll}
\text { C } & 4.682453 & -0.689896 & -1.784416
\end{array} \\
& \text { H } \quad 5.620988-1.138488-2.112283 \\
& \mathrm{H} \quad 4.804183 \quad 0.404055 \quad-1.828814 \\
& \begin{array}{llll}
\text { C } & 3.956836 & -2.367070 & -3.565273
\end{array} \\
& \text { H } \quad 4.362040 \quad-3.154798 \quad-2.920718 \\
& \text { H } \quad 3.119353 \quad-2.785957 \quad-4.135554 \\
& \text { H } \quad 4.739216 \quad-2.079964 \quad-4.278149 \\
& \begin{array}{llll}
\text { C } & 2.224670 & -0.371862 & -0.837116
\end{array} \\
& \begin{array}{llll}
\text { C } & 2.010020 & 0.886473 & -1.453137
\end{array} \\
& \begin{array}{llll}
\text { C } & 1.945799 & 2.095522 & -0.677097
\end{array} \\
& \mathrm{H} \quad 0.556616 \quad 2.036176 \quad 0.469388 \\
& \begin{array}{llll}
\text { C } & -2.172588 & 5.861961 & -1.122467
\end{array} \\
& \text { H } \quad-2.526152 \quad 6.780394 \quad-0.662603 \\
& \begin{array}{llll}
\text { C } & -1.675777 & 5.876206 & -2.426095
\end{array} \\
& \text { H } \quad-1.642665 \quad 6.805646 \quad-2.987298 \\
& \begin{array}{llll}
\text { C } & -1.213378 & 4.691099 & -3.002836
\end{array}
\end{aligned}
$$




$$
\begin{aligned}
& \mathrm{H} \quad-0.812816 \quad 4.692476 \quad-4.012672 \\
& \begin{array}{llll}
\text { C } & -1.948390 & 2.687755 & 3.950858
\end{array} \\
& \begin{array}{llll}
\mathrm{H} & -1.239744 & 2.686839 & 4.774079
\end{array} \\
& \begin{array}{llll}
\text { C } & -3.272276 & 3.073875 & 4.165614
\end{array} \\
& \mathrm{H} \quad-3.596260 \quad 3.378013 \quad 5.156799 \\
& \begin{array}{llll}
\text { C } & -4.181669 & 3.065137 & 3.104156
\end{array} \\
& \begin{array}{llll}
\text { H } & -5.212700 & 3.366663 & 3.266774
\end{array} \\
& \begin{array}{llll}
\text { C } & -1.905138 & -2.604045 & -3.509338
\end{array} \\
& \text { H } \quad-1.758426 \quad-2.219598 \quad-4.514558 \\
& \text { C } \quad-2.612884-3.790429-3.307533 \\
& \text { H } \quad-3.008969-4.338600 \quad-4.157690 \\
& \begin{array}{llll}
\text { C } & -2.816302 & -4.269411 & -2.009678
\end{array} \\
& \text { H } \quad-3.369303 \quad-5.190673 \quad-1.848400 \\
& \begin{array}{llll}
\text { C } & 1.586000 & -4.637005 & 1.267679
\end{array} \\
& \begin{array}{llll}
\text { H } & 1.823139 & -5.615589 & 0.860273
\end{array} \\
& \begin{array}{llll}
\text { C } & 2.232523 & -4.182339 & 2.415997
\end{array} \\
& \text { H } \quad 2.981375 \quad-4.798377 \quad 2.903486 \\
& \begin{array}{llll}
\text { C } & 1.926015 & -2.918560 & 2.927228
\end{array} \\
& \mathrm{H} \quad 2.437618 \quad-2.551310 \quad 3.811611 \\
& \begin{array}{llll}
\text { C } & 1.918620 & 3.481712 & -1.273236
\end{array} \\
& \text { H } \quad 2.955279 \quad 3.804056 \quad-1.445961 \\
& \text { H } \quad 1.381415 \quad 3.525141 \quad-2.217348 \\
& \text { H } \quad 1.467851 \quad 4.198240 \quad-0.581241 \\
& \begin{array}{llll}
\mathrm{H} & 2.505362 & 2.051665 & 0.256724
\end{array}
\end{aligned}
$$

TS4a-trans

B3LYP SCF energy: $\quad 3936.04743005$ a.u.

B3LYP enthalpy: 3935.040006 a.u.

B3LYP free energy: $\quad 3935.206451$ a.u.

M06-L SCF energy in solution: 3937.70698932 a.u.

M06-L enthalpy in solution: $\quad 3936.699565$ a.u. 
M06-L free energy in solution: $\quad 3936.866010$ a.u.

Imaginary frequency: \$TAB \$ $-689.3967 \mathrm{~cm}-1$

Cartesian coordinates
$\begin{array}{llll}\text { ATOM } & \mathrm{X} & \mathrm{Y} & \mathrm{Z}\end{array}$
$\begin{array}{llll}\mathrm{P} & -1.859963 & 1.870336 & -0.266893\end{array}$
$\begin{array}{llll}\mathrm{P} & -0.985101 & -1.419932 & 0.438711\end{array}$
$\begin{array}{llll}\text { O } & -5.810208 & 0.211534 & 1.893833\end{array}$
$\begin{array}{llll}\text { O } & -5.608685 & -0.479314 & 4.094320\end{array}$
O $\quad-5.607726-1.883430 \quad-0.477553$
O $\quad-6.283775 \quad-1.354821 \quad-2.628265$
$\begin{array}{llll}\text { C } & -2.397507 & -1.184170 & 1.602364\end{array}$
$\begin{array}{llll}\text { C } & -3.588539 & -0.545270 & 1.137887\end{array}$
$\begin{array}{llll}\text { C } & -4.590174 & -0.381367 & 2.080755\end{array}$
$\begin{array}{llll}\text { C } & -4.471946 & -0.792531 & 3.408399\end{array}$
$\begin{array}{llll}\text { C } & -3.332121 & -1.413343 & 3.868849\end{array}$
$\begin{array}{llll}\text { C } & -2.297456 & -1.601224 & 2.935407\end{array}$
$\begin{array}{llll}\text { C } & -6.525537 & 0.048133 & 3.126553\end{array}$
$\begin{array}{llll}\text { C } & -3.275963 & 0.941582 & -0.987944\end{array}$
$\begin{array}{llll}\text { C } & -3.710618 & 1.252588 & -2.283816\end{array}$
$\begin{array}{llll}\text { C } & -4.726495 & 0.535476 & -2.937661\end{array}$
$\begin{array}{llll}\text { C } & -5.287180 & -0.511611 & -2.239886\end{array}$
$\begin{array}{llll}\text { C } & -4.874513 & -0.826695 & -0.944356\end{array}$
$\begin{array}{llll}\text { C } & -3.875647 & -0.142491 & -0.270584\end{array}$
$\begin{array}{llll}\text { C } & -6.448199 & -2.300499 & -1.562000\end{array}$
$\begin{array}{llll}\text { C } & -1.681015 & -2.473606 & -0.900019\end{array}$
$\begin{array}{llll}\text { C } & -1.432846 & -2.150772 & -2.242645\end{array}$
$\begin{array}{llll}\text { C } & -2.474406 & -3.594058 & -0.600372\end{array}$
$\begin{array}{llll}\text { C } & 0.205468 & -2.480493 & 1.360505\end{array}$
$\begin{array}{llll}\text { C } & 0.449712 & -3.819871 & 1.024701\end{array}$
$\begin{array}{llll}\text { C } & 0.934622 & -1.902424 & 2.416902\end{array}$
$\begin{array}{llll}\text { C } & -2.422575 & 2.470709 & 1.372917\end{array}$
$\begin{array}{llll}\text { C } & -3.744127 & 2.910581 & 1.561122\end{array}$ 


$$
\begin{aligned}
& \begin{array}{llll}
\text { C } & -1.515810 & 2.548587 & 2.439591
\end{array} \\
& \begin{array}{llll}
\text { C } & -1.121776 & 3.291088 & -2.579779
\end{array} \\
& \text { C } \quad-1.694076 \quad 3.392816 \quad-1.297740 \\
& \begin{array}{llll}
\text { C } & -2.104362 & 4.651897 & -0.834252
\end{array} \\
& \text { H } \quad-3.237751 \quad-1.747001 \quad 4.895977 \\
& \text { H } \quad-1.400554 \quad-2.103596 \quad 3.273500 \\
& \text { H } \quad-6.892866 \quad 1.020385 \quad 3.465484 \\
& \text { H } \quad-7.350679 \quad-0.659405 \quad 2.980739 \\
& \text { H } \quad-3.263415 \quad 2.083698 \quad-2.813032 \\
& \begin{array}{llll}
\mathrm{H} & -5.053777 & 0.797692 & -3.937248
\end{array} \\
& \text { H } \quad-6.134547 \quad-3.293258 \quad-1.904961 \\
& \text { H } \quad-7.492053 \quad-2.302793 \quad-1.236067 \\
& \text { H } \quad-0.856351 \quad-1.263126 \quad-2.490184 \\
& \text { H } \quad-2.697745 \quad-3.841802 \quad 0.433316 \\
& \mathrm{H} \quad-0.090897 \quad-4.288671 \quad 0.210168 \\
& \text { H } \quad-4.462003 \quad 2.843089 \quad 0.749144 \\
& \begin{array}{llll}
\mathrm{H} & -0.496495 & 2.197155 & 2.305440
\end{array} \\
& \begin{array}{llll}
\mathrm{H} & -0.773212 & 2.332970 & -2.954773
\end{array} \\
& \begin{array}{llll}
\mathrm{H} & -2.541152 & 4.759633 & 0.151922
\end{array} \\
& \begin{array}{llll}
\mathrm{Rh} & 0.137364 & 0.622716 & -0.333586
\end{array} \\
& \begin{array}{llll}
\text { O } & 5.204156 & -1.893231 & 1.992097
\end{array} \\
& \begin{array}{llll}
\text { S } & 5.795147 & -1.516087 & 0.704995
\end{array} \\
& \begin{array}{llll}
\mathrm{N} & 4.490515 & -1.105138 & -0.277828
\end{array} \\
& \begin{array}{llll}
\text { O } & 6.616941 & -2.436752 & -0.082274
\end{array} \\
& \begin{array}{llll}
\text { C } & 6.742625 & -0.016304 & 0.960077
\end{array} \\
& \begin{array}{llll}
\text { C } & 6.444723 & 0.812925 & 2.044823
\end{array} \\
& \begin{array}{llll}
\text { C } & 7.757405 & 0.321002 & 0.059351
\end{array} \\
& \begin{array}{llll}
\mathrm{H} & 5.682731 & 0.518065 & 2.758567
\end{array} \\
& \begin{array}{llll}
\text { C } & 7.165140 & 1.994404 & 2.215377
\end{array} \\
& \begin{array}{llll}
\text { C } & 8.466055 & 1.505446 & 0.246994
\end{array} \\
& \text { H } \quad 8.004342 \quad-0.349628 \quad-0.756883 \\
& \begin{array}{llll}
\mathrm{H} & 6.942037 & 2.636339 & 3.063917
\end{array} \\
& \begin{array}{llll}
\text { C } & 8.183894 & 2.360477 & 1.323732
\end{array}
\end{aligned}
$$




$$
\begin{aligned}
& \mathrm{H} \quad 9.261167 \quad 1.765025 \quad-0.447419 \\
& \begin{array}{llll}
\text { C } & 8.986365 & 3.621275 & 1.537555
\end{array} \\
& \begin{array}{llll}
\mathrm{H} & 9.913928 & 3.404543 & 2.083254
\end{array} \\
& \begin{array}{llll}
\mathrm{H} & 9.271356 & 4.082120 & 0.586035
\end{array} \\
& \text { H } \quad 8.428534 \quad 4.359266 \quad 2.122416 \\
& \text { H } \quad 0.770777 \quad-0.860130 \quad 2.680672 \\
& \begin{array}{llll}
\text { C } & 3.318085 & -0.442496 & 0.347385
\end{array} \\
& \begin{array}{llll}
\mathrm{H} & 3.556873 & 0.574979 & 0.669297
\end{array} \\
& \text { H } \quad 3.030339-1.033569 \quad 1.216462 \\
& \begin{array}{llll}
\text { C } & 2.326962 & -1.802007 & -1.479615
\end{array} \\
& \begin{array}{llll}
\text { C } & 3.469303 & -1.587172 & -2.505990
\end{array} \\
& \text { H } \quad 1.397974 \quad-2.057894 \quad-1.997646 \\
& \begin{array}{llll}
\text { C } & 2.878523 & -0.633819 & -3.581416
\end{array} \\
& \begin{array}{llll}
\text { C } & 1.810128 & 0.332394 & -3.043133
\end{array} \\
& \text { H } \quad 3.657455 \quad-0.021237 \quad-4.058511 \\
& \text { H } \quad 2.407313 \quad-1.204687 \quad-4.389834 \\
& \begin{array}{llll}
\text { O } & 0.997650 & 0.872425 & -3.771896
\end{array} \\
& \text { H } \quad 2.558886 \quad-2.616054-0.790545 \\
& \begin{array}{llll}
\text { C } & 4.667766 & -0.920858 & -1.724658
\end{array} \\
& \text { H } \quad 5.605376-1.401002-2.006863 \\
& \text { H } \quad 4.754652 \quad 0.148647 \quad-1.971279 \\
& \begin{array}{llll}
\text { C } & 3.918057 & -2.914147 & -3.132549
\end{array} \\
& \text { H } \quad 4.380656 \quad-3.555632 \quad-2.374522 \\
& \text { H } \quad 3.069179 \quad-3.453408 \quad-3.569257 \\
& \text { H } \quad 4.655292 \quad-2.747081 \quad-3.927189 \\
& \begin{array}{llll}
\text { C } & 2.264772 & -0.473781 & -0.745206
\end{array} \\
& \begin{array}{llll}
\text { C } & 1.942404 & 0.626308 & -1.559753
\end{array} \\
& \begin{array}{llll}
\text { C } & 1.711778 & 1.994837 & -1.123430
\end{array} \\
& \begin{array}{llll}
\mathrm{H} & 0.595734 & 1.999250 & 0.255242
\end{array} \\
& \text { H } \quad 1.271361 \quad 2.591545 \quad-1.919054 \\
& \begin{array}{llll}
\text { C } & 2.642353 & 2.840790 & -0.257414
\end{array} \\
& \text { H } \quad 2.181403 \quad 3.816968 \quad-0.075951 \\
& \text { H } \quad 2.886360 \quad 2.401868 \quad 0.710393
\end{aligned}
$$




$$
\begin{array}{lrrr}
\mathrm{H} & 3.581345 & 3.016170 & -0.798315 \\
\mathrm{C} & -1.951998 & 5.783789 & -1.639314 \\
\mathrm{H} & -2.272335 & 6.751952 & -1.265089 \\
\mathrm{C} & -1.396931 & 5.673352 & -2.914086 \\
\mathrm{H} & -1.283395 & 6.555127 & -3.538142 \\
\mathrm{C} & -0.983464 & 4.423646 & -3.382782 \\
\mathrm{H} & -0.546270 & 4.326209 & -4.372441 \\
\mathrm{C} & -1.918779 & 3.064144 & 3.672770 \\
\mathrm{H} & -1.209091 & 3.117363 & 4.493364 \\
\mathrm{C} & -3.230819 & 3.505795 & 3.850705 \\
\mathrm{H} & -3.544127 & 3.907250 & 4.810171 \\
\mathrm{C} & -4.142131 & 3.427534 & 2.793829 \\
\mathrm{H} & -5.163800 & 3.771853 & 2.927974 \\
\mathrm{C} & -1.950049 & -2.944334 & -3.268667 \\
\mathrm{H} & -1.755694 & -2.680888 & -4.304329 \\
\mathrm{C} & -2.720901 & -4.067187 & -2.962128 \\
\mathrm{H} & -3.119388 & -4.687491 & -3.759968 \\
\mathrm{C} & -2.984426 & -4.389105 & -1.627249 \\
\mathrm{H} & -3.586295 & -5.260564 & -1.384567 \\
\mathrm{C} & 1.399582 & -4.563174 & 1.731726 \\
\mathrm{H} & 1.582932 & -5.597285 & 1.454348 \\
\mathrm{C} & 2.110018 & -3.984446 & 2.782760 \\
\mathrm{H} & 2.854494 & -4.560370 & 3.323482 \\
\mathrm{H} & 1.870483 & -2.651855 & 3.129084 \\
& 2.430174 & -2.191572 & 3.937600
\end{array}
$$

IntE-cis

B3LYP SCF energy: $\quad 3936.07062268$ a.u.

B3LYP enthalpy: 3935.061787 a.u.

B3LYP free energy: 3935.228073 a.u.

M06-L SCF energy in solution: $\quad 3937.72803598$ a.u. 
M06-L enthalpy in solution: 3936.719200 a.u.

M06-L free energy in solution: 3936.885486 a.u.

Cartesian coordinates
ATOM $\mathrm{X} \quad \mathrm{Y} \quad \mathrm{Z}$
P $\quad-1.792145 \quad 1.807740 \quad-0.292571$
P $\quad-0.923070 \quad-1.598291 \quad 0.262229$
$\begin{array}{llll}\text { O } & -5.317040 & 0.333633 & 2.539309\end{array}$
O $\quad-4.798439 \quad-0.458350 \quad 4.653475$
O $\quad \begin{array}{llll}-5.684547 & -1.773059 & 0.181491\end{array}$
O $\quad-6.775253 \quad-1.140077 \quad-1.759429$
C $\quad-2.145765 \quad-1.330940 \quad 1.614337$
C $\quad-3.334600 \quad-0.566636 \quad 1.383816$
C $\quad-4.130713 \quad-0.349590 \quad 2.498071$
C $\quad-3.826736 \quad-0.826626 \quad 3.772531$
C $\quad-2.699241 \quad-1.582749 \quad 4.003760$
$\begin{array}{llll}\text { C } & -1.867084 & -1.821508 & 2.897594\end{array}$
C $\quad \begin{array}{llll}-5.741058 & 0.325703 & 3.909912\end{array}$
C $\quad \begin{array}{llll}-3.376968 & 0.969248 & -0.735750\end{array}$
$\begin{array}{llll}\text { C } & -4.057956 & 1.340477 & -1.903591\end{array}$
$\begin{array}{llll}\text { C } & -5.225311 & 0.694070 & -2.344057\end{array}$
C $\quad \begin{array}{llll}-5.686372 & -0.342122 & -1.562869\end{array}$
$\begin{array}{llll}\text { C } & -5.026506 & -0.717485 & -0.393239\end{array}$
$\begin{array}{llll}\text { C } & -3.869079 & -0.107625 & 0.065657\end{array}$
$\begin{array}{llll}\text { C } & -6.873737 & -1.971205 & -0.595670\end{array}$
C $\quad-1.851552 \quad-2.361376 \quad-1.125334$
$\begin{array}{llll}\text { C } & -1.547474 & -2.001569 & -2.446865\end{array}$
C $\quad-2.840450 \quad-3.330428 \quad-0.888812$
$\begin{array}{llll}\text { C } & 0.211766 & -2.917352 & 0.862439\end{array}$
$\begin{array}{llll}\text { C } & 0.234456 & -4.190968 & 0.273409\end{array}$
C $\quad \begin{array}{llll}1.119546 & -2.635768 & 1.900024\end{array}$
$\begin{array}{llll}\text { C } & -2.076198 & 2.548599 & 1.366788\end{array}$
$\begin{array}{llll}\text { C } & -3.354667 & 2.973173 & 1.764795\end{array}$ 


$$
\begin{aligned}
& \begin{array}{llll}
\text { C } & -0.989686 & 2.758780 & 2.228947
\end{array} \\
& \begin{array}{llll}
\text { C } & -1.336422 & 3.050955 & -2.765550
\end{array} \\
& \begin{array}{llll}
\text { C } & -1.735098 & 3.258231 & -1.433284
\end{array} \\
& \begin{array}{llll}
\text { C } & -2.089409 & 4.553263 & -1.024664
\end{array} \\
& \text { H } \quad-2.467431 \quad-1.980703 \quad 4.985040 \\
& \text { H } \quad-0.985841 \quad-2.426874 \quad 3.059770 \\
& \begin{array}{llll}
\mathrm{H} & -5.745887 & 1.351054 & 4.296009
\end{array} \\
& \text { H } \quad-6.732863 \quad-0.130664 \quad 3.983474 \\
& \text { H } \quad-3.687763 \quad 2.165729 \quad-2.497375 \\
& \text { H } \quad-5.737601 \quad 1.001117 \quad-3.248763 \\
& \text { H } \quad-6.938429-3.018772 \quad-0.901051 \\
& \text { H } \quad-7.750555 \quad-1.674471 \quad-0.007252 \\
& \text { H } \quad-0.787516 \quad-1.250799-2.653799 \\
& \text { H } \quad-3.097748 \quad-3.608353 \quad 0.128879 \\
& \text { H } \quad-0.448184 \quad-4.436850 \quad-0.531640 \\
& \begin{array}{llll}
\text { H } & -4.208037 & 2.812934 & 1.113317
\end{array} \\
& \text { H } \quad 0.004372 \quad 2.435867 \quad 1.933175 \\
& \begin{array}{llll}
\mathrm{H} & -1.013487 & 2.068877 & -3.098429
\end{array} \\
& \mathrm{H} \quad-2.394386 \quad 4.741032-0.001151 \\
& \begin{array}{llll}
\mathrm{Rh} & 0.155211 & 0.327833 & -0.527156
\end{array} \\
& \begin{array}{llll}
\text { O } & 4.970562 & -1.926731 & 2.033043
\end{array} \\
& \text { S } \quad 5.651556 \quad-1.463920 \quad 0.819342 \\
& \text { N } \quad 4.418640 \quad-1.082062 \quad-0.264293 \\
& \begin{array}{llll}
\text { O } & 6.592972 & -2.306621 & 0.080702
\end{array} \\
& \begin{array}{llll}
\text { C } & 6.486376 & 0.071128 & 1.219155
\end{array} \\
& \begin{array}{llll}
\text { C } & 6.041190 & 0.840383 & 2.297783
\end{array} \\
& \begin{array}{llll}
\text { C } & 7.562928 & 0.495823 & 0.434438
\end{array} \\
& \begin{array}{llll}
\mathrm{H} & 5.232001 & 0.478404 & 2.923163
\end{array} \\
& \begin{array}{llll}
\text { C } & 6.676616 & 2.049103 & 2.579300
\end{array} \\
& \begin{array}{llll}
\text { C } & 8.184928 & 1.706228 & 0.731851
\end{array} \\
& \begin{array}{llll}
\text { H } & 7.923291 & -0.128437 & -0.376341
\end{array} \\
& \text { H } \quad 6.338877 \quad 2.644274 \quad 3.424063 \\
& \text { C } \quad 7.754806 \quad 2.501846 \quad 1.805070
\end{aligned}
$$




$$
\begin{aligned}
& \mathrm{H} \quad 9.027769 \quad 2.033359 \quad 0.128180 \\
& \begin{array}{llll}
\text { C } & 8.463698 & 3.791854 & 2.140784
\end{array} \\
& \begin{array}{llll}
\mathrm{H} & 9.344688 & 3.600437 & 2.767173
\end{array} \\
& \mathrm{H} \quad 8.813824 \quad 4.304230 \quad 1.238635 \\
& \mathrm{H} \quad 7.813051 \quad 4.476848 \quad 2.693382 \\
& \text { H } \quad 1.125172 \quad-1.654121 \quad 2.366185 \\
& \begin{array}{llll}
\text { C } & 3.180286 & -0.478491 & 0.275089
\end{array} \\
& \begin{array}{llll}
\mathrm{H} & 3.362005 & 0.534418 & 0.664401
\end{array} \\
& \text { H } \quad 2.849514 \quad-1.111977 \quad 1.093836 \\
& \begin{array}{llll}
\text { C } & 2.398426 & -1.682551 & -1.760766
\end{array} \\
& \begin{array}{llll}
\text { C } & 3.650570 & -1.351968 & -2.619118
\end{array} \\
& \text { H } \quad 1.549331 \quad-1.897732 \quad-2.420643 \\
& \begin{array}{llll}
\text { C } & 3.191978 & -0.288843 & -3.664096
\end{array} \\
& \begin{array}{llll}
\text { C } & 2.032683 & 0.561016 & -3.146630
\end{array} \\
& \begin{array}{llll}
\mathrm{H} & 4.019302 & 0.382285 & -3.937772
\end{array} \\
& \text { H } \quad 2.852392 \quad-0.761090 \quad-4.592224 \\
& \begin{array}{llll}
\text { O } & 1.130254 & 0.963445 & -3.862864
\end{array} \\
& \mathrm{H} \quad 2.564534 \quad-2.569847 \quad-1.146306 \\
& \text { C } \quad 4.744100 \quad-0.755234-1.655886 \\
& \text { H } \quad 5.715846 \quad-1.194723 \quad-1.885244 \\
& \text { H } \quad 4.833734 \quad 0.334828 \quad-1.786312 \\
& \text { C } \quad 4.190546-2.604768 \quad-3.321481 \\
& \text { H } \quad 4.570609 \quad-3.320092 \quad-2.583819 \\
& \text { H } \quad 3.406774 \quad-3.099022 \quad-3.907677 \\
& \text { H } \quad \begin{array}{llll}
5.013044 & -2.354726 & -4.002529
\end{array} \\
& \begin{array}{llll}
\text { C } & 2.176933 & -0.455711 & -0.879232
\end{array} \\
& \begin{array}{llll}
\text { C } & 2.066619 & 0.777462 & -1.648854
\end{array} \\
& \begin{array}{llll}
\text { C } & 1.896803 & 2.024434 & -1.061104
\end{array} \\
& \begin{array}{llll}
\mathrm{H} & 0.558609 & 0.512932 & 0.925562
\end{array} \\
& \begin{array}{llll}
\text { C } & -2.056089 & 5.613984 & -1.933559
\end{array} \\
& \text { H } \quad-2.329672 \quad 6.611534-1.601668 \\
& \begin{array}{llll}
\text { C } & -1.681937 & 5.394276 & -3.259830
\end{array} \\
& \text { H } \quad-1.663634 \quad 6.220002 \quad-3.965353
\end{aligned}
$$




$$
\begin{array}{llll}
\mathrm{C} & -1.322478 & 4.109348 & -3.674053 \\
\mathrm{H} & -1.017045 & 3.929405 & -4.700878 \\
\mathrm{C} & -1.174350 & 3.379952 & 3.465187 \\
\mathrm{H} & -0.324695 & 3.534464 & 4.124118 \\
\mathrm{C} & -2.448170 & 3.799344 & 3.853084 \\
\mathrm{H} & -2.592461 & 4.283512 & 4.814701 \\
\mathrm{C} & -3.536328 & 3.595517 & 3.000748 \\
\mathrm{H} & -4.528514 & 3.927454 & 3.294388 \\
\mathrm{C} & -2.216606 & -2.599170 & -3.516449 \\
\mathrm{H} & -1.975618 & -2.308354 & -4.534674 \\
\mathrm{C} & -3.191217 & -3.568330 & -3.273056 \\
\mathrm{H} & -3.709574 & -4.037865 & -4.104039 \\
\mathrm{C} & -3.500871 & -3.932794 & -1.959579 \\
\mathrm{H} & -4.256965 & -4.689048 & -1.768075 \\
\mathrm{C} & 1.141890 & -5.156824 & 0.716638 \\
\mathrm{H} & 1.148029 & -6.137047 & 0.248555 \\
\mathrm{C} & 2.035908 & -4.866543 & 1.746769 \\
\mathrm{H} & 2.747975 & -5.615212 & 2.080581 \\
\mathrm{C} & 2.022276 & -3.602232 & 2.341596 \\
\mathrm{H} & 2.734216 & -3.357317 & 3.122600 \\
\mathrm{H} & 2.129439 & 2.101933 & -0.000145 \\
\mathrm{H} & 1.805525 & 3.340315 & -1.777312 \\
\mathrm{H} & 1.544907 & 3.233338 & -2.829633 \\
& 1.068981 & 3.996521 & -1.304406 \\
\hline
\end{array}
$$

IntE-trans

B3LYP SCF energy: $\quad 3936.06825593$ a.u.

B3LYP enthalpy: 3935.059154 a.u.

B3LYP free energy: 3935.225344 a.u.

M06-L SCF energy in solution: $\quad 3937.72679418$ a.u. 
M06-L enthalpy in solution: 3936.717692 a.u.

M06-L free energy in solution: 3936.883882 a.u.

Cartesian coordinates

\begin{tabular}{|c|c|c|c|}
\hline \multicolumn{2}{|c|}{ ATOM } & $\mathrm{Y}$ & Z \\
\hline $\mathrm{P}$ & -1.772292 & 1.854228 & -0.357475 \\
\hline $\mathrm{P}$ & -0.958055 & -1.520375 & 0.430207 \\
\hline $\mathrm{O}$ & -5.583458 & 0.432606 & 2.140285 \\
\hline $\mathrm{O}$ & -5.268080 & -0.215754 & 4.340722 \\
\hline $\mathrm{O}$ & -5.645611 & -1.771510 & -0.158757 \\
\hline $\mathrm{O}$ & -6.510186 & -1.244092 & -2.242518 \\
\hline $\mathrm{C}$ & -2.287991 & -1.173130 & 1.654578 \\
\hline $\mathrm{C}$ & -3.467333 & -0.468368 & 1.253019 \\
\hline $\mathrm{C}$ & -4.387365 & -0.223621 & 2.260612 \\
\hline $\mathrm{C}$ & -4.202890 & -0.610935 & 3.587602 \\
\hline $\mathrm{C}$ & -3.075442 & -1.294465 & 3.985750 \\
\hline $\mathrm{C}$ & -2.123541 & -1.567624 & 2.988976 \\
\hline $\mathrm{C}$ & -6.208620 & 0.368638 & 3.429875 \\
\hline $\mathrm{C}$ & -3.294876 & 0.980042 & -0.923308 \\
\hline $\mathrm{C}$ & -3.842730 & 1.290351 & -2.175605 \\
\hline $\mathrm{C}$ & -4.936892 & 0.597639 & -2.720947 \\
\hline $\mathrm{C}$ & -5.460288 & -0.422812 & -1.958223 \\
\hline $\mathrm{C}$ & -4.934633 & -0.736883 & -0.704825 \\
\hline $\mathrm{C}$ & -3.852935 & -0.080209 & -0.138784 \\
\hline $\mathrm{C}$ & -6.660028 & -2.114896 & -1.113258 \\
\hline $\mathrm{C}$ & -1.781126 & -2.483244 & -0.901765 \\
\hline $\mathrm{C}$ & -1.485847 & -2.215711 & -2.247134 \\
\hline $\mathrm{C}$ & -2.703394 & -3.497103 & -0.592746 \\
\hline $\mathrm{C}$ & 0.190873 & -2.693404 & 1.260201 \\
\hline $\mathrm{C}$ & 0.297641 & -4.035423 & 0.865714 \\
\hline $\mathrm{C}$ & 1.028308 & -2.220237 & 2.287502 \\
\hline $\mathrm{C}$ & -2.182001 & 2.556773 & 1.291112 \\
\hline $\mathrm{C}$ & -3.483062 & 3.003577 & 1.577122 \\
\hline
\end{tabular}




$$
\begin{aligned}
& \begin{array}{llll}
\text { C } & -1.177327 & 2.722607 & 2.255193
\end{array} \\
& \begin{array}{llll}
\text { C } & -1.155633 & 3.169567 & -2.767632
\end{array} \\
& \text { C } \quad-1.635910 \quad 3.335665 \quad-1.454950 \\
& \begin{array}{llll}
\text { C } & -1.985799 & 4.623068 & -1.019963
\end{array} \\
& \text { H } \quad-2.931438 \quad-1.612998 \quad 5.011849 \\
& \text { H } \quad-1.240025 \quad-2.121668 \quad 3.277701 \\
& \begin{array}{llll}
\text { H } & -6.458307 & 1.379546 & 3.764045
\end{array} \\
& \begin{array}{llll}
\text { H } & -7.103079 & -0.262681 & 3.373090
\end{array} \\
& \text { H } \quad-3.423540 \quad 2.103290 \quad-2.754398 \\
& \text { H } \quad-5.349999 \quad 0.858877 \quad-3.688539 \\
& \text { H } \quad-6.521944 \quad-3.152786 \quad-1.433509 \\
& \mathrm{H} \quad-7.648721 \quad-1.966234-0.666526 \\
& \mathrm{H} \quad-0.788275 \quad-1.424885 \quad-2.513083 \\
& \mathrm{H} \quad-2.958581 \quad-3.703045 \quad 0.442494 \\
& \begin{array}{llll}
\text { H } & -0.329228 & -4.426639 & 0.072612
\end{array} \\
& \text { H } \quad-4.275060 \quad 2.876664 \quad 0.845494 \\
& \text { H } \quad-0.166658 \quad 2.384299 \quad 2.047513 \\
& \text { H } \quad-0.848509 \quad 2.190434 \quad-3.124300 \\
& \mathrm{H} \quad-2.352322 \quad 4.781575 \quad-0.012226 \\
& \begin{array}{llll}
\mathrm{Rh} & 0.125639 & 0.351718 & -0.522762
\end{array} \\
& \begin{array}{llll}
\text { O } & 5.094301 & -1.825444 & 2.043498
\end{array} \\
& \begin{array}{llll}
\text { S } & 5.722926 & -1.448790 & 0.773489
\end{array} \\
& \text { N } \quad 4.445992 \quad-1.098331 \quad-0.266827 \\
& \begin{array}{llll}
\text { O } & 6.606568 & -2.354277 & 0.037324
\end{array} \\
& \begin{array}{llll}
\text { C } & 6.612197 & 0.084477 & 1.041700
\end{array} \\
& \begin{array}{llll}
\text { C } & 6.244058 & 0.921579 & 2.098421
\end{array} \\
& \begin{array}{llll}
\text { C } & 7.652859 & 0.439326 & 0.177874
\end{array} \\
& \begin{array}{llll}
\mathrm{H} & 5.462495 & 0.614075 & 2.785099
\end{array} \\
& \begin{array}{llll}
\text { C } & 6.920010 & 2.127780 & 2.278034
\end{array} \\
& \begin{array}{llll}
\text { C } & 8.316181 & 1.648417 & 0.373703
\end{array} \\
& \begin{array}{llll}
\mathrm{H} & 7.954349 & -0.236512 & -0.615385
\end{array} \\
& \text { H } \quad 6.642189 \quad 2.775993 \quad 3.105413 \\
& \begin{array}{llll}
\text { C } & 7.963147 & 2.511400 & 1.422807
\end{array}
\end{aligned}
$$




$$
\begin{aligned}
& \mathrm{H} \quad 9.131147 \quad 1.921728 \quad-0.291845 \\
& \begin{array}{llll}
\text { C } & 8.716779 & 3.800254 & 1.647085
\end{array} \\
& \begin{array}{llll}
\mathrm{H} & 9.631757 & 3.620704 & 2.226487
\end{array} \\
& \text { H } \quad 9.019077 \quad 4.258656 \quad 0.699690 \\
& \mathrm{H} \quad 8.115716 \quad 4.526680 \quad 2.202868 \\
& \text { H } \quad 0.970677 \quad-1.180398 \quad 2.599944 \\
& \begin{array}{llll}
\text { C } & 3.236441 & -0.454523 & 0.298405
\end{array} \\
& \begin{array}{llll}
\text { H } & 3.446180 & 0.574071 & 0.613320
\end{array} \\
& \text { H } \quad 2.935863 \quad-1.039249 \quad 1.166424 \\
& \text { C } \quad 2.359477-1.811970-1.588955 \\
& \begin{array}{llll}
\text { C } & 3.553752 & -1.561850 & -2.546171
\end{array} \\
& \text { H } \quad 1.471365 \quad-2.085236 \quad-2.169510 \\
& \begin{array}{llll}
\text { C } & 3.014244 & -0.600856 & -3.647746
\end{array} \\
& \begin{array}{llll}
\text { C } & 1.873904 & 0.288412 & -3.145147
\end{array} \\
& \text { H } \quad 3.807756 \quad 0.055169-4.034810 \\
& \text { H } \quad 2.628349 \quad-1.159729 \quad-4.507318 \\
& \begin{array}{llll}
\text { O } & 0.968871 & 0.680796 & -3.863024
\end{array} \\
& \begin{array}{llll}
\mathrm{H} & 2.565407 & -2.636470 & -0.903167
\end{array} \\
& \begin{array}{llll}
\text { C } & 4.693692 & -0.882311 & -1.696868
\end{array} \\
& \text { H } \quad 5.655710 \quad-1.334178-1.941955 \\
& \text { H } \quad 4.765473 \quad 0.193997 \quad-1.918836 \\
& \begin{array}{llll}
\text { C } & 4.062151 & -2.870864 & -3.164257
\end{array} \\
& \text { H } \quad 4.493728 \quad-3.514138 \quad-2.389537 \\
& \text { H } \quad 3.249128 \quad-3.419804 \quad-3.654113 \\
& \mathrm{H} \quad 4.839152 \quad-2.680168 \quad-3.914461 \\
& \begin{array}{llll}
\text { C } & 2.197593 & -0.504727 & -0.818243
\end{array} \\
& \begin{array}{llll}
\text { C } & 1.986207 & 0.634534 & -1.671758
\end{array} \\
& \begin{array}{llll}
\text { C } & 1.664992 & 1.952804 & -1.295556
\end{array} \\
& \begin{array}{llll}
\mathrm{H} & 0.548268 & 0.666724 & 0.898132
\end{array} \\
& \mathrm{H} \quad 1.310571 \quad 2.557507 \quad-2.127185 \\
& \begin{array}{llll}
\text { C } & 2.147834 & 2.786547 & -0.131928
\end{array} \\
& \begin{array}{llll}
\mathrm{H} & 1.432818 & 3.592010 & 0.064271
\end{array} \\
& \begin{array}{llll}
\mathrm{H} & 2.301913 & 2.231404 & 0.793765
\end{array}
\end{aligned}
$$




$$
\begin{array}{lrrr}
\mathrm{H} & 3.101464 & 3.265604 & -0.398305 \\
\mathrm{C} & -1.864596 & 5.717356 & -1.880542 \\
\mathrm{H} & -2.136030 & 6.707812 & -1.526449 \\
\mathrm{C} & -1.402515 & 5.541307 & -3.184593 \\
\mathrm{H} & -1.312926 & 6.393643 & -3.851939 \\
\mathrm{C} & -1.049887 & 4.263448 & -3.626795 \\
\mathrm{H} & -0.683584 & 4.115035 & -4.638646 \\
\mathrm{C} & -1.464798 & 3.321281 & 3.483042 \\
\mathrm{H} & -0.677820 & 3.441622 & 4.222080 \\
\mathrm{C} & -2.759803 & 3.762662 & 3.759534 \\
\mathrm{H} & -2.983665 & 4.229066 & 4.714636 \\
\mathrm{C} & -3.767100 & 3.603177 & 2.804448 \\
\mathrm{H} & -4.775564 & 3.950115 & 3.012298 \\
\mathrm{C} & -2.092440 & -2.951085 & -3.267032 \\
\mathrm{H} & -1.859840 & -2.728782 & -4.304288 \\
\mathrm{C} & -2.995127 & -3.967660 & -2.950215 \\
\mathrm{H} & -3.462463 & -4.545437 & -3.742420 \\
\mathrm{C} & -3.298917 & -4.239195 & -1.613000 \\
\mathrm{H} & -3.999353 & -5.031225 & -1.362859 \\
\mathrm{C} & 1.219097 & -4.882310 & 1.488081 \\
\mathrm{H} & 1.293476 & -5.917470 & 1.167439 \\
\mathrm{C} & 2.040405 & -4.404532 & 2.508458 \\
\mathrm{H} & 2.763692 & -5.061215 & 2.982056 \\
\mathrm{H} & 1.940609 & -3.070128 & 2.911306 \\
& 2.593005 & -2.685751 & 3.688698
\end{array}
$$

TS5a-cis

B3LYP SCF energy: $\quad 3936.05680509$ a.u.

B3LYP enthalpy: $\quad 3935.049757$ a.u.

B3LYP free energy: 3935.215615 a.u.

M06-L SCF energy in solution: $\quad 3937.71421548$ a.u. 
M06-L enthalpy in solution: $\quad 3936.707167$ a.u.

M06-L free energy in solution: $\quad 3936.873025$ a.u.

Imaginary frequency: \$TAB \$ $-774.3951 \mathrm{~cm}-1$

Cartesian coordinates

\begin{tabular}{lrrr} 
ATOM X & Y Z \\
P & -2.237058 & 1.650967 & 0.341845 \\
P & -0.893258 & -1.439731 & -0.372306 \\
O & -5.527359 & -1.292743 & 2.230196 \\
O & -4.856517 & -2.774809 & 3.878327 \\
O & -5.670293 & -2.254686 & -0.738570 \\
O & -6.845361 & -1.051886 & -2.326789 \\
C & -2.150103 & -1.953380 & 0.873464 \\
C & -3.448827 & -1.350646 & 0.905560 \\
C & -4.249979 & -1.716535 & 1.976650 \\
C & -3.852695 & -2.609953 & 2.971118 \\
C & -2.616070 & -3.215228 & 2.939099 \\
C & -1.774127 & -2.863142 & 1.871182 \\
C & -5.907890 & -1.885390 & 3.479645 \\
C & -3.696651 & 0.862248 & -0.467230 \\
C & -4.420124 & 1.572002 & -1.434951 \\
C & -5.513043 & 1.020848 & -2.125320 \\
C & -5.853432 & -0.274028 & -1.803069 \\
C & -5.146263 & -0.991731 & -0.839307 \\
C & -4.060358 & -0.481562 & -0.144396 \\
C & -6.854745 & -2.254296 & -1.546614 \\
C & -1.679347 & -1.600214 & -2.022732 \\
C & -1.323036 & -0.707952 & -3.044462 \\
C & -2.578196 & -2.642667 & -2.302610 \\
C & 0.400334 & -2.759579 & -0.353273 \\
& 0.507685 & -3.698032 & -1.390869 \\
\hline & -2.631278 & 1.623434 & 2.139074
\end{tabular} 


$$
\begin{aligned}
& \begin{array}{llll}
\text { C } & -3.950639 & 1.7746004 & 2.597525
\end{array} \\
& \begin{array}{llll}
\text { C } & -1.594436 & 1.486460 & 3.073850
\end{array} \\
& \begin{array}{llll}
\text { C } & -1.885724 & 3.794927 & -1.419515
\end{array} \\
& \begin{array}{llll}
\text { C } & -2.370163 & 3.421258 & -0.154763
\end{array} \\
& \begin{array}{llll}
\text { C } & -2.943976 & 4.400062 & 0.671450
\end{array}
\end{aligned}
$$

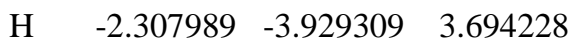

$$
\begin{aligned}
& \begin{array}{llll}
\mathrm{H} & -0.801221 & -3.334432 & 1.825582
\end{array} \\
& \text { H } \quad-6.026029 \quad-1.099535 \quad 4.234758 \\
& \text { H } \quad-6.834122 \quad-2.452334 \quad 3.348611 \\
& \text { H } \quad-4.144839 \quad 2.593196 \quad-1.664015 \\
& \text { H } \quad-6.060218 \quad 1.588781 \quad-2.869124 \\
& \text { H } \quad-6.841493 \quad-3.120714 \quad-2.212233 \\
& \text { H } \quad-7.741038 \quad-2.263783 \quad-0.899589 \\
& \text { H } \quad-0.636288 \quad 0.108099 \quad-2.833304 \\
& \text { H } \quad-2.875078 \quad-3.332296 \quad-1.517897 \\
& \text { H } \quad-0.186091 \quad-3.679936-2.222967 \\
& \mathrm{H} \quad-4.766992 \quad 1.863311 \quad 1.887197 \\
& \mathrm{H} \quad-0.575567 \quad 1.346151 \quad 2.723263 \\
& \text { H } \quad-1.398089 \quad 3.065179 \quad-2.057472 \\
& \begin{array}{llll}
\mathrm{H} & -3.316197 & 4.138119 & 1.655709
\end{array} \\
& \begin{array}{llll}
\mathrm{Rh} & -0.002823 & 0.697058 & -0.050270
\end{array} \\
& \begin{array}{llll}
\text { O } & 4.954504 & -1.784736 & 1.677158
\end{array} \\
& \begin{array}{llll}
\text { S } & 5.503421 & -1.179541 & 0.455591
\end{array} \\
& \begin{array}{llll}
\mathrm{N} & 4.596471 & 0.239766 & 0.213197
\end{array} \\
& \begin{array}{llll}
\text { O } & 5.579934 & -1.970765 & -0.781888
\end{array} \\
& \begin{array}{llll}
\text { C } & 7.138208 & -0.538389 & 0.786752
\end{array} \\
& \begin{array}{llll}
\text { C } & 7.364526 & 0.198762 & 1.954328
\end{array} \\
& \begin{array}{llll}
\text { C } & 8.177670 & -0.824095 & -0.098064
\end{array} \\
& \begin{array}{llll}
\mathrm{H} & 6.549489 & 0.400615 & 2.641764
\end{array} \\
& \begin{array}{llll}
\text { C } & 8.648053 & 0.660105 & 2.222203
\end{array} \\
& \begin{array}{llll}
\text { C } & 9.459114 & -0.350404 & 0.188631
\end{array} \\
& \mathrm{H} \quad 7.983700 \quad-1.413350 \quad-0.987570 \\
& \begin{array}{llll}
\mathrm{H} & 8.830092 & 1.233271 & 3.127784
\end{array}
\end{aligned}
$$




$$
\begin{aligned}
& \begin{array}{llll}
\text { C } & 9.715900 & 0.393951 & 1.346581
\end{array} \\
& \text { H } \quad 10.272080 \quad-0.569360 \quad-0.498403 \\
& \text { C } \quad 11.107282 \quad 0.883582 \quad 1.666601 \\
& \begin{array}{llll}
\mathrm{H} & 11.549768 & 0.296641 & 2.481596
\end{array} \\
& \text { H } \quad 11.771501 \quad 0.801582 \quad 0.801414 \\
& \mathrm{H} \quad 11.097567 \quad 1.929848 \quad 1.991854 \\
& \text { H } \quad 1.268804 \quad-2.099399 \quad 1.517785 \\
& \begin{array}{llll}
\text { C } & 3.247551 & 0.309070 & 0.803314
\end{array} \\
& \begin{array}{llll}
\mathrm{H} & 3.209529 & 1.130812 & 1.528424
\end{array} \\
& \begin{array}{llll}
\mathrm{H} & 3.081219 & -0.619645 & 1.346455
\end{array} \\
& \begin{array}{llll}
\text { C } & 2.649563 & -0.223911 & -1.571952
\end{array} \\
& \begin{array}{llll}
\text { C } & 3.797069 & 0.619017 & -2.190984
\end{array} \\
& \text { H } \quad 1.842889-0.336586 \quad-2.303631 \\
& \begin{array}{llll}
\text { C } & 3.147702 & 1.901387 & -2.776159
\end{array} \\
& \begin{array}{llll}
\text { C } & 1.912803 & 2.394347 & -2.022041
\end{array} \\
& \begin{array}{llll}
\mathrm{H} & 3.867127 & 2.732950 & -2.809041
\end{array} \\
& \text { H } \quad 2.832226 \quad 1.741363 \quad-3.813635 \\
& \begin{array}{llll}
\text { O } & 1.090163 & 3.117983 & -2.556185
\end{array} \\
& \mathrm{H} \quad 2.999155 \quad-1.226891 \quad-1.315403 \\
& \begin{array}{llll}
\text { C } & 4.799475 & 0.990623 & -1.033089
\end{array} \\
& \text { H } \quad 5.832065 \quad 0.843694 \quad-1.360520 \\
& \text { H } \quad 4.711158 \quad 2.053669-0.774436 \\
& \begin{array}{llll}
\text { C } & 4.507671 & -0.154477 & -3.312579
\end{array} \\
& \text { H } \quad 4.999406 \quad-1.046192 \quad-2.915853 \\
& \text { H } \quad 3.792229 \quad-0.465914 \quad-4.083187 \\
& \mathrm{H} \quad 5.265286 \quad 0.473660 \quad-3.798169 \\
& \begin{array}{llll}
\text { C } & 2.207138 & 0.530120 & -0.323630
\end{array} \\
& \begin{array}{llll}
\text { C } & 1.796035 & 1.922031 & -0.588314
\end{array} \\
& \begin{array}{llll}
\text { C } & 1.340875 & 2.733707 & 0.443198
\end{array} \\
& \begin{array}{llll}
\mathrm{H} & 1.036170 & -0.102788 & 0.798528
\end{array} \\
& \begin{array}{llll}
\text { C } & -3.039371 & 5.723136 & 0.233402
\end{array} \\
& \begin{array}{llll}
\text { H } & -3.481919 & 6.471889 & 0.884292
\end{array} \\
& \begin{array}{llll}
\text { C } & -2.573295 & 6.081564 & -1.032557
\end{array}
\end{aligned}
$$




$$
\begin{array}{lrrr}
\mathrm{H} & -2.652232 & 7.110753 & -1.370966 \\
\mathrm{C} & -1.996036 & 5.114243 & -1.858638 \\
\mathrm{H} & -1.615250 & 5.385574 & -2.838906 \\
\mathrm{C} & -1.867167 & 1.509569 & 4.442646 \\
\mathrm{H} & -1.056500 & 1.398310 & 5.157176 \\
\mathrm{C} & -3.179521 & 1.670755 & 4.890783 \\
\mathrm{H} & -3.392125 & 1.691310 & 5.955892 \\
\mathrm{C} & -4.219590 & 1.802300 & 3.966624 \\
\mathrm{H} & -5.242257 & 1.930325 & 4.310932 \\
\mathrm{C} & -1.851362 & -0.856202 & -4.328250 \\
\mathrm{H} & -1.571520 & -0.157553 & -5.111455 \\
\mathrm{C} & -2.737687 & -1.899285 & -4.601481 \\
\mathrm{H} & -3.147615 & -2.016958 & -5.600458 \\
\mathrm{C} & -3.100314 & -2.790477 & -3.587643 \\
\mathrm{H} & -3.790192 & -3.602952 & -3.797849 \\
\mathrm{C} & 1.518773 & -4.662712 & -1.369864 \\
\mathrm{H} & 1.591131 & -5.375994 & -2.185930 \\
\mathrm{C} & 2.432552 & -4.706235 & -0.318150 \\
\mathrm{H} & 3.225672 & -5.447774 & -0.312273 \\
\mathrm{C} & 2.333671 & -3.780951 & 0.724708 \\
\mathrm{H} & 3.055303 & -3.786353 & 1.534753 \\
\mathrm{H} & 0.973969 & 4.185941 & 0.362950 \\
\mathrm{H} & 1.789696 & 4.777030 & 0.804708 \\
\mathrm{H} & 0.801641 & 4.523122 & -0.657084 \\
& 0.078729 & 4.397376 & 0.955929 \\
\mathrm{H} & 1.497876 & 2.365294 & 1.456721
\end{array}
$$

TS5a-trans

B3LYP SCF energy: $\quad 3936.05540860$ a.u.

B3LYP enthalpy: 3935.048271 a.u.

B3LYP free energy: $\quad 3935.214135$ a.u. 
M06-L SCF energy in solution: $\quad 3937.71334653$ a.u.

M06-L enthalpy in solution: $\quad 3936.706209$ a.u.

M06-L free energy in solution: $\quad 3936.872073$ a.u.

Imaginary frequency: \$TAB \$ $-769.6092 \mathrm{~cm}-1$

Cartesian coordinates

\begin{tabular}{|c|c|c|c|}
\hline \multicolumn{2}{|c|}{ ATOM } & $\mathrm{Y}$ & Z \\
\hline $\mathrm{P}$ & -2.221099 & 1.737804 & 0.238912 \\
\hline $\mathrm{P}$ & -0.899105 & -1.435005 & -0.115296 \\
\hline $\mathrm{O}$ & -5.798859 & -1.039043 & 1.894187 \\
\hline $\mathrm{O}$ & -5.356608 & -2.380840 & 3.728541 \\
\hline $\mathrm{O}$ & -5.576275 & -2.184048 & -1.012685 \\
\hline $\mathrm{O}$ & -6.523956 & -1.062513 & -2.802240 \\
\hline $\mathrm{C}$ & -2.282455 & -1.800386 & 1.041893 \\
\hline $\mathrm{C}$ & -3.569894 & -1.203045 & 0.853224 \\
\hline $\mathrm{C}$ & -4.507959 & -1.492820 & 1.832392 \\
\hline $\mathrm{C}$ & -4.246820 & -2.297822 & 2.940961 \\
\hline $\mathrm{C}$ & -3.014508 & -2.883254 & 3.129925 \\
\hline $\mathrm{C}$ & -2.038850 & -2.615470 & 2.155337 \\
\hline $\mathrm{C}$ & -6.390883 & -1.665117 & 3.040525 \\
\hline $\mathrm{C}$ & -3.611604 & 0.930321 & -0.664580 \\
\hline $\mathrm{C}$ & -4.201276 & 1.592717 & -1.748837 \\
\hline $\mathrm{C}$ & -5.205067 & 1.010560 & -2.541597 \\
\hline $\mathrm{C}$ & -5.593069 & -0.266089 & -2.200928 \\
\hline $\mathrm{C}$ & -5.021361 & -0.935779 & -1.119570 \\
\hline $\mathrm{C}$ & -4.025856 & -0.395275 & -0.319680 \\
\hline $\mathrm{C}$ & -6.620101 & -2.242301 & -1.994561 \\
\hline $\mathrm{C}$ & -1.542218 & -1.835264 & -1.789380 \\
\hline $\mathrm{C}$ & -1.182462 & -1.038998 & -2.886745 \\
\hline $\mathrm{C}$ & -2.363101 & -2.956999 & -1.995550 \\
\hline $\mathrm{C}$ & 0.390035 & -2.713944 & 0.222728 \\
\hline $\mathrm{C}$ & 0.601910 & -3.799432 & -0.640034 \\
\hline $\mathrm{C}$ & 1.220719 & -2.576676 & 1.349958 \\
\hline
\end{tabular}




$$
\begin{aligned}
& \begin{array}{llll}
\text { C } & -2.726925 & 1.713920 & 2.007859
\end{array} \\
& \begin{array}{llll}
\text { C } & -4.071114 & 1.890968 & 2.376839
\end{array} \\
& \text { C } \quad-1.757333 \quad 1.564206 \quad 3.009642 \\
& \begin{array}{llll}
\text { C } & -1.749419 & 3.908658 & -1.476788
\end{array} \\
& \begin{array}{llll}
\text { C } & -2.313677 & 3.516959 & -0.249207
\end{array} \\
& \begin{array}{llll}
\text { C } & -2.924201 & 4.489253 & 0.558179
\end{array} \\
& \mathrm{H} \quad-2.808204 \quad-3.521576 \quad 3.981411 \\
& \text { H } \quad-1.067930 \quad-3.078772 \quad 2.275486 \\
& \text { H } \quad-6.804055 \quad-0.898023 \quad 3.701594 \\
& \begin{array}{llll}
\mathrm{H} & -7.167009 & -2.367948 & 2.715311
\end{array} \\
& \text { H } \quad-3.888545 \quad 2.600337 \quad-1.991897 \\
& \mathrm{H} \quad \begin{array}{llll}
-5.651850 & 1.541820 & -3.374247
\end{array}
\end{aligned}
$$

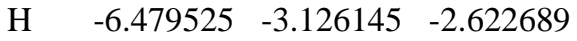

$$
\begin{aligned}
& \mathrm{H} \quad-7.595223 \quad-2.263836 \quad-1.492806 \\
& \text { H } \quad-0.565899 \quad-0.156506 \quad-2.737527 \\
& \text { H } \quad-2.668254-3.571135-1.153526 \\
& \text { H } \quad-0.014755 \quad-3.929568 \quad-1.521658 \\
& \mathrm{H} \quad-4.837055 \quad 1.988890 \quad 1.613387 \\
& \begin{array}{llll}
\mathrm{H} & -0.719257 & 1.410086 & 2.730917
\end{array} \\
& \text { H } \quad-1.235117 \quad 3.186334 \quad-2.103371 \\
& \mathrm{H} \quad-3.358181 \quad 4.216950 \quad 1.513510 \\
& \begin{array}{llll}
\mathrm{Rh} & -0.005937 & 0.733635 & -0.087292
\end{array} \\
& \begin{array}{llll}
\text { O } & 5.036723 & -1.454805 & 1.921967
\end{array} \\
& \begin{array}{llll}
\text { S } & 5.536697 & -1.077264 & 0.593050
\end{array} \\
& \begin{array}{llll}
\mathrm{N} & 4.626877 & 0.289827 & 0.145312
\end{array} \\
& \begin{array}{llll}
\text { O } & 5.558176 & -2.071587 & -0.490669
\end{array} \\
& \begin{array}{llll}
\text { C } & 7.186399 & -0.406306 & 0.740769
\end{array} \\
& \begin{array}{llll}
\text { C } & 7.463528 & 0.520062 & 1.752169
\end{array} \\
& \begin{array}{llll}
\text { C } & 8.187620 & -0.853410 & -0.120928
\end{array} \\
& \begin{array}{llll}
\mathrm{H} & 6.678013 & 0.847684 & 2.425387
\end{array} \\
& \begin{array}{llll}
\text { C } & 8.758893 & 1.006175 & 1.884966
\end{array} \\
& \begin{array}{llll}
\text { C } & 9.481822 & -0.351807 & 0.028210
\end{array} \\
& \text { H } \quad 7.954993 \quad-1.586156 \quad-0.885798
\end{aligned}
$$




$$
\begin{aligned}
& \begin{array}{llll}
\mathrm{H} & 8.980248 & 1.725916 & 2.669005
\end{array} \\
& \begin{array}{llll}
\text { C } & 9.788978 & 0.579472 & 1.027792
\end{array} \\
& \text { H } \quad 10.265138 \quad-0.696242 \quad-0.641603 \\
& \begin{array}{llll}
\text { C } & 11.194372 & 1.101362 & 1.202705
\end{array} \\
& \text { H } \quad 11.665126 \quad 0.664349 \quad 2.092592 \\
& \text { H } \quad 11.823929 \quad 0.857964 \quad 0.341977 \\
& \text { H } \quad 11.202861 \quad 2.188994 \quad 1.335368 \\
& \text { H } \quad 1.078171 \quad-1.742208 \quad 2.032524 \\
& \begin{array}{llll}
\text { C } & 3.305457 & 0.479119 & 0.775618
\end{array} \\
& \text { H } \quad 3.312337 \quad 1.416311 \quad 1.337956 \\
& \text { H } \quad 3.155711 \quad-0.338591 \quad 1.479940 \\
& \begin{array}{llll}
\text { C } & 2.620940 & -0.465581 & -1.440750
\end{array} \\
& \begin{array}{llll}
\text { C } & 3.729683 & 0.249433 & -2.253996
\end{array} \\
& \text { H } \quad 1.783383 \quad-0.714152 \quad-2.099581 \\
& \begin{array}{llll}
\text { C } & 3.037529 & 1.403148 & -3.030430
\end{array} \\
& \begin{array}{llll}
\text { C } & 1.819475 & 2.009222 & -2.331929
\end{array} \\
& \text { H } \quad 3.737886 \quad 2.226277 \quad-3.234871 \\
& \text { H } \quad 2.692589 \quad 1.058253 \quad-4.012052 \\
& \begin{array}{llll}
\text { O } & 0.982990 & 2.649153 & -2.945407
\end{array} \\
& \text { H } \quad 2.990532-1.402909-1.017913 \\
& \begin{array}{llll}
\text { C } & 4.777238 & 0.814633 & -1.220170
\end{array} \\
& \text { H } \quad 5.796702 \quad 0.609701 \quad-1.557293 \\
& \begin{array}{llll}
\text { H } & 4.694647 & 1.906702 & -1.145601
\end{array} \\
& \begin{array}{llll}
\text { C } & 4.396750 & -0.710893 & -3.251035
\end{array} \\
& \text { H } \quad 4.912782 \quad-1.517697 \quad-2.724732 \\
& \text { H } \quad 3.650669-1.154790 \quad-3.921148 \\
& \mathrm{H} \quad 5.126770-0.178665 \quad-3.874101 \\
& \begin{array}{llll}
\text { C } & 2.229248 & 0.507598 & -0.333663
\end{array} \\
& \text { C } \quad 1.762042 \quad 1.802848 \quad-0.830345 \\
& \begin{array}{llll}
\text { C } & 1.177497 & 2.811926 & -0.058082
\end{array} \\
& \begin{array}{llll}
\mathrm{H} & 1.025271 & 0.088564 & 0.895295
\end{array} \\
& \text { H } \quad 0.742335 \quad 3.609631 \quad-0.655110 \\
& \begin{array}{llll}
\text { C } & 1.354474 & 3.169807 & 1.396174
\end{array}
\end{aligned}
$$




$$
\begin{array}{lrrr}
\mathrm{H} & 0.457025 & 3.679763 & 1.760542 \\
\mathrm{H} & 1.554735 & 2.321585 & 2.052236 \\
\mathrm{H} & 2.186059 & 3.883278 & 1.499849 \\
\mathrm{C} & -2.976194 & 5.821582 & 0.141109 \\
\mathrm{H} & -3.448135 & 6.563228 & 0.779366 \\
\mathrm{C} & -2.428483 & 6.198623 & -1.085408 \\
\mathrm{H} & -2.472897 & 7.235183 & -1.407183 \\
\mathrm{C} & -1.815147 & 5.238431 & -1.893999 \\
\mathrm{H} & -1.375662 & 5.521787 & -2.846054 \\
\mathrm{C} & -2.121019 & 1.598787 & 4.357142 \\
\mathrm{H} & -1.361663 & 1.478305 & 5.124643 \\
\mathrm{C} & -3.456936 & 1.784600 & 4.716355 \\
\mathrm{H} & -3.739837 & 1.813298 & 5.764752 \\
\mathrm{C} & -4.430539 & 1.929598 & 3.724338 \\
\mathrm{H} & -5.471733 & 2.074841 & 3.998977 \\
\mathrm{C} & -1.627351 & -1.361299 & -4.170395 \\
\mathrm{H} & -1.347918 & -0.733288 & -5.011400 \\
\mathrm{C} & -2.431101 & -2.484920 & -4.369210 \\
\mathrm{H} & -2.774364 & -2.738165 & -5.368172 \\
\mathrm{C} & -2.798323 & -3.280935 & -3.280544 \\
\mathrm{H} & -3.424602 & -4.155811 & -3.431398 \\
\mathrm{C} & 1.622317 & -4.719067 & -0.381349 \\
\mathrm{H} & 1.779014 & -5.547765 & -1.065888 \\
\mathrm{H} & 2.440697 & -4.570282 & 0.737093 \\
\hline & 2.243194 & -5.276700 & 0.926130 \\
\mathrm{H} & -3.497256 & 1.608960 \\
\mathrm{H} & -3.359399 & 2.468809
\end{array}
$$

IntF-cis

B3LYP SCF energy: $\quad 3936.11283893$ a.u.

B3LYP enthalpy: $\quad 3935.100161$ a.u. 
B3LYP free energy: 3935.266232 a.u.

M06-L SCF energy in solution: $\quad 3937.76047116$ a.u.

M06-L enthalpy in solution: $\quad 3936.747793$ a.u.

M06-L free energy in solution: $\quad 3936.913864$ a.u.

Cartesian coordinates

$\begin{array}{lrrr}\text { ATOM X } & \text { Y Z } \\ \text { P } & -2.454872 & 1.853703 & 0.141485 \\ \text { P } & -1.226420 & -1.329260 & 0.408728 \\ \text { O } & -6.505160 & -0.767147 & 0.400140 \\ \text { O } & -6.845284 & -2.002380 & 2.326752 \\ \text { O } & -5.217955 & -2.045837 & -2.141120 \\ \text { O } & -5.430069 & -0.967039 & -4.178206 \\ \text { C } & -2.948941 & -1.563755 & 1.034100 \\ \text { C } & -4.051573 & -0.994144 & 0.321291 \\ \text { C } & -5.303543 & -1.229036 & 0.868382 \\ \text { C } & -5.514748 & -1.966598 & 2.032885 \\ \text { C } & -4.464972 & -2.530859 & 2.722183 \\ \text { C } & -3.180512 & -2.313596 & 2.195239 \\ \text { C } & -7.511290 & -1.368047 & 1.225984 \\ \text { C } & -3.495248 & 1.078335 & -1.165242 \\ \text { C } & -3.655248 & 1.723280 & -2.397892 \\ \text { C } & -4.298086 & 1.118444 & -3.492017 \\ \text { C } & -4.764707 & -0.163295 & -3.299706 \\ \text { C } & -4.629952 & -0.811707 & -2.071185 \\ \text { C } & -4.007210 & -0.244000 & -0.970419 \\ \text { C } & -5.622802 & -2.218430 & -3.506295 \\ \text { C } & -1.285717 & -2.126124 & -1.253540 \\ \text { C } & -0.610482 & -1.549487 & -2.339218 \\ \text { C } & -1.998534 & -3.321165 & -1.449400 \\ \text { C } & -0.196110 & -2.462449 & 1.440555 \\ \text { C } & 0.459440 & -3.574805 & 0.891285 \\ \text { C } & -0.005159 & -2.173190 & 2.805473\end{array}$




$$
\begin{aligned}
& \begin{array}{llll}
\text { C } & -3.464987 & 1.854645 & 1.673991
\end{array} \\
& \begin{array}{llll}
\text { C } & -4.852950 & 2.064800 & 1.622462
\end{array} \\
& \begin{array}{llll}
\text { C } & -2.836726 & 1.722729 & 2.919314
\end{array} \\
& \begin{array}{llll}
\text { C } & -1.459904 & 4.019281 & -1.361495
\end{array} \\
& \begin{array}{llll}
\text { C } & -2.306339 & 3.637102 & -0.305338
\end{array} \\
& \begin{array}{llll}
\text { C } & -3.010379 & 4.633092 & 0.391204
\end{array} \\
& \text { H } \quad-4.618547 \quad-3.117433 \quad 3.620760 \\
& \text { H } \quad-2.348744 \quad-2.772356 \quad 2.711845 \\
& \text { H } \quad-8.182541 \quad-0.593144 \quad 1.604523 \\
& \text { H } \quad-8.059823 \quad-2.121792 \quad 0.647935 \\
& \text { H } \quad-3.280390 \quad 2.731340 \quad-2.524289 \\
& \text { H } \quad-4.423837 \quad 1.636912 \quad-4.435752 \\
& \text { H } \quad-4.996363 \quad-2.985912 \quad-3.977031 \\
& \text { H } \quad-6.680824 \quad-2.491355 \quad-3.541732 \\
& \text { H } \quad-0.070928 \quad-0.616770 \quad-2.198361 \\
& \text { H } \quad-2.540212 \quad-3.773562-0.624229 \\
& \text { H } \quad 0.334782 \quad-3.820163 \quad-0.157430 \\
& \text { H } \quad-5.354579 \quad 2.158795 \quad 0.663755 \\
& \begin{array}{llll}
\mathrm{H} & -1.764983 & 1.553815 & 2.961951
\end{array} \\
& \text { H } \quad-0.875813 \quad 3.273704 \quad-1.888228 \\
& \text { H } \quad-3.661372 \quad 4.370616 \quad 1.216697 \\
& \begin{array}{llll}
\mathrm{Rh} & -0.316473 & 0.783288 & 0.186565
\end{array} \\
& \begin{array}{llll}
\text { O } & 6.382948 & -2.414172 & 0.684392
\end{array} \\
& \begin{array}{llll}
\text { S } & 6.513338 & -1.296195 & -0.256627
\end{array} \\
& \text { N } \quad 5.283266 \quad-0.198464 \quad 0.166675 \\
& \begin{array}{llll}
\text { O } & 6.507317 & -1.548252 & -1.706727
\end{array} \\
& \begin{array}{llll}
\text { C } & 7.994831 & -0.366557 & 0.115317
\end{array} \\
& \begin{array}{llll}
\text { C } & 8.278761 & -0.027850 & 1.442760
\end{array} \\
& \begin{array}{llll}
\text { C } & 8.876227 & -0.039820 & -0.914554
\end{array} \\
& \text { H } \quad 7.590835 \quad-0.301137 \quad 2.236291 \\
& \begin{array}{llll}
\text { C } & 9.454406 & 0.656569 & 1.728449
\end{array} \\
& \begin{array}{llll}
\text { C } & 10.051188 & 0.648876 & -0.607377
\end{array} \\
& \text { H } \quad 8.645817 \quad-0.329246 \quad-1.933954
\end{aligned}
$$




$$
\begin{aligned}
& \begin{array}{llll}
\text { H } & 9.679744 & 0.922443 & 2.758222
\end{array} \\
& \begin{array}{llll}
\text { C } & 10.360078 & 1.005430 & 0.710856
\end{array} \\
& \text { H } \quad 10.740342 \quad 0.906600 \quad-1.407196 \\
& \begin{array}{llll}
\text { C } & 11.643954 & 1.726304 & 1.043594
\end{array} \\
& \mathrm{H} \quad 12.361565 \quad 1.046230 \quad 1.520220 \\
& \begin{array}{llll}
\mathrm{H} & 12.120324 & 2.133811 & 0.147103
\end{array} \\
& \text { H } \quad 11.469451 \quad 2.551729 \quad 1.742773 \\
& \text { H } \quad-0.499828 \quad-1.314622 \quad 3.252564 \\
& \begin{array}{llll}
\text { C } & 4.106076 & -0.708365 & 0.877246
\end{array} \\
& \text { H } \quad 3.955644 \quad-0.102493 \quad 1.781030 \\
& \mathrm{H} \quad 4.325914 \quad-1.726816 \quad 1.198924 \\
& \begin{array}{llll}
\text { C } & 3.227187 & -0.687627 & -1.489959
\end{array} \\
& \begin{array}{llll}
\text { C } & 3.921573 & 0.650875 & -1.825961
\end{array} \\
& \mathrm{H} \quad 2.353056 \quad-0.827630 \quad-2.138347 \\
& \begin{array}{llll}
\text { C } & 2.848460 & 1.762369 & -1.768544
\end{array} \\
& \begin{array}{llll}
\text { C } & 1.897215 & 1.645937 & -0.603371
\end{array} \\
& \begin{array}{llll}
\mathrm{H} & 3.299168 & 2.764417 & -1.732178
\end{array} \\
& \text { H } \quad 2.237966 \quad 1.751982 \quad-2.682526 \\
& \begin{array}{llll}
\text { O } & 0.943333 & 2.473649 & -0.473240
\end{array} \\
& \mathrm{H} \quad 3.907504 \quad-1.520701 \quad-1.689212 \\
& \begin{array}{llll}
\text { C } & 5.026979 & 0.925943 & -0.737423
\end{array} \\
& \mathrm{H} \quad 5.968096 \quad 1.213770 \quad-1.213537 \\
& \text { H } \quad 4.735073 \quad 1.773217 \quad-0.101180 \\
& \begin{array}{llll}
\text { C } & 4.538216 & 0.616486 & -3.232853
\end{array} \\
& \text { H } \quad \begin{array}{llll}
5.325726 & -0.139415 & -3.286639
\end{array} \\
& \begin{array}{llll}
\text { H } & 3.778559 & 0.377617 & -3.987612
\end{array} \\
& \text { H } \quad 4.972923 \quad 1.589378 \quad-3.495893 \\
& \begin{array}{llll}
\text { C } & 1.936696 & 0.520605 & 0.313375
\end{array} \\
& \begin{array}{llll}
\text { C } & 1.312620 & 0.669984 & 1.596434
\end{array} \\
& \begin{array}{llll}
\text { C } & -2.880668 & 5.975000 & 0.028749
\end{array} \\
& \begin{array}{llll}
\mathrm{H} & -3.431099 & 6.732553 & 0.579254
\end{array} \\
& \begin{array}{llll}
\text { C } & -2.053515 & 6.341510 & -1.033036
\end{array} \\
& \text { H } \quad-1.956116 \quad 7.386301 \quad-1.313796
\end{aligned}
$$




$$
\begin{array}{lrrr}
\mathrm{C} & -1.344353 & 5.359550 & -1.727908 \\
\mathrm{H} & -0.689590 & 5.636424 & -2.549231 \\
\mathrm{C} & -3.580403 & 1.804036 & 4.097971 \\
\mathrm{H} & -3.084062 & 1.701342 & 5.058826 \\
\mathrm{C} & -4.958584 & 2.017572 & 4.040712 \\
\mathrm{H} & -5.537695 & 2.082426 & 4.957405 \\
\mathrm{C} & -5.593012 & 2.147025 & 2.802085 \\
\mathrm{H} & -6.665256 & 2.315468 & 2.754060 \\
\mathrm{C} & -0.639506 & -2.155571 & -3.596651 \\
\mathrm{H} & -0.116419 & -1.696645 & -4.430689 \\
\mathrm{C} & -1.339334 & -3.349609 & -3.779293 \\
\mathrm{H} & -1.356258 & -3.826188 & -4.755339 \\
\mathrm{C} & -2.017570 & -3.931048 & -2.704026 \\
\mathrm{H} & -2.561466 & -4.861572 & -2.840839 \\
\mathrm{C} & 1.279168 & -4.380317 & 1.687992 \\
\mathrm{H} & 1.781011 & -5.235601 & 1.244999 \\
\mathrm{C} & 1.450570 & -4.091424 & 3.041387 \\
\mathrm{H} & 2.085636 & -4.720528 & 3.657998 \\
\mathrm{C} & 0.802248 & -2.986224 & 3.600388 \\
\mathrm{H} & 0.928154 & -2.754739 & 4.654277 \\
\mathrm{H} & 2.812364 & -0.686417 & -0.009877 \\
\mathrm{H} & 2.245869 & -1.593214 & 0.224819 \\
\mathrm{H} & 1.305170 & 1.922902 & 2.445318 \\
& 2.197040 & 1.909323 & 3.090920 \\
\mathrm{H} & 1.310489 & 2.841955 & 1.859820 \\
\mathrm{H} & 0.437109 & 1.940420 & 3.112656 \\
\mathrm{H} & -0.239339 & 2.193993
\end{array}
$$

IntF-trans

B3LYP SCF energy: $\quad 3936.10843031$ a.u.

B3LYP enthalpy: 3935.095698 a.u. 
B3LYP free energy: 3935.262262 a.u.

M06-L SCF energy in solution: 3937.75928204 a.u.

M06-L enthalpy in solution: $\quad 3936.746550$ a.u.

M06-L free energy in solution: $\quad 3936.913114$ a.u.

Cartesian coordinates

$\begin{array}{lrrr}\text { ATOM X } & \text { Y Z } \\ \text { P } & -2.422398 & 1.858961 & 0.158173 \\ \text { P } & -1.256970 & -1.337575 & 0.290256 \\ \text { O } & -6.517609 & -0.683736 & 0.624533 \\ \text { O } & -6.774722 & -2.035189 & 2.484739 \\ \text { O } & -5.459187 & -1.894795 & -2.020660 \\ \text { O } & -5.798586 & -0.740733 & -3.999189 \\ \text { C } & -2.951756 & -1.607358 & 0.978397 \\ \text { C } & -4.081628 & -0.967114 & 0.372671 \\ \text { C } & -5.302463 & -1.203293 & 0.986762 \\ \text { C } & -5.464258 & -2.011918 & 2.110921 \\ \text { C } & -4.392258 & -2.654618 & 2.687554 \\ \text { C } & -3.137390 & -2.433063 & 2.095633 \\ \text { C } & -7.488205 & -1.311327 & 1.472689 \\ \text { C } & -3.576314 & 1.146558 & -1.083512 \\ \text { C } & -3.809610 & 1.835692 & -2.280327 \\ \text { C } & -4.553294 & 1.287089 & -3.338983 \\ \text { C } & -5.045052 & 0.014536 & -3.149202 \\ \text { C } & -4.835510 & -0.677511 & -1.956134 \\ \text { C } & -4.112128 & -0.165392 & -0.889259 \\ \text { C } & -6.000969 & -1.999428 & -3.344784 \\ \text { C } & -1.424051 & -1.924007 & -1.452942 \\ \text { C } & -0.766010 & -1.254917 & -2.495429 \\ \text { C } & -2.182232 & -3.069179 & -1.748757 \\ \text { C } & -0.184176 & -2.620288 & 1.076494 \\ \text { C } & 0.479624 & -3.584193 & 0.299457 \\ \text { C } & 0.037976 & -2.610917 & 2.465800\end{array}$




$$
\begin{aligned}
& \begin{array}{llll}
\text { C } & -3.321847 & 1.872921 & 1.759072
\end{array} \\
& \begin{array}{llll}
\text { C } & -4.713856 & 2.049707 & 1.811749
\end{array} \\
& \begin{array}{llll}
\text { C } & -2.594702 & 1.792011 & 2.954809
\end{array} \\
& \begin{array}{llll}
\text { C } & -1.433000 & 4.019045 & -1.357080
\end{array} \\
& \begin{array}{llll}
\text { C } & -2.248751 & 3.643111 & -0.275150
\end{array} \\
& \begin{array}{llll}
\text { C } & -2.915985 & 4.643688 & 0.450593
\end{array} \\
& \text { H } \quad-4.507486 \quad-3.303774 \quad 3.547993 \\
& \text { H } \quad-2.293732 \quad-2.957316 \quad 2.521042 \\
& \text { H } \quad-8.110445 \quad-0.545764 \quad 1.943007 \\
& \mathrm{H} \quad-8.094760-2.010002 \quad 0.883583 \\
& \mathrm{H} \quad-3.412030 \quad 2.835114 \quad-2.405058 \\
& \text { H } \quad-4.733384 \quad 1.839278 \quad-4.254325 \\
& \text { H } \quad-5.470001 \quad-2.785905 \quad-3.894069 \\
& \mathrm{H} \quad-7.072376 \quad-2.211260 \quad-3.285598 \\
& \text { H } \quad-0.183913 \quad-0.362300 \quad-2.278593 \\
& \text { H } \quad-2.704865 \quad-3.595492 \quad-0.955876 \\
& \mathrm{H} \quad 0.336351 \quad-3.614984 \quad-0.774268 \\
& \text { H } \quad-5.291275 \quad 2.107653 \quad 0.893886 \\
& \mathrm{H} \quad-1.517858 \quad 1.655096 \quad 2.916536 \\
& \text { H } \quad-0.879325 \quad 3.267640 \quad-1.907639 \\
& \text { H } \quad-3.545000 \quad 4.383798 \quad 1.293979 \\
& \begin{array}{llll}
\mathrm{Rh} & -0.318828 & 0.771290 & 0.171731
\end{array} \\
& \begin{array}{llll}
\text { O } & 6.469684 & -2.117736 & 1.236497
\end{array} \\
& \begin{array}{llll}
\text { S } & 6.556190 & -1.232135 & 0.069893
\end{array} \\
& \text { N } \quad \begin{array}{llll}
5.364434 & -0.041503 & 0.312434
\end{array} \\
& \begin{array}{lllll}
\text { O } & 6.462143 & -1.790236 & -1.288954
\end{array} \\
& \begin{array}{llll}
\text { C } & 8.066021 & -0.277568 & 0.148633
\end{array} \\
& \begin{array}{llll}
\text { C } & 8.429423 & 0.336615 & 1.352039
\end{array} \\
& \begin{array}{llll}
\text { C } & 8.889540 & -0.203349 & -0.974119
\end{array} \\
& \begin{array}{llll}
\mathrm{H} & 7.785823 & 0.259422 & 2.222327
\end{array} \\
& \begin{array}{llll}
\text { C } & 9.626299 & 1.040505 & 1.416455
\end{array} \\
& \begin{array}{llll}
\text { C } & 10.086992 & 0.509457 & -0.889610
\end{array} \\
& \begin{array}{llll}
\text { H } & 8.597645 & -0.702845 & -1.891334
\end{array}
\end{aligned}
$$




$$
\begin{aligned}
& \mathrm{H} \quad 9.913116 \quad 1.520083 \quad 2.348976 \\
& \begin{array}{llll}
\text { C } & 10.475046 & 1.138406 & 0.299556
\end{array} \\
& \text { H } \quad 10.731150 \quad 0.571044 \quad-1.762725 \\
& \begin{array}{llll}
\text { C } & 11.782951 & 1.885921 & 0.395868
\end{array} \\
& \text { H } \quad 12.521421 \quad 1.308965 \quad 0.967220 \\
& \begin{array}{llll}
\mathrm{H} & 12.208827 & 2.080287 & -0.592845
\end{array} \\
& \text { H } \quad 11.657888 \quad 2.846121 \quad 0.908653 \\
& \text { H } \quad-0.447257 \quad-1.867061 \quad 3.089402 \\
& \begin{array}{llll}
\text { C } & 4.232292 & -0.348745 & 1.189609
\end{array} \\
& \text { H } \quad 4.110569 \quad 0.491533 \quad 1.885485 \\
& \mathrm{H} \quad 4.496011 \quad-1.228650 \quad 1.776914 \\
& \begin{array}{llll}
\text { C } & 3.234217 & -0.891580 & -1.076051
\end{array} \\
& \begin{array}{llll}
\text { C } & 3.871215 & 0.357647 & -1.723250
\end{array} \\
& \text { H } \quad 2.326169-1.173556 \quad-1.624902 \\
& \begin{array}{llll}
\text { C } & 2.781779 & 1.450887 & -1.811845
\end{array} \\
& \begin{array}{llll}
\text { C } & 1.883147 & 1.552140 & -0.602389
\end{array} \\
& \text { H } \quad 3.211523 \quad 2.446074 \quad-1.991873 \\
& \text { H } \quad 2.124513 \quad 1.261253 \quad-2.672796 \\
& \begin{array}{llll}
\text { O } & 0.959168 & 2.423906 & -0.573562
\end{array} \\
& \text { H } \quad 3.923173 \quad-1.738014 \quad-1.142839 \\
& \begin{array}{llll}
\text { C } & 5.049854 & 0.853737 & -0.803184
\end{array} \\
& \mathrm{H} \quad 5.956384 \quad 1.008974 \quad-1.394051 \\
& \begin{array}{llll}
\mathrm{H} & 4.803487 & 1.827421 & -0.356915
\end{array} \\
& \begin{array}{llll}
\text { C } & 4.387489 & 0.042233 & -3.135914
\end{array} \\
& \text { H } \quad 5.184760 \quad-0.704401 \quad-3.092486 \\
& \text { H } \quad 3.582400 \quad-0.350360 \quad-3.769568 \\
& \text { H } \quad 4.783022 \quad 0.943709 \quad-3.621288 \\
& \begin{array}{llll}
\text { C } & 1.963804 & 0.599492 & 0.496908
\end{array} \\
& \begin{array}{llll}
\text { C } & 1.292811 & 0.997402 & 1.685657
\end{array} \\
& \text { H } \quad 1.059242 \quad 2.059203 \quad 1.770218 \\
& \begin{array}{llll}
\text { C } & 1.444550 & 0.318678 & 3.014591
\end{array} \\
& \begin{array}{llll}
\text { H } & 0.532015 & 0.421025 & 3.611882
\end{array} \\
& \text { H } \quad 1.699652-0.737931 \quad 2.938835
\end{aligned}
$$




$$
\begin{array}{lrrr}
\mathrm{H} & 2.242774 & 0.821865 & 3.579943 \\
\mathrm{C} & -2.778229 & 5.986023 & 0.093084 \\
\mathrm{H} & -3.299609 & 6.747829 & 0.665585 \\
\mathrm{C} & -1.979754 & 6.347570 & -0.992316 \\
\mathrm{H} & -1.875414 & 7.392880 & -1.268716 \\
\mathrm{C} & -1.308848 & 5.360370 & -1.717141 \\
\mathrm{H} & -0.676960 & 5.633687 & -2.557345 \\
\mathrm{C} & -3.245882 & 1.887599 & 4.185602 \\
\mathrm{H} & -2.673602 & 1.825572 & 5.106966 \\
\mathrm{C} & -4.630132 & 2.063585 & 4.231564 \\
\mathrm{H} & -5.137638 & 2.138338 & 5.189049 \\
\mathrm{C} & -5.361836 & 2.144178 & 3.043912 \\
\mathrm{H} & -6.438663 & 2.284925 & 3.076013 \\
\mathrm{C} & -0.862922 & -1.717229 & -3.809519 \\
\mathrm{H} & -0.353311 & -1.186668 & -4.608696 \\
\mathrm{C} & -1.612315 & -2.860189 & -4.093006 \\
\mathrm{H} & -1.682889 & -3.224853 & -5.113800 \\
\mathrm{C} & -2.269529 & -3.535601 & -3.060490 \\
\mathrm{H} & -2.848568 & -4.429538 & -3.275270 \\
\mathrm{C} & 1.330962 & -4.516752 & 0.897550 \\
\mathrm{H} & 1.837129 & -5.251790 & 0.278612 \\
\mathrm{C} & 1.527736 & -4.508931 & 2.278433 \\
\mathrm{H} & 2.185885 & -5.238211 & 2.741255 \\
& 0.875457 & -3.554056 & 3.062320 \\
\mathrm{H} & 1.022009 & -3.538449 & 4.138686 \\
\mathrm{H} & 2.403563 & -1.466983 & 0.835076
\end{array}
$$

$2 \mathrm{a}$

B3LYP SCF energy: $\quad 1378.30226687$ a.u.

B3LYP enthalpy: 1377.894146 a.u. 
B3LYP free energy: 1377.971842 a.u.

M06-L SCF energy in solution: 1378.47381788 a.u.

M06-L enthalpy in solution: $\quad 1378.065697$ a.u.

M06-L free energy in solution: $\quad 1378.143393$ a.u.

Cartesian coordinates

\begin{tabular}{|c|c|c|c|}
\hline & & & $z$ \\
\hline h & 1.010256 & 1.740622 & 1.966825 \\
\hline & 1.080330 & 0.412470 & 1.345324 \\
\hline & -0.121382 & 0.382502 & 0.158375 \\
\hline & 1.037563 & -0.795077 & 2.186911 \\
\hline & 2.576947 & 0.302957 & 0.361354 \\
\hline & 2.875238 & 1.315669 & -0.556071 \\
\hline & 3.454528 & -0.759558 & 0.563931 \\
\hline & 2.187008 & 2.142649 & -0.697875 \\
\hline & 4.059644 & 1.247228 & -1.279743 \\
\hline & 4.639597 & -0.812637 & -0.173158 \\
\hline & 3.209175 & -1.526554 & 1.290325 \\
\hline & 4.293927 & 2.032486 & -1.994637 \\
\hline & 4.960513 & 0.182733 & -1.102121 \\
\hline & 5.325281 & -1.641835 & -0.018454 \\
\hline & 6.241657 & 0.125511 & -1.899276 \\
\hline & 6.857387 & 1.016250 & -1.724846 \\
\hline & 6.839163 & -0.752184 & -1.635743 \\
\hline & 6.037660 & & \\
\hline & -1.265703 & 1.298437 & 0.272438 \\
\hline & -1.394412 & 1.796431 & -0.696725 \\
\hline 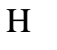 & -1.005176 & 2.061657 & 1.006291 \\
\hline $\mathrm{C}$ & -2.254950 & -0.811146 & 1.267368 \\
\hline $\mathrm{C}$ & -1.612225 & -1.695087 & 0.180901 \\
\hline H & -3.164088 & -1.296638 & 1.644778 \\
\hline & -2.689906 & -1.978158 & -0.885207 \\
\hline & -3.447373 & -0.755110 & -1.401016 \\
\hline
\end{tabular}




$$
\begin{array}{lrrr}
\mathrm{H} & -2.282797 & -2.508237 & -1.754460 \\
\mathrm{H} & -3.451890 & -2.644507 & -0.452075 \\
\mathrm{O} & -3.990912 & -0.796938 & -2.495783 \\
\mathrm{H} & -1.574305 & -0.701225 & 2.117756 \\
\mathrm{C} & -0.422231 & -0.905753 & -0.479493 \\
\mathrm{H} & 0.487172 & -1.512462 & -0.483880 \\
\mathrm{H} & -0.649075 & -0.681778 & -1.530571 \\
\mathrm{C} & -1.111071 & -3.018366 & 0.779326 \\
\mathrm{H} & -0.327409 & -2.833557 & 1.520068 \\
\mathrm{H} & -1.929270 & -3.559642 & 1.270268 \\
\mathrm{H} & -0.702249 & -3.673227 & -0.001186 \\
\mathrm{C} & -3.548872 & 0.446965 & -0.501028 \\
\mathrm{C} & -4.488734 & 1.357791 & -0.821666 \\
\mathrm{C} & -2.596137 & 0.567851 & 0.679260 \\
\mathrm{H} & -3.074767 & 1.171914 & 1.456449 \\
\mathrm{H} & -5.078529 & 1.135762 & -1.710335 \\
\mathrm{C} & -4.822691 & 2.635907 & -0.118293 \\
\mathrm{H} & -4.235886 & 2.806247 & 0.788044 \\
\mathrm{H} & -4.659972 & 3.489955 & -0.790473 \\
\mathrm{H} & -5.887205 & 2.659089 & 0.151045
\end{array}
$$

$2 a^{\prime}$

B3LYP SCF energy: 1378.30051098 a.u.

B3LYP enthalpy: 1377.892474 a.u.

B3LYP free energy: 1377.969831 a.u.

M06-L SCF energy in solution: $\quad 1378.47104720$ a.u.

M06-L enthalpy in solution: $\quad 1378.063010$ a.u.

M06-L free energy in solution: $\quad 1378.140367$ a.u.

Cartesian coordinates

ATOM $\mathrm{X} \quad \mathrm{Y} \quad \mathrm{Z}$ 


$$
\begin{aligned}
& \begin{array}{llll}
\text { O } & 1.139093 & -0.016810 & 2.635834
\end{array} \\
& \text { S } \quad 1.178753 \quad-0.585965 \quad 1.283282 \\
& \begin{array}{llll}
\mathrm{N} & -0.051399 & 0.177703 & 0.412081
\end{array} \\
& \text { O } \quad 1.140745 \quad-2.046311 \quad 1.097006 \\
& \begin{array}{llll}
\text { C } & 2.650778 & 0.002523 & 0.442426
\end{array} \\
& \begin{array}{llll}
\text { C } & 2.939587 & 1.371006 & 0.433328
\end{array} \\
& \begin{array}{llll}
\text { C } & 3.519721 & -0.915529 & -0.142503
\end{array} \\
& \begin{array}{llll}
\mathrm{H} & 2.258431 & 2.075071 & 0.900212
\end{array} \\
& \begin{array}{llll}
\text { C } & 4.105426 & 1.813540 & -0.179927
\end{array} \\
& \begin{array}{llll}
\text { C } & 4.686090 & -0.452548 & -0.755552
\end{array} \\
& \text { H } \quad 3.282399-1.973290 \quad-0.111718 \\
& \text { H } \quad 4.332162 \quad 2.877065-0.189675 \\
& \begin{array}{llll}
\text { C } & 4.997044 & 0.910922 & -0.785387
\end{array} \\
& \text { H } \quad 5.364927 \quad-1.166765 \quad-1.214632 \\
& \begin{array}{llll}
\text { C } & 6.257970 & 1.411895 & -1.448078
\end{array} \\
& \text { H } \quad 6.889702 \quad 1.957891 \quad-0.736883 \\
& \text { H } \quad 6.849677 \quad 0.588454 \quad-1.858766 \\
& \mathrm{H} \quad 6.027855 \quad 2.102385-2.268823 \\
& \begin{array}{llll}
\text { C } & -1.176388 & 0.783582 & 1.137767
\end{array} \\
& \text { H } \quad-1.362073 \quad 1.769108 \quad 0.692256 \\
& \begin{array}{llll}
\mathrm{H} & -0.858037 & 0.942258 & 2.168282
\end{array} \\
& \begin{array}{llll}
\text { C } & -2.150439 & -1.485335 & 0.555060
\end{array} \\
& \begin{array}{llll}
\text { C } & -1.612335 & -1.375266 & -0.885101
\end{array} \\
& \text { H } \quad-3.049369 \quad-2.114890 \quad 0.564250 \\
& \text { C } \quad-2.747246-0.814894 \quad-1.771353 \\
& \begin{array}{llll}
\text { C } & -3.419772 & 0.456727 & -1.250381
\end{array}
\end{aligned}
$$

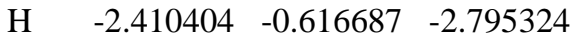

$$
\begin{aligned}
& \mathrm{H} \quad-3.542783 \quad-1.573105 \quad-1.843985 \\
& \begin{array}{llll}
\text { O } & -3.851859 & 1.288165 & -2.037450
\end{array} \\
& \mathrm{H} \quad-1.410450 \quad-1.972902 \quad 1.197431 \\
& \begin{array}{llll}
\text { C } & -0.396520 & -0.375938 & -0.903618
\end{array} \\
& \text { H } \quad 0.490947 \quad-0.859360 \quad-1.319613 \\
& \text { H } \quad-0.616942 \quad 0.481552 \quad-1.553503
\end{aligned}
$$




$$
\begin{array}{lrrr}
\mathrm{C} & -1.173225 & -2.751227 & -1.408543 \\
\mathrm{H} & -0.358004 & -3.150806 & -0.797101 \\
\mathrm{H} & -2.005676 & -3.465079 & -1.382082 \\
\mathrm{H} & -0.825098 & -2.685777 & -2.447526 \\
\mathrm{C} & -3.559933 & 0.583863 & 0.236528 \\
\mathrm{C} & -4.593995 & 1.233028 & 0.808544 \\
\mathrm{C} & -2.486732 & -0.082948 & 1.088927 \\
\mathrm{H} & -2.869317 & -0.166891 & 2.112321 \\
\mathrm{C} & -5.737966 & 1.950263 & 0.158011 \\
\mathrm{H} & -6.683144 & 1.672686 & 0.641830 \\
\mathrm{H} & -5.624154 & 3.035213 & 0.294929 \\
\mathrm{H} & -5.796475 & 1.763129 & -0.913735 \\
\mathrm{H} & -4.620667 & 1.229926 & 1.900769
\end{array}
$$

TS-deCO

B3LYP SCF energy: 3935.99714262 a.u.

B3LYP enthalpy: 3934.990110 a.u.

B3LYP free energy: 3935.166378 a.u.

M06-L SCF energy in solution: 3937.65209988 a.u.

M06-L enthalpy in solution: $\quad 3936.645067$ a.u.

M06-L free energy in solution: $\quad 3936.821335$ a.u.

Imaginary frequency: \$TAB \$ $-241.7189 \mathrm{~cm}-1$

Cartesian coordinates

\begin{tabular}{lccc} 
ATOM X & Y & \multicolumn{2}{l}{ Z } \\
P & -2.101942 & 1.860679 & 0.075101 \\
P & -1.382924 & -1.669384 & -0.353288 \\
O & -5.666192 & 0.008491 & 2.329573 \\
O & -5.150990 & -1.188632 & 4.242482 \\
O & -6.160207 & -1.465833 & -0.522372 \\
O & -7.147963 & -0.294555 & -2.256459
\end{tabular}




$$
\begin{aligned}
& \begin{array}{llll}
\text { C } & -2.599038 & -1.596372 & 1.026828
\end{array} \\
& \begin{array}{llll}
\text { C } & -3.745201 & -0.738210 & 0.977535
\end{array} \\
& \begin{array}{llll}
\text { C } & -4.513581 & -0.702745 & 2.130798
\end{array} \\
& \begin{array}{llll}
\text { C } & -4.209993 & -1.426232 & 3.285165
\end{array} \\
& \begin{array}{llll}
\text { C } & -3.113115 & -2.257241 & 3.344337
\end{array} \\
& \begin{array}{llll}
\text { C } & -2.314377 & -2.324433 & 2.190109
\end{array} \\
& \begin{array}{llll}
\text { C } & -6.015859 & -0.177184 & 3.708245
\end{array} \\
& \begin{array}{llll}
\text { C } & -3.661737 & 1.254834 & -0.695380
\end{array} \\
& \begin{array}{llll}
\text { C } & -4.278110 & 1.955317 & -1.739923
\end{array} \\
& \begin{array}{llll}
\text { C } & -5.468434 & 1.515513 & -2.345785
\end{array} \\
& \begin{array}{llll}
\text { C } & -6.016385 & 0.351767 & -1.852509
\end{array} \\
& \begin{array}{llll}
\text { C } & -5.417813 & -0.350045 & -0.805425
\end{array} \\
& \begin{array}{llll}
\text { C } & -4.237010 & 0.044112 & -0.198465
\end{array} \\
& \begin{array}{llll}
\text { C } & -7.344066 & -1.367902 & -1.326540
\end{array} \\
& \begin{array}{llll}
\text { C } & -2.333073 & -2.033327 & -1.883845
\end{array} \\
& \begin{array}{llll}
\text { C } & -1.870402 & -1.536067 & -3.111481
\end{array} \\
& \text { C } \quad-3.475641-2.849795 \quad-1.865763 \\
& \text { C } \quad-0.380670-3.182756-0.054232 \\
& \text { C } \quad-0.740373-4.423785-0.606662 \\
& \begin{array}{llll}
\text { C } & 0.764228 & -3.103497 & 0.755274
\end{array} \\
& \begin{array}{llll}
\text { C } & -2.620666 & 2.365218 & 1.768431
\end{array} \\
& \begin{array}{llll}
\text { C } & -3.874916 & 2.965026 & 1.976529
\end{array} \\
& \begin{array}{llll}
\text { C } & -1.761905 & 2.180236 & 2.861692
\end{array} \\
& \begin{array}{llll}
\text { C } & -1.325770 & 3.395523 & -2.143846
\end{array} \\
& \begin{array}{llll}
\text { C } & -1.715489 & 3.443024 & -0.790969
\end{array} \\
& \mathrm{H} \quad-2.878930 \quad-2.833983 \quad 4.231813 \\
& \mathrm{H} \quad-1.447361 \quad-2.973188 \quad 2.212076 \\
& \mathrm{H} \quad \begin{array}{llll}
-5.858586 & 0.761967 & 4.252347
\end{array} \\
& \text { H } \quad-7.053922 \quad-0.512022 \quad 3.779319 \\
& \text { H } \quad-3.842861 \quad 2.882021 \quad-2.092735 \\
& \text { H } \quad-5.935556 \quad 2.070026-3.151773 \\
& \text { H } \quad-7.490346 \quad-2.301807 \quad-1.874723 \\
& \text { H } \quad-8.205402 \quad-1.144142 \quad-0.685139
\end{aligned}
$$




$$
\begin{aligned}
& \text { H } \quad-0.983407 \quad-0.908186 \quad-3.145708 \\
& \text { H } \quad-3.860335 \quad-3.228232 \quad-0.923778 \\
& \text { H } \quad-1.609354 \quad-4.510148 \quad-1.248882 \\
& \text { H } \quad-4.556220 \quad 3.103955 \quad 1.142682 \\
& \text { H } \quad-0.796310 \quad 1.707386 \quad 2.727096 \\
& \mathrm{H} \quad-1.330636 \quad 2.447795 \quad-2.678976 \\
& \begin{array}{llll}
\mathrm{Rh} & -0.103624 & 0.358385 & -0.440917
\end{array} \\
& \begin{array}{llll}
\text { O } & 3.765964 & -1.871950 & 0.849849
\end{array} \\
& \begin{array}{llll}
\text { C } & 0.969547 & 0.796146 & 0.970969
\end{array} \\
& \begin{array}{llll}
\text { S } & 4.996301 & -1.730536 & 0.056337
\end{array} \\
& \begin{array}{llll}
\text { O } & 1.464553 & 0.859169 & 2.022738
\end{array} \\
& \text { N } \quad 4.610002 \quad-0.593817 \quad-1.152026 \\
& \begin{array}{llll}
\text { O } & 5.594551 & -2.866219 & -0.647171
\end{array} \\
& \begin{array}{llll}
\text { C } & 6.239883 & -1.037452 & 1.144769
\end{array} \\
& \begin{array}{llll}
\text { C } & 5.617347 & -0.407576 & -2.223138
\end{array} \\
& \begin{array}{llll}
\text { C } & 7.592980 & -1.208486 & 0.839163
\end{array} \\
& \begin{array}{llll}
\text { C } & 5.846089 & -0.334832 & 2.287010
\end{array} \\
& \text { H } \quad 6.110444 \quad-1.373093 \quad-2.365431 \\
& \text { H } \quad 5.073255 \quad-0.208315 \quad-3.152225 \\
& \text { H } \quad 7.886804 \quad-1.779771 \quad-0.034300 \\
& \begin{array}{llll}
\text { C } & 8.553400 & -0.658697 & 1.684530
\end{array} \\
& \begin{array}{llll}
\text { C } & 6.822684 & 0.210278 & 3.118521
\end{array} \\
& \begin{array}{llll}
\mathrm{H} & 4.794145 & -0.242607 & 2.534715
\end{array} \\
& \text { H } \quad 9.606404 \quad-0.797298 \quad 1.452996 \\
& \begin{array}{llll}
\text { C } & 8.187546 & 0.058652 & 2.833939
\end{array} \\
& \text { H } \quad 6.519465 \quad 0.752422 \quad 4.010626 \\
& \begin{array}{llll}
\text { C } & 9.238847 & 0.617701 & 3.762082
\end{array} \\
& \mathrm{H} \quad 8.867880 \quad 1.488325 \quad 4.312109 \\
& \text { H } \quad 9.542522 \quad-0.132489 \quad 4.503970 \\
& \text { H } \quad 10.139658 \quad 0.915520 \quad 3.215757 \\
& \begin{array}{llll}
\text { C } & 1.653541 & 1.809997 & -0.686169
\end{array} \\
& \begin{array}{llll}
\mathrm{H} & 2.219662 & 2.423643 & 0.017535
\end{array} \\
& \text { H } \quad 1.000795 \quad 2.463915 \quad-1.269658
\end{aligned}
$$




$$
\begin{aligned}
& \begin{array}{llll}
\text { C } & 2.459897 & 0.749468 & -1.472304
\end{array} \\
& \begin{array}{llll}
\text { C } & 1.569774 & -0.507622 & -1.389152
\end{array} \\
& \text { H } \quad 1.079893 \quad-2.153746 \quad 1.171993 \\
& \text { H } \quad \begin{array}{llll}
1.197066 & -0.858393 & -2.359017
\end{array} \\
& \begin{array}{llll}
\text { C } & 2.648028 & 1.221567 & -2.927589
\end{array} \\
& \text { H } \quad 3.109610 \quad 0.437405 \quad-3.535495 \\
& \text { H } \quad 1.679681 \quad 1.458668 \quad-3.384960 \\
& \text { H } \quad 3.276875 \quad 2.117733 \quad-2.984895 \\
& \begin{array}{llll}
\text { C } & 3.821353 & 0.571660 & -0.736708
\end{array} \\
& \text { H } \quad 4.420946 \quad 1.483529-0.867175 \\
& \text { H } \quad 3.615338 \quad 0.472753 \quad 0.330359 \\
& \mathrm{H} \quad 2.025224 \quad-1.338285 \quad-0.853692 \\
& \begin{array}{llll}
\text { C } & 6.595777 & 0.660658 & -1.982946
\end{array} \\
& \begin{array}{llll}
\text { C } & 7.390850 & 1.555297 & -1.799605
\end{array} \\
& \begin{array}{llll}
\text { C } & 8.391162 & 2.604313 & -1.586147
\end{array} \\
& \text { H } \quad 8.818097 \quad 2.491494 \quad-0.580297 \\
& \text { H } \quad 7.901200 \quad 3.587350-1.601155 \\
& \begin{array}{llll}
\text { C } & 9.521368 & 2.576479 & -2.633737
\end{array} \\
& \begin{array}{llll}
\mathrm{H} & 9.123300 & 2.721809 & -3.642733
\end{array} \\
& \text { H } \quad 10.243008 \quad 3.374168-2.429791 \\
& \text { H } \quad 10.050466 \quad 1.618785 \quad-2.612489 \\
& \text { C } \quad-0.956666 \quad 4.557094 \quad-2.821623 \\
& \begin{array}{llll}
\mathrm{H} & -0.673284 & 4.504047 & -3.869071
\end{array} \\
& \begin{array}{llll}
\text { C } & -0.951550 & 5.784679 & -2.152956
\end{array} \\
& \text { H } \quad-0.660361 \quad 6.689682 \quad-2.678010 \\
& \text { C } \quad-1.322555 \quad 5.841071 \quad-0.809631 \\
& \mathrm{H} \quad-1.321073 \quad 6.791437 \quad-0.283550 \\
& \begin{array}{llll}
\text { C } & -1.704363 & 4.680169 & -0.130994
\end{array} \\
& \begin{array}{llll}
\mathrm{H} & -1.995408 & 4.744269 & 0.911781
\end{array} \\
& \begin{array}{llll}
\text { C } & -4.252330 & 3.382191 & 3.252947
\end{array} \\
& \begin{array}{llll}
\mathrm{H} & -5.219864 & 3.854291 & 3.399982
\end{array} \\
& \begin{array}{llll}
\text { C } & -3.388103 & 3.197867 & 4.336493
\end{array} \\
& \text { H } \quad-3.683175 \quad 3.524360 \quad 5.329778
\end{aligned}
$$




$$
\begin{array}{llll}
\mathrm{C} & -2.144845 & 2.595114 & 4.139074 \\
\mathrm{H} & -1.471102 & 2.443975 & 4.977445 \\
\mathrm{C} & 0.026510 & -5.558741 & -0.343310 \\
\mathrm{H} & -0.259913 & -6.510928 & -0.780735 \\
\mathrm{C} & 1.159354 & -5.470999 & 0.468298 \\
\mathrm{H} & 1.759978 & -6.355035 & 0.662060 \\
\mathrm{C} & 1.528150 & -4.241631 & 1.015828 \\
\mathrm{H} & 2.423937 & -4.149588 & 1.620214 \\
\mathrm{C} & -4.131574 & -3.166235 & -3.055972 \\
\mathrm{H} & -5.009564 & -3.805753 & -3.031700 \\
\mathrm{C} & -3.661551 & -2.668244 & -4.273760 \\
\mathrm{H} & -4.174907 & -2.916698 & -5.198103 \\
\mathrm{C} & -2.531007 & -1.850002 & -4.300151 \\
\mathrm{H} & -2.161153 & -1.459446 & -5.243787
\end{array}
$$

IntC-deCO

B3LYP SCF energy: $\quad 3936.01379314$ a.u.

B3LYP enthalpy: 3935.004896 a.u.

B3LYP free energy: 3935.181254 a.u.

M06-L SCF energy in solution: $\quad 3937.66841529$ a.u.

M06-L enthalpy in solution: $\quad 3936.659518$ a.u.

M06-L free energy in solution: $\quad 3936.835876$ a.u.

Cartesian coordinates

\begin{tabular}{lccc}
\multicolumn{2}{l}{ ATOM } & X & Y \\
P & 1.548397 & 1.779910 & 0.424090 \\
P & 1.807695 & -1.804764 & -0.316237 \\
O & 5.859393 & 1.576818 & -1.065455 \\
O & 6.140051 & 1.045690 & -3.301550 \\
O & 6.144283 & -0.667679 & 1.271507 \\
O & 6.368472 & 0.038742 & 3.465407
\end{tabular}




$$
\begin{aligned}
& \begin{array}{llll}
\text { C } & 3.188425 & -0.978895 & -1.238812
\end{array} \\
& \begin{array}{llll}
\text { C } & 3.999801 & 0.025981 & -0.614825
\end{array} \\
& \begin{array}{lllll}
\text { C } & 4.952283 & 0.619137 & -1.428417
\end{array} \\
& \begin{array}{llll}
\text { C } & 5.129326 & 0.300360 & -2.775619
\end{array} \\
& \text { C } \quad 4.362276-0.665402 \quad-3.387761 \\
& \begin{array}{llll}
\text { C } & 3.391854 & -1.295401 & -2.590030
\end{array} \\
& \begin{array}{llll}
\text { C } & 6.559490 & 1.942351 & -2.262826
\end{array} \\
& \begin{array}{llll}
\text { C } & 3.008810 & 1.286888 & 1.428799
\end{array} \\
& \begin{array}{llll}
\text { C } & 3.169271 & 1.718651 & 2.750882
\end{array} \\
& \begin{array}{llll}
\text { C } & 4.274545 & 1.347827 & 3.537216
\end{array} \\
& \begin{array}{llll}
\text { C } & 5.213156 & 0.536850 & 2.937844
\end{array} \\
& \begin{array}{llll}
\text { C } & 5.072435 & 0.111087 & 1.616760
\end{array} \\
& \begin{array}{llll}
\text { C } & 3.987108 & 0.440686 & 0.821770
\end{array} \\
& \begin{array}{llll}
\text { C } & 7.037360 & -0.639570 & 2.394457
\end{array} \\
& \begin{array}{llll}
\text { C } & 2.589806 & -2.503489 & 1.194883
\end{array} \\
& \begin{array}{llll}
\text { C } & 1.857599 & -2.628397 & 2.383808
\end{array} \\
& \begin{array}{llll}
\text { C } & 3.901097 & -3.006466 & 1.146207
\end{array} \\
& \text { C } \quad \begin{array}{llll}
1.401722 & -3.302428 & -1.324839
\end{array} \\
& \begin{array}{llll}
\text { C } & 1.743327 & -4.596750 & -0.904771
\end{array} \\
& \begin{array}{llll}
\text { C } & 0.705499 & -3.150937 & -2.539611
\end{array} \\
& \begin{array}{llll}
\text { C } & 2.245574 & 2.867727 & -0.887785
\end{array} \\
& \begin{array}{llll}
\text { C } & 3.269459 & 3.786671 & -0.602353
\end{array} \\
& \begin{array}{llll}
\text { C } & 1.735365 & 2.798867 & -2.192804
\end{array} \\
& \begin{array}{llll}
\text { C } & -0.030114 & 2.414734 & 2.668560
\end{array} \\
& \begin{array}{llll}
\text { C } & 0.565973 & 2.915915 & 1.495463
\end{array} \\
& \text { H } \quad 4.504191 \quad-0.935966 \quad-4.427849 \\
& \mathrm{H} \quad 2.797664 \quad-2.071884-3.052411 \\
& \text { H } \quad 6.294436 \quad 2.969154 \quad-2.539748 \\
& \text { H } \quad 7.635910 \quad 1.835473 \quad-2.103578 \\
& \mathrm{H} \quad 2.432890 \quad 2.378401 \quad 3.192053 \\
& \mathrm{H} \quad 4.387782 \quad 1.693812 \quad 4.558388 \\
& \text { H } \quad 7.268723 \quad-1.663224 \quad 2.701088 \\
& \text { H } \quad \begin{array}{lll}
7.947191 & -0.090523 & 2.124335
\end{array}
\end{aligned}
$$




$$
\begin{aligned}
& \text { H } \quad 0.839438 \quad-2.261323 \quad 2.439036 \\
& \text { H } \quad 4.485283 \quad-2.916709 \quad 0.236059 \\
& \text { H } \quad 2.267237 \quad-4.750382 \quad 0.031176 \\
& \text { H } \quad 3.683239 \quad 3.844246 \quad 0.400020 \\
& \text { H } \quad 0.951183 \quad 2.086432 \quad-2.434780 \\
& \begin{array}{llll}
\mathrm{H} & 0.157387 & 1.391200 & 2.979793
\end{array} \\
& \begin{array}{llll}
\text { Rh } & -0.019625 & -0.078161 & -0.365075
\end{array} \\
& \begin{array}{llll}
\text { O } & -3.930979 & -0.125466 & -2.313382
\end{array} \\
& \begin{array}{llll}
\text { C } & -1.263307 & 1.269894 & -0.874501
\end{array} \\
& \begin{array}{llll}
\text { S } & -5.123722 & -0.790273 & -1.767702
\end{array} \\
& \begin{array}{llll}
\text { O } & -1.938264 & 2.126377 & -1.228891
\end{array} \\
& \mathrm{~N} \quad \begin{array}{llll}
4.593448 & -1.557534 & -0.345489
\end{array} \\
& \begin{array}{llll}
\text { O } & -5.848005 & -1.816323 & -2.519391
\end{array} \\
& \begin{array}{llll}
\text { C } & -6.289209 & 0.504252 & -1.343164
\end{array} \\
& \begin{array}{llll}
\text { C } & -5.556469 & -2.471377 & 0.311786
\end{array} \\
& \begin{array}{llll}
\text { C } & -7.646677 & 0.193612 & -1.222423
\end{array} \\
& \begin{array}{llll}
\text { C } & -5.833052 & 1.809973 & -1.144452
\end{array} \\
& \text { H } \quad-6.153383 \quad-2.928925 \quad-0.481867 \\
& \text { H } \quad-4.977120 \quad-3.283948 \quad 0.762793 \\
& \text { H } \quad-7.993509-0.816650-1.408406 \\
& \begin{array}{llll}
\text { C } & -8.546175 & 1.202433 & -0.887496
\end{array} \\
& \begin{array}{llll}
\text { C } & -6.749013 & 2.805108 & -0.807066
\end{array} \\
& \text { H } \quad-4.784126 \quad 2.047532-1.283286 \\
& \text { H } \quad-9.603339 \quad 0.964247 \quad-0.801430 \\
& \begin{array}{llll}
\text { C } & -8.115512 & 2.520943 & -0.673468
\end{array} \\
& \text { H } \quad-6.397671 \quad 3.823148 \quad-0.658624 \\
& \begin{array}{llll}
\text { C } & -9.106265 & 3.613098 & -0.349238
\end{array} \\
& \begin{array}{llll}
\mathrm{H} & -8.635468 & 4.432317 & 0.203417
\end{array} \\
& \text { H } \quad-9.530862 \quad 4.039649 \quad-1.267474 \\
& \text { H } \quad-9.942181 \quad 3.233177 \quad 0.247196 \\
& \begin{array}{llll}
\text { C } & -1.266355 & -0.532269 & 1.280249
\end{array} \\
& \begin{array}{llll}
\mathrm{H} & -1.674871 & 0.408063 & 1.653314
\end{array} \\
& \text { H } \quad-0.583099 \quad-0.981551 \quad 1.999511
\end{aligned}
$$




$$
\begin{aligned}
& \begin{array}{llll}
\text { C } & -2.235950 & -1.488982 & 0.593776
\end{array} \\
& \begin{array}{llll}
\text { C } & -1.509755 & -1.550662 & -0.765770
\end{array} \\
& \text { H } \quad \begin{array}{llll}
0.398825 & -2.163424 & -2.873628
\end{array} \\
& \text { H } \quad-1.031169-2.508630 \quad-0.944181 \\
& \begin{array}{llll}
\text { C } & -2.278302 & -2.864220 & 1.278193
\end{array} \\
& \mathrm{H} \quad-2.836082 \quad-3.580221 \quad 0.666204 \\
& \text { H } \quad-1.266293 \quad-3.267698 \quad 1.401597 \\
& \text { H } \quad-2.745441 \quad-2.811406 \quad 2.269766 \\
& \begin{array}{llll}
\text { C } & -3.642120 & -0.838431 & 0.514024
\end{array} \\
& \text { H } \quad-4.069713 \quad-0.762895 \quad 1.522709 \\
& \begin{array}{llll}
\mathrm{H} & -3.537124 & 0.184310 & 0.143104
\end{array} \\
& \text { H } \quad-2.117902 \quad-1.237383-1.613583 \\
& \begin{array}{llll}
\text { C } & -6.412170 & -1.851021 & 1.331901
\end{array} \\
& \begin{array}{llll}
\text { C } & -7.104876 & -1.349968 & 2.189285
\end{array} \\
& \begin{array}{llll}
\text { C } & -7.984871 & -0.759820 & 3.201115
\end{array} \\
& \begin{array}{llll}
\text { H } & -8.440695 & 0.151436 & 2.790327
\end{array} \\
& \text { H } \quad-7.387663 \quad-0.439270 \quad 4.065634 \\
& \text { C } \quad-9.091268 \quad-1.725018 \quad 3.669910 \\
& \text { H } \quad-8.660545 \quad-2.627196 \quad 4.115149 \\
& \text { H } \quad-9.722752 \quad-1.238124 \quad 4.420217 \\
& \mathrm{H} \quad-9.724707 \quad-2.030077 \quad 2.831295 \\
& \begin{array}{llll}
\text { C } & -0.845137 & 3.226903 & 3.456533
\end{array} \\
& \begin{array}{llll}
\mathrm{H} & -1.284290 & 2.827610 & 4.366538
\end{array} \\
& \begin{array}{llll}
\text { C } & -1.095468 & 4.548221 & 3.076626
\end{array} \\
& \begin{array}{llll}
\mathrm{H} & -1.733398 & 5.179892 & 3.687882
\end{array} \\
& \begin{array}{llll}
\text { C } & -0.520051 & 5.051051 & 1.910009
\end{array} \\
& \mathrm{H} \quad-0.707790 \quad 6.077314 \quad 1.607555 \\
& \begin{array}{llll}
\text { C } & 0.306943 & 4.244004 & 1.124198
\end{array} \\
& \begin{array}{llll}
\mathrm{H} & 0.748949 & 4.656016 & 0.223874
\end{array} \\
& \begin{array}{llll}
\text { C } & 3.758380 & 4.627772 & -1.602323
\end{array} \\
& \begin{array}{llll}
\mathrm{H} & 4.541102 & 5.344053 & -1.367788
\end{array} \\
& \begin{array}{llll}
\text { C } & 3.239076 & 4.554988 & -2.898719
\end{array} \\
& \begin{array}{llll}
\mathrm{H} & 3.619819 & 5.212500 & -3.675158
\end{array}
\end{aligned}
$$




$\begin{array}{llll}\mathrm{C} & 2.228795 & 3.637859 & -3.193760 \\ \mathrm{H} & 1.824507 & 3.573970 & -4.199763 \\ \mathrm{C} & 1.412874 & -5.706178 & -1.687375 \\ \mathrm{H} & 1.684468 & -6.700423 & -1.344503 \\ \mathrm{C} & 0.741335 & -5.541597 & -2.898099 \\ \mathrm{H} & 0.486336 & -6.406146 & -3.503942 \\ \mathrm{C} & 0.388368 & -4.258529 & -3.324024 \\ \mathrm{H} & -0.145883 & -4.118753 & -4.259178 \\ \mathrm{C} & 4.462070 & -3.617946 & 2.267189 \\ \mathrm{H} & 5.473573 & -4.010945 & 2.213548 \\ \mathrm{C} & 3.726409 & -3.731866 & 3.449817 \\ \mathrm{H} & 4.166026 & -4.208102 & 4.321430 \\ \mathrm{C} & 2.423371 & -3.235983 & 3.506306 \\ \mathrm{H} & 1.844360 & -3.323751 & 4.421028\end{array}$

TS-deCO-RE

B3LYP SCF energy: 3936.01098468 a.u.

B3LYP enthalpy: 3935.003300 a.u.

B3LYP free energy: 3935.180127 a.u.

M06-L SCF energy in solution: $\quad 3937.66518109$ a.u.

M06-L enthalpy in solution: $\quad 3936.657496$ a.u.

M06-L free energy in solution: 3936.834323 a.u.

Imaginary frequency: \$TAB \$ $-168.2913 \mathrm{~cm}-1$

Cartesian coordinates

\begin{tabular}{lccc}
\multicolumn{2}{l}{ ATOM } & X & Y \\
P & 1.671465 & 1.788580 & 0.247953 \\
P & 1.777884 & -1.728235 & -0.148744 \\
O & 6.085226 & 1.164224 & -1.296260 \\
O & 6.402175 & 0.240872 & -3.397541 \\
O & 6.210063 & -0.749240 & 1.263693
\end{tabular}




$$
\begin{aligned}
& \begin{array}{llll}
\text { O } & 6.474851 & 0.156986 & 3.379638
\end{array} \\
& \text { C } \quad 3.241067-1.200505-1.159092 \\
& \begin{array}{llll}
\text { C } & 4.111359 & -0.172533 & -0.673173
\end{array} \\
& \begin{array}{llll}
\text { C } & 5.126250 & 0.216264 & -1.533475
\end{array} \\
& \begin{array}{llll}
\text { C } & 5.323869 & -0.337433 & -2.797401
\end{array} \\
& \begin{array}{llll}
\text { C } & 4.514094 & -1.346197 & -3.267752
\end{array} \\
& \begin{array}{llll}
\text { C } & 3.471048 & -1.761498 & -2.421903
\end{array} \\
& \begin{array}{llll}
\text { C } & 6.887023 & 1.234304 & -2.484231
\end{array} \\
& \begin{array}{llll}
\text { C } & 3.174207 & 1.358640 & 1.219398
\end{array} \\
& \begin{array}{llll}
\text { C } & 3.356521 & 1.902922 & 2.497803
\end{array} \\
& \begin{array}{llll}
\text { C } & 4.446159 & 1.563739 & 3.317872
\end{array} \\
& \begin{array}{llll}
\text { C } & 5.344288 & 0.654692 & 2.803503
\end{array} \\
& \begin{array}{llll}
\text { C } & 5.180658 & 0.112388 & 1.528923
\end{array} \\
& \begin{array}{llll}
\text { C } & 4.115751 & 0.420631 & 0.697339
\end{array} \\
& \begin{array}{llll}
\text { C } & 7.063568 & -0.727685 & 2.416165
\end{array} \\
& \begin{array}{llll}
\text { C } & 2.507219 & -2.224813 & 1.467721
\end{array} \\
& \begin{array}{llll}
\text { C } & 1.921943 & -1.804911 & 2.670440
\end{array} \\
& \begin{array}{llll}
\text { C } & 3.648596 & -3.044494 & 1.511519
\end{array} \\
& \text { C } \quad \begin{array}{llll}
1.228757 & -3.331064 & -0.897953
\end{array} \\
& \text { C } \quad 1.243904 \quad-4.530091 \quad-0.167722 \\
& \begin{array}{llll}
\text { C } & 0.705143 & -3.353769 & -2.206557
\end{array} \\
& \begin{array}{llll}
\text { C } & 2.270850 & 2.529186 & -1.320797
\end{array} \\
& \begin{array}{llll}
\text { C } & 3.448581 & 3.293794 & -1.367489
\end{array} \\
& \begin{array}{llll}
\text { C } & 1.508780 & 2.368400 & -2.488015
\end{array} \\
& \begin{array}{llll}
\text { C } & 0.155408 & 2.905359 & 2.330925
\end{array} \\
& \begin{array}{llll}
\text { C } & 0.921042 & 3.192125 & 1.186188
\end{array} \\
& \text { H } \quad 4.674519-1.805076 \quad-4.236661 \\
& \mathrm{H} \quad 2.845341 \quad-2.568831 \quad-2.773896 \\
& \mathrm{H} \quad 6.782076 \quad 2.226651 \quad-2.936407 \\
& \text { H } \quad 7.930731 \quad 1.020682-2.233850 \\
& \begin{array}{llll}
\mathrm{H} & 2.644527 & 2.623204 & 2.879163
\end{array} \\
& \mathrm{H} \quad 4.574880 \quad 2.000777 \quad 4.301557 \\
& \text { H } \quad \begin{array}{llll}
7.128447 & -1.734983 & 2.839761
\end{array}
\end{aligned}
$$




\begin{tabular}{|c|c|c|c|}
\hline & 3.052724 & -0.352312 & \\
\hline & 1.058544 & -1.147158 & 2.649606 \\
\hline & 4.123742 & -3.363982 & 0.588670 \\
\hline & 1.625620 & -4.549392 & 0.846204 \\
\hline & 4.056354 & 3.413791 & -0.475755 \\
\hline & 0.601606 & 1.769964 & -2.464730 \\
\hline & 0.017514 & 1.873402 & 2.644867 \\
\hline & -0.023571 & 0.003627 & -0.034141 \\
\hline & -3.898419 & 0.496218 & -2.097766 \\
\hline & -1.306200 & 1.398612 & -0.110082 \\
\hline & -5.171085 & -0.204966 & -1.868519 \\
\hline & -2.070870 & 2.251997 & -0.188570 \\
\hline & -4.806137 & -1.426005 & -0.745848 \\
\hline & -5.889924 & -0.873723 & -2.953791 \\
\hline & -6.289147 & 0.976620 & -1.116878 \\
\hline & -5.872452 & -2.415060 & -0.464687 \\
\hline & -7.667742 & 0.756137 & -1.188382 \\
\hline & -5.776272 & & -0.473369 \\
\hline & -6.441806 & -2.541547 & -1.389632 \\
\hline & -5.380982 & -3.375 & -0.276298 \\
\hline & -8.055196 & -0.107717 & -1.716725 \\
\hline & -8.532593 & 1.672971 & -0.596798 \\
\hline & -6.659123 & 3.010387 & 0.114698 \\
\hline & -4.707669 & & \\
\hline & -9.605192 & 1.507229 & -0.658241 \\
\hline & -8.046075 & 2.811731 & 0.063879 \\
\hline & -6.263423 & 3.893424 & 0.610200 \\
\hline & -8.996324 & 3.817614 & 0.667761 \\
\hline & -8.522760 & 4.383493 & 1.476212 \\
\hline & -9.329335 & 4.541772 & -0.087273 \\
\hline & -9.892926 & & 1.068123 \\
\hline & -1.445236 & -1.285881 & 1.106765 \\
\hline & -1.755327 & -0.519961 & 1.818394 \\
\hline
\end{tabular}




$$
\begin{aligned}
& \mathrm{H} \quad-0.766345 \quad-2.027533 \quad 1.518653 \\
& \text { C } \quad-2.505543 \quad-1.850569 \quad 0.194792 \\
& \begin{array}{llll}
\text { C } & -1.618063 & -1.312751 & -0.905569
\end{array} \\
& \text { H } \quad \begin{array}{llll}
0.653258 & -2.439903 & -2.792089
\end{array} \\
& \text { H } \quad-1.013086 \quad-2.069473 \quad-1.386195 \\
& \begin{array}{llll}
\text { C } & -2.640633 & -3.373942 & 0.225011
\end{array} \\
& \text { H } \quad-3.202202-3.728311 \quad-0.645596 \\
& \begin{array}{llll}
\mathrm{H} & -1.657830 & -3.855322 & 0.200700
\end{array} \\
& \text { H } \quad-3.157590 \quad-3.706605 \quad 1.133924 \\
& \text { C } \quad-3.859111 \quad-1.123607 \quad 0.338799
\end{aligned}
$$

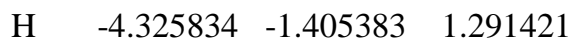

$$
\begin{aligned}
& \begin{array}{llll}
\text { H } & -3.689829 & -0.044319 & 0.381594
\end{array} \\
& \text { H } \quad-2.076082 \quad-0.583458 \quad-1.570298 \\
& \begin{array}{llll}
\text { C } & -6.751775 & -2.080698 & 0.663079
\end{array} \\
& \begin{array}{llll}
\text { C } & -7.462857 & -1.820482 & 1.607842
\end{array} \\
& \begin{array}{llll}
\text { C } & -8.363333 & -1.510469 & 2.721157
\end{array} \\
& \text { H } \quad-8.740401 \quad-0.485651 \quad 2.601171 \\
& \mathrm{H} \quad-7.798501 \quad-1.519524 \quad 3.663281 \\
& \begin{array}{llll}
\text { C } & -9.549518 & -2.489268 & 2.823198
\end{array} \\
& \mathrm{H} \quad-9.198680 \quad-3.514237 \quad 2.978161 \\
& \text { H } \quad-10.193076 \quad-2.214294 \quad 3.665205 \\
& \text { H } \quad-10.150857 \quad-2.471164 \quad 1.909059 \\
& \begin{array}{llll}
\text { C } & -0.427223 & 3.932115 & 3.072122
\end{array} \\
& \begin{array}{llll}
\mathrm{H} & -1.009460 & 3.694956 & 3.958132
\end{array} \\
& \begin{array}{llll}
\text { C } & -0.269180 & 5.261161 & 2.669712
\end{array} \\
& \text { H } \quad-0.730267 \quad 6.061712 \quad 3.240866 \\
& \begin{array}{llll}
\text { C } & 0.478413 & 5.554631 & 1.529508
\end{array} \\
& \text { H } \quad 0.600553 \quad 6.584803 \quad 1.207584 \\
& \begin{array}{llll}
\text { C } & 1.075442 & 4.528497 & 0.792373
\end{array} \\
& \text { H } \quad 1.653226 \quad 4.775966 \quad-0.091180 \\
& \begin{array}{llll}
\text { C } & 3.846060 & 3.894262 & -2.562662
\end{array} \\
& \text { H } \quad 4.752726 \quad 4.492698 \quad-2.588253 \\
& \begin{array}{llll}
\text { C } & 3.078769 & 3.733791 & -3.719952
\end{array}
\end{aligned}
$$




$$
\begin{array}{llll}
\mathrm{H} & 3.391175 & 4.202776 & -4.648634 \\
\mathrm{C} & 1.911240 & 2.969328 & -3.681840 \\
\mathrm{H} & 1.313580 & 2.838543 & -4.579198 \\
\mathrm{C} & 0.774485 & -5.717436 & -0.736926 \\
\mathrm{H} & 0.800087 & -6.633974 & -0.154417 \\
\mathrm{C} & 0.282165 & -5.729874 & -2.041541 \\
\mathrm{H} & -0.077381 & -6.654920 & -2.482469 \\
\mathrm{C} & 0.247971 & -4.542336 & -2.775735 \\
\mathrm{H} & -0.141955 & -4.535765 & -3.789418 \\
\mathrm{C} & 4.177933 & -3.448186 & 2.736657 \\
\mathrm{H} & 5.053306 & -4.091519 & 2.758840 \\
\mathrm{C} & 3.582965 & -3.030433 & 3.931247 \\
\mathrm{H} & 3.997215 & -3.345691 & 4.884655 \\
\mathrm{C} & 2.458429 & -2.205058 & 3.896465 \\
\mathrm{H} & 1.998876 & -1.869794 & 4.821808
\end{array}
$$

ML-CO

B3LYP SCF energy: $\quad 2671.15091818$ a.u.

B3LYP enthalpy: 2670.537696 a.u.

B3LYP free energy: 2670.659122 a.u.

M06-L SCF energy in solution: $\quad 2672.61967628$ a.u.

M06-L enthalpy in solution: $\quad 2672.006454$ a.u.

M06-L free energy in solution: $\quad 2672.127880$ a.u.

Cartesian coordinates

\begin{tabular}{lccc}
\multicolumn{2}{l}{ ATOM X } & Y & \multicolumn{2}{l}{ Z } \\
P & 1.740982 & -0.315637 & -0.245894 \\
P & -1.588006 & -0.997451 & 0.064244 \\
O & -0.056477 & 3.768910 & 1.646409 \\
O & -1.113969 & 3.654195 & 3.704374 \\
O & -1.808177 & 3.572722 & -1.013517
\end{tabular}




$$
\begin{aligned}
& \begin{array}{llll}
\text { O } & -1.029540 & 4.103699 & -3.129250
\end{array} \\
& \begin{array}{llll}
\text { C } & -1.576085 & 0.432816 & 1.194314
\end{array} \\
& \begin{array}{llll}
\text { C } & -0.792499 & 1.567658 & 0.815762
\end{array} \\
& \begin{array}{llll}
\text { C } & -0.721148 & 2.579742 & 1.757476
\end{array} \\
& \begin{array}{llll}
\text { C } & -1.358809 & 2.514226 & 2.999397
\end{array} \\
& \begin{array}{llll}
\text { C } & -2.126217 & 1.429870 & 3.366985
\end{array} \\
& \begin{array}{llll}
\text { C } & -2.219960 & 0.381303 & 2.434162
\end{array} \\
& \begin{array}{llll}
\text { C } & -0.234831 & 4.449699 & 2.898380
\end{array} \\
& \begin{array}{llll}
\text { C } & 0.913309 & 1.072215 & -1.121016
\end{array} \\
& \begin{array}{llll}
\text { C } & 1.362722 & 1.406798 & -2.406438
\end{array} \\
& \begin{array}{llll}
\text { C } & 0.776827 & 2.424949 & -3.176891
\end{array} \\
& \begin{array}{llll}
\text { C } & -0.284020 & 3.093160 & -2.606438
\end{array} \\
& \begin{array}{llll}
\text { C } & -0.745452 & 2.772022 & -1.327743
\end{array} \\
& \begin{array}{llll}
\text { C } & -0.193278 & 1.775663 & -0.538605
\end{array} \\
& \begin{array}{llll}
\text { C } & -1.994344 & 4.457876 & -2.127663
\end{array} \\
& \begin{array}{llll}
\text { C } & -2.505889 & -0.513777 & -1.438290
\end{array} \\
& \begin{array}{llll}
\text { C } & -2.164171 & -1.103054 & -2.665310
\end{array} \\
& \begin{array}{llll}
\text { C } & -3.568299 & 0.402705 & -1.378965
\end{array} \\
& \begin{array}{llll}
\text { C } & -2.520255 & -2.371792 & 0.840836
\end{array} \\
& \begin{array}{llll}
\text { C } & -3.713342 & -2.877913 & 0.298161
\end{array} \\
& \begin{array}{llll}
\text { C } & -1.931986 & -3.029004 & 1.944041
\end{array} \\
& \begin{array}{llll}
\text { C } & 2.329053 & 0.382488 & 1.346635
\end{array} \\
& \begin{array}{llll}
\text { C } & 2.715555 & 1.731407 & 1.430120
\end{array} \\
& \begin{array}{llll}
\text { C } & 2.459062 & -0.440106 & 2.476187
\end{array} \\
& \begin{array}{llll}
\text { C } & 3.154525 & -1.576222 & -2.310805
\end{array} \\
& \begin{array}{llll}
\text { C } & 3.253158 & -0.693584 & -1.220499
\end{array} \\
& \mathrm{H} \quad-2.633043 \quad 1.388973 \quad 4.324318 \\
& \text { H } \quad-2.811699 \quad-0.489095 \quad 2.694438 \\
& \text { H } \quad 0.733988 \quad 4.545620 \quad 3.401137 \\
& \text { H } \quad-0.689285 \quad 5.428519 \quad 2.720065 \\
& \begin{array}{llll}
\text { H } & 2.202119 & 0.872194 & -2.832690
\end{array} \\
& \text { H } \quad 1.143487 \quad 2.671861 \quad-4.166574 \\
& \text { H } \quad-3.002681 \quad 4.327224 \quad-2.532021
\end{aligned}
$$




$$
\begin{aligned}
& \mathrm{H} \quad-1.824545 \quad 5.491348 \quad-1.807811 \\
& \mathrm{H} \quad-1.335776-1.805440-2.715468 \\
& \mathrm{H} \quad-3.831490 \quad 0.872444 \quad-0.435746 \\
& \text { H } \quad-4.173455 \quad-2.390493 \quad-0.554898 \\
& \begin{array}{llll}
\mathrm{H} & 2.608003 & 2.385938 & 0.570912
\end{array} \\
& \text { H } \quad 2.150591 \quad-1.479637 \quad 2.424604 \\
& \text { H } \quad 2.197384 \quad-2.032349 \quad-2.550518 \\
& \begin{array}{llll}
\mathrm{Rh} & 0.456305 & -2.166359 & 0.004385
\end{array} \\
& \begin{array}{llll}
\text { C } & 1.970973 & -3.332487 & 0.238251
\end{array} \\
& \begin{array}{llll}
\text { O } & 2.825558 & -4.075649 & 0.424892
\end{array} \\
& \text { H } \quad-1.018018 \quad-2.636992 \quad 2.385120 \\
& \begin{array}{llll}
\text { C } & 4.278110 & -1.875376 & -3.079960
\end{array} \\
& \mathrm{H} \quad 4.190189 \quad-2.557824 \quad-3.920264 \\
& \begin{array}{llll}
\text { C } & 5.514716 & -1.308064 & -2.761078
\end{array} \\
& \text { H } \quad \begin{array}{llll}
6.392315 & -1.549333 & -3.353659
\end{array} \\
& \begin{array}{llll}
\text { C } & 5.622098 & -0.437997 & -1.675129
\end{array} \\
& \begin{array}{llll}
\text { H } & 6.582818 & -0.000751 & -1.419478
\end{array} \\
& \begin{array}{llll}
\text { C } & 4.498284 & -0.127263 & -0.907026
\end{array} \\
& \mathrm{H} \quad 4.598683 \quad 0.546995 \quad-0.063534 \\
& \begin{array}{llll}
\text { C } & 3.233664 & 2.239095 & 2.622329
\end{array} \\
& \text { H } \quad 3.539196 \quad 3.280434 \quad 2.673961 \\
& \begin{array}{llll}
\text { C } & 3.363482 & 1.412627 & 3.741166
\end{array} \\
& \begin{array}{llll}
\mathrm{H} & 3.766532 & 1.810757 & 4.667933
\end{array} \\
& \begin{array}{llll}
\text { C } & 2.973423 & 0.073879 & 3.666917
\end{array} \\
& \begin{array}{llll}
\text { H } & 3.068899 & -0.571520 & 4.535159
\end{array} \\
& \begin{array}{llll}
\text { C } & -4.312229 & -4.006109 & 0.857741
\end{array}
\end{aligned}
$$

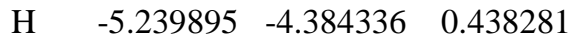

$$
\begin{aligned}
& \text { C } \quad-3.726541 \quad-4.649069 \quad 1.952433 \\
& \text { H } \quad-4.197768 \quad-5.528857 \quad 2.380598 \\
& \begin{array}{llll}
\text { C } & -2.537430 & -4.159891 & 2.495667
\end{array} \\
& \text { H } \quad-2.081368 \quad-4.653462 \quad 3.348813 \\
& \begin{array}{llll}
\text { C } & -4.282829 & 0.715610 & -2.535798
\end{array}
\end{aligned}
$$

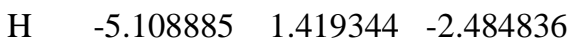




$$
\begin{array}{llll}
\mathrm{C} & -3.941089 & 0.122501 & -3.754434 \\
\mathrm{H} & -4.499500 & 0.368903 & -4.652956 \\
\mathrm{C} & -2.881827 & -0.785726 & -3.818983 \\
\mathrm{H} & -2.612903 & -1.244255 & -4.766044
\end{array}
$$

$2 \mathrm{a}^{\prime \prime}$

B3LYP SCF energy: 1264.89305881 a.u.

B3LYP enthalpy: 1264.498944 a.u.

B3LYP free energy: 1264.579335 a.u.

M06-L SCF energy in solution: $\quad 1265.04972705$ a.u.

M06-L enthalpy in solution: $\quad 1264.655612$ a.u.

M06-L free energy in solution: $\quad 1264.736003$ a.u.

Cartesian coordinates

\begin{tabular}{lrrr} 
ATOM & X & Y & \multicolumn{2}{l}{ Z } \\
O & 1.500819 & -2.227643 & 0.845679 \\
S & 0.675332 & -1.094431 & 1.273376 \\
N & 1.403568 & 0.283454 & 0.627479 \\
O & 0.436278 & -0.796226 & 2.688963 \\
C & -0.932727 & -1.319496 & 0.497681 \\
C & 0.978261 & 1.594823 & 1.153447 \\
C & -2.049464 & -0.654384 & 1.009691 \\
C & -1.051749 & -2.159528 & -0.611410 \\
H & 0.745014 & 1.457381 & 2.212512 \\
H & 1.851813 & 2.254934 & 1.110515 \\
H & -1.950909 & -0.024113 & 1.886293 \\
C & -3.286568 & -0.827642 & 0.394126 \\
C & -2.297282 & -2.318345 & -1.217271 \\
H & -0.183963 & -2.699756 & -0.973818 \\
H & -4.157524 & -0.315939 & 0.796975 \\
C & -3.431787 & -1.658212 & -0.727160
\end{tabular}




$$
\begin{aligned}
& \text { H } \quad-2.390234 \quad-2.974904 \quad-2.079060 \\
& \text { C } \quad-4.785069-1.866163-1.364492 \\
& \text { H } \quad-4.694248 \quad-2.121577 \quad-2.425207 \\
& \text { H } \quad-5.327412 \quad-2.686887 \quad-0.876556 \\
& \text { H } \quad-5.410041 \quad-0.970750 \quad-1.281374 \\
& \begin{array}{llll}
\text { C } & 3.959409 & -0.411852 & -2.151455
\end{array} \\
& \text { H } \quad 3.261460 \quad-0.871638 \quad-2.847844 \\
& \begin{array}{llll}
\mathrm{H} & 4.820617 & 0.044807 & -2.632971
\end{array} \\
& \begin{array}{llll}
\text { C } & 3.406478 & 0.255665 & -0.907375
\end{array} \\
& \begin{array}{llll}
\text { C } & 4.145097 & -1.055662 & -0.797299
\end{array} \\
& \text { H } \quad 5.138634 \quad-1.035896 \quad-0.356190 \\
& \begin{array}{llll}
\text { C } & 4.100901 & 1.509069 & -0.404366
\end{array} \\
& \begin{array}{llll}
\mathrm{H} & 4.000945 & 1.609997 & 0.683123
\end{array} \\
& \text { H } \quad 5.172676 \quad 1.476984 \quad-0.629505 \\
& \begin{array}{llll}
\text { H } & 3.691591 & 2.414277 & -0.873286
\end{array} \\
& \begin{array}{llll}
\text { C } & 1.889374 & 0.230431 & -0.769722
\end{array} \\
& \mathrm{H} \quad 1.444312 \quad 1.079626-1.305569 \\
& \text { H } \quad \begin{array}{llll}
1.502345 & -0.675079 & -1.244248
\end{array} \\
& \text { H } \quad 3.568654 \quad-1.942377 \quad-0.554505 \\
& \begin{array}{llll}
\text { C } & -0.147378 & 2.221508 & 0.443367
\end{array} \\
& \begin{array}{llll}
\text { C } & -1.061797 & 2.737652 & -0.159199
\end{array} \\
& \begin{array}{llll}
\text { C } & -2.190830 & 3.359377 & -0.854751
\end{array} \\
& \text { H } \quad-2.953244 \quad 2.593598 \quad-1.054070 \\
& \text { H } \quad-1.861774 \quad 3.723525 \quad-1.837620 \\
& \begin{array}{llll}
\text { C } & -2.820906 & 4.520319 & -0.061962
\end{array} \\
& \text { H } \quad-2.087546 \quad 5.312261 \quad 0.119554 \\
& \text { H } \quad-3.660274 \quad 4.949119-0.619891 \\
& \begin{array}{llll}
\mathrm{H} & -3.191720 & 4.174702 & 0.908135
\end{array}
\end{aligned}
$$


Spectra

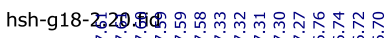

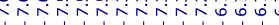

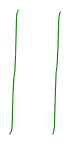

$\int$

${ }^{\mathrm{Me}}$

Ts $-\mathrm{N}-\mathrm{S}$

Me 2a
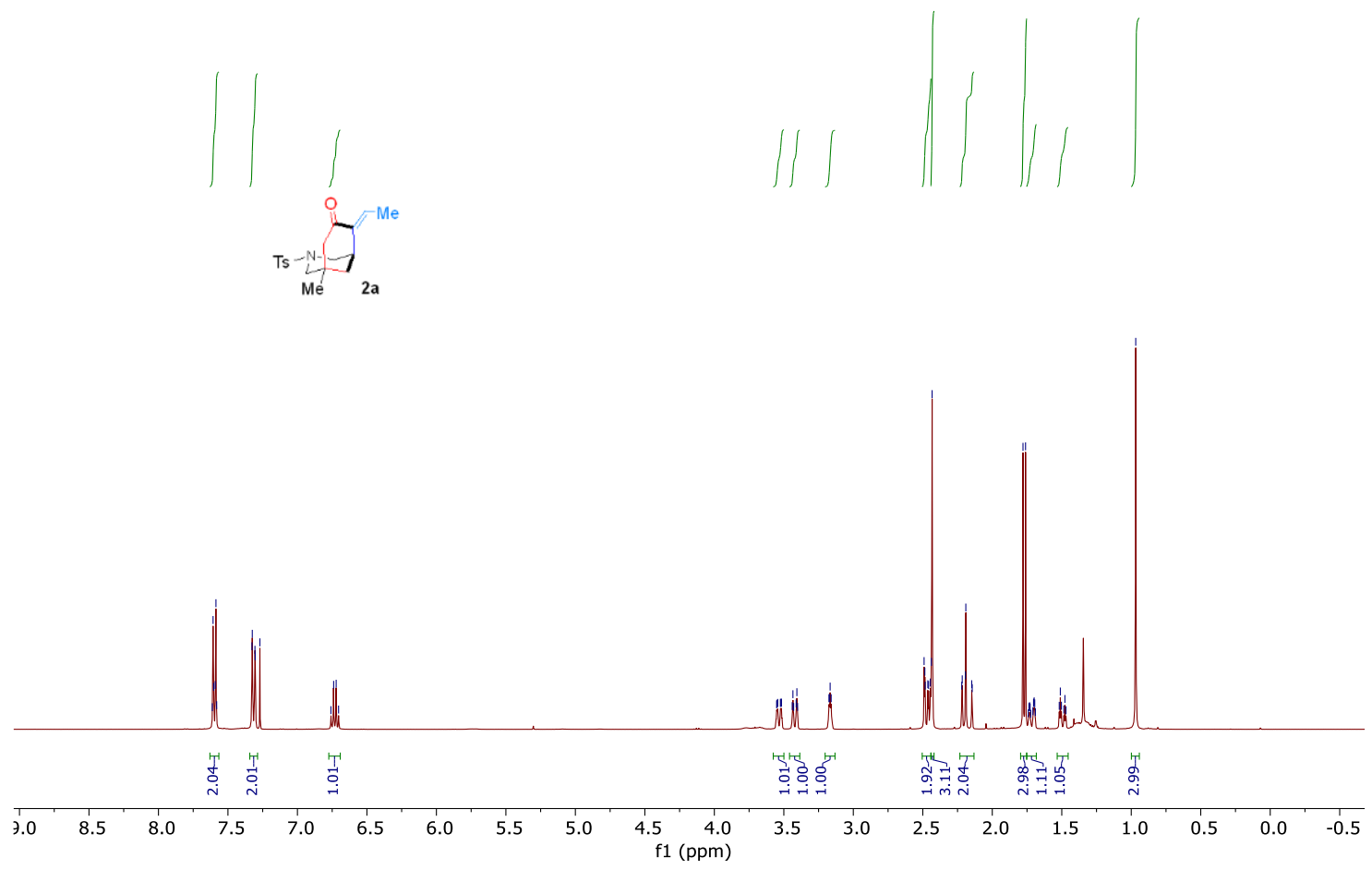

${ }^{13} \mathrm{C}-\mathrm{NMR}$ spectrum of $2 \mathrm{a}$ in $\mathrm{CDCl}_{3}, 101 \mathrm{MHz}$

hsh-g18-2.21.fid

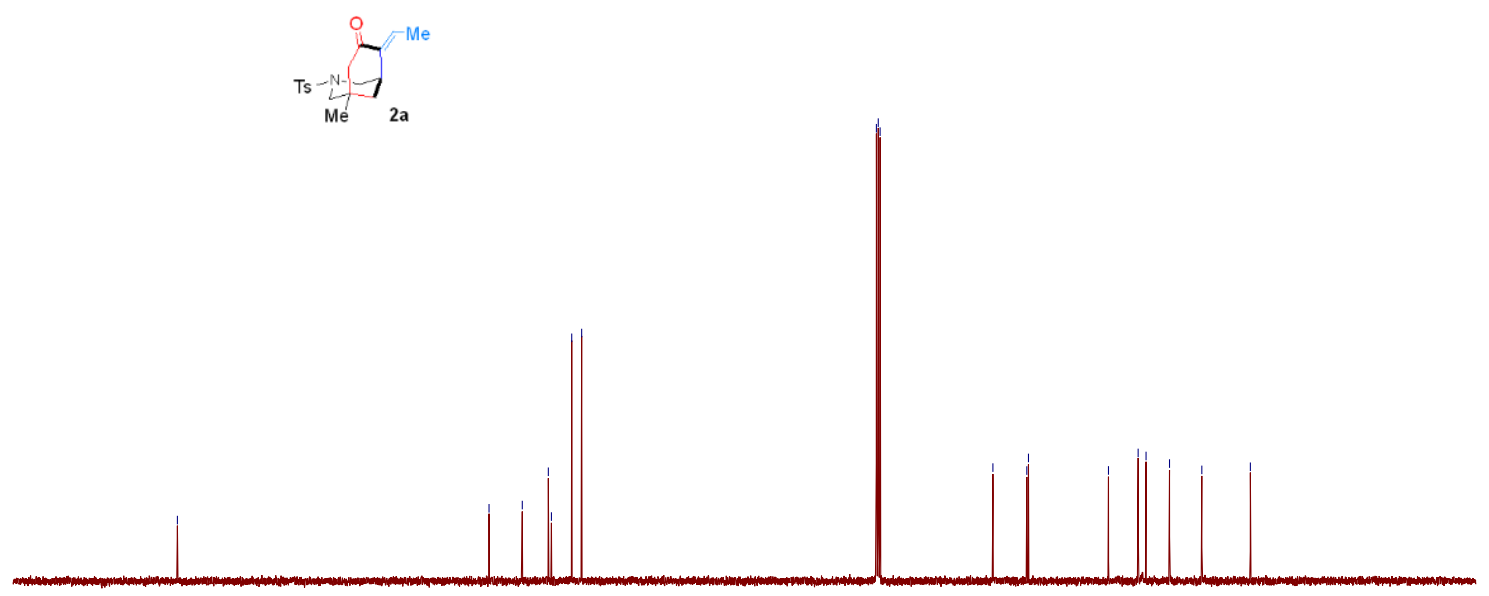

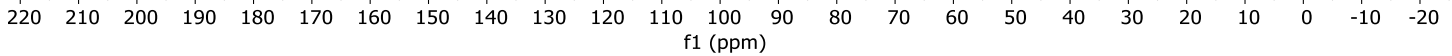




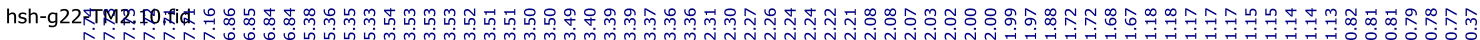

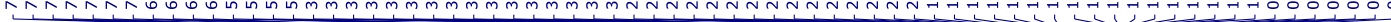

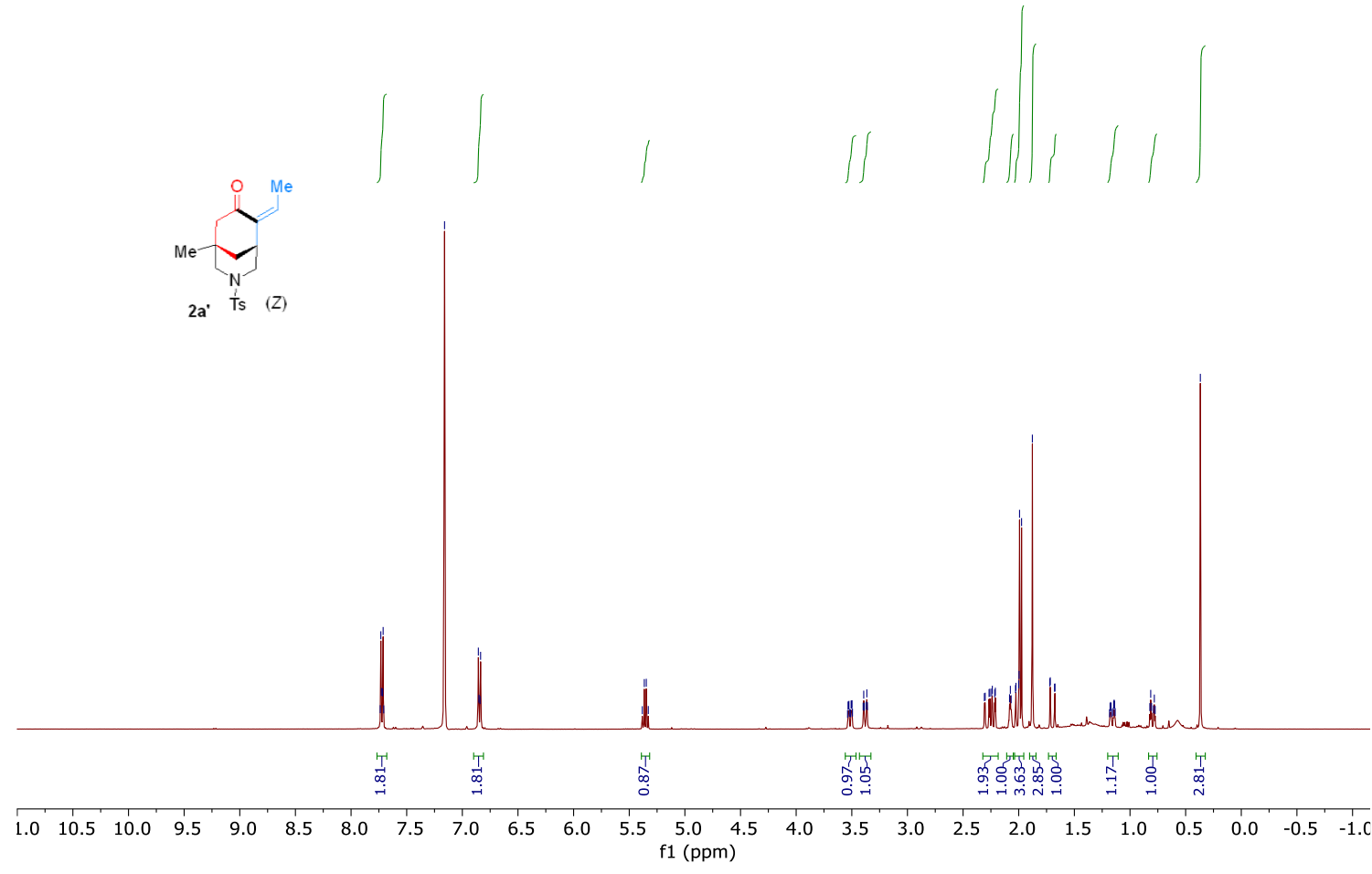

${ }^{13} \mathrm{C}-\mathrm{NMR}$ spectrum of $2 \mathrm{a}^{\prime}$ in $\mathrm{C}_{6} \mathrm{D}_{6}, 101 \mathrm{MHz}$
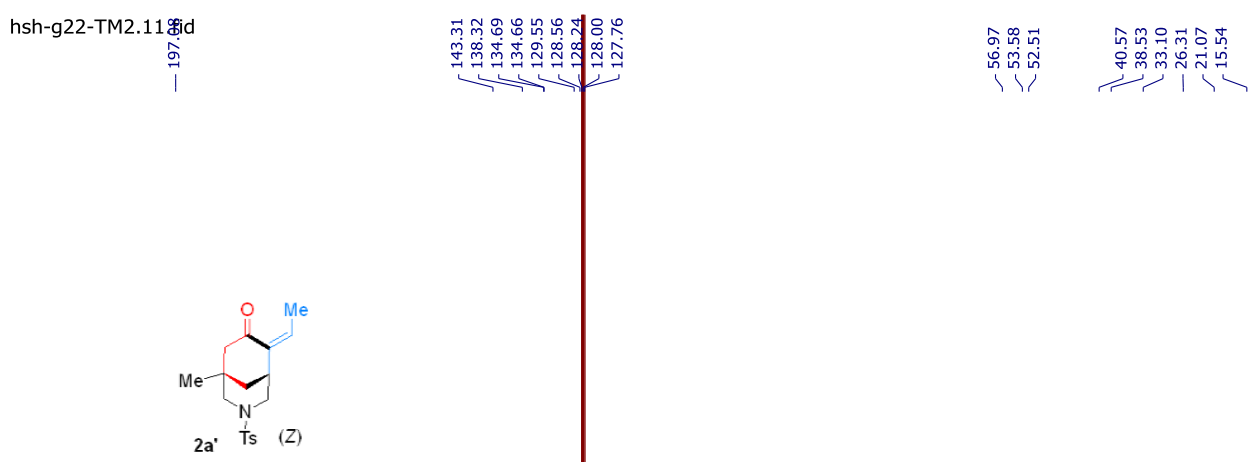

$\begin{array}{lllllllllllllllllllllllllllllllllll}220 & 210 & 200 & 190 & 180 & 170 & 160 & 150 & 140 & 130 & 120 & 110 & 100 & 90 & 80 & 70 & 60 & 50 & 40 & 30 & 20 & 10 & 0 & -10 & -20\end{array}$ 
${ }^{1} \mathrm{H}-\mathrm{NMR}$ spectrum of $2 \mathrm{a}$ " in $\mathrm{CDCl}_{3}, 400 \mathrm{MHz}$

hsh-g164-2.10.fid

i
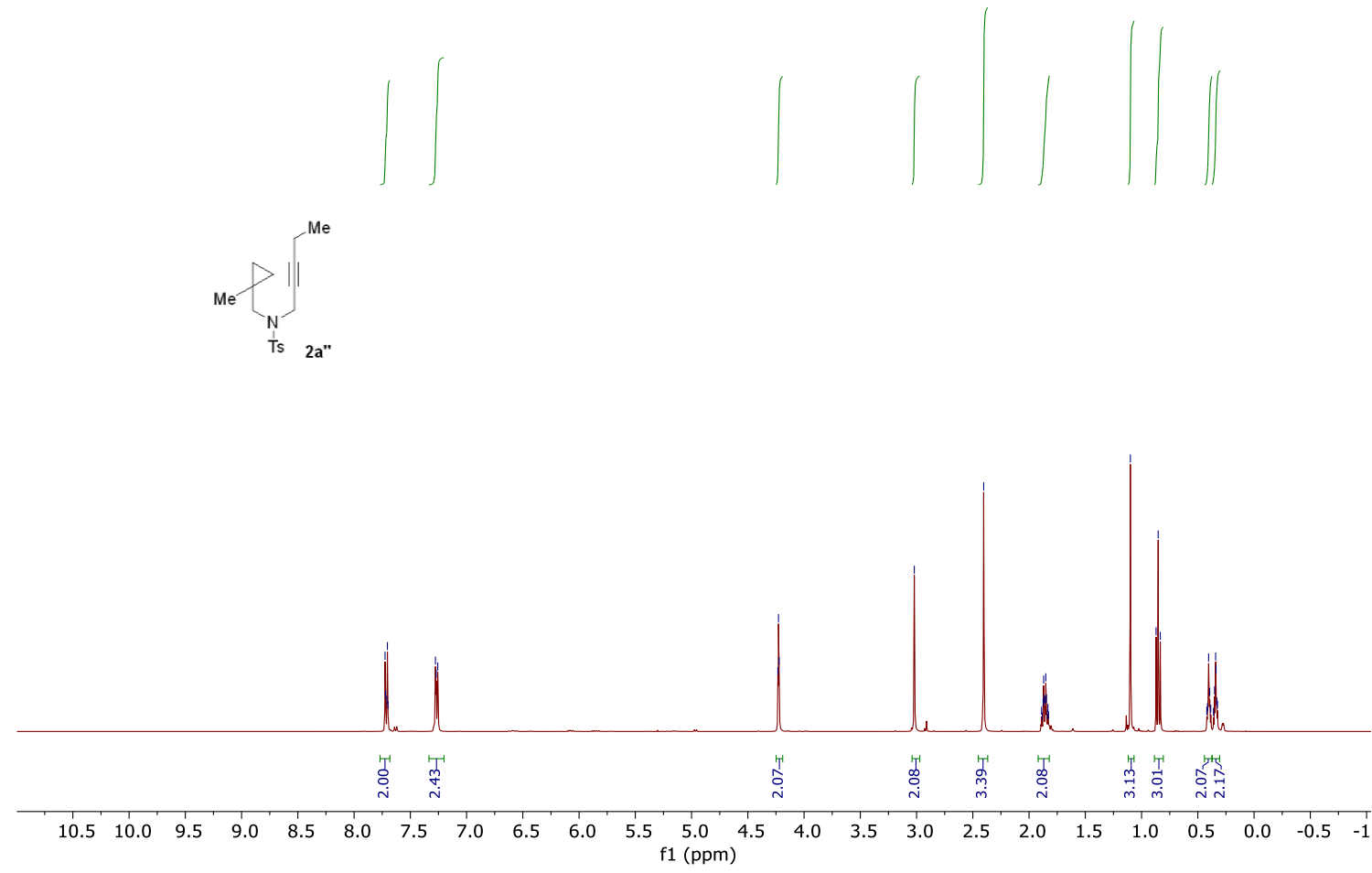

${ }^{13} \mathrm{C}$-NMR spectrum of $2 \mathrm{a}$ " in $\mathrm{CDCl}_{3}, 101 \mathrm{MHz}$

hsh-g164-2.11.fid
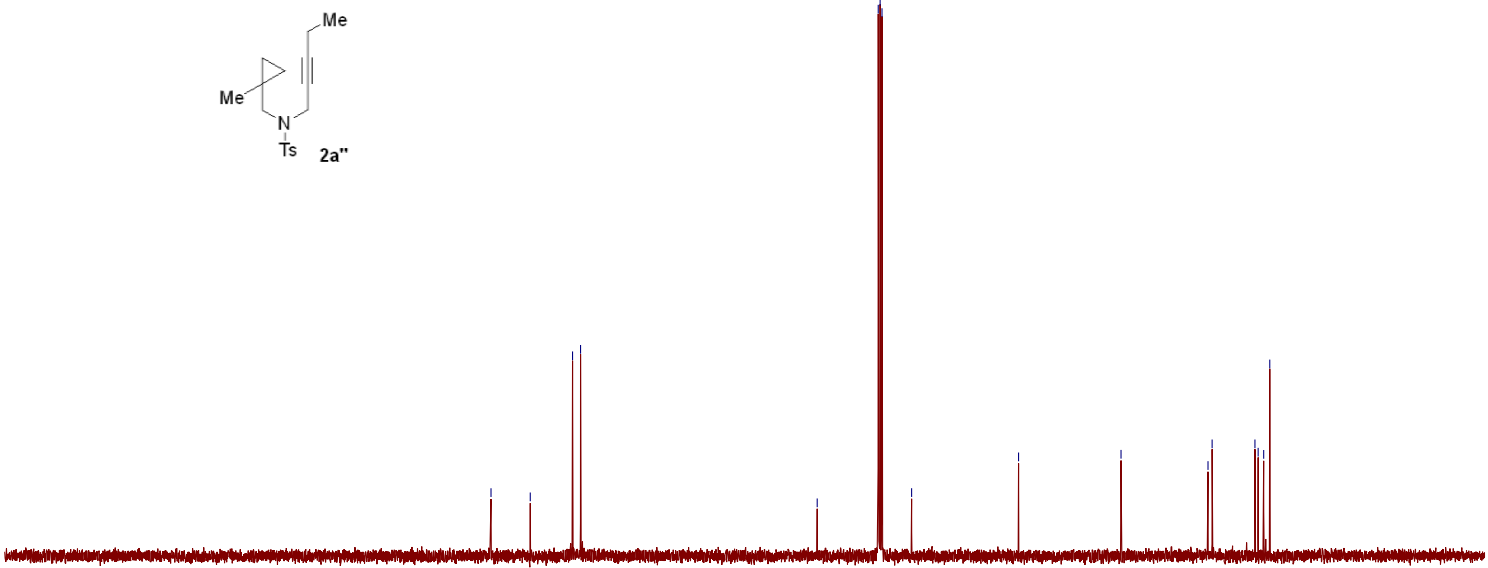

$\begin{array}{lllllllllllllllllllllllll}220 & 210 & 200 & 190 & 180 & 170 & 160 & 150 & 140 & 130 & 120 & 110 & \begin{array}{c}100 \\ \mathrm{f} 1(\mathrm{ppm})\end{array} & 90 & 80 & 70 & 60 & 50 & 40 & 30 & 20 & 10 & 0 & -10 & -20\end{array}$ 


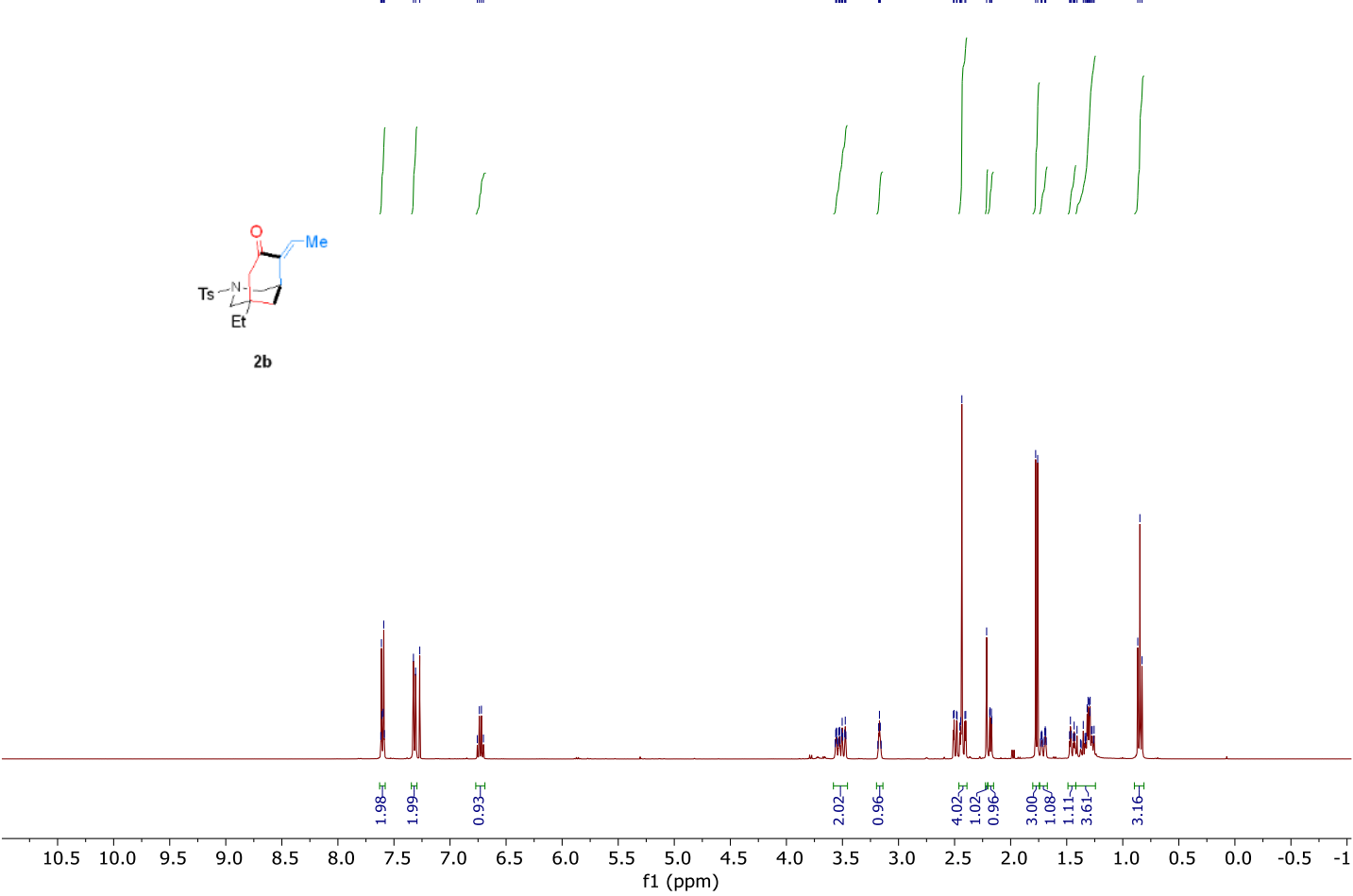

${ }^{13} \mathrm{C}-\mathrm{NMR}$ spectrum of $\mathbf{2} \mathbf{b}$ in $\mathrm{CDCl}_{3}, 101 \mathrm{MHz}$

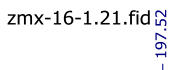
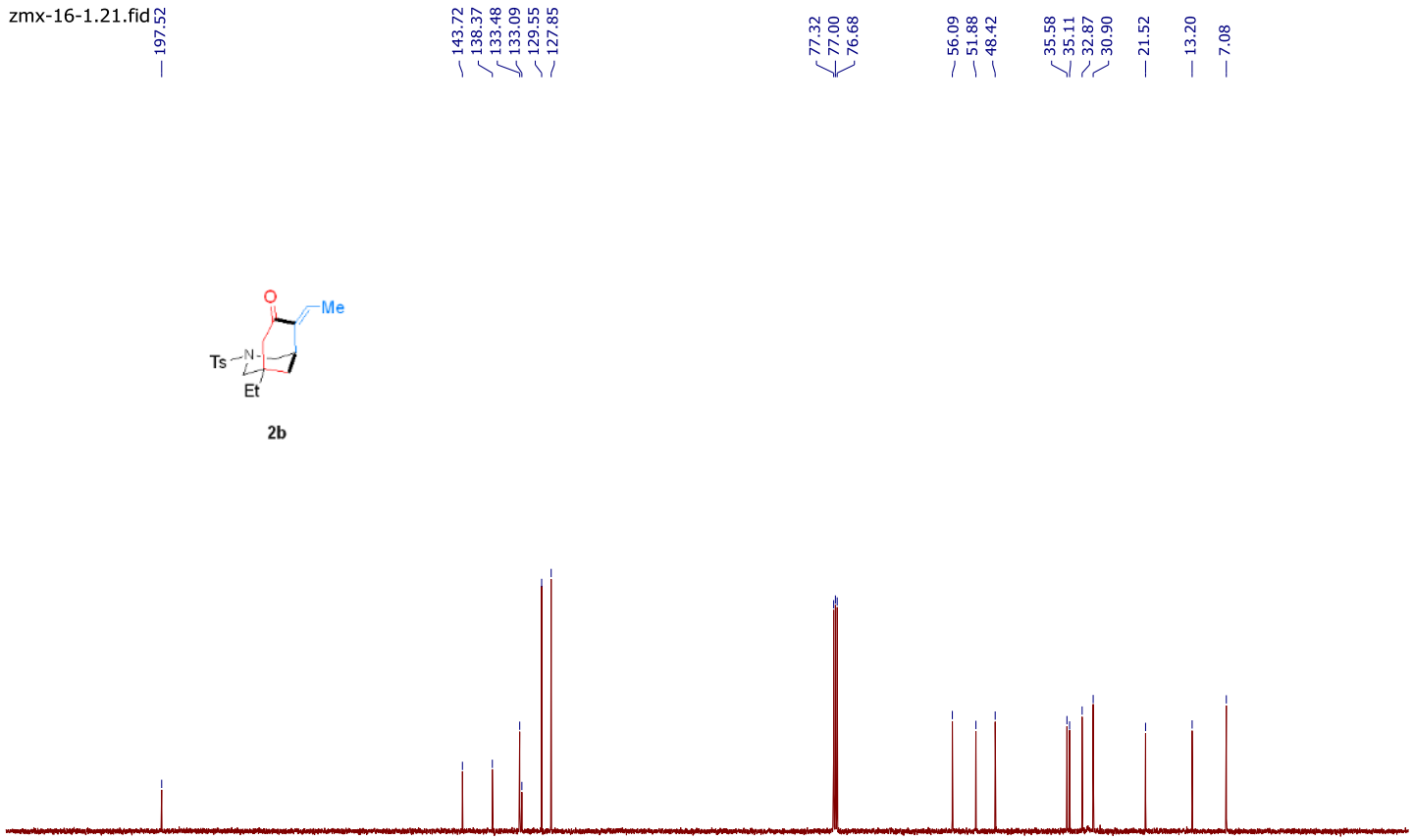

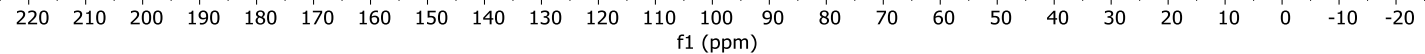




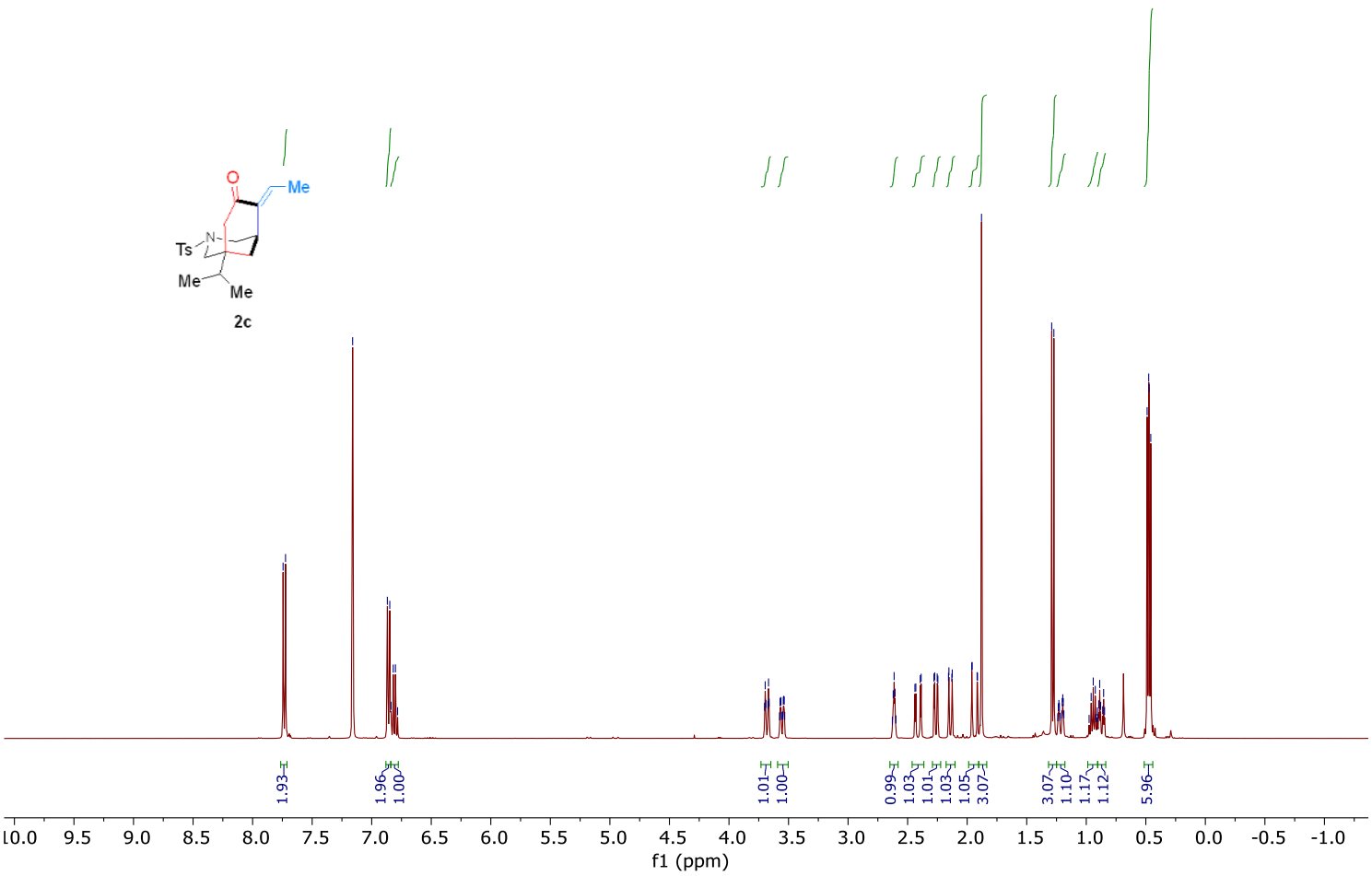

${ }^{13} \mathrm{C}$-NMR spectrum of $2 \mathrm{c}$ in $\mathrm{C}_{6} \mathrm{D}_{6}, 101 \mathrm{MHz}$

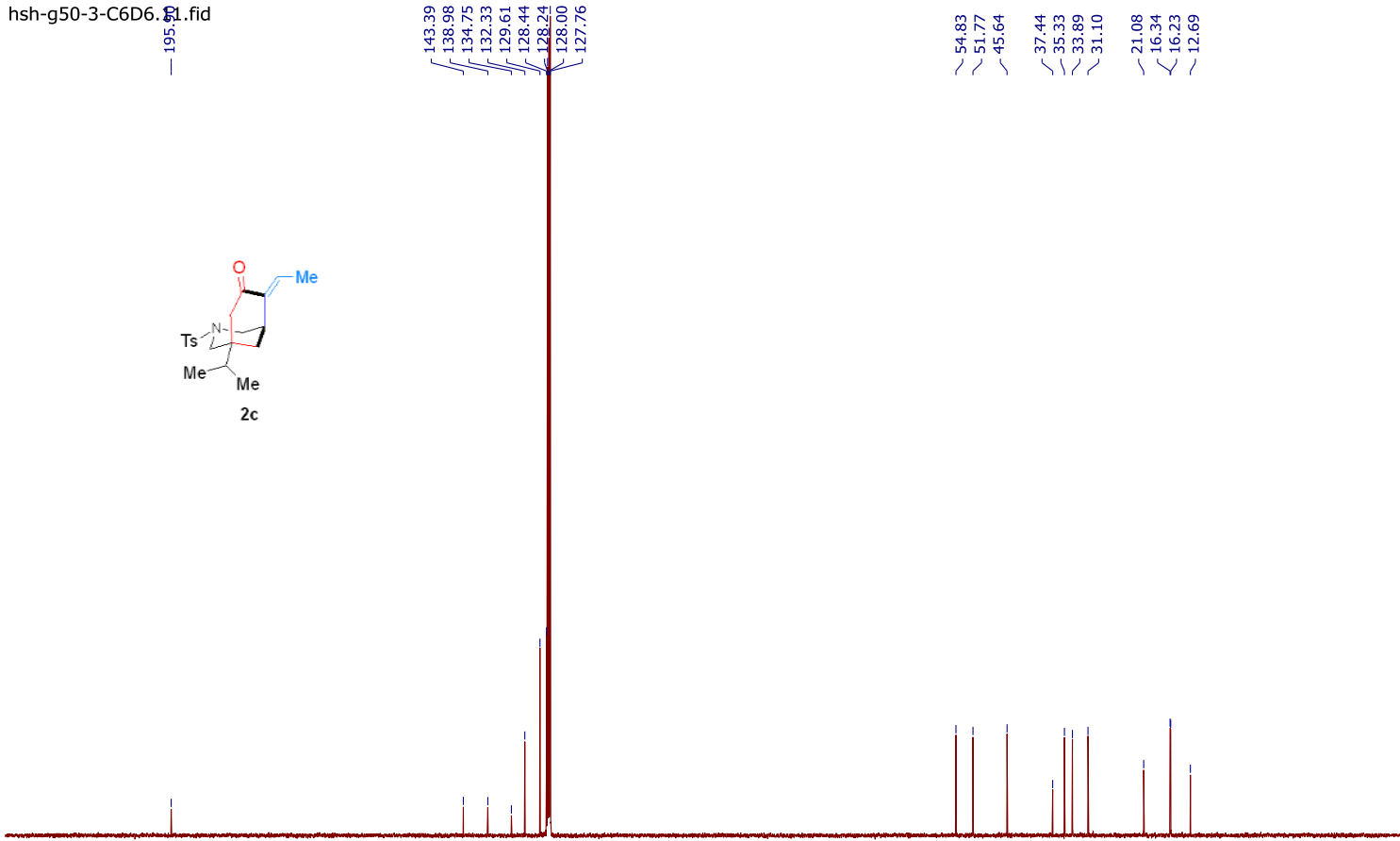

$\begin{array}{llllllllllllllllllllllllllllllllll}220 & 210 & 200 & 190 & 180 & 170 & 160 & 150 & 140 & 130 & 120 & 110 & 100 & 90 & 80 & 70 & 60 & 50 & 40 & 30 & 20 & 10 & 0 & -10 & -20\end{array}$ 


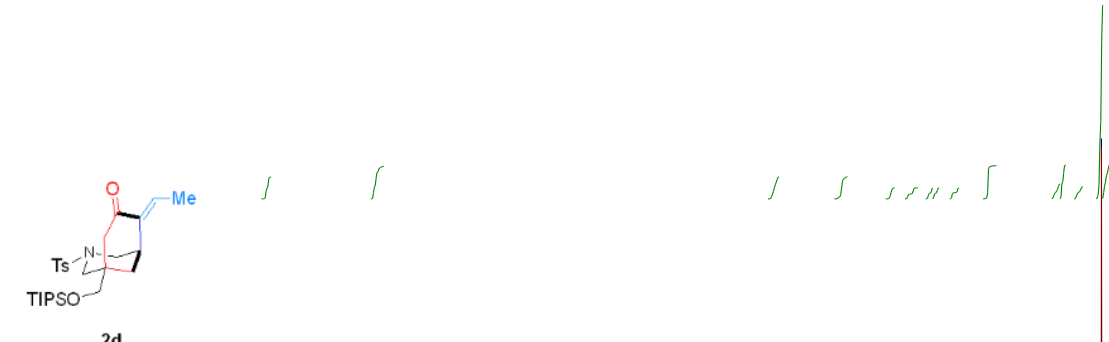

2d

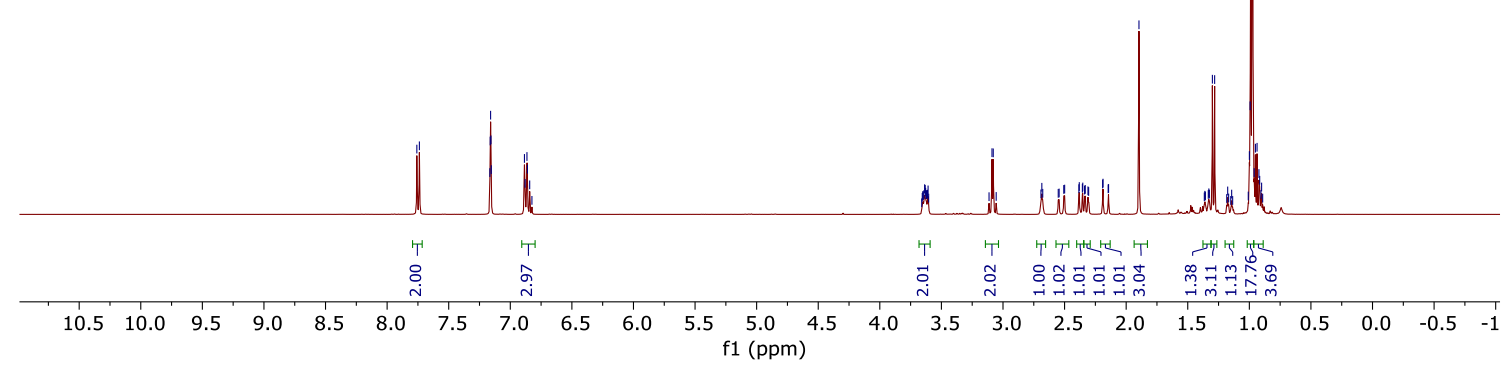

${ }^{13} \mathrm{C}$-NMR spectrum of $\mathbf{2 d}$ in $\mathrm{C}_{6} \mathrm{D}_{6}, 101 \mathrm{MHz}$
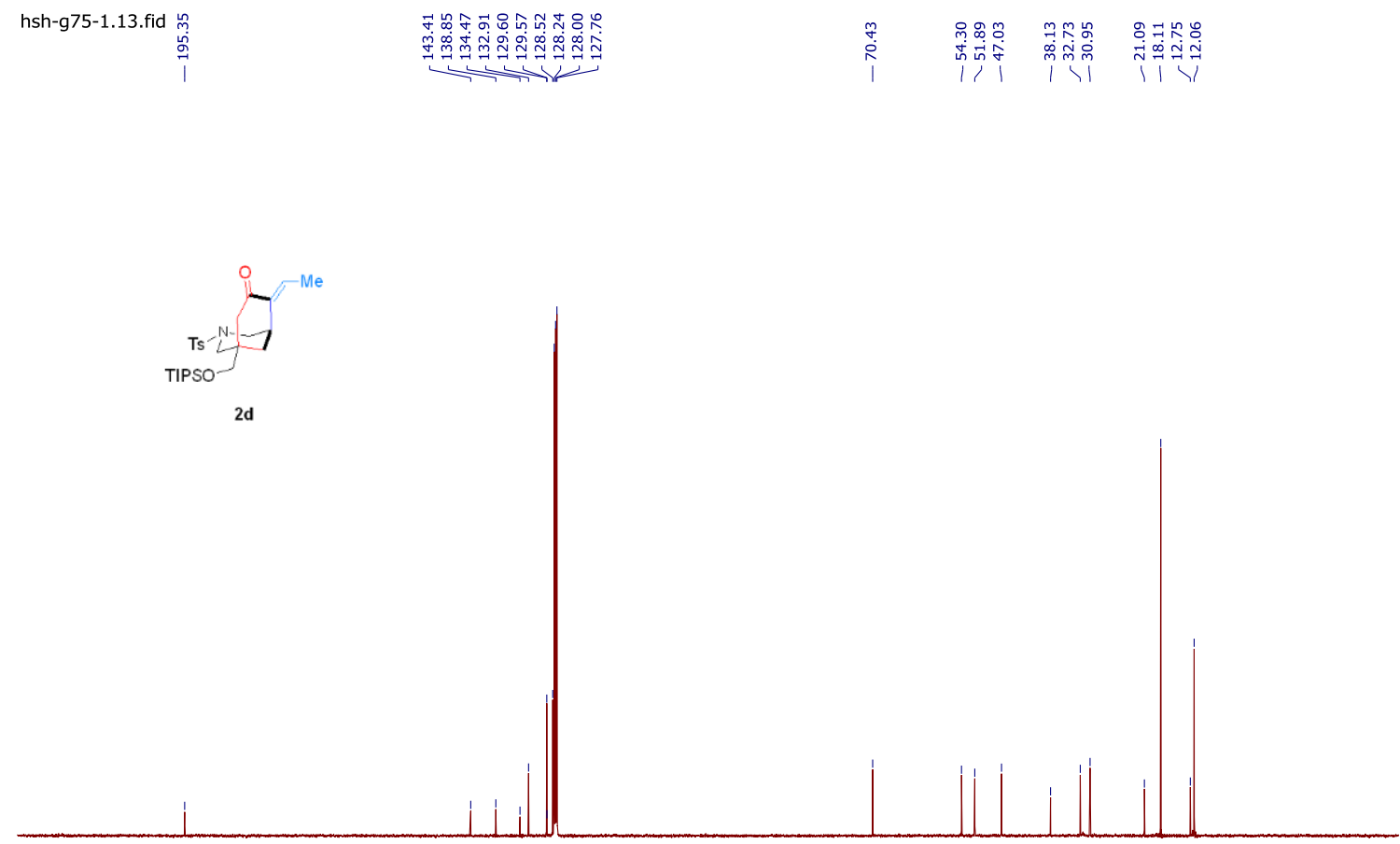

$\begin{array}{llllllllllllllllllllllllllllllllll}220 & 210 & 200 & 190 & 180 & 170 & 160 & 150 & 140 & 130 & 120 & 110 & 100 & 90 & 80 & 70 & 60 & 50 & 40 & 30 & 20 & 10 & 0 & -10 & -20\end{array}$ 
${ }^{1} \mathrm{H}-\mathrm{NMR}$ spectrum of $2 \mathrm{e}$ in $\mathrm{C}_{6} \mathrm{D}_{6}, 400 \mathrm{MHz}$

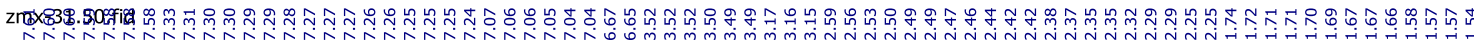

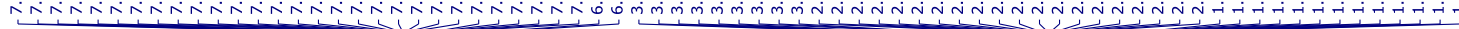
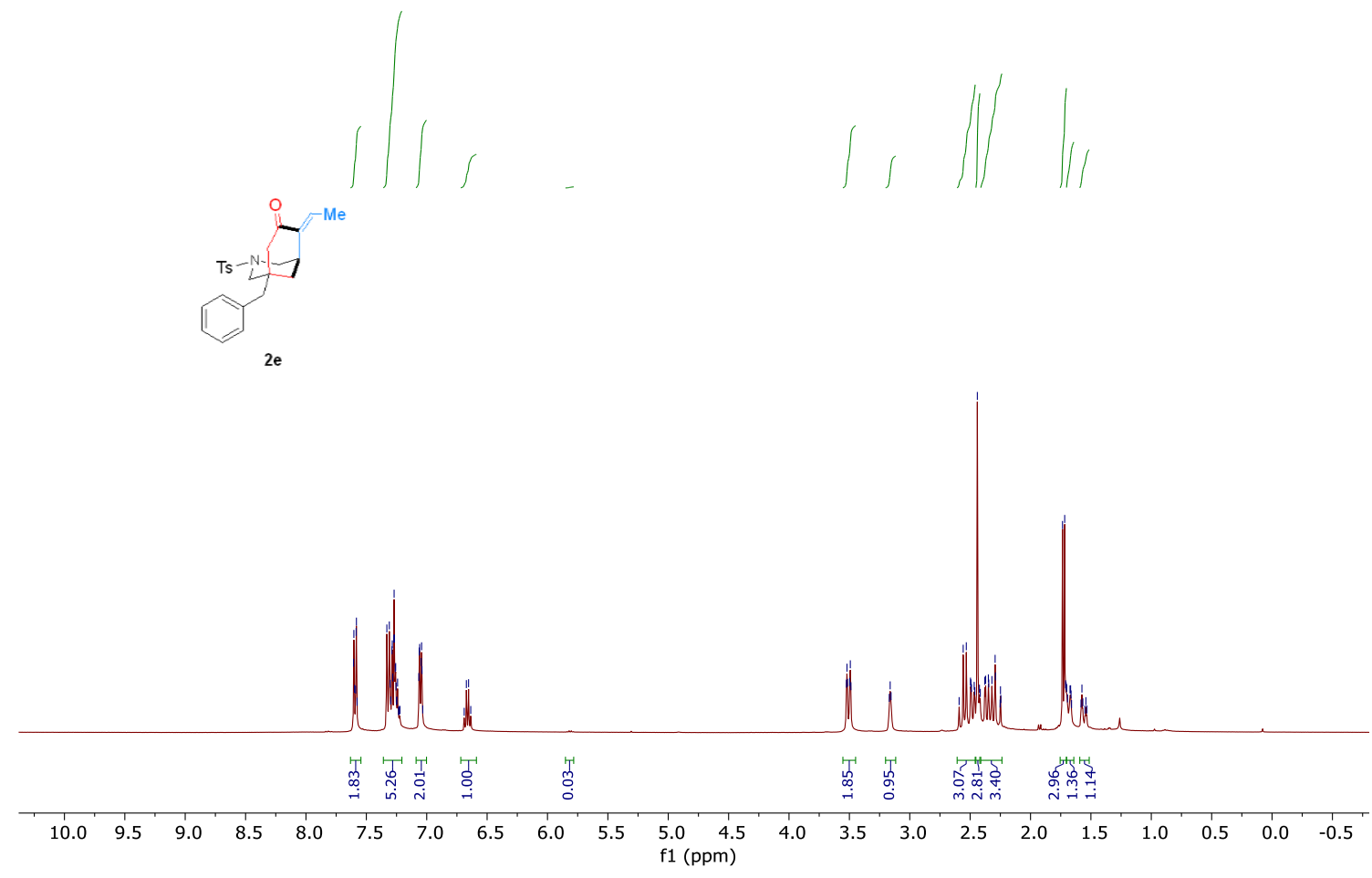

${ }^{13} \mathrm{C}-\mathrm{NMR}$ spectrum of $2 \mathrm{e}$ in $\mathrm{C}_{6} \mathrm{D}_{6}, 101 \mathrm{MHz}$

\begin{tabular}{|c|c|c|c|c|c|}
\hline zmx-31.11.fid & 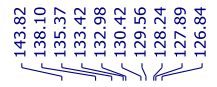 & 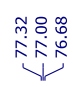 & 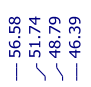 & 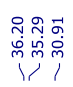 & $\stackrel{\substack{n \\
\sim}}{1}$ \\
\hline
\end{tabular}
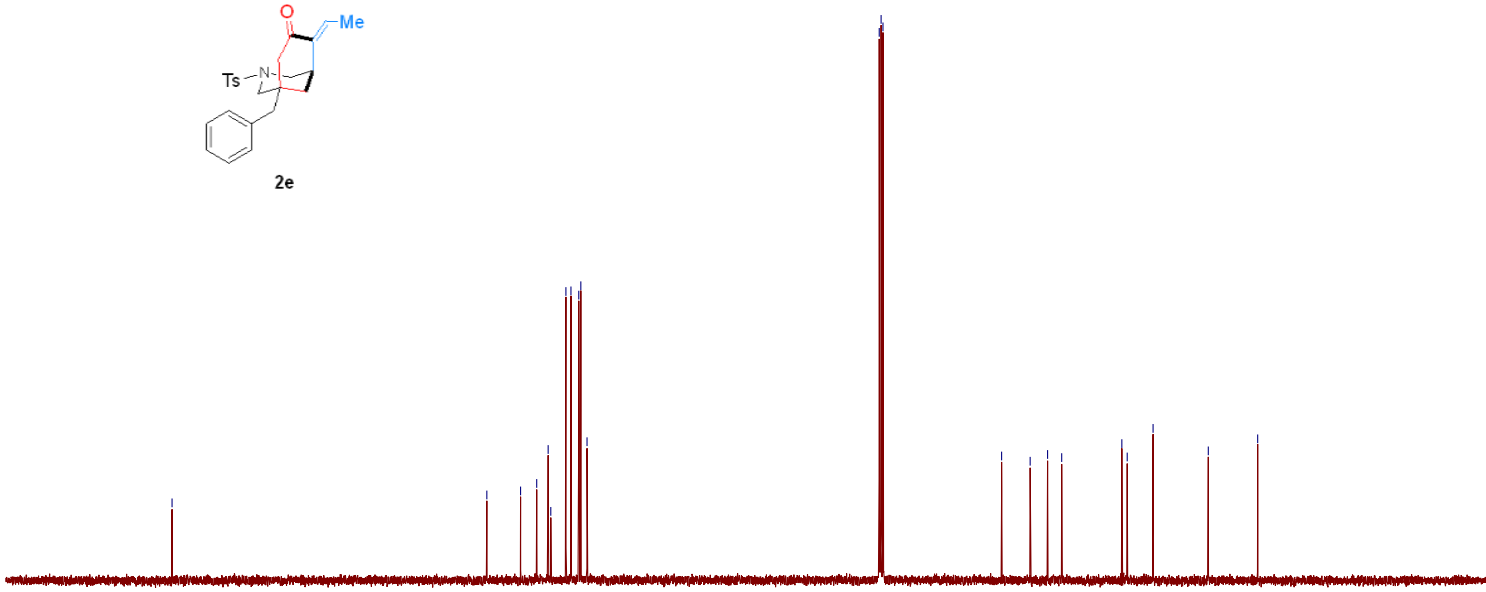

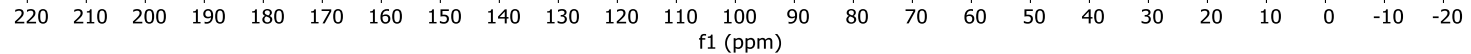




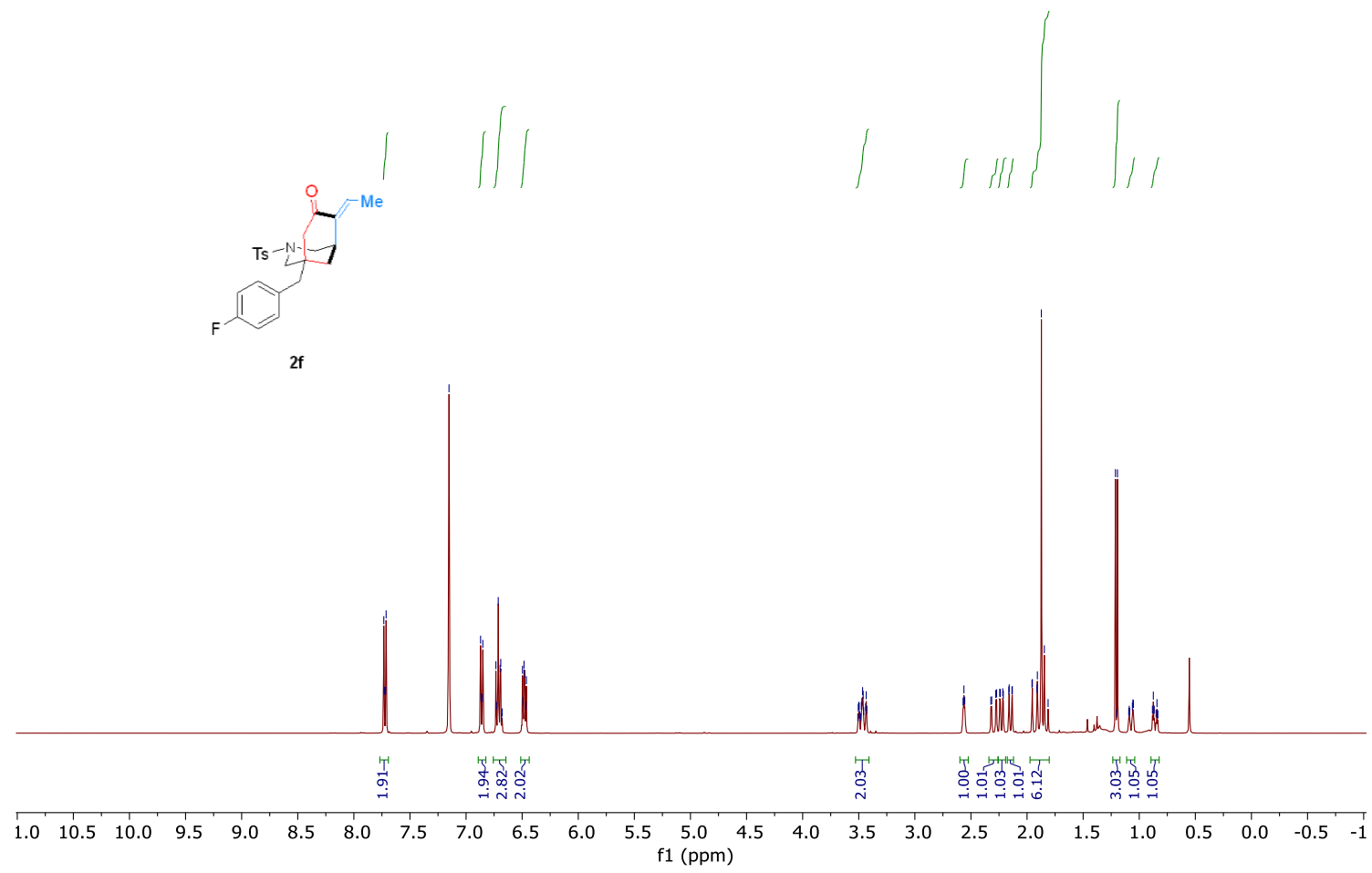

${ }^{13} \mathrm{C}$-NMR spectrum of $2 \mathrm{f}$ in $\mathrm{C}_{6} \mathrm{D}_{6}, 101 \mathrm{MHz}$

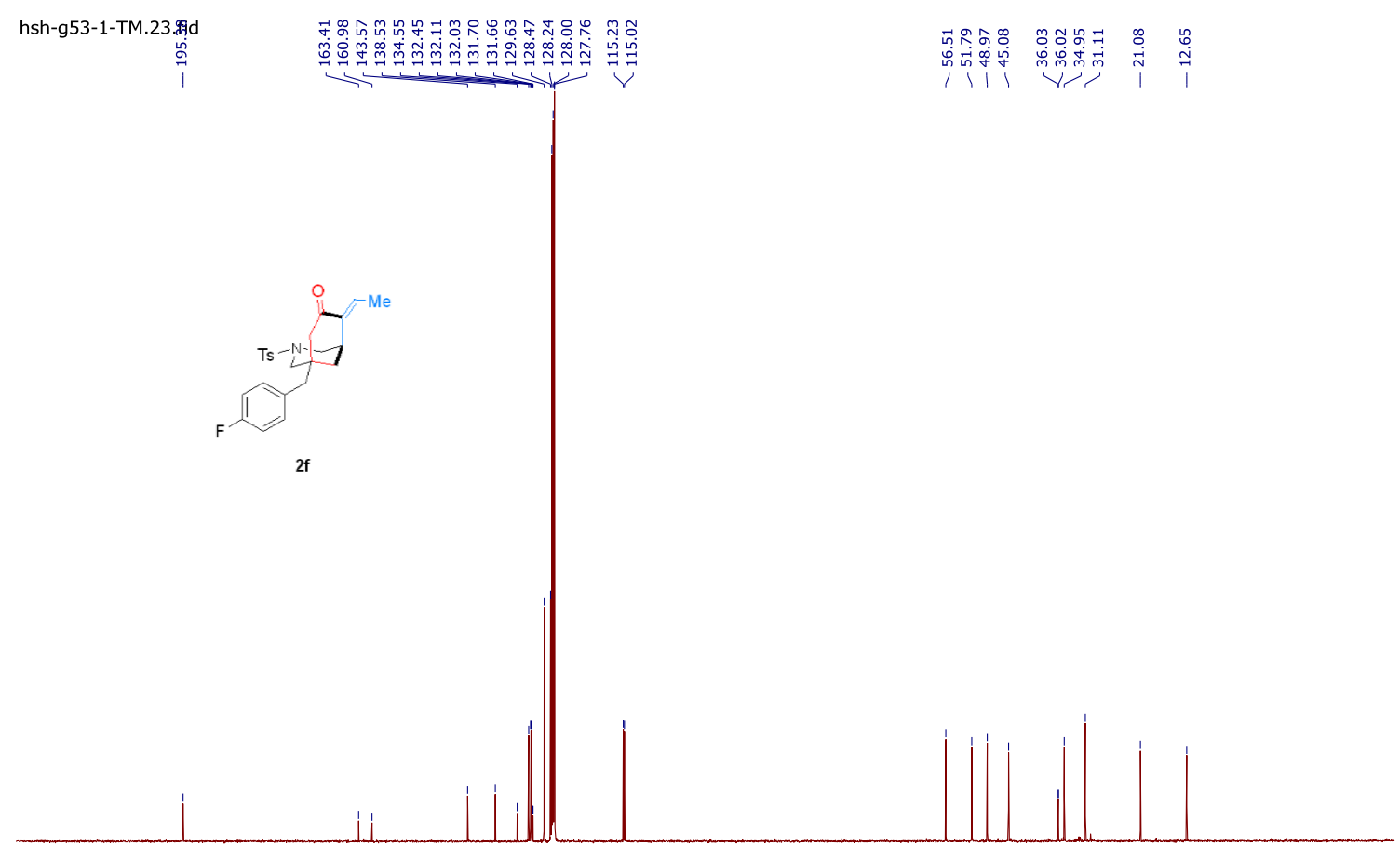

$\begin{array}{llllllllllllllllllllllllllllllllllll}220 & 210 & 200 & 190 & 180 & 170 & 160 & 150 & 140 & 130 & 120 & 110 & 100 & 90 & 80 & 70 & 60 & 50 & 40 & 30 & 20 & 10 & 0 & -10 & -20\end{array}$ 


\section{${ }^{1} \mathrm{H}-\mathrm{NMR}$ spectrum of $\mathbf{2 g}$ in $\mathrm{C}_{6} \mathrm{D}_{6}, 400 \mathrm{MHz}$}

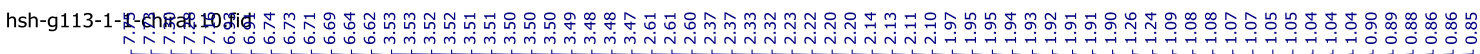

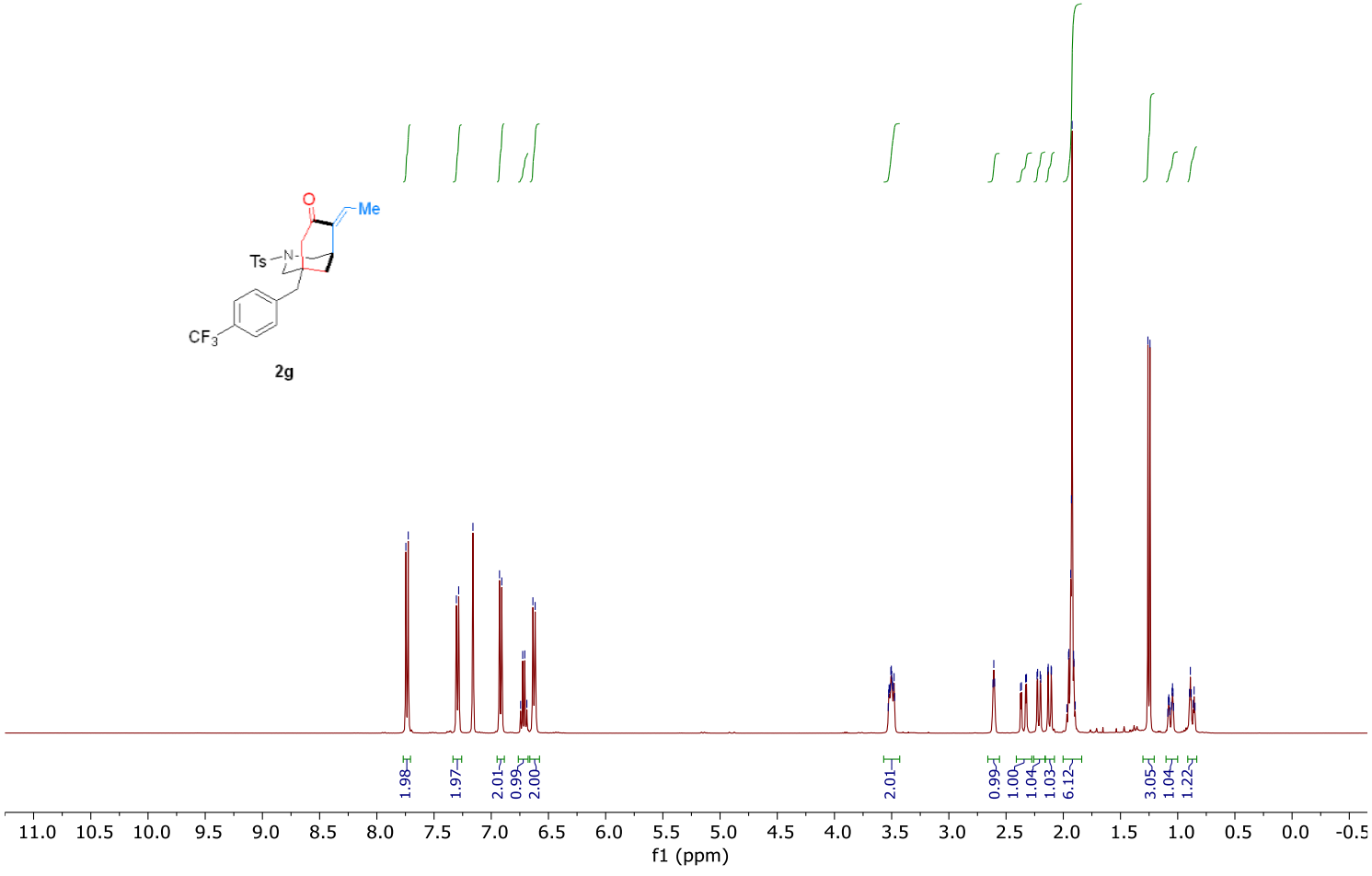

${ }^{19} \mathrm{~F}-\mathrm{NMR}$ spectrum of $\mathbf{2 g}$ in $\mathrm{C}_{6} \mathrm{D}_{6}, 376 \mathrm{MHz}$

hsh-g113-2-rac.12.fid

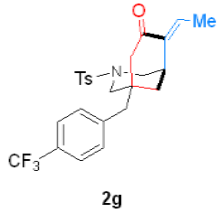

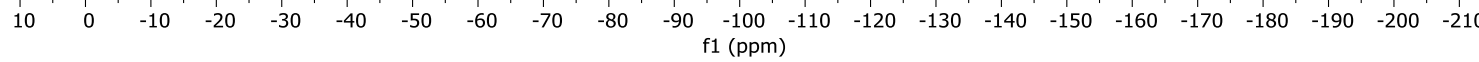


${ }^{13} \mathrm{C}-N M R$ spectrum of $\mathbf{2 g}$ in $\mathrm{C}_{6} \mathrm{D}_{6}, 101 \mathrm{MHz}$
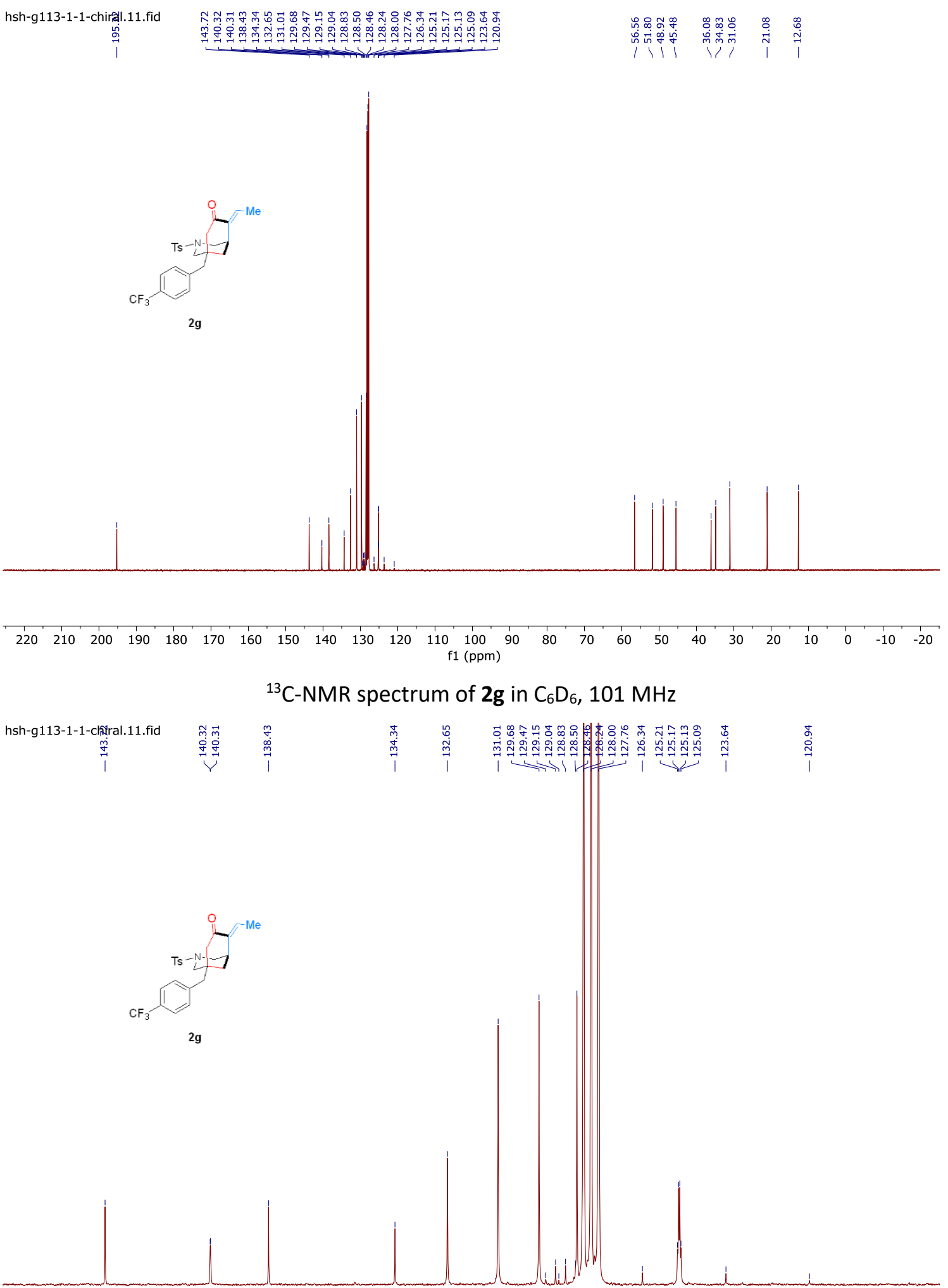

$\begin{array}{llllllllllllllllllllllllllllllll}47 & 146 & 145 & 144 & 143 & 142 & 141 & 140 & 139 & 138 & 137 & 136 & 135 & 134 & 133 & 132 & 131 & 130 & 129 & 128 & 127 & 126 & 125 & 124 & 123 & 122 & 121 & 120 & 119 & 118 & 11\end{array}$ f1 (ppm) 


\section{${ }^{1} \mathrm{H}-\mathrm{NMR}$ spectrum of $\mathbf{2} \mathrm{h}$ in $\mathrm{C}_{6} \mathrm{D}_{6}, 400 \mathrm{MHz}$}

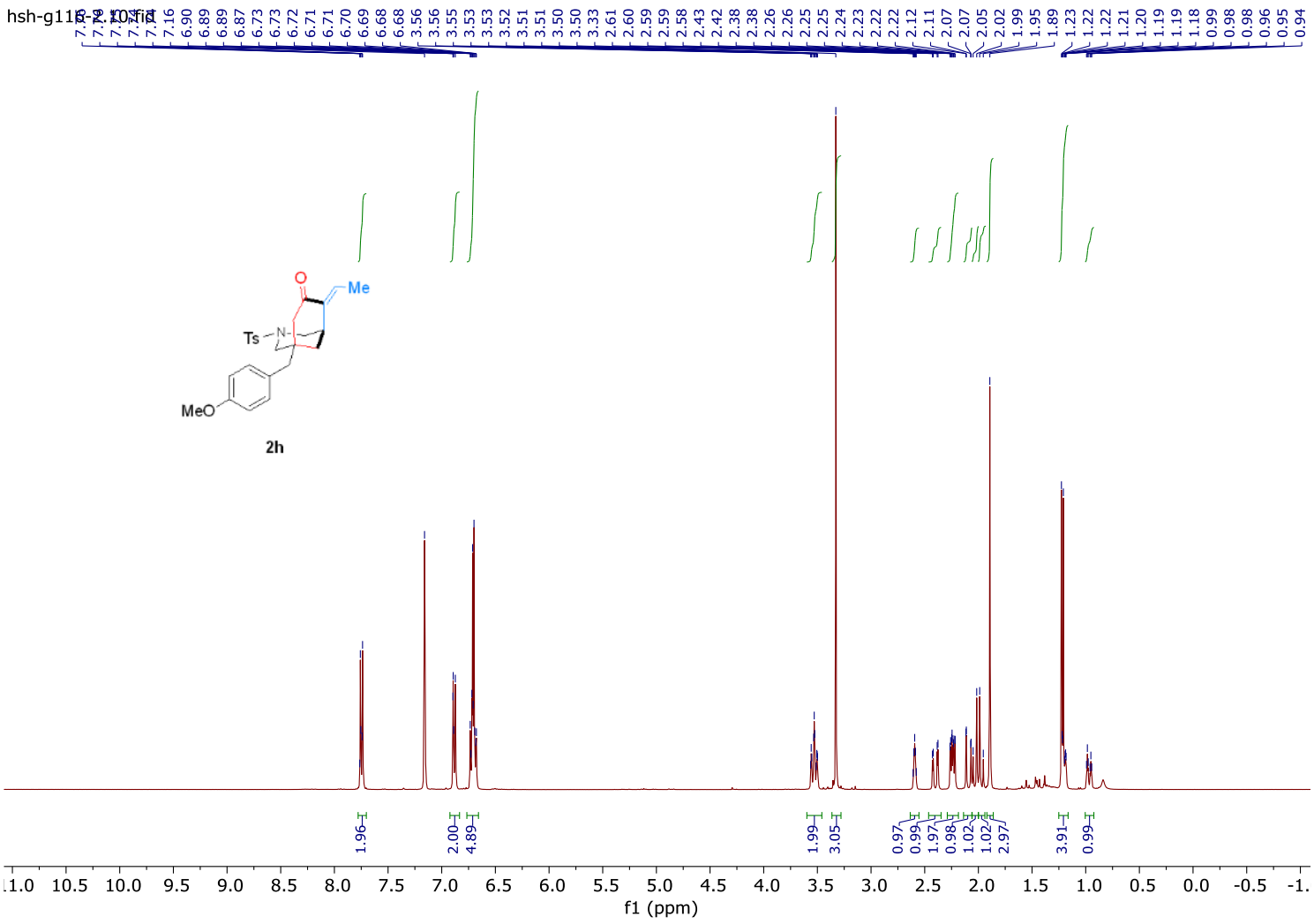

${ }^{13} \mathrm{C}$-NMR spectrum of $\mathbf{2} \mathbf{h}$ in $\mathrm{C}_{6} \mathrm{D}_{6}, 101 \mathrm{MHz}$

hsh-f116-1.12.fidm

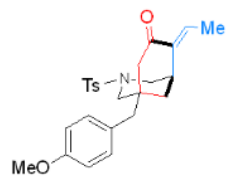

$2 h$

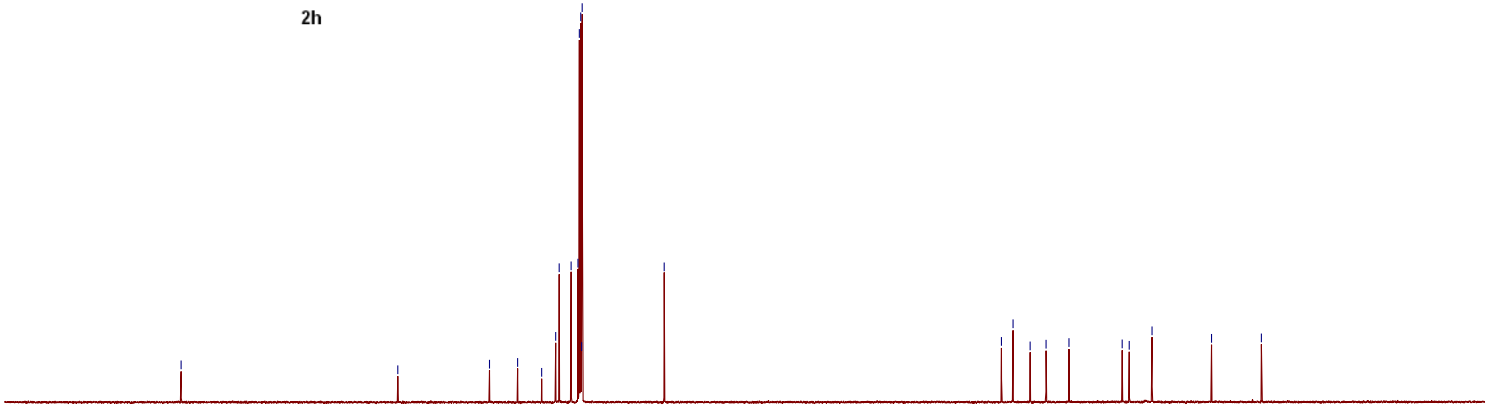

$\begin{array}{llllllllllllllllllllllllllllllll}220 & 210 & 200 & 190 & 180 & 170 & 160 & 150 & 140 & 130 & 120 & 110 & 100 & 90 & 80 & 70 & 60 & 50 & 40 & 30 & 20 & 10 & 0 & -10 & -20\end{array}$ 


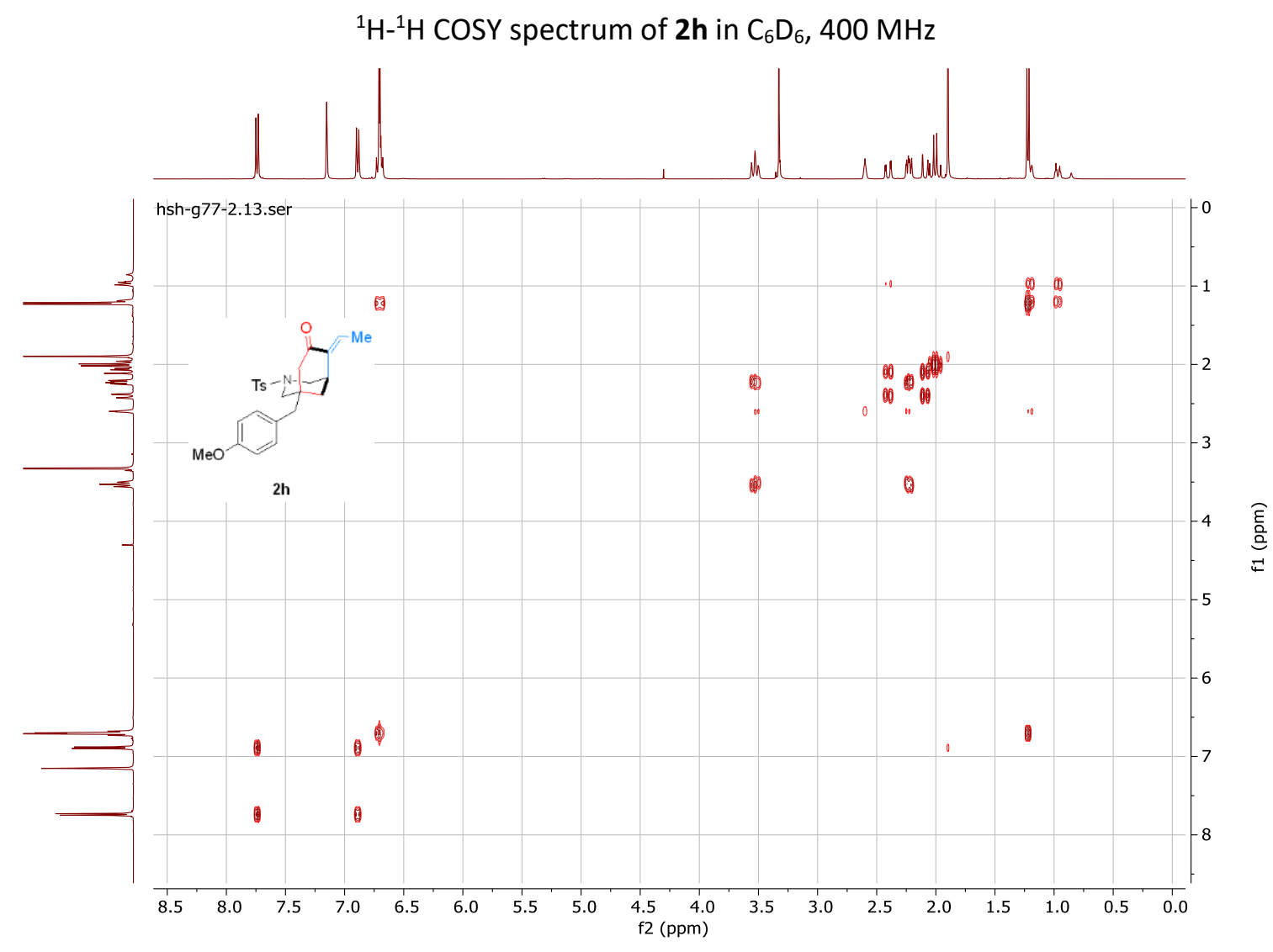

HSQC spectrum of $\mathbf{2} \mathrm{h}$ in $\mathrm{C}_{6} \mathrm{D}_{6}, 400 \mathrm{MHz}$

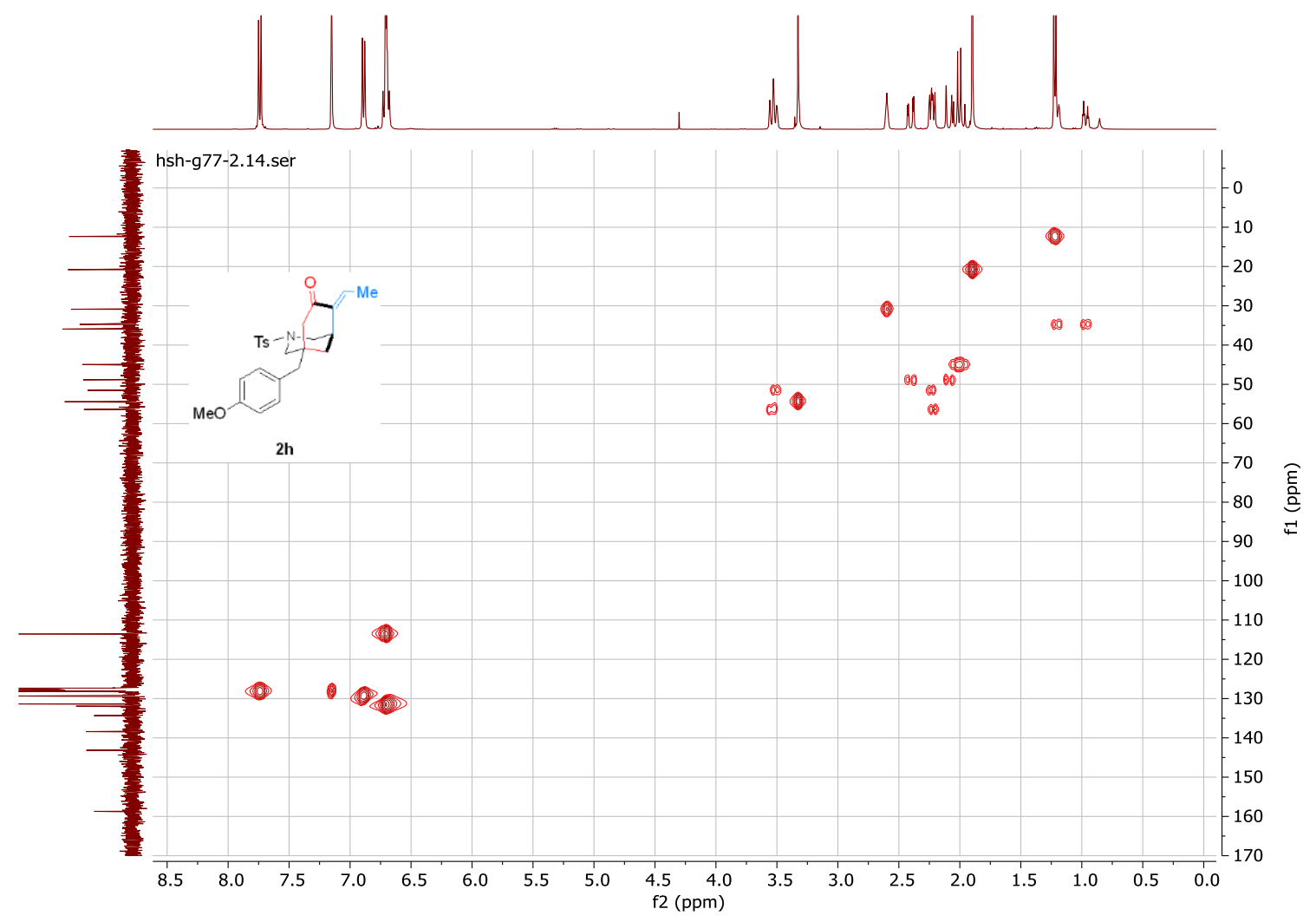




\section{${ }^{1} \mathrm{H}-\mathrm{NMR}$ spectrum of $2 \mathbf{i}$ in $\mathrm{C}_{6} \mathrm{D}_{6}, 400 \mathrm{MHz}$}

hsh-g157-2.10.fid

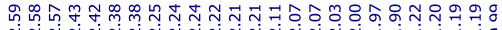
$Y$

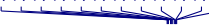

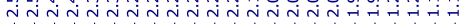

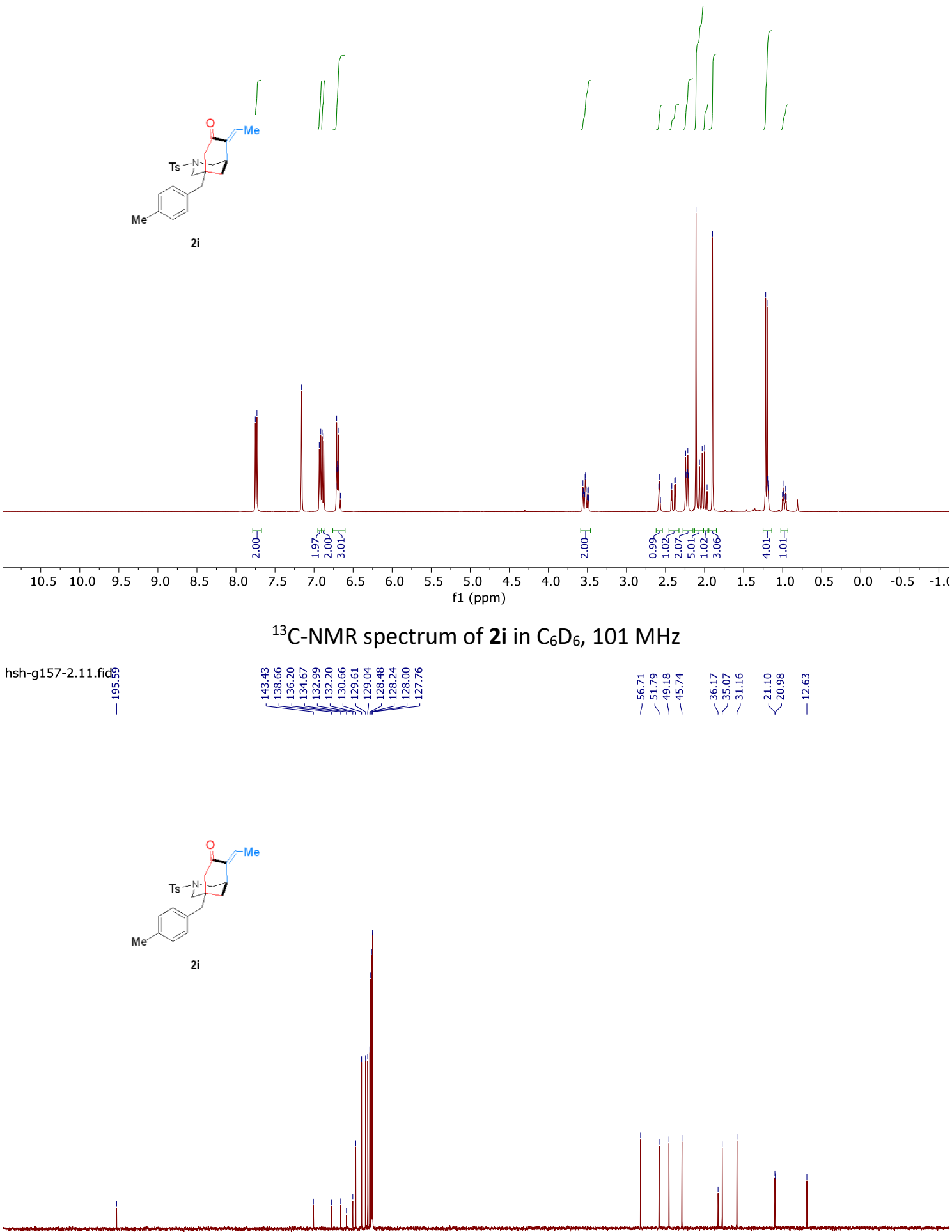

$\begin{array}{llllllllllllllllllllllllllllllllllll}220 & 210 & 200 & 190 & 180 & 170 & 160 & 150 & 140 & 130 & 120 & 110 & 100 & 90 & 80 & 70 & 60 & 50 & 40 & 30 & 20 & 10 & 0 & -10 & -20\end{array}$ 


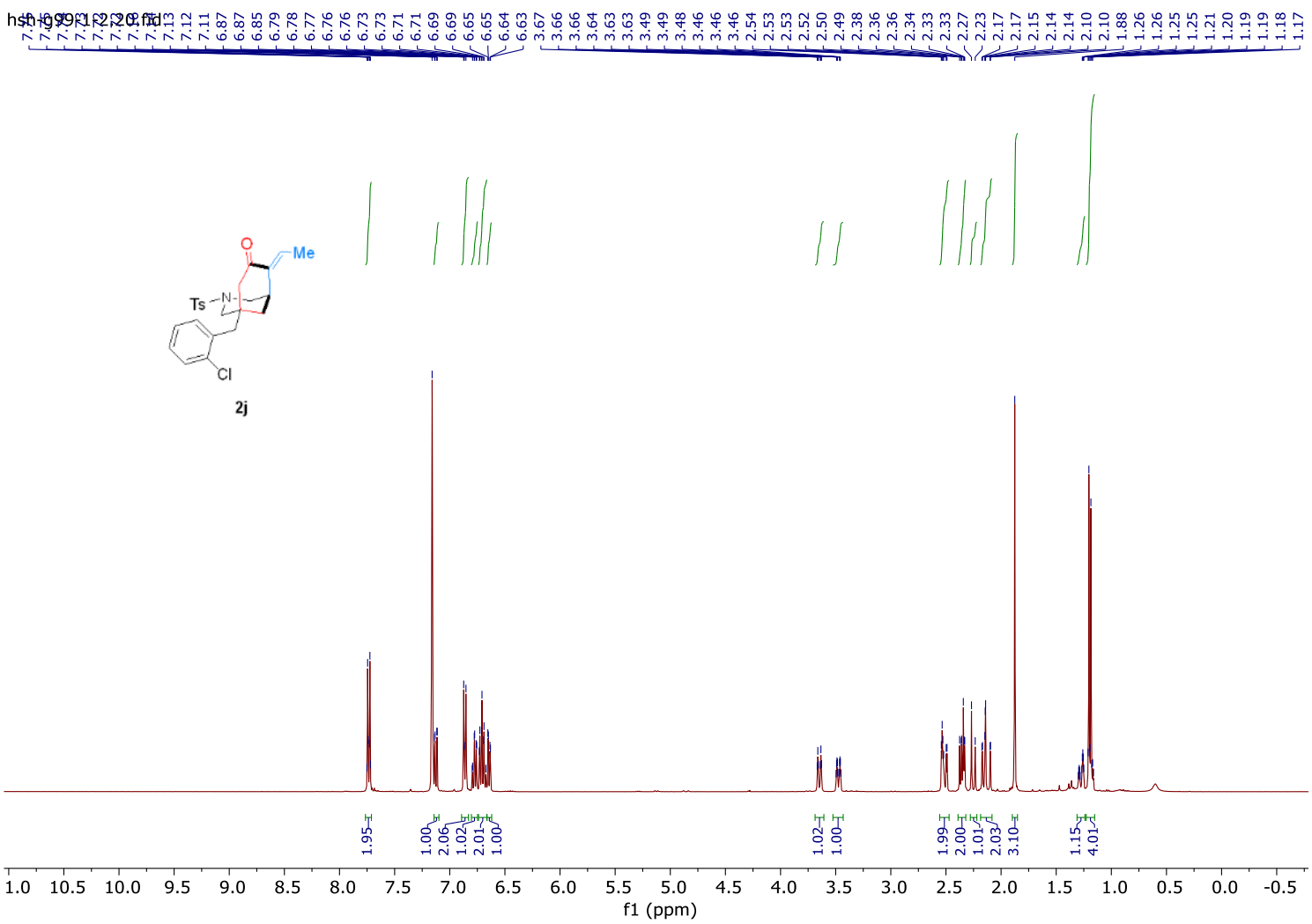

${ }^{13} \mathrm{C}-\mathrm{NMR}$ spectrum of $2 \mathrm{j}$ in $\mathrm{C}_{6} \mathrm{D}_{6}, 101 \mathrm{MHz}$

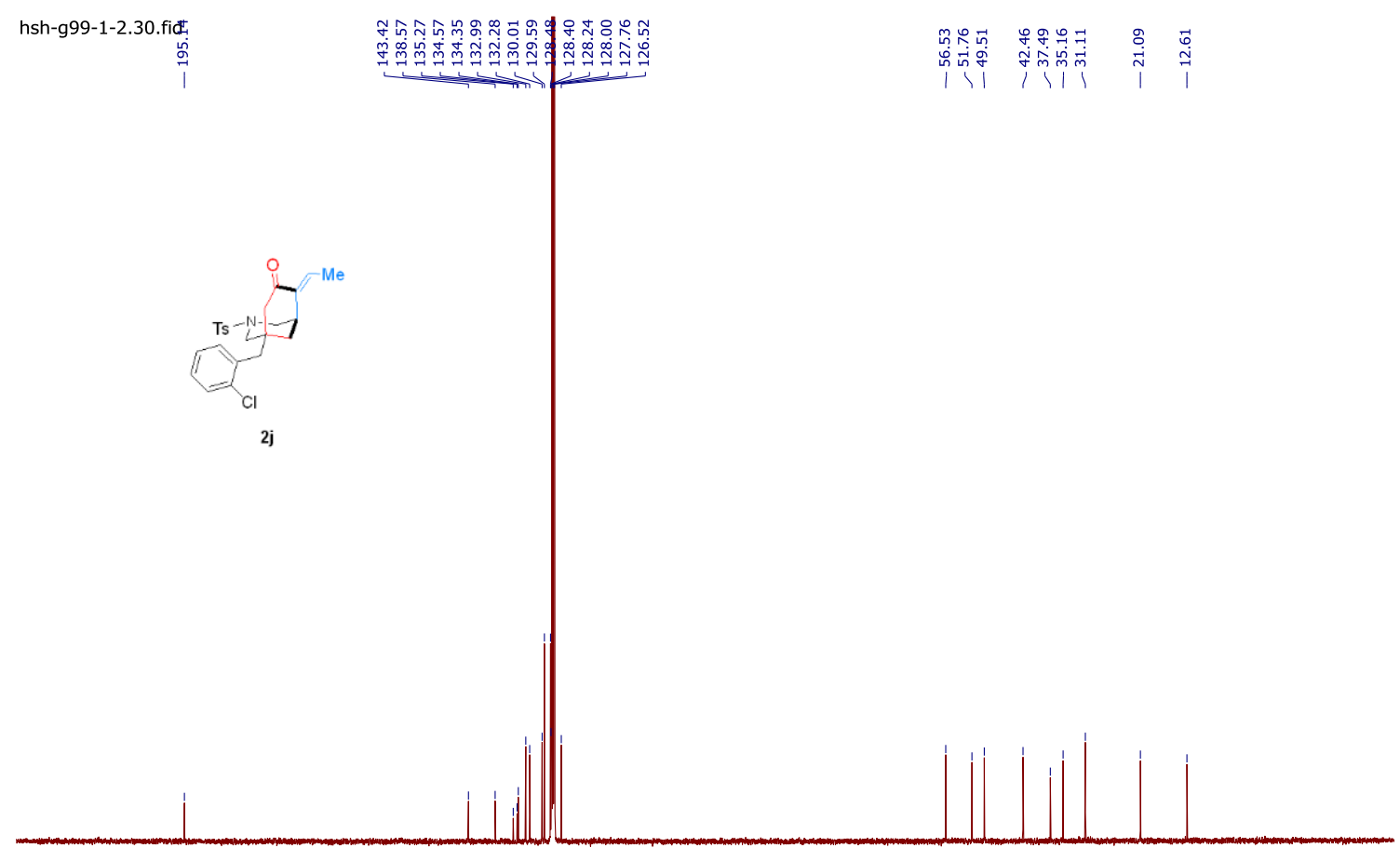

$\begin{array}{llllllllllllllllllllllllllllllllllll}220 & 210 & 200 & 190 & 180 & 170 & 160 & 150 & 140 & 130 & 120 & 110 & 100 & 90 & 80 & 70 & 60 & 50 & 40 & 30 & 20 & 10 & 0 & -10 & -20\end{array}$ 


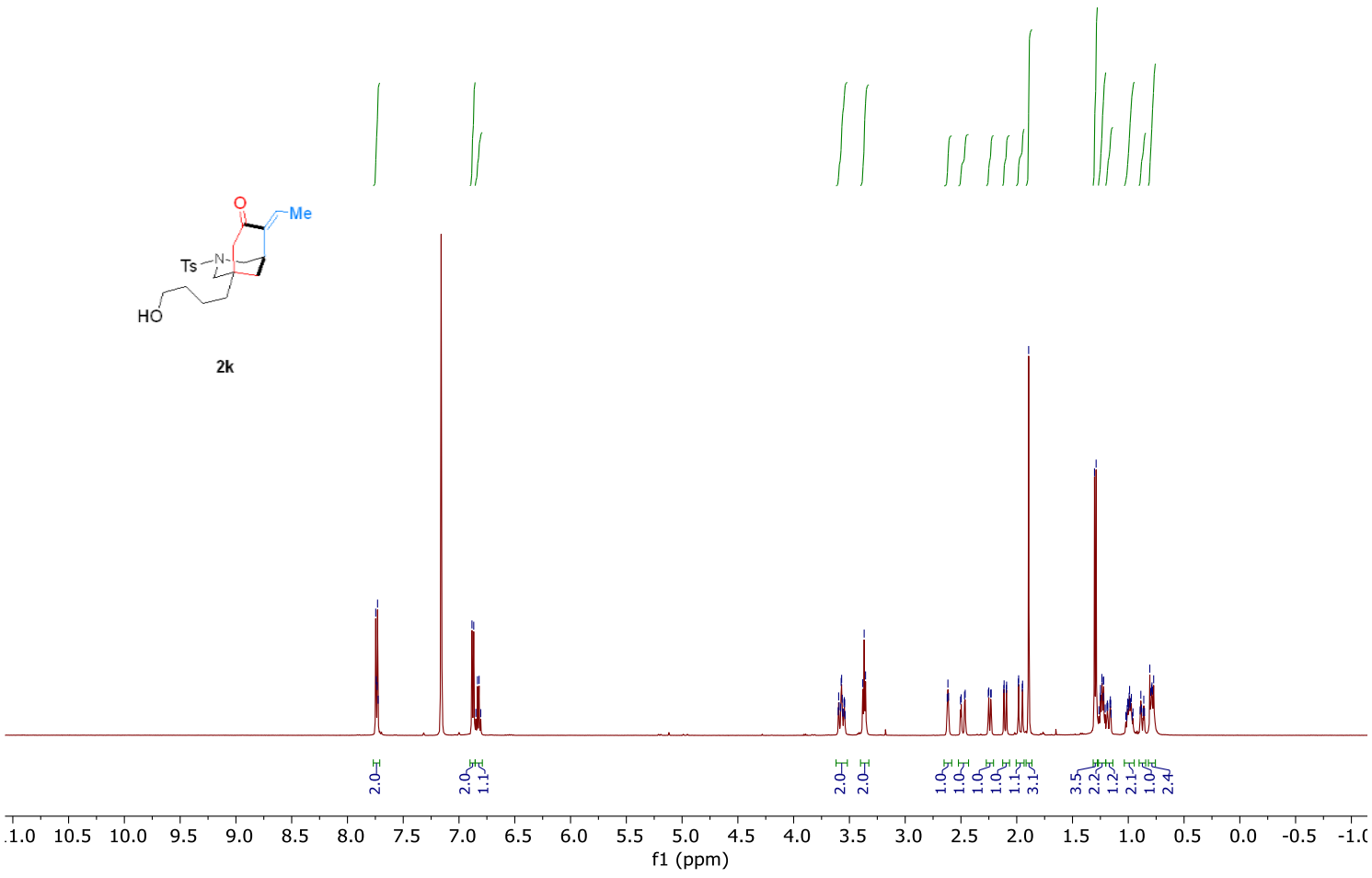

${ }^{13} \mathrm{C}$-NMR spectrum of $\mathbf{2 k}$ in $\mathrm{C}_{6} \mathrm{D}_{6}, 101 \mathrm{MHz}$

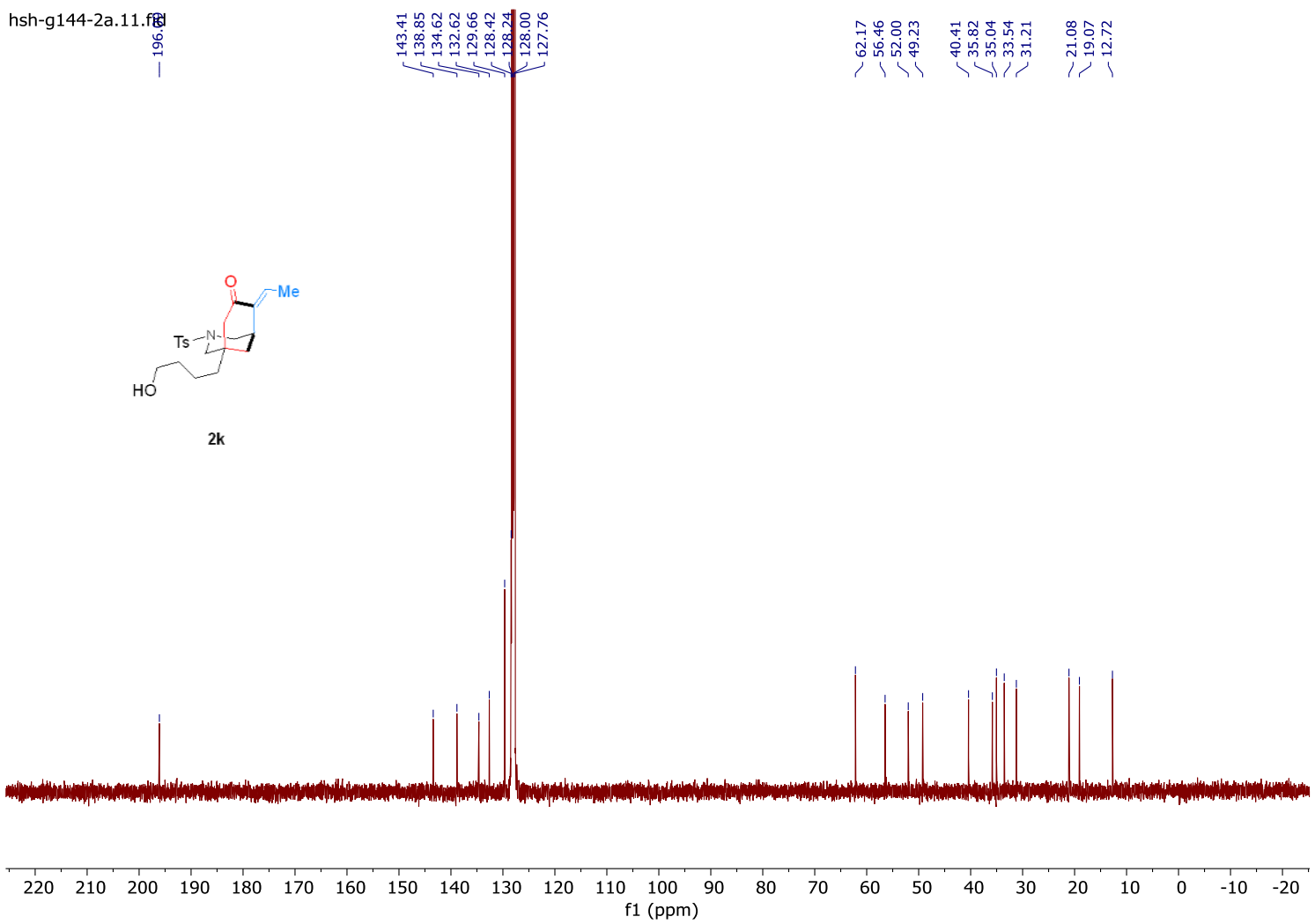


${ }^{1} \mathrm{H}-\mathrm{NMR}$ spectrum of $2 \mathrm{I}$ in $\mathrm{C}_{6} \mathrm{D}_{6}, 400 \mathrm{MHz}$

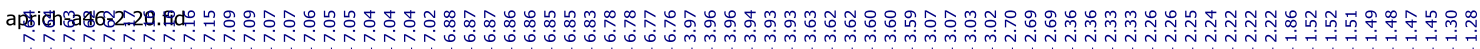

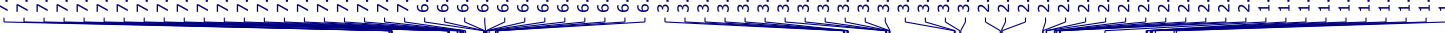

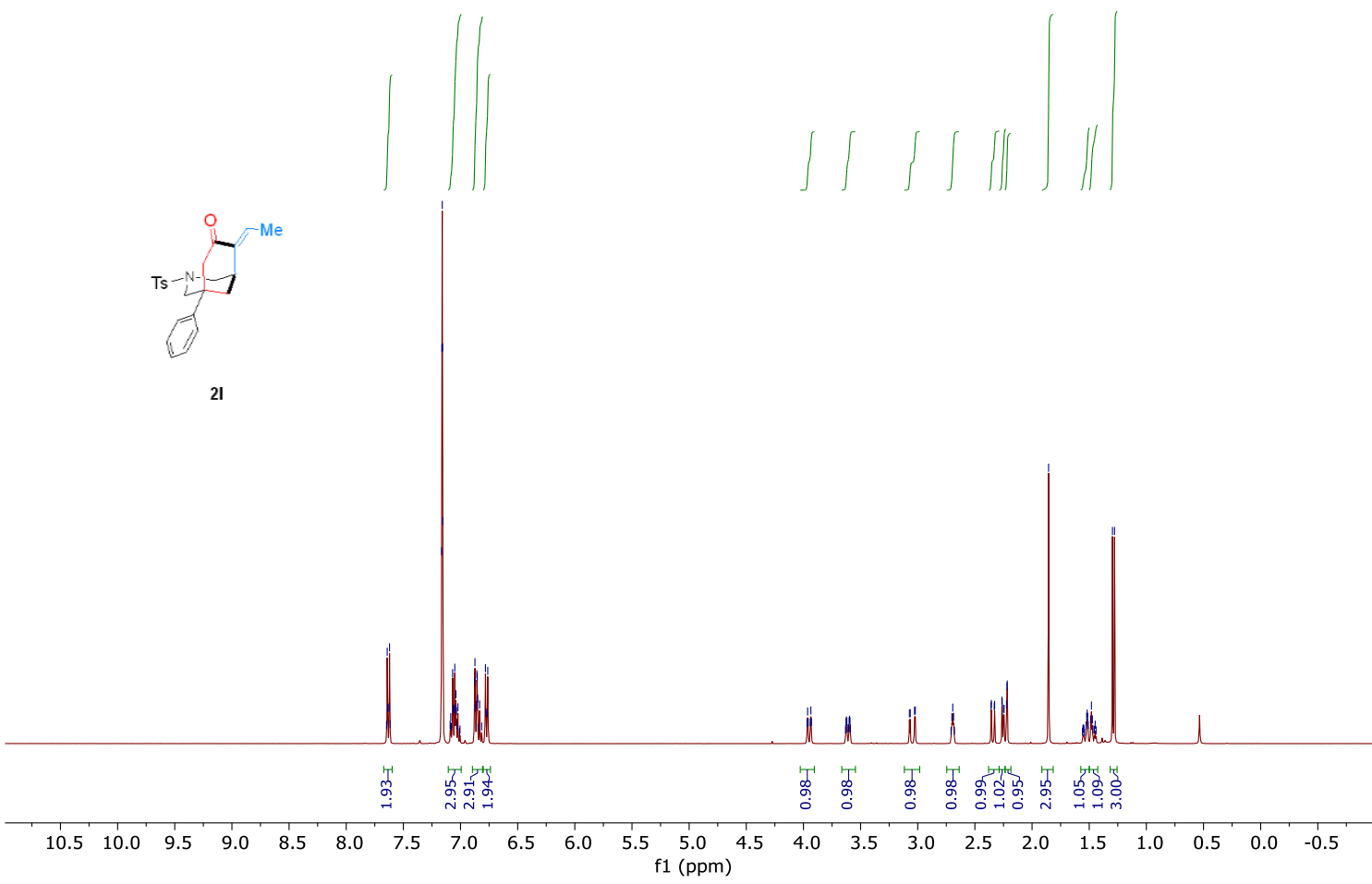

${ }^{13} \mathrm{C}-\mathrm{NMR}$ spectrum of $2 \mathrm{l}$ in $\mathrm{C}_{6} \mathrm{D}_{6}, 101 \mathrm{MHz}$

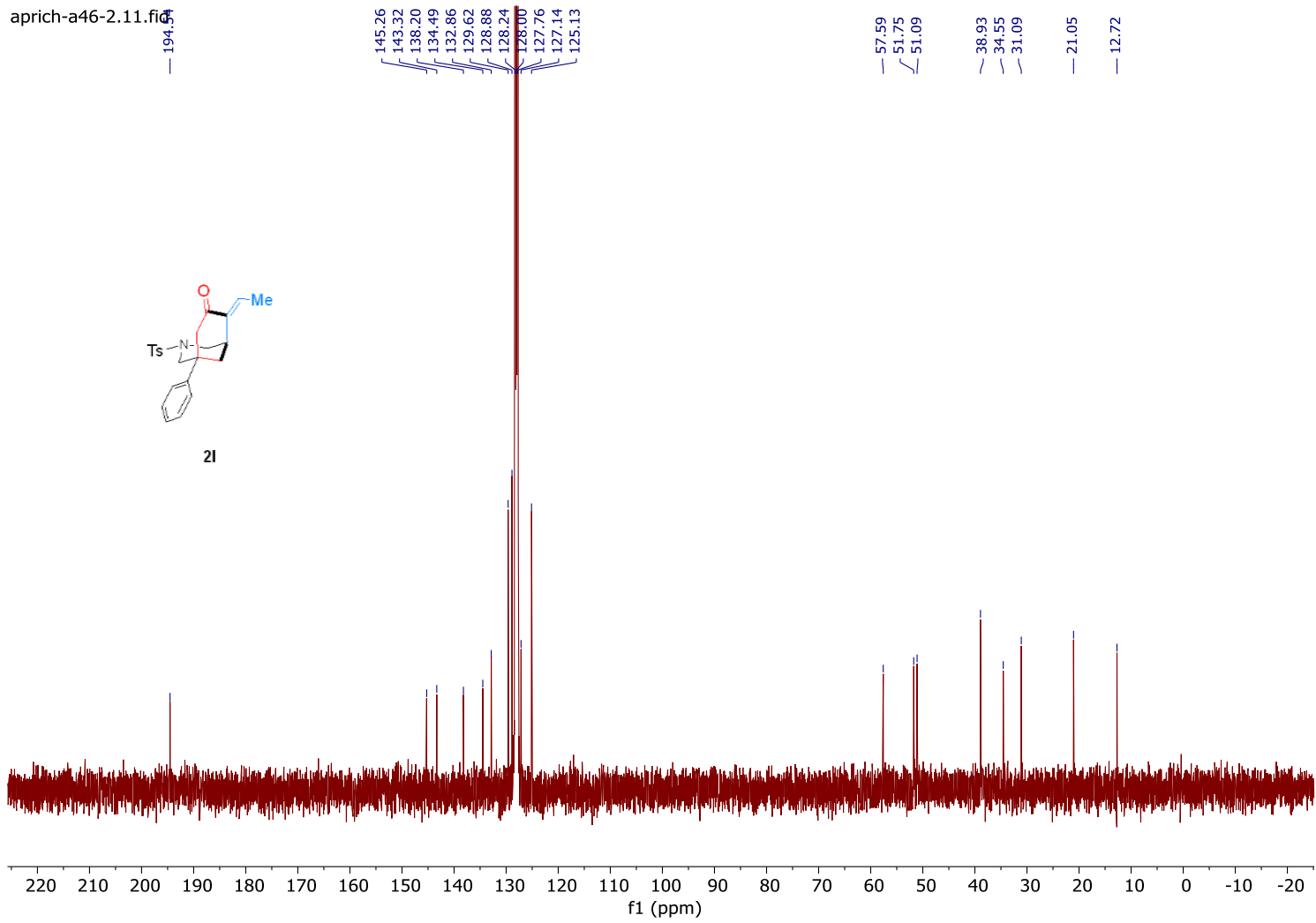




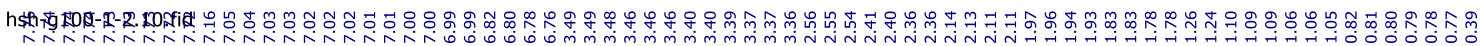

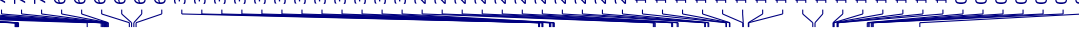

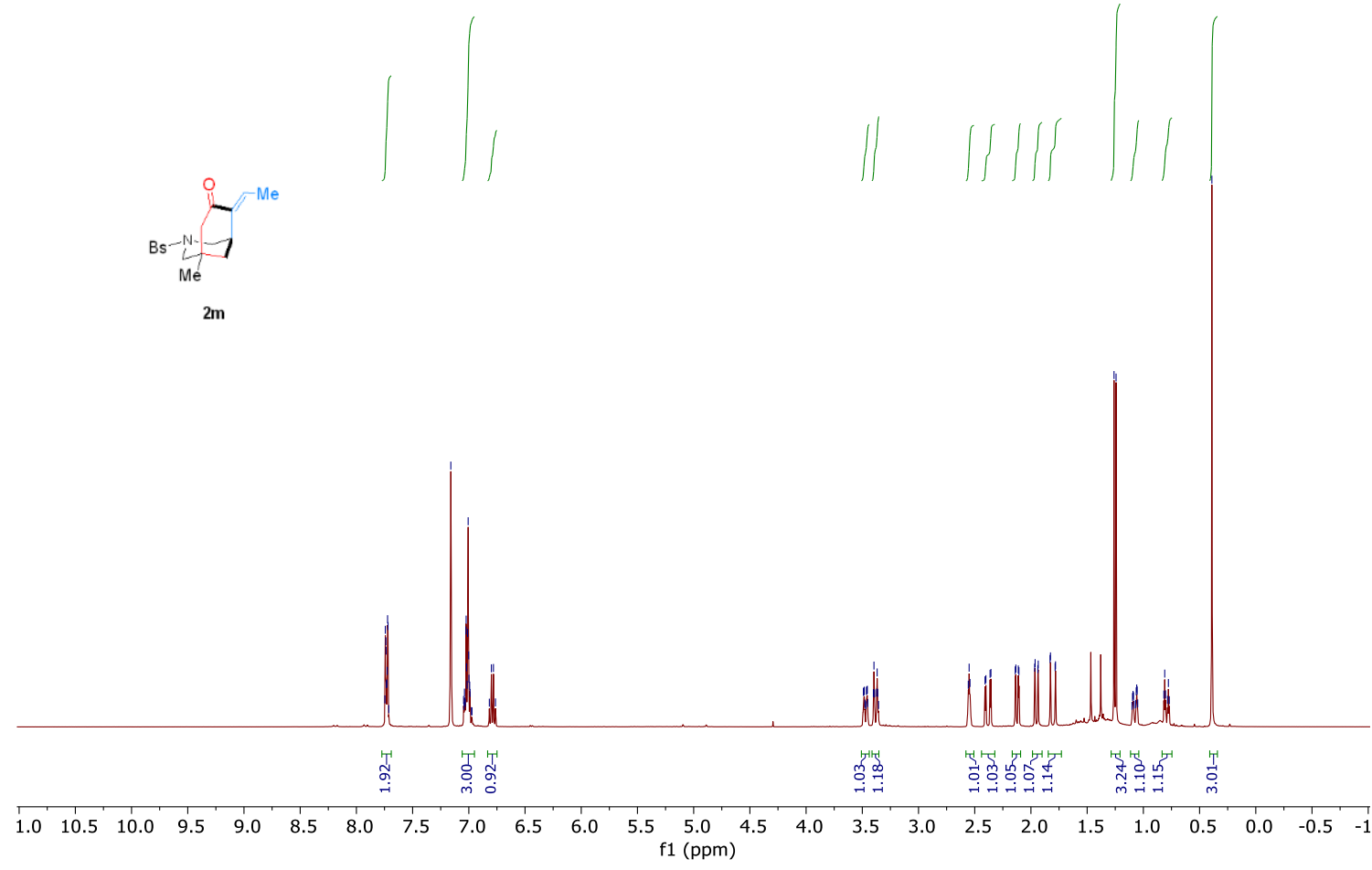

${ }^{13} \mathrm{C}-\mathrm{NMR}$ spectrum of $2 \mathrm{~m}$ in $\mathrm{C}_{6} \mathrm{D}_{6}, 101 \mathrm{MHz}$

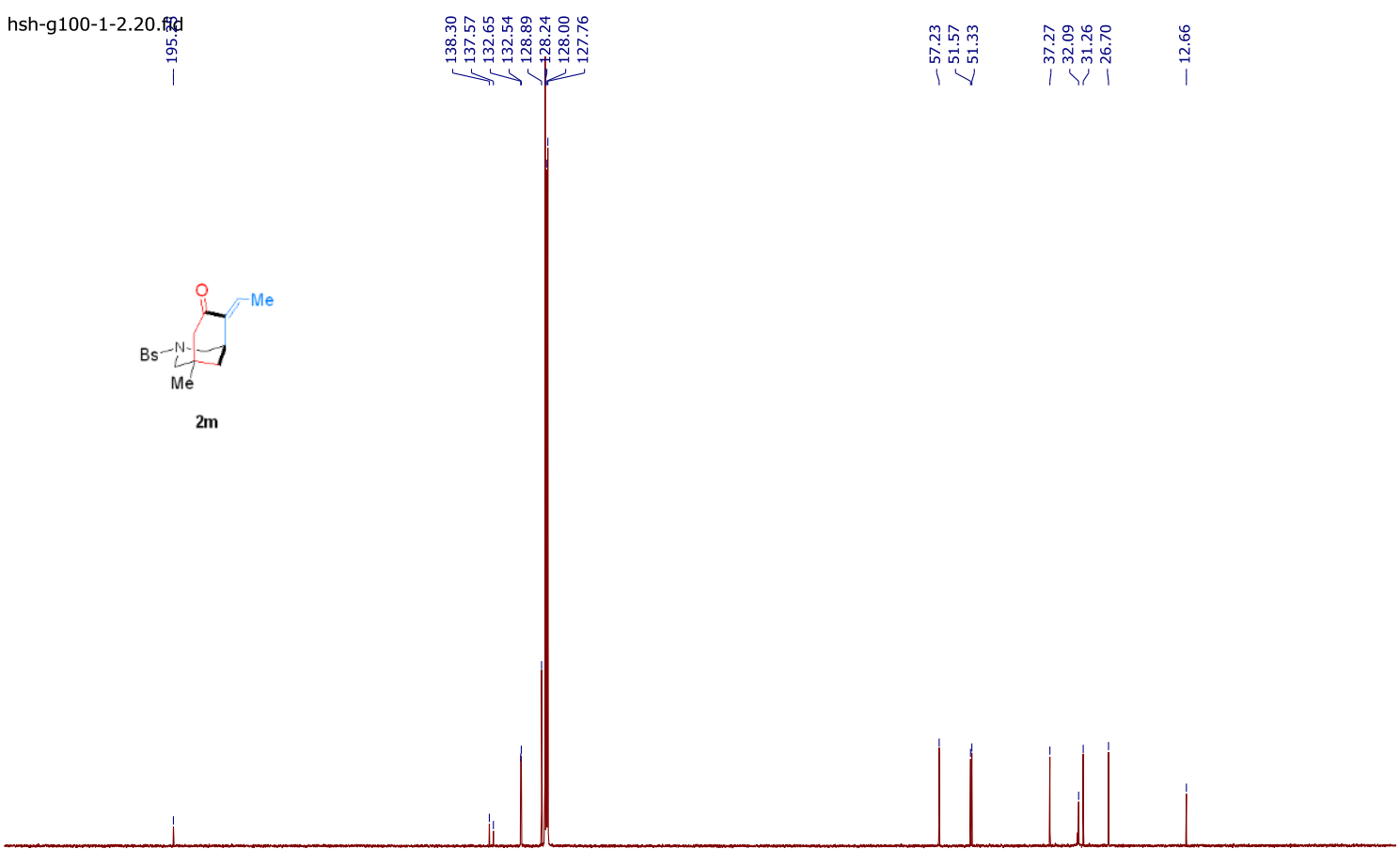

$\begin{array}{lllllllllllllllllllllllllllllllllll}220 & 210 & 200 & 190 & 180 & 170 & 160 & 150 & 140 & 130 & 120 & 110 & 100 & 90 & 80 & 70 & 60 & 50 & 40 & 30 & 20 & 10 & 0 & -10 & -20\end{array}$ 


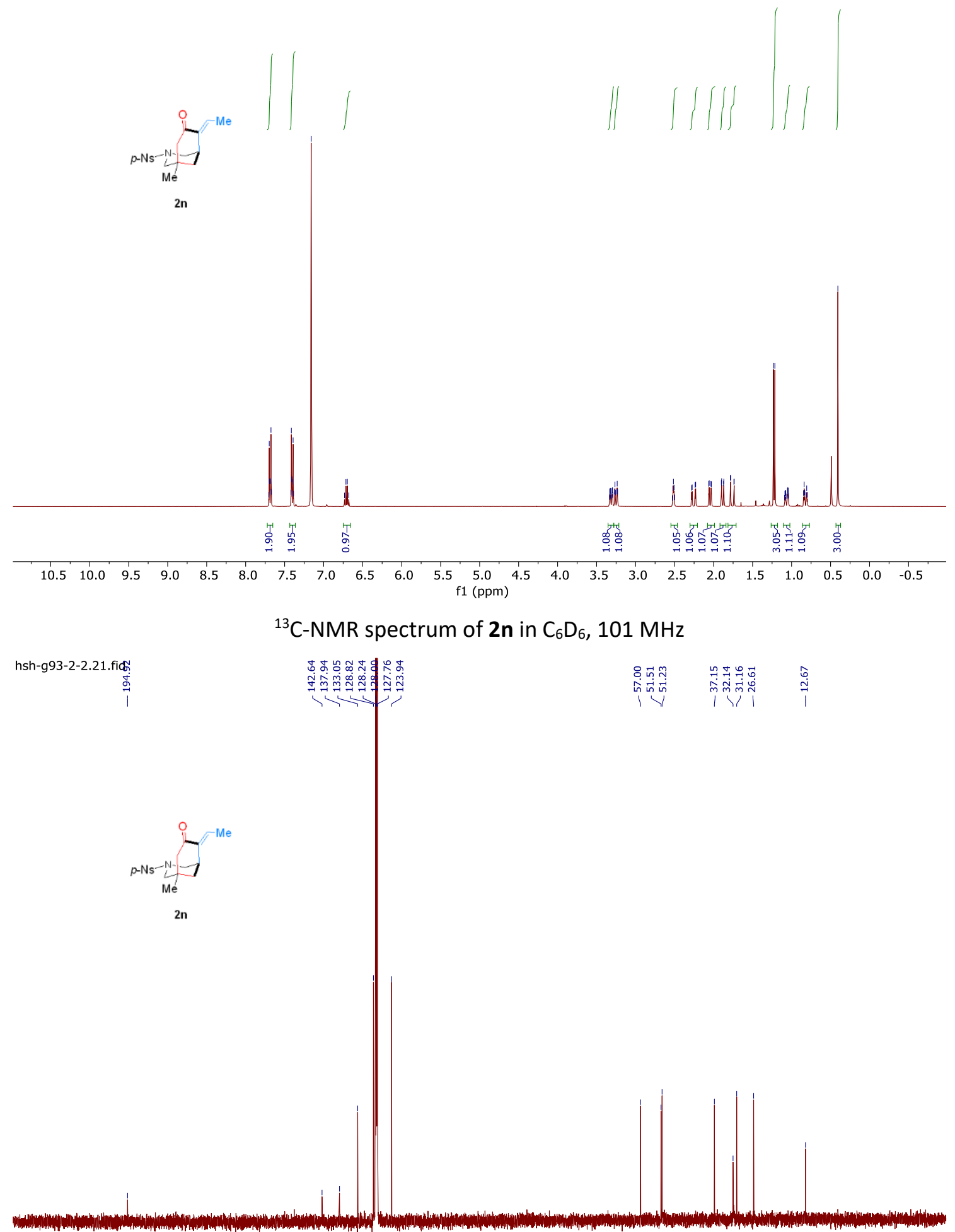

$\begin{array}{lllllllllllllllllllllllllllllllllll}220 & 210 & 200 & 190 & 180 & 170 & 160 & 150 & 140 & 130 & 120 & 110 & 100 & 90 & 80 & 70 & 60 & 50 & 40 & 30 & 20 & 10 & 0 & -10 & -20\end{array}$ 


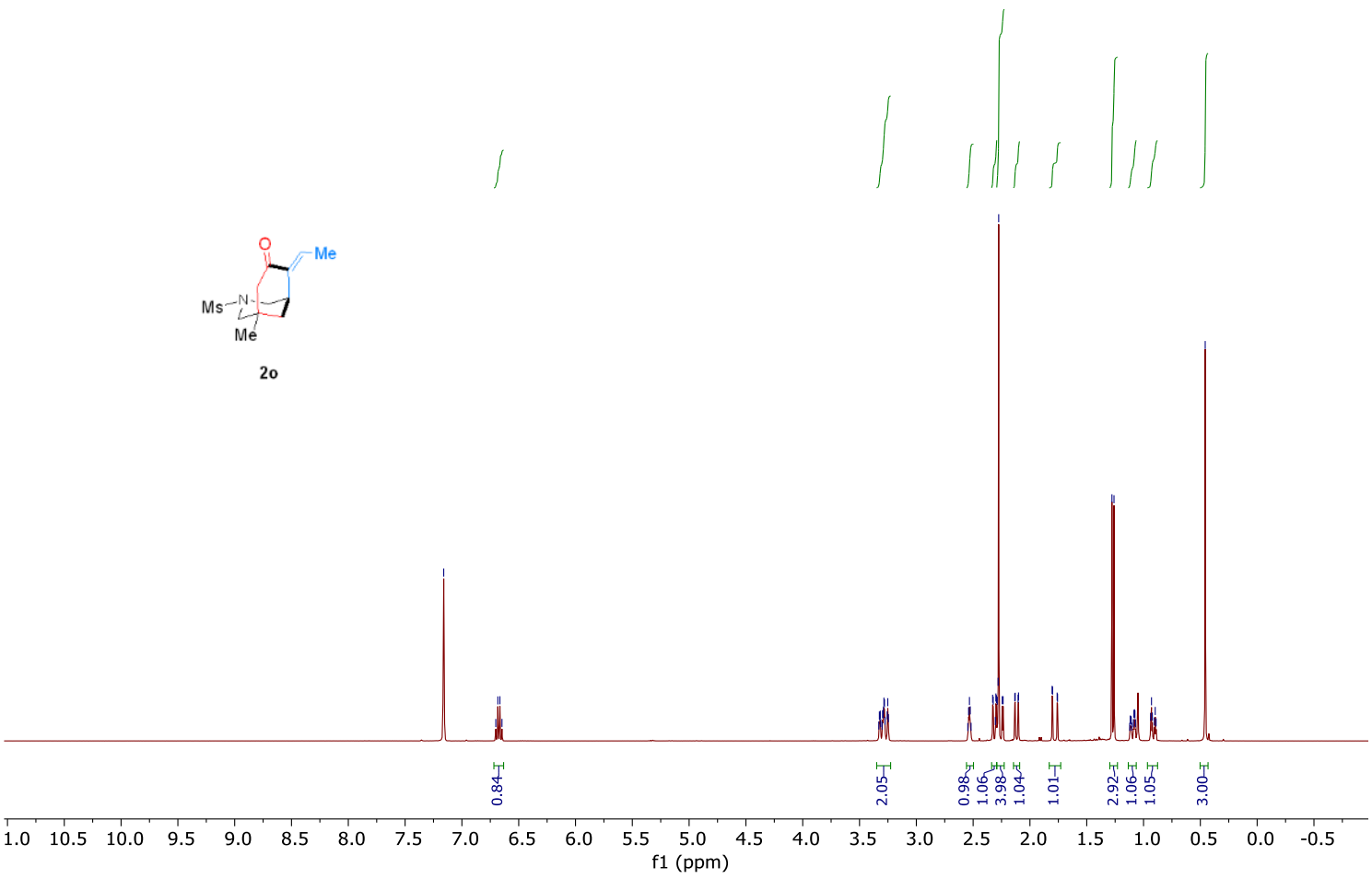

${ }^{13} \mathrm{C}-\mathrm{NMR}$ spectrum of 20 in $\mathrm{C}_{6} \mathrm{D}_{6}, 101 \mathrm{MHz}$

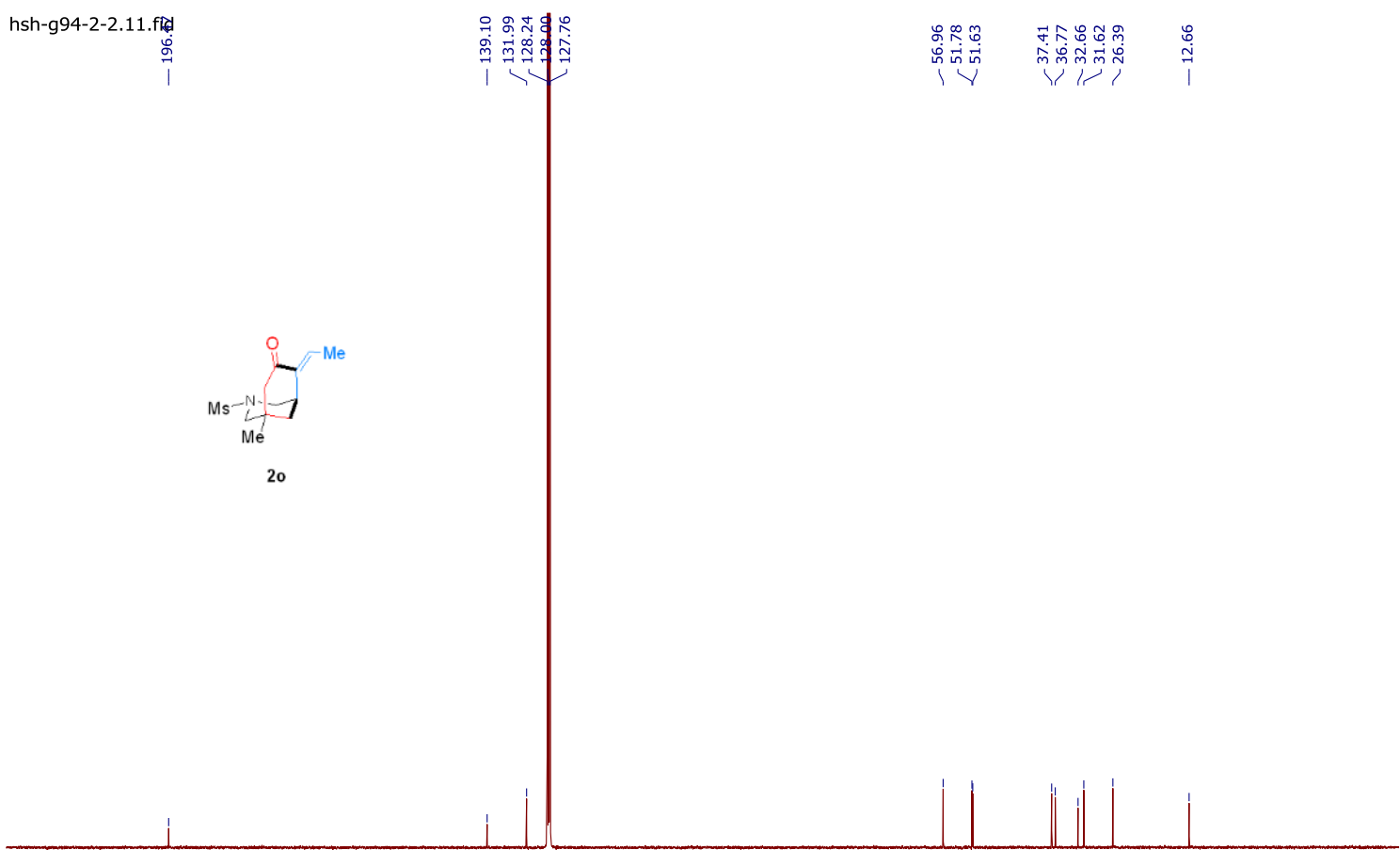

$\begin{array}{llllllllllllllllllllllllllllllllll}220 & 210 & 200 & 190 & 180 & 170 & 160 & 150 & 140 & 130 & 120 & 110 & 100 & 90 & 80 & 70 & 60 & 50 & 40 & 30 & 20 & 10 & 0 & -10 & -20\end{array}$ 
hsh-g118-2-12.10. Ahdo

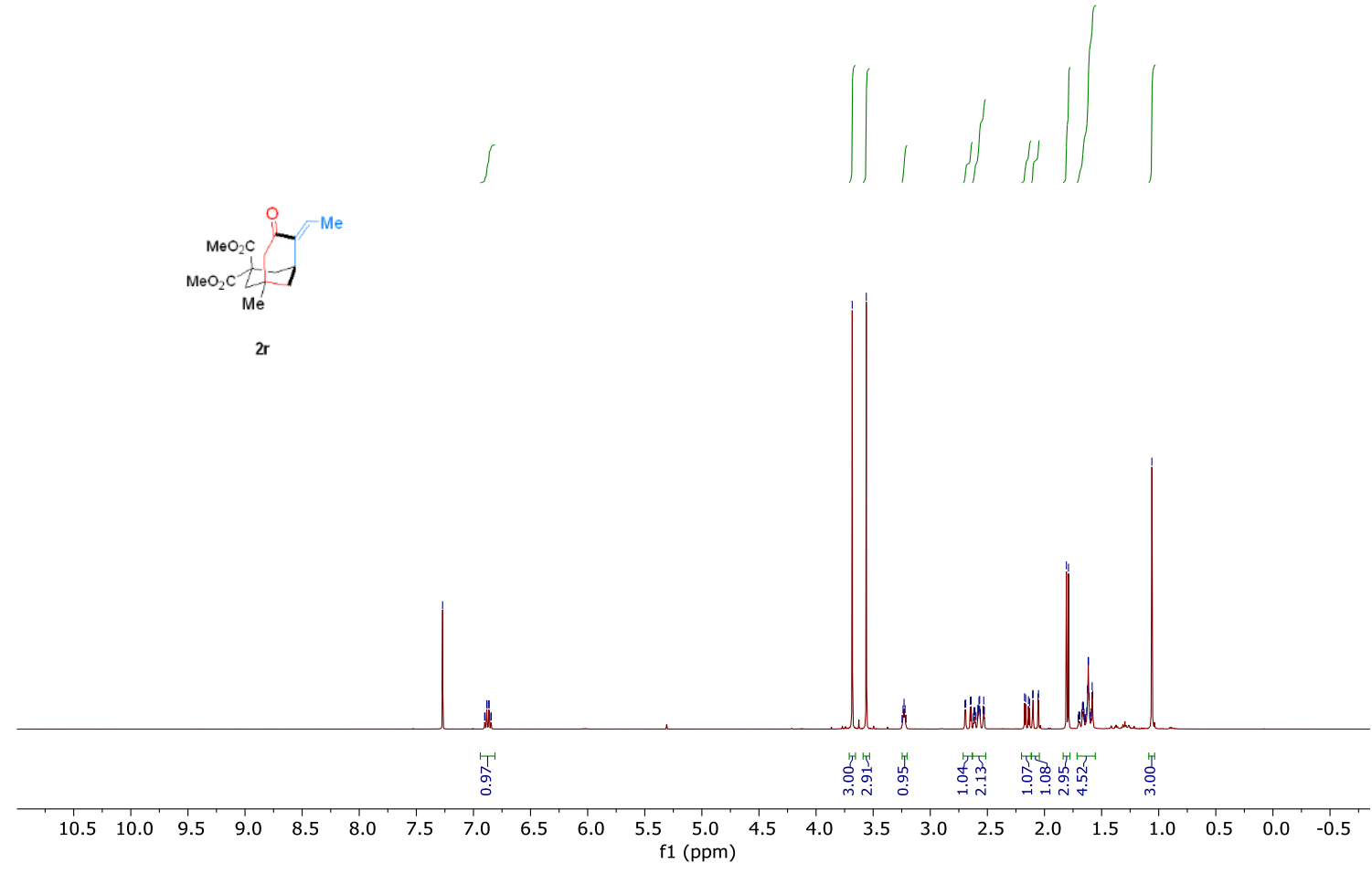

${ }^{13} \mathrm{C}$-NMR spectrum of $2 \mathrm{r}$ in $\mathrm{CDCl}_{3}, 101 \mathrm{MHz}$

\begin{tabular}{|c|c|c|c|c|}
\hline hsh-g118-2-12. \$1.fid & 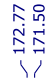 & 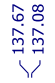 & 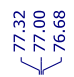 & 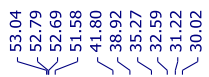 \\
\hline
\end{tabular}

$$
\mathrm{MeO}_{2} \mathrm{C}
$$

$2 \mathrm{r}$

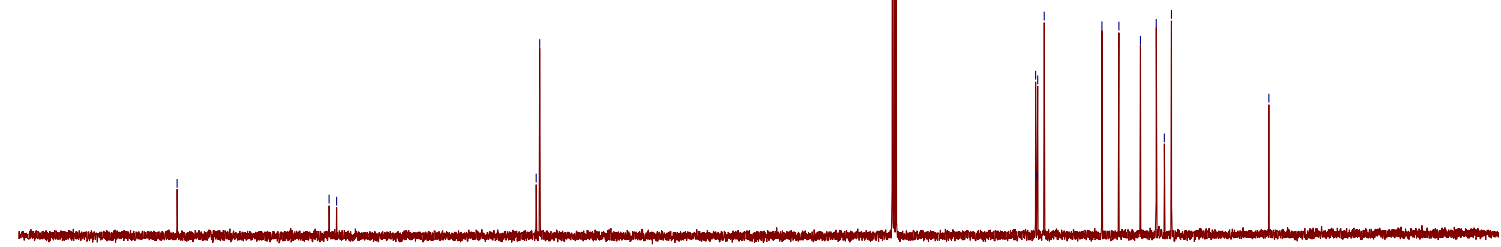

$\begin{array}{lllllllllllllllllllllllllllllllllll}220 & 210 & 200 & 190 & 180 & 170 & 160 & 150 & 140 & 130 & 120 & 110 & 100 & 90 & 80 & 70 & 60 & 50 & 40 & 30 & 20 & 10 & 0 & -10 & -20\end{array}$ 
${ }^{1} \mathrm{H}-\mathrm{NMR}$ spectrum of $2 \mathrm{~s}$ in $\mathrm{CDCl}_{3}, 400 \mathrm{MHz}$
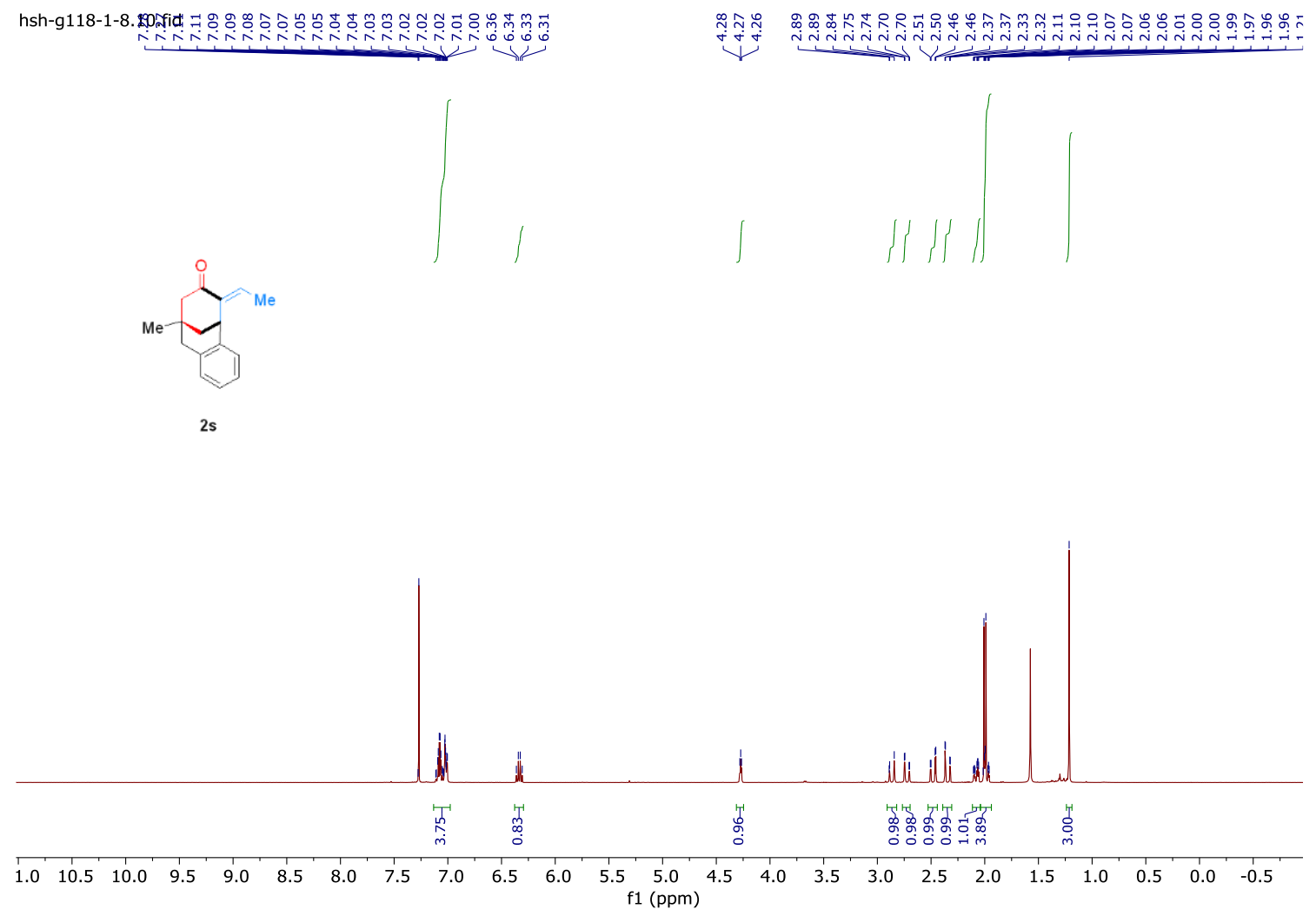

${ }^{13} \mathrm{C}$-NMR spectrum of $2 \mathrm{~s}$ in $\mathrm{CDCl}_{3}, 101 \mathrm{MHz}$

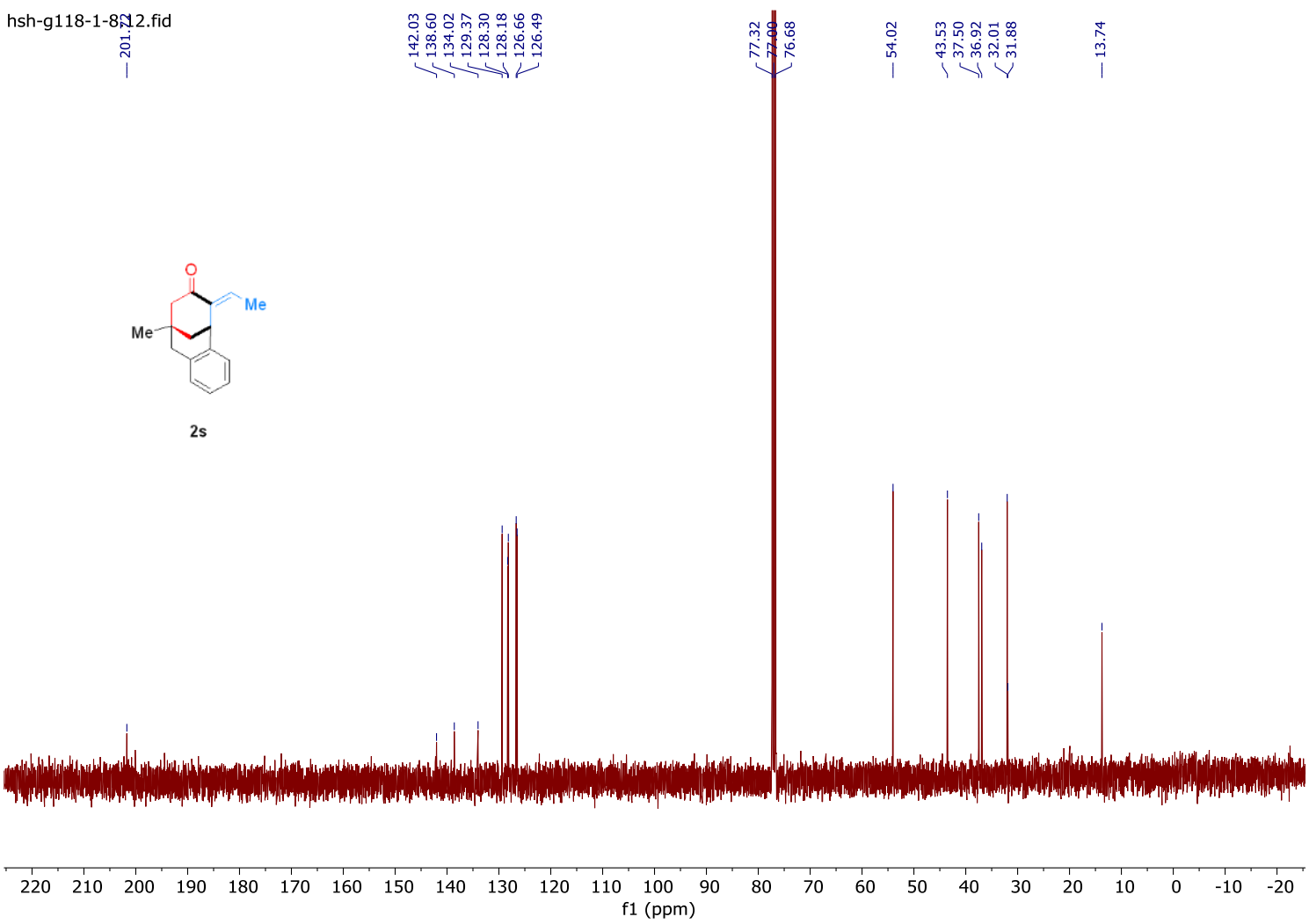




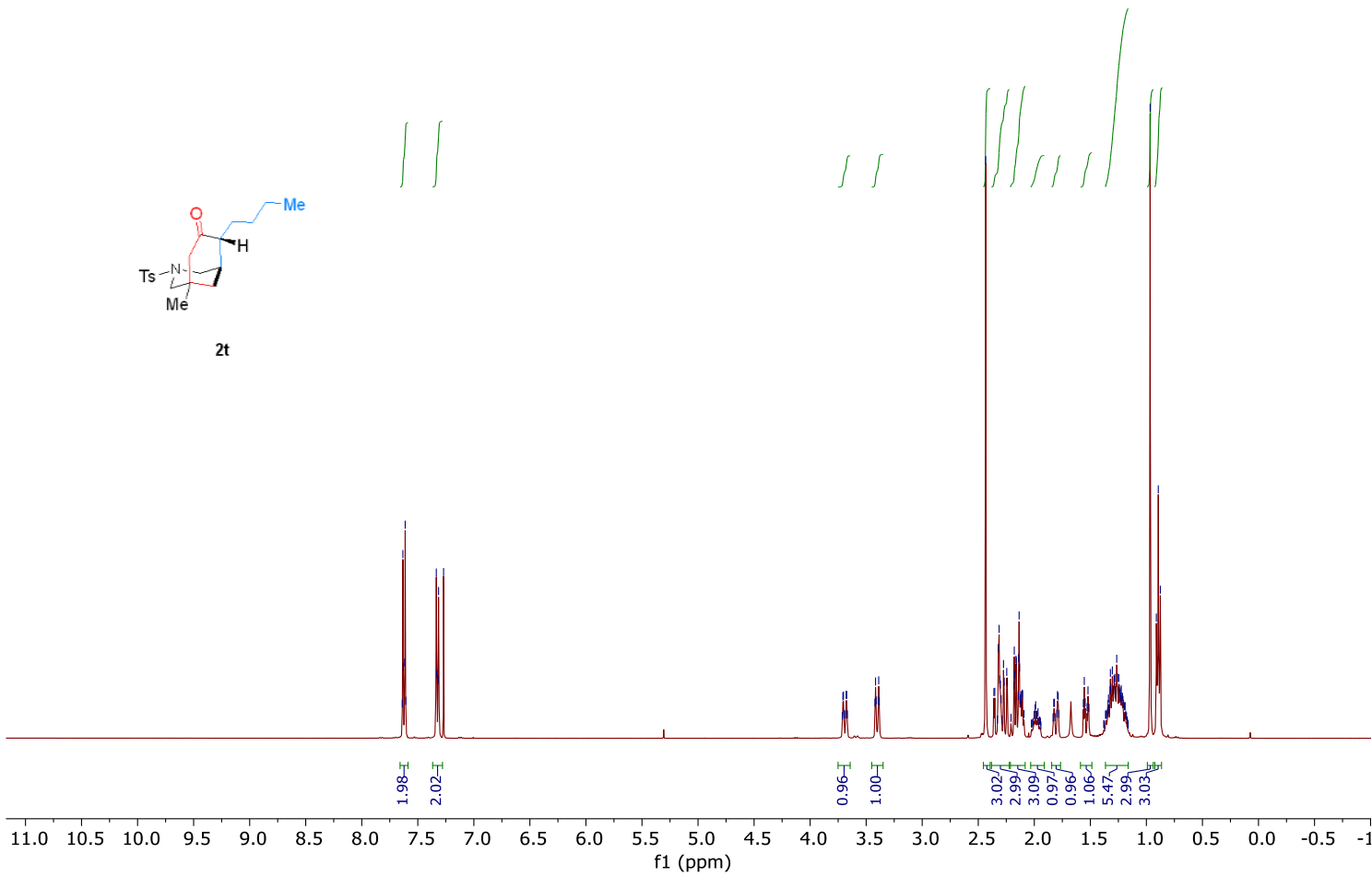

${ }^{13} \mathrm{C}-\mathrm{NMR}$ spectrum of $2 \mathrm{t}$ in $\mathrm{CDCl}_{3}, 101 \mathrm{MHz}$

hsh-g160-27.11.fid

ì
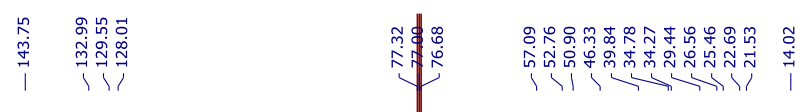

$\mathrm{Ts}_{\mathrm{Me}}^{\mathrm{N}-\mathrm{C}_{\mathrm{H}}^{\mathrm{Me}}}$

$2 t$

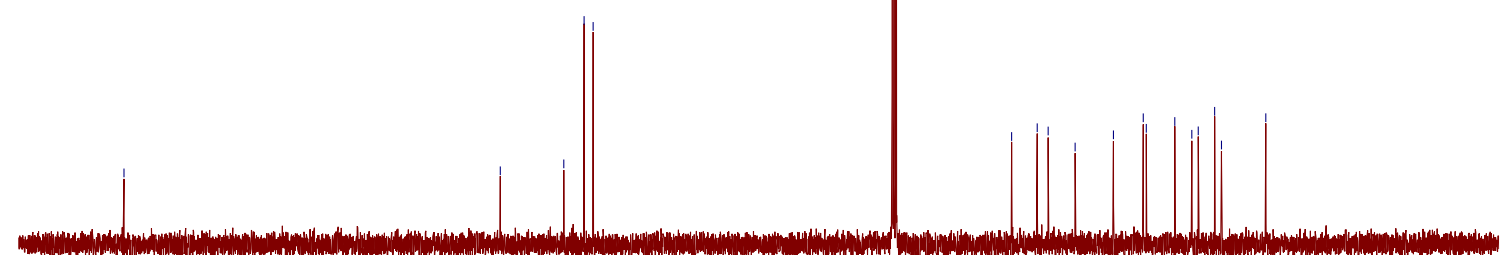

$\begin{array}{lllllllllllllllllllllllllllllllllllll}220 & 210 & 200 & 190 & 180 & 170 & 160 & 150 & 140 & 130 & 120 & 110 & 100 & 90 & 80 & 70 & 60 & 50 & 40 & 30 & 20 & 10 & 0 & -10 & -20\end{array}$ 


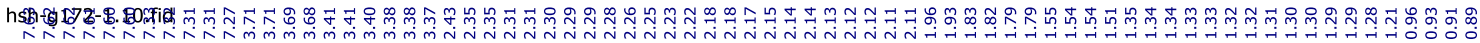
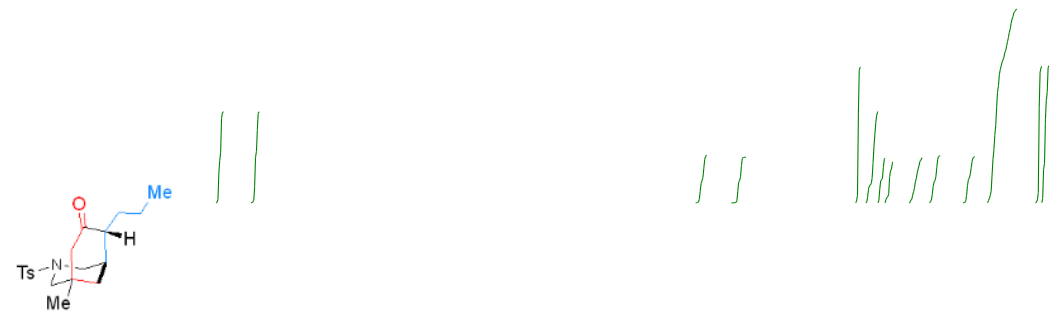

$2 \mathbf{u}$

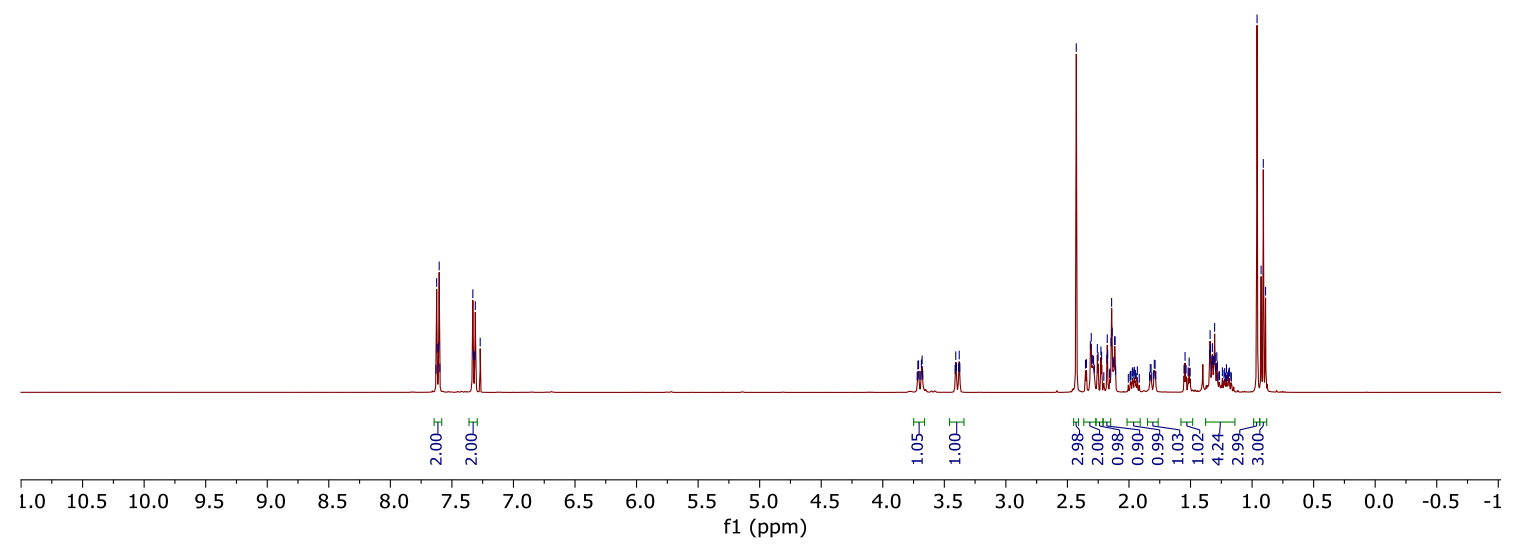

${ }^{13} \mathrm{C}-\mathrm{NMR}$ spectrum of $\mathbf{2 u}$ in $\mathrm{CDCl}_{3}, 101 \mathrm{MHz}$

hsh-g172นึ.11.fid

iे

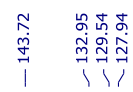

$\underbrace{2}$

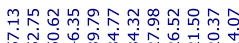

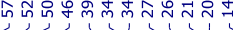

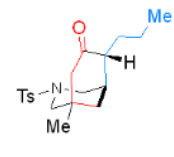

$2 \mathrm{u}$

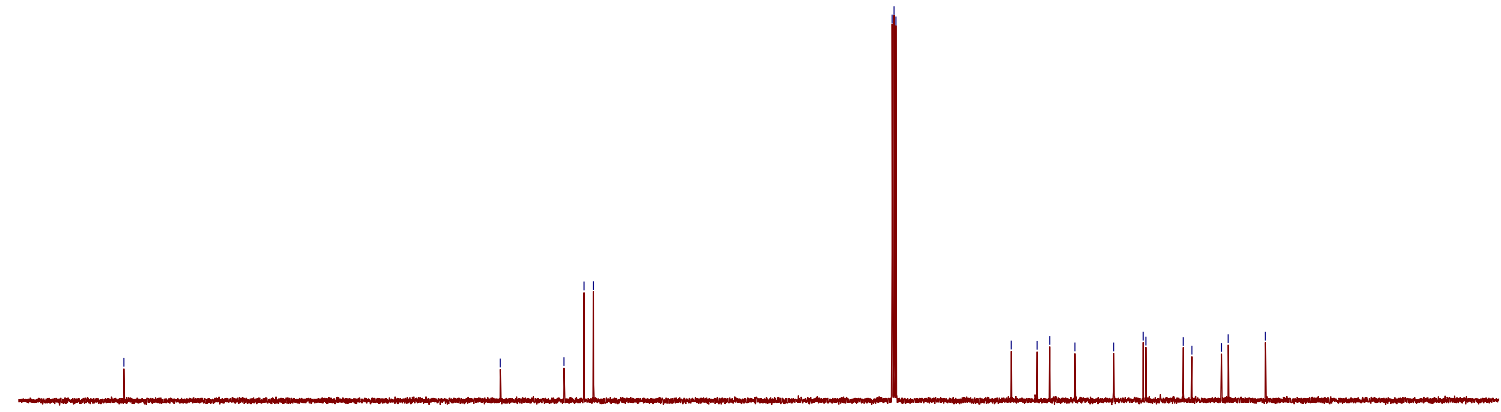

$\begin{array}{lllllllllllllllllllllllllllllllllll}220 & 210 & 200 & 190 & 180 & 170 & 160 & 150 & 140 & 130 & 120 & 110 & 100 & 90 & 80 & 70 & 60 & 50 & 40 & 30 & 20 & 10 & 0 & -10 & -20\end{array}$ 
${ }^{1} \mathrm{H}-\mathrm{NMR}$ spectrum of $\mathbf{2} \mathbf{v}$ in $\mathrm{CDCl}_{3}, 400 \mathrm{MHz}$

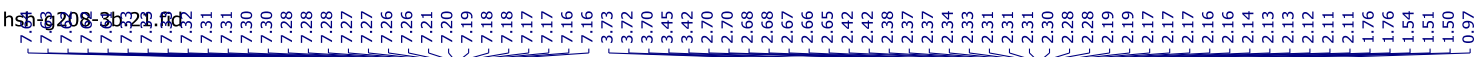
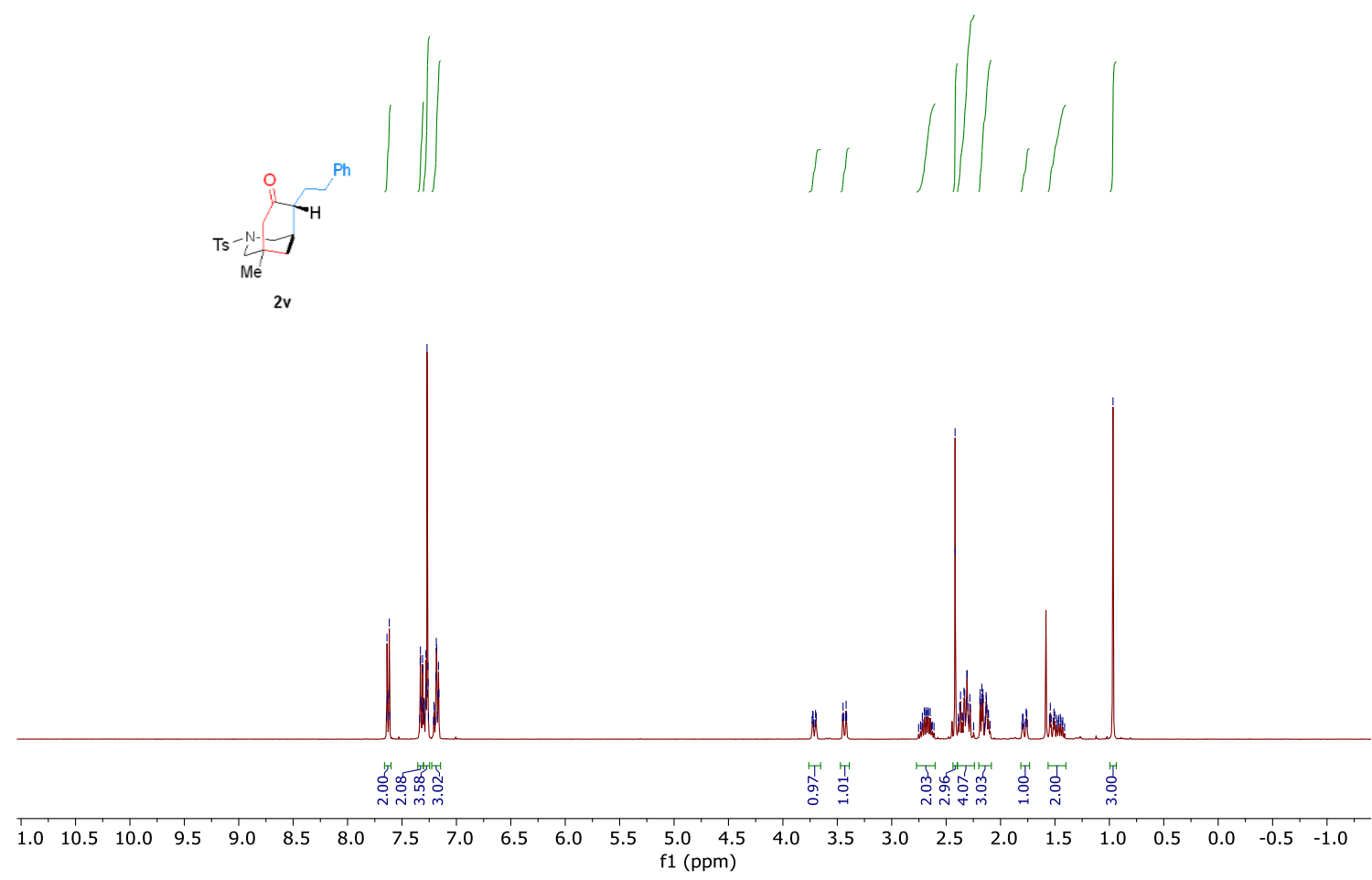

${ }^{13} \mathrm{C}$-NMR spectrum of $\mathbf{2} \mathbf{v}$ in $\mathrm{CDCl}_{3}, 101 \mathrm{MHz}$

hsh-g208-@̣p.12.fid

ì

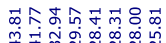

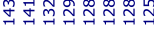

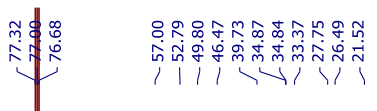

$\underbrace{\mathrm{Ph}}_{\mathrm{Me}}$

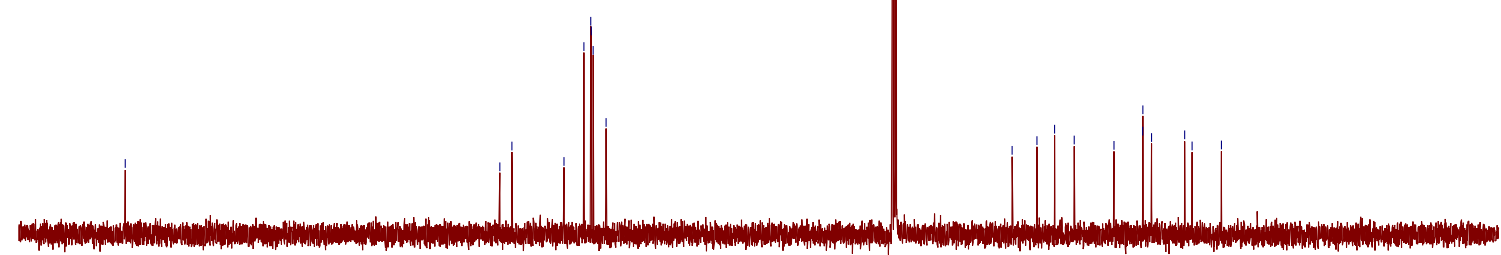

$\begin{array}{lllllllllllllllllllllllllllllllllllll}220 & 210 & 200 & 190 & 180 & 170 & 160 & 150 & 140 & 130 & 120 & 110 & 100 & 90 & 80 & 70 & 60 & 50 & 40 & 30 & 20 & 10 & 0 & -10 & -20\end{array}$ 

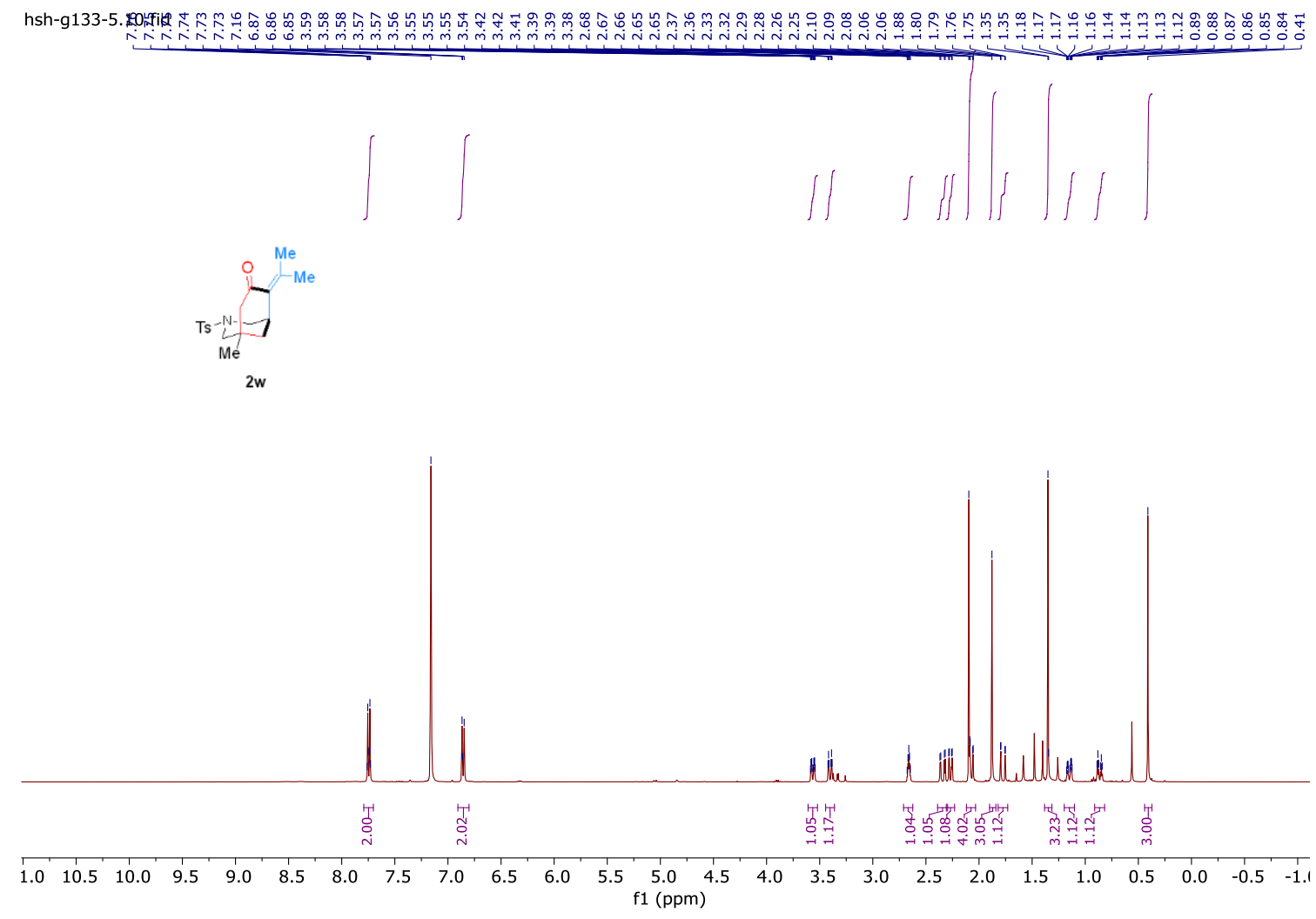

${ }^{13} \mathrm{C}$-NMR spectrum of $\mathbf{2 w}$ in $\mathrm{C}_{6} \mathrm{D}_{6}, 101 \mathrm{MHz}$

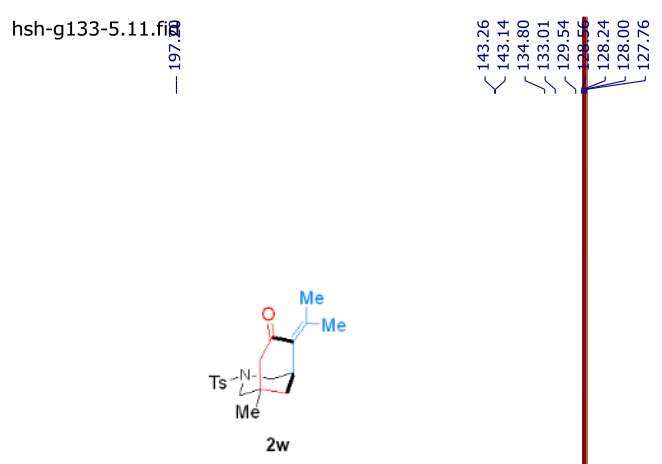

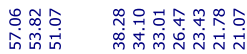

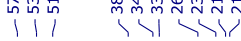

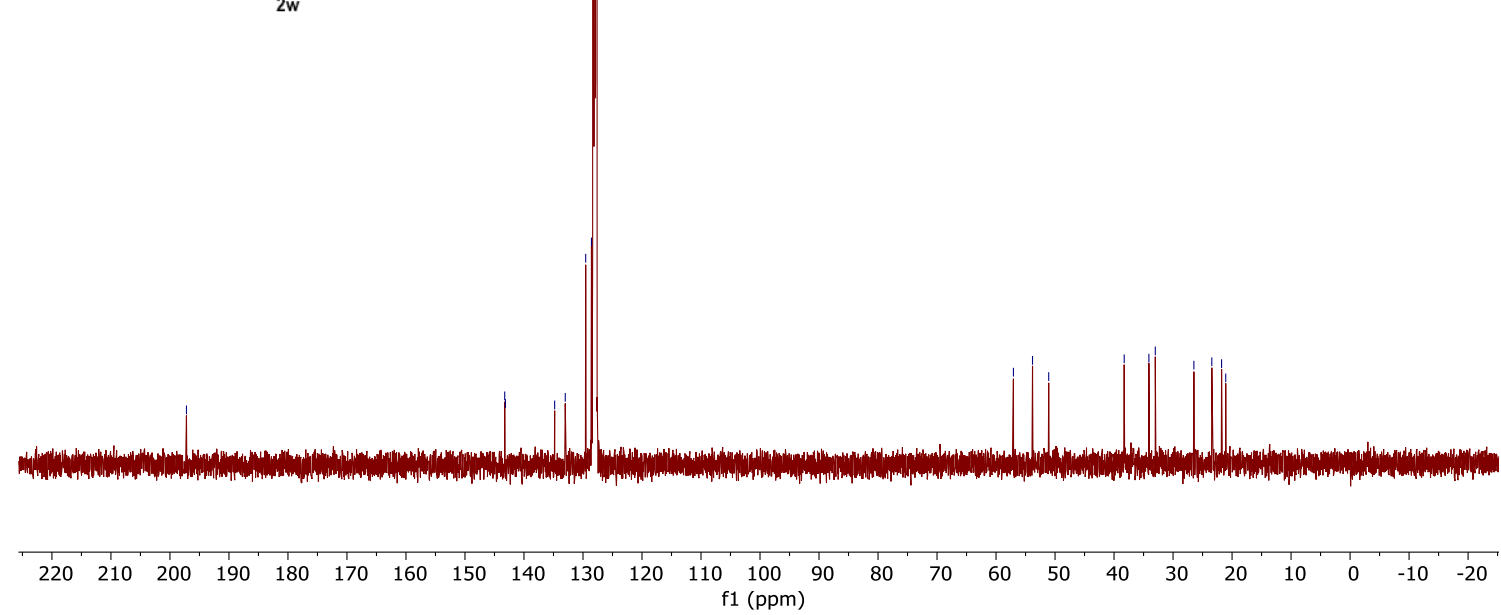


${ }^{1} \mathrm{H}-\mathrm{NMR}$ spectrum of $3 \mathbf{x}$ in $\mathrm{CDCl}_{3}, 400 \mathrm{MHz}$

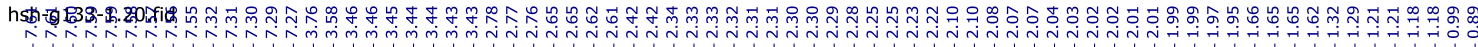
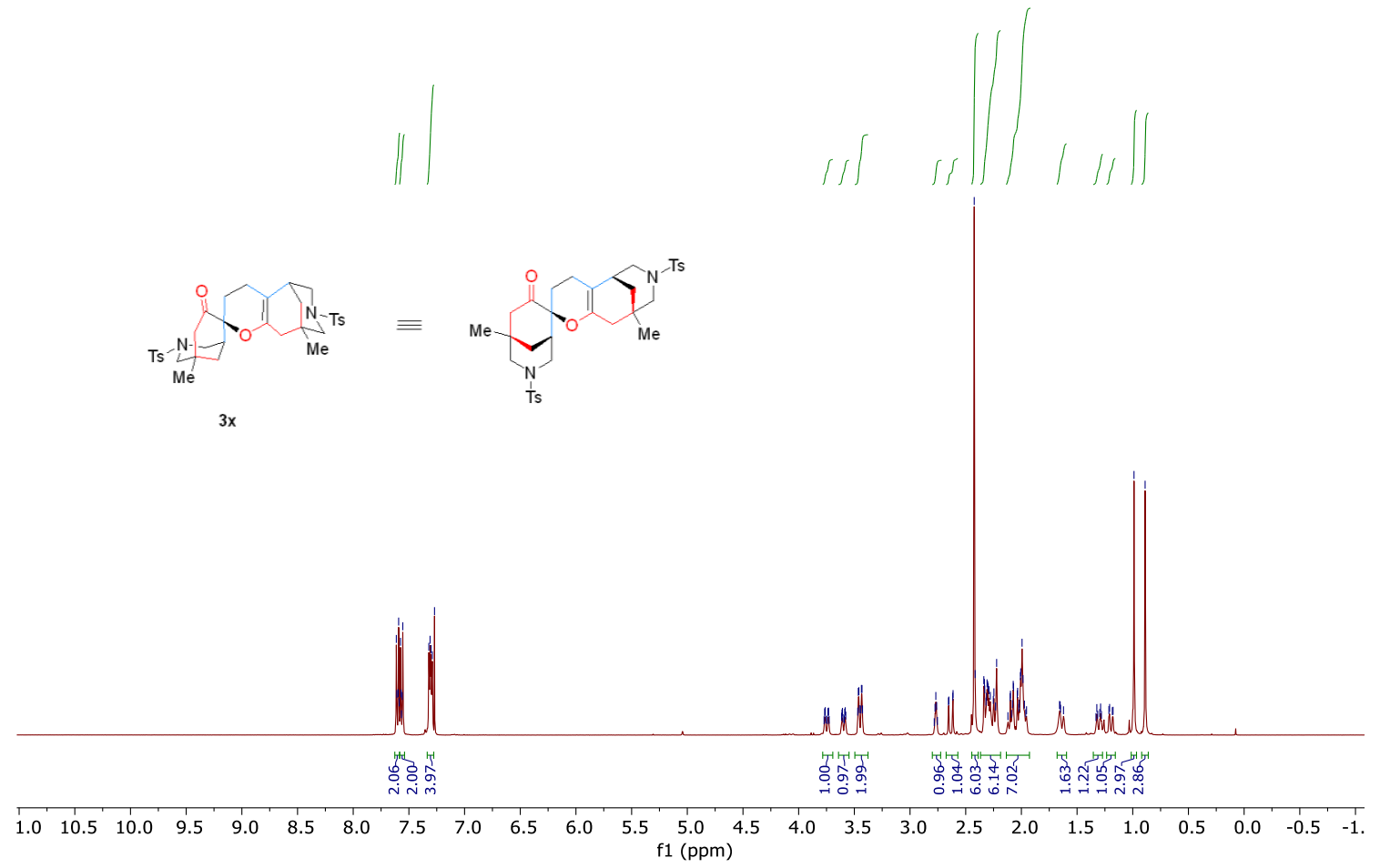

${ }^{13} \mathrm{C}-\mathrm{NMR}$ spectrum of $\mathbf{3 x}$ in $\mathrm{CDCl}_{3}, 101 \mathrm{MHz}$

hsh-g133-1. F1.fid

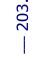
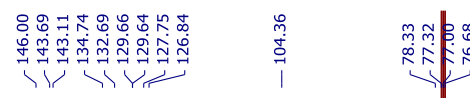

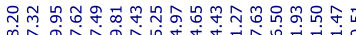

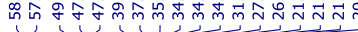

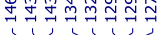

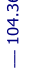

$\checkmark \backslash Y \backslash 1)$
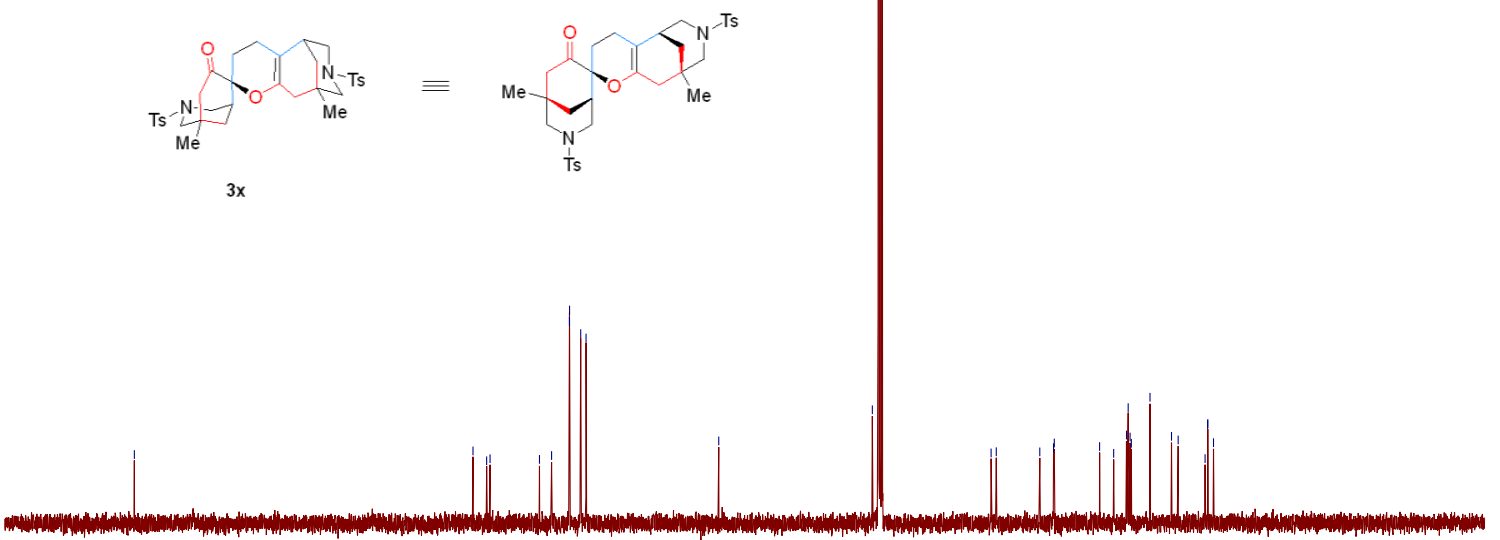

$\begin{array}{llllllllllllllllllllllllllllllllllll}220 & 210 & 200 & 190 & 180 & 170 & 160 & 150 & 140 & 130 & 120 & 110 & 100 & 90 & 80 & 70 & 60 & 50 & 40 & 30 & 20 & 10 & 0 & -10 & -20\end{array}$ 
DEPT spectrum of $\mathbf{3 x}$ in $\mathrm{CDCl}_{3}, 101 \mathrm{MHz}$

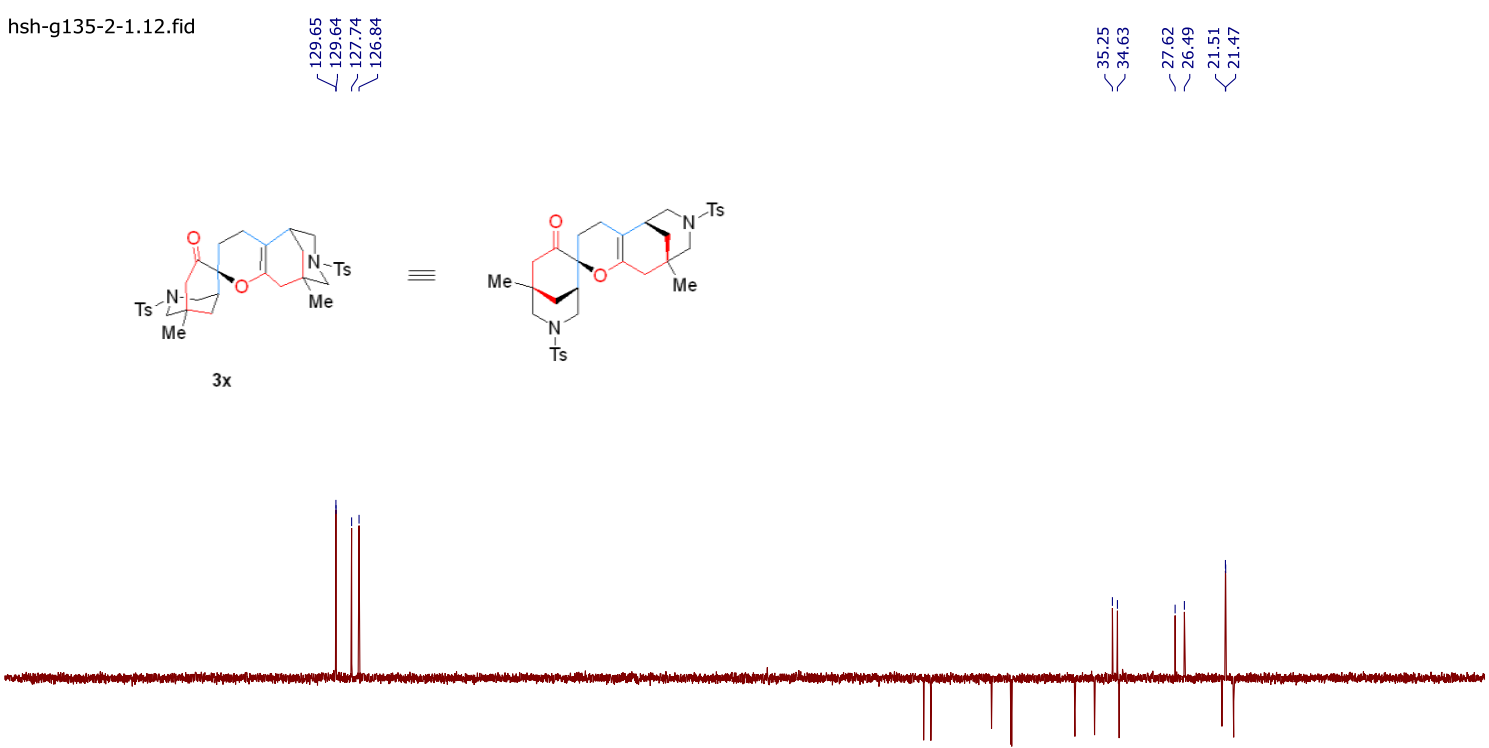

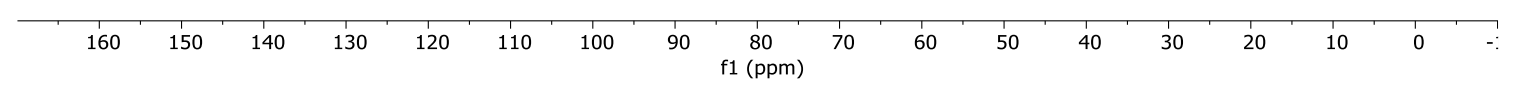

DEPT and ${ }^{13} \mathrm{C}-\mathrm{NMR}$ spectrum of $3 \mathbf{x}$ in $\mathrm{CDCl}_{3}, 101 \mathrm{MHz}$

hsh-g135-2-1.12.fid

$$
{ }_{3 \mathrm{x}}
$$

hsh-g133-1.21.fid

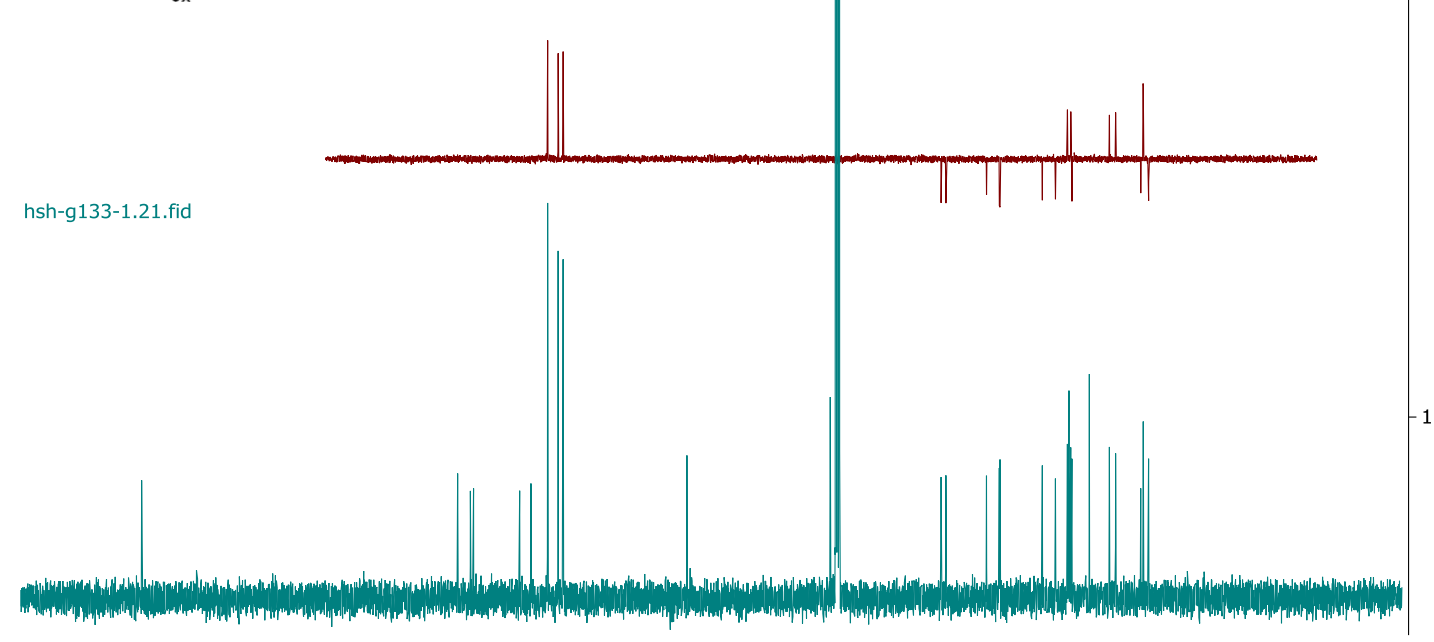

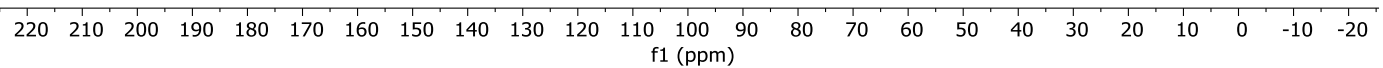


HSQC spectrum of $3 \mathbf{x}$ in $\mathrm{CDCl}_{3}, 400 \mathrm{MHz}$

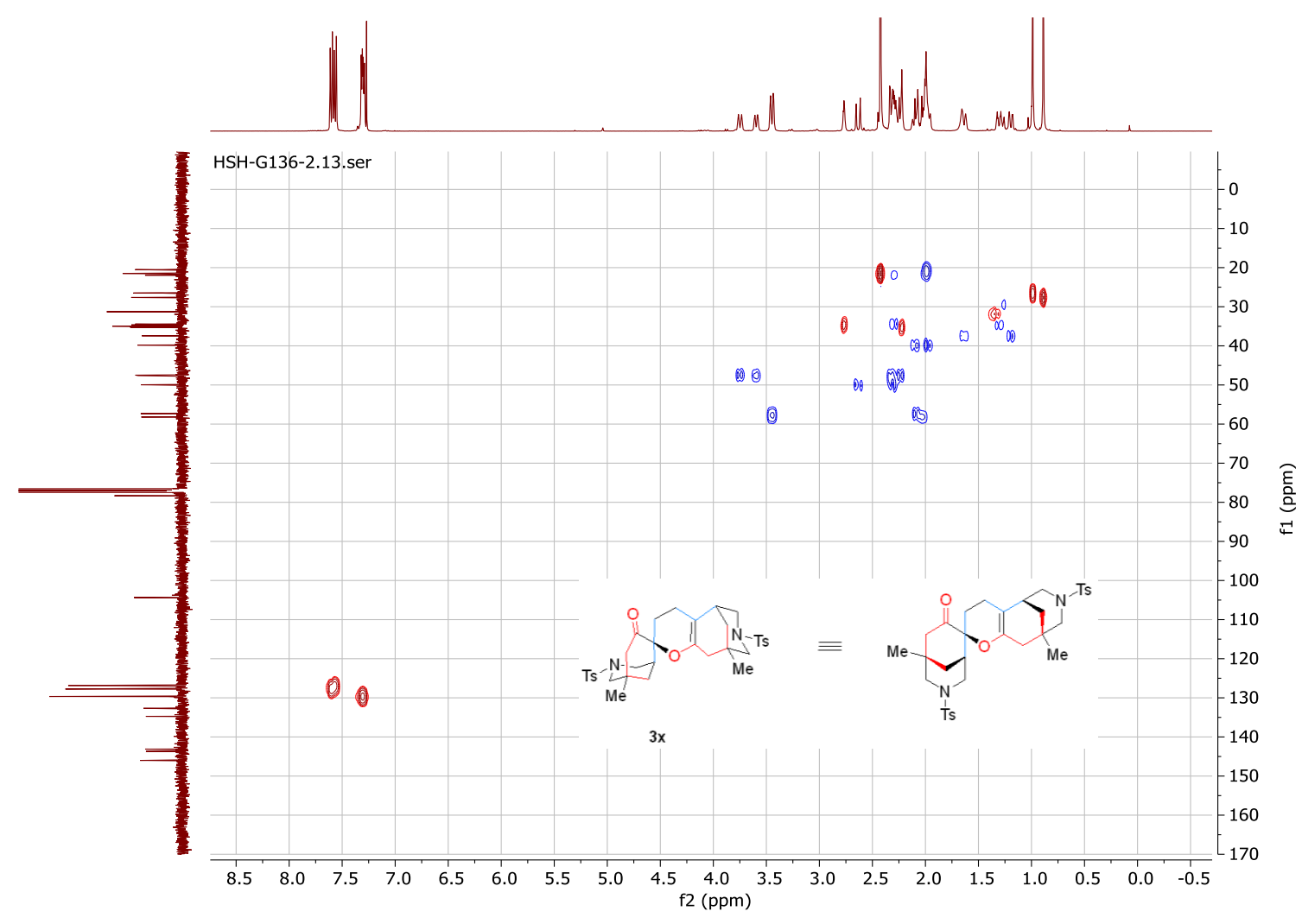

${ }^{1} \mathrm{H}-{ }^{1} \mathrm{H}$ COSY spectrum of $3 \mathbf{x}$ in $\mathrm{CDCl}_{3}, 400 \mathrm{MHz}$

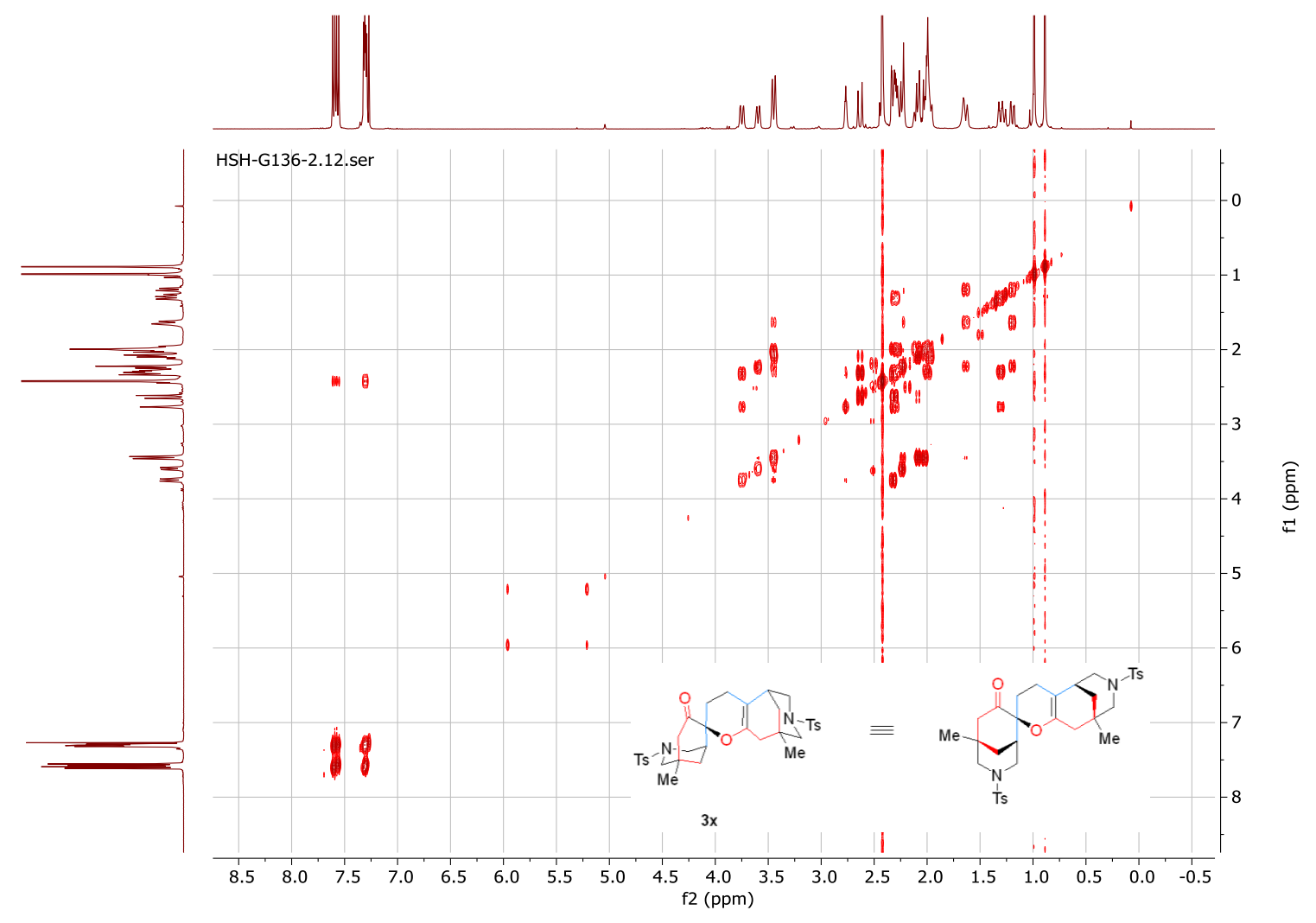



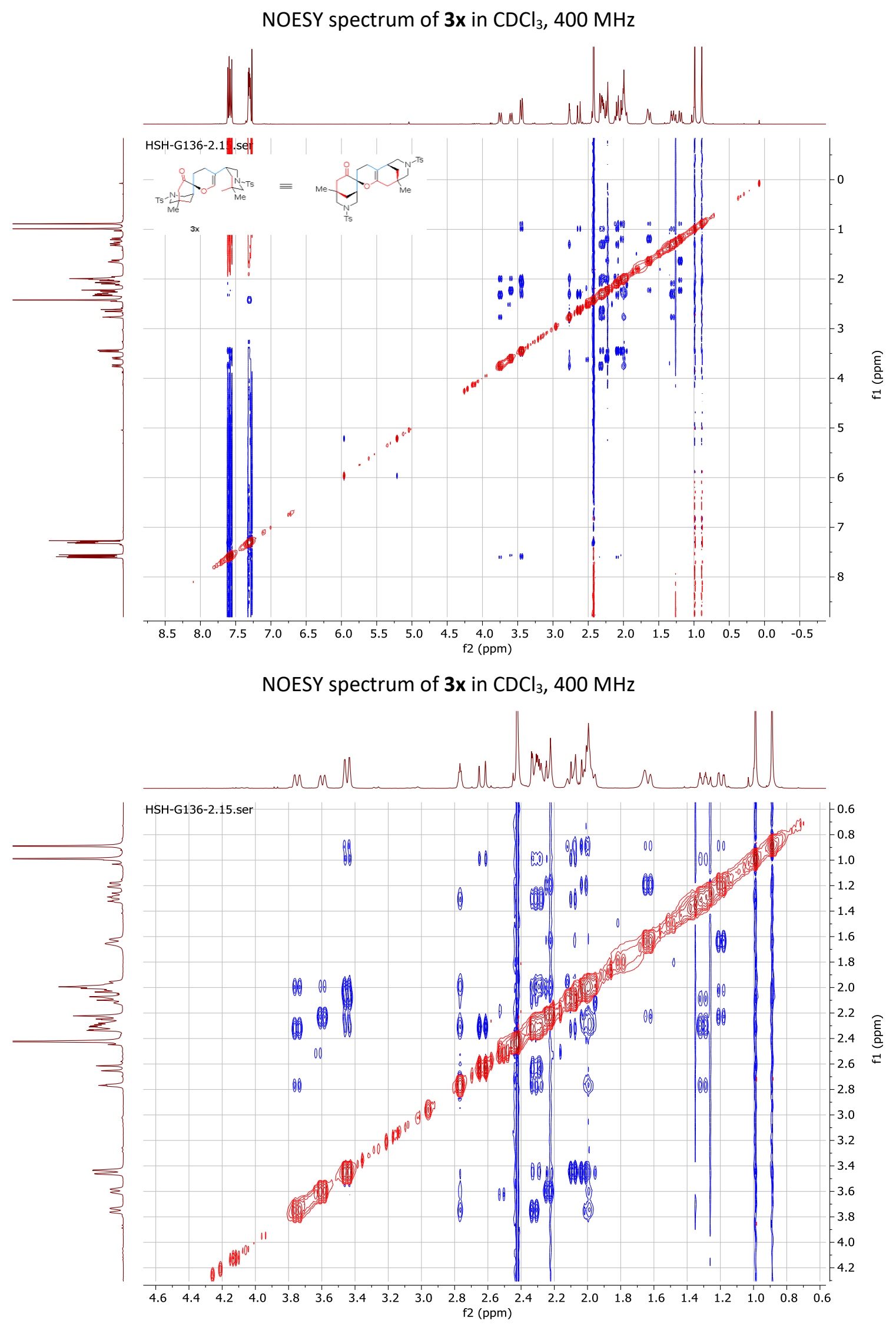
HMBC spectrum of $\mathbf{3 x}$ in $\mathrm{CDCl}_{3}, 400 \mathrm{MHz}$

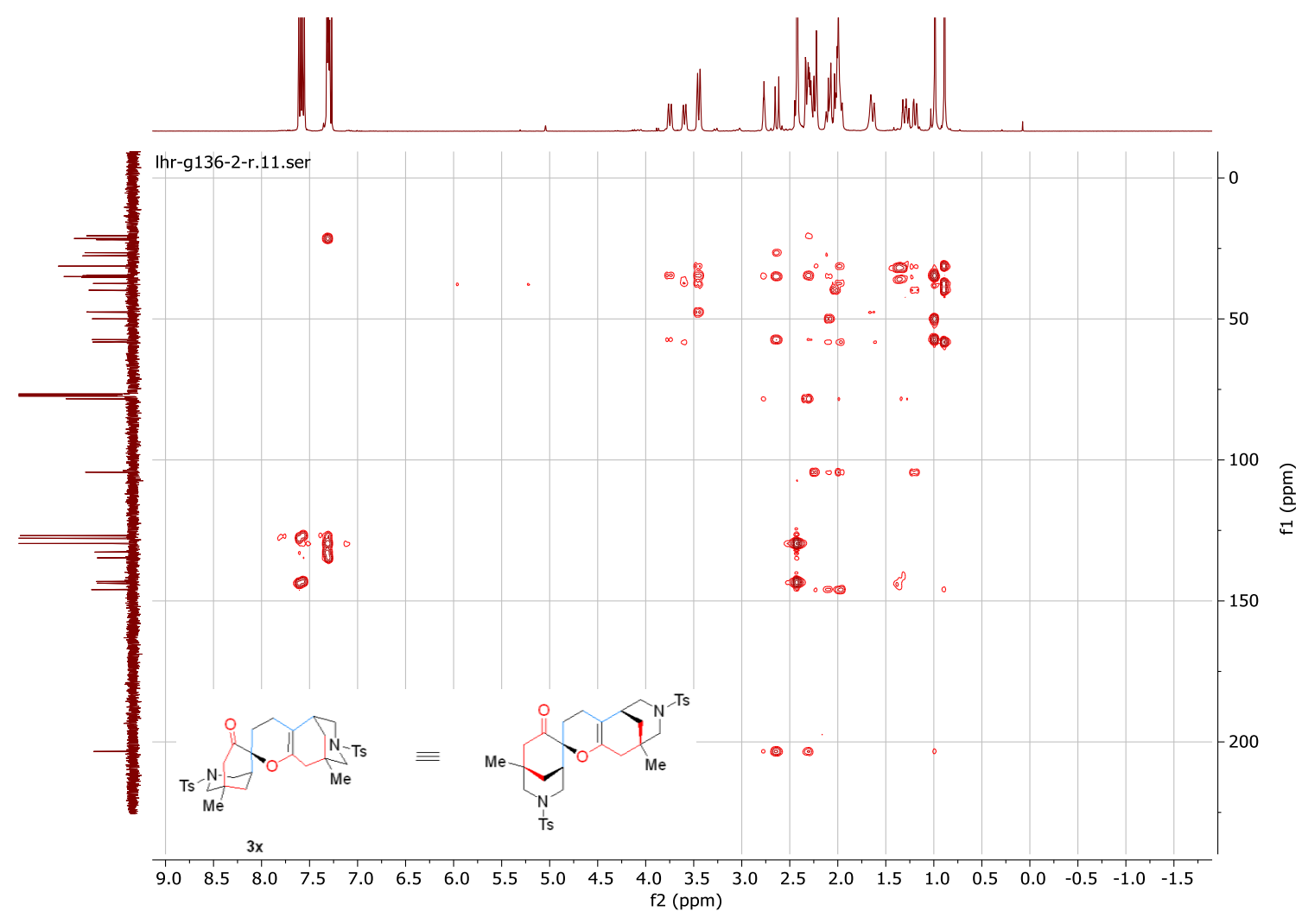

$\mathrm{HMBC}$ spectrum of $\mathbf{3 x}$ in $\mathrm{CDCl}_{3}, 400 \mathrm{MHz}$

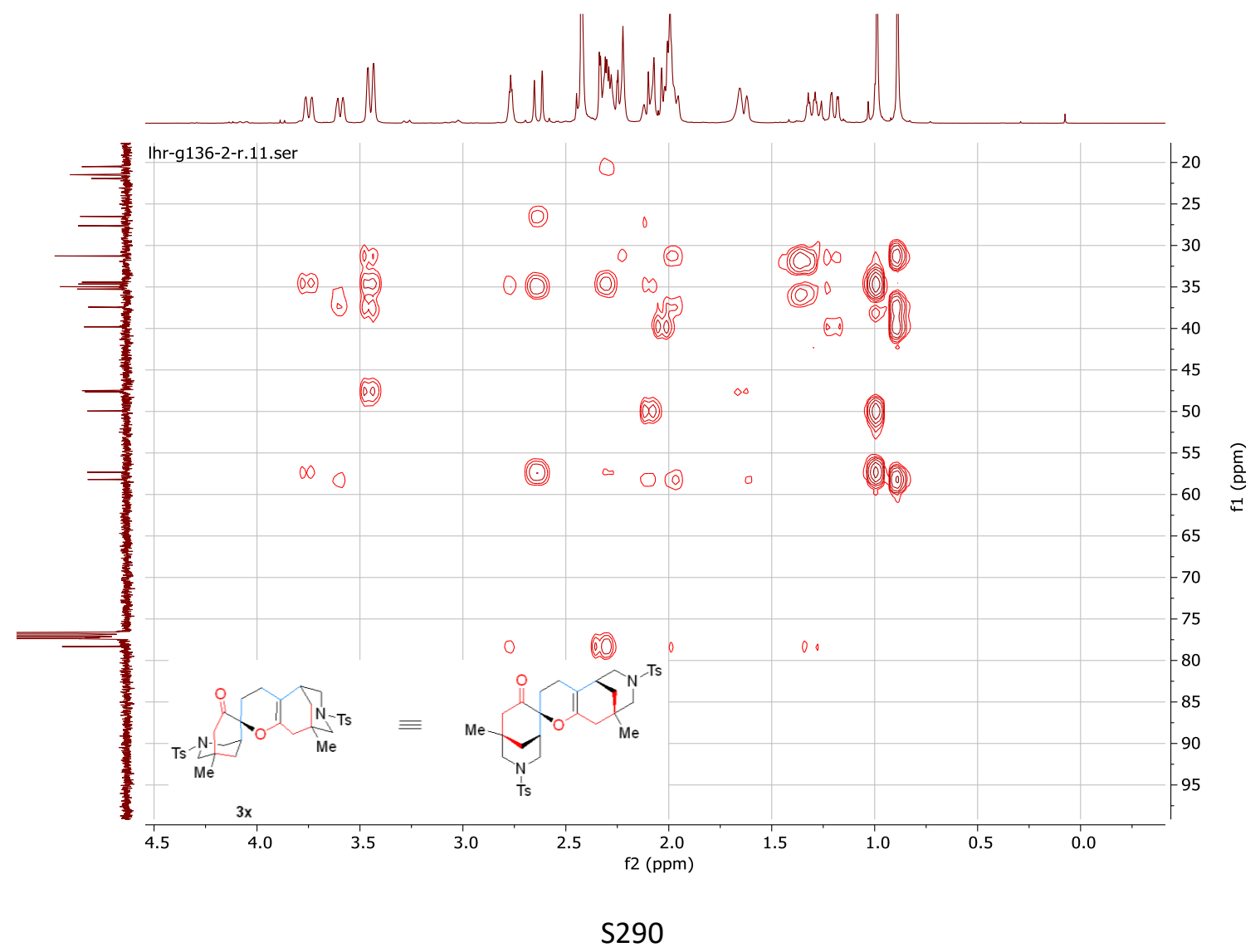



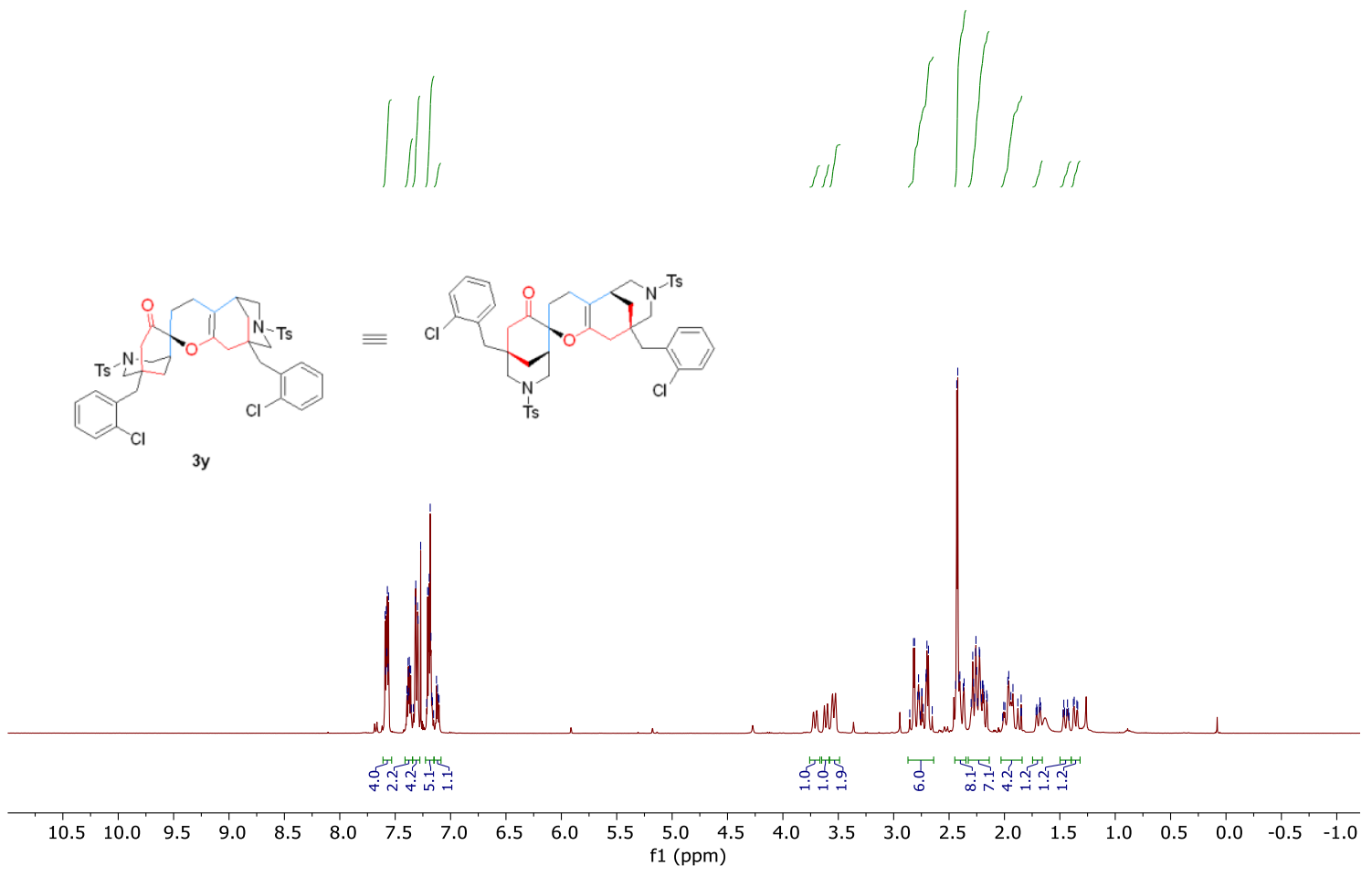

${ }^{13} \mathrm{C}$-NMR spectrum of $\mathbf{3 y}$ in $\mathrm{CDCl}_{3}, 101 \mathrm{MHz}$

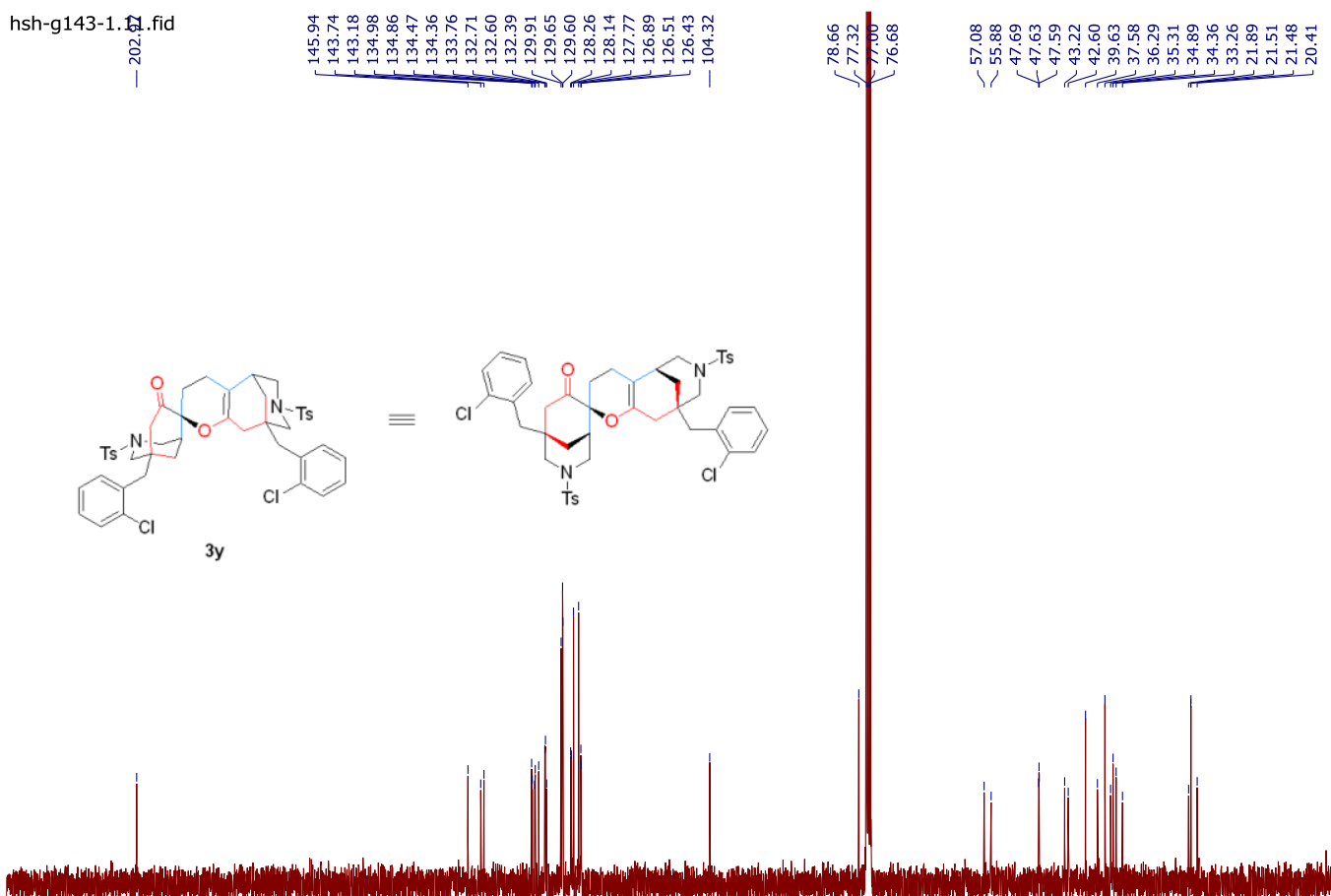

$\begin{array}{lllllllllllllllllllllllllllllllllll}220 & 210 & 200 & 190 & 180 & 170 & 160 & 150 & 140 & 130 & 120 & 110 & 100 & 90 & 80 & 70 & 60 & 50 & 40 & 30 & 20 & 10 & 0 & -10 & -20\end{array}$ 


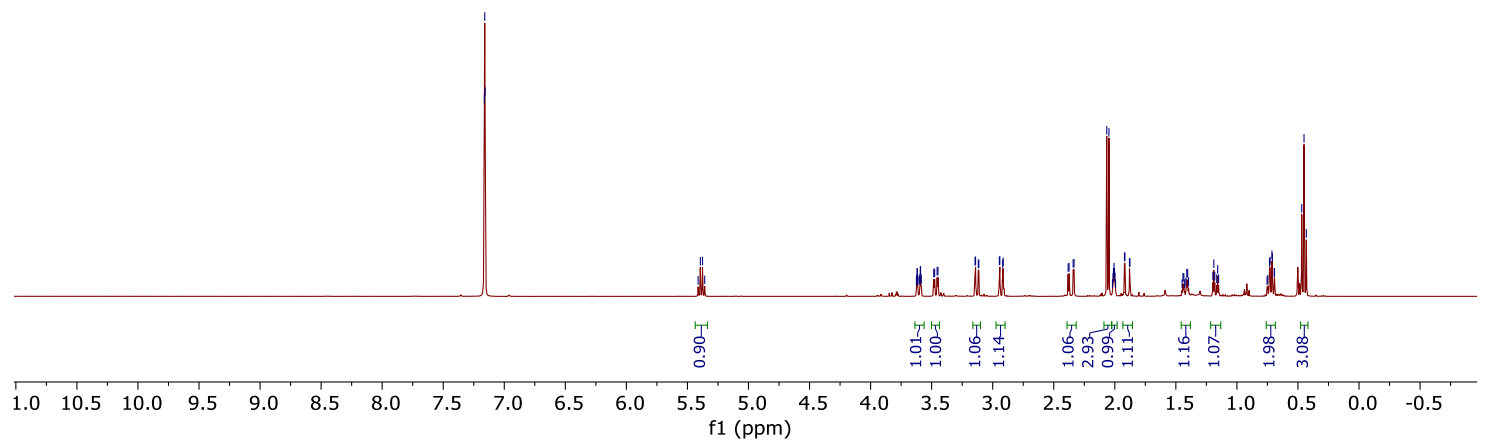

${ }^{13} \mathrm{C}$-NMR spectrum of $\mathbf{2 z}$ in $\mathrm{C}_{6} \mathrm{D}_{6}, 101 \mathrm{MHz}$

hsh-g222-1-chifa्al.11.fid
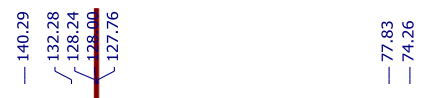

mे
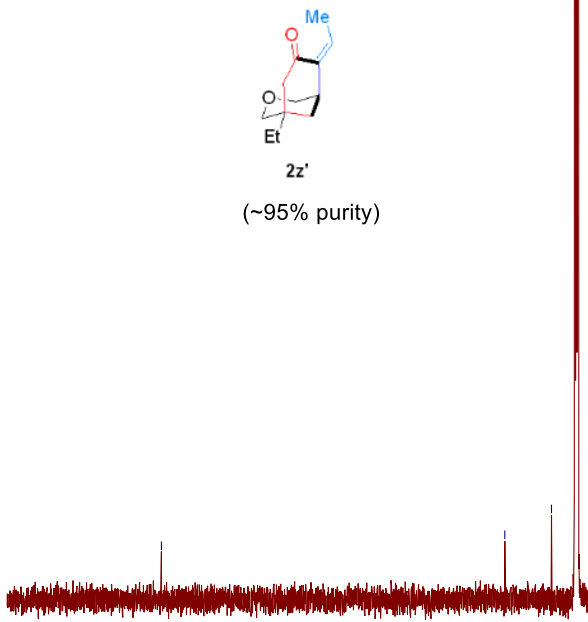

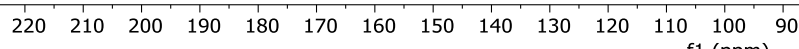

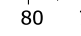

f1 (ppm) 

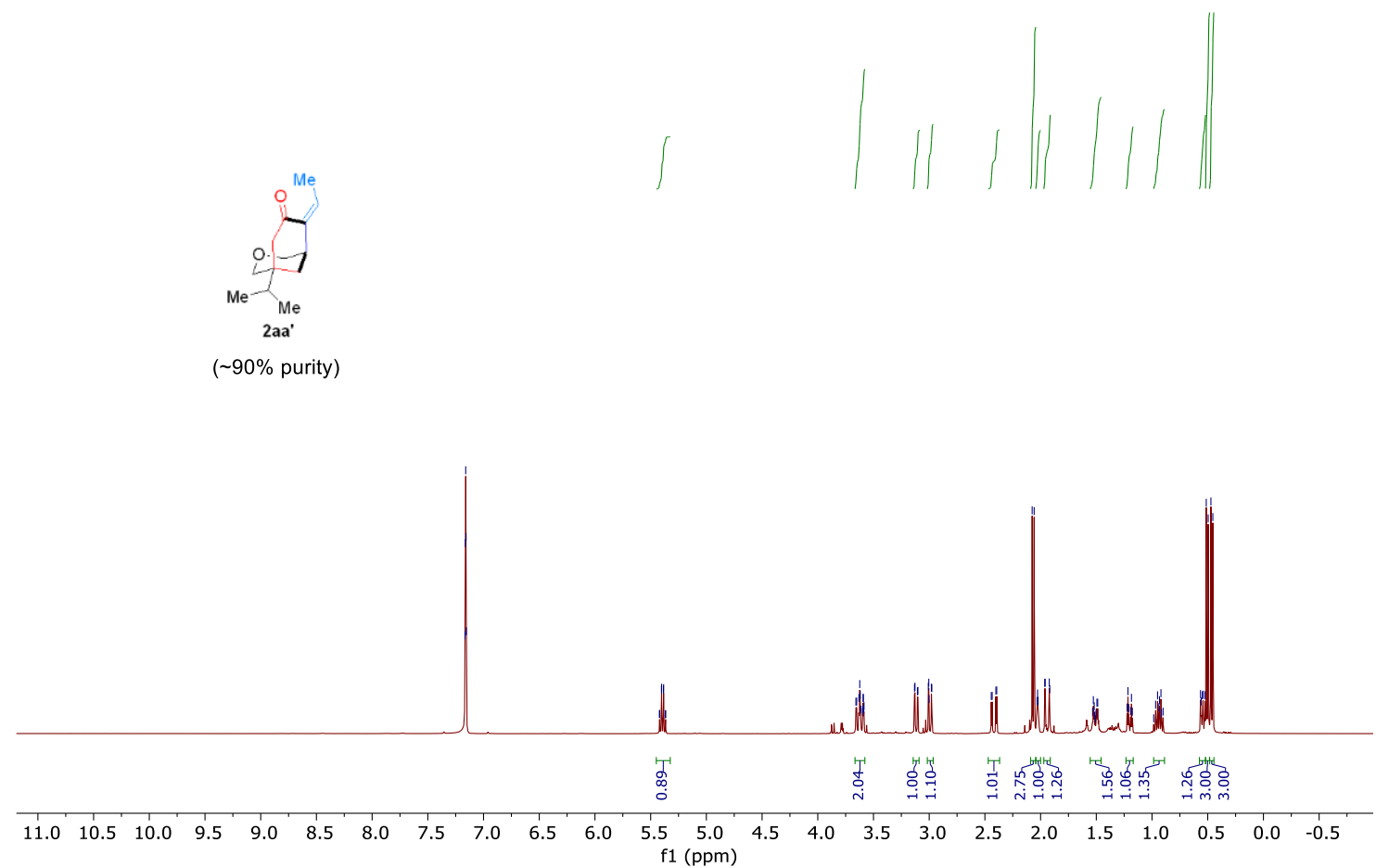

${ }^{13} \mathrm{C}$-NMR spectrum of $\mathbf{2}$ aa' in $\mathrm{C}_{6} \mathrm{D}_{6}, 101 \mathrm{MHz}$

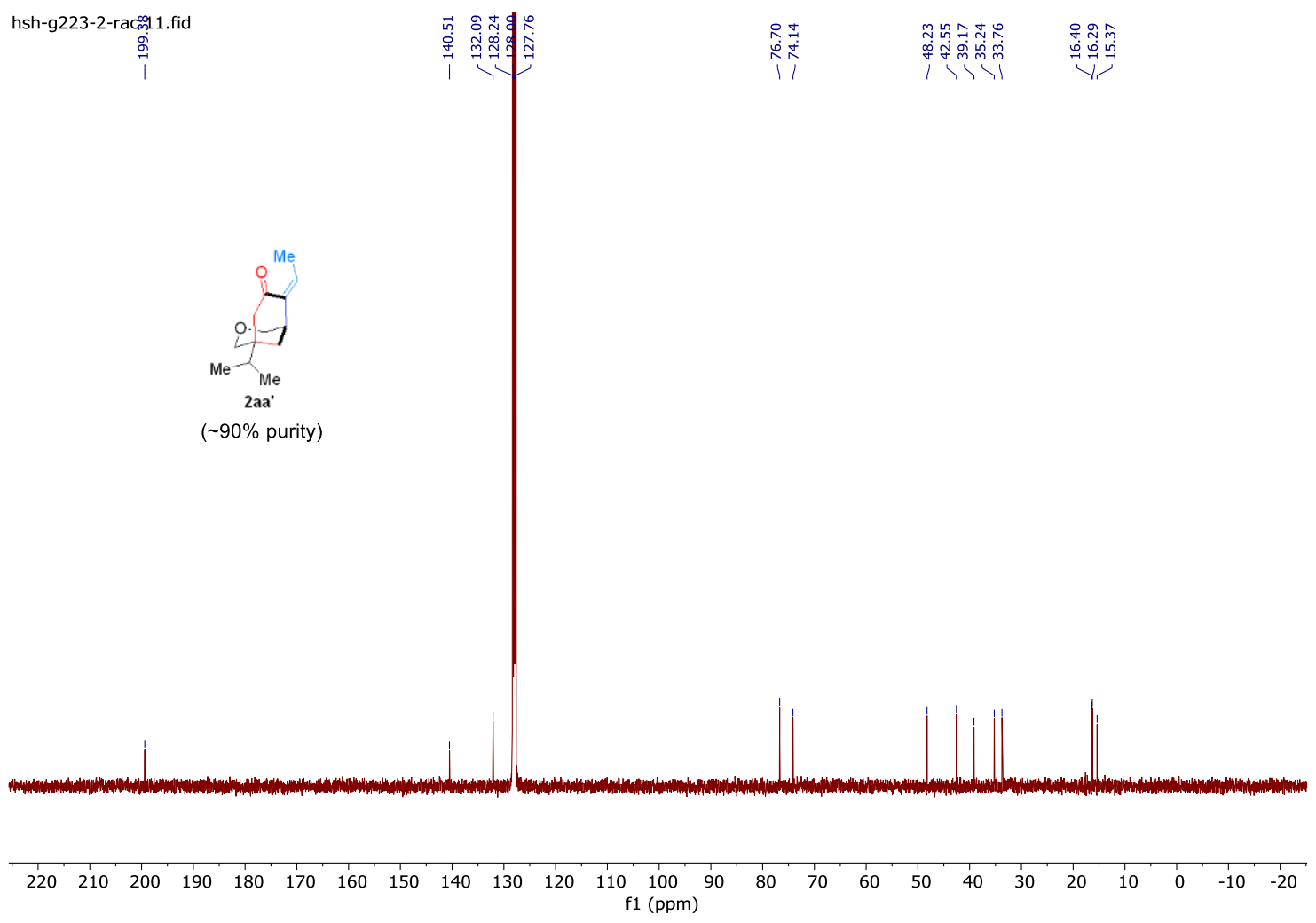


${ }^{1} \mathrm{H}$-NMR spectrum of $2 \mathbf{a b}$ ' in $\mathrm{C}_{6} \mathrm{D}_{6}, 400 \mathrm{MHz}$

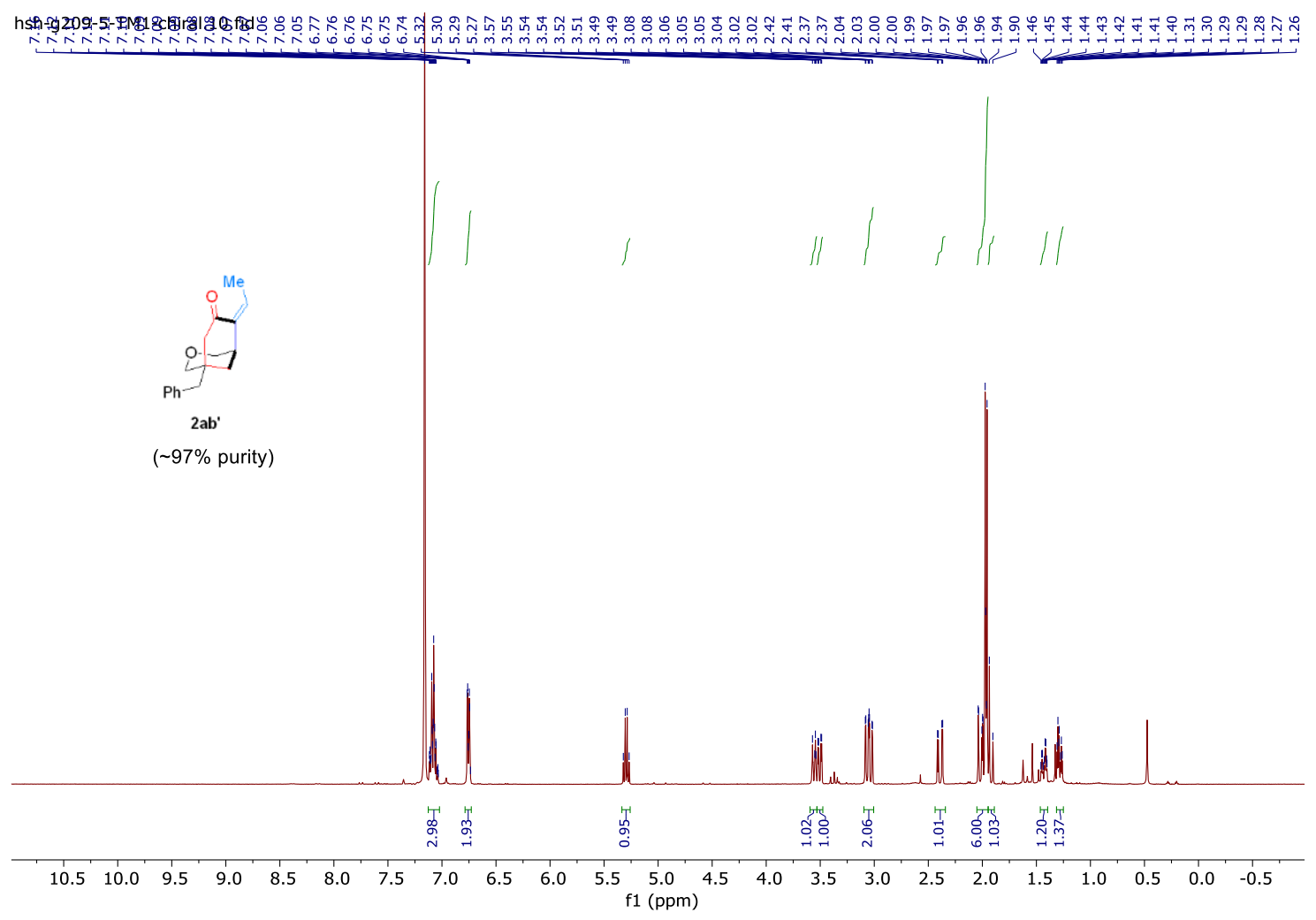

${ }^{13} \mathrm{C}$-NMR spectrum of $\mathbf{2} \mathbf{a b} \mathbf{b}^{\prime}$ in $\mathrm{C}_{6} \mathrm{D}_{6}, 101 \mathrm{MHz}$
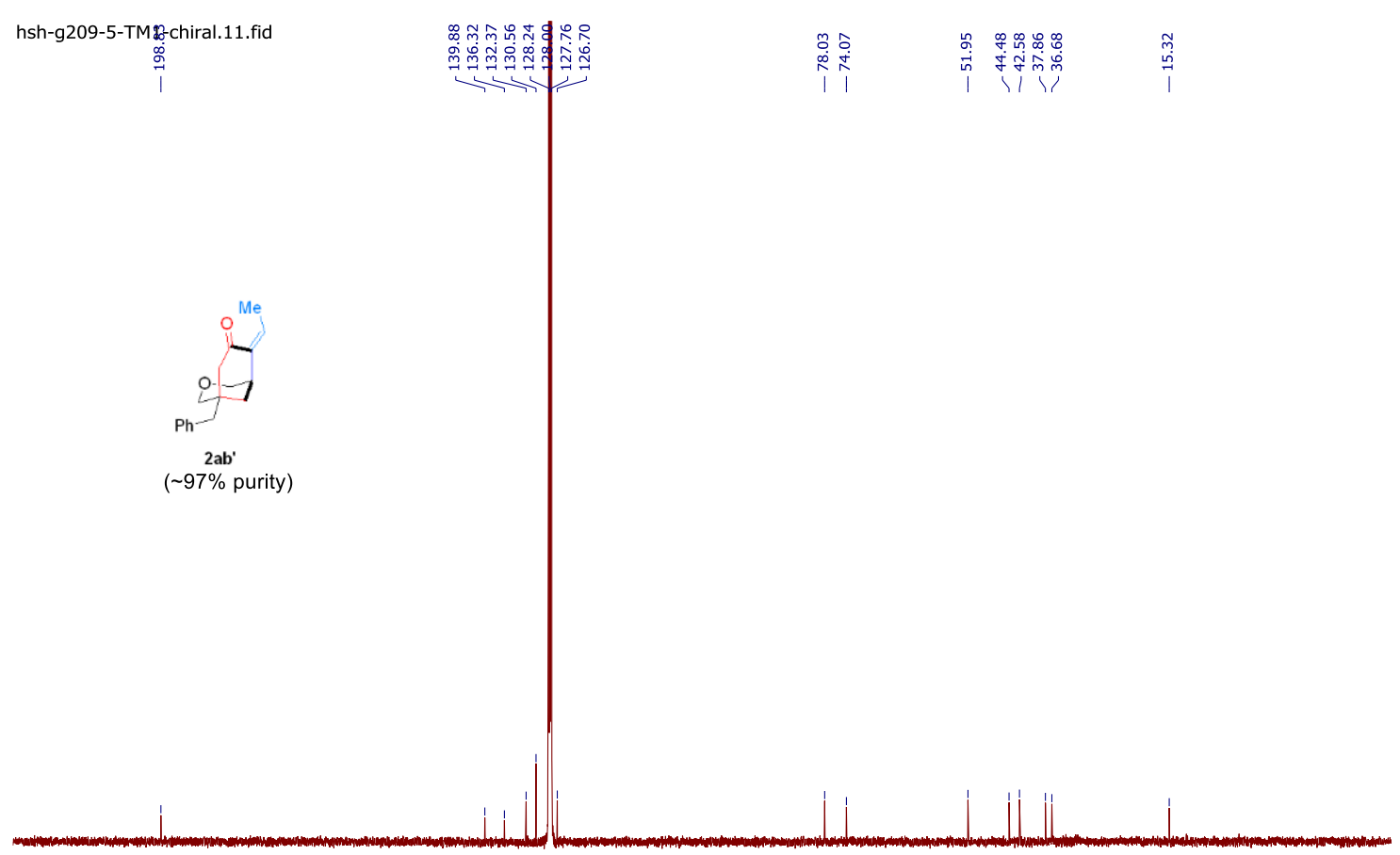

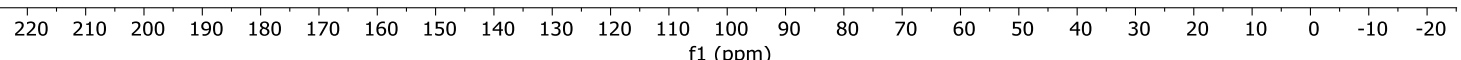




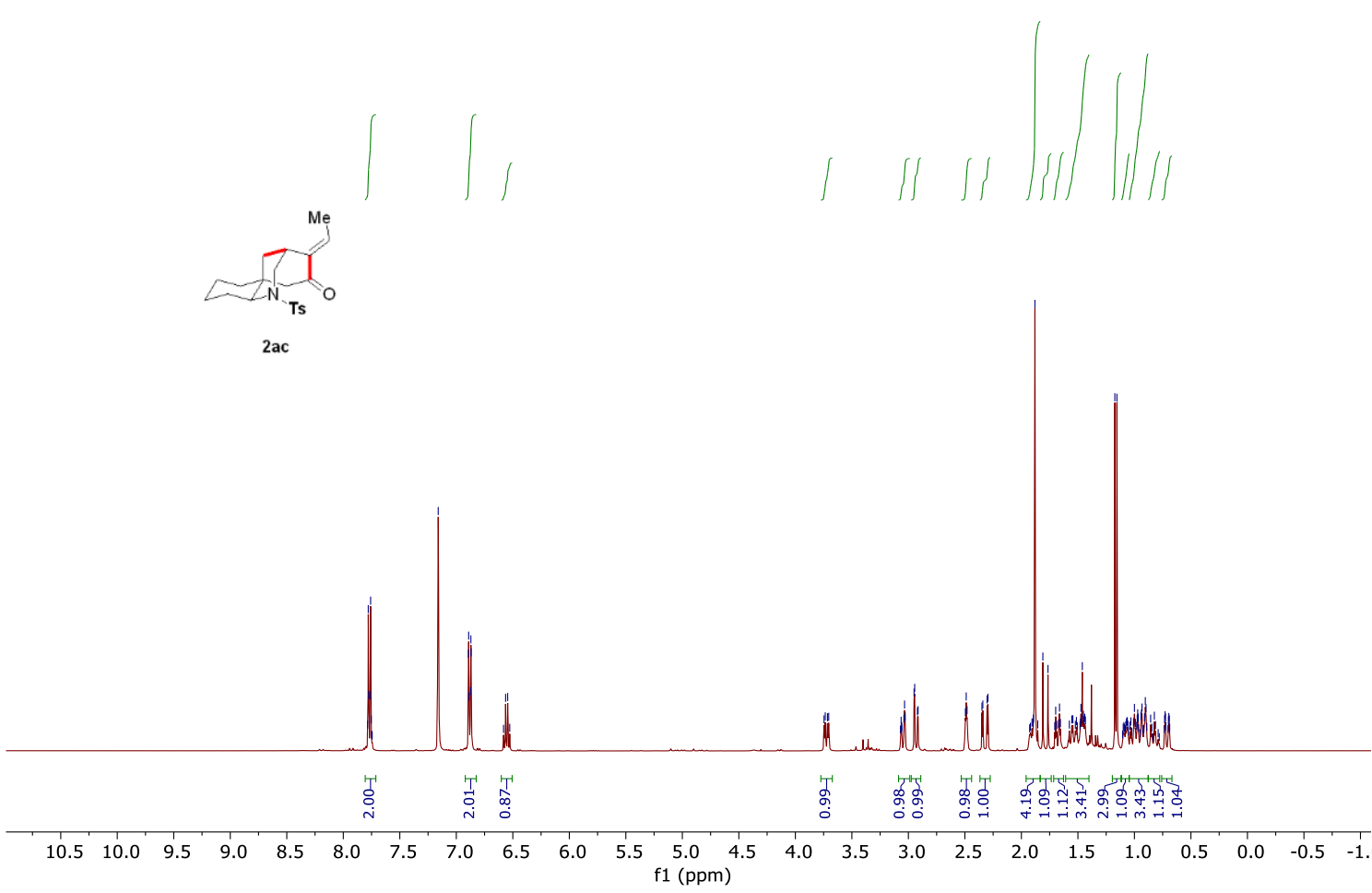

${ }^{13} \mathrm{C}$-NMR spectrum of $2 \mathrm{ac}$ in $\mathrm{C}_{6} \mathrm{D}_{6}, 101 \mathrm{MHz}$

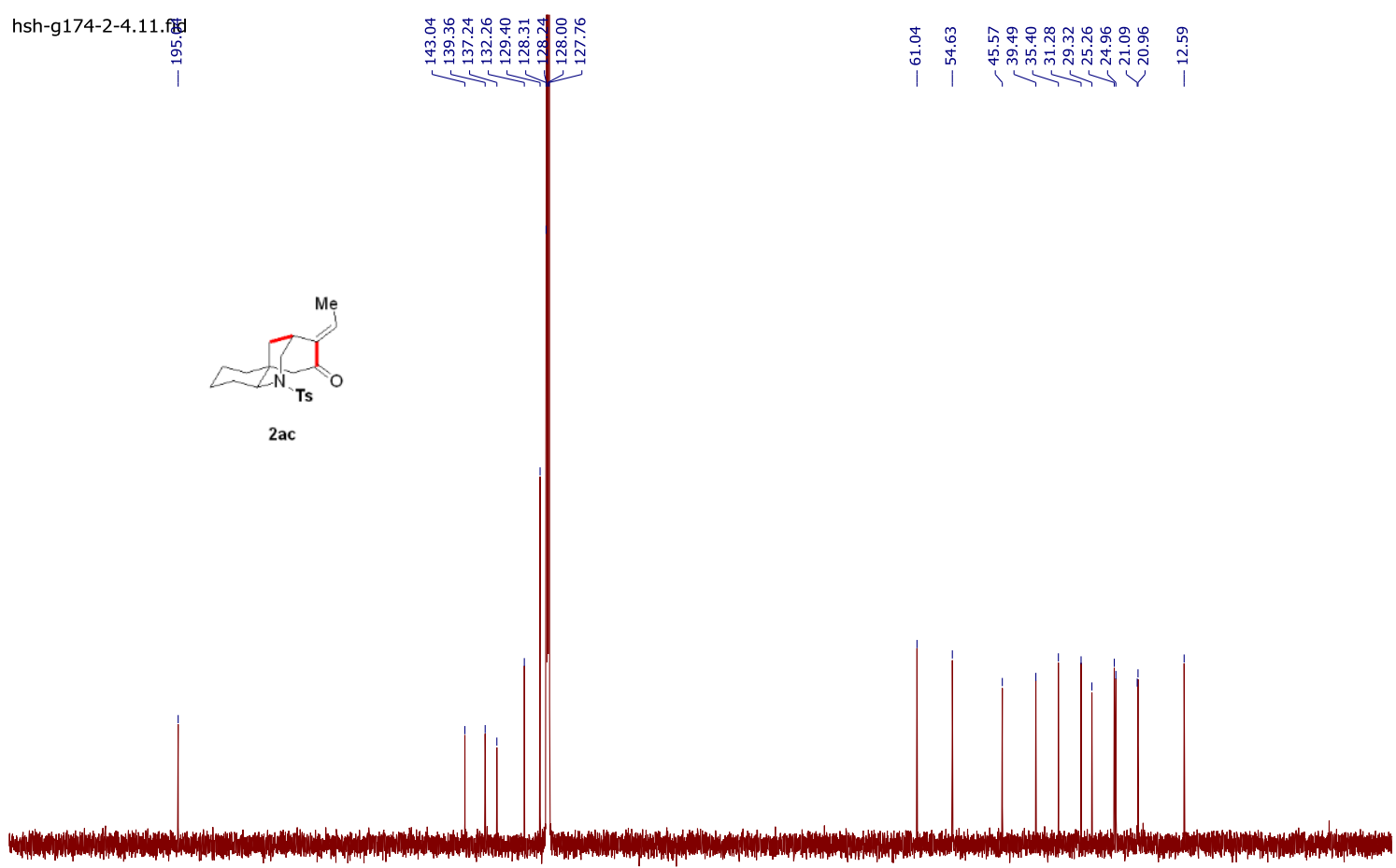

$\begin{array}{lllllllllllllllllllllllllllllllllll}220 & 210 & 200 & 190 & 180 & 170 & 160 & 150 & 140 & 130 & 120 & 110 & 100 & 90 & 80 & 70 & 60 & 50 & 40 & 30 & 20 & 10 & 0 & -10 & -20\end{array}$ 
DEPT spectrum of $2 a c$ in $C_{6} D_{6}, 101 \mathrm{MHz}$

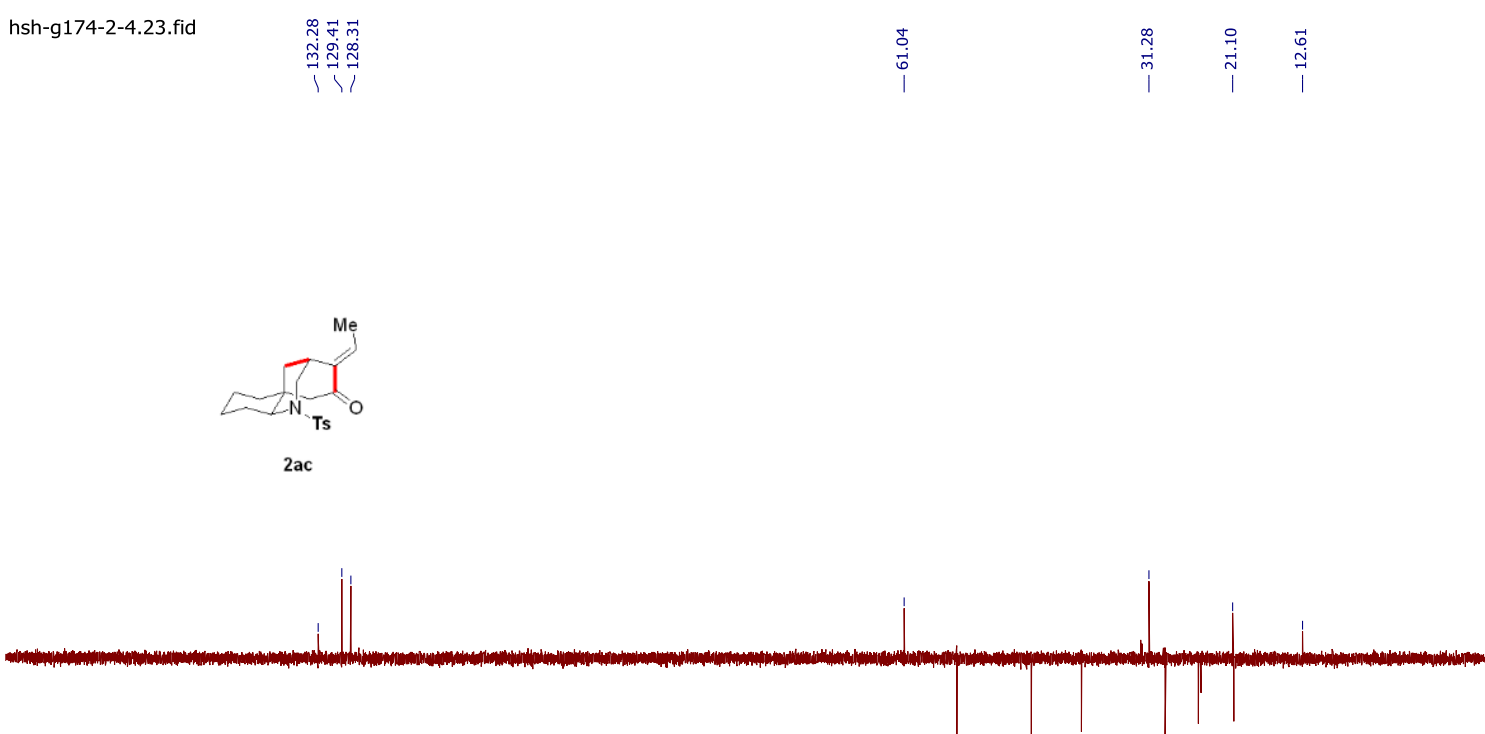

\begin{tabular}{|c|c|c|c|c|c|c|c|c|c|c|c|c|c|c|c|c|c|}
\hline 70 & 160 & 150 & 140 & 130 & 120 & 110 & 100 & 90 & $\begin{array}{c}80 \\
\mathrm{f} 1(\mathrm{ppm})\end{array}$ & 70 & 60 & 50 & 40 & 30 & 20 & 10 & 0 \\
\hline
\end{tabular}

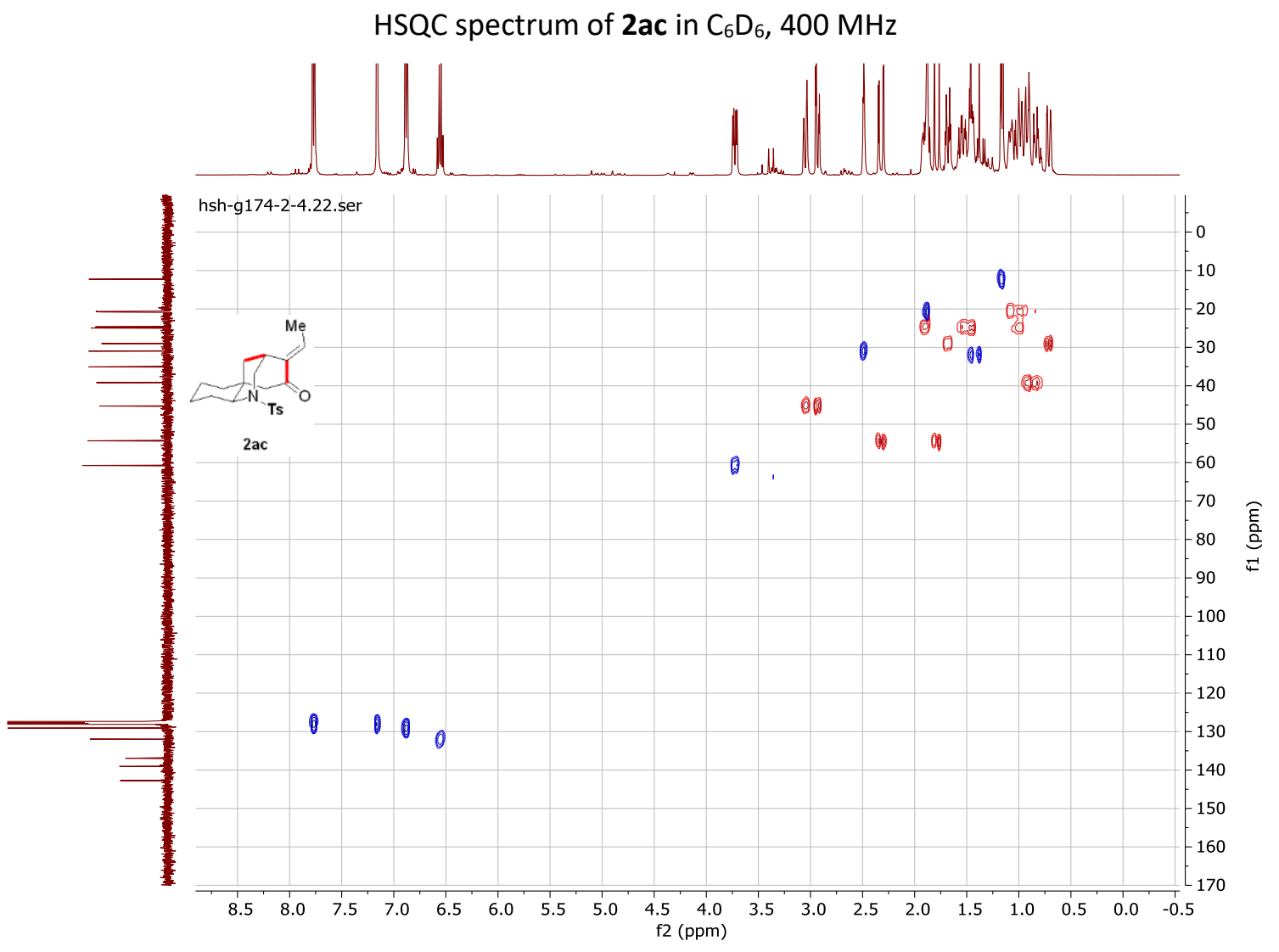




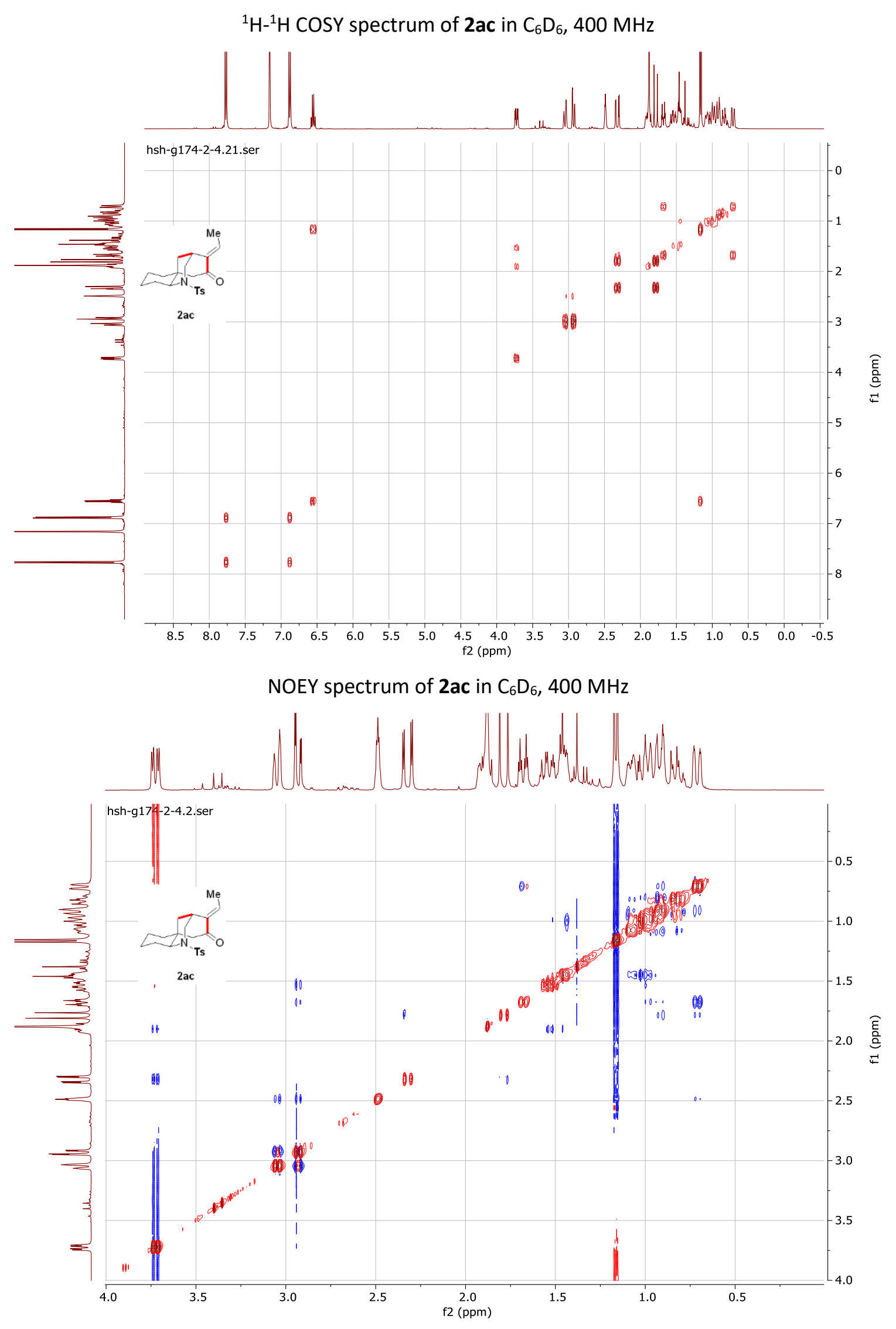




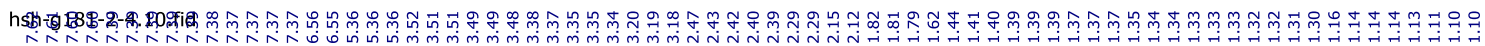

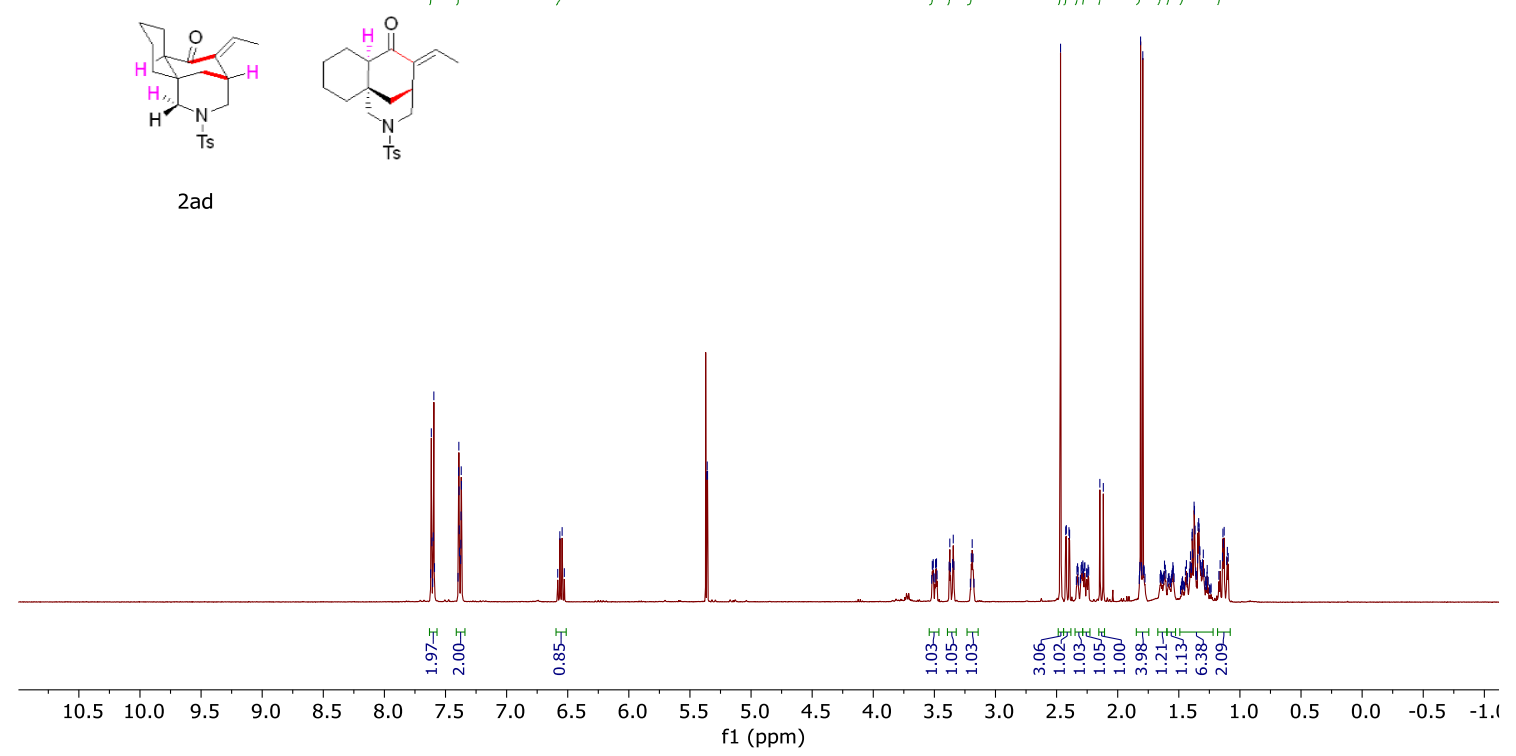

$2 a d$

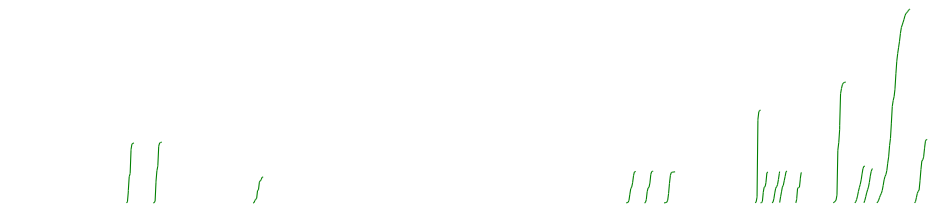

${ }^{13} \mathrm{C}$-NMR spectrum of $2 \mathrm{ad}$ in $\mathrm{CD}_{2} \mathrm{Cl}_{2}, 101 \mathrm{MHz}$

hsh-g181-2-4

ㅇำํํำำำ

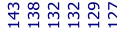

i

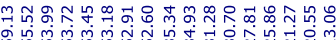
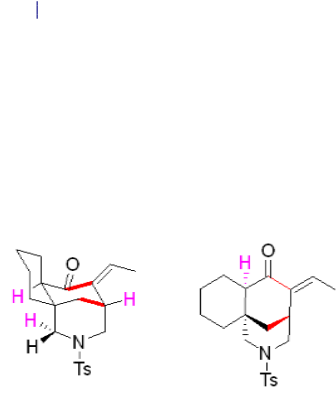

Ts

$2 \mathrm{ad}$

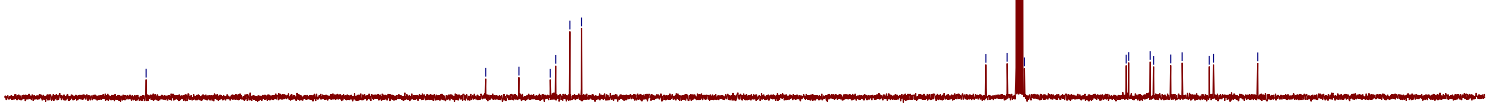

$\begin{array}{llllllllllllllllllllllllllllllllllll}220 & 210 & 200 & 190 & 180 & 170 & 160 & 150 & 140 & 130 & 120 & 110 & 100 & 90 & 80 & 70 & 60 & 50 & 40 & 30 & 20 & 10 & 0 & -10 & -20\end{array}$ 
DEPT spectrum of $2 \mathrm{ad}$ in $\mathrm{CD}_{2} \mathrm{Cl}_{2}, 101 \mathrm{MHz}$

hsh-g181-2-4.3.fid m:
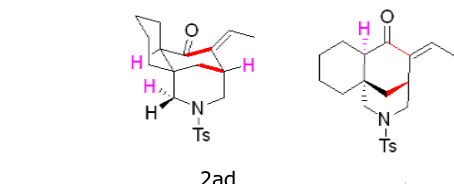

$2 \mathrm{ad}$

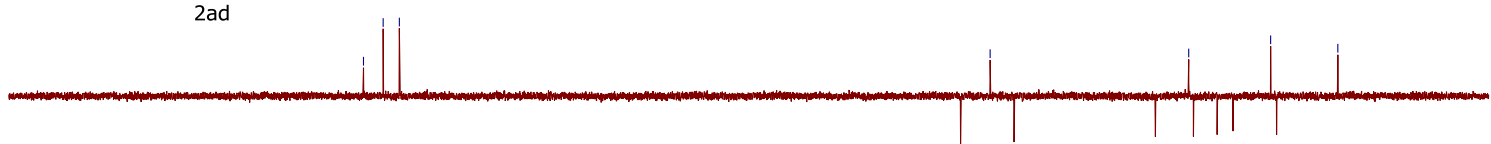

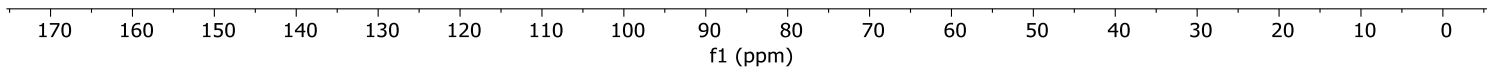

${ }^{1} \mathrm{H}-{ }^{1} \mathrm{H}$ COSY spectrum of 2 ad in $\mathrm{CD}_{2} \mathrm{Cl}_{2}, 400 \mathrm{MHz}$

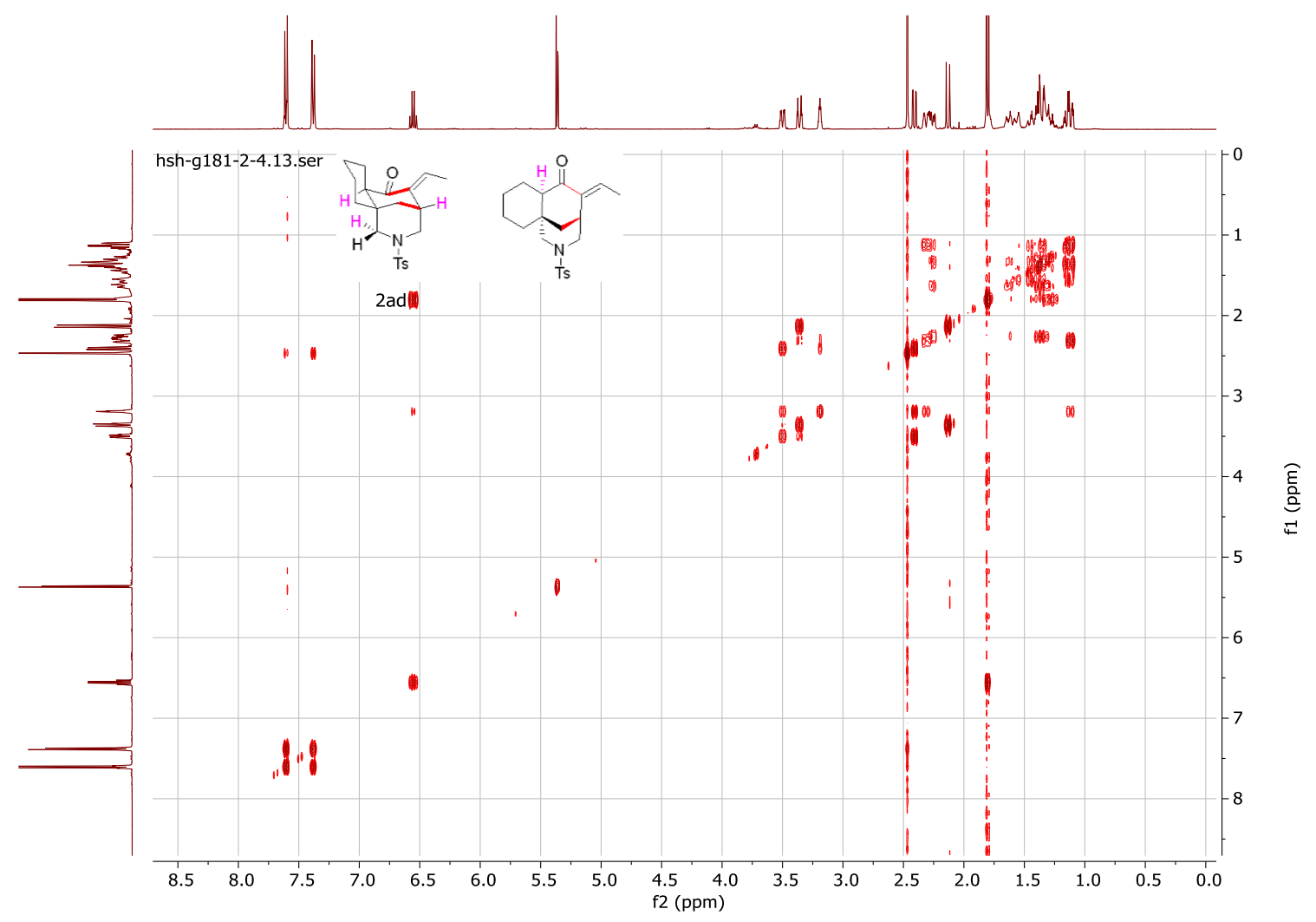


HSQC spectrum of $2 \mathrm{ad}$ in $\mathrm{CD}_{2} \mathrm{Cl}_{2}, 400 \mathrm{MHz}$

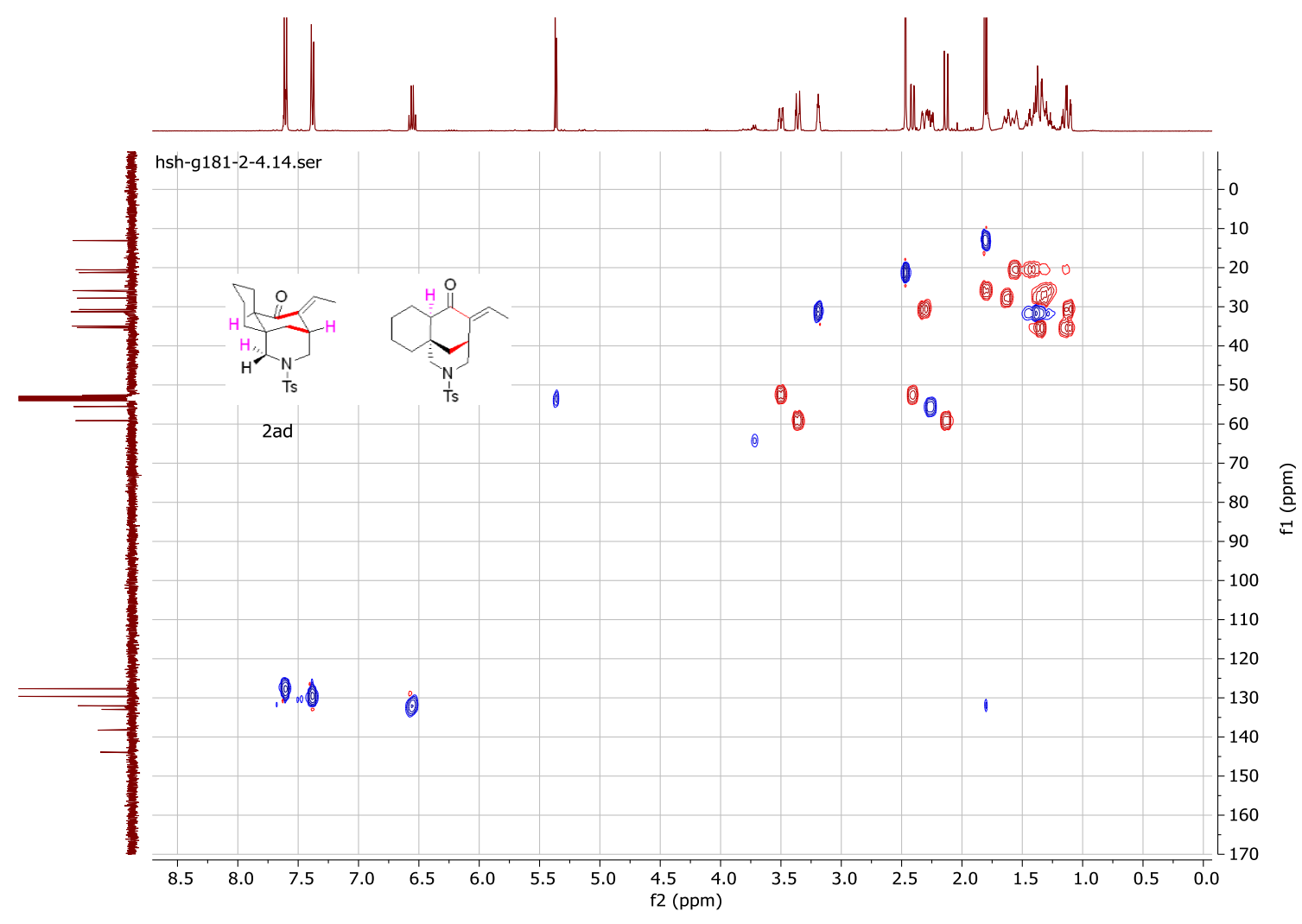

NOEY spectrum of 2ad in $\mathrm{CD}_{2} \mathrm{Cl}_{2}, 400 \mathrm{MHz}$

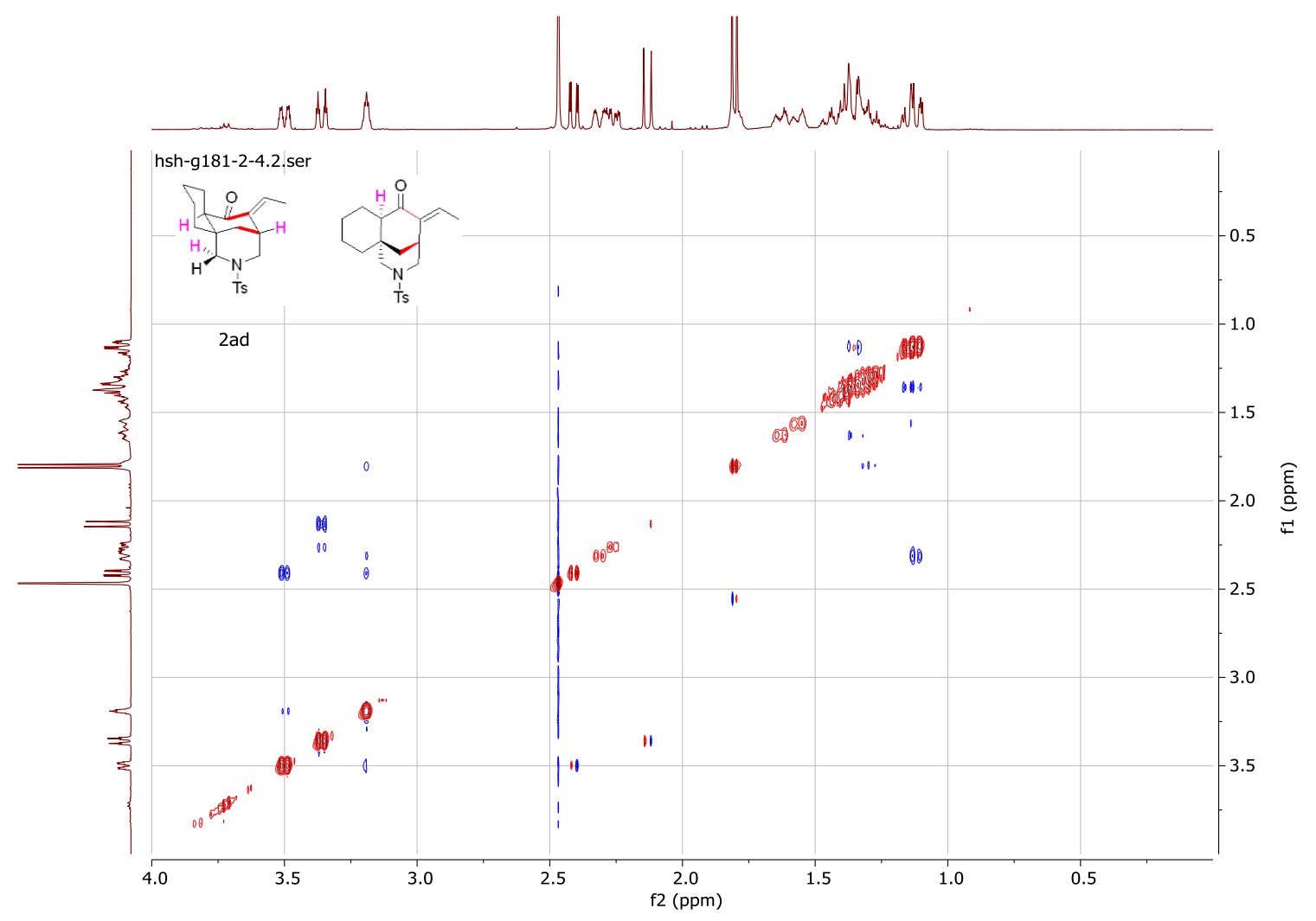




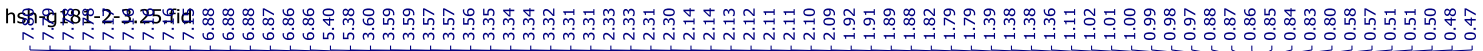

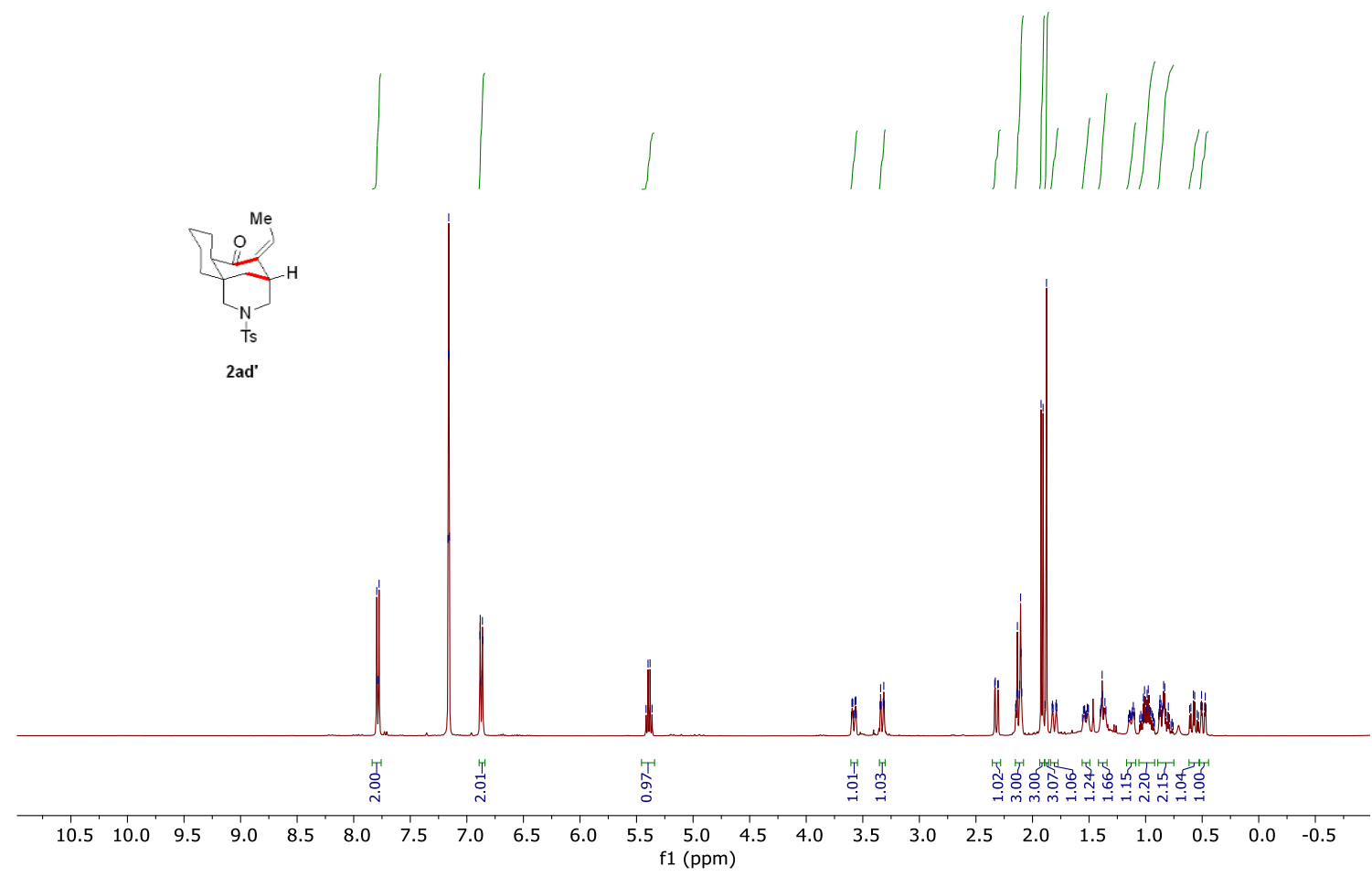

${ }^{13} \mathrm{C}-\mathrm{NMR}$ spectrum of $\mathbf{2 a d}$ ' in $\mathrm{C}_{6} \mathrm{D}_{6}, 101 \mathrm{MHz}$
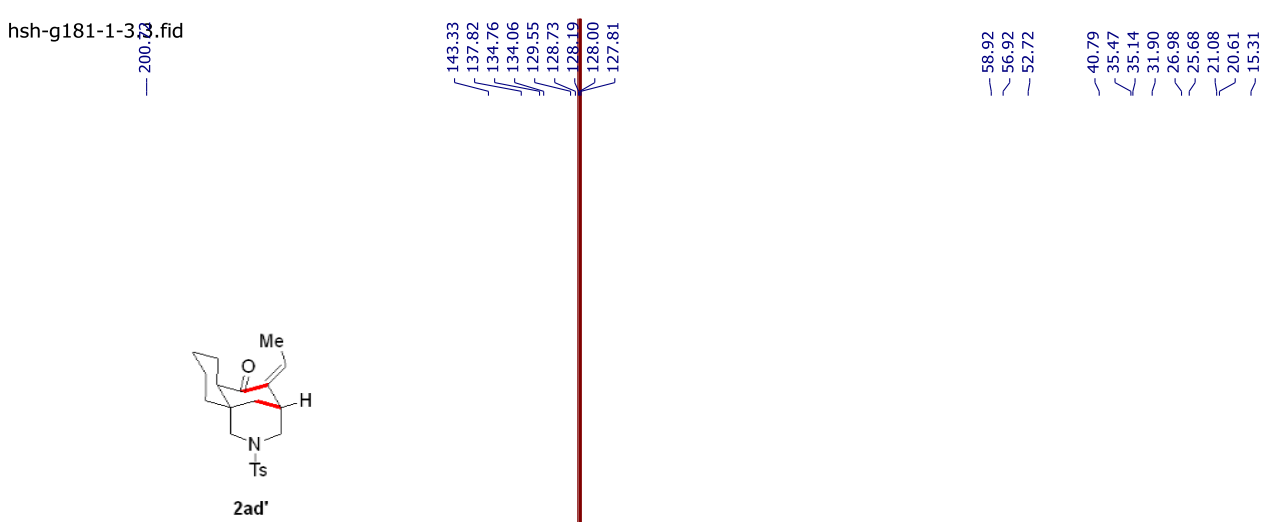

$\begin{array}{llllllllllllllllllllllllllll}220 & 210 & 200 & 190 & 180 & 170 & 160 & 150 & 140 & 130 & 120 & 110 & 100 & 90 & 80 & 70 & 60 & 50 & 40 & 30 & 20 & 10 & 0 & -10 & -20\end{array}$ 


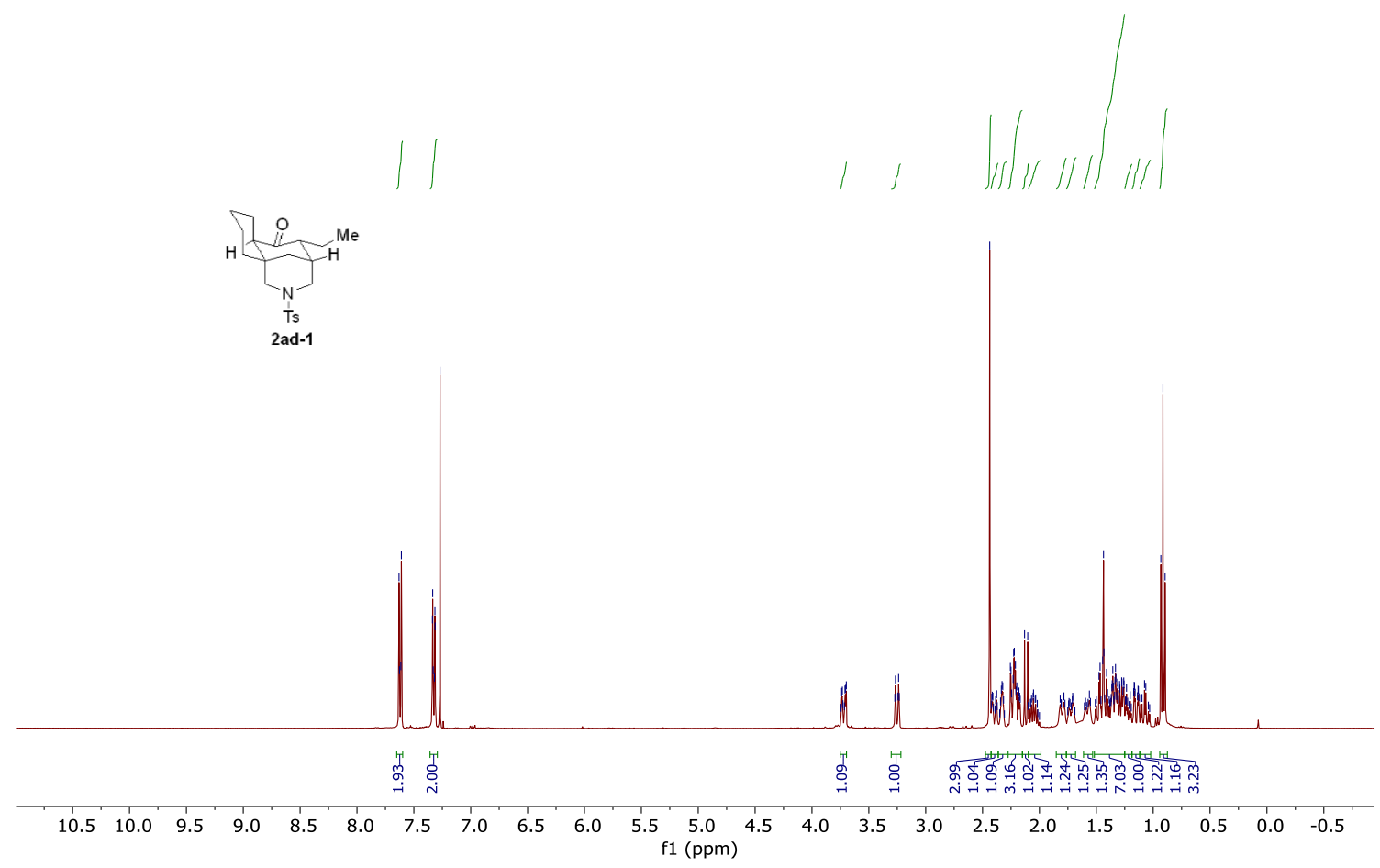

${ }^{13} \mathrm{C}$-NMR spectrum of $\mathbf{2 a d - 1}$ in $\mathrm{CDCl}_{3}, 101 \mathrm{MHz}$

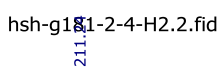

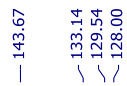

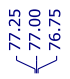

กุ๊

กิ
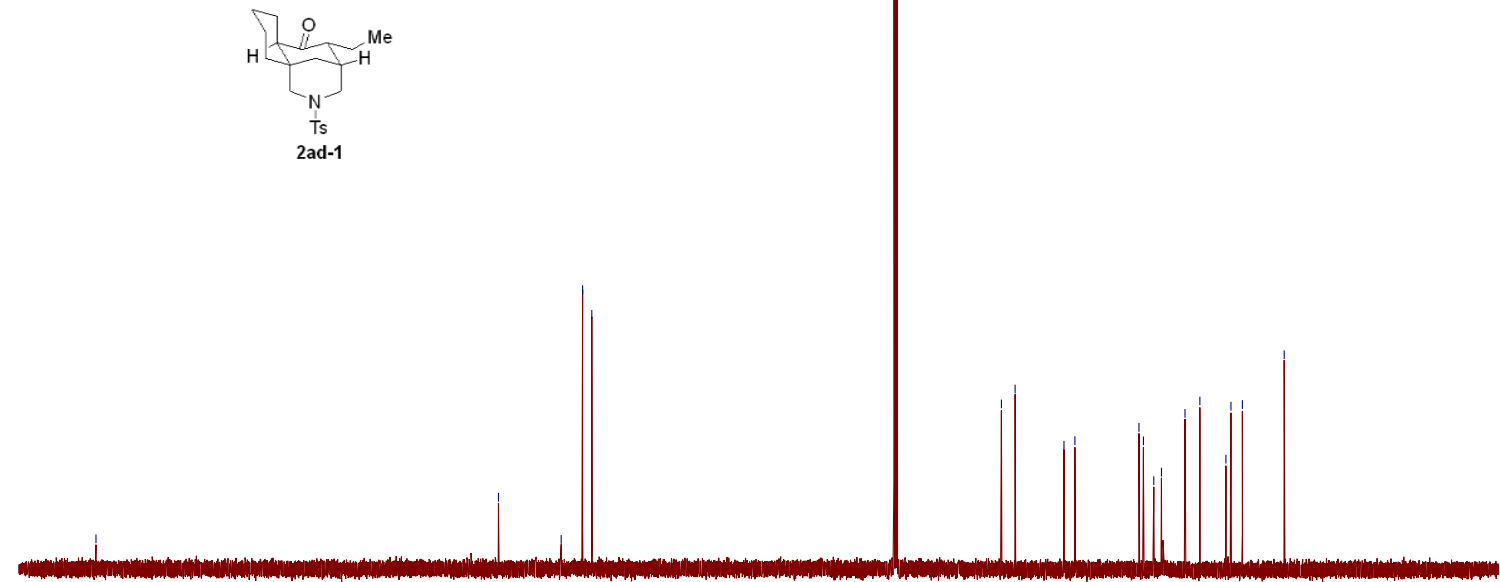

$\begin{array}{llllllllllllllllllllllllllll}220 & 210 & 200 & 190 & 180 & 170 & 160 & 150 & 140 & 130 & 120 & 110 & 100 & 90 & 80 & 70 & 60 & 50 & 40 & 30 & 20 & 10 & 0 & -10 & -20\end{array}$ 


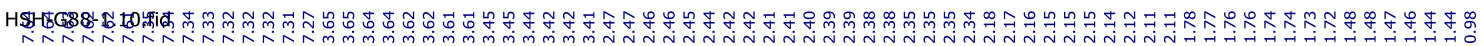

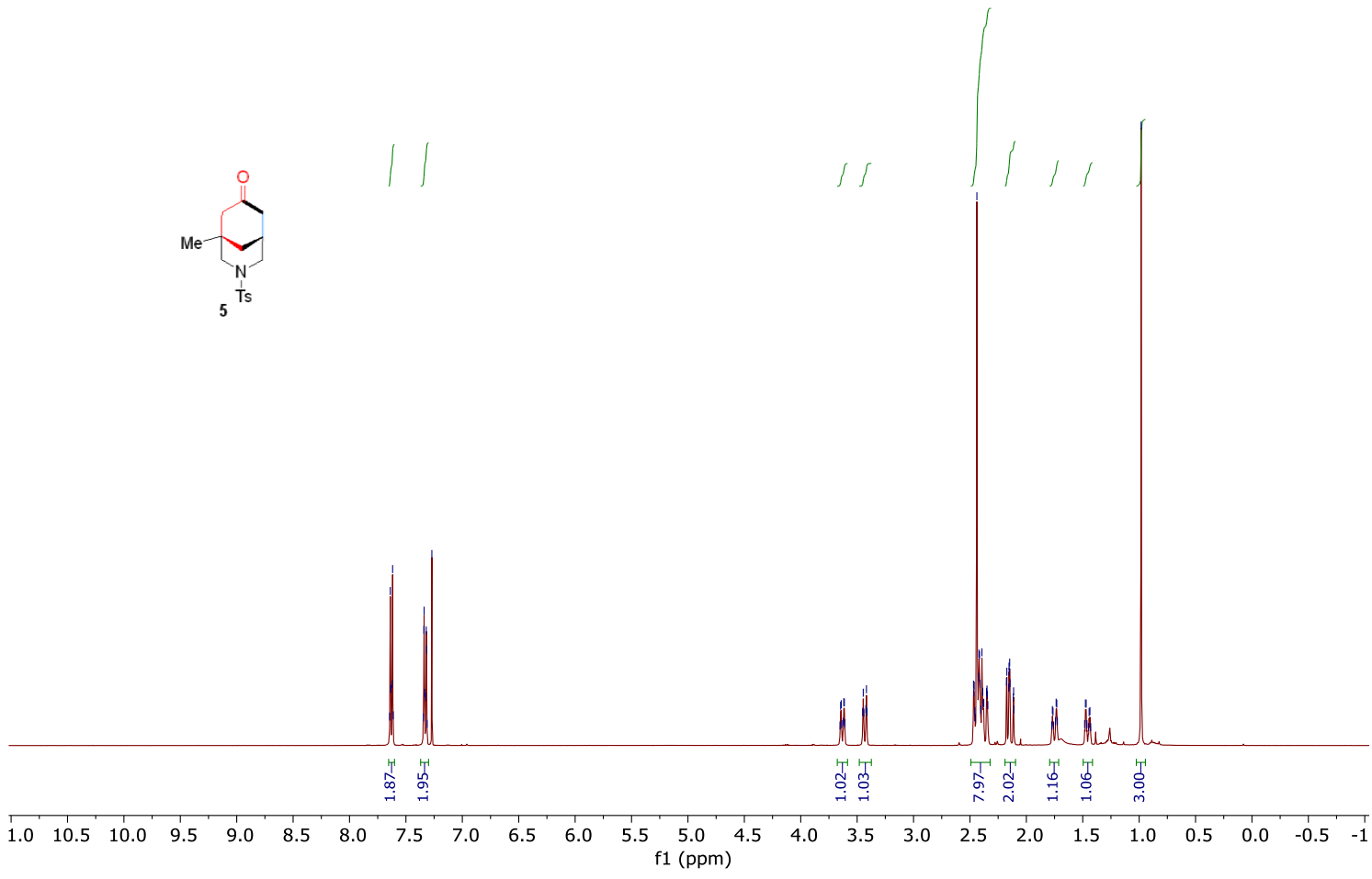

${ }^{13} \mathrm{C}$-NMR spectrum of 5 in $\mathrm{CDCl}_{3}, 101 \mathrm{MHz}$

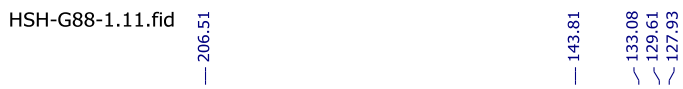
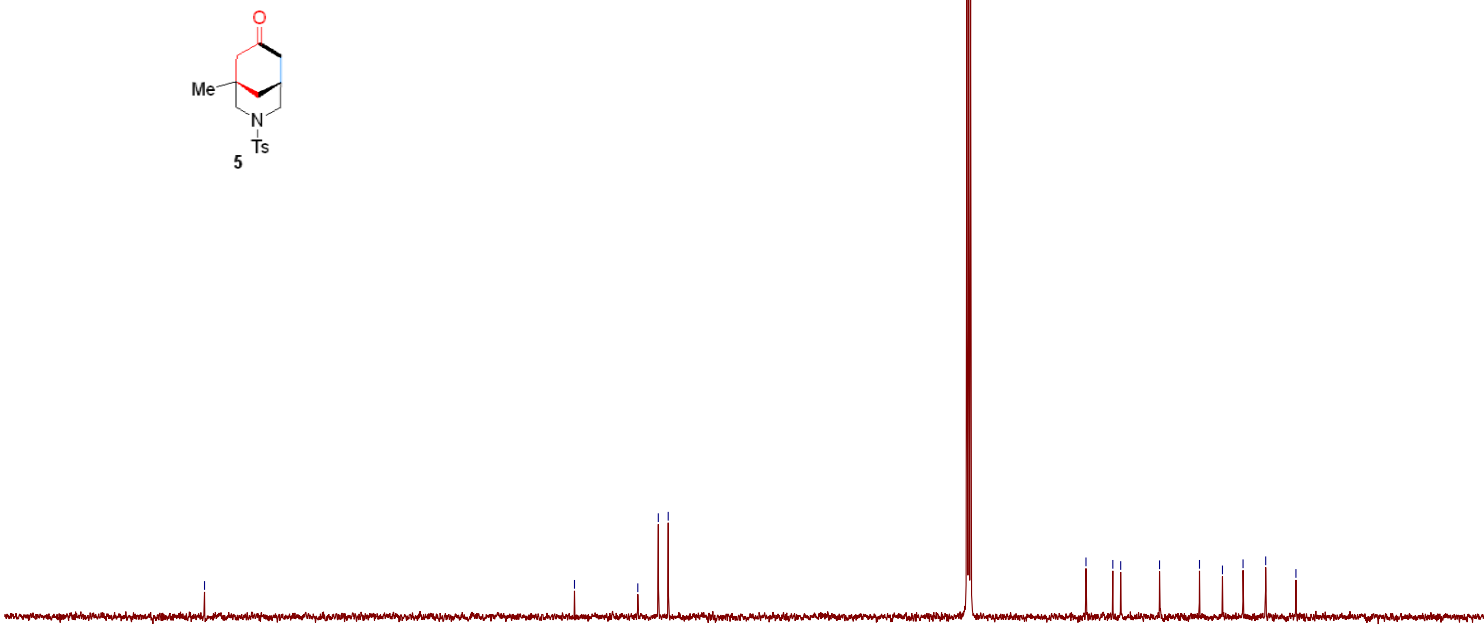

\begin{tabular}{lllllllllllllllllllllllllllll}
\hline 40 & 230 & 220 & 210 & 200 & 190 & 180 & 170 & 160 & 150 & 140 & 130 & 120 & 110 & 100 & 90 & 80 & 70 & 60 & 50 & 40 & 30 & 20 & 10 & 0 & -1 \\
& $f 1(\mathrm{ppm})$
\end{tabular} 
${ }^{1} \mathrm{H}-\mathrm{NMR}$ spectrum of $2 \mathrm{ab}$ in $\mathrm{C}_{6} \mathrm{D}_{6}, 400 \mathrm{MHz}$

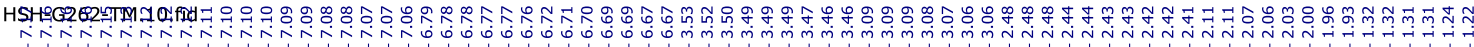

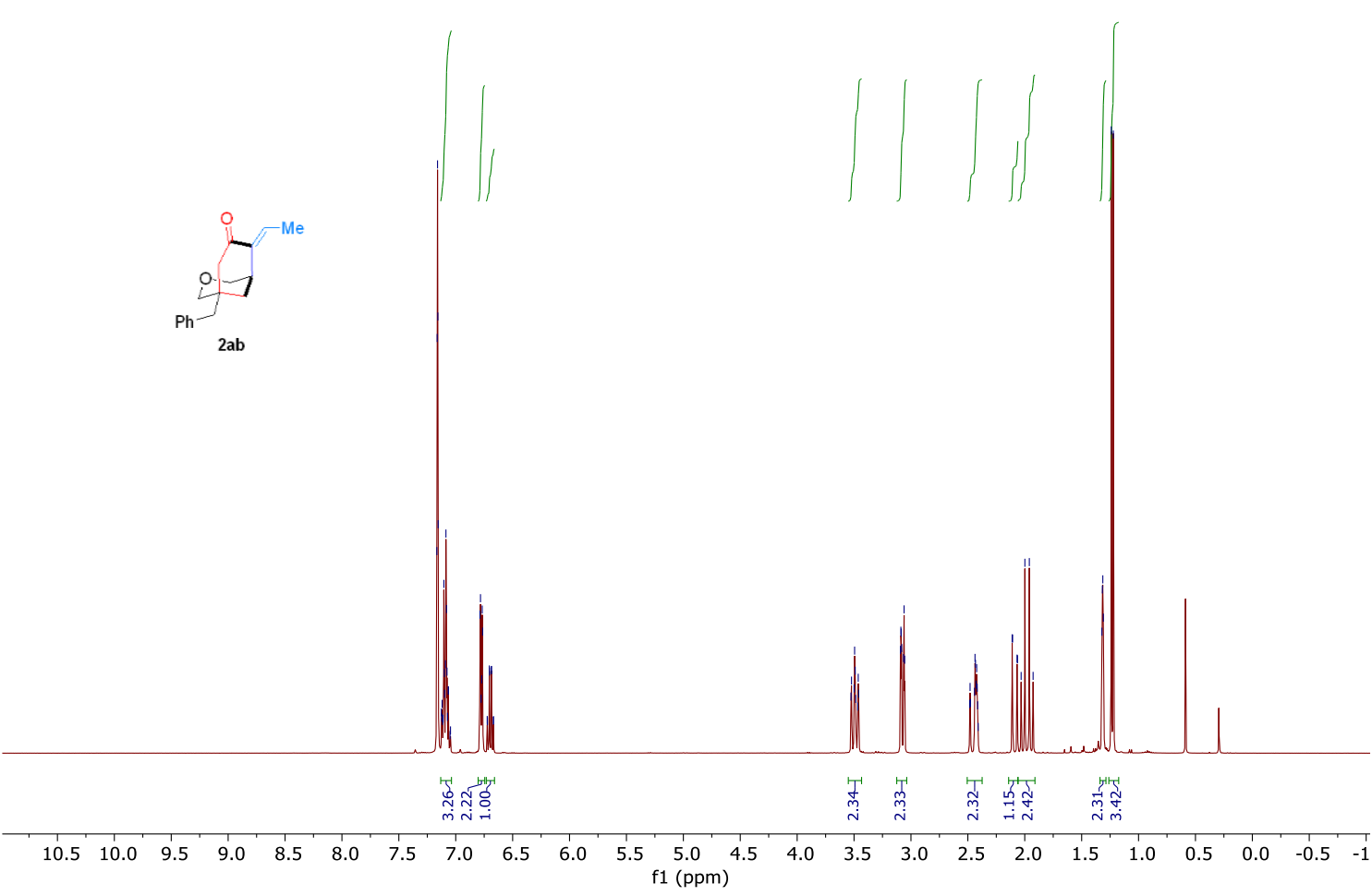

${ }^{13} \mathrm{C}-\mathrm{NMR}$ spectrum of $2 \mathrm{ab}$ in $\mathrm{C}_{6} \mathrm{D}_{6}, 101 \mathrm{MHz}$

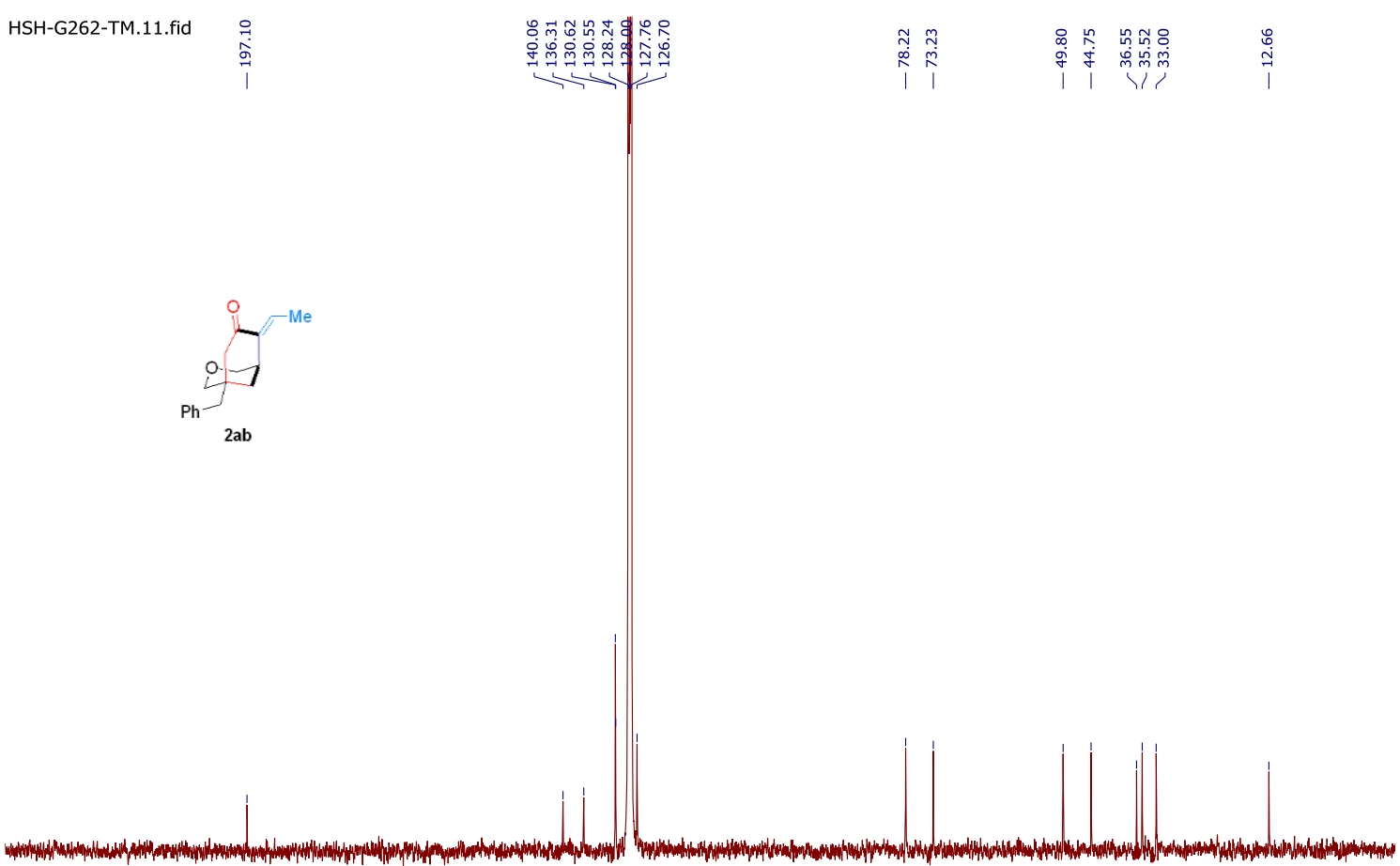

$\begin{array}{llllllllllllllllllllllllllllllllll}40 & 230 & 220 & 210 & 200 & 190 & 180 & 170 & 160 & 150 & 140 & 130 & \begin{array}{l}120 \\ \mathrm{f} 1(\mathrm{ppm})\end{array} & 100 & 90 & 80 & 70 & 60 & 50 & 40 & 30 & 20 & 10 & 0 & -\end{array}$ 
${ }^{1} \mathrm{H}-\mathrm{NMR}$ spectrum of $\mathbf{2 j - 1}$ in $\mathrm{C}_{6} \mathrm{D}_{6}, 400 \mathrm{MHz}$

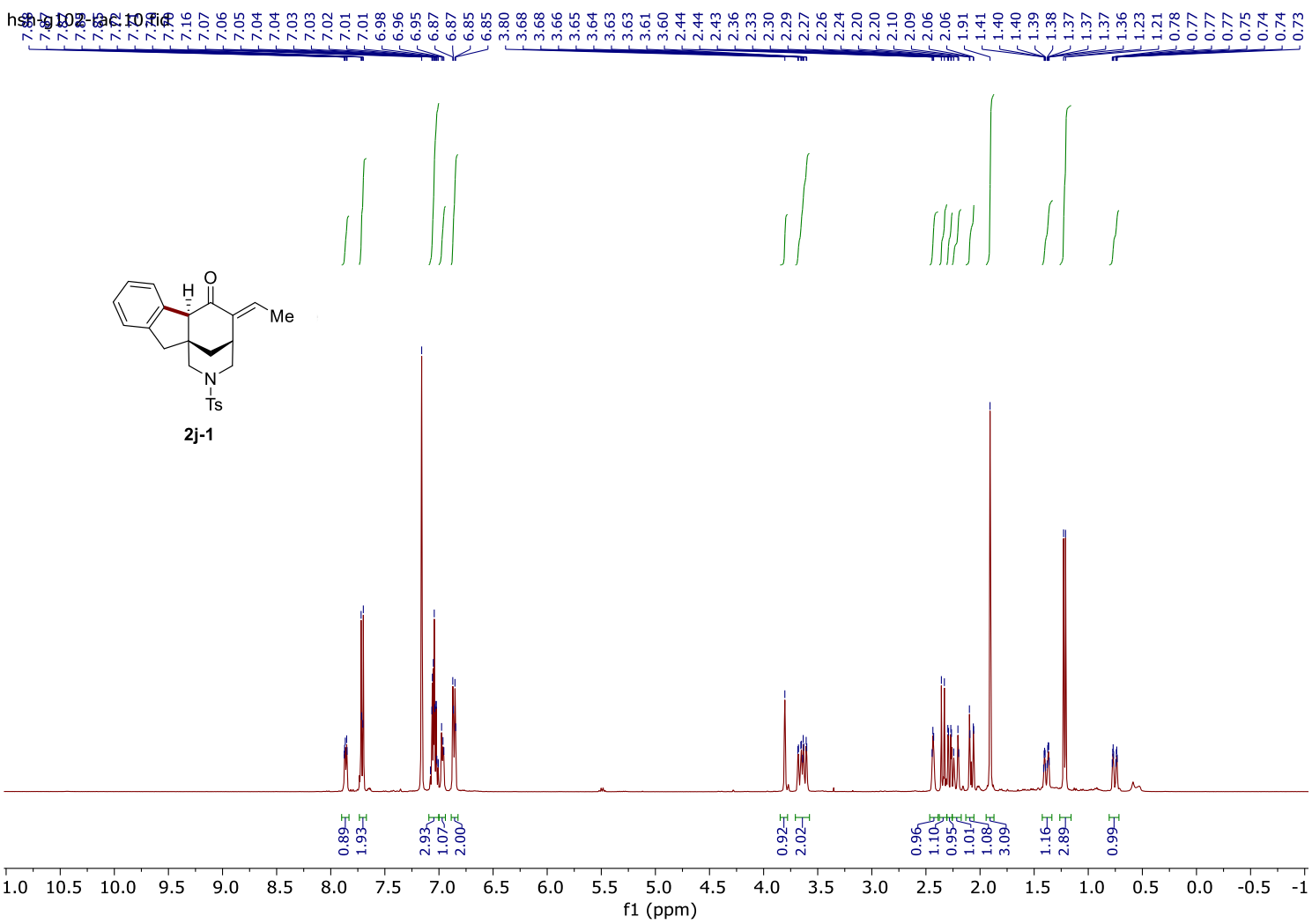

${ }^{13} \mathrm{C}-\mathrm{NMR}$ spectrum of $\mathbf{2 j - 1}$ in $\mathrm{C}_{6} \mathrm{D}_{6}, 101 \mathrm{MHz}$

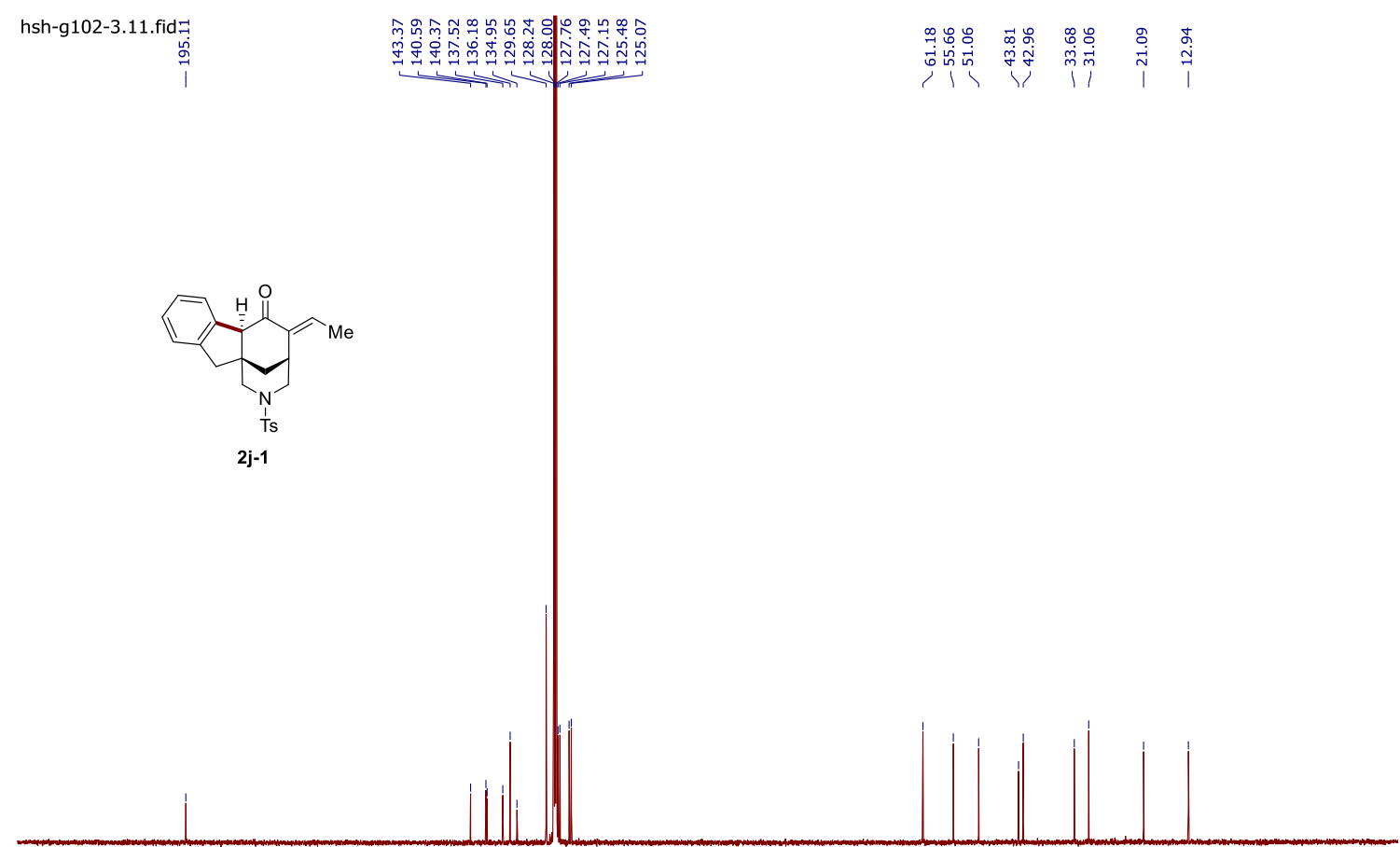

$\begin{array}{lllllllllllllllllllllllllllllllllll}220 & 210 & 200 & 190 & 180 & 170 & 160 & 150 & 140 & 130 & 120 & 110 & 100 & 90 & 80 & 70 & 60 & 50 & 40 & 30 & 20 & 10 & 0 & -10 & -20\end{array}$ 


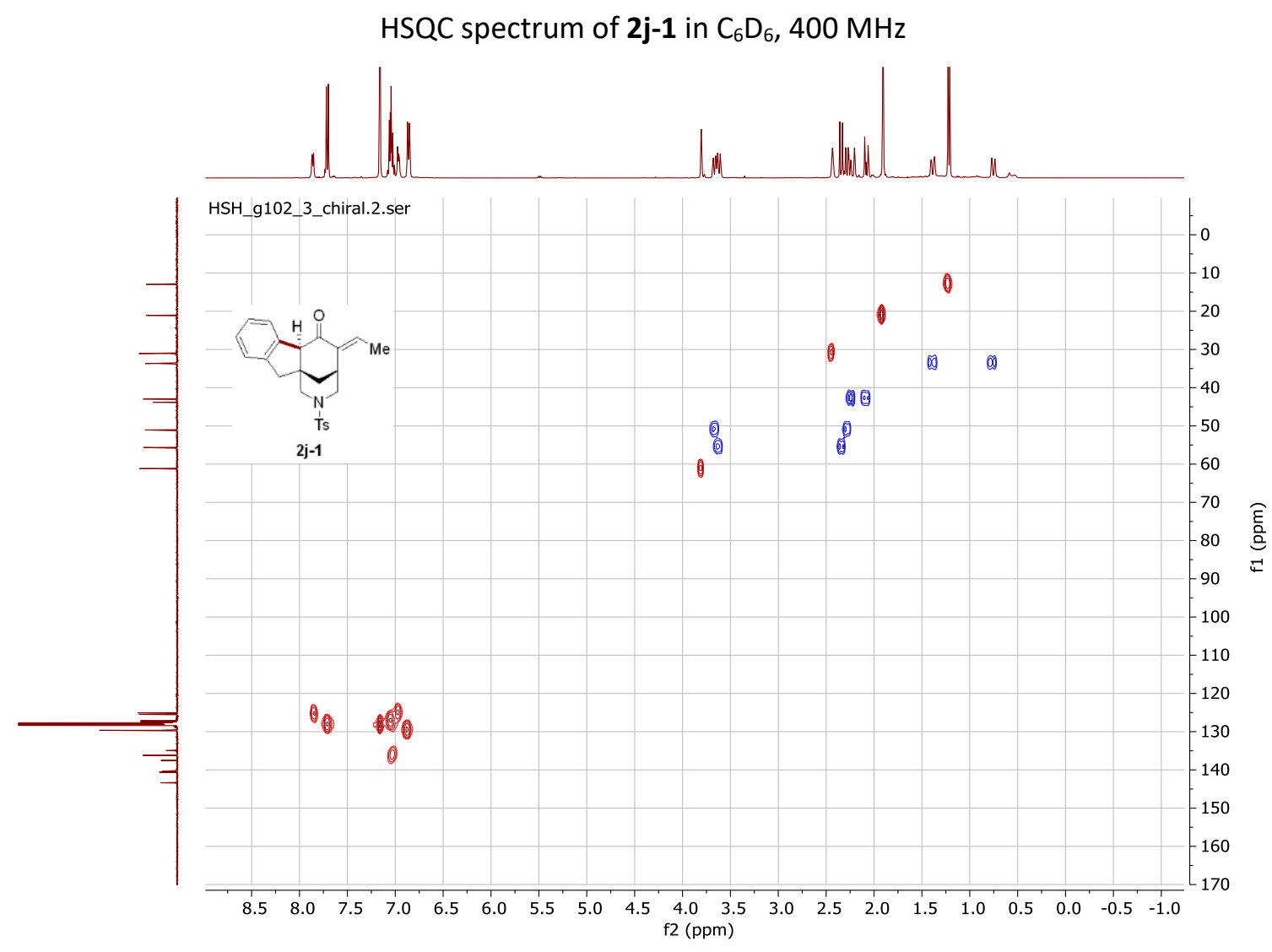

${ }^{1} \mathrm{H}-{ }^{1} \mathrm{H}$ COSY spectrum of $\mathbf{2 j - 1}$ in $\mathrm{C}_{6} \mathrm{D}_{6}, 400 \mathrm{MHz}$

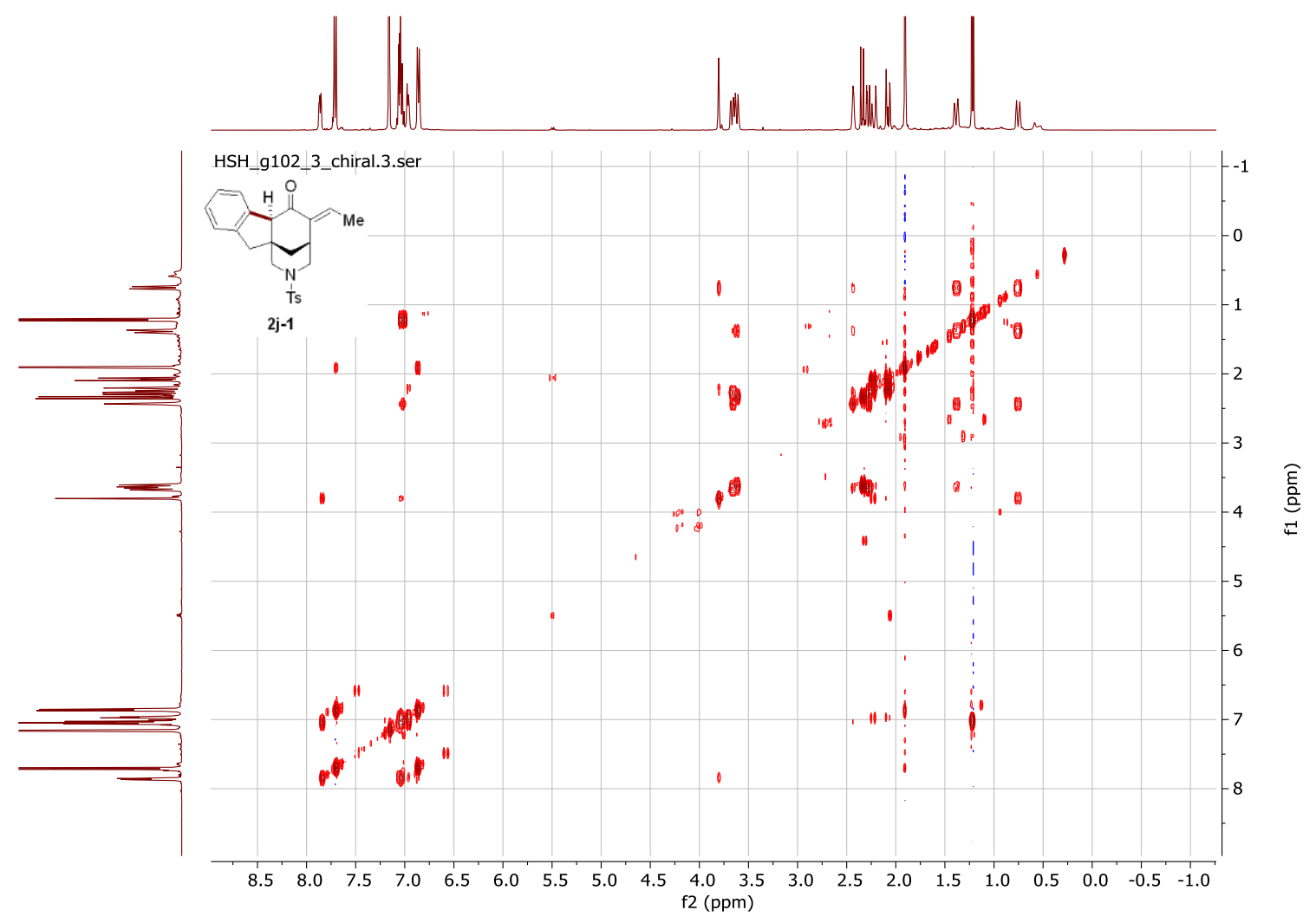




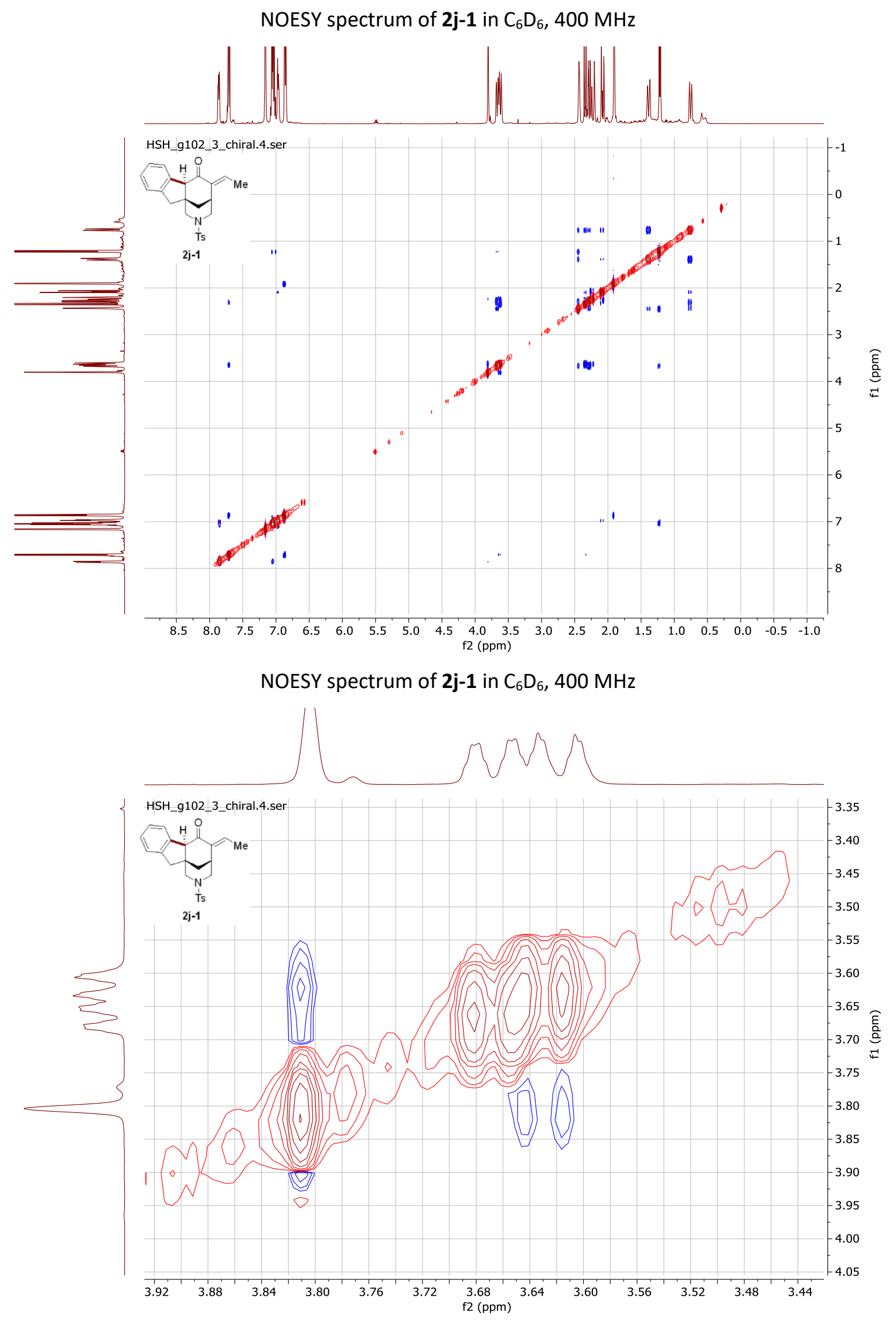


${ }^{1} \mathrm{H}-\mathrm{NMR}$ spectrum of $\mathbf{2 a - 1}$ in $\mathrm{CDCl}_{3}, 400 \mathrm{MHz}$

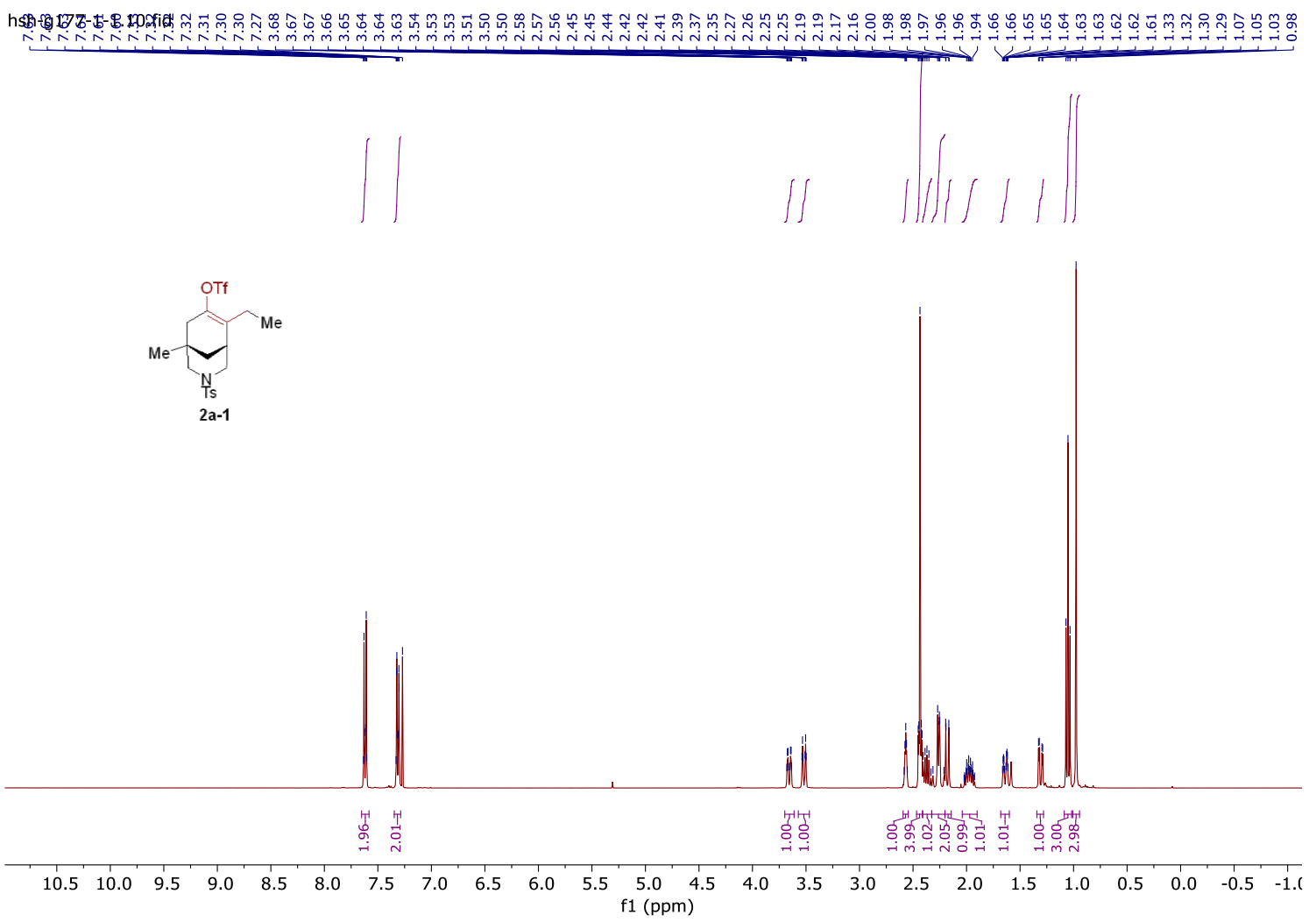

${ }^{19} \mathrm{~F}-\mathrm{NMR}$ spectrum of $\mathbf{2 a - 1}$ in $\mathrm{CDCl}_{3}, 376 \mathrm{MHz}$

hsh-g177-1-1.11.fid

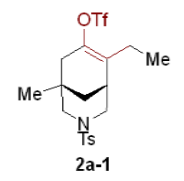

\begin{tabular}{rllllllllllllllllllllll}
\hline 10 & 0 & -10 & -20 & -30 & -40 & -50 & -60 & -70 & -80 & -90 & -100 & -110 & -120 & -130 & -140 & -150 & -160 & -170 & -180 & -190 & -200 & $-21 C$
\end{tabular} 
${ }^{13} \mathrm{C}-\mathrm{NMR}$ spectrum of $\mathbf{2 a - 1}$ in $\mathrm{CDCl}_{3}, 101 \mathrm{MHz}$

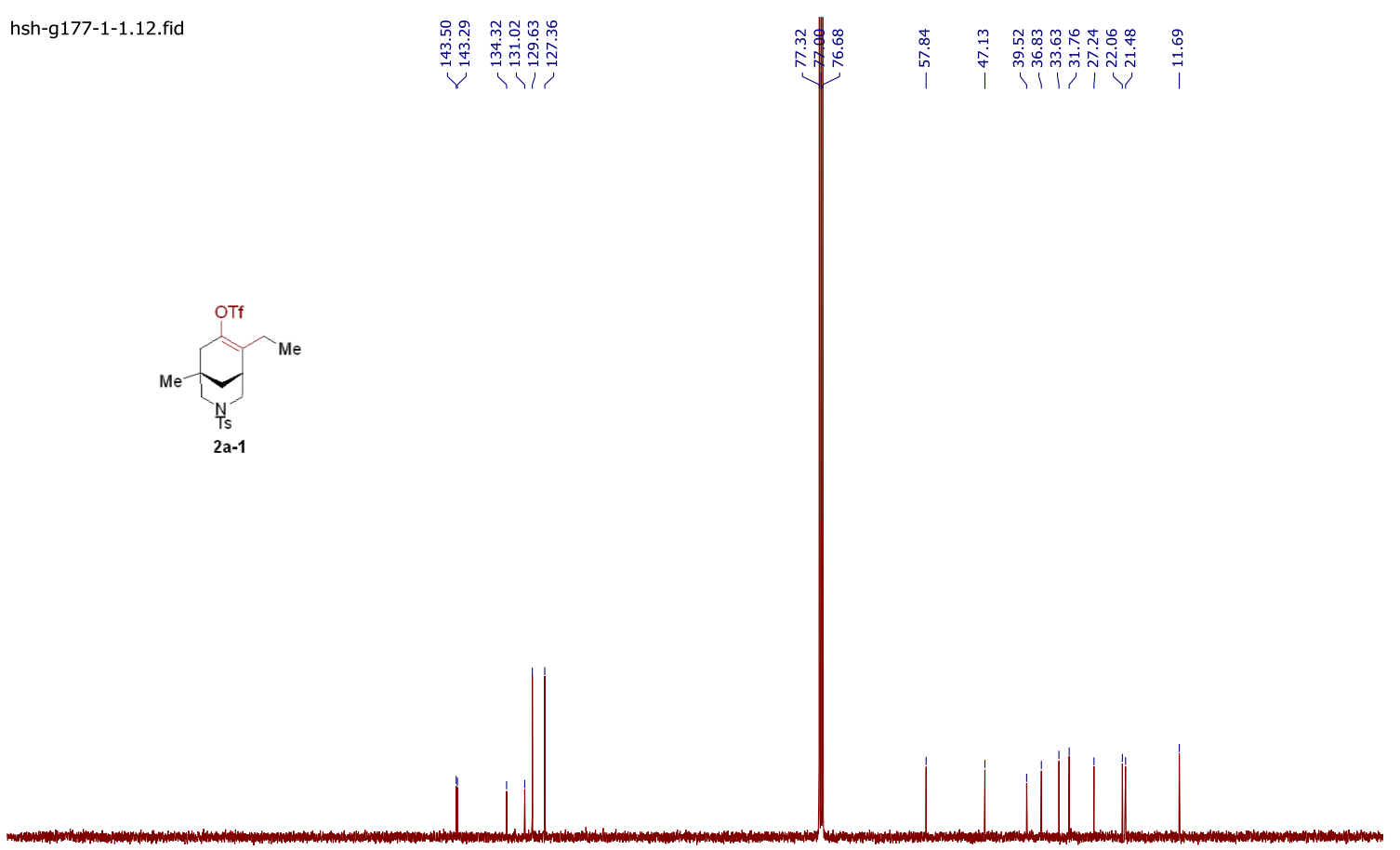

$\begin{array}{llllllllllllllllllllllllllllllllll}220 & 210 & 200 & 190 & 180 & 170 & 160 & 150 & 140 & 130 & 120 & 110 & 100 & 90 & 80 & 70 & 60 & 50 & 40 & 30 & 20 & 10 & 0 & -10 & -20\end{array}$ 
${ }^{1} \mathrm{H}-\mathrm{NMR}$ spectrum of $\mathbf{2 a - 2}$ in $\mathrm{CDCl}_{3}, 400 \mathrm{MHz}$

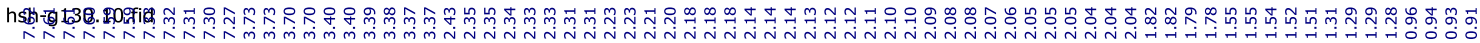

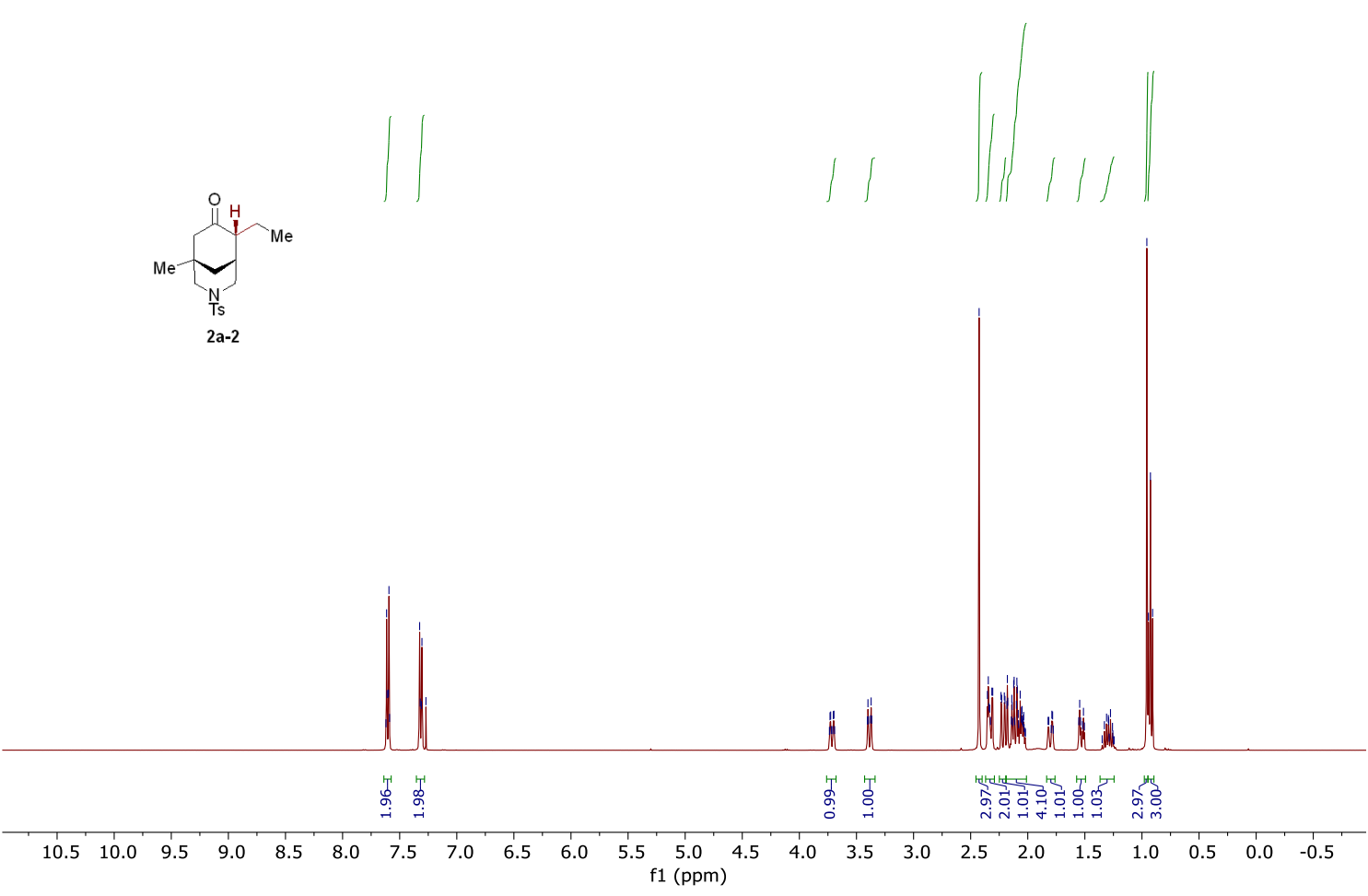

${ }^{13} \mathrm{C}$-NMR spectrum of $\mathbf{2 a - 2}$ in $\mathrm{CDCl}_{3}, 101 \mathrm{MHz}$

hsh-g130.11.fid
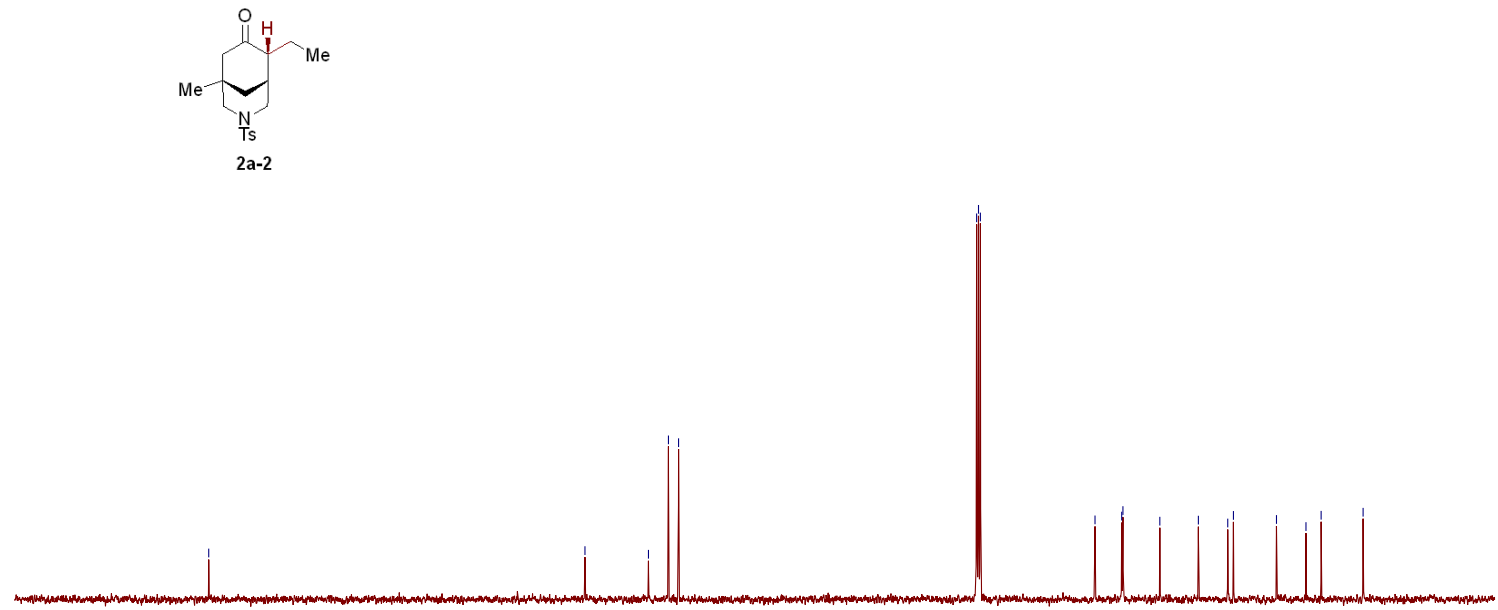

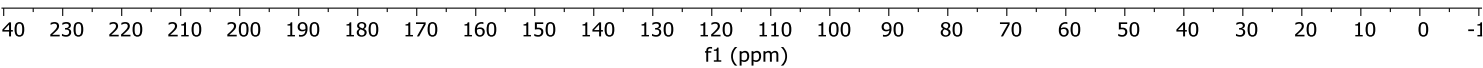


DEPT spectrum of $\mathbf{2 a - 2}$ in $\mathrm{CDCl}_{3}, 101 \mathrm{MHz}$

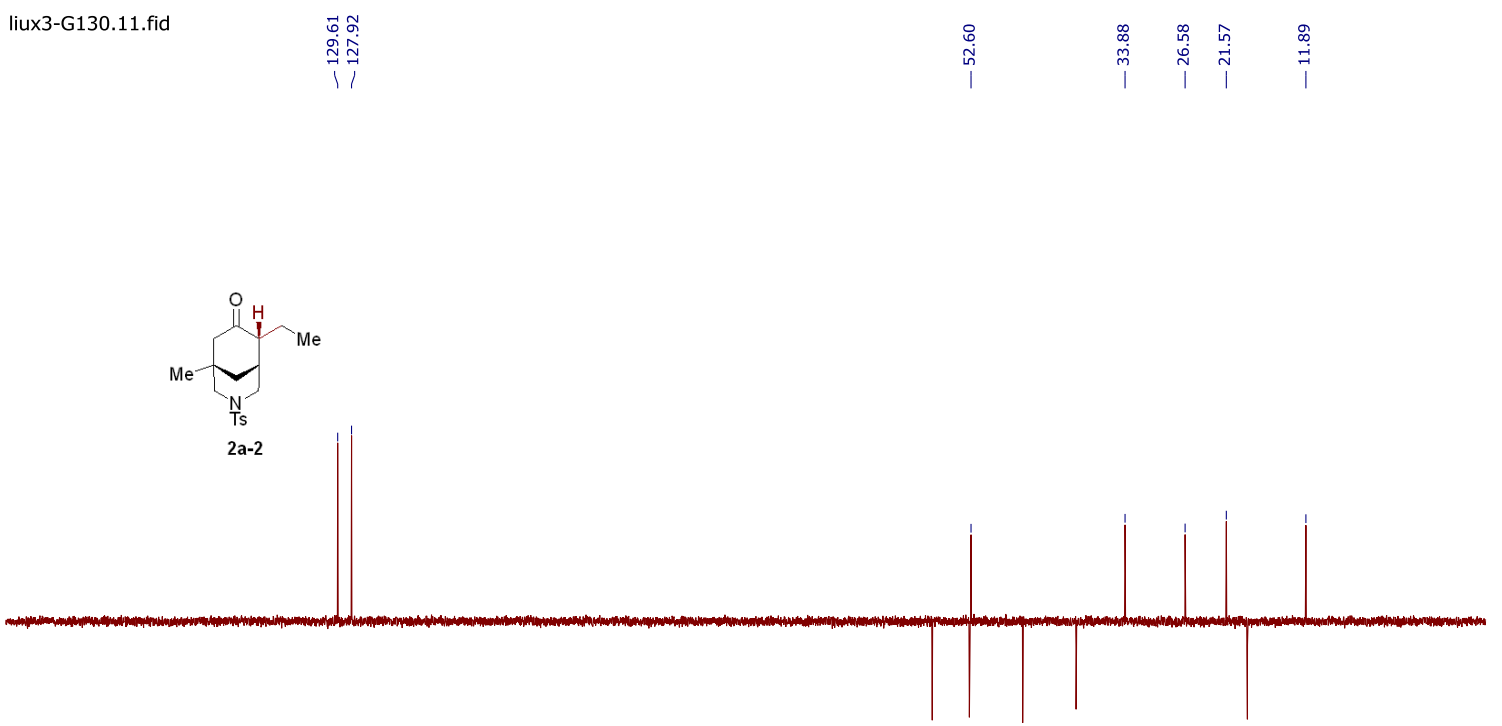

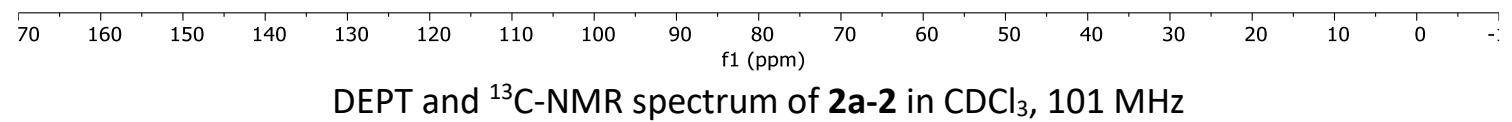

liux3-G130.11.fid

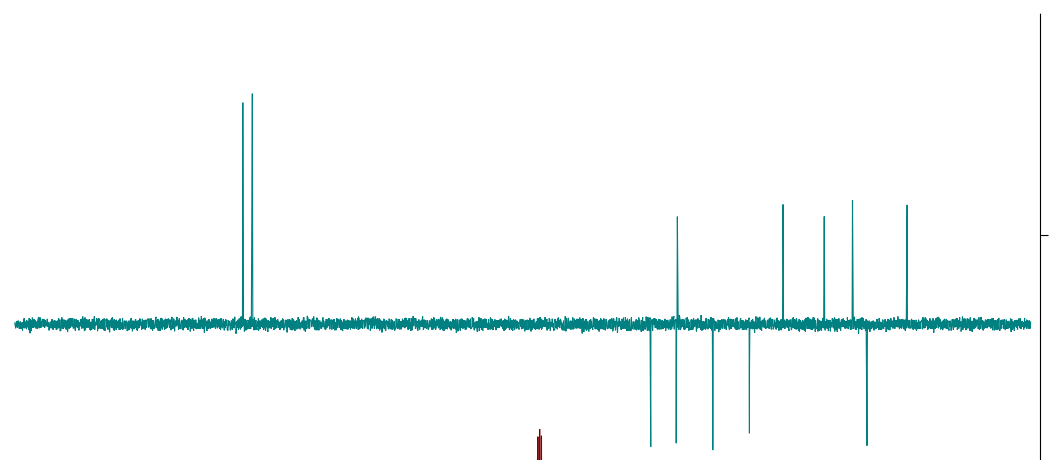

hsh-g130.11.fid

a-2
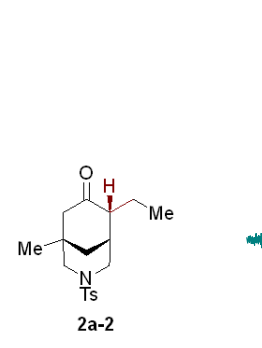

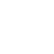
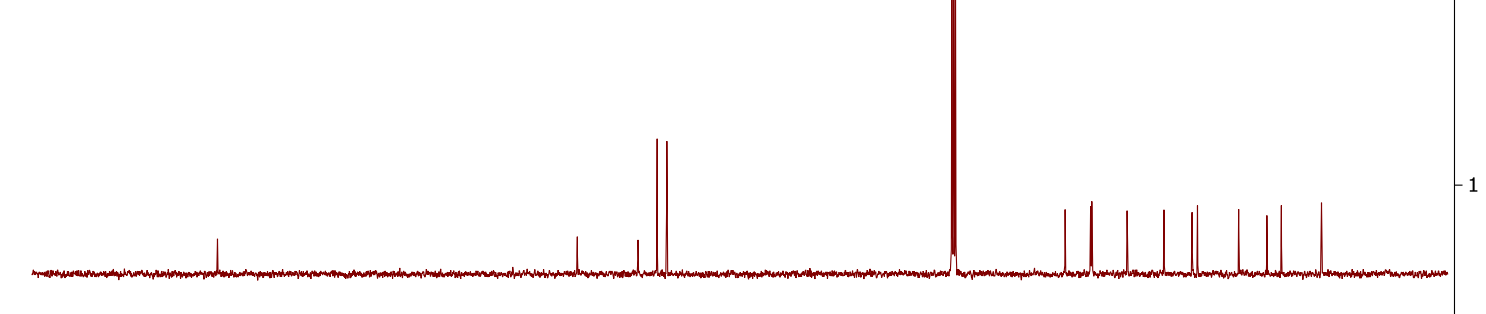

\begin{tabular}{llllllllllllllllllllllllllll}
\hline & 230 & 220 & 210 & 200 & 190 & 180 & 170 & 160 & 150 & 140 & 130 & $\begin{array}{r}120 \\
\mathrm{f} 1(\mathrm{ppm})\end{array}$ & 110 & 100 & 90 & 80 & 70 & 60 & 50 & 40 & 30 & 20 & 10 & 0 & -10
\end{tabular} 
HSQC spectrum of $2 \mathrm{a}-\mathbf{2}$ in $\mathrm{CDCl}_{3}, 400 \mathrm{MHz}$

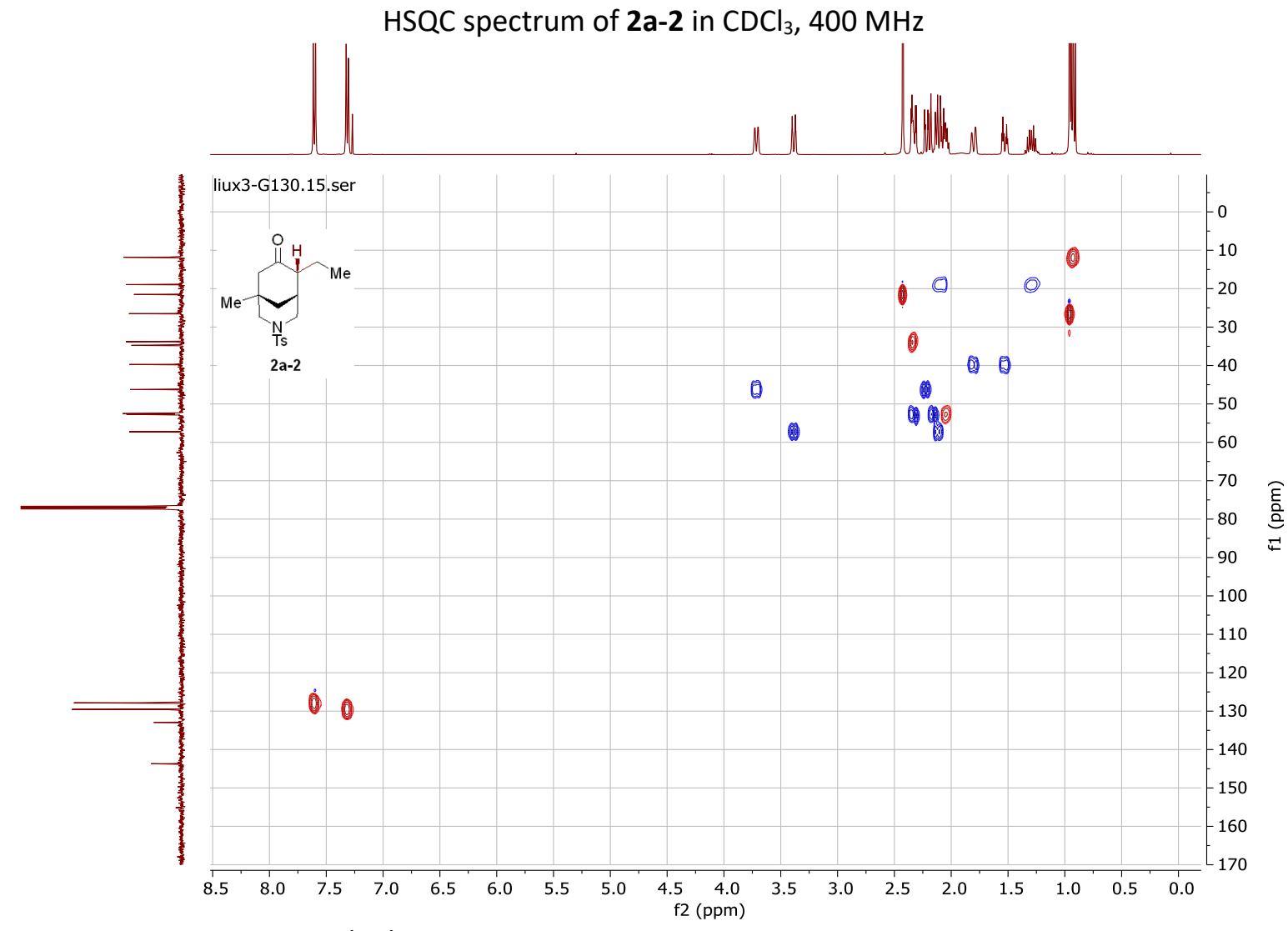

$\underbrace{\widehat{c}}_{\bar{a}}$

${ }^{1} \mathrm{H}-{ }^{1} \mathrm{H}$ COSY spectrum of $\mathbf{2 a - 2}$ in $\mathrm{CDCl}_{3}, 400 \mathrm{MHz}$

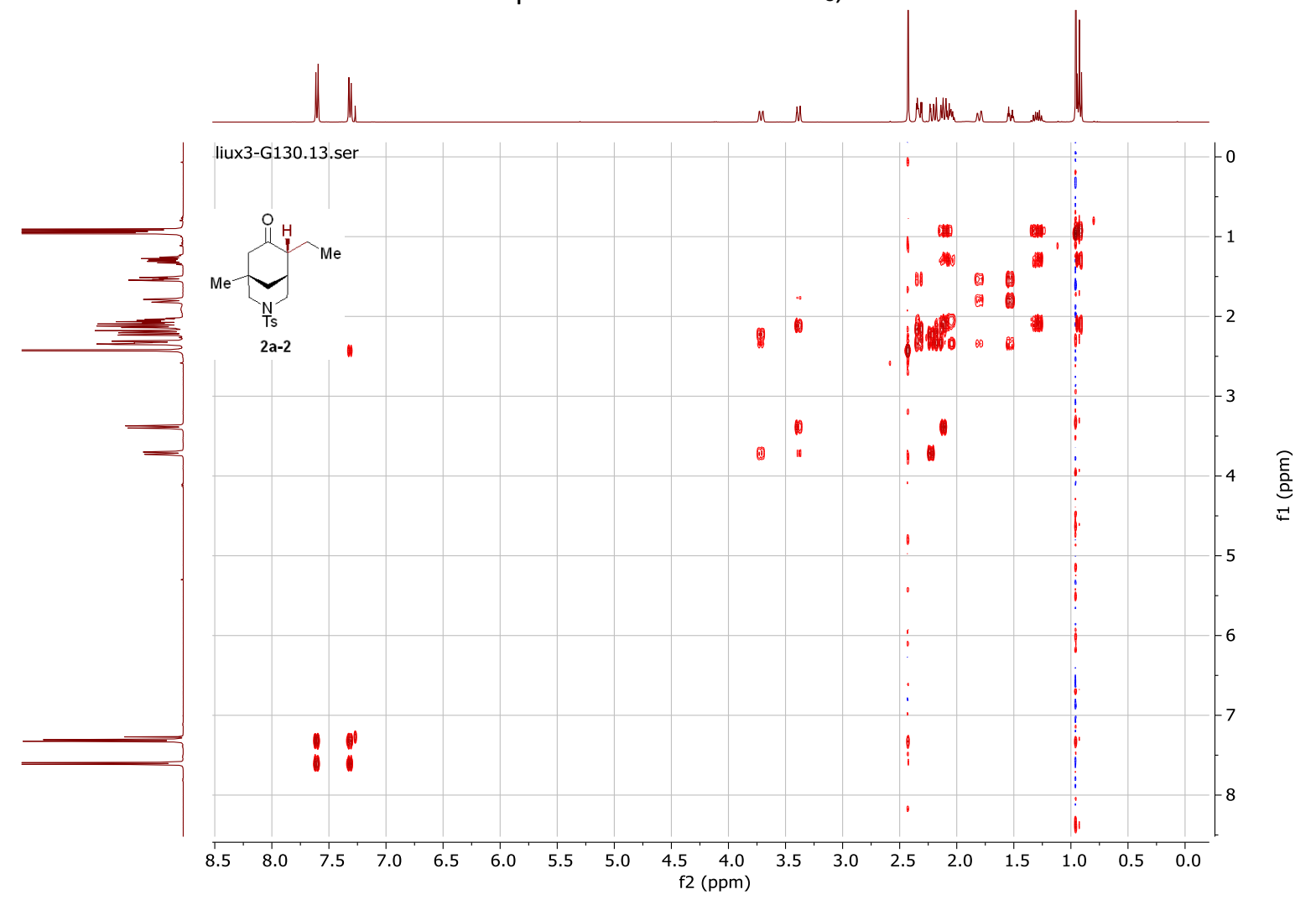




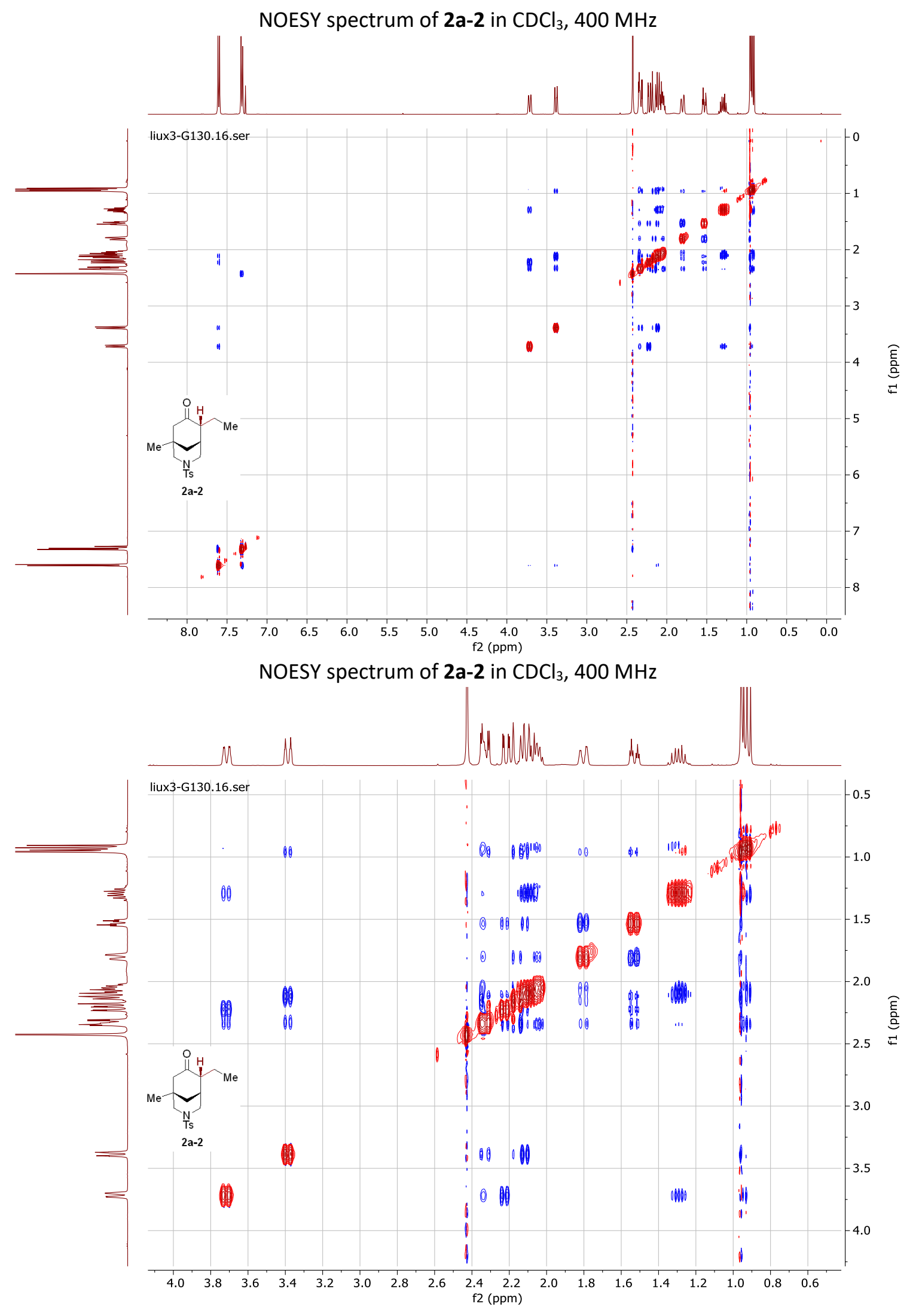


${ }^{1} \mathrm{H}-\mathrm{NMR}$ spectrum of $2 \mathrm{a}-3$ in $\mathrm{CDCl}_{3}, 400 \mathrm{MHz}$

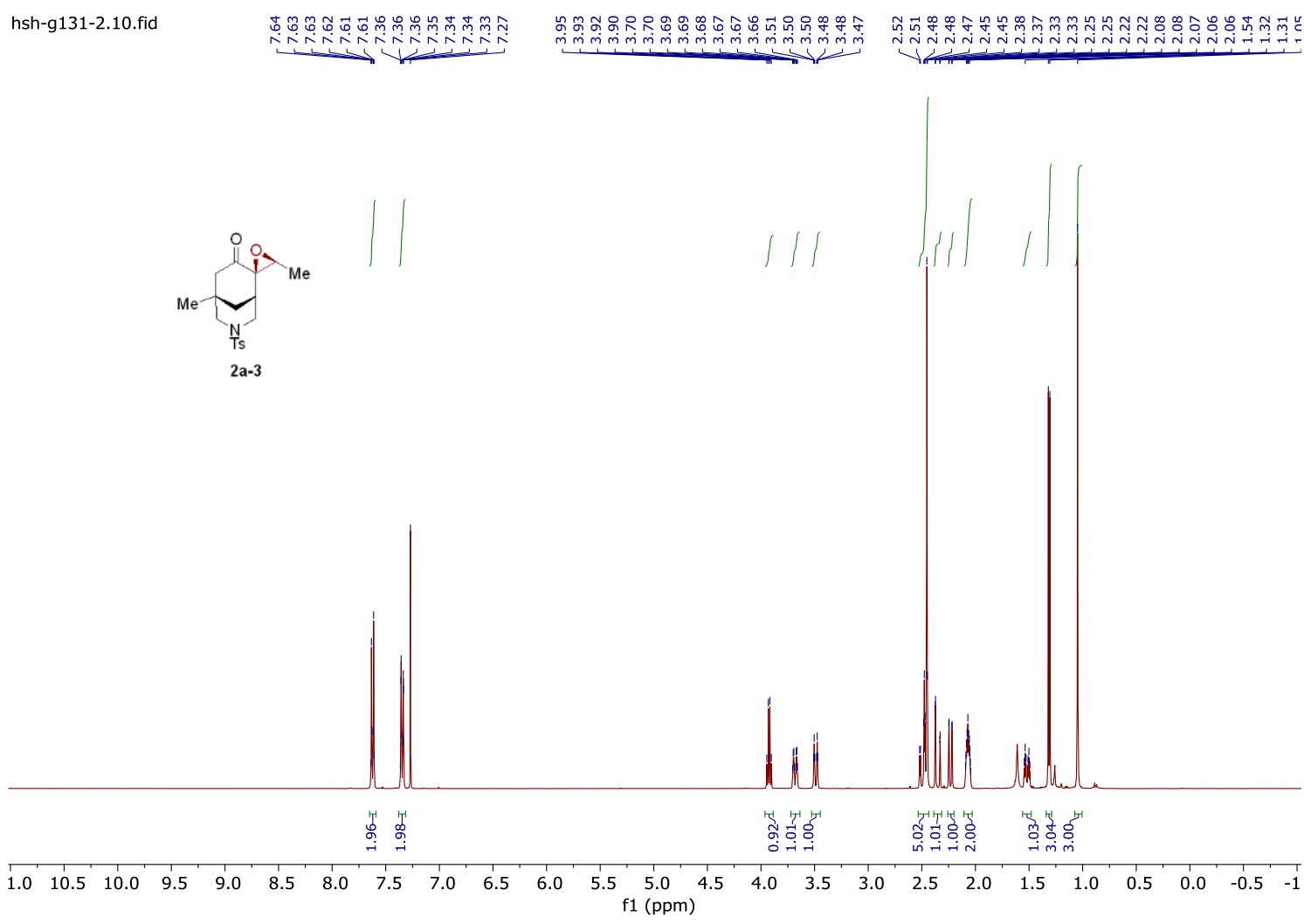

${ }^{13} \mathrm{C}-\mathrm{NMR}$ spectrum of $\mathbf{2 a}-\mathbf{3}$ in $\mathrm{CDCl}_{3}, 101 \mathrm{MHz}$

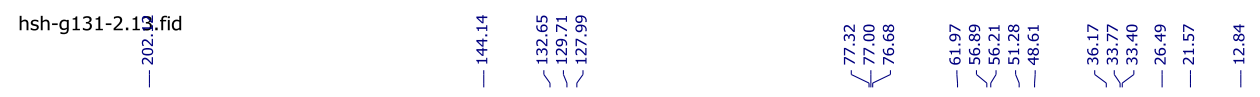
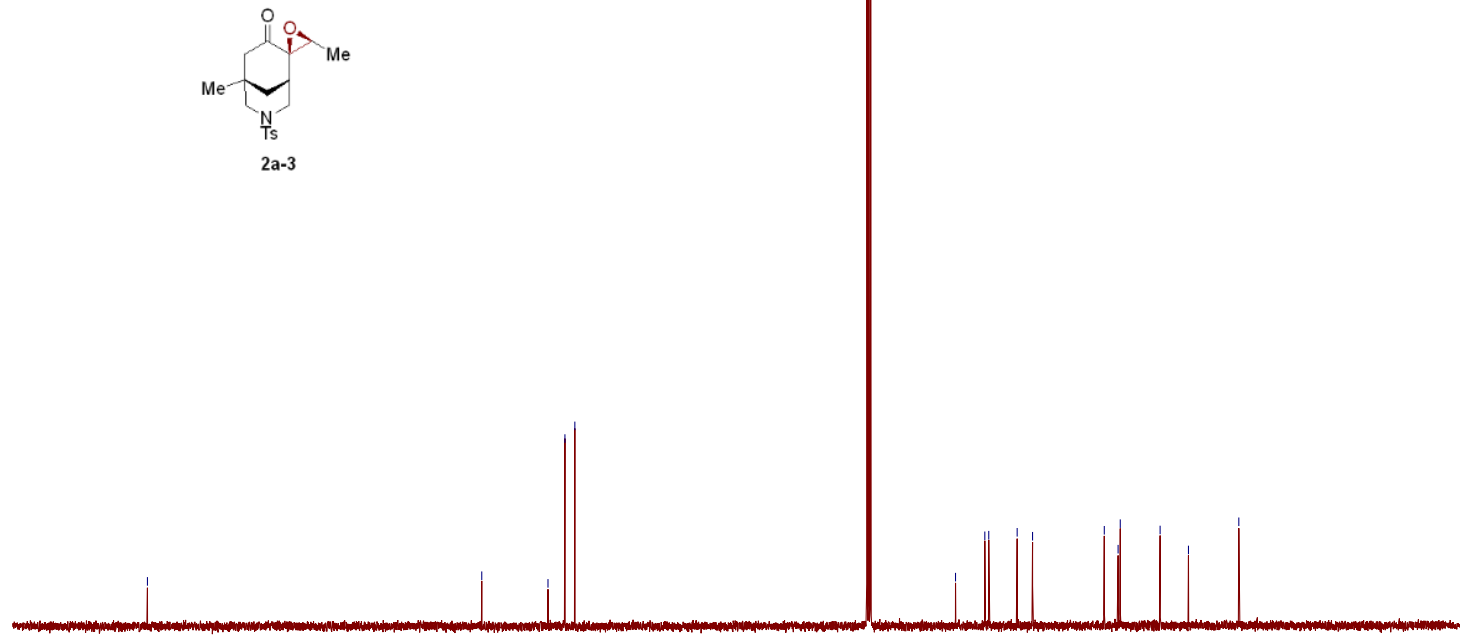

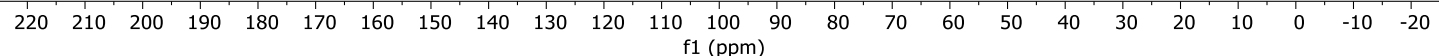


DEPT spectrum of $\mathbf{2 a - 3}$ in $\mathrm{CDCl}_{3}, 101 \mathrm{MHz}$

liux3-G131-2.12.fid
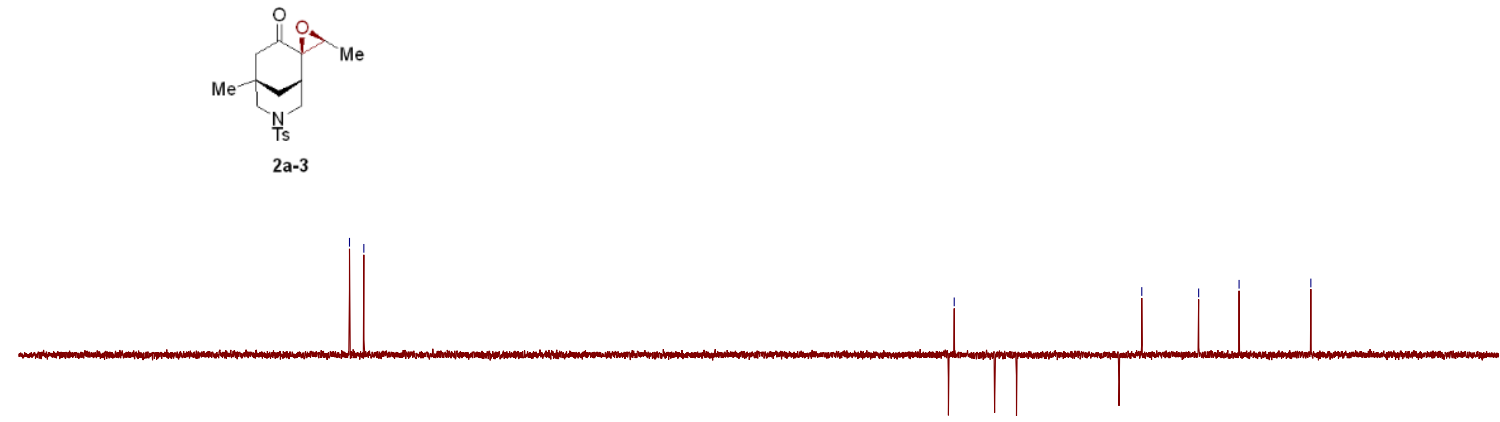

liux3-G131-2.12.fid

hsh-g131-2.13.fid

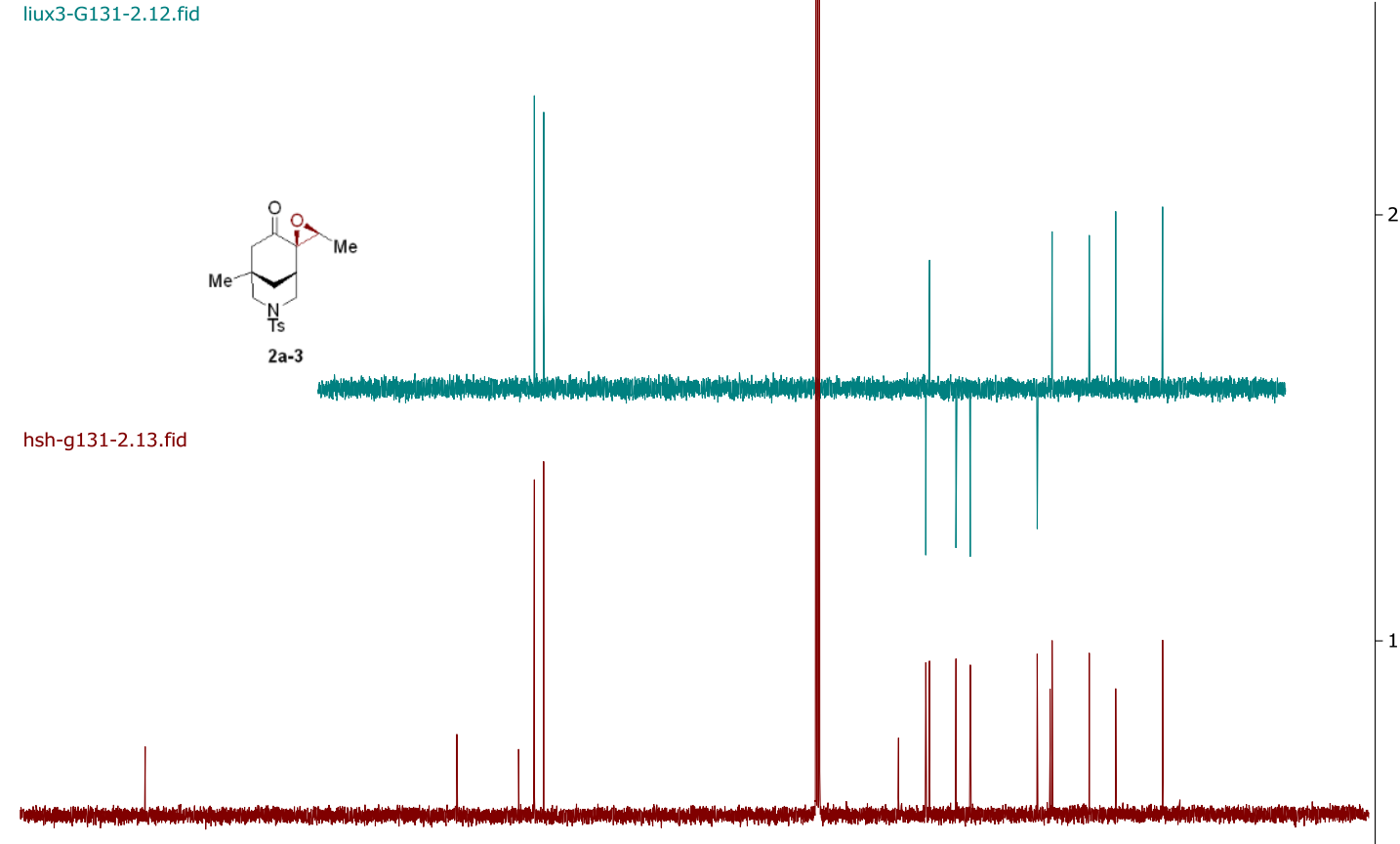

DEPT and ${ }^{13} \mathrm{C}-\mathrm{NMR}$ spectrum of $\mathbf{2 a - 3}$ in $\mathrm{CDCl}_{3}, 101 \mathrm{MHz}$

$\begin{array}{llllllllllllllllllllllllllllllllll}220 & 210 & 200 & 190 & 180 & 170 & 160 & 150 & 140 & 130 & 120 & 110 & 100 & 90 & 80 & 70 & 60 & 50 & 40 & 30 & 20 & 10 & 0 & -10 & -20\end{array}$ 

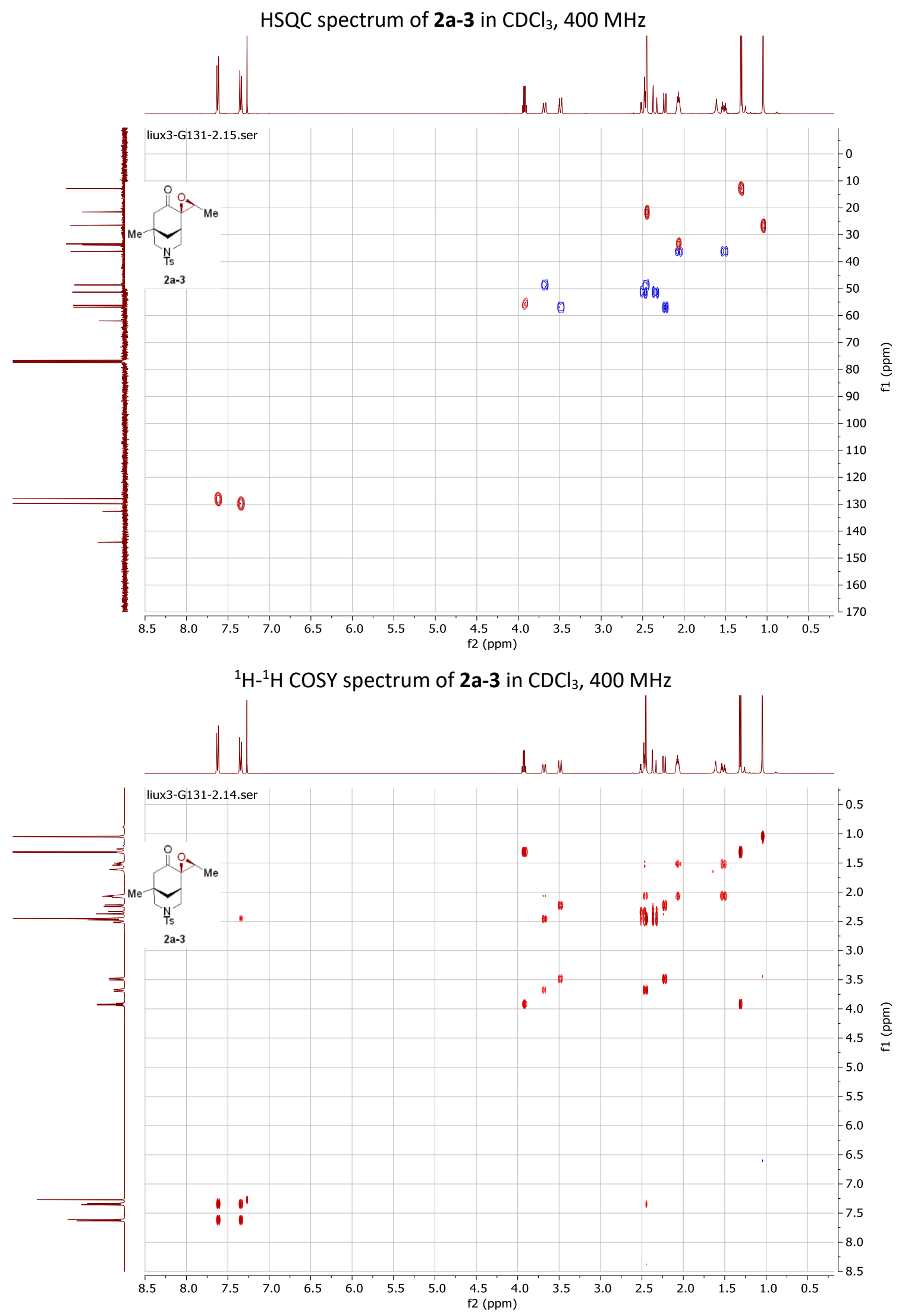

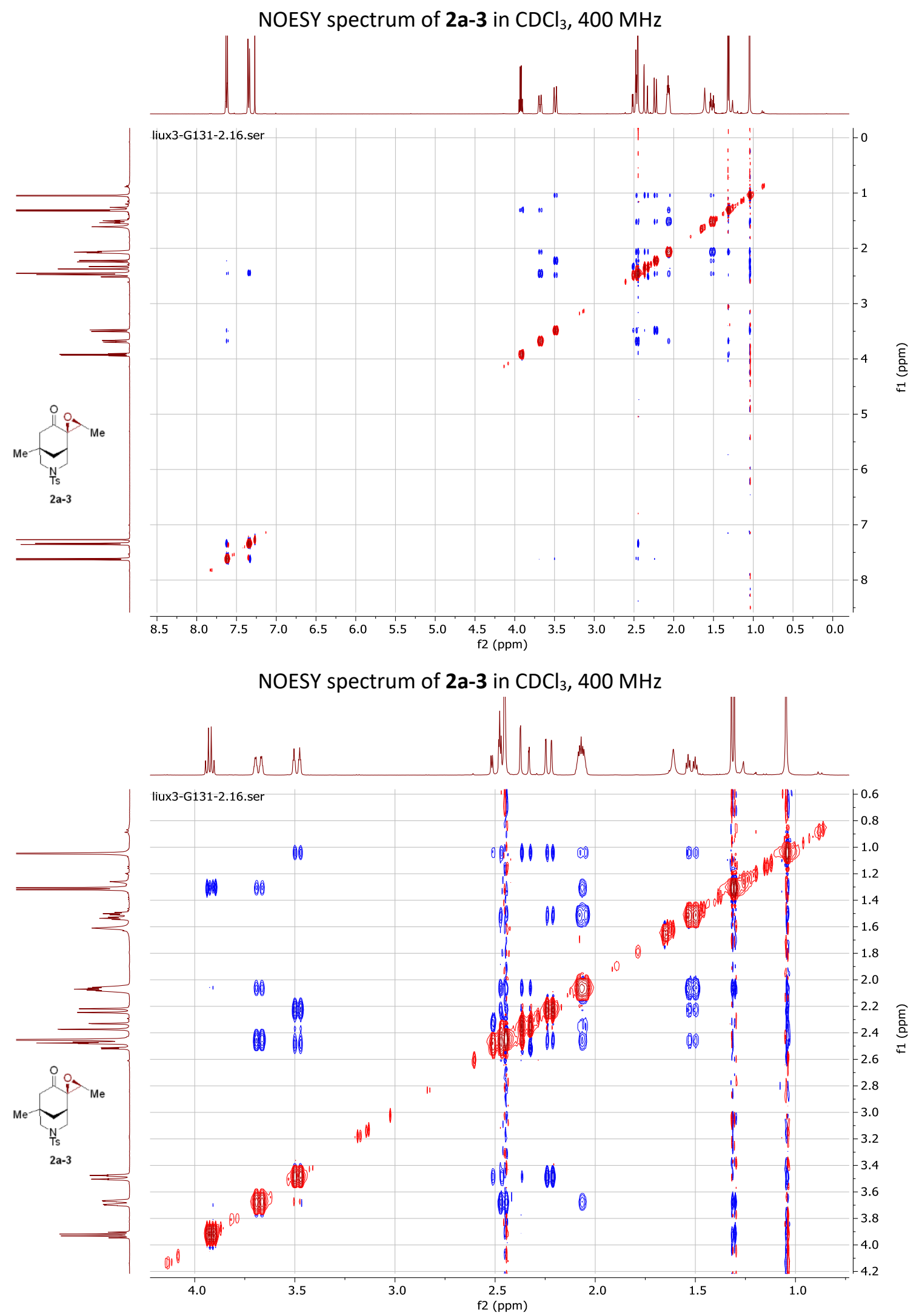
${ }^{1} \mathrm{H}-\mathrm{NMR}$ spectrum of $2 \mathrm{a}-4$ in $\mathrm{CDCl}_{3}, 500 \mathrm{MHz}$

hsकी

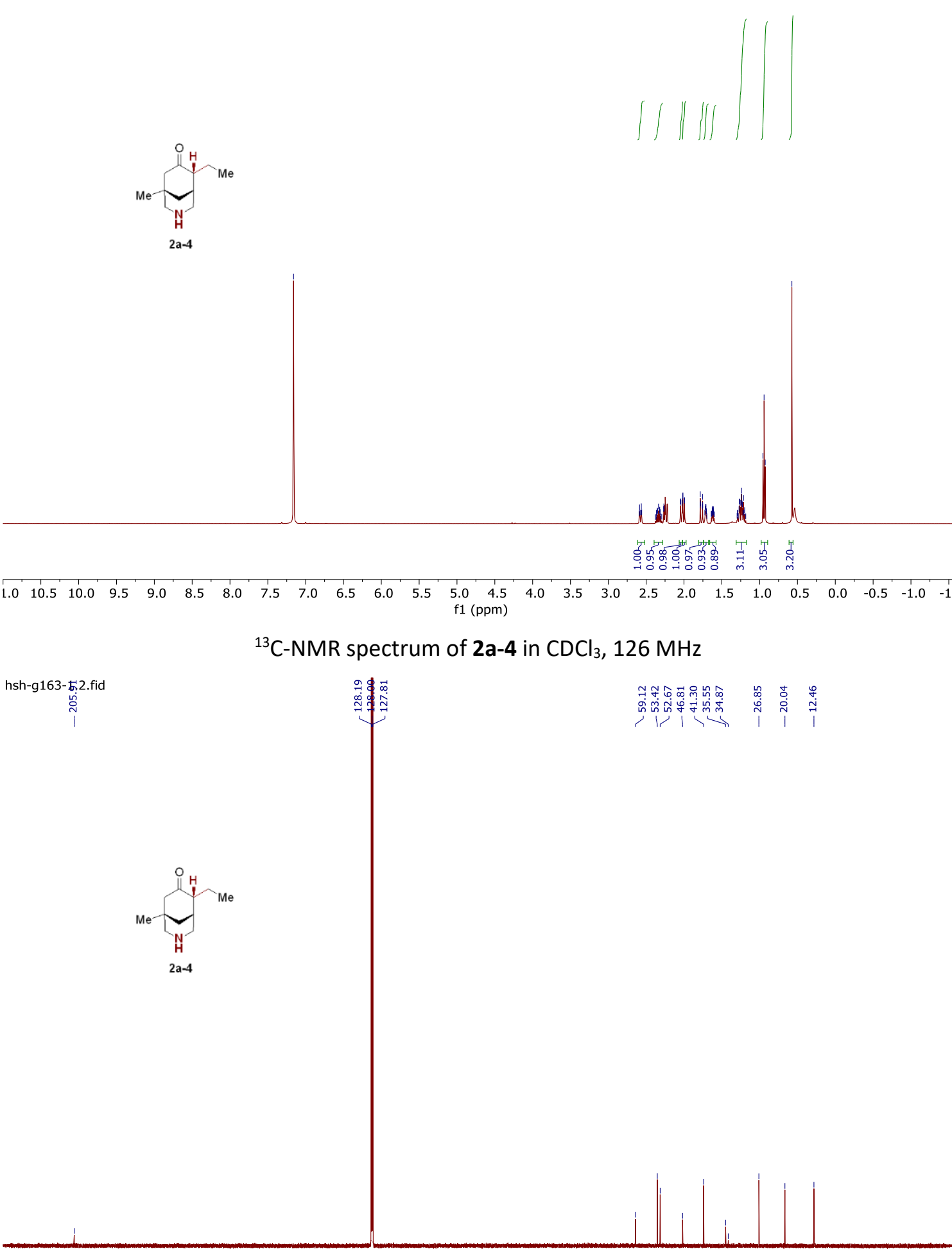

\begin{tabular}{lllllllllllllllllllllllllll}
\hline 220 & 210 & 200 & 190 & 180 & 170 & 160 & 150 & 140 & 130 & 120 & 110 & 100 & 90 & 80 & 70 & 60 & 50 & 40 & 30 & 20 & 10 & 0 & -10 & -20
\end{tabular} 


\section{DEPT spectrum of $2 \mathrm{a}-\mathbf{4}$ in $\mathrm{CDCl}_{3}, 126 \mathrm{MHz}$}

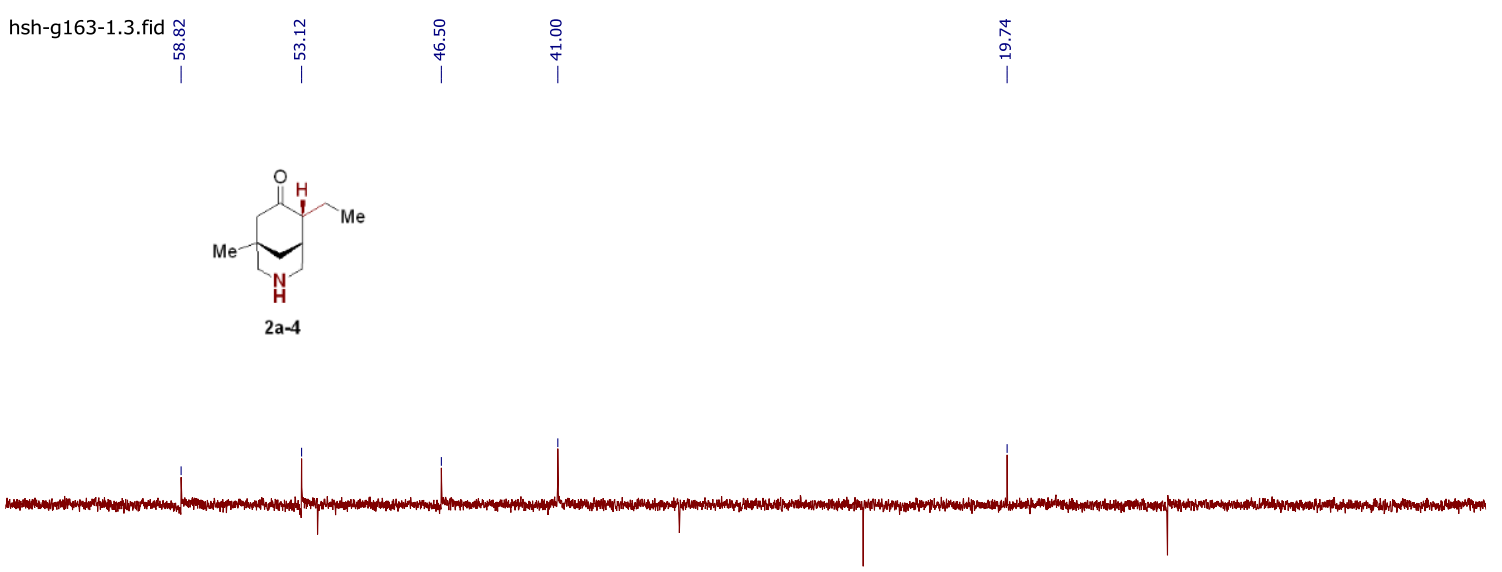

\begin{tabular}{|c|c|c|c|c|c|c|c|c|c|c|c|c|c|}
\hline 65 & 60 & 55 & 50 & 45 & 40 & & & 25 & 20 & 15 & 10 & 5 & 0 \\
\hline
\end{tabular}

\section{DEPT and ${ }^{13} \mathrm{C}-\mathrm{NMR}$ spectrum of $\mathbf{2 a}-\mathbf{4}$ in $\mathrm{CDCl}_{3}, 126 \mathrm{MHz}$}

hsh-g163-1.3.fid

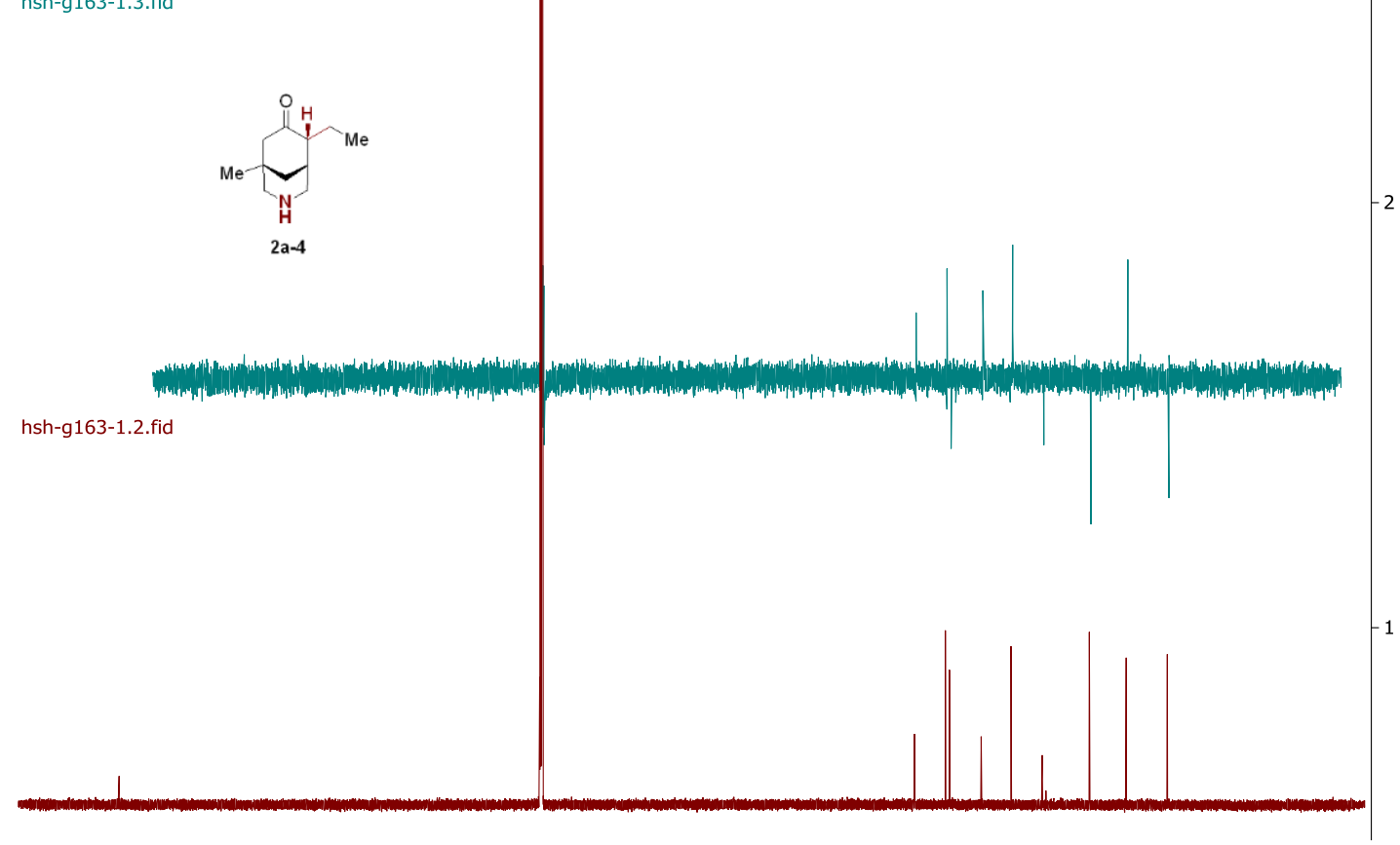

$\begin{array}{lllllllllllllllllllllllllllllllllllll}220 & 210 & 200 & 190 & 180 & 170 & 160 & 150 & 140 & 130 & 120 & 110 & 100 & 90 & 80 & 70 & 60 & 50 & 40 & 30 & 20 & 10 & 0 & -10 & -20\end{array}$ 
HSQC spectrum of $2 \mathrm{a}-\mathbf{4}$ in $\mathrm{CDCl}_{3}, 500 \mathrm{MHz}$

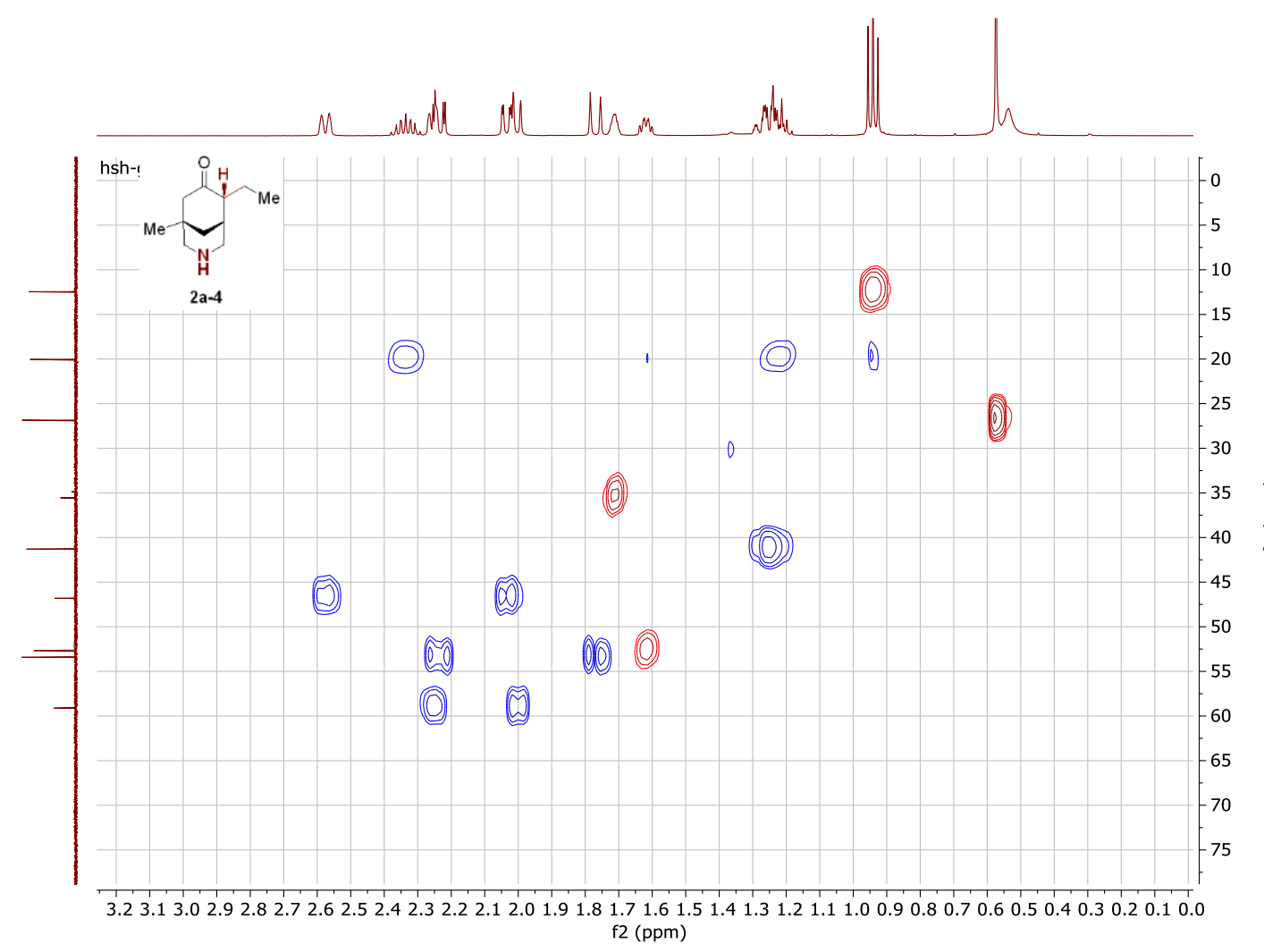

${ }^{1} \mathrm{H}-{ }^{1} \mathrm{H}$ COSY spectrum of $2 \mathrm{a}-4$ in $\mathrm{CDCl}_{3}, 500 \mathrm{MHz}$

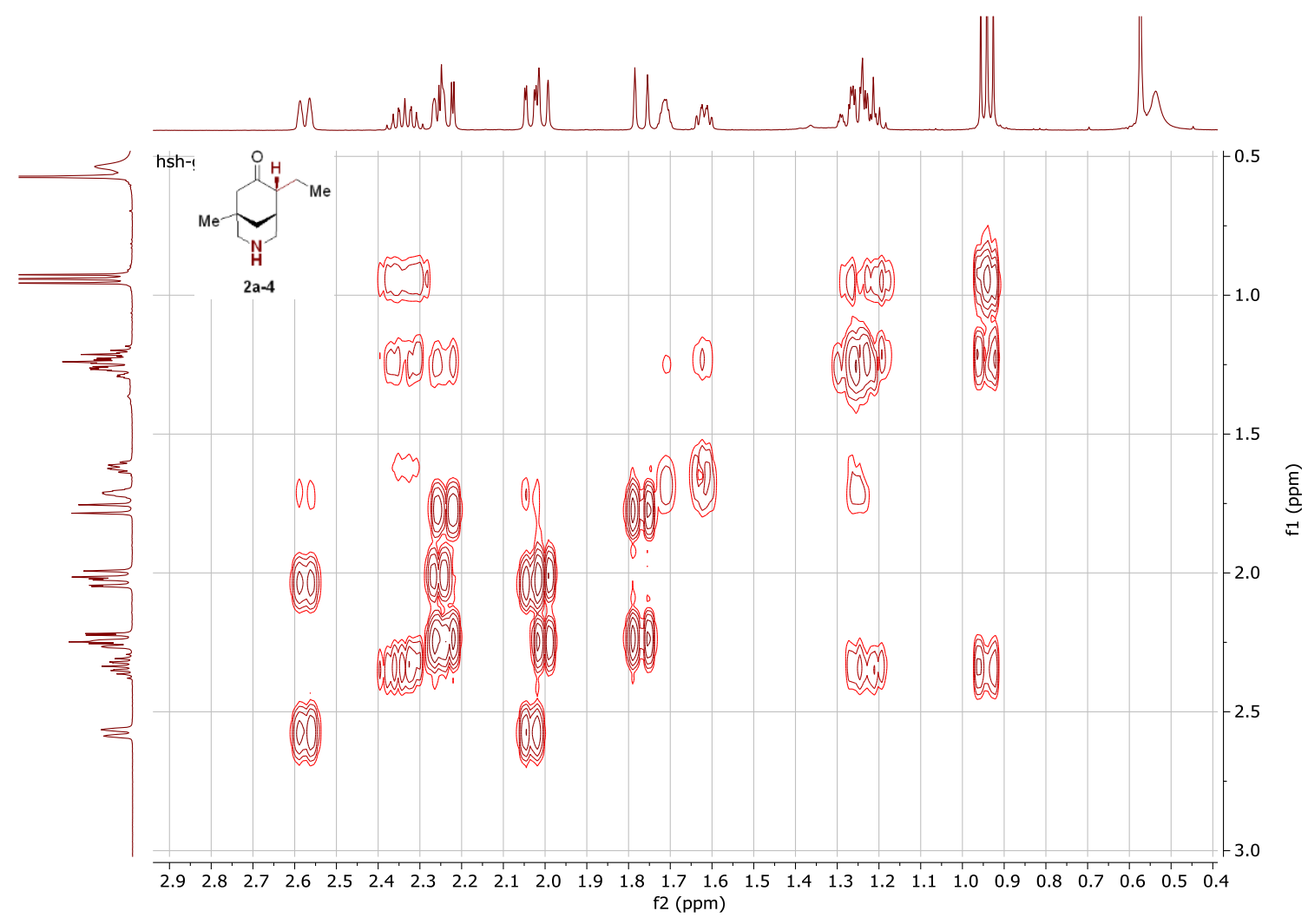




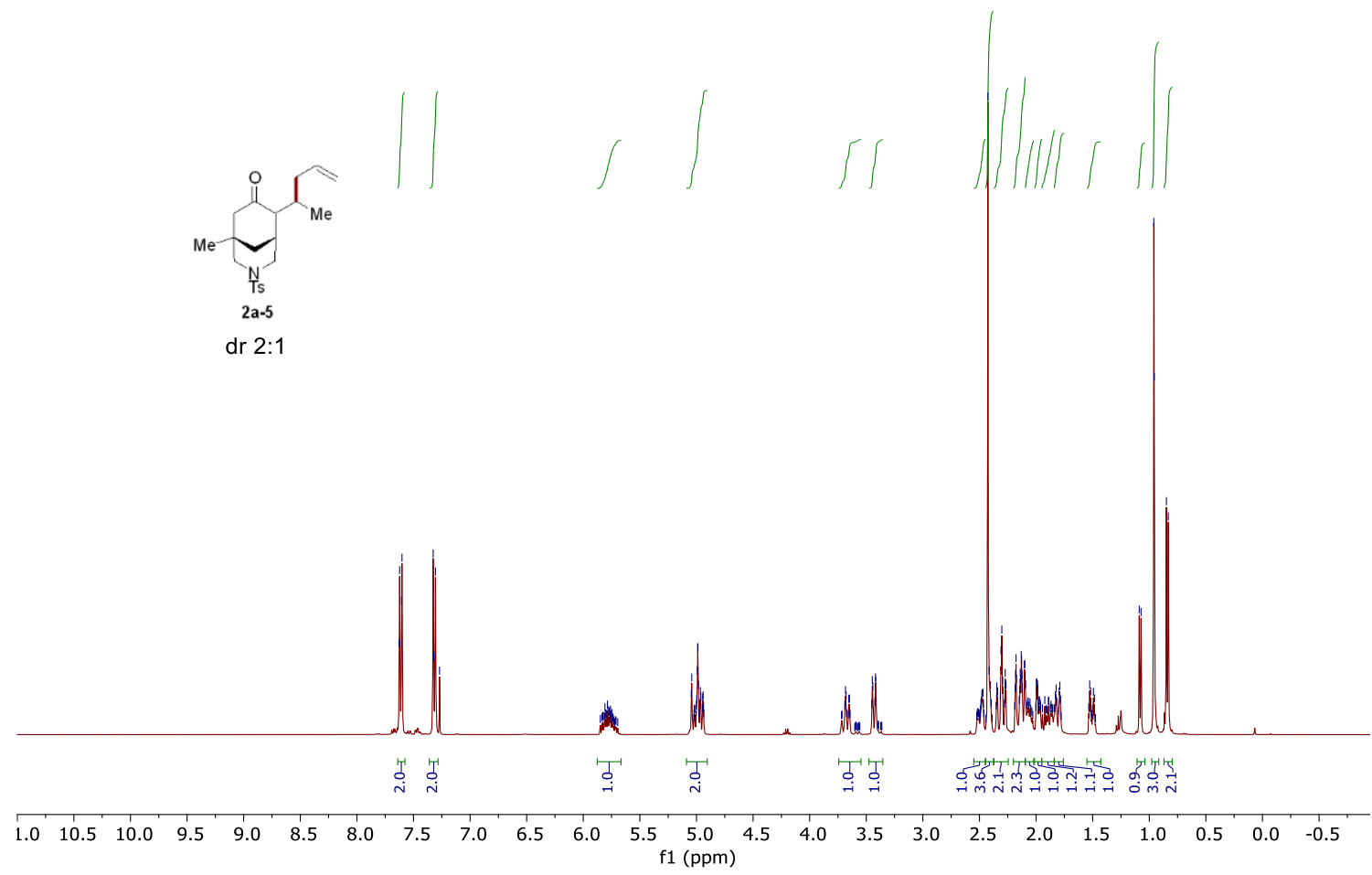

${ }^{13} \mathrm{C}-\mathrm{NMR}$ spectrum of $\mathbf{2 a - 5}$ in $\mathrm{CDCl}_{3}, 101 \mathrm{MHz}$

hsh-g152省

它

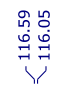

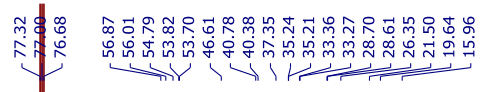

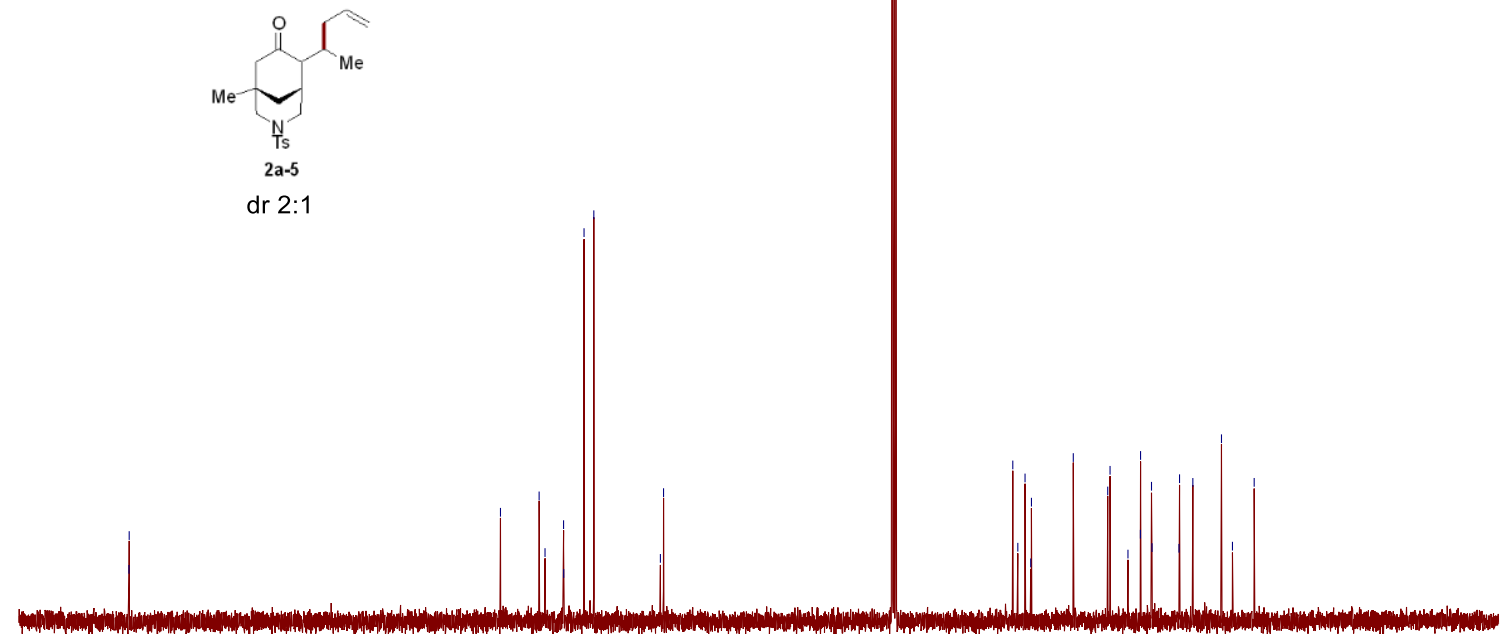

dr 2:1

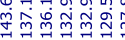

$\begin{array}{llllllllllllllllllllllllllllllllll}220 & 210 & 200 & 190 & 180 & 170 & 160 & 150 & 140 & 130 & 120 & 110 & 100 & 90 & 80 & 70 & 60 & 50 & 40 & 30 & 20 & 10 & 0 & -10 & -20\end{array}$ 


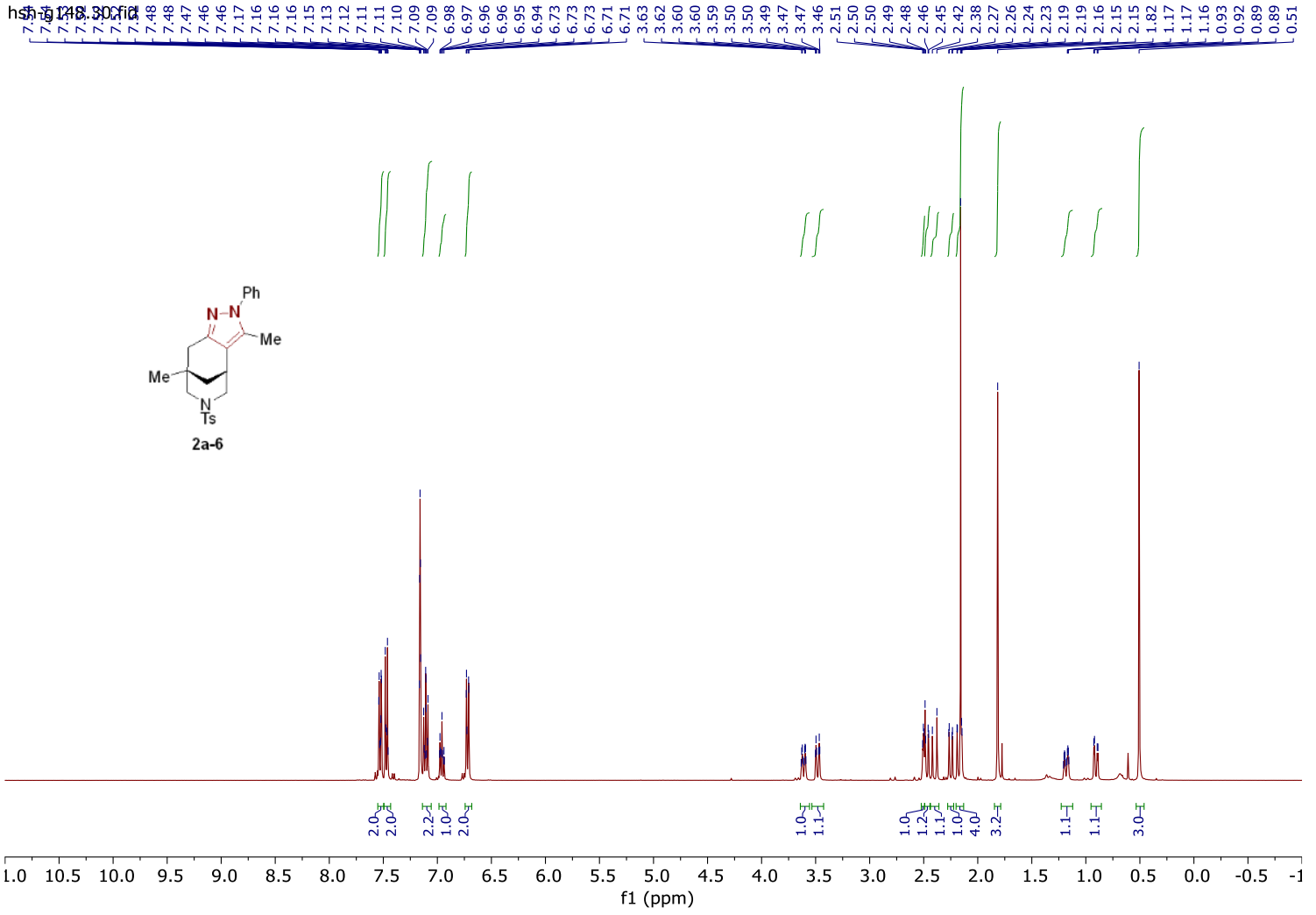

${ }^{13} \mathrm{C}-\mathrm{NMR}$ spectrum of $2 \mathrm{a}-6$ in $\mathrm{C}_{6} \mathrm{D}_{6}, 101 \mathrm{MHz}$

hsh-g148.31.fid
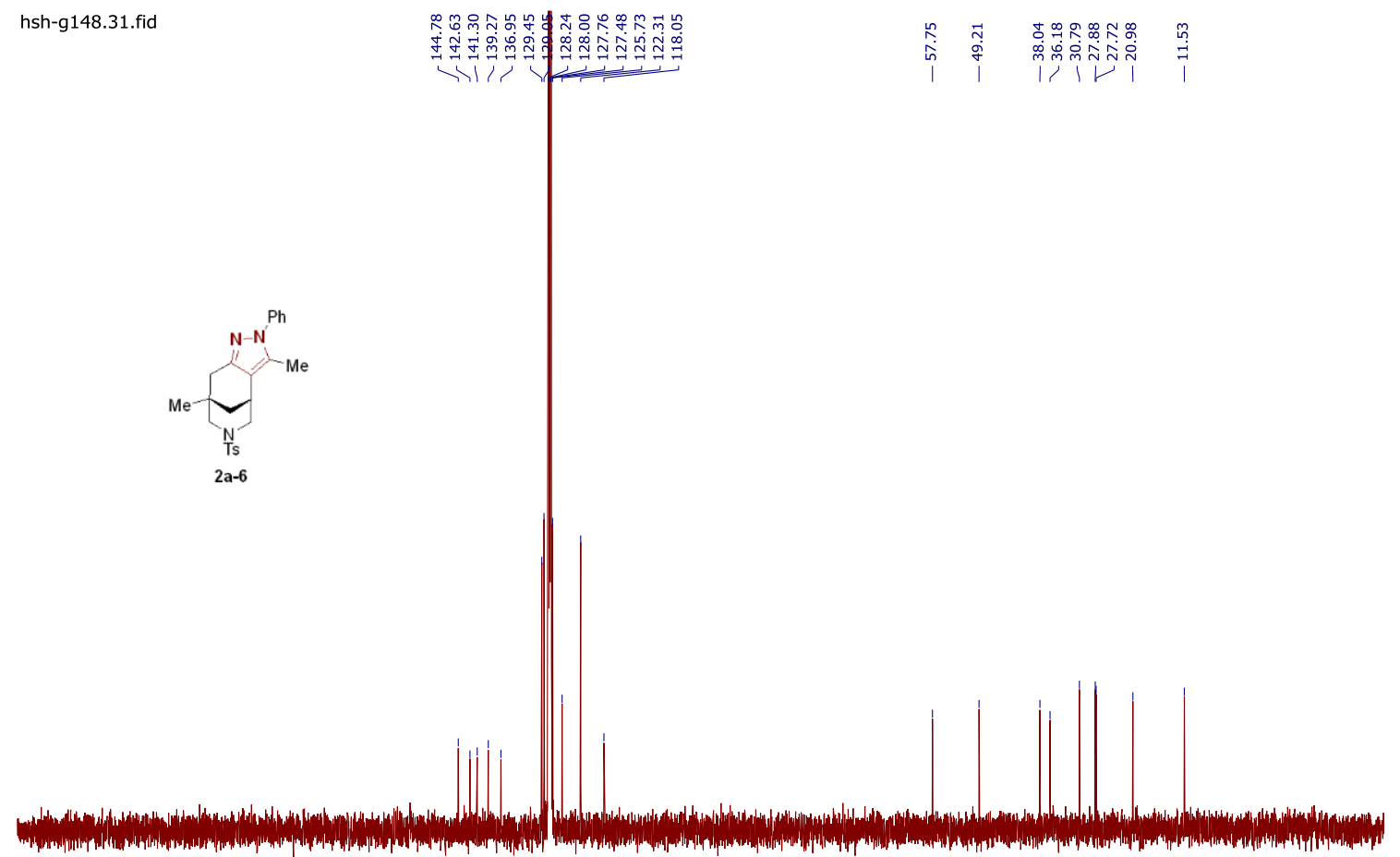

$\begin{array}{lllllllllllllllllllllllllllllllllll}220 & 210 & 200 & 190 & 180 & 170 & 160 & 150 & 140 & 130 & 120 & 110 & 100 & 90 & 80 & 70 & 60 & 50 & 40 & 30 & 20 & 10 & 0 & -10 & -20\end{array}$ 
${ }^{1} \mathrm{H}-\mathrm{NMR}$ spectrum of 6 a in $\mathrm{CDCl}_{3}, 400 \mathrm{MHz}$

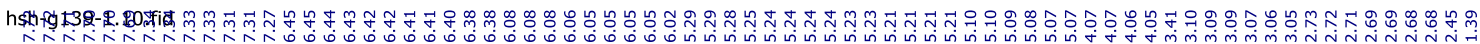
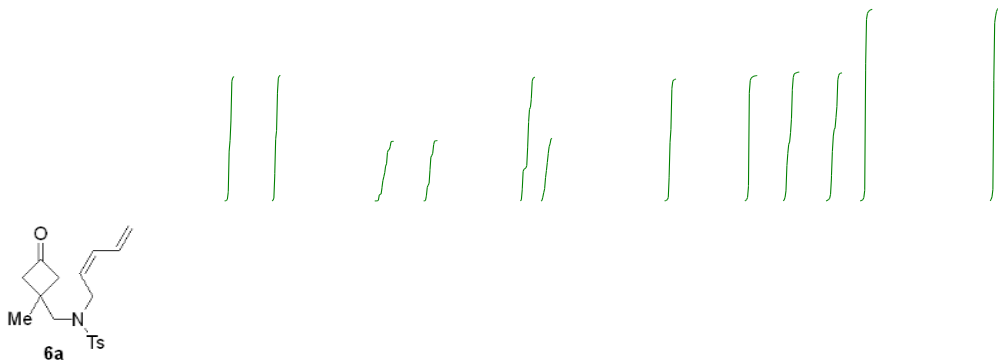

6a Ts

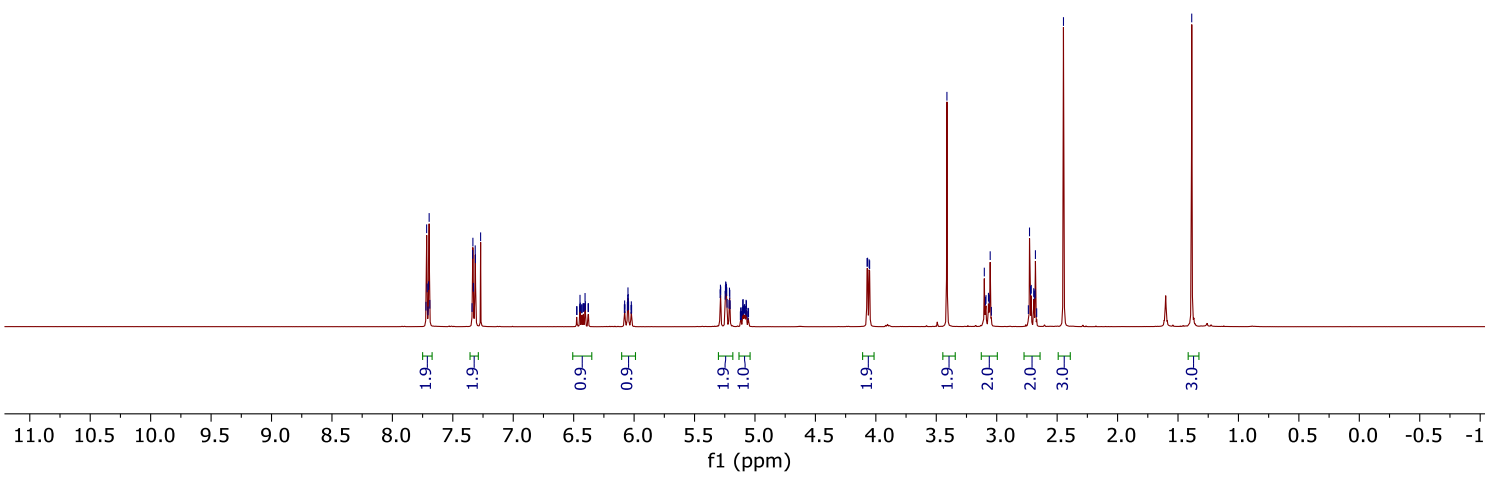

${ }^{13} \mathrm{C}$-NMR spectrum of 6 a in $\mathrm{CDCl}_{3}, 101 \mathrm{MHz}$

hsh-g139-1211.fid

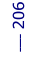

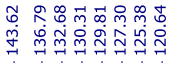

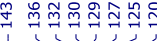

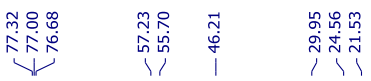

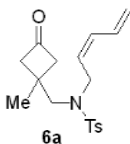

$\begin{array}{llllllllllllllllllllllllllllll}220 & 210 & 200 & 190 & 180 & 170 & 160 & 150 & 140 & 130 & 120 & 110 & 100 & 90 & 80 & 70 & 60 & 50 & 40 & 30 & 20 & 10 & 0 & -10 & -20\end{array}$ 
${ }^{1} \mathrm{H}-\mathrm{NMR}$ spectrum of $\mathbf{2} \mathbf{h}-\mathbf{d}_{\mathbf{5}}$ in $\mathrm{C}_{6} \mathrm{D}_{6}, 400 \mathrm{MHz}$

hsh-g202-2.10.fid

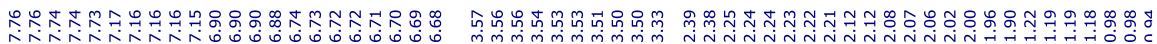

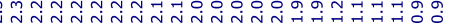

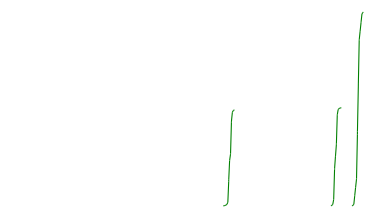

$29 \%$ (D) $\mathrm{H}$ O $\mathrm{D}>95 \%$ (D)

$7 \%(\mathrm{D}) \mathrm{CD}$ (D)

N $D>95 \%$ (D)

$-1$

MeO $2 \mathrm{~h}-\mathrm{d}_{5}$

$2 h-d_{5}$

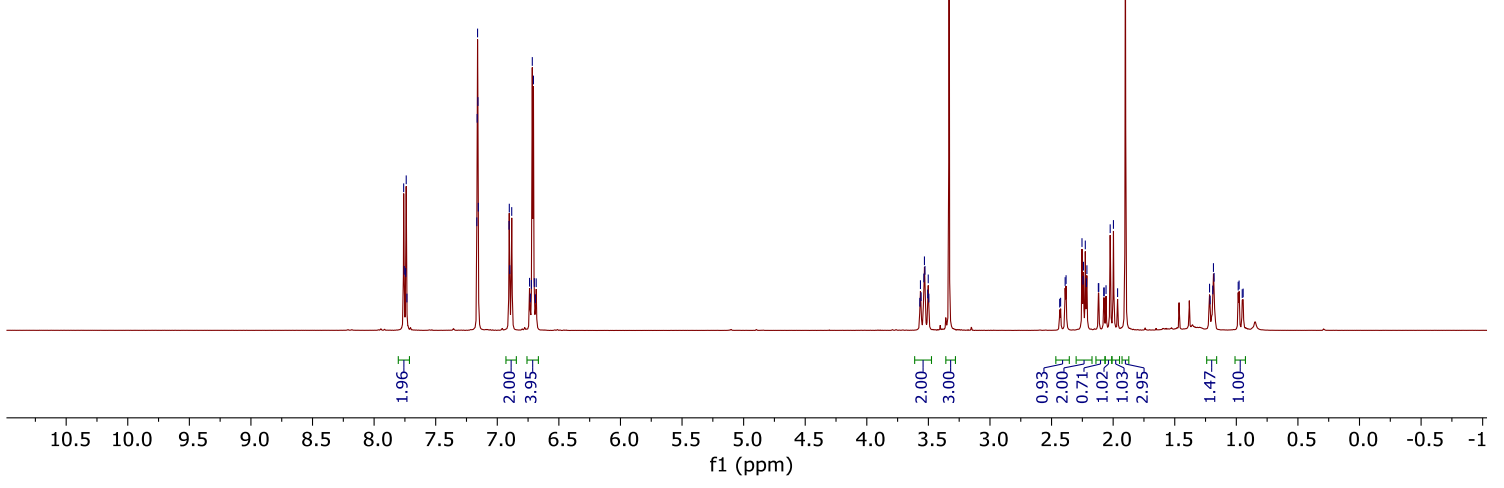

${ }^{13} \mathrm{C}$-NMR spectrum of $\mathbf{2} \mathbf{h}-\mathbf{d}_{\mathbf{5}}$ in $\mathrm{C}_{6} \mathrm{D}_{6}, 101 \mathrm{MHz}$

hsh-g202-2.12.fid

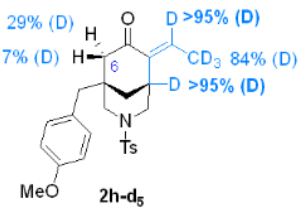

$\begin{array}{llllllllllllll}220 & 210 & 200 & 190 & 180 & 170 & 160 & 150 & 140 & 130 & 120 & 110 & 100 & 90\end{array}$ 
${ }^{1} \mathrm{H}-\mathrm{NMR}$ spectrum of $\mathbf{2} \mathbf{h}$ and $\mathbf{2} \mathbf{h}-\mathbf{d}_{5}$ in $\mathrm{C}_{6} \mathrm{D}_{6}, 400 \mathrm{MHz}$

hsh-g116-2.10.fid
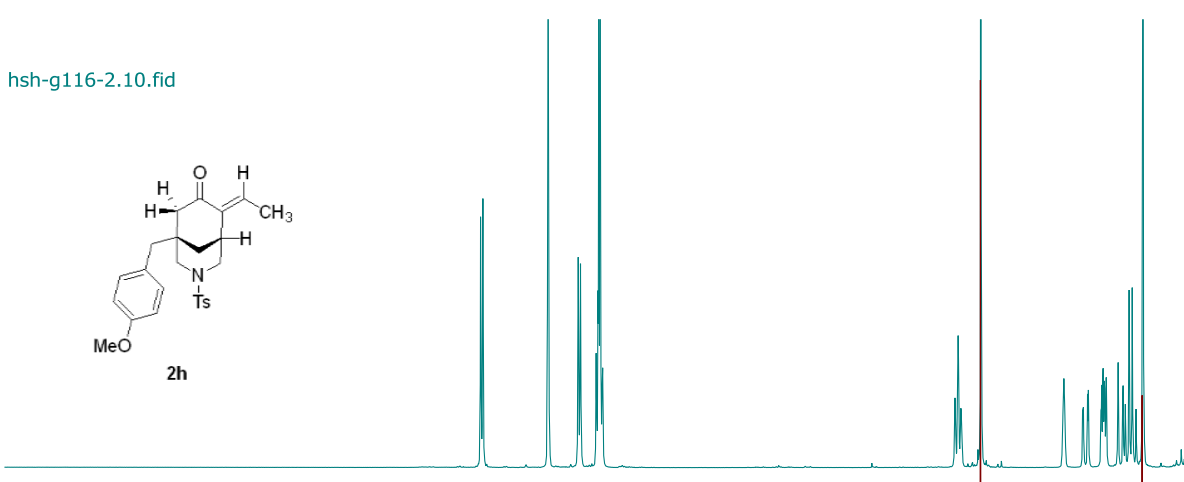

hsh-g202-2.10.fid
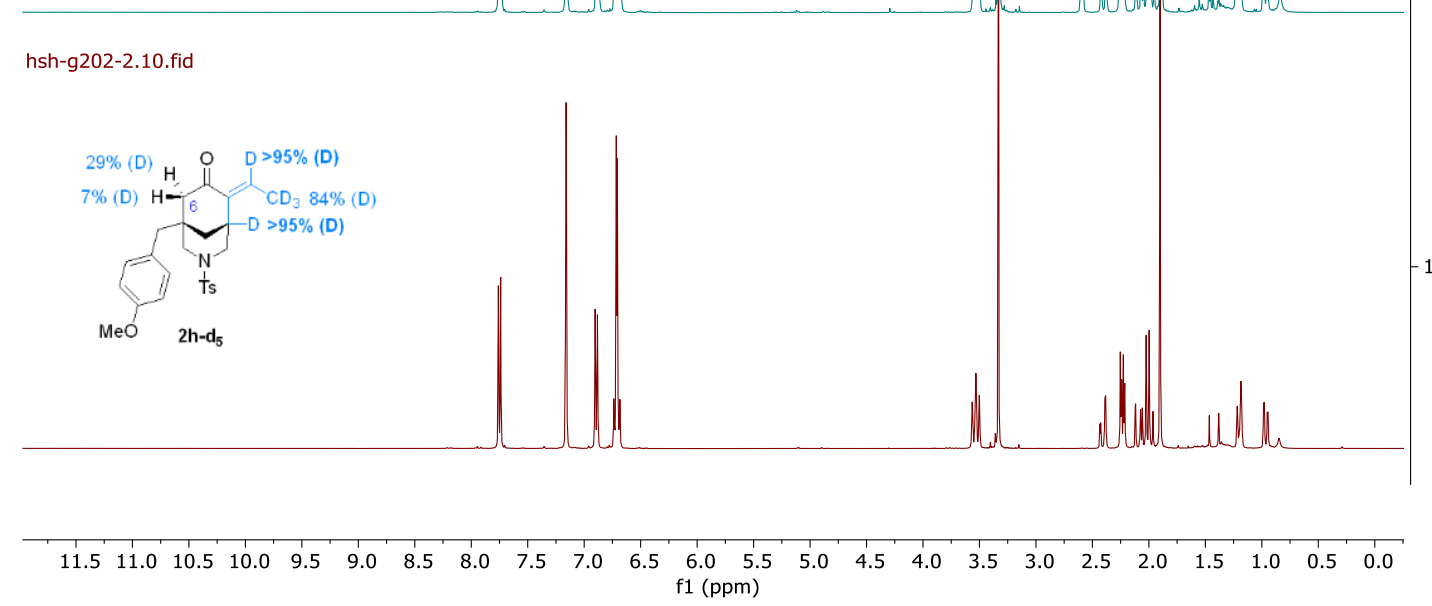

${ }^{13} \mathrm{C}-\mathrm{NMR}$ spectrum of $\mathbf{2} \mathbf{h}$ and $\mathbf{2} \mathbf{h}-\mathbf{d}_{5}$ in $\mathrm{C}_{6} \mathrm{D}_{6}, 101 \mathrm{MHz}$

hsh-f116-1.12.fid
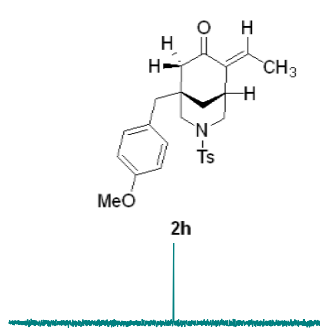

hsh-g202-2.12.fid

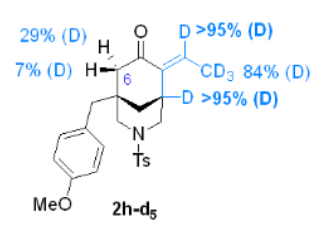

$\begin{array}{llllllllllllllllllllllllllllllllllll}220 & 210 & 200 & 190 & 180 & 170 & 160 & 150 & 140 & 130 & 120 & 110 & 100 & 90 & 80 & 70 & 60 & 50 & 40 & 30 & 20 & 10 & 0 & -10 & -20\end{array}$ 
${ }^{1} \mathrm{H}-\mathrm{NMR}$ spectrum of $\mathbf{2} \mathbf{h}-\mathbf{d}_{\mathbf{5}}$ in $\mathrm{C}_{6} \mathrm{D}_{6}, 400 \mathrm{MHz}$

hsh-g203-4.10.fid

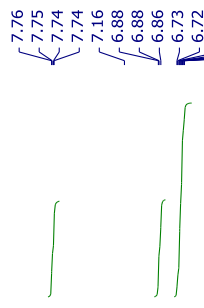

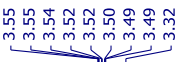

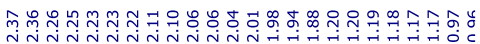

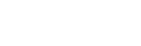
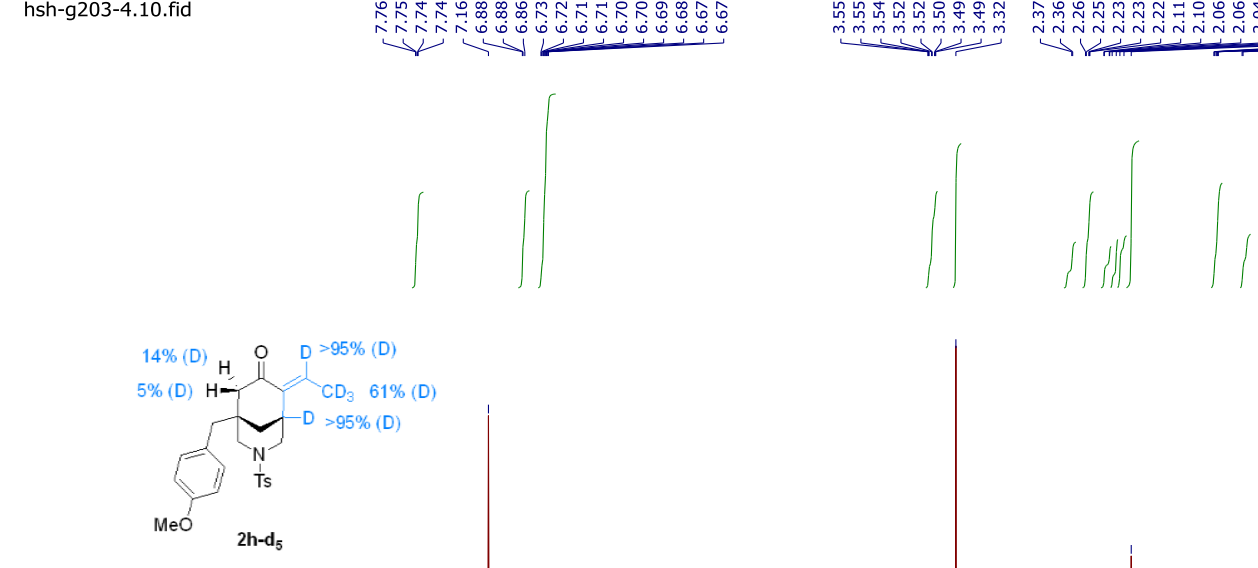

$14 \%$ (D) $\mathrm{H} \quad \mathrm{O} \quad \mathrm{D}>95 \%$ (D)
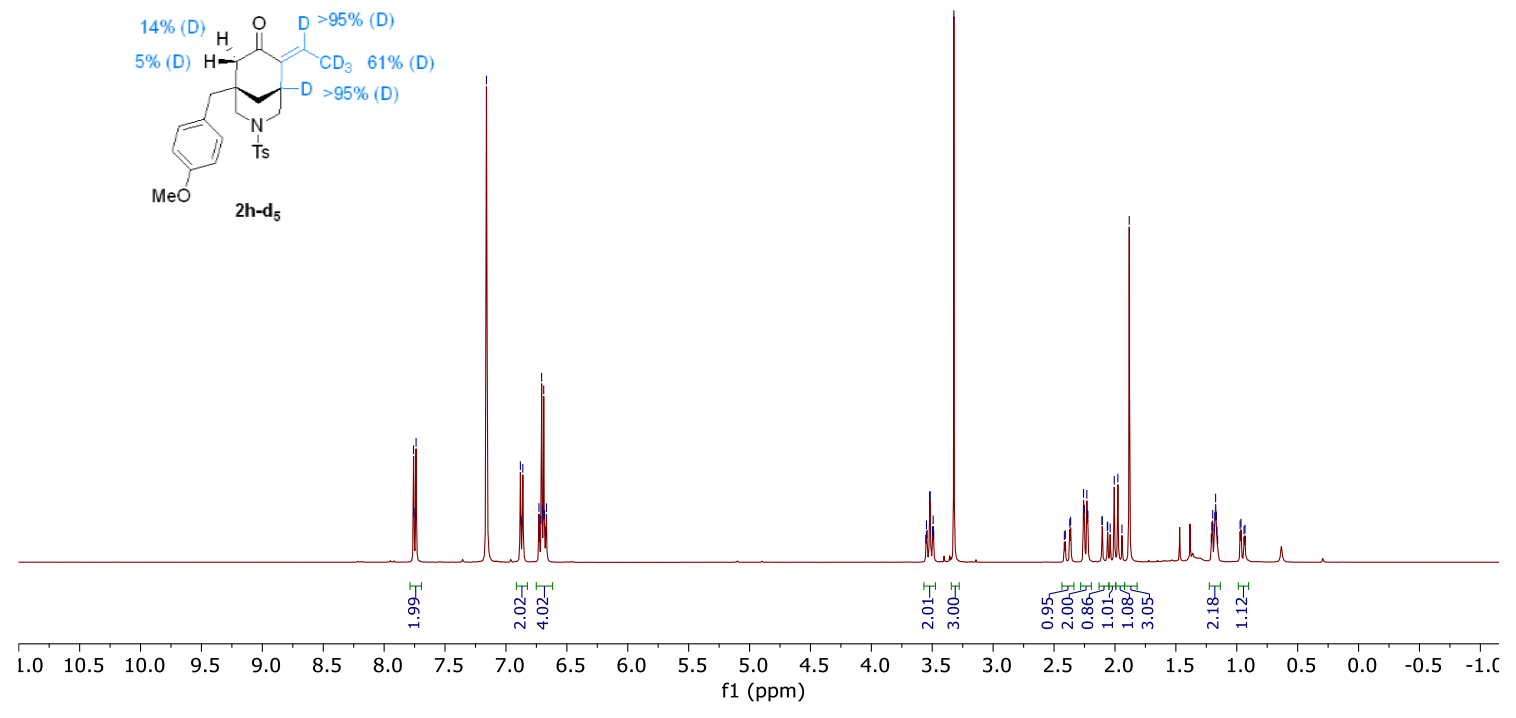

${ }^{1} \mathrm{H}-\mathrm{NMR}$ spectrum of $\mathbf{2} \mathbf{i}$-d in $\mathrm{C}_{6} \mathrm{D}_{6}, 400 \mathrm{MHz}$

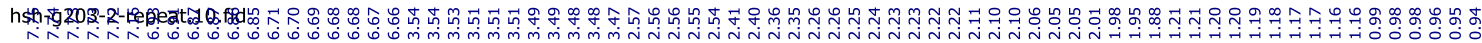

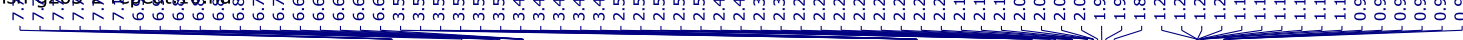

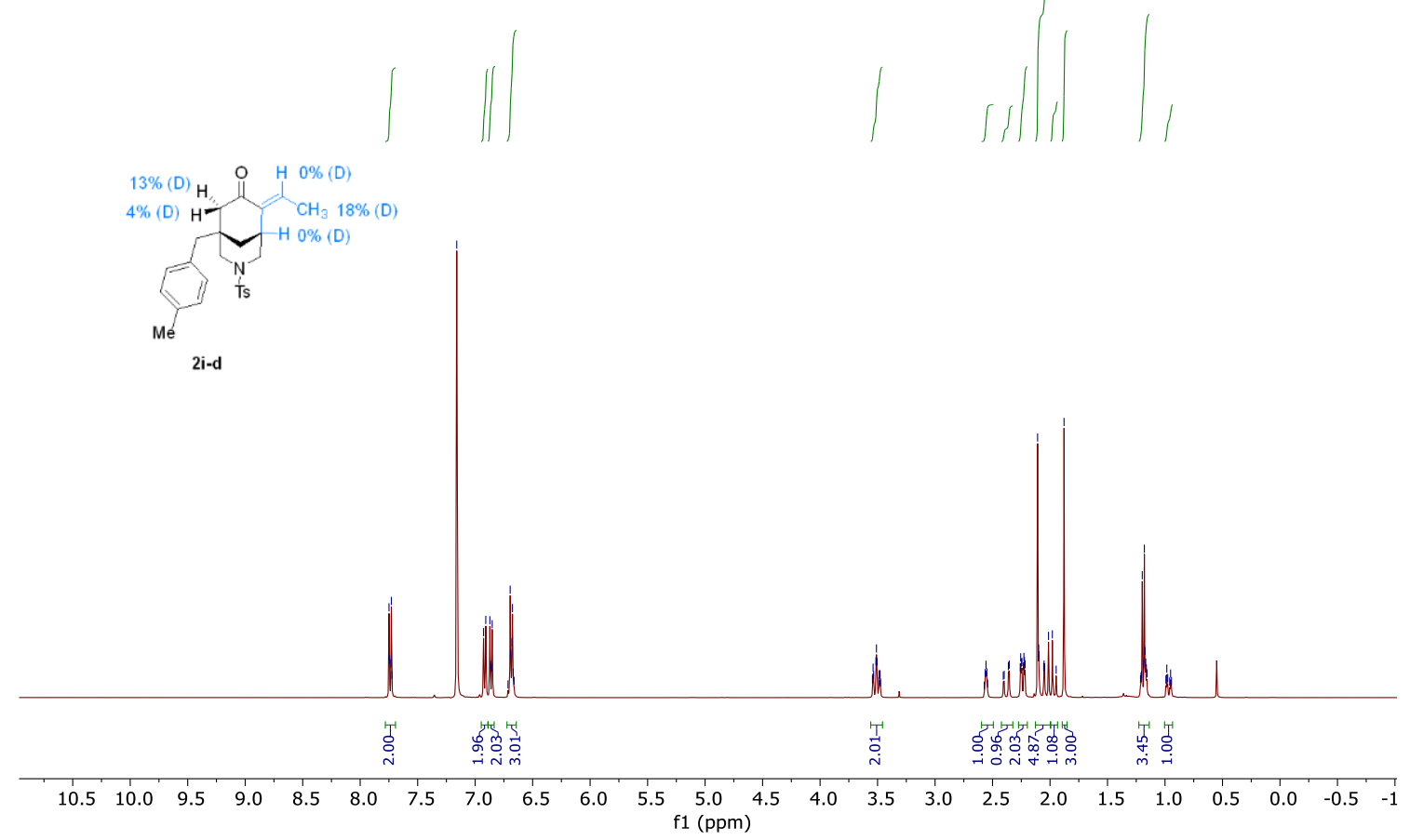


${ }^{13} \mathrm{C}-\mathrm{NMR}$ spectrum of $\mathbf{2} \mathbf{i}-\mathbf{d}$ in $\mathrm{C}_{6} \mathrm{D}_{6}, 101 \mathrm{MHz}$
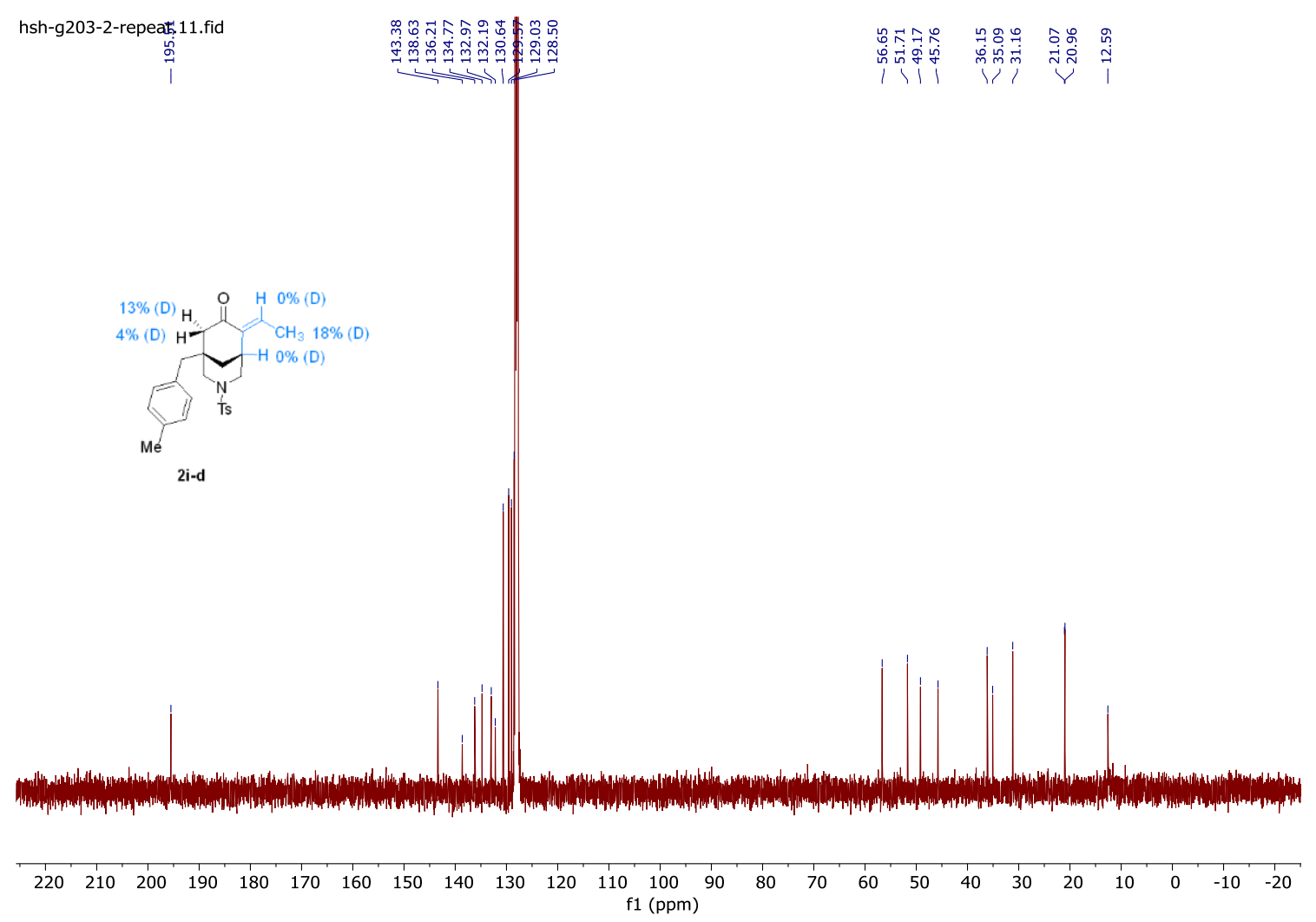

${ }^{1} \mathrm{H}$-NMR spectrum of $\mathbf{2} \mathbf{h}-\mathbf{d}$ in $\mathrm{C}_{6} \mathrm{D}_{6}, 400 \mathrm{MHz}$

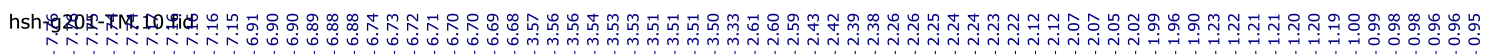
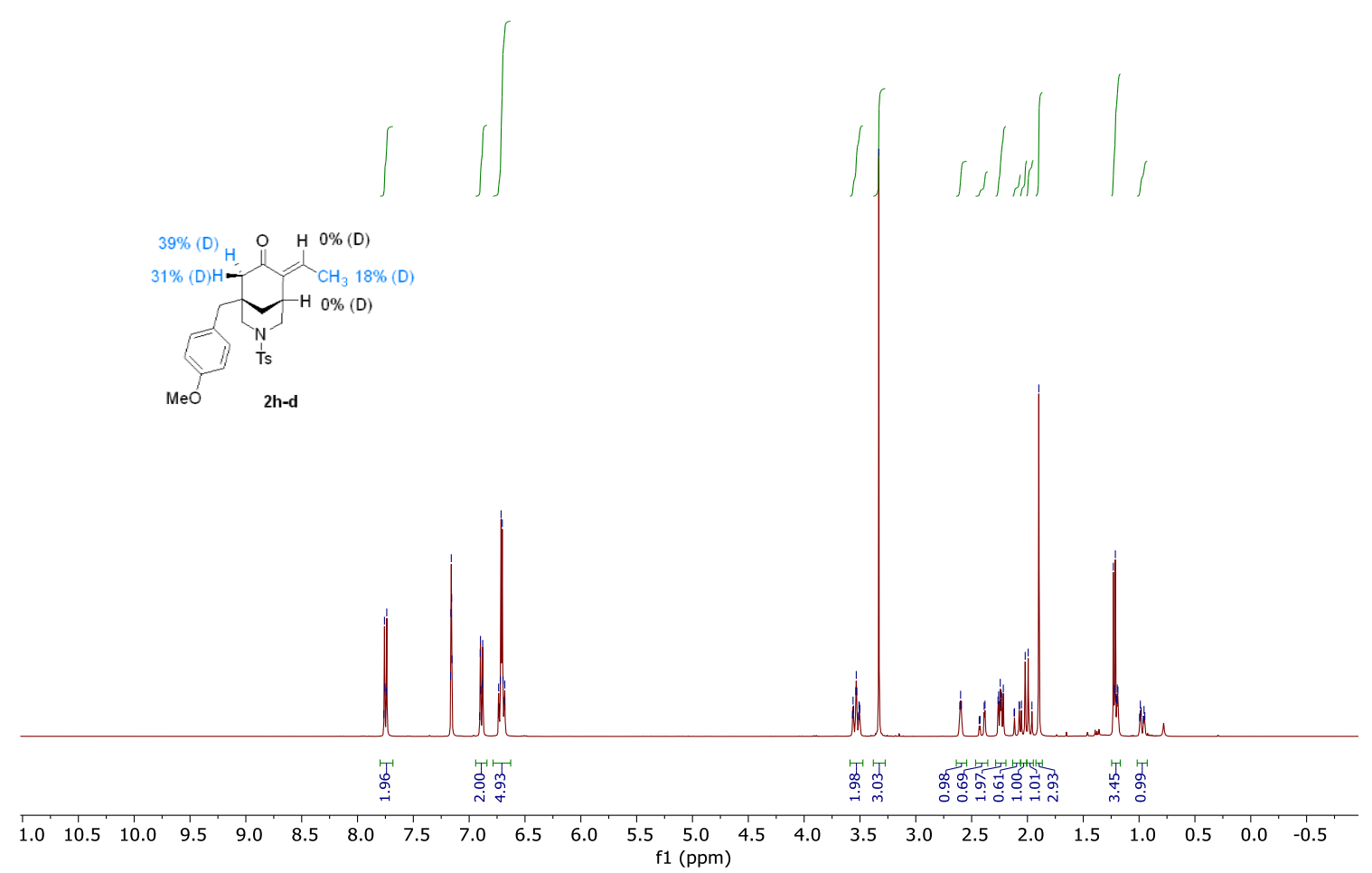
${ }^{1} \mathrm{H}-\mathrm{NMR}$ spectrum of $\mathbf{2} \mathbf{h}$ and $\mathbf{2 h}-\mathbf{d}$ in $\mathrm{C}_{6} \mathrm{D}_{6}, 400 \mathrm{MHz}$

hsh-g116-2.10.fid

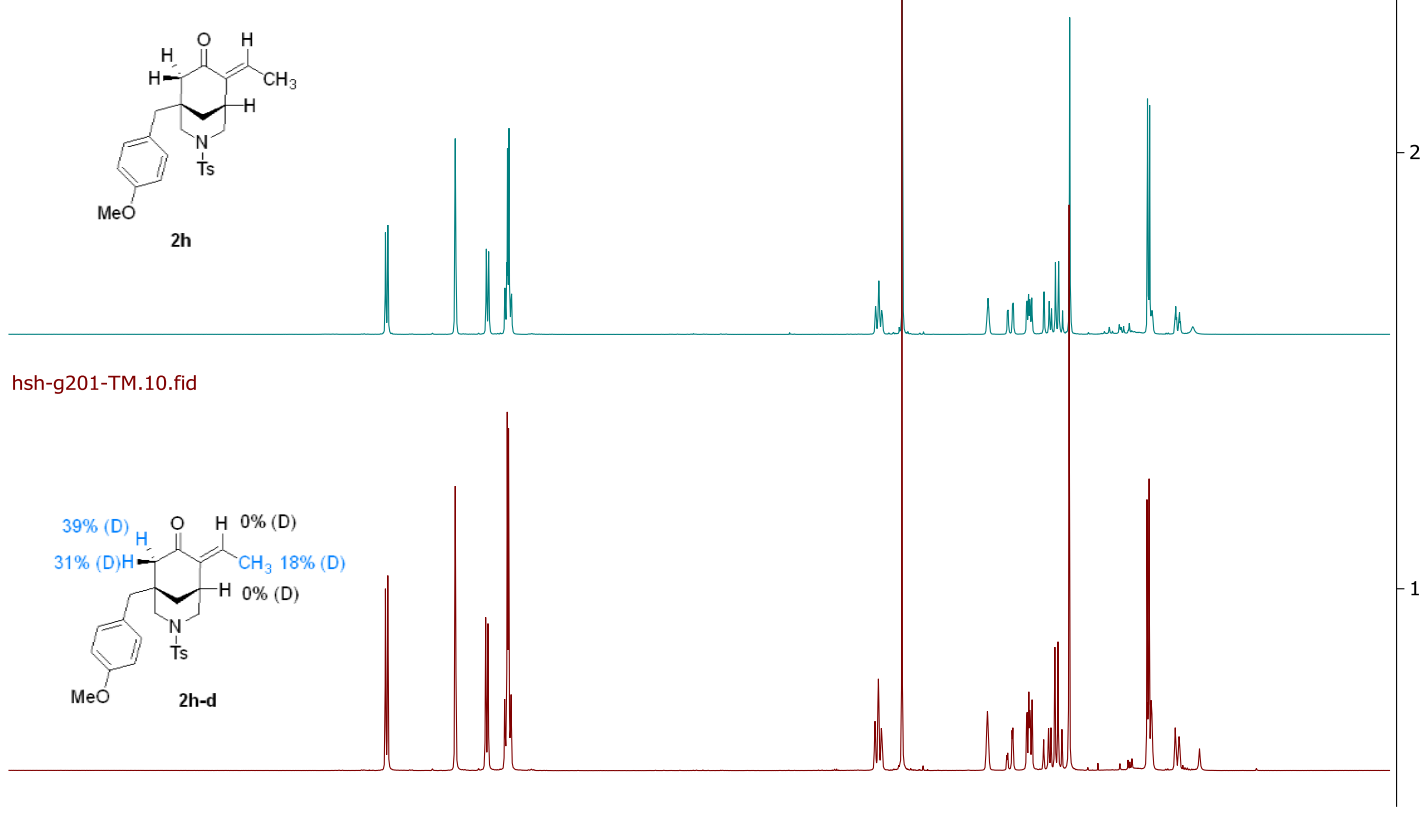

$\begin{array}{lllllllllllllllllllllllllllll}10.5 & 10.0 & 9.5 & 9.0 & 8.5 & 8.0 & 7.5 & 7.0 & 6.5 & 6.0 & 5.5 & 5.0 & 4.5 & 4.0 & 3.5 & 3.0 & 2.5 & 2.0 & 1.5 & 1.0 & 0.5 & 0.0 & -0.5\end{array}$

${ }^{1} \mathrm{H}$-NMR spectrum of $\mathbf{2} \mathbf{h}-\mathbf{d}$ in $\mathrm{C}_{6} \mathrm{D}_{6}, 400 \mathrm{MHz}$

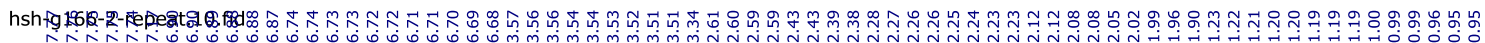

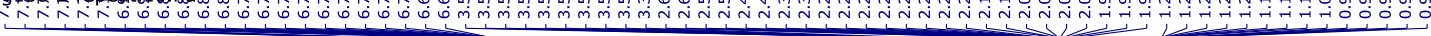

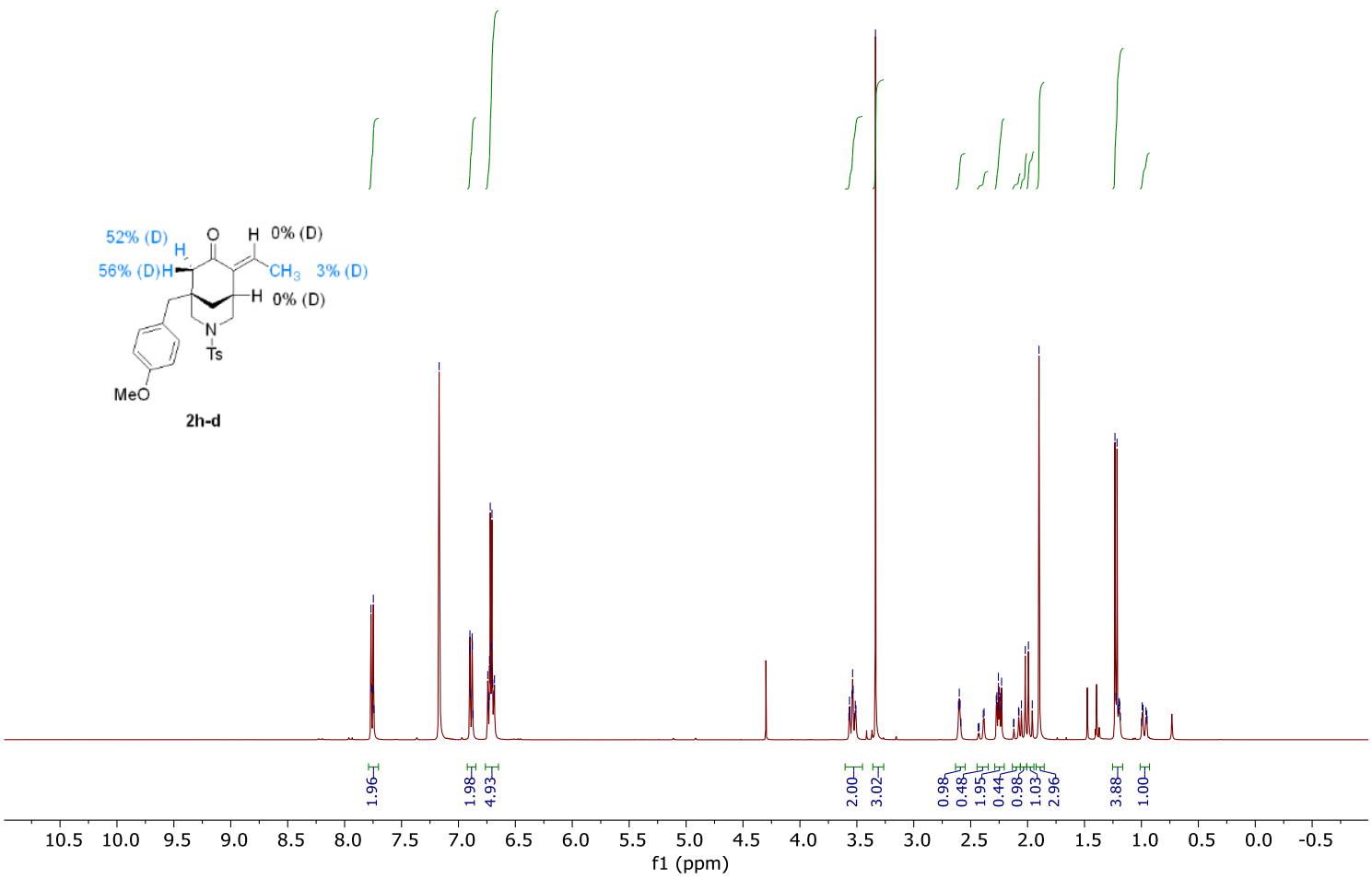




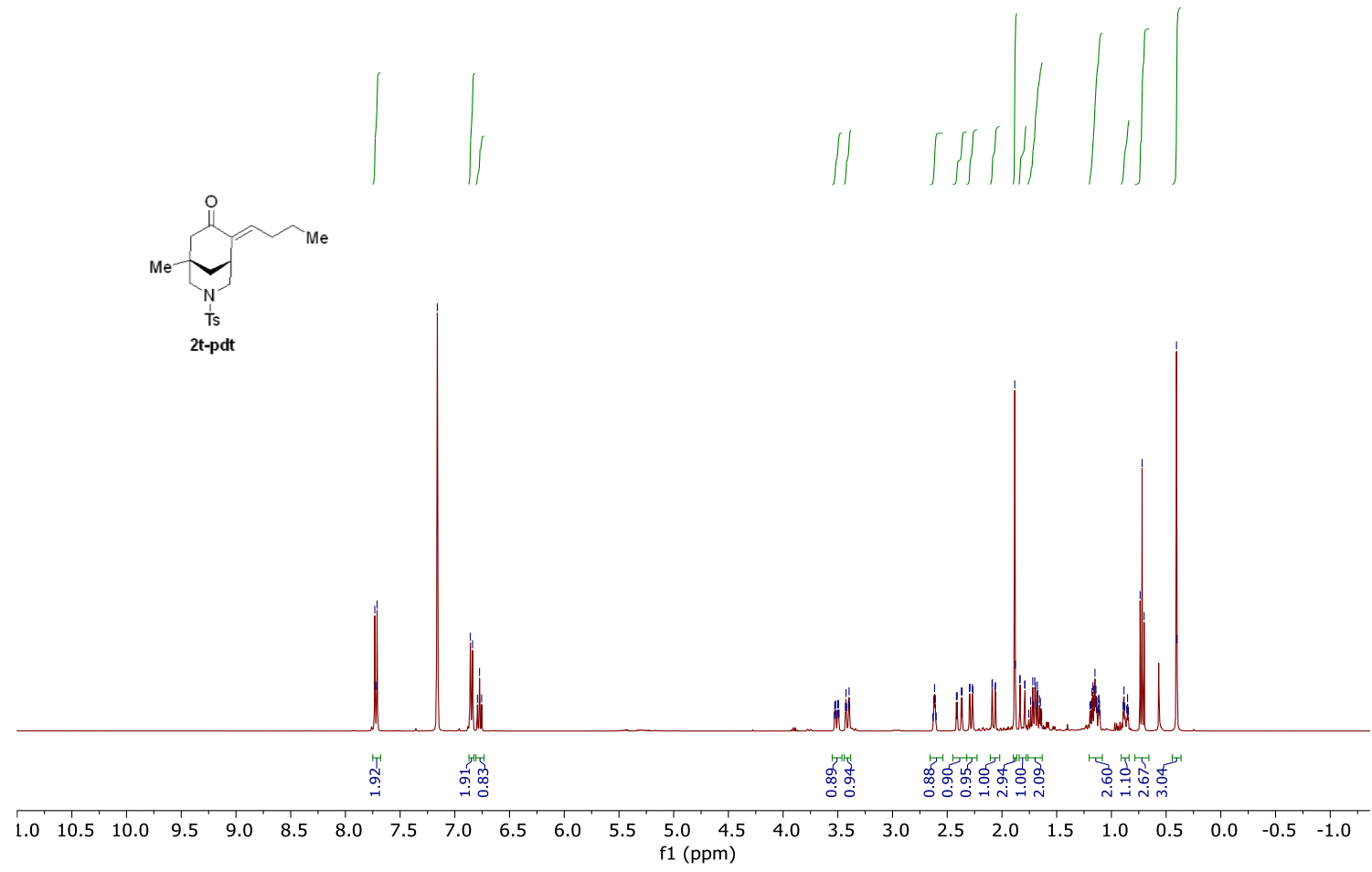

${ }^{13} \mathrm{C}-\mathrm{NMR}$ spectrum of $\mathbf{2 t - d p t}$ in $\mathrm{C}_{6} \mathrm{D}_{6}, 101 \mathrm{MHz}$

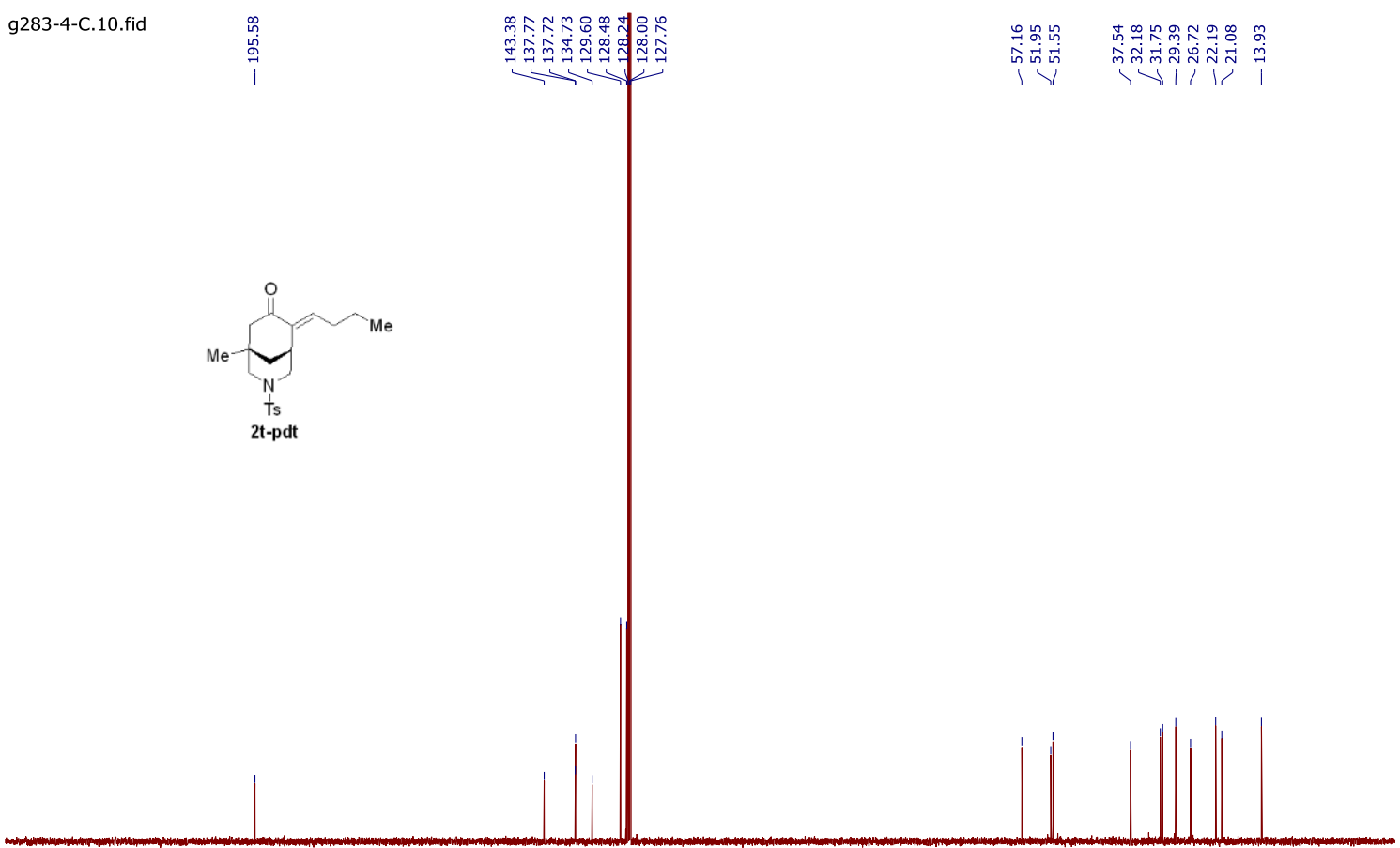

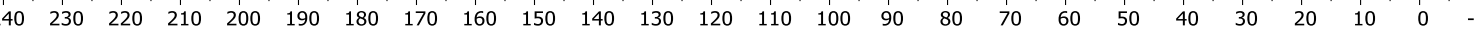




\section{${ }^{1} \mathrm{H}-\mathrm{NMR}$ spectrum of $1 \mathrm{a}$ in $\mathrm{CDCl}_{3}, 400 \mathrm{MHz}$}

hsh-g17.32.fid

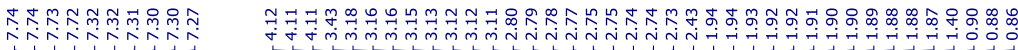

Mè

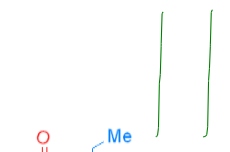

$\int_{n}^{N}$

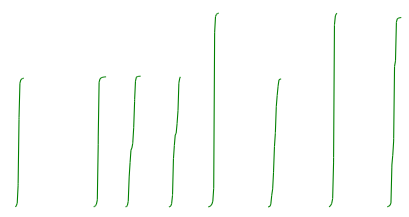

1a ts

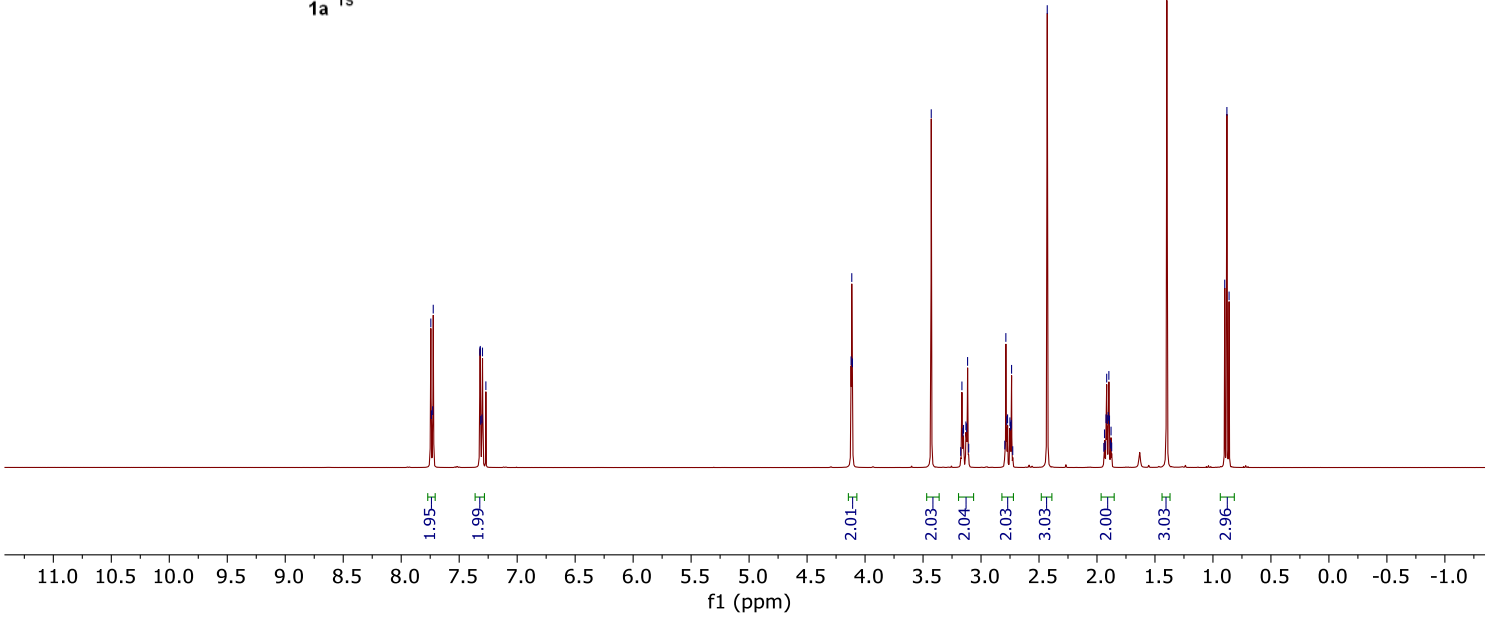

${ }^{13} \mathrm{C}-\mathrm{NMR}$ spectrum of $1 \mathrm{a}$ in $\mathrm{CDCl}_{3}, 101 \mathrm{MHz}$

hsh-g17.3악id

㝏 in
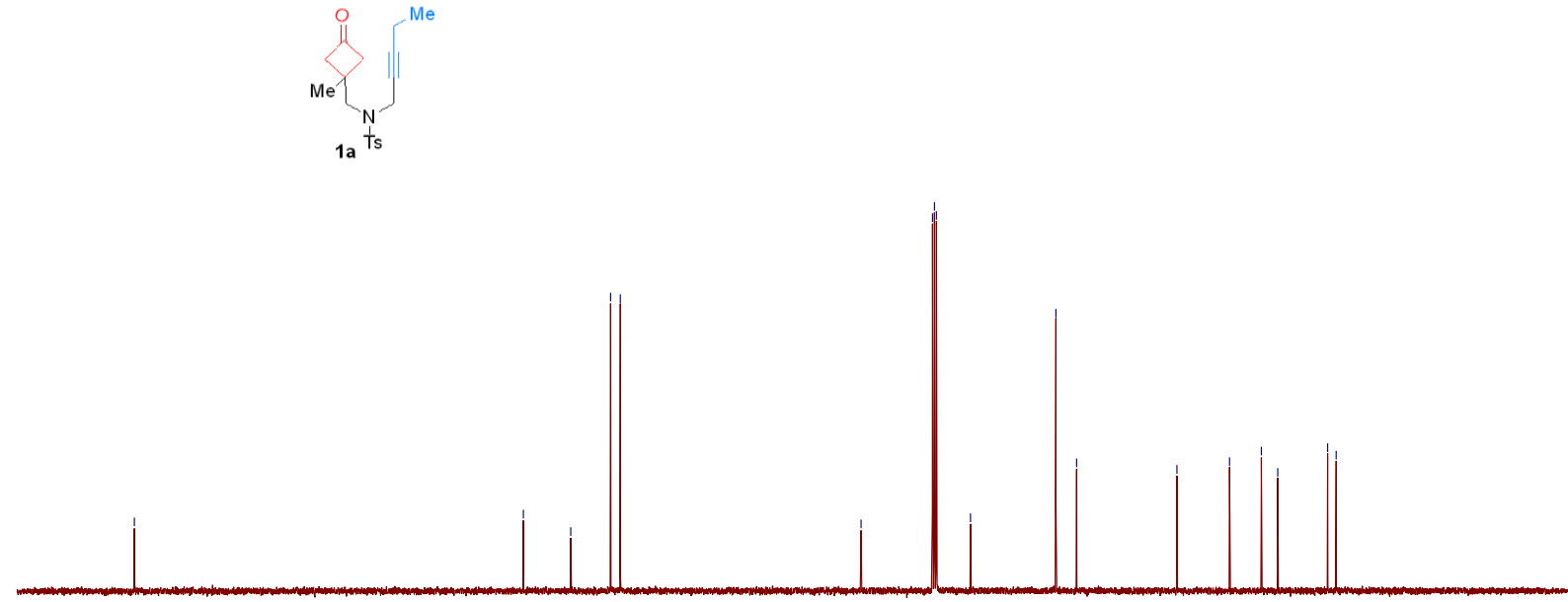

$\begin{array}{lllllllllllllllllllllllllll}220 & 210 & 200 & 190 & 180 & 170 & 160 & 150 & 140 & 130 & 120 & 110 & 100 & 90 & 80 & 70 & 60 & 50 & 40 & 30 & 20 & 10 & 0 & -10 & -20\end{array}$ 
${ }^{1} \mathrm{H}-\mathrm{NMR}$ spectrum of $\mathbf{1 b}$ in $\mathrm{CDCl}_{3}, 400 \mathrm{MHz}$

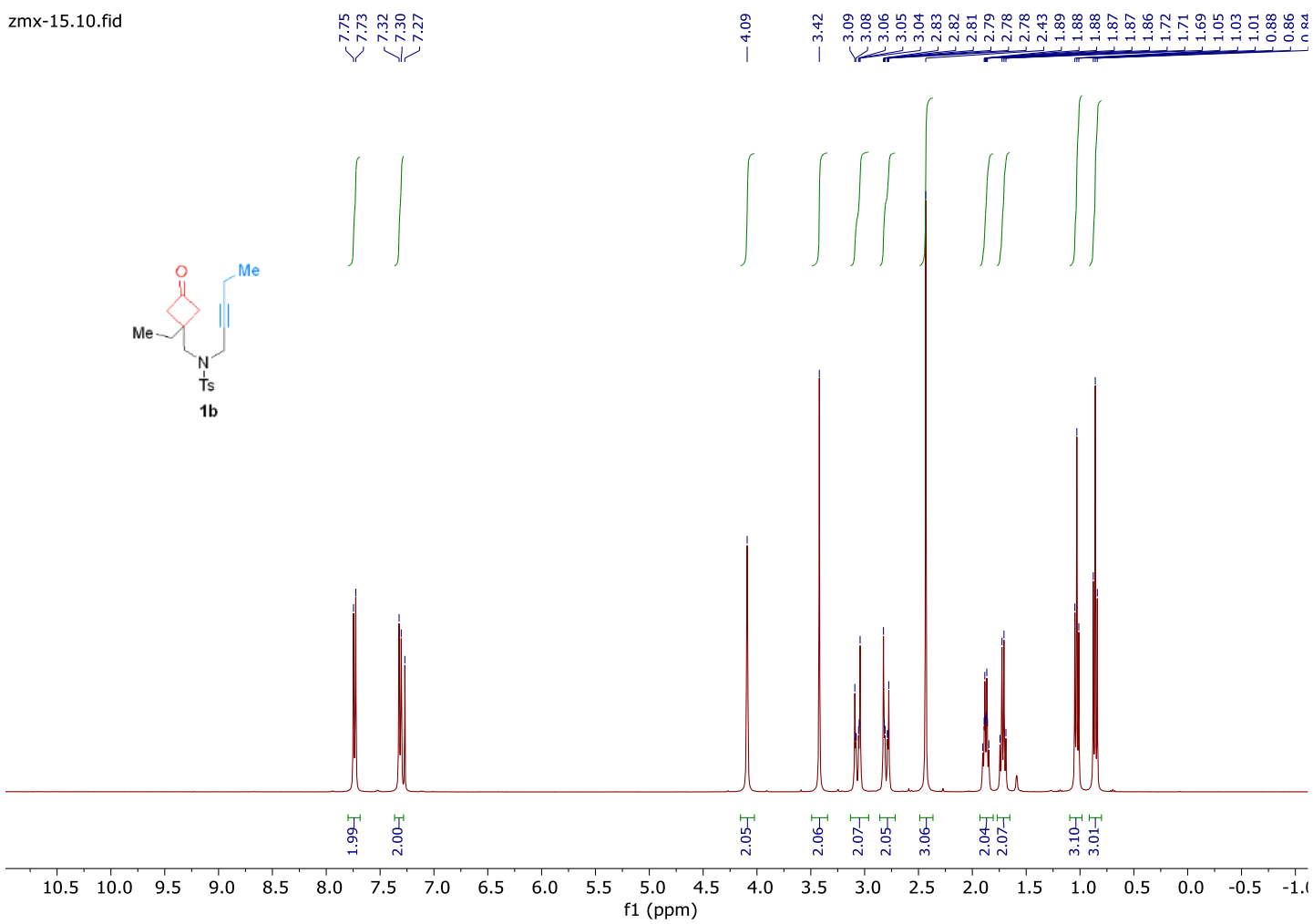

${ }^{13} \mathrm{C}-\mathrm{NMR}$ spectrum of $\mathbf{1 b}$ in $\mathrm{CDCl}_{3}, 101 \mathrm{MHz}$

$\mathrm{zmx}-15.11$ gृid

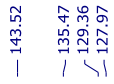

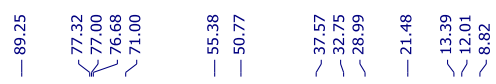
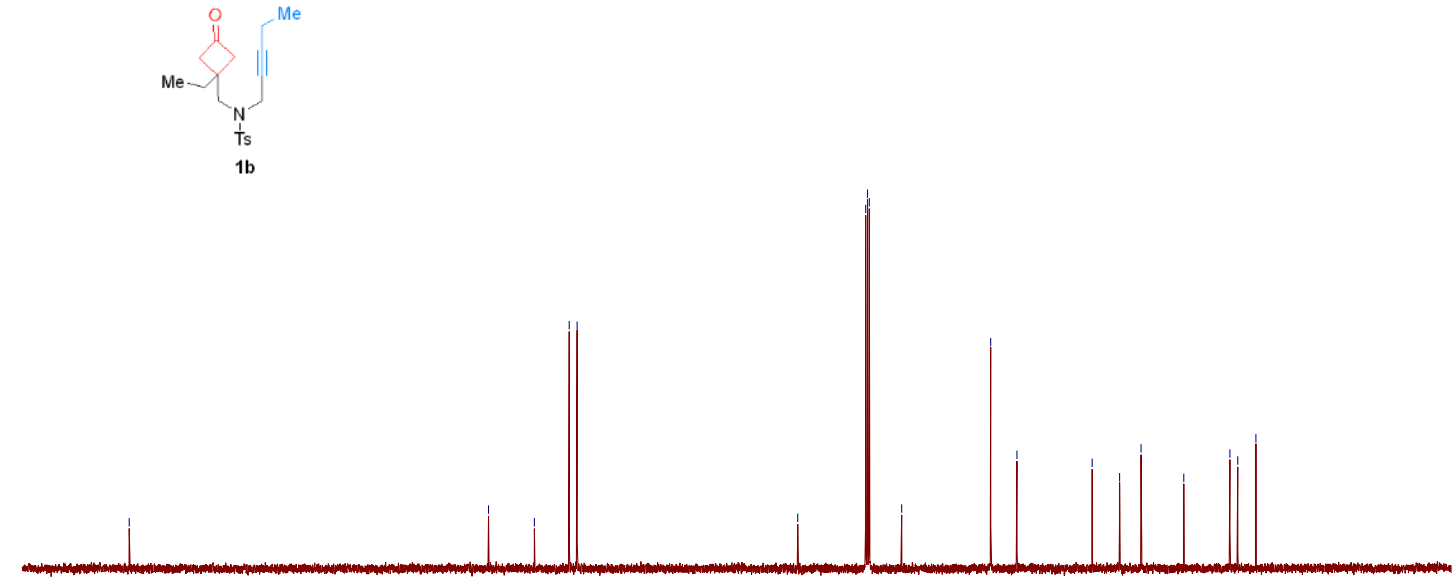

$\begin{array}{lllllllllllllllllllllllll}220 & 210 & 200 & 190 & 180 & 170 & 160 & 150 & 140 & 130 & 120 & 110 & 100 & 90 & 80 & 70 & 60 & 50 & 40 & 30 & 20 & 10 & 0 & -10 & -20\end{array}$ 
${ }^{1} \mathrm{H}-\mathrm{NMR}$ spectrum of $1 \mathrm{c}$ in $\mathrm{CDCl}_{3}, 400 \mathrm{MHz}$

hsh-g50-1.20.fid

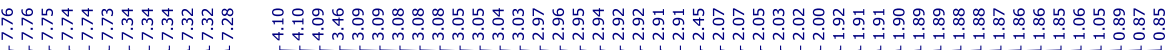
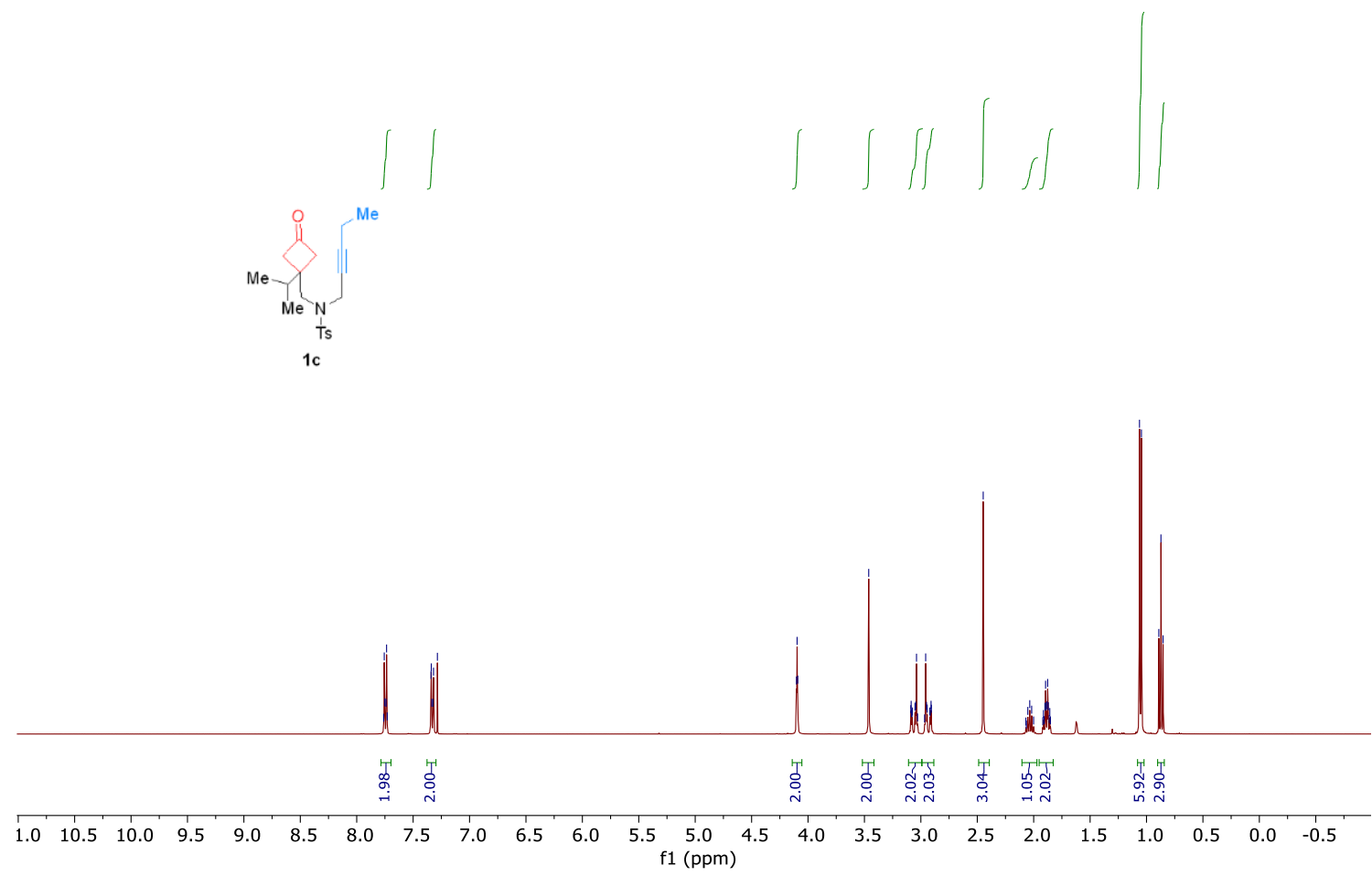

${ }^{13} \mathrm{C}-\mathrm{NMR}$ spectrum of $1 \mathrm{c}$ in $\mathrm{CDCl}_{3}, 101 \mathrm{MHz}$

hsh-g50-191.fid

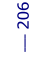

负

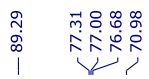

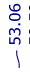

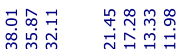

\section{लำ}

$11>1$

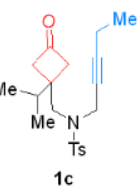

$1 \mathrm{c}$

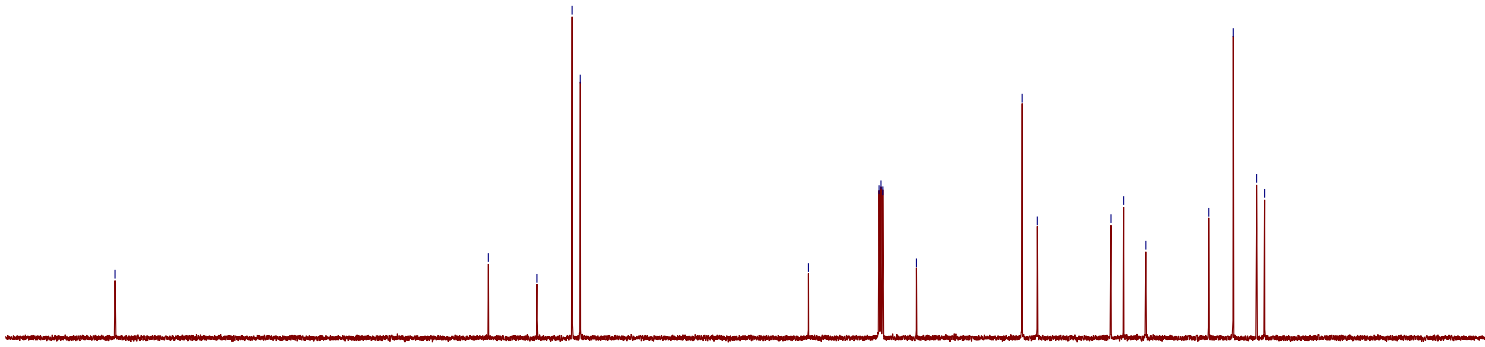

$\begin{array}{lllllllllllllllllllllllll}220 & 210 & 200 & 190 & 180 & 170 & 160 & 150 & 140 & 130 & 120 & 110 & 100 & 90 & 80 & 70 & 60 & 50 & 40 & 30 & 20 & 10 & 0 & -10 & -20\end{array}$ 
${ }^{1} \mathrm{H}-\mathrm{NMR}$ spectrum of $\mathbf{1 d}$ in $\mathrm{CDCl}_{3}, 400 \mathrm{MHz}$

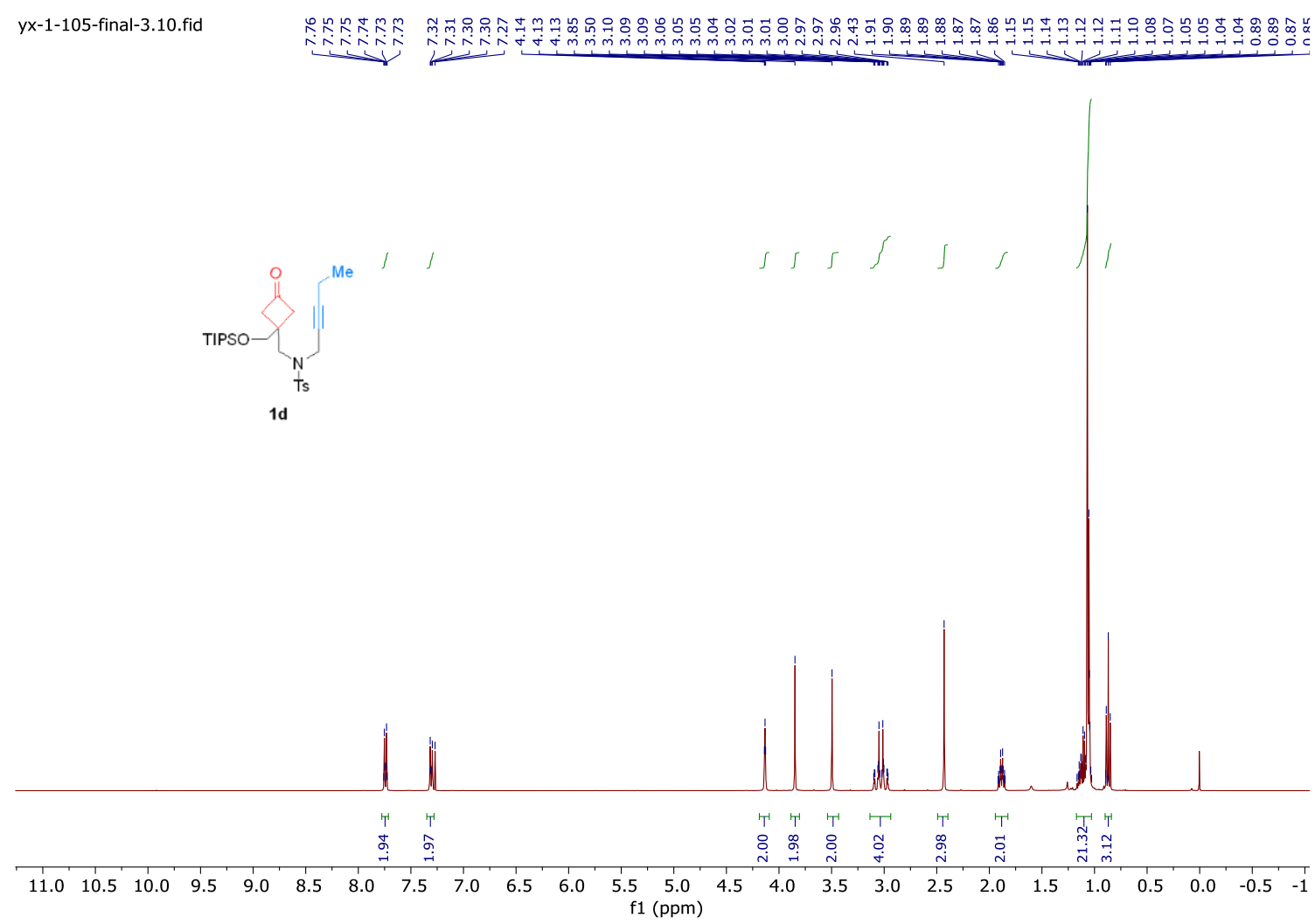

${ }^{13} \mathrm{C}-\mathrm{NMR}$ spectrum of $\mathbf{1 d}$ in $\mathrm{CDCl}_{3}, 101 \mathrm{MHz}$

yx-1-105fingl-C.10.fid

$\stackrel{\text { i }}{\text { I }}$

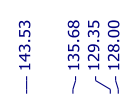

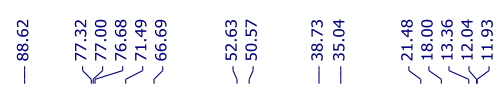
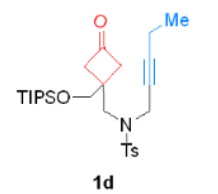

1

$\begin{array}{lllllllllllllll}220 & 210 & 200 & 190 & 180 & 170 & 160 & 150 & 140 & 130 & 120 & 110 & 100 & 90 \\ \mathrm{f} 1(\mathrm{ppm}) & & \end{array}$ 
${ }^{1} \mathrm{H}-\mathrm{NMR}$ spectrum of $1 \mathrm{e}$ in $\mathrm{CDCl}_{3}, 400 \mathrm{MHz}$

$z m x-23.30 . f i d$

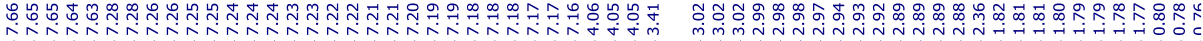
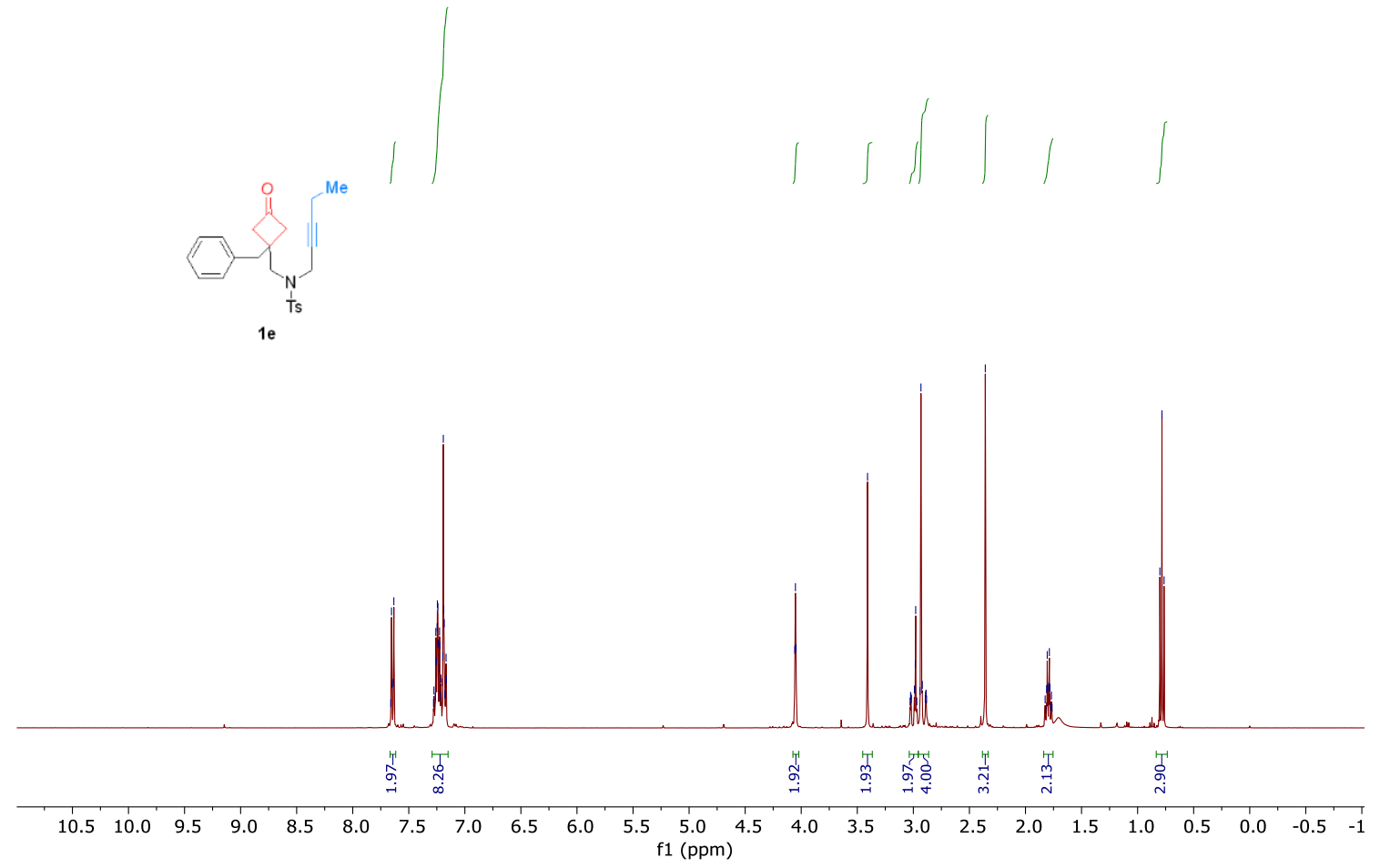

${ }^{13} \mathrm{C}-\mathrm{NMR}$ spectrum of $1 \mathrm{e}$ in $\mathrm{CDCl}_{3}, 101 \mathrm{MHz}$

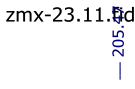

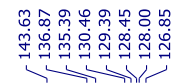

m.
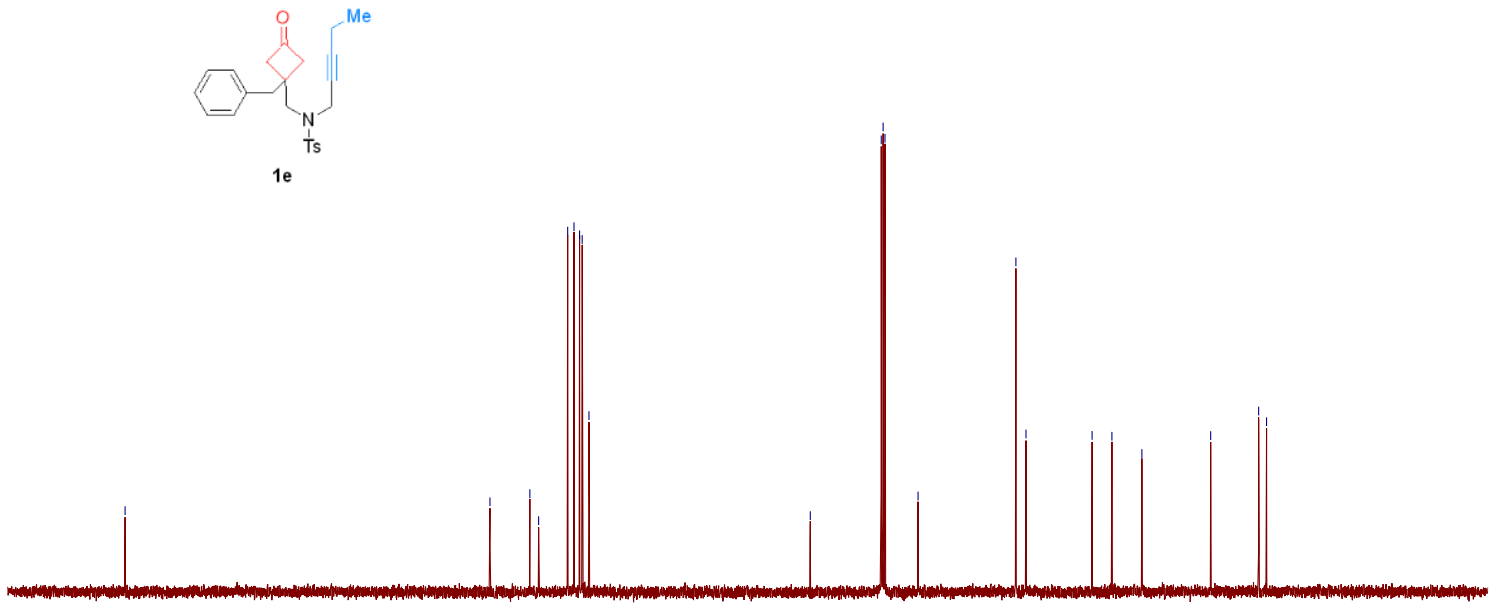

$\begin{array}{lllllllllllllllllllllllllllllllll}220 & 210 & 200 & 190 & 180 & 170 & 160 & 150 & 140 & 130 & 120 & 110 & 100 & 90 & 80 & 70 & 60 & 50 & 40 & 30 & 20 & 10 & 0 & -10 & -20\end{array}$ 

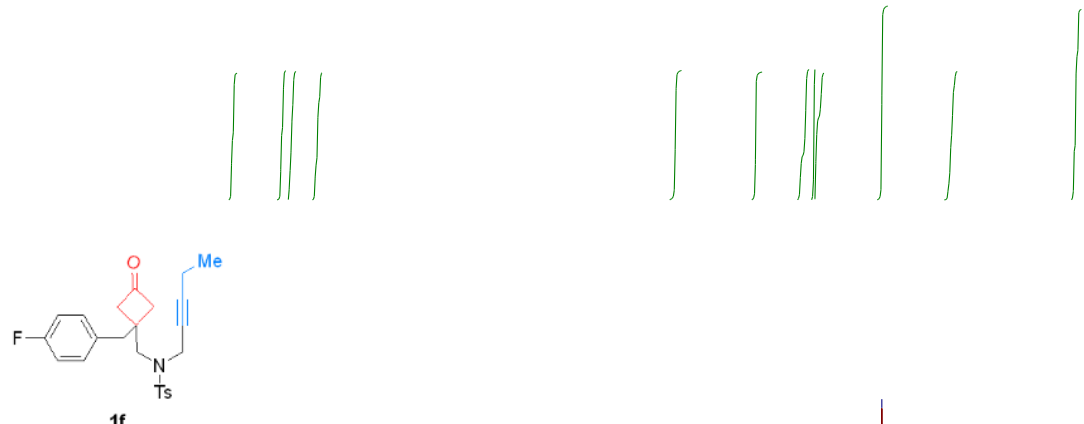

1f

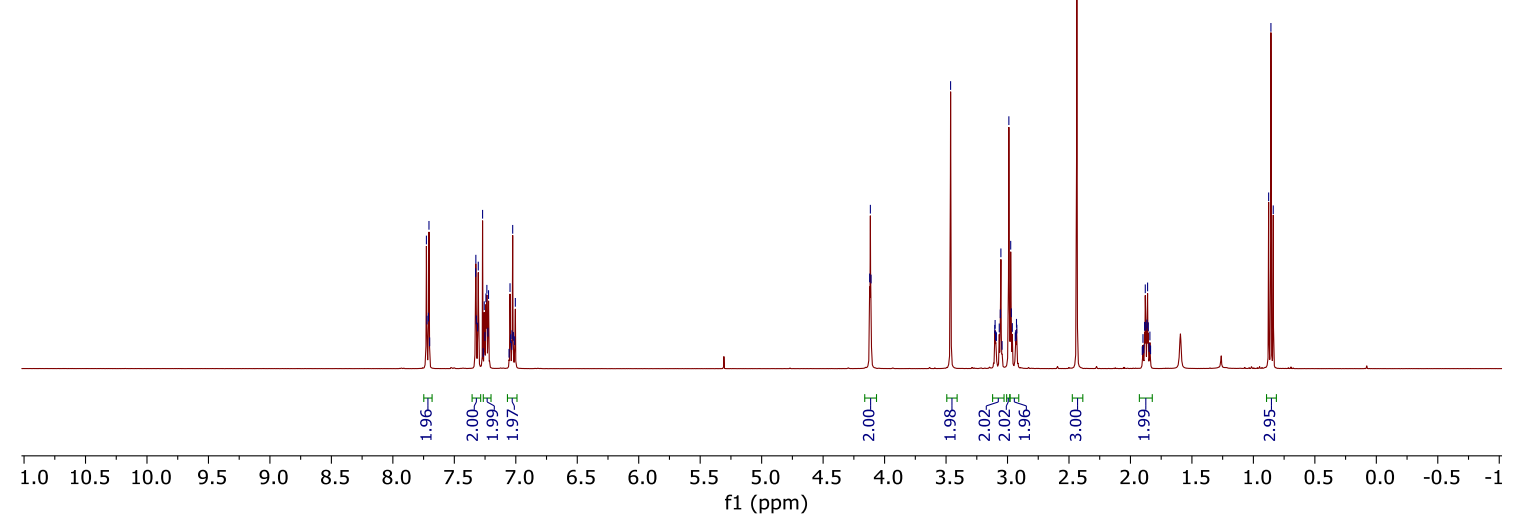

${ }^{13} \mathrm{C}-\mathrm{NMR}$ spectrum of $\mathbf{1 f}$ in $\mathrm{CDCl}_{3}, 101 \mathrm{MHz}$

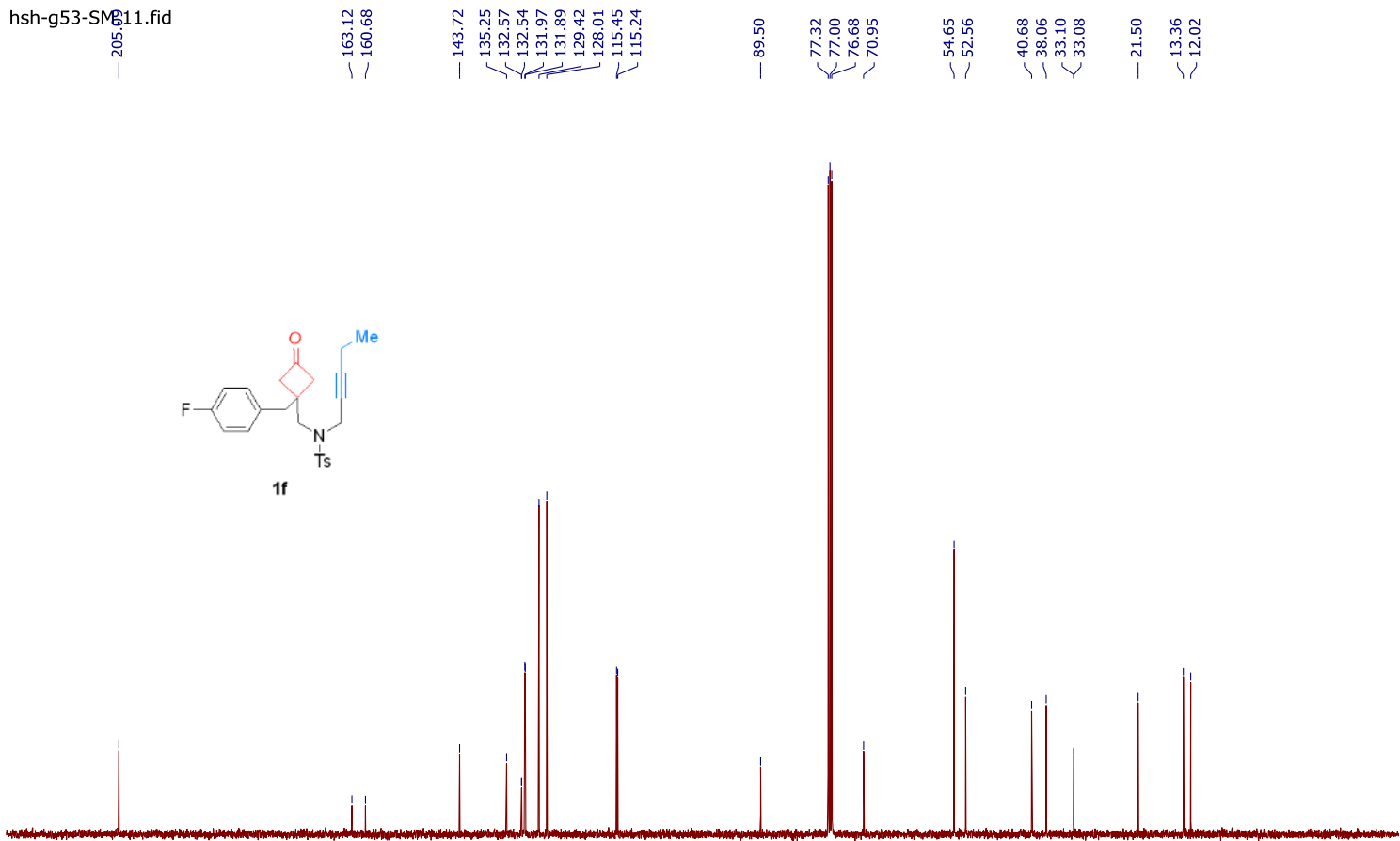

$\begin{array}{lllllllllllllllllllllllllllllll}220 & 210 & 200 & 190 & 180 & 170 & 160 & 150 & 140 & 130 & 120 & 110 & 100 & 90 & 80 & 70 & 60 & 50 & 40 & 30 & 20 & 10 & 0 & -10 & -20\end{array}$ 
${ }^{1} \mathrm{H}-\mathrm{NMR}$ spectrum of $1 \mathrm{~g}$ in $\mathrm{CDCl}_{3}, 400 \mathrm{MHz}$

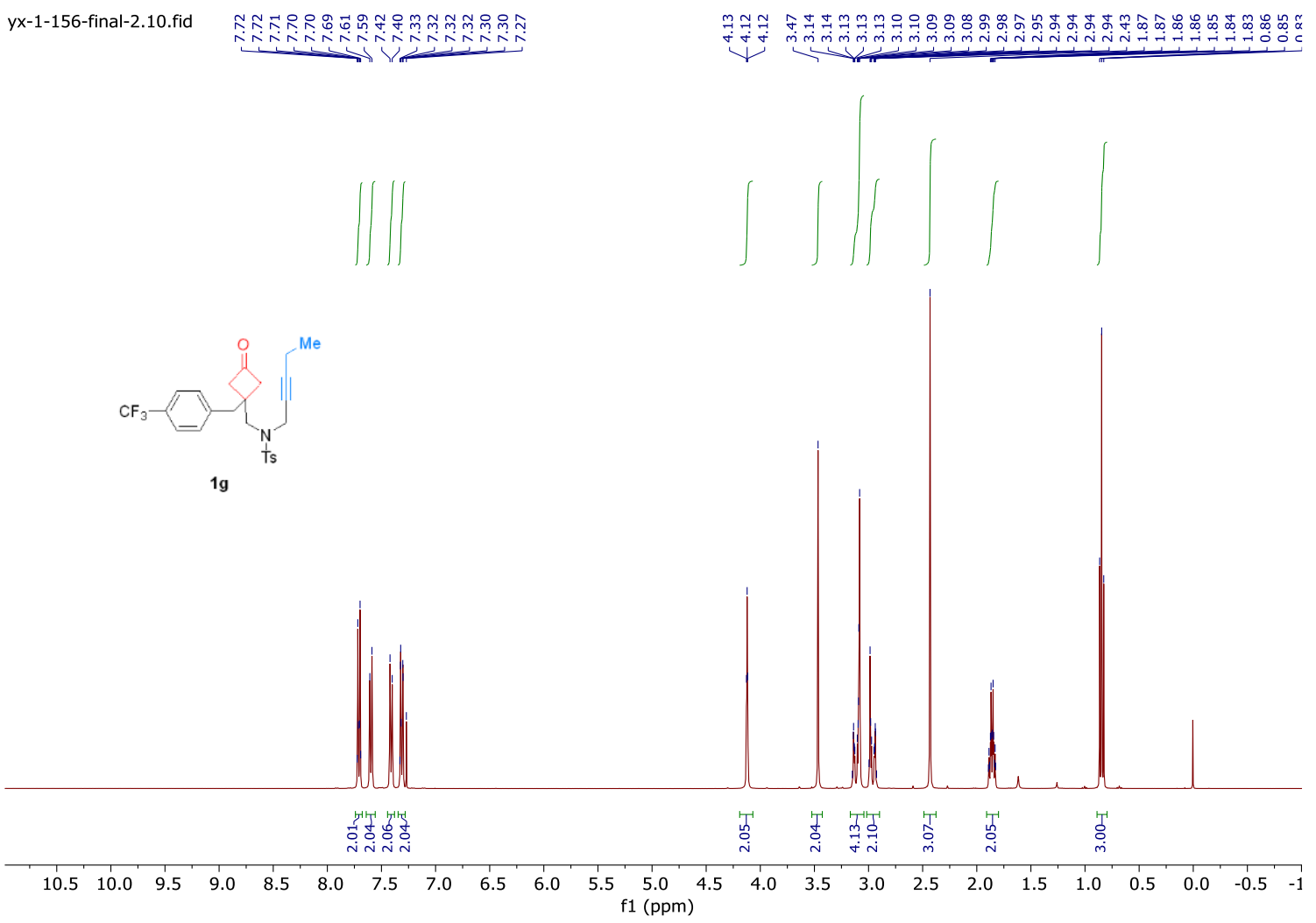

${ }^{19} \mathrm{~F}-\mathrm{NMR}$ spectrum of $\mathbf{1 g}$ in $\mathrm{CDCl}_{3}, 376 \mathrm{MHz}$

yx-1-156final-C.11.fid

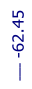

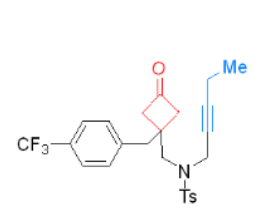

$1 \mathrm{~g}$

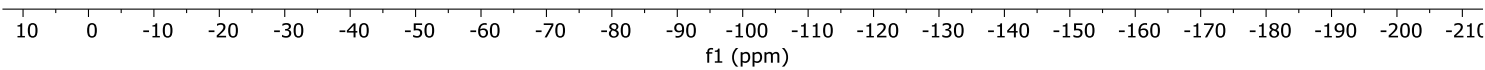


${ }^{13} \mathrm{C}-\mathrm{NMR}$ spectrum of $\mathbf{1 g}$ in $\mathrm{CDCl}_{3}, 101 \mathrm{MHz}$

yx-1-156finक्षै-C.12.fid

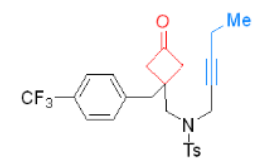

19
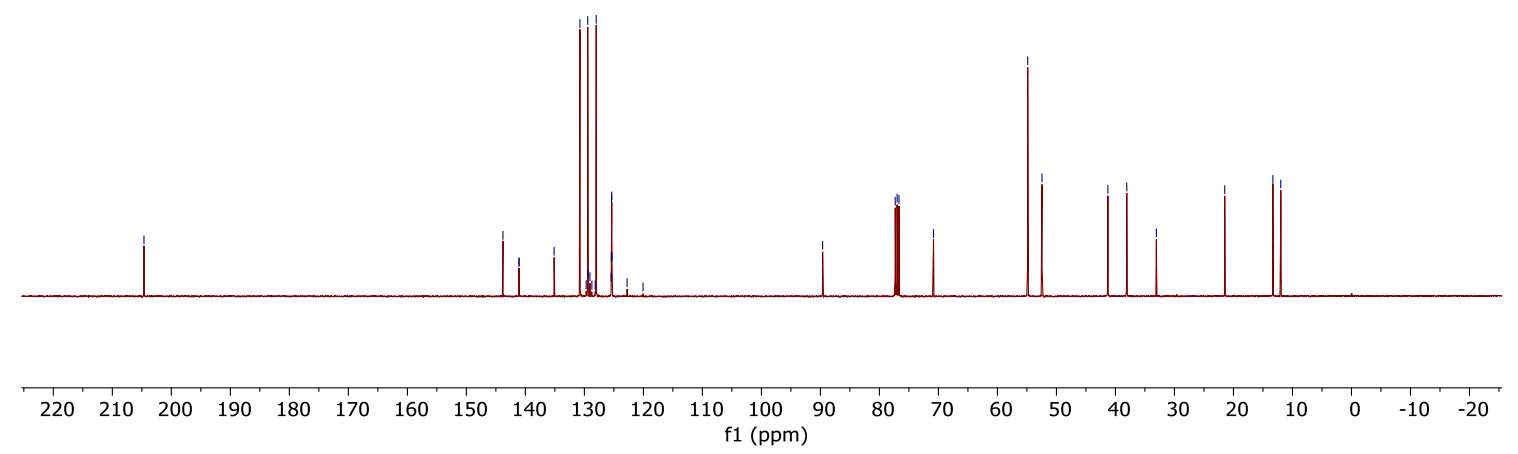

${ }^{13} \mathrm{C}$-NMR spectrum of $\mathbf{1 g}$ in $\mathrm{CDCl}_{3}, 101 \mathrm{MHz}$

yx-1-156final-c.12.fid

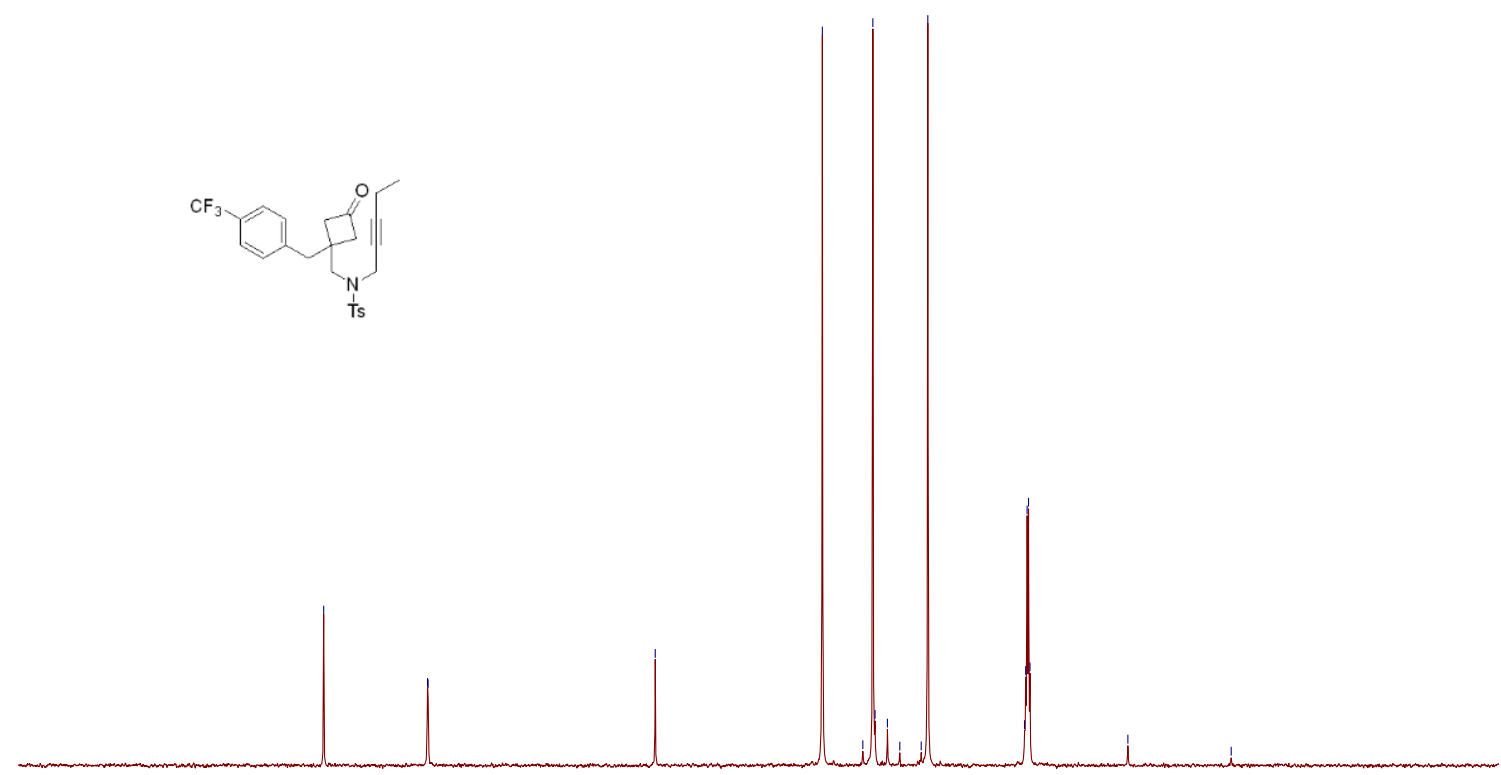

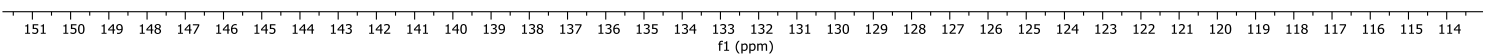


${ }^{1} \mathrm{H}-\mathrm{NMR}$ spectrum of $\mathbf{1 h}$ in $\mathrm{CDCl}_{3}, 400 \mathrm{MHz}$

yx-1-110-final.10.fid

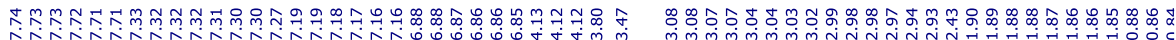

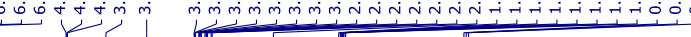
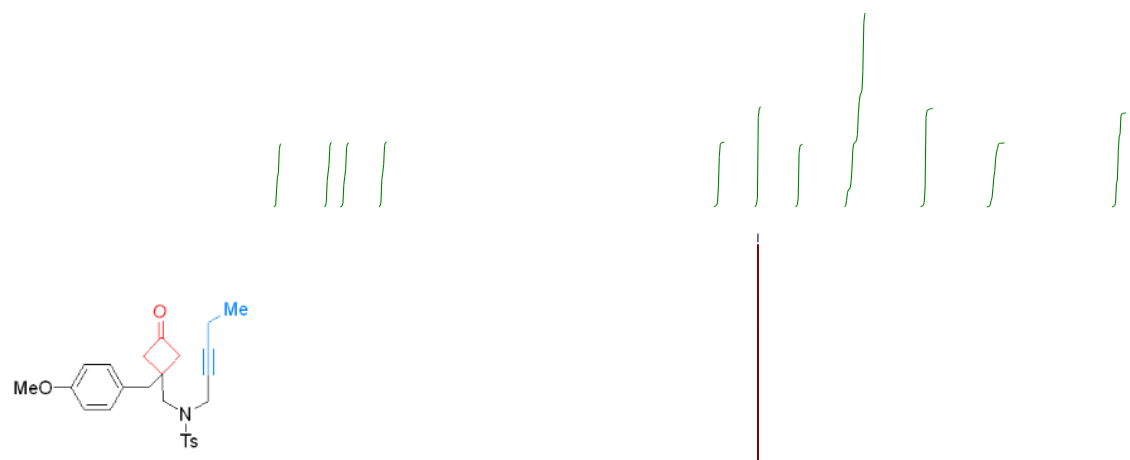

1h

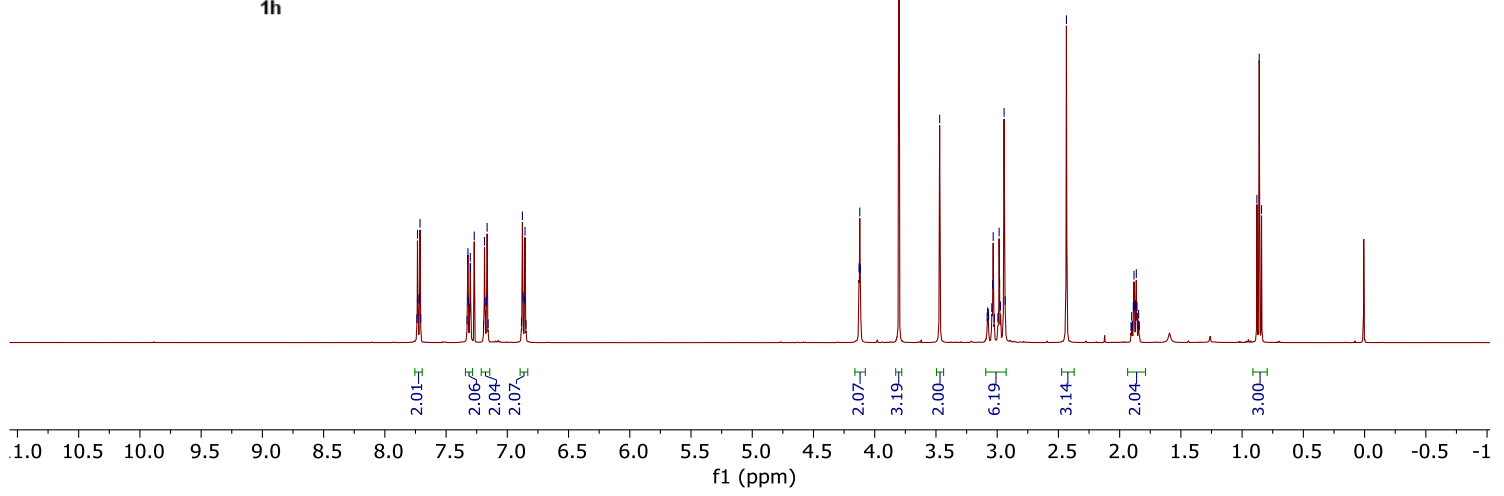

${ }^{13} \mathrm{C}-\mathrm{NMR}$ spectrum of $\mathbf{1 h}$ in $\mathrm{CDCl}_{3}, 101 \mathrm{MHz}$

yx-1-110final-C.10.fid

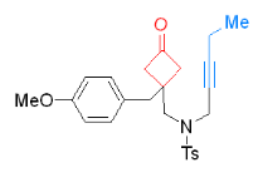

1h

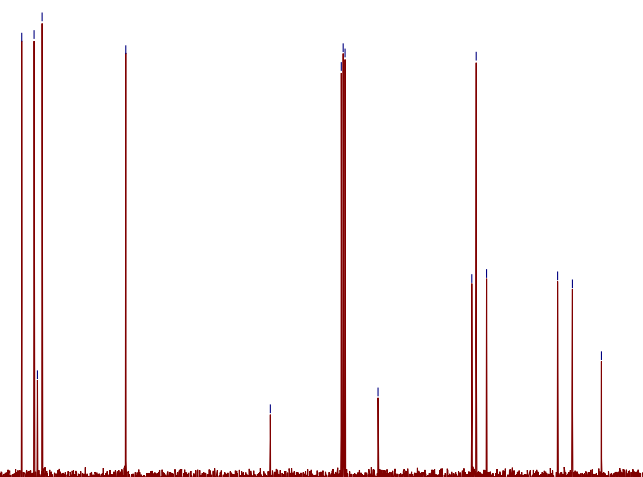


${ }^{1} \mathrm{H}-\mathrm{NMR}$ spectrum of $1 \mathrm{i}$ in $\mathrm{CDCl}_{3}, 400 \mathrm{MHz}$

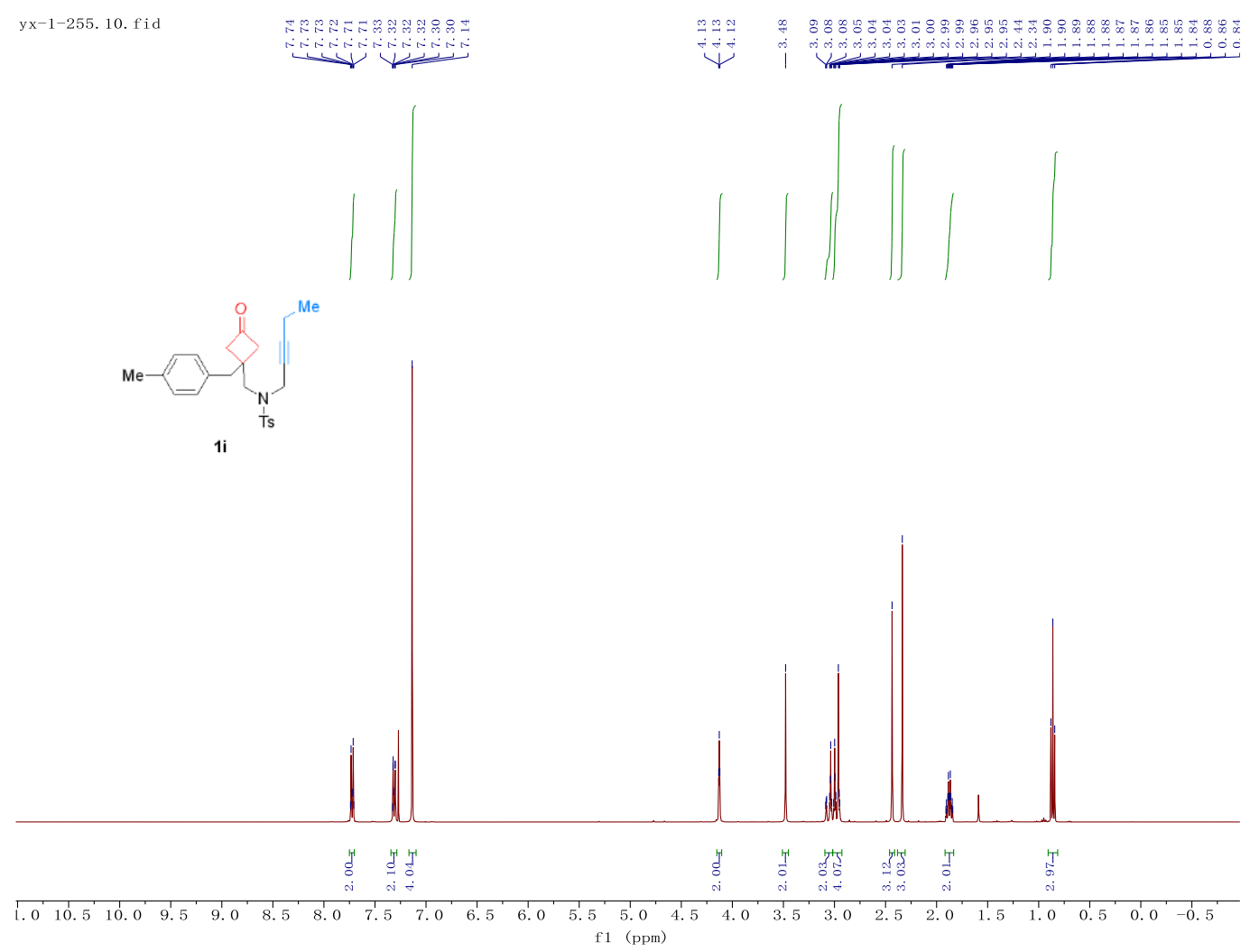

${ }^{13} \mathrm{C}-\mathrm{NMR}$ spectrum of $\mathbf{1 i}$ in $\mathrm{CDCl}_{3}, 101 \mathrm{MHz}$

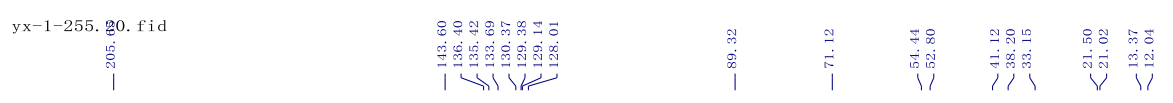

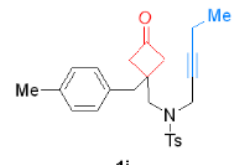

$1 \mathrm{i}$

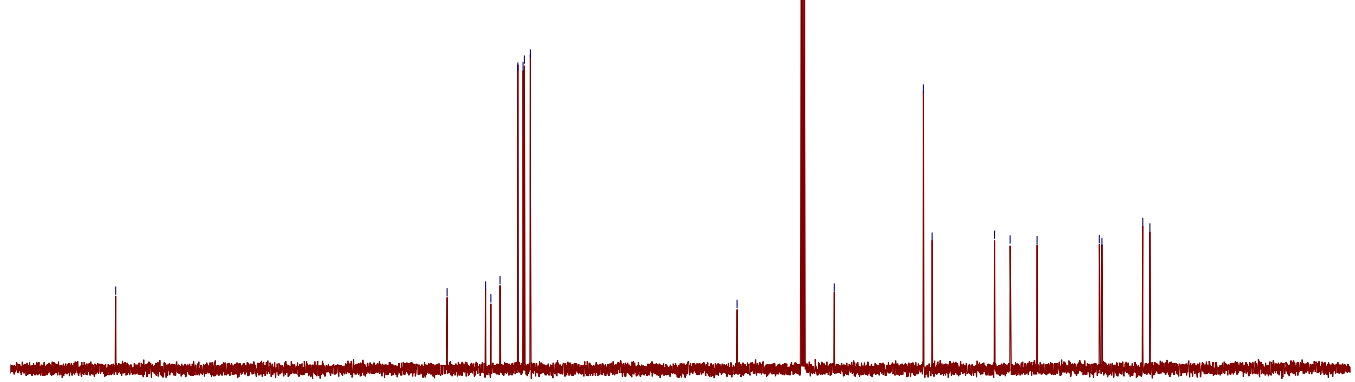

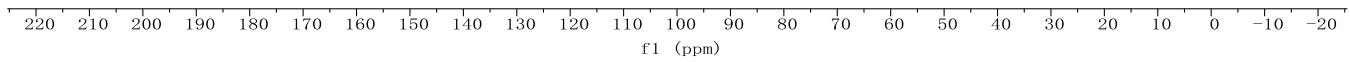


${ }^{1} \mathrm{H}-\mathrm{NMR}$ spectrum of $\mathbf{1 j}$ in $\mathrm{CDCl}_{3}, 400 \mathrm{MHz}$

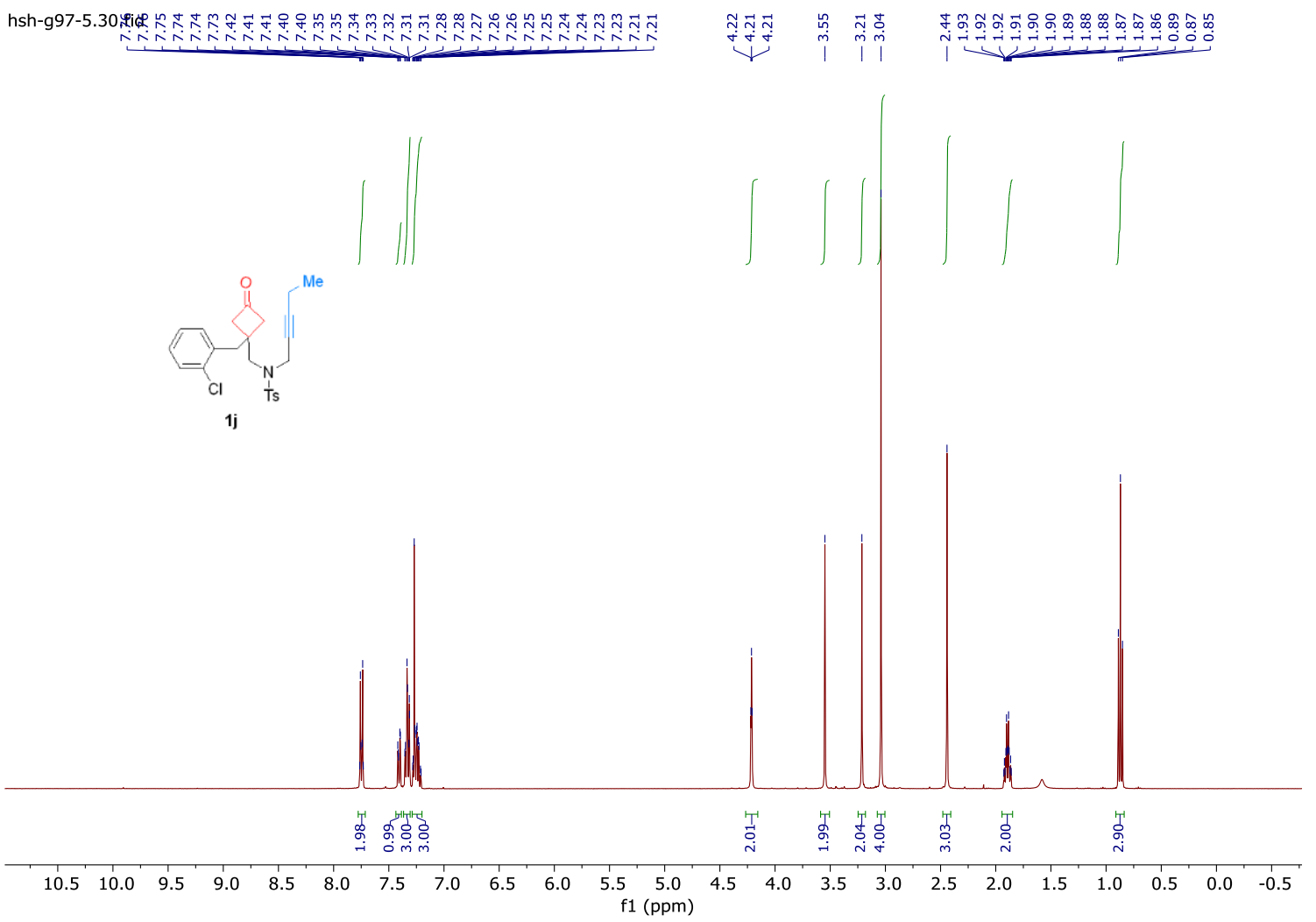

${ }^{13} \mathrm{C}-\mathrm{NMR}$ spectrum of $1 \mathrm{j}$ in $\mathrm{CDCl}_{3}, 101 \mathrm{MHz}$

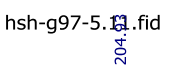

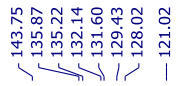

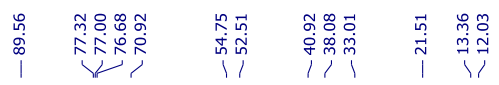

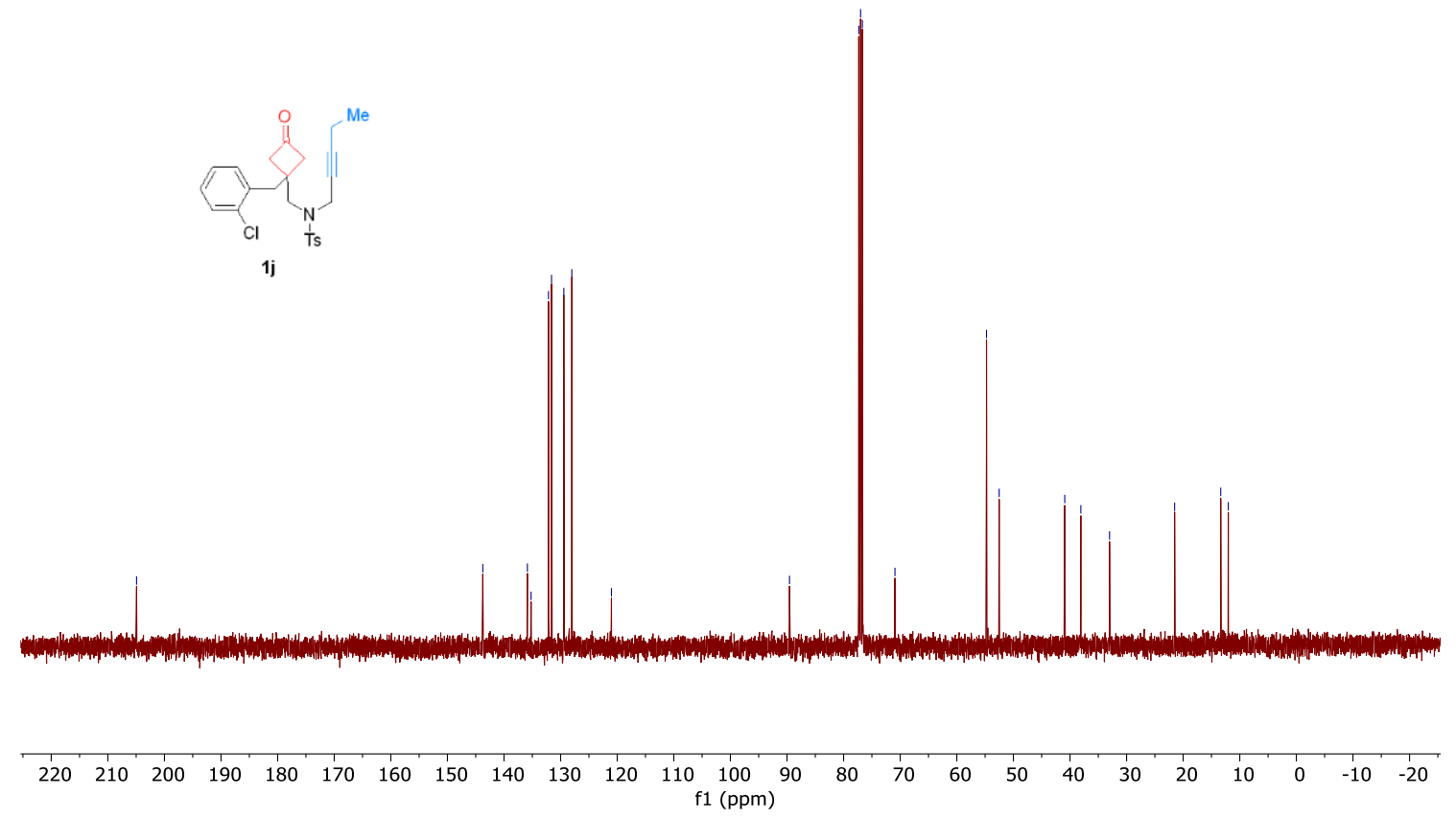




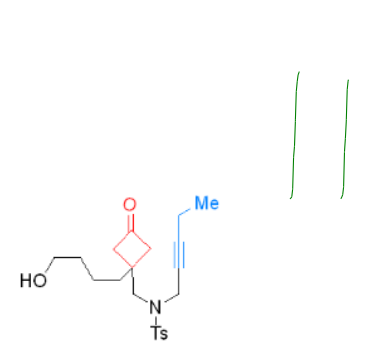

1k
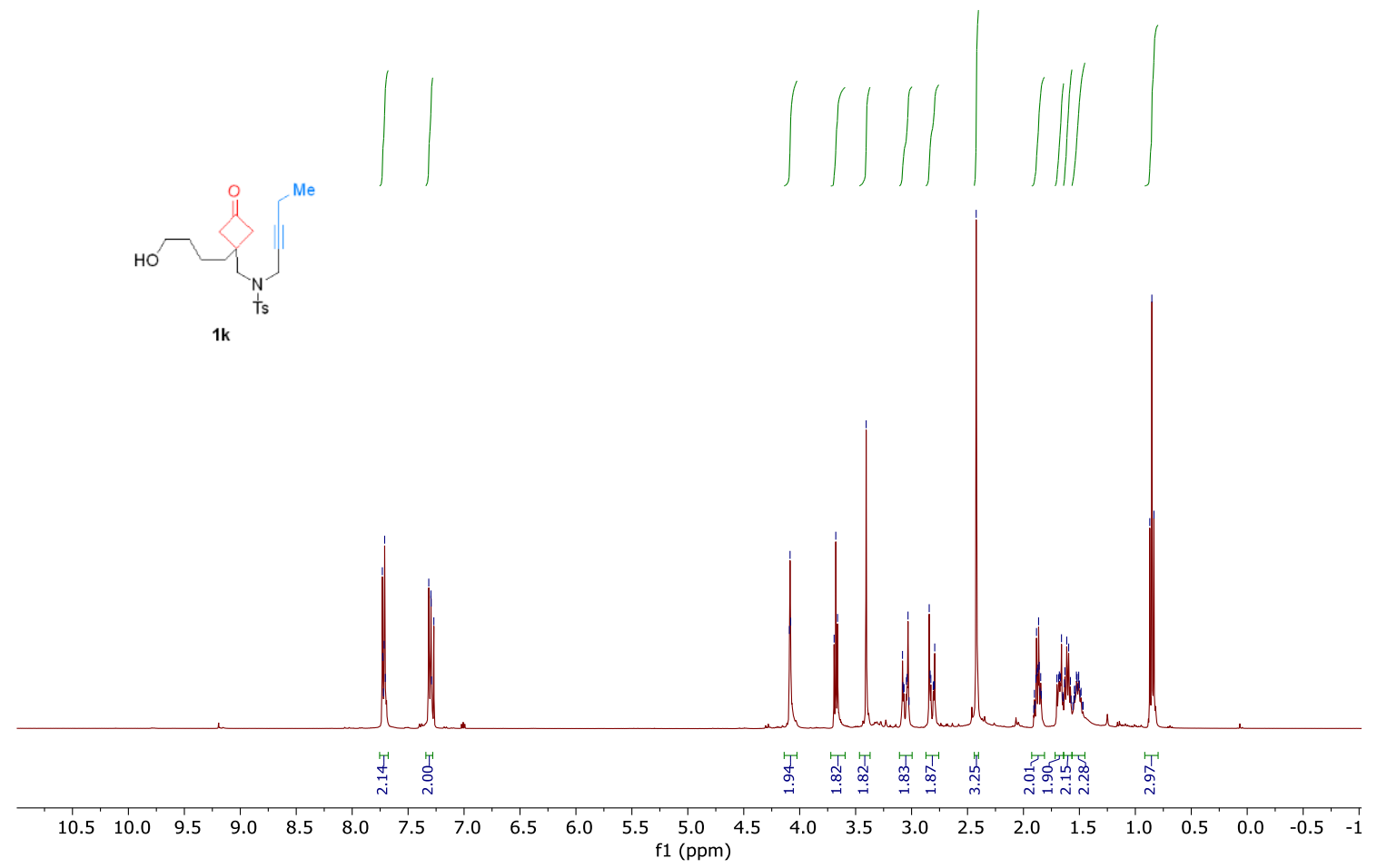

${ }^{13} \mathrm{C}-\mathrm{NMR}$ spectrum of $\mathbf{1} \mathbf{k}$ in $\mathrm{CDCl}_{3}, 101 \mathrm{MHz}$

hsh-g144-2क्षेSM.11.fid

$\stackrel{\text { ¿ }}{\text { i }}$

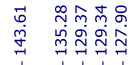

| m.

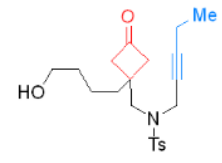

1k

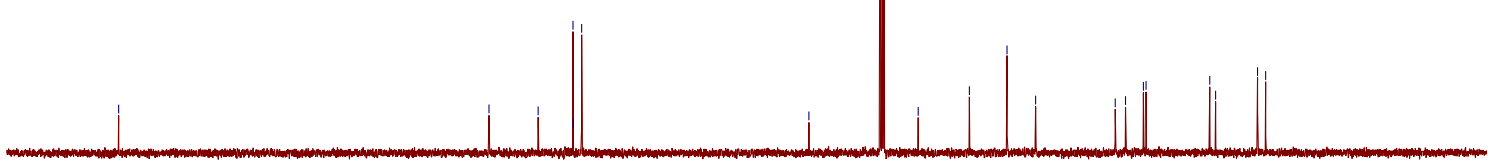

$\begin{array}{llllllllllllllllllllllllllllll}220 & 210 & 200 & 190 & 180 & 170 & 160 & 150 & 140 & 130 & 120 & 110 & 100 & 90 & 80 & 70 & 60 & 50 & 40 & 30 & 20 & 10 & 0 & -10 & -20\end{array}$ 
${ }^{1} \mathrm{H}-\mathrm{NMR}$ spectrum of $1 \mathrm{l}$ in $\mathrm{CDCl}_{3}, 400 \mathrm{MHz}$

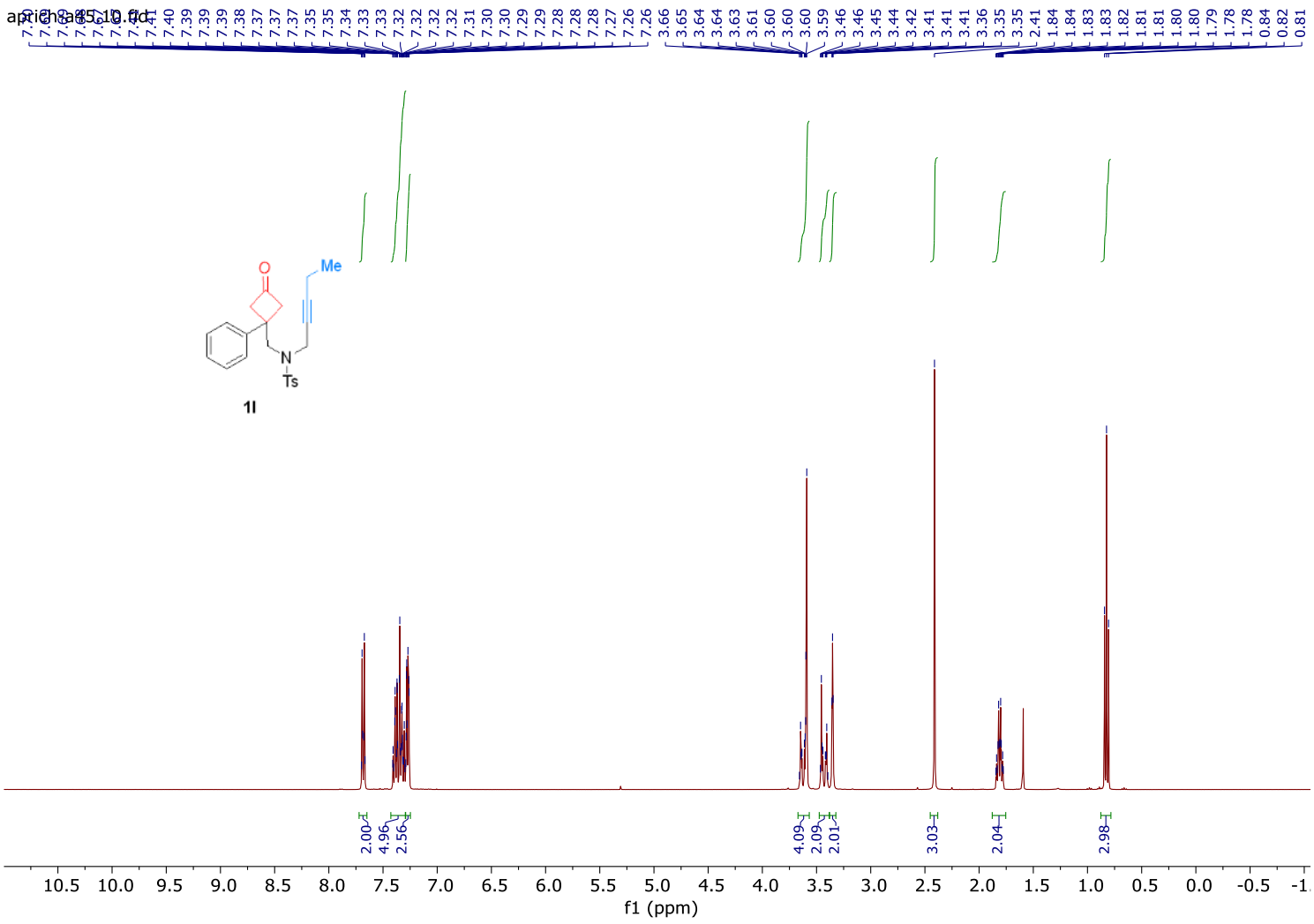

${ }^{13} \mathrm{C}-\mathrm{NMR}$ spectrum of $\mathbf{1 l}$ in $\mathrm{CDCl}_{3}, 101 \mathrm{MHz}$

aprich-a45.\$1.fid

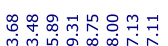

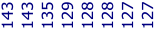

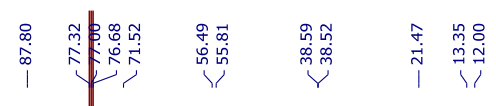

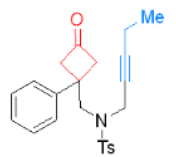

11

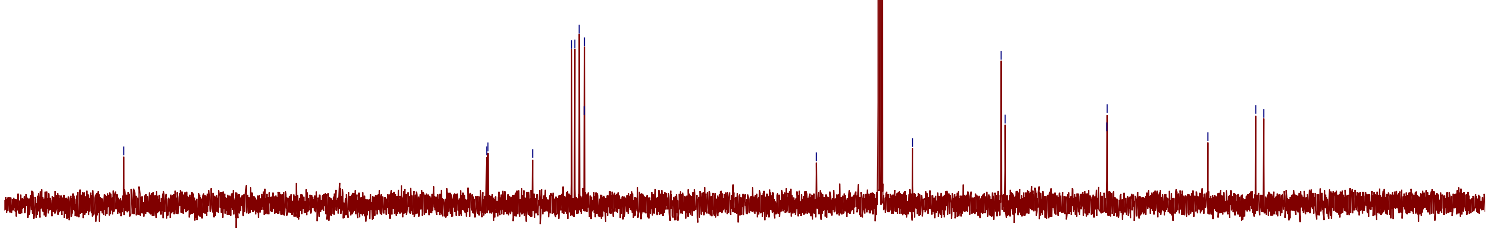

$\begin{array}{llllllllllllllllllllllllllllllllll}220 & 210 & 200 & 190 & 180 & 170 & 160 & 150 & 140 & 130 & 120 & 110 & 100 & 90 & 80 & 70 & 60 & 50 & 40 & 30 & 20 & 10 & 0 & -10 & -20\end{array}$ 
${ }^{1} \mathrm{H}$-NMR spectrum of $1 \mathrm{~m}$ in $\mathrm{CDCl}_{3}, 400 \mathrm{MHz}$

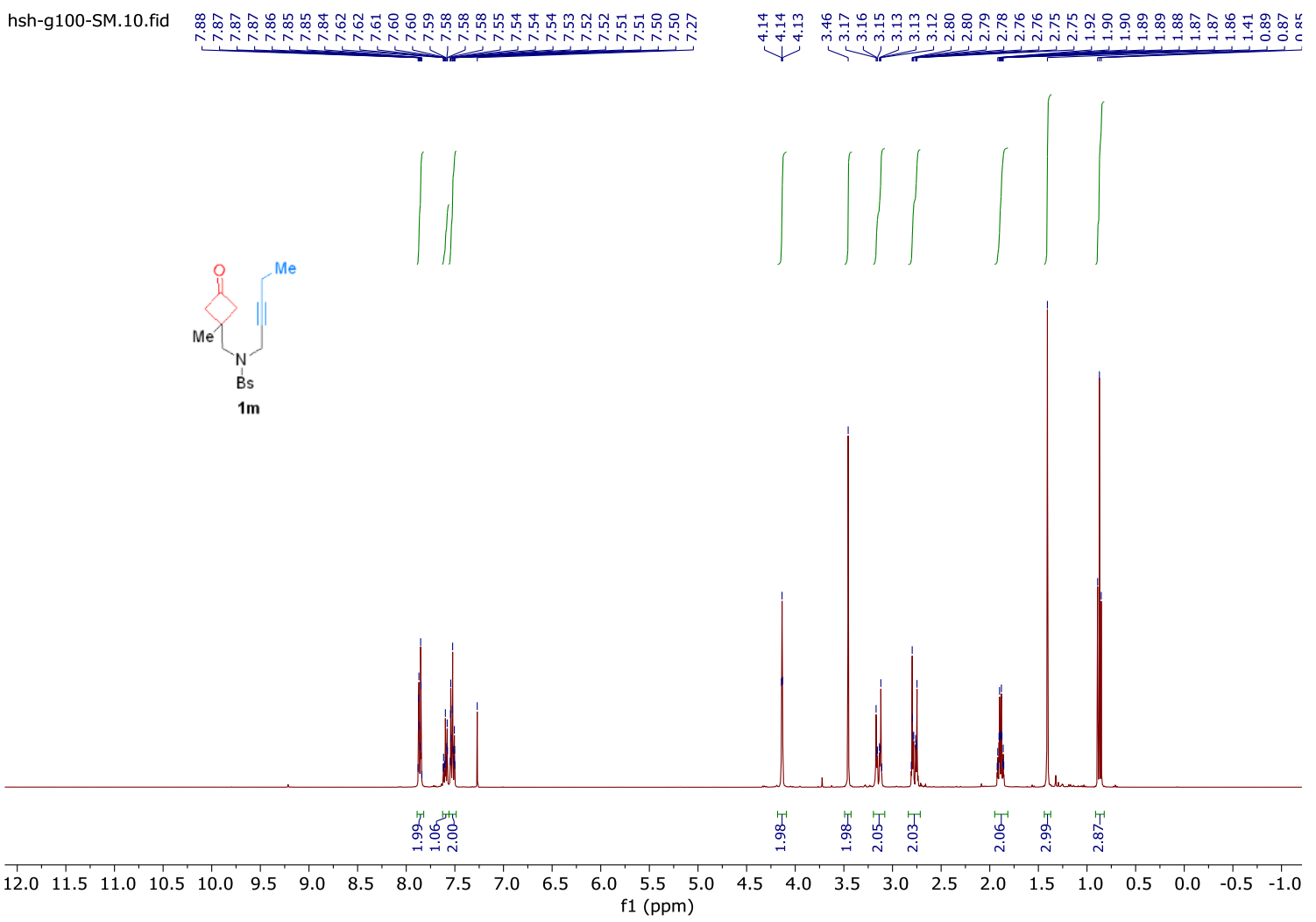

${ }^{13} \mathrm{C}-\mathrm{NMR}$ spectrum of $1 \mathrm{~m}$ in $\mathrm{CDCl}_{3}, 101 \mathrm{MHz}$

hsh-g100-\$M.11.fid

ڤั̀

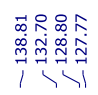

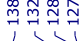

1

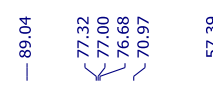

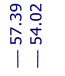

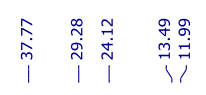

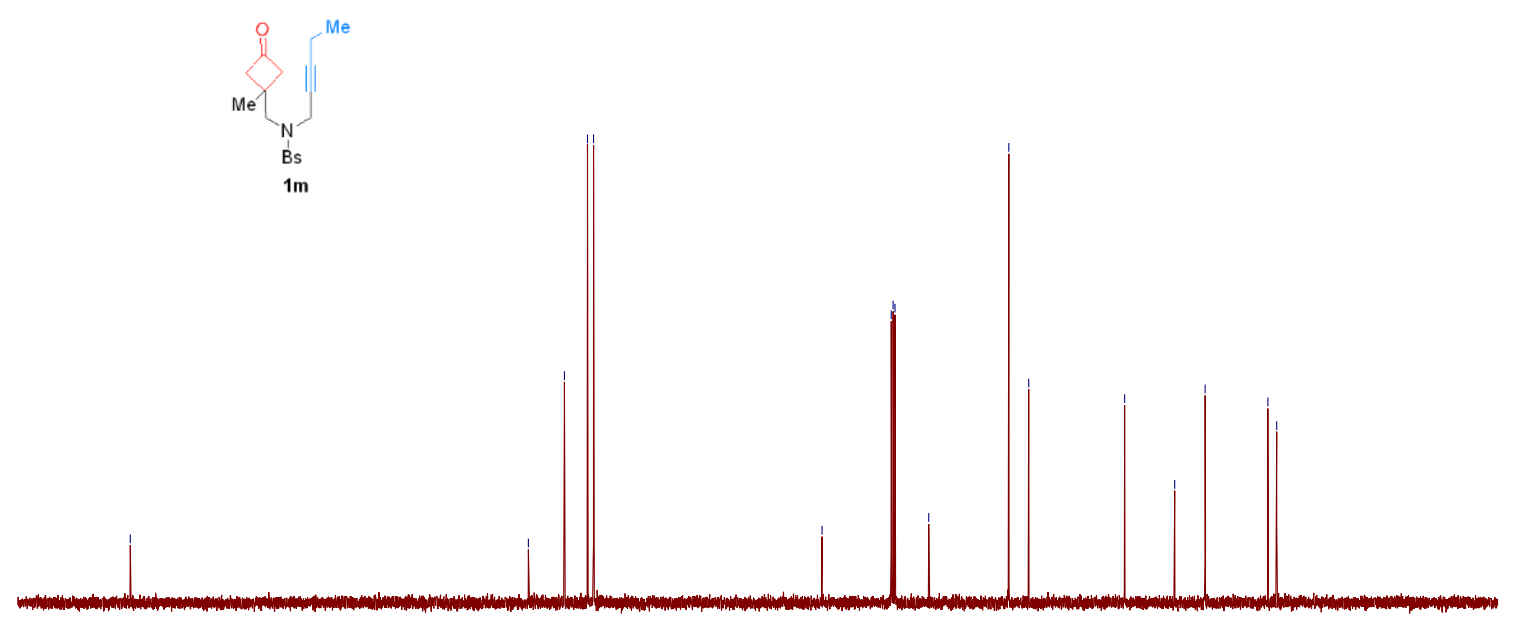

$\begin{array}{lllllllllllllllllllllllllllllllllllll}220 & 210 & 200 & 190 & 180 & 170 & 160 & 150 & 140 & 130 & 120 & 110 & 100 & 90 & 80 & 70 & 60 & 50 & 40 & 30 & 20 & 10 & 0 & -10 & -20\end{array}$ 
${ }^{1} \mathrm{H}$-NMR spectrum of $1 \mathrm{n}$ in $\mathrm{CDCl}_{3}, 400 \mathrm{MHz}$

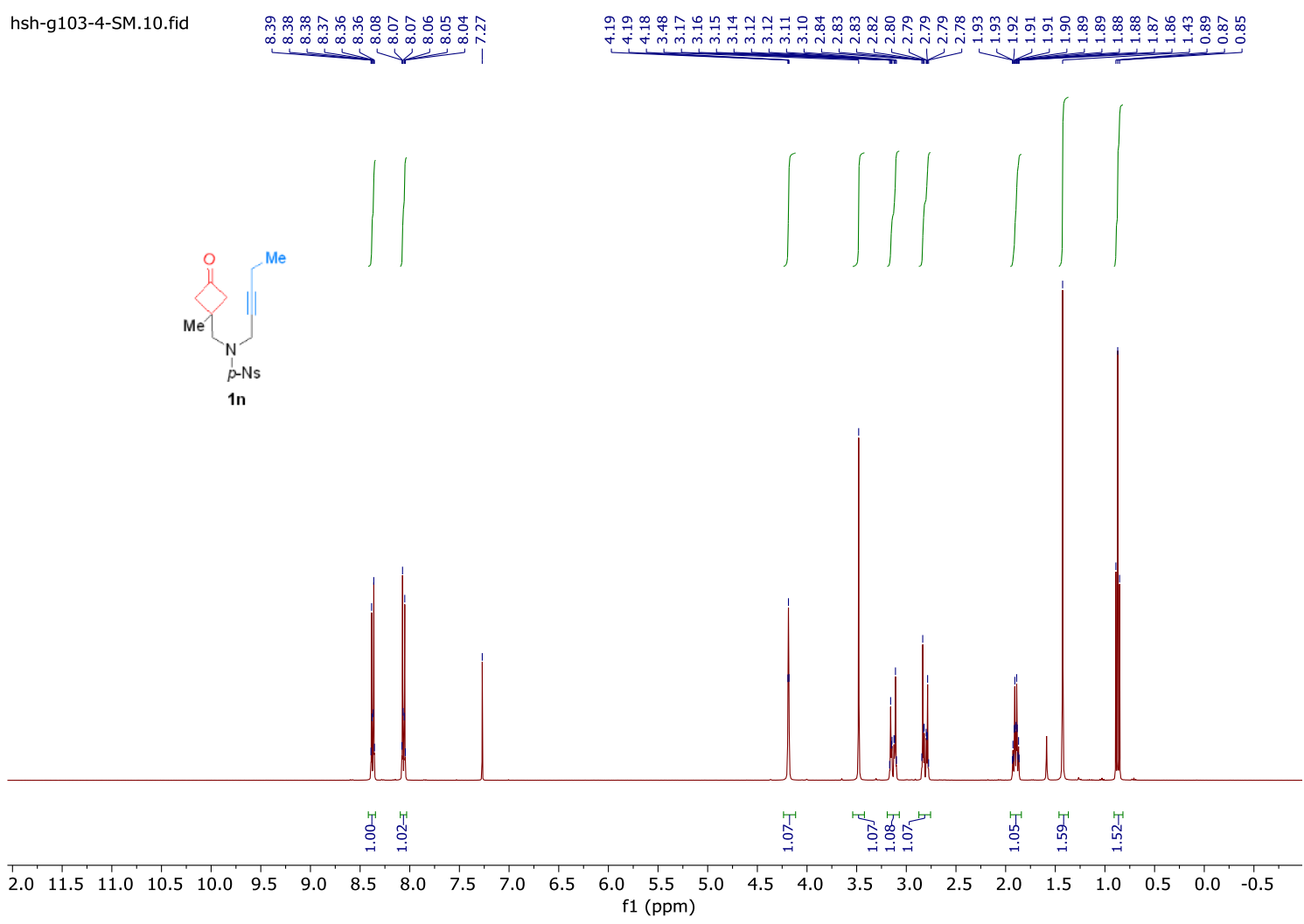

${ }^{13} \mathrm{C}$-NMR spectrum of $\mathbf{1} \mathbf{n}$ in $\mathrm{CDCl}_{3}, 101 \mathrm{MHz}$

hsh-g103-4 TSM.11.fid

iั

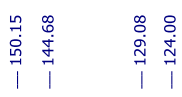

芯

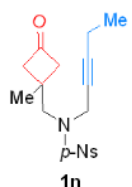

1n

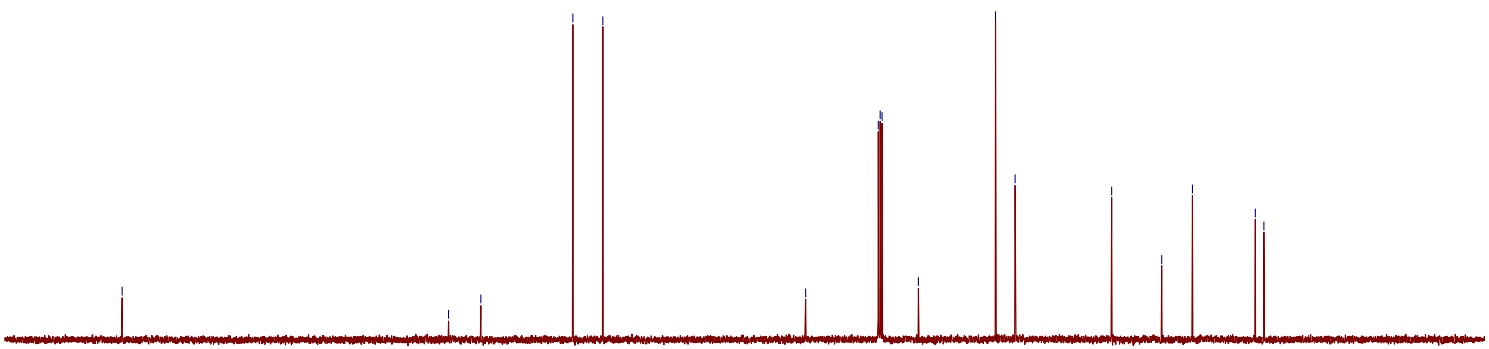

$\begin{array}{llllllllllllllllllllllllllllll}220 & 210 & 200 & 190 & 180 & 170 & 160 & 150 & 140 & 130 & 120 & 110 & 100 & 90 & 80 & 70 & 60 & 50 & 40 & 30 & 20 & 10 & 0 & -10 & -20\end{array}$ 
${ }^{1} \mathrm{H}-\mathrm{NMR}$ spectrum of 10 in $\mathrm{CDCl}_{3}, 400 \mathrm{MHz}$

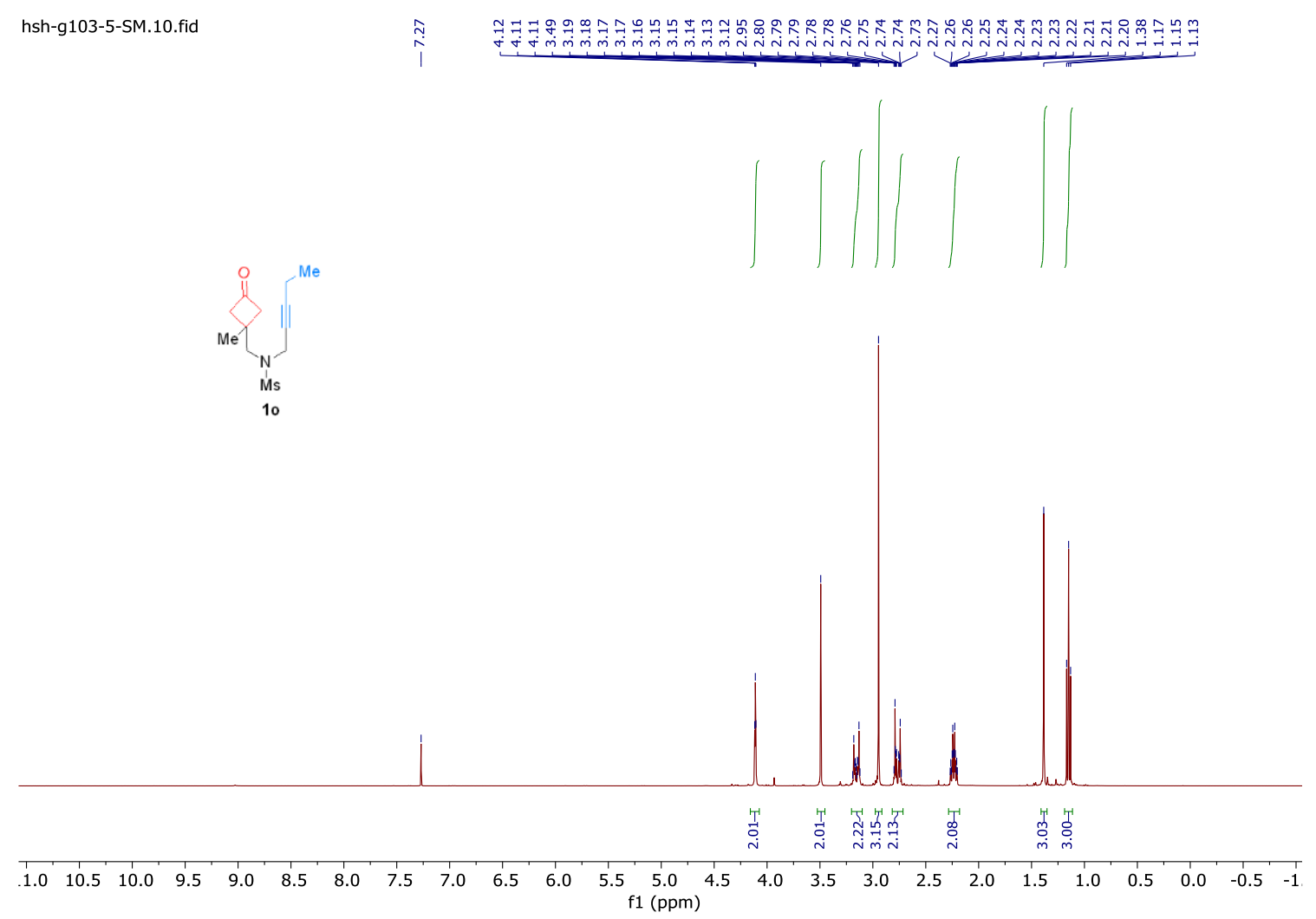

${ }^{13} \mathrm{C}$-NMR spectrum of 10 in $\mathrm{CDCl}_{3}, 101 \mathrm{MHz}$

hsh-g103-55SM.11.fid

ڤั่
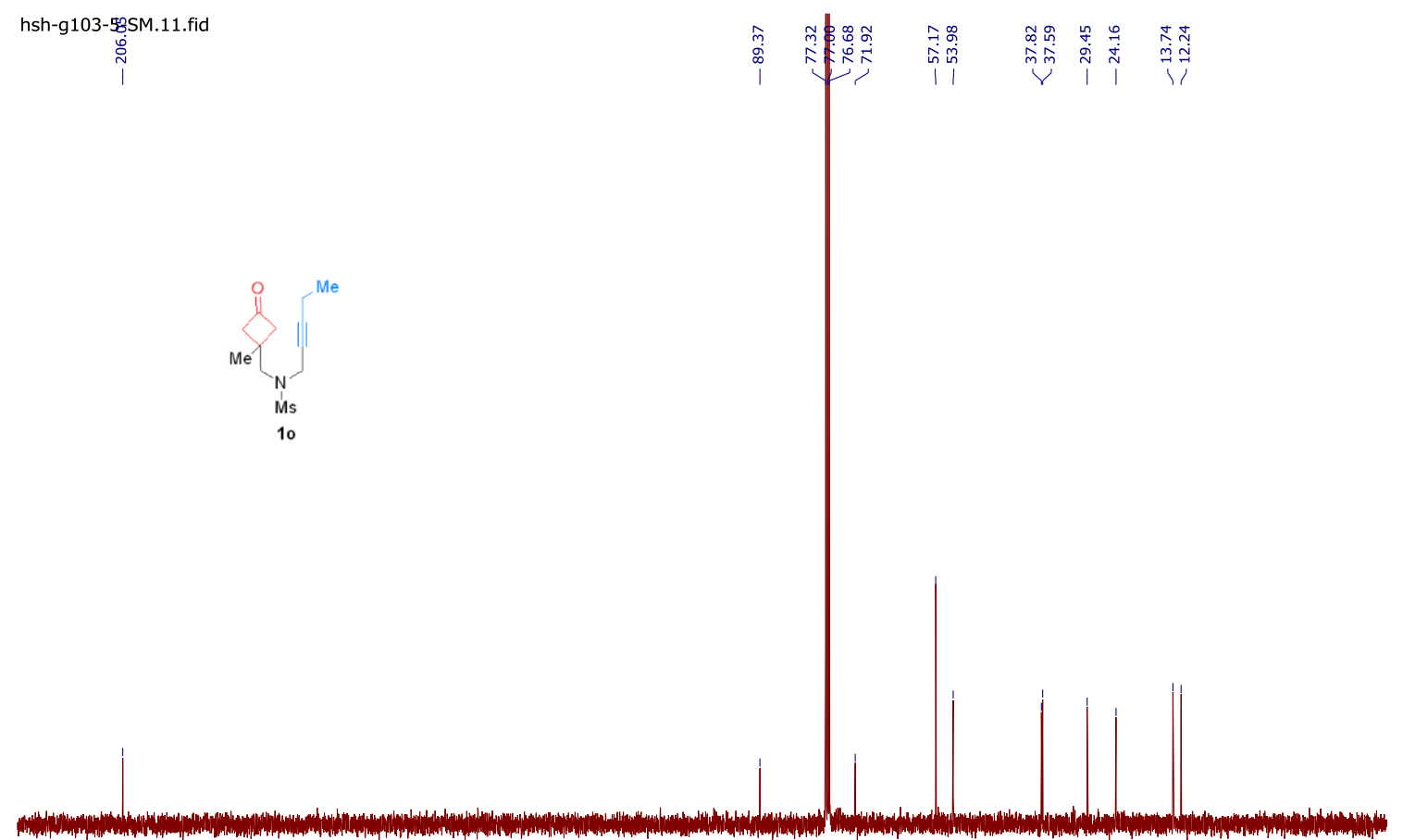

$\begin{array}{lllllllllllllllllllllllllllllllllllll}220 & 210 & 200 & 190 & 180 & 170 & 160 & 150 & 140 & 130 & 120 & 110 & 100 & 90 & 80 & 70 & 60 & 50 & 40 & 30 & 20 & 10 & 0 & -10 & -20\end{array}$ 
${ }^{1} \mathrm{H}-\mathrm{NMR}$ spectrum of $1 \mathrm{p}$ in $\mathrm{CDCl}_{3}, 400 \mathrm{MHz}$

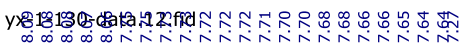

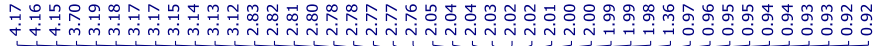
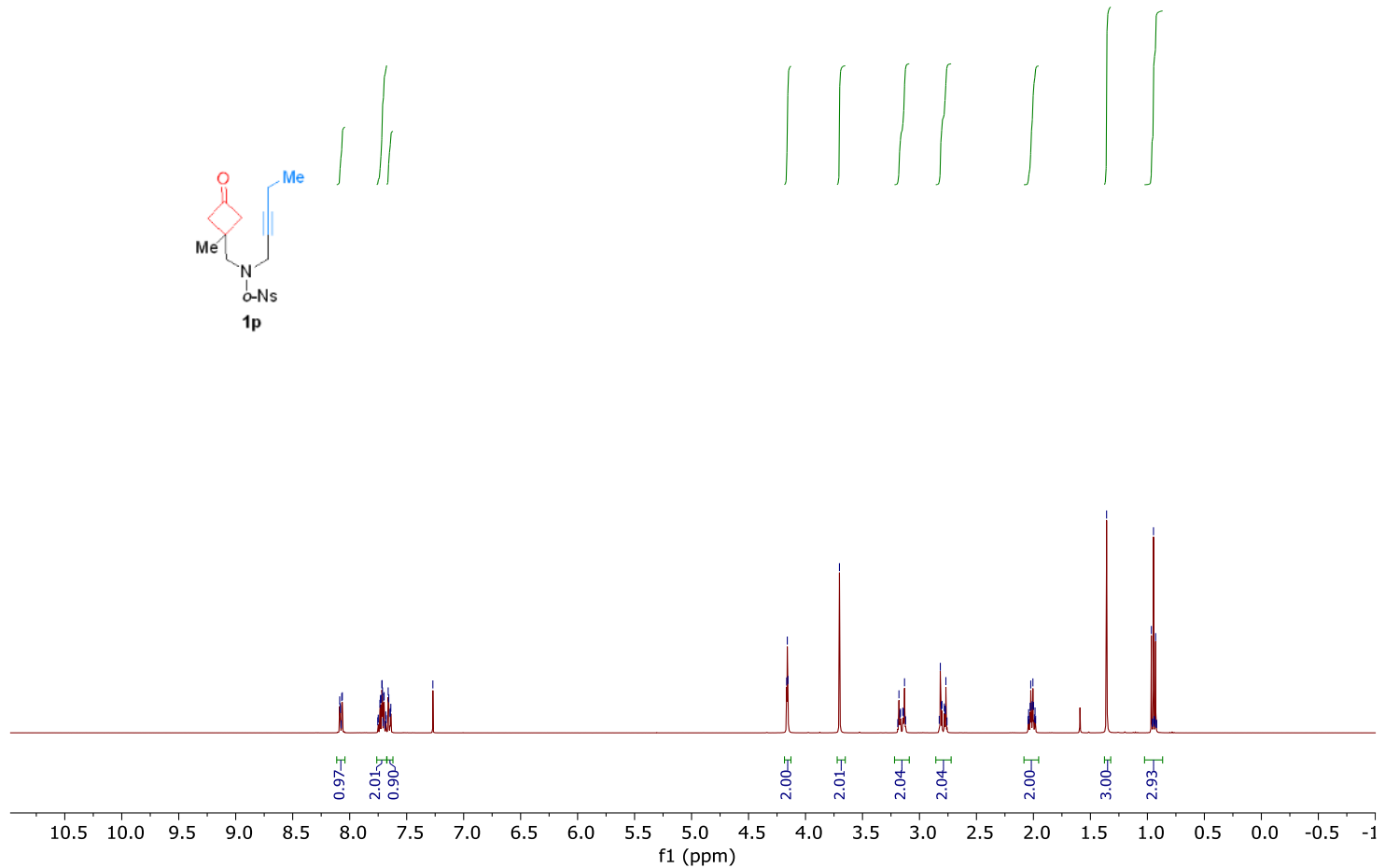

${ }^{13} \mathrm{C}-\mathrm{NMR}$ spectrum of $1 \mathrm{p}$ in $\mathrm{CDCl}_{3}, 101 \mathrm{MHz}$

yx-1-130-data.11.fid i

m

它

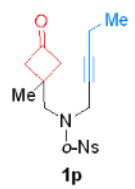

$1 \mathrm{p}$

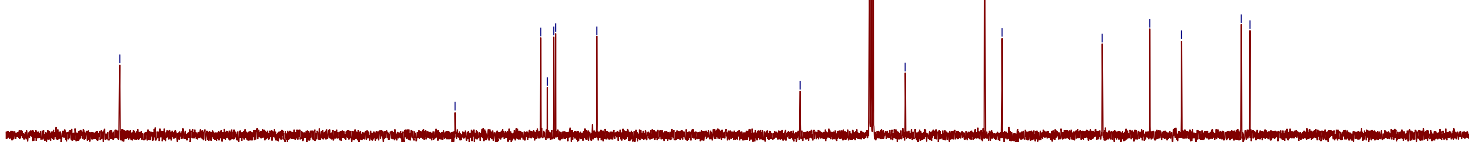

$\begin{array}{lllllllllllllllllllllllll}220 & 210 & 200 & 190 & 180 & 170 & 160 & 150 & 140 & 130 & 120 & 110 & 100 & 90 & 80 & 70 & 60 & 50 & 40 & 30 & 20 & 10 & 0 & -10 & -20\end{array}$ 
${ }^{1} \mathrm{H}-\mathrm{NMR}$ spectrum of $1 \mathrm{q}$ in $\mathrm{CDCl}_{3}, 400 \mathrm{MHz}$

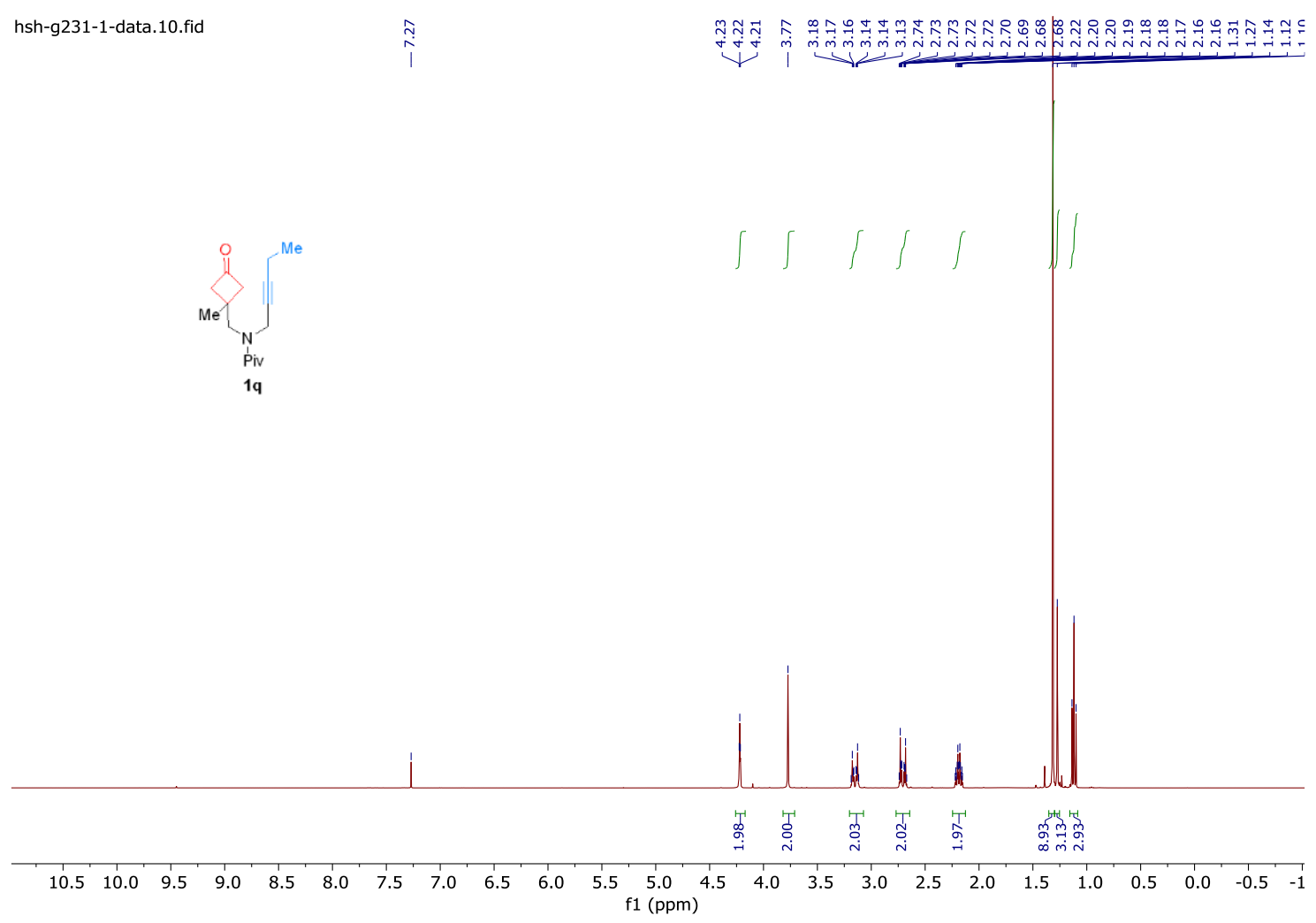

${ }^{13} \mathrm{C}-\mathrm{NMR}$ spectrum of $\mathbf{1 q}$ in $\mathrm{CDCl}_{3}, 101 \mathrm{MHz}$
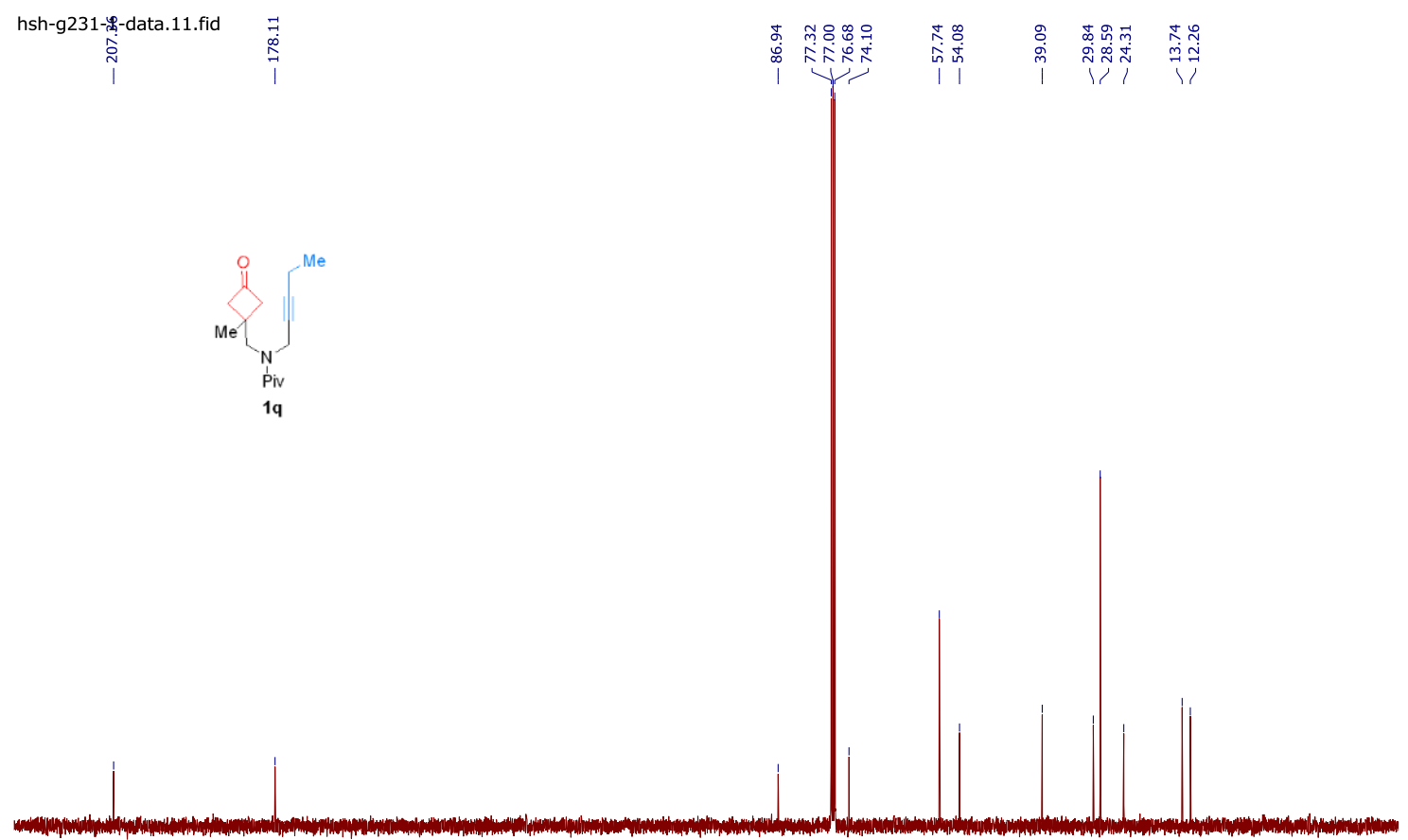

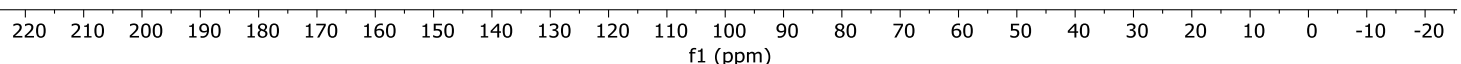


hsh-g193-3-SM.10.fid

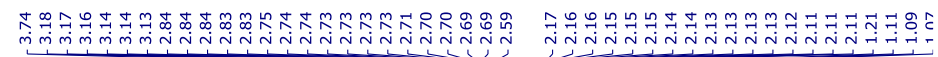
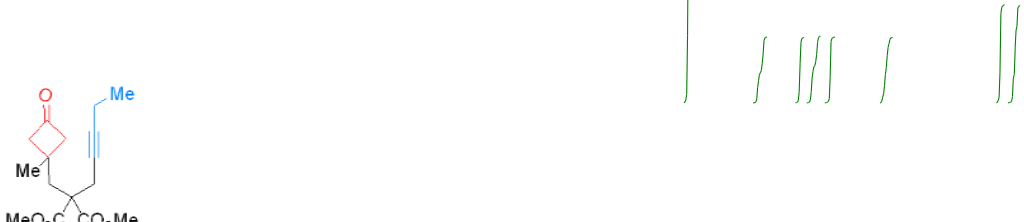

$\mathrm{MeO}_{2} \mathrm{C} \mathrm{CO}_{2} \mathrm{Me}$

1r

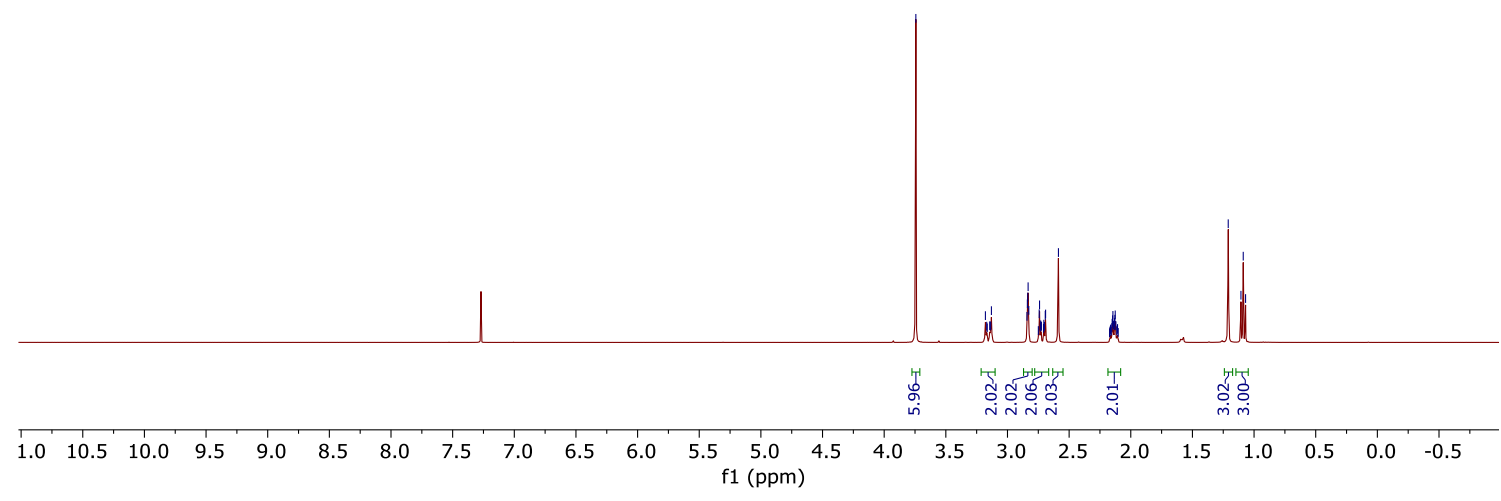

${ }^{13} \mathrm{C}$-NMR spectrum of $\mathbf{1} \mathbf{r}$ in $\mathrm{CDCl}_{3}, 101 \mathrm{MHz}$

hsh-g193\%3-SM.11.fid ì

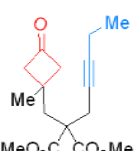

$\mathrm{MeO}_{3} \mathrm{C} \mathrm{CO}_{2} \mathrm{Me}$

$1 \mathrm{r}$

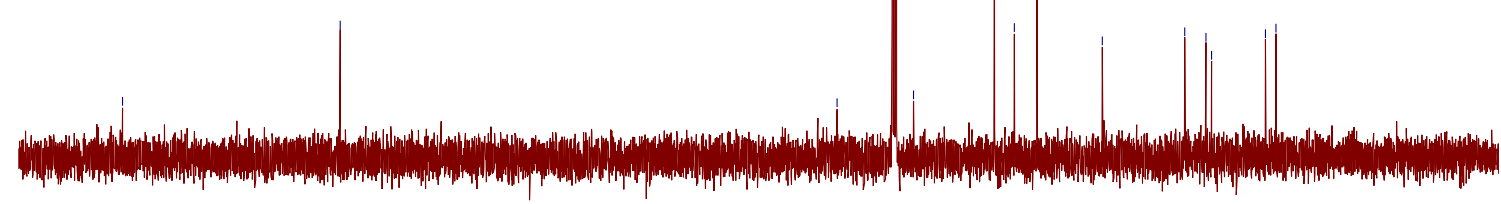

$\begin{array}{lllllllllllllllllllllllllllllllllll}220 & 210 & 200 & 190 & 180 & 170 & 160 & 150 & 140 & 130 & 120 & 110 & 100 & 90 & 80 & 70 & 60 & 50 & 40 & 30 & 20 & 10 & 0 & -10 & -20\end{array}$ 
${ }^{1} \mathrm{H}-\mathrm{NMR}$ spectrum of $1 \mathrm{~s}$ in $\mathrm{CDCl}_{3}, 400 \mathrm{MHz}$
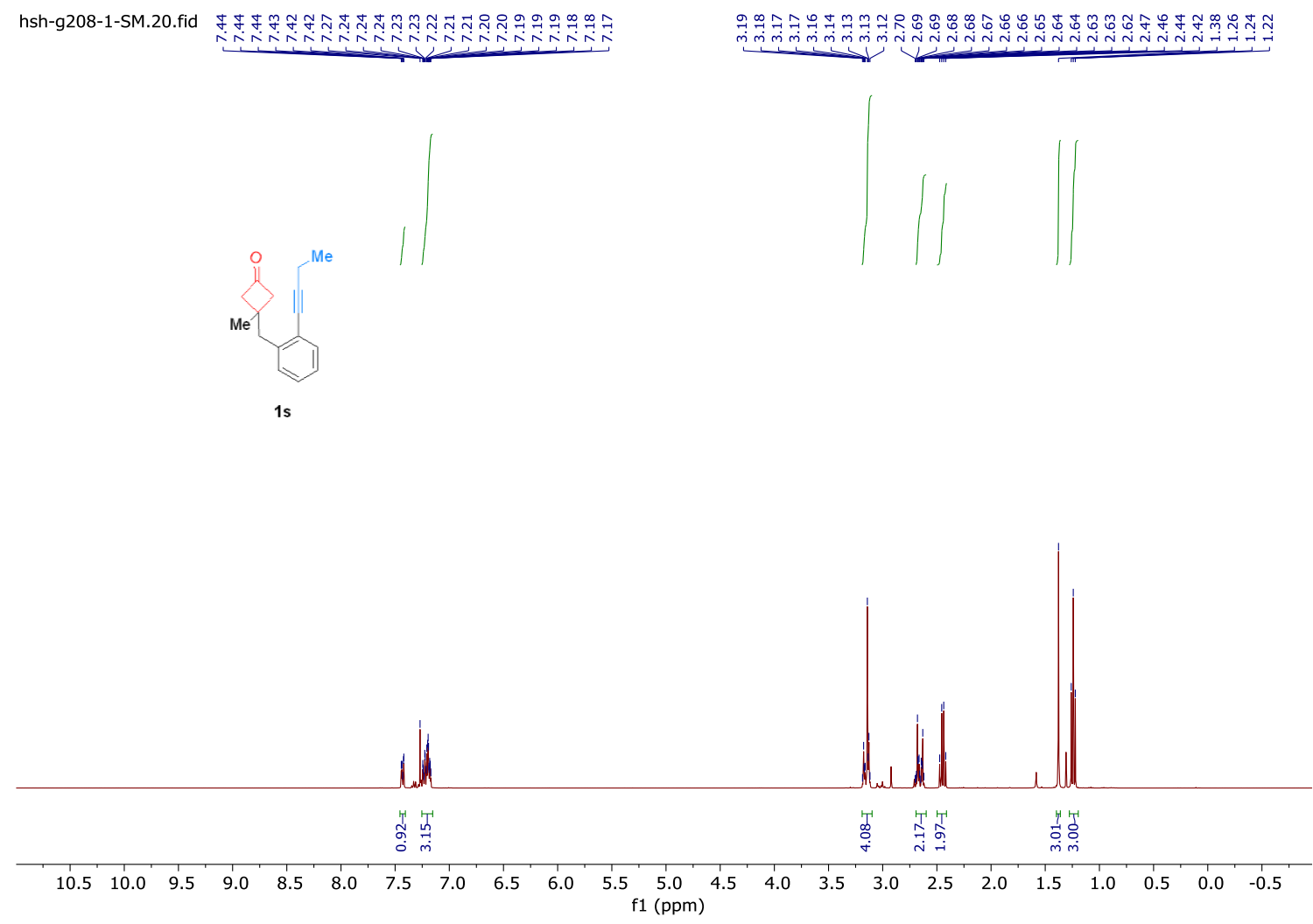

${ }^{13} \mathrm{C}$-NMR spectrum of $1 \mathrm{~s}$ in $\mathrm{CDCl}_{3}, 101 \mathrm{MHz}$

hsh-g208-1-SM.11.99

尔d

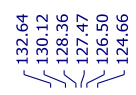

寊

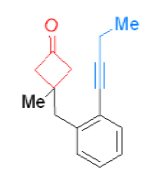

1s

\begin{tabular}{llllllllllllllllllllllllll}
\hline & 230 & 220 & 210 & 200 & 190 & 180 & 170 & 160 & 150 & 140 & 130 & $\begin{array}{c}120 \\
\mathrm{f} 1(\mathrm{ppm})\end{array}$ & 110 & 100 & 90 & 80 & 70 & 60 & 50 & 40 & 30 & 20 & 10 & 0 & -1
\end{tabular} 
hshg169-2.10.fid

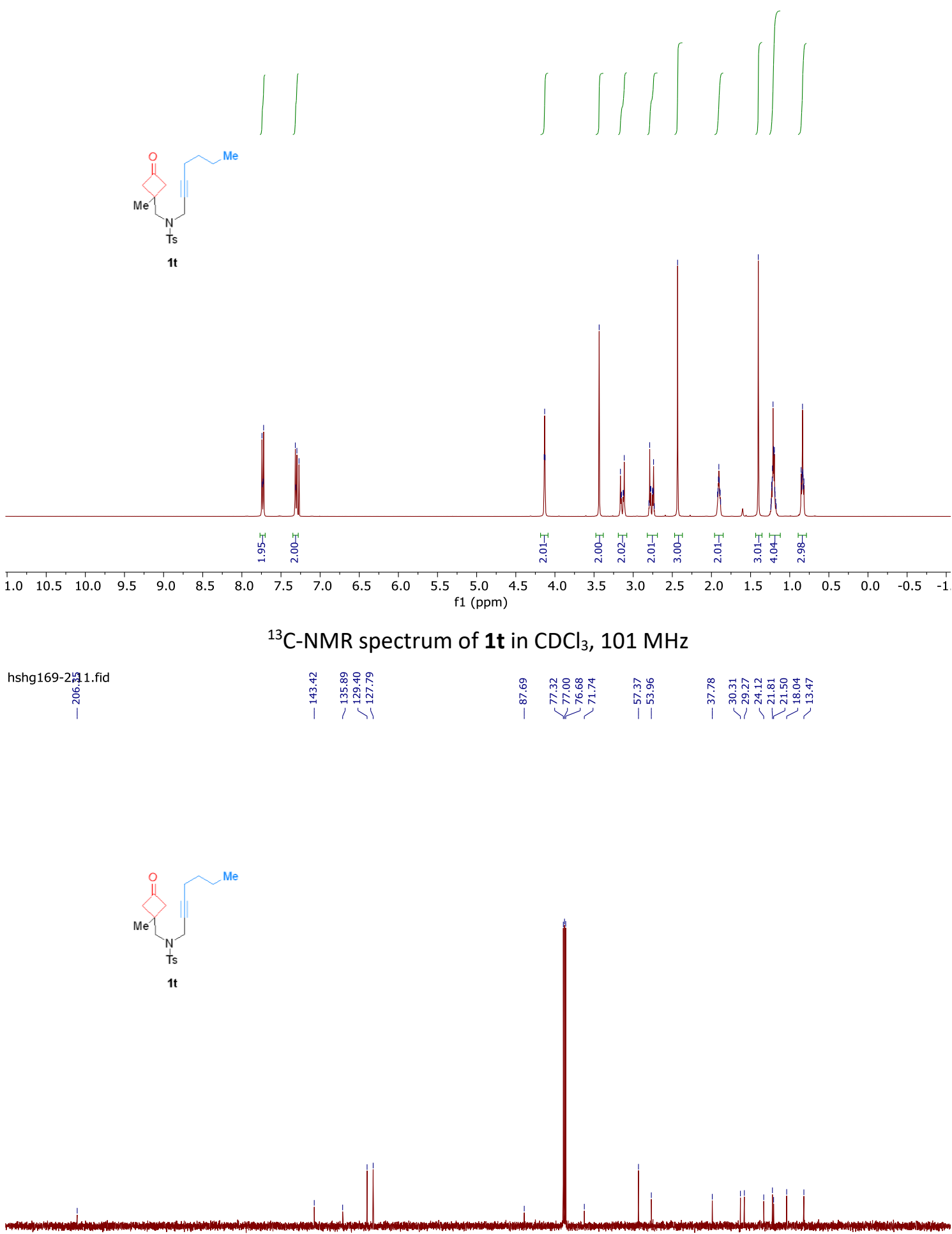

$\begin{array}{lllllllllllllllllllllllllllllllllllll}220 & 210 & 200 & 190 & 180 & 170 & 160 & 150 & 140 & 130 & 120 & 110 & 100 & 90 & 80 & 70 & 60 & 50 & 40 & 30 & 20 & 10 & 0 & -10 & -20\end{array}$ 
${ }^{1} \mathrm{H}-\mathrm{NMR}$ spectrum of $1 \mathbf{u}$ in $\mathrm{CDCl}_{3}, 400 \mathrm{MHz}$

hshg170-2.10.fid
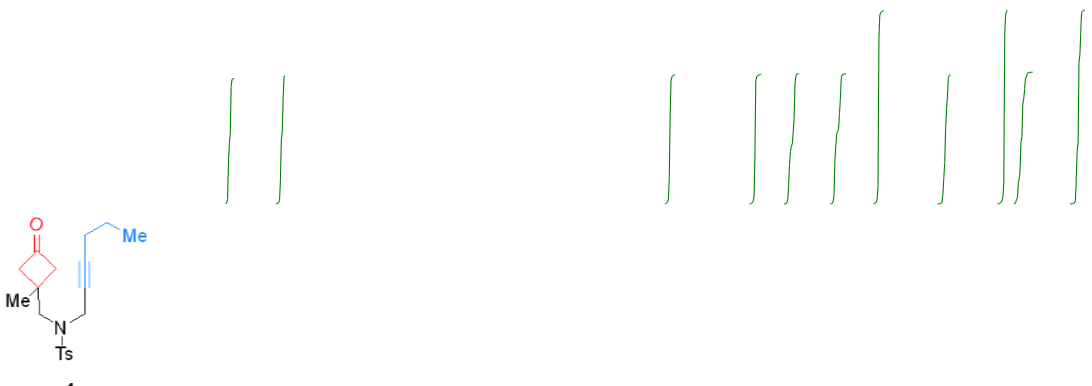

14

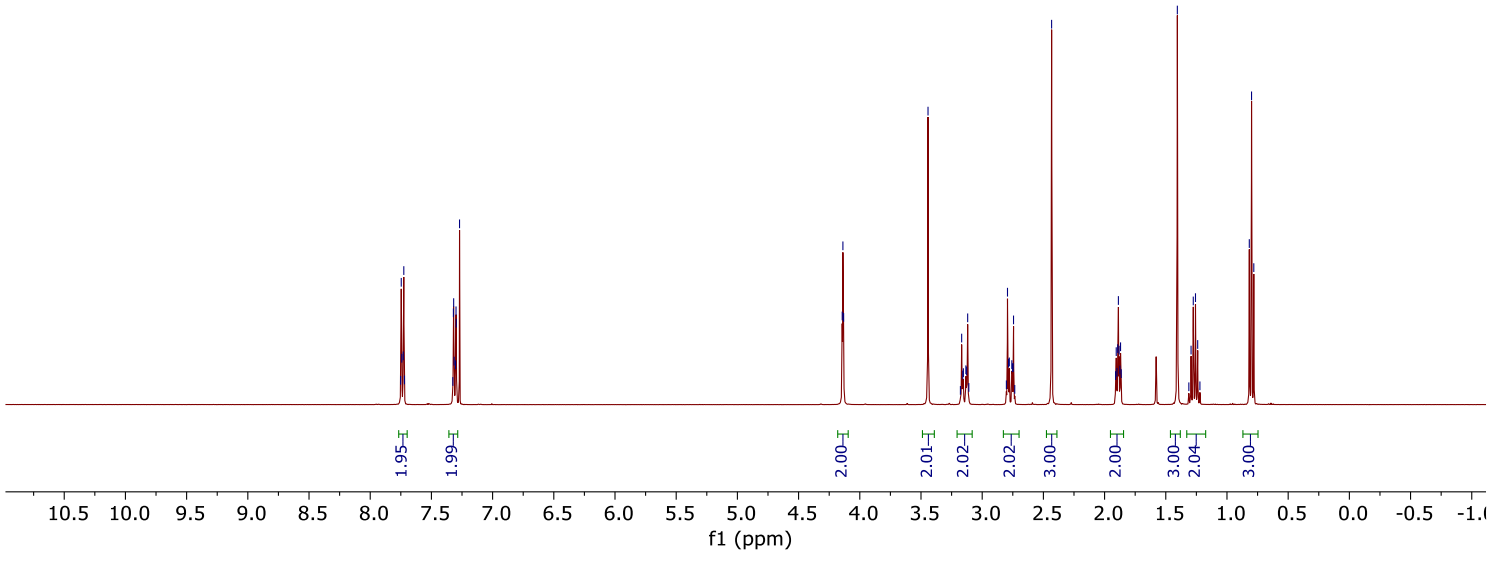

${ }^{13} \mathrm{C}-\mathrm{NMR}$ spectrum of $\mathbf{1} \mathbf{u}$ in $\mathrm{CDCl}_{3}, 101 \mathrm{MHz}$

hshg170-2\%20.fid 
${ }^{1} \mathrm{H}-\mathrm{NMR}$ spectrum of $1 \mathbf{v}$ in $\mathrm{CDCl}_{3}, 400 \mathrm{MHz}$

hsh-g206.10.fid

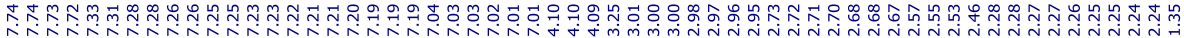

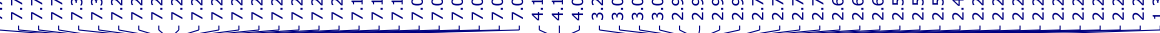
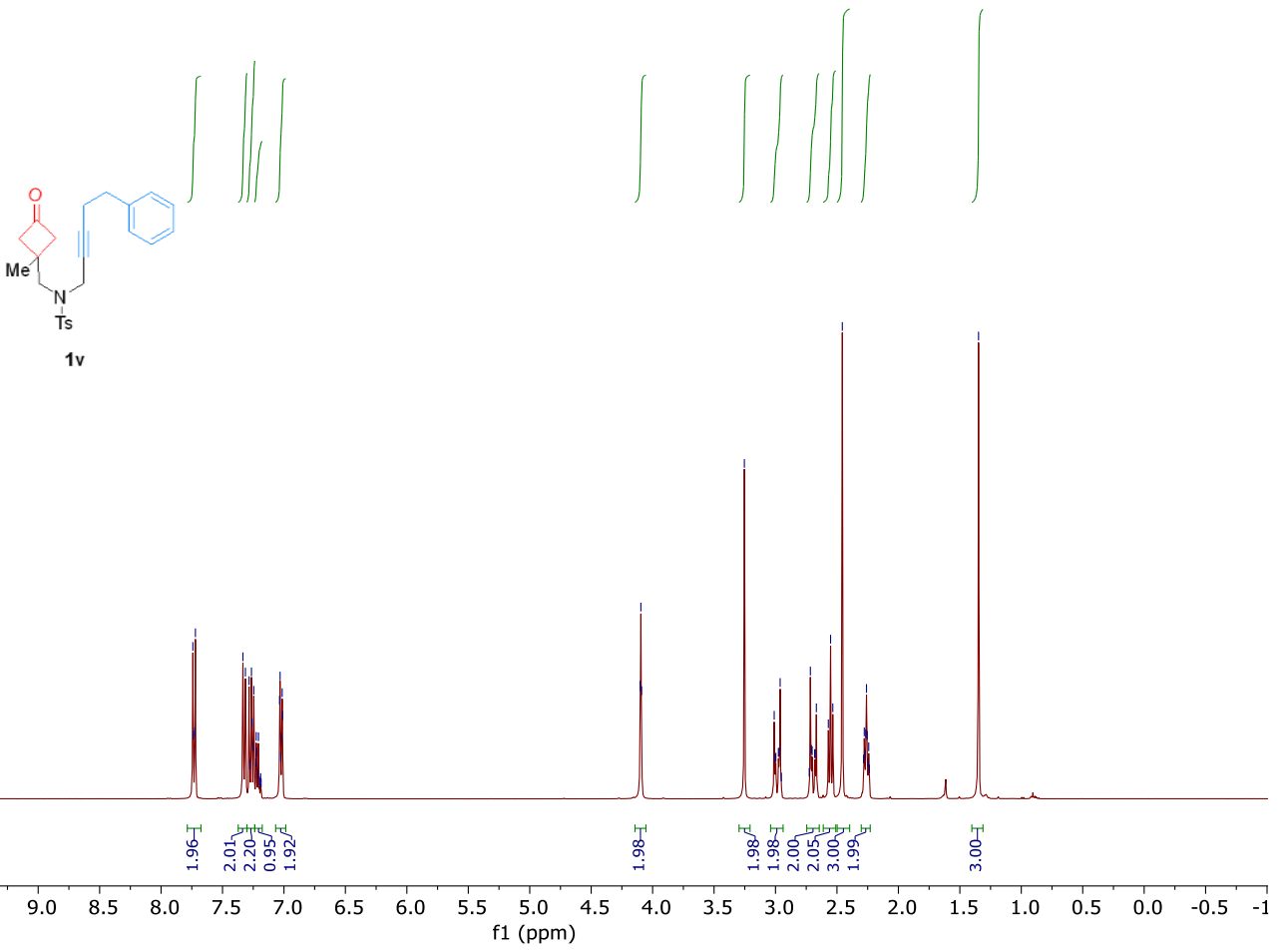

${ }^{13} \mathrm{C}$-NMR spectrum of $1 \mathbf{v}$ in $\mathrm{CDCl}_{3}, 101 \mathrm{MHz}$

hsh-g206. If.fid

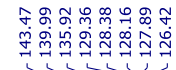

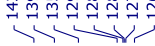

1

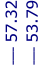

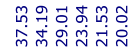

1 (1)

$11<>1$
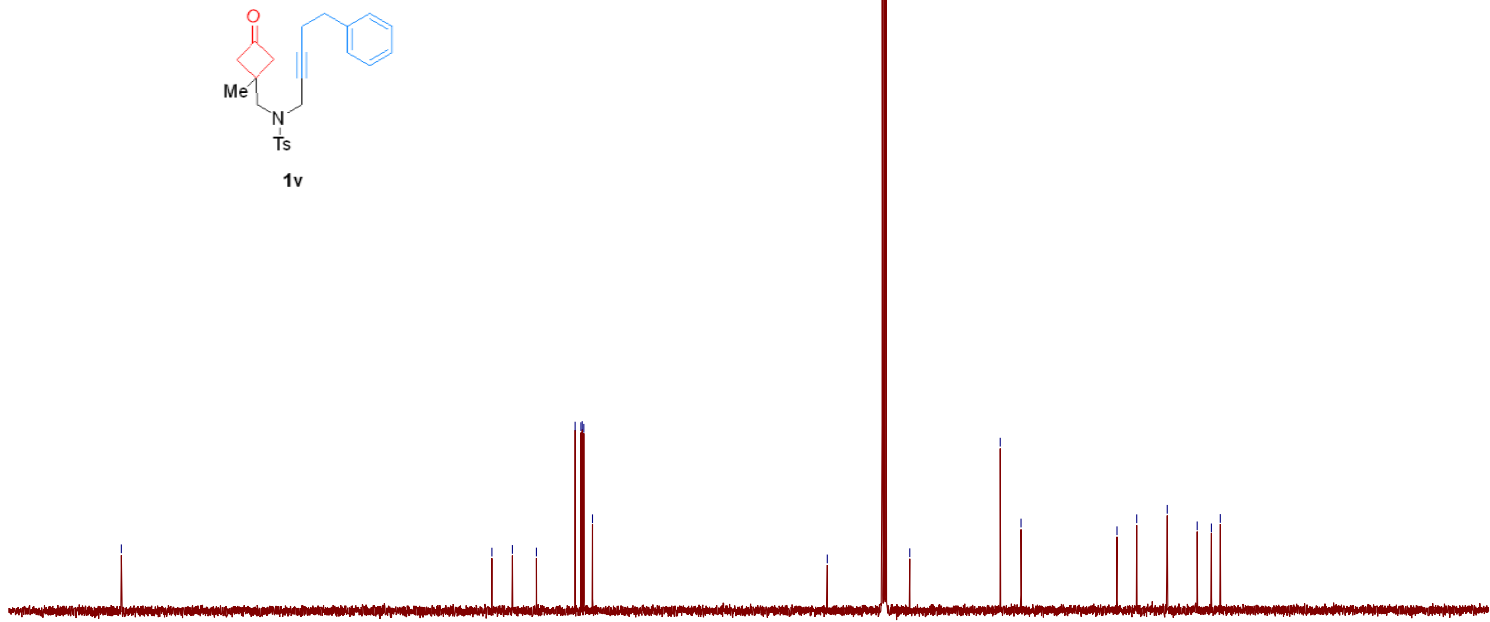

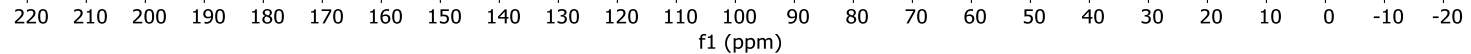


${ }^{1} \mathrm{H}-\mathrm{NMR}$ spectrum of $\mathbf{1 w}$ in $\mathrm{CDCl}_{3}, 400 \mathrm{MHz}$

hsh-g134-2.12.fid

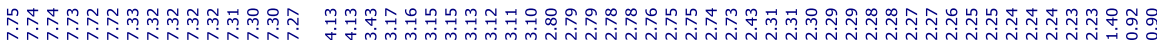

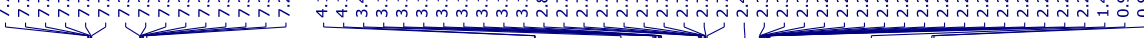

Me

Ts

$1 w$

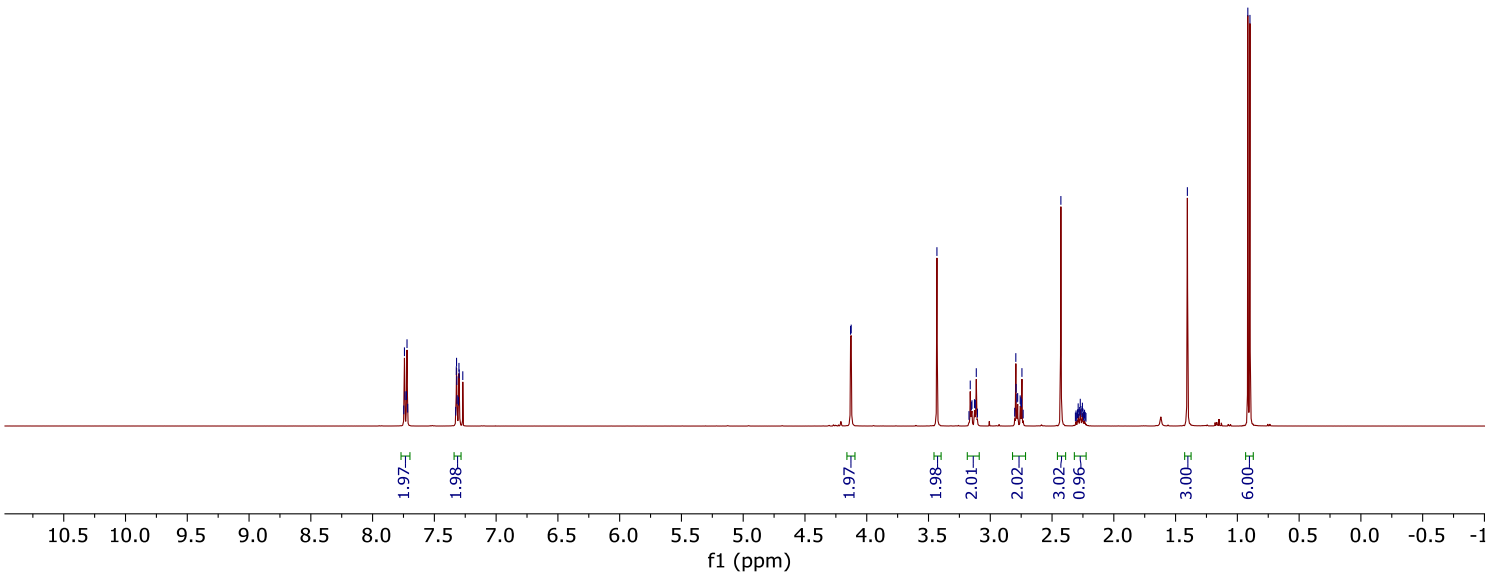

${ }^{13} \mathrm{C}-\mathrm{NMR}$ spectrum of $\mathbf{1} \mathbf{w}$ in $\mathrm{CDCl}_{3}, 101 \mathrm{MHz}$

hsh-g134-2n11.fid

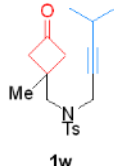

$1 w$

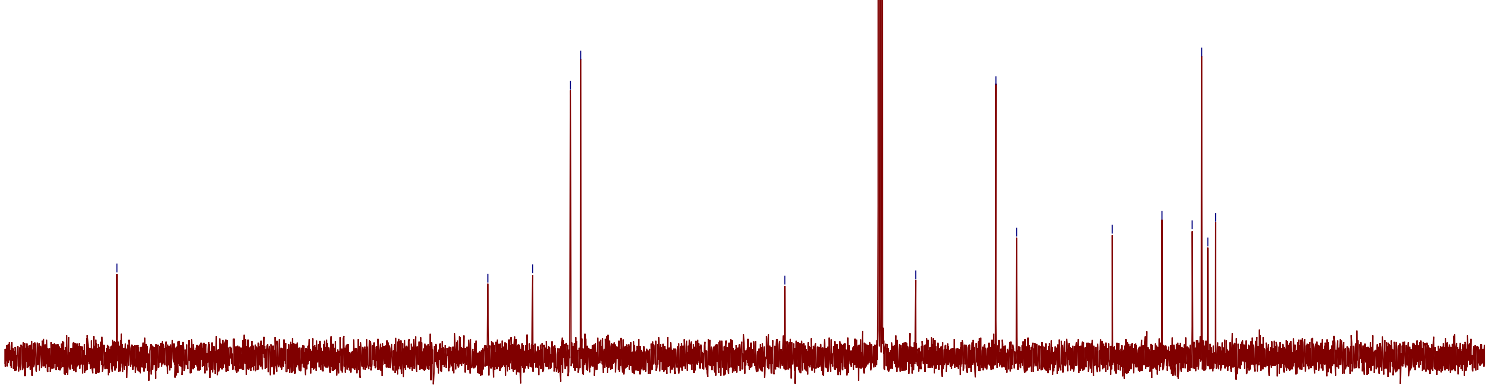

$\begin{array}{lllllllllllllllllllllllllllllllll}220 & 210 & 200 & 190 & 180 & 170 & 160 & 150 & 140 & 130 & 120 & 110 & 100 & 90 & 80 & 70 & 60 & 50 & 40 & 30 & 20 & 10 & 0 & -10 & -20\end{array}$ 
${ }^{1} \mathrm{H}-\mathrm{NMR}$ spectrum of $\mathbf{1 x}$ in $\mathrm{CDCl}_{3}, 400 \mathrm{MHz}$

hsh-g132.10.fid

$\underbrace{2}$

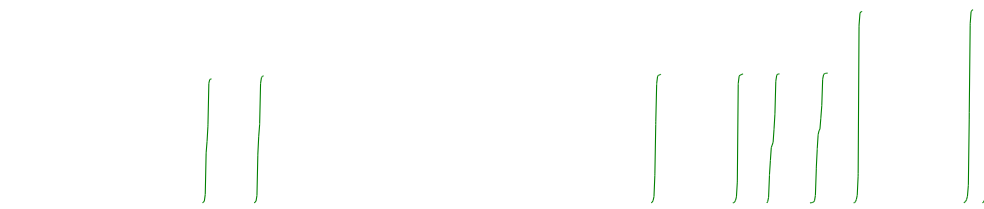

Me
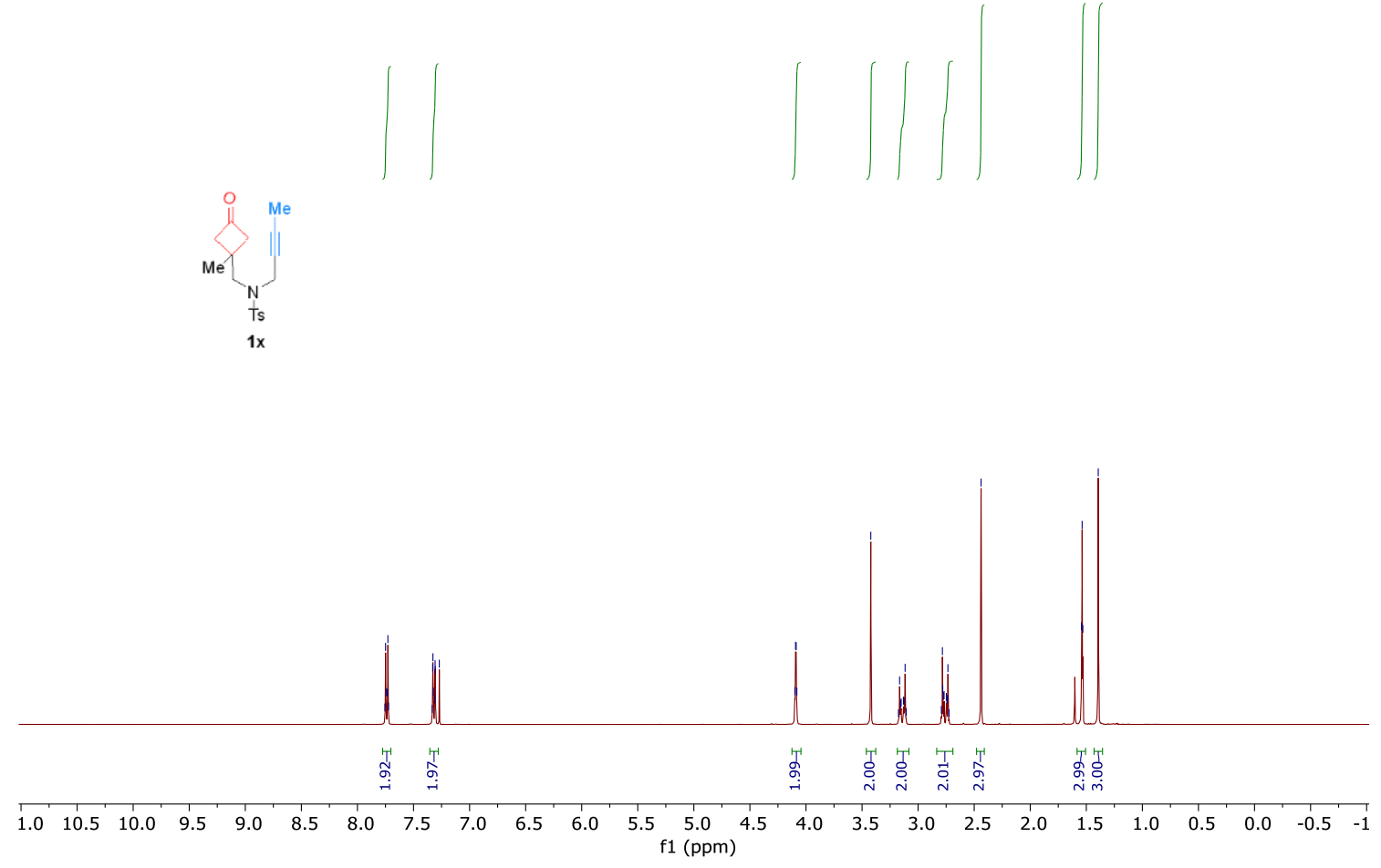

${ }^{13} \mathrm{C}$-NMR spectrum of $\mathbf{1} \mathbf{x}$ in $\mathrm{CDCl}_{3}, 101 \mathrm{MHz}$

hsh-g132. flid

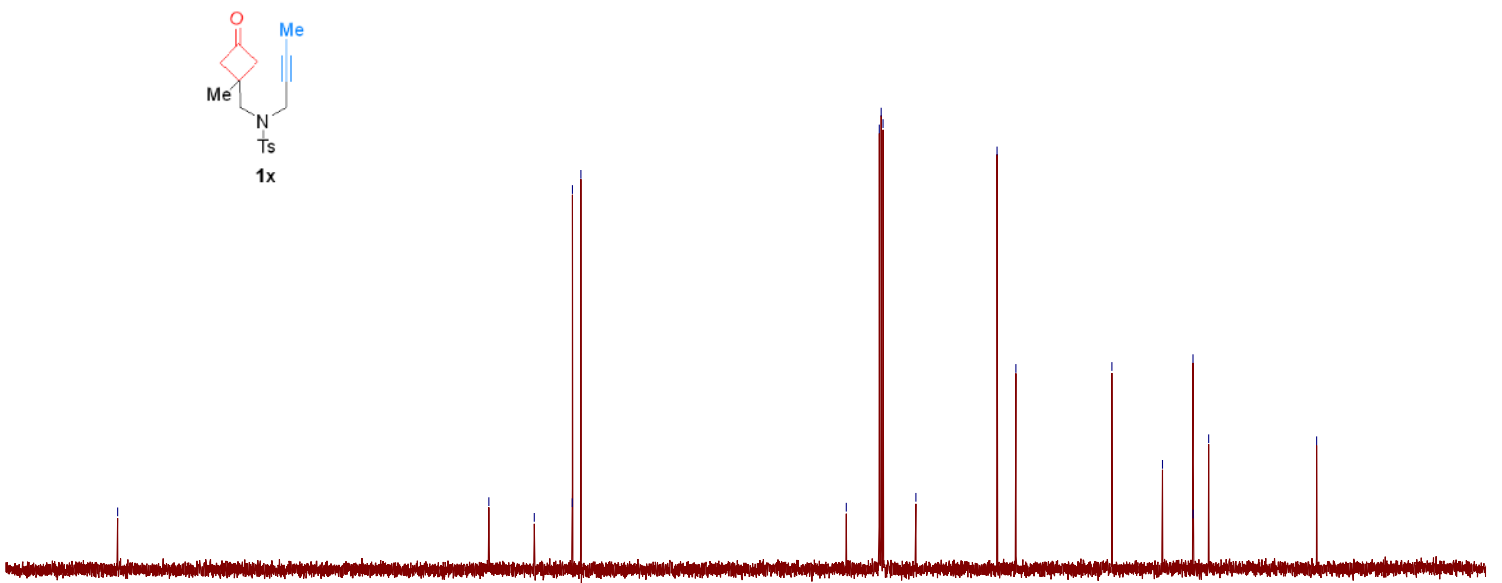

$\begin{array}{lllllllllllllllllllllllllllllllll}220 & 210 & 200 & 190 & 180 & 170 & 160 & 150 & 140 & 130 & 120 & 110 & 100 & 90 & 80 & 70 & 60 & 50 & 40 & 30 & 20 & 10 & 0 & -10 & -20\end{array}$ 
${ }^{1} \mathrm{H}-\mathrm{NMR}$ spectrum of $\mathbf{1 y}$ in $\mathrm{CDCl}_{3}, 400 \mathrm{MHz}$

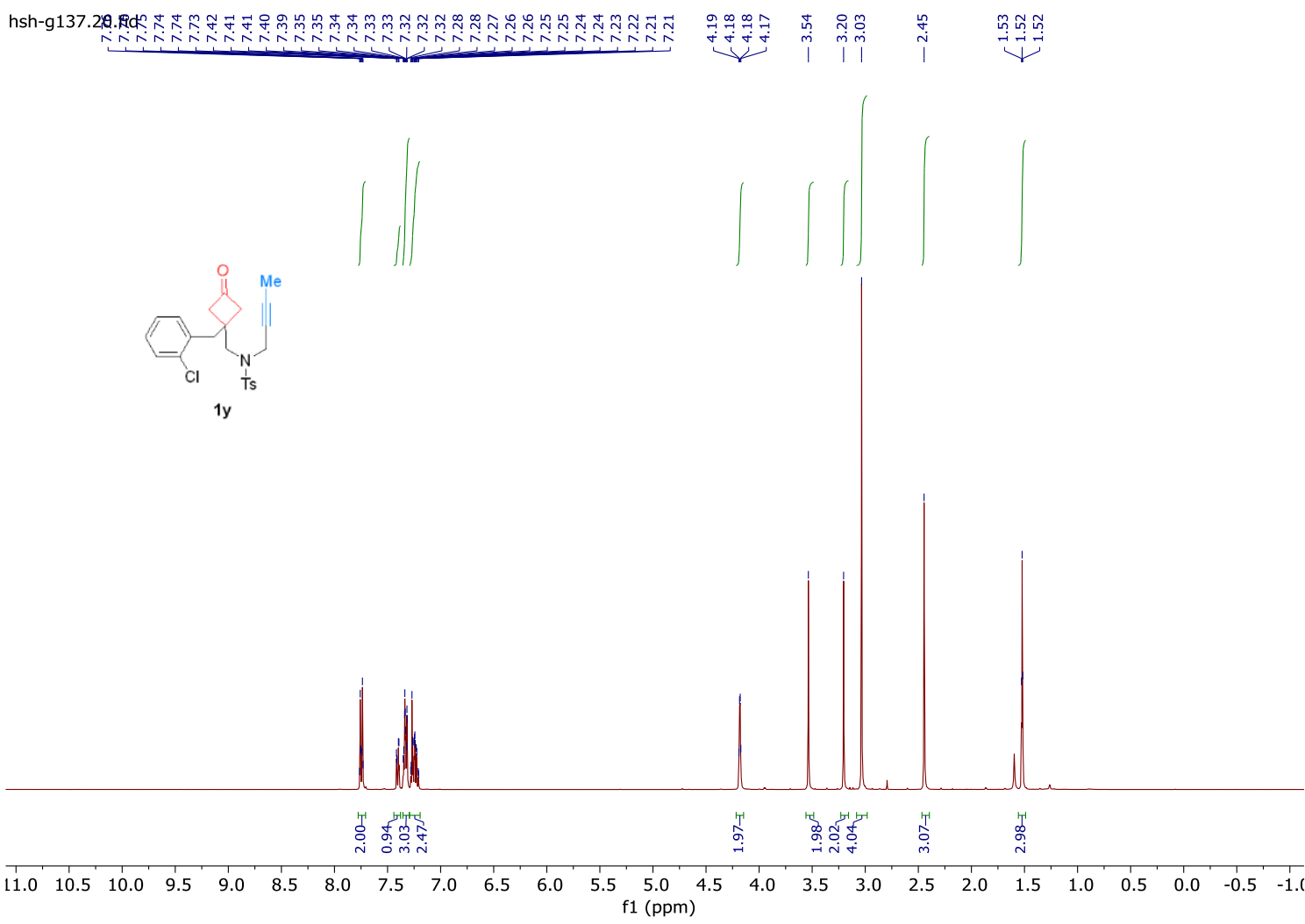

${ }^{13} \mathrm{C}-\mathrm{NMR}$ spectrum of $\mathbf{1 y}$ in $\mathrm{CDCl}_{3}, 101 \mathrm{MHz}$

hsh-g137.2黑.fid
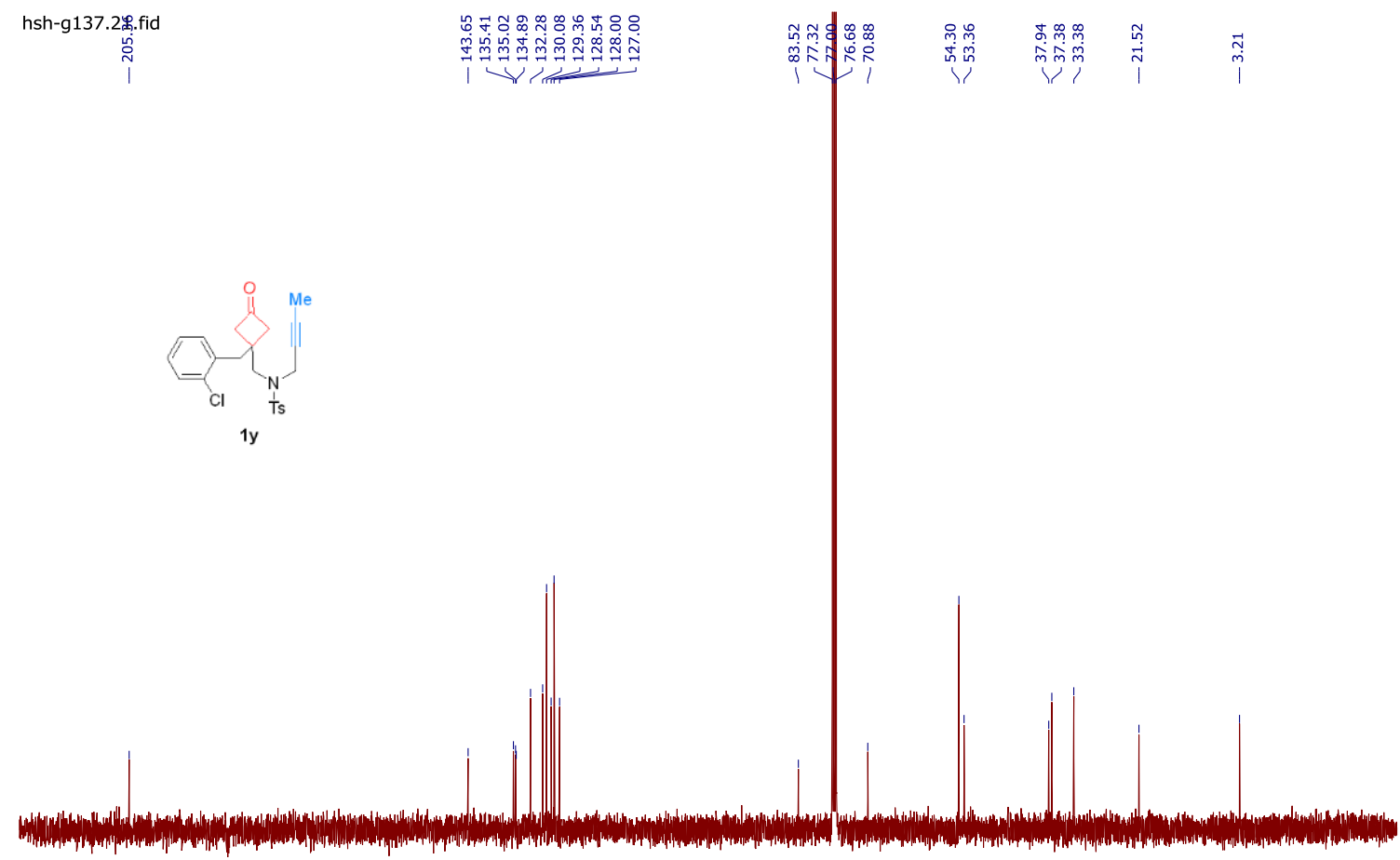

$\begin{array}{llllllllllllllllllllllllllllllllll}220 & 210 & 200 & 190 & 180 & 170 & 160 & 150 & 140 & 130 & 120 & 110 & 100 & 90 & 80 & 70 & 60 & 50 & 40 & 30 & 20 & 10 & 0 & -10 & -20\end{array}$ 


\section{${ }^{1} \mathrm{H}$-NMR spectrum of $1 \mathrm{z}$ in $\mathrm{CDCl}_{3}, 400 \mathrm{MHz}$}

hsh-g40-4.20.fid

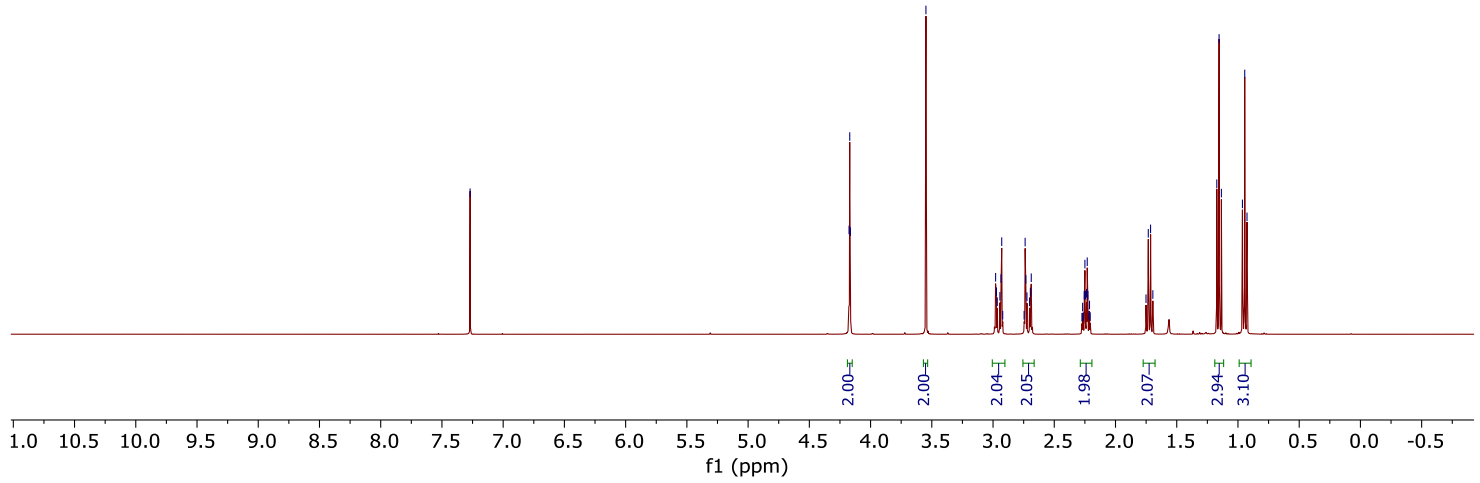

${ }^{13} \mathrm{C}$-NMR spectrum of $\mathbf{1 z}$ in $\mathrm{CDCl}_{3}, 101 \mathrm{MHz}$

hsh-g40-421.fid

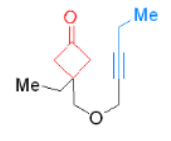

$1 z$

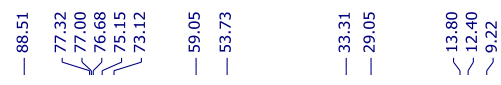

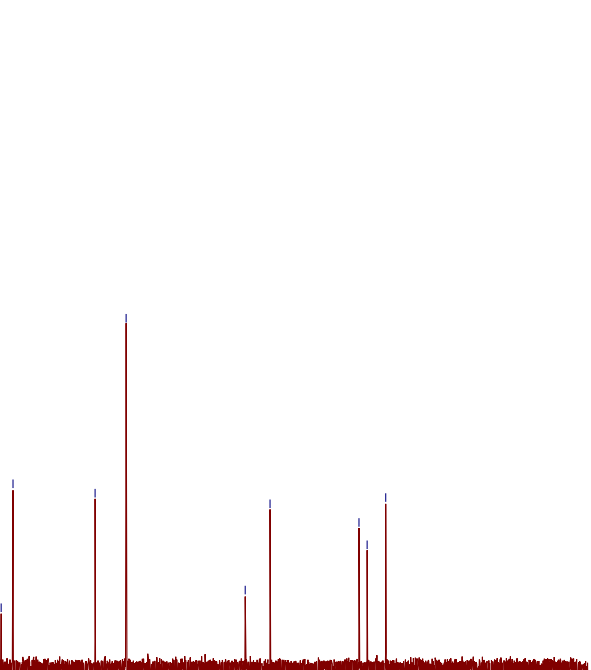

$\begin{array}{llllllllllllllllllllllllll}220 & 210 & 200 & 190 & 180 & 170 & 160 & 150 & 140 & 130 & 120 & 110 & 100 & 90 & 80 & 70 & 60 & 50 & 40 & 30 & 20 & 10 & 0 & -10 & -20\end{array}$ 
${ }^{1} \mathrm{H}-\mathrm{NMR}$ spectrum of 1 aa in $\mathrm{CDCl}_{3}, 400 \mathrm{MHz}$

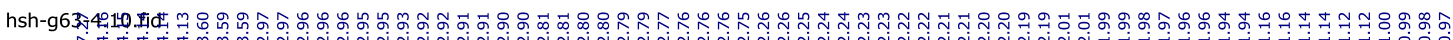

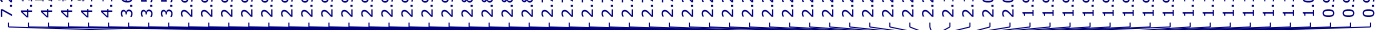

Me $\|^{M}$

Me or

1aa

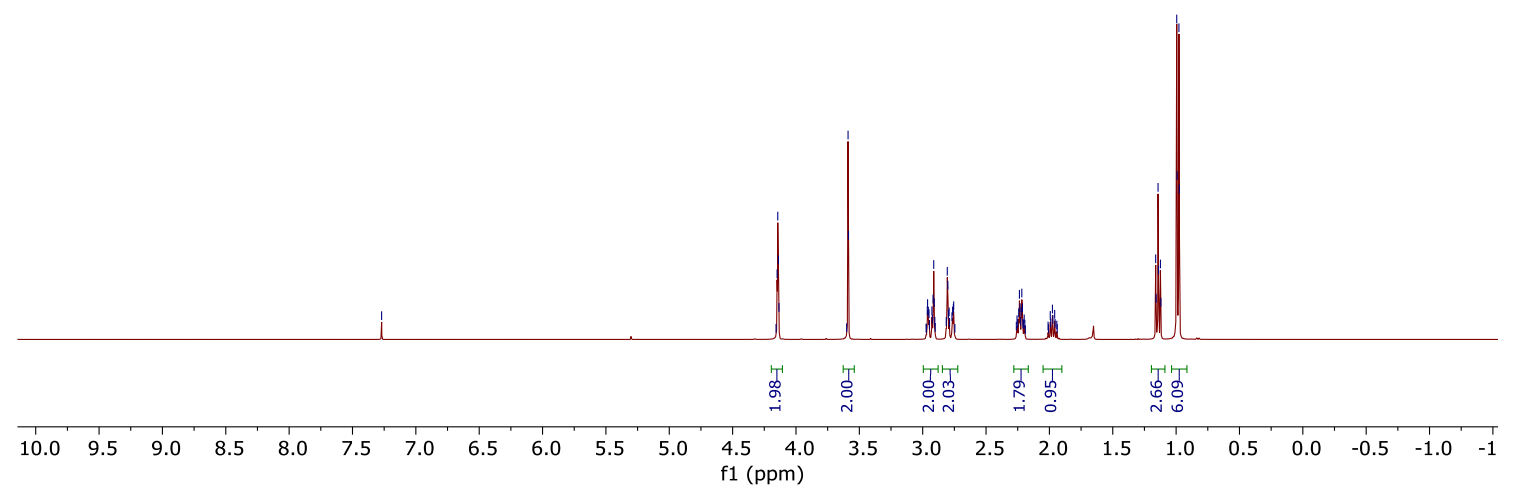

${ }^{13} \mathrm{C}-\mathrm{NMR}$ spectrum of 1aa in $\mathrm{CDCl}_{3}, 101 \mathrm{MHz}$

hsh-g63-\$od1.fid

ì

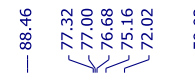

å.

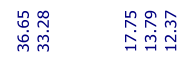

$\sum_{M e} \|^{M e}$

1aa

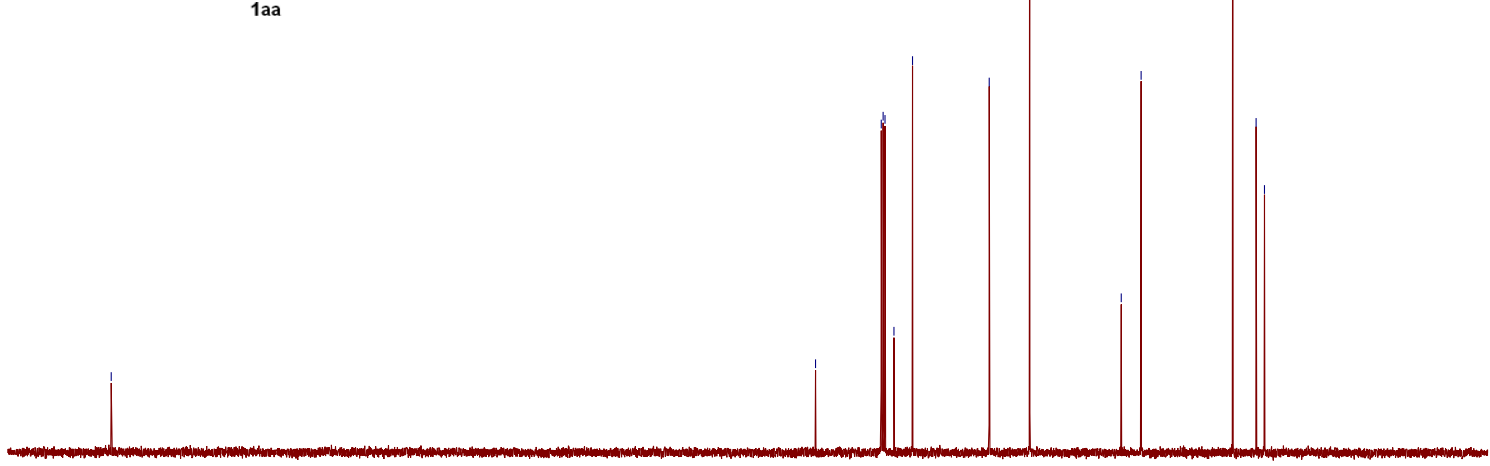

$\begin{array}{lllllllllllllllllllllllll}220 & 210 & 200 & 190 & 180 & 170 & 160 & 150 & 140 & 130 & 120 & \begin{array}{c}110 \\ \mathrm{f} 1(\mathrm{ppm})\end{array} & \begin{array}{c}100 \\ (\mathrm{pm})\end{array} & 70 & 60 & 50 & 40 & 30 & 20 & 10 & 0 & -10 & -20\end{array}$ 
${ }^{1} \mathrm{H}-\mathrm{NMR}$ spectrum of $1 \mathrm{ab}$ in $\mathrm{CDCl}_{3}, 400 \mathrm{MHz}$

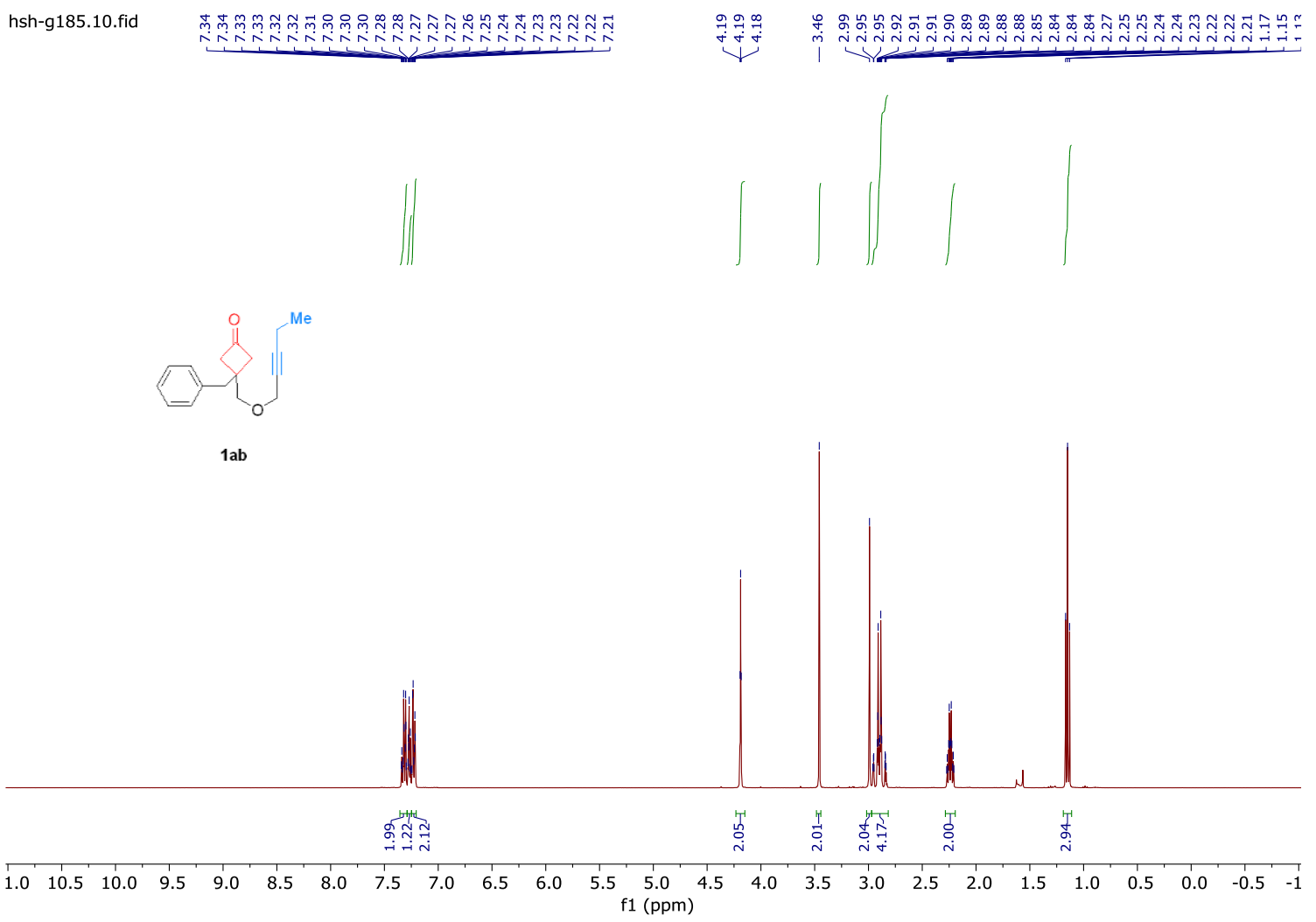

${ }^{13} \mathrm{C}-\mathrm{NMR}$ spectrum of $1 \mathrm{ab}$ in $\mathrm{CDCl}_{3}, 101 \mathrm{MHz}$

hsh-g185. fol.fid

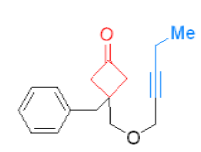

$1 \mathrm{ab}$

$\begin{array}{llllllllllllllllllllllllllllllllll}220 & 210 & 200 & 190 & 180 & 170 & 160 & 150 & 140 & 130 & 120 & 110 & 100 & 90 & 80 & 70 & 60 & 50 & 40 & 30 & 20 & 10 & 0 & -10 & -20\end{array}$ 
${ }^{1} \mathrm{H}-\mathrm{NMR}$ spectrum of $1 \mathrm{ac}$ in $\mathrm{CDCl}_{3}, 400 \mathrm{MHz}$

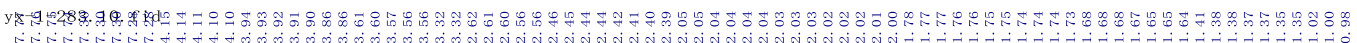

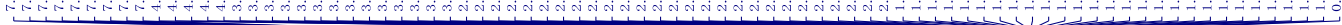

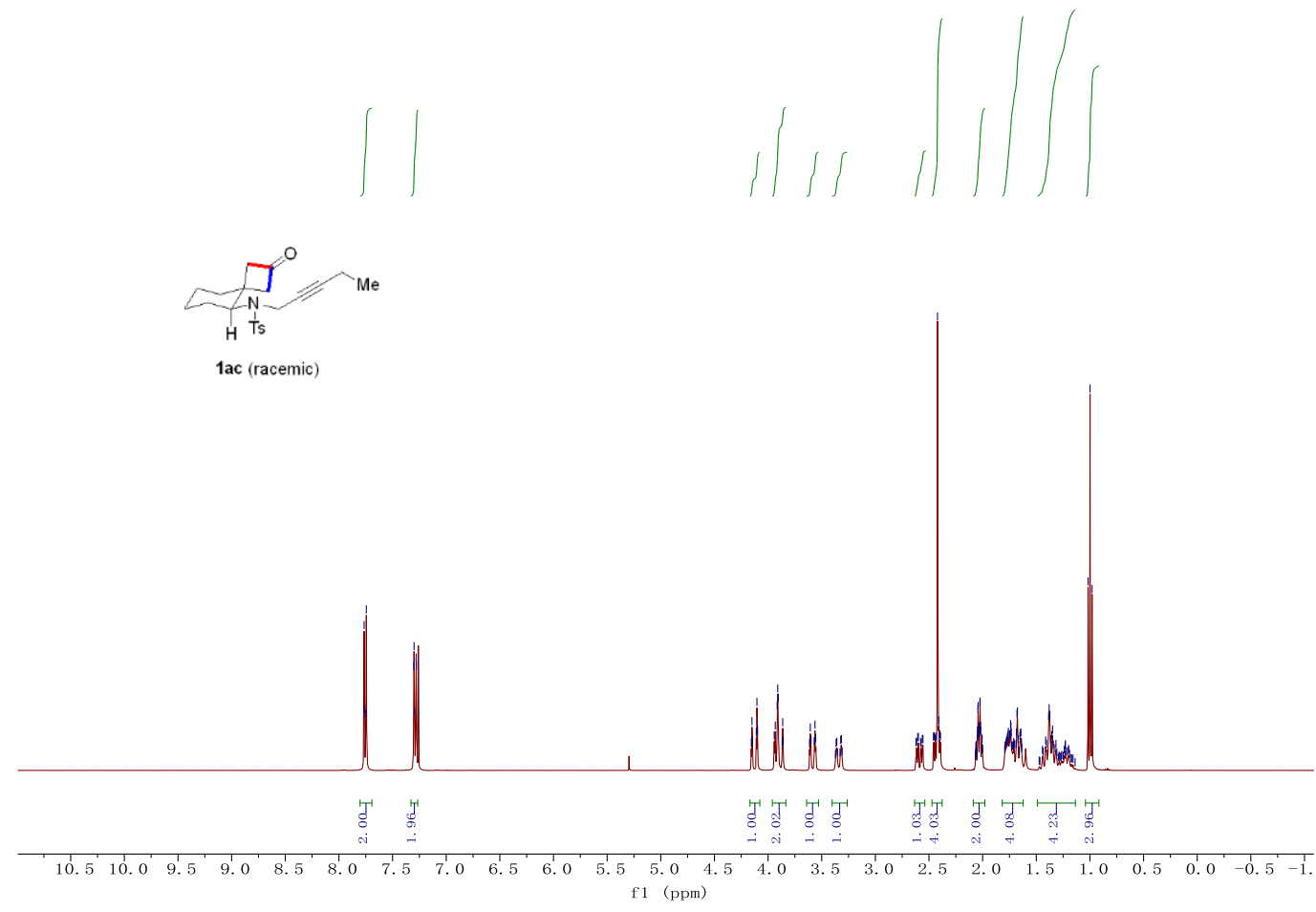

${ }^{13} \mathrm{C}-\mathrm{NMR}$ spectrum of $1 \mathrm{ac}$ in $\mathrm{CDCl}_{3}, 101 \mathrm{MHz}$

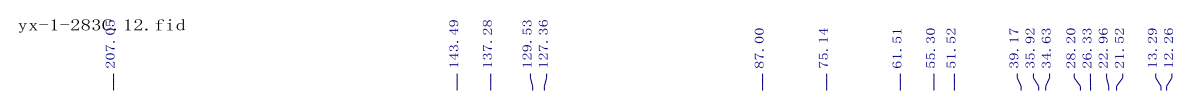
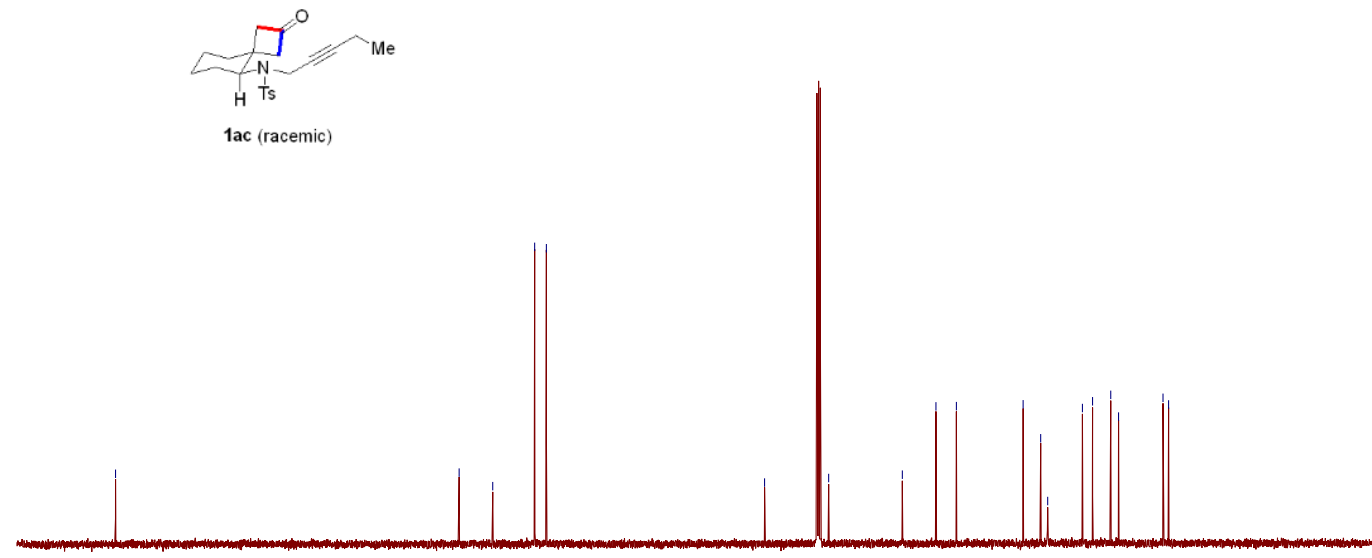

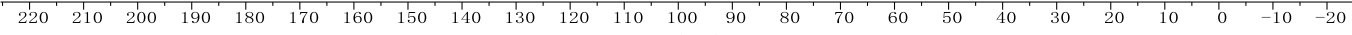
f1 (ppm) 
${ }^{1} \mathrm{H}-\mathrm{NMR}$ spectrum of $1 \mathrm{ad}$ in $\mathrm{CDCl}_{3}, 400 \mathrm{MHz}$

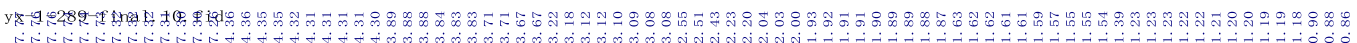

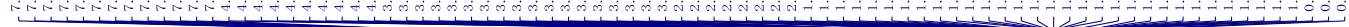

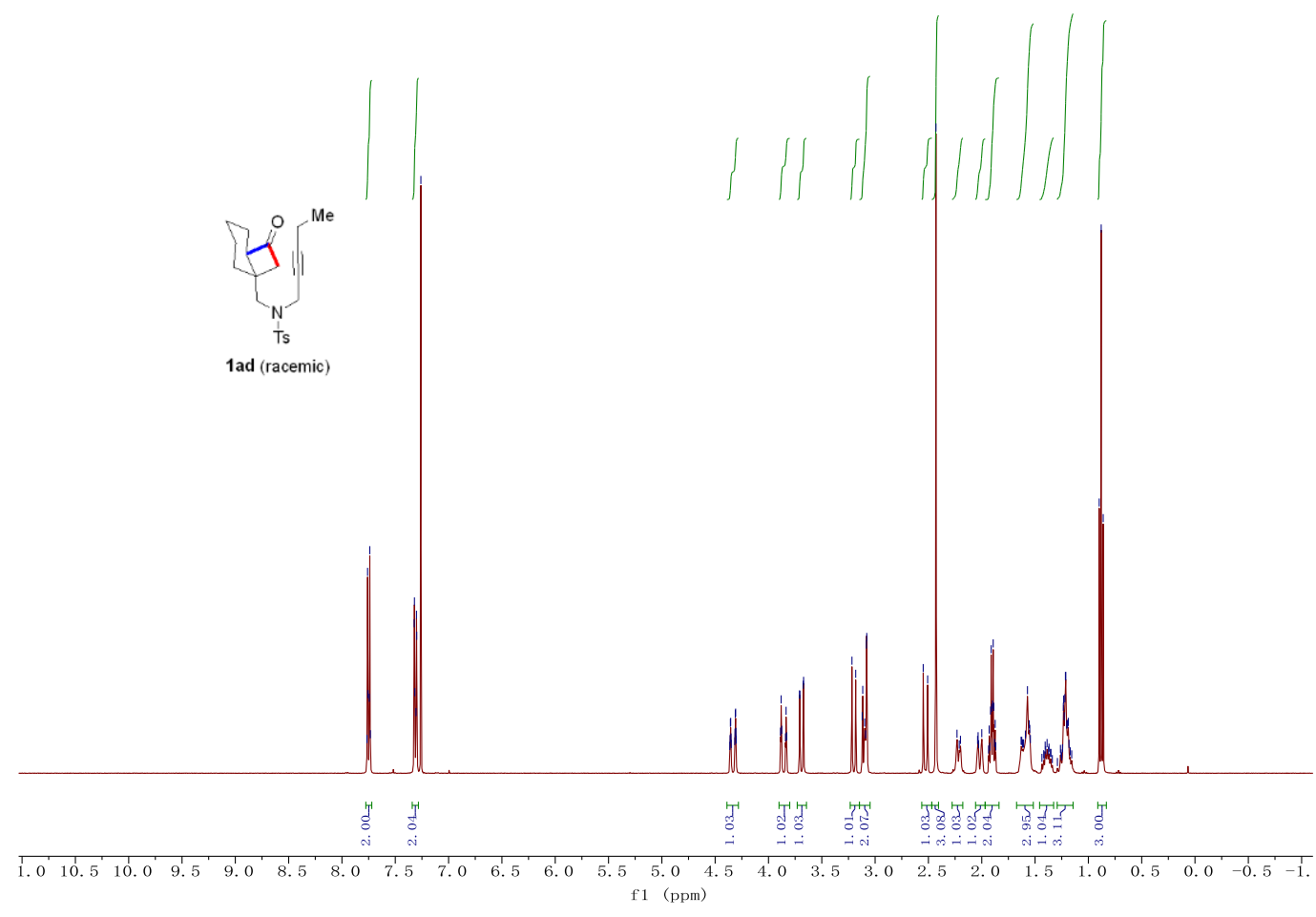

${ }^{13} \mathrm{C}$-NMR spectrum of $1 \mathrm{ad}$ in $\mathrm{CDCl}_{3}, 101 \mathrm{MHz}$

yx-1-289øinal. 11. fid $\stackrel{\stackrel{\leftrightarrow}{\sim}}{1}$

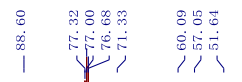

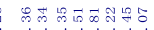

|
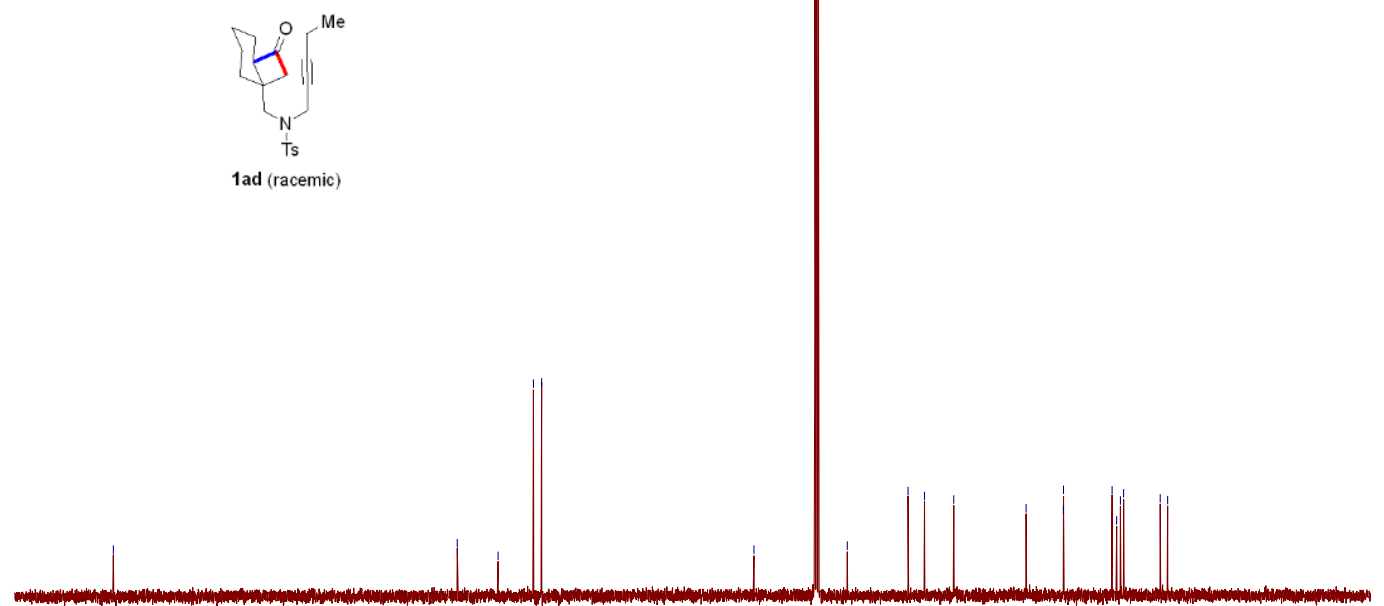

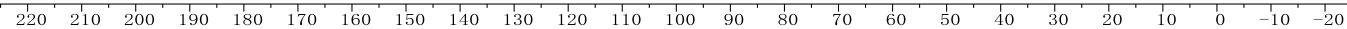
$\mathrm{fl}(\mathrm{ppm})$ 


\section{${ }^{1} \mathrm{H}-\mathrm{NMR}$ spectrum of $\mathbf{S} 16$ in $\mathrm{CDCl}_{3}, 400 \mathrm{MHz}$}

zmx-35-data.10.fid

$\mathrm{MeO}$ OMe
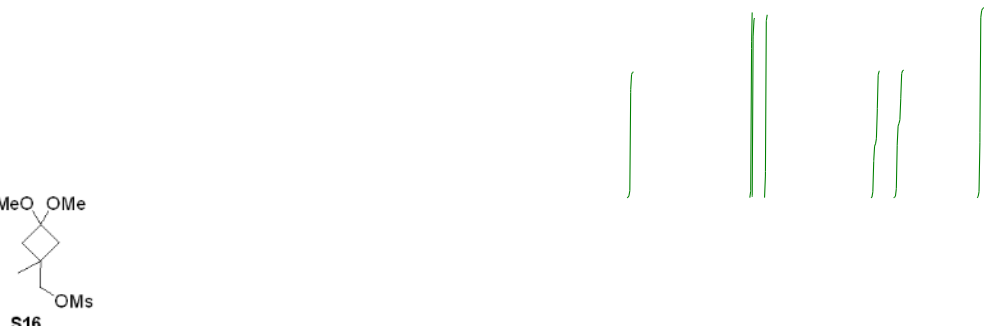

s16

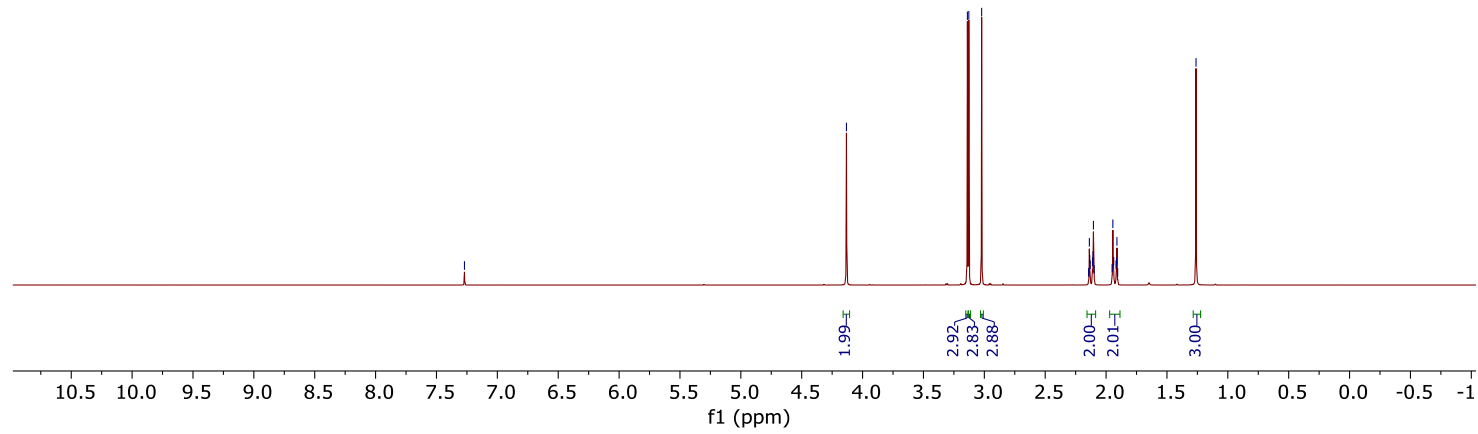

${ }^{13} \mathrm{C}$-NMR spectrum of $\mathbf{S} 16$ in $\mathrm{CDCl}_{3}, 101 \mathrm{MHz}$

zmx-35-data.11.fid
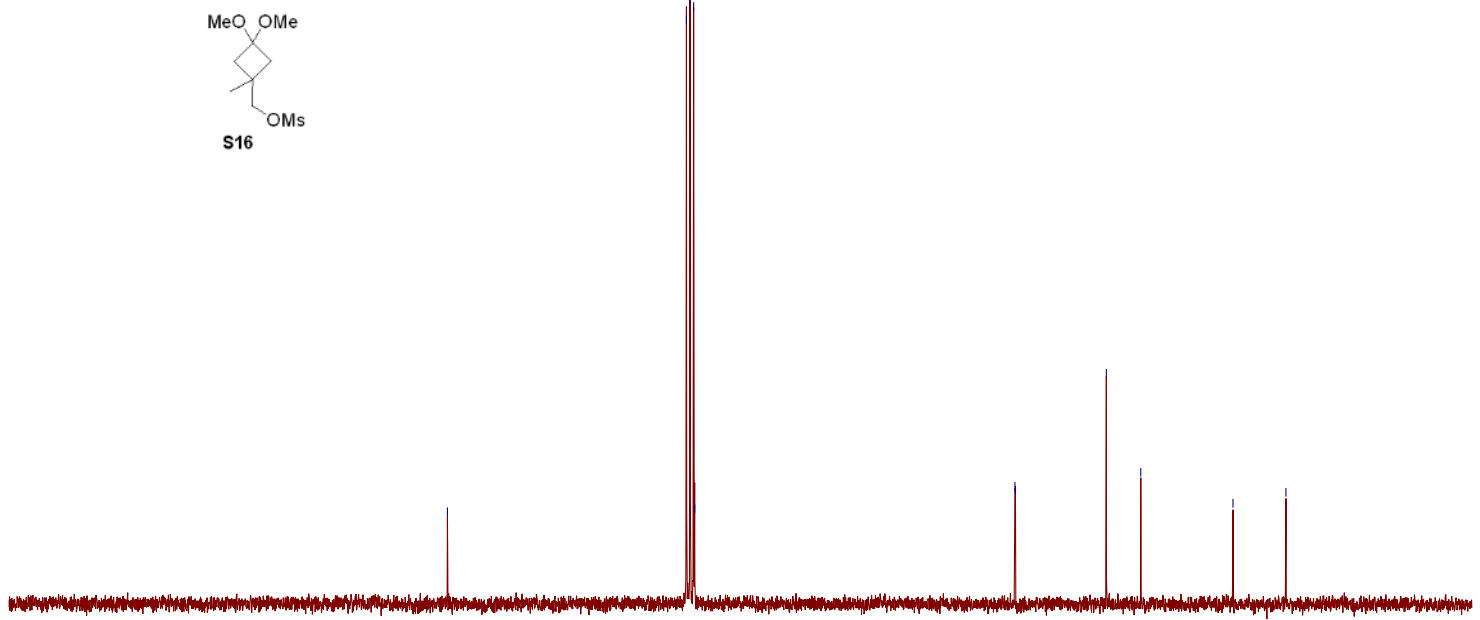

\begin{tabular}{llllllllllllllllllllllllllllllllll}
\hline 135 & 130 & 125 & 120 & 115 & 110 & 105 & 100 & 95 & 90 & 85 & 80 & 75 & 70 & 65 & 60 & 55 & 50 & 45 & 40 & 35 & 30 & 25 & 20 & 15 & 10
\end{tabular} 


\section{${ }^{1} \mathrm{H}-\mathrm{NMR}$ spectrum of $\mathbf{S 1 7}$ in $\mathrm{CDCl}_{3}, 400 \mathrm{MHz}$}

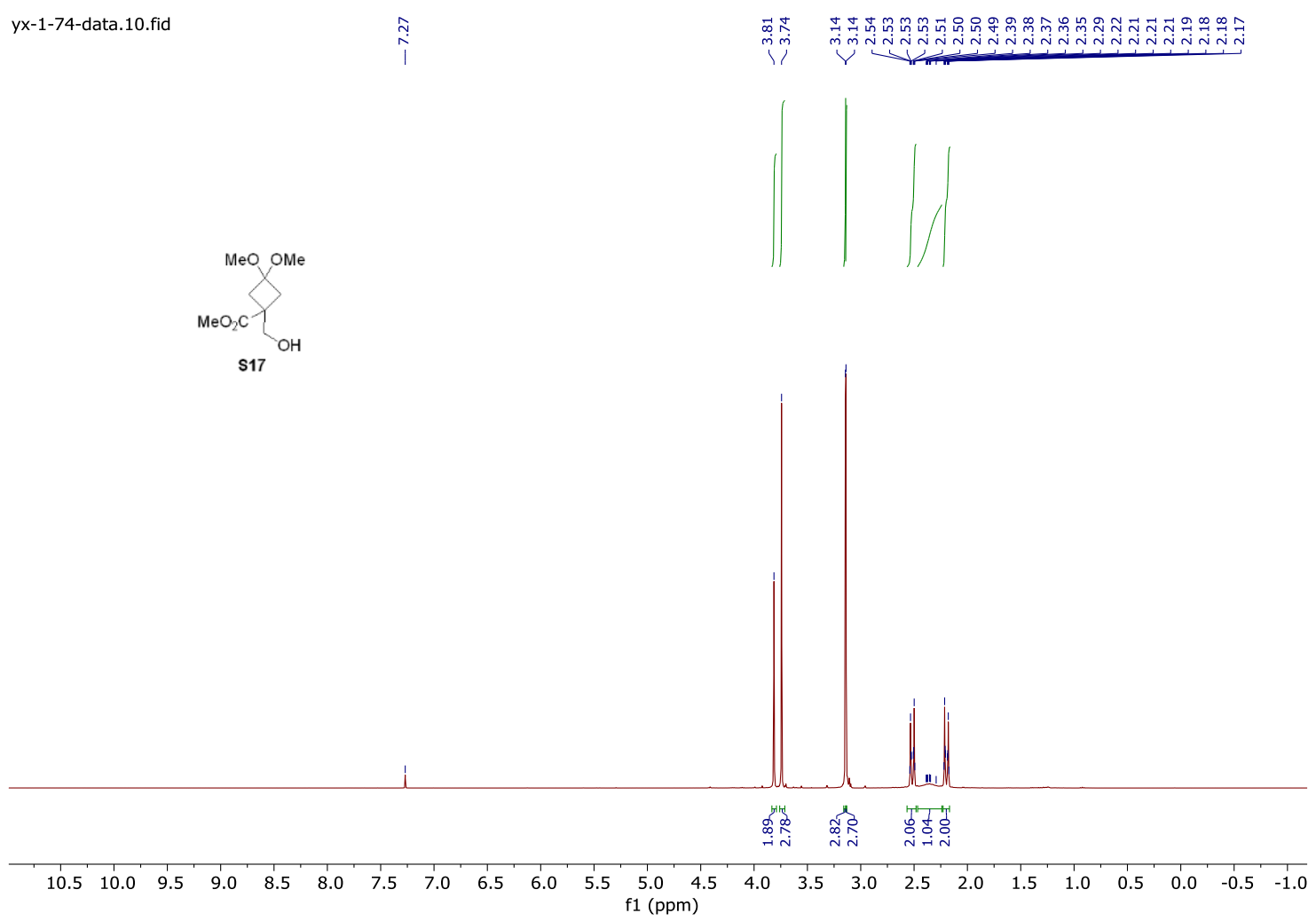

${ }^{13} \mathrm{C}$-NMR spectrum of $\mathbf{S 1 7}$ in $\mathrm{CDCl}_{3}, 101 \mathrm{MHz}$

yx-1-74-data.11.fid

\begin{tabular}{|c|c|c|c|c|}
\hline 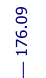 & $\begin{array}{l}\infty \\
\infty \\
\infty \\
\infty \\
\mid\end{array}$ & 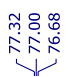 & $\begin{array}{l}\vec{b} \\
\dot{0} \\
1\end{array}$ & 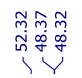 \\
\hline
\end{tabular}
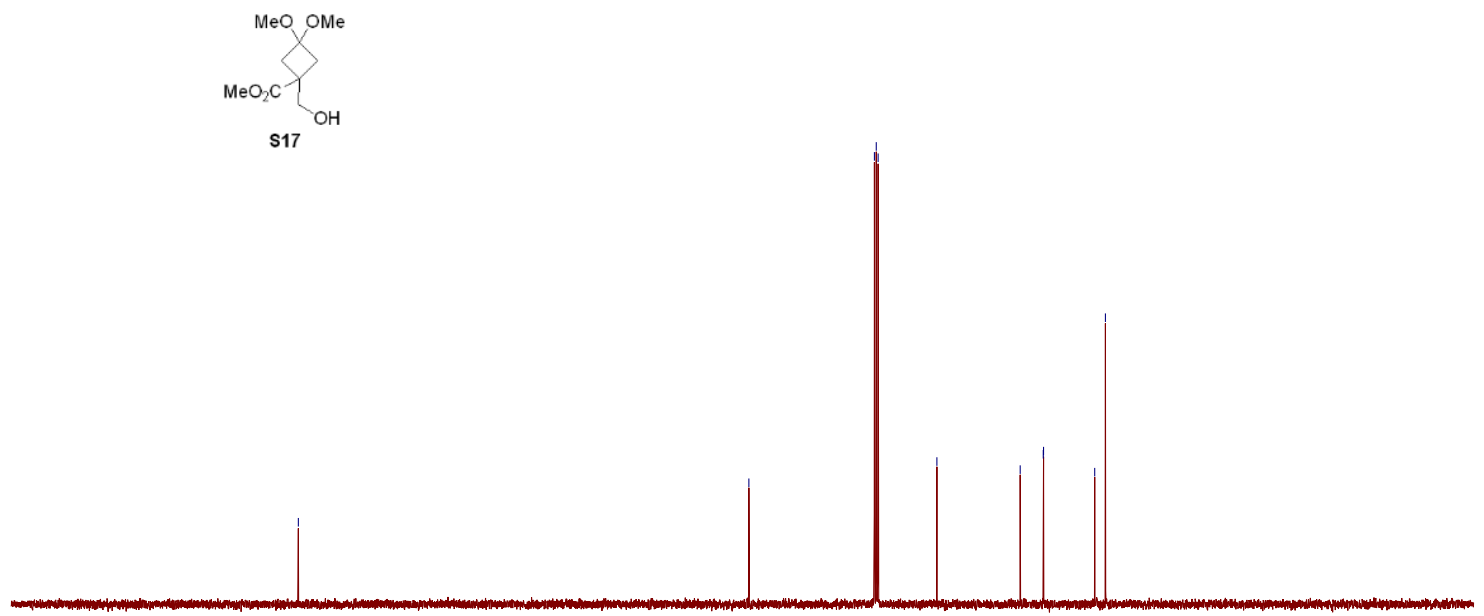

$\begin{array}{lllllllllllllllllllllllllll}220 & 210 & 200 & 190 & 180 & 170 & 160 & 150 & 140 & 130 & 120 & 110 & 100 & 90 & 80 & 70 & 60 & 50 & 40 & 30 & 20 & 10 & 0 & -10 & -20\end{array}$ 


\section{${ }^{1} \mathrm{H}-\mathrm{NMR}$ spectrum of $\mathbf{S} 18$ in $\mathrm{CDCl}_{3}, 400 \mathrm{MHz}$}

yx-1-78-data.10.fid
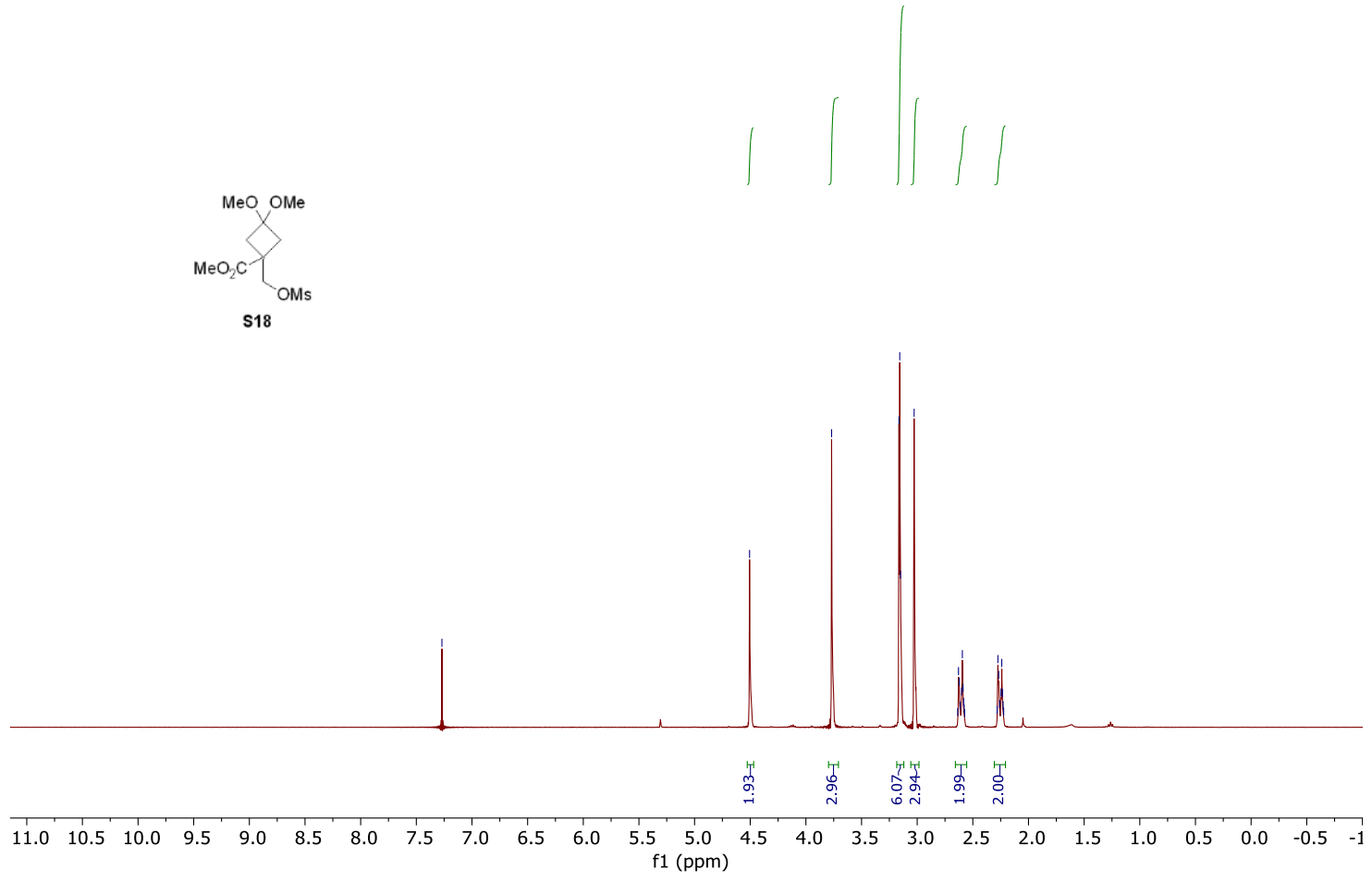

${ }^{13} \mathrm{C}-\mathrm{NMR}$ spectrum of $\mathbf{S 1 8}$ in $\mathrm{CDCl}_{3}, 101 \mathrm{MHz}$

yx-1-78-data.11.fid

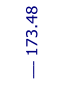

票

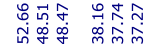

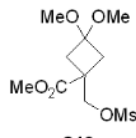

s18 $\begin{array}{lllllllllllll}220 & 210 & 200 & 190 & 180 & 170 & 160 & 150 & 140 & 130 & 120 & 110 & 100 \\ \mathrm{f} 1(\mathrm{ppm})\end{array}$ 


\section{${ }^{1} \mathrm{H}-\mathrm{NMR}$ spectrum of $\mathbf{S 2 0}$ in $\mathrm{CDCl}_{3}, 400 \mathrm{MHz}$}

hsh-g218.10.fid
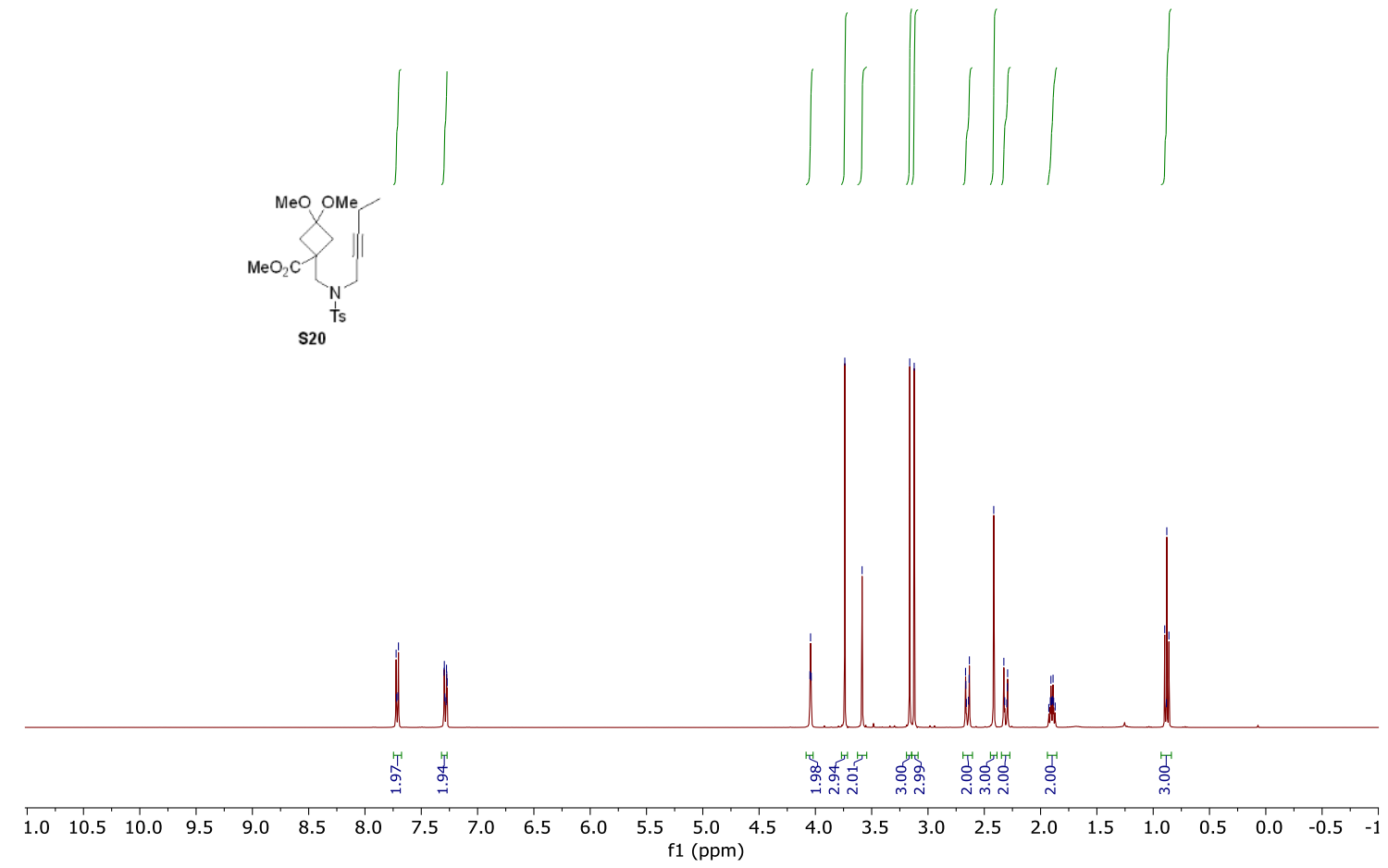

${ }^{13} \mathrm{C}$-NMR spectrum of $\mathbf{S 2 0}$ in $\mathrm{CDCl}_{3}, 101 \mathrm{MHz}$

hsh-g218.11.fid

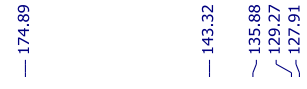

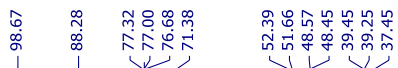

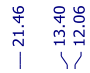
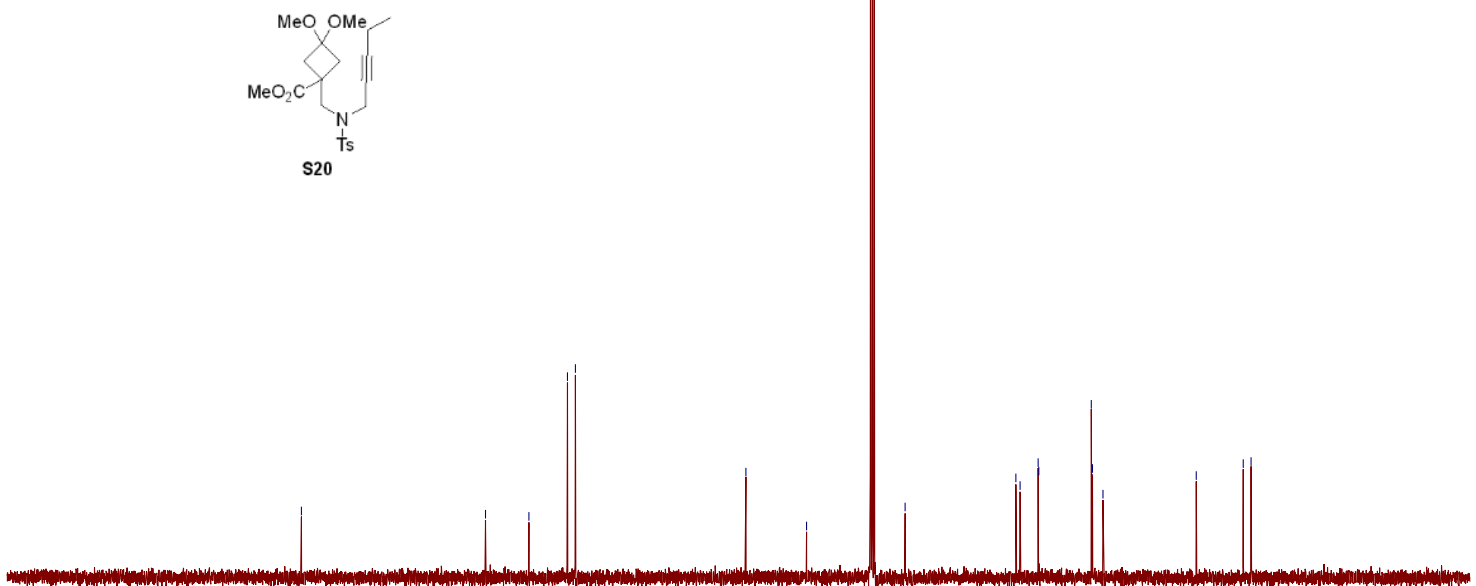

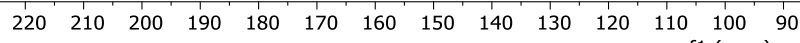
$80 \quad 70 \quad 60$ f1 (ppm) 


\section{${ }^{1} \mathrm{H}-\mathrm{NMR}$ spectrum of $\mathbf{S 2 2}$ in $\mathrm{CDCl}_{3}, 400 \mathrm{MHz}$}

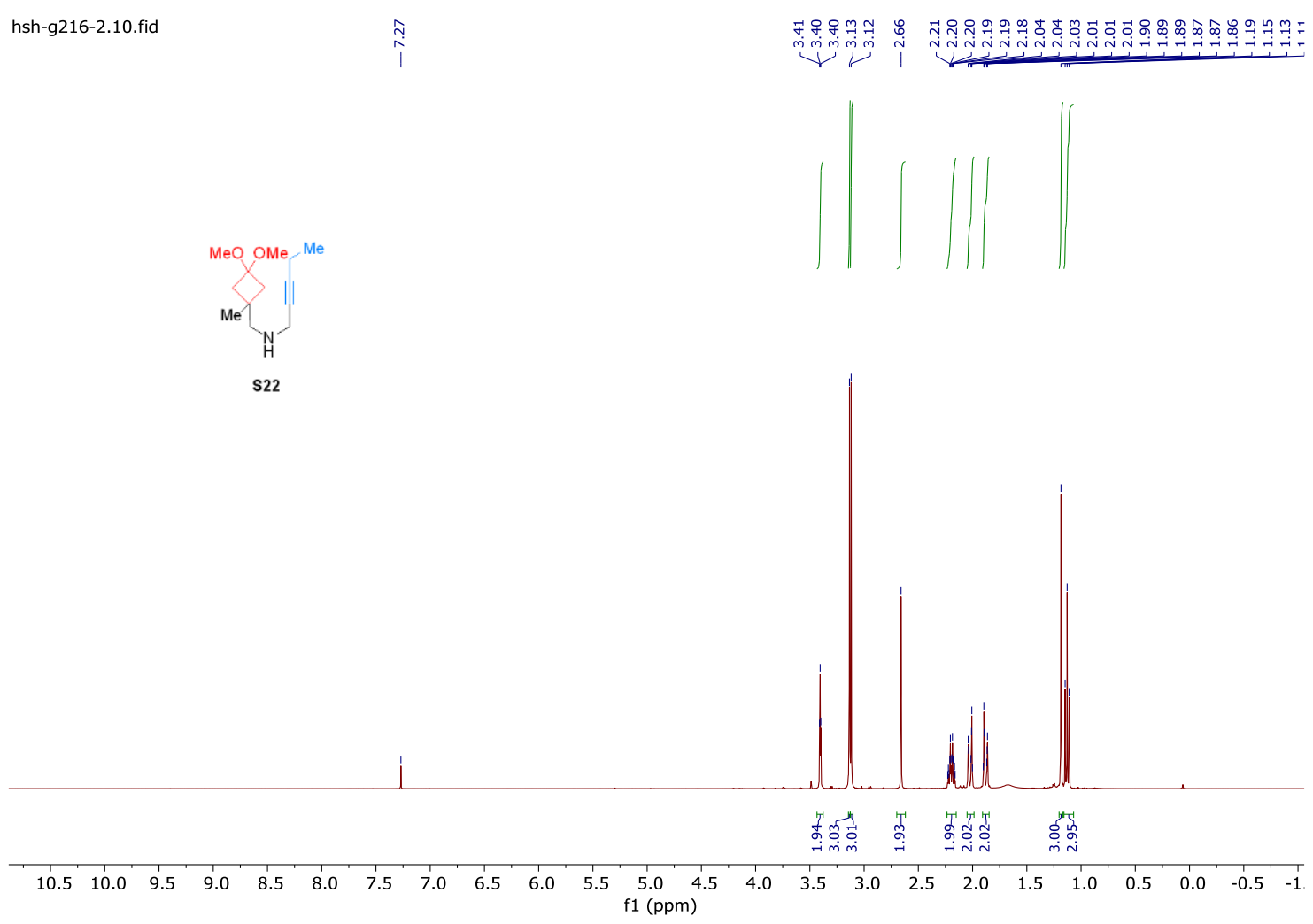

${ }^{13} \mathrm{C}-\mathrm{NMR}$ spectrum of $\mathbf{S 2 2}$ in $\mathrm{CDCl}_{3}, 101 \mathrm{MHz}$

hsh-g216-2.11.fid

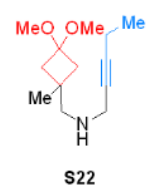

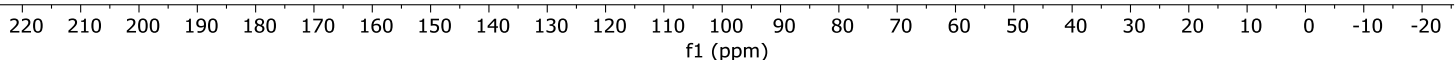


${ }^{1} \mathrm{H}-\mathrm{NMR}$ spectrum of $\mathbf{S 1 5 - 1}$ in $\mathrm{CDCl}_{3}, 400 \mathrm{MHz}$

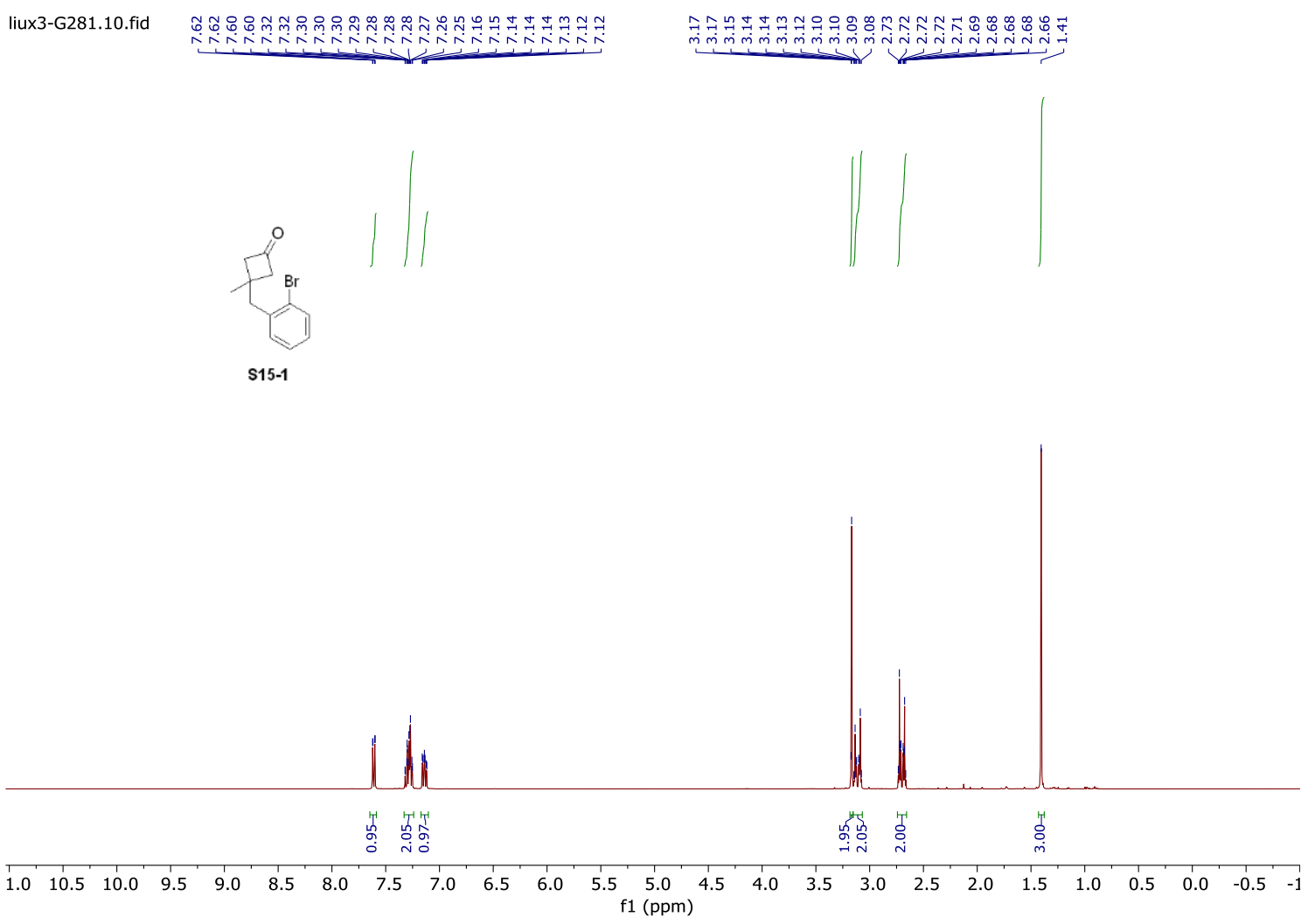

${ }^{13} \mathrm{C}-\mathrm{NMR}$ spectrum of $\mathbf{S 1 5 - 1}$ in $\mathrm{CDCl}_{3}, 101 \mathrm{MHz}$

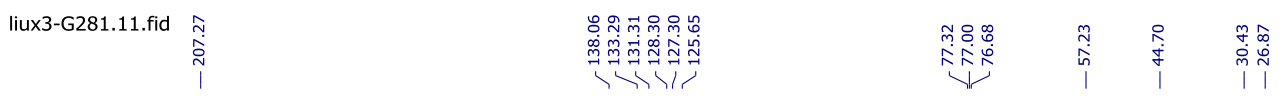

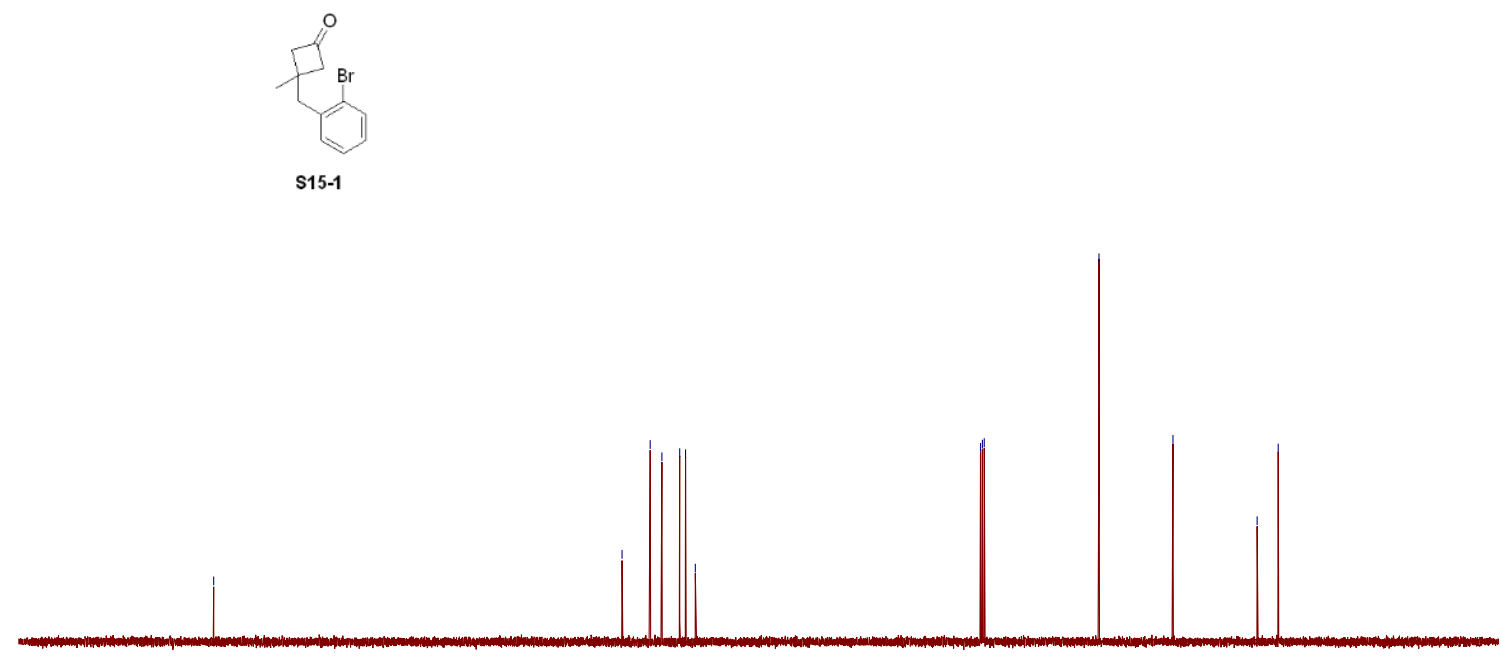

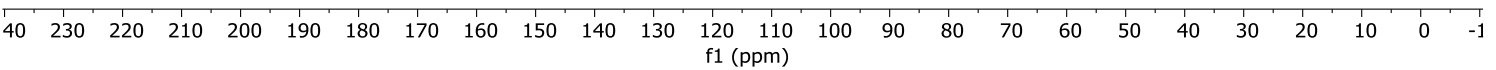


${ }^{1} \mathrm{H}-\mathrm{NMR}$ spectrum of $\mathbf{S 1 5 - 2}$ in $\mathrm{CDCl}_{3}, 400 \mathrm{MHz}$

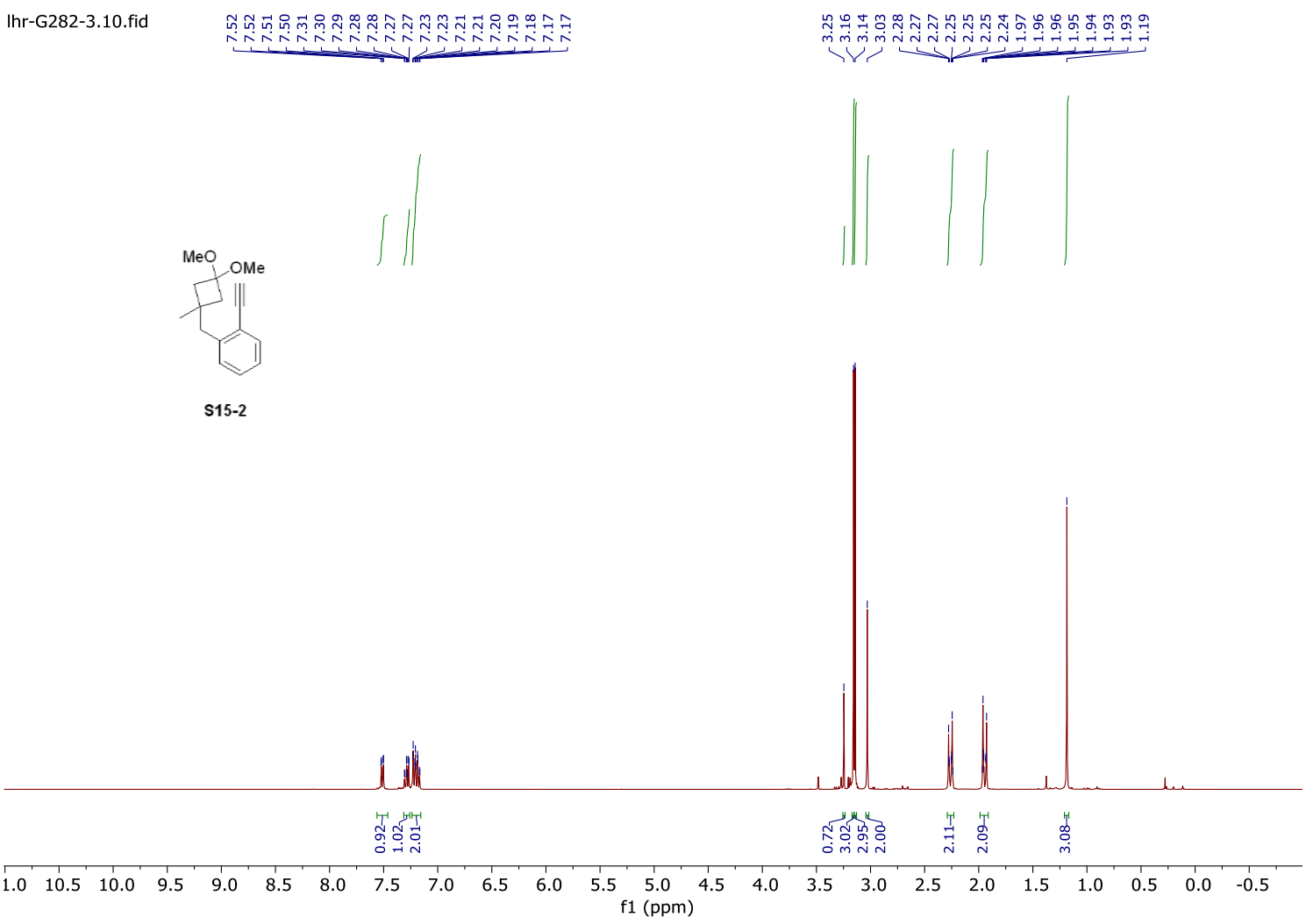

${ }^{13} \mathrm{C}-\mathrm{NMR}$ spectrum of $\mathbf{S 1 5 - 2}$ in $\mathrm{CDCl}_{3}, 101 \mathrm{MHz}$

Ihr-G282-r.10.1.1r

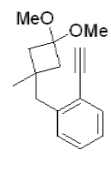

S15-2

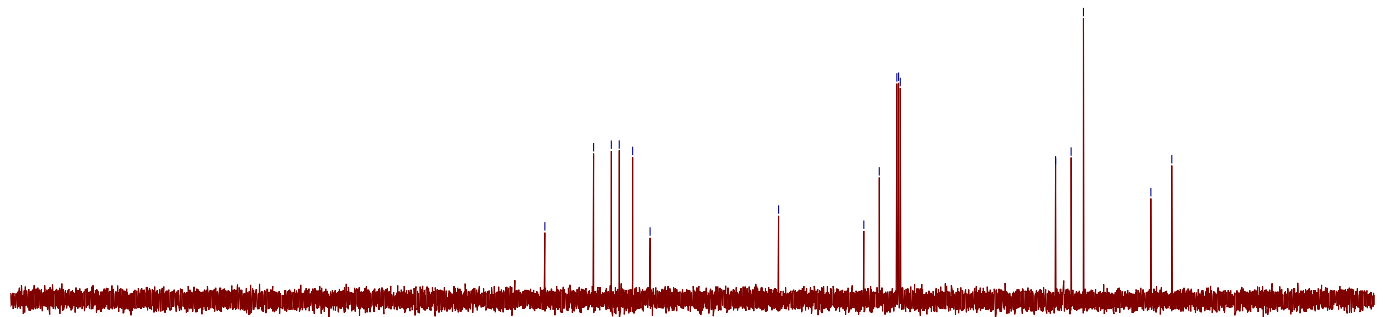

\begin{tabular}{lllllllllllllllllllllllllllllll}
\hline 40 & 230 & 220 & 210 & 200 & 190 & 180 & 170 & 160 & 150 & 140 & 130 & 120 & 110 & 100 & 90 & 80 & 70 & 60 & 50 & 40 & 30 & 20 & 10 & 0 & -1
\end{tabular} f1 (ppm) 
${ }^{1} \mathrm{H}-\mathrm{NMR}$ spectrum of 6 in $\mathrm{CDCl}_{3}, 400 \mathrm{MHz}$

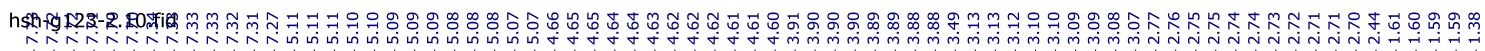

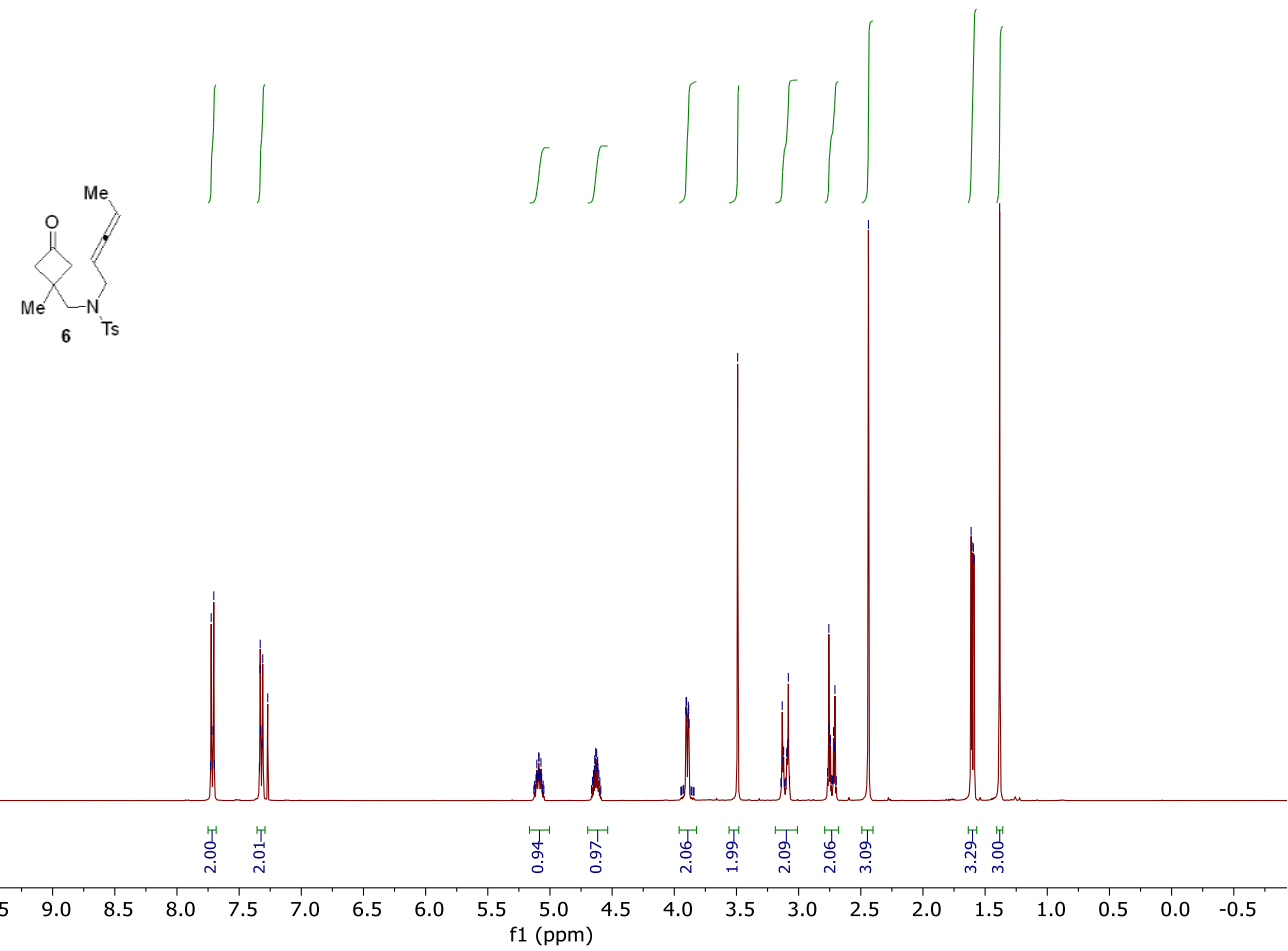

${ }^{13} \mathrm{C}$-NMR spectrum of 6 in $\mathrm{CDCl}_{3}, 101 \mathrm{MHz}$

hsh-g123-2.11.fid

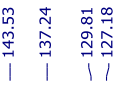

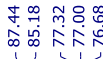

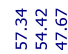

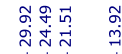
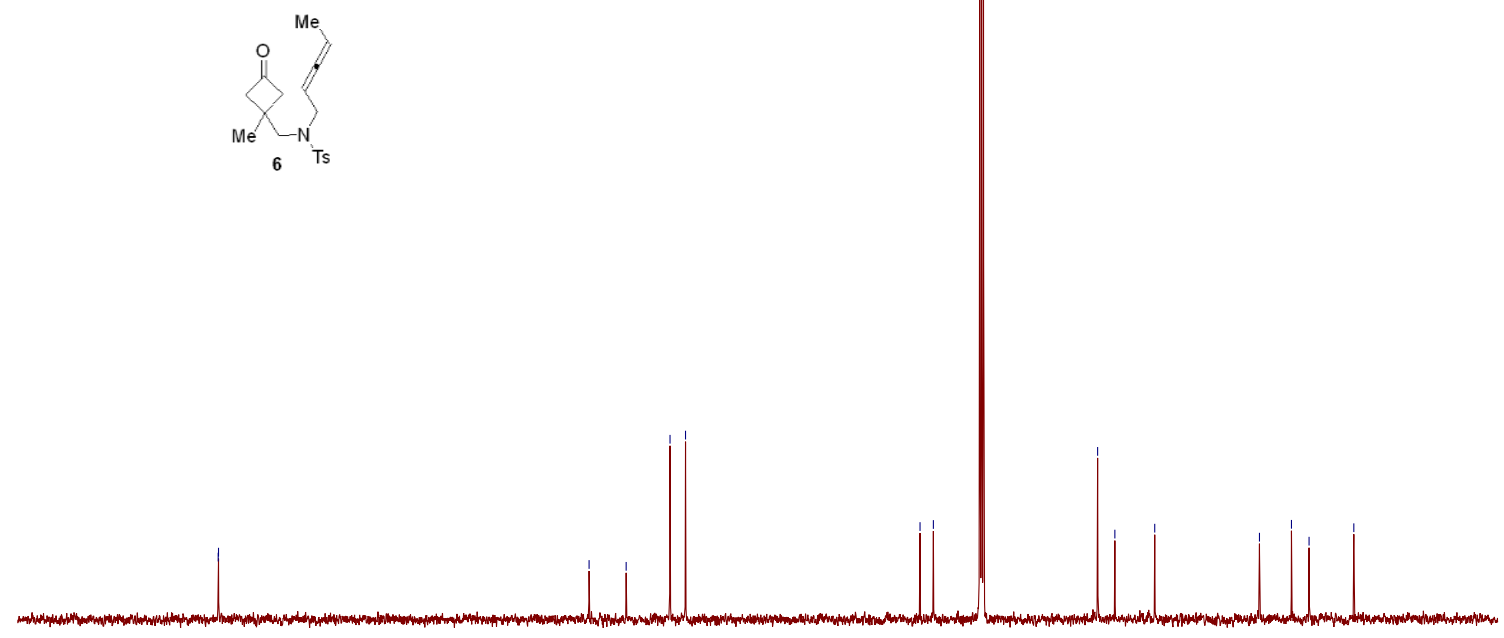

\begin{tabular}{lllllllllllllllllllllllllllllllllllll}
\hline 40 & 230 & 220 & 210 & 200 & 190 & 180 & 170 & 160 & 150 & 140 & 130 & $\begin{array}{c}120 \\
\mathrm{f} 1(\mathrm{ppm})\end{array}$ & 110 & 100 & 90 & 80 & 70 & 60 & 50 & 40 & 30 & 20 & 10 & 0 & -1
\end{tabular} 
${ }^{1} \mathrm{H}-\mathrm{NMR}$ spectrum of $\mathbf{S 2 5}$ in $\mathrm{CDCl}_{3}, 400 \mathrm{MHz}$

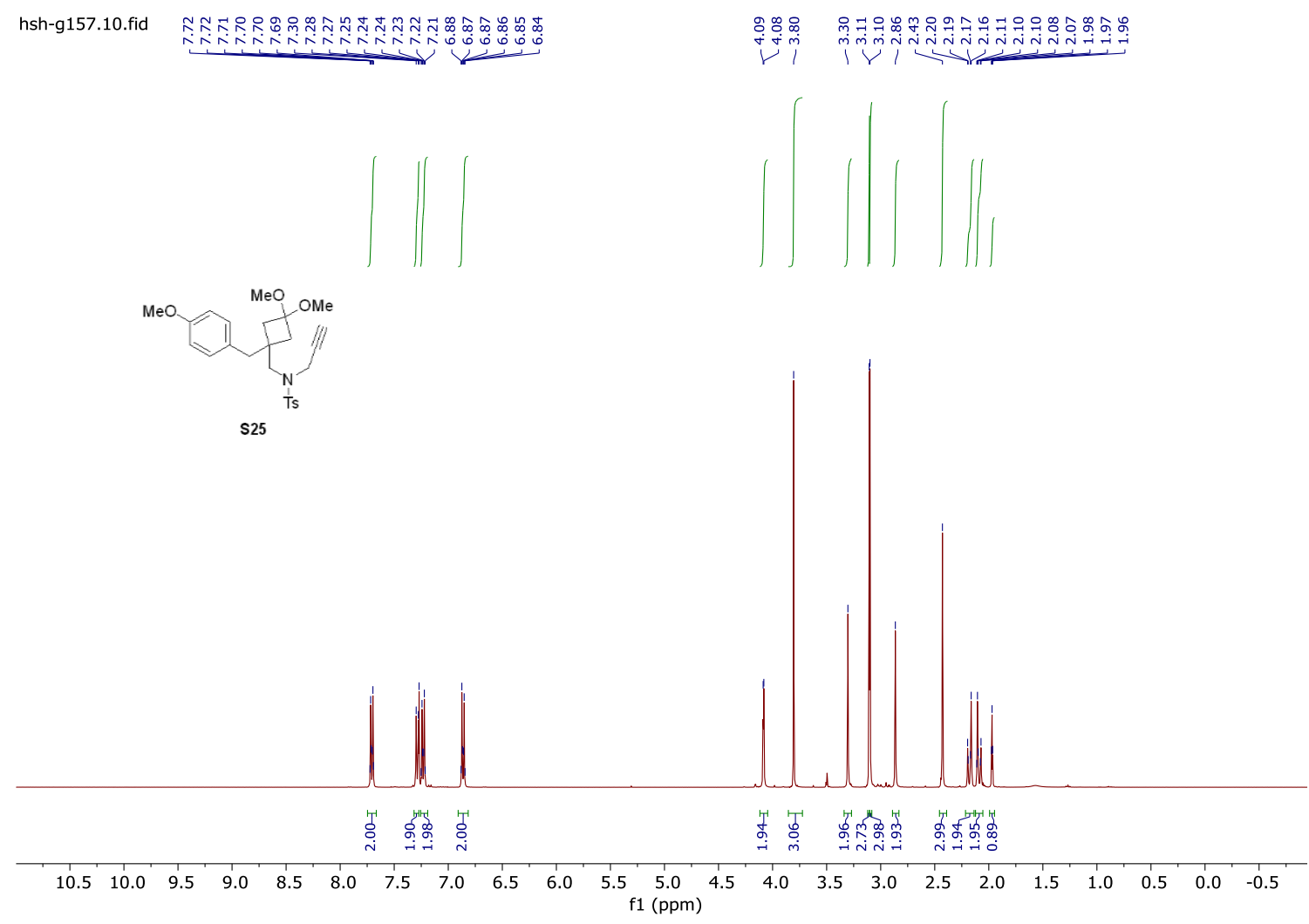

${ }^{13} \mathrm{C}-\mathrm{NMR}$ spectrum of $\mathbf{S} 25$ in $\mathrm{CDCl}_{3}, 101 \mathrm{MHz}$

hsh-g157.11.fid

\begin{tabular}{|c|c|c|c|c|c|}
\hline 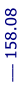 & 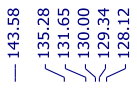 & 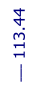 & \begin{tabular}{l}
$\infty$ \\
$\stackrel{0}{\circ}$ \\
\multirow{1}{*}{}
\end{tabular} & 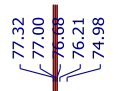 & 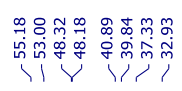 \\
\hline
\end{tabular}
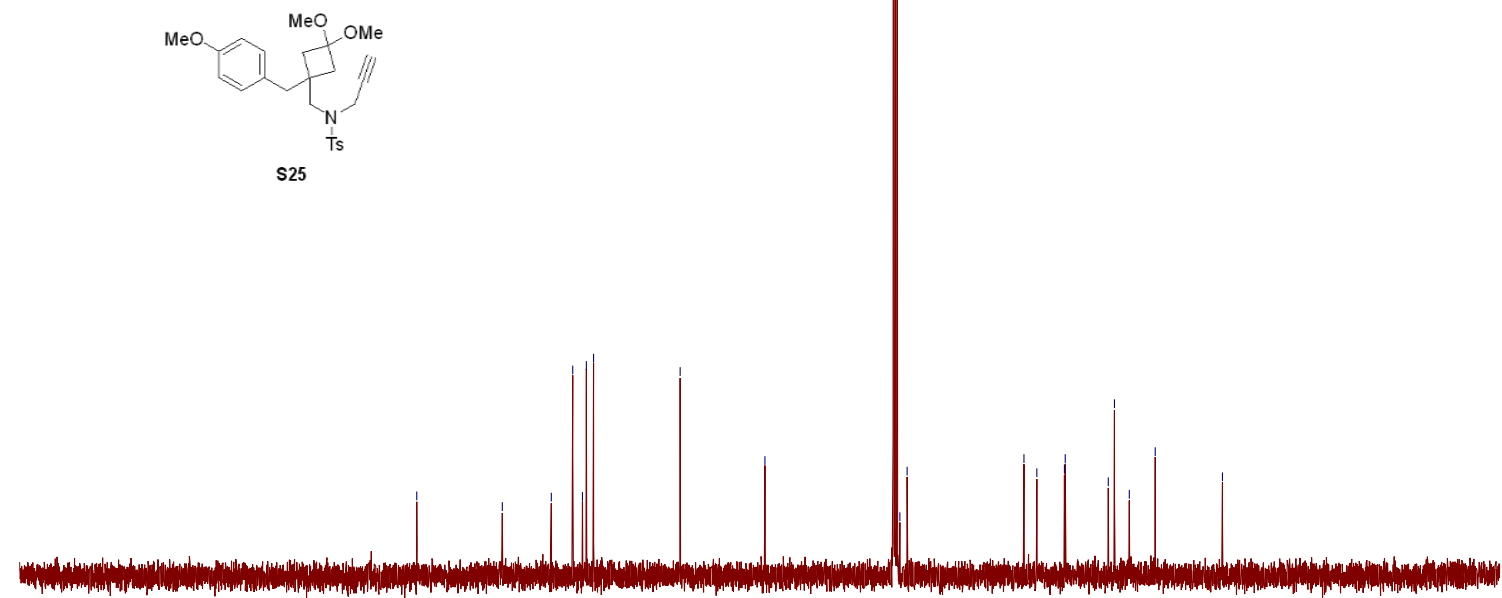

$\begin{array}{llllllllllllllllllllllllllllllllllll}220 & 210 & 200 & 190 & 180 & 170 & 160 & 150 & 140 & 130 & 120 & 110 & 100 & 90 & 80 & 70 & 60 & 50 & 40 & 30 & 20 & 10 & 0 & -10 & -20\end{array}$ 
${ }^{1} \mathrm{H}-\mathrm{NMR}$ spectrum of $\mathbf{1 h}-\mathbf{d}_{\mathbf{5}}$ in $\mathrm{CDCl}_{3}, 400 \mathrm{MHz}$

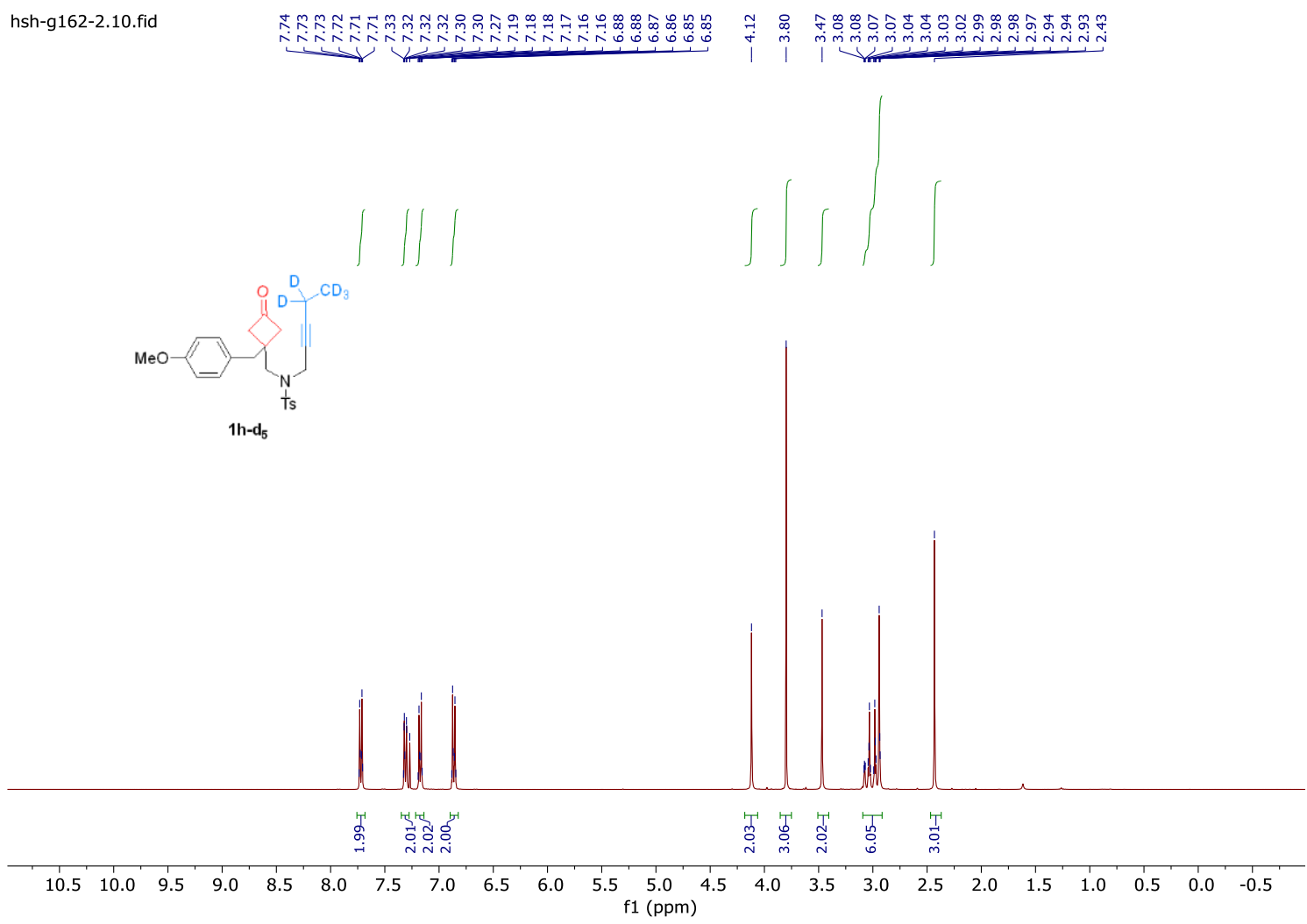

${ }^{2} \mathrm{H}-\mathrm{NMR}$ spectrum of $\mathbf{1} \mathbf{h}-\mathbf{d}_{\mathbf{5}}$ in $\mathrm{CDCl}_{3}, 400 \mathrm{MHz}$

hsh-g162-2-D.1.fid

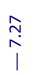
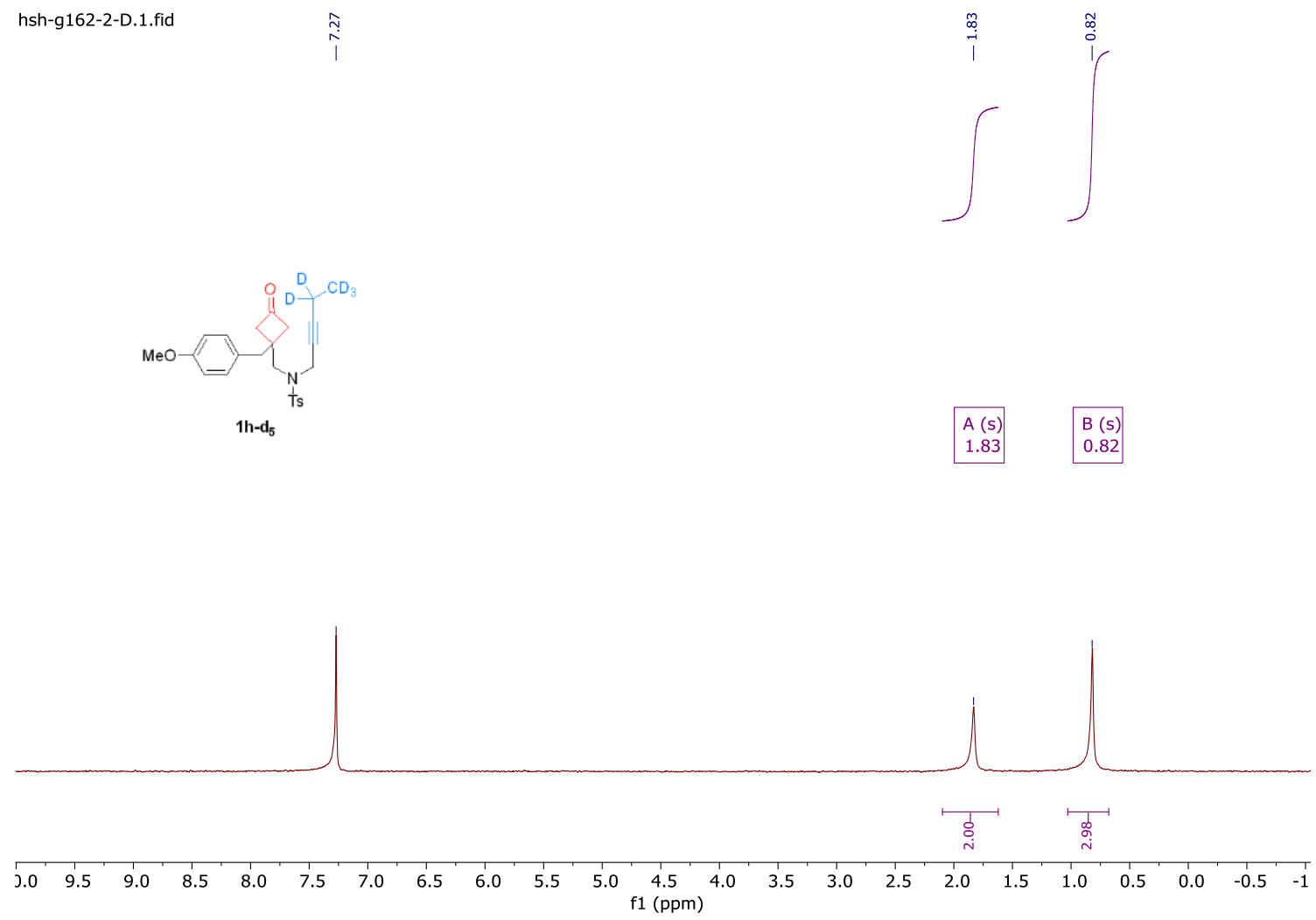
${ }^{13} \mathrm{C}-\mathrm{NMR}$ spectrum of $\mathbf{1} \mathbf{h}-\mathbf{d}_{\mathbf{5}}$ in $\mathrm{CDCl}_{3}, 101 \mathrm{MHz}$

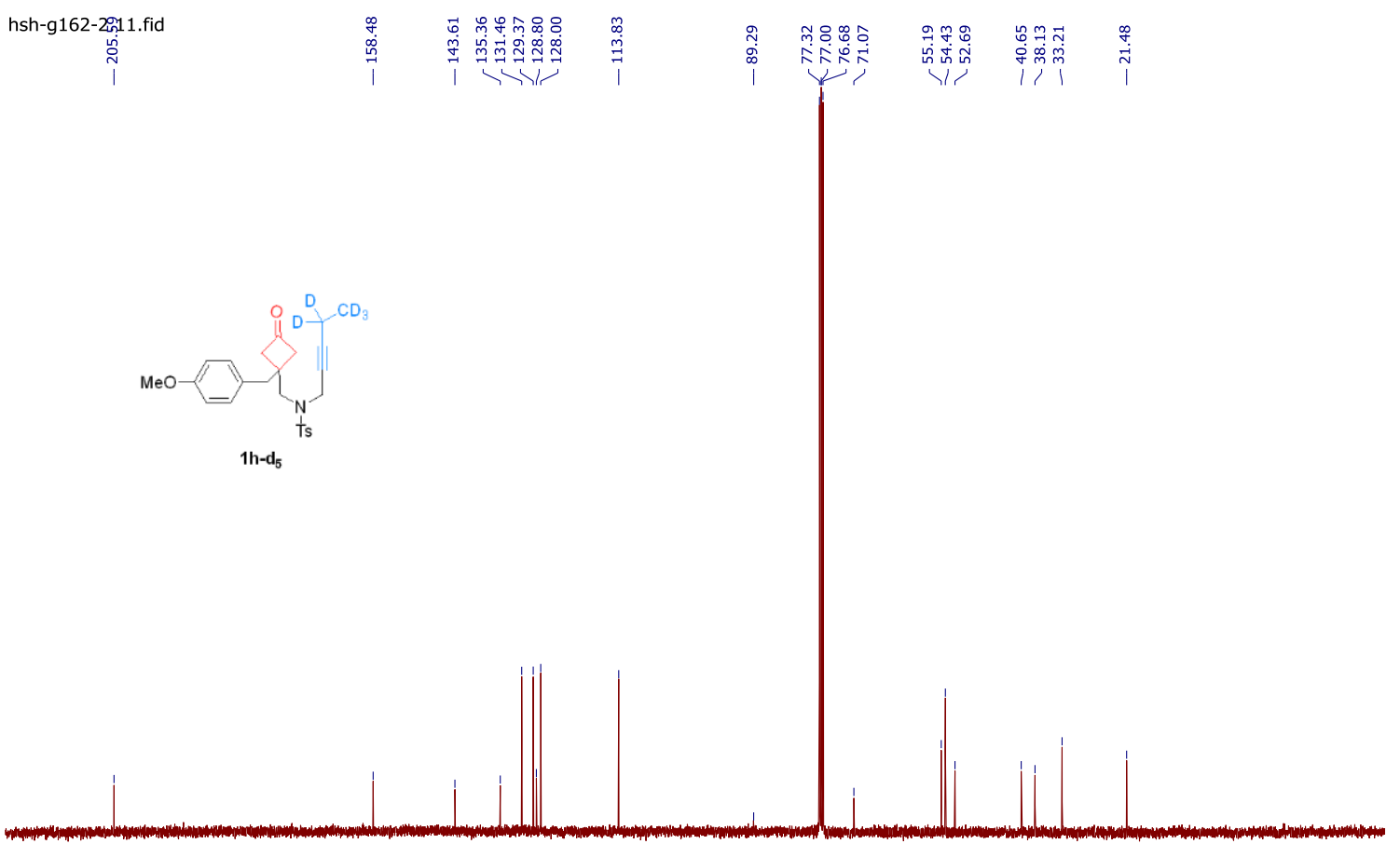

$\begin{array}{lllllllllllllllllllllllll}220 & 210 & 200 & 190 & 180 & 170 & 160 & 150 & 140 & 130 & 120 & 110 & 100 & 90 & 80 & 70 & 60 & 50 & 40 & 30 & 20 & 10 & 0 & -10 & -20\end{array}$ 


\section{References}

${ }^{1}$ Ko, H. M.; Dong, G. Cooperative Activation of Cyclobutanones and Olefins Leads to Bridged Ring Systems by a Catalytic [4+2] Coupling. Nat. Chem. 2014, 6, 739-744

2 (a) Zhou, X.; Dong, G. (4+1) vs (4+2): Catalytic Intramolecular Coupling between Cyclobutanones and Trisubstituted Allenes via C-C Activation. J. Am. Chem. Soc. 2015, 137, 13715-13721. (b) Zhou, X.; Dong, G. Nickel-Catalyzed Chemo- and Enantioselective Coupling between Cyclobutanones and Allenes: Rapid Synthesis of [3.2.2] Bicycles. Angew. Chem. Int. Ed. 2016, 55, 15091-15095. (c) Zhou, X.; Ko, H. M.; Dong, G. Synthesis of Bridged Cyclopentane Derivatives by Catalytic Decarbonylative Cycloaddition of Cyclobutanones and Olefins. Angew. Chem. Int. Ed. 2016, 55, 13867-13871.

${ }^{3}$ Kim-Lee, S.-H.; Alonso, I.; Mauleón, P.; Arrayás, R. G.; Carretero, J. C. Rationalizing the Role of NaOtBu in Copper-Catalyzed Carboboration of Alkynes: Assembly of Allylic All-Carbon Quaternary Stereocenters. ACS Cat. 2018, 8, 8993-9005.

${ }^{4}$ Fager-Jokela, E.; Muuronen, M.; Khaizourane, H.; Vázquez-Romero, A.; Verdaguer, X.; Riera, A.; Helaja, J. Regioselectivity of Intermolecular Pauson-Khand Reaction of Aliphatic Alkynes: Experimental and Theoretical Study of the Effect of Alkyne Polarization. J. Org. Chem. 2014, 79, 10999-11010.

${ }^{5}$ Derosa, J.; Cantu, A. L.; Boulous, M. N.; O’Duill, M. L.; Turnbull, J. L.; Liu, Z.; De La Torre, D. M.; Engle, K. M. Palladium(II)-Catalyzed Directed anti-Hydrochlorination of Unactivated Alkynes with HCl. J. Am. Chem. Soc. 2017, 139, 5183-5193.

6 Yamamoto, Y.; Okude, Y.; Mori, S.; Shibuya, M. Combined Experimental and Computational Study on Ruthenium(II)-Catalyzed Reactions of Diynes with Aldehydes and N,N-Dimethylformamide. J. Org. Chem. 2017, 82, 7964-7973

${ }^{7}$ Glaisyer, E. L.; Watt, M. S.; Booker-Milburn, K. I. Pd(II)-Catalyzed [4 + 2] Heterocyclization Sequence for Polyheterocycle Generation. Org. Lett. 2018, 20 , 5877-5880.

8 Watson, I. D. G.; Ritter, S.; Toste, F. D. Asymmetric Synthesis of Medium-Sized Rings by Intramolecular Au(I)Catalyzed Cyclopropanation. J. Am. Chem. Soc. 2009, 131, 2056-2057.

${ }^{9}$ Stevens, C.; De Kimpe, N. A New Entry into 2-Azabicyclo[2.1.1]hexanes via 3-(Chloromethyl)cyclobutanone. J. Org. Chem. 1996, 61, 2174-2178.

${ }^{10}$ Boutier, A.; Kammerer-Pentier, C.; Krause, N.; Prestat, G.; Poli, G. Pd-Catalyzed Asymmetric Synthesis of NAllenyl Amides and Their Au-Catalyzed Cycloisomerizative Hydroalkylation: A New Route Toward Enantioenriched Pyrrolidones. Chem. Eur. J. 2012, 18, 3840-3844.

${ }^{11}$ Deng, L.; Fu, Y.; Lee, S. Y.; Wang, C.; Liu, P.; Dong, G., Kinetic Resolution via Rh-Catalyzed C-C Activation of Cyclobutanones at Room Temperature. J. Am. Chem. Soc. 2019, 141, 16260-16265.

12 Karad, S. N.; Liu, R.-S. Gold-Catalyzed 1,2-Oxoarylations of Nitriles with Pyridine-Derived Oxides. Angew. Chem. Int. Ed. 2014, 53, 5444-5448

${ }^{13}$ Compound S23 was obtained following the first two steps of general procedure B of preparation of compound S16. 
${ }^{14}$ Gaussian 16, Revision C.01, Frisch, M. J.; Trucks, G. W.; Schlegel, H. B.; Scuseria, G. E.; Robb, M. A.; Cheeseman, J. R.; Scalmani, G.; Barone, V.; Petersson, G. A.; Nakatsuji, H.; Li, X.; Caricato, M.; Marenich, A. V.; Bloino, J.; Janesko, B. G.; Gomperts, R.; Mennucci, B.; Hratchian, H. P.; Ortiz, J. V.; Izmaylov, A. F.; Sonnenberg, J. L.; Williams-Young, D.; Ding, F.; Lipparini, F.; Egidi, F.; Goings, J.; Peng, B.; Petrone, A.; Henderson, T.; Ranasinghe, D.; Zakrzewski, V. G.; Gao, J.; Rega, N.; Zheng, G.; Liang, W.; Hada, M.; Ehara, M.; Toyota, K.; Fukuda, R.; Hasegawa, J.; Ishida, M.; Nakajima, T.; Honda, Y.; Kitao, O.; Nakai, H.; Vreven, T.; Throssell, K.; Montgomery, J. A., Jr.; Peralta, J. E.; Ogliaro, F.; Bearpark, M. J.; Heyd, J. J.; Brothers, E. N.; Kudin, K. N.; Staroverov, V. N.; Keith, T. A.; Kobayashi, R.; Normand, J.; Raghavachari, K.; Rendell, A. P.; Burant, J. C.; Iyengar, S. S.; Tomasi, J.; Cossi, M.; Millam, J. M.; Klene, M.; Adamo, C.; Cammi, R.; Ochterski, J. W.; Martin, R. L.; Morokuma, K.; Farkas, O.; Foresman, J. B.; Fox, D. J. Gaussian, Inc., Wallingford CT, 2016.

15 (a) Becke, A. D. Density-functional thermochemistry 3. The role of exact exchange. J. Chem. Phys. 1993, 98, 5648-5652. (b) Lee, C.; Yang, W.; Parr, R. G. Development of the Colle-Salvetti correlation-energy formula into a functional of the electron density. Phys. Rev. B 1988, 37, 785-789.

${ }^{16}$ Hay, P. J.; Wadt, W. R. Ab initio effective core potentials for molecular calculations - potentials for the transition-metal atoms Sc to Hg. J. Chem. Phys., 1985, 82, 270-83.

17 Zhao, Y.; Truhlar, D. G. A new local density functional for main-group thermochemistry, transition metal bonding, thermochemical kinetics, and noncovalent interactions. J. Chem. Phys., 2006, 125, 194101: 1-18.

18 Fuentealba, P.; Preuss, H.; Stoll, H.; Von Szentpaly, L. A proper'account of core-polarization with pseudopotentials: single valenceelectron alkali compounds. Chem. Phys. Lett. 1982, 89, 418-422.

${ }^{19}$ Marenich, A. V.; Cramer, C. J.; Truhlar, D. G. Universal Solvation Model Based on Solute Electron Density and on a Continuum Model of the Solvent Defined by the Bulk Dielectric Constant and Atomic Surface Tensions. J. Phys. Chem. B 2009, 113, 6378-6396.

${ }^{20}$ CYLview, 1.0b; Legault, C. Y., Université de Sherbrooke, 2009 (http://www.cylview.org). 